\title{
Computational analysis of $\beta$-adrenergic stimulation and its effects on cardiac ventricular electrophysiology
}

Citation for published version (APA):

Heijman, J. (2012). Computational analysis of $\beta$-adrenergic stimulation and its effects on cardiac ventricular electrophysiology. [Doctoral Thesis, Maastricht University]. Maastricht University. https://doi.org/10.26481/dis.20120427jh

Document status and date:

Published: 01/01/2012

DOI:

10.26481/dis.20120427jh

Document Version:

Publisher's PDF, also known as Version of record

\section{Please check the document version of this publication:}

- A submitted manuscript is the version of the article upon submission and before peer-review. There can be important differences between the submitted version and the official published version of record.

People interested in the research are advised to contact the author for the final version of the publication, or visit the DOI to the publisher's website.

- The final author version and the galley proof are versions of the publication after peer review.

- The final published version features the final layout of the paper including the volume, issue and page numbers.

Link to publication

\footnotetext{
General rights rights.

- You may freely distribute the URL identifying the publication in the public portal. please follow below link for the End User Agreement:

www.umlib.nl/taverne-license

Take down policy

If you believe that this document breaches copyright please contact us at:

repository@maastrichtuniversity.nl

providing details and we will investigate your claim.
}

Copyright and moral rights for the publications made accessible in the public portal are retained by the authors and/or other copyright owners and it is a condition of accessing publications that users recognise and abide by the legal requirements associated with these

- Users may download and print one copy of any publication from the public portal for the purpose of private study or research.

- You may not further distribute the material or use it for any profit-making activity or commercial gain

If the publication is distributed under the terms of Article $25 \mathrm{fa}$ of the Dutch Copyright Act, indicated by the "Taverne" license above, 


\section{COMPUTATIONAL ANALYSIS OF $\boldsymbol{\beta}$ -}

ADRENERGIC STIMULATION AND ITS

EFFECTS ON CARDIAC VENTRICULAR

ELECTROPHYSIOLOGY

JORDI HEIJMAN

2012 


\title{
COMPUTATIONAL ANALYSIS OF $\beta$ - ADRENERGIC STIMULATION AND ITS EFFECTS ON CARDIAC VENTRICULAR ELECTROPHYSIOLOGY
}

\author{
ProefsChRIFT \\ ter verkrijging van de graad van doctor aan de Universiteit Maastricht, \\ op gezag van de Rector Magnificus, Prof. mr. G.P.M.F. Mols, \\ volgens het besluit van het College van Decanen, \\ in het openbaar te verdedigen op \\ Vrijdag 27 April 2012 om 10.00 uur
}

door

Jordi Heijman

geboren op 8 September 1985 te Sittard 


\section{PROMOTORES}

Prof. Dr. H.J.G.M. Crijns

Prof. Dr. Ir. R.L.M. Peeters

\section{CO-PROMOTORES}

Dr. P.G.A. Volders

Dr. R.L. Westra

\section{BEOORDELINGSCOMMISSIE}

Prof. Dr. H.J.J. Wellens

Prof. Dr. M.R. Rosen

Prof. Dr. R. Sepulchre

Prof. Dr. H.A.J. Struijker-Boudier

Prof. Dr. A.A.M. Wilde

Prof. Dr. L.J. de Windt
Voorzitter

Columbia University, New York, NY, USA

Université de Liège, Liège, Belgium

Academisch Medisch Centrum, Amsterdam, The Netherlands 
Voor mijn ouders 


\section{Contents}

LIST OF ABBREVIATIONS

8

Chapter 1 11

Introduction

ChAPTER 2

27

Properties and ionic mechanisms of action potential adaptation, restitution, and accommodation in canine epicardium

Keith F. Decker, Jordi Heijman, Jonathan R. Silva, Thomas J. Hund and Yoram Rudy

Am. J. Physiol. Heart Circ. Physiol. 2009, 296:H1017-H1026

\section{CHAPTER 3}

Local control of $\beta$-adrenergic stimulation: Effects on ventricular myocyte

electrophysiology and $\mathrm{Ca}^{2+}$-transient

Jordi Heijman, Paul G.A. Volders, Ronald L. Westra and Yoram Rudy

J. Mol. Cell. Cardiol. 2011, 50:863-871

\section{CHAPTER 4}

Dominant-Negative Control of CAMP-Dependent $I_{\mathrm{Ks}}$ Upregulation in Human Long-QT Syndrome Type 1

Jordi Heijman, Roel L.H.M.G. Spätjens, Sandrine R.M. Seyen, Viola Lentink, Helma J.H. Kuijpers, Inge R. Boulet, Leon J. de Windt, Miren David and Paul G.A. Volders

Circ. Res. 2012, 110:211-219

\section{CHAPTER 5}

$I_{K s}$ restricts excessive beat-to-beat variability of repolarization during betaadrenergic receptor stimulation

Daniel M. Johnson, Jordi Heijman, Chris E. Pollard, Jean-Pierre Valentin, Harry J.G.M. Crijns, Najah Abi-Gerges and Paul G.A. Volders

J. Mol. Cell. Cardiol. 2010, 48:122-130 
Diastolic Spontaneous Calcium Release from the Sarcoplasmic Reticulum Increases Beat-to-Beat Repolarization Variability in Calcium-Overloaded Canine Ventricular Myocytes

Jordi Heijman*, Daniel M. Johnson*, Elizabeth F. Bode, David J. Greensmith, Najah Abi-Gerges, David A. Eisner, Andrew W. Trafford and Paul G.A. Volders

* These authors contributed equally to this work Manuscript in preparation and in part presented at Heart Rhythm Society 2011 \& Cardiac EP Society 2011

\section{CHAPTER 7}

Determinants of beat-to-beat variability of repolarization duration in the canine ventricular myocyte - a computational analysis

Jordi Heijman, Antonio Zaza, Daniel M. Johnson, Yoram Rudy, Ralf L.M. Peeters, Paul G.A. Volders* and Ronald L. Westra*

* These authors contributed equally to this work

Manuscript in preparation and in part accepted for Frontiers in Cardiovascular Biology 2012

CHAPTER 8 201

General discussion

SUMMARY / SAMENVATTING 223

ACKNOWLEDGEMENTS / DANKWOORD 231

Curriculum Vitae / Publications 235

COLOR Figures 241

APPENDIX 257

Model definition and equations 


\section{Abbreviations}

A

AC Adenylyl cyclase

AKAPs A-kinase anchoring protein

AM Atrial myocyte

AP Action potential

APD AP duration

ATXII Sea anemone toxin II

B

BAR $\quad \beta$-adrenergic receptor

$\beta A R S \quad \beta A R$ stimulation

BVR Beat-to-beat variability of repolarization duration

C

CaMKII $\mathrm{Ca}^{2+} /$ Calmodulin kinase II

CAMP 3'-5' cyclic adenosine monophosphate

CaT $\mathrm{Ca}^{2+}$ transient

$\mathrm{CaT}_{\text {AMP }}$ CaT amplitude

CAV Caveolar domain

$\mathrm{CDI} \quad \mathrm{Ca}^{2+}$-dependent inactivation

$\mathrm{CHO}$ Chinese hamster ovary

$\mathrm{CICR} \mathrm{Ca}^{2+}$-induced $\mathrm{Ca}^{2+}$ release

$\mathrm{CL} \quad$ Cycle length

CPVT Catecholaminergic polymorphic ventricular tachycardia

CSQN Calsequestrin

$\mathrm{CTKCl} \quad \mathrm{K}^{+}-\mathrm{Cl}^{-}$co-transporter

$\mathrm{CTNaCl} \mathrm{Na}^{+}-\mathrm{Cl}^{-}$co-transporter

CV Coefficient of variability

CYT Cytosolic domain

D

DAD Delayed afterdepolarization

DI Diastolic interval

E

EAD Early afterdepolarization

ECAV Extracaveolar membrane domain

ECC Excitation-contraction coupling

ECGI Electrocardiographic imaging

EPAC Exchange protein activated by cAMP
$\mathbf{F}$

FRET Fluorescence resonance energy transfer

G

Gi Inhibitory G-protein

Gs Stimulatory G-protein

H

$\mathrm{HH} \quad$ Hodgkin-Huxley

I

IBMX 3-isobutyl-1-methylxanthine; nonspecific PDE inhibitor

$I_{\mathrm{Cab}} \quad$ Background $\mathrm{Ca} 2+$ current

$I_{\text {CaL }} \quad$ L-type $\mathrm{Ca}^{2+}$ current

$\mathrm{I}_{\mathrm{Cl}(\mathrm{Ca})} \quad \mathrm{Ca}^{2+}$-dependent $\mathrm{Cl}^{-}$current

$I_{f} \quad$ Pacemaker ("funny") current

$\mathrm{I}_{\mathrm{K} 1} \quad$ Inward rectifying $\mathrm{K}^{+}$current

$I_{\mathrm{Kp}} \quad$ Plateau K $\mathrm{K}^{+}$current

$\mathrm{I}_{\mathrm{Kr}} \quad$ Rapidly-activating delayed-rectifier $\mathrm{K}^{+}$ current

$\mathrm{I}_{\mathrm{Ks}} \quad$ Slowly-activating delayed-rectifier $\mathrm{K}^{+}$ current

$\mathrm{I}_{\text {Kur }} \quad$ Ultrarapidly-activating delayed rectifier $\mathrm{K}^{+}$ current

$I_{\text {leak }} \quad$ JSR Ca ${ }^{2+}$ leak flux

$\mathrm{I}_{\mathrm{Na}} \quad$ Fast $\mathrm{Na}^{+}$current

$\mathrm{I}_{\mathrm{Nab}} \quad$ Background $\mathrm{Na}^{+}$current

$\mathrm{I}_{\mathrm{NaCa}} \quad \mathrm{Na}^{+}-\mathrm{Ca}^{2+}$ exchange current

$\mathrm{I}_{\mathrm{NaK}} \quad \mathrm{Na}^{+}-\mathrm{K}^{+}$pump current

$\mathrm{I}_{\mathrm{NaL}} \quad$ Late / persistent $\mathrm{Na}^{+}$current

Inh1 Inhibitor protein 1

IP3 Inositol(1,4,5)-triphosphate

$\mathrm{I}_{\mathrm{pCa}} \quad$ Plasmalemmal $\mathrm{Ca}^{2+}$ pump current

IPS Induced pluripotent stem cell

$I_{\text {rel }} \quad J S R \mathrm{Ca}^{2+}$ release flux

ISO Isoproterenol

$\mathrm{I}_{\mathrm{ti}} \quad$ Transient inward current

$\mathrm{I}_{\text {To }} \quad 4$ AP-sensitive transient outward $\mathrm{K}^{+}$ current

Iup $\quad \mathrm{SR} \mathrm{Ca}^{2+}$ uptake flux by SERCA2a

I-V Current-voltage relationship 


\section{J}

JSR Junctional sarcoplasmic reticulum

$\mathbf{L}$

LQT Long QT syndrome

LTV Long-term variability

LV Left ventricular

$\mathbf{N}$

$\mathrm{NCX} \quad \mathrm{Na}^{+}-\mathrm{Ca}^{2+}$ exchanger

NSR Network sarcoplasmic reticulum

\section{OA Okadaic acid}

$\mathbf{P}$

PDE Phosphodiesterase

PF Purkinje fiber

PGE1 Prostaglandin receptor 1

PKA Protein kinase A

PKC Protein kinase $C$

PLB Phospholamban

$P_{0} \quad$ Open probability

PP1 Protein phosphatase 1

PTX Pertussis toxin

\section{$\mathbf{R}$}

RV Right ventricular

RyR Ryanodine receptor

\section{S}

SAN Sino-atrial node

SCR Spontaneous $\mathrm{Ca}^{2+}$ release

SERCA Sarco/endoplasmic reticulum $\mathrm{Ca}^{2+}$-ATPase

SR Sarcoplasmic reticulum

SS Steady state

STV Short-term variability

\section{$\mathbf{T}$}

TA Triggered activity

TdP Torsades des pointes

TnI Troponin I

\section{V}

Vm Membrane potential

VM Ventricular myocyte

Vmax Maximum membrane potential

VPH Virtual physiological human

VT Ventricular tachycardia

\section{W}

WT Wild type 
List of abbreviations 


\section{Chapter 1}

\section{INTRODUCTION}

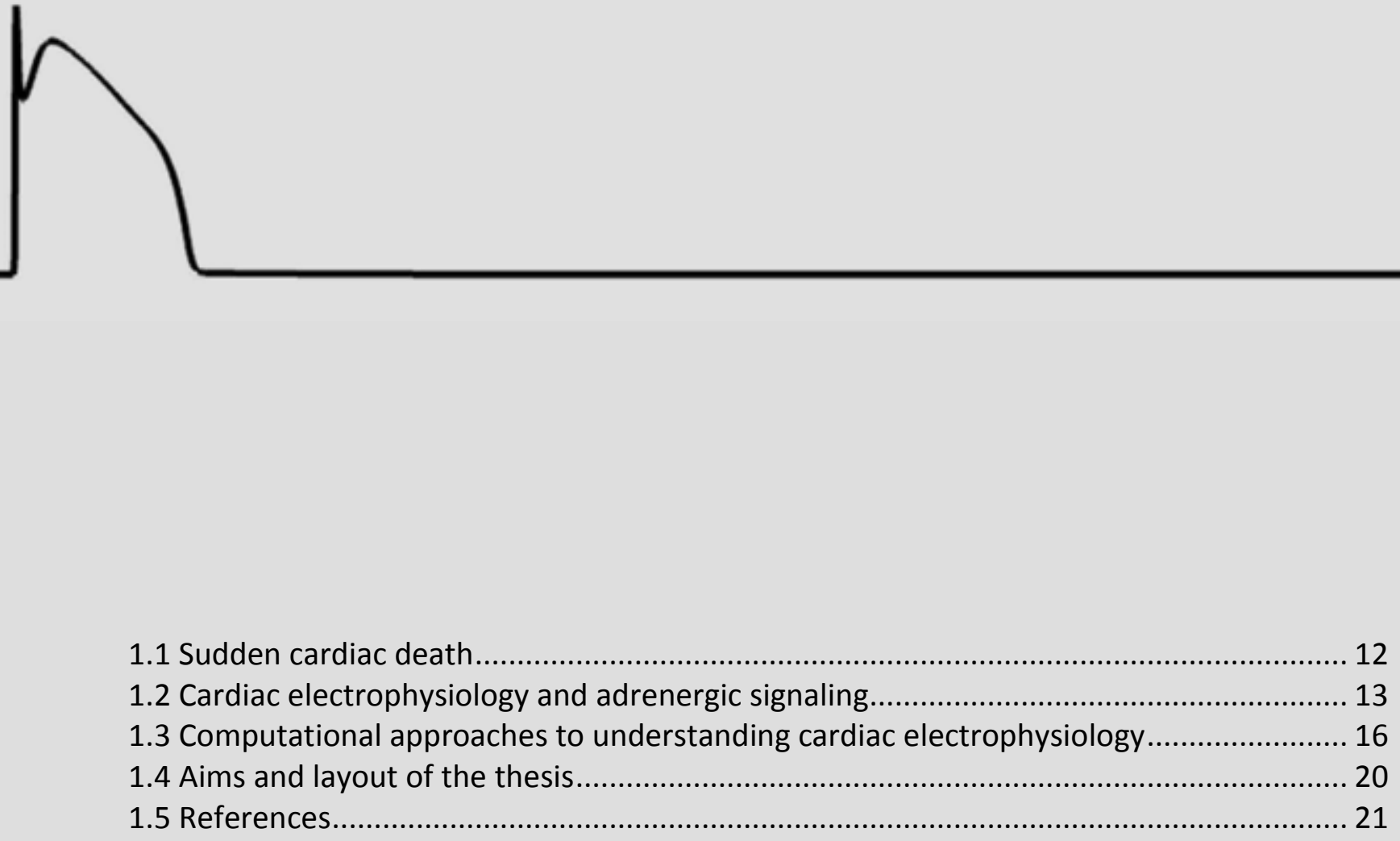




\subsection{SUDDEN CARDIAC DEATH}

Despite significant advances in diagnostic modalities and treatment options, cardiovascular diseases still remain the most common cause of death and morbidity in the developed world. Based on an overall cardiovascular-disease-related death rate of 262.5 per 100,000 inhabitants (USA, 2006), it can be estimated that more than 2300 people die every day from cardiovascular diseases in the United States alone. ${ }^{1}$ In The Netherlands, 176.5 and 84.2 cardiovascular-disease-related deaths occurred per 100,000 inhabitants in 2007 among men and women, respectively, ${ }^{1}$ making it indeed the most common cause of death. ${ }^{2}$ The etiology of the diseases underlying these deaths is diverse. The incidence of sudden cardiac death remains difficult to assess and published numbers vary between 180,000 and 450,000 cases per year in the United States. ${ }^{3}$ However, even the lowest estimate indicates a sizable incidence. In the Maastricht area, the mean yearly incidence of sudden cardiac arrest in the general population (age group 20 to 75 years) was 1 in 1,000 and in approximately half of the cases sudden cardiac arrest was most likely the first manifestation of heart disease. ${ }^{4}$ The relatively high prevalence of sudden cardiac death and its high impact on society give rise to what Zipes and Rubart ${ }^{5}$ called "one of the most important-and vexing-questions in clinical cardiology today": "Why did he die on Tuesday and not on Monday?"

In general, the genesis of arrhythmias involves at least two components: a vulnerable substrate created by the disease and a trigger. For many diseases, and in particular for congenital conditions, it can be assumed that the substrate did not change significantly between Monday and Tuesday. As such, sudden cardiac death can be considered an "electrophysiological accident". ${ }^{6}$ Therefore, part of the answer to the question concerning the origin and control of the proximate precipitator to sudden cardiac death ${ }^{5}$ may be found in a better understanding of the "accident"-specific triggers. Signaling pathways that relay external cues to the heart play a critical role herein. Of these pathways, the autonomic nervous system has been described as a major modulator of arrhythmic events in a wide variety of cardiovascular diseases, including atrial fibrillation ${ }^{7-9}$, heart failure ${ }^{10-12}$, myocardial infarction ${ }^{13,14}$, long-QT syndrome ${ }^{15-17}$ and catecholaminergic polymorphic ventricular tachycardia. ${ }^{18-20}$

In this thesis we present an integrative experimental and computational analysis of the signaling pathways involved in sympathetic stimulation of the heart and their effects on cardiac cellular electrophysiology in physiological and pathophysiological conditions. We focus on conditions in which there is a structurally normal heart. Such a primary electrophysiological abnormality accounts for approximately $3-5 \%$ of the sudden cardiac death cases $^{6}$ and provides an important paradigm to further our understanding of arrhythmogenesis. However, the insights obtained from these studies also provide information that can be applied to the more common acquired conditions such as heart failure, myocardial ischaemia, or digitalis intoxication, in which sympathetic stimulation causes electrophysiological alterations that predispose the development of ventricular arrhythmias. ${ }^{21-24}$ In the remainder of this chapter, we present a brief introduction to cardiac electrophysiology and adrenergic signaling under normal conditions, as well as an overview of computational approaches in electrocardiology. 


\subsection{CARDIAC ELECTROPHYSIOLOGY AND ADRENERGIC SIGNALING}

The "raison d'être" of the human heart is to supply $\mathrm{O}_{2}$ and nutrient-rich blood to the body and pump $\mathrm{CO}_{2}$-rich blood to the lungs. A 'normal' heart does so by beating more than three billion times during an average person's lifetime. However, calling the heart simply a pump would not do justice to the incredible complexity this organ has accrued during millions of years of evolution. The pumping function of the heart is orchestrated through a large number of electrical and biochemical pathways. Under normal conditions, each beat starts from an electrical signal initiated by spontaneouslyactivating cells in the sinoatrial node. The electrical wave spreads across the atria and results in the pumping of blood from the atria to the ventricles. The electrical signal is delayed at the atrioventricular node to allow sufficient time for the ventricles to fill and then propagates through the rapid conduction system to facilitate synchronous activation of the ventricular muscle and pumping of blood to the lungs (from the right ventricle) and other organs (from the left ventricle).

At the level of the single ventricular myocyte, depolarization of the membrane from its resting potential of approximately $-80 \mathrm{mV}$ results in rapid activation of voltage-gated $\mathrm{Na}^{+}$channels (giving rise to the $\mathrm{Na}^{+}$current: $I_{\mathrm{Na}}$ ) to depolarize the membrane (Phase 0 ). At positive potentials the $\mathrm{Na}^{+}$ channels rapidly inactivate, resulting in a peak membrane potential of approximately $30 \mathrm{mV}$. In some cell types, most prominently the epicardial myocytes, this rapid depolarization is followed by a fast partial repolarization (Phase 1$)$ due to the activation of a transient outward $\mathrm{K}^{+}$current $\left(\mathrm{I}_{\mathrm{to}}\right)$ and a $\mathrm{Ca}^{2+}$-activated $\mathrm{Cl}^{-}$current $\left(\mathrm{I}_{\mathrm{CI}(\mathrm{Ca})}\right)$. During phases 0 and 1 , L-type $\mathrm{Ca}^{2+}$ channels are activated, giving rise to $\mathrm{I}_{\mathrm{Cal}}$. The $\mathrm{Ca}^{2+}$ brought into the cell via $\mathrm{I}_{\mathrm{CaL}}$ triggers release of $\mathrm{Ca}^{2+}$ from the intracellular stores (the sarcoplasmic reticulum; SR) through ryanodine receptors (RyRs). The combined free subcellular $\mathrm{Ca}^{2+}$ can bind to the cell's contractile machinery, thereby activating cell contraction. This process is termed excitation-contraction coupling (ECC) and provides the main link between electrical and mechanical properties of the cardiac myocyte. During the plateau phase (Phase 2), there exists a delicate balance between depolarizing currents $\left(I_{\mathrm{CaL}}\right.$, the late component of $\mathrm{I}_{\mathrm{Na}}$ and $\mathrm{Na}^{+}-\mathrm{Ca}^{2+}$ exchange current) and repolarizing currents. A concerted effort of multiple voltage-gated $\mathrm{K}^{+}$ channels (in particular the rapidly and slowly activating delayed rectifiers $I_{\mathrm{kr}}$ and $I_{\mathrm{ks}}$ ), as well as the $\mathrm{Na}^{+}-\mathrm{K}^{+}$pump $\left(\mathrm{I}_{\mathrm{NaK}}\right)$, underlie the final repolarization phase (Phase 3). The resting membrane potential (Phase 4 ) is maintained mainly by the inward rectifier $\mathrm{K}^{+}$current $\left(\mathrm{I}_{\mathrm{K} 1}\right)$ and $\mathrm{I}_{\mathrm{NaK}}$ and is, as such, close to the $\mathrm{K}^{+}$reversal potential. Meanwhile, $\mathrm{Ca}^{2+}$ is pumped back in to the SR by SERCA2a and out of the cell by the $\mathrm{Na}^{+}-\mathrm{Ca}^{2+}$ exchanger (and to a lesser extent the sarcolemmal $\mathrm{Ca}^{2+}$-ATPase) to maintain homeostasis and achieve cell relaxation. For a more complete overview of the molecular constituents of cardiac electrophysiology and ECC, the interested reader is referred to excellent reviews on this topic ${ }^{25-28}$. The time course of membrane depolarization and repolarization is called the cardiac action potential (AP) and AP duration (APD) is the most commonly used quantification. There exist significant differences in AP morphology between different species as well as between cells from different regions of the heart. ${ }^{29-32}$ This thesis mostly concerns the canine (left) ventricular myocyte. A prototypical example of an AP and $\mathrm{Ca}^{2+}$ transient (CaT) from a canine epicardial myocyte, and the main underlying currents, are shown in Figure 1. 


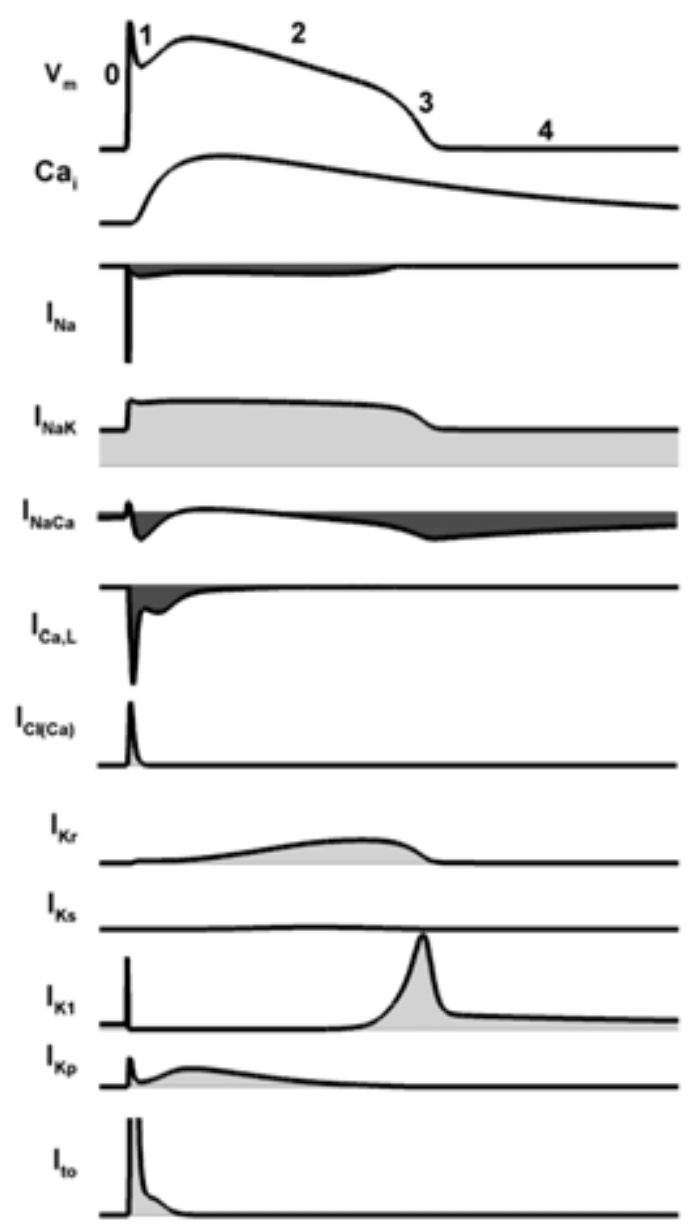

Figure 1. Prototypical epicardial action potential, $\mathrm{Ca}^{2+}$ transient and ionic currents. Numbers indicate the four phases of the action potential. Outward currents are shaded in light grey, inward currents in dark grey. Not to scale.

Of pivotal importance in the operation of the heart is its connection to the autonomic nervous system which can tailor the cardiac performance through the activation of various signaling cascades. The autonomic nervous system is divided into two parts. Activation of the sympathetic nervous system results in a fight-or-flight response characterized by enhanced heart rate (chronotropy), force of contraction (inotropy), rate of relaxation (lusitropy) and conduction (dromotropy). These adaptations greatly increase cardiac output, allowing the body to respond swiftly and strongly to any potential threat. The sympathetic nervous system uses the neurotransmitter norepinephrine to activate signaling cascades via adrenergic receptors. In contrast, the parasympathetic nervous system balances the response to sympathetic stimulation and decreases cardiac output and rate. The parasympathetic nervous system controls the heart via muscarinic receptors and uses acetylcholine as its neurotransmitter. ${ }^{33}$ These two pathways show complex interactions. ${ }^{34}$ 
At the single myocyte level, the effects of sympathetic stimulation are largely mediated by the $\alpha$ adrenergic- and $\beta$-adrenergic receptor ( $\beta A R$ ) signaling pathways (Figure 2 ). Both types are part of the large family of G-protein coupled receptors. ${ }^{21}$ When catecholamines are released from sympathetic nerves they bind to the $\beta A R$, resulting in activation of the receptor-bound stimulatory G-proteins (Gs). Activation of Gs is achieved by dissociation of the $\alpha$ and $\beta \gamma$ subunits resulting from a GTP-GDP exchange on the $\mathrm{Gs}_{\alpha}$ subunit. Active $\mathrm{Gs}_{\alpha}$ can subsequently stimulate adenylyl cyclases to produce the ubiquitous second messenger $3^{\prime}-5^{\prime}$ - cyclic adenosine monophosphate (cAMP), which can be broken down by phosphodiesterases (PDEs). The "traditional" downstream element of CAMP is protein kinase A (PKA). Activation of PKA results in the phosphorylation of many intracellular proteins (PKA substrates). Stimulation of $\alpha$-adrenergic receptors activates Gq-proteins and phospholipase $C$, generating inositol(1,4,5)-triphosphate and diacylglycerol. These molecules in turn mobilize intracellular $\mathrm{Ca}^{2+}$, leading to the activation of protein kinase $\mathrm{C}$ (PKC) and $\mathrm{Ca}^{2+} / \mathrm{Calmodulin}^{2}$ kinase II (CaMKII). ${ }^{35}$

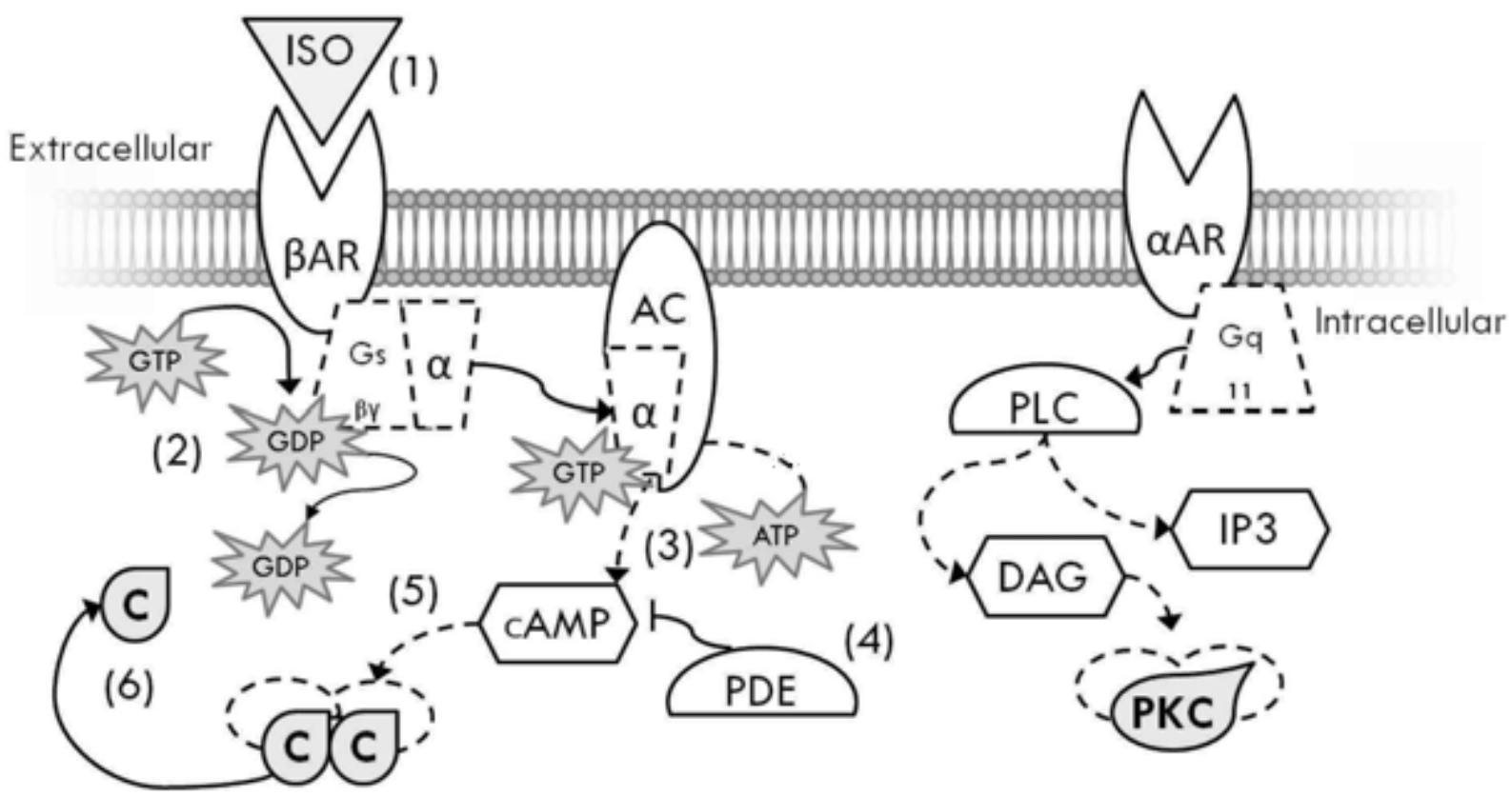

Figure 2. Schematic representation of the $\beta A R$ signaling pathway. Ligand binding (1) causes dissociation of the $\mathrm{Gs}_{\alpha}$ subunit as a result of GDP-GTP exchange (2). Gs ${ }_{\alpha}$ activates adenylyl cyclases (AC) to produce cAMP from ATP (3). Phosphodiesterases degrade cAMP (4). cAMP activates PKA (5) which causes dissociation of the catalytic subunit that can subsequently phosphorylate intracellular proteins (6). The $\alpha$-adrenergic receptor pathway is shown on the right.

In the ventricular myocyte the PKA substrates include, among others, $I_{\mathrm{CaL}}, I_{\mathrm{Ks}}, I_{\mathrm{Na}}, I_{\mathrm{NaK}}$, RyR, phospholamban (PLB) and Troponin I (TnI). Activation of PKA results in increased CaT amplitude and rate of decay, contributing to the inotropic and lusitropic effects of sympathetic stimulation. In sinoatrial node cells, there is evidence both for direct CAMP-dependent modulation of the hyperpolarization-activated ("funny") current $\left(\mathrm{I}_{\mathrm{f}}\right)$ and increased $\mathrm{Ca}^{2+}$ handling via PKA underlying the increased chronotropy during sympathetic stimulation. ${ }^{36}$ PKA-dependent increases in $\mathrm{Ca}^{2+}$ handling 
and $\mathrm{I}_{\mathrm{CaL}}$ in the atrioventricular node are also responsible for the increased dromotropic effects. ${ }^{37}$ In addition to these effects of $\beta A R$ stimulation via CAMP/PKA, recent reports have highlighted interactions with other signaling pathways, including the PKC and CaMKII pathways, leading to a complex 'signalome'. ${ }^{38,39}$ This interaction is established through the PKA-dependent changes in $\mathrm{Ca}^{2+}$ cycling and heart rate as well as through a PKA-independent pathway involving the exchange protein activated by CAMP (EPAC), Phospholipase C, and PKC. ${ }^{38}$ The CaMKII pathway has also been implicated in the adverse effects of long-term adrenergic stimulation in a variety of conditions. ${ }^{40,41}$

Because of its critical importance in the control of cardiac output and rate, the adrenergic signaling pathway is tightly controlled, both temporally and spatially. Several negative feedback loops exist to limit excessive cAMP levels. These temporal control mechanisms include desensitization (and subsequent internalization) of $\beta A R$ due to phosphorylation by PKA- and G-protein-coupled receptor kinases, as well as stimulation of CAMP hydrolysis by the long isoforms of PDE3 and PDE4 after phosphorylation by PKA. ${ }^{21,42}$ Moreover, in recent years it has become increasingly clear that cAMP signaling is highly localized. Localization is essential for different receptors that all affect CAMP to achieve a specific response. For example, it has been shown that stimulation of $\beta 1 A R$ results in a cell-wide response, whereas $\beta 2 A R$ stimulation results in localized elevation of $C A M P .^{43}$ Localized signaling is achieved by macromolecular signaling complexes that are tethered to specific subcellular locations. ${ }^{44}$ One such complex is created by caveolin-3, which is involved in the formation of caveolae, small invaginations in the cell membrane. In addition, it acts as a scaffold protein that groups various channels and transporters as well as signaling molecules together. ${ }^{45}$ In addition, Akinase anchoring proteins bind to various PKA substrates and localize PKA, phosphatases and PDEs to the channel complex. ${ }^{46}$ These localized signaling domains, together with cAMP-limiting actions by PDEs and $\mathrm{G}_{\mathrm{i}}$-proteins, allow for local elevation of cAMP and specific responses to stimulation of different receptors. ${ }^{47}$ Although improved cAMP-imaging techniques have facilitated the analysis of localized signaling domains ${ }^{48,49}$, it remains difficult to experimentally quantify the contributions of individual signaling components to the local and global effects of adrenergic stimulation.

\subsection{COMPUTATIONAL APPROACHES TO UNDERSTANDING CARDIAC ELECTROPHYSIOLOGY}

From the aforementioned description, it will be readily apparent that the heart is a complex, nonlinear system with numerous interacting components and a high degree of synthesis and integration. Since human intuition subsides in face of these complexities, it is virtually impossible to predict the effect of perturbations to this complex system by reasoning alone. Because of this, the field cardiac (electro)physiology has a rich history of computational approaches to promote the understanding of various mechanisms, including arrhythmogenesis. The interaction between experimental and computational cardiology started in the early 1960s and thanks to the advent of more powerful computing hardware, the field has grown ever since. Figure 3 illustrates this growth by showing the number of publications on computational electrocardiology since 1962. Recently, a USA National Institutes of Health / National Heart, Lung and Blood Institute workshop on sudden 
cardiac death recommended to "establish multiscale integrative models, including molecular, cellular, organ-level, animal and computational, relevant to human electrophysiology and disease" and "enhance acceptance and use of such models to achieve improved mechanistic understanding of arrhythmogenesis as well as the effects and implications of new antiarrhythmia therapies." ${ }^{50}$

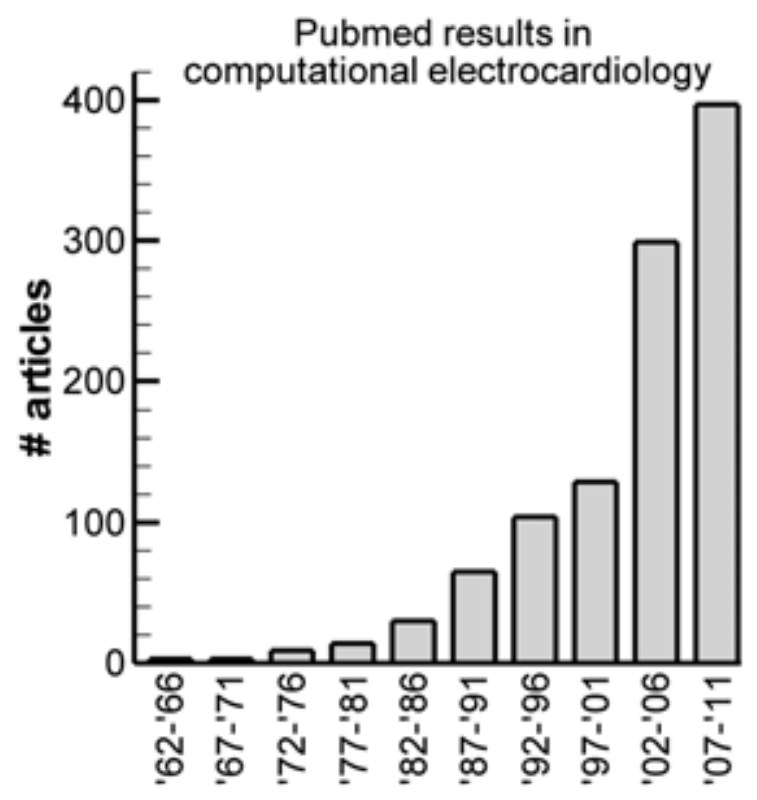

Figure 3. Number of articles published per 5 year period that satisfy the Pubmed query (cardiac $O R$ heart $O R$ ventricle $O R$ ventricular $O R$ atria) AND ("action potential" OR electrical OR electromechanical $O R$ electrophysiological $O R$ electrophysiology) AND (computational OR mathematical OR simulation) AND model (in title and/or abstract).

A brief overview of important milestones and recent research efforts in computational electrocardiology is given below.

The history of computational modeling of excitable cells based on underlying ionic mechanisms started with the seminal work by Hodgkin and Huxley $(\mathrm{HH})$ on the conduction in the giant axon of the squid in $1952 .{ }^{51}$ Noble $^{52}$ was among the first to adapt this work to the cardiac domain by adapting the $\mathrm{HH}$ equations to reproduce the long plateau of the action potential from the cardiac Purkinje fiber. Noble and coworkers subsequently expanded this model ${ }^{53}$ to include seminal experimental discoveries by the laboratories of Carmeliet, ${ }^{54}$ Coraboef, ${ }^{55}$ Trautwein, $^{56}$ Weidmann ${ }^{57}$ and others. This model was later adapted to the first model of a ventricular cell by Beeler and Reuter. ${ }^{58}$ In 1985, DiFrancesco and Noble ${ }^{59}$ created the first model that incorporated dynamic changes in intracellular ion concentrations as well as a formulation for $\mathrm{Ca}^{2+}$ release from the SR. These models are sometimes referred to as "dynamic" or "Phase 2" models. The DiFrancesco-Noble model and its subsequent extension with $\mathrm{Ca}^{2+}$ buffering by Hilgemann and Noble ${ }^{60}$ (for an atrial action potential) provided the starting point for many subsequent simulation studies on $\mathrm{Ca}^{2+}$ handling in cardiac cells. 
The ventricular cell model by Beeler and Reuter was expanded in two seminal studies by Luo and Rudy $^{61,62}$ to include many new experimental data specific for the single ventricular myocyte that had accumulated as a result of improved experimental techniques. Their model provided a very complete overview of the guinea-pig ventricular action potential and remains extensively used to date. This class of models has subsequently been extended by many research groups to describe the cardiac cellular electrophysiology of a wide variety of species (including, recently, human specific) and cell types and represents the most common type of models in computational electrocardiology $y^{63-71}$ (see Winslow et al. ${ }^{72}$ or Chapter 8 for a more complete list).

Several different research directions can be distinguished that have expanded on these initial results. One important direction focuses on a more detailed understanding of ion-channel function and the disturbances that occur as a result of various hereditary channelopathies. To describe these details, models that explicitly reflect a physical state (or aggregate of states) of ion channels were developed. In contrast to the HH formulations, these Markov models also made it possible to represent interdependencies between channel activation and inactivation, consistent with experimental observations. ${ }^{73}$ In the early 1990s the first Markov models were developed to study gating properties of ion channels. ${ }^{74,75}$ However, it was not until the late 90s when the first studies integrated Markov model representations of ion channels into a computational model of the cardiac action potential. A prime example of such a study is that by Clancy and Rudy. ${ }^{76}$ Their models of the wild-type and $\triangle K P Q$-mutant cardiac sodium channel illustrated how Markov models that represent individual channel states can provide mechanistic insight into the effects of genetic mutations that alter channel gating. This technique has since been applied to a wide variety of channels and diseases. Recently, Silva et al. added another level of physiological detail by proposing a model for $\mathrm{I}_{\mathrm{Ks}}$ that combines molecular dynamics and electrostatics to determine a multidimensional, continuous energy landscape that can be used to determine channel transitions and single channel currents. ${ }^{77}$ This approach provides a more mechanistic understanding of channel function than can be obtained with Markov models, where model topology has to be determined manually instead of being based on the physical channel structure. Incremental approaches that extend on Silva et al. ${ }^{77}$ can potentially enhance the understanding of genotype/phenotype relationships in various channelopathies.

Markov models have also played an important role in so called "local control" models that include details of subcellular $\mathrm{Ca}^{2+}$ handling, particularly in the dyadic cleft. The dyadic cleft is a small region between the sarcolemmal invaginations called t-tubules and the opposing intracellular SR. This space is a microdomain for $\mathrm{Ca}^{2+}$ signaling where ECC is initiated via $\mathrm{Ca}^{2+}$-induced $\mathrm{Ca}^{2+}$ release $(\mathrm{CICR}){ }^{78} \mathrm{CICR}$ is a highly localized process characterized by strong positive feedback. The Jafri-Rice-Winslow model was the first to include a more detailed description of CICR using Markov models for the L-type $\mathrm{Ca}^{2+}$ channel and RyR and a subcellular compartment representing the total cellular dyadic space. ${ }^{79}$ In the myocyte, the dyadic cleft is a large cluster of small microdomains. These volumes are so small that only a few L-type $\mathrm{Ca}^{2+}$ channels interact with a few dozen RyRs and under physiological conditions only a small number of $\mathrm{Ca}^{2+}$ ions are present in each domain. With such small numbers involved, models using deterministic ordinary differential equations based on continuum assumptions may 
not be appropriate. ${ }^{80}$ Stochastic descriptions of CICR were first developed by Stern. ${ }^{81}$ He showed that common pool models are not able to reproduce both the high-gain and graded-release properties of CICR observed experimentally. Since then much work has been performed, particularly concerning the question what ends the positive feedback loop that controls CICR. ${ }^{82}$ In 2002, Greenstein and Winslow $^{83}$ were the first to incorporate a stochastic local control model of CICR into a detailed model of the ventricular action potential. Stochastic simulations combined with the nonlinear dynamics of the ventricular action potential have recently been described to result in dynamic temporal repolarization patterns, including the generation of arrhythmogenic early afterdepolarizations $s^{84}$ and the induction of noise-induced chaos ${ }^{85}$. Since temporal variability of repolarization has been described as a marker for proarrhtyhmic risk ${ }^{86}$, future models incorporating local stochastic $\mathrm{Ca}^{2+}$ dynamics may also provide additional insight into the more macroscopic properties of repolarization variability.

At the other end of the spectrum, multicellular tissue or whole-heart models can provide insight into macroscopic, emergent processes such as arrhythmias, which do not exist at the level of the single cell. Multicellular models can be classified as spatially discrete or continuous. In discrete models (also called network models), individual cell models that are coupled electrically are explicitly represented. Although this approach more closely reflects the biological structure of the heart, it is computationally extremely demanding. As such, simulations using this approach are usually confined to 1-dimensional strands or small sheets of 2-dimensional tissue. ${ }^{87,88}$ The first continuum based simulations were performed using a generic excitable media model by Fitzhugh and Nagumo around the same time Noble presented the first ionic cardiac cell model. ${ }^{89}$ Detailed anatomical models that include fiber direction anisotropy were first developed at the University of Auckland, New Zealand, by Nielsen et al. ${ }^{90}$ Later generic models have been extended to reproduce macroscopic properties of cardiac tissue and combined with the anatomical models resulted in the first macroscopic tissue models with realistic geometry. ${ }^{91,}{ }^{92}$ More recent approaches have used detailed ionic cell models incorporated in a monodomain or bidomain framework. Bidomain models use a continuum approach to represent two interpenetrating domains representing cardiac cells and the space surrounding them and include mechanistic descriptions of the ionic currents. Computational hardware is now powerful enough to produce organ level simulations (whole ventricle, whole heart) with detailed ionic models and geometry. Such models have been employed to study many aspects of cardiac electrophysiology, including organization of ventricular fibrillation, ${ }^{93}$ arrhythmogenic effects of heterogeneous restitution of repolarization ${ }^{94}$, effects of pharmacological interventions ${ }^{95}$, human atrial electrophysiology ${ }^{96}$ and synchronization of single cell behavior ${ }^{97}$.

Several models, including some of the whole-heart models described above, also include the mechanical component of the cardiac function. At the subcellular level, contraction models have been designed to reproduce key characteristics of the contractile machinery as well as heterogeneous tension development. ${ }^{98,}{ }^{99}$ Initially, the electrophysiological and mechanical models were developed independently and only weakly coupled. However, in the heart, activation and contraction are strongly coupled (via ECC and mechanoelectrical feedback). ${ }^{100} \mathrm{Kohl}$ et al. provided one of the first computational analyses of mechanical effects on cardiac electrophysiology in a 
multicellular network model of the sinoatrial node. ${ }^{101}$ Later, 3-dimensional models of cardiac electromechanics have been employed to study precordial thump efficacy, ${ }^{102}$ acute regional ischemia, ${ }^{103}$ reentrant wave stability, ${ }^{104}$ biventricular pacing in the failing heart ${ }^{105}$ and stretchinduced initiation of atrial fibrillation, ${ }^{106}$ among other things.

Finally, recent efforts have started to integrate signaling pathways in computational models of cardiac electrophysiology. These include models for the CAMP/PKA pathway, PKC pathway and CaMKII pathway, as well as models of ATP regulation. Although several studies had already simulated the electrophysiological effects of adrenergic stimulation, Saucerman and McCulloch were the first to integrate the CAMP/PKA signaling pathway in a model of the ventricular myocyte. ${ }^{107}$ Recently, lancu et al. ${ }^{108}$ described a model of CAMP signaling that also takes into account localized signaling domains. However, their model did not include downstream effects of CAMP or any electrophysiological targets. In 2004, Hund and Rudy ${ }^{65}$ were the first to integrate the CaMKII signaling pathway in a model of the canine ventricular electrophysiology. Since then, several studies have further investigated the signaling aspects ${ }^{109,}{ }^{110}$, as well as the effects on cardiac electrophysiology ${ }^{111,112}$ of CaMKII. The highly localized effects and the large number of interacting pathways and electrophysiological substrates of these signaling pathways make it difficult to characterize individual components experimentally. The rest of this thesis focuses on the computational analysis of (sub)cellular aspects of the adrenergic signaling pathway and electrophysiological substrates.

\subsection{AIMS AND LAYOUT OF THE THESIS}

Several experimental studies by our group have investigated the role of adrenergic stimulation in the genesis of torsades de pointes-type arrhythmias in the dog at both the in-vivo and in-vitro level. The aim of this thesis was to develop a detailed computational model of localized adrenergic signaling in the canine ventricular myocyte and apply this model, in conjunction with further experimental studies, to investigate the effects of adrenergic stimulation on the cellular electrophysiology and $\mathrm{Ca}^{2+}$ handling in physiological and pathophysiological conditions. This was performed via increasing levels of complexity starting from a model of the canine epicardial electrophysiology (Chapter 2), to adrenergic stimulation under physiological conditions (Chapter 3 ), to the role of adrenergic stimulation in arrhythmogenesis for a specific long-QT type 1 mutation (Chapter 4). Subsequently, we identified the role of IKs in beat-to-beat variability of repolarization duration during $\beta A R$ stimulation (Chapter 5) and the role of abnormal $\mathrm{Ca}^{2+}$ handling in this process (Chapter 6). Finally, we analyzed the ionic contributors to beat-to-beat variability of repolarization during basal conditions and adrenergic stimulation (Chapter 7 ) and discuss the results of this thesis in a broader context (Chapter 8). 


\subsection{REFERENCES}

1. Lloyd-Jones D, Adams RJ, Brown TM, Carnethon M, Dai S, De Simone G, Ferguson TB, Ford E, Furie K, Gillespie C, Go A, Greenlund K, Haase N, Hailpern S, Ho PM, Howard V, Kissela B, Kittner S, Lackland D, Lisabeth L, Marelli A, McDermott MM, Meigs J, Mozaffarian D, Mussolino M, Nichol G, Roger VL, Rosamond W, Sacco R, Sorlie P, Thom T, WasserthielSmoller S, Wong ND, Wylie-Rosett J. Heart disease and stroke statistics--2010 update: a report from the American Heart Association. Circulation. 2010;121:e46-e215.

2. Vaartjes I, Peters RJG, van Dis SJ, Bots ML. Hart- en vaatziekten in Nederland naar geslacht. In: Vaartjes I, van Dis SJ, Peters RJG, Visseren FL, Bots ML, eds. Hart- en vaatziekten in Nederland 2008, cijfers over ziekte en sterfte. Den Haag: Nederlandse Hartstichting; 2008:922.

3. Kong $M H$, Fonarow GC, Peterson ED, Curtis $A B$, Hernandez AF, Sanders GD, Thomas KL, Hayes DL, Al-Khatib SM. Systematic review of the incidence of sudden cardiac death in the United States. J Am Coll Cardiol. 2011;57:794-801.

4. de Vreede-Swagemakers JJ, Gorgels AP, Dubois-Arbouw WI, van Ree JW, Daemen MJ, Houben LG, Wellens HJ. Out-of-hospital cardiac arrest in the 1990's: a population-based study in the Maastricht area on incidence, characteristics and survival. J Am Coll Cardiol. 1997;30:1500-1505.

5. Zipes DP, Rubart M. Neural modulation of cardiac arrhythmias and sudden cardiac death. Heart Rhythm. 2006;3:108-113.

6. Zipes DP, Wellens HJ. Sudden cardiac death. Circulation. 1998;98:2334-2351.

7. Coumel P. Autonomic influences in atrial tachyarrhythmias. I Cardiovasc Electrophysiol. 1996;7:999-1007.

8. Lu Z, Scherlag BJ, Lin J, Yu L, Guo JH, Niu G, Jackman WM, Lazzara R, Jiang H, Po SS. Autonomic mechanism for initiation of rapid firing from atria and pulmonary veins: evidence by ablation of ganglionated plexi. Cardiovasc Res. 2009;84:245-252.

9. Sharifov OF, Fedorov VV, Beloshapko GG, Glukhov AV, Yushmanova AV, Rosenshtraukh LV. Roles of adrenergic and cholinergic stimulation in spontaneous atrial fibrillation in dogs. $J$ Am Coll Cardiol. 2004;43:483-490.

10. Ogawa M, Zhou S, Tan AY, Song J, Gholmieh G, Fishbein MC, Luo H, Siegel RJ, Karagueuzian HS, Chen LS, Lin SF, Chen PS. Left stellate ganglion and vagal nerve activity and cardiac arrhythmias in ambulatory dogs with pacing-induced congestive heart failure. J Am Coll Cardiol. 2007;50:335-343.

11. Liggett SB, Cresci S, Kelly RJ, Syed FM, Matkovich SJ, Hahn HS, Diwan A, Martini JS, Sparks L, Parekh RR, Spertus JA, Koch WJ, Kardia SL, Dorn GW, 2nd. A GRK5 polymorphism that inhibits beta-adrenergic receptor signaling is protective in heart failure. Nat Med. 2008;14:510-517.

12. Triposkiadis F, Karayannis G, Giamouzis G, Skoularigis J, Louridas G, Butler J. The sympathetic nervous system in heart failure physiology, pathophysiology, and clinical implications. J Am Coll Cardiol. 2009;54:1747-1762.

13. Schwartz PJ, Vanoli E, Stramba-Badiale M, De Ferrari GM, Billman GE, Foreman RD. Autonomic mechanisms and sudden death. New insights from analysis of baroreceptor reflexes in conscious dogs with and without a myocardial infarction. Circulation. 1988;78:969-979.

14. Billman GE. Cardiac autonomic neural remodeling and susceptibility to sudden cardiac death: effect of endurance exercise training. Am J Physiol Heart Circ Physiol. 2009;297:H1171-1193.

15. Schwartz PJ, Priori SG, Spazzolini C, Moss AJ, Vincent GM, Napolitano C, Denjoy I, Guicheney $P$, Breithardt G, Keating MT, Towbin JA, Beggs AH, Brink P, Wilde AA, Toivonen L, Zareba W, Robinson JL, Timothy KW, Corfield V, Wattanasirichaigoon D, Corbett C, Haverkamp W, Schulze-Bahr E, Lehmann MH, Schwartz K, Coumel P, Bloise R. Genotype-phenotype 
correlation in the long-QT syndrome: gene-specific triggers for life-threatening arrhythmias. Circulation. 2001;103:89-95.

16. Schwartz PJ, Vanoli E, Crotti L, Spazzolini C, Ferrandi C, Goosen A, Hedley P, Heradien M, Bacchini S, Turco A, La Rovere MT, Bartoli A, George AL, Jr., Brink PA. Neural control of heart rate is an arrhythmia risk modifier in long QT syndrome. J Am Coll Cardiol. 2008;51:920-929.

17. Takenaka K, Ai T, Shimizu W, Kobori A, Ninomiya T, Otani H, Kubota T, Takaki H, Kamakura S, Horie M. Exercise stress test amplifies genotype-phenotype correlation in the LQT1 and LQT2 forms of the long-QT syndrome. Circulation. 2003;107:838-844.

18. Leenhardt A, Lucet V, Denjoy I, Grau F, Ngoc DD, Coumel P. Catecholaminergic polymorphic ventricular tachycardia in children. A 7-year follow-up of 21 patients. Circulation. 1995;91:1512-1519.

19. Rosso R, Kalman JM, Rogowski O, Diamant S, Birger A, Biner S, Belhassen B, Viskin S. Calcium channel blockers and beta-blockers versus beta-blockers alone for preventing exerciseinduced arrhythmias in catecholaminergic polymorphic ventricular tachycardia. Heart Rhythm. 2007;4:1149-1154.

20. Watanabe H, Chopra N, Laver D, Hwang HS, Davies SS, Roach DE, Duff HJ, Roden DM, Wilde AA, Knollmann BC. Flecainide prevents catecholaminergic polymorphic ventricular tachycardia in mice and humans. Nat Med. 2009;15:380-383.

21. Madamanchi A. $\beta$-Adrenergic receptor signaling in cardiac function and heart failure. Mcgill J Med. 2007;10:99-104.

22. El-Armouche $A$, Eschenhagen T. $\beta$-Adrenergic stimulation and myocardial function in the failing heart. Heart Fail Rev. 2009;14:225-241.

23. Chen LS, Zhou S, Fishbein MC, Chen PS. New perspectives on the role of autonomic nervous system in the genesis of arrhythmias. J Cardiovasc Electrophysiol. 2007;18:123-127.

24. Roberts J, Kelliher GJ, Lathers CM. Role of adrenergic influences in digitalis-induced ventricular arrhythmia. Life Sci. 1976;18:665-677.

25. Bers DM. Cardiac excitation-contraction coupling. Nature. 2002;415:198-205.

26. Charpentier F, Merot J, Loussouarn G, Baro I. Delayed rectifier $\mathrm{K}^{+}$currents and cardiac repolarization. J Mol Cell Cardiol. 2010;48:37-44.

27. Nerbonne JM, Kass RS. Molecular physiology of cardiac repolarization. Physiol Rev. 2005;85:1205-1253.

28. Roden DM, Balser JR, George AL, Jr., Anderson ME. Cardiac ion channels. Annu Rev Physiol. 2002;64:431-475.

29. Diego JMD, Sun ZQ, Antzelevitch C. $I_{\text {to }}$ and action potential notch are smaller in left vs. right canine ventricular epicardium. Am J Physiol. 1996;271:H548-H561.

30. Liu DW, Gintant GA, Antzelevitch C. Ionic bases for electrophysiological distinctions among epicardial, midmyocardial, and endocardial myocytes from the free wall of the canine left ventricle. Circ Res. 1993;72:671-687.

31. Guo D, Zhou J, Zhao X, Gupta P, Kowey PR, Martin J, Wu Y, Liu T, Yan GX. L-type calcium current recovery versus ventricular repolarization: preserved membrane-stabilizing mechanism for different QT intervals across species. Heart Rhythm. 2008;5:271-279.

32. Wang ZG, Pelletier LC, Talajic M, Nattel S. Effects of flecainide and quinidine on human atrial action potentials. Role of rate-dependence and comparison with guinea pig, rabbit, and dog tissues. Circulation. 1990;82:274-283.

33. Kang M, Chung KY, Walker JW. G-protein coupled receptor signaling in myocardium: not for the faint of heart. Physiology (Bethesda). 2007;22:174-184.

34. Levy MN. Sympathetic-parasympathetic interactions in the heart. Circ Res. 1971;29:437-445.

35. Volders PGA. Novel insights into the role of the sympathetic nervous system in cardiac arrhythmogenesis. Heart Rhythm. 2010;7:1900-1906.

36. Lakatta EG, DiFrancesco D. What keeps us ticking: a funny current, a calcium clock, or both? J Mol Cell Cardiol. 2009;47:157-170. 
37. Zima A, Martynyuk AE, Seubert CN, Morey TE, Sumners C, Cucchiara RF, Dennis DM. Antagonism of the positive dromotropic effect of isoproterenol by adenosine: role of nitric oxide, cGMP-dependent cAMP-phosphodiesterase and protein kinase G. J Mol Cell Cardiol. 2000;32:1609-1619.

38. Grimm M, Brown JH. $\beta$-Adrenergic receptor signaling in the heart: Role of CaMKII. J Mol Cell Cardiol. 2010;48:322-330.

39. Xiao RP, Zhu W, Zheng M, Cao C, Zhang Y, Lakatta EG, Han Q. Subtype-specific $\alpha 1$ - and $\beta$ adrenoceptor signaling in the heart. Trends Pharmacol Sci. 2006;27:330-337.

40. Wang W, Zhu W, Wang S, Yang D, Crow MT, Xiao R-P, Cheng H. Sustained B1-adrenergic stimulation modulates cardiac contractility by $\mathrm{Ca}^{2+} /$ calmodulin kinase signaling pathway. Circ Res. 2004;95:798-806.

41. Zhang R, Khoo MS, Wu Y, Yang Y, Grueter CE, Ni G, Price EE, Jr., Thiel W, Guatimosim S, Song LS, Madu EC, Shah AN, Vishnivetskaya TA, Atkinson JB, Gurevich VV, Salama G, Lederer WJ, Colbran RJ, Anderson ME. Calmodulin kinase II inhibition protects against structural heart disease. Nat Med. 2005;11:409-417.

42. Houslay MD, Adams DR. PDE4 CAMP phosphodiesterases: modular enzymes that orchestrate signalling cross-talk, desensitization and compartmentalization. Biochem J. 2003;370:1-18.

43. Nikolaev VO, Bunemann M, Schmitteckert E, Lohse MJ, Engelhardt S. Cyclic AMP imaging in adult cardiac myocytes reveals far-reaching $\beta 1$-adrenergic but locally confined $\beta 2$-adrenergic receptor-mediated signaling. Circ Res. 2006;99:1084-1091.

44. Steinberg SF, Brunton LL. Compartmentation of $\mathrm{G}$ protein-coupled signaling pathways in cardiac myocytes. Annu Rev Pharmacol Toxicol. 2001;41:751-773.

45. Balijepalli RC, Kamp TJ. Caveolae, ion channels and cardiac arrhythmias. Prog Biophys Mol Biol. 2008;98:149-160.

46. Mauban JRH, O'Donnell M, Warrier S, Manni S, Bond M. AKAP-Scaffolding Proteins and Regulation of Cardiac Physiology. Physiology (Bethesda). 2009;24:78-87.

47. Fischmeister R, Castro LRV, Abi-Gerges A, Rochais F, Jurevicius J, Leroy J, Vandecasteele G. Compartmentation of cyclic nucleotide signaling in the heart: the role of cyclic nucleotide phosphodiesterases. Circ Res. 2006;99:816-828.

48. Mongillo M, McSorley T, Evellin S, Sood A, Lissandron V, Terrin A, Huston E, Hannawacker A, Lohse MJ, Pozzan T, Houslay MD, Zaccolo M. Fluorescence resonance energy transfer-based analysis of cAMP dynamics in live neonatal rat cardiac myocytes reveals distinct functions of compartmentalized phosphodiesterases. Circ Res. 2004;95:67-75.

49. Zaccolo M, Di Benedetto G, Lissandron V, Mancuso L, Terrin A, Zamparo I. Restricted diffusion of a freely diffusible second messenger: mechanisms underlying compartmentalized cAMP signalling. Biochem Soc Trans. 2006;34:495-497.

50. Fishman GI, Chugh SS, Dimarco JP, Albert CM, Anderson ME, Bonow RO, Buxton AE, Chen PS, Estes $M$, Jouven X, Kwong R, Lathrop DA, Mascette AM, Nerbonne JM, O'Rourke B, Page RL, Roden DM, Rosenbaum DS, Sotoodehnia N, Trayanova NA, Zheng ZJ. Sudden cardiac death prediction and prevention: report from a National Heart, Lung, and Blood Institute and Heart Rhythm Society Workshop. Circulation. 2010;122:2335-2348.

51. Hodgkin AL, Huxley AF. A quantitative description of membrane current and its application to conduction and excitation in nerve. J Physiol. 1952;117:500-544.

52. Noble D. A modification of the Hodgkin-Huxley equations applicable to Purkinje fibre action and pace-maker potentials. J Physiol. 1962;160:317-352.

53. McAllister RE, Noble D, Tsien RW. Reconstruction of the electrical activity of cardiac Purkinje fibres. J Physiol. 1975;251:1-59.

54. Carmeliet E, Lacquet L. Duration of ventricular action potential of the frog in relation to frequency; effects of ionic variations of potassium \& sodium. Arch Int Physiol Biochim. 1958;66:1-21. 
55. Coraboeuf E, Vassort G. Effects of some inhibitors of ionic permeabilities on ventricular action potential and contraction of rat and guinea-pig hearts. J Electrocardiol. 1968;1:19-29.

56. Trautwein A. Membrane currens in cardiac muscle fibers. Physiol Rev. 1973;53:793-835.

57. Draper $\mathrm{MH}$, Weidmann $\mathrm{S}$. Cardiac resting and action potentials recorded with an intracellular electrode. J Physiol. 1951;115:74-94.

58. Beeler GW, Reuter $\mathrm{H}$. Reconstruction of the action potential of ventricular myocardial fibres. J Physiol. 1977;268:177-210.

59. Difrancesco D, Noble D. A model of cardiac electrical activity incorporating ionic pumps and concentration changes. Philos Trans R Soc Lond B Biol Sci. 1985;307:353-398.

60. Hilgemann DW, Noble D. Excitation-contraction coupling and extracellular calcium transients in rabbit atrium: reconstruction of basic cellular mechanisms. Proc $R$ Soc Lond B Biol Sci. 1987;230:163-205.

61. Luo CH, Rudy Y. A model of the ventricular cardiac action potential. Depolarization, repolarization, and their interaction. Circ Res. 1991;68:1501-1526.

62. Luo $\mathrm{CH}$, Rudy Y. A dynamic model of the cardiac ventricular action potential. I. Simulations of ionic currents and concentration changes. Circ Res. 1994;74:1071-1096.

63. Winslow RL, Rice J, Jafri S, Marban E, O'Rourke B. Mechanisms of altered excitationcontraction coupling in canine tachycardia-induced heart failure, II: model studies. Circ Res. 1999;84:571-586.

64. Pandit SV, Clark RB, Giles WR, Demir SS. A mathematical model of action potential heterogeneity in adult rat left ventricular myocytes. Biophys J. 2001;81:3029-3051.

65. Hund TJ, Rudy Y. Rate dependence and regulation of action potential and calcium transient in a canine cardiac ventricular cell model. Circulation. 2004;110:3168-3174.

66. ten Tusscher KH, Noble D, Noble PJ, Panfilov AV. A model for human ventricular tissue. Am J Physiol Heart Circ Physiol. 2004;286:H1573-1589.

67. Bondarenko VE, Szigeti GP, Bett GC, Kim SJ, Rasmusson RL. Computer model of action potential of mouse ventricular myocytes. Am J Physiol Heart Circ Physiol. 2004;287:H13781403.

68. Mahajan A, Shiferaw $Y$, Sato D, Baher A, Olcese R, Xie LH, Yang MJ, Chen PS, Restrepo JG, Karma A, Garfinkel A, Qu Z, Weiss JN. A rabbit ventricular action potential model replicating cardiac dynamics at rapid heart rates. Biophys J. 2008;94:392-410.

69. Li P, Rudy Y. A model of canine purkinje cell electrophysiology and $\mathrm{Ca}^{2+}$ cycling: rate dependence, triggered activity, and comparison to ventricular myocytes. Circ Res. 2011;109:71-79.

70. O'Hara T, Virag L, Varro A, Rudy Y. Simulation of the undiseased human cardiac ventricular action potential: model formulation and experimental validation. PLoS Comput Biol. 2011;7:e1002061.

71. Grandi E, Pandit SV, Voigt N, Workman AJ, Dobrev D, Jalife J, Bers DM. Human atrial action potential and $\mathrm{Ca}^{2+}$ model: sinus rhythm and chronic atrial fibrillation. Circ Res. 2011;109:1055-1066.

72. Winslow RL, Cortassa S, O'Rourke B, Hashambhoy YL, Rice JJ, Greenstein JL. Integrative modeling of the cardiac ventricular myocyte. Wiley Interdiscip Rev Syst Biol Med. 2010.

73. Bezanilla F, Armstrong CM. Inactivation of the sodium channel. I. Sodium current experiments. J Gen Physiol. 1977;70:549-566.

74. Herzig S, Patil P, Neumann J, Staschen CM, Yue DT. Mechanisms of $\beta$-adrenergic stimulation of cardiac $\mathrm{Ca} 2+$ channels revealed by discrete-time Markov analysis of slow gating. Biophys J. 1993;65:1599-1612.

75. Balser JR, Roden DM, Bennett PB. Global parameter optimization for cardiac potassium channel gating models. Biophys J. 1990;57:433-444.

76. Clancy CE, Rudy Y. Linking a genetic defect to its cellular phenotype in a cardiac arrhythmia. Nature. 1999;400:566-569. 
77. Silva JR, Pan H, Wu D, Nekouzadeh A, Decker KF, Cui J, Baker NA, Sept D, Rudy Y. A multiscale model linking ion-channel molecular dynamics and electrostatics to the cardiac action potential. Proc Natl Acad Sci U S A. 2009;106:11102-11106.

78. Ter Keurs HE, Boyden PA. Calcium and arrhythmogenesis. Physiol Rev. 2007;87:457-506.

79. Jafri MS, Rice JJ, Winslow RL. Cardiac $\mathrm{Ca}^{2+}$ dynamics: the roles of ryanodine receptor adaptation and sarcoplasmic reticulum load. Biophys J. 1998;74:1149-1168.

80. Winslow RL, Tanskanen A, Chen M, Greenstein JL. Multiscale modeling of calcium signaling in the cardiac dyad. Ann N Y Acad Sci. 2006;1080:362-375.

81. Stern MD. Theory of excitation-contraction coupling in cardiac muscle. Biophys J. 1992;63:497-517.

82. Williams GS, Smith GD, Sobie EA, Jafri MS. Models of cardiac excitation-contraction coupling in ventricular myocytes. Math Biosci. 2010;226:1-15.

83. Greenstein JL, Winslow RL. An integrative model of the cardiac ventricular myocyte incorporating local control of $\mathrm{Ca}^{2+}$ release. Biophys J. 2002;83:2918-2945.

84. Tanskanen AJ, Greenstein JL, O'Rourke B, Winslow RL. The role of stochastic and modal gating of cardiac L-type Ca2+ channels on early after-depolarizations. Biophys J. 2005;88:8595.

85. Sato D, Xie LH, Nguyen TP, Weiss JN, Qu Z. Irregularly appearing early afterdepolarizations in cardiac myocytes: random fluctuations or dynamical chaos? Biophys J. 2010;99:765-773.

86. Thomsen MB, Volders PG, Beekman JD, Matz J, Vos MA. Beat-to-Beat variability of repolarization determines proarrhythmic outcome in dogs susceptible to drug-induced torsades de pointes. J Am Coll Cardiol. 2006;48:1268-1276.

87. Shaw RM, Rudy Y. The vulnerable window for unidirectional block in cardiac tissue: characterization and dependence on membrane excitability and intercellular coupling. J Cardiovasc Electrophysiol. 1995;6:115-131.

88. Winslow RL, Varghese A, Noble D, Adlakha C, Hoythya A. Generation and propagation of ectopic beats induced by spatially localized Na-K pump inhibition in atrial network models. Proc Biol Sci. 1993;254:55-61.

89. Fitzhugh R. Impulses and Physiological States in Theoretical Models of Nerve Membrane. Biophys J. 1961;1:445-466.

90. Nielsen PM, Le Grice IJ, Smaill BH, Hunter PJ. Mathematical model of geometry and fibrous structure of the heart. Am J Physiol. 1991;260:H1365-1378.

91. Aliev RR, Panfilov AV. Modeling of heart excitation patterns caused by a local inhomogeneity. J Theor Biol. 1996;181:33-40.

92. Fenton $\mathrm{F}$, Karma A. Vortex dynamics in three-dimensional continuous myocardium with fiber rotation: Filament instability and fibrillation. Chaos. 1998;8:20-47.

93. Ten Tusscher KH, Hren R, Panfilov AV. Organization of ventricular fibrillation in the human heart. Circ Res. 2007;100:e87-101.

94. Keldermann RH, ten Tusscher KH, Nash MP, Hren R, Taggart P, Panfilov AV. Effect of heterogeneous APD restitution on VF organization in a model of the human ventricles. Am J Physiol Heart Circ Physiol. 2008;294:H764-774.

95. Benson AP, Aslanidi OV, Zhang H, Holden AV. The canine virtual ventricular wall: a platform for dissecting pharmacological effects on propagation and arrhythmogenesis. Prog Biophys Mol Biol. 2008;96:187-208.

96. Seemann G, Hoper C, Sachse FB, Dossel O, Holden AV, Zhang H. Heterogeneous threedimensional anatomical and electrophysiological model of human atria. Philos Transact $A$ Math Phys Eng Sci. 2006;364:1465-1481.

97. Sato D, Xie LH, Sovari AA, Tran DX, Morita N, Xie F, Karagueuzian H, Garfinkel A, Weiss JN, Qu Z. Synchronization of chaotic early afterdepolarizations in the genesis of cardiac arrhythmias. Proc Natl Acad Sci U S A. 2009;106:2983-2988. 
Introduction

98. Rice JJ, Wang F, Bers DM, de Tombe PP. Approximate model of cooperative activation and crossbridge cycling in cardiac muscle using ordinary differential equations. Biophys J. 2008;95:2368-2390.

99. Campbell SG, Flaim SN, Leem CH, McCulloch AD. Mechanisms of transmurally varying myocyte electromechanics in an integrated computational model. Philos Transact A Math Phys Eng Sci. 2008;366:3361-3380.

100. Kohl P, Sachs F. Mechanoelectric feedback in cardiac cells. Philos Trans $R$ Soc Lond A. 2001;359:1173-1185.

101. Kohl P, Varghese A, Dekanski J, Noble D, Winslow RL. Computational study of the impact of cardiac mechanosensitive fibroblasts on heart rhythm. Exp Clin Cardiol. 1996;1:80-86.

102. Li W, Kohl P, Trayanova N. Myocardial ischemia lowers precordial thump efficacy: an inquiry into mechanisms using three-dimensional simulations. Heart Rhythm. 2006;3:179-186.

103. Jie X, Gurev V, Trayanova N. Mechanisms of mechanically induced spontaneous arrhythmias in acute regional ischemia. Circ Res. 2010;106:185-192.

104. Keldermann RH, Nash MP, Gelderblom H, Wang VY, Panfilov AV. Electromechanical wavebreak in a model of the human left ventricle. Am J Physiol Heart Circ Physiol. 2010;299:H134-143.

105. Kerckhoffs RC, McCulloch AD, Omens JH, Mulligan LJ. Effects of biventricular pacing and scar size in a computational model of the failing heart with left bundle branch block. Med Image Anal. 2009;13:362-369.

106. Kuijpers NH, Potse M, van Dam PM, ten Eikelder HM, Verheule S, Prinzen FW, Schotten U. Mechanoelectrical coupling enhances initiation and affects perpetuation of atrial fibrillation during acute atrial dilation. Heart Rhythm. 2011;8:429-436.

107. Saucerman JJ, Brunton LL, Michailova AP, McCulloch AD. Modeling $\beta$-adrenergic control of cardiac myocyte contractility in silico. J Biol Chem. 2003;278:47997-48003.

108. lancu RV, Jones SW, Harvey RD. Compartmentation of CAMP signaling in cardiac myocytes: a computational study. Biophys J. 2007;92:3317-3331.

109. Chiba H, Schneider NS, Matsuoka S, Noma A. A simulation study on the activation of cardiac CaMKII delta-isoform and its regulation by phosphatases. Biophys J. 2008;95:2139-2149.

110. Saucerman JJ, Bers DM. Calmodulin mediates differential sensitivity of CaMKII and calcineurin to local $\mathrm{Ca}^{2+}$ in cardiac myocytes. Biophys J. 2008;95:4597-4612.

111. Hashambhoy YL, Greenstein JL, Winslow RL. Role of CaMKII in RyR leak, EC coupling and action potential duration: a computational model. J Mol Cell Cardiol. 2010;49:617-624.

112. Soltis AR, Saucerman JJ. Synergy between CaMKII Substrates and beta-Adrenergic Signaling in Regulation of Cardiac Myocyte Ca ${ }^{2+}$ Handling. Biophys J. 2010;99:2038-2047. 


\section{CHAPTER 2}

PROPERTIES AND IONIC MECHANISMS OF ACTION POTENTIAL ADAPTATION, RESTITUTION, AND ACCOMMODATION IN CANINE EPICARDIUM

Keith F. Decker, Jordi Heijman, Jonathan R. Silva, Thomas J. Hund AND YORAM RUDY.

AM. J. PhySIOL. HeART CIRC. PHYSIOL. 2009, 296:H1017-H1026 


\section{ABSTRACT}

Computational models of cardiac myocytes are important tools for understanding ionic mechanisms of arrhythmia. This work presents a new model of the canine epicardial myocyte that reproduces a wide range of experimentally observed rate-dependent behaviors in cardiac cell and tissue, including action potential (AP) duration (APD) adaptation, restitution, and accommodation. Model behavior depends on updated formulations for the 4-aminopyridine-sensitive transient outward current $\left(I_{\text {to1 }}\right)$, the slow component of the delayed rectifier $\mathrm{K}^{+}$current $\left(\mathrm{I}_{\mathrm{Ks}}\right)$, the L-type $\mathrm{Ca}^{2+}$ channel current $\left(\mathrm{I}_{\mathrm{Ca}, \mathrm{L}}\right)$, and the $\mathrm{Na}^{+}-\mathrm{K}^{+}$pump current $\left(I_{\mathrm{NaK}}\right)$ fit to data from canine ventricular myocytes. We found that $I_{\text {to1 }}$ plays a limited role in potentiating peak $\mathrm{I}_{\mathrm{Ca}, \mathrm{L}}$ and sarcoplasmic reticulum $\mathrm{Ca}^{2+}$ release for propagated APs but modulates the time course of APD restitution. $I_{\mathrm{Ks}}$ plays an important role in APD shortening at short diastolic intervals, despite a limited role in AP repolarization at longer cycle lengths. In addition, we found that $\mathrm{I}_{\mathrm{C}, \mathrm{L}}$ plays a critical role in APD accommodation and rate dependence of APD restitution. $\mathrm{Ca}^{2+}$ entry via $\mathrm{I}_{\mathrm{Ca}, \mathrm{L}}$ at fast rate drives increased $\mathrm{Na}^{+}-\mathrm{Ca}^{2+}$ exchanger $\mathrm{Ca}^{2+}$ extrusion and $\mathrm{Na}^{+}$ entry, which in turn increases $\mathrm{Na}^{+}$extrusion via outward $\mathrm{I}_{\text {Nak. }}$. APD accommodation results from this increased outward $I_{\text {Nak. }}$. Our simulation results provide valuable insight into the mechanistic basis of rate-dependent phenomena important for determining the heart's response to rapid and irregular pacing rates (e.g., arrhythmia). Accurate simulation of rate-dependent phenomena and increased understanding of their mechanistic basis will lead to more realistic multicellular simulations of arrhythmia and identification of molecular therapeutic targets. 


\section{INTRODUCTION}

Cardiac arrhythmias and sudden death involve complex myocardial activation patterns, including unidirectional block, reentry, and fibrillation. To understand the relations and transitions between these patterns, the ionic determinants of the response of healthy and diseased cardiac myocytes to complex patterns of excitation must be understood. The single-cell response to such excitation patterns depends on the complex interaction between ionic currents, intracellular ion concentrations, and membrane voltage. Computational cell models provide critical tools for exploring these interactions, allowing the development and testing of hypotheses about underlying ionic mechanisms based on careful integration of available experimental data. ${ }^{1}$ The dog is a common animal model for studying cell electrophysiology in a range of disease states. Our group and others have developed detailed mathematical models of the canine action potential (AP). ${ }^{2-4}$ Although these models have been used to study arrhythmia mechanisms after heart failure ${ }^{4}$ and myocardial infarction, ${ }^{5,6}$ as well as ionic mechanisms of alternans, ${ }^{2,7}$ they are limited in their ability to simulate important rate-dependent phenomena, ${ }^{8}$ including the dependence of steady-state AP duration (APD) on pacing cycle length (CL; i.e., APD adaptation), the dependence of APD on diastolic interval (DI; i.e., APD restitution), and the time course of the adjustment of APD to changes in rate (shortterm memory ${ }^{9}$ or APD accommodation ${ }^{10}$ ). These limitations extend to ionic models of other species, including the human. ${ }^{8}$ We hypothesized that canine epicardial APD adaptation, restitution, and accommodation could be simulated and understood on the basis of available descriptions of subcellular ionic processes. We incorporated updated and validated formulations of the 4aminopyridinesensitive transient outward current $\left(\mathrm{I}_{\text {to1 }}\right)$, the slow component of the delayed rectifier $\mathrm{K}^{+}$current $\left(\mathrm{I}_{\mathrm{Ks}}\right)$, the L-type $\mathrm{Ca}^{2+}$ channel current $\left(\mathrm{I}_{\mathrm{Ca}, \mathrm{L}}\right)$, and the $\mathrm{Na}^{+}-\mathrm{K}^{+}$pump current $\left(\mathrm{I}_{\mathrm{NaK}}\right)$ into a previously published model of the canine epicardial myocyte., ${ }^{3,7}$ Model behavior was examined in single cell and multicellular (strand) simulations. Our work provides new insight into ionic mechanisms underlying important rate-dependent AP properties, including APD restitution, adaptation, and accommodation. Specifically, our studies highlight the importance of $I_{\text {to1 }}$ and $I_{\mathrm{Ks}}$ in APD restitution and the role of $I_{\mathrm{Ca}, \mathrm{L}}$ and $\mathrm{I}_{\mathrm{NaK}}$ in APD accommodation and rate-dependent APD restitution. 


\section{METHODS}

\section{Model}

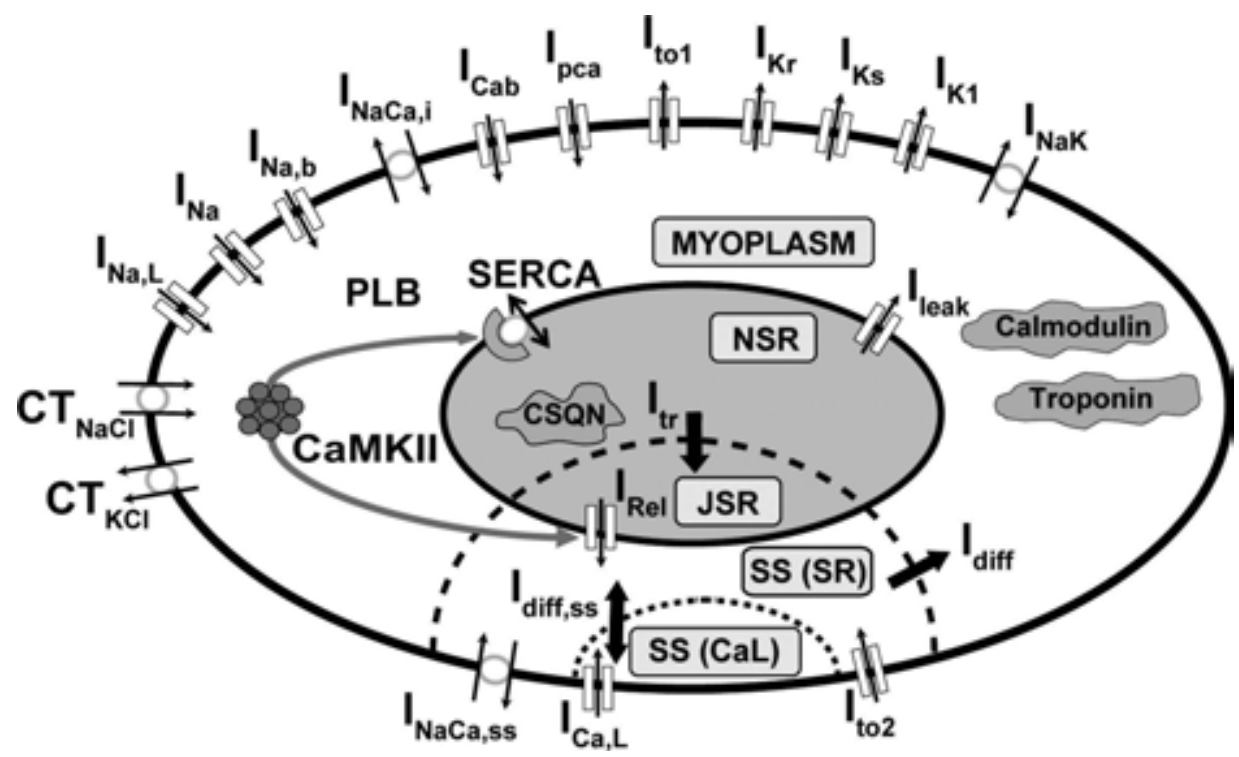

Figure 1. Hund-Rudy dynamic model of the canine epicardial myocyte. SR, sarcoplasmic reticulum; $\mathrm{CTKCl}, \mathrm{K}^{+}-\mathrm{Cl}^{-}$cotransporter; $\mathrm{CTNaCl}, \mathrm{Na}^{+}-\mathrm{Cl}^{-}$cotransporter; $\mathrm{I}_{\mathrm{NaL}}$, slowly activating late $\mathrm{Na}^{+}$current; $\mathrm{I}_{\mathrm{Na}}$, $\mathrm{Na}^{+}$current; $I_{\mathrm{Nab}}$, background $\mathrm{Na}^{+}$current; $\mathrm{I}_{\mathrm{NaCa}, \mathrm{i}}, \mathrm{Na}^{+} / \mathrm{Ca}^{2+}$ exchanger (localized to myoplasm); $I_{\mathrm{Cab}}$, background $\mathrm{Ca}^{2+}$ current; $I_{\text {pca }}$ sarcolemmal $\mathrm{Ca}^{2+}$ pump; $\mathrm{I}_{\text {to1 }}$, 4-aminopyridine-sensitive transient outward current; $I_{\mathrm{Kr}}$, fast component of delayed rectifier $\mathrm{K}^{+}$current; $\mathrm{I}_{\mathrm{Ks}}$, slow component of delayed rectifier $\mathrm{K}^{+}$current; $I_{\mathrm{K} 1}$, time-dependent $\mathrm{K}^{+}$current; $I_{\mathrm{NaK}}, \mathrm{Na}^{+}-\mathrm{K}^{+}$pump current; $I_{\text {leak, }}, \mathrm{NSR}$ leak flux; $I_{\text {diff, }}$, ion diffusion, myoplasm-to-SR subspace; $I_{\mathrm{tr}}, \mathrm{Ca}^{2+}$ transfer, NSR to JSR; $I_{\text {rel, }}$ JSR release flux; $I_{\text {diff,ss, }}$ ion diffusion, subspace-to-local $I_{\mathrm{CaL}}$ subspace; $I_{\mathrm{NaCa}, \mathrm{ss}}, \mathrm{Na}^{+} / \mathrm{Ca}^{+}$exchanger (localized to SR subspace); $I_{\mathrm{Ca}, \mathrm{L}}$ L-type $\mathrm{Ca}^{2+}$ current; $\mathrm{I}_{\mathrm{to} 2}, \mathrm{Ca}^{2+}$-dependent transient outward $\mathrm{Cl}^{-}$current $\left(\mathrm{I}_{\mathrm{CI}(\mathrm{Ca})}\right)$; SS $(\mathrm{CaL}), \mathrm{I}_{\mathrm{CaL}}$ subspace; SS(SR), SR subspace; PLB, phospholamban; SERCA, sarco(endo)plasmic reticulum $\mathrm{Ca}^{2+}$-ATPase; CSQN, calsequestrin; CaMKII, $\mathrm{Ca}^{2+}$ /calmodulin-dependent kinase; NSR, network SR; JSR, junctional SR. (For additional model details, see Refs. ${ }^{3}$ and ${ }^{7}$, the appendix of this thesis, and http://rudylab.wustl.edu/.)

The Hund-Rudy dynamic model (Figure 1) of the canine epicardial myocyte ${ }^{3,6,7}$ serves as the basis for these simulations. Updates to intracellular $\mathrm{Ca}^{2+}\left(\left[\mathrm{Ca}^{2+}\right]_{\mathrm{i}}\right)$ handling in a recent study of APD and $\left[\mathrm{Ca}^{2+}\right]_{i}$ transient $(\mathrm{CaT})$ alternans are included. ${ }^{7}$ Ion channel formulations, including $\mathrm{I}_{\mathrm{Ca}, \mathrm{L}}, \mathrm{I}_{\mathrm{to1}}, \mathrm{I}_{\mathrm{Ks}}$, and $I_{\text {NaK }}$, have been updated on the basis of additional data from canine ventricular myocytes. Model parameters were fit to experimental data from the literature (Figure 2). 
A

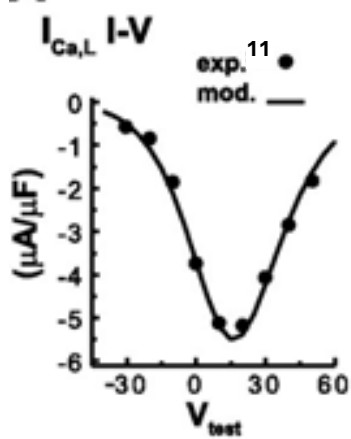

E

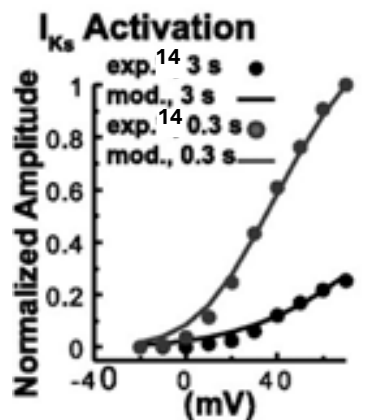

B

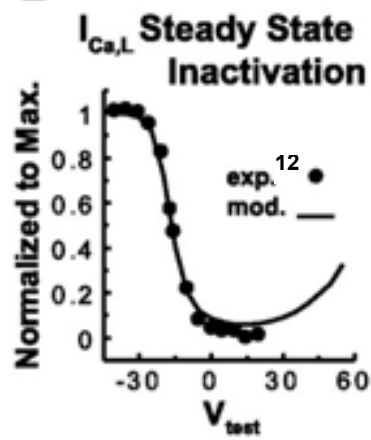

F

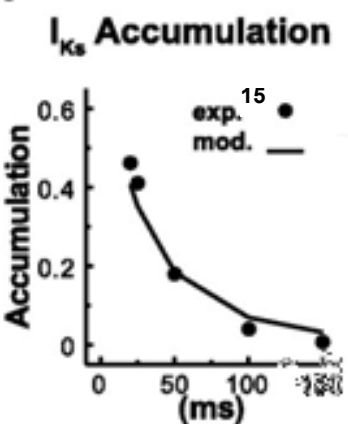

C $I_{\text {to } 1}$ I-V

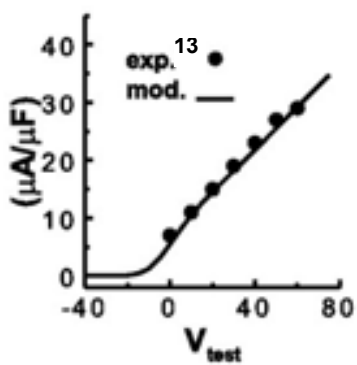

G

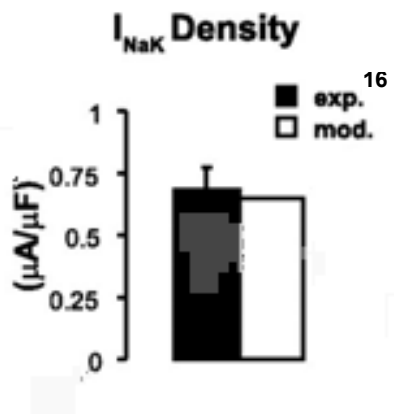

D $\mathrm{I}_{\text {to1 }}$ Time to Priak

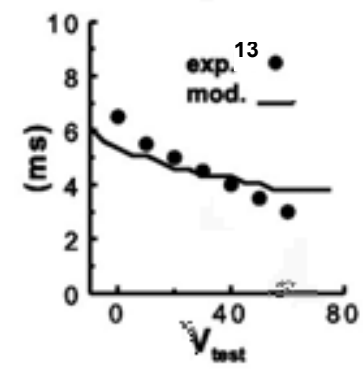

H Steady State [ $\left[\mathrm{Na}^{+}\right]$

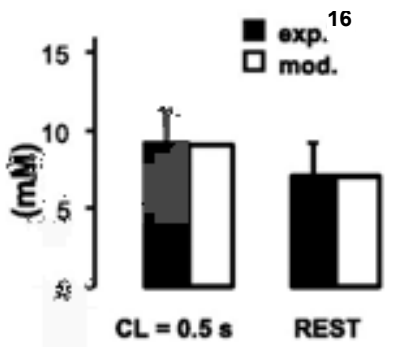

Figure 2. $\mathrm{A}$ and $\mathrm{B}$ : model $\mathrm{I}_{\mathrm{C}, \mathrm{L}}$ current-voltage $(\mathrm{I}-\mathrm{V})$ relationship and steady-state inactivation fit to experimental data from canine epicardial myocytes. ${ }^{11,12} \mathrm{C}$ and $\mathrm{D}$ : model $\mathrm{I}_{\mathrm{to} 1} \mathrm{I}-\mathrm{V}$ curve and time to peak fit to data from canine epicardial myocytes. ${ }^{13} \mathrm{E}$ and $\mathrm{F}$ : model $\mathrm{I}_{\mathrm{Ks}}$ activation and accumulation fit to data from canine ventricular myocytes. ${ }^{14,15} \mathrm{G}$ and $\mathrm{H}$ : model $\mathrm{I}_{\mathrm{NaK}}$ density and steady-state intracellular $\mathrm{Na}^{+}$concentration $\left(\left[\mathrm{Na}^{+}\right]_{\mathrm{i}}\right)$ fit to data from canine ventricular myocytes. ${ }^{16} \mathrm{~V}_{\text {test }}$, test potential; $\mathrm{CL}$, cycle length.

A new Markov model of $\mathrm{I}_{\mathrm{Ca}, \mathrm{L}}$ was formulated to reproduce a wide range of experimental data while maintaining computational tractability to facilitate long-term multicellular simulations. The state structure (Figure $3 \mathbf{C}$ ) of the model reflects the hypothesis that $\mathrm{Ca}^{2+}$ binding to calmodulin removes a "brake" and speeds up $I_{\mathrm{Ca}, \mathrm{L}}$ voltage-dependent inactivation. ${ }^{17,}{ }^{18}$ The model reproduces the $\mathrm{I}_{\mathrm{Ca}, \mathrm{L}}$ current-voltage (I-V) relationship ${ }^{11}$ (Figure 2A), steady-state inactivation ${ }^{12}$ (Figure 2B), time constant of voltage-dependent inactivation, ${ }^{19}$ and recovery from inactivation ${ }^{20}$ in canine ventricular myocytes. A model of $\mathrm{I}_{\text {to } 1}$ was developed and fit to the I-V relationship (Figure 2C), time to peak (Figure 2D), time constant of inactivation, ${ }^{13}$ and slow time constant of recovery from inactivation ${ }^{21}$ in canine epicardial myocytes. A Markov model of $\mathrm{I}_{\mathrm{Ks}}{ }^{22}$ previously developed in our laboratory was adopted and fit to data from canine ventricular myocytes. The model accurately reproduces the kinetics of $I_{\mathrm{Ks}}$ based on underlying voltage sensor transitions and has provided insight into the role of $I_{\mathrm{Ks}}$ in APD adaptation in the guinea pig and humans. ${ }^{22}$ The model fits canine ventricular data on the time course of activation ${ }^{15}$ (Figure 2E) and time dependence of current accumulation ${ }^{14}$ (Figure 2F). $\mathrm{I}_{\mathrm{Ks}}$ density was scaled to fit data from canine epicardial myocytes. ${ }^{23} \mathrm{~A}$ recently developed formulation of $\mathrm{I}_{\mathrm{NaK}}$ based on data from canine ventricular myocytes ${ }^{16}$ was also incorporated into the model. Model $I_{\text {Nak }}$ density matches epicardial myocyte data (Figure 2G) and results in intracellular $\mathrm{Na}^{+}\left(\left[\mathrm{Na}^{+}\right]_{i}\right)$ at rest and during pacing $(\mathrm{CL}=0.5 \mathrm{~s}$ ) consistent with experimental results (Figure $2 \mathrm{H})$. See the appendix of this thesis for complete model equations and more detailed descriptions. 


\section{Notes on Experimental Data for Model Fits}

In general, experimental data that were chosen for model fitting and comparison represent either the consensus of a wide range of experimental results or the most complete experimental study. Data sets chosen for reproducing ICaL (I-V relationship, steady state inactivation, recovery from inactivation) and Ito1 behavior (I-V relationship, time to peak, time constant of inactivation, recovery from inactivation) are representative of multiple available experiments in canine ventricular myocytes (Figures 3-4). Model formulations for INaK and IKs are based on recent experimental studies that provide the most detailed information available on these currents in canine epicardial myocytes. Details are provided in the appendix of this thesis.

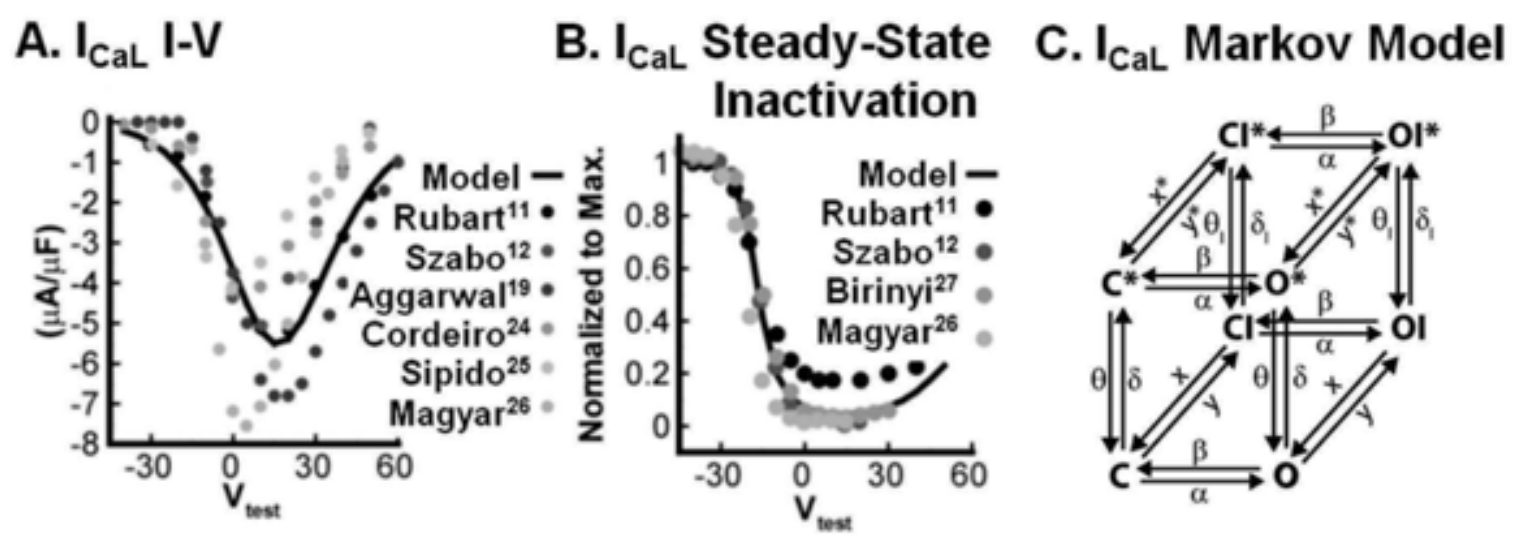

Figure 3. A. Model $\mathrm{I}_{\mathrm{CaL}}$ current-voltage (I-V) relationship and B. steady-state inactivation compared to multiple data sets from canine ventricular cells. ${ }^{11,12,19,24-27}$ For color figure, see page 242.

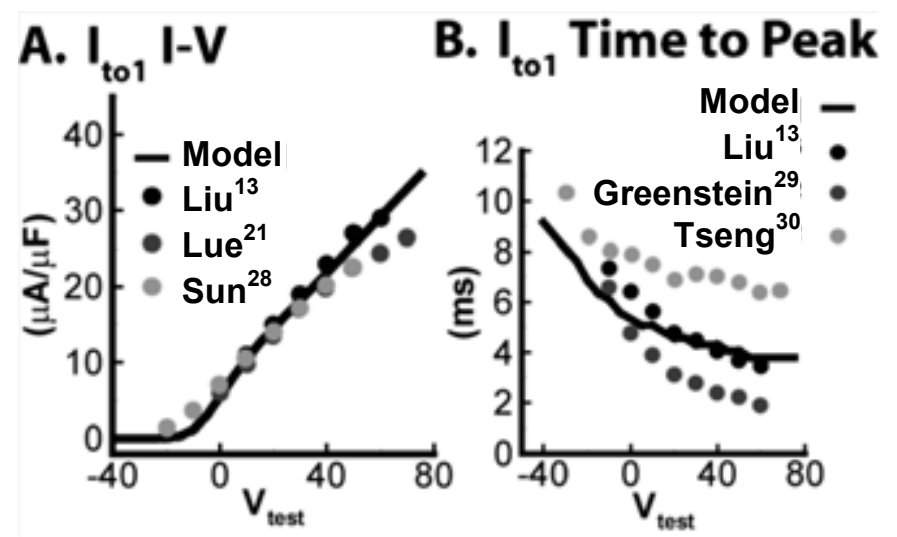

Figure 4. A. Model $\mathrm{I}_{\mathrm{to1} 1} \mathrm{I}-\mathrm{V}$ curve. B. Time to peak compared to multiple data sets from canine left ventricular epicardial cells. ${ }^{13,21,28-30}$ For color figure, see page 242. 


\section{Single-cell simulations}

Steady-state results are shown after $1,800 \mathrm{~s}$ of pacing at a given $\mathrm{CL}$ with a conservative $\mathrm{K}^{+}$stimulus. ${ }^{31}$ This simulation time resulted in a beat-to-beat change in APD and maximum intracellular $\mathrm{Na}^{+}$ $\left(\left[\mathrm{Na}^{+}\right]_{i, \text { max }}\right)$ and $\mathrm{Ca}^{2+}\left(\left[\mathrm{Ca}^{2+}\right]_{i, \max }\right)<0.1 \%$. Restitution results were obtained for an additional beat after pacing to steady state as described. Accommodation simulations involved pacing to steady state at a given $\mathrm{CL}\left(\mathrm{CL}_{\mathrm{s} 1}\right)$ followed by pacing to steady state at a different $\mathrm{CL}\left(\mathrm{CL}_{\mathrm{s} 2}\right)$.

\section{Strand simulations}

A detailed description of computational methods for strand simulations is included in previous work from our laboratory. ${ }^{32}$ Briefly, propagation in a strand of serially connected HRd model cells was simulated via a finite difference approximation of the cable equation (see appendix of this thesis). No flux boundary conditions $(\delta \mathrm{V} / \delta \mathrm{x}=0)$ were set at the beginning and end of the strand. A 1-ms stimulus $(-200 \mu \mathrm{A} / \mu \mathrm{F})$ was applied to cell 1 . One-dimensional simulations of propagation were performed in a strand of 96 cells following previous work from our laboratory. ${ }^{32}$ Cell 1 was directly stimulated, and simulation results are shown for a central cell (cell 48) where end effects were negligible. Simulation protocols were similar to those used for single cells (1,800 s of pacing), resulting in beat-to-beat changes in APD and $\left[\mathrm{Na}^{+}\right]_{\text {, max }}$ and $\left[\mathrm{Ca}^{2+}\right]_{\text {, max }}<0.1 \%$. Gap junction conductance of $2.5 \mu \mathrm{S}$ gave a maximum upstroke velocity $(\mathrm{dV} / \mathrm{dtmax})^{33}$ and conduction velocity ${ }^{34}$ consistent with experimental results in canine epicardial tissue.

\section{RESULTS}

\section{APD rate dependence in a single cell and a multicellular strand}

Figure 5 shows the rate dependence of APD in a cell and a strand during different pacing protocols. The rate dependence of simulated steady-state APs is shown in Figure 5A, left. Cell and strand simulations exhibit the spike-and-dome morphology characteristic of canine epicardium ${ }^{21}$ (Figure 5A, right). Steady-state APs in the strand show a reduced upstroke amplitude and velocity compared with the single cell due to electronic load during propagation. Maximum upstroke voltage $\left(\mathrm{V}_{\max }\right)$ and $\mathrm{dV} / \mathrm{dt}_{\max }$ match experiments in canine ventricular epicardium at $\mathrm{CL}=0.8 \mathrm{s:}:^{33}$ experimental $\mathrm{V}_{\max }=13.1$ $\pm 4.7 \mathrm{mV}, \mathrm{dV} / \mathrm{dt}_{\max }=151.8 \pm 39.8 \mathrm{~V} / \mathrm{s}$; model $\mathrm{V}_{\max }=9.24 \mathrm{mV}, \mathrm{dV} / \mathrm{dt}_{\max }=162.6 \mathrm{~V} / \mathrm{s}$. The model reproduces the decreased rate dependence of the depth of the phase 1 notch generally observed in tissue $^{21,33,35}$ (Figure 5A). The cause of different AP spike-and-dome morphology in different tissue experiments is unknown, but possibilities include heterogeneity in the density and recovery kinetics of $\mathrm{I}_{\text {to } 1}$ due to age, ${ }^{38}$ sex $^{39}$ and precise apicobasal or transmural location. ${ }^{21,40}$ We next characterized APD restitution in our single-cell and strand models. After pacing to steady state at a given $C L\left(C L_{S 1}\right)$, additional stimuli (S2) scanning the DI were applied to generate restitution curves plotting APD vs. S2 coupling interval $\left(\mathrm{Cl}_{\mathrm{S}_{2}}\right)$ (Figure 5B, left). As the basic pacing $\mathrm{CL}\left(\mathrm{CL}_{\mathrm{S}_{1}}\right)$ decreases, restitution curves in the single-cell and strand models shift toward shorter APD, consistent with experimental measurements ${ }^{8,36,41,42}$ (Figure 5B, right). The model also reproduces tissue AP restitution kinetics as a function of DI (see below and Figures 8-9). Figure 5C (left) shows the time course of APD in a model cell and a strand after pacing to steady state at $\mathrm{CL}_{\mathrm{s} 1}=1 \mathrm{~s}$ followed by sustained pacing at $\mathrm{CL}_{\mathrm{s} 2}$ $=0.5 \mathrm{~s}$ starting at time 0 . APD at the new $\mathrm{CL}$ approaches steady state after several minutes of pacing, 
a process referred to as accommodation, ${ }^{10}$ consistent with experiments in canine ventricle ${ }^{37}$ (Figure $5 C$, right).

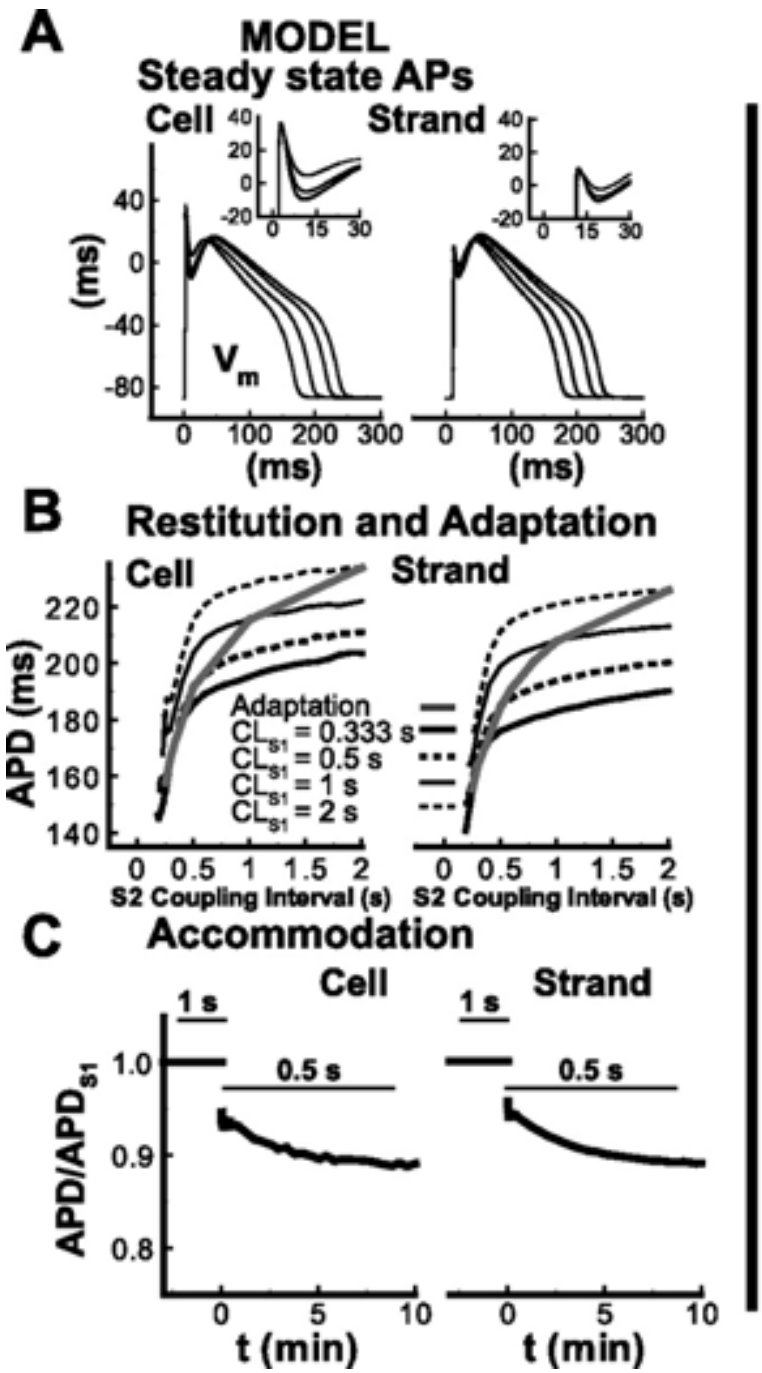

\section{EXPERIMENT}

Cell ${ }_{\text {Liu et al. }}^{21}$ Tissue ${ }^{21}$ Diu et al. ${ }^{21}$ Diego et al. Balati et al. ${ }^{33,35}$
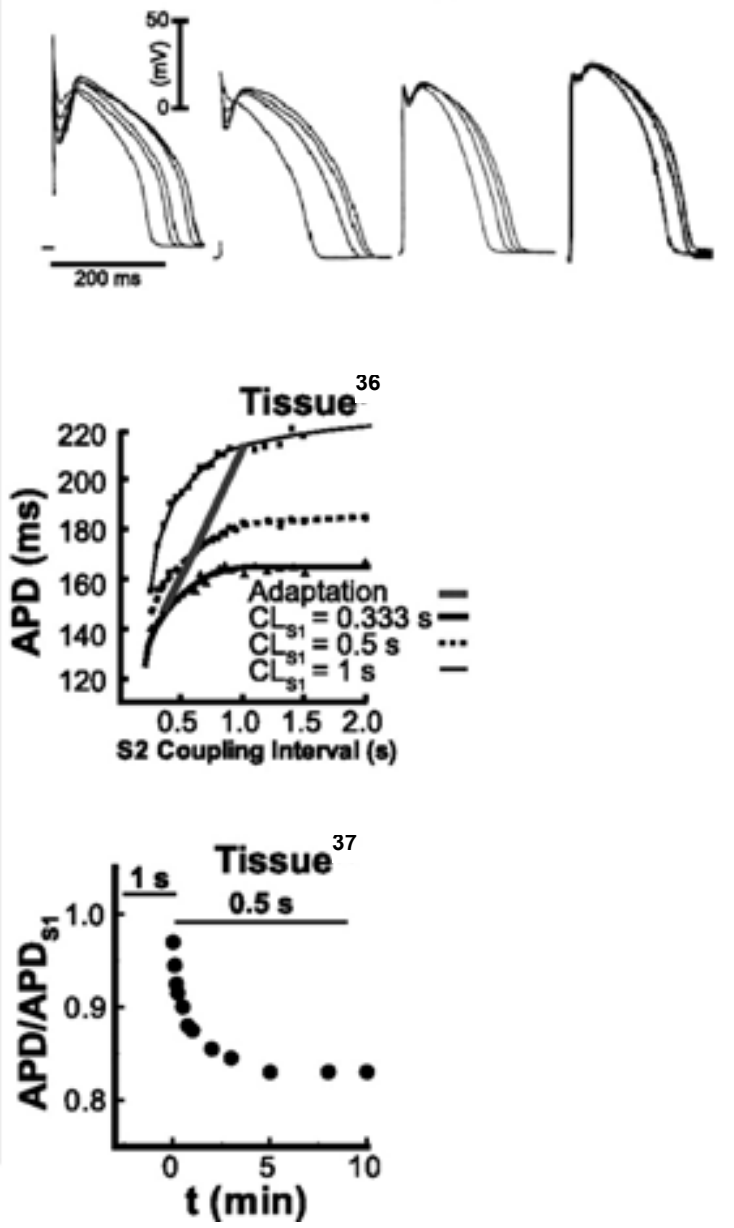

Figure 5. A, left: model steady-state action potentials (APs) at $\mathrm{CL}=0.3,0.5,1$, and $2 \mathrm{~s}$ in single-cell and strand simulations. $V_{m}$, membrane potential. Inset: peak upstroke voltage and rate dependence of notch depth. A, right: experimental steady-state APs in a single cell and 3 tissue preparations. Experimental data for a single cell are from Ref. 21 (cell $C L=0.3,0.5,0.8,2$, and $8 \mathrm{~s}$ ); experimental data for tissue are from Ref. 21 (tissue $C L=0.3,1,2$, and $5 \mathrm{~s}$ ), Ref. 33 (tissue $\mathrm{CL}=0.3,0.5,0.8$, and 2 s), and Ref. 35 (tissue $C L=0.3,1$, and $5 \mathrm{~s}$ ). $\mathrm{B}$, left: model $\mathrm{CL}_{\mathrm{s} 1}$ dependence of AP duration (APD) restitution in single cell and strand. Gray traces denote adaptation ( $\mathrm{S} 2$ coupling interval $=\mathrm{CL}_{S_{1}}$ ). $\mathrm{B}$, right: experimental results from study of $\mathrm{CL}_{S 1}$ dependence of APD restitution in right ventricle of open-chest dogs ${ }^{36} \mathrm{C}$, left: model accommodation of APD after change from $\mathrm{CL}=1 \mathrm{~s}$ to $\mathrm{CL}=0.5 \mathrm{~s}$ in single cell and strand. C, right: APD accommodation in canine ventricular muscle fiber experiments. ${ }^{37}$ 


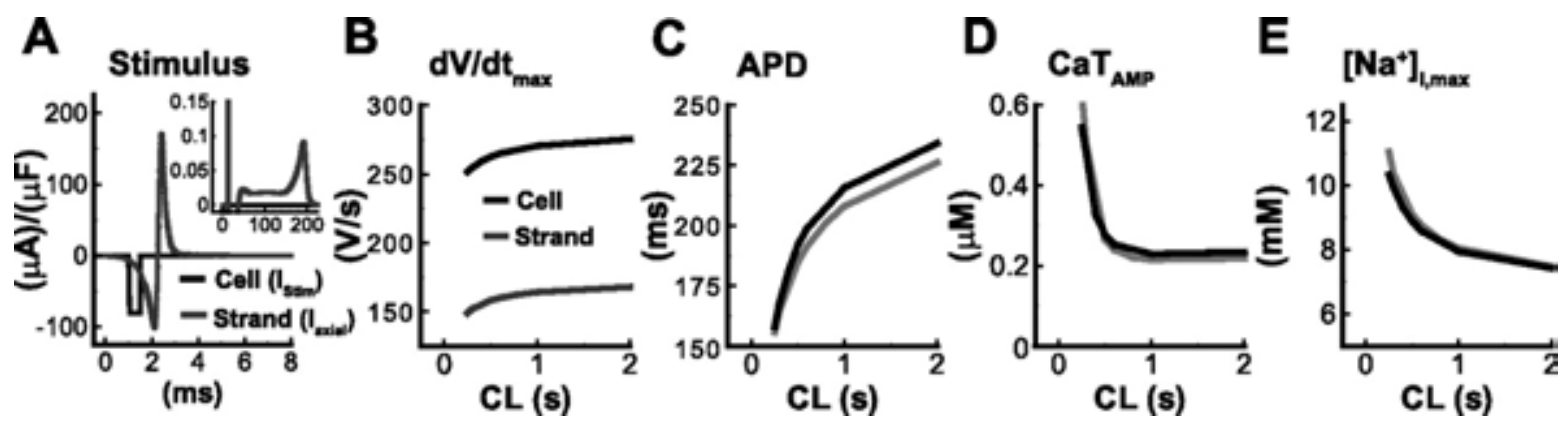

Figure 6. A. isolated single-cell stimulus (black trace, $\mathrm{I}_{\text {stim }}$ ) and strand axial current (gray trace, laxial). Inset: sustained repolarizing axial current. $\mathrm{B}-\mathrm{E}$. $\mathrm{CL}_{\mathrm{s} 1}$ dependence of maximum upstroke velocity $\left(\mathrm{dV} / \mathrm{dt}_{\mathrm{max}}\right), \mathrm{APD}, \mathrm{Ca}^{2+}$ transient amplitude $\left(\mathrm{CaT}_{\mathrm{AMP}}\right)$, and maximal intracellular $\mathrm{Na}^{+}$concentration $\left(\left[\mathrm{Na}^{+}\right]_{i, \max }\right)$ in single-cell and strand simulations.

Previous simulation studies ${ }^{8,43}$ showed that direct stimulation in single cells and electrotonic loading in tissue can lead to different AP dynamics. Figure 6 compares cell and tissue steady-state APs and ion accumulation in the new model. A $-80 \mathrm{pA} / \mathrm{pF}, 0.5-\mathrm{ms}$ stimulus was used in single-cell simulations (Figure 6A). In the strand, excitatory axial current in well-coupled tissue (Figure 6A) consists of 1 ) an initial transient depolarizing current, 2) a transient repolarizing current as the cell supplies charge to depolarizing downstream cells, and 3 ) a small sustained repolarizing current (Figure 6A, inset). The biphasic axial current in the tissue reduces AP upstroke velocity relative to its value in the single cell (Figure 6B). In addition, steady-state tissue APD is reduced slightly (Figure $6 \mathrm{C}$ ) as a result of the sustained repolarizing axial current (Figure 6A, inset). APD adaptation in cell and strand simulations is consistent with experimental results ${ }^{21}$. Cell and strand simulations show an increase in $\mathrm{Ca}^{2+}$ transient amplitude $\left(\mathrm{CaT}_{\mathrm{Amp}} \text {; Figure } 6 \mathrm{D}\right)^{25}$ and maximum $\left[\mathrm{Na}^{+}\right]_{i}\left(\left[\mathrm{Na}^{+}\right]_{i \text {,max; }} \text { Figure } 6 \mathrm{E}\right)^{16}$ with pacing rate, consistent with experimental results. Differences in $\mathrm{CaT}_{\mathrm{Amp}}$ and $\left[\mathrm{Na}^{+}\right]_{i, \max }$ in cell and strand simulations are minimal. Subsequent results are from strand simulations unless otherwise specified.

$I_{\text {to1, }}$ peak $I_{\mathrm{Ca}, \mathrm{L}}$ and $\mathrm{CaT}_{\mathrm{Amp}}$ in cell and strand
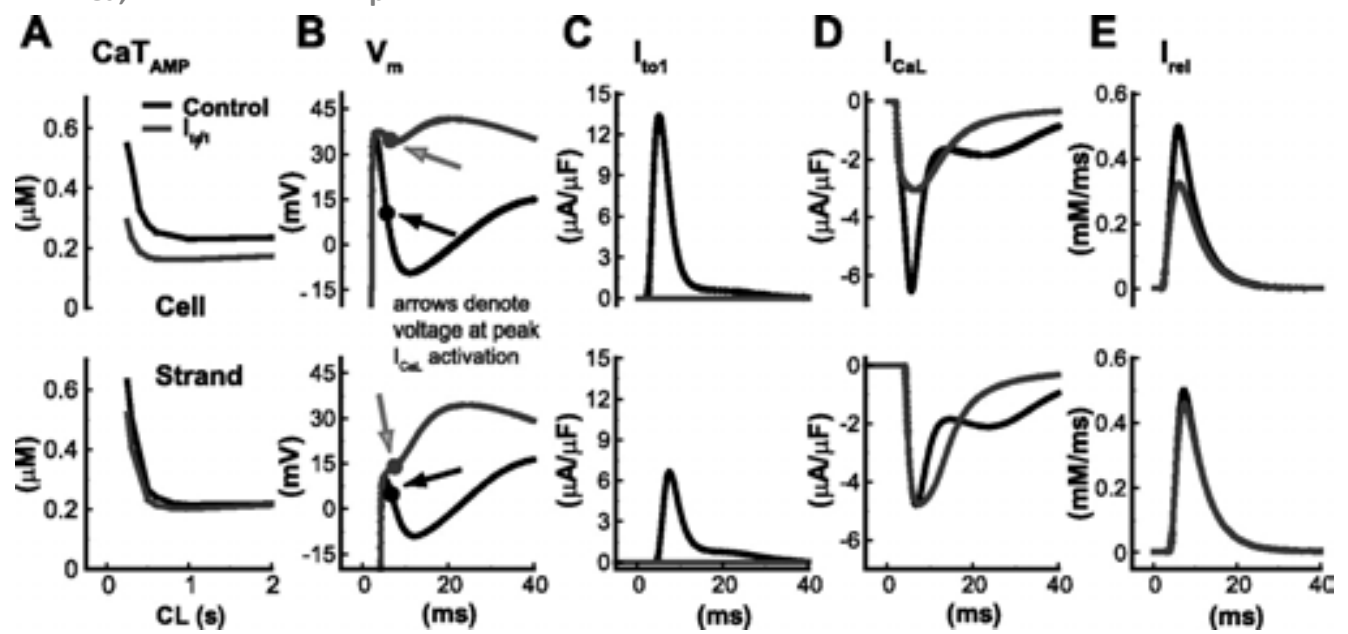

Figure 7. $\mathrm{CaT}_{\mathrm{AMP}}(\mathrm{A}), \mathrm{V}_{\mathrm{m}}(\mathrm{B}), \mathrm{I}_{\mathrm{to1}}(\mathrm{C}), \mathrm{I}_{\mathrm{Ca}, \mathrm{L}}(\mathrm{D})$, and $\mathrm{I}_{\mathrm{rel}}(\mathrm{E})$ in control and after $100 \% \mathrm{I}_{\text {to1 }}$ block in single-cell (top) and strand (bottom) simulations. In $B-E, C L_{S 1}=1 \mathrm{~s}$. Arrows in $B$ denote time and $V_{m}$ at peak $I_{C a, L}$. 
Experimental $^{44}$ and computational ${ }^{29}$ studies have suggested that $I_{\text {to1 }}$, by controlling phase 1 repolarization, is an important modulator of $\mathrm{I}_{\mathrm{Ca}, \mathrm{L}}$ activation and sarcoplasmic reticulum (SR) $\mathrm{Ca}^{2+}$ release. We examined differences in the effect of $\mathrm{I}_{\mathrm{to} 1}$ on $\mathrm{I}_{\mathrm{Ca}, \mathrm{L}}$ activation and $\mathrm{SR} \mathrm{Ca}^{2+}$ release in cell and strand simulations. Figure 7A shows that although blockade of $\mathrm{I}_{\text {to1 }}$ leads to a significant reduction in $\mathrm{CaT}_{\mathrm{Amp}}$ in cell simulations, reduction of $\mathrm{CaT}_{\mathrm{Amp}}$ after $\mathrm{I}_{\text {to1 }}$ blockade in tissue simulations is minimal. The interplay between membrane potential $\left(\mathrm{V}_{\mathrm{m}}\right), \mathrm{I}_{\mathrm{to} 1}, \mathrm{I}_{\mathrm{Ca}, \mathrm{L}}$, and $\mathrm{SR} \mathrm{Ca}^{2+}$ release in a cell and a strand is examined in Figure 7, B-E. We note several important differences between simulated APs in the strand and in the isolated cell. These unique features of the strand AP include 1) reduced peak upstroke voltage (Figure 7B), 2) reduced $I_{\text {to1 }}$ activation (Figure 7C), and 3) minimal dependence of peak $I_{C a, L}$ on $I_{t o 1}$ activation (Figure 7D). Reduced peak upstroke voltage in the strand leads to a reduction in peak $I_{\text {to1 }}$ due to the approximately linear dependence of $I_{\text {to1 }}$ activation on $V_{m}$. This reduction in $\mathrm{I}_{\text {to } 1}$ activation leads to a reduction in phase 0 repolarization. Arrows in Figure 7B denote $V_{m}$ at the peak of $I_{C a, L}$ activation in a cell and a strand, respectively. In cell simulations, a large $I_{\text {to } 1}$ repolarizes $V_{m}$ toward the peak of the $I_{C a, L} I-V$ relationship (Figure $7 B$ ), promoting $I_{C a, L}$ activation and $S R \mathrm{Ca}^{2+}$ release. When $\mathrm{I}_{\mathrm{to1}}$ is blocked in the cell, $\mathrm{I}_{\mathrm{Ca}, \mathrm{L}}$ activation occurs at a $\mathrm{V}_{\mathrm{m}}$ that is far from the peak of the I-V curve (Figure 7B), decreasing peak $I_{C a, L}$ and $S R \mathrm{Ca}^{2+}$ release. For a propagating action potential, however, increased load decreases peak up-stroke voltage, so that it is near the peak of the $I_{C a, L} I-V$ curve (Figure 7B, $+10 \mathrm{mV}$ ). Maximal $I_{C a, L}$ activation occurs shortly after the peak upstroke $V_{m}$, such that the depth of the phase 1 notch plays little role in determining $V_{m}$ as $I_{C a, L}$ is peaking. Consequently, blockade of $I_{\text {to1 }}$ in the strand has little effect on peak $I_{\mathrm{Ca}_{\mathrm{L}} \mathrm{L}}$ (Figure 7D) and SR Ca ${ }^{2+}$ release. Furthermore, reduced phase 1 repolarization due to reduced $I_{\text {to1 }}$ activation also suggests a diminished importance of $\mathrm{I}_{\mathrm{to} 1}$ in the strand relative to the cell.

\section{$I_{\text {to1 }}$ and APD restitution}

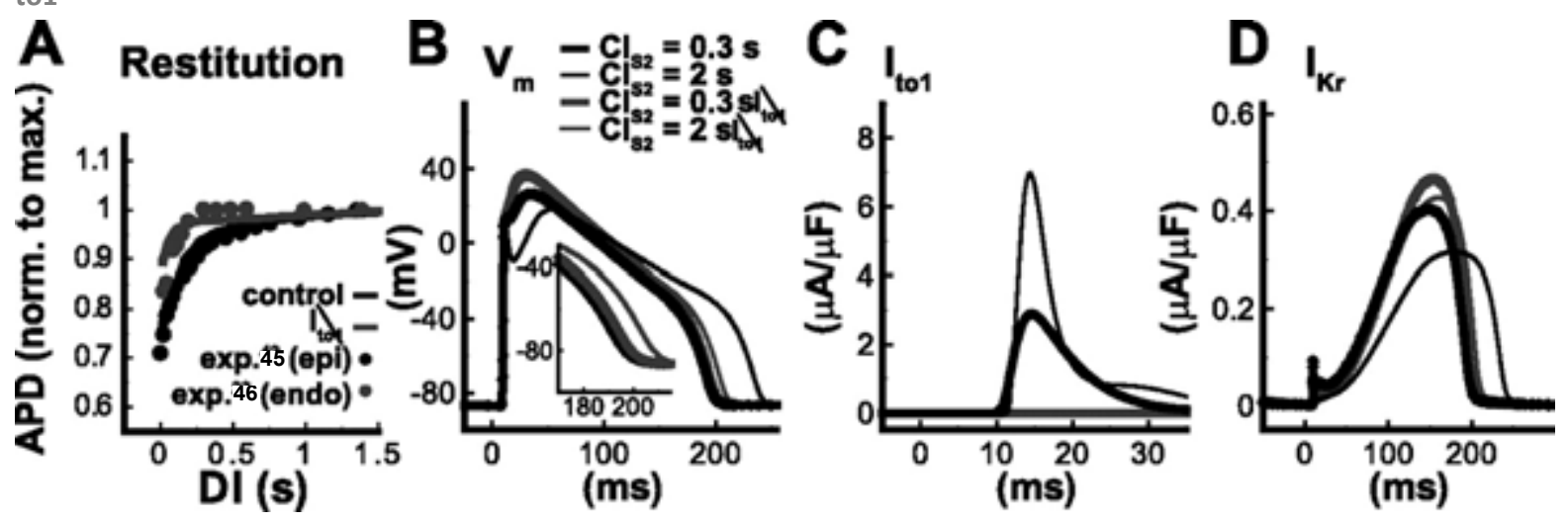

Figure 8. A. APD (normalized to maximum) as a function of diastolic interval (DI) during restitution at $\mathrm{CL}_{\mathrm{S}_{1}}=2 \mathrm{~s}$ in control and after $100 \% \mathrm{I}_{\text {to }}$ block in strand simulations. Results are compared with data from canine epicardial ${ }^{45}$ and endocardial ${ }^{46}$ tissue experiments. B-D. $V_{m}, I_{t o}$, and $I_{k r}$ during APD restitution at $\mathrm{S} 2$ coupling intervals $\left(\mathrm{Cl}_{\mathrm{S}_{2}}\right)$ of 0.3 and $2 \mathrm{~s}$ in control and after $\mathrm{I}_{\text {to }}$ block in the strand. Inset in B shows very similar AP after $\mathrm{I}_{\text {to }}$ block at short CIS2 (thick gray trace) and long $\mathrm{Cl}_{52}$ (thin gray trace). 
Although our results suggest a limited role for $\mathrm{I}_{\text {to1 }}$ in regulation of peak $\mathrm{I}_{\mathrm{Ca}, \mathrm{L}}$ and $\mathrm{SR} \mathrm{Ca}{ }^{2+}$ release in multicellular strands, $I_{\text {to1 }}$ was found to play an important role in APD restitution. Figure 8A shows that the time course of APD restitution is consistent with experiments in epicardial tissue. ${ }^{45}$ (See

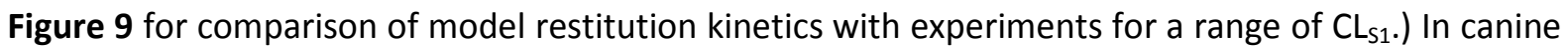
epicardium, APD gradually increases as DI increases beyond $0.3 \mathrm{~s}$. After $100 \% \mathrm{I}_{\text {to } 1}$ block, the increase in APD with increasing DI is more abrupt, as observed in canine endocardium, ${ }^{46}$ consistent with the absence of $I_{\text {to1 }}$ in this tissue. Figure 8, B-D, examines the role of $I_{\text {to1 }}$ in APD restitution time course. At short $\mathrm{Cl}_{\mathrm{s} 2}, \mathrm{I}_{\text {to1 }}$ has not fully recovered from inactivation, resulting in minimal phase 1 repolarization (Figure 8B). As $\mathrm{Cl}_{\mathrm{s} 2}$ increases, notch depth increases as $\mathrm{I}_{\text {to1 }}$ recovers from inactivation. Since activation of the fast component of the delayed rectifier $\mathrm{K}^{+}$current $\left(\mathrm{I}_{\mathrm{Kr}}\right)$ is slower at more negative voltages, increasing notch depth slows $I_{k r}$ activation (Figure 8D). The gradual increase in APD during restitution follows the slow time course of $\mathrm{I}_{\text {to1 }}$ recovery and the suppression of $\mathrm{I}_{\mathrm{Kr}}$ activation due to increasing notch depth. When $\mathrm{I}_{\text {to1 }}$ is absent, increase in $\mathrm{Cl}_{\mathrm{s} 2}$ beyond $0.3 \mathrm{~s}$ results in minimal phase 1 voltage change (Figure $\mathbf{8 B}$, overlapping gray traces), constant $I_{\mathrm{kr}}$ activation, and minimal APD increase (Figure 8B inset, gray traces). This small increase in APD at $\mathrm{Cl}_{\mathrm{S2}}=0.3 \mathrm{~s}$ results in a restitution curve resembling that recorded in endocardium. ${ }^{46}$

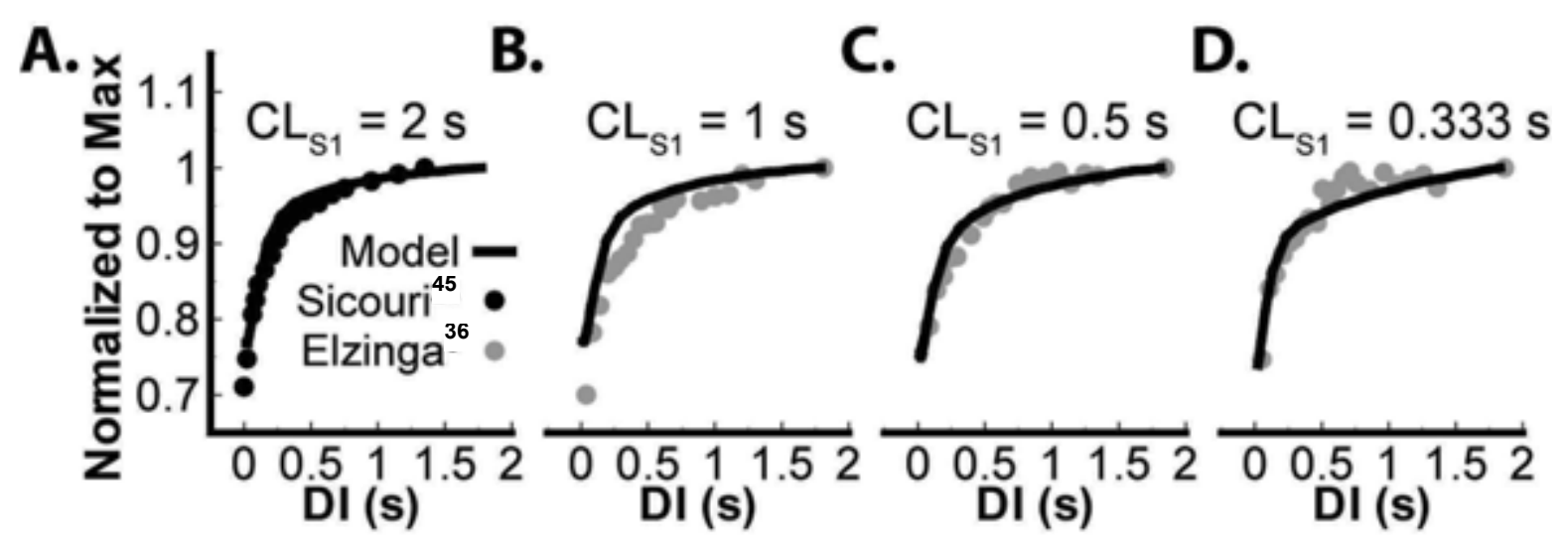

Figure 9. Model and experimental APD (normalized to maximum) as a function of diastolic interval (DI) during restitution at (left-to-right) $(A) C_{s 1}=2 \mathrm{~s}$, (B) $C_{s 1}=1 \mathrm{~s}$, (C) $C_{s 1}=0.5 \mathrm{~s}$ and (D) $C_{L_{s 1}}=0.333$ s. Experimental data in Panel $A$ are from canine left ventricle, ${ }^{45}$ while data in Panels B-D are from canine right ventricle. ${ }^{36}$ The time course of APD restitution in the model strand is consistent with experiments in canine epicardial tissue ${ }^{36,45}$ for a range of $\mathrm{CL}_{s 1}$. Comparison at $\mathrm{CL}_{s 1}=2 \mathrm{~s}$ is to left ventricular (LV) epicardial tissue experiment, ${ }^{36,45}$ while $\mathrm{CL}_{\mathrm{s} 1}=1 \mathrm{~s}, 0.5 \mathrm{~s}$ and $0.333 \mathrm{~s}$ is to right ventricular (RV) epicardial tissue experiment ${ }^{36,45}$ because left ventricular data at these rates are not available. Model restitution kinetics show a modest dependence on $\mathrm{CL}_{S_{1}}$, in part due to the effects of rate dependent $\mathrm{SR} \mathrm{Ca}{ }^{2+}$ loading and $\mathrm{Ca}^{2+}$ release on APD, but this phenomenon was not addressed in the present study. Differences in restitution kinetics between the model and the RV experiments are relatively small and may be due to differences in LV and $\mathrm{RV} \mathrm{I}_{\text {to1 }}$ expression and kinetics, ${ }^{33} \mathrm{Ca}^{2+}$ handling or other heterogeneities. 


\section{$I_{\text {Ks }}$ and APD adaptation}

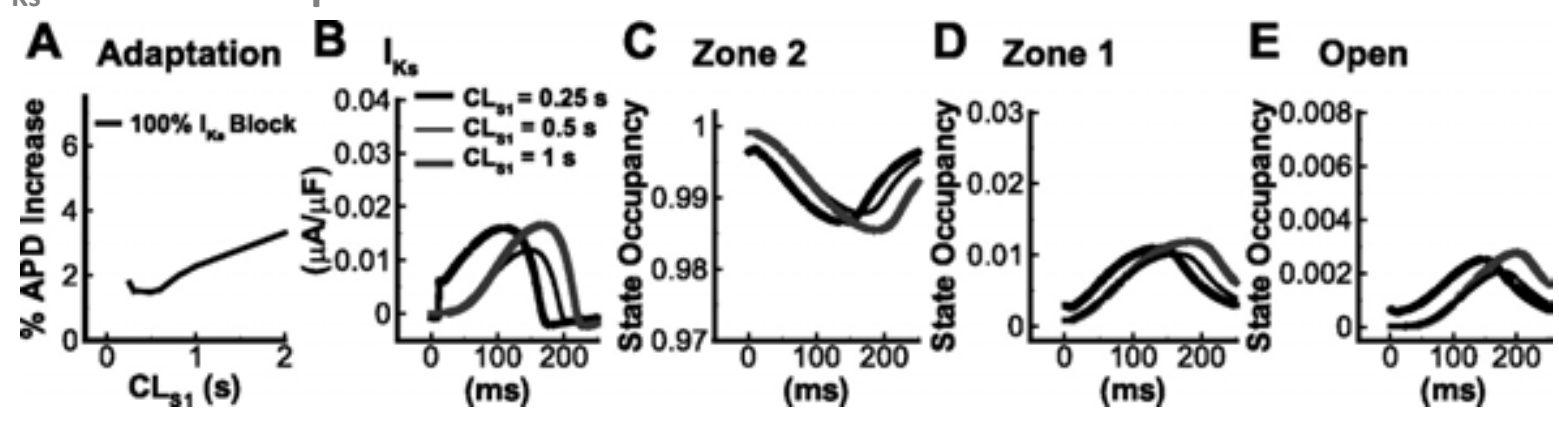

Figure 10. A. percent increase in steady-state APD after $100 \% \mathrm{I}_{\mathrm{Ks}}$ block as a function of $\mathrm{CL}_{\mathrm{S} 1}$. B-E. $\mathrm{I}_{\mathrm{Ks}}$ current density and state occupancy of zone 2 , zone 1 , and open state at steady-state for $\mathrm{CL}_{s 1}=0.25$, 0.5 , and $1 \mathrm{~s}$.

The role of $I_{\mathrm{Ks}}$ in canine APD adaptation is examined in Figure 10. As shown in Figure 10A, 100\% block of $I_{\mathrm{Ks}}$ leads to a modest prolongation of APD at steady state. Percent APD prolongation after $I_{\mathrm{Ks}}$ blockade decreases as $\mathrm{CL}$ decreases ("use-dependent" block), except at short $\mathrm{CL}$, where percent prolongation begins to increase again. The biphasic effect of $I_{\mathrm{Ks}}$ block on APD is consistent with the rate dependence of $I_{\mathrm{Ks}}$ activation shown in Figure 10B. $I_{\mathrm{Ks}}$ activation is smallest at $\mathrm{CL}_{\mathrm{s} 1}=0.5 \mathrm{~s}$, corresponding to the smallest percent prolongation of APD after $I_{\mathrm{Ks}}$ block in Figure 10A. $I_{\mathrm{Ks}}$ activation at $C_{S_{11}}=0.25$ and $1 \mathrm{~s}$ is increased relative to $C_{L_{11}}=0.5 \mathrm{~s}$, leading to a relative increase in percent $A P D$ prolongation after $I_{\mathrm{Ks}}$ block at these rates. Figure 10, C-E, examines the rate dependence of $I_{\mathrm{Ks}}$ state occupancy, following previously published analysis in the guinea pig. ${ }^{22}$ The 17 -state $I_{\mathrm{ks}}$ model (Figure 11) can be divided into two open states, five zone 1 closed states that can activate rapidly, and 10 "deep" zone 2 closed states, which require slow transitions to zone 1 before activation is possible. At $\mathrm{CL}_{\mathrm{s} 1}=0.5$ and $1 \mathrm{~s}$, all channels reside in zone 2 at the start of the AP (Figure 10C) and activate slowly upon depolarization (Figure 10B). Slow activation from zone 2 and small peak density in the dog dictate that $\mathrm{I}_{\mathrm{Ks}}$ plays a limited role in AP repolarization at longer $\mathrm{CL}$. At $\mathrm{CL}_{\mathrm{S} 1}=0.25 \mathrm{~s}$, the $\mathrm{DI}$ is insufficient for activated channels to return to zone 2 . Channels activated during the previous beat remain in zone 1 (Figure 10D) and the open state (Figure 10E) as the next stimulus is applied, resulting in more rapid channel activation and an increased role for $I_{\mathrm{Ks}}$ in APD shortening at fast rate.

\section{$I_{\mathrm{Ks}}$ and APD restitution}

We also examined the role of $I_{\mathrm{Ks}}$ in APD restitution (Figure 12). Simulation results show that the percent increase in APD after $100 \% \mathrm{I}_{\mathrm{Ks}}$ block is largest at the shortest $\mathrm{Cl}_{\mathrm{S} 2}$ (Figure 12B). At $\mathrm{Cl}_{\mathrm{S2}}=0.26$ and $0.3 \mathrm{~s}$, a significant fraction of channels activated during the previous AP remain in the open state (Figure 12D), while an additional fraction occupies zone 1 (Figure 12F). When a premature stimulus is applied, channels that are already open and those moving rapidly from zone 1 to the open state combine to yield prominent $I_{\mathrm{Ks}}$ activation (Figure 12C). Peak activation of $\mathrm{I}_{\mathrm{Ks}}$ at $\mathrm{Cl}_{\mathrm{S} 2}=0.26 \mathrm{~s}$ is markedly increased relative to peak density during the $\mathrm{S} 1$ beat. This prominent $\mathrm{I}_{\mathrm{Ks}}$ activation due to accumulation in the open state and zone 1 is required to achieve APD shortening at short DI consistent with experimental results. ${ }^{45} \mathrm{APD}$ at $\mathrm{Cl}_{\mathrm{s} 2}=0.26 \mathrm{~s}$ is increased by $+7.5 \%$ after $\mathrm{I}_{\mathrm{ks}}$ block. As DI increases, channels move from zone 1 and the open state to zone 2, resulting in less prominent $I_{\mathrm{Ks}}$ activation for more mature stimuli and reduced (+2.5\%) APD prolongation after $\mathrm{I}_{\mathrm{Ks}}$ block. 
First voltage sensor transition $\rightarrow$

$$
\begin{aligned}
& \mathrm{C}_{1} \underset{\beta}{\stackrel{4 \alpha}{\rightleftarrows}} \mathrm{C}_{2} \underset{2 \beta}{\stackrel{3 \alpha}{\rightleftarrows}} \mathrm{C}_{3} \underset{3 \beta}{\stackrel{2 \alpha}{\rightleftarrows}} \mathrm{C}_{4} \underset{4 \beta}{\stackrel{\alpha}{\rightleftarrows}} \mathrm{C}_{5}
\end{aligned}
$$

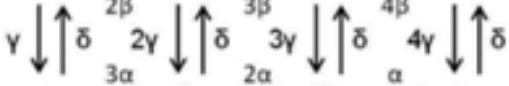

$$
\begin{aligned}
& \mathrm{C}_{6} \underset{\beta}{\stackrel{3 \alpha}{\rightleftarrows}} \mathrm{C}_{7} \underset{2 \beta}{\stackrel{2 \alpha}{\rightleftarrows}} \mathrm{C}_{8} \underset{3 \beta}{\stackrel{\alpha}{\rightleftarrows}} \mathrm{C}_{9} \\
& \gamma \downarrow \uparrow 2 \delta_{2 \alpha}^{2 \beta} 2 \gamma \mid \uparrow 2 \delta_{\alpha}^{3 \beta} 3 \gamma \downarrow \uparrow 2 \delta \\
& \mathrm{C}_{10} \underset{\beta}{\stackrel{2 \alpha}{\rightleftarrows}} \mathrm{C}_{11} \underset{2 \beta}{\stackrel{\alpha}{\rightleftarrows}} \mathrm{C}_{1} \\
& \gamma\left|\uparrow 3{ }^{2 \beta} 2 \gamma\right| \uparrow 3 \delta \\
& \begin{aligned}
C_{13} \stackrel{\alpha}{\stackrel{\alpha}{\rightleftarrows}} C_{14} \\
\gamma \mid \uparrow 4 \delta \\
\varepsilon_{15}
\end{aligned} \\
& \theta \downarrow \uparrow \eta \\
& \mathrm{O}_{2} \underset{\psi}{\stackrel{\theta}{\rightleftarrows}} \mathrm{O}_{1}
\end{aligned}
$$

Figure 11. $I_{\mathrm{Ks}}$ Markov state diagram.

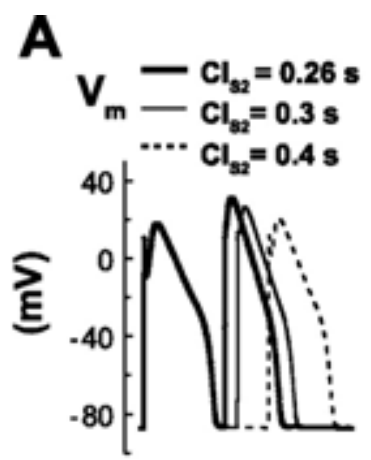

B
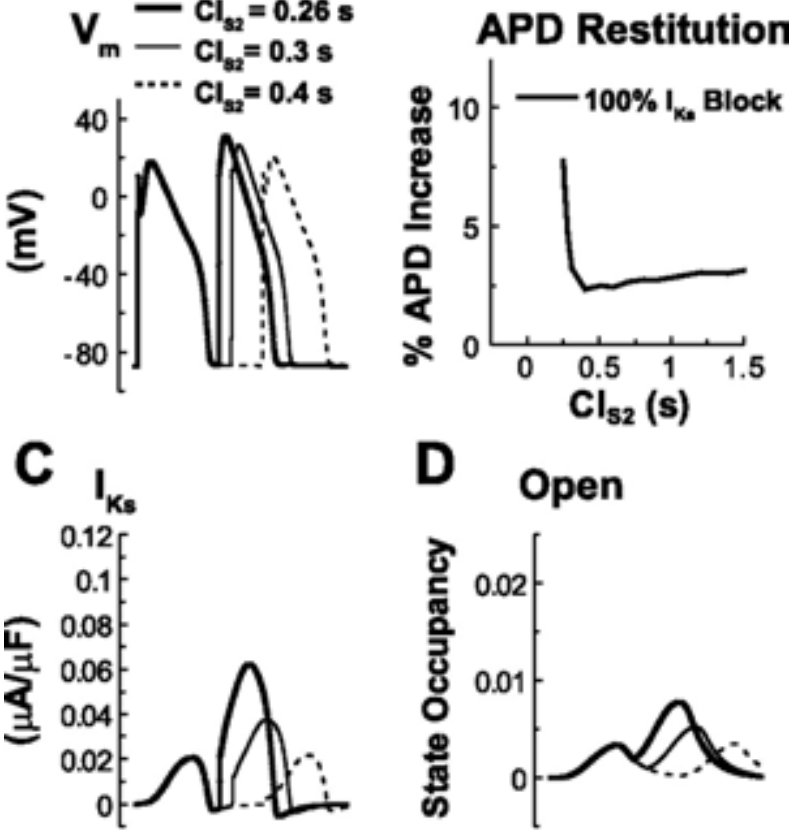

D Open
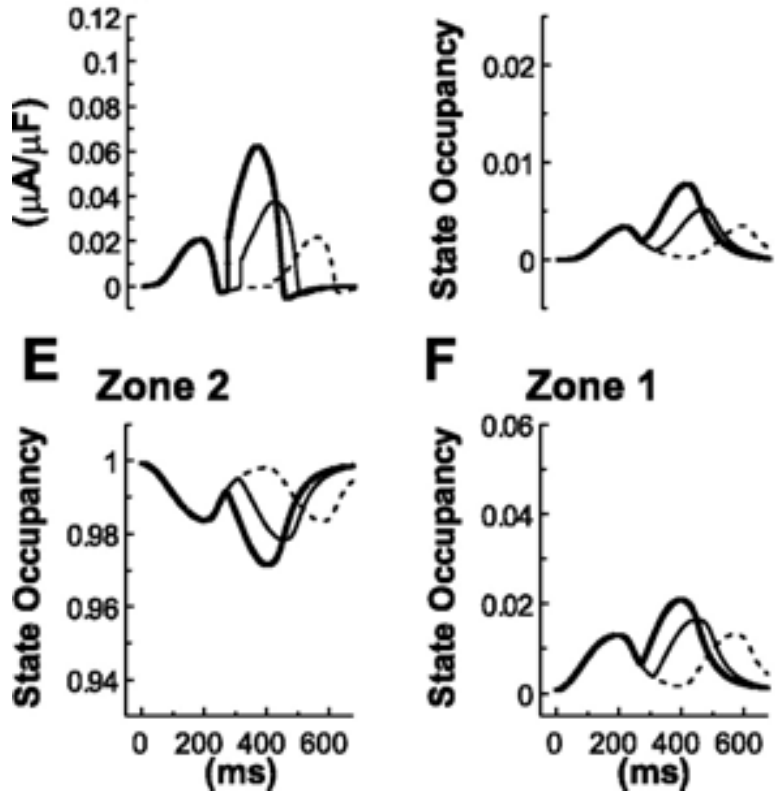
Figure 12. A. premature APs at $\mathrm{S} 2$ coupling intervals $\left(\mathrm{Cl}_{\mathrm{s}_{2}}\right)$ of $0.26,0.3$, and $0.4 \mathrm{~s}$ for $\mathrm{CL}_{\mathrm{s} 1}=2 \mathrm{~s}$. B. percent increase after $100 \% I_{\mathrm{Ks}}$ block as a function of coupling interval. C-F. $I_{\mathrm{Ks}}$ activation and occupancy of open state, zone 2 , and zone 1 for restitution protocol in A.

\section{APD accommodation}
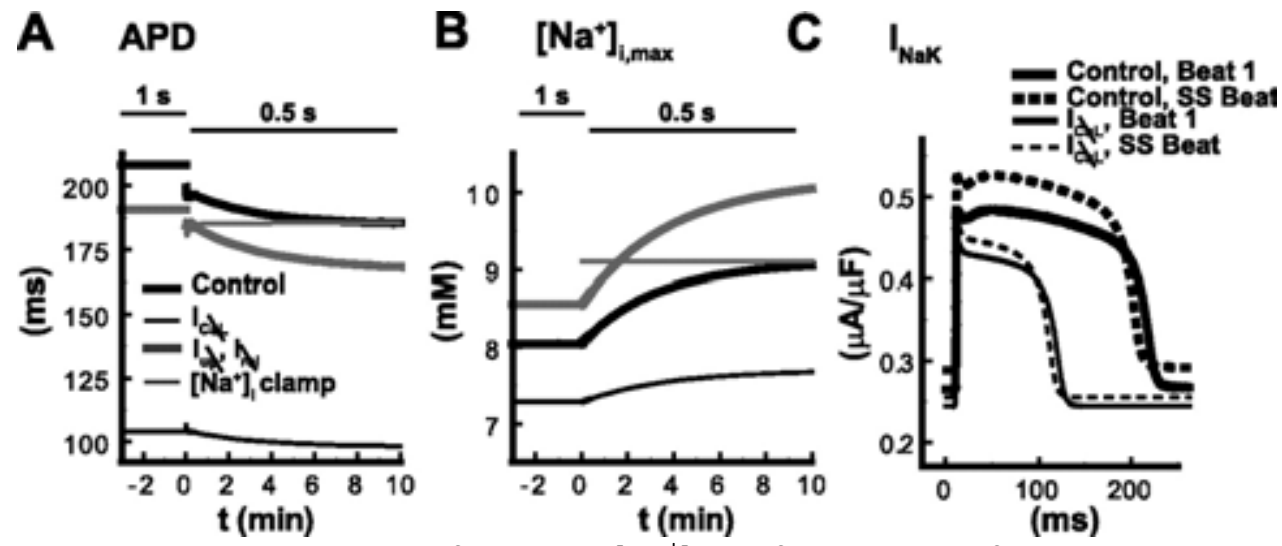

Figure 13. $A$ and $B$. accommodation of $A P D$ and $\left[\mathrm{Na}^{+}\right]_{, \text {, max }}$ after a change from $\mathrm{CL}=1 \mathrm{~s}$ to $\mathrm{CL}=0.5 \mathrm{~s}$ in control, after $\mathrm{I}_{\mathrm{Ca}, \mathrm{L}}$ block, after block of SR $\mathrm{Ca}^{2+}$ release $\left(\mathrm{I}_{\text {rel }}\right)$ and uptake $\left(\mathrm{I}_{\mathrm{up}}\right)$, and with $\left[\mathrm{Na}^{+}\right]_{\mathrm{i}}$ clamped to its steady-state value at $\mathrm{CL}=0.5 \mathrm{~s}$. $\mathrm{C}$. $\mathrm{I}_{\mathrm{NaK}}$ for the $1 \mathrm{st}$ beat and a steady-state (SS) beat after change from $C L=1 \mathrm{~s}$ to $\mathrm{CL}=0.5 \mathrm{~s}$.

We sought to gain insight into the ionic mechanism of APD accommodation by examining underlying ionic currents and concentrations. The transition from steady state at $\mathrm{CL}=1 \mathrm{~s}$ to steady state at $\mathrm{CL}=$ $0.5 \mathrm{~s}$ is examined in Figure 13. APD accommodation exhibits several properties consistent with experimental observations. Equilibration of APD after a change in CL occurs after several minutes of pacing, consistent with experiments in canine ventricular tissue. ${ }^{37}$ Consistent with the effect of nisoldipine on rabbit ventricular myocytes, ${ }^{47} 100 \%$ block of $I_{C a, L}$ in the model diminishes APD accommodation. In addition, accommodation persists after $100 \%$ block of SR $\mathrm{Ca}^{2+}$ uptake (uptake current) and release (release current), consistent with effects of ryanodine and thapsigargin on rabbit myocytes. ${ }^{47}$ Figure 13B shows changes in $\left[\mathrm{Na}^{+}\right]_{i}$ during APD accommodation. In control and when $S R$ release and uptake are blocked, accumulation of $\left[\mathrm{Na}^{+}\right]_{i}$ occurs with a time course similar to that of APD shortening. When $\mathrm{I}_{\mathrm{C}, \mathrm{L}}$ is blocked, APD shortening and $\left[\mathrm{Na}^{+}\right]_{\mathrm{i}}$ accumulation are diminished. When $\left[\mathrm{Na}^{+}\right]_{i}$ is clamped to its steady-state value at $\mathrm{CL}=0.5 \mathrm{~s}$, APD accommodation is eliminated. Figure ${ }^{13 C}$ demonstrates that, in control, $\left[\mathrm{Na}^{+}\right]_{i}$ accumulation leads to a large increase in outward $I_{\text {Nak }}$ from the first beat to the steady-state beat after a change in CL. After $I_{\mathrm{Ca}, \mathrm{L}}$ block, there is a smaller difference in $I_{\mathrm{NaK}}$ from the first beat to the steady-state beat. Collectively, these simulations suggest that $I_{C a, L}$ is responsible for the increases in $\left[\mathrm{Na}^{+}\right]_{i}$ and outward $I_{\text {Nak }}$ that underlie APD accommodation. 

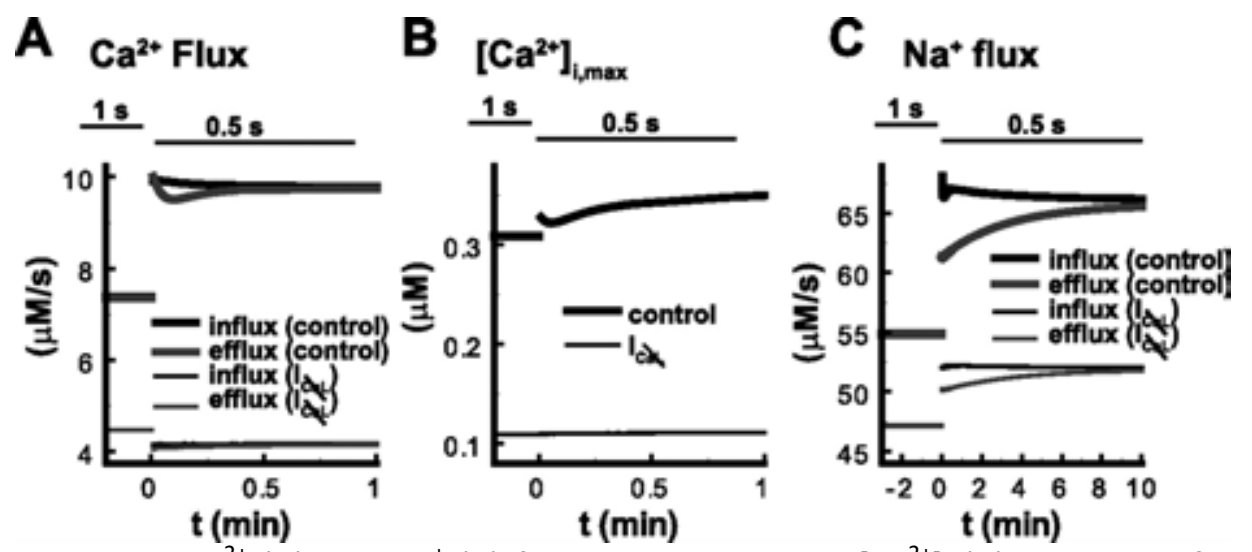

Figure 14. Integrated $\mathrm{Ca}^{2+}(\mathrm{A})$ and $\mathrm{Na}^{+}(\mathrm{C})$ fluxes and maximum $\left[\mathrm{Ca}^{2+}\right]_{\mathrm{i}}(\mathrm{B})$ calculated for each beat during transition from steady state at $C L=1 \mathrm{~s}$ to steady state at $\mathrm{CL}=0.5 \mathrm{~s}$. Integrated sarcolemmal $\mathrm{Ca}^{2+}$ influx was calculated as $\mathrm{I}_{\mathrm{Ca}, \mathrm{L}}+\mathrm{I}_{\mathrm{Cab}}$ and efflux as $\mathrm{I}_{\mathrm{NaCa}}+\mathrm{I}_{\mathrm{pca}}$. Integrated $\mathrm{Na}^{+}$influx was calculated as $I_{\mathrm{NaCa}}+I_{\mathrm{Na}}+I_{\mathrm{NaL}}+I_{\mathrm{Nab}}+\mathrm{CTNaCl}$ and $\mathrm{Na}^{+}$efflux as $I_{\mathrm{NaK}}$.

To understand the link between $\mathrm{I}_{\mathrm{Ca}, \mathrm{L}}\left[\mathrm{Na}^{+}\right]_{\mathrm{i}}$, and accommodation, we examined the sarcolemmal $\mathrm{Ca}^{2+}$ and $\mathrm{Na}^{+}$flux $(\mu \mathrm{M} / \mathrm{s})$ via currents, pumps, and exchangers after a change from $\mathrm{CL}=1 \mathrm{~s}$ to $\mathrm{CL}=0.5 \mathrm{~s}$. Fluxes were calculated at steady state at $\mathrm{CL}=1 \mathrm{~s}$ and for each subsequent beat after a change to $\mathrm{CL}$ $=0.5 \mathrm{~s}$. At steady-state $\mathrm{CL}=1 \mathrm{~s}, \mathrm{Ca}^{2+}$ influx (primarily $\mathrm{I}_{\mathrm{Ca}, \mathrm{L}}$ ) and efflux (primarily $\mathrm{I}_{\mathrm{NaCa}}$ ) are balanced (Figure 14A). After a change to $C L=0.5 \mathrm{~s}$, the shorter $\mathrm{DI}$ does not provide sufficient time for $I_{\mathrm{NaCa}}$ to extrude $\mathrm{Ca}^{2+}$ that entered via $\mathrm{I}_{\mathrm{Ca}, \mathrm{L}}$. Excess $\mathrm{Ca}^{2+}$ influx leads to $\left[\mathrm{Ca}^{2+}\right]_{i}$ accumulation after the first beat and with continued pacing at $\mathrm{CL}=0.5 \mathrm{~s}$ (Figure 14B). $\left[\mathrm{Ca}^{2+}\right]_{\mathrm{i}}$ accumulation provides feedback (autoregulation ${ }^{48}$ ) by decreasing $\left[\mathrm{Ca}^{2+}\right]_{\mathrm{i}}$ influx (through $\mathrm{Ca}^{2+}$-dependent inactivation of $\mathrm{I}_{\mathrm{Ca}, \mathrm{L}} \mathrm{L}$ ) and increasing $\left[\mathrm{Ca}^{2+}\right]_{i}$ efflux (through forward-mode $I_{\mathrm{NaC}}$ ). This feedback promotes decreased net $\mathrm{Ca}^{2+}$ entry per cycle, so that $\left[\mathrm{Ca}^{2+}\right]_{i}$ approaches equilibrium within the $1^{\text {st }}$ minute of pacing at the new $\mathrm{CL}$.

$\mathrm{Na}^{+}$influx exceeds efflux after change from $\mathrm{CL}_{s 1}=1 \mathrm{~s}$ to $\mathrm{CL}_{\mathrm{s} 2}=0.5 \mathrm{~s}$ (Figure 15C). As with $\mathrm{Ca}^{2+}$, the shortened DI provides insufficient time for complete extrusion of $\mathrm{Na}^{+}$that entered during the AP, primarily via forward-mode $I_{\mathrm{NaCa}}$ and the fast $\mathrm{Na}^{+}$current $\left(\mathrm{I}_{\mathrm{Na}}\right)$. Increased forward-mode $\mathrm{I}_{\mathrm{NaCa}}$ as $\left[\mathrm{Ca}^{2+}\right]_{\mathrm{i}}$ accumulates further increases $\mathrm{Na}^{+}$influx. The imbalance of $\mathrm{Na}^{+}$influx and efflux drives $\left[\mathrm{Na}^{+}\right]_{i}$ accumulation (Figure 13B) until $\mathrm{Na}^{+}$efflux via $\mathrm{I}_{\mathrm{Nak}}$ increases to match influx after minutes of pacing (Figure 14C). The time course for equilibration of $\mathrm{Na}^{+}$influx and efflux in our simulations (on the order of minutes) agrees with the slow time course of $\mathrm{Na}^{+}$accumulation seen experimentally. ${ }^{16,49}$ The increasingly outward $I_{\text {Nak }}$ as $\mathrm{Na}^{+}$influx and efflux approach equilibrium is the primary cause of APD shortening during accommodation. This ionic mechanism by which $I_{C a, L}$ drives $\left[\mathrm{Na}^{+}\right]_{i}$ accumulation and $I_{\mathrm{NaK}}$ increase via $I_{\mathrm{NaCa}}$ is consistent with effects of nisoldipine and ryanodine/thapsigargin on rabbit ventricular myocytes. ${ }^{47}$ Block of $\mathrm{I}_{\mathrm{Ca}, \mathrm{L}}$ (nisoldipine) eliminates a critical driving force for $\left[\mathrm{Na}^{+}\right]_{i}$ accumulation (greater forward-mode $\mathrm{I}_{\mathrm{NaCa}}$, which promotes $\mathrm{Ca}^{2+}$ extrusion and $\mathrm{Na}^{+}$entry). After $\mathrm{I}_{\mathrm{Ca}, \mathrm{L}}$ blockade, $\mathrm{Ca}^{2+}$ and $\mathrm{Na}^{+}$influx barely exceeds $\mathrm{Ca}^{2+}$ and $\mathrm{Na}^{+}$efflux after a change to $\mathrm{CL}=0.5 \mathrm{~s}$. $\left[\mathrm{Ca}^{2+}\right]_{\mathrm{i}}$ (Figure $14 \mathrm{~A}, \mathrm{~B}$ ) and $\left[\mathrm{Na}^{+}\right]_{\mathrm{i}}$ (Figure 13A,B) accumulation after $\mathrm{I}_{\mathrm{Ca}, \mathrm{L}}$ block are diminished, resulting in diminished APD accommodation. Block of $S R \mathrm{Ca}^{2+}$ release (ryanodine) and SR Ca ${ }^{2+}$ uptake (thapsigargin) eliminates SR Ca ${ }^{2+}$ cycling, but the mechanism of $\mathrm{I}_{\mathrm{Ca}, L^{-}}$ 
driven increase in $\left[\mathrm{Na}^{+}\right]_{i}$ remains intact. Although block of $\mathrm{SR}$ function eliminates $\mathrm{Ca}^{2+}$ transients, intact $I_{\mathrm{Ca}, \mathrm{L}}$ leads to an increase in cell $\left[\mathrm{Ca}^{2+}\right]_{\text {. }}$. Forward-mode $\mathrm{I}_{\mathrm{NaCa}}$ must increase to extrude $\mathrm{Ca}^{2+}$, and $\left[\mathrm{Na}^{+}\right]_{i}$ accumulation and APD accommodation persist.

\section{Dependence of APD restitution on pacing rate}
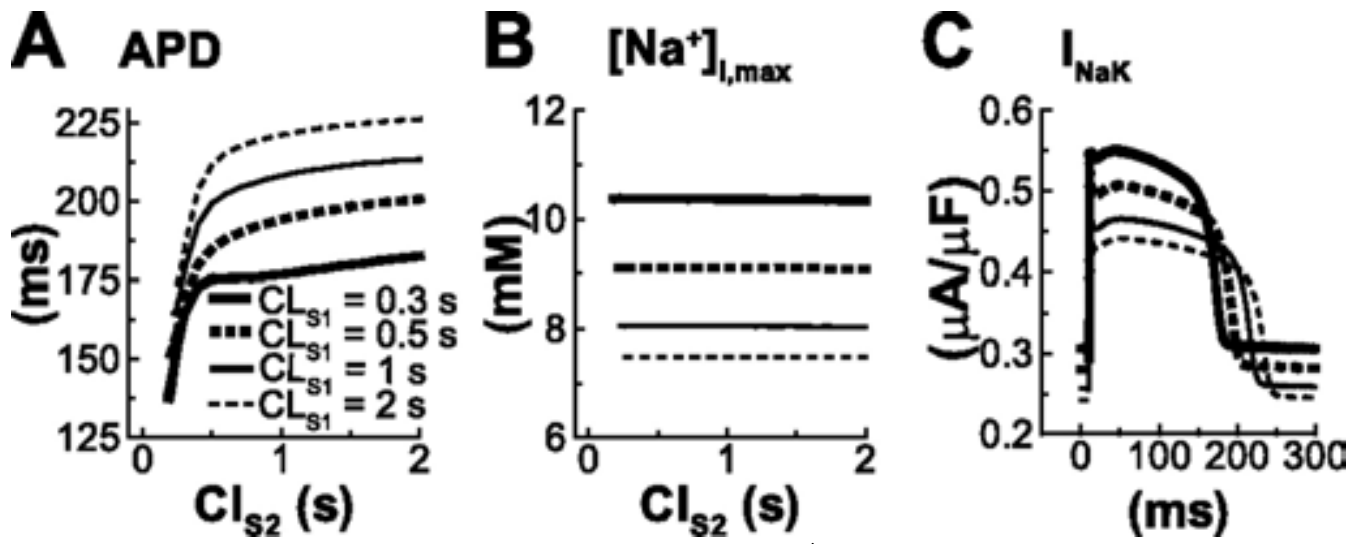

Figure 15. $A$ and $B$ : dependence of $A P D$ restitution and $\left[\mathrm{Na}^{+}\right]_{i, \max }$ on pacing rate $\left(\mathrm{CL}_{S_{1}}\right)$. C: dependence of $\mathrm{I}_{\mathrm{NaK}}$ on pacing rate $\left(\mathrm{CL}_{\mathrm{S} 1}\right)$ at constant $\mathrm{S} 2$ coupling interval $\left(\mathrm{Cl}_{\mathrm{S2}}=2 \mathrm{~s}\right)$.

Several experimental studies have shown that the APD restitution curve shifts to shorter APD as basic pacing $\mathrm{CL}$ decreases (shorter $\mathrm{CL}_{\mathrm{S} 1}$ ). $8,36,41,42$ Consistent with these experimental observations, our model reproduces the dependence of restitution on the rate of S1 pacing (Figure 15). These simulations also provide insight into the underlying ionic mechanism. Fast pacing (short $\mathrm{CL}_{s 1}$; Figure 15A) is accompanied by $\left[\mathrm{Na}^{+}\right]_{\mathrm{i}}$ accumulation (Figure 15B), as described above in the case of accommodation (Figure 13). $\left[\mathrm{Na}^{+}\right]_{i}$ accumulation, in turn, promotes an increase in repolarizing $I_{\mathrm{NaK}}$ (Figure 15C), which decreases APD, thereby shifting the APD restitution curve to shorter APDs.

\section{DISCUSSION}

The rate-dependent phenomena examined in this study are thought to play an important role in the dynamics of arrhythmia. ${ }^{50-52}$ The model presented here reproduces experimentally observed APD adaptation, restitution, and accommodation in cell and tissue. Although the rate dependence of model APD, CaT, and $\left[\mathrm{Na}^{+}\right]_{i}$ accumulation is similar in cell and strand, our simulation results show that the effects of $I_{\text {to1 }}$ block on ion accumulation differ. Other simulation results ${ }^{43}$ have suggested that APD restitution kinetics and the transition to APD alternans and more complex excitation patterns in cell and tissue also differ. Taken together, these results suggest that experimental findings in isolated myocytes should be extrapolated to the multicellular tissue with caution.

Recent studies have emphasized limitations in our mechanistic understanding of the rate dependence of APD. ${ }^{8}$ The detailed, physiologically based mathematical descriptions of critical ionic currents, pumps, and exchangers incorporated into the model lead to novel insight into underlying ionic mechanisms. Specific mechanistic insights generated by our study include the following. 1) $I_{\text {to1 }}$ 
potentiates $\mathrm{I}_{\mathrm{Ca}, \mathrm{L}}$ and $\mathrm{SR} \mathrm{Ca}{ }^{2+}$ release during early AP repolarization in isolated cells, but not in multicellular tissue. 2) $I_{\text {to } 1}$ plays an important role in APD restitution because of its slow recovery kinetics. As DI increases, $I_{\text {to1 }}$ recovery and phase 1 notch depth increase, suppressing $I_{\mathrm{kr}}$ activation and lengthening APD. 3) $I_{\mathrm{Ks}}$ plays a limited role in repolarization of paced APs but plays an important role in APD shortening for premature stimuli. 4) $I_{C a, L}$ plays a critical role in APD accommodation and memory. As a response to $\mathrm{Ca}^{2+}$ entry via $\mathrm{I}_{\mathrm{Ca}, \mathrm{L}}$ at fast rates, forward-mode $\mathrm{I}_{\mathrm{NaCa}}$ increases, leading to increased $\mathrm{Ca}^{2+}$ efflux and $\mathrm{Na}^{+}$influx. $\mathrm{Na}^{+}$accumulates and repolarizing $I_{\text {Nak }}$ increases, leading to APD shortening during accommodation. 5) $I_{C_{a},-}$-dependent increase in $\left[\mathrm{Na}^{+}\right]_{\mathrm{i}}$ and $I_{\text {Nak }}$ at fast rates is responsible for the shift in APD restitution curves toward shorter APD.

Previous experiments and simulation studies have suggested that $I_{\text {to1 }}$, by increasing phase 1 repolarization, increases peak $I_{C a, L}$ (the trigger for SR release) and, consequently, SR Ca ${ }^{2+}$ release. ${ }^{29,44}$ These studies showed enhanced $I_{C a, L}$ and $S R \mathrm{Ca}^{2+}$ release when phase 1 repolarization increases the $I_{C a, L}$ driving force. Although this scenario is relevant to an isolated, directly stimulated myocyte, model simulations indicate that it does not apply to the situation in vivo, where electrotonic loading from neighboring cells weakens the AP upstroke. Our simulations suggest that, for propagating APs, $I_{\text {to1 }}$-dependent phase 1 repolarization has little effect on peak $I_{C a, L}$ and SR $\mathrm{Ca}^{2+}$ release. In addition, simulations show that the weakened AP upstroke in the tissue decreases $I_{\text {to } 1}$ activation and phase 1 repolarization. $I_{\text {to1 }}$ has been accorded a role in conduction ${ }^{53,54}$ and in electrophysiological remodeling in various diseased states. ${ }^{13,55,56}$ Our results suggest that predictions of the effect of $I_{\text {to1 } 1}$ and $I_{\text {to } 1}$ block based on single-cell simulations and experiments may not apply to the in vivo situation. In contrast, model simulations predict that $I_{\text {to1 }}$ plays a significant role in APD restitution. The interplay between $I_{\mathrm{kr}}$ activation and the slow recovery kinetics of $I_{\text {to1 }}$ leads to a gradual increase in APD during restitution, whereas $I_{\text {to1 }}$ block results in a more abrupt APD increase. This result provides a mechanistic explanation for the correlation between notch depth and APD observed in canine ventricular tissue. ${ }^{46}$ The time course of APD restitution is thought to play an important role in the stability of ventricular arrhythmias ${ }^{51}$. Our results suggest that experimentally observed heterogeneity in $\mathrm{I}_{\text {to } 1}$ density and recovery kinetics ${ }^{57,58}$ may play a role in the stability of arrhythmias in different species, regions of tissue, or pathophysiological states. Canine experiments have shown transmural heterogeneity in $\mathrm{I}_{\mathrm{NaK}},{ }^{59} \mathrm{I}_{\mathrm{to} 1},{ }^{21} \mathrm{I}_{\mathrm{Ks}}{ }^{23}$ and $\mathrm{I}_{\mathrm{NaCa}}{ }^{60}$ The epicardial model accurately reproduces endocardial restitution kinetics after blocking only $I_{\text {to1 }}$, suggesting that $I_{\text {to1 }}$ is the dominant determinant of heterogeneity in restitution between the two cell types.

Experimental measurements of the effect of $I_{\mathrm{Ks}}$ block on APD in canine ventricle have ranged from near $0^{14}$ to as much as $30 \% .{ }^{61}$ Varro et al. ${ }^{62}$ report a modest prolongation (3-7\%) and biphasic $\mathrm{CL}$ dependence after chromanol block, consistent with our simulation results. Although the role of $I_{k_{s}}$ in repolarization remains controversial, LQT mutations linked to genes for $I_{\mathrm{Ks}} \alpha$ - and $\beta$-subunits ${ }^{63}$ argue for an important role in repolarization. Our simulations demonstrate that slow activation and relatively low density limit the role of $I_{K s}$ in AP repolarization at physiological heart rates in the absence of $\beta$-adrenergic stimulation, consistent with our previous modeling studies. ${ }^{3}$ Simulations with a detailed Markov model of $I_{\mathrm{Ks}}$ predict an important role for $I_{\mathrm{Ks}}$ in APD restitution. During deactivation, channels accumulate in closed states (zone 1), where rapid activation in response to a 
premature stimulus is possible, leading to an important role for $I_{K_{s}}$ in APD restitution at short DI. A role for $I_{\mathrm{Ks}}$ in APD shortening at fast $\mathrm{CL}_{\mathrm{S}_{1}}$ via accumulation in closed states has been shown in the guinea pig and humans ${ }^{22}$. Despite major differences between $I_{\mathrm{Ks}}$ density and kinetics in the guinea pig and dog, the mechanism for enhanced participation of $\mathrm{I}_{\mathrm{Ks}}$ at fast $\mathrm{CL}_{\mathrm{S} 1}$ during adaptation and at short DI during restitution are similar in the two species. Importantly, although large density in the guinea pig ensures an important role for $I_{\mathrm{Ks}}$ at all rates, we find that $I_{\mathrm{Ks}}$ plays a major role in canine restitution at short $\mathrm{DI}$, despite a limited role at longer physiological $\mathrm{CL}$. Although the effect of $\mathrm{I}_{\mathrm{Ks}}$ block on paced APs has been studied experimentally, no studies on the role of $\mathrm{I}_{\mathrm{Ks}}$ in restitution have been reported. $I_{\mathrm{Ks}}$ is also likely to play an important role in repolarization in the presence of $\beta$ adrenergic stimulation ${ }^{15}$ or for abnormally prolonged APD. ${ }^{62}$

Recent simulations have addressed the role of APD accommodation and short-term memory in the dynamics of arrhythmia. ${ }^{50,52}$ Simulations ${ }^{50}$ and experiments ${ }^{47}$ have emphasized uncertainty about the ionic mechanisms underlying this phenomenon. The results presented here predict a major role for $I_{C a, L}$-driven $\left[\mathrm{Na}^{+}\right]_{i}$ accumulation in this phenomenon. A role for $\left[\mathrm{Na}^{+}\right]_{i}$ and $I_{\mathrm{NaK}}$ in gradual APD changes during long-term pacing has been proposed. ${ }^{41,64,65}$ Similarities between the effect of block of $I_{C a, L}$ and $S R$ function in simulations and experiments ${ }^{47}$ support for this hypothesis. Although a similar time course of APD accommodation after a $\mathrm{CL}$ change has been observed in a range of preparations, APD accommodation in other species and preparations is often more rapid. ${ }^{9,47,66}$ This suggests that other mechanisms may play a role or, alternatively, that additional experiments are required to improve our understanding of equilibration of $\left[\mathrm{Na}^{+}\right]_{i}$ and $\left[\mathrm{Ca}^{2+}\right]_{i}$ during long-term pacing. A role for $I_{C a, L}$ in the dynamics of arrhythmia has been proposed by many investigators, usually through effects on APD restitution. ${ }^{41,67}$ Our simulations suggest that the effect of $\mathrm{I}_{\mathrm{Ca}, \mathrm{L}}$ on $\left[\mathrm{Na}^{+}\right]_{i}$ and APD accommodation should also be considered. The importance of $\left[\mathrm{Na}^{+}\right]_{i}$ in determining the rate dependence of APD also points to an important limitation of simplified computational models, where intracellular ion concentrations are often held constant.

The predictive value of multicellular simulations of arrhythmia depends on the ability of cell models to reproduce experimental observation. Despite variability across species, cell type, and preparation, APD adaptation, restitution, and accommodation are qualitatively similar in many species. Our model examines the ionic basis of these rate-dependent phenomena in the dog but may provide insight into ionic mechanisms in other species, including humans. In addition, our model will provide a valuable tool for linking initiation and maintenance of arrhythmia to underlying cellular processes and ionic currents through multicellular simulations.

\section{ACKNOWLEDGEMENTS}

We thank Dr. Gregory Faber, Thomas O'Hara, Dr. Leonid Livshitz, Namit Gaur, and Dr. Ali Nekouzadeh for helpful discussions. 


\section{GRANTS}

This research was supported by National Heart, Lung, and Blood Institute Merit Award R37-HL-33343 and Grant RO1-HL-49054 (to Y. Rudy) and by a Fondation Leducq Award to the Alliance for CaMK Signaling in Heart Disease (to Y. Rudy). Y. Rudy is the Fred Saigh Distinguished Professor at Washington University in St. Louis.

\section{REFERENCES}

1. Rudy Y, Silva JR. Computational biology in the study of cardiac ion channels and cell electrophysiology. Q Rev Biophys. 2006;39:57-116.

2. Fox JJ, McHarg JL, Gilmour RF, Jr. Ionic mechanism of electrical alternans. Am J Physiol Heart Circ Physiol. 2002;282:H516-530.

3. Hund TJ, Rudy Y. Rate dependence and regulation of action potential and calcium transient in a canine cardiac ventricular cell model. Circulation. 2004;110:3168-3174.

4. Winslow RL, Rice J, Jafri S, Marban E, O'Rourke B. Mechanisms of altered excitationcontraction coupling in canine tachycardia-induced heart failure, II: model studies. Circ Res. 1999;84:571-586.

5. Cabo C, Boyden PA. Electrical remodeling of the epicardial border zone in the canine infarcted heart: a computational analysis. Am J Physiol Heart Circ Physiol. 2003;284:H372384.

6. Hund TJ, Decker KF, Kanter E, Mohler PJ, Boyden PA, Schuessler RB, Yamada KA, Rudy Y. Role of activated CaMKII in abnormal calcium homeostasis and $I_{N a}$ remodeling after myocardial infarction: insights from mathematical modeling. J Mol Cell Cardiol. 2008;45:420-428.

7. Livshitz $L M$, Rudy $Y$. Regulation of $\mathrm{Ca}^{2+}$ and electrical alternans in cardiac myocytes: role of CAMKII and repolarizing currents. Am J Physiol Heart Circ Physiol. 2007;292:H2854-2866.

8. Cherry EM, Fenton FH. A tale of two dogs: analyzing two models of canine ventricular electrophysiology. Am J Physiol Heart Circ Physiol. 2007;292:H43-55.

9. Franz MR, Swerdlow CD, Liem LB, Schaefer J. Cycle length dependence of human action potential duration in vivo. Effects of single extrastimuli, sudden sustained rate acceleration and deceleration, and different steady-state frequencies. J Clin Invest. 1988;82:972-979.

10. Watanabe MA, Koller ML. Mathematical analysis of dynamics of cardiac memory and accommodation: theory and experiment. Am J Physiol Heart Circ Physiol. 2002;282:H15341547.

11. Rubart M, Lopshire JC, Fineberg NS, Zipes DP. Changes in left ventricular repolarization and ion channel currents following a transient rate increase superimposed on bradycardia in anesthetized dogs. J Cardiovasc Electrophysiol. 2000;11:652-664.

12. Szabó G, Szentandrássy N, Bíró T, Tóth BI, Czifra G, Magyar J, Bányász T, Varró A, Kovács L, Nánási PP. Asymmetrical distribution of ion channels in canine and human left-ventricular wall: epicardium versus midmyocardium. Pflugers Arch. 2005;450:307-316.

13. Lue WM, Boyden PA. Abnormal electrical properties of myocytes from chronically infarcted canine heart. Alterations in Vmax and the transient outward current. Circulation. 1992;85:1175-1188.

14. Stengl M, Volders PGA, Thomsen MB, Spätjens RLHMG, Sipido KR, Vos MA. Accumulation of slowly activating delayed rectifier potassium current $\mathrm{I}_{\mathrm{Ks}}$ in canine ventricular myocytes. $J$ Physiol. 2003;551:777-786.

15. Volders PGA, StengI M, van Opstal JM, Gerlach U, Spätjens RL, Beekman JD, Sipido KR, Vos MA. Probing the contribution of $I_{\mathrm{Ks}}$ to canine ventricular repolarization: key role for $\beta$ adrenergic receptor stimulation. Circulation. 2003;107:2753-2760.

16. Gao J, Wang W, Cohen IS, Mathias RT. Transmural gradients in $\mathrm{Na} / \mathrm{K}$ pump activity and $\left[\mathrm{Na}^{+}\right]_{\mathrm{i}}$ in canine ventricle. Biophys J. 2005;89:1700-1709. 
17. Mahajan A, Shiferaw $Y$, Sato D, Baher A, Olcese R, Xie LH, Yang MJ, Chen PS, Restrepo JG, Karma A, Garfinkel A, Qu Z, Weiss JN. A rabbit ventricular action potential model replicating cardiac dynamics at rapid heart rates. Biophys J. 2008;94:392-410.

18. Pitt GS. Calmodulin and CaMKII as molecular switches for cardiac ion channels. Cardiovasc Res. 2007;73:641-647.

19. Aggarwal $\mathrm{R}$, Boyden PA. Diminished $\mathrm{Ca}^{2+}$ and $\mathrm{Ba}^{2+}$ currents in myocytes surviving in the epicardial border zone of the 5-day infarcted canine heart. Circ Res. 1995;77:1180-1191.

20. Tseng GN. Calcium current restitution in mammalian ventricular myocytes is modulated by intracellular calcium. Circ Res. 1988;63:468-482.

21. Liu DW, Gintant GA, Antzelevitch C. Ionic bases for electrophysiological distinctions among epicardial, midmyocardial, and endocardial myocytes from the free wall of the canine left ventricle. Circ Res. 1993;72:671-687.

22. Silva J, Rudy $Y$. Subunit interaction determines $I_{\mathrm{ks}}$ participation in cardiac repolarization and repolarization reserve. Circulation. 2005;112:1384-1391.

23. Liu DW, Antzelevitch C. Characteristics of the delayed rectifier current $\left(I_{\mathrm{Kr}}\right.$ and $\left.I_{\mathrm{Ks}}\right)$ in canine ventricular epicardial, midmyocardial, and endocardial myocytes. A weaker $I_{\mathrm{Ks}}$ contributes to the longer action potential of the M cell. Circ Res. 1995;76:351-365.

24. Cordeiro JM, Greene L, Heilmann C, Antzelevitch D, Antzelevitch C. Transmural heterogeneity of calcium activity and mechanical function in the canine left ventricle. Am J Physiol Heart Circ Physiol. 2004;286:H1471-1479.

25. Sipido KR, Volders PGA, de Groot SHM, Verdonck F, Van de Werf F, Wellens HJJ, Vos MA. Enhanced $\mathrm{Ca}^{2+}$ release and $\mathrm{Na} / \mathrm{Ca}$ exchange activity in hypertrophied canine ventricular myocytes: potential link between contractile adaptation and arrhythmogenesis. Circulation. 2000;102:2137-2144.

26. Magyar J, Szentandrassy N, Banyasz T, Fulop L, Varro A, Nanasi PP. Effects of thymol on calcium and potassium currents in canine and human ventricular cardiomyocytes. $\mathrm{Br} J$ Pharmacol. 2002;136:330-338.

27. Birinyi P, Acsai K, Banyasz T, Toth A, Horvath B, Virag L, Szentandrassy N, Magyar J, Varro A, Fulop F, Nanasi PP. Effects of SEA0400 and KB-R7943 on Na+/Ca2+ exchange current and Ltype $\mathrm{Ca} 2+$ current in canine ventricular cardiomyocytes. Naunyn Schmiedebergs Arch Pharmacol. 2005;372:63-70.

28. Sun X, Wang HS. Role of the transient outward current (Ito) in shaping canine ventricular action potential--a dynamic clamp study. J Physiol. 2005;564:411-419.

29. Greenstein JL, Wu R, Po S, Tomaselli GF, Winslow RL. Role of the calcium-independent transient outward current $I_{\text {to1 }}$ in shaping action potential morphology and duration. Circ Res. 2000;87:1026-1033.

30. Tseng GN, Hoffman BF. Two components of transient outward current in canine ventricular myocytes. Circ Res. 1989;64:633-647.

31. Hund TJ, Kucera JP, Otani NF, Rudy Y. lonic charge conservation and long-term steady state in the Luo-Rudy dynamic cell model. Biophys J. 2001;81:3324-3331.

32. Shaw RM, Rudy Y. Ionic mechanisms of propagation in cardiac tissue. Roles of the sodium and L-type calcium currents during reduced excitability and decreased gap junction coupling. Circ Res. 1997;81:727-741.

33. Diego JMD, Sun ZQ, Antzelevitch C. $\mathrm{I}_{\text {to }}$ and action potential notch are smaller in left vs. right canine ventricular epicardium. Am J Physiol. 1996;271:H548-H561.

34. Spach MS, Heidlage JF, Dolber PC, Barr RC. Electrophysiological effects of remodeling cardiac gap junctions and cell size: experimental and model studies of normal cardiac growth. Circ Res. 2000;86:302-311.

35. Baláti B, Varró A, Papp JG. Comparison of the cellular electrophysiological characteristics of canine left ventricular epicardium, M cells, endocardium and Purkinje fibres. Acta Physiol Scand. 1998;164:181-190. 
36. Elzinga G, Lab MJ, Noble MI, Papadoyannis DE, Pidgeon J, Seed A, Wohlfart B. The actionpotential duration and contractile response of the intact heart related to the preceding interval and the preceding beat in the dog and cat. J Physiol. 1981;314:481-500.

37. Saitoh H, Bailey JC, Surawicz B. Alternans of action potential duration after abrupt shortening of cycle length: differences between dog Purkinje and ventricular muscle fibers. Circ Res. 1988;62:1027-1040.

38. Pacioretty LM, Gilmour RF, Jr. Developmental changes of action potential configuration and $I_{\text {to }}$ in canine epicardium. Am J Physiol. 1995;268:H2513-2521.

39. Xiao L, Zhang L, Han W, Wang Z, Nattel S. Sex-based transmural differences in cardiac repolarization and ionic-current properties in canine left ventricles. Am J Physiol Heart Circ Physiol. 2006;291:H570-580.

40. Szentadrassy N, Banyasz T, Biro T, Szabo G, Toth BI, Magyar J, Lazar J, Varro A, Kovacs L, Nanasi PP. Apico-basal inhomogeneity in distribution of ion channels in canine and human ventricular myocardium. Cardiovasc Res. 2005;65:851-860.

41. Boyett MR, Jewell BR. A study of the factors responsible for rate-dependent shortening of the action potential in mammalian ventricular muscle. J Physiol. 1978;285:359-380.

42. Han J, Moe GK. Cumulative effects of cycle length on refractory periods of cardiac tissues. Am J Physiol. 1969;217:106-109.

43. Qu Z. Dynamical effects of diffusive cell coupling on cardiac excitation and propagation: a simulation study. Am J Physiol Heart Circ Physiol. 2004;287:H2803-2812.

44. Sah R, Ramirez RJ, Backx PH. Modulation of $\mathrm{Ca}^{2+}$ release in cardiac myocytes by changes in repolarization rate: role of phase-1 action potential repolarization in excitation-contraction coupling. Circ Res. 2002;90:165-173.

45. Sicouri S, Antzelevitch C. A subpopulation of cells with unique electrophysiological properties in the deep subepicardium of the canine ventricle. The M cell. Circ Res. 1991;68:1729-1741.

46. Litovsky SH, Antzelevitch C. Rate dependence of action potential duration and refractoriness in canine ventricular endocardium differs from that of epicardium: role of the transient outward current. J Am Coll Cardiol. 1989;14:1053-1066.

47. Tolkacheva EG, Anumonwo JM, Jalife J. Action potential duration restitution portraits of mammalian ventricular myocytes: role of calcium current. Biophys J. 2006;91:2735-2745.

48. Eisner DA, Trafford AW, Díaz ME, Overend CL, O'Neill SC. The control of Ca release from the cardiac sarcoplasmic reticulum: regulation versus autoregulation. Cardiovasc Res. 1998;38:589-604.

49. Wang DY, Chae SW, Gong QY, Lee CO. Role of aiNa in positive force-frequency staircase in guinea pig papillary muscle. Am J Physiol. 1988;255:C798-807.

50. Baher A, Qu Z, Hayatdavoudi A, Lamp ST, Yang MJ, Xie F, Turner S, Garfinkel A, Weiss JN. Short-term cardiac memory and mother rotor fibrillation. Am J Physiol Heart Circ Physiol. 2007;292:H180-189.

51. Karma A. Electrical alternans and spiral wave breakup in cardiac tissue. Chaos. 1994;4:461472.

52. Mironov S, Jalife J, Tolkacheva EG. Role of conduction velocity restitution and short-term memory in the development of action potential duration alternans in isolated rabbit hearts. Circulation. 2008;118:17-25.

53. Huelsing DJ, Pollard AE, Spitzer KW. Transient outward current modulates discontinuous conduction in rabbit ventricular cell pairs. Cardiovasc Res. 2001;49:779-789.

54. Wang YG, Wagner MB, Kumar R, Goolsby WN, Joyner RW. Fast pacing facilitates discontinuous action potential propagation between rabbit atrial cells. Am J Physiol Heart Circ Physiol. 2000;279:H2095-2103.

55. Kääb S, Nuss HB, Chiamvimonvat N, O'Rourke B, Pak PH, Kass DA, Marban E, Tomaselli GF. lonic mechanism of action potential prolongation in ventricular myocytes from dogs with pacing-induced heart failure. Circ Res. 1996;78:262-273. 
56. Yu H, McKinnon D, Dixon JE, Gao J, Wymore R, Cohen IS, Danilo P, Jr., Shvilkin A, Anyukhovsky EP, Sosunov EA, Hara M, Rosen MR. Transient outward current, $\mathrm{I}_{\text {to1 }}$, is altered in cardiac memory. Circulation. 1999;99:1898-1905.

57. Oudit GY, Kassiri Z, Sah R, Ramirez RJ, Zobel C, Backx PH. The molecular physiology of the cardiac transient outward potassium current $\left(\mathrm{I}_{\mathrm{to}}\right)$ in normal and diseased myocardium. J Mol Cell Cardiol. 2001;33:851-872.

58. Patel SP, Campbell DL. Transient outward potassium current, 'Ito', phenotypes in the mammalian left ventricle: underlying molecular, cellular and biophysical mechanisms. J Physiol. 2005;569:7-39.

59. Zygmunt AC, Eddlestone GT, Thomas GP, Nesterenko VV, Antzelevitch C. Larger late sodium conductance in $\mathrm{M}$ cells contributes to electrical heterogeneity in canine ventricle. $\mathrm{Am} \mathrm{J}$ Physiol Heart Circ Physiol. 2001;281:H689-697.

60. Zygmunt AC, Goodrow RJ, Antzelevitch C. $I_{\mathrm{NaCa}}$ contributes to electrical heterogeneity within the canine ventricle. Am J Physiol Heart Circ Physiol. 2000;278:H1671-1678.

61. Shimizu W, Antzelevitch C. Cellular basis for the ECG features of the LQT1 form of the longQT syndrome: effects of beta-adrenergic agonists and antagonists and sodium channel blockers on transmural dispersion of repolarization and torsade de pointes. Circulation. 1998;98:2314-2322.

62. Varró A, Lathrop DA, Papp JG. Role of the delayed rectifier component $I_{\mathrm{Ks}}$ in cardiac repolarization. J Cardiovasc Electrophysiol. 2001;12:1204-1206.

63. Splawski I, Tristani-Firouzi M, Lehmann MH, Sanguinetti MC, Keating MT. Mutations in the

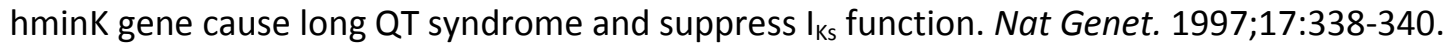

64. Faber GM, Rudy Y. Action potential and contractility changes in $\left[\mathrm{Na}^{+}\right]_{\mathrm{i}}$ overloaded cardiac myocytes: a simulation study. Biophys J. 2000;78:2392-2404.

65. Hund TJ, Rudy Y. Determinants of excitability in cardiac myocytes: mechanistic investigation of memory effect. Biophys J. 2000;79:3095-3104.

66. Williams BA, Dickenson DR, Beatch GN. Kinetics of rate-dependent shortening of action potential duration in guinea-pig ventricle; effects of $I_{K 1}$ and $I_{\mathrm{Kr}}$ blockade. Br J Pharmacol. 1999;126:1426-1436.

67. Qu Z, Weiss JN, Garfinkel A. Cardiac electrical restitution properties and stability of reentrant spiral waves: a simulation study. Am J Physiol. 1999;276:H269-283. 


\section{CHAPTER 3}

LOCAL CONTROL OF $\beta$-ADRENERGIC STIMULATION: EFFECTS ON VENTRICULAR MYOCYTE ELECTROPHYSIOLOGY AND

$$
\mathrm{CA}^{2+} \text {-TRANSIENT }
$$

Jordi Heijman, Paul G.A. Volders, Ronald L. Westra and Yoram Rudy.

J. Mol. Cell. CARDIOL. 2011, 50:863-871 


\section{ABSTRACT}

Local signaling domains and numerous interacting molecular pathways and substrates contribute to the whole-cell response of myocytes during $\beta$-adrenergic stimulation (BARS). We aimed to elucidate the quantitative contribution of substrates and their local signaling environments during $\beta A R S$ to the canine epicardial ventricular myocyte electrophysiology and calcium transient (CaT). We present a computational compartmental model of $\beta$ ARS and its electrophysiological effects. Novel aspects of the model include localized signaling domains, incorporation of $\beta 1$ and $\beta 2$ receptor isoforms, a detailed population-based approach to integrate the $\beta A R$ and $\mathrm{Ca}^{2+} /$ Calmodulin kinase (CaMKII) signaling pathways and their effects on a wide range of substrates that affect whole-cell electrophysiology and CaT. The model identifies major roles for phosphodiesterases, adenylyl cyclases, PKA and restricted diffusion in the control of local cAMP levels and shows that activation of specific CAMP domains by different receptor isoforms allows for specific control of action potential and CaT properties. In addition, the model predicts increased CaMKII activity during $\beta A R S$ due to rate-dependent accumulation and increased $\mathrm{Ca}^{2+}$ cycling. CaMKII inhibition, reduced compartmentation, and selective blockade $\beta 1 A R$ are predicted to reduce the occurrence of delayed afterdepolarizations during $\beta A R S$. Finally, the relative contribution of each PKA substrate to wholecell electrophysiology is quantified by comparing simulations with and without phosphorylation of each target. In conclusion, this model enhances our understanding of localized $\beta A R$ signaling and its whole-cell effects in ventricular myocytes by incorporating receptor isoforms, multiple pathways and a detailed representation of multiple-target phosphorylation; it provides a basis for further studies of $\beta$ ARS under pathological conditions. 


\section{INTRODUCTION}

Maximal $\beta$-adrenergic receptor ( $\beta A R$ ) stimulation ( $\beta A R S$ ) in ventricular myocytes activates the $\beta A R$ / G-protein / adenylyl cyclase (AC) / cyclic AMP (cAMP) / protein kinase A (PKA) pathway that results in the phosphorylation of numerous intracellular proteins ("substrates") including the L-type $\mathrm{Ca}^{2+}$ channel $\left(I_{C a, L}\right)$, slowly activating delayed rectifier $K^{+}$channel $\left(I_{K s}\right)$, phospholamban (PLB) and the inhibitory troponin subunit (Tnl). In addition, there are several feedback loops, such as $\beta A R$ desensitization, that control the temporal response of $\beta A R S .{ }^{1,2}$ Observations in living myocytes using fluorescent techniques have demonstrated the importance of localized signaling domains in $\beta A R$ cascade function. Moreover, many interdependencies were identified between PKA substrates that influence the cardiac action potential (AP) and calcium transient (CaT). ${ }^{3,4}$

Localized signaling results from physical subcellular domains with restricted diffusion of the second messenger cAMP (e.g., caveolae), from specific subcellular localization of signaling molecules (e.g., CAMP and phosphodiesterases; PDEs) and from targeting of PKA to specific substrates by A-kinase anchoring proteins (AKAPs).$^{5-7}$ Localized signaling in subcellular domains is essential for precise specific regulation ("local control"); it allows multiple signaling cascades that affect cAMP to target specific substrates. This local organization may change in pathology such as heart failure. ${ }^{8}$ The relative contribution of each local signaling domain and substrate to the whole-cell electrophysiological response during $\beta A R S$ is extremely difficult to determine experimentally, especially in large mammals. Moreover, an increasing number of interactions between the $\beta A R$ signaling cascade and other signaling cascades, most prominently the $\mathrm{Ca}^{2+} /$ Calmodulin kinase (CaMKII), are discovered to play an important role in normal physiology and in pathology. ${ }^{9}$ Currently, our understanding of the complex interactions between signaling cascades and substrates and their effects on cellular electrophysiology is rather limited.

Computational approaches have been employed to enhance our understanding of various (patho)physiological processes. ${ }^{10}$ The computational approach is particularly useful when the system under investigation is complex, highly interactive and nonlinear, as is the case with the $\beta A R$ cascade and its effects on cellular electrophysiology. Quantitative studies of cAMP signaling have been performed ${ }^{11-13}$ but did not include electrophysiological effects. Several studies have modeled different aspects of the adrenergic system in the ventricular myocyte in various levels of detail. ${ }^{14-18}$ However, none of these studies described the effects of localized signaling domains or included all interacting PKA substrates. Here, we present the first detailed computational model of $\beta A R S$ in the canine ventricular myocyte that includes both localized signaling domains and a wide range of electrophysiological substrates. We use this model to study the local effects of $\beta A R S$ on subcellular targets and their consequences in terms of whole-cell electrophysiology and CaT. 


\section{METHODS}

Details of the computational model (Figure 1) are provided in the appendix of this thesis.
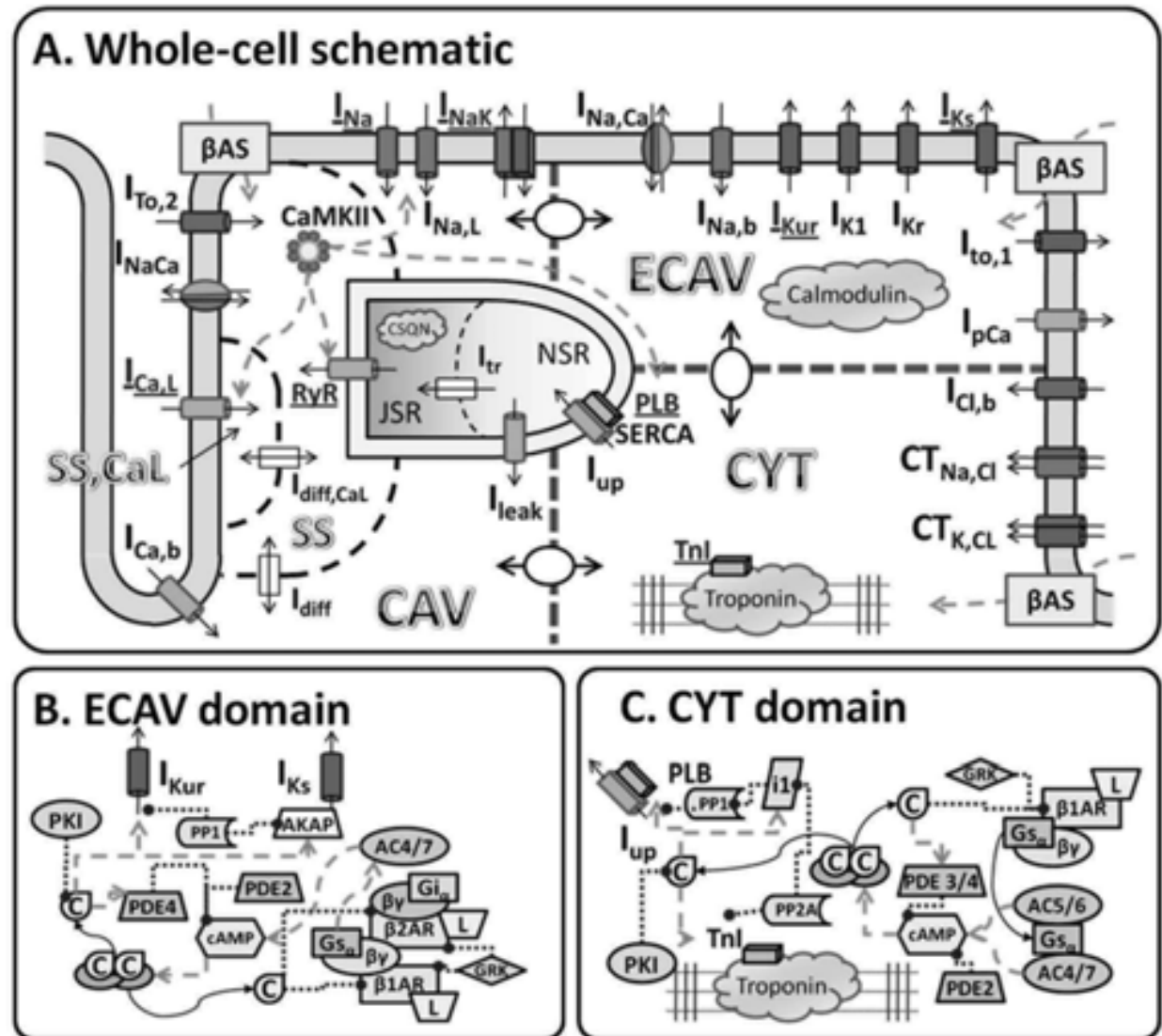

C. CYT domain

D. CAV domain
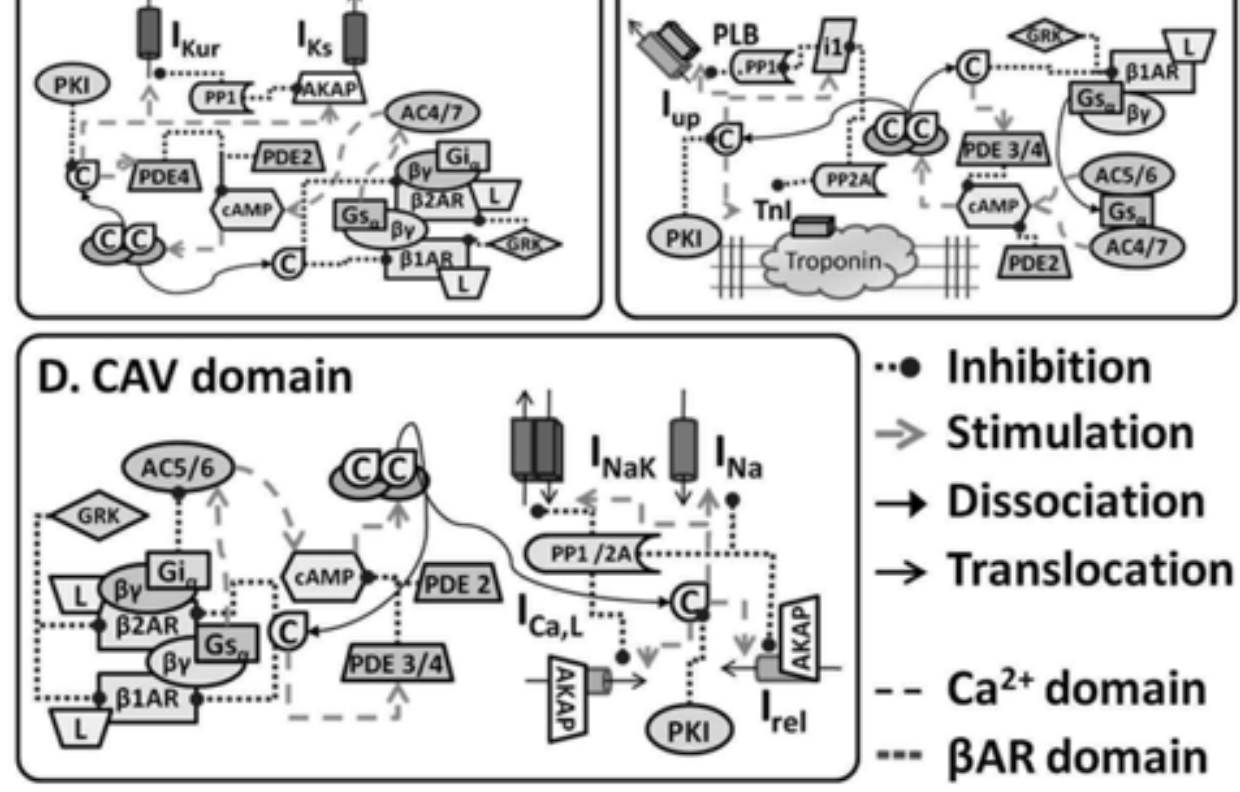

Figure 1. A. Schematic of the canine ventricular cell model including $\mathrm{Ca}^{2+}$ handling, electrophysiology, CaMKII signaling pathway and $\beta A R$ signaling domains. Substrates modulated by PKA phosphorylation are indicated. Model CaMKII targets include PLB/Serca and RyR. B-D, Detailed schematics of the interactions in the extracaveolar (ECAV; B), cytosolic (CYT; C) and caveolar (CAV; D) signaling domains. Abbreviations are defined in the appendix of this thesis. Model code is provided in the Research Section of http://rudylab.wustl.edu. For color figure, see page 243.

\section{BAR signaling cascade}

A recent compartmental model of CAMP signaling in ventricular myocytes ${ }^{11}$ served as starting point for the $\beta A R$-signaling model of this study. Three functional compartments represent the caveolar 
(CAV), extracaveolar membrane (ECAV) and cytosolic (CYT) signaling domains. Each compartment contains signaling proteins at local concentrations. We extend the CAMP signaling cascade with differential localization of $\beta_{1} A R$ and $\beta_{2} A R$, local PKA activation (high affinity PKAI in CYT, lower affinity PKAll in CAV and ECAV), PKA-dependent feedback loops, PKA targeting to $I_{\text {CaL, }} l_{\mathrm{Ks}}$ and RyR by AKAPS, and eight substrates of direct PKA-dependent phosphorylation. Figure 1 B-D shows schematics of the compartmental signaling cascades in the model.

All biochemical reactions were modeled either as differential equations (e.g., cAMP production, PKA activation, receptor desensitization) or as algebraic equations based on steady-state assumptions for reactions occurring much faster than changes in CAMP and substrate phosphorylation. Timedependent concentrations of the $\beta A R$ agonist isoproterenol (ISO, in combination with blockade of either $\beta_{1} A R$ or $\beta_{2} A R$ in some simulations) and/or the PDE inhibitor IBMX served as inputs to the adrenergic signaling cascade.

\section{Choice and localization of PKA substrates}

The model contains eight PKA substrates which are included in specific compartments of the signaling model (Table 1) based on experimental evidence (in particular association with caveolin-3 for CAV substrates). It is important to note that our compartments are defined as functional entities. For example, although caveolae are small cellular invaginations of less than $100 \mathrm{nM}$ in size, ${ }^{19}$ we still consider the ryanodine receptor part of this compartment because of its intimate interaction with $\mathrm{I}_{\mathrm{CaL}}$, even though it will not physically reside in this small invagination, but is localized at the opposing SR membrane surface. L-type $\mathrm{Ca}^{2+}$ channels are known to reside predominantly in T-tubules in the ventricular myocyte. ${ }^{20}$ However, since caveolin has been implicated in T-tubular biogenesis ${ }^{21}$ and Ltype $\mathrm{Ca}^{2+}$ channels have indeed been shown to colocalize with caveolin, ${ }^{22}$ we decided to include both $I_{\text {Cat }}$ and RyR in the functional caveolar compartment.

\section{Substrate $\quad$ Compartment $\quad$ Evidence}

\begin{tabular}{|c|c|c|}
\hline$I_{\mathrm{CaL}}$ & CAV & Colocalization with caveolin-3. ${ }^{22}$ \\
\hline $\mathrm{I}_{\mathrm{Ks}}$ & ECAV & $\begin{array}{l}\text { Based on membrane targeting with yotiao, but no } \mathrm{K}^{+} \text {channels } \\
\text { associate with caveolin }{ }^{22} \text {. Although certain studies have found } \\
\text { that Kv7.1 (KCNQ1, the } \alpha \text { subunit of the } \mathrm{I}_{\mathrm{Ks}} \text { channel) localizes to } \\
\text { low density membrane fractions, no co-immunoprecipitation of } \\
\text { Kv7.1 and caveolin-3 has been shown so far. }{ }^{23}\end{array}$ \\
\hline$I_{\text {Kur }}$ & ECAV & Membrane ion channel, not found in caveolae in dog. ${ }^{24}$ \\
\hline$I_{\mathrm{Na}}$ & CAV & Colocalization with caveolin-3. ${ }^{22}$ \\
\hline$I_{\mathrm{Na}, \mathrm{K}}$ & CAV & $\begin{array}{l}\text { Predominantly located in caveolae, depending on subunit } \\
\text { composition. }{ }^{25}\end{array}$ \\
\hline RyR & CAV & $\begin{array}{l}\text { Based on close association with } \mathrm{I}_{\mathrm{Ca}, \mathrm{L}} \text {, and regulation of RyR by } \\
\text { caveolin-associated eNOS. }{ }^{26}\end{array}$ \\
\hline PLB & CYT & Influenced by cytosolic protein Inh1, ${ }^{27}$ phosphorylated by PKAl. ${ }^{14}$ \\
\hline $\mathrm{Tnl}$ & CYT & \\
\hline
\end{tabular}

Table 1: Localization of PKA substrates. 
The following targets are not included in the current model: $I_{\mathrm{Cl}, \text { cAMP }}$ is a cAMP activated $\mathrm{Cl}^{-}$current that is present in guinea pig, but not canine ventricular myocytes. ${ }^{28,}{ }^{29}$ Karle and co-workers found evidence that $\mathrm{I}_{\mathrm{Kr}}$ is modulated by PKA in guinea pig ventricular myocytes ${ }^{30}$ and similarly a small but significant alteration in $\mathrm{I}_{\mathrm{kr}}$ was found recently in response to IBMX and forskolin in $\mathrm{CHO}$ cells ${ }^{31}$. However, no such modulation was found in dogs. ${ }^{32}$ Because of the absence in dog, and the small effects in $\mathrm{CHO}$ cells, $\mathrm{I}_{\mathrm{kr}}$ modulation by PKA was not incorporated. Similarly, no consensus exists concerning the modulating effect of PKA on $\mathrm{Na}^{+}-\mathrm{Ca}^{2+}$ exchanger $\mathrm{I}_{\mathrm{Na}, \mathrm{Ca}}{ }^{33,}{ }^{34}$ Relatively little is known about the adrenergic modulation of $\mathrm{I}_{\mathrm{TO}}$, however Van der Heyden et al. argue that only $\alpha$-adrenergic modulation has a significant impact on $\mathrm{I}_{\mathrm{TO}_{\mathrm{O}}}{ }^{35}$ As a result, we do not include direct modulation of $\mathrm{I}_{\mathrm{TO}_{\mathrm{O}}}$ in our model of $\beta A R$ stimulation, although it should be noted that $I_{T o}$ is known to be modulated by $\mathrm{CaMKII}^{36}$ which shows increased activity during $\beta A R$ stimulation (see below). Finally, many disparate results exist concerning the modulation of the inward rectifier potassium current $I_{K 1}$ by PKA, which has a consensus phosphorylation site on $\mathrm{S} 425 .{ }^{37}$ Although recent studies suggest a role for $\mathrm{I}_{\mathrm{K} 1}$ mutations that alter its adrenergic responsiveness in catecholaminergic polymorphic ventricular tachycardia (CPVT)-like arrhythmias, the major differences in WT $I_{K 1}$ in the presence and absence of adrenergic stimulation occur at potentials below the normal physiological range, with no difference at $-80 \mathrm{mV} .^{38}$ This is further strengthened by the unaltered resting membrane potential in canine ventricular myocytes in the presence of adrenergic stimulation. ${ }^{39,40}$ As such, adrenergic stimulation of $I_{k 1}$ is not included in the model.

\section{Definition of the level of phosphorylation of PKA substrates}

The effective level of phosphorylation of a PKA substrate $X$, denoted by $\hat{\mathrm{f}}_{\mathrm{X}}^{\mathrm{p}}$ is defined relative to the baseline phosphorylation level. That is, the level in the absence of catecholamine stimulation is defined as $\hat{\mathrm{f}}_{\mathrm{X}}^{\mathrm{p}}=0$, although in an actual myocyte there may be a certain number of phosphorylated proteins under basal conditions. Similarly, for the research described here, the phosphorylation level is defined to be 1 under a saturating concentration of isoproterenol (although this does not necessarily mean that all channels are phosphorylated). This functional definition facilitates the use of a population-based approach to link the signaling cascade and the electrophysiology. Although a cocktail of agonists may increase CAMP beyond the levels observed with maximal ISO stimulation, this will not be reflected in the electrophysiological properties of the model, because no substrate data were available under these conditions and hence no extrapolations to higher CAMP levels could be made. When these electrophysiological data become available, the population-based approach (see below) makes it possible to set a new upper limit and have a larger range of cAMP levels for which the electrophysiological properties can be simulated. It should also be noted that although this functional definition is a simplification, it does not prevent simulation of other adrenergic agonists (e.g., Forskolin or PDE inhibition with IBMX), particularly for CAMP levels within the range observed with ISO stimulation. This is consistent with the observations by Hohl and Li, who showed that various adrenergic agonists over a wide range of concentrations produced a similar range of changes in peak amplitude and decay time of the calcium transient. ${ }^{41}$ 


\section{Analysis of cAMP compartmentation}

Experimentally, compartmentation of cAMP is revealed by differential effects of signaling pathways that affect CAMP on phosphorylation of cytosolic versus sarcolemmal proteins, ${ }^{5,42}$ and by differences in cytosolic cAMP and subsarcolemmal cAMP levels measured with fluorescent probes and cyclic nucleotide-gated channels, ${ }^{43}$ respectively. This has illustrated that selective augmentation of cAMP in specific compartments occurs and is critical for specific regulation of subcellular targets. Here, we focus on the effects of $\beta A R S$. Other signaling pathways affecting cAMP (e.g., the prostaglandin receptor PGE1) are not included. Compartmentation has also been shown through differences in $\beta A R$ isoform stimulation. In mammalian ventricle, three isoforms have been reported. In canine, approximately $80 \%$ are $\beta_{1} A R{ }^{44}$ Functional effects of $\beta_{2} A R$ stimulation are highly dependent on species and experimental conditions. In rodents, $\beta_{2} A R S$ only affects local cAMP concentrations, whereas CAMP increases elicited by $\beta_{1}$ ARS diffuse throughout the cell. ${ }^{45}, 46$ Studies in the failing canine ventricle show a reduction in $\beta_{1} A R$ without changes in $\beta_{2} A R$ and consequently an important role of $\beta_{2}$ ARS. ${ }^{47}$ Under normal physiological conditions, differential effects of $\beta_{2}$ ARS on whole cell behavior have been reported. ${ }^{47-49}$ The model includes $\beta_{1} A R$ and $\beta_{2} A R$ with cell-wide and CAV localization respectively, based on experimental data. ${ }^{8}$ The functional significance of $\beta_{3} A R$ in large mammals is incompletely understood and not included here. ${ }^{50}$ Virtual knock-out models of selected signaling proteins were created by setting their concentration to 0 . Reduced compartmentation was simulated by increasing the CAMP diffusion rates by a factor 1000 .

\section{Definition of the soluble and particulate fractions}

The model contains 3 compartments that provide the localized signaling characteristics that are vital for efficient and specific signaling: CAV, ECAV CYT. In lancu et al. it is stated that ${ }^{51}$ : “... the only information available on the subcellular location of the different PDE isoforms describes their presence in membrane (particulate) and/or cytosolic (soluble) fractions. How the membrane associated PDE activity is distributed between caveolar and extracaveolar domains is not known." In accordance with this description, we define the particulate fraction as made up of the CAV and ECAV compartments and let the soluble fraction correspond to the CYT compartment. In order to relate protein activities that are expressed as pmol / $\mathrm{mg}$ cell protein (e.g. cAMP from Hohl and $\mathrm{Li}^{41}$ ) to the intracellular concentrations used in the model, we compare the values normalized to the total cellular activity, thereby taking into account the relative contribution of the compartments based on their volume.

\section{CaMKII signaling cascade}

The baseline model ${ }^{52}$ (Chapter 2 ) includes phosphorylation of PLB and RyR by CaMKII. We included populations for CaMKII-dependent phosphorylation of $I_{\mathrm{CaL}}, I_{\mathrm{Na}}$ and late $I_{\mathrm{Na}}\left(I_{\mathrm{Na}, \mathrm{L}}\right)$ based on recently published formulations ${ }^{53}$, as well as CaMKII-dependent changes in $\mathrm{I}_{\mathrm{To}}$ and $\mathrm{I}_{\mathrm{K} 1}$ (see Appendix of this thesis). CaMKII is activated by localized $\mathrm{Ca}^{2+}$ in the $\mathrm{Ca}^{2+}$ subspace, consistent with previous modeling studies, but no further localization is included. Additional crosstalk between $\beta A R S$ and CaMKII occurs via PKA-dependent activation of Inhibitor 1 and subsequent deactivation of PP1 that controls CaMKII deactivation, consistent with experimental observations. ${ }^{9}$ 


\section{Changes to electrophysiological substrates after phosphorylation}

Phosphorylation effects were incorporated by including populations of phosphorylated and nonphosphorylated channels with distinct properties for the eight substrates affected by BARS. Properties of non-phosphorylated (baseline) substrates were based on the recently published model of the canine epicardial ventricular myocyte ${ }^{52}$ (Chapter 2) and properties of phosphorylated channels were determined from experimental data in the presence of a saturating dose of ISO. At each time instant, the current/flux for a given substrate is determined for both (phosphorylated and non-phosphorylated) populations. The fraction of phosphorylated channels, which is determined by the $\beta A R$ cascade, is then used to interpolate between these values to determine the net current/flux. We assume that phosphorylation by PKA and CaMKII is independent and have integrated the $\beta A R$ and CaMKII cascades using four populations for these targets (PKA phosphorylated, CaMKII phosphorylated, PKA and CaMKII phosphorylated and non-phosphorylated).

The population-based approach is based on the assumption that phosphorylation is a binary process that is independent of channel gating. It captures the distinct conformations of phosphorylated and non-phosphorylated channels as they coexist in the ventricular myocyte. Most previous modeling approaches employed only one homogeneous population and shifted model parameters (such as the $\mathrm{V}_{1 / 2}$ of activation) based on phosphorylation levels. ${ }^{14,17}$ Although both approaches coincide at phosphorylation levels of $0 \%$ and $100 \%$, they are different for other levels (Figure 2).

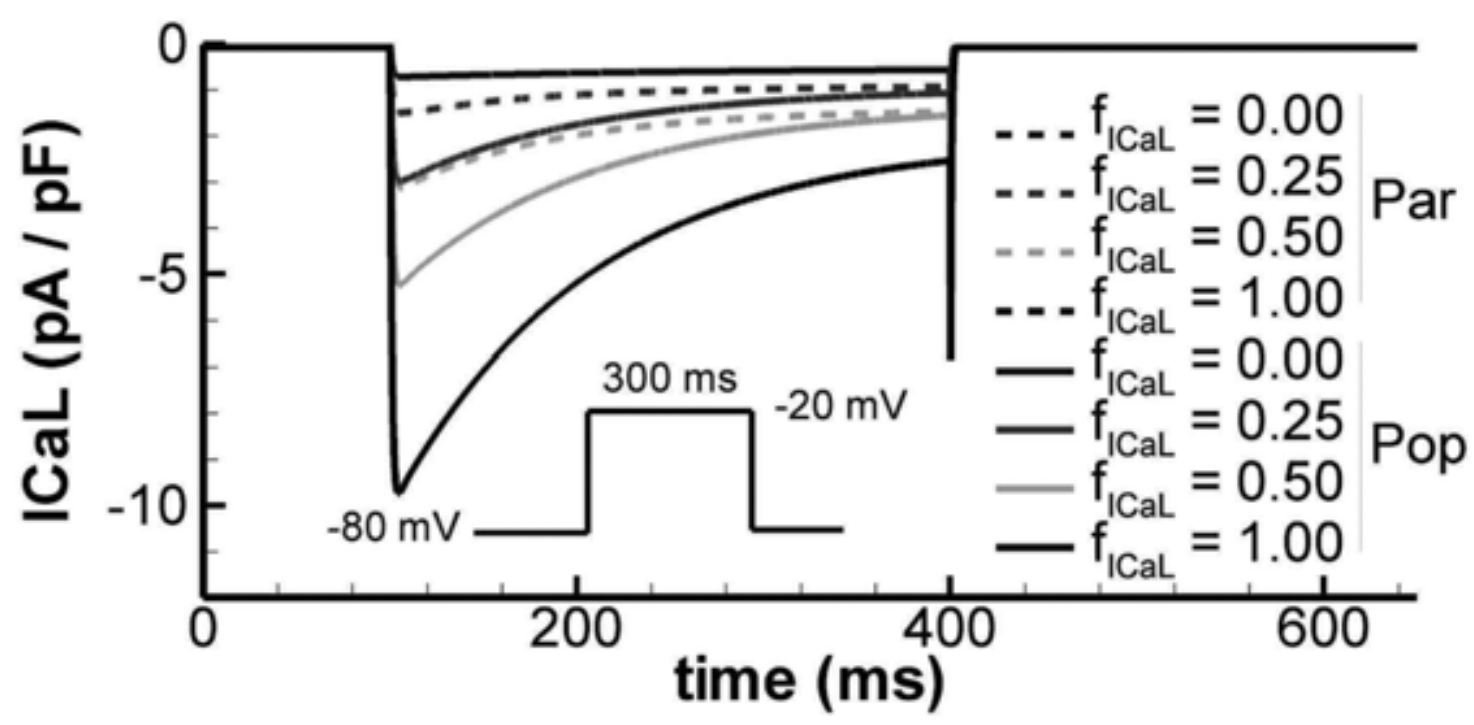

Figure 2. $I_{\text {CaL }}$ traces at various levels of phosphorylation elicited during a $300 \mathrm{~ms}$ depolarizing pulse to $-20 \mathrm{mV}$ using the population based approach (Pop) with the specified fraction ( $\left.f_{\mid C a L}\right)$ of channels phosphorylated (solid lines), or using a shift of parameters in a single homogeneous population (Par; dashed lines). Results are identical only at $0 \%$ and $100 \%$ phosphorylation, but differ for intermediate phosphorylation levels. $\mathrm{f}_{\mathrm{ICaL}}$ indicates the fraction of phosphorylated L-type $\mathrm{Ca}^{2+}$ channels. 


\section{Parameter estimation}

Parameters for the model were obtained either directly from the cited literature source (possibly after conversion of units), or derived using minimization of the squared difference between experimental data and model output. Initial exploration of the parameter space was performed using evolutionary algorithms. Bounds on parameters were determined based on physiological considerations (e.g., requirement of positive concentrations and rate constants), or centered around values used in existing models. Highest scoring parameter sets were further optimized using the Nelder-Meade simplex algorithm. All optimizations were performed in Matlab (Mathworks ${ }^{\circledR}$, Natick, MA). In some optimizations of electrophysiological substrates the Matlab optimizations were connected to the custom $\mathrm{C}++$ software to generate model output.

A number of reparametrizations were performed to facilitate the optimization process by reducing the number of ill-constrained parameters. Let $[\mathrm{P}]^{\mathrm{CAV}},[\mathrm{P}]^{\mathrm{ECAV}},[\mathrm{P}]^{\mathrm{CYT}}$ denote the 3 parameters describing the concentrations of protein $\mathrm{P}$ in the caveolar, extracaveolar and cytosolic compartments respectively. Furthermore, let $[\mathrm{P}]^{\mathrm{CELL}}, \mathrm{f}_{\mathrm{P}}^{\mathrm{CAV}}, \mathrm{f}_{\mathrm{P}}^{\mathrm{ECAV}}$, represent the total cellular concentration of $\mathrm{P}$, the fraction of $\mathrm{P}$ located in the caveolar compartment and the fraction of $\mathrm{P}$ located in the extracaveolar compartment respectively. The following transformations are applied:

$$
\begin{aligned}
& {[\mathrm{P}]^{\mathrm{CAV}}=\mathrm{f}_{\mathrm{P}}^{\mathrm{CAV}} \cdot[\mathrm{P}]^{\mathrm{CELL}} \cdot \frac{\mathrm{V}^{\mathrm{CELL}}}{\mathrm{V}^{\mathrm{CAV}}}} \\
& {[\mathrm{P}]^{\mathrm{ECAV}}=\mathrm{f}_{\mathrm{P}}^{\mathrm{ECAV}} \cdot[\mathrm{P}]^{\mathrm{CELL}} \cdot \frac{\mathrm{V}^{\mathrm{CELL}}}{\mathrm{V}^{\mathrm{ECAV}}}} \\
& {[\mathrm{P}]^{\mathrm{CYT}}=\left(1-\mathrm{f}_{\mathrm{P}}^{\mathrm{CAV}}-\mathrm{f}_{\mathrm{p}}^{\mathrm{ECAV}}\right) \cdot[\mathrm{P}]^{\mathrm{CELL}} \cdot \frac{\mathrm{V}^{\mathrm{CELL}}}{\mathrm{V}^{\mathrm{CYT}}}}
\end{aligned}
$$

Here $V^{x}$ represents the volume of compartment $x$. This has as an advantage that the value of $[\mathrm{P}]^{\mathrm{CELL}}$ can be much better constrained based on the available literature (using $1 \mathrm{pmol} / \mathrm{mg}$ cell protein $=1.084 \mu \mathrm{mol} / \mathrm{L}^{2}$ whenever necessary). In a similar fashion we use the forward rate constant $k_{f}$ and the equilibrium constant $K_{e}$ to define a general reversible reaction. The backward rate constant is then given by $k_{b}=k_{f} \cdot K_{e}$. The equilibrium constant is better constrained based on available experimental data.

Using these parametrizations, a parameter estimation procedure was followed, designed to minimize parameter uncertainties (Figure 3). After fixing the parameters that were directly available from literature (e.g., $\beta A R$ affinities for ISO), parameters were first estimated for direct steps in the adrenergic cascade (e.g., adenylyl cyclase activity as a function of G-protein activation). These estimations produce, in general, a well defined, unique set of parameters within the physiological range. Subsequently more global data (e.g., adenylyl cyclase activity as function of isoproterenol) that cover multiple steps of the cascade were used to determine missing parameter values. Finally, the parameters of the PKA substrates were fit based on the intracellular cAMP concentrations resulting from the previously estimated parameters and the substrate isoproterenol dose-response and time-dependence of phosphorylation curves. The combined global exploration and subsequent 
local optimization of promising parameter sets should reduce the risks of getting trapped in a local minimum. For the electrophysiological properties of the phosphorylated population, parameter values were either set directly based on literature (e.g., $\mathrm{K}_{\mathrm{m}}$, half-maximal activation voltage, etc.) or resulted from a Nelder-Meade simplex optimization to the experimental data starting from the properties of the non-phosphorylated population.

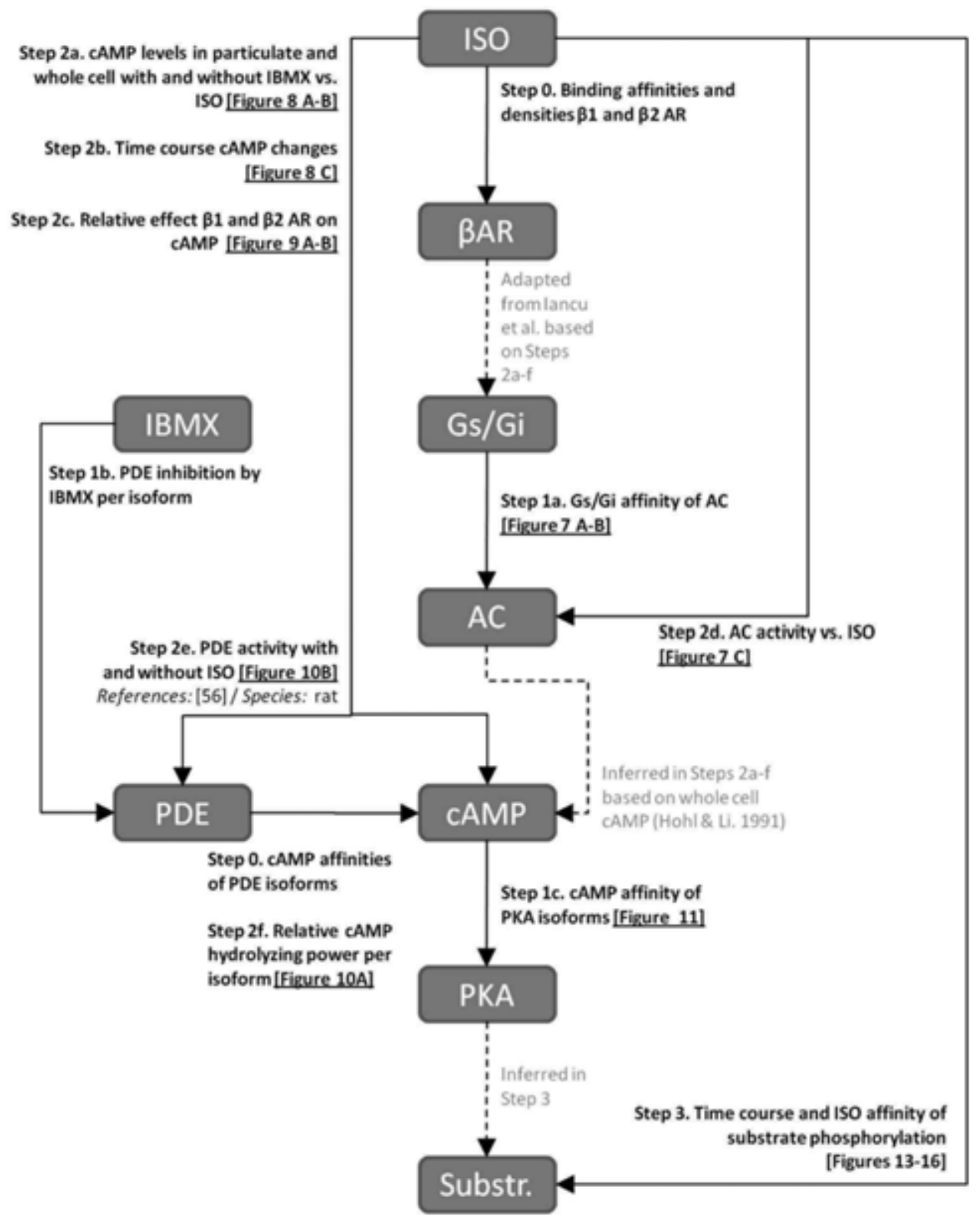

Figure 3. Simplified signaling schematic indicating parameter estimation procedure. Parameters that were directly available in the experimental literature or characterized carefully based on experimental data in previous modeling studies were fixed (Step 0). Subsequently, data representing direct steps in the cascade were used whenever possible to determine missing parameter values (Steps 1a-c, each of which could be performed independently due to the direct relation between quantities). Thereafter, more global aspects (e.g., the relationship between ISO and cAMP levels) were used simultaneously to constrain remaining parameters (Step 2a-c). Finally, the substrate parameters were inferred using the experimental ISO concentration response curve for each substrate and the PKA activity obtained in steps 1 and 2. In Figures 7-24 the relation between 
experimental and simulation data is presented and the experimental source reference and species information are indicated. All signaling validation was performed in the absence of electrophysiological changes (all state variables for the electrophysiological model were fixed).

\section{Parameter sensitivity analysis}

For the parameters in the $\beta A R$ signaling cascade, a one-factor-at-a-time sensitivity analysis was performed to investigate the individual contribution of each parameter. Each parameter $X_{i}$ was altered to $99 \%$ and $101 \%$ of its original value and the changes in output measures were evaluated:

$\Delta f_{i}^{(k)}=f^{(k)}\left(\left[\begin{array}{c}X_{1} \\ \vdots \\ X_{i-1} \\ 1.01 \cdot X_{i} \\ X_{i+1} \\ \vdots \\ X_{n}\end{array}\right]\right)-f^{(k)}\left(\left[\begin{array}{c}X_{1} \\ \vdots \\ X_{i-1} \\ 0.99 \cdot X_{i} \\ X_{i+1} \\ \vdots \\ X_{n}\end{array}\right]\right)$

Because of the modular structure of the $\beta A R$ signaling cascade, properties of the endpoints of the cascade that connect with the electrophysiological module were chosen as output measures. The ISO affinity of each substrate was determined for each parameter set using a fit of the Hill equation to steady-state phoshorylation levels over a wide range of ISO concentrations. The other two output measures that were determined are the time to half maximal activation of a substrate and the amount of desensitization (difference between maximum and steady-state levels) in the presence of an intermediate dose of ISO $(20 \mathrm{nmol} / \mathrm{L})$ for each substrate. The 10 parameters with the highest impact (based on the mean $\Delta f_{i}^{(k)}$ over all eight substrates) and the 10 with the lowest impact are shown in Figures 4-6. 
A.

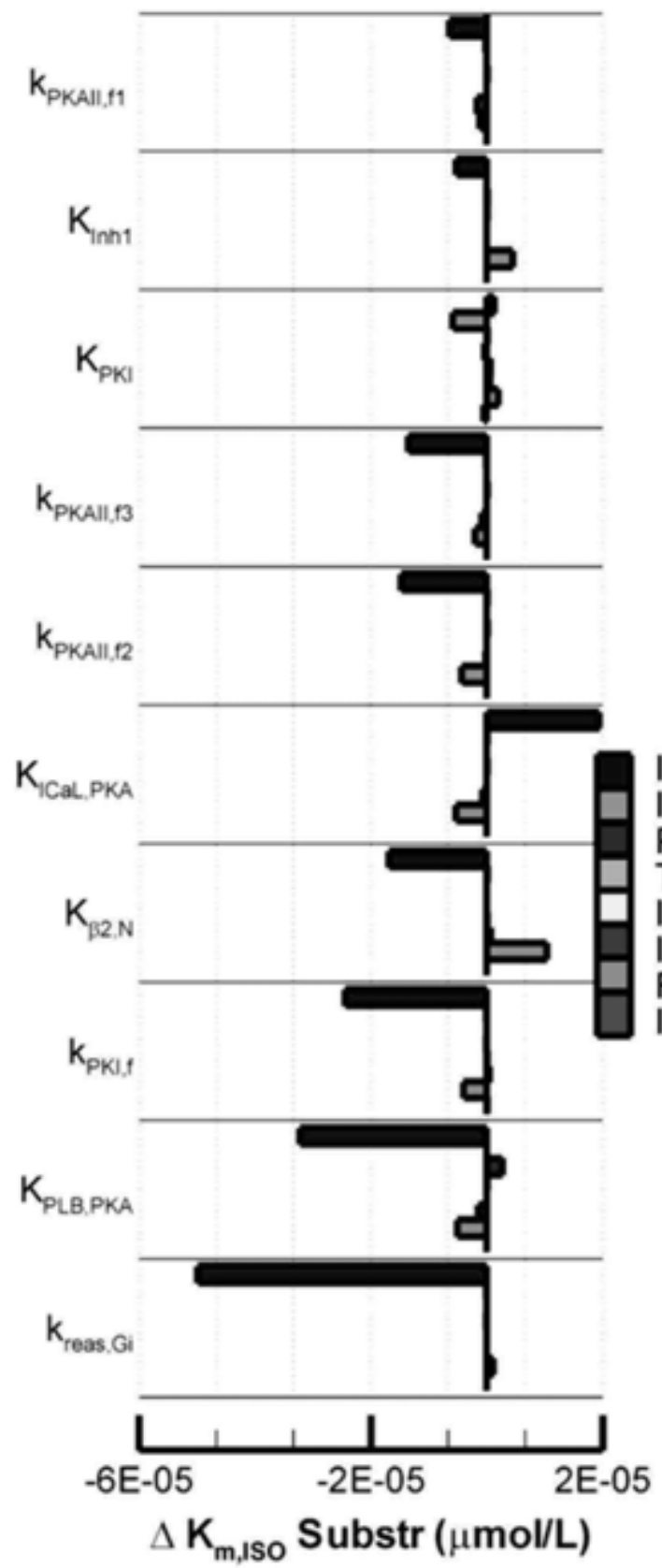

B.

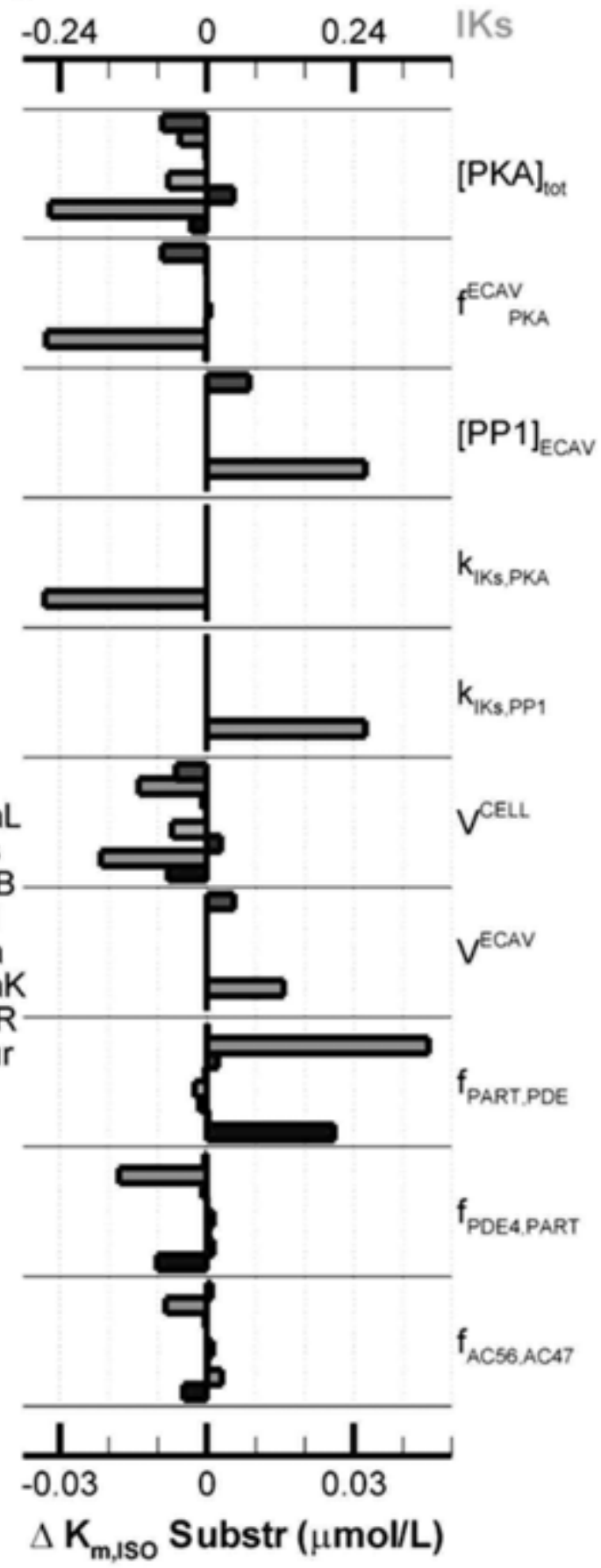

Figure 4. The 10 parameters with the smallest (A) and largest (B) impact on the mean change in ISO affinity over all substrates. Note different impact scale in (B) for $I_{\mathrm{Ks}}$. For color figure, see page 244. 
A.

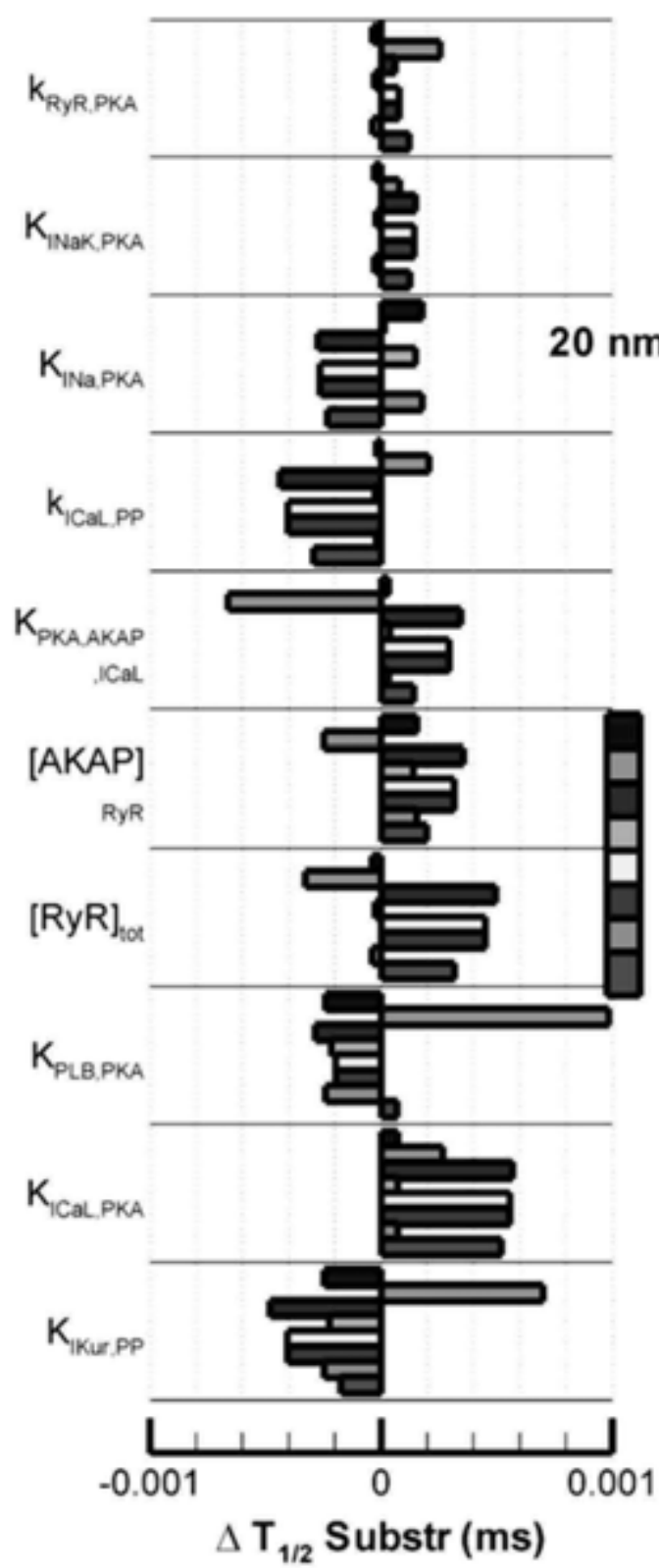

B.

\begin{tabular}{rrr}
-50 & 0 & 50 PLB \\
\hline & 0 & -1
\end{tabular}
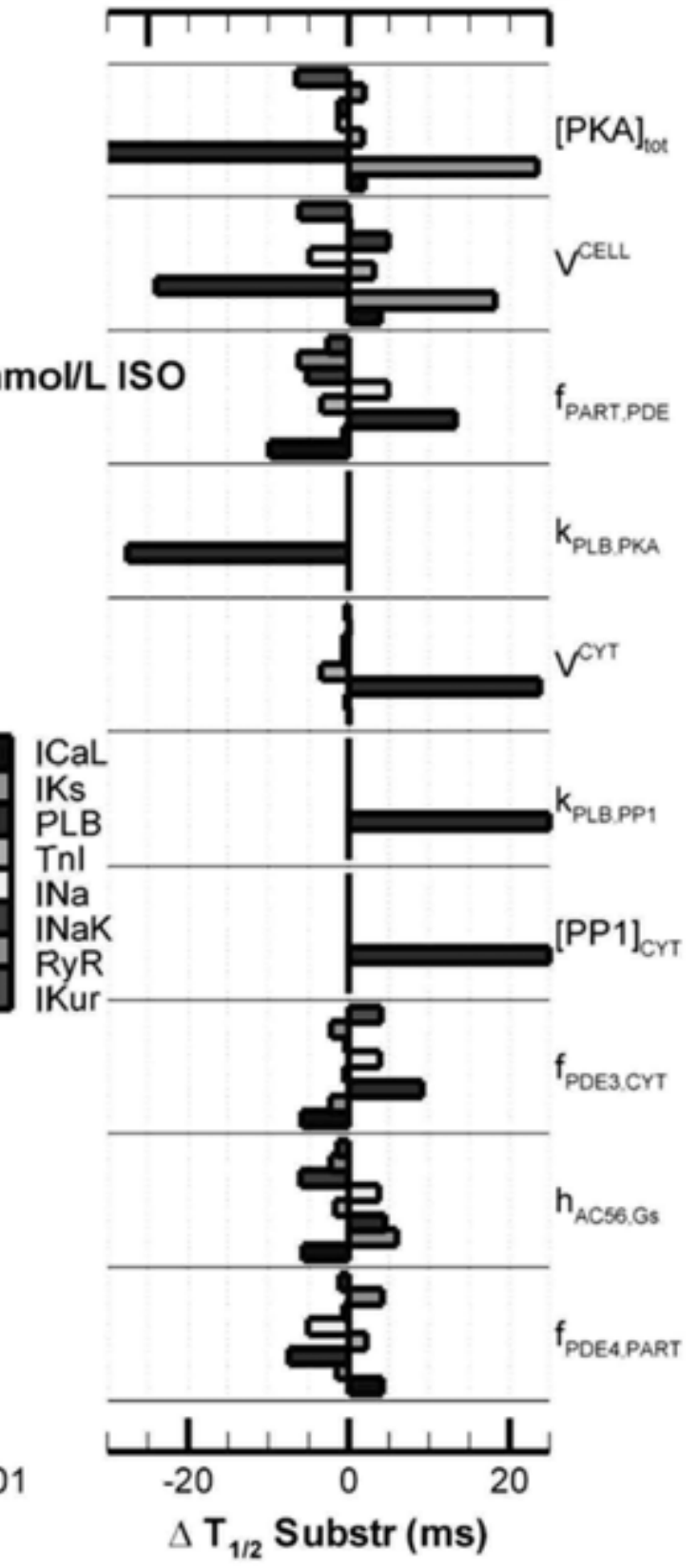

Figure 5. The 10 parameters with the smallest (A) and largest (B) impact on the mean change in time to half maximal activation in the presence of $20 \mathrm{nmol} / \mathrm{L}$ ISO over all substrates. Note different impact scale in (B) for PLB. For color figure, see page 245. 


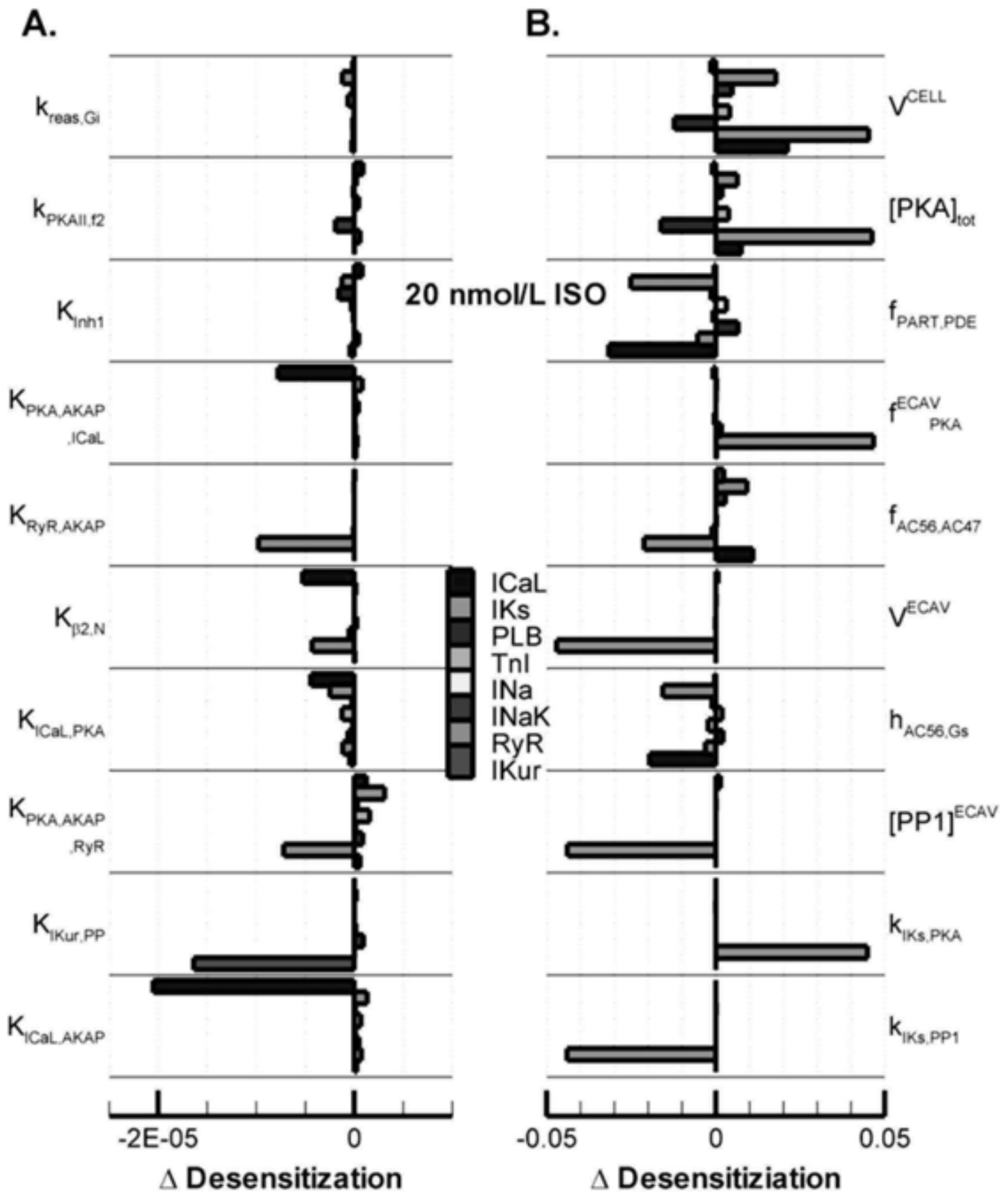

Figure 6. The 10 parameters with the smallest (A) and largest (B) impact on the mean change in the amount of desensitization (difference between peak phosphorylation level and level after $30 \mathrm{~min}$ ) in the presence of $20 \mathrm{nmol} / \mathrm{L}$ ISO over all substrates. For color figure, see page 246.

The sensitivity analysis is based on a $2 \%$ change in parameter values $(+1 \%$ and $-1 \%$ from the values reported here) using a one-factor-at-a-time approach. Simulations with $+5 \%$ and $-5 \%$ indicated a similar ordering of parameters with respect to their effect on the three output measures: ISO affinity, time to half maximal phosphorylation (in the presence of $20 \mathrm{nmol} / \mathrm{L} \mathrm{ISO}$ ) and amount of desensitization. These three outputs were determined for all eight substrates. 
From the sensitivity analysis it is clear that the extracaveolar compartment and in particular the affinity and desensitization of $I_{\mathrm{Ks}}$ are the most sensitive to parameter changes. This may be explained by the high basal cAMP levels in the ECAV $(10 \mu \mathrm{mol} / \mathrm{L}$, within the micromolar range reported by lancu et al. ${ }^{11}$ ), which result in small dynamic range of PKA activity. With respect to the phosphorylation time-course, PLB is the most sensitive substrate. PLB is also the substrate with the fastest kinetics, based on experimental data (Figure 16), which may partly explain this sensitivity. Additionally, it becomes clear that parameters that affect whole cell properties (and hence multiple substrates) have a large impact on these output measures. Such parameters include cell volume, whole cell PKA levels and distribution of PKA. Consistent with experimental observations, ${ }^{5,43}$ these simulations also further emphasize a major role for PDEs and their specific subcellular localization in controlling substrate phosphorylation levels, as evident from the large impact of parameters such as $f_{\text {PART,PDE. }}$

The sensitivity analysis also indicates aspects of the model that could be simplified. In particular, the dynamics of cAMP/PKA binding as well as PKI-dependent inhibition of PKA appear to have little impact on phosphorylation. Indeed, the reaction rates for these interactions are significantly faster than CAMP production or substrate phosphorylation. This indicates that the CAMP/PKA pathway could also be modeled as a set of algebraic equations. Although this would significantly reduce the number of states (by 15 / 57 states in the signaling module), the reduction in the number of parameters would be modest (by 6 parameters). Finally, the parameters representing the affinities between substrates and AKAPs and between AKAPs and signaling molecules are determined to have a low impact. Experimentally, AKAPs are considered critical elements in the localization of PKA close to specific substrates. ${ }^{7}$ Their small effect in these simulations can be explained by the fact that the binding affinities involved are very high. Thus, a small change in affinity does not significantly affect the distribution of bound/free substrates.

The parameter sensitivity analysis described here provides insight into the contribution of individual parameters. It is possible that alteration of a subset of parameters is able to compensate for changes in a different parameter. This would provide further possibilities to simplify the model. However, such a combinatorial examination was found to be too demanding in terms of computation time and data storage.

\section{Validation}

The model is validated (Figures 7-24) with a wide range of experimental data at the levels of the signaling cascade, individual electrophysiological components and whole-cell response. 

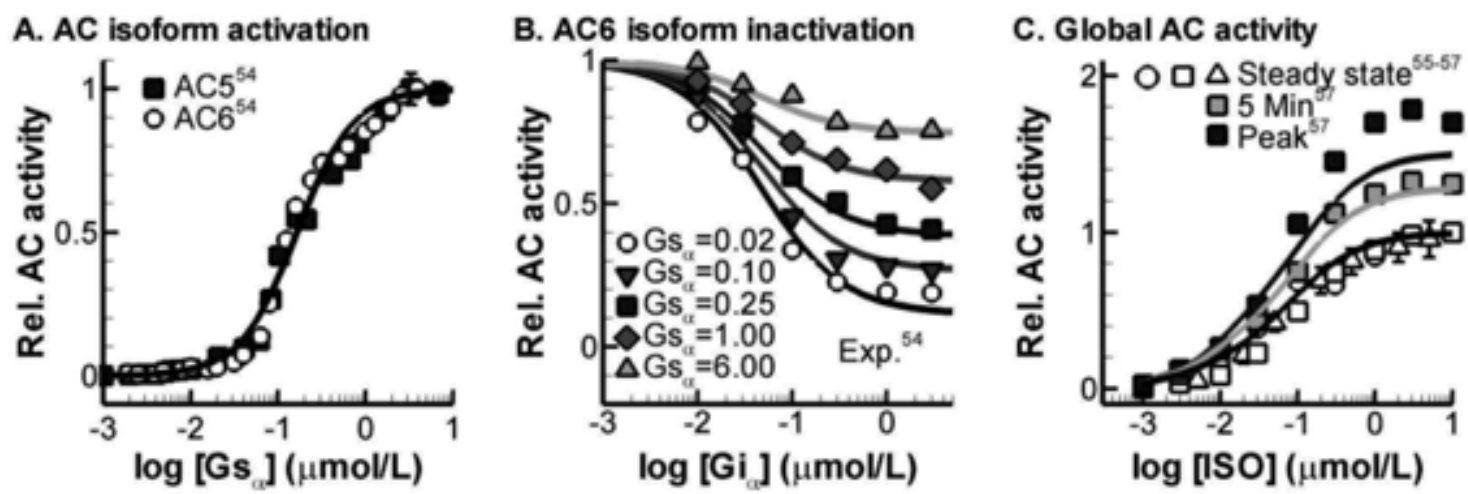

Figure 7. Comparison between adenylyl cyclase (AC) properties of model (lines) and experimental data (symbols). A. Gs-dependence of AC 5/6 in experiments and model. B. Inactivation of AC6 by Gi at various levels of Gs-stimulation in experiments ${ }^{54}$ and model. No error bars provided in experimental source. C. Simulated and measured whole-cell AC activity: (i) at peak (red), (ii) after 5 $\min$ (green) and (iii) at steady-state (white symbols; black line) in canine ventricular myocytes ${ }^{55,56}$ (circles, triangles) and Chinese hamster fibroblasts with human $\beta 1 A R s$ expressed (squares; no error bars provided in experimental source $)^{57}$. For color figure, see page 247.
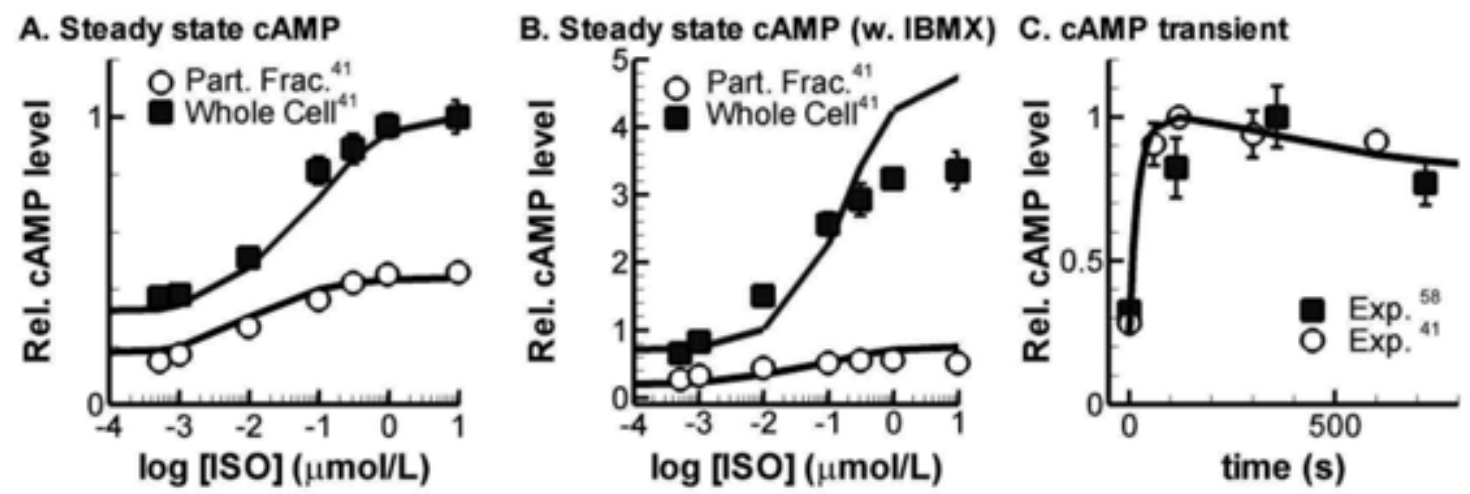

Figure 8. Comparison between CAMP properties of model (lines) and experimental data (symbols). A. Simulated and measured ${ }^{41}$ cAMP levels in whole-cell (filled) and particulate fraction (defined as CAV and ECAV compartmens in the model; open) of canine ventricular myocytes as function of [ISO]. B. Same as $A$ in the presence of $10 \mu \mathrm{mol} / L$ of the PDE inhibitor IBMX. Values in $A$ and $B$ are normalized to the maximal whole-cell cAMP level in the absence of IBMX. C. Temporal response of whole cell cAMP levels during stimulation with ISO in the model (line), canine $^{41}$ (circles) and rat $^{58}$ (squares) ventricular myocytes. 
A. Whole cell cAMP

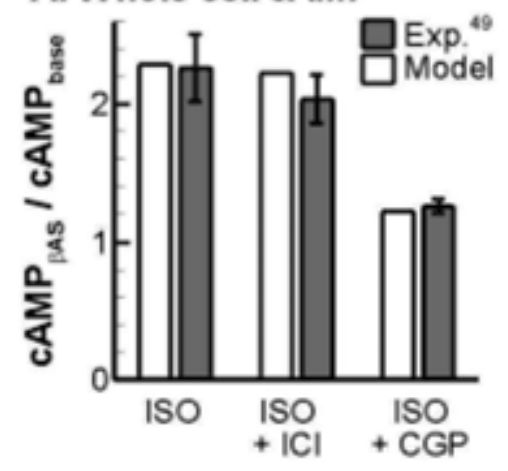

B. Localized cAMP

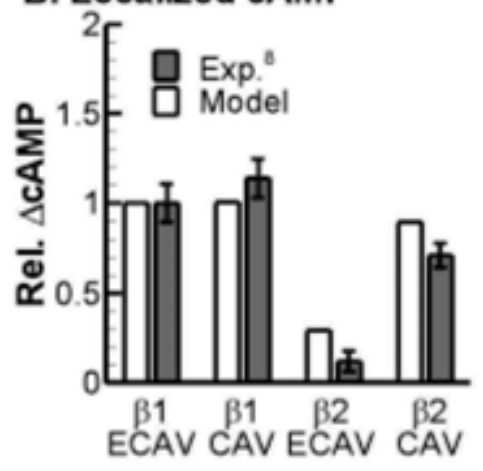

C. PLB Phosph. w. B2AR Stim.

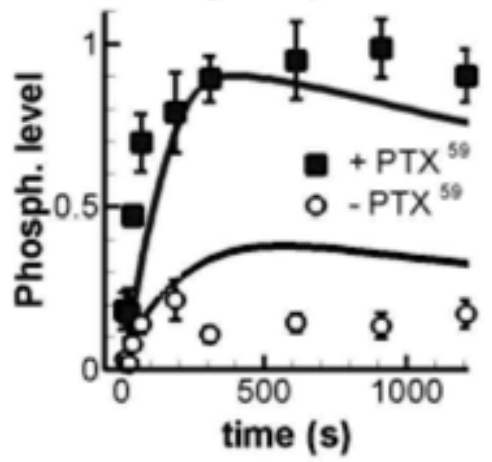

Figure 9. Comparison between effects elicited by $\beta 1 A R$ or $\beta 2 A R$ stimulation in model (white bars and lines) and experiments (shaded bars, symbols). A. Fractional increase in whole-cell cAMP compared to baseline elicited by $100 \mathrm{nmol} / \mathrm{L}$ ISO (left), ISO + $\beta 2 A R$ antagonist (ICl; middle) and ISO + $\beta 1 \mathrm{AR}$ antagonist (CGP; right) in model and canine ventricular myocytes ${ }^{49}$. B. FRET changes of the HCN cAMP sensor in T-tubule or cell-membrane crest in response to local stimulation of $\beta 1 A R(I S O+I C I)$ or $\beta 2 A R$ (ISO + CGP) in rat ventricular myocytes ${ }^{8}$ compared to CAMP changes after stimulation of CAV domain only (corresponding to T-tubule; e.g., containing $I_{\text {CaL }}$ channels) or ECAV domain only (corresponding to cell crest) of model. Values are normalized to ECAV response with $\beta 1 A R$ stimulation. $\beta 1 A R$ stimulation produces a cell wide signal, whereas $\beta 2 A R$ response is local. Magnitude differs because of species differences and nonlinearity of the sensor. $C$. Time course of PLB phosphorylation during $\beta 2 A R$ stimulation (with zinterol) in rat ventricular myocytes in the absence (white) or presence (red) of pertussis toxin (PTX) (19 $^{59}$ compared to $\beta 2 A R$ stimulation in the model $(0.1 \mu \mathrm{mol} / \mathrm{L}$ ISO + CGP). Treatment with PTX is simulated by setting the concentration of Gi to 0.0 . Both in model and experiments, stimulation of $\beta 2 A R$ does not result in phosphorylation of cytosolic proteins unless PTX is added.
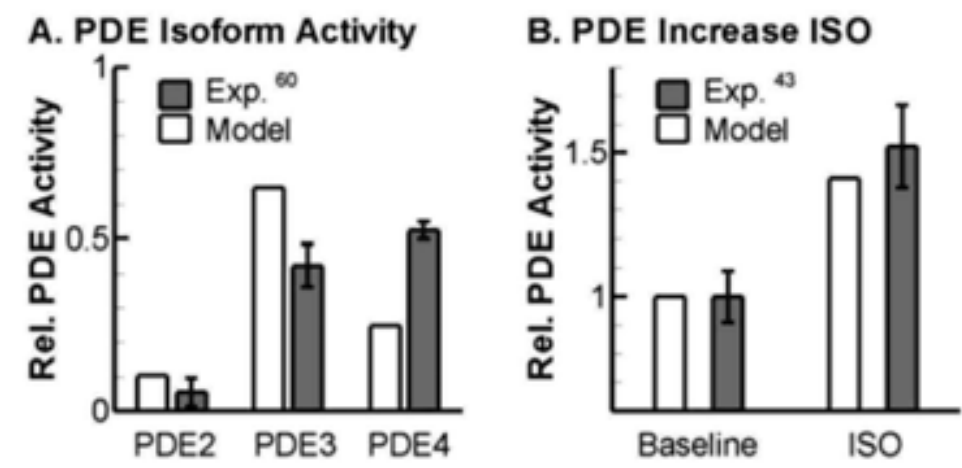

Figure 10. Validation of PDE properties. A. Relative PDE activity under basal conditions in model (white bars) and adult rat ventricular myocytes ${ }^{60}$ (grey bars) in the presence of $1 \mu \mathrm{mol} / \mathrm{L}$ of CAMP as assessed by specific inhibition of each isoform. Quantitative differences between contribution of PDE3 and PDE4 can be explained by species differences. ${ }^{61} \mathrm{~B}$. Relative whole-cell PDE activity under baseline conditions or in the presence of ISO in model (white) and adult rat ventricular myocytes ${ }^{43}$ (blue) showing increased PDE activity resulting from PKA-dependent phosphorylation. 

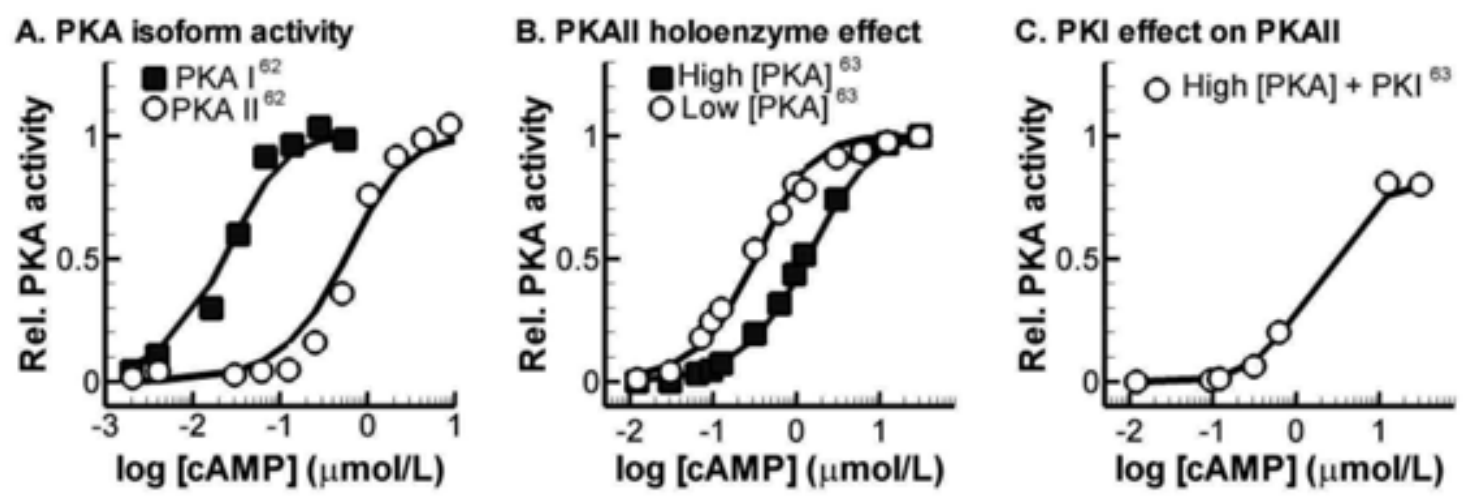

Figure 11. Comparison between PKA properties of model (lines) and experimental data (symbols). A. Simulated and measured ${ }^{62}$ cAMP dependence of activation of PKA type I (filled symbols) and II (open symbols). B. cAMP dependence of high (red) and low (black) concentrations of PKA type II holoenzyme. C. Effect of PKI on PKAll activity in the presence of high concentration of holoenzyme. No error bars provided in experimental sources. ${ }^{63}$
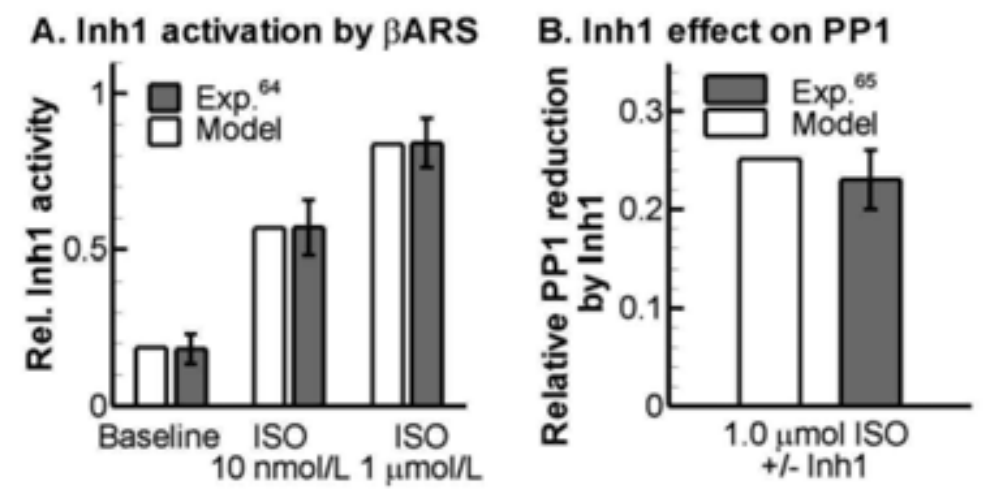

Figure 12. Validation of protein phosphatase inhibitor 1 (Inh1) properties. A. Relative Inh1 activity (compared to total Inh1 concentration) at baseline, during stimulation with $10 \mathrm{nmol} / \mathrm{L} \mathrm{ISO}$ and during stimulation with $1 \mu \mathrm{mol} / \mathrm{L}$ ISO in model (white bars) and experiments in guinea-pig ventricular preparations (grey bars) ${ }^{64}$. B. Relative reduction in PP1 by Inh1 in the presence of $1 \mu \mathrm{mol} / \mathrm{L}$ ISO. Yaxis shows fractional reduction relative to value in absence of $\operatorname{lnh} 1$ in model (white bar) and mouse ventricle (grey bar) ${ }^{65}$. 
A. ICaL Phosphorylation

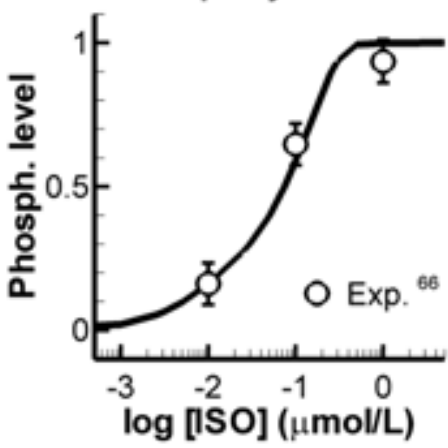

C. INa Phosphorylation

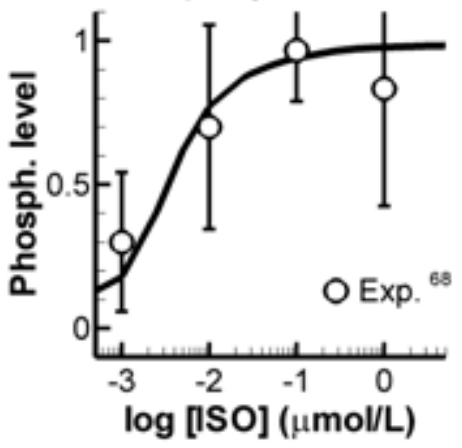

B. RyR Phosphorylation

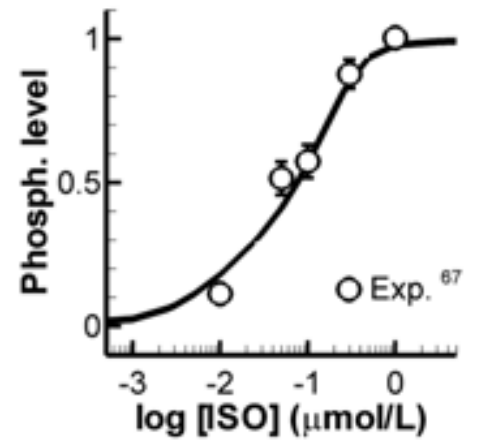

D. INaK Phosphorylation

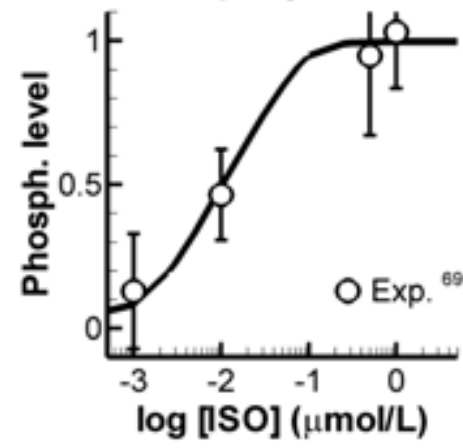

Figure 13. Relative phosphorylation level of CAV substrates as function of ISO in model (lines) and experiments (symbols). A. I Iat phosphorylation compared to relative increase in peak I-V during voltage-clamp experiments in canine-ventricular myocytes. ${ }^{66}$ B. RyR phosphorylation compared to intensity obtained with a Ser 2030 phosphospecific RyR antibody in rat cardiac myocytes. ${ }^{67} \mathrm{C}$. I Ia phosphorylation compared to relative increase in peak $I_{\mathrm{Na}}$ during voltage-clamp to $0 \mathrm{mV}$ in rat papillary muscle. ${ }^{68} \mathrm{D}$. $I_{\text {Nak }}$ phosphorylation dose-response compared to relative increase in pump current (DHO sensitive at $-60 \mathrm{mV}$ ) in guinea-pig ventricular myocytes. ${ }^{69}$
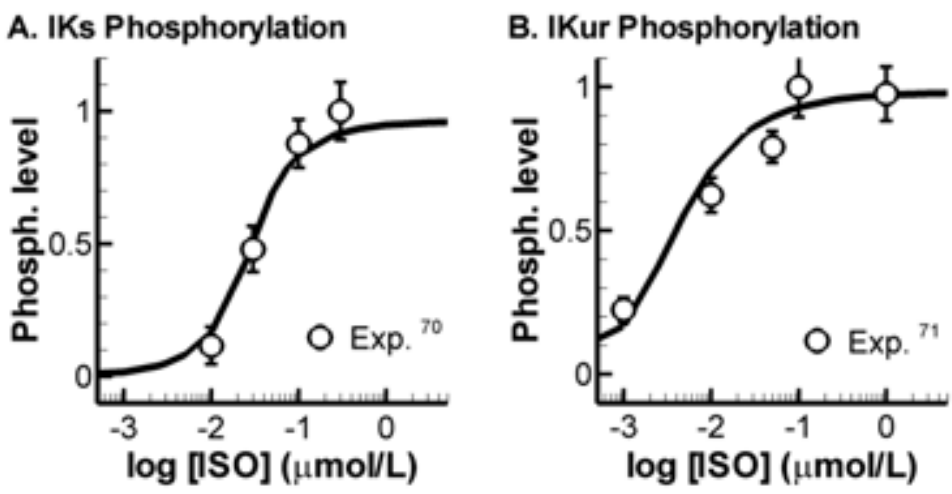

Figure 14. Relative phosphorylation level of ECAV substrates as function of ISO in model (lines) and experiments (symbols). A. $I_{\mathrm{Ks}}$ phosphorylation in model and relative $I_{\mathrm{Ks}}$ (HMR1556 sensitive) increase in canine ventricular myocytes during $3 \mathrm{~s}$. depolarizing pulses to $+50 \mathrm{mV}^{70}$. B. I $I_{\text {Kur }}$ phosphorylation compared to relative change in $I_{\text {Kur }}$ (separated from $I_{\text {To }}$ by an inactivating prepulse) during $140 \mathrm{~ms}$ depolarizing pulses to $+50 \mathrm{mV}$ in canine atrial myocytes. ${ }^{71}$ 

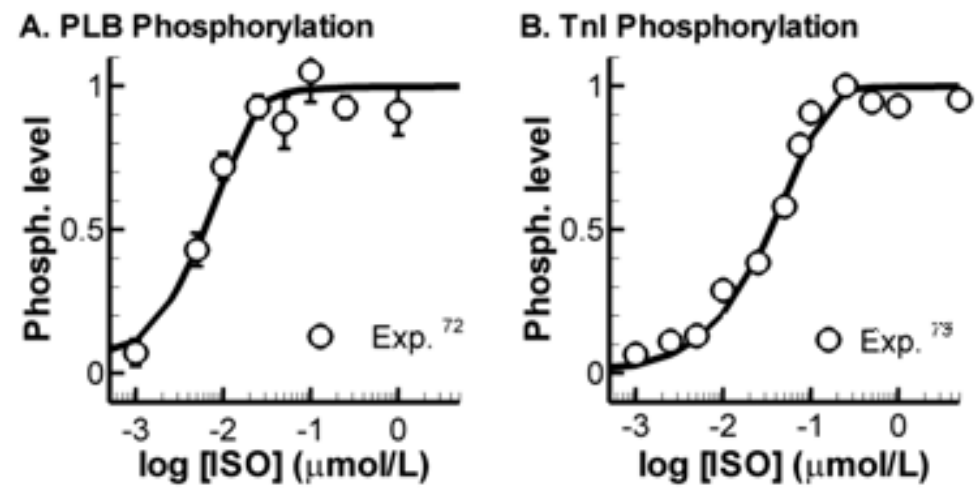

Figure 15. Relative phosphorylation level of CYT substrates as function of ISO in model (lines) and experiments (symbols). A. PLB phosphorylation compared to relative intensity obtained with a Ser 16 phosphospecific PLB antibody in rat heart preparations after treatment with various doses of ISO. ${ }^{72}$ B. Tnl phosphorylation compared to radioblots of ${ }^{32} \mathrm{P}$ incorporation in $\mathrm{Tnl}$ in rat cardiac myocytes (no error bars provided in experimental source). ${ }^{73}$
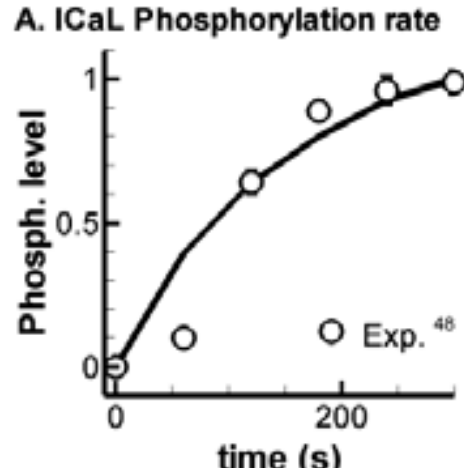
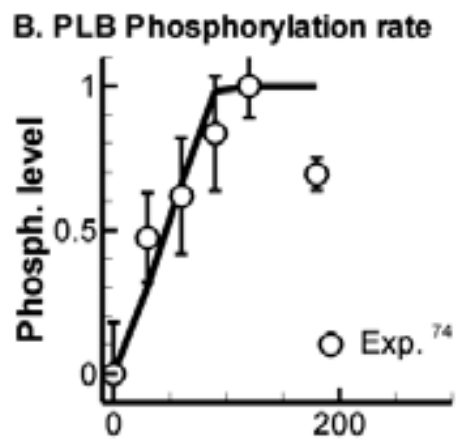

time (s)

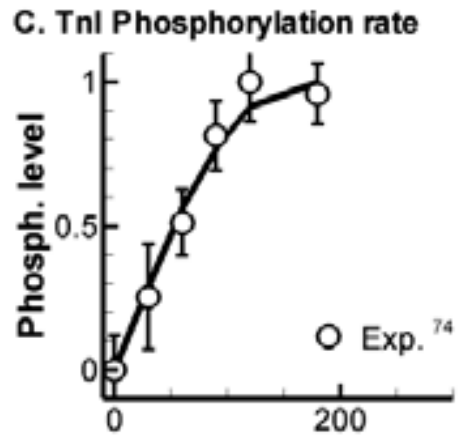

time (s)

Figure 16. Time-dependence of substrate phoshorylation. A. Phosphorylated fraction of $I_{\text {Cat }}$ channels in unpaced model $\left(\hat{\mathrm{f}}_{\mathrm{Ca}, \mathrm{L}}^{\mathrm{P}}\right.$; line) compared to relative increase in peak $\mathrm{I}_{\mathrm{CaL}}$ during repetitive voltageclamp commands to $0 \mathrm{mV}$, elicited every 60 seconds in canine ventricular myocytes in the presence of $0.5 \mu \mathrm{mol} / \mathrm{L}$ ISO. ${ }^{48}$ B. Fraction of phosphorylated PLB ( $f_{\text {up }}^{\mathrm{PKA}}$ ) in unpaced model (line) compared to relative ${ }^{32} \mathrm{P}$ incorporation in PLB in unpaced mouse ventricular myocytes treated with $1.0 \mu \mathrm{mol} / \mathrm{L}$ ISO. ${ }^{74} \mathrm{C}$. Same as (B) for TnI. The time-instant $t=0$ indicates the start of $\beta$ AR stimulation. All other substrates $\left(I_{\mathrm{Na}}, I_{\mathrm{NaK}}, I_{\mathrm{Ks}}, I_{\mathrm{Kur}}, \mathrm{RyR}\right)$ for which no rate of phosphorylation was available, were given the same phosphorylation time-course as $\mathrm{I}_{\mathrm{CaL}}$. Validation of CaMKII-dependent phosphorylation timecourses can be found in Figure 24. 
A. ICaL I-V

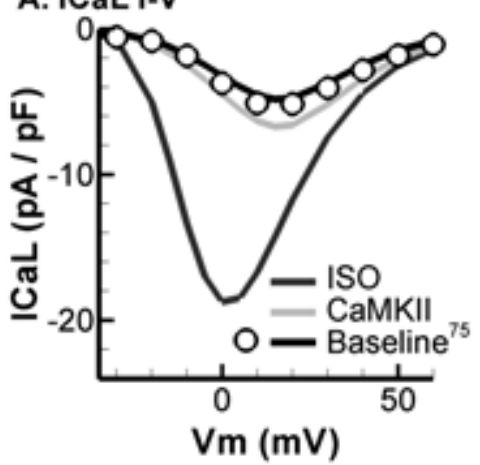

D. ICaL recovery from inactivation

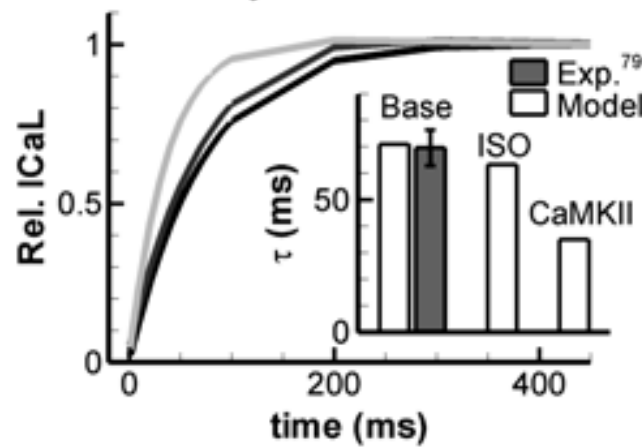

B. ICaL inactivation

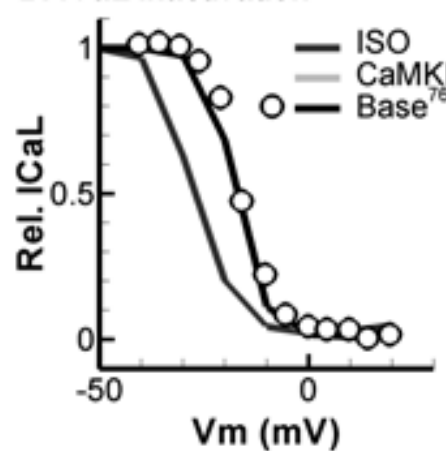

C. ICaL modulation

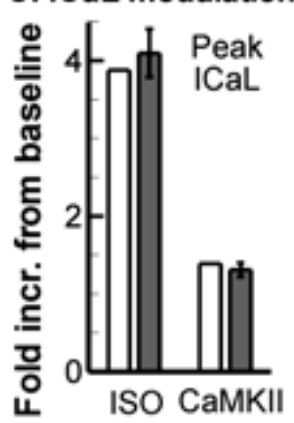

E. ICaL time course of inactivation

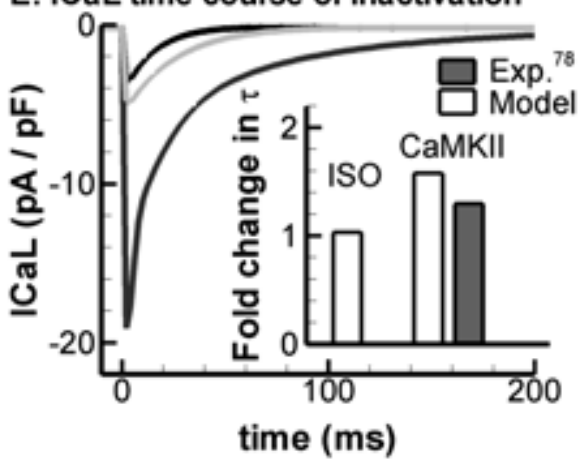

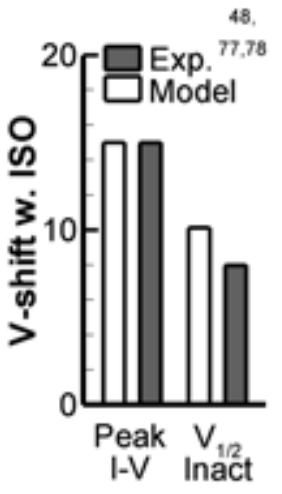

Figure 17. Simulated characteristics of PKA phosphorylated (red), CaMKII phosphorylated (orange) and non-phosphorylated (black) populations of $\mathrm{I}_{\mathrm{CaL}}$. Experimental data are shown as symbols, model output as lines. A. Peak I-V relationship. ${ }^{75} \mathrm{~B}$. Voltage dependence of steady-state $\mathrm{I}_{\mathrm{CaL}}$ inactivation. ${ }^{76} \mathrm{C}$. Fold increase in peak $I_{\text {CaL }} I-V$ with ISO or maximal CaMKII activation (left) and shift in peak I-V and midpoint of inactivation ( $\mathrm{V}_{1 / 2}$ Inact; right) during stimulation with ISO in model (white) and experiments ${ }^{48,77,78}$ (blue). D. Recovery from inactivation. Inset shows time-constants under baseline, ISO or CaMKII conditions based on a mono-exponential fit in model (white bars) and canine epicardial myocytes (blue bar). ${ }^{79} \mathrm{E}$. Time course of $\mathrm{I}_{\mathrm{Ca}}$ inactivation during a $200 \mathrm{~ms}$ depolarizing pulse to $0 \mathrm{mV}$. Inset shows the relative change in time constant of inactivation with $1.0 \mu \mathrm{mol} / \mathrm{L}$ ISO or maximal CaMKII activation. CaMKII-dependent effects are consistent with experiments in rabbit ventricular myocytes. ${ }^{78}$ For color figure, see page 247.
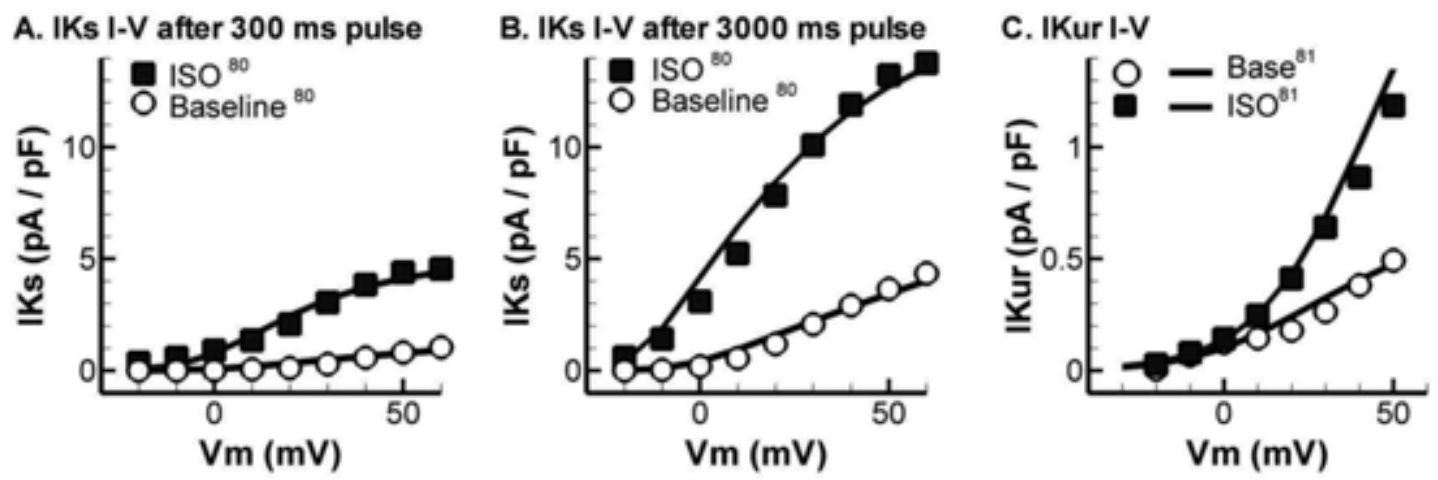

Figure 18. Simulated characteristics of phosphorylated (filled symbols) and non-phosphorylated (open symbols) populations of $I_{\mathrm{Ks}}$ and $I_{\mathrm{Kur}}$. Experimental data are shown as symbols, model output as 
lines. A. $I_{\mathrm{ks}}$ tail-current amplitude after 300 -ms depolarizing pulses in model and canine ventricular myocytes. ${ }^{80} \mathrm{~B}$. Same as A, after 3000-ms depolarizing pulses. C. I Kur I-V in canine ventricular myocytes (in response to $100 \mathrm{nmol} / \mathrm{L} \mathrm{ISO})^{81}$ and model.
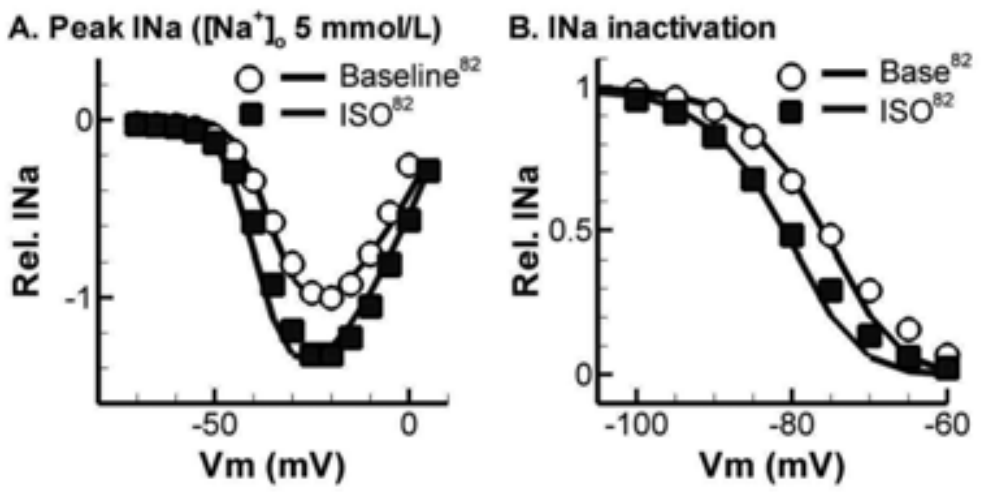

Figure 19. Simulated characteristics of phosphorylated (filled symbols) and non-phosphorylated (open symbols) populations of $I_{\text {Na. }}$. Experimental data are shown as symbols, model output as lines. A. $I_{\mathrm{Na}}$ peak I-V relationship in model and canine ventricular myocytes. ${ }^{82}$ B. Steady-state $I_{\mathrm{Na}}$ inactivation.
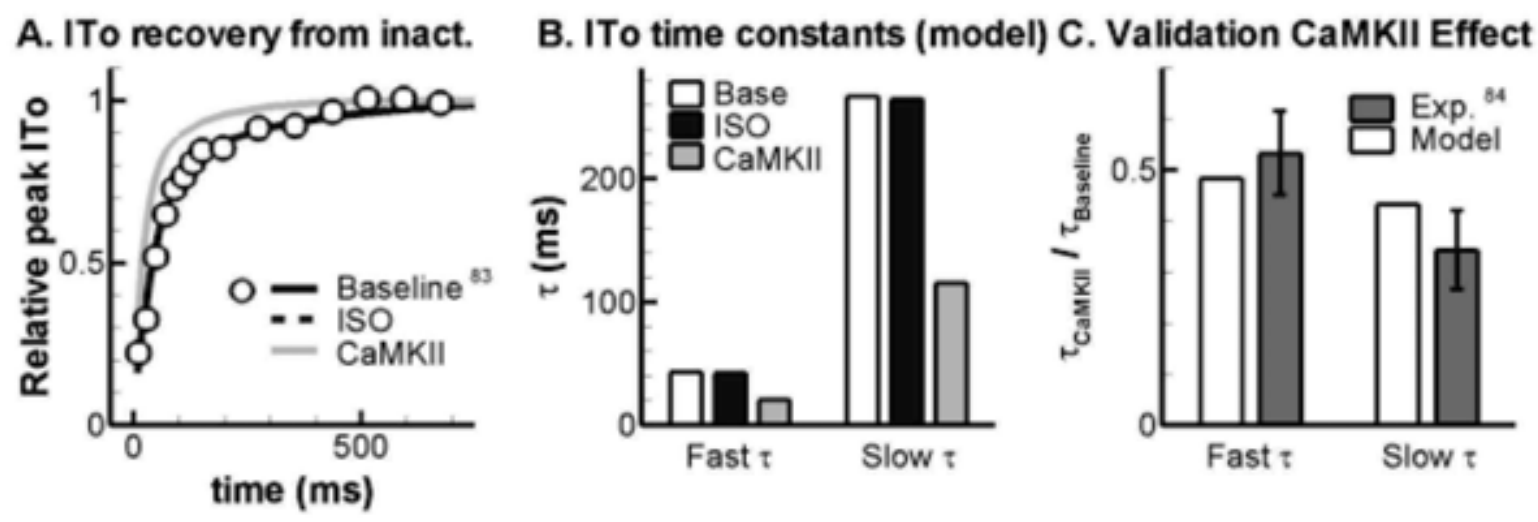

Figure 20. Characteristics of $I_{\text {To }}$ recovery from inactivation at baseline (black), with maximal CaMKII activity (orange) or in the presence of $1.0 \mu \mathrm{mol} / \mathrm{L}$ ISO (red; identical to baseline). A. Simulated (line) time course of $\mathrm{I}_{\text {To }}$ recovery from inactivation (assessed using a double-pulse protocol to $+40 \mathrm{mV}$ for $200 \mathrm{~ms}$ from a holding potential of $-80 \mathrm{mV}$ ) compared to experimental data (white circles) from canine ventricular myocytes under baseline conditions. ${ }^{83}$ B. Comparison of model fast and slow recovery time constants (based on a biexponential fit to the data in A). C. Validation of the effect of CaMKII on $\mathrm{I}_{\text {To }}$ recovery from inactivation in model (white) compared to data in rabbit ventricular myocytes acutely overexpressing CaMKII (blue).$^{84}$ For color figure see page 248. 

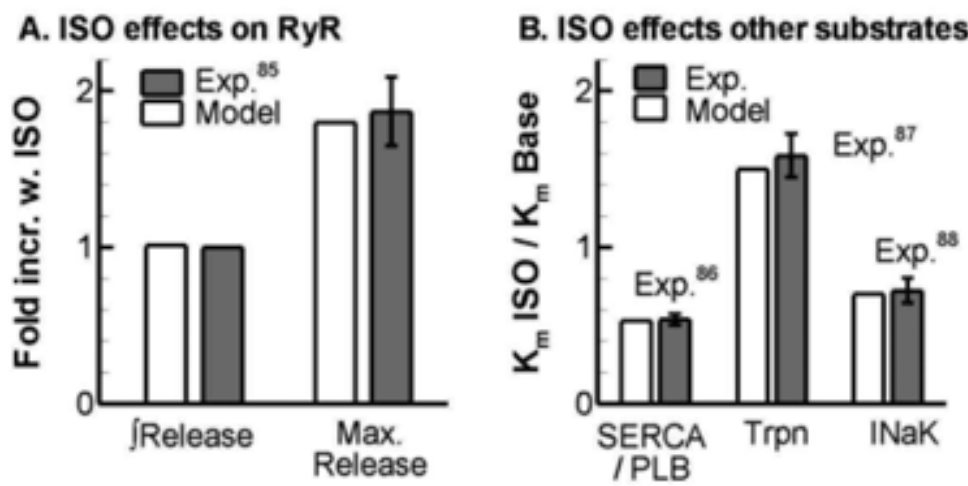

Figure 21. Effects of ISO on RyR, PLB, TnI and $I_{\mathrm{Nak}}$. A. Simulated (white bars) relative change in total ( $S$ Release; left) and maximum SR-Ca ${ }^{2+}$-release flux (Max. Release; right) for phosphorylated RyRs compared to baseline (no $\beta A R S$ ) under conditions of identical SR- $\mathrm{Ca}^{2+}$ loading and $\mathrm{I}_{\mathrm{Cat}}$ trigger strength. Corresponding experimental data ${ }^{85}$ (from rabbit ventricular myocytes) are shown in grey bars. B. Relative change in PLB Ca ${ }^{2+}$ affinity (left), troponin $\mathrm{Ca}^{2+}$ affinity (middle) and $\mathrm{I}_{\mathrm{Nak}}$ intracellular $\mathrm{Na}^{+}$affinity (right) in the presence of ISO (compared to baseline without ISO). Simulation shown in white bars; experimental data in grey bars. ${ }^{86-88}$

A. APD

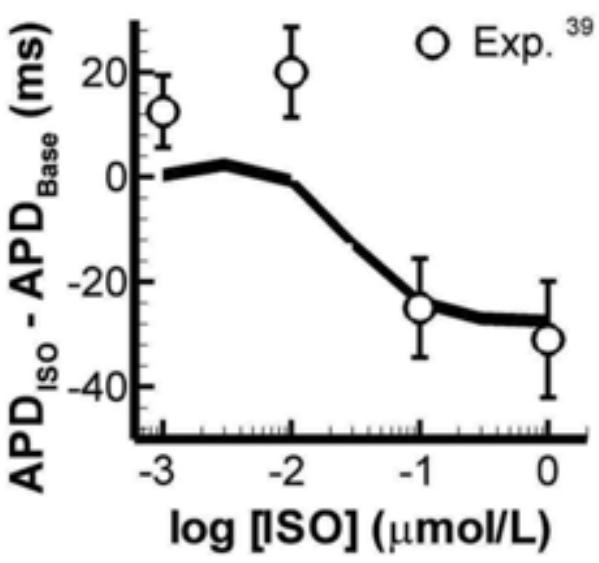

\section{B. CaT}

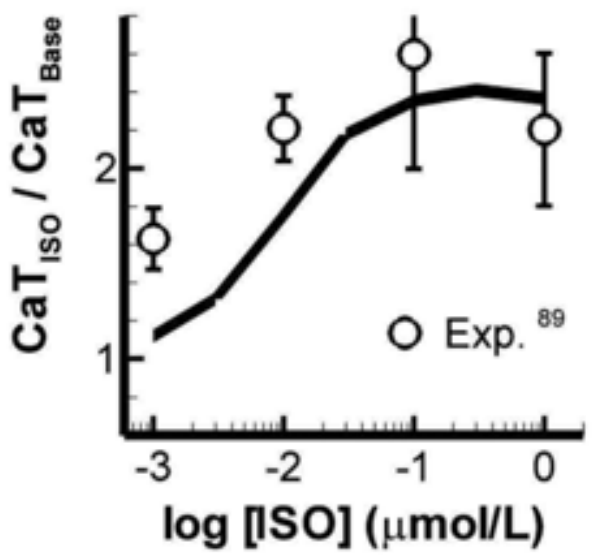

\section{Time course APD}

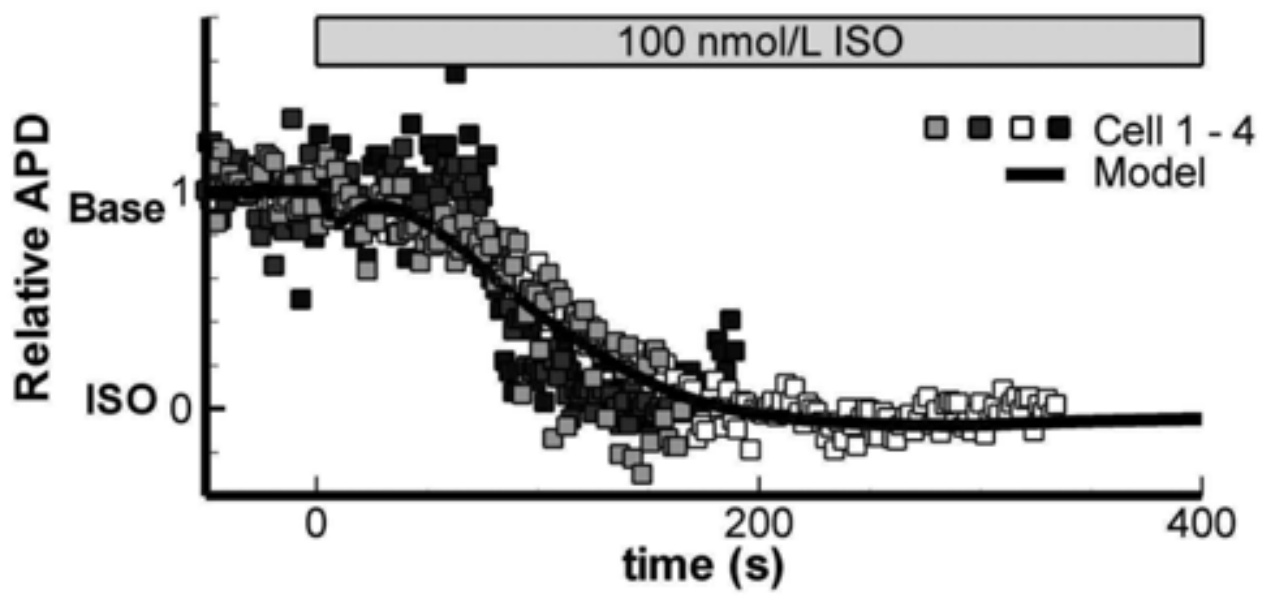


Figure 22. Concentration-dependent effects of ISO on APD (A; $C L=1000 \mathrm{~ms})$ and CaT amplitude (B; $\mathrm{CL}=2000 \mathrm{~ms}$ ) in canine ventricular myocytes (symbols ${ }^{39,89}$ ) and model (lines). C. Time course of APD changes during application of $100 \mathrm{nmol} / \mathrm{L}$ ISO in 4 canine ventricular myocytes (symbols) and model (line). APDs were normalized between 1 (baseline) and 0 (maximal response to ISO) to account for baseline cell to cell differences (see also Figure 26). $\mathrm{CL}=1000 \mathrm{~ms}$. For experimental protocol, see Johnson et al. ${ }^{90}$ For color figure see page 248.

\section{A. Experiment $0.0 \mu \mathrm{mol} / \mathrm{L} \mathrm{ISO}^{91}$}
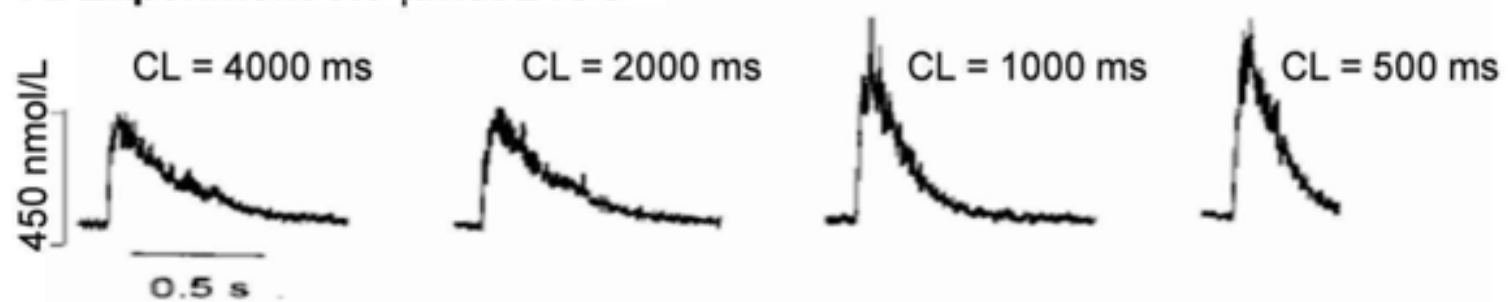

\section{B. Model $0.0 \mu \mathrm{mol} / \mathrm{L}$ ISO}
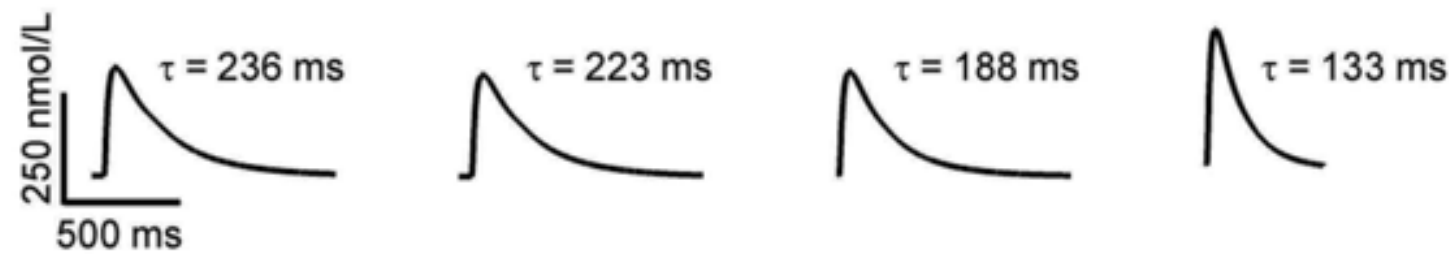

\section{Model $1.0 \mu \mathrm{mol} / \mathrm{L}$ ISO}
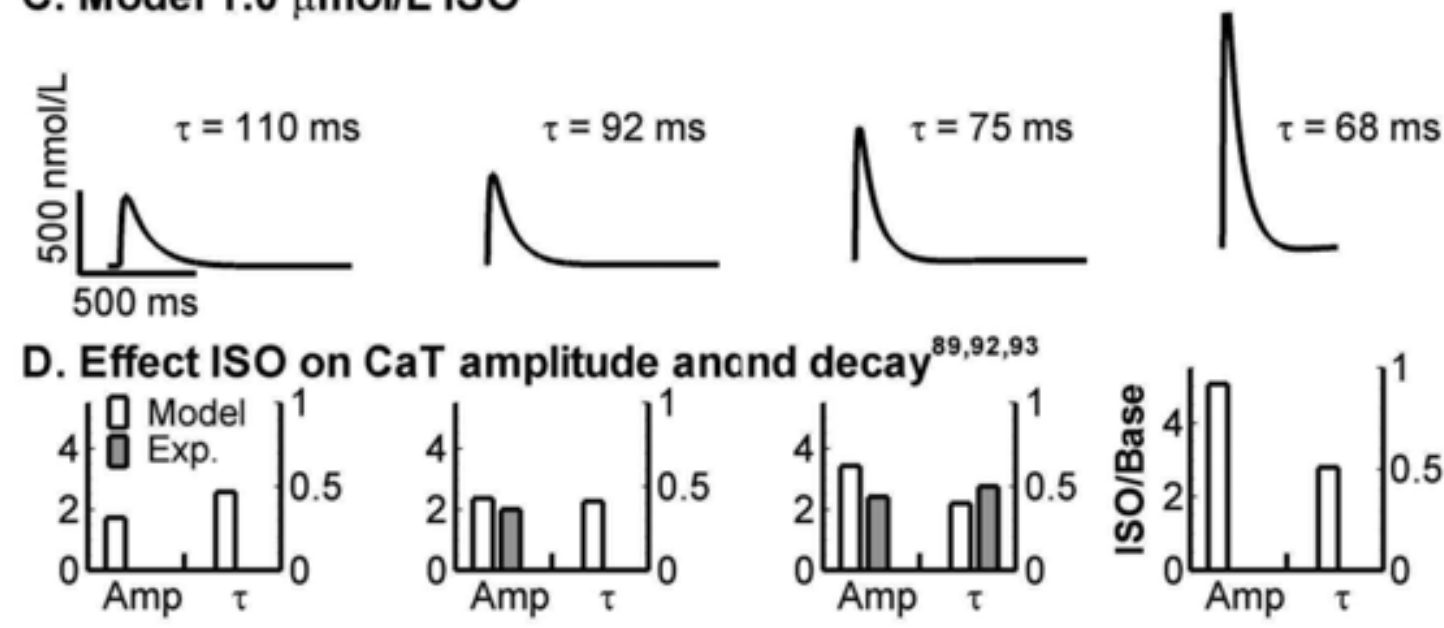

$\mathrm{CL}=4000 \mathrm{~ms}$
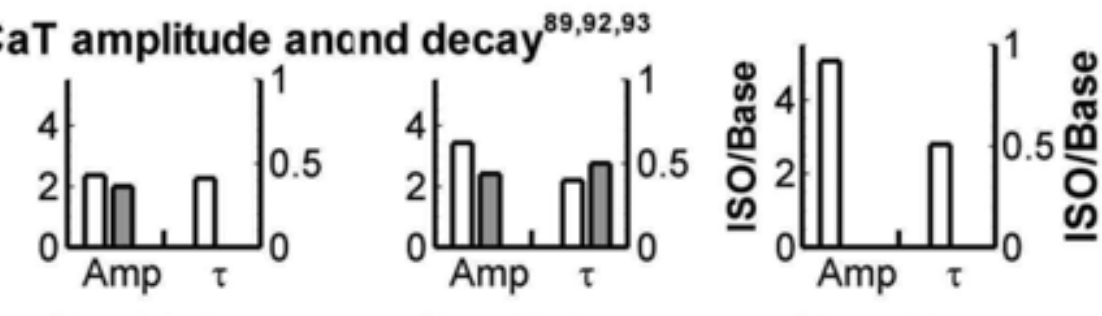

Figure 23. A. CaT recorded in canine ventricular myocytes in the absence of adrenergic stimulation at $\mathrm{CL}$ of $4000 \mathrm{~ms}, 2000 \mathrm{~ms}, 1000 \mathrm{~ms}$ and $500 \mathrm{~ms}$. Reproduced from Ref. ${ }^{91}$, with permission. B. CaT in the model under similar conditions shows a comparable increase with rate in CaT amplitude and rate of decay. C. CaT in the model during maximal adrenergic stimulation shows increased CaT amplitude and rate of decay at all CLs. D. Quantification of the effect of ISO on CaT amplitude and rate of decay expressed as the ratio of CaT amplitude (Amp; left bars) and time-constant (of a mono-exponential fit; $\tau$, right bars) in the presence and absence of $1.0 \mu \mathrm{mol} / \mathrm{L}$ ISO at all (same) CLs. Experimental data ${ }^{89}$, 92,93 are shown in grey, model data in white. 

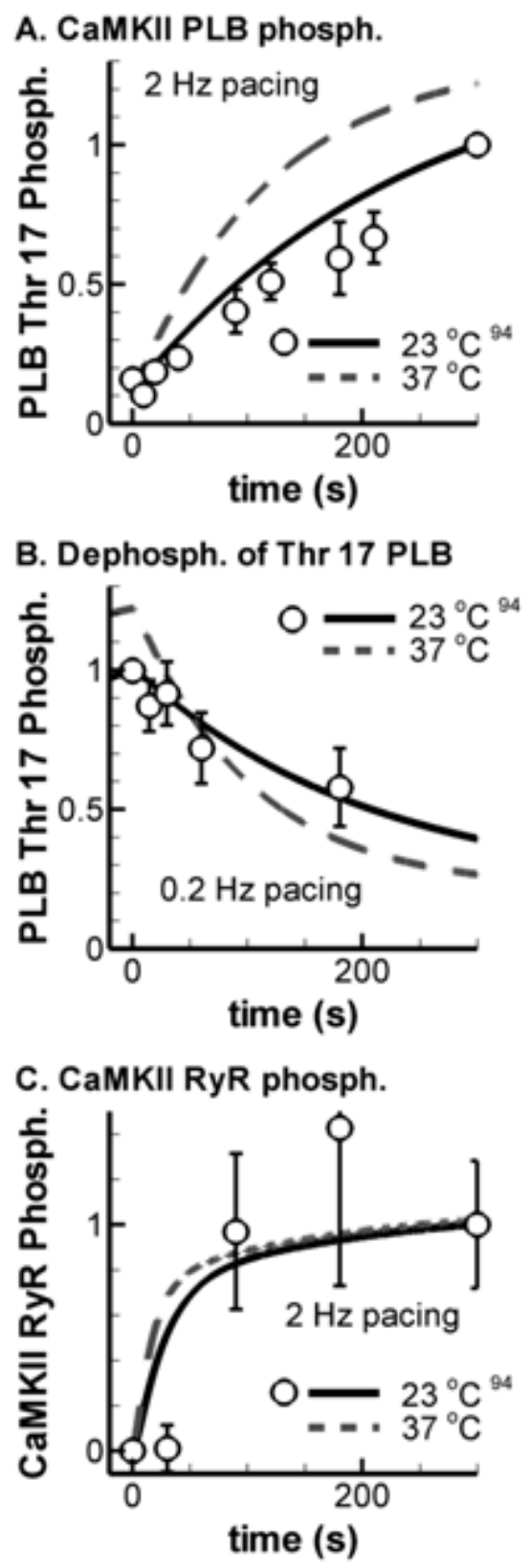

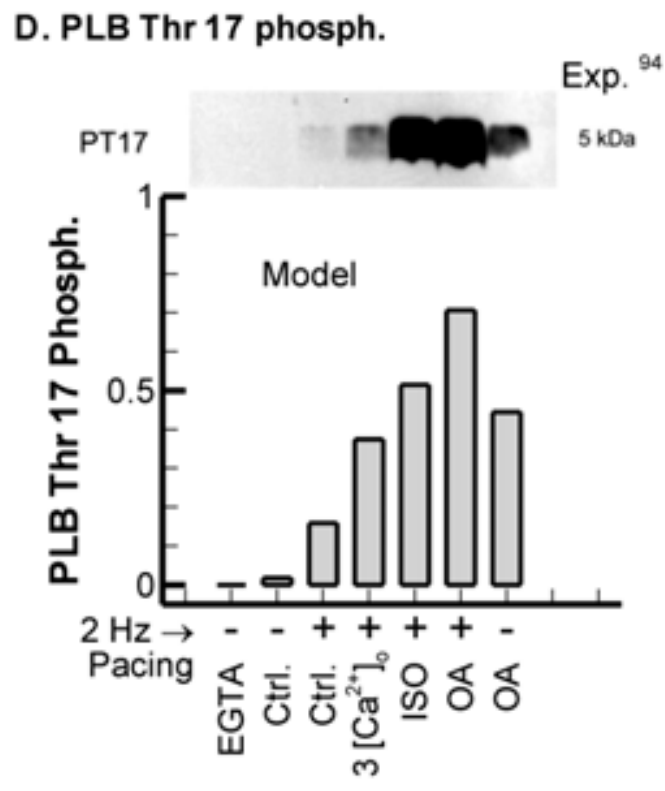

E. CaMKII autophosphorylation

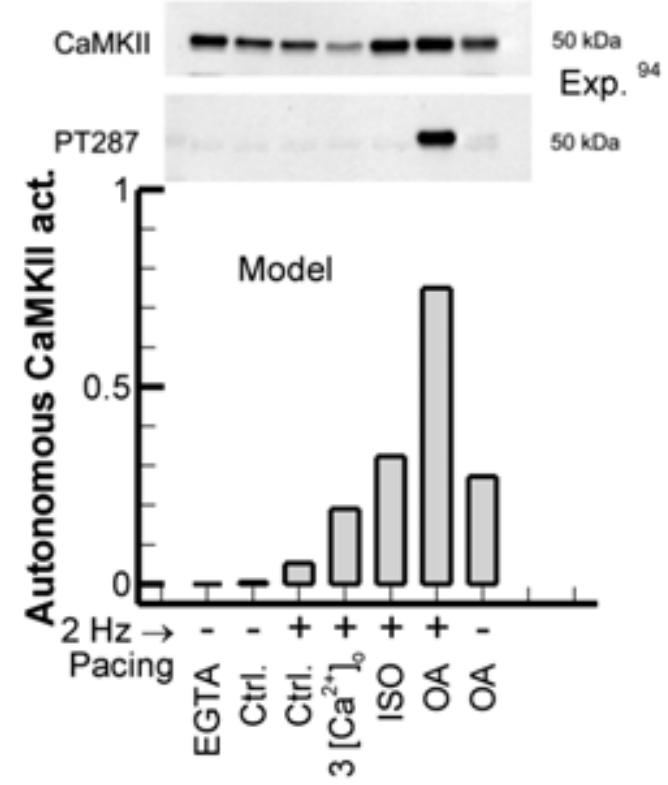

Figure 24. Validation of CaMKII signaling and phosphorylation. A. Time course of CaMK phosphorylation of PLB at Thr 17 during pacing at $2 \mathrm{~Hz}$ under baseline conditions. Model data (solid black line) were determined based on data from experiments in rat ventricular myocytes using a Thr 17 phosphospecific antibody after $2 \mathrm{~Hz}$ pacing at room temperature (symbols). ${ }^{94}$ Time constant of phosphorylation was subsequently adjusted by a factor of 2.0 to perform simulations at $37^{\circ} \mathrm{C}$ (dashed lines). All data were normalized to the maximum PLB phosphorylation at room temperature. B. Similar to panel A for deactivation of PLB Thr 17 phosphorylation at $0.2 \mathrm{~Hz}$ after pacing for $5 \mathrm{~min}$ at $2 \mathrm{~Hz}$. C. Similar to panel A for CaMKII phosphorylation of RyR during pacing at $2 \mathrm{~Hz}$, compared to experimental data obtained with a Ser 2815 phosphospecific RyR antibody. D. CaMKII dependent phosphorylation of PLB at steady state under various conditions in model (bars) compared to experimental Western blots with an antibody against phosphorylated PLB at Thr $17 .{ }^{94}$ The model shows strong CaMKII-dependent PLB phosphorylation during pacing $(2 \mathrm{~Hz})$ in the presence of high $\left[\mathrm{Ca}^{2+}\right]_{0}$ or ISO, or with phosphatase inhibition (with okadaic acid, OA, simulated as $80 \%$ reduction in 
whole cell PP1); consistent with experimental observations. E. Western blots showing CaMKII autophosphorylation at Thr 287 that occurs only in the presence of OA and pacing. ${ }^{94}$ The model shows qualitatively similar behavior of CaMKtrap, although lower levels of autonomous CaMKII activity can also be observed with ISO or high $\left[\mathrm{Ca}^{2+}\right]_{0}$ during pacing, or with $\mathrm{OA}$ in the absence of pacing.

\section{Simulation protocols}

The model consists of a coupled system of 134 ordinary differential equations governed by the equations described in the appendix of this thesis. Numerical integration was performed in custom C++ software using a forward Euler method with variable time step. The Matlab (The Mathworks ${ }^{\circledR}$, Natick, MA) ODE15s steady-state solution and our custom $\mathrm{C}++$ forward Euler method produced identical action potentials and calcium transients.

The model was paced for 2000 seconds at any given setting (cycle length (CL) and ISO concentration) with a conservative $\mathrm{K}^{+}$stimulus $^{95}$ to achieve a pseudo steady-state in which APD (determined at $95 \%$ of repolarization) and maximum intracellular ion concentrations change less than $0.05 \%$ on a beatto-beat basis. Restitution data were obtained for one additional beat after pacing to steady-state for a range of $S 2$ coupling intervals. Temporal effects of $\beta A R$ stimulation were determined by pacing to steady-state in the absence of ISO, followed by pacing in the presence of a given ISO concentration. ISO levels ranged between $0 \mu \mathrm{mol} / \mathrm{L}$ (no $\beta A R$ stimulation) and $1.0 \mu \mathrm{mol} / \mathrm{L}$ (maximum $\beta A R$ stimulation), with $0.02 \mu \mathrm{mol} / \mathrm{L}$ producing approximately half-maximal effects. 


\section{RESULTS}

\section{Whole-cell effects of $\beta$ ARS on APD and CaT}
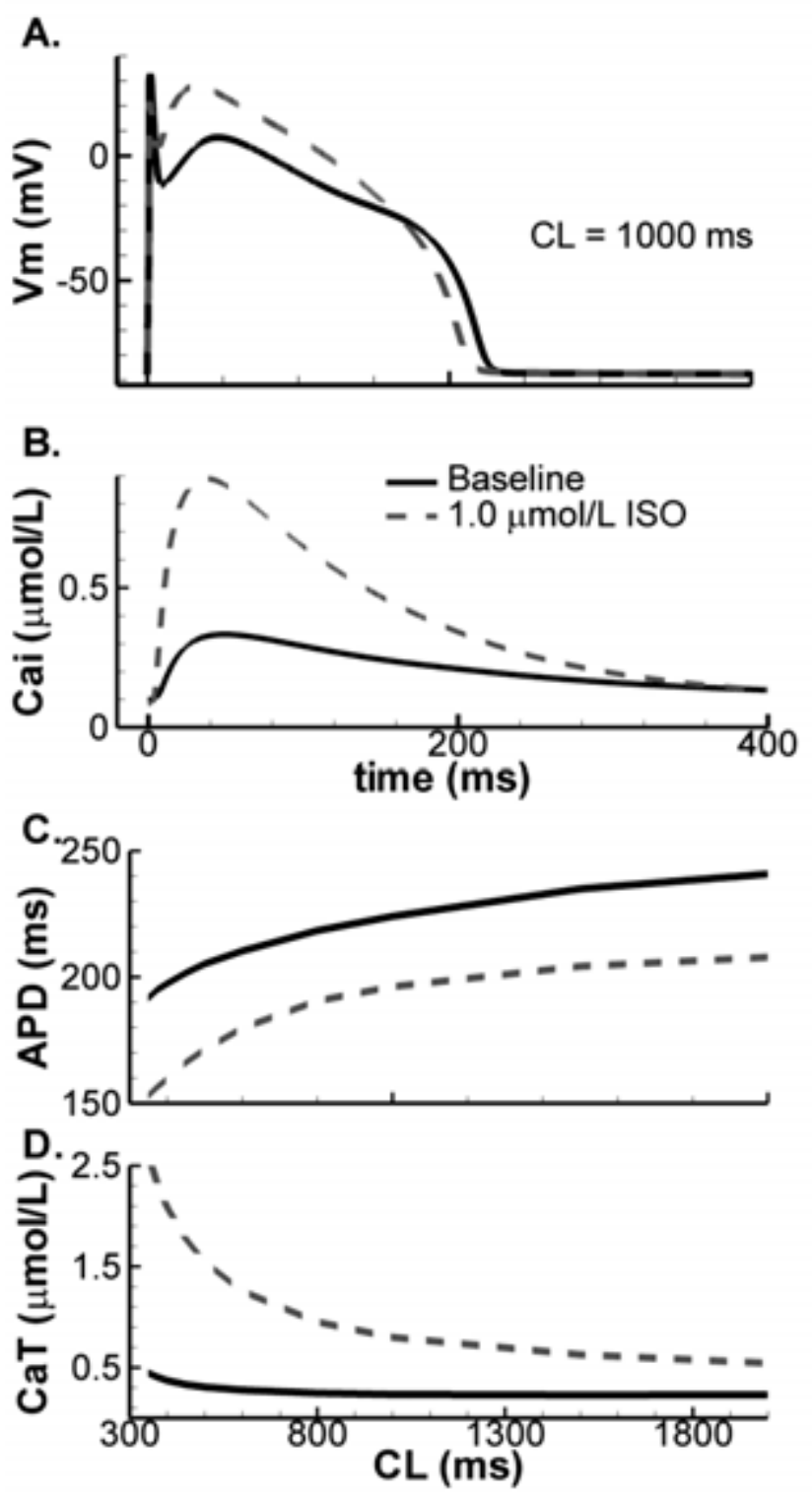

Figure 25. Effect of $\beta A R S$ on whole-cell AP and CaT. Baseline shown in solid lines, maximal $\beta A R S$ in dashed lines. A. Steady-state $A P$ at $C L=1000$. B. Simulated calcium transient at $C L=1000 \mathrm{~ms}$ in the presence of $\beta A R S$ shows increased amplitude and rate of relaxation compared to baseline. $C$. Steadystate APD as a function of pacing CL. D. Steady-state CaT amplitude rate-dependence.

Subcellular alterations of various substrates in response to $\beta A R S$ combine at the cellular level to change the AP morphology and CaT. Figure 25A shows that $\beta A R S$ results in shortening of APD and increase in AP plateau amplitude, consistent with experimental data. ${ }^{80}$ Experimentally, there is considerable cell-to-cell variation in the magnitude of the response (Figure 26) and model output is within this range. At $\mathrm{CL}=1000 \mathrm{~ms}$, CaT amplitude is significantly enhanced and CaT decay is faster, as observed experimentally (Figure 23; Figure 25B). We analyzed the rate-dependence of these APD 
and $\mathrm{CaT}$ changes by pacing the model to steady-state in absence or presence of a saturating dose of ISO at various CL. APD shortening and increased CaT amplitude are observed at all CL (Figure 25C,D). Changes in CaT amplitude and $\mathrm{Ca}^{2+}$ loading are most pronounced at fast rates.
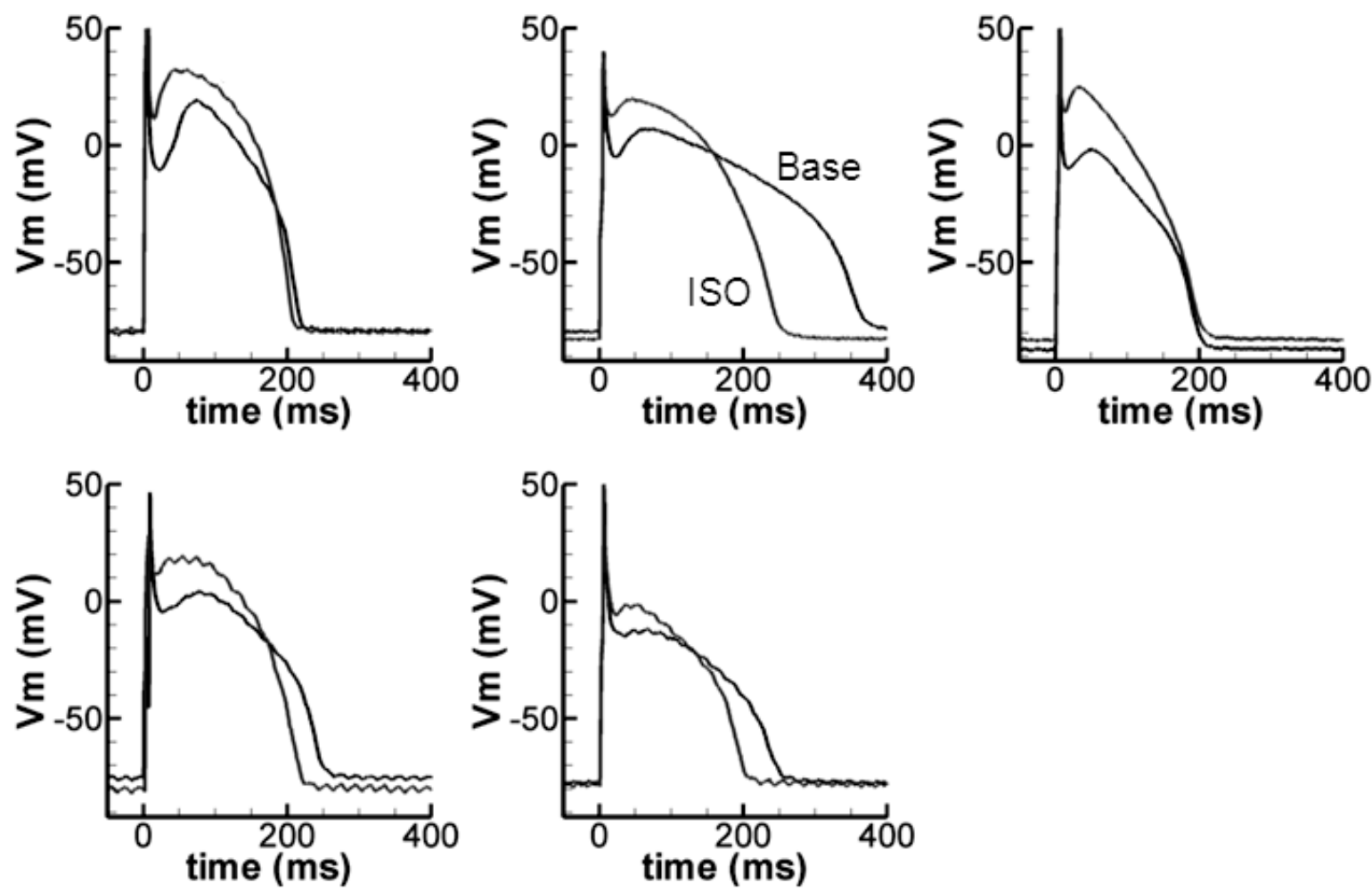

Figure 26. Examples of action potentials recorded in the absence (Base) or presence (ISO) of $\beta$ adrenergic stimulation with ISO in 5 different canine ventricular myocytes at $\mathrm{CL}=1000 \mathrm{~ms}$. Stimulation with ISO increases plateau amplitude and shortens APD but there is considerable cell-tocell variation in the magnitude of the response. For experimental protocol, see Johnson et al. ${ }^{90}$ 

in response to $\beta$ ARS
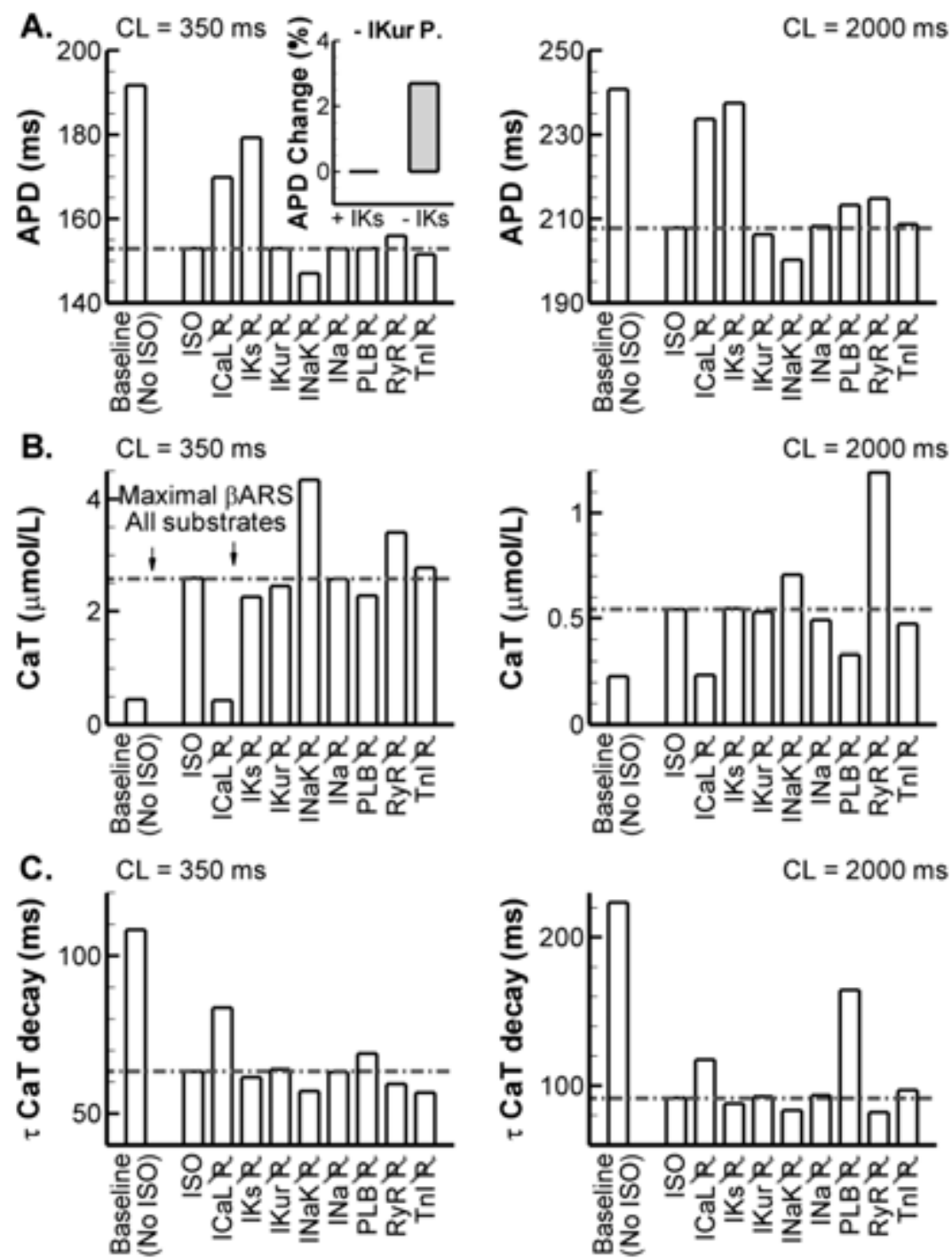

Figure 27. Summary of contributions of individual substrates to APD and CaT changes in the presence of ISO (complete data are provided in Figure 28). A. Steady-state APD at CL $=350 \mathrm{~ms}$ (left) and $\mathrm{CL}=2000 \mathrm{~ms}$ (right) at baseline (no ISO), in the presence of maximal $\beta A R S$ and during maximal BARS when PKA-dependent phosphorylation of a specific substrate is disabled (e.g., indicated by ICaL $P$ for inhibition of $I_{\text {CaL }}$ phosphorylation). CaMKII phosphorylation remained intact. The horizontal dashed-dotted line indicates the reference value during maximal $\beta A R S$ with all substrates phosphorylated. Inset shows the $\%$ increase in APD when $I_{\text {Kur }}$ phosphorylation is disabled (versus when it is present) in the presence or absence of $I_{\text {Ks. }}$ B. Similar to (A) for CaT amplitude. C. Similar to (A) for the time-constant of CaT decay based on a monoexponential fit.

The relative contribution of a substrate was analyzed by pacing the model to steady-state in the absence of $\beta A R S$, in the presence of $1.0 \mu \mathrm{mol} / \mathrm{L}$ ISO and with $1.0 \mu \mathrm{mol} / \mathrm{L}$ ISO but with the phosphorylation of the substrate of interest disabled. Figure 27 summarizes the effects of phosphorylation of each substrate on APD (Figure 27A), CaT amplitude (Figure 27B) and CaT rate of 
decay (Figure 27C) at $\mathrm{CL}=350 \mathrm{~ms}$ (left) and $\mathrm{CL}=2000 \mathrm{~ms}$ (right). $\mathrm{AP}$ and $\mathrm{CaT}$ traces underlying these data can be found in Figure 28.
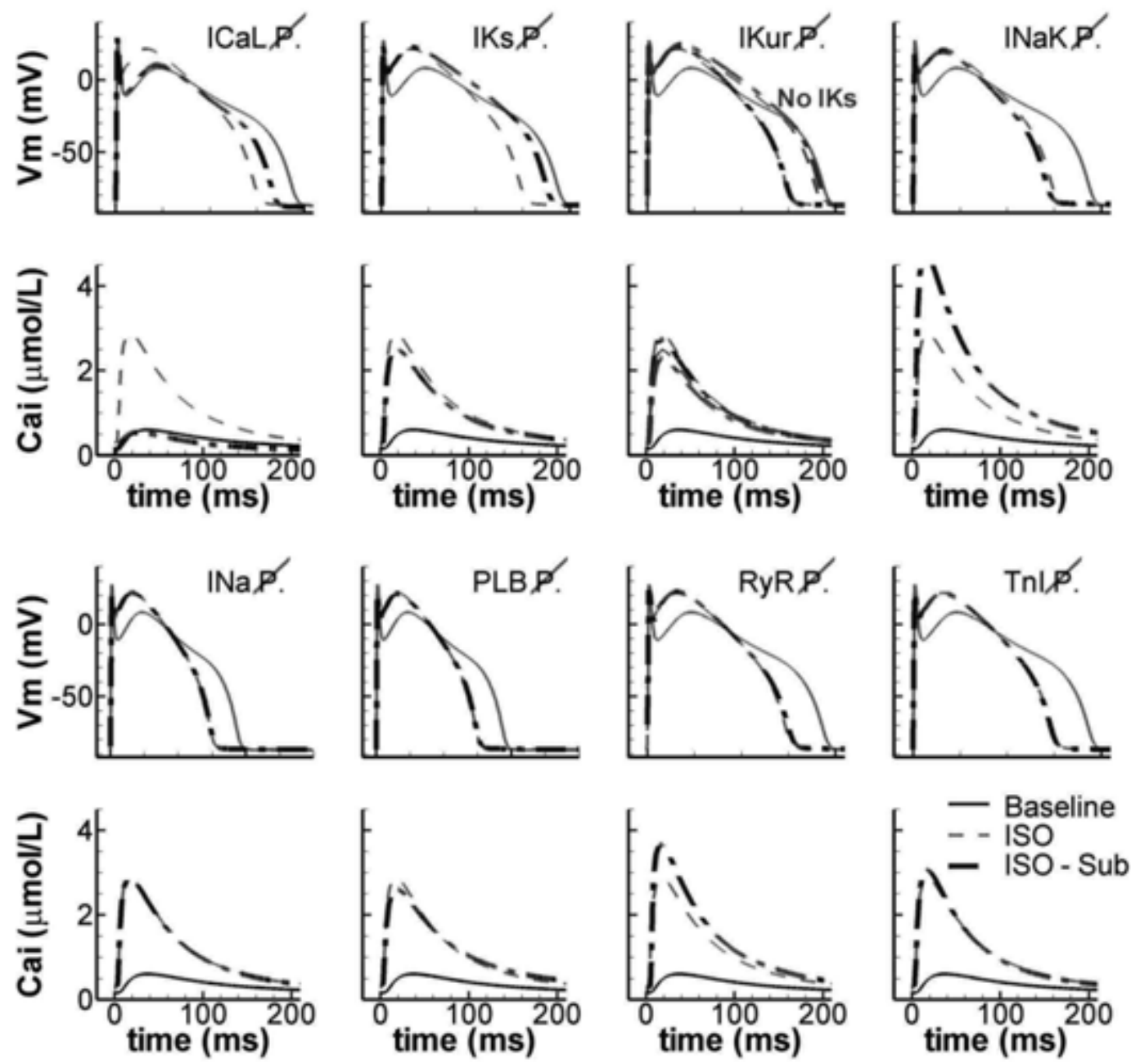

Figure 28. Contribution of individual substrates to $A P D$ and $C a T$ changes in response to $\beta A R$ stimulation at $\mathrm{CL}=350 \mathrm{~ms}$. Steady-state $\mathrm{AP}$ and $\mathrm{CaT}$ are shown in the absence of $\beta A R$ stimulation (black solid line), in the presence of a saturating dose of ISO (gray dashed line), or in the presence of $\beta A R$ stimulation without PKA-dependent phosphorylation (red dashed-dotted line) of $I_{\mathrm{CaL}}, I_{\mathrm{Ks}}, I_{\mathrm{Kur}}$, or $I_{\mathrm{NaK}}$ (top two rows; left to right) or without PKA-dependent phosphorylation of $\mathrm{I}_{\mathrm{Na}}$, PLB, RyR or TnI (bottom two rows; left to right). For color figure, see page 249.

$\mathrm{I}_{\mathrm{Ks}}$ has only a small impact on steady-state APD under baseline conditions ${ }^{52}$ (Chapter 2 ) but plays a major role in APD shortening during $\beta A R S$, consistent with experimental observations. ${ }^{80}$ Previous studies have identified kinetic channel states (termed available reserve) that modulate the ratedependent contribution of $I_{\mathrm{Ks}}$ to repolarization ${ }^{96,97}$ and their alteration by $\beta A R S .{ }^{98,99}$ Consistent with these studies, the model predicts an increased available reserve during $\beta A R S$ in the canine ventricular myocyte (Figure 29). $I_{\mathrm{ks}}$ channel gating is characterized by two major conformational changes of the voltage-sensors of each of the four channel subunits, followed by a final voltage- 
independent cooperative step to open the channel. ${ }^{97,100}$ This structural information is reflected in the 17-state Markov model described by Silva and Rudy. ${ }^{97}$ Because the second voltage-sensor transition is significantly faster than the first, two zones of closed-states are distinguished. "Zone 2" of deep closed states consists of all states in which at least one voltage-sensor still needs to make a slow transition, whereas "Zone 1 " is the available reserve which contains those states that only require the second, faster transitions. In the presence of $\beta A R S$, there is significant increase of $I_{\mathrm{Ks}}$ of a premature beat elicited after a short diastolic interval compared to longer diastolic intervals or to baseline conditions in the absence of $\beta$ ARS (Figure 29D). The mechanisms underlying this larger $I_{\mathrm{Ks}}$ at the level of channel gating kinetics are analyzed by plotting the (fractional) zone occupancy of all $I_{\mathrm{Ks}}$ channels at a diastolic interval of $25 \mathrm{~ms}$ (left) and $125 \mathrm{~ms}$ (right) under baseline conditions (solid lines) and in the presence of $\beta A R S$ (dashed-dotted lines; Figure 29E). Decreased deactivation rates of the phosphorylated $I_{\mathrm{KS}}$ channel due to $\beta A R S$ result in a larger fraction of channels in Zone 1 (greater available reserve) from which they can open quickly to generate a large $I_{\mathrm{Ks}}$ current during the AP repolarization phase. The increased $I_{\mathrm{Ks}}$ offsets the increased $I_{\text {Cal }}$ resulting from reduced $\mathrm{Ca}^{2+}$ dependent inactivation due to the reduced $\mathrm{CaT}$ at short diastolic intervals (Figure 29C) as a result of incomplete JSR refilling. This ensures appropriate APD shortening at short diastolic intervals in the presence of adrenergic stimulation. These simulations of $I_{\mathrm{Ks}}$ gating during $\beta$ ARS are consistent with results reported previously ${ }^{98,99}$ and show that similar mechanisms apply in the canine ventricular myocyte. 

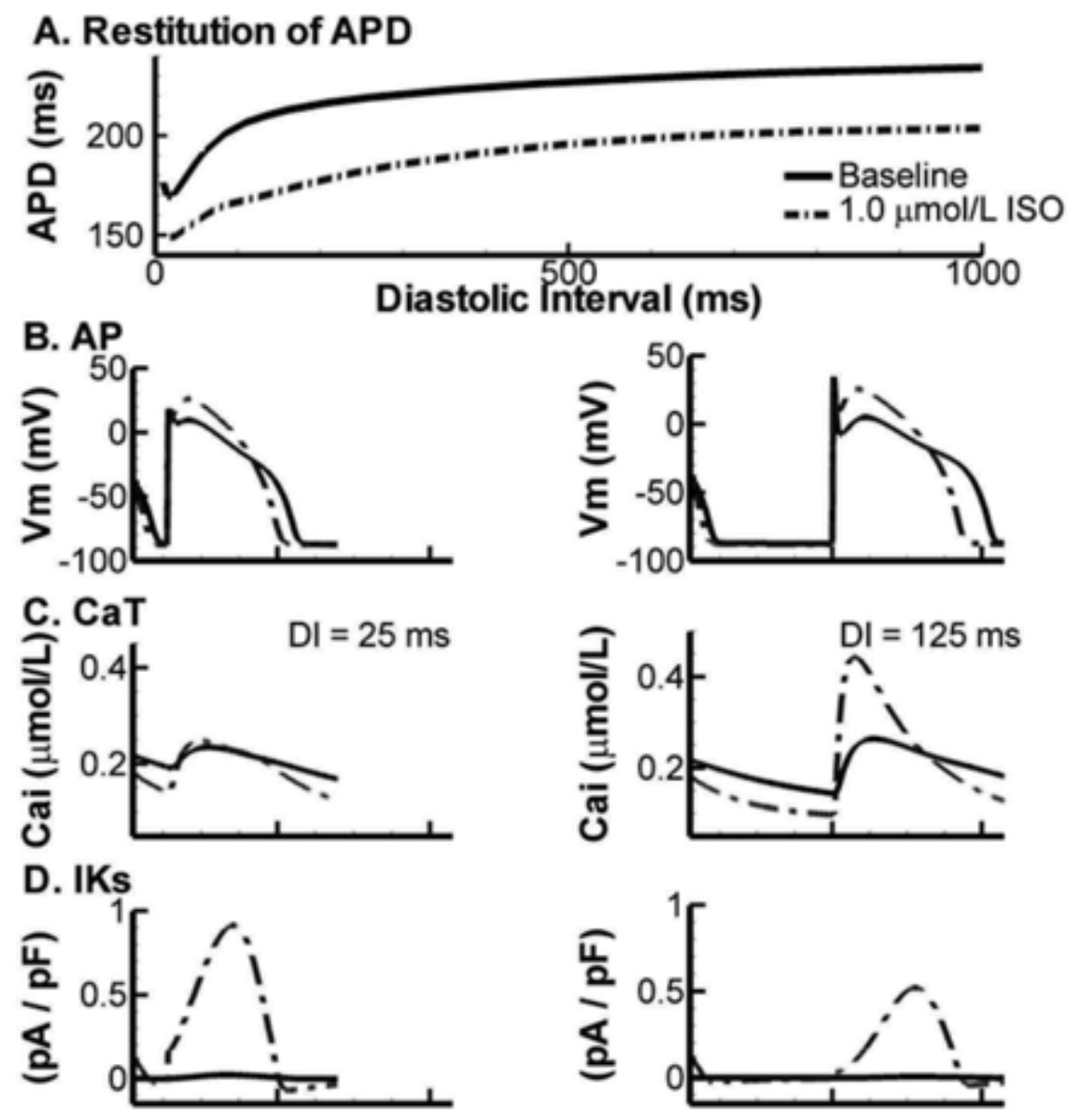

\section{E. IKs zone occupancy}
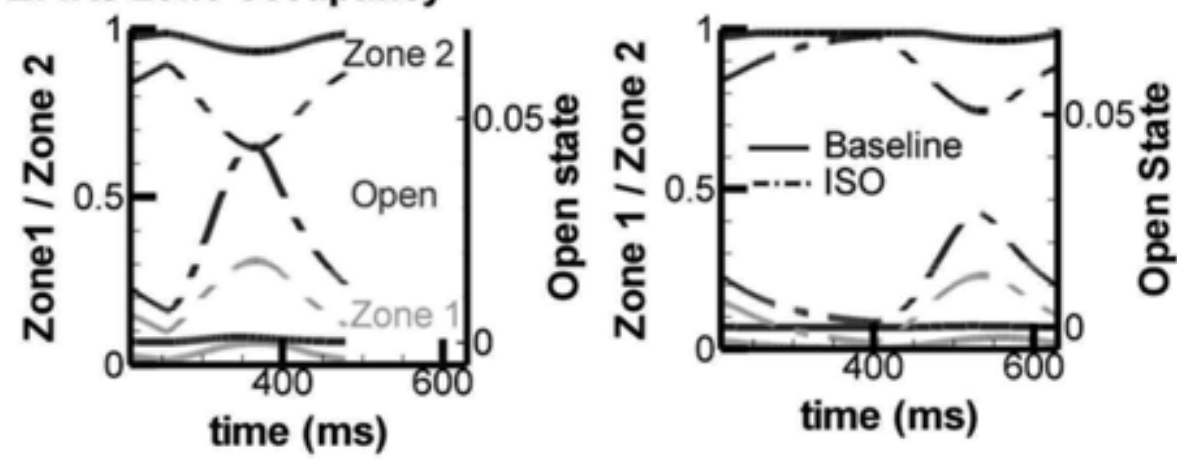

Figure 29. Kinetic changes of $I_{\mathrm{Ks}}$ due to $\beta$ ARS result in altered gating modes and increased available reserve for channel opening. A. APD restitution as function of diastolic interval after pacing to steady-state at $\mathrm{CL}=2000 \mathrm{~ms}$ in the absence (solid black) or presence (dash-dotted red) of $1.0 \mu \mathrm{mol} / \mathrm{L}$ ISO. B. AP of premature beat at diastolic intervals of $25 \mathrm{~ms}$ (left) or $125 \mathrm{~ms}$ (right). C. CaT corresponding to these APs. D. Corresponding $I_{\mathrm{Ks}}$. E. Corresponding zone occupancies of $\mathrm{I}_{\mathrm{Ks}}$ channels during the AP. Zone 2 (red) represents the deep closed states, Zone 1 (green) represents the closed states adjacent to the open state, termed "available reserve". Open state occupancy is shown in blue on a separate y-axis. Data in the absence of ISO are shown in solid lines, data in the presence of ISO in dashed-dotted lines. For color figure, see page 250. 
Consistent with simpler models ${ }^{16}$ and experimental evidence ${ }^{40,98}$, the model predicts a major role for $I_{\text {CaL }}$ in the increased AP plateau potentials and increased inotropy observed during BARS. PLB phosphorylation is primarily responsible for the increased rate of CaT decay, particularly at slow rates. At fast rates, CaMKII phosphorylation (which is not inhibited in Figure 27) reduces the impact of PKA-dependent phosphorylation. $\beta A R S$ increases $\mathrm{Ca}^{2+}$ leak from the $\mathrm{SR}^{101}$ Consistent with this observation, disabling RyR phosphorylation increases CaT, particularly at long CL.

The model provides new insights into the contribution of other substrates. Inhibition of $I_{\text {Kur }}$ was recently predicted to have a significant APD-prolonging effect in canine ventricular myocytes ${ }^{81}$ and, as such, augmentation of $I_{\text {Kur }}$ by phosphorylation is expected to result in APD shortening. Interestingly, we do not find any effect on APD and only a modest effect on AP plateau amplitude when comparing normal $\beta A R S$ to $\beta A R S$ in the absence of $I_{\text {Kur }}$ phosphorylation (Figures 27-28). However, in the absence of $I_{\mathrm{Ks}}$, inhibition of $I_{\mathrm{Kur}}$ phosphorylation results in a significant additional prolongation of APD (2.7\% increase compared to $I_{\mathrm{Ks}}$ block alone versus $<0.1 \%$ increase in the presence of $I_{\mathrm{Ks}}$, Figure 27A, inset), as expected based on the dependence of APD prolongation on initial APD. ${ }^{102}$ As such, $I_{\text {Kur }}$ phosphorylation may still have important effects in pathological conditions and provide "repolarization reserve" when $\mathrm{I}_{\mathrm{Ks}}$ is compromised by disease or drugs.

Figure 27B indicates that phosphorylation of $I_{\mathrm{NaK}}$ (through the accessory protein phospholemman) reduces cardiac inotropy (i.e., inhibition of $I_{\text {Nak }}$ phosphorylation increases CaT amplitude). This was previously shown in mouse ventricular myocytes by Despa et al. ${ }^{103}$ and we show that similar mechanisms apply in the dog, despite significant differences in $\mathrm{Ca}^{2+}$ handling between these species. The increase in CaT results from increased steady-state intracellular $\mathrm{Na}^{+}$levels that reduce $\mathrm{Ca}^{2+}$ extrusion via $\mathrm{Na}^{+}-\mathrm{Ca}^{2+}$ exchange. Similar $\mathrm{Na}^{+}$accumulation resulting from $\beta$ ARS in the absence of $\mathrm{I}_{\mathrm{NaK}}$ phosphorylation was previously shown in a model of the guinea-pig ventricular myocyte, although CaT amplitude was not quantified. ${ }^{17}$ Interestingly, despite a reduction in repolarizing $I_{\text {Nak }}$ when phosphorylation is inhibited, the increased CaT is accompanied by a shortening of APD (Figure 27A). This occurs via reduced forward mode $I_{\mathrm{NaCa}}$ and enhanced $\mathrm{Ca}^{2+}$-dependent inactivation of $\mathrm{I}_{\mathrm{Cal}}$. When $\mathrm{Ca}^{2+}$-dependent inactivation was disabled for the final beat after pacing to steady state, APD shortening in the absence of $I_{\text {Nak }}$ phosphorylation was reduced from $7.7 \mathrm{~ms}$ to $2.4 \mathrm{~ms}$ (Figure 30). Similarly, inhibition of $I_{\text {NaCa }}$ reduced APD shortening to $2.5 \mathrm{~ms}$. When both CDI and $I_{\text {NaCa }}$ were disabled, APD in the absence of $I_{\text {NaK }}$ was prolonged compared to the presence of $I_{\text {Nak }}$ phosphorylation (by $1 \mathrm{~ms}$, not shown). Thus, it appears that both $\mathrm{CDI}$ and $\mathrm{I}_{\mathrm{Naca}}$ contribute to the APD shortening observed in the absence of $I_{\mathrm{NaK}}$ phosphorylation. $I_{\mathrm{NaC}}$ and/or CDI were only disabled for a single beat to prevent secondary changes in $\mathrm{Ca}^{2+}$ loading or $\left[\mathrm{Na}^{+}\right]_{\text {. }}$. 

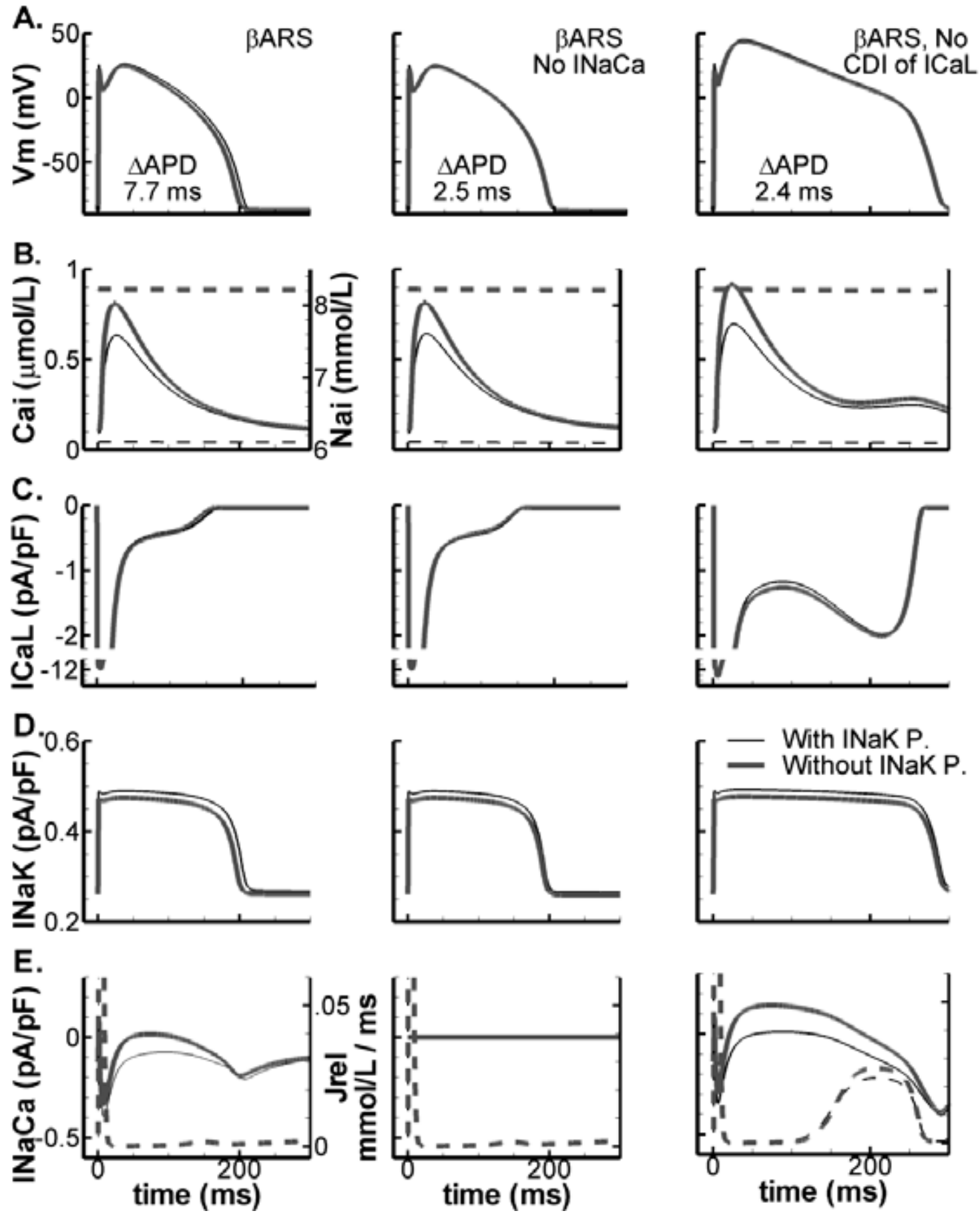

Figure 30. Effect of inhibition of $I_{\text {NaK }}$ phosphorylation in the presence of $\beta$ ARS (left), $\beta A R S$ with $I_{\text {NaCa }}$ inhibition (right) or $\beta A R S$ without $\mathrm{Ca}^{2+}$-dependent inactivation (CDI) of $\mathrm{I}_{\mathrm{CaL}}$ (right). Results are shown at steady state during pacing at $\mathrm{CL}=2000 \mathrm{~ms}$ in the presence (thin black lines) or absence (thick grey lines) of $I_{\text {Nak }}$ phosphorylation. A. Membrane potential. B. CaT (solid lines) and intracellular $\mathrm{Na}^{+}$ (dashed lines). C. I $I_{\mathrm{CaL}}$ D. I I $\mathrm{IaK}_{\mathrm{K}} \mathrm{E}$. $\mathrm{I}_{\mathrm{NaCa}}$ (solid lines) and SR Ca ${ }^{2+}$ release ( $\mathrm{J}_{\text {rel; }}$ dashed lines). CDI was only blocked for the last beat shown to prevent secondary changes in $\mathrm{Ca}^{2+}$ loading. INaK P. denotes $I_{\mathrm{NaK}}$ phosphorylation. 

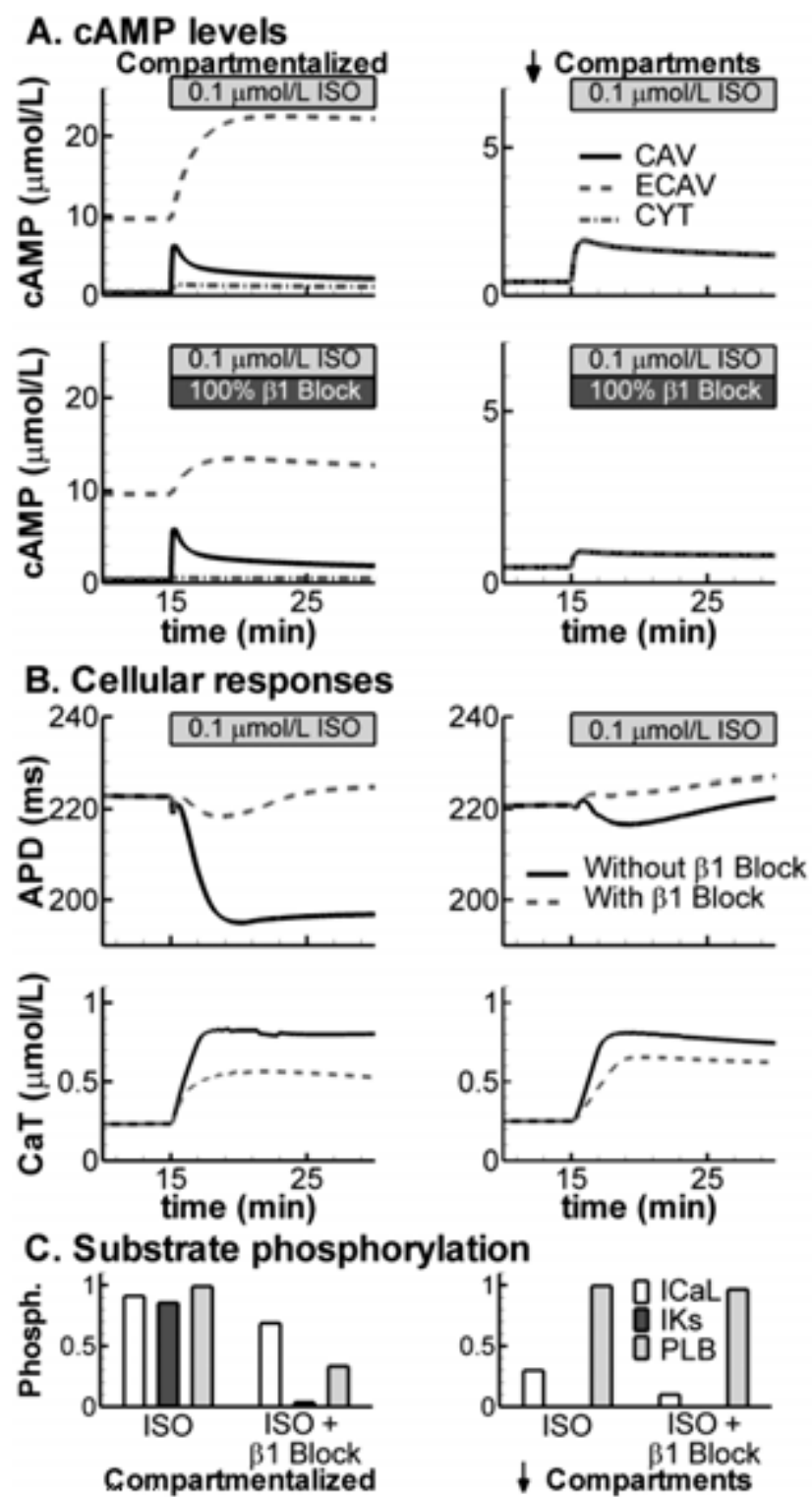

Figure 31. Compartmentation of $\beta A R S$ in the control model with normal compartments (left) or in simulations with reduced compartmentation (right). A. Time dependent changes in cAMP levels in CAV (solid line), ECAV (dashed) and CYT (dash-dotted) during stimulation with $100 \mathrm{nmol} / \mathrm{L}$ ISO (top) or in the presence of $\beta_{2} A R$ stimulation only (100 nmol/L ISO and $100 \%$ block of $\beta_{1} A R$; bottom). B. Differential effects of combined $\left(\beta_{1} A R+\beta_{2} A R\right)$ stimulation (solid) or $\beta_{2} A R S$ only (dashed) on APD (top) and CaT amplitude (bottom) at a cycle length of $1000 \mathrm{~ms}$. C. Steady-state $I_{\text {CaL, }} I_{\mathrm{ks}}$ and PLB phosphorylation levels during the simulations in (B).

Experimentally, it is established that CAMP levels are localized in subdomains. The control model with normal compartmentation shows distinct CAMP concentrations in CAV, ECAV and CYT domains at baseline, as well as in response to $\beta A R S$ (Figure 31A; top-left panel). The relative distribution of CAMP at baseline (micromolar range for ECAV, high nanomolar range for CAV and CYT) is consistent with that reported by lancu et al. ${ }^{11}$ Activation of different signaling pathways can elicit specific 
subcellular cAMP responses. For example, $\beta_{2} A R$ are predominantly located in caveolae ${ }^{8}$ and simulated $\beta_{2} A R S$ elevates CAV cAMP with only modest effects on CAMP in ECAV and CYT domains (Figure 31A; bottom-left panel). In contrast, when CAMP diffusion barriers are reduced (e.g., simulating application of $\mathrm{M} \beta \mathrm{C}$, a cholesterol depleting agent that, among other things, disrupts local cAMP domains), cAMP concentrations become uniform throughout the cell and $\beta A R S$ results in cellwide increase in CAMP independent of the activated receptor isoform, consistent with experiment ${ }^{8}$ (Figure 31A; right panels). In the control (compartmental) model (left panels), in contrast to APD shortening with whole-cell $\beta A R S$, selective stimulation of $\beta_{2} A R$ prolongs APD (Figure 32B; top-left panel). However, increases in CaT are present for both types of $\beta A R S$ (bottom-left panel). When compartmentation is reduced (right), the subsarcolemmal CAMP levels in CAV and ECAV are too small to allow regulation of APD or CaT, which show a similar time-dependent change, independent of the receptor isoform that is stimulated (Figure 31B; right panels). The mechanism underlying the distinct responses to whole cell $\left(\beta_{1}+\beta_{2}\right) \beta A R S$ and $\beta_{2}$ ARS-only in the compartmental model is shown in Figure 31C. Whole cell $\beta$ ARS phosphorylates substrates throughout the cell, including $I_{K s}$, which is responsible for APD shortening (Figure 27). In contrast, $\beta_{2} A R S$ does not phosphorylate $I_{K s}$ or PLB (Figure 31C; left panel). When compartmentation is reduced, the differential control of phosphorylation by the two isoforms is lost (Figure 31C; right panel). Since $\mathrm{M} \beta C$ application alters the distribution of other signaling molecules in addition to CAMP diffusion, it is difficult to relate the predicted functional responses to experimental measurements in the presence of $\mathrm{MBC}{ }^{46}$
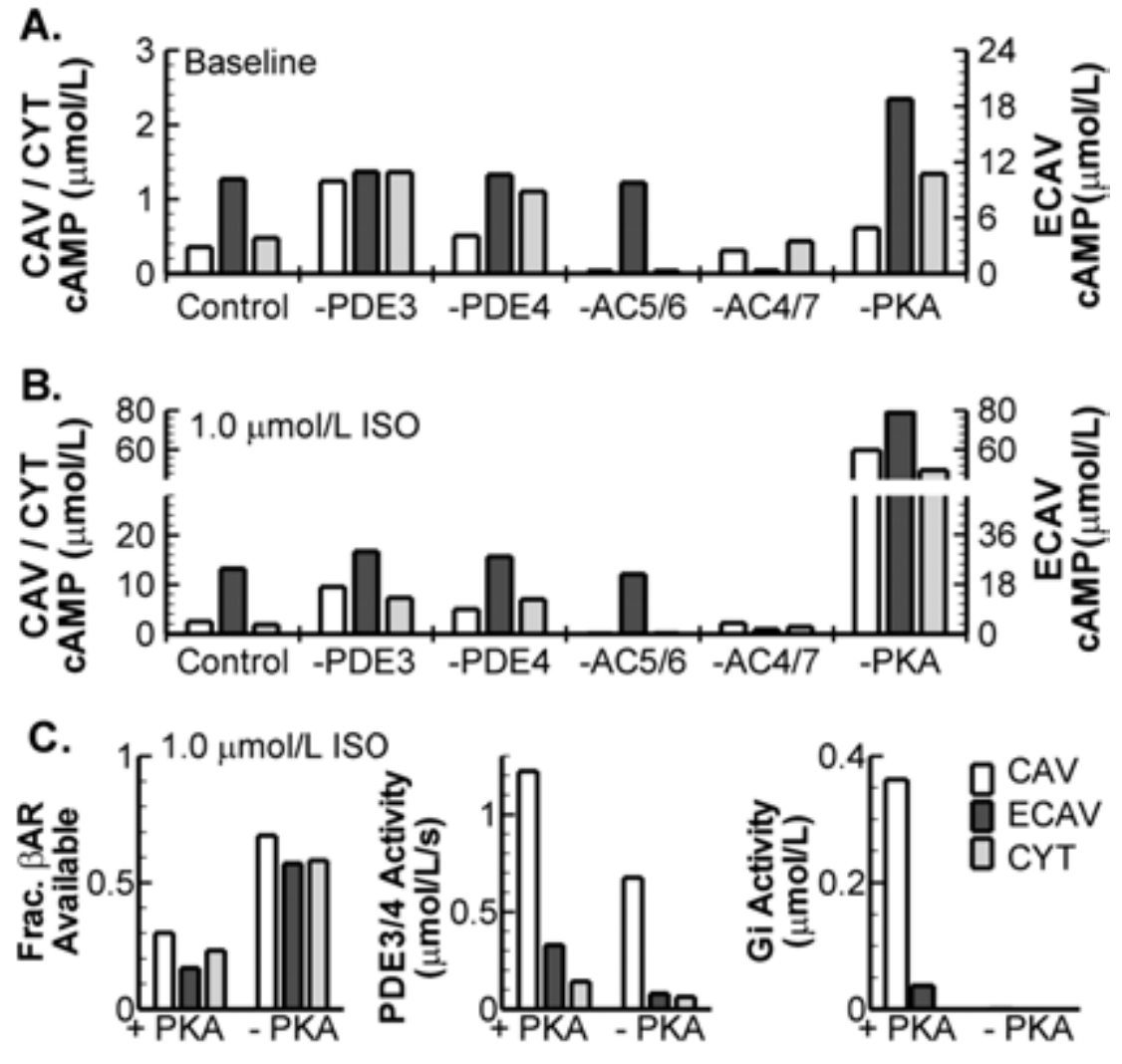

Figure 32. Contribution of $\beta A R$ signaling components to $C A M P$ compartmentation. A. Effect of virtual knock-outs of PDE3, PDE4, AC5/6, AC4/7 or PKA on steady-state cAMP concentration in CAV, ECAV and $C Y T$ domains in the absence of $\beta A R S$. B. Identical to $A$, in the presence of $1.0 \mu \mathrm{mol} / \mathrm{L}$ ISO. Note 
separate $y$-scale for cAMP in ECAV and different y-scales in (A) and (B). C. Mechanisms behind prominent CAMP increase resulting from virtual PKA knock-out in the presence of $\beta A R S$. Difference in $\beta A R$ availability (left), PDE3/4 Activity (at $1.0 \mu \mathrm{mol} / \mathrm{L}$ cAMP; middle) or Gi activity (right) in the presence and absence of PKA during stimulation with $1.0 \mu \mathrm{mol} / \mathrm{L}$ ISO for each compartment.

To identify the main signaling components involved in establishing local cAMP levels, virtual knockouts of these components were simulated in the absence and presence of $\beta A R S$ (Figure 32). Specific sub-cellular distributions of PDEs, ACs and PKA cause inter-domain differences in local CAMP concentrations. In the absence of $\beta A R S$, PDE3 (and to a lesser extent PDE4) and AC5/6 predominantly determine CAMP in CYT and CAV, while AC4/7 determine CAMP in ECAV. As expected, cAMP levels increase in all compartments upon $\beta A R S$ in control conditions and in all virtual knockouts. In general, the localized differences occurring in knock-outs remain present during $\beta A R S$ (Figure 32B). Several negative feedback loops exist in the $\beta A R$ signaling cascade. In these loops PKA activated by CAMP modifies upstream elements in the cascade to limit excessive CAMP production. The model predicts that PKA-dependent feedback loops have important effects on steady-state CAMP levels and that this occurs in a localized manner (right-most bar graphs). There is a prominent increase in CAMP in the absence of PKA during $B A R S$ (Figure 32B; far-right). This increase occurs via several feedback loops (Figure 32C). Without PKA there is increased availability of $\beta A R$ (Figure 32C; left panel), due to reduced $\beta A R$ desensitization. Moreover, there is reduction in CAMP hydrolyzing activity of PDE3 and PDE4 (Figure 32C; middle panel). PDE3 and PDE4 can be phosphorylated by PKA, thereby increasing their activity. In the PKA knock-out model this negative-feedback on cAMP is removed, resulting in increased CAMP levels. Finally, there is no activation of Gi (due to $\beta 2 A R$ phosphorylation) in the absence of PKA, reducing inhibition of AC5/6. 


\section{Downstream effects of $\beta$ ARS on CaMKII}
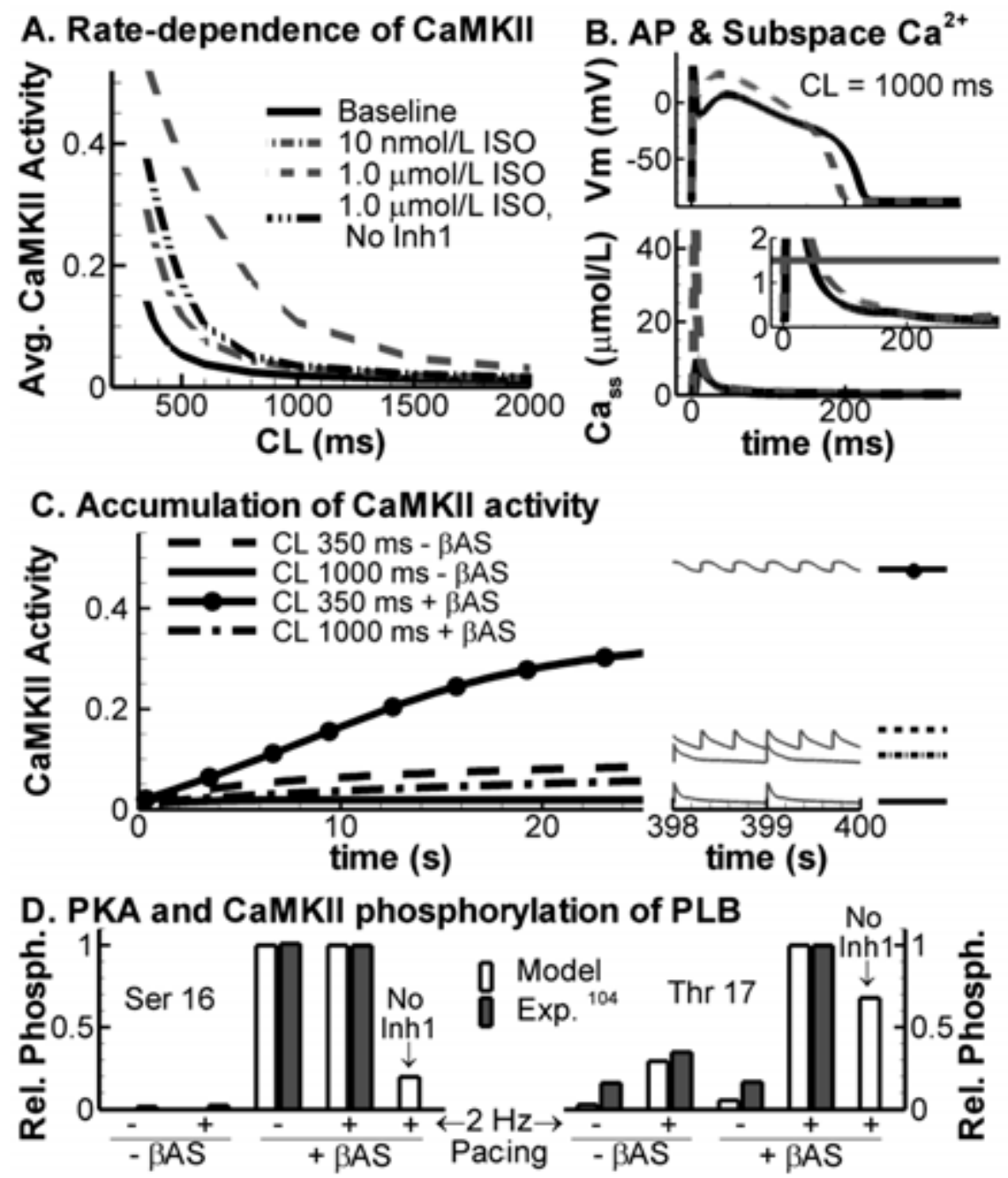

Figure 33. A. Rate-dependence of steady-state CaMKII activity averaged per beat at baseline (solid black) and in the presence of $10 \mathrm{nmol} / \mathrm{L}$ ISO (dash-dotted, grey), $1.0 \mu \mathrm{mol} / \mathrm{L}$ ISO (dashed, grey) or 1.0 $\mu \mathrm{mol} / \mathrm{L}$ ISO with virtual knock-out of Inh1 (dash-double dot, black). B. Steady-state AP and subsarcolemmal $\mathrm{Ca}^{2+}$ transient in the dyadic cleft at baseline (solid black) and during maximal $\beta A R S$ (dashed grey) at $\mathrm{CL}=1000 \mathrm{~ms}$. Horizontal line in magnified inset indicates the half maximal $\mathrm{Ca}^{2+}$ level for CaMKII activation. C. Accumulation of CaMKII activity during the first 25 seconds of pacing in the absence and presence of $\beta$ ARS at $C L=350 \mathrm{~ms}$ and $\mathrm{CL}=1000 \mathrm{~ms}$. Black lines indicate the average CaMKII per beat. Right part shows corresponding steady state unaveraged CaMKII traces. D. Phosphorylation of PLB by PKA at Ser 16 (left) and CaMKII at Thr 17 (right) at rest and during pacing in the absence or presence of adrenergic stimulation in the model (white bars) and in rat ventricular myocytes $^{104}$ (shaded bars).

In addition to cAMP compartmentation, $\mathrm{Ca}^{2+}$ signaling is also highly localized. High subsarcolemmal $\mathrm{Ca}^{2+}$ concentrations in dyadic clefts $\left(\mathrm{Cass}^{2+}\right)$ activate the CaMKII signaling pathway which phosphorylates several substrates that are also targeted by PKA. CaMKII activity in the model is ratedependent, consistent with experimental observations ${ }^{105,}$ 106. ISO increases CaMKII activity in a concentration-dependent manner, particularly at fast rates (Figure 33A). Increased CaMKII is caused 
by a larger peak $\mathrm{Ca}_{\mathrm{ss}}{ }^{2+}$ transient and a larger duration with $\mathrm{Ca}_{\mathrm{ss}}{ }^{2+}$ levels close to CaMKII affinity (Figure 33B). In addition, there is an important role for reduction in PP1 via PKA-dependent activation of Inh1, since virtual knock-out of Inh1 significantly reduces CaMKII activity during maximal $\beta$ ARS (Figure 33A; dash-double-dotted line), consistent with the effect of PP1 inhibition by okadaic acid in rat ventricular myocytes. ${ }^{94}$ The rate-dependent increase in CaMKII is more pronounced than the increase due to enhanced $\mathrm{Ca}^{2+}$ cycling with $\beta$ ARS (e.g., CaMKII activity at $\mathrm{CL}=$ 350 ms without $\beta A R S$ is 0.15 , compared to 0.06 at $C L=1500 \mathrm{~ms}$ with $\beta A R S)$. Therefore, the slow rate of CaMKII deactivation (time constant of deactivation is approximately $1500 \mathrm{~ms}^{105}$ ), which results in accumulation of active CaMKII at fast rates (Figure 33C), has a larger impact on steady-state CaMKII activity than enhanced activation due to $\beta A R S-d e p e n d e n t$ increases in $\mathrm{Ca}^{2+}$ cycling. PLB is phosphorylated by PKA on Ser16 and by CaMKII on Thr17. Hagemann et al. ${ }^{104}$ analyzed the rate- and adrenergic-stimulation dependent modulation of PLB phosphorylation in rat ventricular myocytes. The simulation results in Figure 33D show an important role of pacing rate for CaMKII-dependent phosphorylation of PLB, whereas PKA-dependent phosphorylation is rate-independent, in agreement with Hagemann's experimental observations. Knock-out of Inh1 increases PP1 activity and reduces both Ser16 and Thr17 PLB phosphorylation.

\section{Calcium overload and delayed afterdepolarizations}

For the final set of simulations, the model was extended with a formulation for store-overload induced SR Ca ${ }^{2+}$ release. After cessation of pacing at $\mathrm{CL}=500 \mathrm{~ms}$ in the presence of $1.0 \mu \mathrm{mol} / \mathrm{L} \mathrm{ISO}$, the model shows four $\mathrm{Ca}^{2+}$ after- transients and corresponding delayed afterdepolarizations (DADs; Figure 34A). DAD characteristics are consistent with those measured in canine ventricular myocytes $^{39}$ (Figure 34B). The effects of $\beta A R$ isoforms, compartmental signaling and CaMKII on DADs are shown in Figure $34 \mathrm{C}$ for various pacing CLs. At faster $\mathrm{CL}, \mathrm{Ca}^{2+}$ load increases, more DADs are generated, their average amplitude increases (Figure 34C) and coupling interval decreases (not shown). Inhibition of $\beta 1 A R$ and, to a lesser extent, reduced compartmentation (via increased cAMP diffusion) reduce the number of DADs, in line with the lower CaT amplitudes observed in Figure 31, although DADs were still observed at the fastest rates. Virtual knock-out of CaMKII, on the other hand, prevented DADs even at the fastest rate $(3 \mathrm{~Hz})$ investigated. Although all conditions reduce the incidence of DADs, this occurs via different mechanisms. $\beta 1 A R$ blockade predominantly affects PKA-dependent phosphorylation of PLB, reduced compartmentation modestly reduces PKAdependent phosphorylation of $\mathrm{I}_{\mathrm{CaL}}$ and RyR, and CaMKII inhibition abolishes CaMKII-dependent phosphorylation of all substrates without altering the PKA-dependent phosphorylation (Figure 34D). $\beta 1 A R$ block or CaMKII inhibition strongly reduce the maximum JSR $\mathrm{Ca}^{2+}$ levels (Figure 34E). This reduction in JSR Ca ${ }^{2+}$ explains the low incidence of DADs in these conditions since the threshold for spontaneous release is reached less often. To investigate which substrate has the largest impact on JSR $\mathrm{Ca}^{2+}$ levels and the incidence of DADs, we selectively inhibited either the PKA- or CaMKIIdependent phosphorylation of these 3 targets individually. The model predicts that PKA-dependent phosphorylation of $\mathrm{I}_{\mathrm{CaL}}$ (in line with the strong reduction in CaT amplitude shown in Figure 27), and both PKA- and CaMKII-dependent modulation of SR $\mathrm{Ca}^{2+}$ uptake (via SERCA2a \& PLB phosphorylation) have the largest impact (not shown). Consistent with previous modeling studies ${ }^{107}$, when an effect of RyR phosphorylation (by CaMKII and/or PKA) to lower the threshold for spontaneous $\mathrm{Ca}^{2+}$ release is included, this target also has a significant impact, enhancing DAD 
incidence (not shown). However, the functional consequences of RyR phosphorylation by both kinases are still actively debated in the literature. ${ }^{108}$

A. DAD Occurence

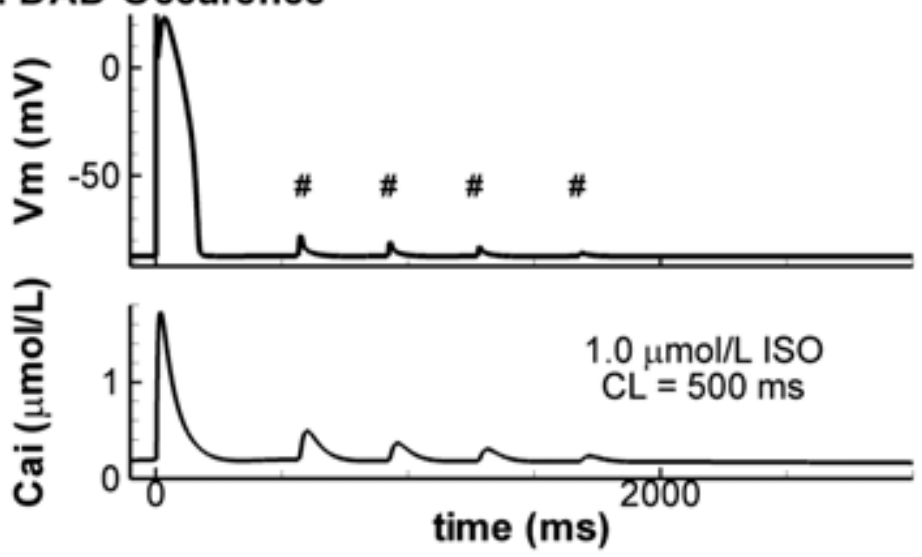

B. Validation of DAD properties

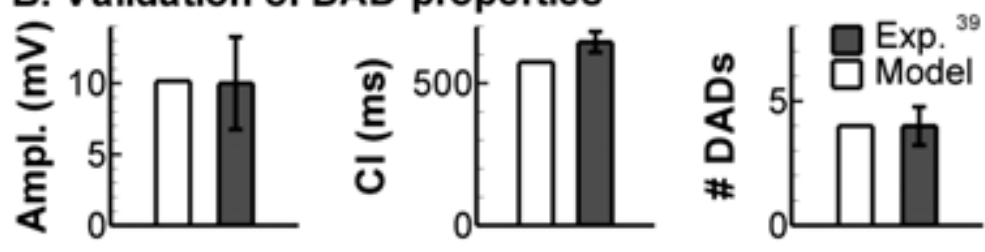

C. Modulation of DAD properties

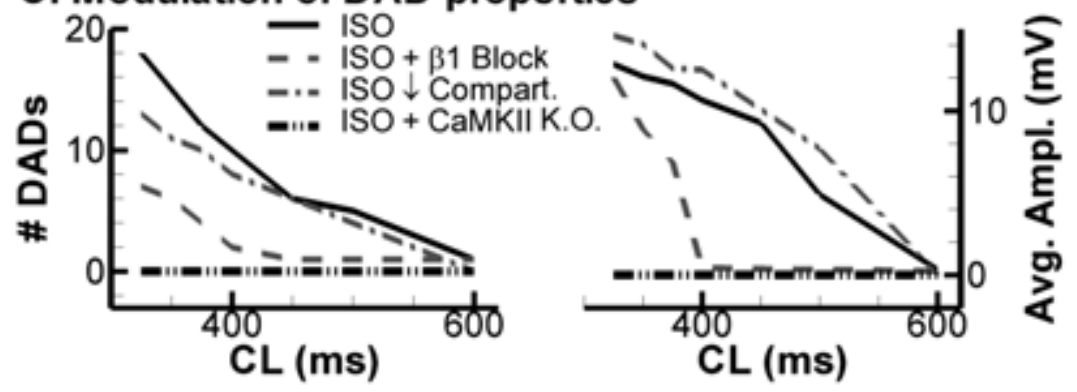

D. Substrate phosphorylation

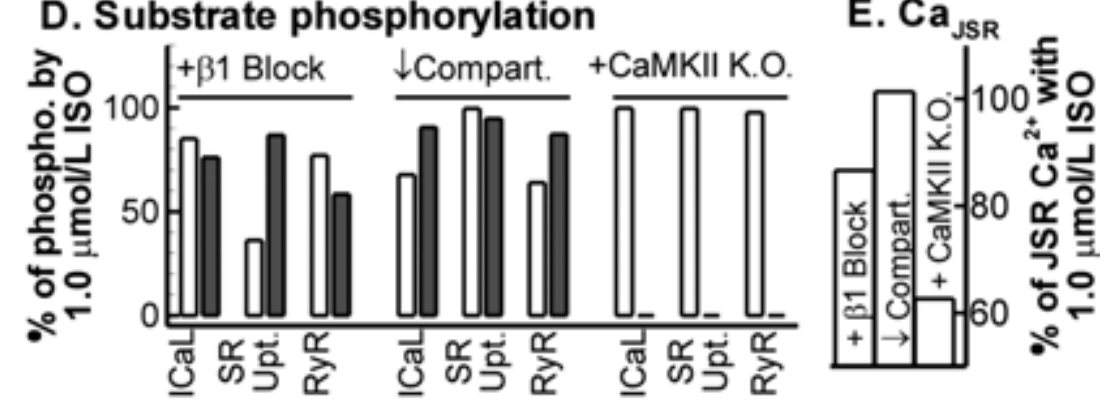

Figure 34. A. DADs (top, indicated with \#) and $\mathrm{Ca}^{2+}$ after- transients (bottom) after cessation of pacing $(\mathrm{CL}=500 \mathrm{~ms}$; last paced beat shown) in the presence of $1.0 \mu \mathrm{mol} / \mathrm{L} \mathrm{ISO}$. B. Comparison of DAD properties from $A$ to experimental data from canine ventricular myocytes. ${ }^{39}$ DAD amplitude (left), DAD coupling interval (middle) and number of DADs (right). C. Modulation of number of DADs (left) and DAD amplitude (right) at different CLs by $1.0 \mu \mathrm{mol} / \mathrm{L}$ ISO (solid line), ISO + $\beta 1$ AR blockade (dashed), ISO with reduced compartmentation (dash-dotted), or ISO + CaMKII inhibition (dashdouble dot). D. Phosphorylation of I CaL, SERCa2a \& PLB ("SR Upt."), and RyR by PKA (white bars) or CaMKII (shaded bars) relative to $1.0 \mu \mathrm{mol} / \mathrm{L} \mathrm{ISO}$ at $\mathrm{CL}=500 \mathrm{~ms}$, for the conditions in $\mathrm{C}$. E. Maximum 
JSR Ca ${ }^{2+}$ levels during $\beta 1 A R$ blockade, reduced compartmentation, or CaMKII inhibition, relative to $1.0 \mu \mathrm{mol} / \mathrm{L}$ ISO at $\mathrm{CL}=500 \mathrm{~ms}$. K.O.= knock out.

\section{Discussion}

This study investigates the interplay between compartmentalization of the $\beta$-adrenergic signaling cascade and the different isoforms of the $\beta A R$ in determining responses of whole-cell electrophysiology and calcium transient to $\beta A R S$. The main novel mechanistic insights include: (i) compartmentation of CAMP is mainly controlled by PDEs, AC and PKA and allows for selective effects of $\beta_{1} A R S$ and $\beta_{2} A R S$ (see below); (ii) I $I_{\text {Nak }}$ modulation by phospholemman phosphorylation alters [ $\left.\mathrm{Na}^{+}\right]_{1}$ and consequently $\left[\mathrm{Ca}^{2+}\right]_{i}$ to limit inotropy in large mammals; this alters APD via $I_{\mathrm{NaCa}}$ and $\mathrm{Ca}^{2+}-$ dependent inactivation of $I_{\mathrm{CaL}}$; (iii) $I_{\mathrm{Kur}}$ phosphorylation enhances repolarization reserve when $I_{\mathrm{Ks}}$ is reduced, but does not affect APD for control $I_{\mathrm{Ks}}$; (iv) inhibition of CaMKII, blockade of $\beta_{1} A R$ and reduced compartmentation reduce the incidence of DADs during $\beta A R S$ at fast rates.

Substrates experience local signaling environments depending on their subcellular localization. The model identifies restricted diffusion and localized expression of PDEs, AC and PKA as the main elements controling CAMP compartmentation. Knock-out of PKA has a particularly strong effect on CAMP during $\beta A R S$ via disruption of 3 different negative-feedback loops ( $\beta A R$ desensitization, PDE stimulation and $\mathrm{Gi}$ activation via $\beta 2 \mathrm{AR}$ phosphorylation). Increased cAMP after PKA inhibition was also reported by Rochais et al. in rat ventricular myocytes, ${ }^{109}$ although their increase is less pronounced. Differences between specific PKA knock-out and (possibly incomplete) PKA inhibition with $\mathrm{H} 89$ may explain this difference. Interestingly, $\mathrm{H} 89$ has also been shown to block $\beta A R$ in human airway cells, ${ }^{110}$ which would disable the disruption of one of the negative feedback loops.

Activation of specific cAMP domains by localized G-protein-coupled receptors can modulate wholecell responses such as APD and CaT selectively. For example, activation of $\beta_{2} A R$ alone does not phosphorylate $I_{\mathrm{Ks}}$ or PLB and prolongs APD, but has a modest positive inotropic effect. However, $\beta_{1}$ AR stimulation has a cell-wide response resulting in decreased APD and increased CaT amplitude. This specific control is lost when compartmentation is removed. Knowing which signaling components are affected by which receptors, and which cAMP pools and downstream effectors they control, is a critical first step to develop pharmaceutical compounds that act locally, thereby ensuring their specificity and avoiding arrhythmogenic side-effects.

We integrated the novel compartmental cAMP/PKA pathway with the CaMKII pathway present in the baseline model ${ }^{52,105}$ (Chapter 2) via a physiological, population based approach. Soltis and Saucerman ${ }^{107}$ recently described a computational model that includes both CaMKII and PKA pathways and identified a synergistic effect of these pathways on CaT amplitude. Consistent with their data, we find that $\beta A R S$ enhances CaMKII activity and phosphorylation of CaMKII substrates. 
This enhanced activity is due to enhanced $\mathrm{Ca}^{2+}$ cycling. In addition, we show that this effect is dosedependent and more pronounced at fast rates. Enhanced CaMKII activity with increasing pacing rate (due to the accumulation of CaMKII activity) is larger than that due to $\beta A R S$ at a given rate, consistent with the concept that CaMKII is predominantly a frequency sensor. ${ }^{106}$ Because $\beta A R S$ has important chronotropic effects in-vivo, a synergistic increase in CaMKII activity via both mechanisms is predicted. Experimental techniques for real-time monitoring of CaMKII activity in specific myocyte compartments are needed to fully characterize the BARS-CaMKII interactions and their mechanisms. Other interactions, such as those via CAMP-dependent activation of EPAC which has downstream effects on CaMKII, may be important. Recently, Mangmool et al. showed that this interaction is $\beta 1 A R$-specific and involves translocation of EPAC and CaMKII to the receptor complex via $\beta$ arrestin. ${ }^{111}$ When more quantitative aspects of EPAC-dependent CaMKII activation become available, the compartmental model presented here will be able to include these localized and isoform specific interactions.

\section{Comparison to existing models}

Many simulation studies have included functional effects of $\beta A R S$ on cardiac electrophysiology (see Table 2 for model comparisons). Most studies included only functional effects on substrates via a shift in parameters for a single pathway. Saucerman et al. ${ }^{14}$ were the first to integrate a global model of the cAMP signaling cascade into a model of ventricular cell electrophysiology. Recently, these authors expanded their model ${ }^{107}$ by combining the effects of CaMKII and CAMP/PKA on electrophysiological targets. lancu et al. ${ }^{11}$ were the first to develop a model which included localized aspects of cAMP signaling but not electrophysiological effects. These pioneering studies formed the basis for the model presented here, whose main novel characteristics include: (i) compartmental model of $\beta A R S$ and its effects on electrophysiology and CaT in canine ventricular myocyte; (ii) incorporation of both $\beta_{1} A R$ and $\beta_{2} A R$ isoforms to study physiological effects of compartmentation; (iii) a detailed population-based approach to integrate the CAMP/PKA and CaMKII pathways and their effects on a wide range of substrates. 


\begin{tabular}{|c|c|c|c|c|c|c|c|c|}
\hline $\begin{array}{l}\stackrel{\mathscr{u}}{\pi} \\
\stackrel{\varpi}{n}\end{array}$ & 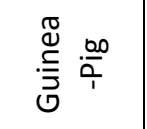 & 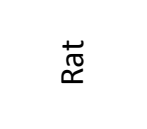 & $\stackrel{\text { on }}{\circ}$ & 乫 & 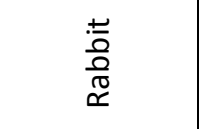 & 离 & 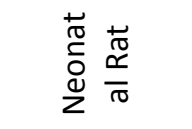 & 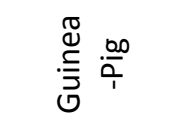 \\
\hline 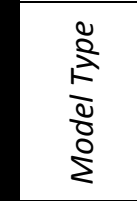 & 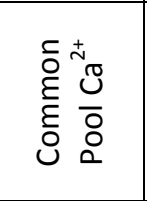 & 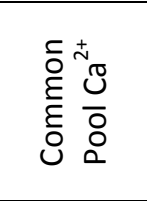 & 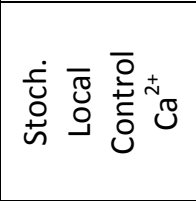 & 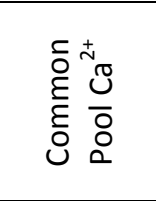 & 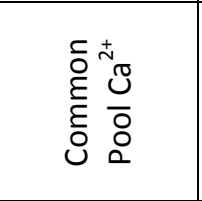 & 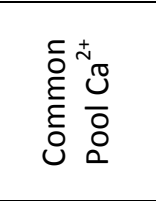 & 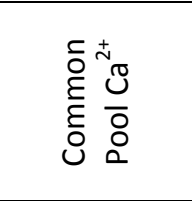 & 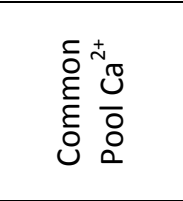 \\
\hline $\bar{\Xi}$ & ' & & ' & 总总 & & & . & ' \\
\hline $\begin{array}{l}\text { शे } \\
\text { जे } \\
\text { जे }\end{array}$ & ' & & ' & 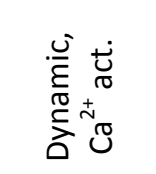 & & & & \\
\hline 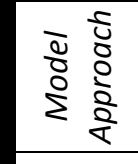 & & & ' & 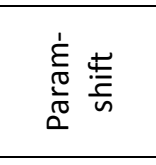 & & & & , \\
\hline 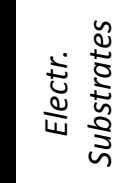 & ' & & ' & 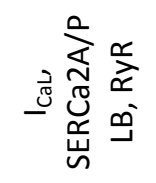 & & & & \\
\hline 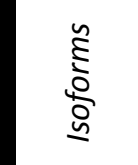 & ' & 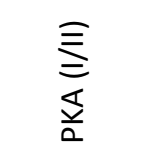 & ' & ' & 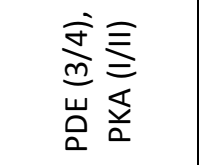 & $\sum_{\substack{\frac{1}{a}\\
}}$ & & \\
\hline 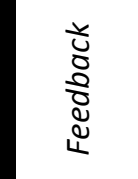 & ' & 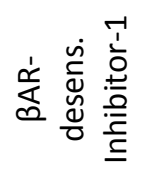 & ' & ' & 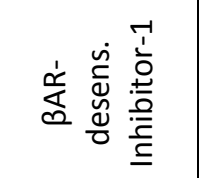 & 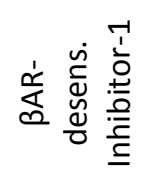 & 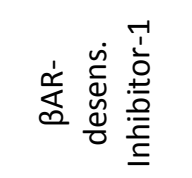 & , \\
\hline $\bar{\Xi}$ & ' & $\begin{array}{l}\bar{\pi} \\
\frac{\pi}{0} \\
\frac{0}{0}\end{array}$ & ' & ' & 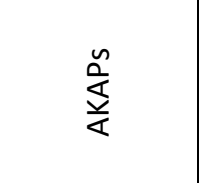 & $\begin{array}{l}\bar{\pi} \\
\frac{0}{0} \\
\text { O }\end{array}$ & 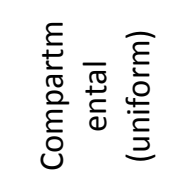 & \\
\hline $\begin{array}{l}\text { शे } \\
\text { जे } \\
\text { जे }\end{array}$ & 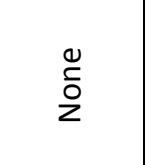 & 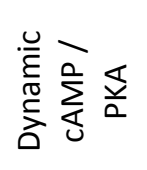 & 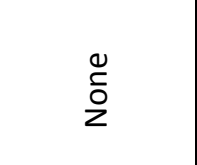 & ' & 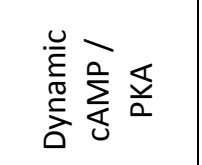 & 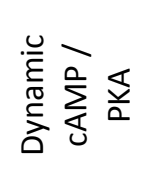 & 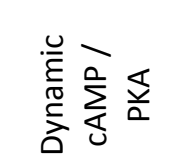 & $\begin{array}{l}0 \\
\stackrel{0}{0} \\
z\end{array}$ \\
\hline 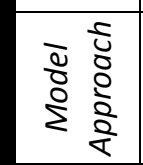 & 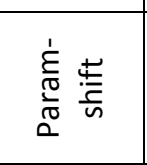 & 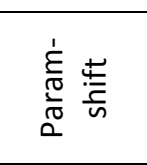 & 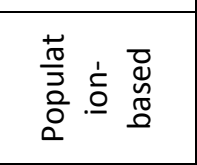 & ' & 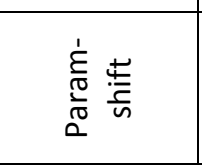 & 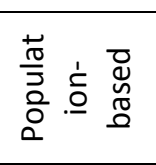 & 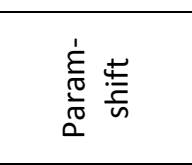 & 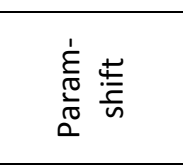 \\
\hline 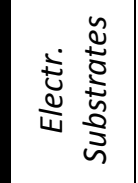 & 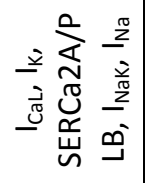 & 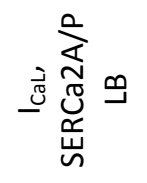 & 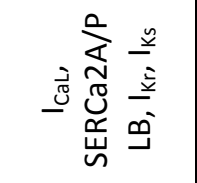 & . & 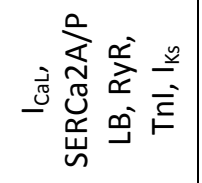 & 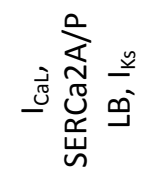 & 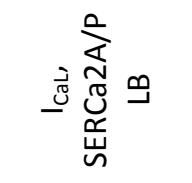 & 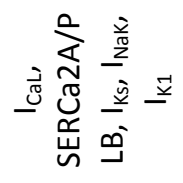 \\
\hline 旁 & 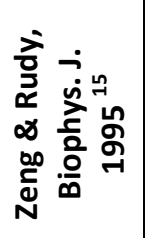 & 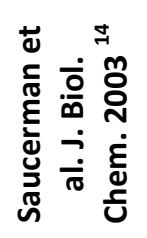 & 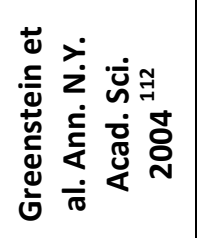 & 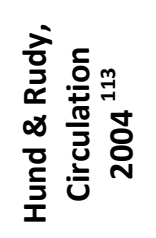 & 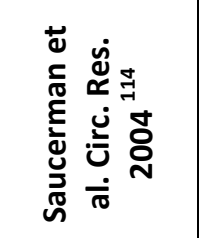 & 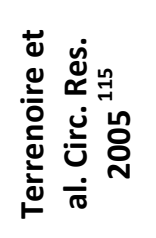 & 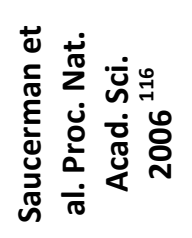 & 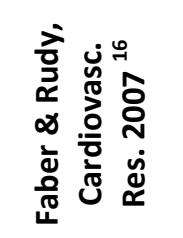 \\
\hline
\end{tabular}




\begin{tabular}{|c|c|c|c|c|c|c|c|c|}
\hline $\begin{array}{l}\stackrel{\mathscr{U}}{\tilde{\Xi}} \\
\stackrel{\Delta}{n}\end{array}$ & 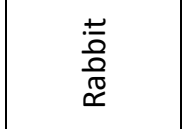 & 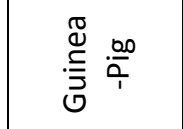 & 蔦 & 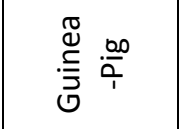 & . & 乫 & 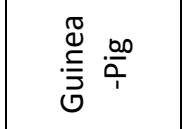 & $\begin{array}{l}\text { 䓂 } \\
\frac{0}{0} \\
\stackrel{\pi}{x}\end{array}$ \\
\hline$\frac{\tilde{z}}{\frac{\Sigma}{8}}$ & 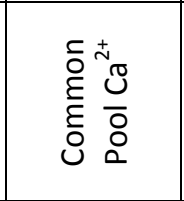 & 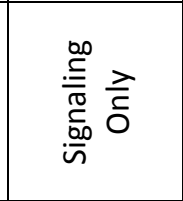 & 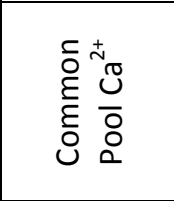 & 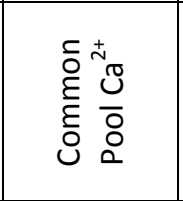 & 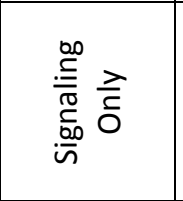 & 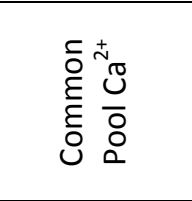 & 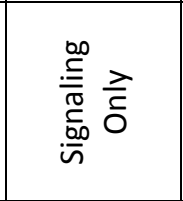 & 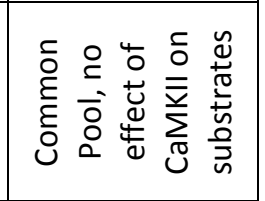 \\
\hline$\overline{\bar{g}}$ & ' & & , & & ' & 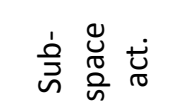 & & 意总 \\
\hline $\begin{array}{l}\text { ò } \\
\text { के } \\
\text { जे }\end{array}$ & 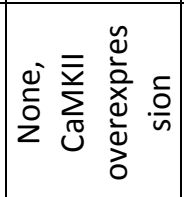 & & , & & 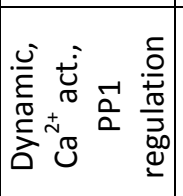 & 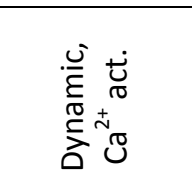 & & 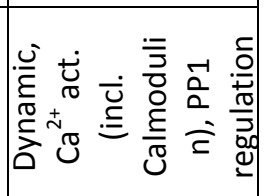 \\
\hline 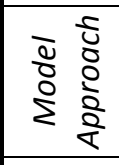 & 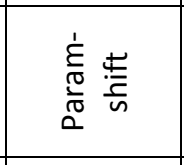 & & ' & & ' & 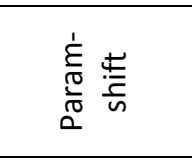 & & ' \\
\hline 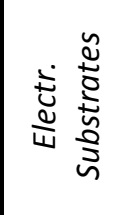 & 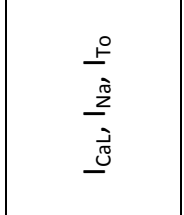 & & ' & & ' & 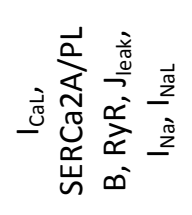 & & ' \\
\hline 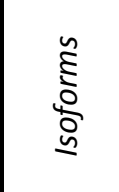 & ' & 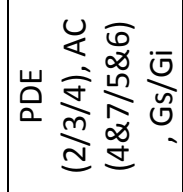 & ' & $\begin{array}{l}0 \\
\stackrel{0}{0}\end{array}$ & ' & , & 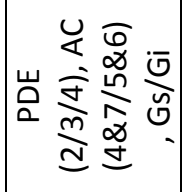 & ' \\
\hline 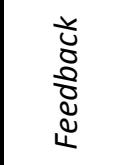 & , & $\begin{array}{l}0 \\
\tilde{0} \\
2\end{array}$ & , & 它悹㝘 & ' & ' & & , \\
\hline $\bar{g}$ & ' & 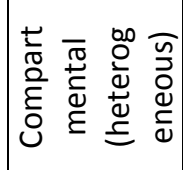 & ' & 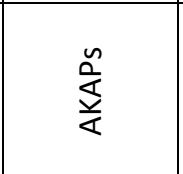 & ' & , & 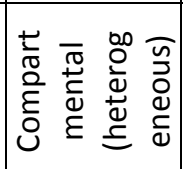 & , \\
\hline $\begin{array}{l}\text { o } \\
\text { 咅 } \\
\text { 离 } \\
\text { in }\end{array}$ & ' & 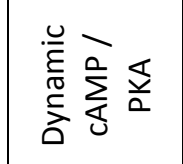 & $\begin{array}{l}\stackrel{0}{0} \\
\stackrel{0}{2}\end{array}$ & 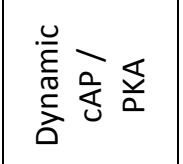 & ' & , & 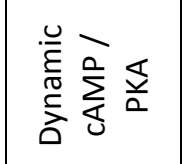 & ' \\
\hline 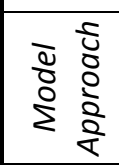 & , & & 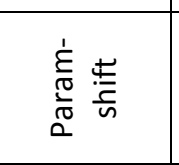 & 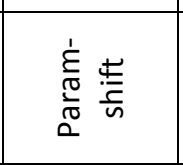 & , & , & , & ' \\
\hline 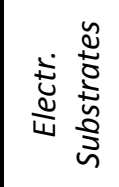 & ' & & 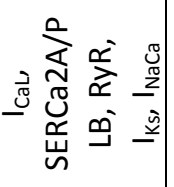 & 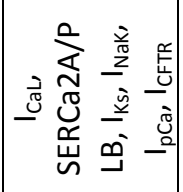 & ' & , & & . \\
\hline 竧 & 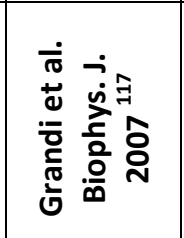 & 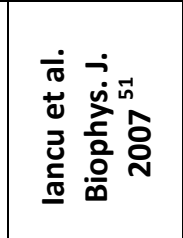 & 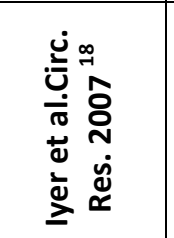 & 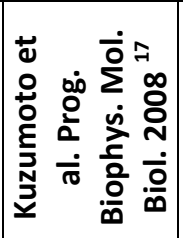 & 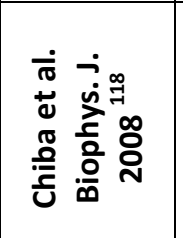 & 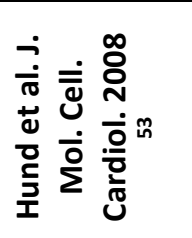 & 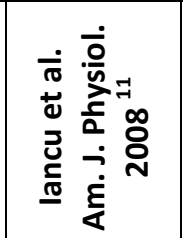 & 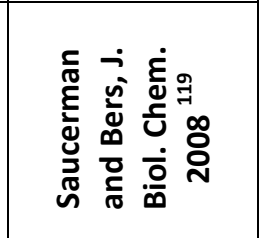 \\
\hline
\end{tabular}




\begin{tabular}{|c|c|c|c|c|c|c|c|c|}
\hline 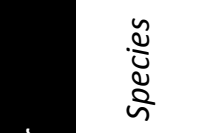 & 疍 & 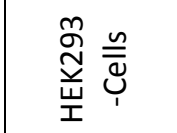 & 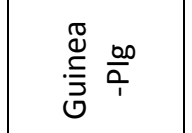 & \&) & 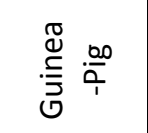 & 吕 & 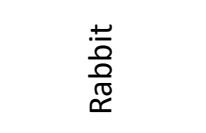 & வ0 \\
\hline 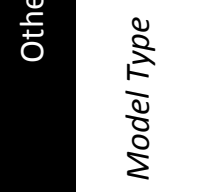 & 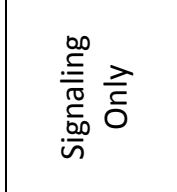 & 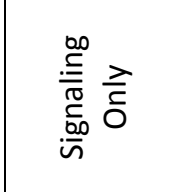 & 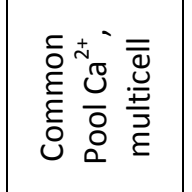 & 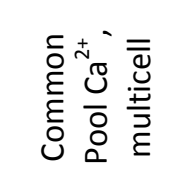 & 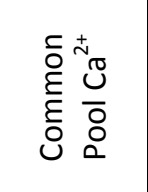 & 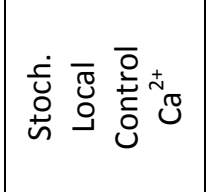 & 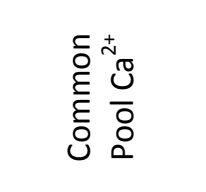 & 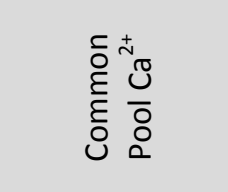 \\
\hline $\bar{\Xi}$ & ' & ' & ' & 㝘惫莕 & ' & 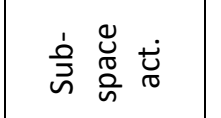 & ن̀ & 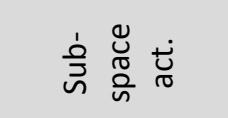 \\
\hline $\begin{array}{l}\text { oे } \\
\text { जิ } \\
\text { जे } \\
\text { जे }\end{array}$ & I & ' & ' & 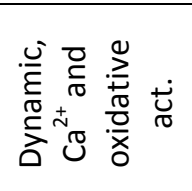 & ' & 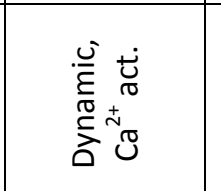 & 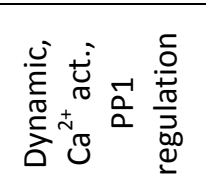 & 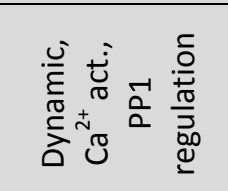 \\
\hline 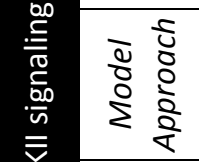 & & ' & ' & 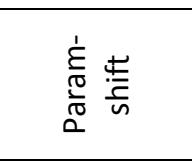 & ' & 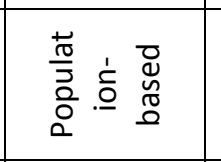 & 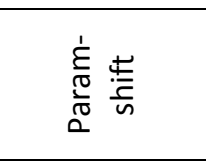 & 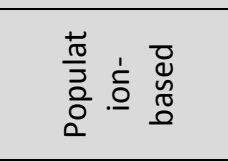 \\
\hline 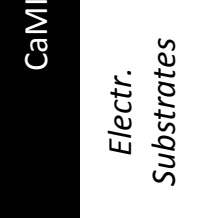 & 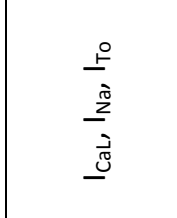 & ' & ' & 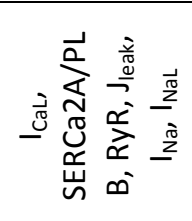 & ' & 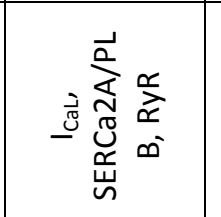 & 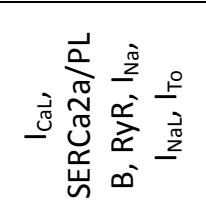 & 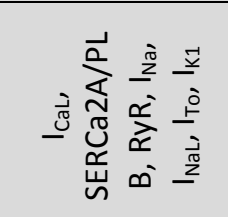 \\
\hline 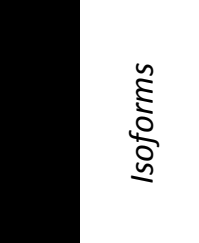 & 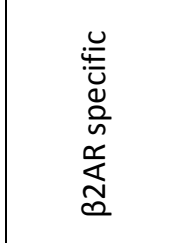 & 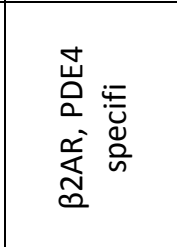 & 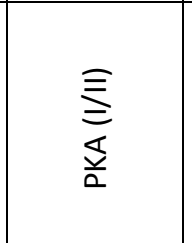 & ' & ' & ' & 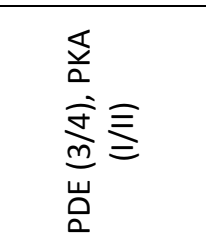 & 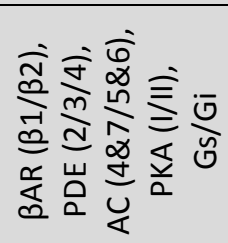 \\
\hline 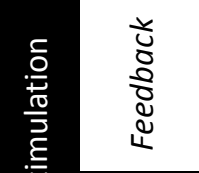 & 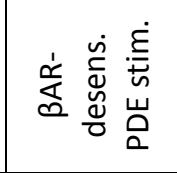 & 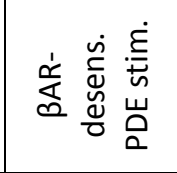 & 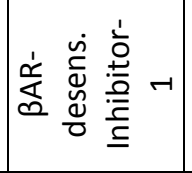 & ' & ' & ' & 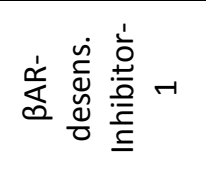 & 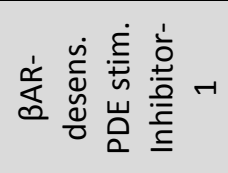 \\
\hline $\bar{\Xi}$ & $\begin{array}{l}\bar{\pi} \\
\frac{0}{0}\end{array}$ & $\begin{array}{l}\bar{\pi} \\
\frac{0}{0}\end{array}$ & ' & ' & ' & ' & $\frac{n}{\frac{a}{4}}$ & 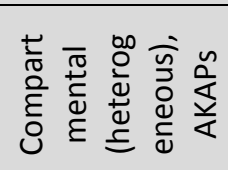 \\
\hline 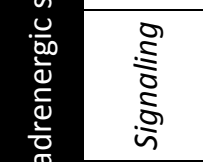 & 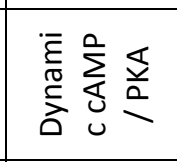 & 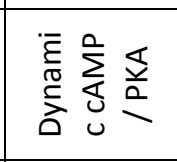 & $\begin{array}{l}\frac{\pi}{0} \\
\frac{0}{0} \\
\frac{0}{1}\end{array}$ & , & $\frac{0}{0}$ & ' & 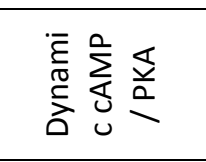 & 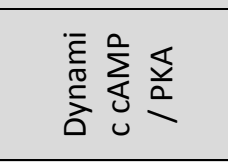 \\
\hline 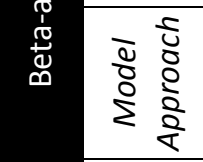 & & ' & 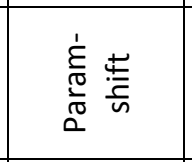 & ' & 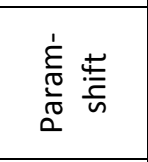 & ' & $\begin{array}{l}\frac{\dot{\varepsilon}}{\pi} \\
\frac{\pi}{\pi} \\
\frac{\pi}{2} \\
\frac{\pi}{n}\end{array}$ & 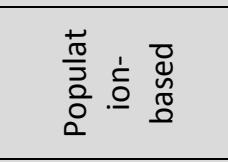 \\
\hline 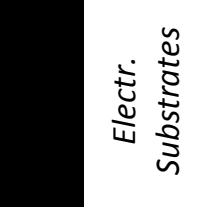 & & ' & 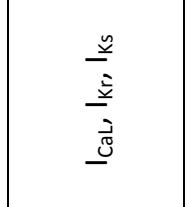 & ' & 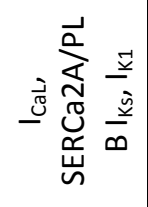 & ' & 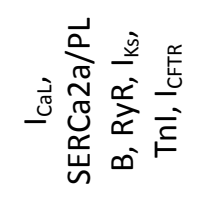 & 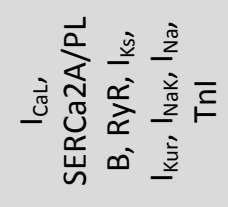 \\
\hline 흘 & 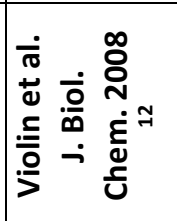 & 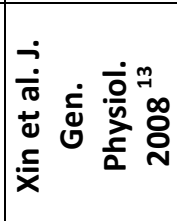 & 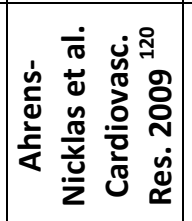 & 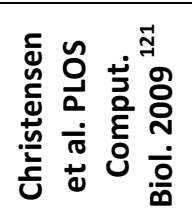 & 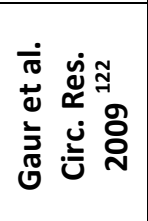 & 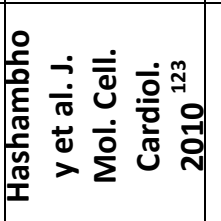 & 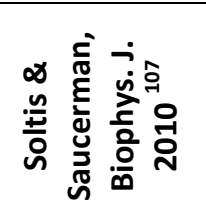 & 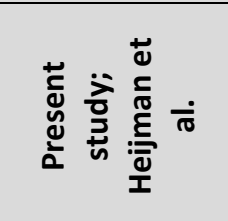 \\
\hline
\end{tabular}

Table 2. Comparison of current study to existing models. 


\section{Limitations}

Due to the inherent complexity of the simulated system, the resulting model is complex and involves many parameters. This is because it is based on the actual mechanistic interactions between many proteins for both the signaling pathway and the electrophysiological model. Parameter sensitivity analysis (Figures 4-6) provides insight into the contributions and relative importance of individual parameters. To facilitate application of this model, model code, including validation protocols, is available online in the Research Section of http://rudylab.wustl.edu. In addition, the model can be decomposed into two simpler modules (signaling and electrophysiology) that are connected through the phosphorylation levels. Thus, in investigations involving only electrophysiological measures, the phosphorylation levels can simply be fixed and only the electrophysiological module can be used. Similarly, other investigations may only require the signaling pathway.

Not all parameters are currently available from experimental data. In addition, certain characteristics of the $\beta A R$ signaling cascade are only available for rodents or are determined in biochemical reaction systems. However, major elements of the cascade ( $\beta A R$ density, AC activity, cAMP levels, $I_{\text {cat }}$ and $I_{\mathrm{Ks}}$ ISO concentration-dependence) and most electrophysiological properties of PKA substrates are available from canine ventricular myocytes. Nonetheless, a model based entirely on canine-specific experimental data is currently not feasible. Importantly, all parameters have a physiological interpretation and can in principle be determined experimentally. The parameter estimation procedure was designed such that parameters were determined from data reflecting direct steps in the $\beta A R$ cascade whenever possible, in order to minimize uncertainties.

The exact effect of phosphorylation is still debated for some substrates, particularly the RyR. ${ }^{124}$ Because the substrate data are based on whole-cell properties in the presence of maximal ISO stimulation, we do not distinguish between specific phosphorylation sites per substrate. For example, the $I_{\text {CaL }}$ channel has multiple subunits with various PKA phosphorylation sites. The model reproduces the net effect on $I_{\text {cat }}$ of all phosphorylation sites combined. However, we do distinguish between the phosphorylation mechanisms (i.e., CaMKII versus PKA) using separate populations. Once electrophysiological and signaling data become available for individual phosphorylation sites, the population-based approach introduced here can be readily extended to incorporate this level of detail.

Long pacing sequences must be simulated to capture the phosphorylation time course, which means that it is computationally prohibitive to simulate microscopic $\mathrm{Ca}^{2+}$ events (e.g., $\mathrm{Ca}^{2+}$ sparks). As such, the model is limited to reproducing macroscopic $\mathrm{Ca}^{2+}$ properties. Similarly, the model employs a macroscopic, threshold-based SR store-overload induced $\mathrm{Ca}^{2+}$-release mechanism. 


\section{ACKNOWLEDGEMENTS}

We thank Dr. Leonid Livshitz, Dr. Ali Nekouzadeh, Dr. Keith Decker, Namit Gaur, Tom O'Hara and Jiajing $\mathrm{Xu}$, Washington University in St. Louis, and Daniel M. Johnson, Maastricht University, The Netherlands for helpful discussions.

\section{GRANTS}

This work was supported by the National Institutes of Health-National Heart, Lung, and Blood Institute (Grants R01-HL049054-18 and R01-HLR01033343-26) and Fondation Leducq Award to the Alliance for Calmodulin Kinase Signaling in Heart Disease (Grant 08CVD01) to Y. Rudy. This material is also based in part upon work supported by the National Science Foundation under Grant No. CBET0929633 to Y. Rudy. Any opinions, findings and conclusions or recommendations expressed in this material are those of the authors and do not necessarily reflect the views of the National Science Foundation (NSF). Support by the Hein J.J. Wellens Foundation to J Heijman is acknowledged. P.G.A. Volders is supported by The Netherlands Heart Foundation (NHS 2007T51); and The Netherlands Organization for Scientific Research (Grant ZonMw Vidi 91710365). Y. Rudy is the Fred Saigh Distinguised Professor at Washington University in St. Louis and the Distinguished Hein J.J. Wellens Visiting Professor 2008-2009 at Maastricht University.

\section{REFERENCES}

1. Rapundalo ST. Cardiac protein phosphorylation: functional and pathophysiological correlates. Cardiovasc Res. 1998;38:559-588.

2. Bers DM. Excitation-contraction coupling and cardiac contractile force. 2nd ed. ed. Dordrecht ; London: Kluwer Academic; 2001.

3. Scott JD, Pawson T. Cell signaling in space and time: where proteins come together and when they're apart. Science. 2009;326:1220-1224.

4. Kang M, Chung KY, Walker JW. G-protein coupled receptor signaling in myocardium: not for the faint of heart. Physiology (Bethesda). 2007;22:174-184.

5. Steinberg SF, Brunton LL. Compartmentation of $\mathrm{G}$ protein-coupled signaling pathways in cardiac myocytes. Annu Rev Pharmacol Toxicol. 2001;41:751-773.

6. Vandecasteele $G$, Rochais F, Abi-Gerges A, Fischmeister R. Functional localization of cAMP signalling in cardiac myocytes. Biochem Soc Trans. 2006;34:484-488.

7. Mauban JRH, O'Donnell M, Warrier S, Manni S, Bond M. AKAP-Scaffolding Proteins and Regulation of Cardiac Physiology. Physiology (Bethesda). 2009;24:78-87.

8. Nikolaev VO, Moshkov A, Lyon AR, Miragoli M, Novak P, Paur H, Lohse MJ, Korchev YE, Harding SE, Gorelik J. $\beta 2$-adrenergic receptor redistribution in heart failure changes CAMP compartmentation. Science. 2010;327:1653-1657.

9. Grimm M, Brown JH. $\beta$-Adrenergic receptor signaling in the heart: Role of CaMKII. J Mol Cell Cardiol. 2010;48:322-330.

10. Rudy $Y$, Silva JR. Computational biology in the study of cardiac ion channels and cell electrophysiology. Q Rev Biophys. 2006;39:57-116.

11. Iancu RV, Ramamurthy G, Warrier S, Nikolaev VO, Lohse MJ, Jones SW, Harvey RD. Cytoplasmic cAMP concentrations in intact cardiac myocytes. Am J Physiol Cell Physiol. 2008;295:C414-C422.

12. Violin JD, DiPilato LM, Yildirim N, Elston TC, Zhang J, Lefkowitz RJ. $\beta 2$-adrenergic receptor signaling and desensitization elucidated by quantitative modeling of real time CAMP dynamics. J Biol Chem. 2008;283:2949-2961. 
13. Xin W, Tran TM, Richter W, Clark RB, Rich TC. Roles of GRK and PDE4 activities in the regulation of $\beta 2$ adrenergic signaling. J Gen Physiol. 2008;131:349-364.

14. Saucerman JJ, Brunton LL, Michailova AP, McCulloch AD. Modeling $\beta$-adrenergic control of cardiac myocyte contractility in silico. J Biol Chem. 2003;278:47997-48003.

15. Zeng J, Rudy Y. Early afterdepolarizations in cardiac myocytes: mechanism and rate dependence. Biophys J. 1995;68:949-964.

16. Faber GM, Rudy Y. Calsequestrin mutation and catecholaminergic polymorphic ventricular tachycardia: a simulation study of cellular mechanism. Cardiovasc Res. 2007;75:79-88.

17. Kuzumoto $M$, Takeuchi A, Nakai H, Oka C, Noma A, Matsuoka S. Simulation analysis of intracellular $\mathrm{Na}+$ and $\mathrm{Cl}$ - homeostasis during $\beta 1$-adrenergic stimulation of cardiac myocyte. Prog Biophys Mol Biol. 2008;96:171-186.

18. Iyer V, Hajjar RJ, Armoundas AA. Mechanisms of abnormal calcium homeostasis in mutations responsible for catecholaminergic polymorphic ventricular tachycardia. Circ Res. 2007;100:e22-e31.

19. Razani B, Woodman SE, Lisanti MP. Caveolae: from cell biology to animal physiology. Pharmacol Rev. 2002;54:431-467.

20. Gao T, Puri TS, Gerhardstein BL, Chien AJ, Green RD, Hosey MM. Identification and subcellular localization of the subunits of L-type calcium channels and adenylyl cyclase in cardiac myocytes. J Biol Chem. 1997;272:19401-19407.

21. Parton RG, Way M, Zorzi N, Stang E. Caveolin-3 associates with developing T-tubules during muscle differentiation. J Cell Biol. 1997;136:137-154.

22. Shibata EF, Brown TLY, Washburn ZW, Bai J, Revak TJ, Butters CA. Autonomic regulation of voltage-gated cardiac ion channels. J Cardiovasc Electrophysiol. 2006;17 Suppl 1:S34-S42.

23. Balijepalli RC, Kamp TJ. Caveolae, ion channels and cardiac arrhythmias. Prog Biophys Mol Biol. 2008;98:149-160.

24. Eldstrom J, Van Wagoner DR, Moore ED, Fedida D. Localization of Kv1.5 channels in rat and canine myocyte sarcolemma. FEBS Lett. 2006;580:6039-6046.

25. Liu L, Askari A. Beta-subunit of cardiac Na+-K+-ATPase dictates the concentration of the functional enzyme in caveolae. Am J Physiol Cell Physiol. 2006;291:C569-578.

26. Lim G, Venetucci L, Eisner DA, Casadei B. Does nitric oxide modulate cardiac ryanodine receptor function? Implications for excitation-contraction coupling. Cardiovasc Res. 2008;77:256-264.

27. Herzig S, Neumann J. Effects of serine/threonine protein phosphatases on ion channels in excitable membranes. Physiol Rev. 2000;80:173-210.

28. Hiraoka M, Kawano S, Hirano $Y$, Furukawa T. Role of cardiac chloride currents in changes in action potential characteristics and arrhythmias. Cardiovasc Res. 1998;40:23-33.

29. Sorota S, Siegal MS, Hoffman BF. The isoproterenol-induced chloride current and cardiac resting potential. J Mol Cell Cardiol. 1991;23:1191-1198.

30. Karle CA, Zitron E, Zhang W, Kathöfer S, Schoels W, Kiehn J. Rapid component I(Kr) of the guinea-pig cardiac delayed rectifier $\mathrm{K}(+)$ current is inhibited by beta(1)-adrenoreceptor activation, via CAMP/protein kinase A-dependent pathways. Cardiovasc Res. 2002;53:355362.

31. Zankov DP, Yoshida H, Tsuji K, Toyoda F, Ding WG, Matsuura H, Horie M. Adrenergic regulation of the rapid component of delayed rectifier $\mathrm{K}+$ current: implications for arrhythmogenesis in LQT2 patients. Heart Rhythm. 2009;6:1038-1046.

32. Obreztchikova MN, Sosunov EA, Anyukhovsky EP, Moïse NS, Robinson RB, Rosen MR. Heterogeneous ventricular repolarization provides a substrate for arrhythmias in a German shepherd model of spontaneous arrhythmic death. Circulation. 2003;108:1389-1394.

33. Ginsburg KS, Bers DM. Isoproterenol does not enhance $\mathrm{Ca}$-dependent $\mathrm{Na} / \mathrm{Ca}$ exchange current in intact rabbit ventricular myocytes. J Mol Cell Cardiol. 2005;39:972-981. 
34. Zhang $\mathrm{YH}$, Hancox JC. Regulation of cardiac $\mathrm{Na}^{+}-\mathrm{Ca}^{2+}$ exchanger activity by protein kinase phosphorylation--still a paradox? Cell Calcium. 2009;45:1-10.

35. van der Heyden MA, Wijnhoven TJ, Opthof T. Molecular aspects of adrenergic modulation of the transient outward current. Cardiovasc Res. 2006;71:430-442.

36. Li J, Marionneau C, Zhang R, Shah V, Hell JW, Nerbonne JM, Anderson ME. Calmodulin kinase II inhibition shortens action potential duration by upregulation of $\mathrm{K}^{+}$currents. Circ Res. 2006;99:1092-1099.

37. Lopatin AN, Nichols CG. Inward rectifiers in the heart: an update on $\mathrm{I}_{\mathrm{K} 1}$. J Mol Cell Cardiol. 2001;33:625-638.

38. Vega AL, Tester DJ, Ackerman MJ, Makielski JC. Protein kinase A-dependent biophysical phenotype for V227F-KCNJ2 mutation in catecholaminergic polymorphic ventricular tachycardia. Circ Arrhythm Electrophysiol. 2009;2:540-547.

39. Priori SG, Corr PB. Mechanisms underlying early and delayed afterdepolarizations induced by catecholamines. Am J Physiol. 1990;258:H1796-H1805.

40. Volders PGA, Kulcsár A, Vos MA, Sipido KR, Wellens HJ, Lazzara R, Szabo B. Similarities between early and delayed afterdepolarizations induced by isoproterenol in canine ventricular myocytes. Cardiovasc Res. 1997;34:348-359.

41. Hohl CM, Li QA. Compartmentation of CAMP in adult canine ventricular myocytes. Relation to single-cell free Ca2+ transients. Circ Res. 1991;69:1369-1379.

42. Warrier S, Ramamurthy G, Eckert RL, Nikolaev VO, Lohse MJ, Harvey RD. cAMP microdomains and L-type $\mathrm{Ca}^{2+}$ channel regulation in guinea-pig ventricular myocytes. $J$ Physiol. 2007;580:765-776.

43. Leroy J, Abi-Gerges A, Nikolaev VO, Richter W, Lechêne P, Mazet J-L, Conti M, Fischmeister R, Vandecasteele G. Spatiotemporal dynamics of $\beta$-adrenergic cAMP signals and L-type $\mathrm{Ca}^{2+}$ channel regulation in adult rat ventricular myocytes: role of phosphodiesterases. Circ Res. 2008;102:1091-1100.

44. Madamanchi A. $\beta$-Adrenergic receptor signaling in cardiac function and heart failure. Mcgill J Med. 2007;10:99-104.

45. Nikolaev VO, Bunemann M, Schmitteckert E, Lohse MJ, Engelhardt S. Cyclic AMP imaging in adult cardiac myocytes reveals far-reaching $\beta 1$-adrenergic but locally confined $\beta 2$-adrenergic receptor-mediated signaling. Circ Res. 2006;99:1084-1091.

46. Calaghan S, Kozera L, White E. Compartmentalisation of cAMP-dependent signalling by caveolae in the adult cardiac myocyte. J Mol Cell Cardiol. 2008;45:88-92.

47. He JQ, Balijepalli RC, Haworth RA, Kamp TJ. Crosstalk of $\beta$-adrenergic receptor subtypes through $\mathrm{Gi}$ blunts beta-adrenergic stimulation of L-type $\mathrm{Ca}^{2+}$ channels in canine heart failure. Circ Res. 2005;97:566-573.

48. Nagykaldi Z, Kem D, Lazzara R, Szabo B. Canine ventricular myocyte $\beta 2$-adrenoceptors are not functionally coupled to L-type calcium current. J Cardiovasc Electrophysiol. 1999;10:1240-1251.

49. Altschuld RA, Starling RC, Hamlin RL, Billman GE, Hensley J, Castillo L, Fertel RH, Hohl CM, Robitaille PM, Jones LR, et al. Response of failing canine and human heart cells to $\beta 2$ adrenergic stimulation. Circulation. 1995;92:1612-1618.

50. Rozec B, Gauthier C. $\beta 3$-adrenoceptors in the cardiovascular system: putative roles in human pathologies. Pharmacol Ther. 2006;111:652-673.

51. lancu RV, Jones SW, Harvey RD. Compartmentation of CAMP signaling in cardiac myocytes: a computational study. Biophys J. 2007;92:3317-3331.

52. Decker KF, Heijman J, Silva JR, Hund TJ, Rudy Y. Properties and ionic mechanisms of action potential adaptation, restitution, and accommodation in canine epicardium. Am J Physiol Heart Circ Physiol. 2009;296:H1017-1026. 
53. Hund TJ, Decker KF, Kanter E, Mohler PJ, Boyden PA, Schuessler RB, Yamada KA, Rudy Y. Role of activated CaMKII in abnormal calcium homeostasis and $I_{\mathrm{Na}}$ remodeling after myocardial infarction: insights from mathematical modeling. J Mol Cell Cardiol. 2008;45:420-428.

54. Chen-Goodspeed M, Lukan AN, Dessauer CW. Modeling of Galpha(s) and Galpha(i) regulation of human type V and VI adenylyl cyclase. J Biol Chem. 2005;280:1808-1816.

55. Steinberg SF, Zhang H, Pak E, Pagnotta G, Boyden PA. Characteristics of the $\beta$-adrenergic receptor complex in the epicardial border zone of the 5-day infarcted canine heart. Circulation. 1995;91:2824-2833.

56. Yu X, Zhang M, Kyker K, Patterson E, Benovic JL, Kem DC. Ischemic inactivation of G proteincoupled receptor kinase and altered desensitization of canine cardiac $\beta$-adrenergic receptors. Circulation. 2000;102:2535-2540.

57. Freedman NJ, Liggett SB, Drachman DE, Pei G, Caron MG, Lefkowitz RJ. Phosphorylation and desensitization of the human $\beta 1$-adrenergic receptor. Involvement of $G$ protein-coupled receptor kinases and cAMP-dependent protein kinase. J Biol Chem. 1995;270:17953-17961.

58. Vila Petroff MG, Egan JM, Wang X, Sollott SJ. Glucagon-like peptide-1 increases CAMP but fails to augment contraction in adult rat cardiac myocytes. Circ Res. 2001;89:445-452.

59. Kuschel M, Zhou YY, Cheng H, Zhang SJ, Chen Y, Lakatta EG, Xiao RP. G(i) protein-mediated functional compartmentalization of cardiac $\beta 2$-adrenergic signaling. J Biol Chem. 1999;274:22048-22052.

60. Rochais F, Abi-Gerges A, Horner K, Lefebvre F, Cooper DM, Conti M, Fischmeister R, Vandecasteele G. A specific pattern of phosphodiesterases controls the cAMP signals generated by different Gs-coupled receptors in adult rat ventricular myocytes. Circ Res. 2006;98:1081-1088.

61. Osadchii OE. Myocardial phosphodiesterases and regulation of cardiac contractility in health and cardiac disease. Cardiovasc Drugs Ther. 2007;21:171-194.

62. Dostmann WR, Taylor SS. Identifying the molecular switches that determine whether (Rp)CAMPS functions as an antagonist or an agonist in the activation of CAMP-dependent protein kinase I. Biochemistry. 1991;30:8710-8716.

63. Beavo JA, Bechtel PJ, Krebs EG. Activation of protein kinase by physiological concentrations of cyclic AMP. Proc Natl Acad Sci U S A. 1974;71:3580-3583.

64. Neumann J, Gupta RC, Schmitz W, Scholz H, Nairn AC, Watanabe AM. Evidence for isoproterenol-induced phosphorylation of phosphatase inhibitor-1 in the intact heart. Circ Res. 1991;69:1450-1457.

65. Carr AN, Schmidt AG, Suzuki Y, del Monte F, Sato Y, Lanner C, Breeden K, Jing SL, Allen PB, Greengard P, Yatani A, Hoit BD, Grupp IL, Hajjar RJ, DePaoli-Roach AA, Kranias EG. Type 1 phosphatase, a negative regulator of cardiac function. Mol Cell Biol. 2002;22:4124-4135.

66. Yatani A, Shen YT, Yan L, Chen W, Kim SJ, Sano K, Irie K, Vatner SF, Vatner DE. Down regulation of the L-type Ca2+ channel, GRK2, and phosphorylated phospholamban: protective mechanisms for the denervated failing heart. J Mol Cell Cardiol. 2006;40:619-628.

67. Xiao B, Zhong G, Obayashi M, Yang D, Chen K, Walsh MP, Shimoni Y, Cheng H, Keurs HT, Chen SRW. Ser-2030, but not Ser-2808, is the major phosphorylation site in cardiac ryanodine receptors responding to protein kinase $A$ activation upon beta-adrenergic stimulation in normal and failing hearts. Biochem J. 2006;396:7--16.

68. Kirstein $\mathrm{M}$, Eickhorn $\mathrm{R}$, Kochsiek K, Langenfeld $\mathrm{H}$. Dose-dependent alteration of rat cardiac sodium current by isoproterenol: results from direct measurements on multicellular preparations. Pflugers Arch. 1996;431:395-401.

69. Gao J, Mathias RT, Cohen IS, Baldo GJ. Isoprenaline, $\mathrm{Ca}^{2+}$ and the $\mathrm{Na}^{+}-\mathrm{K}^{+}$pump in guinea-pig ventricular myocytes. J Physiol. 1992;449:689-704.

70. StengI M, Ramakers C, Donker DW, Nabar A, Rybin AV, Spatjens RLHMG, van der Nagel T, Wodzig WKWH, Sipido KR, Antoons G, Moorman AFM, Vos MA, Volders PGA. Temporal 
patterns of electrical remodeling in canine ventricular hypertrophy: focus on $I_{\mathrm{ks}}$ downregulation and blunted beta-adrenergic activation. Cardiovasc Res. 2006;72:90-100.

71. Yue L, Feng J, Wang Z, Nattel S. Adrenergic control of the ultrarapid delayed rectifier current in canine atrial myocytes. J Physiol. 1999;516 (Pt 2):385-398.

72. Kuschel $M$, Karczewski $P$, Hempel $P$, Schlegel WP, Krause EG, Bartel S. Ser16 prevails over Thr17 phospholamban phosphorylation in the $\beta$-adrenergic regulation of cardiac relaxation. Am J Physiol. 1999;276:H1625-H1633.

73. Sulakhe PV, Vo XT. Regulation of phospholamban and troponin-I phosphorylation in the intact rat cardiomyocytes by adrenergic and cholinergic stimuli: roles of cyclic nucleotides, calcium, protein kinases and phosphatases and depolarization. Mol Cell Biochem. 1995;149150:103--126.

74. Li L, Desantiago J, Chu G, Kranias EG, Bers DM. Phosphorylation of phospholamban and troponin I in beta-adrenergic-induced acceleration of cardiac relaxation. Am J Physiol Heart Circ Physiol. 2000;278:H769-779.

75. Rubart M, Lopshire JC, Fineberg NS, Zipes DP. Changes in left ventricular repolarization and ion channel currents following a transient rate increase superimposed on bradycardia in anesthetized dogs. J Cardiovasc Electrophysiol. 2000;11:652-664.

76. Szabó G, Szentandrássy N, Bíró T, Tóth BI, Czifra G, Magyar J, Bányász T, Varró A, Kovács L, Nánási PP. Asymmetrical distribution of ion channels in canine and human left-ventricular wall: epicardium versus midmyocardium. Pflugers Arch. 2005;450:307-316.

77. Antoons G, Volders PGA, Stankovicova T, Bito V, Stengl M, Vos MA, Sipido KR. Window $\mathrm{Ca}^{2+}$ current and its modulation by $\mathrm{Ca}^{2+}$ release in hypertrophied cardiac myocytes from dogs with chronic atrioventricular block. J Physiol. 2007;579:147-160.

78. Kohlhaas $M$, Zhang T, Seidler T, Zibrova D, Dybkova N, Steen A, Wagner S, Chen L, Brown JH, Bers DM, Maier LS. Increased sarcoplasmic reticulum calcium leak but unaltered contractility by acute CaMKII overexpression in isolated rabbit cardiac myocytes. Circ Res. 2006;98:235244.

79. Xiao L, Zhang L, Han W, Wang Z, Nattel S. Sex-based transmural differences in cardiac repolarization and ionic-current properties in canine left ventricles. Am J Physiol Heart Circ Physiol. 2006;291:H570-580.

80. Volders PGA, StengI M, van Opstal JM, Gerlach U, Spätjens RL, Beekman JD, Sipido KR, Vos MA. Probing the contribution of $I_{\mathrm{Ks}}$ to canine ventricular repolarization: key role for $\beta$ adrenergic receptor stimulation. Circulation. 2003;107:2753-2760.

81. Sridhar A, da Cunha DN, Lacombe VA, Zhou Q, Fox JJ, Hamlin RL, Carnes CA. The plateau outward current in canine ventricle, sensitive to 4-aminopyridine, is a constitutive contributor to ventricular repolarization. Br J Pharmacol. 2007;152:870-879.

82. Baba S, Dun $W$, Boyden PA. Can PKA activators rescue $\mathrm{Na}^{+}$channel function in epicardial border zone cells that survive in the infarcted canine heart? Cardiovasc Res. 2004;64:260267.

83. Liu DW, Gintant GA, Antzelevitch C. Ionic bases for electrophysiological distinctions among epicardial, midmyocardial, and endocardial myocytes from the free wall of the canine left ventricle. Circ Res. 1993;72:671-687.

84. Wagner S, Hacker E, Grandi E, Weber SL, Dybkova N, Sossalla S, Sowa T, Fabritz L, Kirchhof P, Bers DM, Maier LS. Ca/calmodulin kinase II differentially modulates potassium currents. Circ Arrhythm Electrophysiol. 2009;2:285-294.

85. Ginsburg KS, Bers DM. Modulation of excitation-contraction coupling by isoproterenol in cardiomyocytes with controlled SR $\mathrm{Ca}^{2+}$ load and $\mathrm{Ca}^{2+}$ current trigger. J Physiol. 2004;556:463-480.

86. Odermatt A, Kurzydlowski $\mathrm{K}$, MacLennan $\mathrm{DH}$. The Vmax of the $\mathrm{Ca}^{2+}$-ATPase of cardiac sarcoplasmic reticulum (SERCA2a) is not altered by $\mathrm{Ca}^{2+} /$ calmodulin-dependent 
phosphorylation or by interaction with phospholamban. J Biol Chem. 1996;271:1420614213.

87. Robertson SP, Johnson JD, Holroyde MJ, Kranias EG, Potter JD, Solaro RJ. The effect of troponin I phosphorylation on the $\mathrm{Ca}^{2+}$-binding properties of the $\mathrm{Ca}^{2+}$-regulatory site of bovine cardiac troponin. J Biol Chem. 1982;257:260-263.

88. Despa S, Bossuyt J, Han F, Ginsburg KS, Jia L-G, Kutchai H, Tucker AL, Bers DM. Phospholemman-phosphorylation mediates the $\beta$-adrenergic effects on $\mathrm{Na} / \mathrm{K}$ pump function in cardiac myocytes. Circ Res. 2005;97:252-259.

89. Yamada KA, Corr PB. Effects of $\beta$-adrenergic receptor activation on intracellular calcium and membrane potential in adult cardiac myocytes. J Cardiovasc Electrophysiol. 1992;3:209-224.

90. Johnson DM, Heijman J, Pollard CE, Valentin JP, Crijns HJ, Abi-Gerges N, Volders PGA. I $\mathrm{Ks}$ restricts excessive beat-to-beat variability of repolarization during beta-adrenergic receptor stimulation. J Mol Cell Cardiol. 2010;48:122-130.

91. Sipido KR, Volders PGA, de Groot SHM, Verdonck F, Van de Werf F, Wellens HJJ, Vos MA. Enhanced $\mathrm{Ca}^{2+}$ release and $\mathrm{Na} / \mathrm{Ca}$ exchange activity in hypertrophied canine ventricular myocytes: potential link between contractile adaptation and arrhythmogenesis. Circulation. 2000;102:2137-2144.

92. Waggoner JR, Ginsburg KS, Mitton B, Haghighi K, Robbins J, Bers DM, Kranias EG. Phospholamban overexpression in rabbit ventricular myocytes does not alter sarcoplasmic reticulum Ca transport. Am J Physiol Heart Circ Physiol. 2009;296:H698--H703.

93. Chakir K, Daya SK, Aiba T, Tunin RS, Dimaano VL, Abraham TP, Jaques-Robinson KM, Lai EW, Pacak K, Zhu WZ, Xiao RP, Tomaselli GF, Kass DA. Mechanisms of enhanced $\beta$-adrenergic reserve from cardiac resynchronization therapy. Circulation. 2009;119:1231-1240.

94. Huke S, Bers DM. Temporal dissociation of frequency-dependent acceleration of relaxation and protein phosphorylation by CaMKII. J Mol Cell Cardiol. 2007;42:590-599.

95. Hund TJ, Kucera JP, Otani NF, Rudy Y. lonic charge conservation and long-term steady state in the Luo-Rudy dynamic cell model. Biophys J. 2001;81:3324-3331.

96. Rocchetti M, Besana A, Gurrola GB, Possani LD, Zaza A. Rate dependency of delayed rectifier currents during the guinea-pig ventricular action potential. J Physiol. 2001;534:721-732.

97. Silva J, Rudy $Y$. Subunit interaction determines $I_{\mathrm{Ks}}$ participation in cardiac repolarization and repolarization reserve. Circulation. 2005;112:1384-1391.

98. Rocchetti M, Freli V, Perego V, Altomare C, Mostacciuolo G, Zaza A. Rate dependency of $\beta$ adrenergic modulation of repolarizing currents in the guinea-pig ventricle. J Physiol. 2006;574:183-193.

99. Severi S, Corsi C, Rocchetti M, Zaza A. Mechanisms of beta-adrenergic modulation of $I_{\mathrm{Ks}}$ in the guinea-pig ventricle: insights from experimental and model-based analysis. Biophys $J$. 2009;96:3862-3872.

100. Silva JR, Pan H, Wu D, Nekouzadeh A, Decker KF, Cui J, Baker NA, Sept D, Rudy Y. A multiscale model linking ion-channel molecular dynamics and electrostatics to the cardiac action potential. Proc Natl Acad Sci U S A. 2009;106:11102-11106.

101. Curran J, Hinton MJ, Ríos E, Bers DM, Shannon TR. $\beta$-Adrenergic enhancement of sarcoplasmic reticulum calcium leak in cardiac myocytes is mediated by calcium/calmodulindependent protein kinase. Circ Res. 2007;100:391-398.

102. Banyasz T, Horvath B, Virag L, Barandi L, Szentandrassy N, Harmati G, Magyar J, Marangoni S, Zaza A, Varro A, Nanasi PP. Reverse rate dependency is an intrinsic property of canine cardiac preparations. Cardiovasc Res. 2009;84:237-244.

103. Despa S, Tucker AL, Bers DM. Phospholemman-mediated activation of Na/K-ATPase limits $[\mathrm{Na}]_{i}$ and inotropic state during $\beta$-adrenergic stimulation in mouse ventricular myocytes. Circulation. 2008;117:1849-1855. 
104. Hagemann D, Kuschel M, Kuramochi T, Zhu W, Cheng H, Xiao RP. Frequency-encoding Thr17 phospholamban phosphorylation is independent of Ser16 phosphorylation in cardiac myocytes. J Biol Chem. 2000;275:22532-22536.

105. Livshitz LM, Rudy Y. Regulation of $\mathrm{Ca}^{2+}$ and electrical alternans in cardiac myocytes: role of CAMKII and repolarizing currents. Am J Physiol Heart Circ Physiol. 2007;292:H2854-2866.

106. De Koninck $\mathrm{P}$, Schulman $\mathrm{H}$. Sensitivity of $\mathrm{CaM}$ kinase II to the frequency of $\mathrm{Ca}^{2+}$ oscillations. Science. 1998;279:227-230.

107. Soltis AR, Saucerman JJ. Synergy between CaMKII Substrates and beta-Adrenergic Signaling in Regulation of Cardiac Myocyte Ca ${ }^{2+}$ Handling. Biophys J. 2010;99:2038-2047.

108. Kashimura T, Briston SJ, Trafford AW, Napolitano C, Priori SG, Eisner DA, Venetucci LA. In the RyR2(R4496C) mouse model of CPVT, beta-adrenergic stimulation induces Ca waves by increasing SR Ca content and not by decreasing the threshold for Ca waves. Circ Res. 2010;107:1483-1489.

109. Rochais F, Vandecasteele G, Lefebvre F, Lugnier C, Lum H, Mazet J-L, Cooper DMF, Fischmeister R. Negative feedback exerted by CAMP-dependent protein kinase and cAMP phosphodiesterase on subsarcolemmal cAMP signals in intact cardiac myocytes: an in vivo study using adenovirus-mediated expression of CNG channels. J Biol Chem. 2004;279:5209552105.

110. Murray AJ. Pharmacological PKA inhibition: all may not be what it seems. Sci Signal. 2008;1:re4.

111. Mangmool S, Shukla AK, Rockman HA. beta-Arrestin-dependent activation of $\mathrm{Ca}^{2+} /$ calmodulin kinase II after $\beta_{1}$-adrenergic receptor stimulation. J Cell Biol. 2010;189:573587.

112. Greenstein JL, Tanskanen AJ, Winslow RL. Modeling the actions of $\beta$-adrenergic signaling on excitation--contraction coupling processes. Ann N Y Acad Sci. 2004;1015:16-27.

113. Hund TJ, Rudy Y. Rate dependence and regulation of action potential and calcium transient in a canine cardiac ventricular cell model. Circulation. 2004;110:3168-3174.

114. Saucerman JJ, Healy SN, Belik ME, Puglisi JL, McCulloch AD. Proarrhythmic consequences of a KCNQ1 AKAP-binding domain mutation: computational models of whole cells and heterogeneous tissue. Circ Res. 2004;95:1216-1224.

115. Terrenoire C, Clancy CE, Cormier JW, Sampson KJ, Kass RS. Autonomic control of cardiac action potentials: role of potassium channel kinetics in response to sympathetic stimulation. Circ Res. 2005;96:e25-e34.

116. Saucerman JJ, McCulloch AD. Cardiac beta-adrenergic signaling: from subcellular microdomains to heart failure. Ann N Y Acad Sci. 2006;1080:348-361.

117. Grandi E, Puglisi JL, Wagner S, Maier LS, Severi S, Bers DM. Simulation of Ca-calmodulindependent protein kinase II on rabbit ventricular myocyte ion currents and action potentials. Biophys J. 2007;93:3835-3847.

118. Chiba H, Schneider NS, Matsuoka S, Noma A. A simulation study on the activation of cardiac CaMKII delta-isoform and its regulation by phosphatases. Biophys J. 2008;95:2139-2149.

119. Saucerman JJ, Bers DM. Calmodulin mediates differential sensitivity of CaMKII and calcineurin to local $\mathrm{Ca}^{2+}$ in cardiac myocytes. Biophys J. 2008;95:4597-4612.

120. Ahrens-Nicklas RC, Clancy CE, Christini DJ. Re-evaluating the efficacy of beta-adrenergic agonists and antagonists in long QT-3 syndrome through computational modelling. Cardiovasc Res. 2009;82:439-447.

121. Christensen MD, Dun W, Boyden PA, Anderson ME, Mohler PJ, Hund TJ. Oxidized calmodulin kinase II regulates conduction following myocardial infarction: a computational analysis. PLoS Comput Biol. 2009;5:e1000583.

122. Gaur N, Rudy Y, Hool L. Contributions of ion channel currents to ventricular action potential changes and induction of early afterdepolarizations during acute hypoxia. Circ Res. 2009;105:1196-1203. 
Local control of $\beta$-adrenergic stimulation

123. Hashambhoy YL, Greenstein JL, Winslow RL. Role of CaMKII in RyR leak, EC coupling and action potential duration: a computational model. J Mol Cell Cardiol. 2010;49:617-624.

124. Bridge JHB, Savio-Galimberti E. What are the consequences of phosphorylation and hyperphosphorylation of ryanodine receptors in normal and failing heart? Circ Res. 2008;102:995-997. 


\title{
ChAPTER 4
}

Dominant-Negative Control of CAMP-DePENDENT $I_{\text {KS UPREGULATION IN HUMAN LONG-QT SYNDROME }}$

\author{
TYPE 1
}

Jordi HeIJMan, Roel L.H.M.G. SpätJens, SANdRine R.M. Seyen, Viola Lentink, HeLma J.H. KuIJPERS, INGE R. BOULET, LEON J. DE Windt, Miren DAVID AND PAUL G.A. VOLDERS.

CIRC. RES. 2012, 110:211-219. 


\section{ABSTRACT}

The mutation A341V in the S6 transmembrane segment of KCNQ1, the $\alpha$-subunit of the slowlyactivating delayed-rectifier $\mathrm{K}^{+}\left(\mathrm{I}_{\mathrm{Ks}}\right)$ channel, predisposes to a severe long-QT1 syndrome with sympathetic-triggered ventricular tachyarrhythmias and sudden cardiac death. Several genetic riskmodifiers have been identified in A341V patients, but the molecular mechanisms underlying the pronounced repolarization phenotype, particularly during $\beta$-adrenergic receptor stimulation, remain unclear. We aimed to elucidate these mechanisms and provide new insights into control of CAMPdependent modulation of $I_{\mathrm{Ks}}$. We characterized the effects of A341V on the $I_{\mathrm{Ks}}$ macromolecular channel complex in transfected Chinese-hamster ovary $(\mathrm{CHO})$ cells and found a dominant-negative suppression of cAMP-dependent Yotiao-mediated $\mathrm{I}_{\mathrm{Ks}}$ upregulation on top of a dominant-negative reduction in basal current. Phosphomimetic substitution of the N-terminal position S27 with aspartic acid rescued this loss of upregulation. Western blot analysis showed reduced phosphorylation of KCNQ1 at S27, even for heterozygous A341V, suggesting that phosphorylation defects in some (mutant) KCNQ1 subunits can completely suppress $I_{\mathrm{Ks}}$ upregulation. Functional analyses of heterozygous KCNQ1 WT : G589D and heterozygous KCNQ1 WT : S27A, a phosphorylation-inert substitution, also showed such suppression. Immunoprecipitation of Yotiao with KCNQ1-A341V (in the presence of KCNE1) was not different from WT. Our results indicate the involvement of the KCNQ1-S6 region at/or around A341 in CAMP-dependent stimulation of $\mathrm{I}_{\mathrm{Ks}}$, a process which is under strong dominant-negative control, suggesting that tetrameric KCNQ1 phosphorylation is required. Specific long-QT1 mutations, including heterozygous A341V, disable this regulation. 


\section{INTRODUCTION}

The slowly-activating delayed-rectifier $\mathrm{K}^{+}$current $\left(\mathrm{I}_{\mathrm{Ks}}\right)$ contributes importantly to cardiac repolarization. In large mammals, including humans, it has small amplitude under basal isolatedmyocyte conditions, but forms a sizable repolarization reserve that is recruited when the actionpotential duration (APD) prolongs, and during $\beta$-adrenergic receptor ( $\beta A R$ ) stimulation. ${ }^{1,2}$

$\mathrm{I}_{\mathrm{Ks}}$ is carried by a macromolecular channel complex consisting of a homotetramer of pore-forming $\alpha$ subunits encoded by KCNQ1 (Kv7.1), KCNE1 $\beta$-subunits ${ }^{3,4}$ and the regulatory A-kinase anchoring protein Yotiao, which binds to the KCNQ1 C-terminus. ${ }^{5}$ There are also multiple interactions with other proteins. During $\beta A R$ stimulation, when CAMP levels rise, phosphorylation of KCNQ1 at Nterminal position S27 is controlled by protein kinase A (PKA) and protein phosphatase 1 that are localized to the complex by Yotiao, thereby providing local control of $\mathrm{I}_{\mathrm{Ks}}$ enhancement. ${ }^{5}$ Anchored PKA also phosphorylates Yotiao itself, thereby further enhancing $\mathrm{I}_{\mathrm{Ks}}{ }^{6}$ An intact C-terminus of KCNE1 is critical for the PKA-dependent upregulation of $\mathrm{IKs}{ }^{7}$

Congenital defects (long-QT syndrome types 1 and 5; LQT1, LQT5; Jervell and Lange-Nielsen syndrome), pharmacological inhibition ${ }^{8}$ and acquired loss of $\mathrm{I}_{\mathrm{Ks}}{ }^{9}$ can all result in QT-interval prolongation and enhanced susceptibility to ventricular tachyarrhythmias, notably torsades de pointes. These arrhythmias occur predominantly during conditions of exercise and/or sympathetic stress. ${ }^{10}$ In 2005, the loss-of-function mutation A341V in KCNQ1 (S6 transmembrane segment) was reported in a large South-African founder population. ${ }^{11}$ This hot-spot LQT1 mutation, found globally, results in an unusually severe clinical phenotype compared to other LQT1 mutations, irrespective of their amino-acid location (transmembrane versus N/C terminal) or dominant-negative characteristics. ${ }^{12}$ Brisk variations in autonomic response increase the arrhythmic risk in A341V LQT1 patients. ${ }^{13}$ Also, polymorphisms in $\alpha$ - and $\beta$-adrenergic receptors (enhancing the autonomic response) are found more often in symptomatic than non-symptomatic KCNQ1-A341V carriers. ${ }^{13}$

In this study, we investigated the molecular control of $I_{\mathrm{Ks}}$ during $\beta A R$ stimulation, hypothesizing that A341V provides a useful case to advance understanding of the $I_{\mathrm{Ks}}$ macromolecular signaling complex, and to obtain novel insights into the determinants of phenotypic severity by A341V and other LQT1 mutations.

\section{METHODS}

\section{Molecular Biology}

Wild-type (WT) human KCNQ1 (also known as KVLQT1 or Kv7.1) in a pIRES2-GFP vector and human KCNE1 (minK, IsK) in a pBK-CMV vector, as well as human $K C N H 2$ were kindly provided by Prof. Dirk J. Snyders, University of Antwerp, Antwerp, Belgium. Yotiao was subcloned in a pGW1 vector (a gift 
from Prof. Robert S. Kass, Columbia University, New York, NY, USA). KCNH2 was subcloned in a pcDNA3 vector.

The KCNQ1 mutations A341V, K557E and G589D were generated by site-directed mutagenesis using the QuickChange $®$ II XL system (Stratagene, La Jolla, CA, USA). Oligonucleotide design and reaction conditions (extension time of 2 minutes $/ \mathrm{kb}$ ) were according to the manufacturer's instructions (Stratagene). All constructs were completely sequenced (BigDye ${ }^{\circledR}$ Terminator Ready kit v1.1, Applied Biosystems) and analyzed on an ABI PRISM® 3100 Genetic Analyzer. Plasmid DNA for mammalian expression was grown in DH5 $\alpha$ cells (Invitrogen Co, Carlsbad, CA, USA) and then isolated from the bacterial cells using a miniprep spin kit (Qiagen). Similar procedures were used to combine the phosphomimetic substitutions S27D and S27A in WT KCNQ1 or A341V-KCNQ1.

The KCNQ1-myc construct was generated by inserting the c-myc epitope (N-EQKLISEEDL-C) in the S1S2 external loop of KCNQ1 after nucleotide position 438 (between glutamate 146 and glutamine 147). Two initial PCR reactions were performed to generate c-myc containing KCNQ1 products (PCR 1 and 2 in Table 1). Once c-myc-epitope-containing KCNQ1 complementary cDNA strands were synthesized, the conditions were set for further PCR amplification: $95{ }^{\circ} \mathrm{C}$ for $2 \mathrm{~min}, 1 \mathrm{~min}$ at the corresponding annealing temperature (see Table 1), and $70{ }^{\circ} \mathrm{C}$ for 1 and $2 \mathrm{~min}$, respectively. These settings were applied for 25 cycles. The amplified PCR products were subsequently hybridized using a fusion PCR (PCR 3 in Table 1). After fusion-PCR product purification, KCNQ1-myc construct was subcloned into PGEMT-easy cloning vector (Promega Corp. Madison, WI, USA) using Spe I and BamH1 restriction sites for ligation and was sequenced as described above. For expression in Chinese Hamster Ovary (CHO-K1) cells (CHO cells), the KCNQ1-myc construct was subcloned into a pBK-CMV vector. In all PCR reactions, KOD Hot Start DNA polymerase Kit (Novagen, EMD Biosciences, La Jolla, CA, USA) was used according to the manufacturer's instructions. Restriction enzymes were from Fermentas (Fermentas GmbH, Germany).

\begin{tabular}{|ccccc|}
\hline PCR & Primer & Sequence & $\begin{array}{c}\text { Length } \\
\text { (bp) }\end{array}$ & $\begin{array}{c}\text { Annealing } \\
\text { ( }\end{array}$ \\
\hline PCR 1
\end{tabular}

Table 1. PCR settings for the generation of KCNQ1-myc construct. All PCRs were performed in the KCNQ1 gene with accession number NM_000218. 


\section{Cell Culture and Transfection for Electrophysiology Experiments}

$\mathrm{CHO}$ cells were cultured at $37{ }^{\circ} \mathrm{C} / 5 \% \mathrm{CO}_{2}$ in Ham's F-12 medium (Gibco, Invitrogen, Co.) supplemented with $10 \%$ fetal calf serum and $1 \%$ penicillin/streptomycin. For patch-clamp recordings in WT or homozygous mutant experiments KCNQ1 ( $0.5 \mu \mathrm{g}$; WT or mutant of interest) and KCNE1 (1 $\mu \mathrm{g})$ cDNA were transiently co-transfected in CHO cells using Fugene 6 (Roche Diagnostics Nederland B.V., Almere, The Netherlands). In coexpression studies (examining heterozygous conditions) $0.25 \mu \mathrm{g}$ WT KCNQ1 and $0.25 \mu \mathrm{g}$ mutant KCNQ1 were co-transfected with $1.0 \mu \mathrm{g}$ KCNE1. In addition, Yotiao (2 $\mu \mathrm{g})$ was co-transfected in all patch-clamp experiments in which WT or mutant $\mathrm{I}_{\mathrm{Ks}}$ was studied, unless specified otherwise. In experiments assessing the $\alpha$ - $\alpha$-subunit interactions between KCNQ1 and $K C N H 2,0.5 \mu \mathrm{g}$ WT or A341V KCNQ1 was co-transfected with $0.5 \mu \mathrm{g} K C N H 2$. Cells were used for analysis $24 \mathrm{hrs}$ after transfection.

\section{Electrophysiology}

For electrophysiological studies, $\mathrm{CHO}$ cells were harvested from the $35-\mathrm{mm}$ culture dish by brief trypsinization, washed twice with culture medium (Ham's F-12 medium supplemented with 10\% fetal calf serum and $1 \%$ penicillin/streptomycin) and placed in a perfusion chamber on an inverted microscope that was continuously perfused with external Tyrode solution containing (in $\mathrm{mmol} / \mathrm{L}$ ): $\mathrm{NaCl} 132, \mathrm{KCl} 4.8, \mathrm{MgCl}_{2} 1.2, \mathrm{CaCl}_{2} 2$, HEPES 10, and glucose $5(\mathrm{pH}=7.4$ with $\mathrm{NaOH}$ ). Pipettes were filled with pipette solution containing (in mmol/L): K-aspartate 110, ATP- $\mathrm{K}_{2} 5, \mathrm{MgCl}_{2}$ 1, $\mathrm{CaCl}_{2}$ 1, EGTA 11, HEPES $10(\mathrm{pH}=7.3$ with $\mathrm{KOH})$. In some experiments, $0.2 \mu \mathrm{mol} / \mathrm{L}$ okadaic acid (OA; EMD Biosciences, La Jolla, CA, USA) and $200 \mu \mathrm{mol} / \mathrm{L}$ cAMP (Sigma-Aldrich Chemie B.V. Zwijndrecht, The Netherlands), indicated as +CAMP/OA throughout, were added to the pipette solution to activate PKA and thereby mimic stimulation of $\beta$-adrenergic receptors. In KCNQ1 (WT or A341V) / KCHN2 cotransfections HMR1556 (500 nmol/L in DMSO; a kind gift of Dr. H. Gögelein, Sanofi-Aventis, Frankfurt am Main, Germany) was used to selectively inhibit the current carried by KCNQ1. Experiments were performed by the whole-cell patch-clamp technique at room temperature $(23 \pm 1$ ${ }^{\circ} \mathrm{C}$ ) using an Axopatch 200B amplifier and pCLAMP software (Axon Instruments, Union City, CA, USA). Patch pipettes were pulled using a DMZ-Universal-Puller (Zeitz-Instruments Vertriebs GmbH, Martinsried, Germany), using $2 \mathrm{~mm}$ borosilicate glass capillaries (World Precision Instruments Germany $\mathrm{GmbH}$, Berlin, Germany). Pipette resistance ranged between 1.5 and $3 \mathrm{M} \Omega$. Access resistance was accepted when below $8 \mathrm{M} \Omega$ and was electronically compensated at $60-80 \%$ series resistance. Currents were sampled at $2 \mathrm{kHz}$ after low-pass filtering at $1 \mathrm{kHz}$ through a Digidata $1322 \mathrm{~A}$ data acquisition system (Axon Instruments). Current densities $(\mathrm{pA} / \mathrm{pF})$ were obtained after normalization to cell capacitance determined with Clampex 9.

For WT and mutant $I_{\mathrm{Ks}}$ studies, the following voltage-clamp protocols were used: (i) a cAMP/OAwash-in protocol started after membrane rupture and correction for cell capacitance and consisted of 20 pulses (given at 15 -second intervals for a total of 5 minutes) to $+60 \mathrm{mV}$ for 2 seconds (from a holding potential of $-70 \mathrm{mV}$ ) followed by 2 seconds at a holding potential of $-40 \mathrm{mV}$ to measure tail currents during wash in of CAMP and OA; (ii) an activation protocol consisting of a 5-s depolarizing pulse (between $-50 \mathrm{mV}$ and $+90 \mathrm{mV}, 10 \mathrm{mV}$ increments) to determine peak currents and activation kinetics followed by $6 \mathrm{~s}$ at $-40 \mathrm{mV}$ to measure tail currents, and (iii) a deactivation protocol of $4 \mathrm{~s}$ at + 
$60 \mathrm{mV}$ followed by repolarization to a potential between $-120 \mathrm{mV}$ and $-30 \mathrm{mV}$ (in steps of $10 \mathrm{mV}$ ). For $K C N H 2 / H E R G$ studies, 1-s depolarizing pulses from $-50 \mathrm{mV}$ to $+50 \mathrm{mV}$ were used, followed by a 2-s step to $-50 \mathrm{mV}$.

\section{Phosphorylation Assays, Protein Extracts, Immunoprecipitation and Western Blots}

Phosphorylation of KCNQ1 channels in $\mathrm{CHO}$ cells was induced by incubating cells at $37^{\circ} \mathrm{C}$ under $5 \%$ $\mathrm{CO}_{2}$ atmosphere with $300 \mu \mathrm{mol} / \mathrm{L}$ CPT-cAMP (Biaffin GmbH \& Co, Kassel, Germany) plus $0.2 \mu \mathrm{mol} / \mathrm{L}$ OA (EMD Biosciences, La Jolla, CA, USA), or with vehicle solution, diluted in F12 medium without serum and without antibiotics during $10 \mathrm{~min}$. For total protein extraction, cells were washed twice in chilled phosphate-buffered saline (PBS) and centrifuged at 3,000 $\times \mathrm{g}$ for $10 \mathrm{~min}$ to separate cells from PBS. The pellet was then lysed on ice with HES buffer $(20 \mathrm{mmol} / \mathrm{L} \mathrm{HEPES} \mathrm{pH} \mathrm{7.4,} 1 \mathrm{mmol} / \mathrm{L}$ EDTA, $255 \mathrm{mmol} / \mathrm{L}$ sucrose), supplemented with Complete Protease Inhibitor Cocktail Tablets (Roche Diagnostics Nederland B.V., Almere, The Netherlands) and lysates were homogenized by repeated passing (10 times) through a $25 \mathrm{G}(0.45 \times 16 \mathrm{~mm})$ needle.

Total protein extracts $(20 \mu \mathrm{g})$ were resuspended in SDS $4 \times(40 \%$ glycerol, $5 \% \beta$-mercaptoethanol, $8 \%$ $\mathrm{SDS}, 0.04 \%$ bromophenol blue, $240 \mathrm{mmol} / \mathrm{L} \mathrm{Tris} / \mathrm{HCl} \mathrm{pH} \mathrm{6.8)}$ and heated at $55{ }^{\circ} \mathrm{C}$ during $5 \mathrm{~min}$. Samples were then centrifuged during $5 \mathrm{~min}$ at 13,000 rpm at room temperature and $20 \mu \mathrm{l}$ of the protein extract was loaded and run in SDS-PAGE (6\% acrylamide/bisacrylamide) with Mini-Protean Tetra-Cell (Bio-Rad, Hercules, CA, USA) and transferred to a PVDF membrane (Amersham, GE Healthcare, Fairfield, CT, USA) using Mini Trans-Blot cell (Bio-Rad).

For co-immunoprecipitation studies, CHO cells were transfected with KCNQ1-WT or KCNQ1-A341V (in $\mathrm{pBK}$ vector) + KCNE1 + Yotiao. $\mathrm{CHO}$ cells were lysed in $150 \mathrm{mmol} / \mathrm{L} \mathrm{NaCl}, 20 \mathrm{mmol} / \mathrm{L}$ Tris (pH 7.5), $5 \mathrm{mmol} / \mathrm{L}$ EDTA and 1\% Triton X-100 supplemented with Complete Protease Inhibitor Cocktail Tablets (Roche Diagnostics Nederland B.V., Almere, The Netherlands). Cell extracts were incubated for $30 \mathrm{~min}$ at $4{ }^{\circ} \mathrm{C}$ under agitation. The cell lysates were centrifuged for $15 \mathrm{~min}$ at 13,000 rpm and supernatants were separated. Protein G-sepharose beads ( $40 \mu \mathrm{l}$; Santa Cruz Biotech., CA, USA) were washed 3 times with $500 \mu \mathrm{l}$ IPB buffer $(150 \mathrm{mmol} / \mathrm{L} \mathrm{NaCl}, 1 \% \mathrm{NP} 40,10 \mathrm{mmol} / \mathrm{L} \mathrm{HEPES}, 10 \%$ glycerol; $\mathrm{pH}$ 7.8), centrifuged for $1 \mathrm{~min}$ at $5000 \mathrm{rpm}$ and subsequently incubated with $2 \mu \mathrm{g}$ of anti-KCNQ1 antibody (Santa Cruz Biotech. CA, USA) under rotation for $2 \mathrm{~h}$ at $4{ }^{\circ} \mathrm{C}$. After incubation, the beads were centrifuged ( $5000 \mathrm{rpm}$ for $1 \mathrm{~min}$ ), washed 3 times with $500 \mu \mathrm{l}$ IPB and incubated overnight with $300 \mu \mathrm{g}$ of protein extract under orbital shaking. After centrifugation (5000 rpm for $1 \mathrm{~min}$ ) and washing with $500 \mu \mathrm{I} \mathrm{IPB}$ at $4{ }^{\circ} \mathrm{C}$ ( 3 times), the beads were resuspended in $30 \mu \mathrm{l}$ SDS $1 \mathrm{x}$, denaturated at $55{ }^{\circ} \mathrm{C}$ for $5 \mathrm{~min}$ and centrifuged at $5000 \mathrm{rpm}$ for $5 \mathrm{~min} .20 \mu \mathrm{l}$ of the immunocomplex was then loaded and run in SDS-PAGE (7\% acrylamide/bisacrylamide) with Mini-Protean Tetra-Cell (Bio-Rad, Hercules, CA, USA) and transferred to a PVDF membrane (Amersham, GE Healthcare, Fairfield, CT, USA) using Mini Trans-Blot cell (Bio-Rad). 
Mouse monoclonal anti-KCNQ1 (1:1000, Neuromab, UCDavis, Ca, USA) and rabbit polyclonal antiKCNQ1-S27-phospho (1:500, kindly provided by Prof. Robert S. Kass, University of Columbia, NY, USA) were used to test KCNQ1-channel phosphorylation at Serine 27. Anti-KCNQ1 (Neuromab) and anti-Yotiao (Santa Cruz Biotecht. CA, USA) were used for co-immunoprecipitation studies. Secondary antibodies were horseradish peroxidase linked rabbit anti-mouse (1:2500; DAKO, Glostrup, Denmark) or goat anti-rabbit (1:2500; Santa Cruz Biotech., CA, USA). Proteins were detected using ECL-Plus Western blotting reagent (Amersham, GE Healthcare, Fairfield, CT, USA). The Yotiao band at $230 \mathrm{kD}$ and the KCNQ1-monomer band of approximately $70 \mathrm{kD}$ were quantified using Image J (http://rsb.info.nih.gov/iij; developed by Dr. Wayne Rasband, National Institutes of Health, Bethesda, $\mathrm{MD}$ ), in both S27-phospho and total KCNQ1 blots and the fraction of Yotiao interaction or phosphorylated KCNQ1 was quantified.

\section{Confocal Imaging}

CHO cells seeded in sterilized coverslips were cultured in Ham's F12 medium containing 10\% FBS and $1 \%$ penicilin/streptomycin antibiotics until they reached $60 \%$ confluency. Cells were then transiently transfected with KCNQ1-GFP, KCNQ1-A341V-GFP or, KCNQ1-myc + A341V-GFP + KCNE1 + Yotiao cDNAs using Lipofectamine LLTX and PLUS Reagent (Invitrogen Co.). 48 Hours after transfection, cells were incubated in vivo, under $5 \% \mathrm{CO}_{2}$ atmosphere and at $37{ }^{\circ} \mathrm{C}$ during 1 hour with 1:500 mouse monoclonal anti-myc antibody (Sigma-Aldrich). Cells were washed 3 times with PBS and fixed with 4\% paraformaldehyde in PBS for 10 min. Coverslips were further incubated with Texas Red conjugated goat anti-mouse antibody (1:80, ITK Southern Biotech, Uithoorn, The Netherlands) during $45 \mathrm{~min}$. Stainings with a secondary antibody were performed at room temperature and cells were washed 3 times in PBS after all of the steps. No permeabilization was applied in the immunostainings. Finally, coverslips were mounted in $75 \%$ glycerol, $0.02 \mathrm{~mol} / \mathrm{L} \mathrm{TRIS}-\mathrm{HCl}(\mathrm{pH}=8.0)$, $0.8 \% \mathrm{NaN}_{3}, 2 \%$ 1,4-diazabicyclo[2.2.2] octane (DABCO; $\mathrm{pH}=8.0$ ).

Confocal analysis was performed at least 24 hours after the stainings, and the immunostained samples were visualized using a Leica TCS-SPE (Leica Microsystems GmbH, Wetzlar, Germany) confocal microscope and further analyzed by Image J. Transfection efficiency was determined by counting the fraction of cells that showed both GFP signal and DAPI staining. Determination of cells showing membrane GFP signal was performed independently by multiple investigators who were blinded with respect to transfection type (WT versus A341V). For cells showing clear membrane expression, fluorescence intensity at the membrane was subsequently determined at 3 separate membrane sites per cell for identical settings of the microscope using ImageJ. 


\section{Computational Modeling}

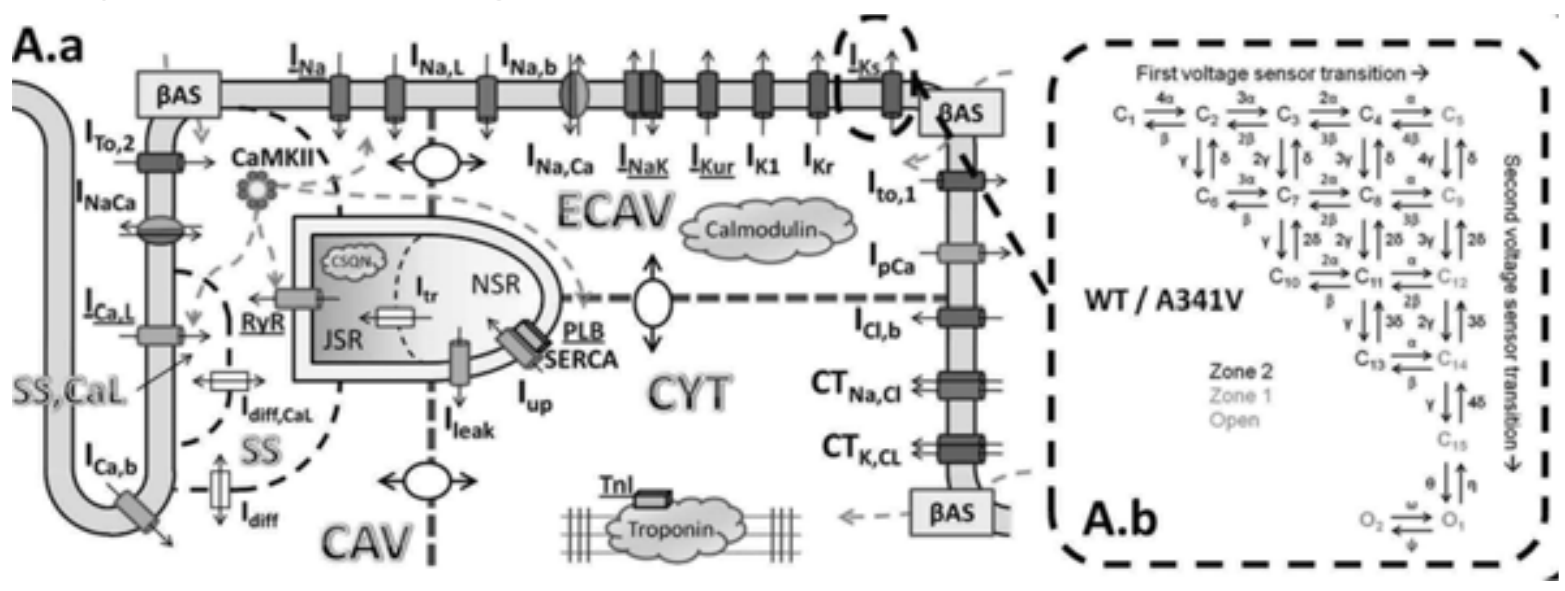

Figure 1. Computational modeling approach. A.a. Schematic overview of the structure of a single cell model, including all ion channels, pumps, exchangers, $\mathrm{Ca}^{2+}$-handling proteins, $\mathrm{Ca}^{2+} / \mathrm{Calmodulin}$ dependent kinase and compartmental $\beta A R$ signaling pathway, as published recently (Chapter 3). ${ }^{14}$ A.b. Structure of the 17-state Markov model that was fitted to WT $I_{\mathrm{Ks}}$, heterozygous or homozygous mutant channel characteristics in the absence and presence of $\beta A R$ stimulation. For color figure, see page 251.

Parameters of the 17-state $I_{\mathrm{Ks}}$ Markov model proposed by Silva and Rudy ${ }^{15}$ (Figure 1A.b) were fitted to the normalized I-V relationship, time constants of activation and time constants of deactivation obtained in $\mathrm{CHO}$ cells expressing WT or mutant $\mathrm{I}_{\mathrm{Ks}}$ channels in the absence or presence of CAMP/OA (Figure 2). Quantitative values of time constants of activation and deactivation of WT unstimulated $\mathrm{I}_{\mathrm{Ks}}$ were scaled to those obtained in canine ventricular myocytes ${ }^{16,17}$ at $37^{\circ} \mathrm{C}$ (at $+20 \mathrm{mV}$ and $-80 \mathrm{mV}$, respectively) to compensate for differences due to temperature and effects of the expression system. We determined one correction factor for the activation time-constant and one for deactivation for $W T I_{\mathrm{Ks}}$ in our $\mathrm{CHO}$ cells at room temperature at these holding potentials to obtain similar half-maximal activation and deactivation times. All WT time constants were then scaled by these values, to maintain the voltage dependence of activation and deactivation measured in $\mathrm{CHO}$ cells. The adjusted time constants were used as input in the parameter fitting procedure. The time constants in the presence of adrenergic stimulation and/or A341V mutation were subsequently scaled to produce a similar relative difference as observed in the heterologous expression systems (e.g., the time constant of activation at $+20 \mathrm{mV}$ is 2.2 fold slower in A341V compared to WT). Similarly, baseline current amplitude of WT unstimulated $I_{\mathrm{Ks}}$ was fit to that in canine epicardial myocytes. ${ }^{18}$ The approximately $60 \%$ increase in current amplitude observed with cAMP/OA stimulation in $\mathrm{CHO}$ cells was scaled to resemble the approximately $200 \%$ increase in $\mathrm{I}_{\mathrm{Ks}}$ observed in ventricular myocytes during $\beta$-adrenergic stimulation ${ }^{17}$ and the relative difference in tail current amplitude between WT and A341V observed in $\mathrm{CHO}$ cells was preserved. $\mathrm{I}_{\mathrm{Ks}}$ model parameters are given in the appendix of this thesis. The adjustments were necessary to apply the results obtained in the heterologous expression system in the model. The phosphorylated and non-phosphorylated $\mathrm{I}_{\mathrm{ks}}$ models were incorporated into a recently developed model of the canine epicardial myocyte incorporating detailed localized effects of $\beta$-adrenergic stimulation ${ }^{14}$ (Figure 1A.a; Chapter 3). This model uses a population-based approach to distinguish between phosphorylated and non- 
phosphorylated channels. The net current is the sum of the currents produced by the channels in each population. The WT $I_{\mathrm{Ks}}$ profile during the cardiac action potential in the presence or absence of $\beta$-adrenergic stimulation was similar to that of the original model, based entirely on canine data (not shown).
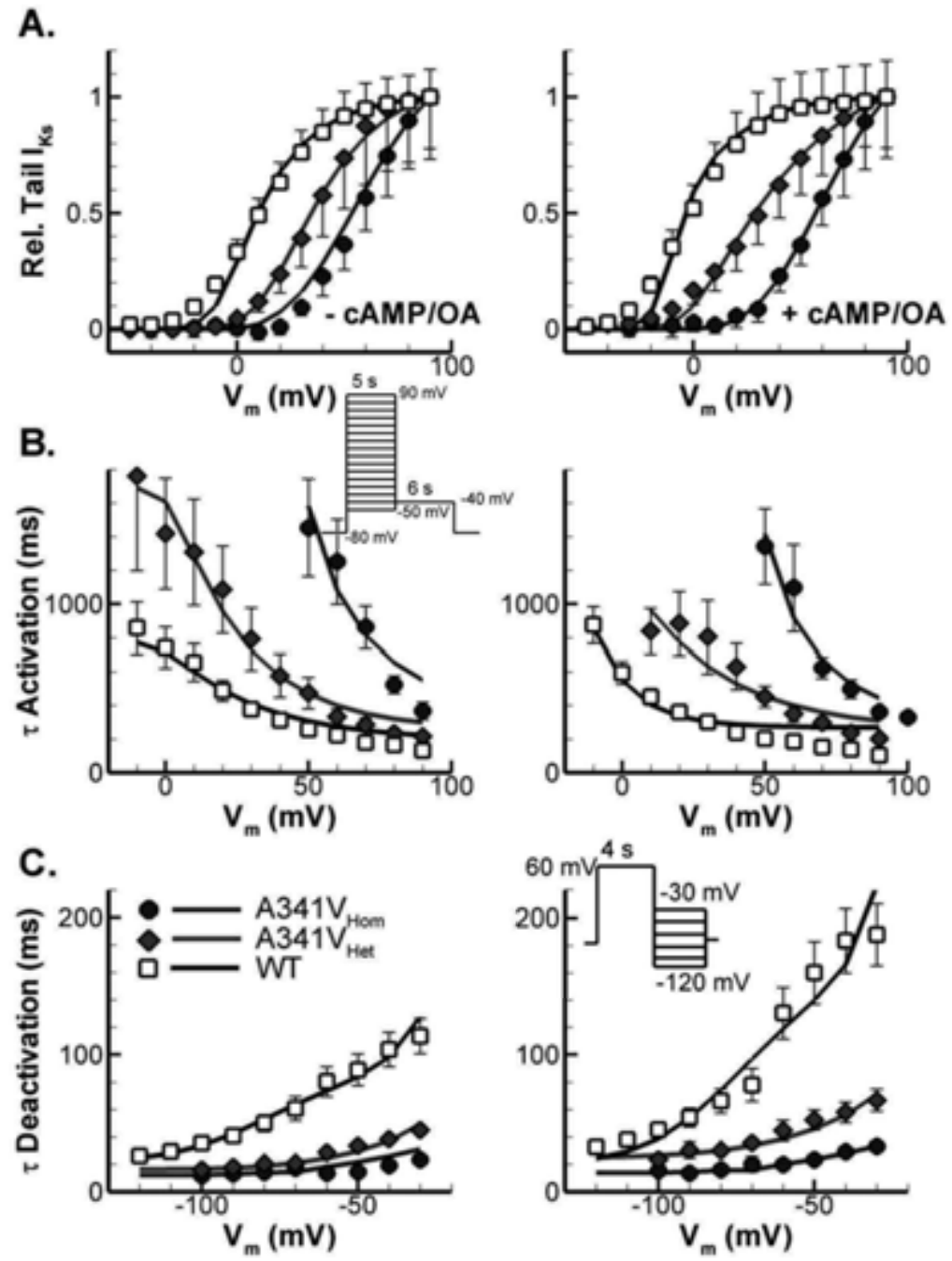

Figure 2. Validation of $I_{\mathrm{Ks}}$ model properties. A. Relative $I_{\mathrm{Ks}}$-tail amplitude in WT (squares), heterozygous A341V (diamonds) and homozygous A341V (circles) conditions in the absence (left) and presence (right) of cAMP stimulation in experiments (symbols) and model (lines). B. Same as panel A for time constants of activation based on a mono-exponential fit. C. Same as panel A for time constants of deactivation. Time constants were scaled to match values found in canine ventricular myocytes at $37^{\circ} \mathrm{C}$. Insets show voltage-clamp protocols used.

The complete model consists of a coupled system of ordinary differential equations. Numerical integration was performed in custom $\mathrm{C}++$ software using a forward Euler method with adaptive time step. The Matlab (The Mathworks ${ }^{\circledR}$, Natick, MA) ODE15s steady-state solution and C++ forward Euler method produced identical action potentials and calcium transients. 
The model was paced for 2000 seconds at any given setting (cycle length and isoproterenol concentration) with a conservative $\mathrm{K}^{+}$stimulus $^{19}$ to achieve a pseudo steady-state in which actionpotential duration (determined at $95 \%$ of repolarization) and maximum intracellular ion concentrations change less than $0.05 \%$ on a beat-to-beat basis. Isoproterenol levels ranged between $0 \mu \mathrm{mol} / \mathrm{L}$ (no $\beta$-adrenergic stimulation) and $1.0 \mu \mathrm{mol} / \mathrm{L}$ (maximum stimulation). Simulations were performed using $W T$ and mutant $I_{\mathrm{Ks}}$ formulation in the absence and presence of adrenergic stimulation.

\section{Data Analysis}

The voltage dependence of activation (evaluated from normalized tail-current amplitudes) was fitted with a Boltzmann equation, $G / G_{\max }\left(\right.$ or $\left.I / I_{\max }\right)=1 /\left(1+\exp \left(\left(V-V_{1 / 2}\right) / k\right)\right.$ to determine the membrane potential for half-maximal activation $\left(V_{1 / 2}\right)$ and the slope factor $(k)$. The time courses of activation and deactivation were fitted with a single exponential function $I / I_{\max }=A \times(1-\exp (-\mathrm{t} / \tau))+\mathrm{C}$ where $A$ is amplitude and $\tau$ is time constant. Data are expressed as mean \pm SEM. Unpaired Student's $t$-test or 1 way ANOVA with Tukey's Post Hoc test were used to assess differences between groups. $P<0.05$ was considered statistically significant.

\section{RESULTS}

KCNQ1-A341V Results in a Dominant-Negative Suppression of CAMPDependent Upregulation of $I_{\mathrm{Ks}}$ on Top of a Dominant-Negative Reduction in Basal Current

The KCNQ1-A341 residue is located in the middle of the 56-transmembrane domain, just before the PAG 'hinge' motif ${ }^{20}$ and is extremely well conserved among the families of voltage-gated $\mathrm{K}^{+}$channels (Figure 3). 
A.

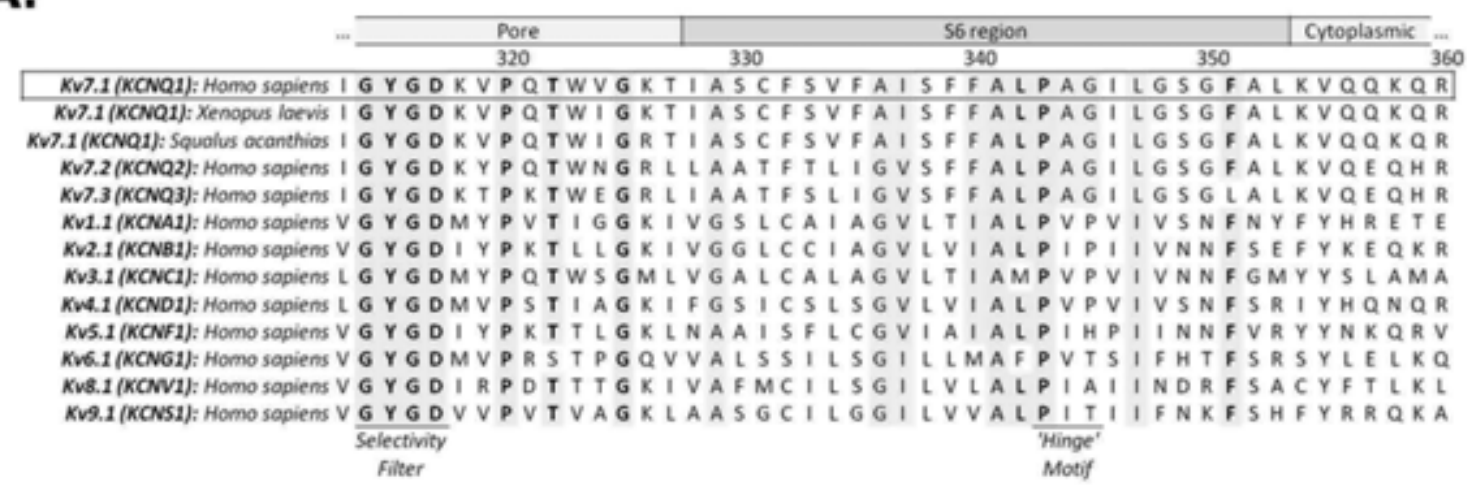

B.
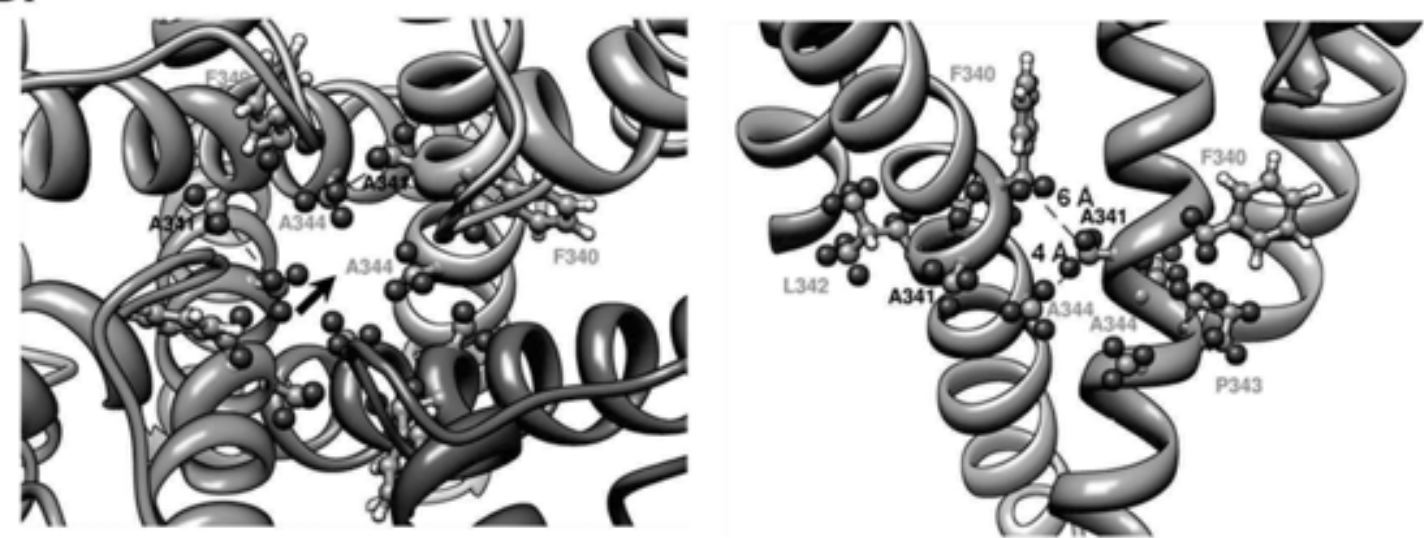

Figure 3. Location and conservation of A341 in KCNQ1. A. Multiple sequence alignment of the KCNQ1-S6 region with other members of the Kv7.x family and other voltage-gated potassium channels. Conserved residues are bold and highlighted in blue, partially conserved residues are highlighted in green and red. The conserved $\mathrm{K}^{+}$selectivity filter and the PAG (in Kv7.x) or PXP (in other Kv channels) hinge motifs are indicated below the alignment. A341 is indicated in red and is extremely conserved among all Kv channels. B. Location of A341 in the closed-state tetrameric KCNQ1 homology model described by Kang et al. ${ }^{21}$ Left panel shows top view from the extracellular side. Residues F340, A341 and A344 are shown as 'ball-and-stick' models. Black arrow indicates the viewing direction for the right panel. Right panel shows front view of two KCNQ1-subunits (only S5, pore and S6 region are shown). Residues F340 - A344 are shown. Distances between residue A341 of the right subunit and its two nearest residues are indicated with dashed lines. For color figure, see page 251. 
A.

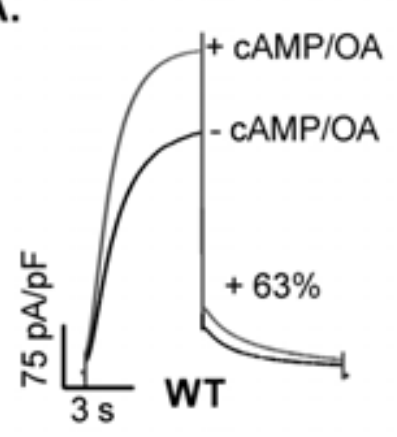

B.

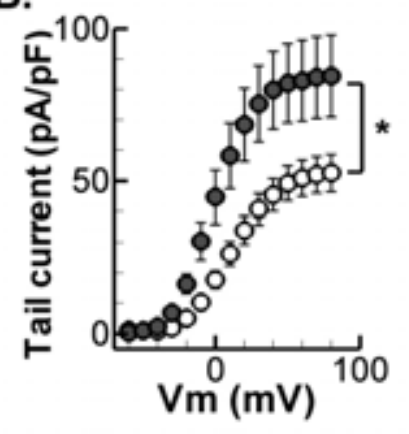

C.

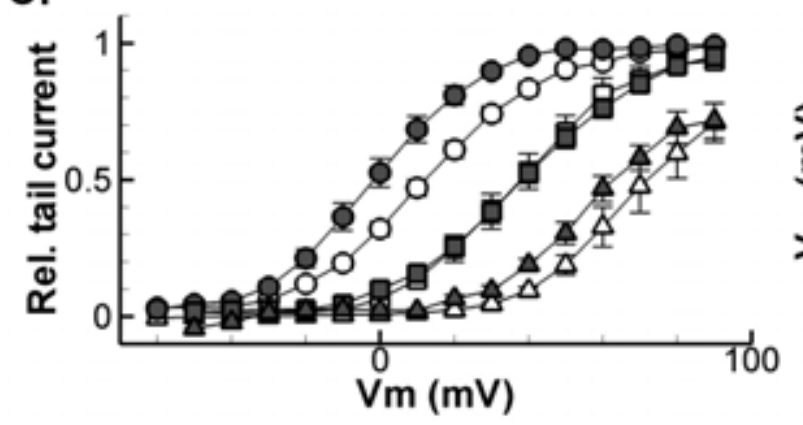

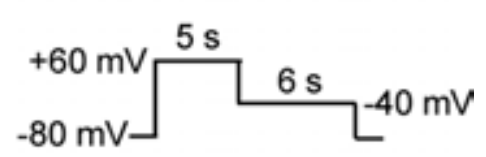
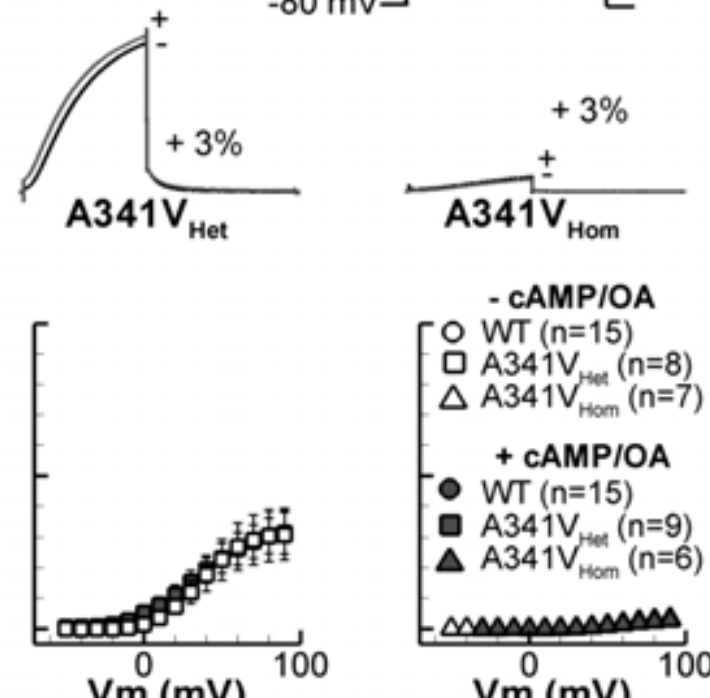

- CAMPIOA

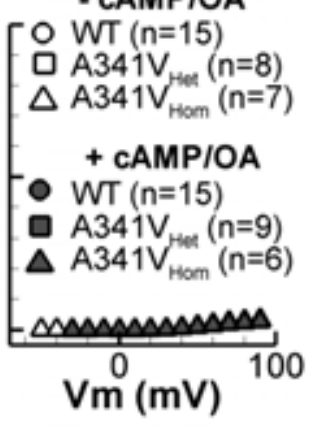

D.

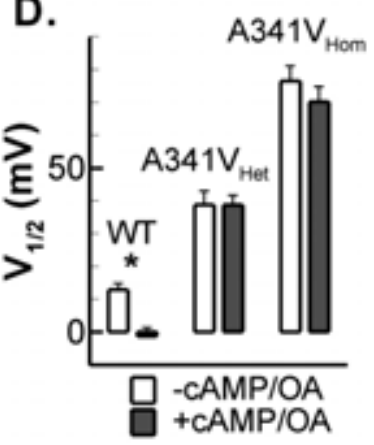

Figure 4. Dominant-negative suppression of CAMP-dependent $I_{\mathrm{Ks}}$ stimulation on top of dominantnegative suppression of basal current by the KCNQ1 mutation A341V. A. Averaged current traces of WT (left), $A 341 V_{\text {Het }}$ (middle) or $A 341 V_{\text {Hom }}$ (right) channels during a 5-s depolarizing pulse to $+60 \mathrm{mV}$ in the absence (-) or presence (+) of intrapipette CAMP/OA. Inset shows voltage-clamp protocol. WT $\mathrm{I}_{\mathrm{Ks}}$, but not $\mathrm{A} 341 \mathrm{~V}$, is upregulated in response to stimulation with cAMP/OA. B. Tail I-V relationship after 5-s depolarizing pulses from $-50 \mathrm{mV}$ to $+90 \mathrm{mV}$ in the absence (open symbols) or presence (filled symbols) of stimulation with CAMP/OA for the conditions in panel A. C. Normalized tail currents for WT (circles), $A 341 V_{\text {Het }}$ (squares) and $A 341 V_{\text {Hom }}$ (triangles) with (open) or without (filled symbols) CAMP/OA. D. Membrane potential resulting in half-maximal activation based on data in panel $\mathrm{C}$. CAMP responsiveness of $\mathrm{WT} \mathrm{I}_{\mathrm{Ks}}$ is characterized by a significant leftward shift in half-maximal activation $(*, P<0.05)$ that is not present in A341V.

Consistent with previous results, ${ }^{11}$ we found that the KCNQ1-A341V mutation resulted in a significant reduction of $\mathrm{I}_{\mathrm{Ks}}$. The $\mathrm{I}_{\mathrm{Ks}}$-tail amplitude after 5 -s depolarizing pulses at $+60 \mathrm{mV}$ was reduced by $96 \%$ and $46 \%$ in homozygous $\left(A 341 V_{\text {Hom }}\right.$ ) and heterozygous $\left(A 341 V_{\text {Het }}\right)$ conditions, respectively (Figure 4A,B). A significant rightward shift in channel activation was also observed (Figure 4C,D). In addition, there was a significant slowing of activation kinetics (time constants at $+60 \mathrm{mV}$ were 
$1306 \pm 156 \mathrm{~ms}, 1947 \pm 265 \mathrm{~ms}$ and $7303 \pm 1486 \mathrm{~ms}$ in $\mathrm{WT}, \mathrm{A} 341 \mathrm{~V}_{\text {Het }}$ and $\mathrm{A} 341 \mathrm{~V}_{\text {Hom, }}$, respectively) as well as faster deactivation (time constants at $-80 \mathrm{mV}$ : $501 \pm 61 \mathrm{~ms}, 205 \pm 14 \mathrm{~ms}$ and $144 \pm 24 \mathrm{~ms} ; P<0.05$ vs. WT for both $A 341 V_{\text {Het }}$ and $A 341 V_{\text {Hom }}$ ). Together, these altered kinetics contributed to the dominantnegative reduction in repolarizing current for depolarizations of physiological duration in $A 341 \mathrm{~V}_{\text {Het }}$ (74\% reduction in peak $I_{\mathrm{Ks}}$ after $300-\mathrm{ms}$ depolarizing pulses, not shown). No differences were found in the number of cells showing membrane expression or the fluorescence intensity at the membrane between $W T$ and $A 341 V_{\text {Hom }}$ (Figure 5), indicating that the observed reduction in $I_{\mathrm{Ks}}$ is unlikely to be due to reduced expression. We also found no differences in the effect of WT and $A 341 V_{\text {Hom }}$ on KCHN2 current, which has been reported for other trafficking-deficient KCNQ1 mutations (Figure 6).
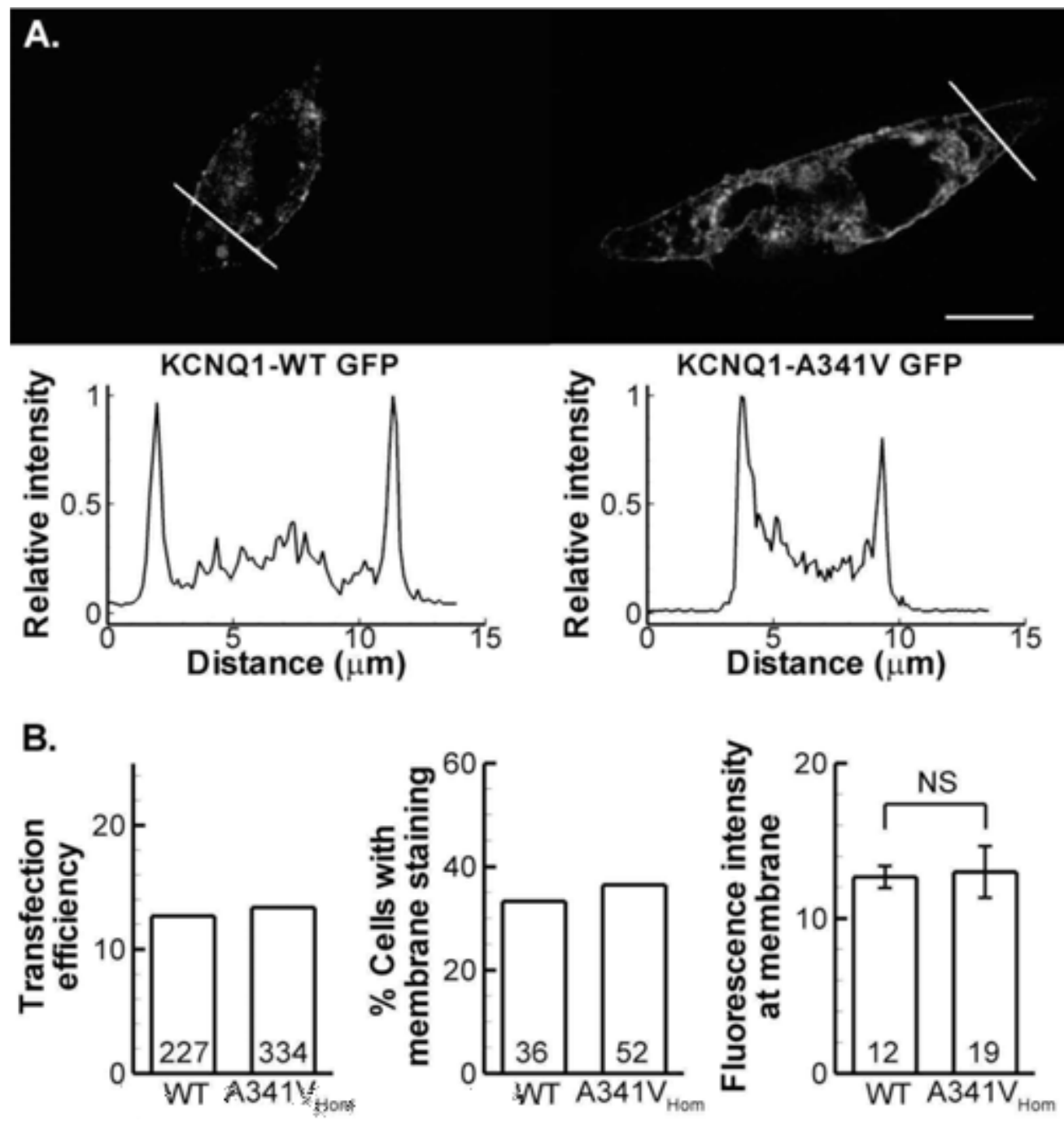

Figure 5. Membrane expression of KCNQ1-WT and KCNQ1-A341V. A. Confocal image of a representative CHO cell expressing KCNQ1-WT-GFP (left panel) or KCNQ1-A341V-GFP (right panel). The white scale bar at the bottom of the right panel indicates $10 \mu \mathrm{m}$. Bottom panels show relative fluorescence intensity along the cross sections indicated with white lines. Both signals show increased intensities at the cell boundaries, indicating membrane expression. B. Average data from confocal images of KCNQ1-WT or KCNQ1-A341V cells for transfection efficiency (left panel, determined as percentage of cells showing GFP signal compared to the number showing DAPI 
staining), percentage of cells with clear membrane signal (middle panel), and fluorescence intensity at the membrane for cells with clear membrane signal. Numbers in bars indicate the number of cells analyzed. There is no difference in transfection efficiency, the number of cells showing a clear membrane signal, or the fluorescence intensity at the membrane between WT and A341V.

To study the effect of simulated $\beta A R$ (as part of sympathetic) stimulation on WT and A341V $I_{\mathrm{Ks}}$ channels, transfected $\mathrm{CHO}$ cells were stimulated with CAMP/OA in the pipette solution. ${ }^{5} \mathrm{WT} \mathrm{I}_{\mathrm{Ks}}$ was significantly upregulated in the presence of CAMP/OA (Figure 4A, left panel; 63\% increase in tailcurrent amplitude). In contrast, both $\mathrm{A} 341 \mathrm{~V}_{\text {Het }}$ and $\mathrm{A} 341 \mathrm{~V}_{\text {Hom }}$ channels showed a negligible increase in current amplitude (Figure $\mathbf{4 A}, \mathbf{B}$ ). The leftward shift in I-V relationship that occurred during CAMPdependent stimulation for $W T I_{K_{s}}$ channels was also absent (Figure 4C,D). This cAMP desensitivity, even in $A 341 V_{H e t}$, suggested that $I_{K s}$ modulation is under dominant-negative control, whereby presence of one defect subunit may be sufficient to abolish $I_{\mathrm{Ks}}$ upregulation.

A.
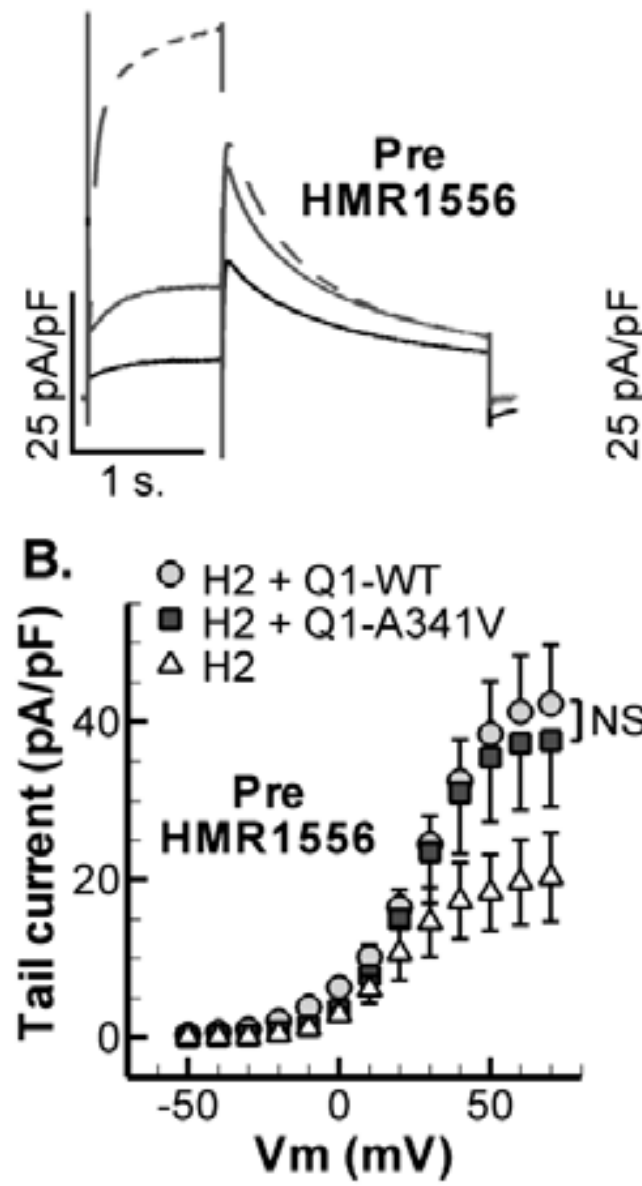
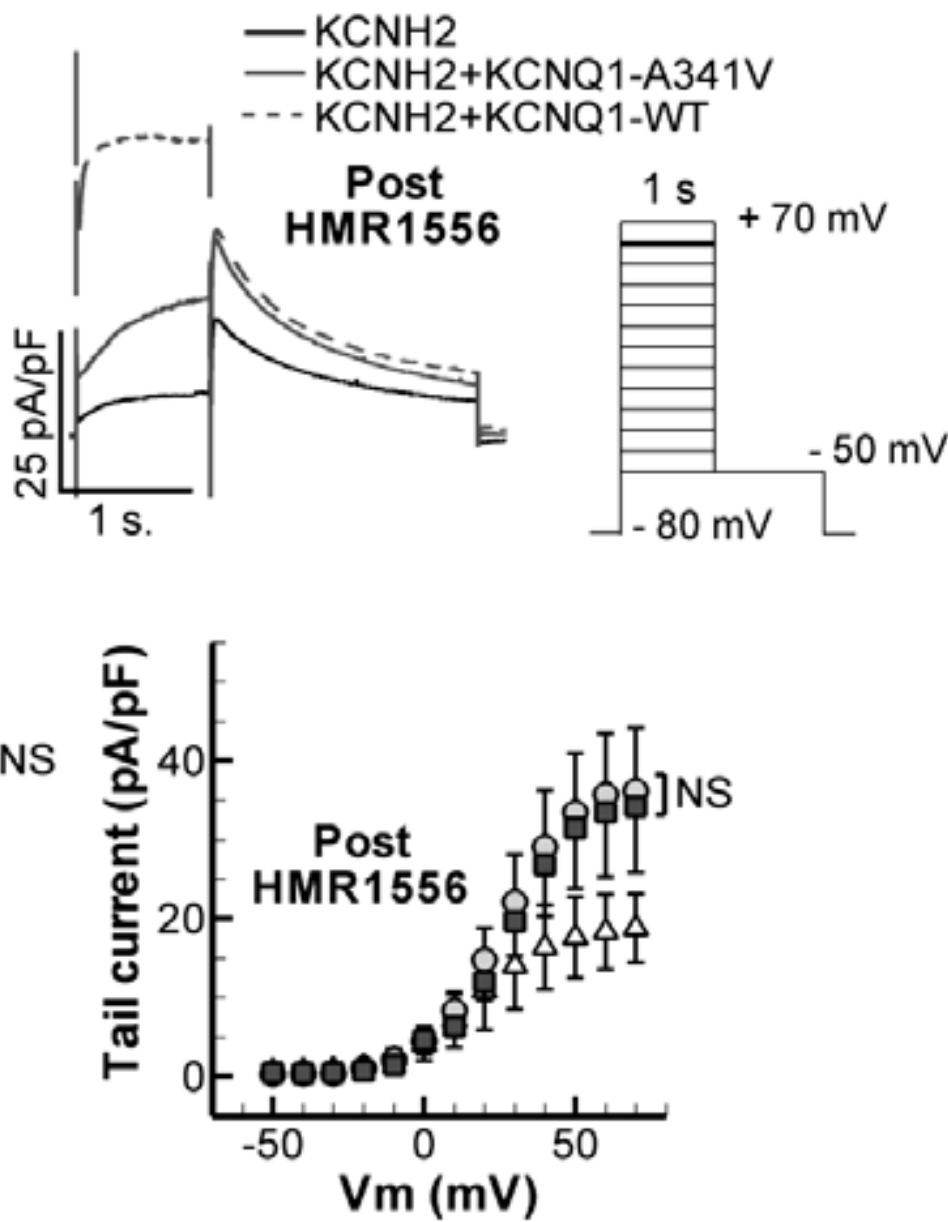

Figure 6. A341V phenotype is not mediated by effects on $\mathrm{KCNH} 2$. A. Averaged current traces during depolarization to $+60 \mathrm{mV}$ in $\mathrm{CHO}$ cells transfected with $\mathrm{KCNH} 2$ alone (solid black line), $\mathrm{KCNH} 2+$ KCNQ1-WT (dashed grey line) or KCNH2 + KCNQ1-A341V (solid grey line) before application of HMR1556 (left) or after application of HMR1556 (right; giving the HMR-resistant KCNH2 current). 
Inset on the right shows voltage-clamp protocol used. B. Tail I-V relationships before (left) or after (right) application of HMR1556 for the conditions in panel (A). KCNH2-only current is indicated with triangles, $\mathrm{KCNH} 2+\mathrm{KCNQ} 1-\mathrm{WT}$ with circles and $\mathrm{KCNH} 2+\mathrm{KCNQ} 1-\mathrm{A} 341 \mathrm{~V}$ with squares. Cotransfection of $\mathrm{KCNQ1-WT}$ increased the $\mathrm{KCNH} 2$ tail current, consistent with previous results. ${ }^{22} \mathrm{~A}$ similar increase in KCNH2 could be observed with KCNQ1-A341V.

\section{Effect of Combined Dominant-Negative A341V Effects on Ventricular Repolarization}

Patients carrying the A341V mutation can show pronounced QTc prolongation (see Figure 7; adapted from Shimizu ${ }^{23}$ ), and QTc is longer among symptomatic than among asymptomatic carriers. ${ }^{11}$ Moreover, QTC duration can prolong during exercise, despite an increase in heart rate (Figure 7, right panel${ }^{23}$ ).
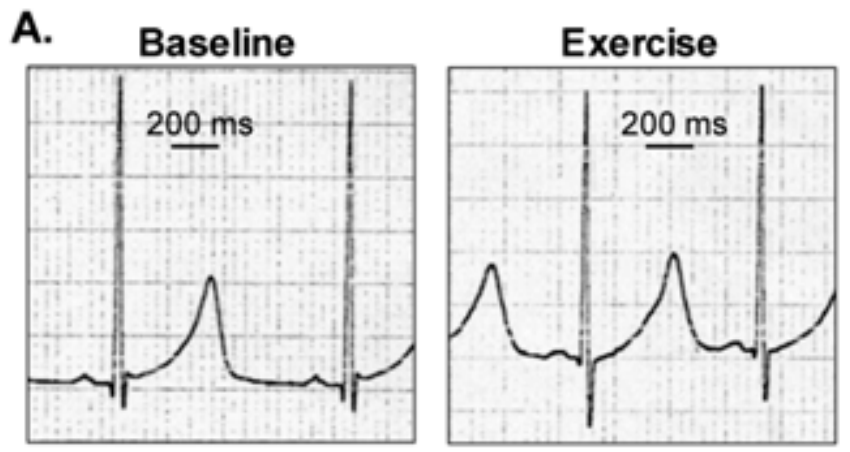

\section{A341V Carrier}

Baseline

$\mathrm{QTc}=513 \mathrm{~ms}$

cTpeak-end $=98 \mathrm{~ms}$

\section{Exercise}

$\mathrm{QTc}=597 \mathrm{~ms}$

cTpeak-end $=152 \mathrm{~ms}$
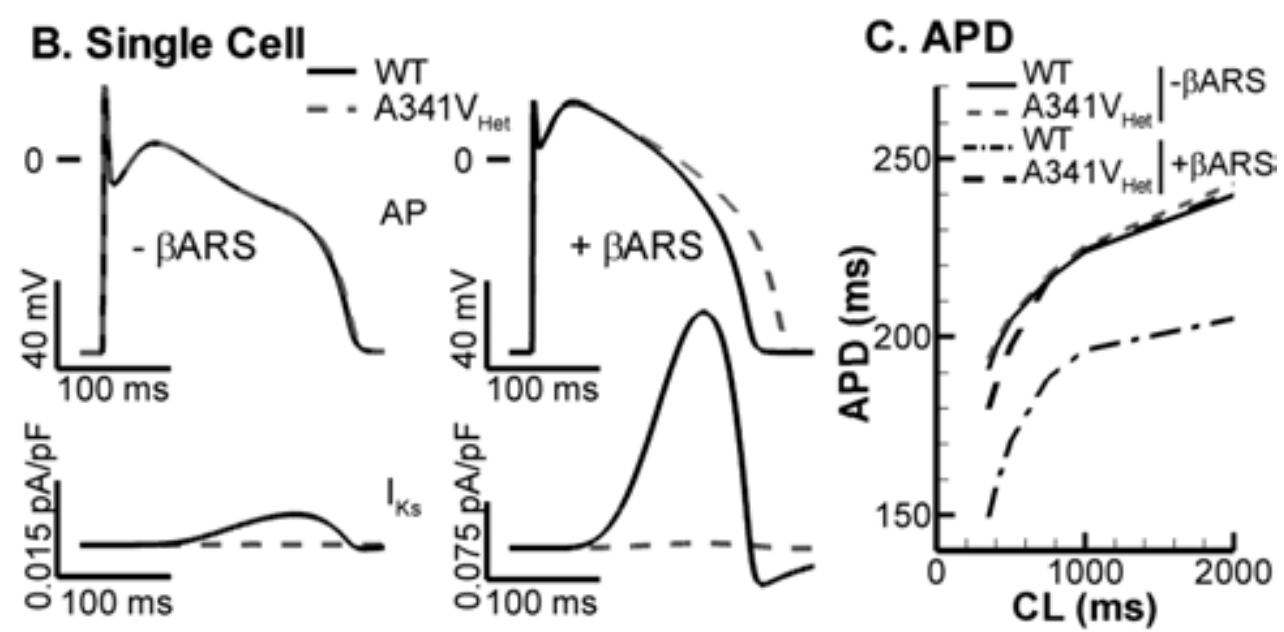

Figure 7. Effect of A341V on ventricular repolarization in the absence or presence of increased sympathetic tone. A. Body-surface ECGs (lead V5) from a 12-year old male A341V carrier at rest (left) and during exercise (right); adapted from Shimizu ${ }^{23}$ with permission. QTC and cTpeak-end are increased during exercise. B. Steady-state action potential (top) and $\mathrm{I}_{\mathrm{ks}}$ (bottom) in the absence (left) or presence (right) of $\beta A R$ stimulation ( $\beta A R S$ ) in a computational model of the canine ventricular myocyte electrophysiology, ${ }_{1}^{14}$ simulating $\mathrm{WT}$ (solid line) or $\mathrm{A} 341 \mathrm{~V}_{\text {Het }}$ (dashed line) conditions at $\mathrm{CL}=$ $1000 \mathrm{~ms}$. Note different scale for $I_{\mathrm{Ks}}$ in the presence of $\beta$ AR stimulation. C. APD rate dependence 
with or without $\beta A R$ stimulation. APD is significantly prolonged in $A 341 V_{\text {Het }}$ conditions during $\beta A R$ stimulation compared to WT.

We examined the effect of $A 341 \mathrm{~V}$ on ventricular repolarization using a computational model of $\beta A R$ stimulation in the canine ventricular myocyte (Figure 1; Chapter 3). ${ }^{14}$ The A341V mutation caused a major reduction in $\mathrm{I}_{\mathrm{Ks}}$ amplitude during the action potential, even in heterozygous conditions (Figure 7B, bottom panels). Under basal conditions $I_{\mathrm{Ks}}$ plays a minor role in ventricular repolarization and, consistently, loss of $\mathrm{I}_{\mathrm{Ks}}$ by A341V resulted only in minimal APD prolongation (Figure 7B, top left; $\triangle A P D=2.6 \mathrm{~ms})$. In contrast, during $\beta A R$ stimulation $A P D$ was significantly prolonged in $A 341 V_{\text {Het }}$ versus WT (Figure 7B, top right; $\triangle A P D=23 \mathrm{~ms}$ ) at all pacing cycle lengths ( $C L$; Figure $7 \mathrm{C}$ ). Thus, these modeling studies confirmed that the biophysical alterations to $I_{\mathrm{Ks}}$ by $A 341 \mathrm{~V}$ in heterozygous conditions result in a pronounced cellular-action-potential phenotype, predominantly during $\beta A R$ stimulation, in line with the ECG changes observed in A341V carriers.

\section{Mechanisms Underlying the Suppressed cAMP Responsiveness of A341V I $\mathrm{Ks}_{\mathrm{s}}$}

The A341V mutation could disrupt the $\beta A R$ modulation of $I_{\mathrm{Ks}}$ through various mechanisms, including: (i) disruption of the conformational changes occurring after phosphorylation of KCNQ1-S27; (ii) disruption of KCNQ1 interaction with Yotiao thereby reducing local PKA availability; or (iii) disruption of phosphorylation of S27, even in the presence of Yotiao (e.g., by a defective transfer of PKA from C-terminal Yotiao to N-terminal S27). 

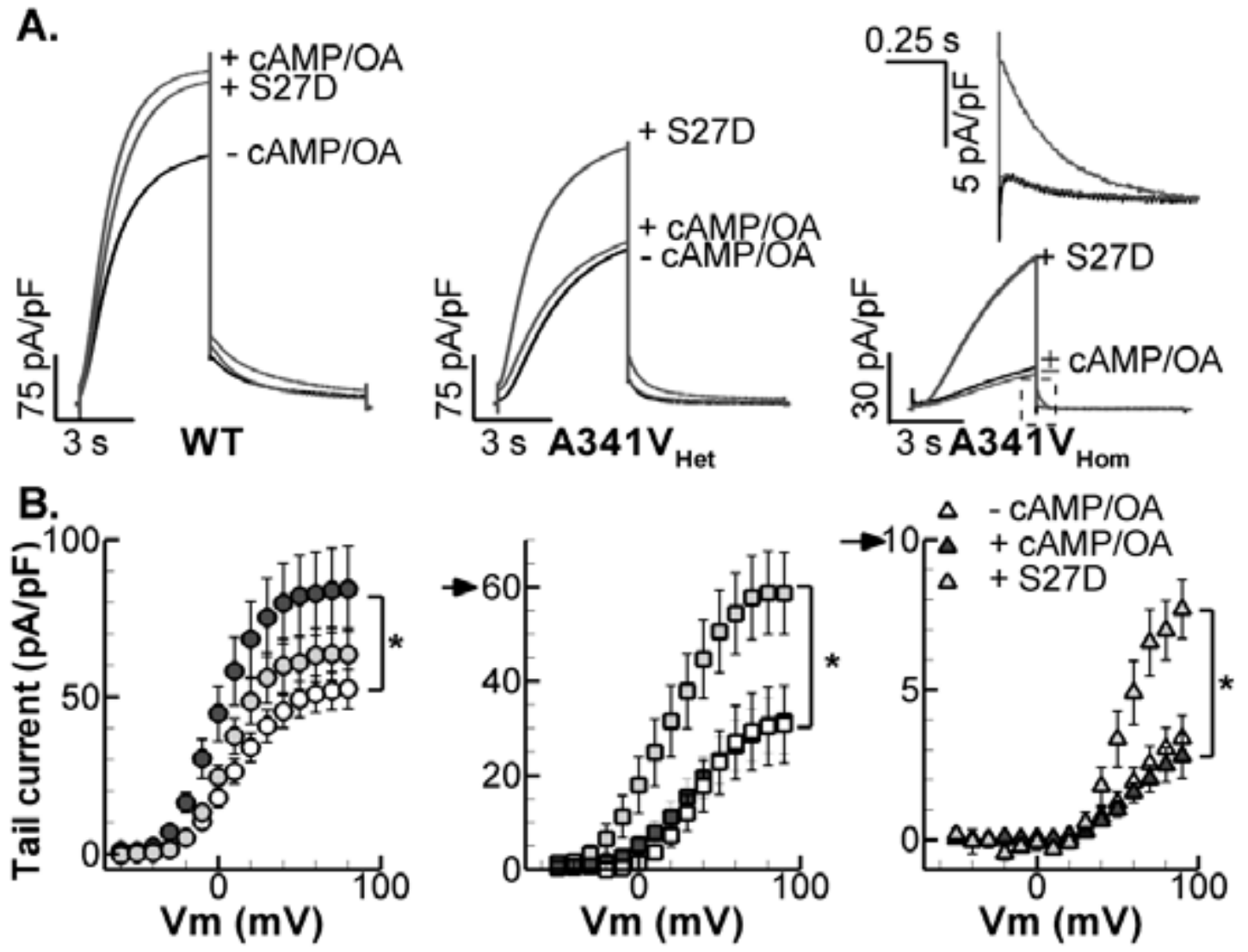

C.

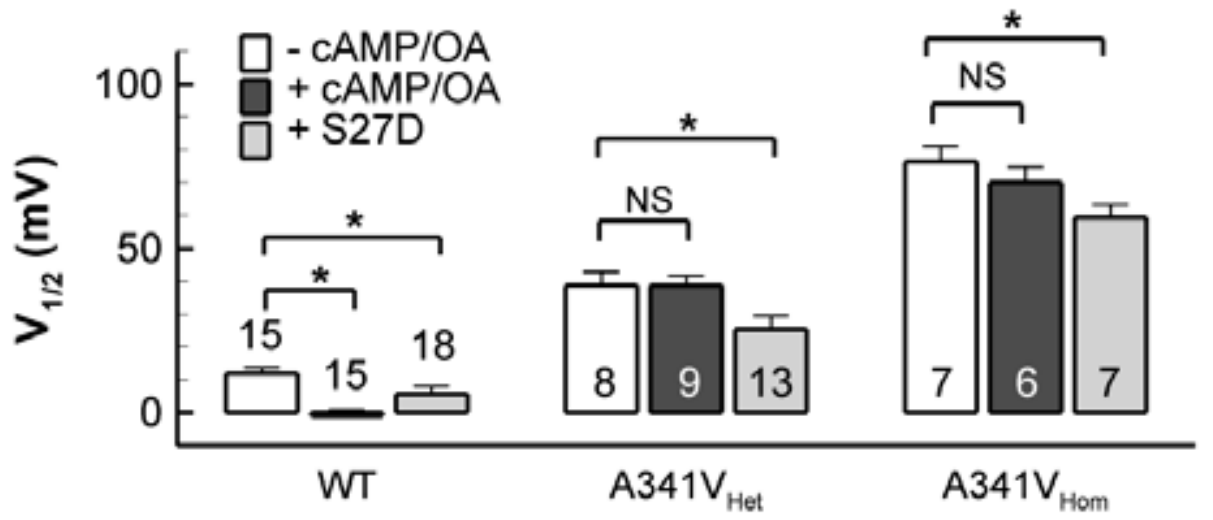

Figure 8. The phosphomimetic substitution S27D 'rescues' cAMP-dependent upregulation of the A341V mutation. A. Averaged current traces of WT (left), $A 341 V_{\text {Het }}$ (middle) or $A 341 V_{\text {Hom }}$ (right) channels cotransfected with KCNE1 and Yotiao in the absence or presence of stimulation with intrapipette CAMP/OA, or in the presence of the phosphomimetic substitution S27D. Voltage-clamp protocol as in Figure 4. B. Tail I-V relationship for the conditions in panel A. Note different $y$-scales (indicated with arrows). C. Membrane potential resulting in half-maximal activation. CAMP responsiveness of $W T I_{\mathrm{Ks}}$ is characterized by a significant leftward shift in half-maximal activation (*, $P<0.05$ ) that is not present in $A 341 V_{\text {Het }}$ or $A 341 V_{\text {Hom }}$. In contrast, the S27D substitution results in significant shifts for all conditions.

Mechanism (i) was tested using the phosphomimetic substitution KCNQ1-S27D. Kurokawa et al. ${ }^{24}$ have previously shown that the S27D substitution reproduces most of the effects of $\beta$ AR stimulation 
on $I_{\mathrm{ks}}$. Indeed, in WT conditions, S27D led to an upregulation of $\mathrm{I}_{\mathrm{Ks}}$-tail amplitudes (Figure 8A,B, left panels) and a significant leftward-shift of the half-maximal activation potential, similar to that observed after the application of cAMP/OA (Figure 8C). In contrast to CAMP/OA, the S27D substitution resulted in a significant upregulation of $A 341 \mathrm{~V}$ in both heterozygous and homozygous conditions (Figure $\mathbf{8 A}, \mathbf{B}$ middle and right panels), as well as a significant leftward shift in halfmaximal activation potential (Figure $\mathbf{8 C}$ ). These data refuted hypothesized mechanism (i) to explain the disrupted cAMP responsiveness of A341V.

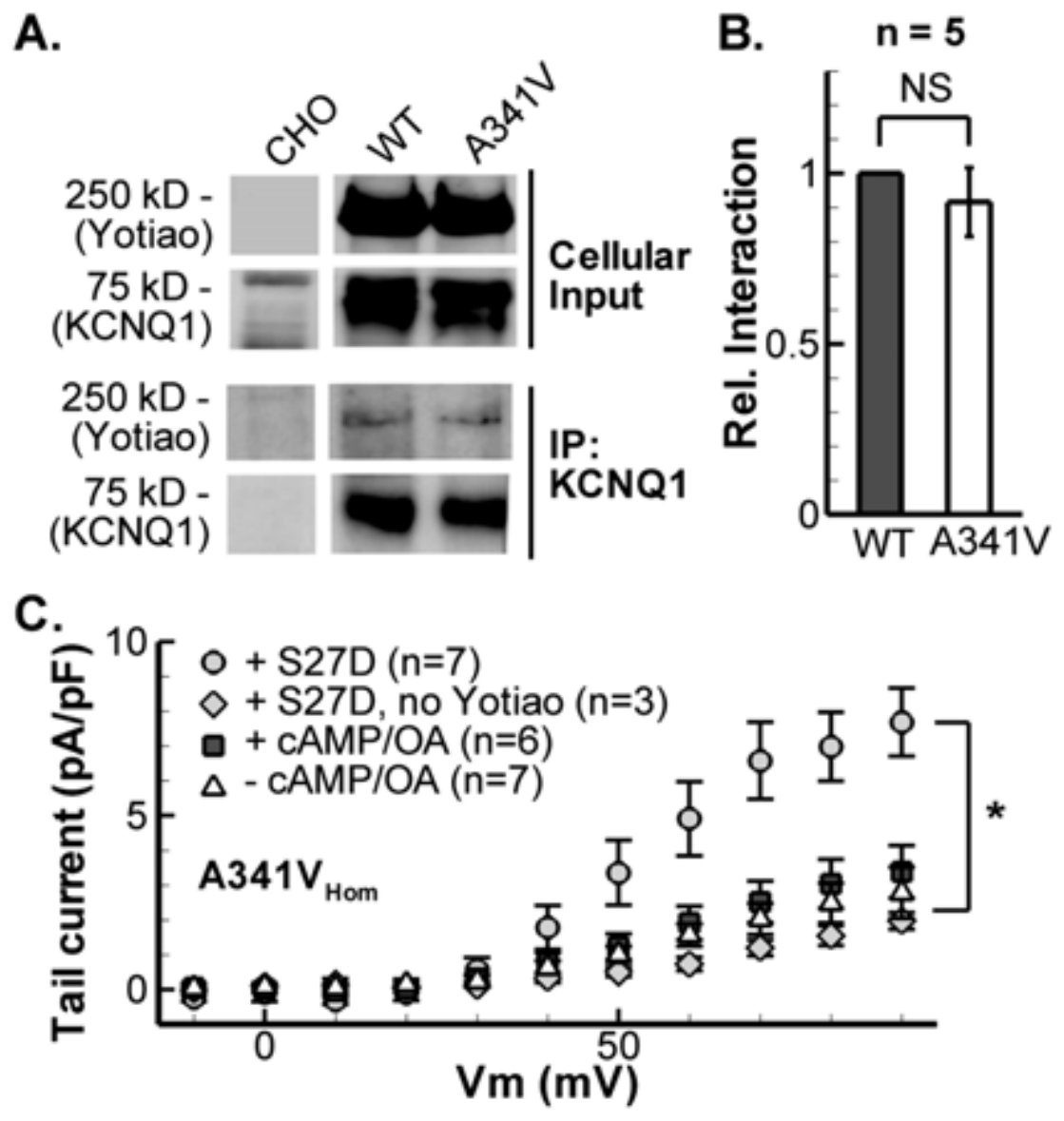

Figure 9. KCNQ1/Yotiao interaction remains intact in the presence of the A341V mutation. A. Representative Western blots of Yotiao and KCNQ1 in lysates (top) of non-transfected CHO cells, CHO cells transfected with KCNQ1-WT + KCNE1 + Yotiao (WT) or KCNQ1-A341V + KCNE1 + Yotiao (A341V). Bottom blots are probed with anti-Yotiao and anti-KCNQ1 antibodies after precipitation of the immunocomplex from lysates using an anti-KCNQ1 antibody in the same conditions. B.

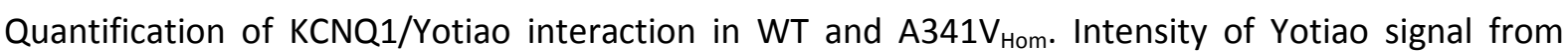
immunocomplexes was corrected for KCNQ1-signal intensity in all conditions and results were normalized to KCNQ1-WT + KCNE1 + Yotiao. C. I-V relationship for A341 $\mathrm{V}_{\text {Hom }}$ currents in the absence of cAMP/OA, presence of CAMP/OA, presence of the S27D phosphomimetic substitution, or S27D without co-transfection of Yotiao (empty vector). Voltage-clamp protocol is identical to Figure 4. Increase in tail-current amplitude by the S27D substitution occurs only in the presence of Yotiao. 
Yotiao serves a dual function: not only is it required for phosphorylation of S27 by PKA, it also actively participates in the conformational changes of the channel complex after phosphorylation of S27. ${ }^{24}$ We investigated the interaction between KCNQ1 and Yotiao via co-immunoprecipitation experiments in CHO cells expressing KCNQ1-WT or KCNQ1-A341V + KCNE1 + Yotiao. Whole-cell lysates or immunocomplexes precipated from lysates using an anti-KCNQ1 antibody were probed with anti-KCNQ1 and anti-Yotiao antibodies (Figure 9A). Yotiao could be detected in both WT and A341V immunocomplexes, but not in negative controls (non-transfected $\mathrm{CHO}$ cells or cells transfected with KCNQ1-WT + KCNE1 but without Yotiao). The intensity of the Yotiao signal from immunocomplexes was corrected by the intensity of the KCNQ1 signal and results were normalized to WT conditions. In the presence of A341V, KCNQ1/Yotiao interaction was not statistically different from WT $(0.92 \pm 0.10$ of $W T$; Figure $9 B)$. Moreover, whereas A341V-mutant $I_{\mathrm{Ks}}$ was markedly increased by the S27D substitution when Yotiao was co-transfected, there was no such increase in the absence of the anchoring protein (Figure 9C). We found no difference between basal WT $I_{\mathrm{Ks}}$ in the presence or absence of Yotiao (not shown), indicating that the observed increase in KCNQ1A341V-S27D was not due to enhanced expression and suggesting that Yotiao can indeed modulate $I_{\mathrm{Ks}}$ in the presence of A341V-S27D. This indicated that hypothesized mechanism (ii) cannot explain the dominant-negative reduction in CAMP-dependent $\mathrm{I}_{\mathrm{Ks}}$ upregulation by the $\mathrm{A} 341 \mathrm{~V}$ mutation.

To test hypothesis (iii), cells transfected with KCNQ1-WT+KCNE1+Yotiao or KCNQ1A341V+KCNE1+Yotiao were incubated with either $300 \mu \mathrm{mol} / \mathrm{L}$ membrane-permeable cAMP and 0.2 $\mu \mathrm{mol} / \mathrm{L}$ OA for $10 \mathrm{~min}$, or with external solution. Lysates were run on SDS-PAGE, transferred onto PVDF membranes, and probed with anti-phospho-S27 KCNQ1 and anti-KCNQ1 antibodies. Nontransfected $\mathrm{CHO}$ cells were used as negative controls. A significant increase in the fraction of phosphorylated KCNQ1 was observed in response to stimulation with CAMP/OA in KCNQ1-WT (Figure 10A). Although increased phosphorylation was also observed in A341V-expressing cells, the fraction of phosphorylated $I_{\mathrm{KS}}$ channels was significantly lower than that in WT cells (by $25 \%$ ), both for $\mathrm{A} 341 \mathrm{~V}_{\mathrm{Hom}}$ and $\mathrm{A} 341 \mathrm{~V}_{\text {Het }}$ (Figure 10A,B). Thus, defective phosphorylation of KCNQ1-A341V is responsible, at least partly, for the loss of cAMP-dependent $I_{\mathrm{Ks}}$ upregulation.

A.

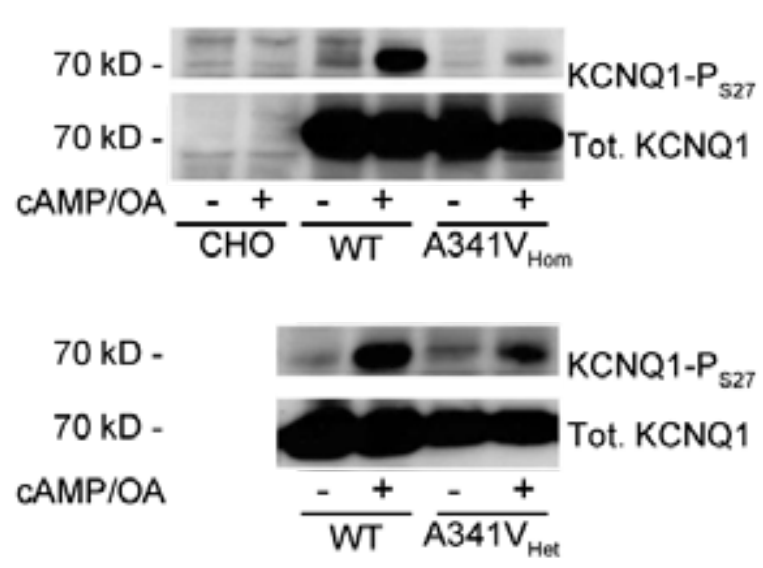

B.

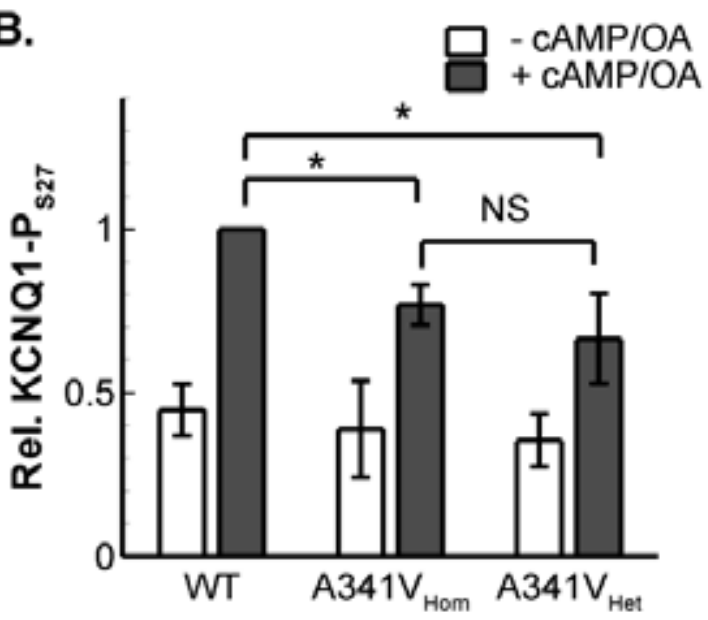


Figure 10. The A341V mutation results in reduced phosphorylation of S27 during cAMP-dependent stimulation. A. Representative Western blot of lysates of non-transfected $\mathrm{CHO}$ cells, $\mathrm{CHO}$ cells transfected with KCNQ1-WT + KCNE1 + Yotiao (WT) or KCNQ1-A341V + KCNE1 + Yotiao (A341V $\left.\mathrm{V}_{\text {Hom }}\right)$, incubated in the absence or presence of 8-CPT-CAMP and OA, and probed with anti-phosphoKCNQ1-S27 (top blot) or anti-KCNQ1 (bottom blot) antibodies. The lower two blots show similar results for WT and heterozygous co-transfection of WT and A341V. B. Quantification of fractions of KCNQ1-P $\mathrm{P}_{\text {S27 }}$ (relative to total KCNQ1) for $\mathrm{WT}, \mathrm{A} 341 \mathrm{~V}_{\text {Hom }}$ (5 blots from 4 samples) or $\mathrm{A} 341 \mathrm{~V}_{\text {Het }}$ (9 blots from 3 different samples) in the absence or presence of stimulation with 8-CPT-cAMP/OA. Results are normalized to KCNQ1-WT + 8-CPT-CAMP/OA. There is a significant reduction of KCNQ1 phosphorylation at $\mathrm{S} 27$ in the presence of A341V.

\section{Dominant-negative suppression of cAMP/PKA-dependent $I_{\mathrm{Ks}}$ upregulation in}

\section{LQT1}

The reduction in KCNQ1-A341V phosphorylation is similar to that observed by Chen et al. ${ }^{25}$ for the Yotiao mutation S1570L. They also show a complete loss of $\mathrm{I}_{\mathrm{Ks}}$ enhancement (in this case due to reduced KCNQ1-Yotiao interaction). Combined, our data and those by Chen et al. ${ }^{25}$ suggest that cAMP-dependent $\mathrm{I}_{\mathrm{Ks}}$ upregulation is under strong dominant-negative control of KCNQ1-S27 phosphorylation, which is further supported by the functional finding of a striking loss of cAMPdependent upregulation of heterozygous KCNQ1-A341V.

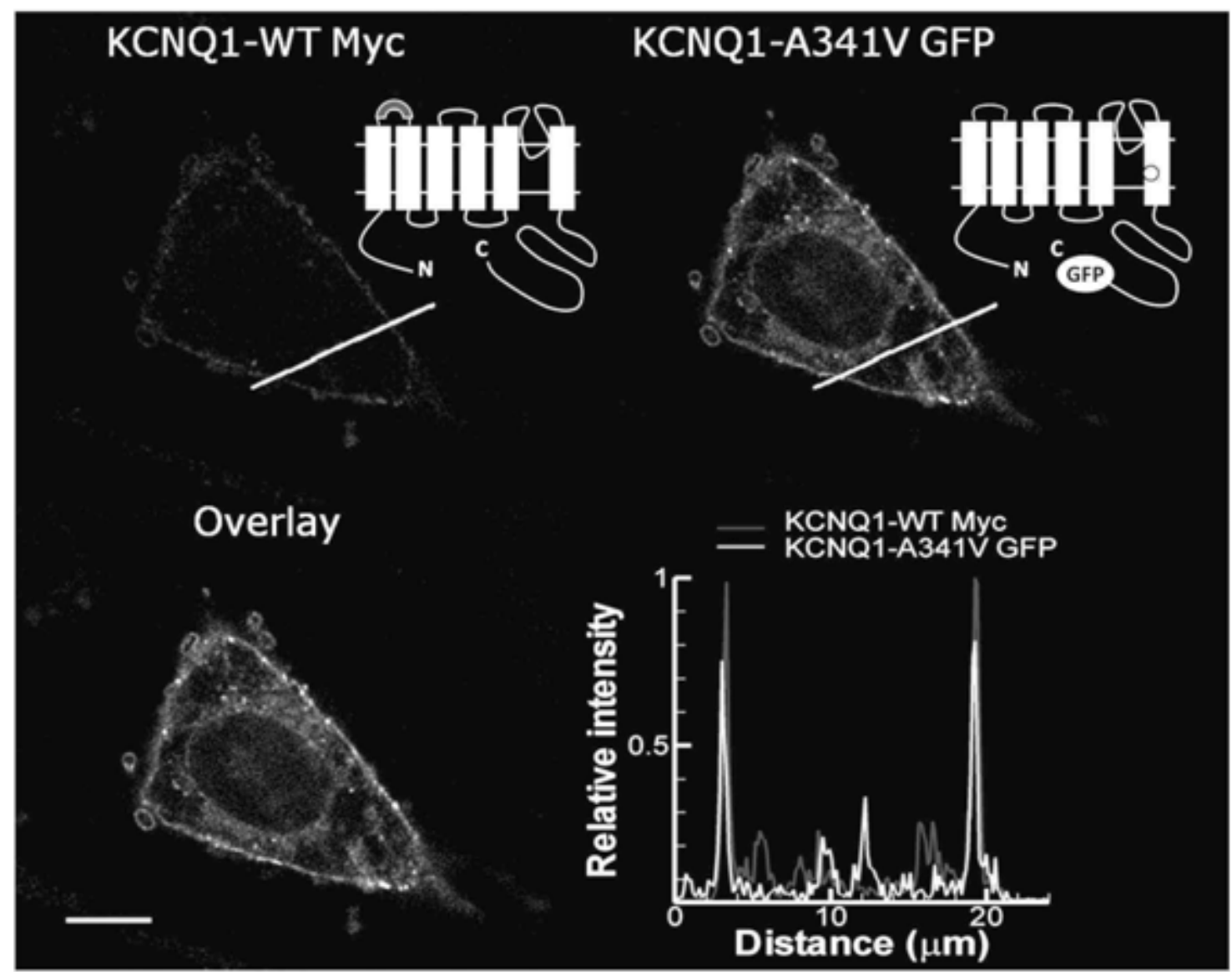


Figure 11. Localization of KCNQ1-WT and KCNQ1-A341V after co-transfection of both subunits with KCNE1 and Yotiao. A c-Myc epitope was inserted in the extracellular loop between transmembrane segments 1 and 2 of KCNQ1-WT and the KCNQ1-A341V was tagged with GFP at the C-terminus. Topleft panel shows a confocal image of KCNQ1-WT obtained with an anti-c-Myc antibody in a representative $\mathrm{CHO}$ cell. Top-right panel shows GFP signal in the same cell. When the two signals are overlayed (bottom-left panel), a clear colocalization of WT and A341V KCNQ1 subunits can be observed at the cell membrane (yellow), confirming "heterozygous" channel expression. The bottom-right panel shows the relative signal-intensity profiles of KCNQ1-WT and KCNQ1-A341V along the cross-sections at the light-green line. Both signals show increased activity at the cell boundaries. The lower-left white scale-bar indicates $10 \mu \mathrm{m}$. For color figure, see page 252.

To further investigate the disruption of upregulation in heterozygous conditions, we first confirmed that KCNQ1-WT and KCNQ1-A341V are both expressed in the membrane by immunolocalization. Coexpression of KCNQ1-WT-C-Myc and KCNQ1-A341V-GFP constructs allowed simultaneous detection of WT (using an anti c-Myc antibody) and A341V subunits. Figure 11 shows individual signals of KCNQ1-WT (top-left panel) and KCNQ1-A341V (top-right panel). The overlay of these signals (Figure 11, bottom-left panel) indicates strong colocalization (yellow color) of WT and A341V subunits in the cell membrane. This is confirmed by the cross-section profile of both signal intensities (Figure 11, bottom-right panel), which shows enhanced intensity at the membrane. Correlation between c-Myc and GFP signal intensity profiles was $0.81 \pm 0.01$ in 50 cells of 4 transfections.

We next compared the loss of cAMP-dependent upregulation by KCNQ1-A341V with the LQT1 mutations KCNQ1-A344V, ${ }^{26}$ KCNQ1-G589D ${ }^{27}$ and KCNQ1-K557E. Suppression of $\mathrm{I}_{\mathrm{Ks}}$ upregulation has previously been reported for the KCNQ1-G589D (KCNQ1-Fin) mutation, which disrupts the binding of Yotiao to the KCNQ1 C-terminus. ${ }^{5}$ We have recently reported on the LQT1 mutation K557E found in a Dutch family with a relatively mild clinical phenotype in which cAMP-dependent $I_{\mathrm{Ks}}$ enhancement is intact. ${ }^{28}$ Responsiveness to CAMP was quantified by an increased tail-current amplitude over the entire population of cells studied (Figure 12A) as well as an increase in current amplitude for each individual cell during a 5-min cAMP/OA wash-in protocol (Figure 12B,C). WT, heterozygous and homozygous $\mathrm{K} 557 \mathrm{E}$, and heterozygous and homozygous $\mathrm{A} 344 \mathrm{~V}$ all showed a pronounced responsiveness to CAMP. In contrast, A341V and G589D I $\mathrm{Ks}_{\text {s }}$ were unresponsive to stimulation, even when co-expressed with WT $I_{\mathrm{Ks}}$ (Figure 12). These results demonstrate that the loss of upregulation observed by A341V does not apply to the very similar mutation A344V (same amino-acid substitution, only three residues apart and, based on the KCNQ1 homology model, in close proximity to A341; Figure 3). Nonetheless, dominant-negative suppression of cAMP-dependent $I_{\mathrm{Ks}}$ upregulation is not exclusive for A341V, as indicated by our heterozygous G589D data. 


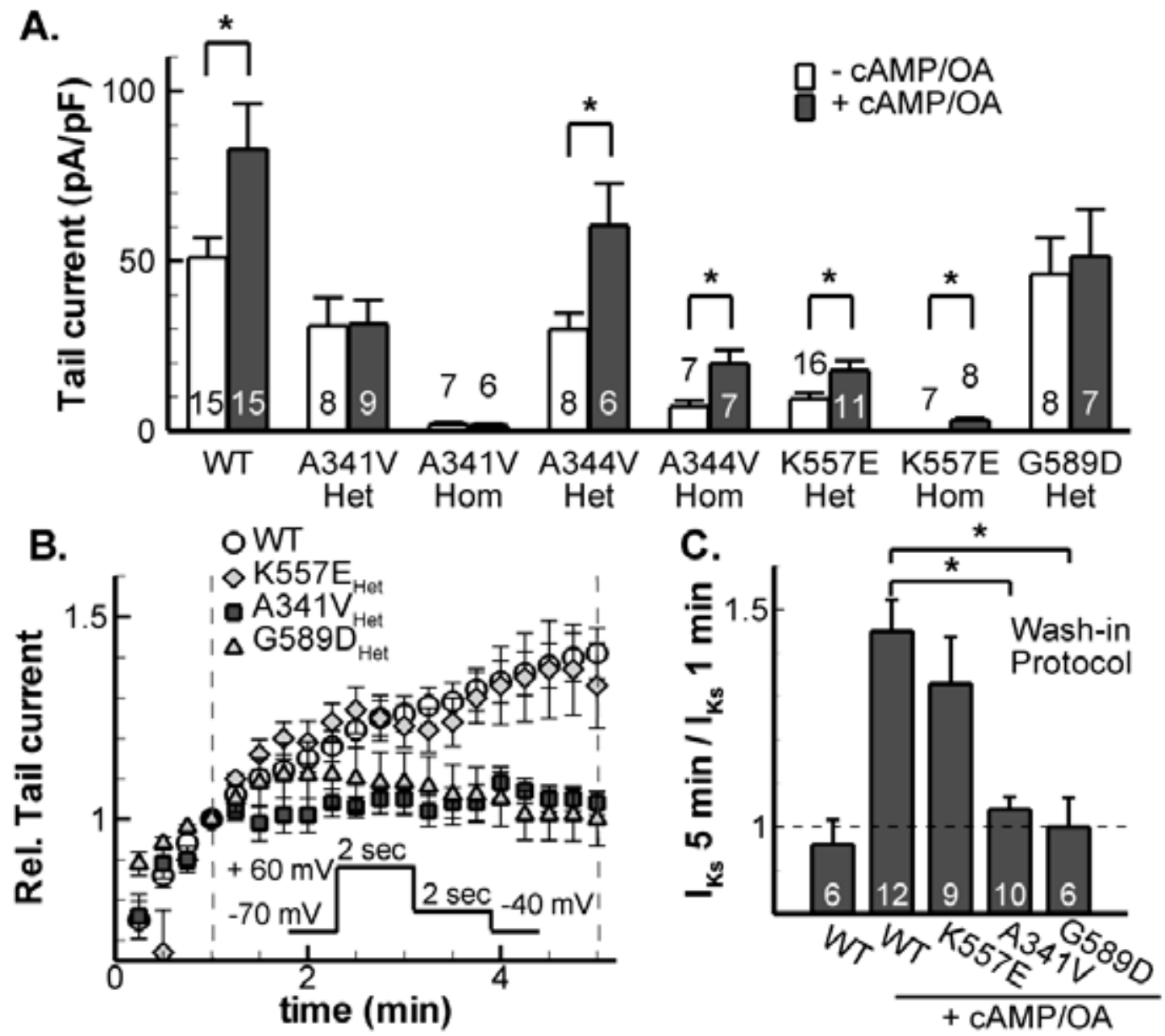

Figure 12. Comparison of cAMP-dependent upregulation of WT and A341V-, A344V-, K557E- or G589D-mutated $I_{\mathrm{Ks}}$. A. Tail-current amplitudes after 5 -s depolarizing pulses to $+60 \mathrm{mV}$ (voltage-clamp protocol identical to Figure 1) in the absence or presence of cAMP/OA for WT current and heterozygous or homozygous expression of mutant $I_{\mathrm{ks}}$. Numbers in/above bars indicate number of cells for each condition. $B$. Time course of $I_{\mathrm{Ks}}$ augmentation during application of CAMP/OA in WT,

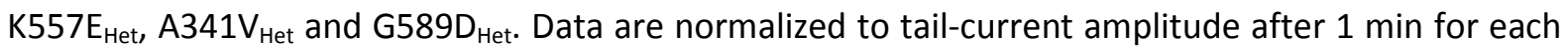
condition. Inset: voltage-clamp protocol. C. $I_{\mathrm{Ks}}$ increase in WT or heterozygous K557E, A341V or G589D conditions after $5 \mathrm{~min}$ of CAMP/OA, relative to $1 \mathrm{~min}$ (vertical dashed lines in panel B). K557E and A344V are significantly upregulated by CAMP/OA. In contrast, A341V and G589D which disrupt BAR sensitivity in homozygous conditions, have dominant-negative loss of CAMP-dependent upregulation. In the absence of $\mathrm{CAMP} / \mathrm{OA}, \mathrm{I}_{\mathrm{Ks}}$ was stable and no significant difference in current amplitude between 5 min and 1 min was found (left-most bar).

To determine if the dominant-negative suppression of cAMP-dependent upregulation by A341V and G589D could be due to their common effects on KCNQ1-S27, and to investigate if alterations in KCNQ1-S27 alone can exert dominant-negative control of CAMP/PKA-dependent $I_{\mathrm{Ks}}$ upregulation, independent of other known pathogenic mutations, we analyzed a 1:1 co-expression of WT KCNQ1 with KCNQ1-S27A (together with KCNE1 and Yotiao). This heterozygous S27A condition will disable the PKA phosphorylation site in approximately half of the KCNQ1 subunits. In contrast to the 
pronounced increase observed with cAMP/OA treatment in WT $I_{\mathrm{Ks}}$, there was no significant increase in $\mathrm{Kk}_{\mathrm{ks}}$-tail current amplitude in heterozygous KCNQ1-S27A cells stimulated with CAMP/OA (Figure 13). Although there was still a small (but non-significant) increase in $I_{\mathrm{Ks}}$ in heterozygous S27A, this increase was also present in cells with homozygous S27A expression after treatment with cAMP/OA (19\% increase in tail current after 5-s depolarizing pulses to $+90 \mathrm{mV}$ in both cases, not shown) and no significant differences were found between heterozygous S27A in the absence or presence of cAMP (Figure 13).
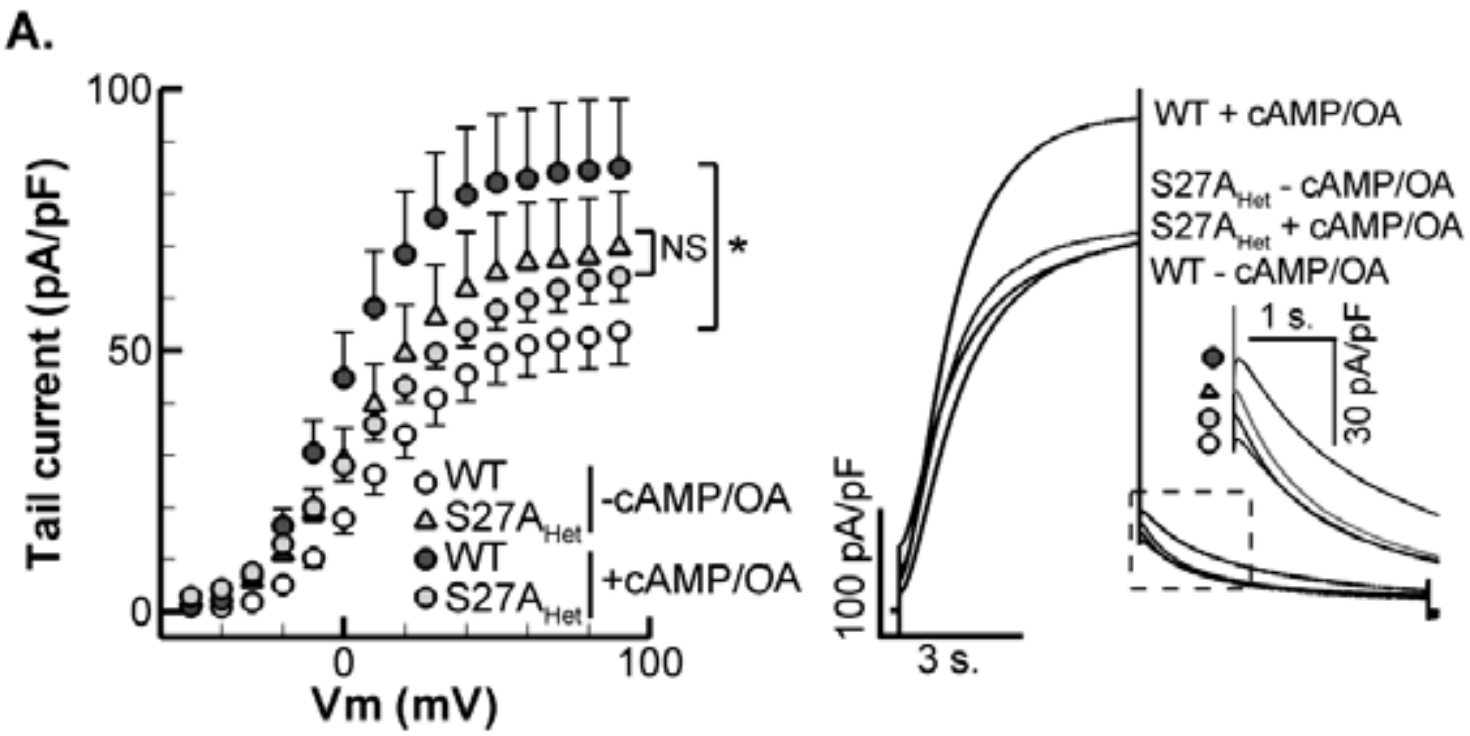

Figure 13. Loss of cAMP-dependent $I_{\mathrm{Ks}}$ upregulation with heterozygous KCNQ1-S27A. Left, tail I-V relationship (voltage-clamp protocol as in Figure 1). Right, averaged current traces at $+60 \mathrm{mV}$ for WT $\mathrm{I}_{\mathrm{Ks}}$ or heterozygous expression of KCNQ1-S27A ( $\mathrm{n}=9$ and $\mathrm{n}=11$ ) in the absence or presence of cAMP/OA, after co-transfection of KCNE1 and Yotiao. Heterozygous KCNQ1-S27A results in a dominant-negative reduction of CAMP-dependent $I_{\mathrm{Ks}}$ upregulation that is indistinguishable from homozygous KCNQ1-S27A (not shown).

\section{DISCUSSION}

In the present study we provide novel mechanistic insights into cAMP-dependent upregulation of $\mathrm{I}_{\mathrm{Ks}}$ and its alteration by the S6 transmembrane LQT1 mutation A341V. We show that A341V confers a dominant-negative suppression of current upregulation. This loss of cAMP sensitivity is not due to a restriction of the conformational changes that occur after S27 phosphorylation, but instead is due to the inability of PKA to phosphorylate KCNQ1-S27 despite the presence of Yotiao. The combined dominant-negative reduction in basal current and loss of cAMP sensitivity result in a pronounced prolongation of repolarization during $\beta A R$ stimulation in a computational model. Moreover, we show that dominant-negative suppression of upregulation is shared by other LQT1 mutations that show loss of $\beta A R$ modulation in homozygous conditions (e.g., KCNQ1-G589D ${ }^{5}$ ) and by heterozygous substitutions at $\mathrm{S} 27$. Our results suggest that tetrameric phosphorylation of KCNQ1 is required. This 
finding has important implications for the clinical severity of KCNQ1 mutations similar to A341V, because genetically heterozygous carriers will exhibit a 'homozygous phenotype' with respect to $\beta A R$ modulation. The present data stress the importance of investigating molecular signaling besides basal function of $I_{\mathrm{Ks}}$ in the human congenital LQT syndrome.

\section{Requirement of a Macromolecular Signaling Complex for $\mathrm{I}_{\mathrm{KS}}$ Modulation}

Specific macromolecular signaling is required for the modulation of voltage-gated ion channels. ${ }^{29}$ Modulation of $\mathrm{I}_{\mathrm{ks}}$ by PKA requires a complex comprising $K C N E 1,{ }^{7}$ Yotiao, ${ }^{5}$ and $\beta$-tubulin, ${ }^{30}$ besides the pore-forming $\alpha$-subunit KCNQ1. In pathological conditions, the composition of the macromolecular complex is even further complicated by the fact that most LQT1 mutations (including A341V) are inherited in an autosomal-dominant mode. In these conditions, variable mixtures of normal and mutant KCNQ1-subunits interact in the membrane-expressed $\mathrm{I}_{\mathrm{Ks}}$ channels.

KCNE1 and $\beta$-tubulin appear to operate downstream of channel phosphorylation in mediating the conformational changes that increase $I_{K s}$, since no difference in $K C N Q 1$ phosphorylation was found after disruption of $K C N E 1^{31}$ or microtubular interaction, ${ }^{30}$ despite altered $I_{\mathrm{Ks}}$ regulation. Interactions between KCNQ1 and KCNE1 have been reported for the extracellular, transmembrane and intracellular parts of the subunits. ${ }^{32,}{ }^{33}$ Panaghie et al. ${ }^{32}$ have shown that KCNE1 interacts with the KCNQ1 pore region through residues F339 and F340. Based on a homology model of the structure of KCNQ1, Smith et al. ${ }^{34}$ determined that A341V does not face the lipid bilayer but instead interacts with S6-residues of other subunits (Figure 3). This makes it unlikely that A341 interacts directly with KCNE1. However, the A341V mutation may induce conformational changes in the KCNQ1-S6 segment that indirectly alter KCNQ1/KCNE1 interactions. Our S27D data show a clear upregulation in the presence of $\mathrm{A} 341 \mathrm{~V}$, indicating that the interaction between KCNQ1 and KCNE1 is preserved, at least partly. In agreement, a recent study showed that KCNQ1-A341V in the absence of KCNE1 or in the presence of the mutant KCNE1-T58A results in non-functional channels. ${ }^{35}$ However, the exact role of $K C N E 1$ in influencing $A 341 \mathrm{~V}$-mutated $I_{\mathrm{Ks}}$ and its upregulation requires further study.

Our S27D data also indicate that the Yotiao contribution downstream of phosphorylation is intact. Previously, Kurokawa et al. ${ }^{24}$ showed that S27D is unable to upregulate WT $\mathrm{I}_{\mathrm{Ks}}$ when Yotiao is not included. Here we confirm these results for A341V-mutant $I_{\mathrm{Ks}}$.

Analysis of KCNQ1 with a phospho-sensitive antibody indicated that, despite the presence of Yotiao, phosphorylation of KCNQ1 is reduced in the presence of A341V. The exact molecular mechanism by which this occurs is currently unclear but may involve a reduced functional interaction between the KCNQ1 C-terminal-coupled Yotiao and the $\mathrm{N}$-terminal phosphorylation site S27. Interestingly, however, the A341V mutation is located in the S6 transmembrane spanning domain of KCNQ1, a region that has not previously been reported to play a role in cAMP-dependent modulation of $\mathrm{I}_{\mathrm{Ks}}$. However, the intact upregulation in the presence of the very similar mutation A344V suggests that 
the role of $\mathrm{S} 6$ in cAMP-dependent $\mathrm{I}_{\mathrm{ks}}$ upregulation involves specific residues. The fact that $\mathrm{A} 341$ is an extremely conserved residue located just prior to the $\mathrm{S} 6$ 'hinge domain' ${ }^{20}$ (Figure 3 ), indicates that even small perturbations of the amino-acid sequence at this position may cause pronounced alterations of channel structure.

Marx et al. ${ }^{5}$ have shown that the KCNQ1-G589D mutation in homozygous conditions results in loss of CAMP-dependent $I_{\mathrm{Ks}}$ upregulation due to disruption of the KCNQ1-Yotiao interaction. Here we report that this abolishment of upregulation occurs also in heterozygous conditions. This illustrates that at least two KCNQ1 mutations (G589D and A341V), in different parts of the channel, show such dominant-negative suppression.

Marx et al. ${ }^{5}$ and Yang et al. ${ }^{36}$ have demonstrated that the substitution KCNQ1-S27A disables the Nterminal phosphorylation site and reduces CAMP-dependent $I_{\mathrm{Ks}}$ upregulation. Yang et al. ${ }^{36}$ also observed a contribution of the 'atypical' PKA sites S468/T470, whereas Marx et al. ${ }^{5}$ demonstrate a complete loss of cAMP-dependent $\mathrm{I}_{\mathrm{Ks}}$ upregulation in the presence of S27A, consistent with our data. These differences may be related to the absence of Yotiao co-transfection in the experiments of Yang et al., but further studies are clearly required to explore this. We found that the phosphomimetic substitutions KCNQ1-S468D/T470D were unable to upregulate $I_{\mathrm{Ks}}$ (not shown), further indicating that S27 is the main phosphorylation site involved in CAMP-dependent $I_{\mathrm{Ks}}$ upregulation. Importantly, we show here that the lack of cAMP-dependent $\mathrm{I}_{\mathrm{Ks}}$ upregulation was also observed upon heterozygous KCNQ1-S27A substitution (a condition in which half of the N-terminal phosphorylation sites are disabled). In heterozygous conditions, a binomial distribution of subunit composition is expected in which $93.75 \%$ of the channels will have at least one mutation in the KCNQ1 tetramer. The complete lack of upregulation in this case indicates that PKA-dependent channel upregulation is under dominant-negative control by alterations at S27 in which a single defective subunit is sufficient to abolish upregulation and suggests a requirement for tetrameric phosphorylation or a strong cooperation between the four subunits in CAMP-dependent channel modulation.

\section{Mechanisms Underlying Phenotypic Severity of A341V and Role of Genetic Risk Modifiers}

Schwartz et al. ${ }^{13}$ found that increased autonomic sensitivity, e.g., resulting from polymorphisms in adrenergic receptors, was an arrhythmia-risk modifier in A341V mutations. This observation is in line with our computational simulations that indicate the most pronounced APD prolongation during high levels of $\beta A R$ stimulation. The data presented here readily explain why genetic variants of adrenergic receptors that boost sympathetic responsiveness of the heart are risk modifiers in A341V carriers due to the combined loss of basal $\mathrm{I}_{\mathrm{ks}}$ and its CAMP-dependent upregulation. 
Both A341V and G589D show complete loss of cAMP-dependent upregulation in heterozygous conditions but have different clinical severities. Although there are important differences in peak basal currents at physiological potentials for 300 -ms depolarizations $(1.03 \pm 0.27 \mathrm{pA} / \mathrm{pF}$ vs $3.07 \pm 0.30$ $\mathrm{pA} / \mathrm{pF}$ at $20 \mathrm{mV}$ for $\mathrm{A} 341 \mathrm{~V}_{\text {Het }}$ and $\mathrm{G} 89 \mathrm{D}_{\text {Het, }}$, respectively; $P<0.05$ ), which may contribute to their phenotypic differences, it is clear that ionic mechanisms within $I_{\mathrm{ks}}$ itself do not provide the complete picture of repolarization instability in vivo. Variants in NOS1AP have been shown to affect QT duration in the normal population ${ }^{37}$ and have been reported as risk modifiers in A341V carriers. Chang et al. have shown that NOS1AP overexpression affects repolarization through $\mathrm{I}_{\mathrm{kr}}$ and $\mathrm{I}_{\mathrm{Cal}}$. Previously, we have shown that the effect of reduced $I_{\mathrm{Ks}}$ is larger when repolarization is impaired by pharmacological blockade of $\mathrm{I}_{\mathrm{kr} .}{ }^{17}$ Thus, NOS1AP variants likely function next to KCNQ1 mutations, affecting among other things $I_{k r}$. The important role of $I_{K s}$ as a repolarization reserve ${ }^{15,17}$ may then partly explain their role as risk modifiers in A341V carriers. $^{38}$

\section{Conclusions}

Loss of cAMP-dependent upregulation of $\mathrm{I}_{\mathrm{Ks}}$, even under heterozygous conditions, on top of a dominant-negative reduction in basal current, is a biophysical characteristic of KCNQ1-A341V that may contribute to its phenotypic severity in patients. This loss of upregulation is related to reduced phosphorylation of KCNQ1 at S27. Moreover, we show in general that CAMP/PKA-dependent $I_{\mathrm{Ks}}$ upregulation is under strong dominant-negative control of KCNQ1 phosphorylation at S27. These data have important implications for future studies of LQT1 mutations, because they highlight the importance of studying the effects of heterozygous expression of mutations, similar to their occurrence in most patients, and they indicate that baseline I-V characteristics alone are insufficient to determine the electrophysiological consequences of LQT1 mutations. Finally, our data point to an as yet unknown involvement of the $S 6$ region of KCNQ1 at and/or around A341 in mediating $\beta A R$ modulation of $I_{\mathrm{ks}}$.

\section{ACKNOWLEDGEMENTS}

The authors thank the members of the Volders lab for helpful discussions. The advice by Prof. Robert S. Kass and Drs. Lei Chen and Cecile Terrenoire, Columbia University, NY on the stimulation with CAMP/OA, cpt-cAMP and their gift of the KCNQ1-S27 phospho-specific antibody are gratefully acknowledged. Human KCNQ1, KCNE1 and KCNH2 constructs were a kind gift from Prof. Dirk J. Snyders, University of Antwerp, Belgium. The authors thank Prof. Yoram Rudy, Washington University in St. Louis, MO for ongoing collaboration and use of his computational resources.

\section{FUNDING SOURCES}

P.G.A.V. is supported by The Netherlands Heart Foundation (NHS2010B216) and a Vidi grant from the Netherlands Organization for Scientific Research (ZonMw 91710365). M.D. is the recipient of a Research Grant of the European Society of Cardiology (2010). 


\section{REFERENCES}

1. Volders PG, StengI M, van Opstal JM, Gerlach U, Spätjens RL, Beekman JD, Sipido KR, Vos MA. Probing the contribution of $I_{\mathrm{Ks}}$ to canine ventricular repolarization: key role for $\beta$ adrenergic receptor stimulation. Circulation. 2003;107:2753-2760.

2. Jost N, Virág L, Bitay M, Takács J, Lengyel C, Biliczki P, Nagy Z, Bogáts G, Lathrop DA, Papp JG, Varró A. Restricting excessive cardiac action potential and QT prolongation: a vital role for $\mathrm{I}_{\mathrm{Ks}}$ in human ventricular muscle. Circulation. 2005;112:1392-1399.

3. Sanguinetti MC, Curran ME, Zou A, Shen J, Spector PS, Atkinson DL, Keating MT. Coassembly of $\mathrm{K}_{\mathrm{V}} \mathrm{LQT1}$ and minK (IsK) proteins to form cardiac $\mathrm{I}_{\mathrm{Ks}}$ potassium channel. Nature. 1996;384:80-83.

4. Nakajo K, Ulbrich MH, Kubo Y, Isacoff EY. Stoichiometry of the KCNQ1 - KCNE1 ion channel complex. Proc Natl Acad Sci U S A. 2010;107:18862-18867.

5. Marx SO, Kurokawa J, Reiken S, Motoike H, D'Armiento J, Marks AR, Kass RS. Requirement of a macromolecular signaling complex for $\beta$ adrenergic receptor modulation of the KCNQ1KCNE1 potassium channel. Science. 2002;295:496-499.

6. Chen L, Kurokawa J, Kass RS. Phosphorylation of the A-kinase-anchoring protein Yotiao contributes to protein kinase A regulation of a heart potassium channel. J Biol Chem. 2005;280:31347-31352.

7. Kurokawa J, Bankston J, Kaihara A, Chen L, Furukawa T, Kass R. KCNE variants reveal a critical role of the $\beta$ subunit carboxyl terminus in PKA-dependent regulation of the $I_{\mathrm{Ks}}$ potassium channel. Channels (Austin). 2009;3:16-24.

8. Gallacher DJ, Van de Water A, van der Linde H, Hermans AN, Lu HR, Towart R, Volders PGA. In vivo mechanisms precipitating torsades de pointes in a canine model of drug-induced long-QT1 syndrome. Cardiovasc Res. 2007;76:247-256.

9. Volders PGA, Sipido KR, Vos MA, Spätjens RLHMG, Leunissen JDM, Carmeliet E, Wellens HJJ. Downregulation of delayed rectifier $\mathrm{K}^{+}$currents in dogs with chronic complete atrioventricular block and acquired torsades de pointes. Circulation. 1999;100:2455-2461.

10. Schwartz PJ, Priori SG, Spazzolini C, Moss AJ, Vincent GM, Napolitano C, Denjoy I, Guicheney P, Breithardt G, Keating MT, Towbin JA, Beggs AH, Brink P, Wilde AA, Toivonen L, Zareba W, Robinson JL, Timothy KW, Corfield V, Wattanasirichaigoon D, Corbett C, Haverkamp W, Schulze-Bahr E, Lehmann MH, Schwartz K, Coumel P, Bloise R. Genotype-phenotype correlation in the long-QT syndrome: gene-specific triggers for life-threatening arrhythmias. Circulation. 2001;103:89-95.

11. Brink PA, Crotti L, Corfield V, Goosen A, Durrheim G, Hedley P, Heradien M, Geldenhuys G, Vanoli E, Bacchini S, Spazzolini C, Lundquist AL, Roden DM, George AL, Jr., Schwartz PJ. Phenotypic variability and unusual clinical severity of congenital long-QT syndrome in a founder population. Circulation. 2005;112:2602-2610.

12. Crotti L, Spazzolini C, Schwartz PJ, Shimizu W, Denjoy I, Schulze-Bahr E, Zaklyazminskaya EV, Swan H, Ackerman MJ, Moss AJ, Wilde AA, Horie M, Brink PA, Insolia R, De Ferrari GM, Crimi $\mathrm{G}$. The common long-QT syndrome mutation KCNQ1/A341V causes unusually severe clinical manifestations in patients with different ethnic backgrounds: toward a mutation-specific risk stratification. Circulation. 2007;116:2366-2375.

13. Schwartz PJ, Vanoli E, Crotti L, Spazzolini C, Ferrandi C, Goosen A, Hedley P, Heradien M, Bacchini S, Turco A, La Rovere MT, Bartoli A, George AL, Jr., Brink PA. Neural control of heart rate is an arrhythmia risk modifier in long QT syndrome. J Am Coll Cardiol. 2008;51:920-929.

14. Heijman J, Volders PGA, Westra RL, Rudy Y. Local control of $\beta$-adrenergic stimulation: Effects on ventricular myocyte electrophysiology and $\mathrm{Ca}^{2+}$-transient. J Mol Cell Cardiol. 2011;50:863871.

15. Silva J, Rudy $\mathrm{Y}$. Subunit interaction determines $I_{\mathrm{ks}}$ participation in cardiac repolarization and repolarization reserve. Circulation. 2005;112:1384-1391. 
16. Stengl M, Volders PGA, Thomsen MB, Spätjens RLHMG, Sipido KR, Vos MA. Accumulation of slowly activating delayed rectifier potassium current $\mathrm{I}_{\mathrm{Ks}}$ in canine ventricular myocytes. $J$ Physiol. 2003;551:777-786.

17. Volders PGA, StengI M, van Opstal JM, Gerlach U, Spätjens RL, Beekman JD, Sipido KR, Vos $\mathrm{MA}$. Probing the contribution of $\mathrm{I}_{\mathrm{Ks}}$ to canine ventricular repolarization: key role for $\beta$ adrenergic receptor stimulation. Circulation. 2003;107:2753-2760.

18. Liu DW, Antzelevitch C. Characteristics of the delayed rectifier current $\left(\mathrm{I}_{\mathrm{Kr}}\right.$ and $\left.\mathrm{I}_{\mathrm{Ks}}\right)$ in canine ventricular epicardial, midmyocardial, and endocardial myocytes. A weaker $I_{\mathrm{Ks}}$ contributes to the longer action potential of the M cell. Circ Res. 1995;76:351-365.

19. Hund TJ, Kucera JP, Otani NF, Rudy Y. Ionic charge conservation and long-term steady state in the Luo-Rudy dynamic cell model. Biophys J. 2001;81:3324-3331.

20. Boulet IR, Labro AJ, Raes AL, Snyders DJ. Role of the S6 C-terminus in KCNQ1 channel gating. J Physiol. 2007;585:325-337.

21. Kang C, Tian C, Sonnichsen FD, Smith JA, Meiler J, George AL, Jr., Vanoye CG, Kim HJ, Sanders CR. Structure of KCNE1 and implications for how it modulates the KCNQ1 potassium channel. Biochemistry. 2008;47:7999-8006.

22. Biliczki P, Girmatsion Z, Brandes RP, Harenkamp S, Pitard B, Charpentier F, Hébert TE, Hohnloser SH, Baró I, Nattel S, Ehrlich JR. Trafficking-deficient long QT syndrome mutation KCNQ1-T587M confers severe clinical phenotype by impairment of KCNH2 membrane localization: evidence for clinically significant $\mathrm{I}_{\mathrm{kr}} \mathrm{I}_{\mathrm{Ks}} \alpha$-subunit interaction. Heart Rhythm. 2009;6:1792-1801.

23. Shimizu W. The long QT syndrome: therapeutic implications of a genetic diagnosis. Cardiovasc Res. 2005;67:347-356.

24. Kurokawa J, Motoike HK, Rao J, Kass RS. Regulatory actions of the A-kinase anchoring protein Yotiao on a heart potassium channel downstream of PKA phosphorylation. Proc Natl Acad Sci U S A. 2004;101:16374-16378.

25. Chen L, Marquardt ML, Tester DJ, Sampson KJ, Ackerman MJ, Kass RS. Mutation of an Akinase-anchoring protein causes long-QT syndrome. Proc Natl Acad Sci U $S$ A. 2007;104:20990-20995.

26. Choi G, Kopplin LJ, Tester DJ, Will ML, Haglund CM, Ackerman MJ. Spectrum and frequency of cardiac channel defects in swimming-triggered arrhythmia syndromes. Circulation. 2004;110:2119-2124.

27. Piippo K, Swan H, Pasternack M, Chapman H, Paavonen K, Viitasalo M, Toivonen L, Kontula K. A founder mutation of the potassium channel KCNQ1 in long QT syndrome: implications for estimation of disease prevalence and molecular diagnostics. J Am Coll Cardiol. 2001;37:562568.

28. Boulet IR, Bebarova M, Spätjens RL, Geelen JL, Jongbloed RJ, Arens YH, Geraedts JP, Crijns HJ, Volders PG. LQT1 mutation K557E: clinical and biophysical characteristics including cAMP-mediated regulation (Abstract). Heart Rhythm. 2009;6:S449.

29. Dai S, Hall DD, Hell JW. Supramolecular assemblies and localized regulation of voltage-gated ion channels. Physiol Rev. 2009;89:411-452.

30. Nicolas CS, Park KH, El Harchi A, Camonis J, Kass RS, Escande D, Mérot J, Loussouarn G, Le Bouffant $\mathrm{F}$, Baró $\mathrm{I}$. $\mathrm{I}_{\mathrm{Ks}}$ response to protein kinase A-dependent KCNQ1 phosphorylation requires direct interaction with microtubules. Cardiovasc Res. 2008;79:427-435.

31. Kurokawa J, Chen L, Kass RS. Requirement of subunit expression for cAMP-mediated regulation of a heart potassium channel. Proc Natl Acad Sci U S A. 2003;100:2122-2127.

32. Panaghie G, Tai KK, Abbott GW. Interaction of KCNE subunits with the KCNQ1 $\mathrm{K}^{+}$channel pore. J Physiol. 2006;570:455-467.

33. Haitin Y, Wiener R, Shaham D, Peretz A, Cohen EB, Shamgar L, Pongs O, Hirsch JA, Attali B. Intracellular domains interactions and gated motions of $I_{\mathrm{KS}}$ potassium channel subunits. EMBO J. 2009;28:1994-2005. 
34. Smith JA, Vanoye CG, George AL, Jr., Meiler J, Sanders CR. Structural models for the KCNQ1 voltage-gated potassium channel. Biochemistry. 2007;46:14141-14152.

35. Mikuni I, Torres CG, Bienengraeber MW, Kwok WM. Partial restoration of the long QT syndrome associated KCNQ1 A341V mutant by the KCNE1 beta-subunit. Biochim Biophys Acta. 2011; In Press.

36. Yang $T$, Kanki $H$, Roden DM. Phosphorylation of the $I_{\mathrm{Ks}}$ channel complex inhibits drug block: novel mechanism underlying variable antiarrhythmic drug actions. Circulation. 2003;108:132-134.

37. Arking DE, Pfeufer A, Post W, Kao WH, Newton-Cheh C, Ikeda M, West K, Kashuk C, Akyol M, Perz S, Jalilzadeh S, Illig T, Gieger C, Guo CY, Larson MG, Wichmann HE, Marbán E, O'Donnell CJ, Hirschhorn JN, Kääb S, Spooner PM, Meitinger T, Chakravarti A. A common genetic variant in the NOS1 regulator NOS1AP modulates cardiac repolarization. Nat Genet. 2006;38:644-651.

38. Crotti L, Monti MC, Insolia R, Peljto A, Goosen A, Brink PA, Greenberg DA, Schwartz PJ, George AL, Jr. NOS1AP is a genetic modifier of the long-QT syndrome. Circulation. 2009;120:1657-1663. 
Loss of $\mathrm{I}_{\mathrm{Ks}}$ Upregulation by CAMP in LQT1 Syndrome

$132 \mid$ 


\section{ChAPTER 5}

\section{$I_{\text {KS }}$ RESTRICTS EXCESSIVE BEAT-TO-BEAT VARIABILITY OF REPOLARZIATION DURING BETA-ADRENERGIC RECEPTOR STIMULATION}

Daniel M. Johnson, Jordi Heijman, Chris E. Pollard, Jean-Pierre Valentin, harry J.g.M. Crijns, Najah Abi-Gerges and paul G.A. VOLDERS

J. Mol. Cell. CARDiol. 2010 48:122-130 


\section{ABSTRACT}

In vivo studies have suggested that increased beat-to-beat variability of ventricular repolarization duration (BVR) is a better predictor of drug-induced torsades de pointes than repolarization prolongation alone. Cellular BVR and its dynamics before proarrhythmic events are poorly understood. We investigated differential responses of BVR in single myocytes during $I_{\mathrm{Ks}}$ blockade versus $I_{\mathrm{kr}}$ blockade and late- $I_{\mathrm{Na}}$ augmentation, under the influence of $\beta$-adrenergic receptor stimulation. Transmembrane action potentials were recorded from isolated canine left-ventricular midmyocytes at various pacing rates. $I_{\mathrm{Ks}}$ was blocked by HMR1556, $I_{\mathrm{Kr}}$ by dofetilide. Late $I_{\mathrm{Na}}$ was augmented by sea anemone toxin-II. Isoproterenol was added for $\beta$-adrenergic receptor stimulation. BAPTA-AM buffered intracellular $\mathrm{Ca}^{2+}$. SEA0400 partially inhibited the $\mathrm{Na}^{+}-\mathrm{Ca}^{2+}$ exchanger. BVR was quantified as variability of action-potential duration at $90 \%$ repolarization: $\Sigma(\mid A P D 90 ; i+1$ minus APD90; $i \mid) /[$ nbeats $\times V 2]$ for 30 consecutive action potentials. Baseline BVR was significantly increased by $I_{\mathrm{kr}}$ blockade and late- $I_{\mathrm{Na}}$ augmentation, especially at slow pacing rates. $\beta$-adrenergic stimulation restabilized these BVR changes. In contrast, $I_{\mathrm{Ks}}$ blockade caused very little change in repolarization when compared to baseline conditions, but predisposed the myocyte to increased BVR during $\beta$ adrenergic stimulation, especially at fast rates. BAPTA-AM and SEA0400 reduced this excessive BVR and eliminated early afterdepolarizations. In conclusion, $\beta$-adrenergic receptor stimulation exaggerates BVR during $I_{\mathrm{Ks}}$ blockade, indicating a BVR-stabilizing role of $\beta$-adrenergic-sensitive $I_{\mathrm{Ks}}$. Loss of $I_{\mathrm{Ks}}$ plus overriding of $\mathrm{Ca}^{2+}$-dependent membrane currents, including inward $\mathrm{Na}^{+}-\mathrm{Ca}^{2+}$ exchange current, conspire to proarrhythmic BVR under these conditions. 


\section{INTRODUCTION}

Beat-to-beat variability of ventricular repolarization duration (BVR) occurs as an apparently random alteration of the repolarization duration (measured from transmembrane or monophasic action potentials (APs), local activation-recovery intervals or QT intervals) in consecutive heart beats at stable rates. Proarrhythmic conditions caused by $I_{\mathrm{kr}}$ blockade, ${ }^{1-3} I_{\mathrm{kr}}$-plus- $I_{\mathrm{Ks}}$ blockade ${ }^{3}$ and late- $I_{\mathrm{Na}}$ augmentation ${ }^{4}$ are characterized by significant increases of BVR. When increased, BVR is a better predictor of drug-induced torsades de pointes $(T \mathrm{dP})$ than repolarization prolongation alone, at least in canine and rabbit models, ${ }^{1-3}$ and in selected human patients. ${ }^{5}$

In the case of selective $I_{K s}$ inhibition in vivo, BVR remains unchanged despite mild QT prolongation., 6 However, upon the addition of intense $\beta$-adrenergic receptor stimulation ( $\beta A S$ ) pronounced repolarization instability occurs, as evident from amplified BVR, and this precedes the triggering of early afterdepolarizations (EADs), ventricular extrasystoles and TdP. ${ }^{6}$ Various antiarrhythmic interventions (e.g., intravenous $\mathrm{KCl}$ administration, $I_{K, A T P}$ activation, steady-state ventricular pacing) that prevent TdP are characterized by stabilization of BVR when often the QT interval is still prolonged. ${ }^{7}$ Collectively, these data indicate that a prominent rise of BVR heralds torsadogenic instability of the heart, whereas its decline is an expression of restabilization of electrical activity.

Cellular studies have revealed that the mechanisms underlying BVR reside, at least partly, in the cardiac myocyte. Under baseline conditions at fixed-rate pacing, isolated ventricular myocytes express temporal (i.e., beat-to-beat) variability of the action-potential duration (APD). ${ }^{1,8}$ A direct relationship exists between rate-dependent $A P D$ and $B V R$, even during random changes in pacing rate. ${ }^{9}$ Pharmacological interventions to influence ion channels that operate during the AP plateau can markedly alter baseline BVR. $\mathrm{I}_{\mathrm{Kr}}$ blockade $^{1,8,10}$ not only increases the cellular APD, but also BVR, while promoting the generation of EADs. Similar effects were noted for late- $\mathrm{I}_{\mathrm{Na}}$ augmentation with sea anemone toxin-II (ATXII). ${ }^{11}$ Conversely, the blockade of late-I ${ }_{\mathrm{Na}}$ with tetrodotoxin ${ }^{8}$ or ranolazine ${ }^{11 \text {, }}$ ${ }^{12}$ largely suppresses these proarrhythmic repolarization changes. Finally, intracellular $\mathrm{Ca}^{2+}$ chelation with EGTA reduces BVR in myocytes. ${ }^{8}$

In contrast, little is known about the contribution of the $\mathrm{K}^{+}$current $\mathrm{I}_{\mathrm{Ks}}$ to BVR stability in cardiac myocytes. $I_{\mathrm{Ks}}$ function is prominent during $\beta A S$ when it promotes AP shortening by increased protein-kinase-A-dependent activation, and during $I_{\mathrm{Kr}}$ block when it limits AP prolongation by timedependent activation, ${ }^{10,13,14}$ thus providing critical "repolarization reserve" when other repolarizing currents are impaired. ${ }^{15,}{ }^{16}$ BVR exaggerates significantly when $I_{\mathrm{Ks}}$ is inhibited after AP preprolongation with the $\mathrm{I}_{\mathrm{Kr}}$ blocker almokalant. ${ }^{10}$

In this study we investigated the properties of BVR in single canine left-ventricular (LV) myocytes during superfusion with standardbuffer solution containing physiological concentrations of ions (baseline) and the differential responses of BVR during selective $I_{\mathrm{Ks}}$ blockade versus $\mathrm{I}_{\mathrm{Kr}}$ blockade and 
late- $I_{\mathrm{Na}}$ augmentation, in the absence or presence of $\beta A S$. Our findings indicate a protective role of $I_{\mathrm{Ks}}$ in preventing excessive BVR and EAD generation during $B A S$, and a mechanistic contribution of inward $\mathrm{Na}^{+}-\mathrm{Ca}^{2+}$ exchange to these proarrhythmic sequelae.

\section{METHODS}

This investigation conformed with the Guide for the Care and Use of Laboratory Animals published by the US National Institutes of Health (NIH Publication No. 85-23, revised 1996). Animal handling was in accordance with the European Directive for the Protection of Vertebrate Animals Used for Experimental and Other Scientific Purposes (86/609/EU).

\section{Cell-isolation procedure}

Twenty-three adult female beagle dogs weighing 12.8 $\pm 0.3 \mathrm{~kg}$ (range: 10.5-15.0 kg; 9-31 months of age) were used for the myocyte isolations. Anesthesia was induced with $45 \mathrm{mg} / \mathrm{kg}$ pentobarbital. Once full anesthesia was reached, the chest was opened via a left thoracotomy and the heart was excised and placed in an $\mathrm{O}_{2}$-gassed $\mathrm{Ca}^{2+}$-free standard buffer solution at approximately $4{ }^{\circ} \mathrm{C}$. The cell isolation procedure was the same as previously described. ${ }^{17}$ Briefly, the left anterior descending coronary artery was cannulated and perfused. After $\sim 20 \mathrm{~min}$ of collagenase perfusion and subsequent washout of the enzyme, the epicardial surface layer was removed from the LV wedge until a depth of $\geq 3 \mathrm{~mm}$ was reached. Softened tissue samples were collected from the midmyocardial layer underneath while contamination with the endocardium was avoided. Samples were gently agitated, filtered and washed. LV midmyocytes were stored at room temperature in standard buffer solution and only quiescent rod-shaped cells with clear cross-striations were used for the experiments.

\section{Cellular recordings}

Transmembrane APs were recorded at $37{ }^{\circ} \mathrm{C}$ bath temperature using high-resistance (30-60 M $\Omega$ ) glass microelectrodes filled with $3 \mathrm{~mol} / \mathrm{L} \mathrm{KCl}$. Intracellular pacing was done at various cycle lengths (CLs) and only cells that showed a spike-and-dome AP morphology were accepted for the experiments. Myocyte contractions were recorded with a video edge motion detector (Crescent Electronics, Sandy, UT, USA).

\section{Solutions and agents}

The standard buffer solution used for the experiments was composed of (in mmol/L): $\mathrm{NaCl} 145, \mathrm{KCl}$ 4.0, $\mathrm{CaCl}_{2}$ 1.8, $\mathrm{MgCl}_{2}$ 1.0, glucose 11 and HEPES 10, pH 7.4 with $\mathrm{NaOH}$ at $37^{\circ} \mathrm{C}$. HMR1556 ((3R,4S)(+)-N-[3-hydroxy-2,2-di methyl-6-(4,4,4-tri fluo robutoxy)chroman-4-yl]-N-methyl methane sulfon amide) was used to block $I_{\mathrm{ks}}$. At $500 \mathrm{nmol} / \mathrm{L}$ it blocks the current completely, ${ }^{10}$ while exerting minimal, if any, effects on $I_{\mathrm{kr}}$ and other ion currents. ${ }^{6,18,19} \mathrm{I}_{\mathrm{kr}}$ was selectively blocked by $1 \mu \mathrm{mol} / \mathrm{L}$ dofetilide (Apin Chemicals, Abingdon, United Kingdom). Late $I_{N a}$ was augmented by $20 \mathrm{nmol} / \mathrm{L}$ ATXII (Sigma-Aldrich, Zwijndrecht, The Netherlands). Intracellular $\mathrm{Ca}^{2+}$ was buffered with $5 \mu \mathrm{mol} / \mathrm{L}$ BAPTA- 
AM (Invitrogen, Breda, The Netherlands). The $\mathrm{Na}^{+}-\mathrm{Ca}^{2+}$ exchanger was inhibited by $300 \mathrm{nmol} / \mathrm{L}$ or 1 $\mu \mathrm{mol} / \mathrm{L}$ SEA0400. At these concentrations SEA0400 has little effect on $\mathrm{I}_{\mathrm{Ca}}$, the cytosolic $\mathrm{Ca}^{2+}$ transient or contraction of canine ventricular myocytes, whereas it blocks $\mathrm{Na}^{+}-\mathrm{Ca}^{2+}$ exchange by $28-80 \%$ (reverse mode \& forward mode) depending on cytosolic $\mathrm{Ca}^{2+}$ concentration. $^{20,}{ }^{21}$ HMR1556, dofetilide, ATXII, BAPTA-AM and SEA0400 were initially dissolved in dimethyl sulfoxide and then diluted so that the concentration of the solvent was $\sim 0.1 \%$ in the superfusate, a concentration that has no measurable effects on AP or ionic currents. $\beta$ AS was applied as $100 \mathrm{nmol} / \mathrm{L}$ isoproterenol. This agent was originally dissolved in distilled water containing $30 \mu \mathrm{mol} / \mathrm{L}$ ascorbic acid and then stored in the dark at $4{ }^{\circ} \mathrm{C}$ until use.

\section{Data analysis and statistics}

BVR was quantified as variability of $A P D$ at $90 \%$ repolarization $\left(\mathrm{APD}_{90}\right): \Sigma\left(\mid A P D_{90} ; \mathrm{i}+1\right.$ minus $A \mathrm{AD}_{90}$; i|)/[nbeats $\times \sqrt{2}$ ] for 30 (or a minimum of 10 ) consecutive APs. ${ }^{1}$ In addition, we calculated the coefficient of variability $(\mathrm{CV})$ of $A P D_{90}$ as the percentage of standard deviation/mean $A P D_{90}{ }^{8}$ Quantitative data are expressed as mean \pm SEM. Intergroup comparisons were made with Student's $t$ test for unpaired and paired data groups, after testing for the normality of distribution. Differences were considered statistically significant if $\mathrm{P}<0.05$.

\section{RESULTS}

\section{Baseline characteristics of BVR in canine LV myocytes}

A

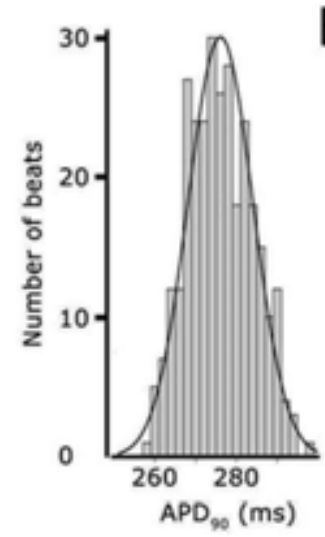

C

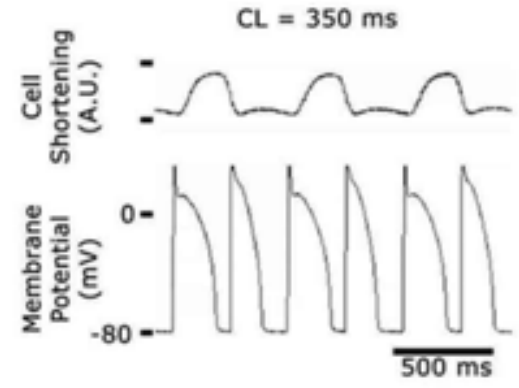

B

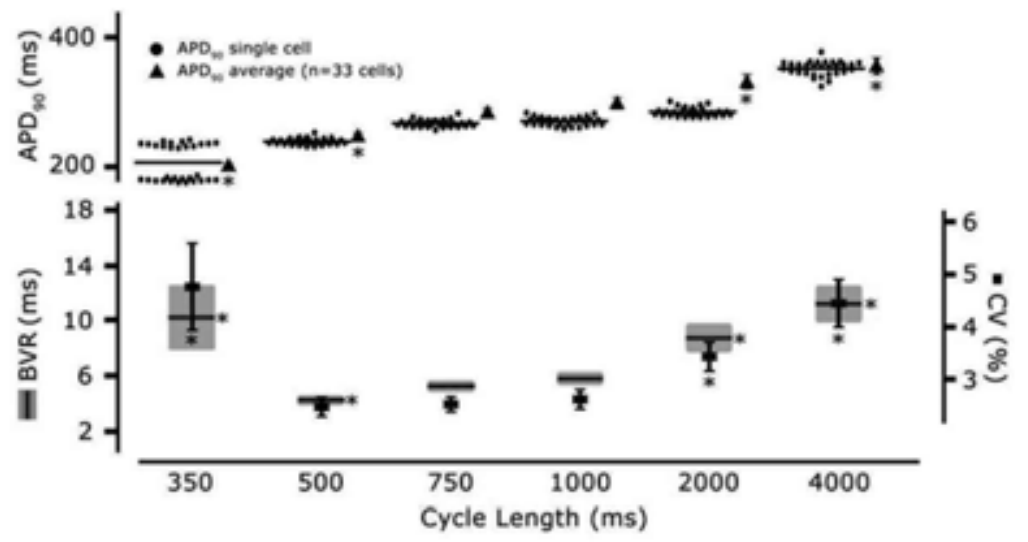

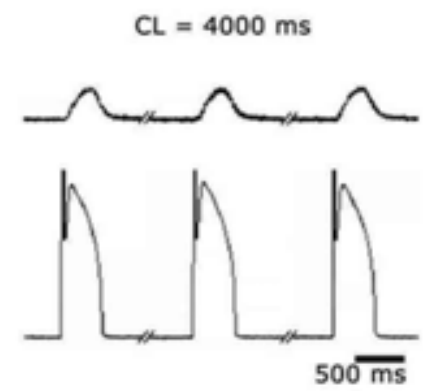

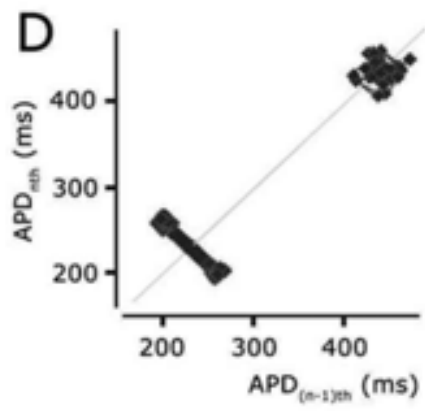


Figure 1. Baseline characteristics of BVR in canine LV myocytes. A. Histogram of $A P D_{90}$ from 300 consecutive cycles in a representative cell with Gaussian fit (solid line) to the histogram data. Bins of 2 ms width. B. Single-cell (30 APs) and average $A P D_{90}$ as a function of pacing $C L$ (Top panel). Bottom panel illustrates the $\mathrm{CL}$ dependence of both BVR and CV. Average parameters are shown $\pm S E M$. C. Representative AP and contraction recordings from the same cell paced at $350 \mathrm{~ms}$ and $4000 \mathrm{~ms} \mathrm{CL}$. D. Poincaré plots of $A P D_{90}$ from the cell shown in $C$ at the $C L$ of $350 \mathrm{~ms}$ (left) and $4000 \mathrm{~ms}$ (right). Of note is the repetitive behavior seen at $350 \mathrm{~ms}$, whereas at $4000 \mathrm{~ms}$ the recordings are more chaotic. $A P D$ represents $A P D_{90} . C V$, coefficient of variability. $C L$, cycle length. A.U., arbitrary units.

Figure $1 \mathrm{~A}$ shows a histogram of 300 consecutive $A P D_{90} \mathrm{~S}$ in a representative contracting myocyte during pacing at a $\mathrm{CL}$ of $1000 \mathrm{~ms}$. $\mathrm{APD}_{90}$ was normally distributed about a mean of $276 \mathrm{~ms}$ with a SEM of $0.5 \mathrm{~ms}$. Similar results were obtained from 13 cells. A correlation coefficient of 0.90 was calculated between the histogram and a normal Gaussian fit. This indicated that $A P D_{90}$ variability within the canine myocyte occurs randomly, which is consistent with previous data from guinea pig. ${ }^{8}$ In 33 cells, pacing rate was varied between CLs of 350 and 4000 ms. APD 90 increased on slowing of the pacing rate, as expected. ${ }^{22}$ Single representative and pooled $A P D_{90}$ data are shown in Figure $\mathbf{1 B}$.

BVR averaged 5.9 $\pm 0.5 \mathrm{~ms}$ at CL $1000 \mathrm{~ms}$ (Figure 1B). Significant increases were calculated at both the fastest and slowest pacing rates; at CL 350 ms BVR was $10.3 \pm 2.3 \mathrm{~ms}$ and at $\mathrm{CL} 4000 \mathrm{~ms} 11.3 \pm 1.3 \mathrm{~ms}$ (both $\mathrm{P}<0.05$ vs. CL $1000 \mathrm{~ms}$ ). Similar patterns were found for CV. At CL $1000 \mathrm{~ms}$ CV averaged $2.6 \pm 0.2 \%$, increasing to $4.7 \pm 0.8 \%$ and $4.4 \pm 0.5 \%$ at the same extreme rates (both $P<0.05$ vs. $C L 1000$ ms; Figure 1B). Thus, at (very) fast pacing, rate-dependent adaptation of APD90 (further decrease) was clearly discordant with that of both BVR and CV (increases). Beat-to-beat alternans of both $A P D_{90}$ and contraction amplitude explained this discordance, as demonstrated in Figure 1C, left panel. $\mathrm{APD}_{90}$ alternans occurred as a very ordered process (interchanging between two values), whereas BVR was apparent as a more random $A$ PD $_{90}$ behavior, generally at slower rates (Figure 1C, right panel; same cell). This is further exemplified in the Poincaré plots of Figure 1D. These data suggest distinct mechanisms for $B V R$ and $A P D_{90}$ alternans.

Both BVR and CV expressed similar patterns of rate-dependent repolarization variability. As BVR incorporates the beat-to-beat consecutiveness of $A P D_{90}$ in its formulation whereas $C V$ does not, we used BVR in the remaining analyses. 


\section{Influences of hyper- and hypokalemia on BVR}

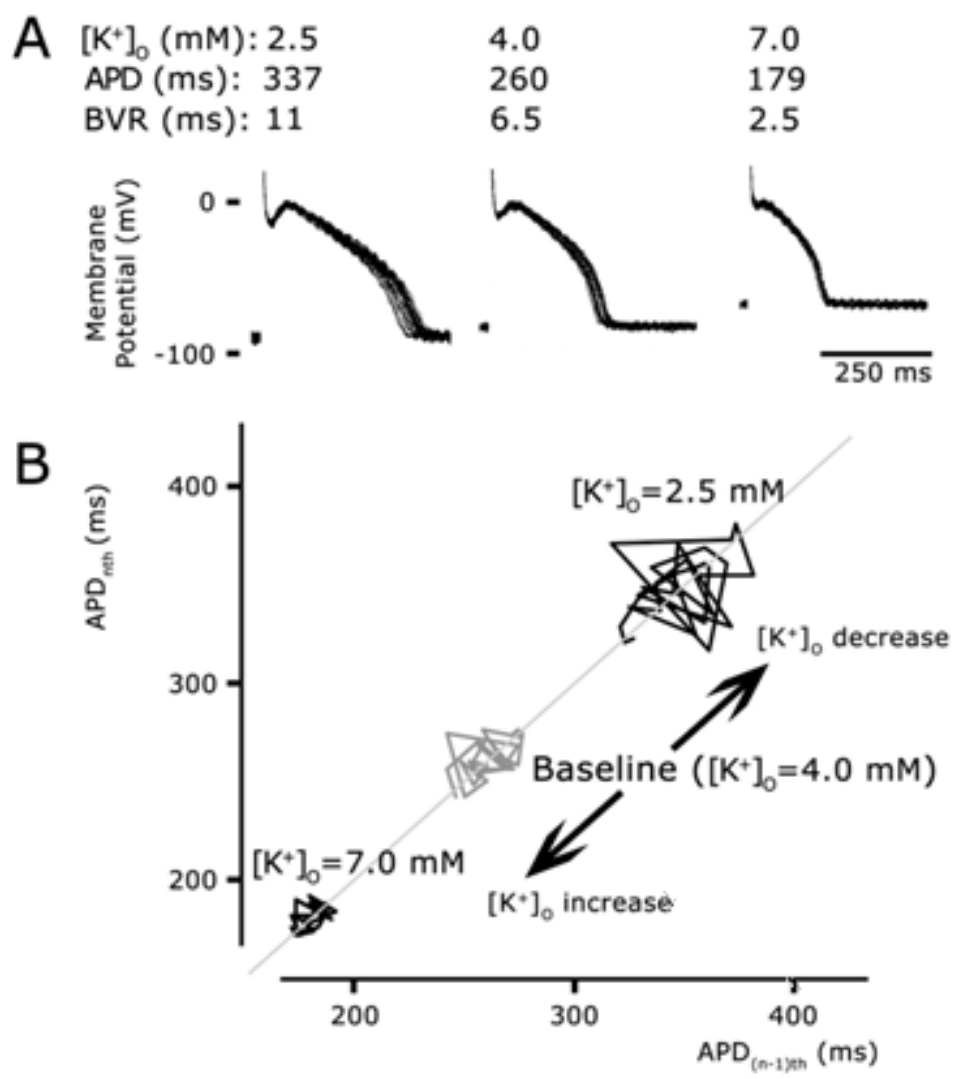

Figure 2. Response of APD and BVR to changes in $\left[\mathrm{K}^{+}\right]_{0}$. A. APs recorded from 30 consecutive cycles in a single canine LV myocyte paced at a cycle length of $2000 \mathrm{~ms}$ under various $\left[\mathrm{K}^{+}\right]_{0}$. BVR and APD values and $\left[\mathrm{K}^{+}\right]_{0}$ are indicated above. B. Poincaré plots of $A P D_{90}$ of each of these traces are shown. An increase in BVR is seen during a state of hypokalemia, and a decrease in a state of hyperkalemia.

Next, we examined the influences of hyper- and hypokalemia. Representative results are shown in Figure 2. Myocytes ( $n=4)$ were constantly paced at $C L 2000 \mathrm{~ms}$. For hyperkalemia, $\left[\mathrm{K}^{+}\right]_{0}$ was raised to $7.0 \mathrm{mmol} / \mathrm{L}$ in the superfusate. Along with $\mathrm{APD}_{90}$ shortening ( $-15 \%$ from $264 \pm 35 \mathrm{~ms}$ ), BVR decreased from $5.3 \pm 1.2 \mathrm{~ms}$ (normokalemia) to $3.0 \pm 0.3 \mathrm{~ms}(\mathrm{P}<0.05)$. For hypokalemia, $\left[\mathrm{K}^{+}\right]_{\text {。 }}$ was lowered to 2.5 $\mathrm{mmol} / \mathrm{L}$. Now, $\mathrm{APD}_{90}$ prolonged $(+26 \%)$ and $\mathrm{BVR}$ increased to $9.0 \pm 0.8 \mathrm{~ms}(\mathrm{P}<0.05)$. No EADs were observed. Resting membrane potentials varied with the $\left[\mathrm{K}^{+}\right]_{0}$ changes, as expected (Figure $2 \mathrm{~A}$ ). 


\section{BVR instability and EAD generation during $\mathrm{I}_{\mathrm{Kr}}$ blockade or late- $\mathrm{I}_{\mathrm{Na}}$}

\section{augmentation, and rescue by $\beta A S$}
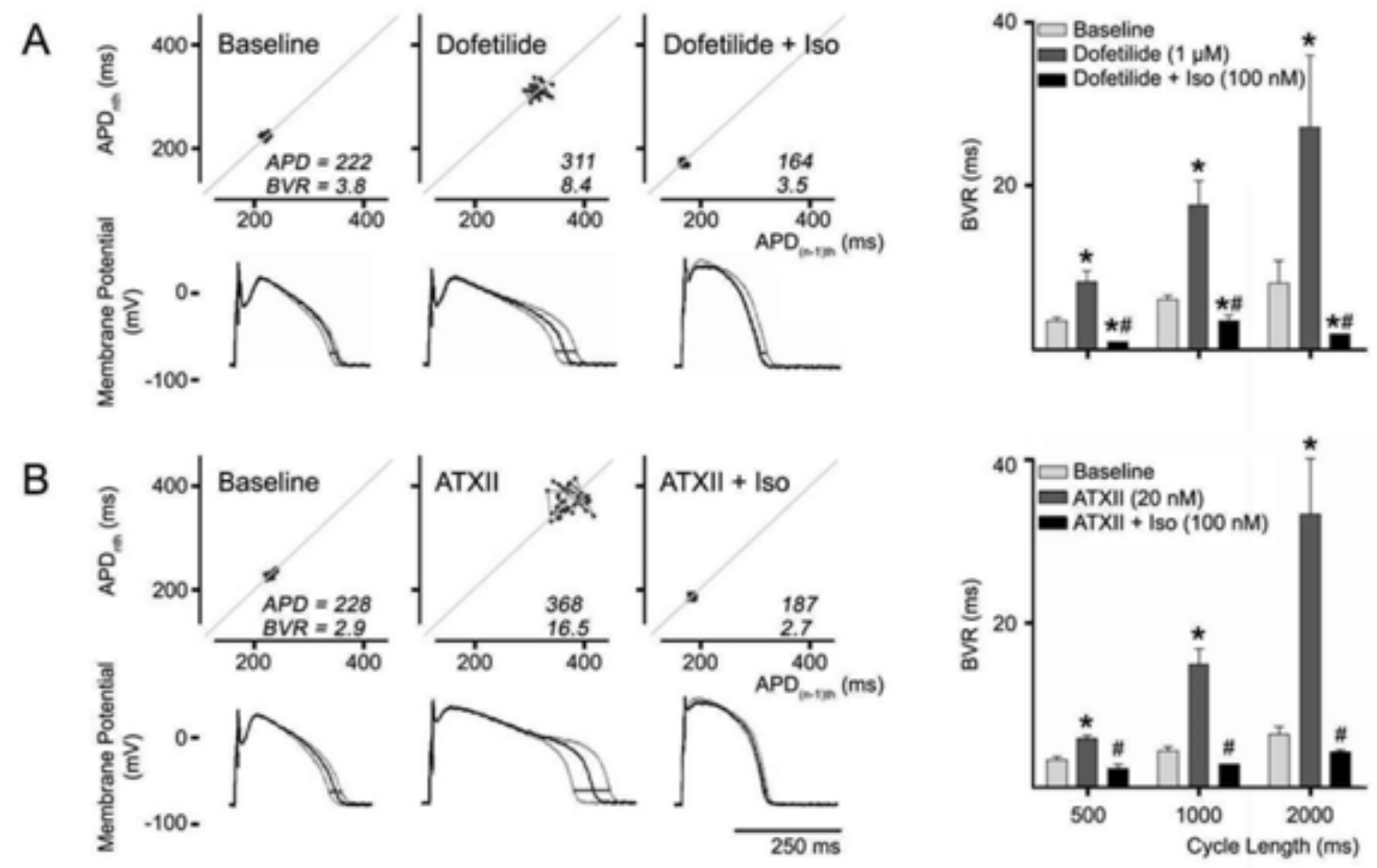

Figure 3. Increases in repolarization instability caused by both $I_{\mathrm{kr}}$ blockade and late- $I_{\mathrm{Na}}$ augmentation and rescue by $\beta A S$. Representative examples showing the effects of dofetilide $1 \mu \mathrm{mol} / L$ (panel $A$ ) or ATX II $20 \mathrm{nmol} / \mathrm{L}$ (panel B) on BVR at $1000 \mathrm{~ms} \mathrm{CL}$ and rescue by $\beta A S$. AP recordings showing the minimum, maximum and median beats are shown for each condition and the $A D P_{90}$ and $B V R$ are indicated (left). Poincaré plots for each condition are also shown. Group data from all cells is shown on the right. Data is shown as mean \pm SEM. ${ }^{*} \mathrm{P}<0.05$ versus baseline; ${ }^{\sharp} \mathrm{P}<0.05$ versus drug challenge.

$\mathrm{I}_{\mathrm{Kr}}$ blockade with dofetilide $(1 \mu \mathrm{mol} / \mathrm{L})$ and late- $\mathrm{I}_{\mathrm{Na}}$ augmentation with ATXII $(20 \mathrm{nmol} / \mathrm{L})$ increased $A P D_{90}$ and BVR at pacing CLs of 500, 1000 and 2000 ms, but most significantly at the slower rate. This is shown in Figure 3. In the case of dofetilide and for $\mathrm{CL} 1000 \mathrm{~ms}$, BVR changed from $5.6 \pm 0.5 \mathrm{~ms}$ at baseline to $16.1 \pm 2.9 \mathrm{~ms}\left(\mathrm{P}<0.05 ; 19\right.$ cells; $A P D_{90}+37 \%$ from $\left.297 \pm 15 \mathrm{~ms}\right)$, as calculated fromAPD ${ }_{90} \mathrm{~S}$ before the first occurrence of EADs (if present; see below). Similarly, in the case of ATXII, BVR increased from $4.4 \pm 0.5 \mathrm{~ms}$ to $15.5 \pm 1.8 \mathrm{~ms}\left(\mathrm{P}<0.05 ; 12\right.$ cells; $A P D_{90}+47 \%$ from $255 \pm 17 \mathrm{~ms}$ ). $A P D_{90}$ histograms remained normally distributed during both dofetilide and ATXII (data not shown). During these drug challenges, the addition of $\beta A S$ (isoproterenol; $100 \mathrm{nmol} / \mathrm{L}$ ) drastically reduced $A P D_{90}$ and BVR at all cycle lengths, even to values below baseline (Figure 3). EADs were also abolished. For dofetilide at $C L 1000 \mathrm{~ms}$, BVR decreased from $16.1 \pm 2.9 \mathrm{~ms}$ to $3.5 \pm 0.7 \mathrm{~ms}\left(P<0.05 ; A D_{90}-102 \%\right.$ to $201 \pm 23 \mathrm{~ms}$ ). For $A T X I I$ at the same $C L, B V R$ was reduced from $15.5 \pm 1.8 \mathrm{~ms}$ to $2.8 \pm 0.3 \mathrm{~ms}(\mathrm{P}<0.05$; $A P D_{90}-40 \%$ to $180 \pm 10 \mathrm{~ms}$ ). In some cells, delayed afterdepolarizations (DADs) appeared during $\beta A S$ and drug challenge, however these did not reach the threshold for triggering APs. 


\section{BVR instability and EAD generation by $\beta A S$ during IKs blockade}
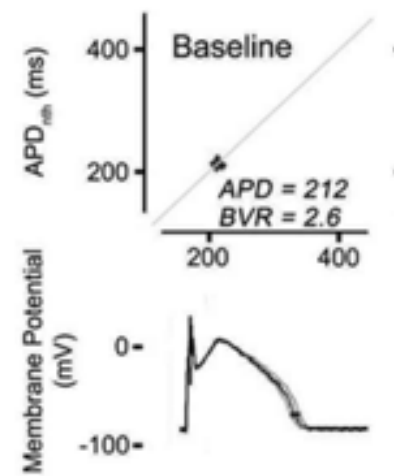
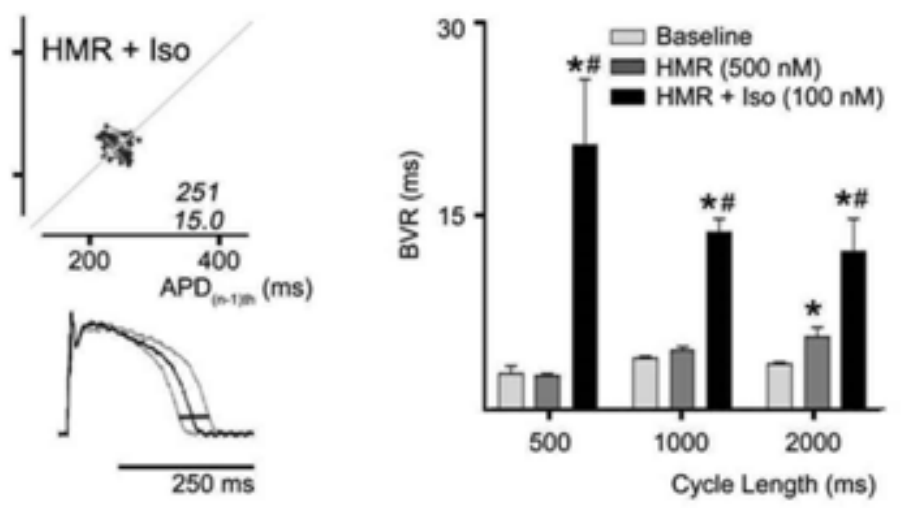

Figure 4. $\beta A S$ unmasks repolarization instability caused by $I_{K_{s}}$ blockade. Representative traces showing the minimum, maximum and median AP under baseline conditions, $I_{\mathrm{Ks}}$ blockade and $I_{\mathrm{Ks}}$ blockade plus $\beta A S$ during pacing at 1000 ms. Poincaré plots, $A P D_{90}$ and $B V R$ values for the particular cell are also shown. Group data from all cells are shown on the right showing mean \pm SEM. ${ }^{*} P<0.05$ versus baseline; ${ }^{\sharp} \mathrm{P}<0.05$ versus drug challenge.

$\mathrm{I}_{\mathrm{Ks}}$ blockade with HMR1556 (500 nmol/L) did not affect APD go $_{90}$ at pacing CLs of 500, 1000 and 2000 $\mathrm{ms}$, in line with previous results. ${ }^{10,23}$ For example, at CL $1000 \mathrm{~ms}$ APD90 was $249 \pm 8 \mathrm{~ms}$ at baseline compared to $250 \pm 8 \mathrm{~ms}$ under HMR1556. Likewise, BVR remained unaltered at CLs of 500 and 1000 ms. However at CL 2000 ms, it increased moderately but significantly (Figure 4, bar graph; n=16). $A P D_{90}$ histograms remained normally distributed during HMR1556. No EADs or DADs were observed during HMR1556 only.

The addition of $\beta A S$ during $I_{\mathrm{ks}}$ blockade changed this picture drastically: BVR increased at all CLs, but most apparently at CL 500 ms and during the window of DAD occurrence (Figures 4 and 5). DADs were generated in all cells $(n=10)$, and EADs in most of them. At pacing CL $1000 \mathrm{~ms}$, BVR increased to $5.7 \pm 0.3 \mathrm{~ms}$ during HMR1556 plus isoproterenol before the occurrence of DADs from a value of 4.2 \pm 0.2 under HMR1556 alone. During the subsequent phase with DADs, BVR increased further to $14.8 \pm 1.5 \mathrm{~ms}(\mathrm{P}<0.05)$. $A \mathrm{PD}_{90}$ also showed a significant increase at all $\mathrm{CLs}$. For example at $\mathrm{CL} 1000 \mathrm{~ms}$, $\mathrm{APD}_{90}$ averaged $291 \pm 15 \mathrm{~ms}$ (+17\% versus baseline) during HMR1556 plus isoproterenol in the phase with DADs. Obviously, EADs exaggerated BVR and $A P D_{90}$ even further (Figure 5). BVR and $A P D_{90}$ changes, and the generation of DADs and EADs, were completely reversible upon washout of isoproterenol. 

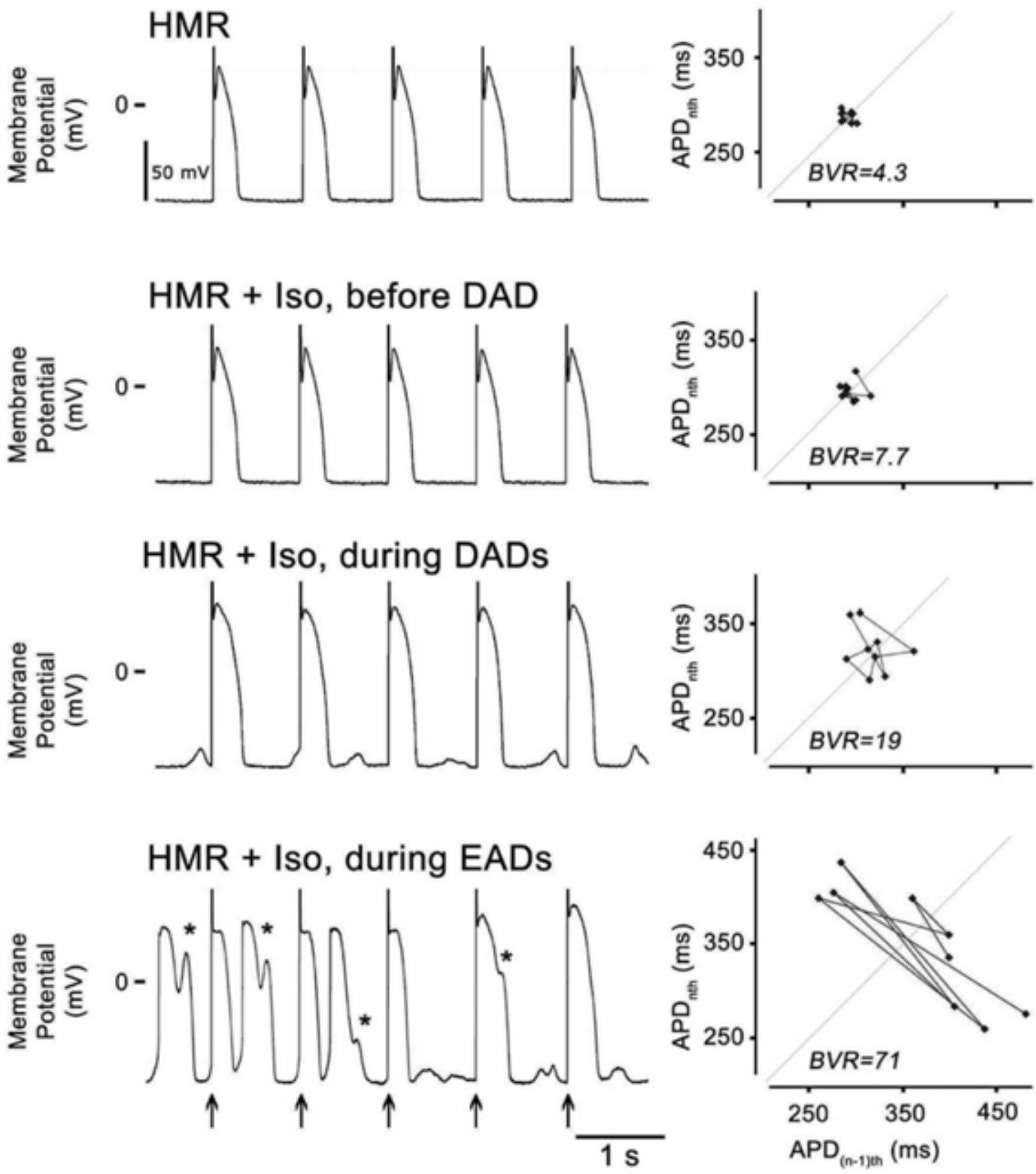

Figure 5. Time course of increased repolarization instability during $I_{\mathrm{Ks}}$ blockade plus $\beta A S$. Upper panel shows consecutively paced APs at $1000 \mathrm{~ms} C L$, together with Poincaré plots during initial perfusion of HMR1556. Addition of $\beta A S$ increases APD and BVR before DADs are seen (2nd panel from above). The occurrence of DADs (3rd panel) increases APD and BVR further, and DAD-triggered APs and EADs are subsequently observed (4th panel). Arrows indicate timings of paced beats, and * indicate EADs. Low amplitude EADs are also generated under these conditions. 


\section{Differential magnitudes of BVR in cells with or without EAD generation, and} rate dependence of EADs under different conditions

A

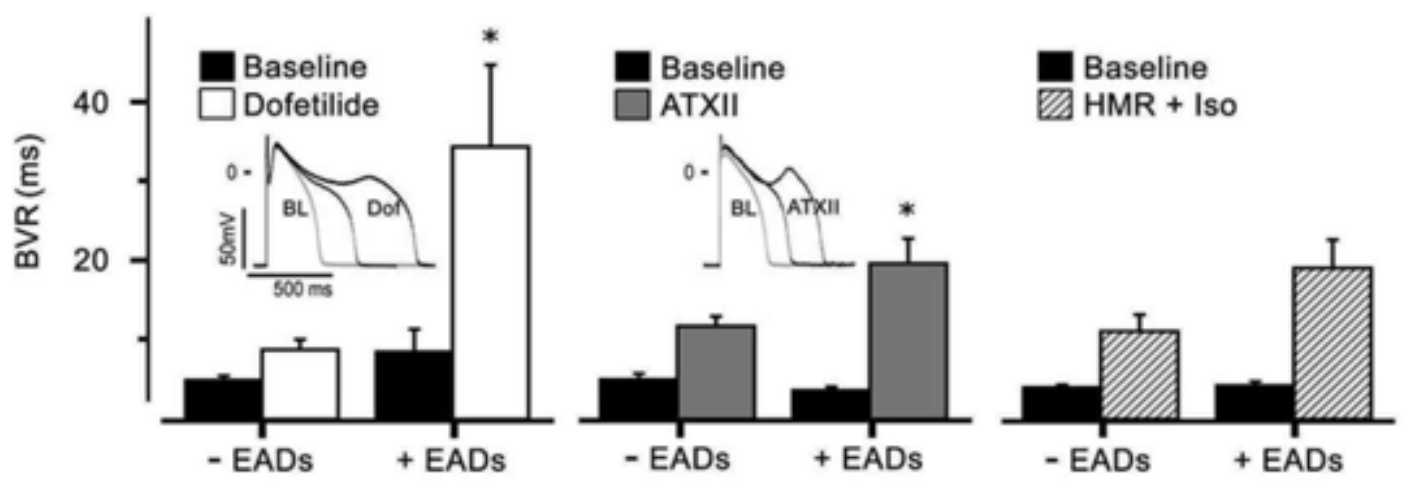

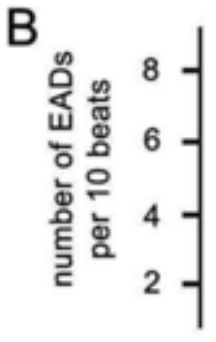

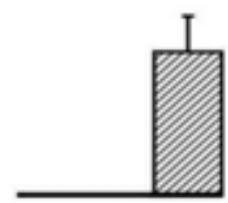

500

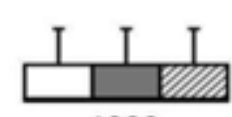

1000

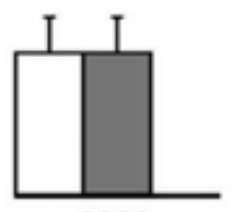

2000

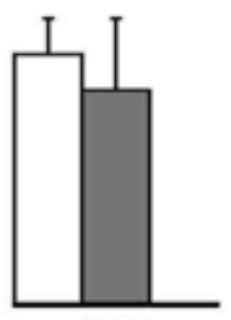

4000

Cycle length (ms)

Figure 6. Magnitude of BVR in cells with or without EADs, and rate dependence of EADs under various conditions. Magnitude of BVR in cells with or without EADs, and rate dependence of EADs under various conditions. A. Bar graph showing BVR values in cells that show EADs compared to those that did not during various drug treatments. Insets show examples of EADs caused by $I_{\mathrm{Kr}}$ block and late-I $I_{\mathrm{Na}}$ augmentation together with the preceding beat and the baseline AP. Mean data shown $\pm S E M$; BL represents baseline. ${ }^{*} P<0.05$ versus BVR in cells with no EADs. $B$. Bar graph showing the rate dependence of EADs. Number of EADs per 10 beats are plotted at various CLs during all 3 treatment groups. Mean data shown+SEM.

During $I_{\mathrm{Kr}}$ blockade and late- $I_{\mathrm{Na}}$ augmentation, BVR increases (most pronounced at slow rates) were significantly larger in myocytes in which EADs ensued, even though BVR was calculated before the first EAD occurred, ( $n=8 / 19$ for dofetilide and $n=5 / 12$ for ATXII) versus those cells in which no EADs were seen ( $n=11 / 19$ and $n=7 / 12$, respectively). This is shown in Figure 6A (for $C L 2000 \mathrm{~ms}$ ). In contrast, EADs generated during $\mathrm{I}_{\mathrm{ks}}$ blockade plus $\beta A S$ were clearly fast-rate dependent (Figure 6B). Under these conditions, BVR was not statistically different for myocytes with ensuing EADs ( $n=5 / 10$ ) versus those without $(n=5 / 10)$. $\beta A$-stimulated EADs were always preceded by DADs and were always accompanied by early aftercontractions (Figures $\mathbf{7}$ and 8, middle panels). Early aftercontractions usually took off before EAD upstrokes (inset, Figure 7, middle panel) and, in fact, occurred often without concrete EADs. Collectively, these data indicated that EADs and DADs during $\mathrm{I}_{\mathrm{Ks}}$ blockade plus $\beta A S$ are generated as the consequence of cellular $\mathrm{Ca}^{2+}$ overload and spontaneous $\mathrm{Ca}^{2+}$ release from the sarcoplasmic reticulum, and they suggested a mechanistic contribution of inward $\mathrm{Na}^{+}-\mathrm{Ca}^{2+}$ exchange. ${ }^{24,25}$ 


\section{Influences of BAPTA-AM and SEA0400 on BVR instability and EAD generation}

\section{during $I_{\mathrm{Ks}}$ blockade plus $\beta A S$}
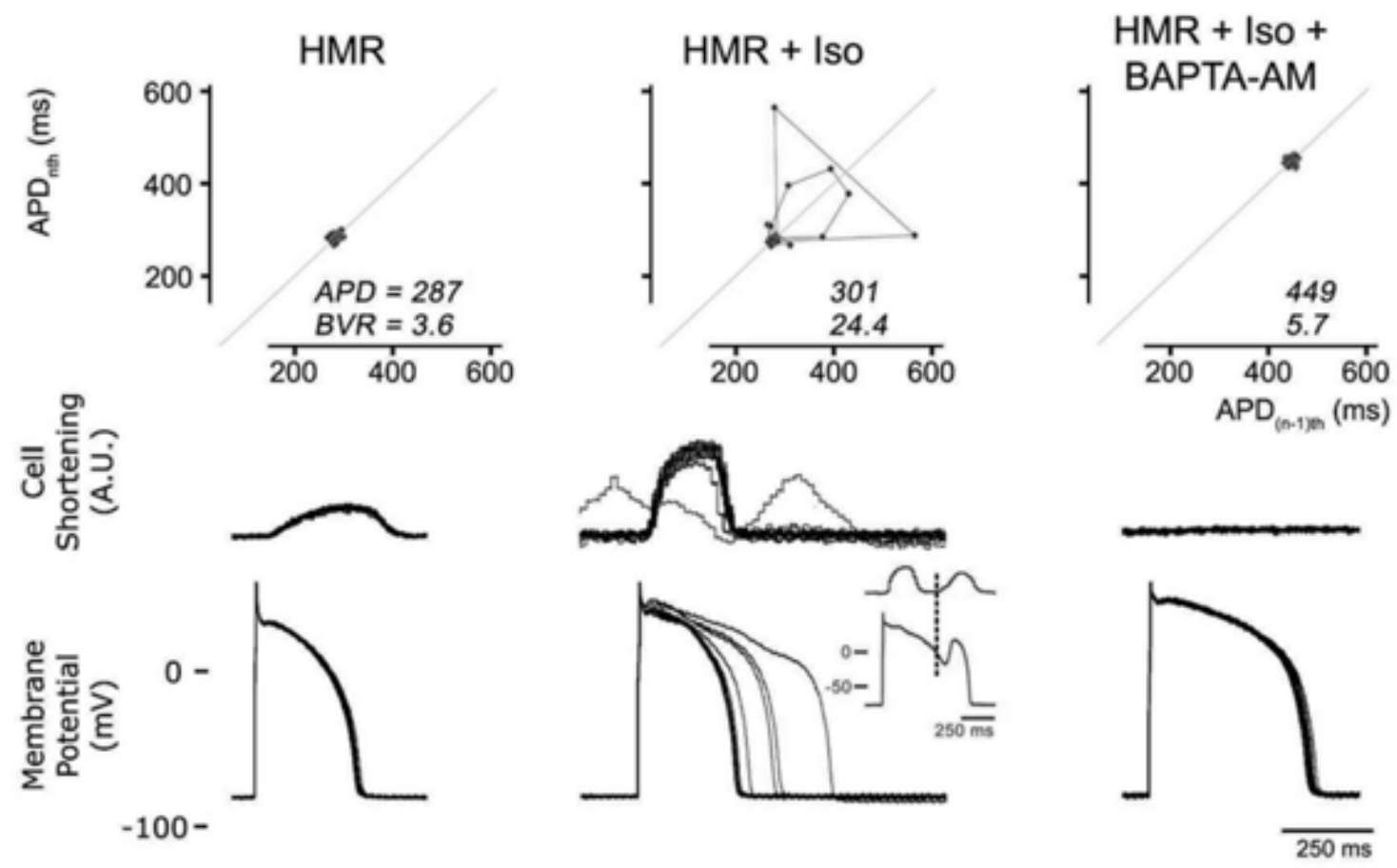

Figure 7. $\mathrm{Ca}^{2+}$ chelator BAPTA-AM rescues from excessive BVR during $I_{\mathrm{Ks}}$ blockade plus $\beta A S$. Representative traces showing 30 consecutive AP and contraction recordings overlapped from the same cell under $I_{\mathrm{Ks}}$ blockade (left panel), plus $\beta A S$ (middle panel) and together with $5 \mu \mathrm{mol} / \mathrm{L}$ BAPTAAM (right panel) while being paced at $1000 \mathrm{~ms}$. Inset shows an example EAD caused by $\mathrm{I}_{\mathrm{ks}}$ blockade plus $\beta A S$. EADs are eliminated during intracellular calcium buffering despite APD lengthening. Dashed line indicates the initiation of the early aftercontraction which preceded the EAD upstroke. Poincaré plots, $\mathrm{APD}_{90}$ and BVR values for this particular cell are also shown.

As a final series of experiments, we examined the effects of the intracellular $\mathrm{Ca}^{2+}$ chelator BAPTA-AM $(5 \mu \mathrm{mol} / \mathrm{L})$ and the $\mathrm{Na}^{+}-\mathrm{Ca}^{2+}$ exchange blocker SEA0400 (300 nmol/L or $\left.1 \mu \mathrm{mol} / \mathrm{L}\right)$ on BVR instability and EAD generation by $\beta A S$ during $I_{\mathrm{ks}}$ blockade. Data were obtained in 6 cells. As shown in Figure 7, BAPTA-AM significantly reduced excessive BVR and eliminated all EADs (and DADs). At pacing CL $1000 \mathrm{~ms}$, BVR changed from 12.9 $\pm 1.6 \mathrm{~ms}$ during HMR1556 plus isoproterenol (before first EADs) to $4.2 \pm 0.1 \mathrm{~ms}(\mathrm{P}<0.05)$ during additional BAPTA-AM, even despite extra AP prolongation $\left(\mathrm{APD}_{90}+56 \%\right)$. The latter is likely caused by a reduction in $\mathrm{I}_{\mathrm{CaL}}$ inactivation ${ }^{26}$ and an $\mathrm{I}_{\mathrm{Kr}}$ blocking side-effect of BAPTAAM. ${ }^{27}$ BVR restabilization was maximal when all (after)contractile activity was inhibited by the $\mathrm{Ca}^{2+}$ buffer. Figure 8 illustrates the effects of SEA0400 (300 nmol/L) at CL $500 \mathrm{~ms}$. BVR decreased significantly from $12.2 \pm 1.5$ ms during HMR1556 plus $\beta A S$ to $2.4 \pm 0.2 \mathrm{~ms}(P<0.05)$ during additional SEA0400. Effects were less pronounced at CLs 1000 and 2000 ms. All EADs were eliminated upon the addition of SEA0400. BVR effects were quantitatively similar for $300 \mathrm{nmol} / \mathrm{L}$ and $1 \mu \mathrm{mol} / \mathrm{L} \mathrm{SEA0400,}$ as were reductions of $A P D_{90}$ and contraction amplitude. At $C L 500 \mathrm{~ms}, A P D_{90}$ decreased from $246 \pm 16$ ms during HMR1556 plus $\beta A S$ to $201 \pm 13$ ms $(P<0.05)$ during additional SEA0400, while contraction amplitudes were decreased by $52 \pm 5 \%$. 

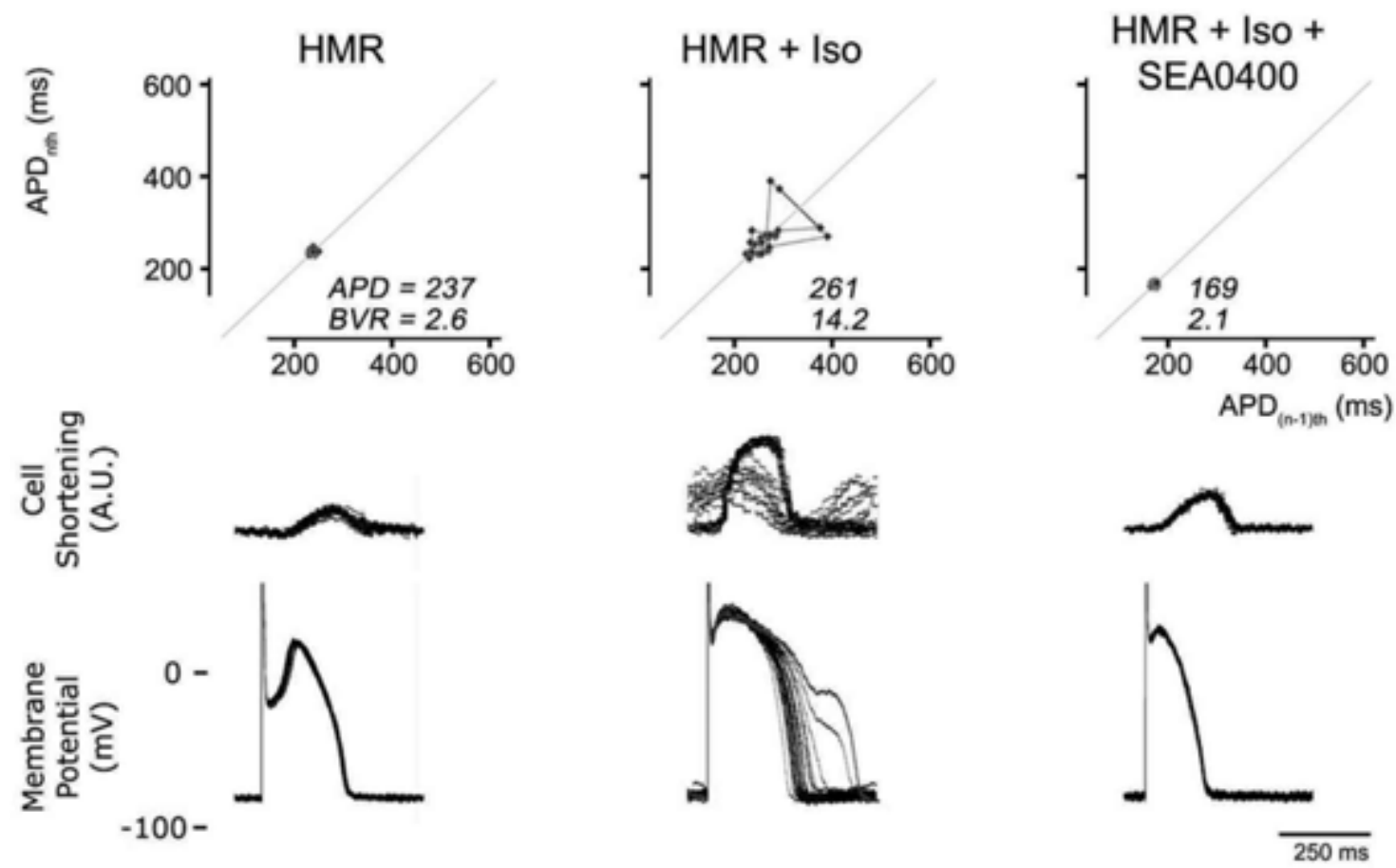

Figure 8. $\mathrm{Na}^{+}-\mathrm{Ca}^{2+}$ exchange inhibitor SEA0400 rescues from excessive BVR during $\mathrm{I}_{\mathrm{Ks}}$ blockade plus $\beta A S$. Representative traces showing 30 consecutive AP and contraction recordings from the same cell under $I_{\mathrm{Ks}}$ blockade (left panel) plus $\beta A S$ (middle panel) plus $300 \mathrm{nmol} / \mathrm{L}$ SEA0400 (right panel) at $\mathrm{CL} 500 \mathrm{~ms}$. EADs are seen under $\mathrm{I}_{\mathrm{Ks}}$ blockade plus $\beta A S$ which are eliminated during application of SEA0400. Poincaré plots, $A P D_{90}$ and BVR values for this particular cell are also shown.

\section{DISCUSSION}

The mechanisms underlying BVR at the single-cell level are poorly understood. In the present study, we used canine ventricular myocytes to investigate BVR responses at baseline and during various proarrhythmic challenges. Our data indicate that $I_{\mathrm{Ks}}$ stabilizes $B V R$ at slow pacing rates under baseline conditions, despite the absence of changes in $A P D_{90}$. $\beta A S$ of $I_{K s}$ rescues from excessive repolarization instability and EAD generation during $\mathrm{I}_{\mathrm{kr}}$ block and late- $\mathrm{I}_{\mathrm{Na}}$ augmentation. Furthermore, our results support the notion that $\beta A S$ during drug-induced $I_{\mathrm{Ks}}$ block promotes altered $\mathrm{Ca}^{2+}$ handling and enhanced activation of $\mathrm{Ca}^{2+}$-dependent membrane currents, including inward $\mathrm{Na}^{+}-$ $\mathrm{Ca}^{2+}$ exchange, thus exaggerating BVR. Although single myocyte BVR is not the sole contributor to invivo QT variability, our novel insights are crucial for the understanding of BVR at the whole organ level such as seen after $I_{\mathrm{kr}}$ blockade and $\beta A S^{6}{ }^{6}$

\section{Distinction between BVR and repolarization alternans}

At baseline, BVR is mainly dependent on APD which in turn is reliant on cycle length. At very fast rates, however, $\mathrm{APD}_{90}$ and $\mathrm{BVR}$ become discordant as the result of beat-to-beat repolarization alternans (Figure 1). The latter is an ordered process, characterized by interchanging between two 
APDs and concomitant contraction amplitudes. Although repolarization alternans is considered arrhythmogenic, EADs are not normally seen under these conditions, at least not in our experiments. Repolarization and contraction alternans arise from beat-to-beat alteration in cellular $\mathrm{Ca}^{2+}$ cycling. $^{28}$ $B V R$, on the other hand, is characterized by an apparently random behavior of APD, generally at intermediate and slow rates. Even during pronounced BVR instability, e.g., by hypokalemia, $I_{\mathrm{kr}}$ blockade and late- ${ }_{\mathrm{Na}}$ augmentation, contraction amplitudes remain relatively stable (contraction data not shown). Our data thus indicate that in the majority of cases BVR and $A P D_{90}$ show parallel changes. However, in specific conditions they can deviate, for example during $I_{\mathrm{ks}}$ blockade plus $\beta A S$, and during $I_{\mathrm{ks}}$ blockade plus $\beta A S$ and BAPTA-AM. This suggests that BVR is not necessarily dependent on $\mathrm{APD}_{90}$ at all times, which has major mechanistic implications.

Cellular BVR likely results from the stochastic activities of transmembrane ion transporters, including ion channels, during the AP plateau phase. During this phase, around the $0 \mathrm{mV}$ level, the membrane resistance is very high, ${ }^{8}$ meaning that minute changes of the ion fluxes across the membrane can significantly affect the membrane potential which in turn will alter the repolarization duration. ${ }^{29}$ Interventions that reduce net outward current will cause a prolongation of this vulnerable phase, meaning that there is an extended period at which the voltage is around the $0-\mathrm{mV}$ level. Stochastic behavior of the ion channels may then increase BVR. It is also possible that this stochastic behavior results indirectly from subsarcolemmal $\mathrm{Ca}^{2+}$ fluctuations that lead to modification of $\mathrm{Ca}^{2+}$-dependent ion currents. Modeling data has shown that during isoproterenol stochastic $I_{\text {CaL }}$ gating in mode 2 results in APD variability as well as EAD generation, ${ }^{30}$ and this could contribute to the BVR seen in this study.

\section{Cellular BVR during $\beta A S$}

In the present study, we have shown that BVR is drastically increased under conditions of $I_{\mathrm{kr}}$ blockade or late $I_{\mathrm{Na}}$ augmentation in isolated canine LV midmyocytes. The addition of $\beta A S$, however, causes a major reduction in BVR and eliminates EAD activity (Figure 3 ). As $I_{\mathrm{ks}}$ is the main $\beta A$-sensitive outward current in the canine ventricular $A P$, this current appears responsible for the restabilization of repolarization. If true, $\beta$ AS would provoke BVR instability during $I_{\mathrm{Ks}}$ blockade, as $I_{\mathrm{Ks}}$ could no longer compensate for the increased $I_{\text {cal }}$ caused by $\beta A S$, which leads to increased cellular $\mathrm{Ca}^{2+}$ load and $A P D_{90}$. This was indeed the case: BVR increased significantly, despite only moderate increases in APD90 (Figure 4). In this phase, $\beta A S$ also evoked DADs and EADs (Figure 5). BVR increased after the first generation of DADs (even before EADs occurred; Figure 5), which supports the concept that dynamic subsarcolemmal $\mathrm{Ca}^{2+}$ fluxes during cellular $\mathrm{Ca}^{2+}$ overload and diastolic spontaneous $\mathrm{Ca}^{2+}$ release from the sarcoplasmic reticulum influence the subsequent (paced) APs and BVR via mechanisms yet to be elucidated. Our finding that BVR and EAD generation by $I_{\mathrm{ks}}$ blockade and $\beta A S$ were most pronounced at fast pacing rates (as opposed to $I_{\mathrm{Kr}}$ blockade and late- $I_{\mathrm{Na}}$ augmentation; Figure 6) fits with this concept. Additional support comes from the result that intracellular $\mathrm{Ca}^{2+}$ chelation with BAPTA-AM reduces beat-to-beat repolarization instability (Figure 7). Moreover, in preliminary experiments in which we used ryanodine $(1 \mu \mathrm{mol} / \mathrm{L})$ during $I_{\mathrm{Ks}}$ blockade and $\beta A S$ (data not shown), blockade of sarcoplasmic-reticulum $\mathrm{Ca}^{2+}$ release reduced excessive BVR (while inhibiting 
the generation of afterdepolarizations), again supporting that $\mathrm{Ca}^{2+}$-dependent membrane currents contribute to BVR.

Our results on afterdepolarization occurrence are partially in agreement with data from the canine LV-wedge preparation. ${ }^{31}$ Burashnikov and Antzelevitch ${ }^{31}$ reported that under combined $\mathrm{I}_{\mathrm{Ks}}$ blockade and $\beta A S$, DADs were induced in LV epi-, mid- and endocardium. Transmural dispersion of repolarization was accentuated. However, no EAD activity was seen. This apparent discrepancy with our observations in isolated myocytes could be explained by the difference in cell-to-cell electrotonic coupling. The latter, which acts in the LV wedge preparation but not in the isolated cell, has been shown to reduce BVR. ${ }^{8}$

\section{The $\mathrm{Na}^{+}-\mathrm{Ca}^{2+}$ exchange inhibitor SEA0400 attenuates excessive BVR and EADs during $I_{\mathrm{Ks}}$ blockade plus $\beta A S$}

Various groups have used the $\mathrm{Na}^{+}-\mathrm{Ca}^{2+}$ exchange inhibitor SEA0400 in attempts to prevent TdP under conditions of $I_{\mathrm{Kr}}$ block $^{32,33}$ and $I_{\mathrm{Na}}$ augmentation ${ }^{32}$ with mixed results. Milberg et al. ${ }^{32}$ showed that the application of SEA0400 $(1 \mu \mathrm{mol} / \mathrm{L})$ in the presence of sotalol or veratridine resulted in a significant decrease of monophasic APD and an attenuation of EADs in paced Langendorff-perfused rabbit hearts with complete atrioventricular block, supporting the hypothesis that the $\mathrm{Na}^{+}-\mathrm{Ca}^{2+}$ exchanger is an important mediator of EADs. ${ }^{25}$ However in the study of Farkas et al. ${ }^{33}$ SEA0400 (1 $\mu \mathrm{mol} / \mathrm{L}$ ) did not suppress dofetilide-induced TdP nor decrease QT variability in unpaced Langendorffperfused rabbit hearts with complete atrioventricular block. The major difference between these two studies was that in the study of Milberg et al. ${ }^{32}$ hearts were paced at constant cycle lengths, whereas in the alternate study ${ }^{33}$ hearts were allowed to beat at their intrinsic ventricular rate. Upon administering SEA0400 in addition to dofetilide, the rate instability increased, and this additional factor could have increased the susceptibility to TdP, at variance with the results of Milberg et al. ${ }^{32}$

To the best of our knowledge this report is the first to describe the use of $\mathrm{Na}^{+}-\mathrm{Ca}^{2+}$ exchange inhibition in the presence of $I_{\mathrm{ks}}$ block combined with $\beta$ AS (as such mimicking long-QT syndrome type 1 (LQT1)). In line with Milberg et al., ${ }^{32}$ the application of SEA0400 resulted in a significant decrease of transmembrane APD and an attenuation of EADs (Figure 8). Moreover, SEA0400 rescued from excessive BVR, suggesting a repolarization-destabilizing role of inward $\mathrm{Na}^{+}-\mathrm{Ca}^{2+}$ exchange under conditions of $I_{\mathrm{ks}}$ blockade combined with $\beta A S^{24}$

\section{Clinical perspectives}

In LQT1 patients, sympathetic stimulation and epinephrine challenges prolong the QT interval, ${ }^{34}$ increase beat-to-beat QT variability ${ }^{35}$ and promote EAD generation. ${ }^{34}$ Our present cellular results may thus have important clinical implications, as they support the use of $\mathrm{Ca}^{2+}$ antagonists, ${ }^{36} \beta \mathrm{A}$ receptor blockers ${ }^{34,36}$ and even $I_{K, A T P}$ openers ${ }^{34}$ to restrain BVR and reduce the risk of TdP in these patients. Indeed, $\beta A$-receptor blockade, the cornerstone of pharmacological treatment in LQT1, 
decreases epinephrine-prolonged monophasic APD and transmural dispersion of repolarization in LQT1. ${ }^{34}$ Likewise, $\beta A$-receptor blockade can decrease transmural dispersion of repolarization in LQT1 patients, but causes it to increase in LQT2 patients. ${ }^{37}$ Whether the $\mathrm{Na}^{+} . \mathrm{Ca}^{2+}$ exchanger is a suitable target for antiarrhythmic treatment in LQT1 patients is currently under debate.

\section{Conclusions}

The repolarizing potassium current $\mathrm{I}_{\mathrm{Ks}}$ stabilizes BVR in single canine LV myocytes. During conditions of $I_{\mathrm{Kr}}$ blockade and late- $I_{\mathrm{Na}}$ augmentation, $\beta A S$ of $I_{\mathrm{Ks}}$ rescues from excessive BVR, AP prolongation and EAD generation. However, during $I_{\mathrm{Ks}}$ blockade $\beta A S$ provokes excessive BVR, while promoting the generation of DADs and EADs. The loss of $\mathrm{I}_{\mathrm{Ks}}$ plus overriding of $\mathrm{Ca}^{2+}$-dependent inward currents, including inward $\mathrm{Na}^{+}-\mathrm{Ca}^{2+}$ exchange current, likely conspire to cellular proarrhythmia under these conditions.

\section{ACKNOWLEDGEMENTS}

The authors wish to thank Dr. Heinz Gogelein, Sanofi-Aventis Germany GmbH, Frankfurt, Germany for supplying HMR1556, and Dr. Andras Varro, University of Szeged, Szeged, Hungary for supplying SEA0400. Technical support by Roel L.H.M.G. Spatjens, BSc, Cardiovascular Research Institute Maastricht, The Netherlands and Ann J. Woods, Safety Pharmacology, SAUK, Astrazeneca R\&D, UK is greatly appreciated.

\section{SOURCES OF FUNDING}

P.G.A.V. is supported by a Vidi grant from the Netherlands Organization for Scientific Research (ZonMw 91710365). D.M.J. was financially supported by AstraZeneca Ltd, UK.

\section{REFERENCES}

1. Thomsen MB, Verduyn SC, Stengl M, Beekman JD, de Pater G, van Opstal J, Volders PGA, Vos MA. Increased short-term variability of repolarization predicts $d$-sotalol-induced torsades de pointes in dogs. Circulation. 2004;110:2453-2459.

2. Schneider J, Hauser R, Andreas JO, Linz K, Jahnel U. Differential effects of human ether-a-gogo-related gene (HERG) blocking agents on QT duration variability in conscious dogs. Eur J Pharmacol. 2005;512:53-60.

3. Lengyel C, Varro A, Tábori K, Papp JG, Baczk I. Combined pharmacological block of $\mathrm{I}_{\mathrm{Kr}}$ and $\mathrm{I}_{\mathrm{Ks}}$ increases short-term QT interval variability and provokes torsades de pointes. $\mathrm{Br} \mathrm{J}$ Pharmacol. 2007;151:941-951.

4. Wu L, Shryock JC, Song Y, Belardinelli L. An increase in late sodium current potentiates the proarrhythmic activities of low-risk QT-prolonging drugs in female rabbit hearts. J Pharmacol Exp Ther. 2006;316:718-726. 
5. Hinterseer $M$, Thomsen MB, Beckmann BM, Pfeufer A, Schimpf R, Wichmann HE, Steinbeck G, Vos MA, Kaab S. Beat-to-beat variability of QT intervals is increased in patients with druginduced long-QT syndrome: a case control pilot study. Eur Heart J. 2008;29:185-190.

6. Gallacher DJ, Van de Water A, van der Linde H, Hermans AN, Lu HR, Towart R, Volders PGA. In vivo mechanisms precipitating torsades de pointes in a canine model of drug-induced long-QT1 syndrome. Cardiovasc Res. 2007;76:247-256.

7. Thomsen MB, Volders PG, Beekman JD, Matz J, Vos MA. Beat-to-Beat variability of repolarization determines proarrhythmic outcome in dogs susceptible to drug-induced torsades de pointes. J Am Coll Cardiol. 2006;48:1268-1276.

8. Zaniboni $M$, Pollard $A E$, Yang $L$, Spitzer $K W$. Beat-to-beat repolarization variability in ventricular myocytes and its suppression by electrical coupling. Am J Physiol Heart Circ Physiol. 2000;278:H677-687.

9. Zaniboni M, Cacciani F, Salvarani N. Temporal variability of repolarization in rat ventricular myocytes paced with time-varying frequencies. Exp Physiol. 2007;92:859-869.

10. Volders PGA, StengI M, van Opstal JM, Gerlach U, Spätjens RL, Beekman JD, Sipido KR, Vos MA. Probing the contribution of $I_{\mathrm{Ks}}$ to canine ventricular repolarization: key role for $\beta$ adrenergic receptor stimulation. Circulation. 2003;107:2753-2760.

11. Song Y, Shryock JC, Wu L, Belardinelli L. Antagonism by ranolazine of the pro-arrhythmic effects of increasing late $I_{\text {Na }}$ in guinea pig ventricular myocytes. J Cardiovasc Pharmacol. 2004;44:192-199.

12. Undrovinas Al, Belardinelli L, Undrovinas NA, Sabbah HN. Ranolazine improves abnormal repolarization and contraction in left ventricular myocytes of dogs with heart failure by inhibiting late sodium current. J Cardiovasc Electrophysiol. 2006;17 Suppl 1:S169-S177.

13. Varró A, Baláti B, lost N, Takács J, Virág L, Lathrop DA, Csaba L, Tálosi L, Papp JG. The role of the delayed rectifier component $I_{K s}$ in dog ventricular muscle and Purkinje fibre repolarization. J Physiol. 2000;523 Pt 1:67-81.

14. Jost N, Virág L, Bitay M, Takács J, Lengyel C, Biliczki P, Nagy Z, Bogáts G, Lathrop DA, Papp JG, Varró $A$. Restricting excessive cardiac action potential and QT prolongation: a vital role for $I_{\mathrm{Ks}}$ in human ventricular muscle. Circulation. 2005;112:1392-1399.

15. Carmeliet E. Repolarization Reserve in Cardiac Cells. J Med Biol Eng. 2006;26:97-105.

16. Roden DM. Taking the "idio" out of "idiosyncratic": predicting torsades de pointes. Pacing Clin Electrophysiol. 1998;21:1029-1034.

17. Volders PGA, Sipido KR, Carmeliet E, Spätjens RLHMG, Wellens HJ, Vos MA. Repolarizing $\mathrm{K}^{+}$ currents $\mathrm{I}_{\mathrm{TO} 1}$ and $\mathrm{I}_{\mathrm{Ks}}$ are larger in right than left canine ventricular midmyocardium. Circulation. 1999;99:206-210.

18. Gögelein $\mathrm{H}$, Bruggemann A, Gerlach $U$, Brendel J, Busch AE. Inhibition of $\mathrm{I}_{\mathrm{KS}}$ channels by HMR 1556. Naunyn Schmiedebergs Arch Pharmacol. 2000;362:480-488.

19. Thomas GP, Gerlach U, Antzelevitch C. HMR 1556, a potent and selective blocker of slowly activating delayed rectifier potassium current. J Cardiovasc Pharmacol. 2003;41:140-147.

20. Birinyi P, Acsai K, Banyasz T, Toth A, Horvath B, Virag L, Szentandrassy N, Magyar J, Varro A, Fulop F, Nanasi PP. Effects of SEA0400 and KB-R7943 on $\mathrm{Na}^{+} / \mathrm{Ca}^{2+}$ exchange current and Ltype $\mathrm{Ca}^{2+}$ current in canine ventricular cardiomyocytes. Naunyn Schmiedebergs Arch Pharmacol. 2005;372:63-70.

21. Birinyi P, Toth A, Jona I, Acsai K, Almassy J, Nagy N, Prorok J, Gherasim I, Papp Z, Hertelendi Z, Szentandrassy N, Banyasz T, Fulop F, Papp JG, Varro A, Nanasi PP, Magyar J. The $\mathrm{Na}^{+} / \mathrm{Ca}^{2+}$ exchange blocker SEA0400 fails to enhance cytosolic $\mathrm{Ca}^{2+}$ transient and contractility in canine ventricular cardiomyocytes. Cardiovasc Res. 2008;78:476-484.

22. Volders PG, Sipido KR, Vos MA, Kulcsar A, Verduyn SC, Wellens HJ. Cellular basis of biventricular hypertrophy and arrhythmogenesis in dogs with chronic complete atrioventricular block and acquired torsade de pointes. Circulation. 1998;98:1136-1147. 
23. Stengl M, Volders PGA, Thomsen MB, Spätjens RLHMG, Sipido KR, Vos MA. Accumulation of slowly activating delayed rectifier potassium current $\mathrm{I}_{\mathrm{Ks}}$ in canine ventricular myocytes. $J$ Physiol. 2003;551:777-786.

24. Volders PGA, Kulcsár A, Vos MA, Sipido KR, Wellens HJ, Lazzara R, Szabo B. Similarities between early and delayed afterdepolarizations induced by isoproterenol in canine ventricular myocytes. Cardiovasc Res. 1997;34:348-359.

25. Volders PG, Vos MA, Szabo B, Sipido KR, de Groot SH, Gorgels AP, Wellens HJ, Lazzara R. Progress in the understanding of cardiac early afterdepolarizations and torsades de pointes: time to revise current concepts. Cardiovasc Res. 2000;46:376--392.

26. Linz KW, Meyer R. Control of L-type calcium current during the action potential of guinea-pig ventricular myocytes. J Physiol. 1998;513 ( Pt 2):425-442.

27. Tang $Q$, Jin MW, Xiang JZ, Dong MQ, Sun HY, Lau CP, Li GR. The membrane permeable calcium chelator BAPTA-AM directly blocks human ether a-go-go-related gene potassium channels stably expressed in HEK 293 cells. Biochem Pharmacol. 2007;74:1596-1607.

28. Wan X, Laurita KR, Pruvot EJ, Rosenbaum DS. Molecular correlates of repolarization alternans in cardiac myocytes. J Mol Cell Cardiol. 2005;39:419-428.

29. Kass RS. Genetically induced reduction in small currents has major impact. Circulation. 1997;96:1720-1721.

30. Tanskanen AJ, Greenstein JL, O'Rourke B, Winslow RL. The role of stochastic and modal gating of cardiac L-type Ca2+ channels on early after-depolarizations. Biophys J. 2005;88:8595.

31. Burashnikov A, Antzelevitch C. Block of $I_{\mathrm{Ks}}$ does not induce early afterdepolarization activity but promotes $\beta$-adrenergic agonist-induced delayed afterdepolarization activity. J Cardiovasc Electrophysiol. 2000;11:458-465.

32. Milberg P, Pott C, Fink M, Frommeyer G, Matsuda T, Baba A, Osada N, Breithardt G, Noble D, Eckardt L. Inhibition of the $\mathrm{Na}^{+} / \mathrm{Ca}^{2+}$ exchanger suppresses torsades de pointes in an intact heart model of long QT syndrome-2 and long QT syndrome-3. Heart Rhythm. 2008;5:14441452.

33. Farkas AS, Makra P, Csik N, Orosz S, Shattock MJ, Fulop F, Forster T, Csanady M, Papp JG, Varro A, Farkas A. The role of the $\mathrm{Na}^{+} / \mathrm{Ca}^{2+}$ exchanger, $\mathrm{I}_{\mathrm{Na}}$ and $\mathrm{I}_{\mathrm{CaL}}$ in the genesis of dofetilideinduced torsades de pointes in isolated, AV-blocked rabbit hearts. $\mathrm{Br} J$ Pharmacol. 2009;156:920-932.

34. Shimizu W, Kurita T, Matsuo K, Suyama K, Aihara N, Kamakura S, Towbin JA, Shimomura K. Improvement of repolarization abnormalities by a $\mathrm{K}^{+}$channel opener in the LQT1 form of congenital long-QT syndrome. Circulation. 1998;97:1581-1588.

35. Satomi K, Shimizu W, Takaki H, Suyama K, Kurita T, Aihara N, Kamakura S. Response of beatby-beat QT variability to sympathetic stimulation in the LQT1 form of congenital long QT syndrome. Heart Rhythm. 2005;2:149-154.

36. Shimizu W, Ohe T, Kurita T, Kawade M, Arakaki Y, Aihara N, Kamakura S, Kamiya T, Shimomura K. Effects of verapamil and propranolol on early afterdepolarizations and ventricular arrhythmias induced by epinephrine in congenital long QT syndrome. J Am Coll Cardiol. 1995;26:1299-1309.

37. Khositseth A, Nemec J, Hejlik J, Shen WK, Ackerman MJ. Effect of phenylephrine provocation on dispersion of repolarization in congenital long QT syndrome. Ann Noninvasive Electrocardiol. 2003;8:208-214. 


\section{ChAPTER 6}

\section{Diastolic Spontaneous Calcium Release from the SARCOPLASMic Reticulum InCREASES BEAT-TO-BEAT REPOLARIZATION VARIABILITY IN CALCIUM-OVERLOADED CANINE VENTRICULAR MYOCYTES}

JoRd Heijman*, Daniel M. Johnson*, Elizabeth F. Bode, David J. Greensmith, NAJAH Abi-Gerges, DaVid A. EISNeR, ANDREW W. TRAFFord AND PAUL G.A. VOLDERS

* THeSe AUthors CONTRIBUted EQUAlly to this WORK

MANUSCRIPT IN PREPARATION AND IN PART PRESENTED AT MEETINGS OF THE HEART RHythm SOCIETY 2011 and the CARDiac Electrophysiology SOCIETY 2011. 


\section{ABSTRACT}

Spontaneous $\mathrm{Ca}^{2+}$ release from the sarcoplasmic reticulum (SCR) can cause delayed afterdepolarizations (DADs) and triggered activity, contributing to arrhythmogenesis during $\mathrm{Ca}^{2+}$ overload. Excessive beat-to-beat variability of repolarization duration (BVR) is a proarrhythmic marker. Previous research has shown that BVR is increased during $\mathrm{Ca}^{2+}$ overload in isolated myocytes. We aimed to determine ionic mechanisms controlling BVR during abnormal $\mathrm{Ca}^{2+}$ handling. Membrane potentials and cell shortening or $\mathrm{Ca}^{2+}$ transients were recorded from isolated canine leftventricular myocytes in the presence of the $\beta$-adrenergic agonist isoproterenol to induce $\mathrm{Ca}^{2+}$ overload. Action-potential (AP) durations following DADs were significantly prolonged. Addition of $\mathrm{I}_{\mathrm{Ks}}$ blockade led to further AP prolongation after SCR and this strongly correlated with exaggerated BVR. Suppressing SCR via inhibition of ryanodine receptors, CaMKII inhibition, or by using $\mathrm{Mg}^{2+}$ or flecainide eliminated DADs and decreased BVR, which was independent of effects on AP duration. Caffeine led to a similar decrease in BVR, due to the occurrence of SCR before every AP. Computational analyses and voltage-clamp experiments measuring $I_{\mathrm{CaL}}$ with and without prior SCR demonstrated that $\mathrm{I}_{\mathrm{CaL}}$ was increased during $\mathrm{Ca}^{2+}$-induced $\mathrm{Ca}^{2+}$ release after $\mathrm{SCR}$, contributing to AP prolongation. SCR promotes BVR by interspersed prolongation of myocyte AP duration, which is exacerbated during $\mathrm{I}_{\mathrm{Ks}}$ blockade. Attenuation of $\mathrm{Ca}^{2+}$-induced $\mathrm{Ca}^{2+}$ release by SCR underlies AP prolongation via increased $I_{\text {CaL. }}$. These data provide insight into arrhythmogenic mechanisms during $\mathrm{Ca}^{2+}$ overload besides triggered activity, as well as illustrating the importance of $\mathrm{I}_{\mathrm{Ks}}$ function in preventing excessive BVR. 


\section{INTRODUCTION}

Enhanced cellular $\mathrm{Ca}^{2+}$ load, for example during $\beta$-adrenergic receptor ( $\left.\beta A R\right)$ stimulation, results in augmentation of $\mathrm{Ca}^{2+}$ release from the sarcoplasmic reticulum (SR), larger $\mathrm{Ca}^{2+}$ transients (CaT), and enhanced contractile force. ${ }^{1}$ Under certain conditions $\mathrm{Ca}^{2+}$ load is increased beyond a certain threshold, leading to spontaneous $\mathrm{Ca}^{2+}$ release (SCR) from the SR during diastole. ${ }^{2}$ In turn, this diastolic leak causes a transient inward current $\left(I_{\mathrm{t}}\right)$, causing delayed afterdepolarizations (DADs), mainly due to activation of the electrogenic $\mathrm{Na}^{+}-\mathrm{Ca}^{2+}$ exchanger (NCX), with the $\mathrm{Ca}^{2+}$-activated $\mathrm{Cl}^{-}$ current $\left(\mathrm{I}_{\mathrm{CI} C \mathrm{C}}\right.$ ) contributing in some species. ${ }^{3,4}$ Previous work has also shown that both DADs and early afterdepolarizations (EADs) may share a common mechanism, at least during $\beta A R$ stimulation and $\mathrm{Ca}^{2+}$ overload, namely SCR-induced currents. ${ }^{5}$ Both types of afterdepolarizations have been incriminated in the formation of ventricular tachycardia (VT), via triggered activity (TA) and/or by increasing dispersion of repolarization. ${ }^{6}$

Beat-to-beat variability of repolarization duration (BVR) occurs as an apparently random alteration in repolarization duration, and can be observed at all levels of cardiac repolarization, from the action potential (AP) of the single cardiac myocyte to the QT interval on the body surface..$^{7-9}$ Exaggerated BVR has been reported to be a more reliable indicator of arrhythmogenic risk than repolarization prolongation per se, at least in several experimental VT model ${ }^{10-12}$ and in selected human subjects, for example in patients with acquired or inherited long QT syndrome, or nonischemic heart failure. ${ }^{8}$ 13

Mechanisms underlying BVR at the single-cell level remain poorly understood. Pharmacological interventions influencing ion channels that operate during the AP plateau can markedly alter BVR. ${ }^{\text {, }}$ 14 Recently, we have shown that during $\mathrm{Ca}^{2+}$ overload in myocytes subjected to blockade of the slowly-activating delayed-rectifier $\mathrm{K}^{+}$current $\left(\mathrm{I}_{\mathrm{Ks}}\right)$ in combination with $\beta A R$ stimulation, $\mathrm{BVR}$ is significantly enhanced, even before the occurrence of EADs and TA. ${ }^{14}$

In the present study, we investigated the relationship between SCR and BVR using a combined experimental and computational approach in canine ventricular myocytes subject to $\beta A R$ stimulation. We show that SCRs not only lead to $I_{t i}$ and DAD formation, but also to a prolonged duration of the following AP via increased L-type $\mathrm{Ca}^{2+}$ current $\left(\mathrm{I}_{\mathrm{CaL}}\right)$, which in turn leads to increased BVR when analyzing multiple consecutive APs. Pharmacological interventions that inhibit SCR (either with reduced or preserved systolic contraction) prevent this SCR-associated AP prolongation and reduce BVR.

\section{MetHODS}

This investigation conformed with the Guide for the Care and Use of Laboratory Animals published by the US National Institutes of Health (NIH Publication No. 85-23, revised 1996). Animal handling 
was in accordance with the European Directive for the Protection of Vertebrate Animals Used for Experimental and Other Scientific Purposes (86/609/EU).

\section{Cell-isolation procedure}

Adult female beagle dogs were used for the myocyte isolations. Anesthesia was induced with 45 $\mathrm{mg} / \mathrm{kg}$ pentobarbital. Once full anesthesia was reached, the chest was opened via a left thoracotomy and the heart was excised and placed in an $\mathrm{O}_{2}$-gassed $\mathrm{Ca}^{2+}$-free standard buffer solution at approximately $4{ }^{\circ} \mathrm{C}$. The cell-isolation procedure was the same as previously described. ${ }^{15}$ Briefly the left anterior descending coronary artery was cannulated and perfused. After $\sim 20 \mathrm{~min}$ of collagenase perfusion and subsequent washout of the enzyme, the epicardial surface layer was removed from the LV wedge until a depth of $\geq 3 \mathrm{~mm}$ was reached. Softened tissue samples were collected from the midmyocardial layer underneath while contamination with the endocardium was avoided. Samples were gently agitated, filtered and washed. LV midmyocytes were stored at room temperature in standard buffer solution (vide infra) and only quiescent rod-shaped cells with clear cross-striations were used for the experiments. Cells were used within $48 \mathrm{~h}$ of isolation.

\section{Sharp-electrode action potential recordings}

Transmembrane action potentials (APs) were recorded at $37{ }^{\circ} \mathrm{C}$ using high-resistance (30-60 M $\Omega$ ) glass microelectrodes filled with $3 \mathrm{M} \mathrm{KCl}$ with a microelectrode amplifier (Axoclamp-2B, Axon Instruments, Inc). Intracellular pacing was done at various cycle lengths (CLs; $500 \mathrm{~ms}-2000 \mathrm{~ms}$ ). Only cells showing a stable spike-and-dome AP morphology and resting membrane potential were accepted for the experiments. Myocyte contractions were recorded with a video edge motion detector (Crescent Electronics, Sandy, UT, USA).

\section{$\mathrm{Ca}^{2+}$ measurements}

Isolated myocytes were loaded with the acetoxymethyl ester of indicator Fluo-3 or Fura-2 (Molecular Probes; $5 \mu \mathrm{mol} / \mathrm{L}, 5 \mathrm{~min}$ loading and $30 \mathrm{~min}$ de-esterification). Electrophysiological control for current clamp and voltage clamp experiments with simultaneous $\left[\mathrm{Ca}^{2+}\right]_{i}$ measurements was achieved using the perforated patch clamp technique with amphotericin-B $(240 \mu \mathrm{g} / \mathrm{ml})$. The switch-clamp facility (frequency $1-3 \mathrm{kHz}$ and gain $1-3$ ) of the Axoclamp-2B voltage clamp amplifier (Axon Instruments, CA, USA) was used to overcome the access resistance of the perforated patch. All experiments were performed at $37^{\circ} \mathrm{C}$.

\section{Drugs and experimental solutions}

The standard buffer solution used for the experiments was composed of (in mmol/L): $\mathrm{NaCl} 145, \mathrm{KCl}$ 4.0, $\mathrm{CaCl}_{2}$ 1.8, $\mathrm{MgCl}_{2}$ 1.0, glucose 11 and HEPES 10, pH 7.4 with $\mathrm{NaOH}$ at $37^{\circ} \mathrm{C}$. In a subset of experiments, extracellular $\left[\mathrm{Mg}^{2+}\right]$ was increased to $5 \mathrm{mmol} / \mathrm{L}$ by the addition of $4 \mathrm{mmol} / \mathrm{L} \mathrm{MgSO}_{4}$. In other experiments, extracellular $\left[\mathrm{Ca}^{2+}\right]$ was increased to $3.6 \mathrm{mmol} / \mathrm{L}$ to further increase cellular $\mathrm{Ca}^{2+}$ loading. 
$500 \mathrm{nmol} / \mathrm{L}$ HMR1556 ((3R,4S)-(+)-N-[3-hydroxy-2,2-dimethyl-6-(4,4,4-trifluorobutoxy)chroman-4yl]-N-methylmethanesulfonamide; a gift from Dr. H. Gögelein, Sanofi-Aventis Germany GmbH, Frankfurt, Germany) was used to selectively and completely block $\mathrm{I}_{\mathrm{Ks}}{ }^{16-18}$ Sarcoplasmic reticulum (SR) $\mathrm{Ca}^{2+}$ release through the ryanodine receptor (RyR) was inhibited with $1 \mu \mathrm{mol} / \mathrm{L}$ ryanodine (RBI, Natick, MA, USA) or stabilized using $5 \mu \mathrm{mol} / \mathrm{L}$ tetracaine (Sigma-Aldrich Zwijndrecht, The Netherlands). Caffeine (Sigma-Aldrich Zwijndrecht, The Netherlands; maximally $500 \mu \mathrm{mol} / \mathrm{L}$ ) was used to increase RyR open probability. $\mathrm{Ca}^{2+} /$ Calmodulin-dependent kinase (CaMKII) activity was modulated using the CaMKII inhibitor KN93 (Sigma-Aldrich Zwijndrecht, The Netherlands; $5 \mu \mathrm{mol} / \mathrm{L}$ ) and the calmodulin inhibitor W7 (Tocris Bioscience, Bristol, UK; $1 \mu \mathrm{mol} / \mathrm{L}$ ). The class-1C antiarrhythmic agent flecainide (MedaPharma, Amstelveen, The Netherlands) was employed in a subset of experiments. At $6 \mu \mathrm{mol} / \mathrm{L}$, flecainide results in a $40 \%$ decrease in RyR activity ${ }^{19}$ as well as affecting multiple other targets (40\% inhibition of $\mathrm{I}_{\mathrm{To}}{ }^{20}$, significant inhibition of (late) $\mathrm{I}_{\mathrm{Na}}{ }^{21}$ and $~ 50 \%$ inhibition of HERG $\mathrm{K}^{+}$channels ${ }^{22}$ ).

For perforated patch experiments the patch pipette solution contained (in mmol/L): $\mathrm{KCH}_{3} \mathrm{O}_{3} \mathrm{~S} 125$, $\mathrm{KCl} 20, \mathrm{NaCl} 10, \mathrm{HEPES} 10, \mathrm{MgCl}_{2} 5, \mathrm{~K}_{2}$ EGTA 0.1 , titrated to 7.2 with $\mathrm{KOH}$ with patch pipette resistance being 2-3 $\mathrm{M} \Omega$. In voltage clamp experiments, $\mathrm{K}^{+}$currents were blocked by addition of 500 $\mathrm{nmol} / \mathrm{L} \mathrm{HMR} 1556,5 \mathrm{mmol} / \mathrm{L}$ 4-aminopyridine and $100 \mathrm{nmol} / \mathrm{L} \mathrm{BaCl}_{2}$ to the standard buffer solution. Extracellular $\mathrm{Ca}^{2+}$ was raised (to 3.6 or $5.0 \mathrm{mmol} / \mathrm{L}$ ) to further enhance cellular $\mathrm{Ca}^{2+}$ loading.

HMR1556, ryanodine, tetracaine, caffeine, W7 and KN93 were initially dissolved in dimethyl sulfoxide (DMSO) and then diluted so that the concentration of the solvent was maximally $0.1 \%$ in the superfusate, a concentration that has no measurable effects on AP or ionic currents.

BAS was applied as $100 \mathrm{nmol} / \mathrm{L}$ isoproterenol in all experiments. This agent was originally dissolved in distilled water containing $30 \mu \mathrm{mol} / \mathrm{L}$ ascorbic acid and then stored in the dark at $4{ }^{\circ} \mathrm{C}$ until use.

\section{Computational Analysis}

A recent model of the canine ventricular myocyte electrophysiology including $\beta A R$ stimulation ${ }^{23}$ (Chapter 3) was extended with a method to induce diastolic SR Ca ${ }^{2+}$ release in a controlled fashion, similar to a recent approach by Xie et al. ${ }^{24}$ Both timing (start, duration) and amplitude of diastolic SR $\mathrm{Ca}^{2+}$ release could be controlled. 


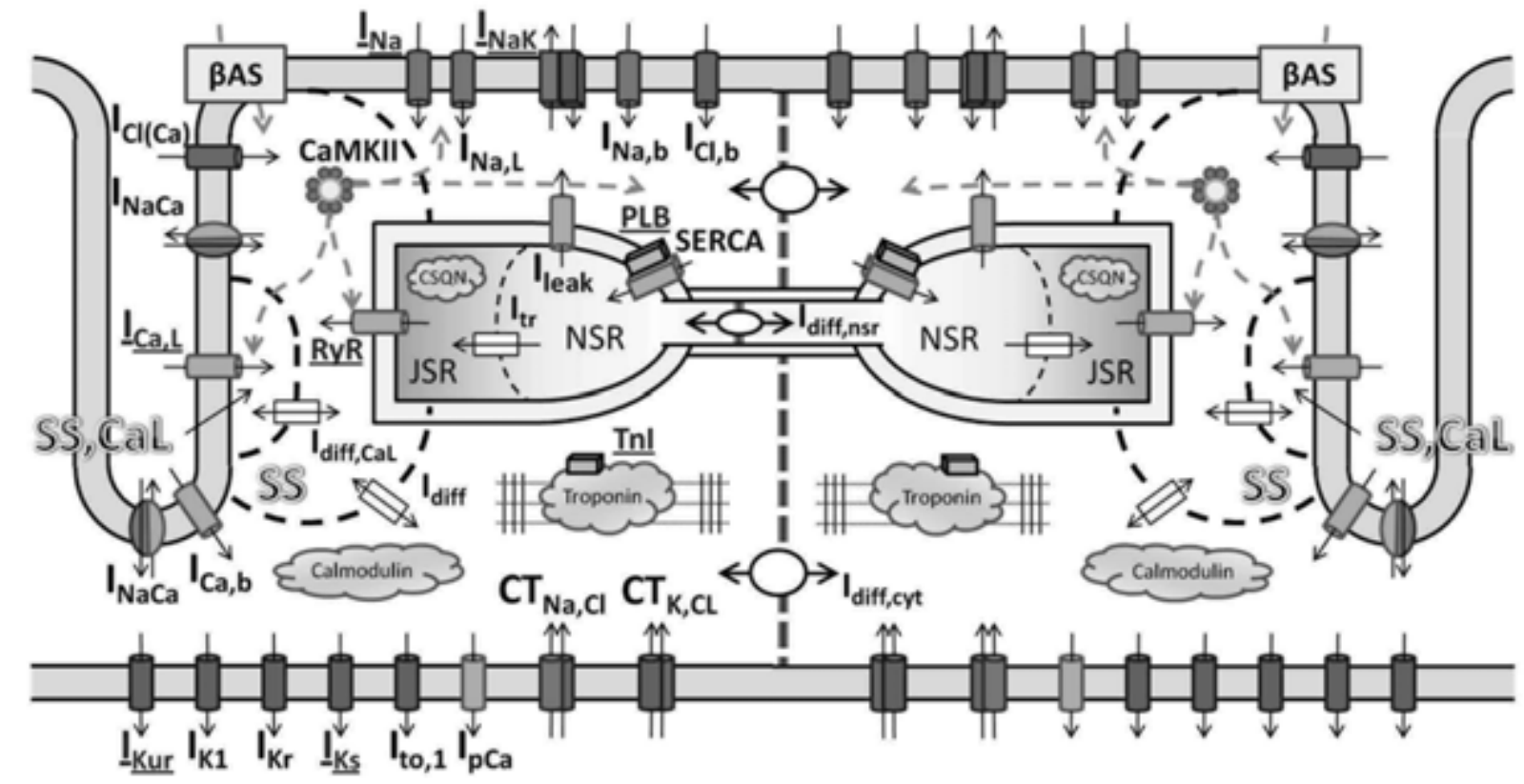

Figure 1. Schematic overview of the computational model of the canine ventricular myocyte. Adapted from Heijman et al. ${ }^{23}$ (Chapter 3). The model was divided into two identical domains to simulate the local origin of SCR. Only components on the left side of the model are labeled for clarity, identical components are located on the right side. The domains are coupled via diffusion of $\mathrm{Ca}^{2+}\left(I_{\text {diff,cyt }}\right.$ and $\left.I_{\text {diff,nst }}\right)$. All other abbreviations are as previously described. ${ }^{23}$ For color figure, see page 253.

In particular, the model was divided into two identical domains (Figure 1) coupled by $\mathrm{Ca}^{2+}$ diffusion between cytosol and network SR to simulate the local origin of SCR. Diffusion time constants were based on the local control model by Restrepo et al..$^{25}$ and diffusion was significantly slower in SR compared to cytosol, consistent with experimental observations ${ }^{26}$. The late component of $I_{\mathrm{Na}}\left(I_{\mathrm{NaL}}\right)$ was increased in the model to simulate the midmyocardial origin of the myocytes used in our experiments, consistent with experimental data from Zygmunt et al. ${ }^{27}$ Model APD rate dependence at baseline, in the presence of ISO and in the presence of ISO+HMR1556 (simulated as 100\% inhibition of $I_{K s}$ ) was consistent with experimental data (Figure 2A). Parameters of the L-type $\mathrm{Ca}^{2+}$ current were adjusted based on the experiments in the presence of ryanodine to obtain quantitative agreement on the amount of APD prolongation in the absence of $\mathrm{SR} \mathrm{Ca}^{2+}$ release. 

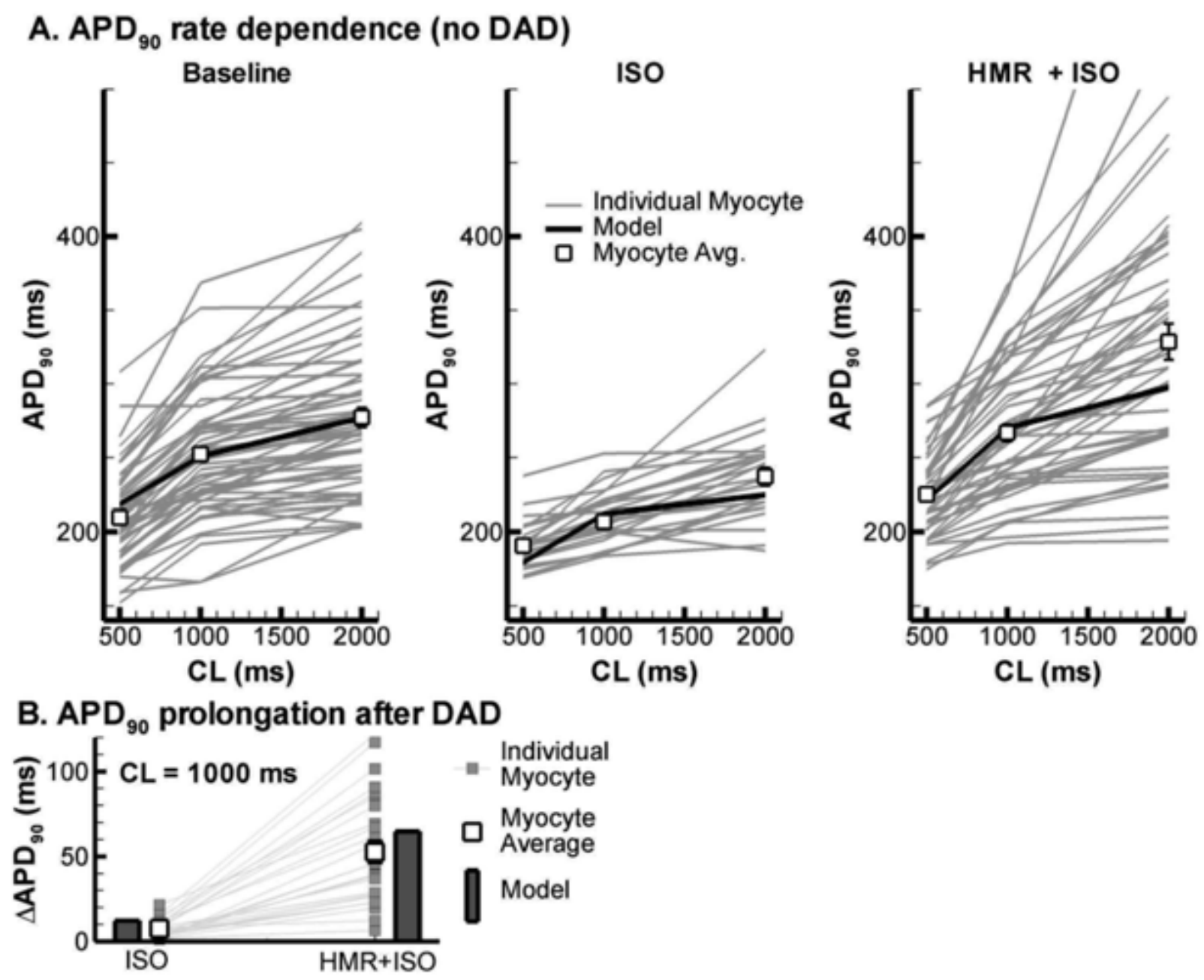

Figure 2. Validation of model properties. A. Steady-state APD-rate dependence under baseline conditions (left panel), in the presence of ISO (middle panel) or in the presence of ISO + HMR1556 (right panel) for APs without prior DAD. Average APD for each cell is shown in blue. Average APD over all myocytes is indicated with symbols. Model APD is shown in black. Model APD-rate dependence falls within experimental range and is close to the experimental average for all conditions and CLs. B. Average APD difference between APs preceded by a DAD and those without prior DAD in the presence of ISO or ISO + HMR1556. Average APD difference for each cell is shown in grey. Average group data is indicated with symbols. Model APD prolongation after SCR is indicated with grey bars. Model APD prolongation is consistent with experimental observations.

To initiate SCR, steady-state RyR activation was set to a constant value in one of the two domains for the interval of diastolic SR $\mathrm{Ca}^{2+}$ release such that the desired reduction in local JSR $\mathrm{Ca}^{2+}$ was achieved. When steady-state RyR activation was set to $0.375\left(\mathrm{SCR}_{\text {level }}=0.375\right)$ from $\mathrm{t}=925 \mathrm{~ms}$ until $\mathrm{t}=950$ $\mathrm{ms}$ (at $\mathrm{CL}=1000 \mathrm{~ms}$ ), the SCR-induced prolongation of APD was consistent with experimental observations (Figure 2B). 
The model was paced to steady state (2000 seconds of pacing) in the presence of $\beta A R$ stimulation with or without $I_{\mathrm{ks}}$ inhibition, to mimic experimental conditions. The effect of diastolic SR $\mathrm{Ca}^{2+}$ release was examined by determining APD of a single beat at steady-state as a function of the timing and amplitude of the preceding diastolic $\mathrm{Ca}^{2+}$ release. In some simulations other currents / fluxes $\left(I_{\mathrm{CaL}}, I_{\mathrm{NaCa}}, I_{\mathrm{Cl}(\mathrm{Ca})}, \mathrm{J}_{\mathrm{rel}}\right)$ were blocked for the duration of this final beat.

\section{Data Analysis and Statistical Comparisons}

APD was quantified at $90 \%$ repolarization. BVR was quantified as variability of APD using the formula $\Sigma\left(\left|A P D_{i+1}-A P D_{i}\right|\right) /\left[n_{\text {beats }} \times V 2\right]$ for 30 consecutive APs in the absence of TA. ${ }^{10}$ Detection of EADs, DADs and determination of APD, BVR and DAD parameters was done in a semi-automated fashion using a custom Matlab (The Mathworks, Natick, MA, USA) script. Briefly, Axon (Axon Instruments, CA, USA) data files were loaded into Matlab. AP upstroke was detected based on peaks in the numerically calculated $\mathrm{dV} / \mathrm{dt}$, and $A \mathrm{AP}_{90}$ was determined. Subsequently, the diastolic interval between $A P D_{90}$ of one beat and the point of maximum $\mathrm{dV} / \mathrm{dt}$ of the next beat was processed for all beats. The signal was low-pass filtered at $30 \mathrm{~Hz}$ to reduce noise. All peaks in the filtered signal were determined and resting membrane potential was defined as the average value between the smallest (most negative) local maximum and local minimum. The amplitude of all other peaks was determined relative to this resting membrane potential. DADs were identified as a local maximum in the filtered signal of more than $2 \mathrm{mV}$ in amplitude and manually confirmed by the investigators. EADs were detected as positive local maxima in the low-pass filtered $\mathrm{dV} / \mathrm{dt}$ signal preceded and followed by negative local minima. Detection of EADs was manually validated.

Where applicable, data were reported as mean \pm standard error of the mean (SEM) of $n$ experiments, and significance tested with either a t-test or 1 way ANOVA. Differences were considered statistically significant if $P<0.05$. 


\section{RESULTS}

\section{DADs Prolong Subsequent APs and Increase BVR}
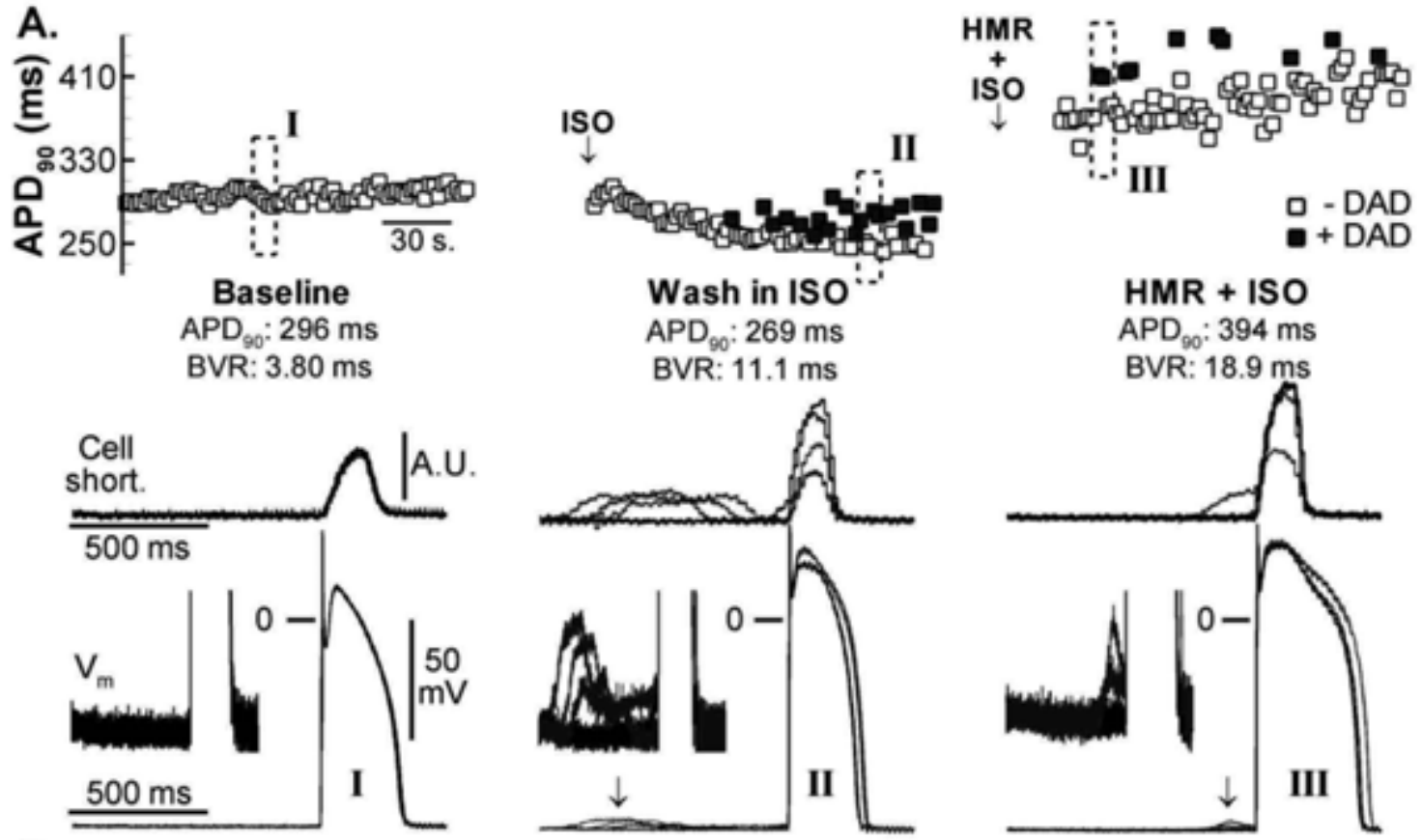

B.

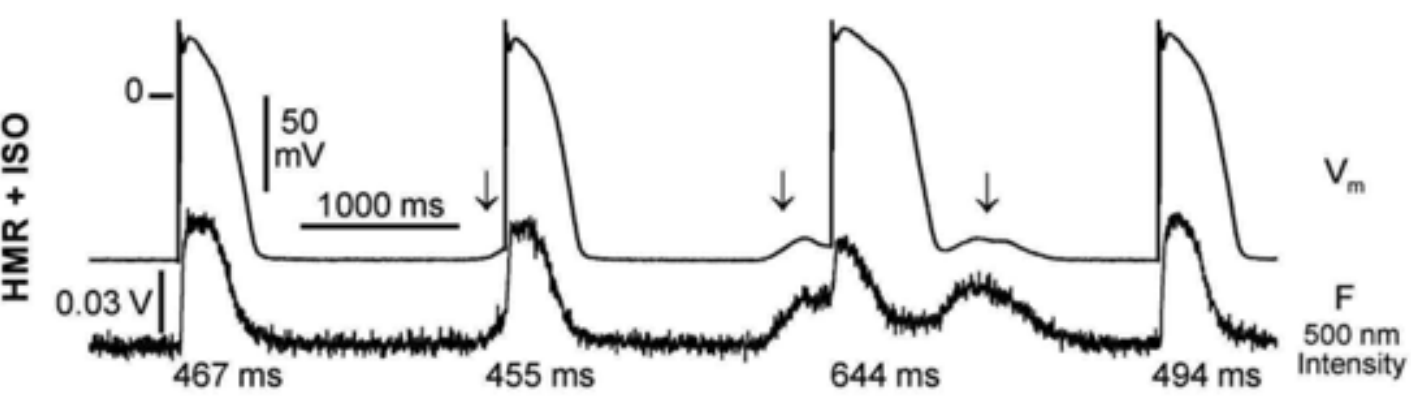

Figure 3. Occurrence of DADs in canine ventricular myocytes is associated with prolongation of subsequent APs and increased BVR. A. APD ${ }_{90}$ for 75 consecutive beats at 2000-ms CL at baseline (left panel: I), during wash-in of ISO (middle panel: II) and in the presence of HMR1556 and ISO (right panel: III). APDs preceded by a DAD are indicated with filled symbols, APDs without DADs depicted by open symbols. Dashed rectangle indicates a set of 5 beats for which cell shortening and membrane potential are depicted below. Insets show diastolic potentials at an expanded scale. Beats without prior SCR are indicated in black, beats with prior SCR in red. B. Membrane potential (top) and $\left[\mathrm{Ca}^{2+}\right]_{i}$ for four consecutive beats. $\mathrm{APD}_{90} \mathrm{~S}$ are indicated below each AP. SCR is indicated with arrows. For color figure, see page 253.

Under baseline conditions, a small beat-to-beat APD variability was observed (Figure 3A, left panel). BAR stimulation with isoproterenol (ISO) shortened APD and enhanced contraction (Figure 3A, middle panel). In the majority of cells, $100 \mathrm{nmol} / \mathrm{L}$ ISO caused DADs during pacing at $1000 \mathrm{~ms} \mathrm{CL}$. APs following a DAD were prolonged, leading to increased BVR due to interspersed occurrence of normal and prolonged APs (Figure 3A, middle panel). Pharmacological inhibition of $I_{\mathrm{Ks}}$ by HMR1556 
(500 nmol/L) in the presence of ISO further prolonged APD and increased BVR (Figure 3A, right panel). In separate experiments, $\mathrm{Ca}^{2+}$ transients were recorded together with APs after HMR1556 and ISO. APs following SCR were prolonged and had a reduced systolic $\mathrm{Ca}^{2+}$-transient amplitude compared to those where no diastolic release preceded the $\mathrm{AP}$, indicating reduced $\mathrm{SR} \mathrm{Ca}{ }^{2+}$ load at the start of the AP (Figure $3 B$ ), consistent with the reduced cell shortening following an aftercontraction (Figure 3A).
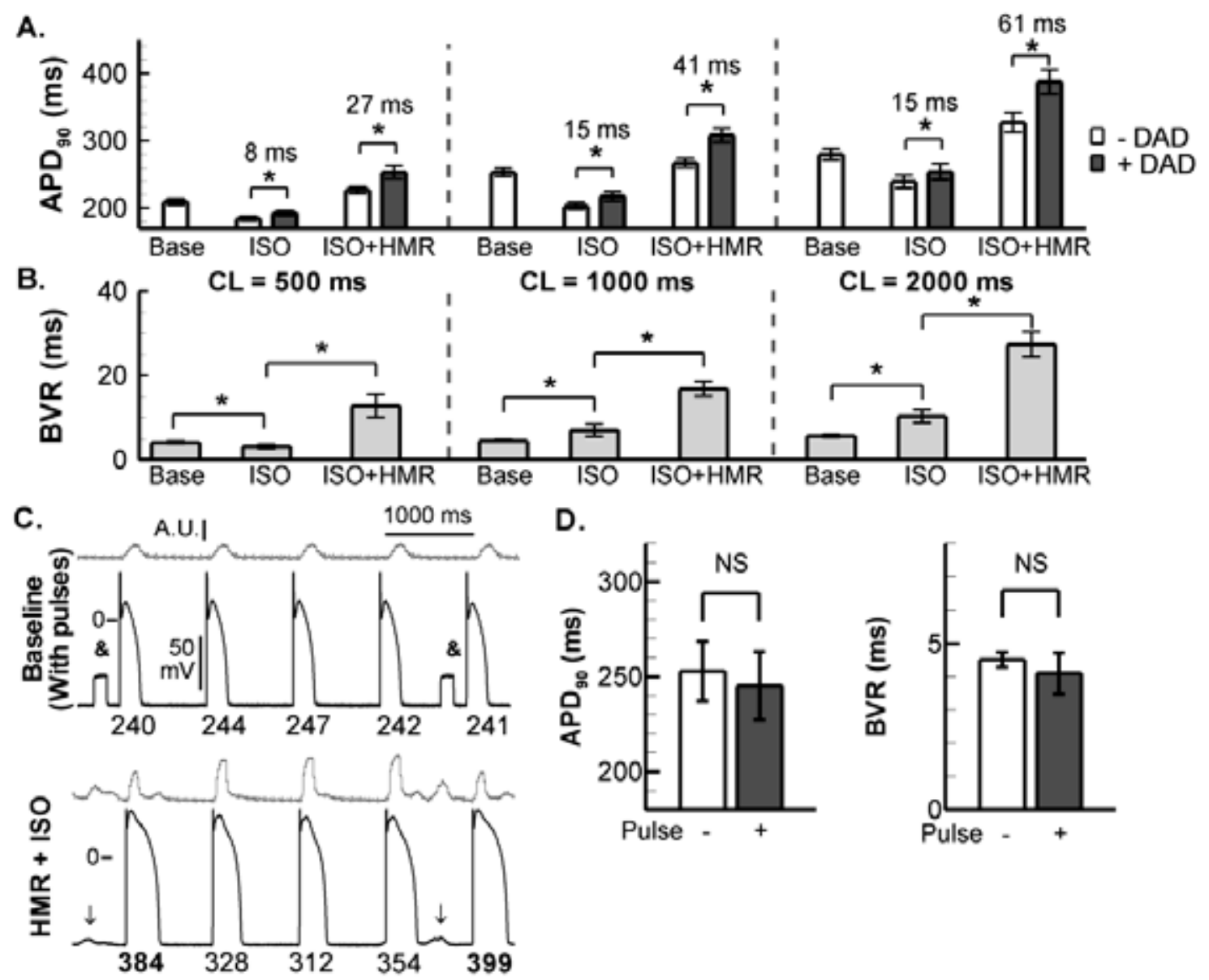

D.
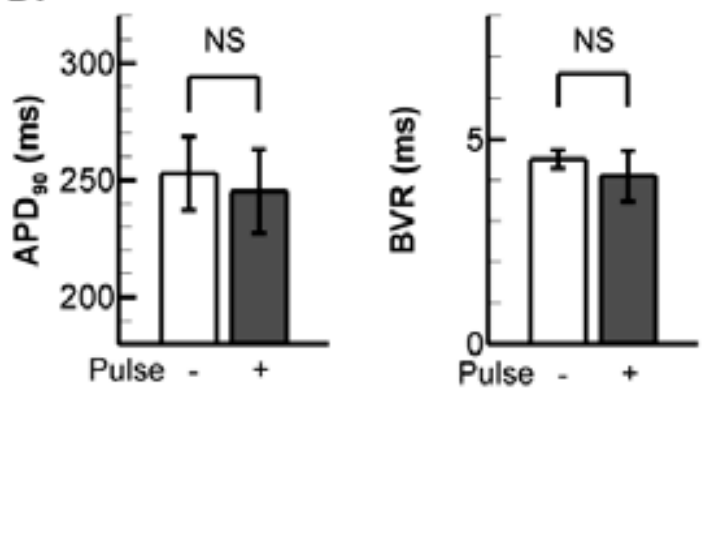

Figure 4. A. Group data for $A P D_{90}$ under baseline conditions, in the presence of ISO, or in the presence of ISO + HMR1556 at CLs of 500, 1000 and 2000 ms. DADs cause a significant prolongation during ISO and ISO + HMR1556 treatment. APD ${ }_{90}$ differences are indicated above each pair of bars. *; $P<0.05$ B. BVR values for the conditions in panel A. C. Cell shortening (top trace) and membrane potential (bottom trace) under baseline conditions with DAD-like electrical pulses (in the absence of SCR) applied every $4^{\text {th }}$ beat (top panel) or with DADs after ISO + HMR1556 in the same cell (bottom panel). $A P D_{90}$ is indicated below each beat. D. Subthreshold diastolic pulses do not alter $A P D_{90}$ (left panel) nor BVR (right panel).

A significant APD prolongation after the occurrence of DADs was observed at all CLs in the presence of ISO and ISO + HMR1556 (Figure 4A). APD prolongation was significantly more pronounced in the presence of ISO + HMR1556 compared to ISO alone at all CLs (e.g., $41 \pm 6 \mathrm{~ms}$ vs. $15 \pm 4 \mathrm{~ms}$ at $\mathrm{CL}=1000$ 
ms, $P<0.05$, Figure 4A). Moreover, a larger APD difference between beats with and without prior DAD was strongly associated with a larger BVR (Figure 4B). As such, HMR1556 was added in all subsequent experiments in the presence of ISO to maximize effects on APD, in effect creating a pharmacological cellular model of drug-induced long-QT1 (LQT1) syndrome, as previously described. ${ }^{14}$

To determine whether DAD-like membrane-potential changes alone (i.e., without underlying alterations in intracellular $\mathrm{Ca}^{2+}$ ), influenced the subsequent AP, small diastolic voltages $(25 \mathrm{mV}$ amplitude, 150 ms duration) were injected under baseline conditions (Figure $4 \mathrm{C}$ top panel). There was no significant difference between APDs in the absence or presence of these diastolic pulses (Figure 4D), independent of the timing of the pulse (not shown). In contrast, when these cells were stimulated with HMR1556 + ISO, DADs occurred and subsequent APD was significantly prolonged (Figure $4 \mathrm{C}$ bottom panel), indicating that alterations in the subsequent APD were influenced by DAD-related events other than membrane-potential alterations per se.

\section{Coupling Between the Occurrence of DADs and EADs During $\mathrm{Ca}^{2+}$ Overload}

A.

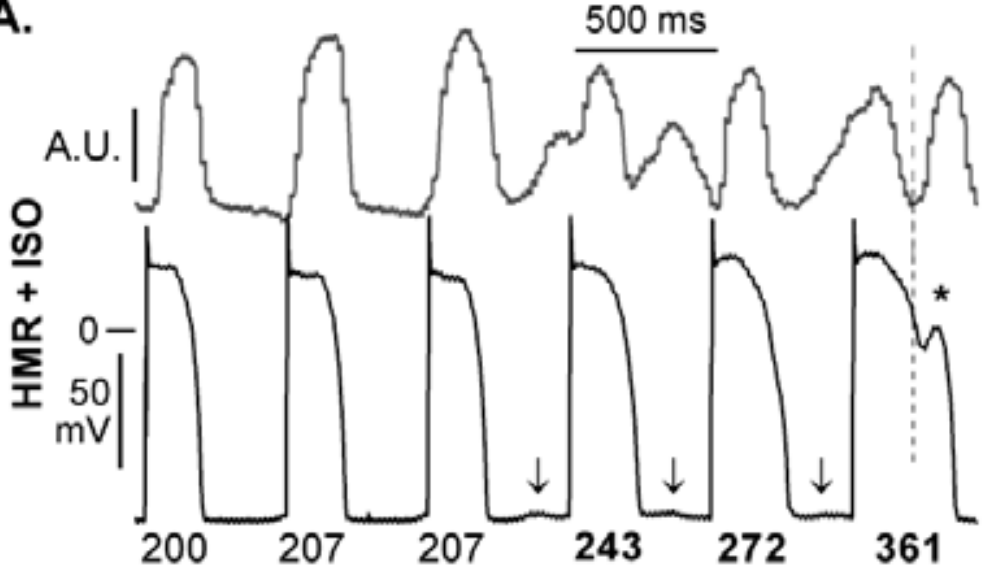

AP \# (relative to EAD)
B.

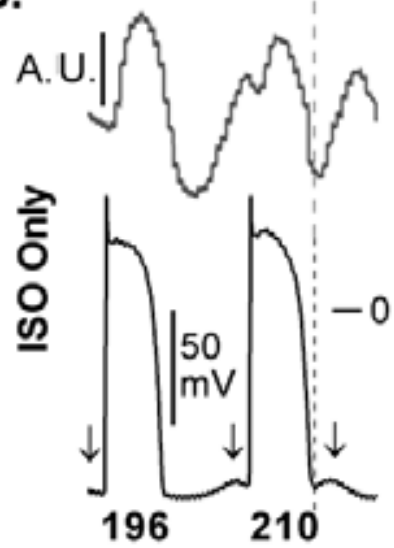

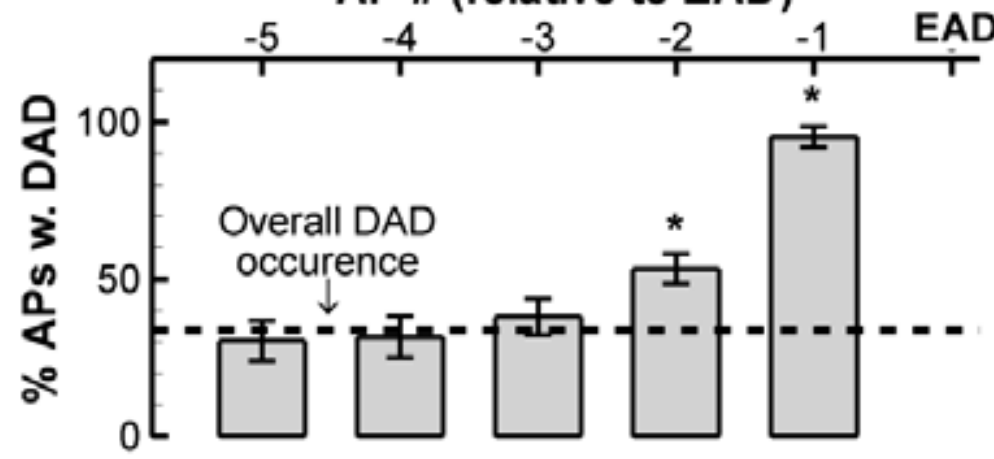

Figure 5. Relationship between diastolic SCR and EADs during $\mathrm{Ca}^{2+}$ overload. A.Top panel: Cell shortening (top trace) and membrane potential (bottom trace) for 5 consecutive APs followed by an AP with EAD (indicated with ${ }^{*}$ ) in a representative cell at 500-ms CL in the presence of HMR1556 + ISO. $A P D_{90}$ is given below each beat and DADs are indicated with arrows. Vertical line indicates 
timing of start of aftercontraction. Bottom panel indicates the percentages of APs that showed a DAD in the 5 beats preceding every EAD. Horizontal dashed line indicates overall DAD occurrence at 500-ms CL in the presence of HMR1556 + ISO, independent of EADs. The beat before an EAD has a DAD $95 \%$ of the time. ${ }^{*}: P<0.05$ compared to overall DAD occurrence. B. Similar to panel A (top) for 2 beats in the presence of ISO only. A similar contraction pattern can be observed but APD prolongation is insufficient to promote EAD formation.

In addition to DADs, EADs were observed during $\mathrm{Ca}^{2+}$ overload. EADs occurred predominantly at 500 ms $\mathrm{CL}$ and exclusively in the presence of HMR1556 + ISO. Consistent with previous findings, we observed early aftercontractions preceding EAD upstrokes (Figure 5A), suggesting a primary mechanistic role of SCR for EAD formation under these specific conditions. ${ }^{5}$ We quantified the occurrence of DADs in the beats preceding an EAD and found that DADs were significantly more likely to occur in the 2 beats directly preceding an EAD ( $53 \%$ and $95 \%$ of APs) compared to the average DAD occurrence for all beats (34\%; Figure $5 \mathrm{~A}$ bottom panel). When APD was not sufficiently prolonged, as was often the case under conditions of ISO alone, a short-coupled DAD could be observed, but no EAD (Figure 5B).

\section{Blockade of (Spontaneous) SR Ca ${ }^{2+}$ Release Reduces BVR}

A.

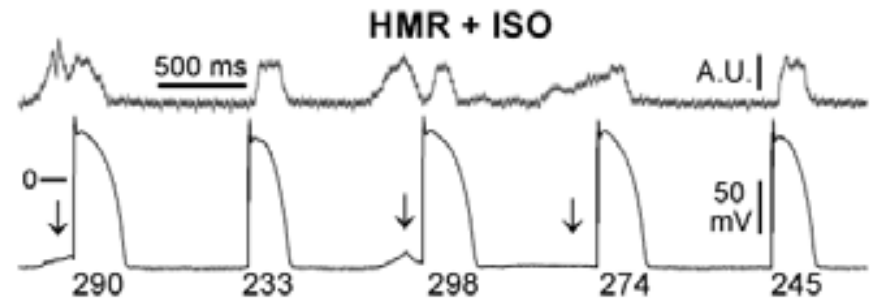

B.
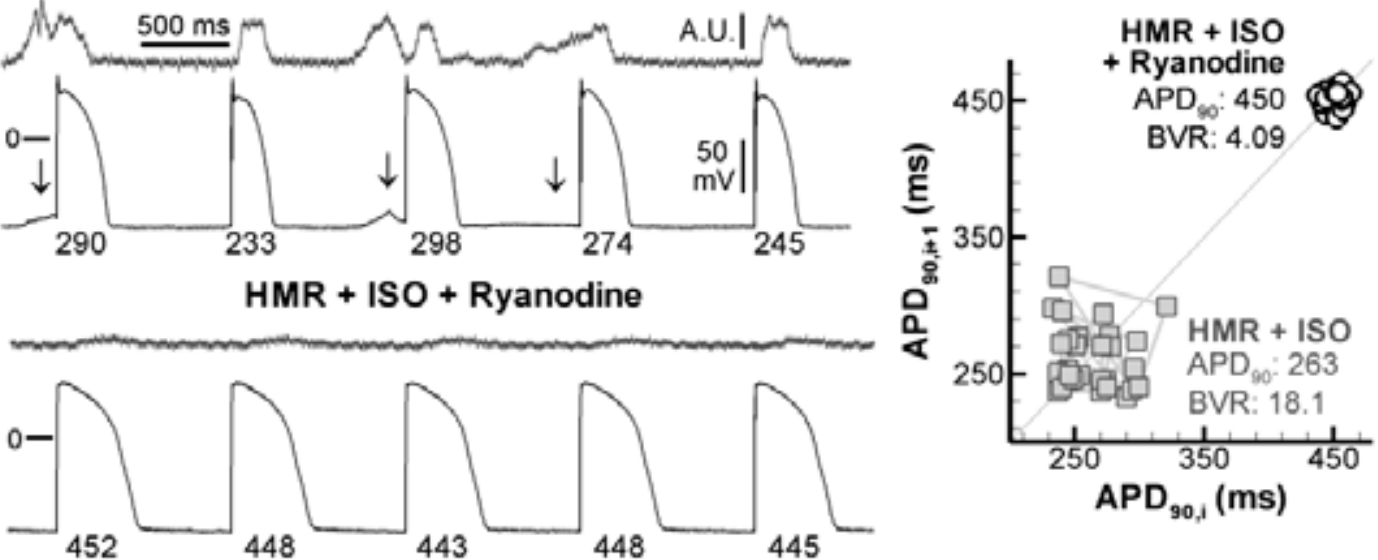

C.

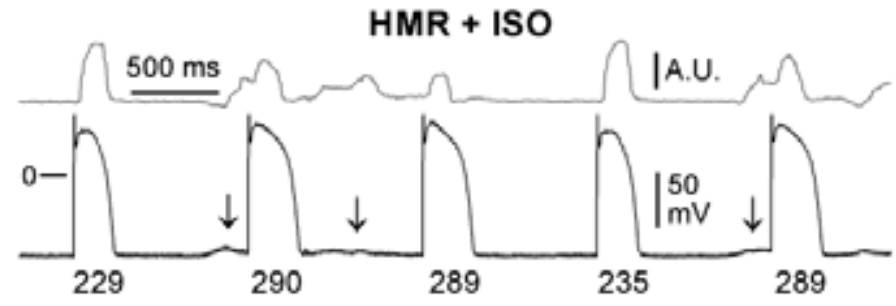

D.

HMR + ISO + Tetracaine
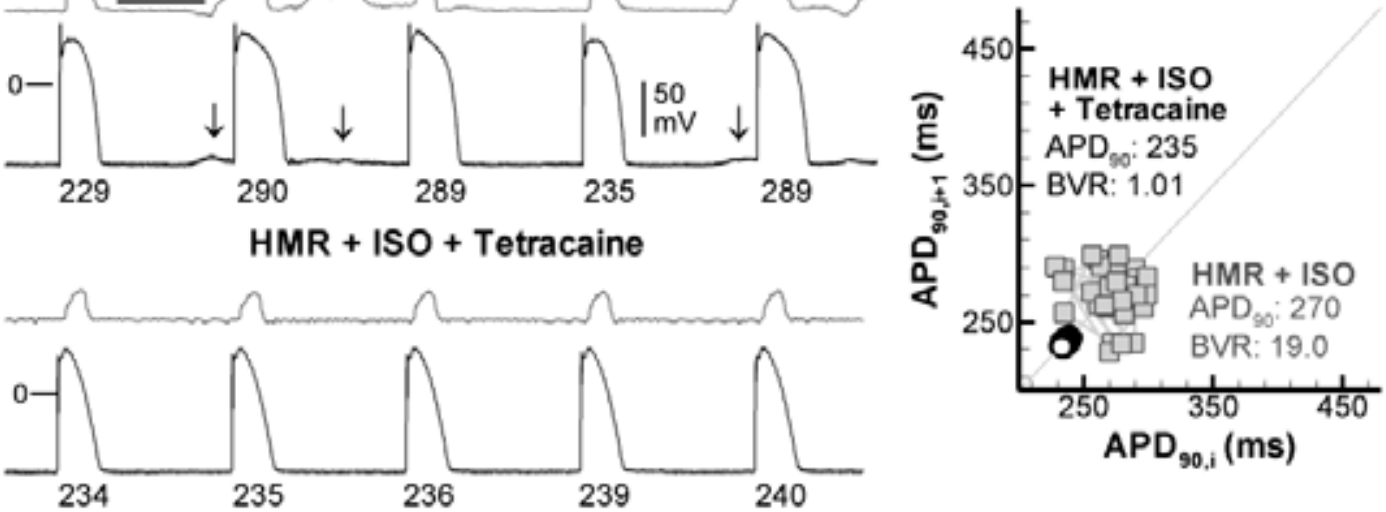
Figure 6. Inhibition of (diastolic) SCR abolishes DADs and reduces BVR. Cell shortening (top panels; arbitrary units) and membrane potential (bottom panels) for 5 representative beats during treatment with ISO + HMR1556 (A), or ISO + HMR1556 + ryanodine $(1 \mu \mathrm{mol} / \mathrm{L})$ (B). APD ${ }_{90}$ is indicated below each beat and mean $A P D_{90}$ and $B V R$ are shown for 30 beats in the Poincaré plot on the right. DADs are indicated with arrows. Panels $C$ and $D$ show similar data for treatment with tetracaine (5 $\mu \mathrm{mol} / \mathrm{L})$. ISO + HMR1556 significantly enhances BVR and DAD occurrence. Ryanodine and tetracaine abolish DADs and reduce BVR.

$\mathrm{Ca}^{2+}$ buffering with BAPTA-AM has previously been show to eliminate DADs and BVR during conditions of $\mathrm{Ca}^{2+}$ overload. ${ }^{14}$ We sought to further investigate how eliminating SCR, as opposed to buffering all cellular $\mathrm{Ca}^{2+}$, would affect APD and BVR. Blockade of the ryanodine receptor (RyR) with ryanodine eliminated DADs and led to a drastic reduction in BVR, despite leading to an increased APD (Figure 6A,B). Results were similar at all tested CLs. APD prolongation under these conditions was not associated with any arrhythmogenic events. A drastic reduction in systolic contraction was also seen, as expected, indicating that blockade of RyRs was achieved effectively.

Tetracaine reduces the open probability $\left(P_{0}\right)$ of RyR leading to a decrease in the frequency of SCR in unstimulated rat ventricular myocytes ${ }^{28}$ and an abolishment of ISO-induced SCR in stimulated voltage-clamped rat ventricular myocytes ${ }^{29}$. Similar to ryanodine, tetracaine led to an elimination of DADs in our experiments, and a reduction in BVR at all CLs. However, unlike ryanodine, cell shortening remained present, indicating that systolic $\mathrm{Ca}^{2+}$ release was still intact (Figure 6C,D). 


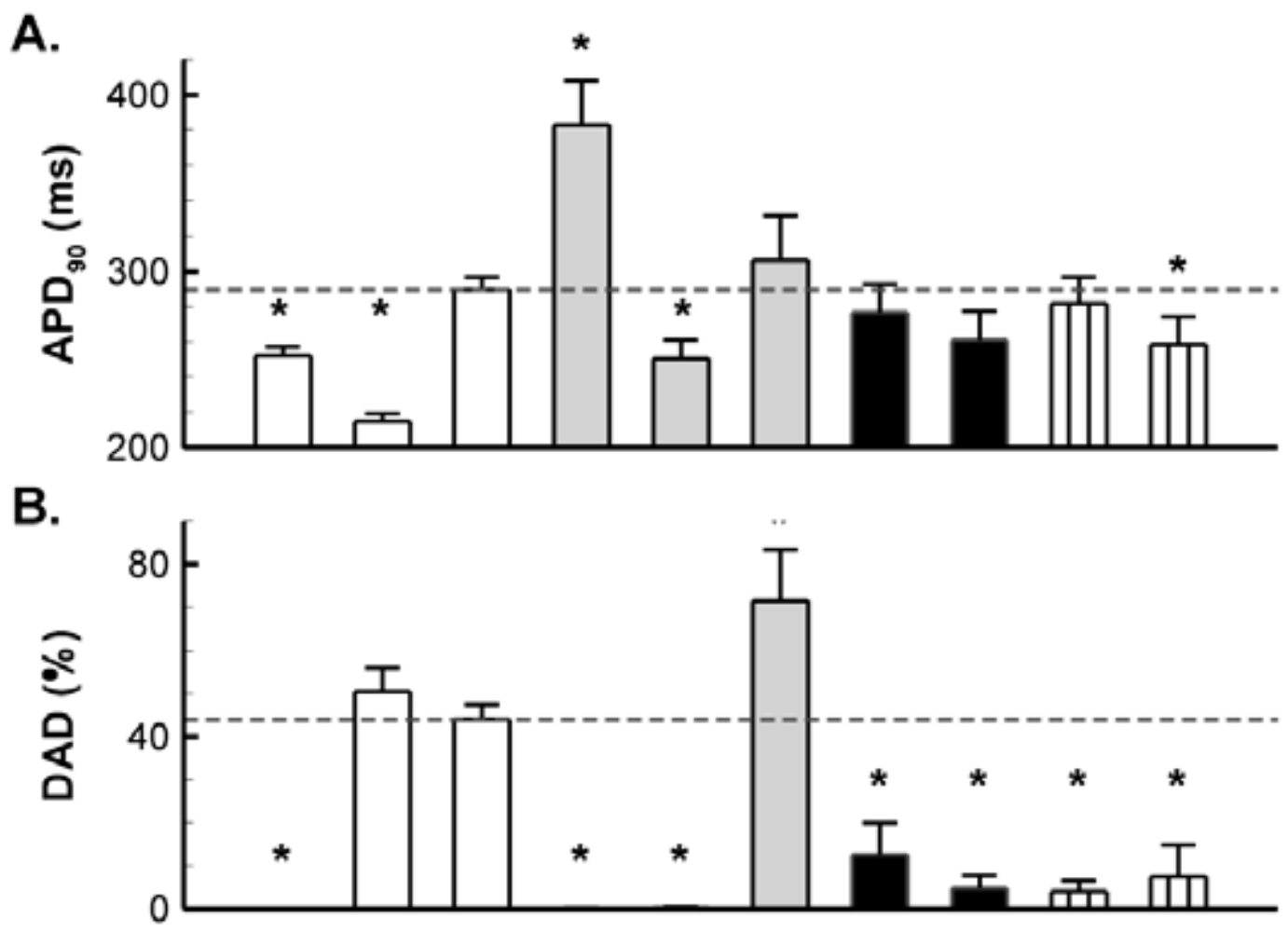

C.

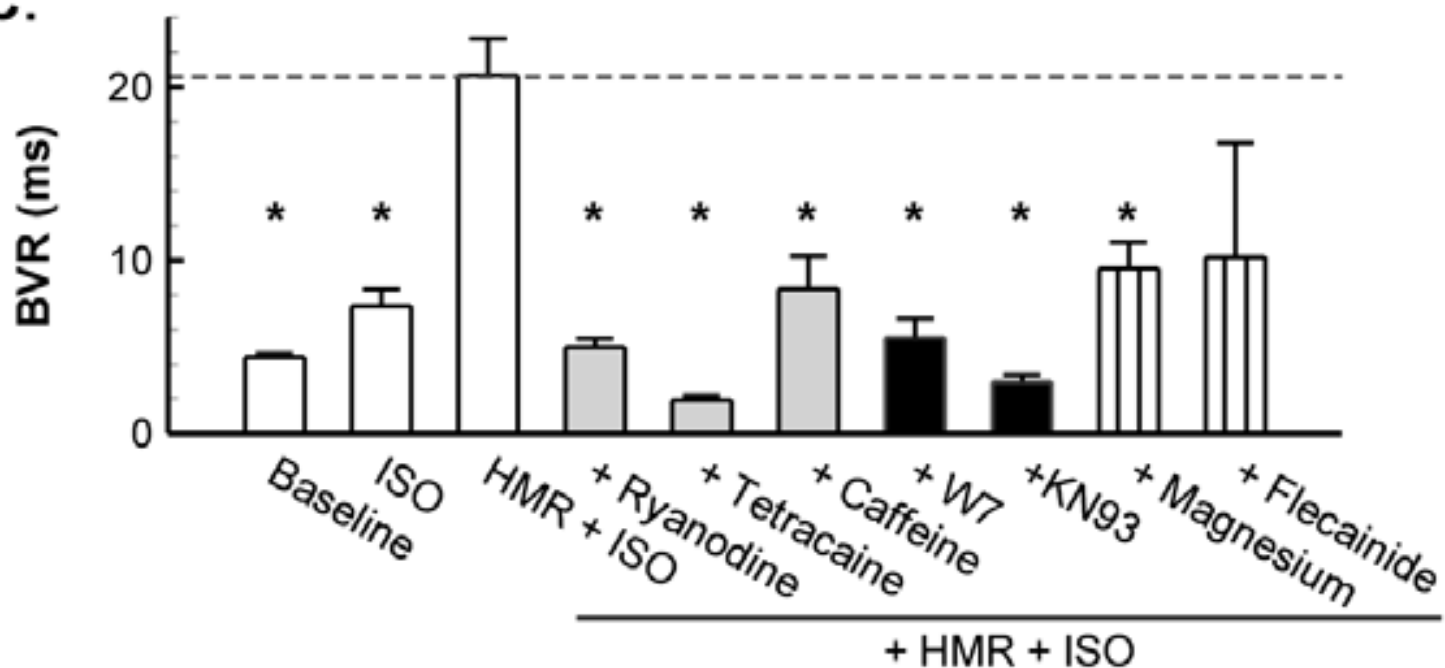

Figure 7. Interventions that abolish or homogenize SCR lower BVR. A. APD $D_{90}$. B. DAD occurrence. C. BVR at $\mathrm{CL}=1000 \mathrm{~ms}$ at baseline, in the presence of ISO, HMR1556 + ISO, or HMR1556 + ISO combined with interventions to modulate SCR. Interventions are grouped by type (RyR modulation, grey; Calmodulin/CaMKII modulation, black; or agents also used clinically; striped). All interventions except caffeine result in a significant lowering of DAD incidence and a concomitant reduction of BVR, with increased (ryanodine), decreased (tetracaine, flecainide $(6 \mu \mathrm{mol} / \mathrm{L}))$, or unaltered (W7 (1 $\left.\mu \mathrm{mol} / \mathrm{L}), \mathrm{KN} 93(5 \mu \mathrm{mol} / \mathrm{L}),\left[\mathrm{Mg}^{+}\right](5 \mathrm{mmol} / \mathrm{L})\right) \mathrm{APD}_{90}$. Caffeine (maximally $500 \mu \mathrm{mol} / \mathrm{L}$ ) regularizes SCR occurrence, increasing DAD occurrence but lowering BVR without changing $\mathrm{APD}_{90} .{ }^{*} ; P<0.05$ versus HMR1556 + ISO. 
To determine the effects of increasing the $P_{\circ}$ of RyRs, as opposed to decreasing it, we used low concentrations of caffeine (maximally $500 \mu \mathrm{mol} / \mathrm{L}$ ). ${ }^{30}$ Under these conditions the percentage of APs showing DADs was increased at all CLs. Interestingly, this led to a significant decrease in BVR compared to HMR1556 and ISO alone, since DADs occurred before every AP (Figure 7, right-most grey bars).

Several studies have shown that $\mathrm{Ca}^{2+} / \mathrm{CaMKII}$ is an important signaling molecule affecting $\mathrm{Ca}^{2+}$ induced $\mathrm{Ca}^{2+}$ release $(\mathrm{CICR})$ and that its inhibition can be antiarrhythmic. ${ }^{31}$ Curran et al. ${ }^{32}$ demonstrated that SR $\mathrm{Ca}^{2+}$ leak during $\beta A R$ stimulation is mediated by CaMKII. In our experiments, inhibition of calmodulin using W7 reduced DAD incidence in all cells (from $44 \%$ to $8 \%$ at $1000 \mathrm{~ms} \mathrm{CL}$, $P<0.05$ ) and completely abolished them in $3 / 7$ cells. The decrease in DAD occurrence was paralleled by a decrease in BVR. Average APD was unaltered by W7, and systolic contractions remained intact. Application of the CaMKII inhibitor KN93 led to similar results (Figure 7, black bars).

$\mathrm{Mg}^{2+}$ has been shown suppress both EADs and DADs in different models ${ }^{33,34}$ and is used clinically as a first-line agent against torsades de pointes. Increasing extracellular $\left[\mathrm{Mg}^{2+}\right]$ from $1 \mathrm{mmol} / \mathrm{l}$ to 5 $\mathrm{mmol} / \mathrm{I}$ abolished DADs induced after HMR1556 and ISO in 4/7 cells. In the remaining 3 cells, DAD incidence was significantly reduced. A concomitant reduction in BVR was seen. APDs were not significantly altered, and although systolic contractions were slightly reduced compared to beats that did not show SCR events before $\mathrm{Mg}^{2+}$, they remained stable, indicating that the SR content remained constant (not shown). Similar results (albeit with reduced APD) were obtained with the class-IC antiarrhythmic agent flecainide (Figure 7, striped bars), which has recently been shown to be effective in preventing catecholaminergic polymorphic VT due to its effects on RyR and $\mathrm{Na}^{+}$ channels. $^{35}$ 
Increases in $\left[\mathrm{Ca}^{2+}\right]_{0}$ Can Reinstate SCR Only When (Residual) RyR Function Is Available

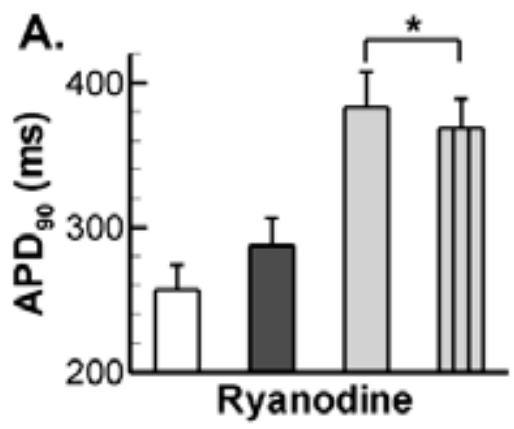

B.
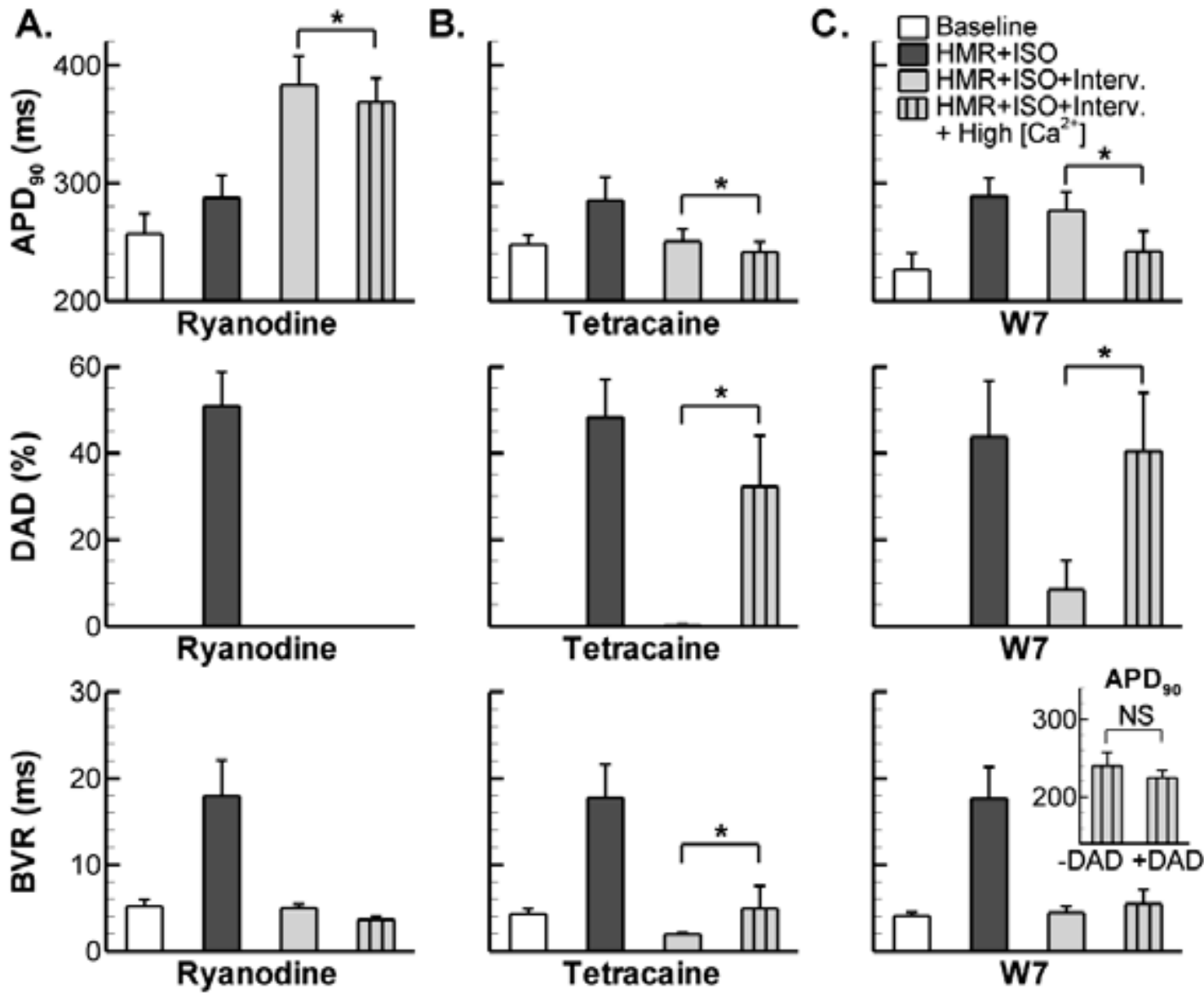

Figure 8. Effect of increased $\left[\mathrm{Ca}^{2+}\right]_{0}$ on $\mathrm{APD}_{90}$ (top panels), DAD occurence (middle panels), and BVR (bottom panels) under baseline conditions, HMR1556 + ISO, HMR1556 + ISO + Intervention or HMR1556 + ISO + Intervention + $3.6 \mathrm{mmol} / \mathrm{L}\left[\mathrm{Ca}^{2+}\right]_{0}$. A. Ryanodine. B. Tetracaine. C. W7. * indicates $P<0.05$. Only significance of intervention versus intervention in the presence of high $\left[\mathrm{Ca}^{2+}\right]_{0}$ is indicated for clarity reasons. Inset in lower-right panel shows $A P D_{90}$ in the presence of HMR1556 + ISO $+\mathrm{W} 7+$ increased $\left[\mathrm{Ca}^{2+}\right]_{0}$ for beats in the absence or presence of a preceding DAD.

We hypothesized that increases in $\left[\mathrm{Ca}^{2+}\right]_{0}$ after interventions that reduced DAD occurrence would lead to a recurrence of SCR, DADs and increase BVR. Therefore, we raised the $\left[\mathrm{Ca}^{2+}\right]_{0}$ from 1.8 $\mathrm{mmol} / \mathrm{L}$ to $3.6 \mathrm{mmol} / \mathrm{L}$ after application of ryanodine, tetracaine, or $\mathrm{W} 7$. This led to a reinduction of DADs after tetracaine or W7, and a significant decrease of APD (Figure 8A-C). However, raising $\left[\mathrm{Ca}^{2+}\right]_{0}$ after ryanodine did not lead to DADs, nor did it alter BVR, indicating that RyR function is required for SCR and without this, BVR cannot be altered under conditions of $\mathrm{Ca}^{2+}$ overload. Interestingly, BVR was not significantly reincreased after W7 and high $\left[\mathrm{Ca}^{2+}\right]_{0}$, whereas after tetracaine and high $\left[\mathrm{Ca}^{2+}\right]_{0}$ it was, despite DADs being reinstated under both conditions (Figure 8B,C). In the presence of W7, APs following SCR were no longer prolonged (Figure $8 \mathrm{C}$, inset). These data 
suggest that calmodulin inhibition has a direct effect on the coupling between SCR and APD, possibly via $I_{\text {Cal }}$.

\section{Reduced $\mathrm{Ca}^{2+}$-Dependent Inactivation of $\mathrm{I}_{\mathrm{CaL}}$ Underlies APD Prolongation} After SCR

A.
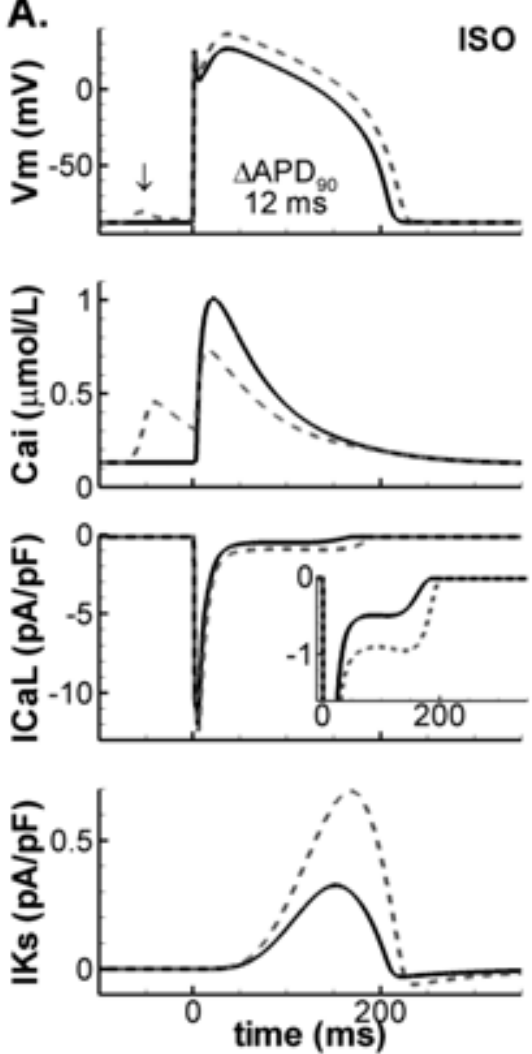

C.

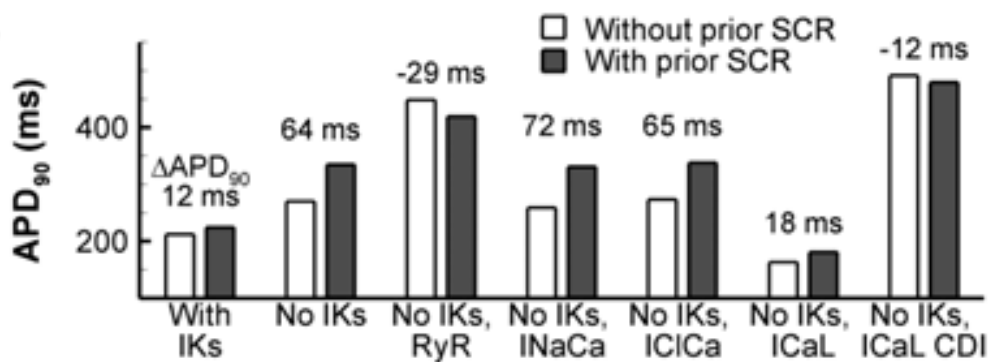

B.
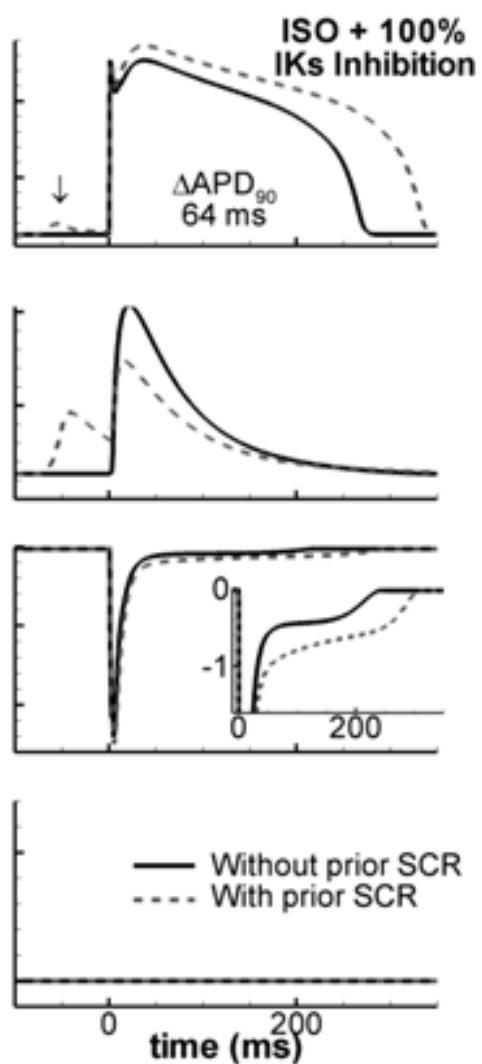

$2 \mathrm{~ms}$

Figure 9. Computational model of the canine ventricular myocyte implicates reduced $\mathrm{SR} \mathrm{Ca}^{2+}$ dependent inactivation of $\mathrm{I}_{\mathrm{CaL}}$ to cause APD prolongation. A. AP, intracellular $\mathrm{Ca}^{2+}, \mathrm{I}_{\mathrm{CaL}}$ and $\mathrm{I}_{\mathrm{Ks}}$ (top to bottom) with (dashed lined) or without (solid lines) prior SR $\mathrm{Ca}^{2+}$ release for a single beat after pacing to steady state in the presence of ISO at $\mathrm{CL}$ of $1000 \mathrm{~ms}$. The DAD resulting from the SCR is indicated by an arrow. Inset in $3^{\text {rd }}$ panel shows $I_{\text {cat }}$ during AP plateau on an expanded scale. $B$. Similar to panel $A$ in the presence of ISO and complete inhibition of $I_{\mathrm{Ks}}$. C. After pacing to steady state in the presence of ISO, SCR was triggered (shaded bars) or not (white bars) and individual currents / fluxes were blocked for one final beat. $A P D_{90}$ differences are indicated above each bar. Only inhibition of $\mathrm{SR} \mathrm{Ca}^{2+}$ release (RyR) or $\mathrm{I}_{\mathrm{CaL}} \mathrm{CDI}$ was sufficient to completely abolish the APD prolongation after SCR. 
We employed a computational model of the canine ventricular myocyte to investigate in more detail the ionic basis of the coupling between SCR and APD prolongation. After pacing to steady state, a partial release of SR $\mathrm{Ca}^{2+}$ was induced during diastole. After this release, APD was prolonged, consistent with the experimental observations (Figure 9A; $\mathrm{CL}=1000 \mathrm{~ms}$ in the presence of ISO). The prolonged APD was associated with a decreased $\mathrm{CaT}$, an increase in the sustained component of $\mathrm{I}_{\mathrm{CaL}}$ and increased $I_{\mathrm{Ks}}$. The increase in $I_{\mathrm{Ks}}$ was due to the increased plateau potential resulting from the larger $I_{\text {Cat }}$ and counteracted the repolarization delay. As such, APD prolongation was significantly larger when $I_{\mathrm{Ks}}$ was inhibited (Figure 9B). An increase in plateau $V_{m}$ was also observed in experimental recordings (maximum amplitude of plateau is $114 \pm 1 \mathrm{mV}$ without prior DAD and $118 \pm 1$ $\mathrm{mV}$ following DADs at $1000 \mathrm{~ms} \mathrm{CL}$ in the presence of HMR1556 + ISO, $P<0.05$ ).

Several currents underlying the ventricular AP are modified by intracellular $\mathrm{Ca}^{2+}$. To determine the relative contribution of each component, we used the computational model to selectively inhibit each current for one final beat at steady state following either a normal diastole or SCR. The main currents activated by SCR in the dog are $I_{\mathrm{NaCa}}$ and $\mathrm{I}_{\mathrm{CICa}}{ }^{3,}{ }^{36}$ However, inhibition of these currents only had a minor impact on the APD differences in the presence and absence of SCR (Figure 9C). In contrast, inhibition of $\mathrm{I}_{\mathrm{CaL}}$ or RyR was able to significantly reduce the DAD-provoked APD prolongation. More specifically, when only $\mathrm{Ca}^{2+}$-dependent inactivation (CDI) of $\mathrm{I}_{\mathrm{CaL}}$ was inhibited, APD after SCR was no longer prolonged compared to APD without prior SCR (Figure 9C). Further investigation showed any differences in APD in these conditions were due to 'priming' of CDI due to a transition of L-type $\mathrm{Ca}^{2+}$ channels to the $\mathrm{Ca}^{2+}$-dependent tier of the Markov model induced by the $\mathrm{Ca}^{2+}$ that was released during the SCR, prior to activation of inhibition.

Combined, our data implicate reduced SR $\mathrm{Ca}^{2+}$-release-dependent inactivation of $\mathrm{I}_{\text {Cat }}$ as the mechanism underlying APD prolongation after SCR. This prediction was confirmed under voltageclamp conditions in native canine ventricular myocytes when the extracellular solution was modified to isolate $I_{\text {CaL }}$ (Figure 10). During a voltage step to $+10 \mathrm{mV}$ from a holding potential of $-80 \mathrm{mV} \mathrm{a}$ significant increase in the integral of $I_{\mathrm{CaL}}$ was observed after SCR: $\int I_{\mathrm{CaL}}=27.9 \pm 3.5 \mathrm{nC}$ vs $33.0 \pm 3.5 \mathrm{nC}$ in the absence or presence of SCR, respectively $(P<0.05)$. 


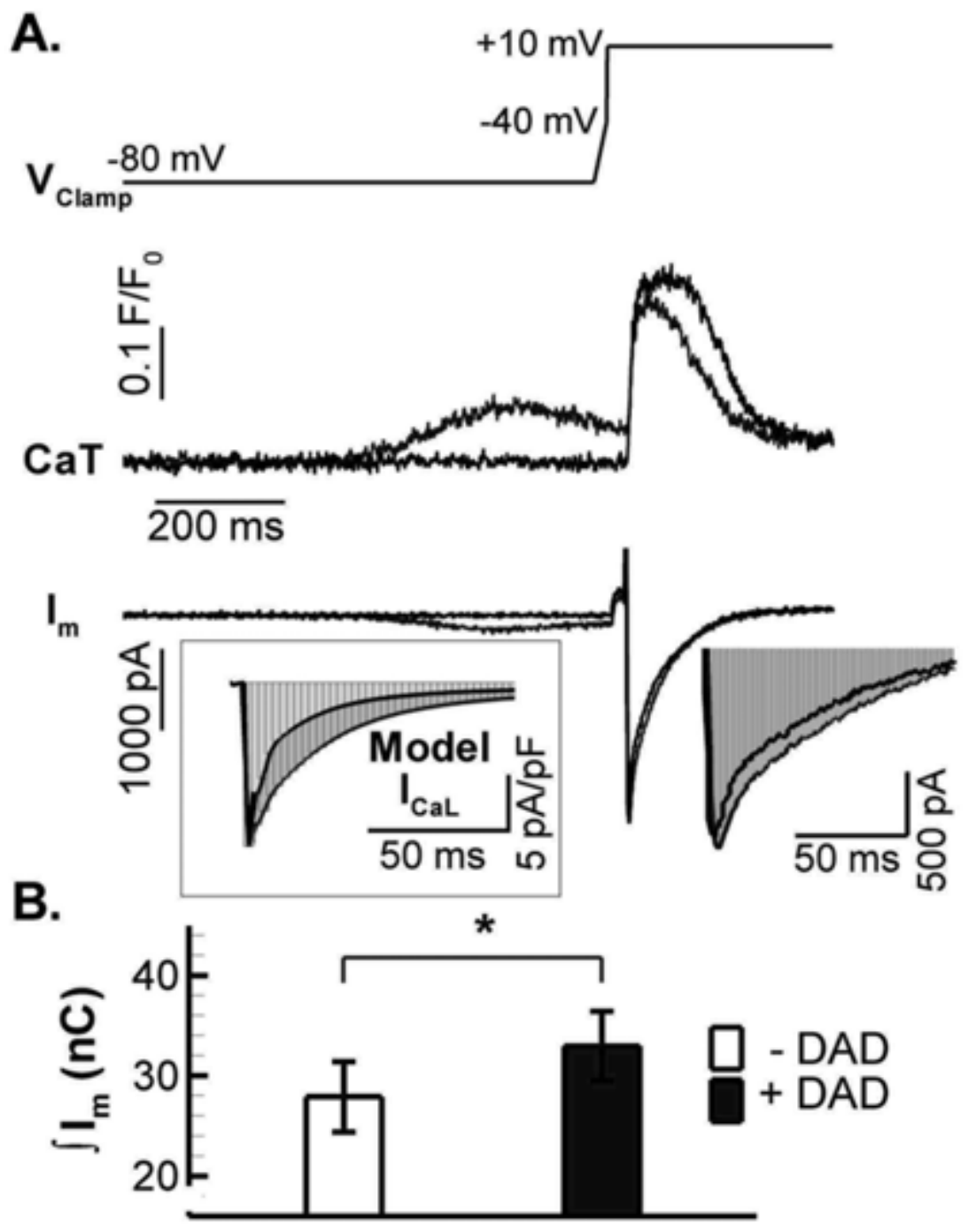

Figure 10. $I_{\text {Cat }}$ inactivation is slowed after SCR. A. Voltage-clamp protocol (top panel), intracellular $\mathrm{Ca}^{2+}$ (middle panel) and membrane current (bottom panel) in a representative canine ventricular myocyte in the presence of HMR1556 + ISO + increased $\left[\mathrm{Ca}^{2+}\right]_{0}(3.6$ or $5.0 \mathrm{mmol} / \mathrm{L}$ ) in the absence (black) or presence of prior SCR (red). Right inset shows membrane current at an expanded scale. 4AP $(5.0 \mathrm{mmol} / \mathrm{L})$ and $\mathrm{BaCl}_{2}(0.1 \mathrm{mmol} / \mathrm{L})$ were used to isolate $\mathrm{I}_{\mathrm{CaL}}$. Left inset shows model results under similar conditions. B. Quantification of average membrane current integral (shaded area in right inset of panel $A$ ) in 6 canine ventricular myocytes in the absence or presence of SCR. Prior SCR significantly increases total inward current (*: P<0.05). For color figure, see page 254.

\section{DISCUSSION}

In this study, we elucidated the relationship between SCR, AP prolongation and BVR in canine leftventricular midmyocytes. We demonstrated that during conditions of ISO-induced $\mathrm{Ca}^{2+}$ overload, SCR occurs over a wide range of pacing CLs and that APD following SCR is significantly prolonged. Reduced CICR-dependent inactivation of $\mathrm{I}_{\mathrm{CaL}}$ after SCR underlies this AP prolongation. The increase in $B V R$ observed during $\mathrm{Ca}^{2+}$ overload was strongly dependent on the degree of APD prolongation after 
SCR. Previous studies ${ }^{37-39}$ have shown that large CaTs result in abbreviation of APD, whereas a small $\mathrm{CaT}$ after SR $\mathrm{Ca}^{2+}$ depletion corresponds to prolonged APD. In agreement, we find substantial APD prolongation after application of ryanodine due to reduced CDI of $\mathrm{I}_{\mathrm{Cat}}$. Moreover, our data indicate

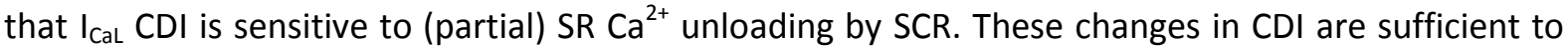
modulate APD on a beat-to-beat basis over a wide range of CLs. Interestingly, Spencer and Sham reported the opposite effect in guinea-pig ventricular myocytes: APD was shortened by inhibition of CICR with ryanodine. ${ }^{40}$ These authors attributed this to a reduced forward-mode NCX activity. Reduced CaT amplitude after SCR can also reduce forward-mode NCX. Thus, the net effect of SCR on APD will depend on the balance between reduced $N C X$ and increased $I_{\text {Cal }}$. This may explain why caffeine, which induces SCR on almost every beat, only results in a mild (non-significant) APD prolongation.

\section{The Importance of $I_{\mathrm{Ks}}$ Blockade}

We have previously shown that during $\beta A R$ stimulation and $I_{\mathrm{Ks}}$ blockade (via KCNQ1 inhibition; ${ }^{41}$ mimicking LQT1), BVR is significantly increased and that this is, at least partly, dependent on $\left[\mathrm{Ca}^{2+}\right]_{\mathrm{i}}{ }^{14}$ Here we extend this by determining an important role for $\mathrm{CDI}$ of $\mathrm{I}_{\mathrm{CaL}}$, at least in the presence of SCR. During $\beta A R$ stimulation, the role of $I_{\mathrm{Ks}}$ becomes more prominent, and hence inhibition of $I_{\mathrm{Ks}}$ leads to APD prolongation. ${ }^{14}$ Barandi et al. ${ }^{42}$ have shown that the degree of APD prolongation induced by pharmacological block of repolarizing currents or augmentation of depolarizing currents, depends on baseline APD. Consistent with these results, we find a more pronounced APD prolongation after SCR at slow $\mathrm{CL}$ and in the presence of $\mathrm{I}_{\mathrm{Ks}}$ blockade. In our experiments, APD prolongation after SCR is particularly pronounced because the increase in $\mathrm{I}_{\mathrm{CaL}}$ also elevates the plateau potential which increases $I_{\mathrm{ks}}$ activation and offsets the prolongation induced by $I_{\mathrm{CaL}}$ (Figure $9 \mathrm{~A}$, bottom panel). This compensating mechanism is absent when $I_{\mathrm{Ks}}$ is inhibited. Thus, $\mathrm{I}_{\mathrm{Ks}}$ inhibition exacerbates the effect of SCR on APD prolongation.

\section{Arrhythmogenesis During Conditions of $\mathrm{Ca}^{2+}$ Overload}

It has long been established that SCR-induced triggered activity is a major arrhythmogenic mechanism during $\mathrm{Ca}^{2+}$ overload and its occurrence is increased in various pathological conditions. ${ }^{6}$,

${ }^{43}$ Here, we show that as diastolic SCR prolongs the subsequent APD, even SCR below the threshold for triggered activity has important cellular electrophysiological effects that may be proarrhythmic. Regional prolongation of APD after SCR in the intact heart may cause increased spatial dispersion of repolarization between regions with the highest $\mathrm{Ca}^{2+}$ load (generating SCR) and regions with lower $\mathrm{Ca}^{2+}$ loading. Regional heterogeneities in ion-channel expression or intercellular conduction may further amplify this dispersion. Combined, these mechanisms can promote functional reentry. Stabilization of $\mathrm{Ca}^{2+}$ handling may therefore not only reduce the incidence of arrhythmogenic triggers but also prevent their reentrant perpetuation. We have also shown that $\mathrm{Ca}^{2+}$ overload by the combination of ISO and fast pacing can give rise to diastolic SCR which prolongs APD sufficiently such that the next SCR occurs before the end of repolarization, generating an EAD. The common dependence of DADs and EADs on SCR in the presence of ISO has previously been described. ${ }^{5,44}$ Our results provide novel mechanistic insights on the coupling between $S C R, A P D$ prolongation and EAD 
occurrence and show that diastolic SCR is a central element in both triggered activity and repolarization instability.

APD prolongation after SCR results from increased $I_{\text {CaL }}$, which enhances $\mathrm{Ca}^{2+}$ loading via increased sarcolemmal $\mathrm{Ca}^{2+}$ influx. Enhanced loading along with reduced $\mathrm{Ca}^{2+}$ efflux serve to restore and finetune SR Ca ${ }^{2+}$ content to maintain CICR efficacy, as has previously been described in rat ventricular myocytes after SR depletion. ${ }^{37,}{ }^{39}$ However, during $\mathrm{Ca}^{2+}$ overload these mechanisms will readjust, promoting $\mathrm{Ca}^{2+}$ extrusion via NCX, which facilitates the occurrence of afterdepolarizations. Consistent with this reasoning, we found that the probability of observing a DAD was not significantly altered by the presence of a DAD on the previous beat. This strongly implies that, under our experimental conditions, the reduction in myocyte $\mathrm{Ca}^{2+}$ load during a DAD is overcome by the increased sarcolemmal $\mathrm{Ca}^{2+}$ influx during the following prolonged AP. Thus, APD prolongation after $\mathrm{SCR}$ contributes to the vicious cycle of $\mathrm{Ca}^{2+}$ overload.

We investigated the utility of several interventions to restabilize $\mathrm{Ca}^{2+}$ handling and repolarization variability during $\mathrm{Ca}^{2+}$ overload. Inhibition of SCR by ryanodine and tetracaine has previously been described, ${ }^{29,44}$ and the results presented here agree with those data. We extend these observations by showing a concomitant decrease in BVR. Both ryanodine and tetracaine are useful for mechanistic studies but due to their deleterious effects in vivo cannot be used as therapeutic agents. In contrast, both magnesium and flecainide are commonly used antiarrhythmic agents. Here we show that both these agents can stabilize $\mathrm{Ca}^{2+}$ handling in the overloaded single myocyte, and that this leads to a reduction in BVR and arrhythmogenic events. Similarly, we confirm the usefulness of modulating Calmodulin/CaMKII during $\mathrm{Ca}^{2+}$ overload as an antiarrhythmic strategy. ${ }^{31}$ CaMKII phosphorylation has been shown to induce a different gating mode (mode 2) of the $I_{\text {Cat }}$ channel, thereby reducing inactivation, favoring $\mathrm{Ca}^{2+}$ entry and the occurrence of EADs and DADs. ${ }^{45}$ In this regard, the development of selective CaMKII modulators suitable for antiarrhtyhmic interventions in humans is awaited.

\section{Conclusions}

We have shown that reduced CICR-dependent inactivation of $\mathrm{I}_{\mathrm{Ca}}$ after SCR underlies APD prolongation and contributes to increased BVR during ISO-induced $\mathrm{Ca}^{2+}$ overload. The degree of APD prolongation is exacerbated by inhibition of $I_{\mathrm{Ks}}$. Pharmacological interventions that regularize SCR or inhibit SCR with or without preserved systolic contractions reduce BVR. Our data provide novel insights into arrhythmogenic mechanisms during $\mathrm{Ca}^{2+}$ overload and suggest novel antiarrhythmic strategies.

\section{ACKNOWLEDGEMENTS}

The authors thank Drs. Chris Pollard and Jean-Pierre Valentin, Department of Safety Pharmacology, Safety Assessment UK, AstraZeneca R\&D, Alderley Park, UK, for active collaboration and for 
providing cardiac myocytes. Ongoing collaborations with Dr. Yoram Rudy, Washington University in St. Louis, MO and use of his computational resources are gratefully acknowledged.

\section{SOURCES OF FUNDING}

P.G.A.V. is supported by a Vidi grant from the Netherlands Organization for Scientific Research (ZonMw 91710365). D.A.E. and A.W.T. are supported by the British Heart Foundation. D.M.J. was financially supported by AstraZeneca Ltd, UK.

\section{REFERENCES}

1. Bers DM. Cardiac excitation-contraction coupling. Nature. 2002;415:198-205.

2. Stern MD, Capogrossi MC, Lakatta EG. Spontaneous calcium release from the sarcoplasmic reticulum in myocardial cells: mechanisms and consequences. Cell Calcium. 1988;9:247-256.

3. Zygmunt $A C$, Goodrow RJ, Weigel $\mathrm{CM}$. $\mathrm{I}_{\mathrm{NaCa}}$ and $\mathrm{I}_{\mathrm{CI}(\mathrm{Ca})}$ contribute to isoproterenol-induced delayed afterdepolarizations in midmyocardial cells. Am J Physiol. 1998;275:H1979-H1992.

4. Bers DM. Calcium cycling and signaling in cardiac myocytes. Annu Rev Physiol. 2008;70:2349.

5. Volders PGA, Kulcsár A, Vos MA, Sipido KR, Wellens HJ, Lazzara R, Szabo B. Similarities between early and delayed afterdepolarizations induced by isoproterenol in canine ventricular myocytes. Cardiovasc Res. 1997;34:348-359.

6. Ter Keurs HE, Boyden PA. Calcium and arrhythmogenesis. Physiol Rev. 2007;87:457-506.

7. Zaniboni $M$, Pollard $A E$, Yang $L$, Spitzer $K W$. Beat-to-beat repolarization variability in ventricular myocytes and its suppression by electrical coupling. Am J Physiol Heart Circ Physiol. 2000;278:H677-687.

8. Hinterseer $M$, Beckmann $B M$, Thomsen $M B$, Pfeufer $A$, Ulbrich $M$, Sinner MF, Perz $S$, Wichmann HE, Lengyel C, Schimpf R, Maier SK, Várro A, Vos MA, Steinbeck G, Kääb S. Usefulness of short-term variability of QT intervals as a predictor for electrical remodeling and proarrhythmia in patients with nonischemic heart failure. Am J Cardiol. 2010;106:216220.

9. Tereshchenko LG, Han L, Cheng A, Marine JE, Spragg DD, Sinha S, Dalal D, Calkins H, Tomaselli GF, Berger RD. Beat-to-beat three-dimensional ECG variability predicts ventricular arrhythmia in ICD recipients. Heart Rhythm. 2010;7:1606-1613.

10. Thomsen MB, Verduyn SC, Stengl M, Beekman JD, de Pater G, van Opstal J, Volders PGA, Vos MA. Increased short-term variability of repolarization predicts $d$-sotalol-induced torsades de pointes in dogs. Circulation. 2004;110:2453-2459.

11. Gallacher DJ, Van de Water A, van der Linde H, Hermans AN, Lu HR, Towart R, Volders PGA. In vivo mechanisms precipitating torsades de pointes in a canine model of drug-induced long-QT1 syndrome. Cardiovasc Res. 2007;76:247-256.

12. Jacobson I, Carlsson L, Duker G. Beat-by-beat QT interval variability, but not QT prolongation per se, predicts drug-induced torsades de pointes in the anaesthetised methoxaminesensitized rabbit. J Pharmacol Toxicol Methods. 2011;63:40-46.

13. Hinterseer $M$, Beckmann BM, Thomsen MB, Pfeufer A, Dalla Pozza R, Loeff $M$, Netz $H$, Steinbeck G, Vos MA, Kääb S. Relation of increased short-term variability of QT interval to congenital long-QT syndrome. Am J Cardiol. 2009;103:1244-1248.

14. Johnson DM, Heijman J, Pollard CE, Valentin JP, Crijns HJ, Abi-Gerges N, Volders PGA. I restricts excessive beat-to-beat variability of repolarization during beta-adrenergic receptor stimulation. J Mol Cell Cardiol. 2010;48:122-130. 
15. Volders PGA, Sipido KR, Carmeliet E, Spätjens RLHMG, Wellens HJ, Vos MA. Repolarizing K ${ }^{+}$ currents $I_{\mathrm{TO} 1}$ and $\mathrm{I}_{\mathrm{Ks}}$ are larger in right than left canine ventricular midmyocardium. Circulation. 1999;99:206-210.

16. Volders PGA, StengI M, van Opstal JM, Gerlach U, Spätjens RL, Beekman JD, Sipido KR, Vos MA. Probing the contribution of $I_{\mathrm{Ks}}$ to canine ventricular repolarization: key role for $\beta$ adrenergic receptor stimulation. Circulation. 2003;107:2753-2760.

17. Gögelein $\mathrm{H}$, Bruggemann A, Gerlach $U$, Brendel J, Busch AE. Inhibition of $I_{\mathrm{Ks}}$ channels by HMR 1556. Naunyn Schmiedebergs Arch Pharmacol. 2000;362:480-488.

18. Thomas GP, Gerlach U, Antzelevitch C. HMR 1556, a potent and selective blocker of slowly activating delayed rectifier potassium current. J Cardiovasc Pharmacol. 2003;41:140-147.

19. Hwang HS, Hasdemir C, Laver D, Mehra D, Turhan K, Faggioni M, Yin H, Knollmann BC. Inhibition of cardiac $\mathrm{Ca}^{2+}$ release channels (RyR2) determines efficacy of class I antiarrhythmic drugs in catecholaminergic polymorphic ventricular tachycardia. Circ Arrhythm Electrophysiol. 2011;4:128-135.

20. Rosati B, Pan Z, Lypen S, Wang HS, Cohen I, Dixon JE, McKinnon D. Regulation of KChIP2 potassium channel $\beta$ subunit gene expression underlies the gradient of transient outward current in canine and human ventricle. J Physiol. 2001;533:119-125.

21. Wang GK, Russell C, Wang SY. State-dependent block of wild-type and inactivation-deficient $\mathrm{Na}+$ channels by flecainide. J Gen Physiol. 2003;122:365-374.

22. Paul AA, Witchel HJ, Hancox JC. Inhibition of the current of heterologously expressed HERG potassium channels by flecainide and comparison with quinidine, propafenone and lignocaine. Br J Pharmacol. 2002;136:717-729.

23. Heijman J, Volders PGA, Westra RL, Rudy Y. Local control of $\beta$-adrenergic stimulation: Effects on ventricular myocyte electrophysiology and $\mathrm{Ca}^{2+}$-transient. J Mol Cell Cardiol. 2011;50:863871.

24. Xie Y, Sato D, Garfinkel A, Qu Z, Weiss JN. So little source, so much sink: requirements for afterdepolarizations to propagate in tissue. Biophys J. 2010;99:1408-1415.

25. Restrepo JG, Weiss JN, Karma A. Calsequestrin-mediated mechanism for cellular calcium transient alternans. Biophys J. 2008;95:3767-3789.

26. Swietach $\mathrm{P}$, Spitzer KW, Vaughan-Jones RD. Modeling calcium waves in cardiac myocytes: importance of calcium diffusion. Front Biosci. 2010;15:661-680.

27. Zygmunt AC, Eddlestone GT, Thomas GP, Nesterenko VV, Antzelevitch C. Larger late sodium conductance in $\mathrm{M}$ cells contributes to electrical heterogeneity in canine ventricle. $\mathrm{Am} \mathrm{J}$ Physiol Heart Circ Physiol. 2001;281:H689-697.

28. Overend CL, Eisner DA, O'Neill SC. The effect of tetracaine on spontaneous $\mathrm{Ca}^{2+}$ release and sarcoplasmic reticulum calcium content in rat ventricular myocytes. J Physiol. 1997;502 ( Pt 3):471-479.

29. Venetucci LA, Trafford AW, Diaz ME, O'Neill SC, Eisner DA. Reducing ryanodine receptor open probability as a means to abolish spontaneous $\mathrm{Ca}^{2+}$ release and increase $\mathrm{Ca}^{2+}$ transient amplitude in adult ventricular myocytes. Circ Res. 2006;98:1299-1305.

30. Trafford AW, Sibbring GC, Diaz ME, Eisner DA. The effects of low concentrations of caffeine on spontaneous Ca release in isolated rat ventricular myocytes. Cell Calcium. 2000;28:269276.

31. Anderson ME. Multiple downstream proarrhythmic targets for calmodulin kinase II: moving beyond an ion channel-centric focus. Cardiovasc Res. 2007;73:657-666.

32. Curran J, Hinton MJ, Ríos E, Bers DM, Shannon TR. $\beta$-Adrenergic enhancement of sarcoplasmic reticulum calcium leak in cardiac myocytes is mediated by calcium/calmodulindependent protein kinase. Circ Res. 2007;100:391-398.

33. Kaseda S, Gilmour RF, Jr., Zipes DP. Depressant effect of magnesium on early afterdepolarizations and triggered activity induced by cesium, quinidine, and 4aminopyridine in canine cardiac Purkinje fibers. Am Heart J. 1989;118:458-466. 
34. Aomine $\mathrm{M}$, Tatsukawa $\mathrm{Y}$, Yamato $\mathrm{T}$, Yamasaki S. Antiarrhythmic effects of magnesium on rat papillary muscle and guinea pig ventricular myocytes. Gen Pharmacol. 1999;32:107-114.

35. Watanabe H, Chopra N, Laver D, Hwang HS, Davies SS, Roach DE, Duff HJ, Roden DM, Wilde AA, Knollmann BC. Flecainide prevents catecholaminergic polymorphic ventricular tachycardia in mice and humans. Nat Med. 2009;15:380-383.

36. Trafford AW, Diaz ME, Eisner DA. Ca-activated chloride current and $\mathrm{Na}$-Ca exchange have different timecourses during sarcoplasmic reticulum $\mathrm{Ca}$ release in ferret ventricular myocytes. Pflugers Arch. 1998;435:743-745.

37. Trafford AW, Diaz ME, Negretti N, Eisner DA. Enhanced $\mathrm{Ca}^{2+}$ current and decreased $\mathrm{Ca}^{2+}$ efflux restore sarcoplasmic reticulum $\mathrm{Ca}^{2+}$ content after depletion. Circ Res. 1997;81:477484.

38. Papp Z, Peineau N, Szigeti G, Argibay J, Kovacs L. Calcium-dependent modulation of the plateau phase of action potential in isolated ventricular cells of rabbit heart. Acta Physiol Scand. 1999;167:119-129.

39. Takamatsu H, Nagao $\mathrm{T}$, Ichijo $\mathrm{H}$, Adachi-Akahane $\mathrm{S}$. L-type $\mathrm{Ca}^{2+}$ channels serve as a sensor of the $\mathrm{SR} \mathrm{Ca}{ }^{2+}$ for tuning the efficacy of $\mathrm{Ca}^{2+}$-induced $\mathrm{Ca}^{2+}$ release in rat ventricular myocytes. $J$ Physiol. 2003;552:415-424.

40. Spencer $\mathrm{Cl}$, Sham JS. Effects of $\mathrm{Na}^{+} / \mathrm{Ca}^{2+}$ exchange induced by $\mathrm{SR} \mathrm{Ca}^{2+}$ release on action potentials and afterdepolarizations in guinea pig ventricular myocytes. Am J Physiol Heart Circ Physiol. 2003;285:H2552-2562.

41. Lerche C, Seebohm G, Wagner Cl, Scherer CR, Dehmelt L, Abitbol I, Gerlach U, Brendel J, Attali B, Busch AE. Molecular impact of MinK on the enantiospecific block of I(Ks) by chromanols. Br J Pharmacol. 2000;131:1503-1506.

42. Barandi L, Virag L, Jost N, Horvath Z, Koncz I, Papp R, Harmati G, Horvath B, Szentandrassy N, Banyasz T, Magyar J, Zaza A, Varro A, Nanasi PP. Reverse rate-dependent changes are determined by baseline action potential duration in mammalian and human ventricular preparations. Basic Res Cardiol. 2010;105:315-323.

43. Sipido KR, Volders PGA, de Groot SHM, Verdonck F, Van de Werf F, Wellens HJJ, Vos MA. Enhanced $\mathrm{Ca}^{2+}$ release and $\mathrm{Na} / \mathrm{Ca}$ exchange activity in hypertrophied canine ventricular myocytes: potential link between contractile adaptation and arrhythmogenesis. Circulation. 2000;102:2137-2144.

44. Priori SG, Corr PB. Mechanisms underlying early and delayed afterdepolarizations induced by catecholamines. Am J Physiol. 1990;258:H1796-H1805.

45. Hashambhoy YL, Winslow RL, Greenstein JL. CaMKII-induced shift in modal gating explains Ltype $\mathrm{Ca}^{2+}$ current facilitation: a modeling study. Biophys J. 2009;96:1770-1785. 


\title{
ChAPTER 7
}

\author{
DETERMINANTS OF BEAT-TO-BEAT VARIABILITY OF \\ Repolarization DURATION in the CANINE Ventricular \\ MYOCYTE - A COMPUTATIONAL ANALYSIS
}

Jordi heijman, ANTONio ZaZA, DANiel M. Johnson, Yoram Rudy, RalF L.M. Peeters, Paul G.A. Volders* and Ronald L. Westra*

* TheSe Authors CONTRIBUted EQUally to this WORK

MANUSCRIPT IN PREPARATION AND IN PART ACCEPTED FOR FRONTIERS IN CARDIOVASCULAR BIOLOGY 2012 


\section{ABSTRACT}

Beat-to-beat variability of repolarization duration (BVR) is an intrinsic characteristic of cardiac function. When increased by repolarization-undermining influences, it is a better marker of proarrhythmia than repolarization prolongation alone. The ionic mechanisms underlying baseline $B V R$ in physiological conditions, the rate dependence of $B V R$, and the factors contributing to increased BVR in pathological conditions, all remain incompletely understood. Here, we employed computer modeling to provide novel insights into the subcellular mechanisms of BVR under physiological conditions and during simulated drug-induced repolarization prolongation, mimicking long-QT syndromes type 1, 2 and 3. We developed stochastic implementations of 13 major ionic currents and fluxes in a model of the canine ventricular myocyte electrophysiology. Combined stochastic gating of all targets resulted in short- and long-term BVR consistent with experimental data from isolated canine ventricular myocytes. The model indicated that the magnitude of stochastic fluctuations is rate dependent due to the rate dependence of action-potential (AP) duration (APD). Both this active process and the intrinsic nonlinear relation between membrane current and APD contribute to rate dependence of BVR. We identified a major role for stochastic gating of the (persistent) $\mathrm{Na}^{+}$current $\left(\mathrm{I}_{\mathrm{Na}}\right)$ and rapidly-activating delayed-rectifier $\mathrm{K}^{+}$current $\left(\mathrm{I}_{\mathrm{Kr}}\right)$ under physiological conditions. Consistent with experimental results, inhibition of $\mathrm{I}_{\mathrm{kr}}$ or augmentation of $I_{\mathrm{Na}}$ significantly increased BVR whereas subsequent $\beta$-adrenergic receptor stimulation reduced it, similar to findings in isolated myocytes. In contrast, $\beta$-adrenergic stimulation increased BVR in simulated long-QT syndrome type 1. In addition to stochastic channel gating, APD, AP morphology and beat-to-beat variations in $\mathrm{Ca}^{2+}$ were found to modulate single-cell BVR. Cell-tocell coupling was able to decrease BVR and this was more pronounced when a cell with increased BVR was coupled to a cell with normal BVR. In conclusion, our results provide new insights into the ionic mechanisms underlying BVR and suggest that BVR reflects multiple potentially proarrhythmic parameters, including increased ion channel stochasticity, APD, AP morphology and abnormal $\mathrm{Ca}^{2+}$ handling. 


\section{INTRODUCTION}

Beat-to-beat variability of repolarization duration (BVR) is an intrinsic characteristic of cardiac function that can be observed at various levels of integration, from temporal variations in action potential (AP) duration (APD) of the single cardiac myocyte to instability of the QT interval on the body surface. ${ }^{1-3}$ When increased by repolarization-undermining influences, it is a better marker of proarrhythmia than repolarization prolongation per se in various experimental models of torsadesde-pointes ventricular tachycardia ${ }^{4-6}$ and in human cardiac pathology. ${ }^{2,7}$ Recently, we described an important role for (abnormal) $\mathrm{Ca}^{2+}$ handling and the slowly-activating delayed-rectifier rectifier $\mathrm{K}^{+}$ current $\left(I_{K_{S}}\right)$ in the increased BVR observed during $\beta$-adrenergic stimulation in single canine ventricular myocytes. ${ }^{8}$ However, the exact mechanisms underlying BVR and its rate dependence under physiological conditions, as well as the various factors contributing to exaggerated BVR in pathological conditions, remain incompletely understood.

Computational models of cardiac myocyte electrophysiology have a rich history, dating back more than 50 years. ${ }^{9}$ Recent models have provided detailed descriptions of various cardiac cell types in different species. They have created insight into the role of the different ion channels in ratedependent alterations in repolarization, ${ }^{10-14}$ have helped to elucidate arrhythmogenic mechanisms in various pathological conditions, ${ }^{15-19}$ and have facilitated analysis of the integration of regulatory pathways and electrophysiological responses. ${ }^{20-23}$ However, to date most computational models are deterministic and have an APD that converges to a fixed steady state or a limit cycle (e.g., APD alternans) for a given pacing cycle length (CL). As such, these models are unsuitable for the study of BVR.

Tanskanen et al. ${ }^{24}$ were among the first to investigate the effect of stochastic properties of local control $\mathrm{Ca}^{2+}$ models on ventricular repolarization. They showed that a variable occurrence of arrhythmogenic early afterdepolarizations (EADs) could be explained by the stochastic gating of the L-type $\mathrm{Ca}^{2+}$ channel. In contrast, Sato et al. ${ }^{25}$ described temporal repolarization variability due to the chaotic occurrence of EADs in a deterministic model of the $\mathrm{H}_{2} \mathrm{O}_{2}$-treated rabbit ventricular myocyte. These authors provided strong evidence that noise-induced transitions between states were insufficient to account for the large APD fluctuations observed under their experimental conditions. ${ }^{26}$ However, none of these studies addressed BVR under physiological or pathological conditions in the absence of EADs.

Recently, the role of stochastic ion-channel gating in repolarization variability under physiological conditions has been described by Lemay et al. ${ }^{27}$ and Pueyo et al. ${ }^{28}$ These studies provide an important basis for modeling cardiac repolarization variability. Here we significantly extended these studies by developing a stochastic version of our recently published model of the canine ventricular myocyte including adrenergic stimulation. ${ }^{22}$ Stochastic formulations of 13 major ion channels and active ion-transporters were included, and APD dynamics were compared to results obtained in isolated canine ventricular myocytes. We employed the model to obtain novel insights into the 
quantitative contribution of individual electrophysiological processes to cellular BVR under physiological conditions and the factors contributing to increased BVR during pathological conditions.

\section{MetHODS}

\section{Adaptations to the HRdßAR model}

\section{Markov model formulation of the rapidly-activating delayed-rectifier $\mathrm{K}^{+}$current $\left(\mathrm{I}_{\mathrm{Kr}}\right)$}

The original Hodgkin-Huxley based formulation of $\mathrm{I}_{\mathrm{kr}}$ was replaced with a 10-state Markov model based on the model structure described by Silva and Rudy ${ }^{29}$ extended with state-dependent block of $\mathrm{I}_{\mathrm{Kr}}$ channels by dofetilide (Figure 1A), based on the approach by Brennan et al. ${ }^{30}$ Model parameters were adjusted to reproduce $I_{\mathrm{kr}}$ peak and tail I-V relationships, time constants of activation and time constants of deactivation measured in canine ventricular myocytes ${ }^{31}$ (Figure 1B-D). Channels can enter the blocked mode via open and inactivated states, based on a $\mathrm{V}_{\mathrm{m}}$-dependent modulation of the dofetilide $\mathrm{EC}_{50 .}{ }^{32}$ The model reproduces dofetilide dose-response relationship measured in rabbit ventricular myocytes, ${ }^{33}$ use-dependence of relative inhibition as determined in AT-1 cells, ${ }^{32}$ and $V_{m}$ dependence of dofetilide $\mathrm{EC}_{50}{ }^{32}$ (Figure 1E-G).

A. Model Schematic
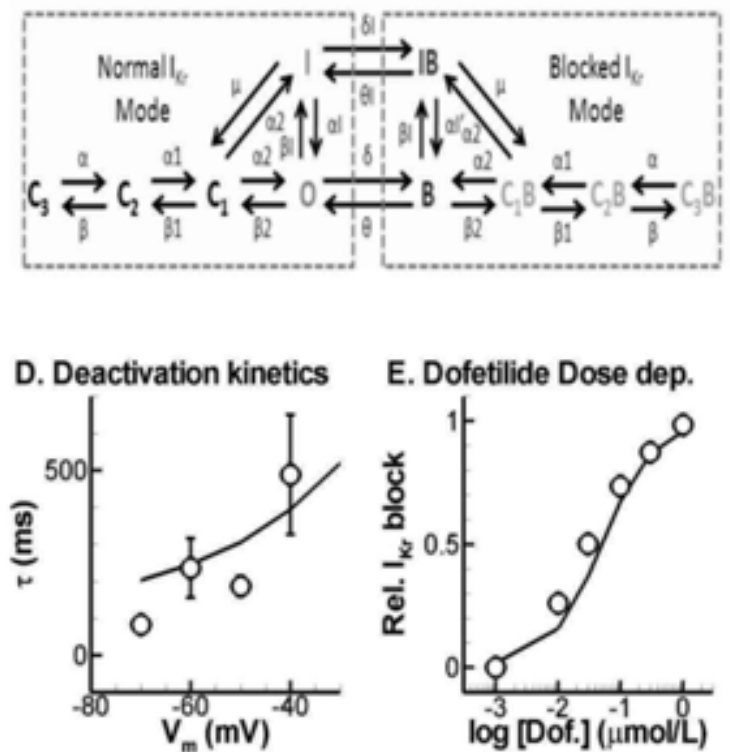

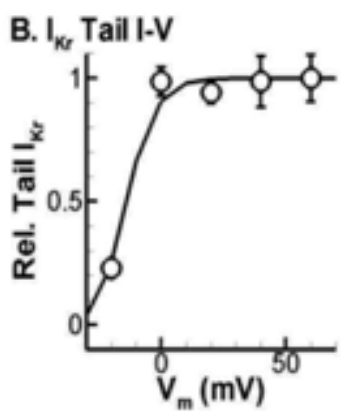

E. Dofetilide Dose dep.

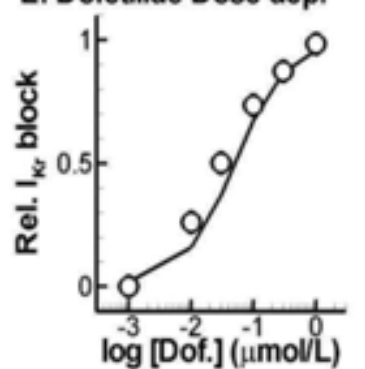

F. Dofetilide Use dep.

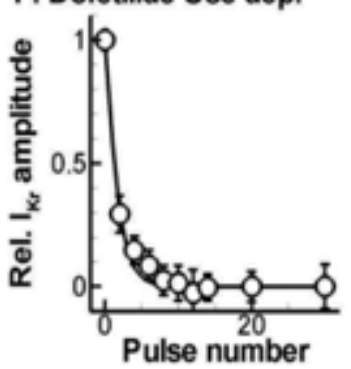

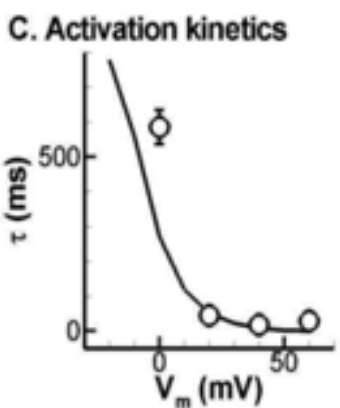

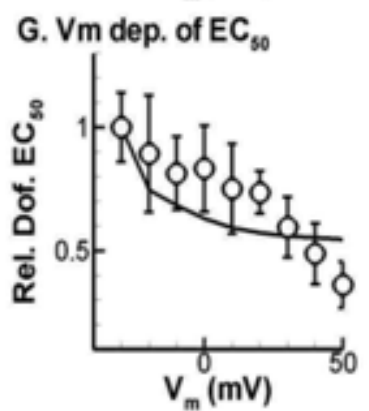

Figure 1. Structure and validation of $\mathrm{I}_{\mathrm{Kr}}$ Markov model properties. A. Model structure. B. Tail I-V relationship in model and canine ventricular myocytes. ${ }^{31} \mathrm{C}$. Time constant of activation based on a single-exponential fit in model and canine ventricular myocytes. ${ }^{31} \mathrm{D}$. Time constant of deactivation in model and canine ventricular myocytes. ${ }^{31} \mathrm{E}$. Dose-response curve of $\mathrm{I}_{\mathrm{kr}}$ block by dofetilide (experimental data from rabbit ventricular myocytes ${ }^{33}$ ). F. Use-dependent block of $\mathrm{I}_{\mathrm{kr}}$ by dofetilide in 
AT-1 cells ${ }^{32}$ and model. G. Vm dependence of dofetilide concentration required for half-maximal $\mathrm{I}_{\mathrm{kr}}$ inhibition (relative to $-30 \mathrm{mV}$ ) in model and AT-1 cells. ${ }^{32}$

\section{Markov model formulation of the $\mathrm{Na}^{+}$current $\left(\mathrm{I}_{\mathrm{Na}}\right)$}

The Markov model structure of Clancy and Rudy ${ }^{16}$ was employed for both normal inactivation and slow inactivation populations (Figure 2). Model parameters were adjusted to reproduce characteristics of $I_{\mathrm{Na}}$ and $I_{\mathrm{NaL}}$ from the original Hodgkin-Huxley-based formulation, including PKA- and CaMKII-dependent alterations. ${ }^{22}$ Model $I_{\text {Na }}$ peak I-V relationship and steady-state inactivation are consistent with those measured in isolated canine ventricular myocytes. ${ }^{34}$

A.
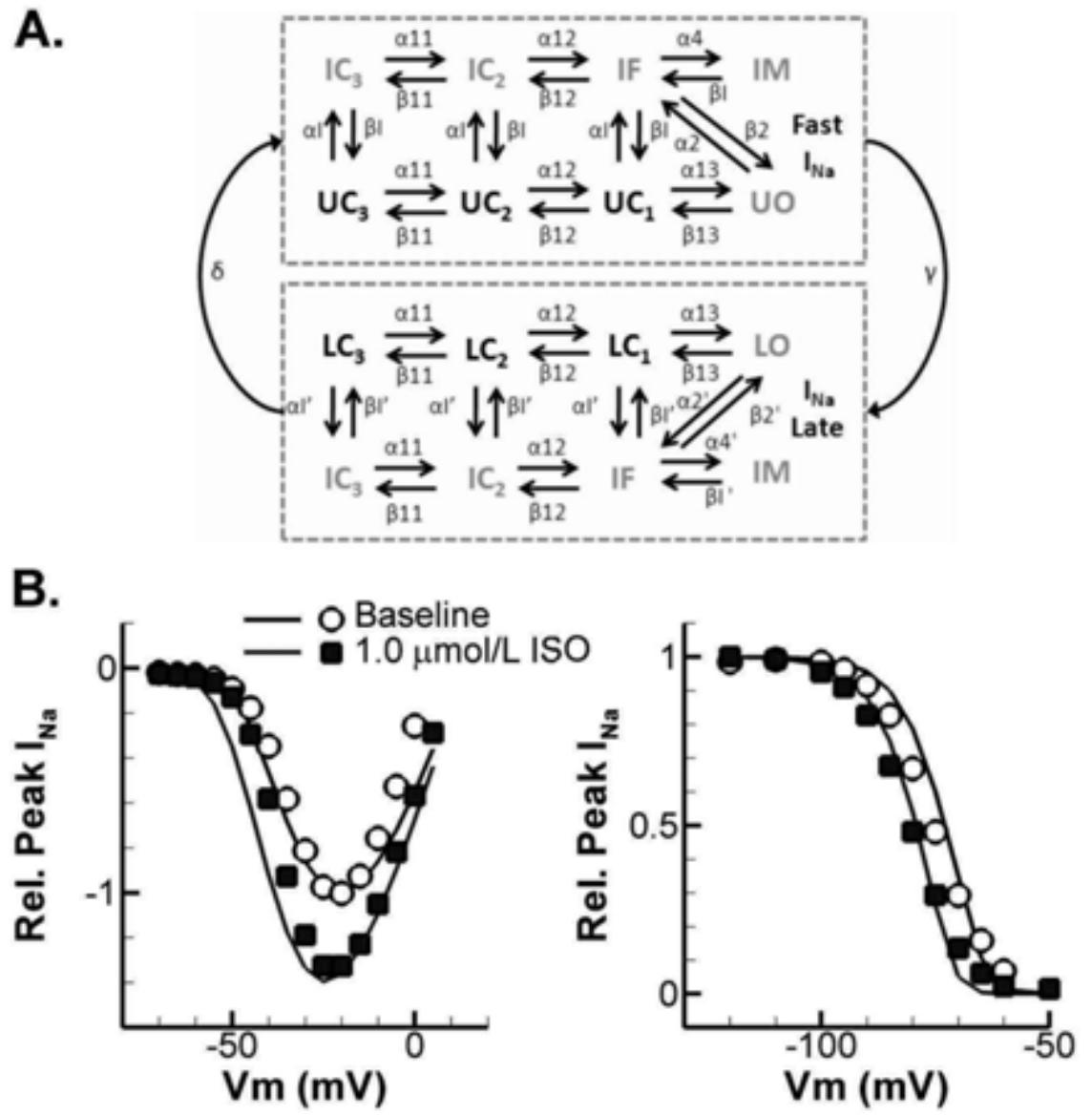

Figure 2. Structure and validation of $I_{\mathrm{Na}}$ Markov model properties. A. Model schematic of fast and late (persistent) $I_{\mathrm{Na}}$ components. B. Peak I-V relationship (left panel) and steady-state inactivation (right panel) in model (lines) and canine ventricular myocytes (symbols) at baseline or in the presence of $\beta$-adrenergic stimulation.

\section{Markov model formulation of sarcoplasmic reticulum (SR) $\mathrm{Ca}^{2+}$ release}

$\mathrm{Ca}^{2+}$-induced $\mathrm{Ca}^{2+}$ release (CICR) in the original HRdßAR model depends directly on the L-type $\mathrm{Ca}^{2+}$ current $\left(I_{\mathrm{CaL}}\right)$ based on the formulation by Livshitz et al. ${ }^{35}$ It is difficult to create a stochastic version of this formulation since it does not exhibit the strong positive feedback characteristic for CICR (where the $\mathrm{Ca}^{2+}$ released by some ryanodine receptors (RyRs) may activate other RyRs in the same 
microdomain), which likely affects its stochastic properties. As such, the original phosphorylated and non-phosphorylated CICR formulations were replaced by two 4-state Markov Models proposed by Restrepo et al. in their local control model of $\mathrm{Ca}^{2+}$ handling in the rabbit ventricular myocyte. ${ }^{36}$ Model parameters were adjusted to reproduce rate-dependent $\mathrm{Ca}^{2+}$ transient (CaT) properties (amplitude, time to peak, and time constant of decay) of the original HRdBAR model in the absence or presence of $\beta$-adrenergic stimulation ( $\beta A R S$ ).

\section{Additional alterations in model parameters}

Parameters of the non-phosphorylated and phosphorylated $\mathrm{I}_{\mathrm{CaL}}$ formulations were adjusted slightly compared to Heijman et al. ${ }^{22}$ to reduce the sensitivity of the model for the generation of EADs in response to $I_{\mathrm{kr}}$ blockade (requiring $80 \% \mathrm{I}_{\mathrm{kr}}$ reduction to generate EADs compared to $25 \%$ in the absence of these changes) and to match the amount of APD prolongation after $I_{\mathrm{Ks}}$ blockade in the presence of adrenergic stimulation, while maintaining peak I-V and inactivation characteristics similar to experimental recordings.

\section{Stochastic formulations of model components}

\section{Random number generation}

The Mersenne-Twister random number generator ${ }^{37}$ was used in all stochastic simulations to generate random variables from uniform and normal distributions. Binomial random variables $B(n, \mathrm{p})$ were generated using the Inverse Function Method or approximated from a normal distribution $N(n \cdot p, n \cdot p \cdot(1-p))$ when $\left|\frac{1}{\sqrt{n}} \cdot\left(\sqrt{\frac{1-p}{p}}-\sqrt{\frac{p}{1-p}}\right)\right|<0.3$.

\section{Langevin-term-based stochastic simulations}

In a subset of experiments, stochastic ion-current behavior was simulated using stochastic differential equations through the addition of a Langevin term to all differential equations controlling the gating of that current: ${ }^{38}$

$\frac{\mathrm{dX}}{\mathrm{dt}}=\mathrm{f}(\mathrm{X})+\alpha \cdot \sqrt{\mathrm{X}_{\mathrm{i}}} \cdot N(0,1)$

The noise amplitude parameter $\alpha$ was scaled to obtain similar APD variability as that observed with stochastic channel gating.

\section{Stochastic formulations of Markov models}

Stochastic simulations of Markov models of ion currents were performed as previously described for local control models. ${ }^{24,36,39}$ Briefly, for a given state i in the Markov model with a state occupancy of $\mathrm{n}_{i}$ channels at time $\mathrm{t}$, the probability for any channel to move to state $\mathrm{j}$ in the time interval $\Delta \mathrm{t}$ is 
given by $r_{i, j} \cdot \Delta t$, where $r_{i, j}$ is the transition rate (in $\mathrm{ms}^{-1}$ ) of the deterministic ODE model. Thus, the number of channels leaving state $\mathrm{i}$ in the interval $\Delta \mathrm{t}$ can be obtained from a multinomial distribution $M\left(n_{i}, \mathrm{r}_{\mathrm{i}, 1} \cdot \Delta \mathrm{t}, \ldots, \mathrm{r}_{\mathrm{i}, \mathrm{i}-1} \cdot \Delta \mathrm{t}, 1-\Delta \mathrm{t} \cdot \sum \mathrm{r}_{\mathrm{i}, \mathrm{j}}, \mathrm{r}_{\mathrm{i}, \mathrm{i}+1} \cdot \Delta \mathrm{t}, \mathrm{r}_{\mathrm{ik}} \cdot \Delta \mathrm{t}\right)$. For sufficiently small $\Delta \mathrm{t}$, the transition probabilities are small and can be assumed to be independent. In this case, the number of channels leaving state $\mathrm{i}$ can be approximated based on $\mathrm{k}$ independent binomial distributions $B\left(n_{i}, \mathrm{r}_{\mathrm{i}, \mathrm{j}} \cdot \Delta \mathrm{t}\right)$, significantly reducing the computational complexity. ${ }^{36}$ The number of channels in state $i$ is then updated at every time step by:

$$
\mathrm{n}_{\mathrm{i}}(\mathrm{t}+\Delta \mathrm{t})=\mathrm{n}_{\mathrm{i}}(\mathrm{t})+\sum_{\mathrm{j} \neq \mathrm{i}} B\left(\mathrm{n}_{\mathrm{j}}(\mathrm{t}), \mathrm{r}_{\mathrm{j}, \mathrm{i}}(\mathrm{t}) \cdot \Delta \mathrm{t}\right)-\sum_{\mathrm{j} \neq \mathrm{i}} B\left(\mathrm{n}_{\mathrm{i}}(\mathrm{t}), \mathrm{r}_{\mathrm{i}, \mathrm{j}}(\mathrm{t}) \cdot \Delta \mathrm{t}\right)
$$

This approach was employed for the Markov models of $I_{\mathrm{CaL}}, I_{\mathrm{Kr}}, I_{\mathrm{Ks}}, I_{\mathrm{Na}}$ and RyR. In the case of simulations involving $\beta$-adrenergic stimulation, both the non-phosphorylated and phosphorylated channel populations ${ }^{22}$ were simulated using this stochastic formulation.

\section{Stochastic formulations of Hodgkin-Huxley-based models and instantaneous currents}

Hodgkin-Huxley-based models are a subset of the class of Markov models with only independent transitions. ${ }^{40}$ As such, the methodology for stochastic simulation of Markov models was also applied to the Markov representation of the equivalent Hodgkin-Huxley model for $I_{\text {To }}$ and $I_{\text {CI|Ca). For }}$ 'instantaneous' currents that are defined by an algebraic equation in the deterministic model, a simple 2-state (Open and Closed) model was used. The steady state distribution between the two states was defined by the original algebraic equation and a global time constant of $0.1 \mathrm{~ms}$ was used to determine the forward and backward rates between the two states. This approach was employed for stochastic simulations of $I_{K 1}$ and $I_{K u r}$.

\section{Stochastic formulations of pumps and exchangers}

In contrast to channels, where gating is a stochastic process but the flux of ions through an open channel is instantaneous, ion transport through pumps and exchangers is an active process. A single iteration of the pump or exchanger transports a given number of ions across the electrochemical gradient. As such, there is not a single 'open state' that defines the flux of ions but instead there is a state transition (or combination of transitions) that determines the flux of ions. Because of this, the throughput of a single pump is much lower than that of a channel. However, the expression of pumps is much higher than that of channels, ${ }^{41}$ thereby ensuring that an ionic balance can be maintained.

The SR and sarcolemmal $\mathrm{Ca}^{2+}$-ATPases (underlying $\mathrm{I}_{\mathrm{up}}$ and $\mathrm{I}_{\mathrm{pCa}}$, respectively) were simulated using the two-state, four-transition model structure of Tran et al. ${ }^{42}$ Parameters of the models were adjusted to reproduce the $\mathrm{Ca}^{2+}$ dependence of the deterministic formulations. We assumed that the state distribution changes more rapidly than the changes in ionic concentrations on both sides of the pump, resulting in a quasi-equilibrium situation in which individual rates are decoupled. The number 
of ions transported via each transition was derived from a binomial distribution and the net ion flux (or current) in the interval $\Delta \mathrm{t}$ was determined.

Smith and Crampin have previously described a detailed model of the $\mathrm{Na}^{+}-\mathrm{K}^{+}-\mathrm{ATPase}$ based on a consecutive ("ping-pong") model for $\mathrm{Na}^{+}$and $\mathrm{K}^{+}$binding. ${ }^{43}$ In their model, 'slippage' of ions is negligible due to a tight coupling between conformational changes of the pump and ion binding, thereby ensuring a strict $3 \mathrm{Na}^{+}: 2 \mathrm{~K}^{+}$stoichiometry. Based on these assumptions and since both pumps belong to the family of P-type cation transporters, ${ }^{43}$ we employed a similar 2-state model structure as that used for SERCA. We set the 'backward' (counter-clockwise) rates to zero to ensure the strict stoichiometry. Forward rates were fitted to reproduce $\mathrm{Na}^{+}, \mathrm{K}^{+}$and $\mathrm{V}_{\mathrm{m}}$ dependence of the original $I_{\mathrm{Nak}}$ formulation for both the non-phosphorylated and phosphorylated populations.

\section{Number of channels / transporters}

The appropriate number of channels, pumps or exchangers of each type was estimated based on the whole-cell conductance in the model and experimentally-determined single-channel conductance (Table 1). It should be noted that $\overline{\mathrm{N}}_{\mathrm{x}}$ represents the maximum number of effective channels that can open (i.e., the number of channels open when open probability equals 1.0). There may be additional channels in a myocyte that do not open. In particular, if the deterministic formulation of a current $x$ is adjusted such that the open probability is halved and the whole-cell conductance is doubled (giving the same net current), then $\overline{\mathrm{N}}_{\mathrm{x}}$ as given below would be increased by a factor of 2.0. However, because open probability is reduced, the number of channels that participate during the AP would not change.

\begin{tabular}{|c|c|c|}
\hline Current & \# Channels & Source \\
\hline $\mathrm{I}_{\mathrm{CaL}}$ & $\overline{\mathrm{N}}_{\mathrm{CaL}}=110,000$ & $\begin{array}{l}\text { Based on a single-channel conductance of } 3.0 \mathrm{pS} \text { (at } 2.0 \mathrm{mmol} / \mathrm{L} \\
\text { extracellular } \mathrm{Ca}^{2+} \text { in rat ventricular myocytes. }{ }^{44} \text { Consistent with } \\
\sim 80,000 \text { channels as in Restrepo et al. }{ }^{36}\end{array}$ \\
\hline$I_{C \mid(C a)}$ & $\overline{\mathrm{N}}_{\mathrm{Cl}(\mathrm{Ca})}=47,500$ & $\begin{array}{l}\text { Based on a single-channel conductance of } 0.91 \mathrm{pS} \text { (at } 150 \\
\mathrm{mmol} / \mathrm{L} \text { extracellular } \mathrm{Cl}^{-} \text {and } 5.0 \mathrm{mmol} / \mathrm{L} \text { intracellular } \mathrm{Cl}^{-} \text {in canine } \\
\text { ventricular myocytes. }\end{array}$ \\
\hline $\mathrm{I}_{\mathrm{K} 1}$ & $\overline{\mathrm{N}}_{\mathrm{K} 1}=15,500$ & $\begin{array}{l}\text { A single-channel conductance of approximately } 30 \mathrm{pS} \text { has been } \\
\text { reported at high (150 mmol/L) }\left[\mathrm{K}^{+}\right]_{\circ} \text { in a number of species, } \\
\text { including canine ventricular myocytes. }{ }^{46} \text { Based on a square-root } \\
\text { dependence of channel conductance on }\left[\mathrm{K}^{+}\right]_{\mathrm{o}} \text {, a single-channel } \\
\text { conductance of approximately } 5.0 \mathrm{pS} \text { is obtained at physiological } \\
{\left[\mathrm{K}^{+}\right]_{\mathrm{o}} \text {. }}\end{array}$ \\
\hline $\mathrm{I}_{\mathrm{Kr}}$ & $\overline{\mathrm{N}}_{\mathrm{Kr}}=2,200$ & $\begin{array}{l}\text { Using a single-channel conductance of } 2.25 \mathrm{pS} \text { as observed in } \\
\text { mouse }^{47} \text { and } \text { rabbit }^{48} \text { ventricular myocytes, after a similar }\end{array}$ \\
\hline
\end{tabular}




\begin{tabular}{|c|c|c|}
\hline & & correction as applied for $I_{K 1}$. \\
\hline $\mathrm{I}_{\mathrm{Ks}}$ & $\overline{\mathrm{N}}_{\mathrm{Ks}}=10,500$ & $\begin{array}{l}\text { Based on a single-channel conductance of } 4.50 \mathrm{pS} \text { obtained in } \\
\text { Xenopus oocytes expressing KCNQ1 and KCNE1. }{ }^{49} \text { There are, to } \\
\text { the best of our knowledge, no data available from cardiac } \\
\text { myocytes. Estimates in heterologous expression systems range } \\
\text { from } 0.58 \mathrm{ps}^{50} \text { to } 16 \mathrm{ps}^{51}\end{array}$ \\
\hline$I_{\text {Kur }}$ & $\overline{\mathrm{N}}_{\mathrm{Kur}}=100$ & $\begin{array}{l}\text { Yue et al. determined a single-channel conductance of } 20.3 \mathrm{pS} \text { in } \\
\text { canine atrial myocytes at } 5.4 \mathrm{mmol} / \mathrm{L}\left[\mathrm{K}^{+}\right]_{0}{ }^{52} \text { The low number of } \\
\text { channels is consistent with the presence, but very low } \\
\text { expression of an } I_{\text {Kur-like channel in canine ventricle. }{ }^{53}}\end{array}$ \\
\hline$I_{\mathrm{Na}}$ & $\overline{\mathrm{N}}_{\mathrm{Na}}=160,000$ & $\begin{array}{l}\text { Using a single-channel conductance of } 20.1 \mathrm{pS} \text { as observed in } \\
\text { canine, rabbit and guinea-pig ventricular myocytes. }{ }^{54}\end{array}$ \\
\hline $\mathrm{I}_{\mathrm{NaCa}}$ & $\overline{\mathrm{N}}_{\mathrm{NaCa}}=2,000,000$ & Based on a density of $200-400$ exchangers per $\mu \mathrm{m}^{255}$ \\
\hline$I_{\mathrm{NaK}}$ & $\overline{\mathrm{N}}_{\mathrm{NaK}}=10,000,000$ & $\begin{array}{l}\text { Pump-density estimates based on ouabain-sensitive transient } \\
\text { currents range between } 1000 \text { and } 3200 \text { exchangers per } \mu \mathrm{m}^{2} \text { in } \\
\text { ventricular myocytes. }{ }^{56} \text { We employ the ratio of approximately } 1 \\
\mathrm{NaCa}: 5 \mathrm{NaK} \text { that follows from these density estimates. }\end{array}$ \\
\hline$I_{\mathrm{pCa}}$ & $\overline{\mathrm{N}}_{\mathrm{pCa}}=2,000,000$ & Assumed to be similar to $\overline{\mathrm{N}}_{\mathrm{NaCa}}$. \\
\hline $\mathrm{I}_{\text {To }}$ & $\overline{\mathrm{N}}_{\mathrm{To}}=5,900$ & $\begin{array}{l}\text { Based on a single-channel conductance of } 13.0 \mathrm{pS} \text { as measured } \\
\text { by Fedida and Giles in rabbit ventricular myocytes. }{ }^{57}\end{array}$ \\
\hline$I_{\text {rel }}$ & $\overline{\mathrm{N}}_{\mathrm{RyR}}=1,100,000$ & $\begin{array}{l}\text { RyR density is } 10 \text { fold larger than } \overline{\mathrm{N}}_{\mathrm{CaL}} \text {, consistent with } \\
\text { previously published local control models. }{ }^{36,39}\end{array}$ \\
\hline$I_{\text {up }}$ & $\overline{\mathrm{N}}_{\text {SERCa }}=2,200,000$ & Assumed to be of similar magnitude as $\overline{\mathrm{N}}_{\mathrm{RyR}}$. \\
\hline
\end{tabular}

Table 1. Number of channels/transporters simulated in the stochastic model.

\section{Experimental recordings in isolated canine ventricular myocytes}

This investigation conformed with the Guide for the Care and Use of Laboratory Animals published by the US National Institutes of Health (NIH Publication No. 85-23, revised 1996). Animal handling was in accordance with the European Directive for the Protection of Vertebrate Animals Used for Experimental and Other Scientific Purposes (86/609/EU).

Transmembrane APs were recorded from isolated left-ventricular canine midmyocardial myocytes as previously described. ${ }^{8}{ }^{58}$ Briefly, the left anterior descending coronary artery was cannulated and perfused. After $20 \mathrm{~min}$ of collagenase perfusion and subsequent washout of the enzyme, the 
epicardial surface layer was removed from the LV wedge until a depth of $\geq 3 \mathrm{~mm}$ was reached. Softened tissue samples were collected from the midmyocardial layer underneath while contamination with the endocardium was avoided. Samples were gently agitated, filtered and washed. Cells were stored at room temperature in standard buffer solution. Only quiescent rodshaped cells with clear cross-striations were used for the experiments within $48 \mathrm{~h}$ of isolation. APs were recorded at $37{ }^{\circ} \mathrm{C}$ using high-resistance (30-60 M $\Omega$ ) glass microelectrodes filled with $3 \mathrm{M} \mathrm{KCl}$ with a microelectrode amplifier (Axoclamp-2B, Axon Instruments, Inc). Intracellular pacing was applied at various cycle lengths ( $C L s ; 500 \mathrm{~ms}-2000 \mathrm{~ms}$ ). Only cells showing a stable spike-and-dome AP morphology and resting membrane potential were accepted for the experiments.

\section{RESULTS}

\section{Stochastic channel gating contributes to BVR}

AP recordings from isolated canine ventricular myocytes showed beat-to-beat variability in APD (Figure 3A, top panel) consistent with previous reports. ${ }^{1,8}$ In contrast, under physiological conditions, APD in the deterministic model converged to a steady-state without APD variability (Figure 3A, second panel). In previous research, stochastic processes were simulated using either stochastic differential equations, ${ }^{28,38}$ or by simulating stochastic state-transitions in the Markov models of various ion channels. ${ }^{24,36,39}$ Application of both methodologies to the Markov model of $\mathrm{I}_{\mathrm{kr}}$ resulted in BVR. However, these two approaches showed different temporal dynamics (Figure 3B). Poincaré plots of $A P D_{i+1}$ versus $A P D_{i}$ have a circular shape under these conditions in experimental recordings and simulations with stochastic channel gating, indicating similar magnitudes of short(STV) and long-term variability (LTV; average distance perpendicular to and along the line of identity, respectively; Figure 3B, inset). In contrast, BVR in simulations employing stochastic differential equations of gating variables (based on a Langevin term) was predominantly long-term, resulting in a STV to LTV ratio that was markedly different from experimental recordings (Figure $3 C$ ). As such, stochastic differential equations do not appear to be suitable to model BVR. All subsequent results are from simulations employing stochastic channel gating. 
A.
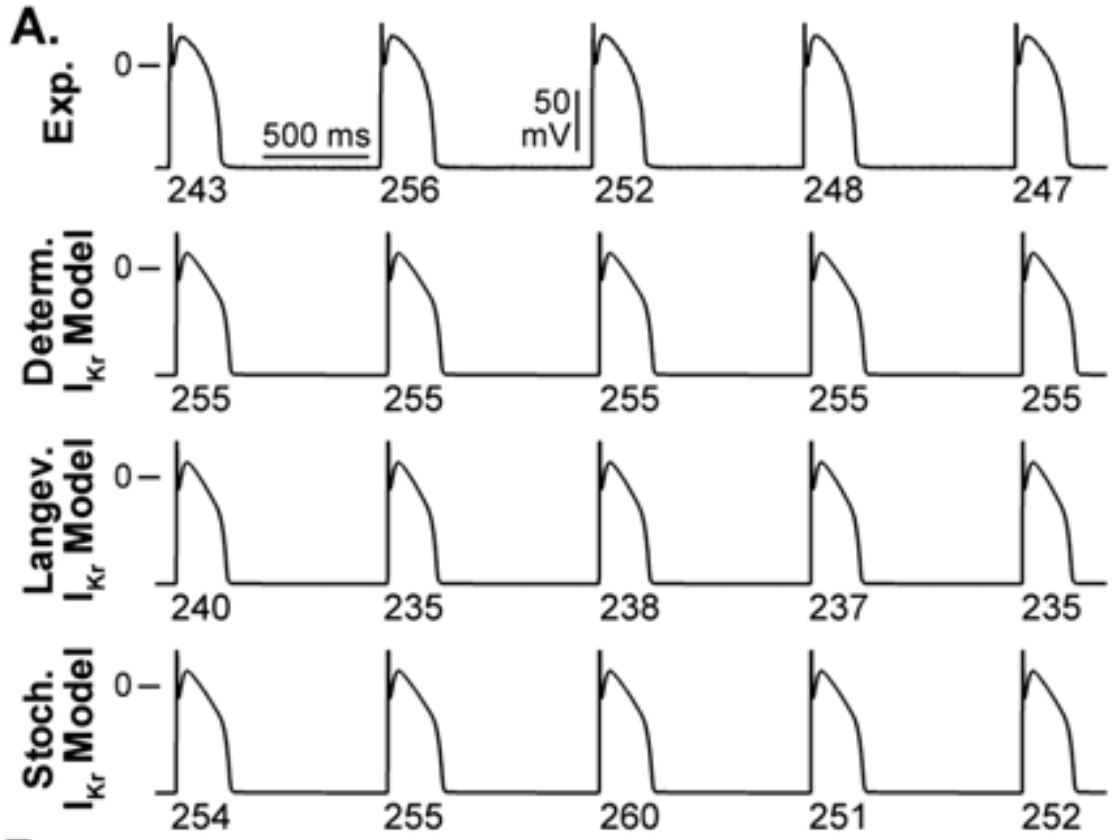

B.
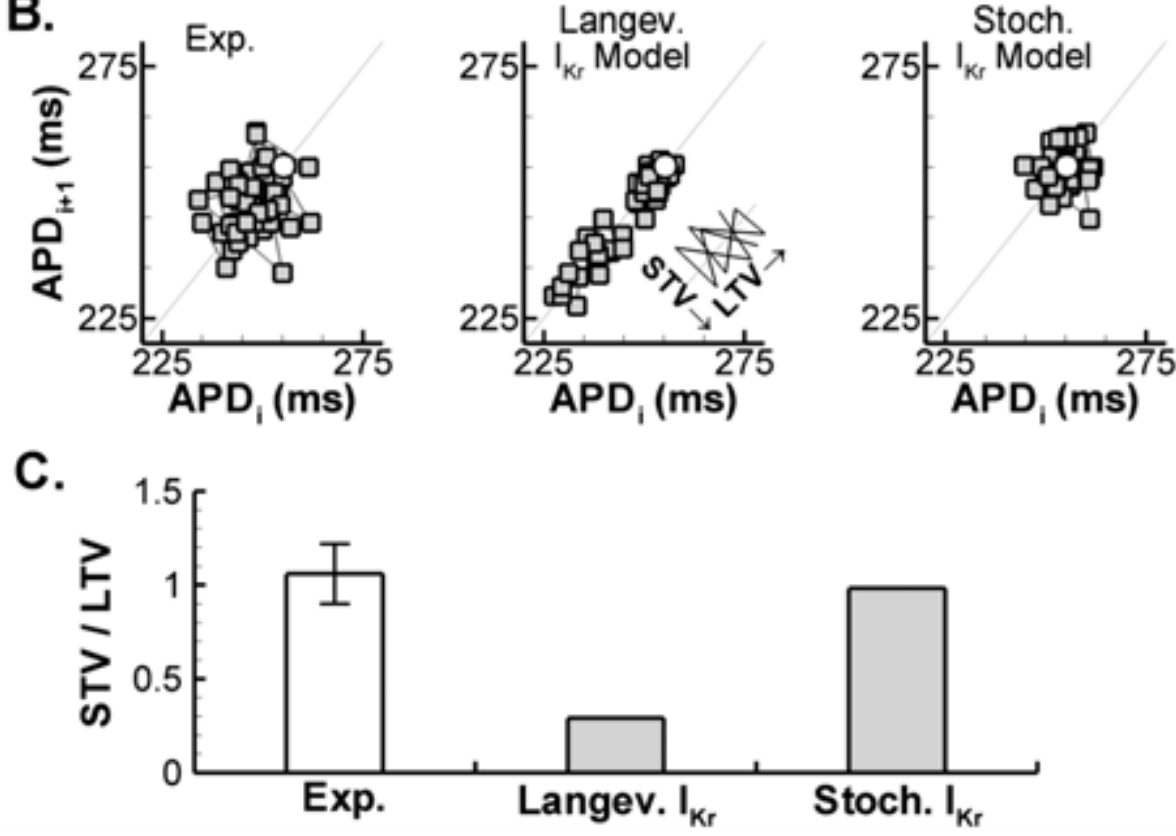

Figure 3. Stochastic channel gating contributes to BVR in a computational model of the canine ventricular myocyte. A. 5 consecutive APs in a representative canine ventricular midmyocardial cell, the deterministic computational model, the model with Langevin $I_{\mathrm{kr}}$ formulation, or the model with a stochastic Markov formulation of $\mathrm{I}_{\mathrm{Kr}}$ (top to bottom) at 1000-ms pacing CL. APD (ms) is indicated below each beat. B. Poincaré plot of 45 consecutive APDs for the conditions in (A). The white circle in each panel indicates the steady-state APD of the deterministic model. C. Relative amount of STV versus LTV in experiments, with Langevin $I_{\mathrm{kr}}$ or with stochastic Markov formulation of $I_{\mathrm{kr}}$. Langevin and stochastic Markov formulations resulted in different compositions of BVR. 


\section{Ionic contributors to BVR in physiological conditions}

A.
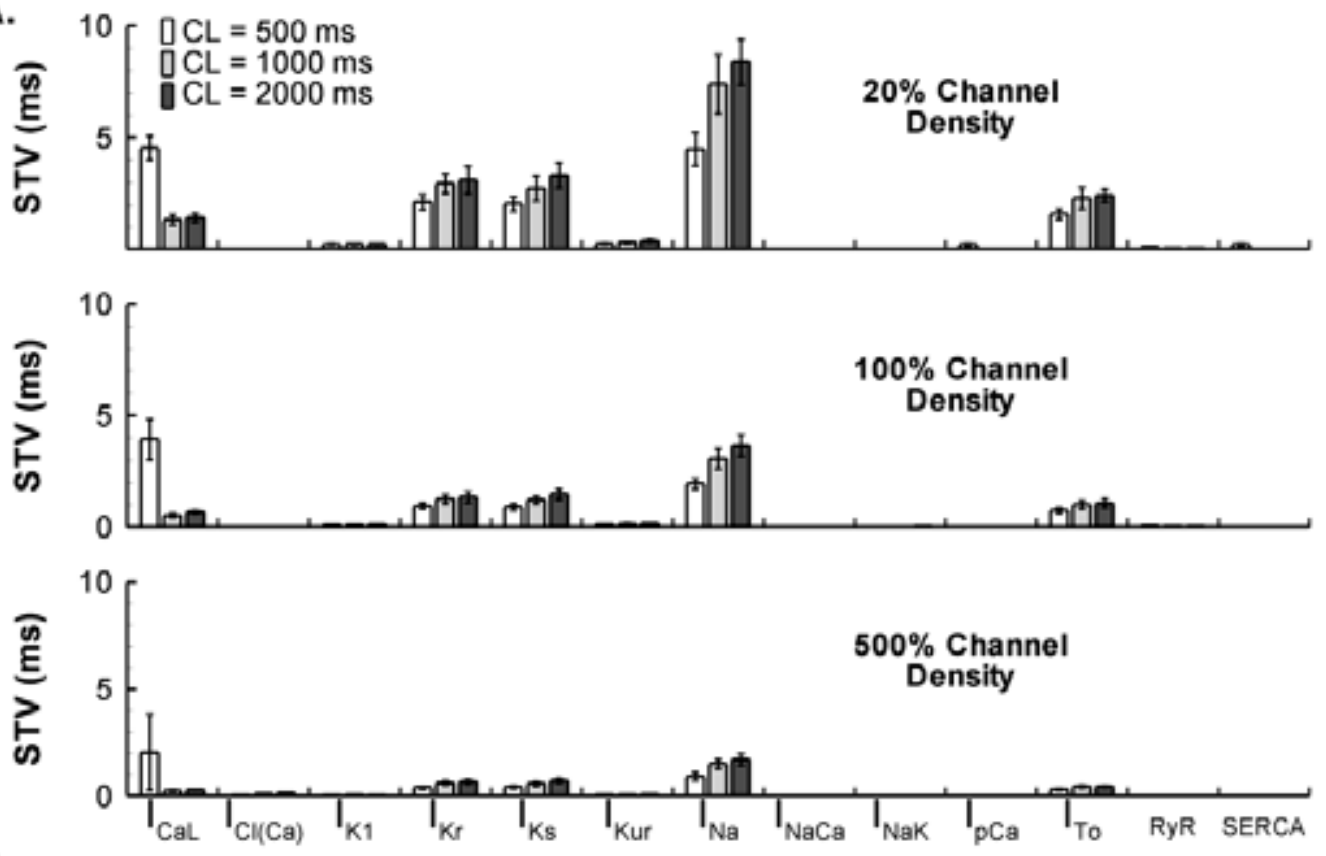

B.
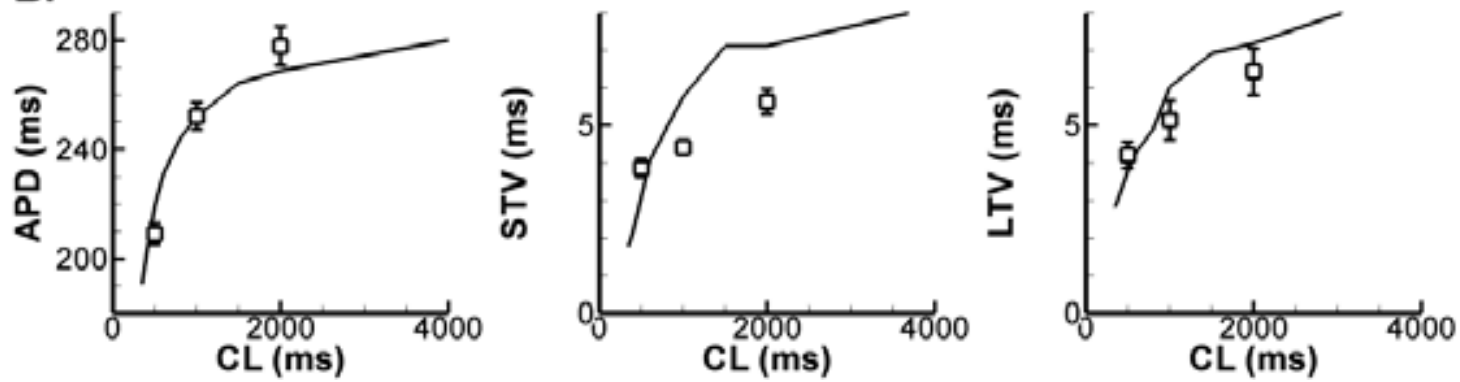

Figure 4. Contribution of channel density and conductance of individual stochastic ion currents to BVR, and rate dependence. A. STV magnitude induced by stochastic channel gating of individual currents in an otherwise deterministic model at CL of $500 \mathrm{~ms}, 1000 \mathrm{~ms}$ or $2000 \mathrm{~ms}$. Top panel shows 5 -fold reduction in channel density (with 5 -fold increase in single-channel conductance), middle panel shows channel density based on estimates from experimental data (Table 1 ) and bottom panel shows 5-fold increase in channel density with reduced single-channel conductance. B. Rate dependence of average APD (left), STV (middle) and LTV (right) in experiments and model with stochastic gating of all 13 targets combined at $100 \%$ channel density.

To obtain insight in the direct contribution of the stochastic gating of ion currents/fluxes to wholecell BVR, we performed simulations with stochastic formulations of each individual channel/pump/transporter in an otherwise deterministic model at cycle lengths (CLs) of 500, 1000 and $2000 \mathrm{~ms}$ (Figure 4A). The number of channels underlying each current was estimated based on experimentally obtained single-channel conductance and whole-cell conductance in the model (Table 1). To investigate the sensitivity of this parameter, we simulated normal channel density as well as a 5-fold increase or decrease in channel density (offset by a reciprocal change in singlechannel conductance to maintain the same total current). A lower channel density (with larger single-channel conductance) resulted in a larger STV for all stochastic simulations. Interestingly, 
however, a large difference between the impacts of individual ion currents could be observed with the largest contribution by $I_{\mathrm{Na}}$ and $I_{\mathrm{Kr}}$ to STV under these conditions (Figure $4 \mathrm{~A}$ ). $I_{\mathrm{Ks}}$ also had a substantial impact on BVR, despite its small effect on APD under basal, isolated-myocyte conditions, ${ }^{59}$ consistent with results by Pueyo et al. ${ }^{28}$ In contrast, pumps and exchangers, which have relatively low individual throughput but high expression density, ${ }^{41}$ contributed little to BVR. In general, BVR increased with increasing CL. When all 13 stochastic targets were combined, STV was larger than that obtained with any individual stochastic formulation but the results were not additive, indicating that certain stochastic fluctuations cancel each-other out. The stochastic model showed APD and BVR rate dependence similar to that observed in canine ventricular myocytes (Figure 4B), indicating that stochastic channel gating (particularly of $I_{\mathrm{Na}}$ and $I_{\mathrm{Kr}}$ channels) is a major contributor to the 'baseline' BVR observed in physiological conditions.

In addition to a direct impact on $\mathrm{V}_{\mathrm{m}}$ fluctuations, stochastic channel gating may modulate STV indirectly (e.g., via fluctuations in intracellular ion concentrations which affect other currents). To quantify the overall contribution of individual ion currents to BVR in the fully stochastic model, we employed the linear regression method recently proposed by Sarkar and Sobie. ${ }^{60}$ We simulated 300 parameter sets in which the current density of each of the currents was scaled based on a Gaussian distribution with mean 1.0 and standard deviation 0.3. For each parameter set, mean APD, STV and LTV were determined at steady-state during pacing at 1000-ms CL (Figure 5). The contribution of each current was determined by performing a linear regression on the parameter settings and output measures (Figure 5A). Consistent with the results based on the direct stochastic impact shown in Figure 4, the linear regression analysis identified major roles for $I_{\mathrm{Na}}$ and $I_{\mathrm{kr}}$ in modulating both APD and STV (Figure 5B). In addition, this approach also identified a substantial impact of $I_{\text {Nak, }}$ $I_{\mathrm{NaCa}}$ and SERCA on STV. Because the stochastic gating of these currents/fluxes did not result in significant BVR when simulated in an otherwise deterministic model, it follows that (cell-to-cell) variations in the maximal conductance of these targets affect STV via other parameters (e.g., APD, intracellular ion concentrations, etc.), which remains to be confirmed experimentally. 
A.

Parameters

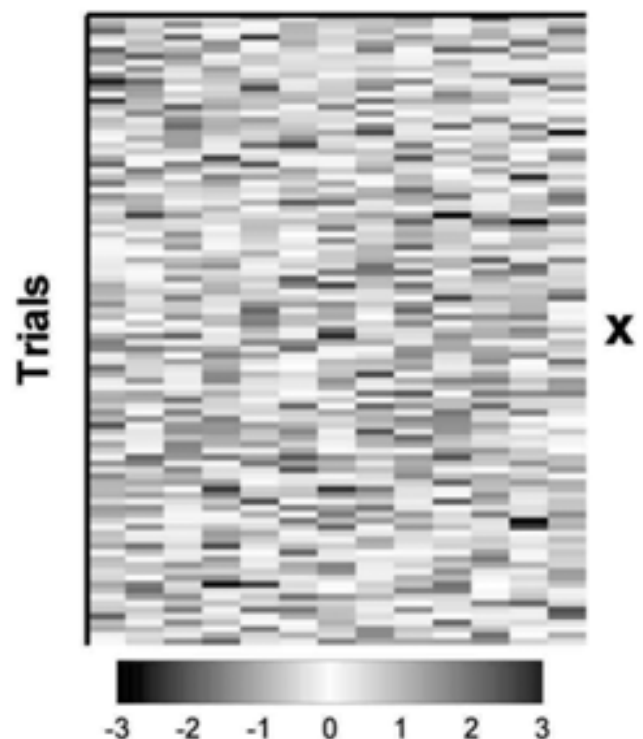

B.

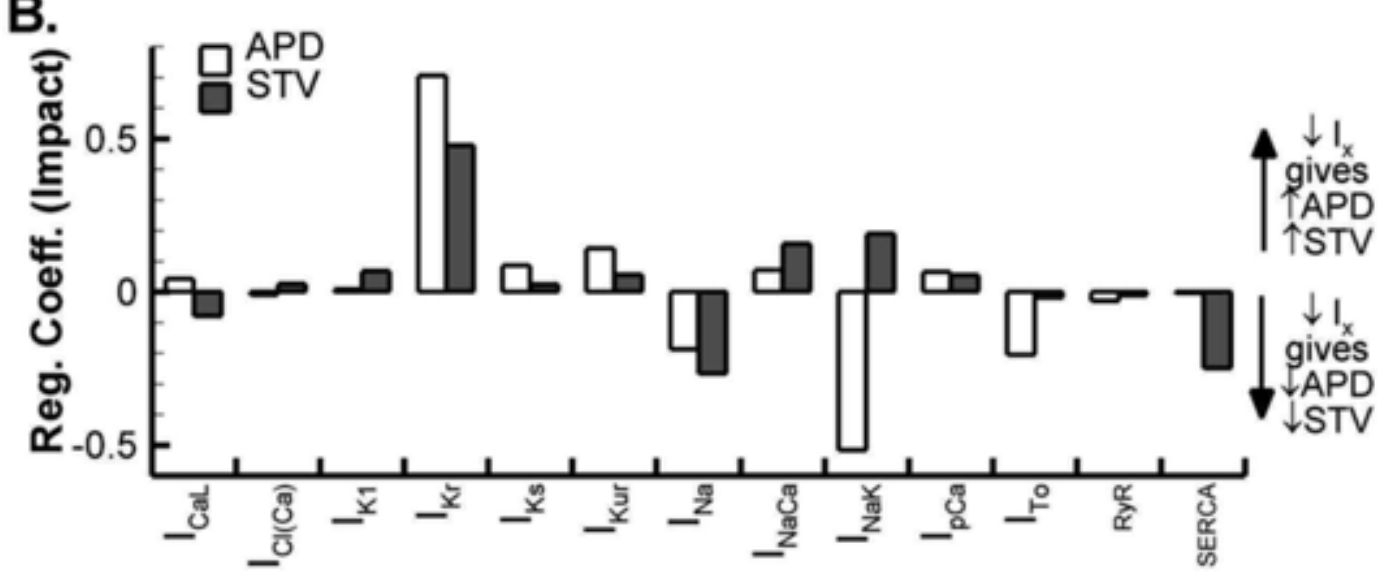

Figure 5. Contribution of currents to BVR determined via linear regression. A. Relative changes in the maximal conductance of the 13 currents/fluxes (lanes correspond to the column-pairs in panel B) for 100 (out of 300) trials (left panel) and corresponding changes in outputs (APD, STV and LTV) during steady-state pacing at $\mathrm{CL}=1000 \mathrm{~ms}$ (right panel). Middle panel shows the coefficients that indicate the contribution of each current to every output measure as determined via linear regression. B. Bar plot of the magnitude of the coefficients from panel A regarding their impact on APD (white bars) or STV (shaded bars). $I_{\mathrm{kr}}$ and $I_{\mathrm{Na}}$ have a large impact on both APD and BVR, consistent with the results from Figure 4. In addition, $I_{\text {Nak }}$ and $\mathrm{Ca}^{2+}$ uptake (SERCA) also affect STV. LTV showed similar pattern as STV and was not shown for the sake of clarity. For color figure, see page 255.

Zaniboni et al. ${ }^{1}$ have previously shown that the electrical coupling of two myocytes reduced their temporal variability, which was confirmed in the modeling study by Lemay et al. ${ }^{27}$ When identical cells were coupled, the overall temporal variability (coefficient of variance: $100 \% \times \operatorname{std}(A P D) /$ mean(APD)) in the model was reduced from $2.02 \%$ to $1.57 \%$, quantitatively similar to that observed by Zaniboni et al. in guinea-pig ventricular myocytes $(2.3 \pm 1.2 \%$ in uncoupled cells vs. $1.5 \pm 0.6 \%$ in 
cell-pairs). We observed a slight reduction in BVR (of $0.7 \mathrm{~ms}$ ) in cell-pairs compared to uncoupled cells when two identical cells were coupled (Figure 6A). Gap junction conductance did not influence BVR over the range of values that would result in successful propagation in a 1-dimensional strand. ${ }^{61}$ Interestingly, Zaniboni et al. also reported that there was an asymmetrical redistribution of APD when a cell with a long APD was coupled to a cell with short APD, whereby the long APD shortened more than the short APD prolonged. ${ }^{1}$ We hypothesized that this asymmetrical response may also apply to BVR and prolonged APD in one of the two cells through the injection of a constant, deterministic current for the duration of the APD (Figure 6B). BVR was larger in the prolonged cell, thereby increasing the average BVR. When the two cells were coupled, spatial APD dispersion was lost. Although BVR remained larger than that of the regular cell-pair, the decrease in average BVR compared to the uncoupled situation was more pronounced (1.0 ms; Figure 6B, inset). These data suggest that cell-to-cell coupling not only reduces spatial dispersion of repolarization but may also limit excessive BVR of vulnerable regions. As such, conditions in which coupling is reduced (e.g., in ischemia) may lead to increased BVR.
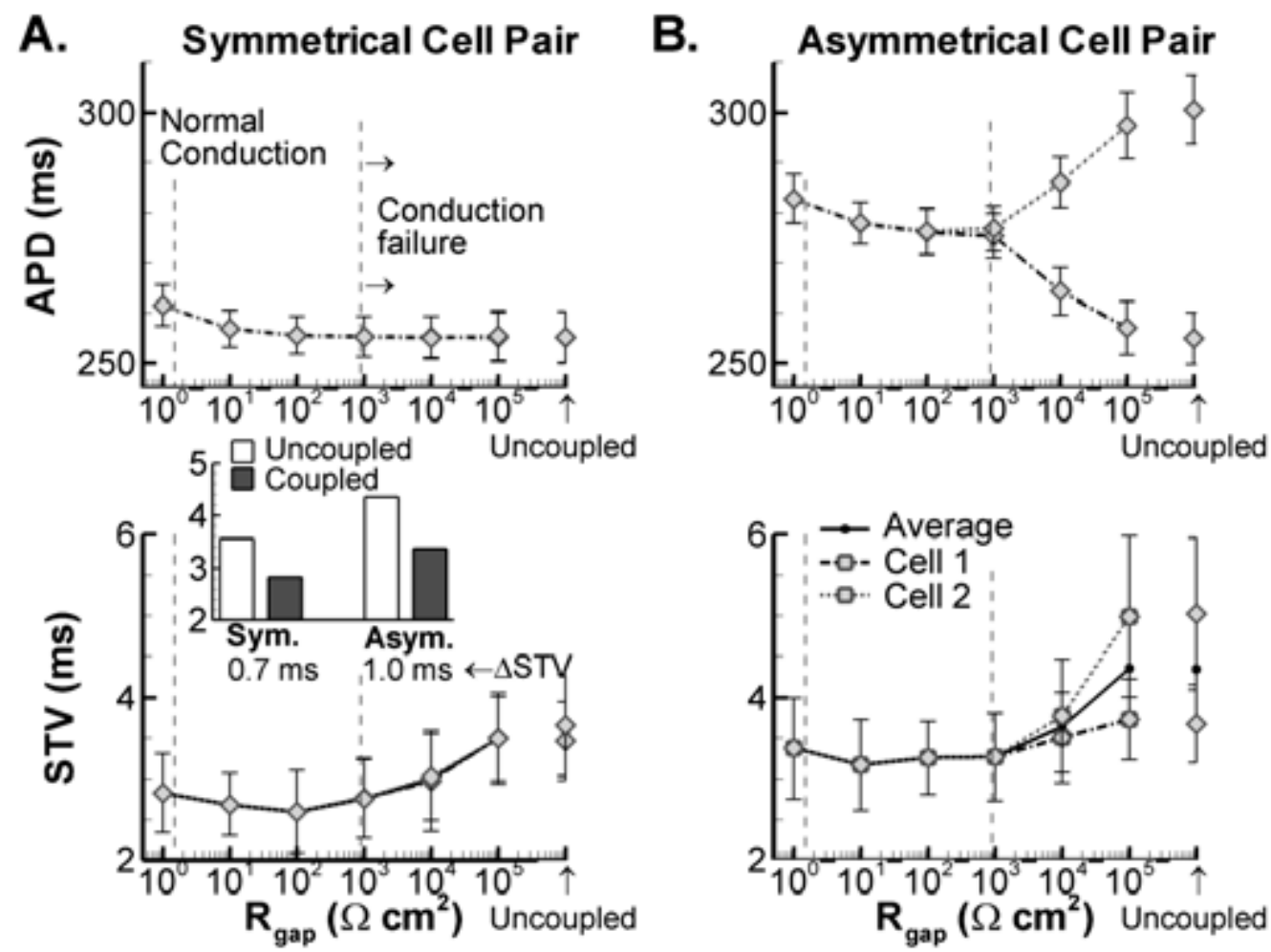

Figure 6. Effect of cell-to-cell coupling on BVR. A. APD (top panel) and STV (bottom panel) of two identical cells for various degrees of electrical coupling. Normal coupling (left vertical dashed line) and critical coupling for successful conduction in a one-dimensional strand of virtual myocytes (right vertical dashed line) are indicated. B. Similar to panel A for two cells of which one is prolonged via current injection. Cell-to-cell coupling causes a mild decrease in average STV that is more pronounced in the case of an asymmetrical cell pair. 


\section{BVR rate dependence}

Recent research has shown that APD reverse rate dependence is an intrinsic property of canine cardiac preparations. ${ }^{62}$ We hypothesized that the reverse rate dependence of BVR (Figure 4B) could be due to at least two components: (i) rate-dependent changes in the magnitude of stochastic channel gating ("active component") and/or (ii) dependence on APD and the intrinsic reverse ratedependence of APD ("intrinsic component"). ${ }^{62}$ To investigate the contribution of these components, membrane potential and net membrane current $\left(V_{m}\right.$ and $\left.I_{m}\right)$ were recorded for 30 beats at steady state and the standard deviation over these 30 beats was determined at each time point. The magnitude of $I_{m}$ variation was $C L$ dependent (Figure 7A,B), suggesting a role for the active component. Moreover, when the $\operatorname{Std}\left(I_{m}\right)-V_{m}$ relationship at $1000-m s C L$ was used to generate a stochastic $I_{m}$ in the deterministic model paced at various $C L, B V R$ rate dependence was blunted (Figure $7 \mathrm{C}$ ) but not eliminated. The remaining BVR rate dependence reflects the intrinsic component (i.e., even with the same amount of 'noise' BVR is larger at long CL). BVR increased when APD was prolonged through injection of a (deterministic) current in either the stochastic model (Figure 7D) or the deterministic model with fixed $I_{m}$ stochastics (not shown). Interestingly however, there were no clear differences in BVR between CL of 500, 1000 or $2000 \mathrm{~ms}$ for any given APD (Figure 7D). This indicated that although both $\mathrm{CL}$ dependence of stochastic properties and intrinsic components contribute to reverse rate dependence of BVR, the former was also strongly dependent on the intrinsically longer APD at slow CL.
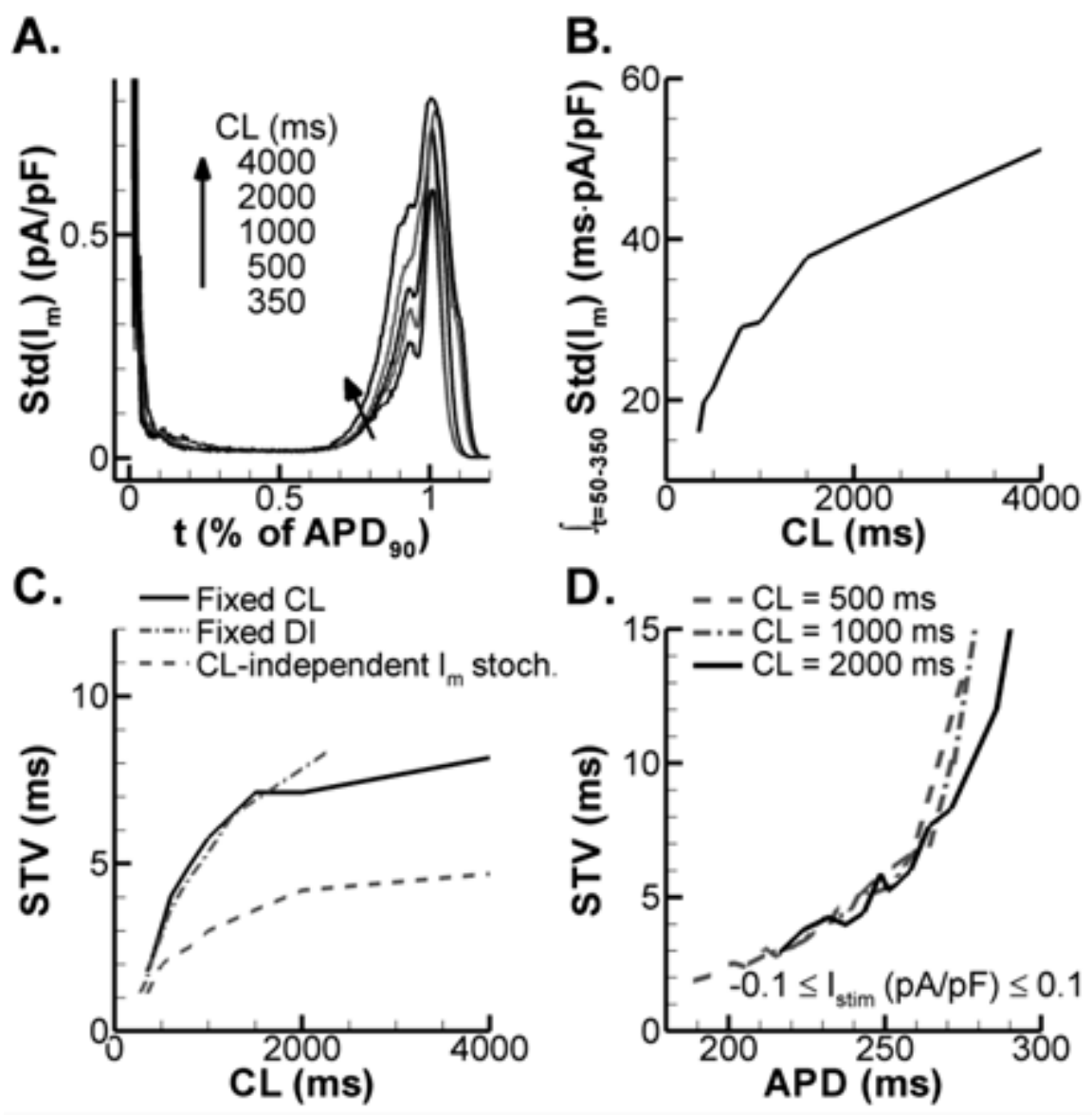
Figure 7. Role of APD and stochastic gating in BVR reverse rate dependence. A. Magnitude of channel gating stochastics (assessed by $\operatorname{Std}\left(\mathrm{I}_{\mathrm{m}}\right)$ for 50 beats) over time for $\mathrm{CL}$ of $350-4000 \mathrm{~ms}$ in the fully stochastic model under baseline conditions. B. Rate dependence of total magnitude of $I_{m}$ fluctuations (given by area under $\operatorname{Std}\left(I_{m}\right)$ curve). C. STV rate dependence in the fully stochastic model during fixed-CL pacing (solid line) or fixed-DI pacing (dash-dotted line), or in the deterministic model during fixed-CL pacing with a $\mathrm{CL}$-independent stochastic term added to $\mathrm{I}_{\mathrm{m}}$ (dashed line). CLindependent stochastic behavior results in a blunted STV rate dependence. D. STV vs. APD relationship at CLs of $500 \mathrm{~ms}$ (dashed line), $1000 \mathrm{~ms}$ (dash-dotted line) or $2000 \mathrm{~ms}$ (solid line). APD was varied through injection of a deterministic stimulus current between -0.1 and $0.1 \mathrm{pA} / \mathrm{pF}$ for the duration of the AP.

In the chaotic models of Sato et al. beat-to-beat APD differences occur through a steep APD/diastolic interval (DI) relationship. ${ }^{25}$ To assess the importance of restitution for BVR in the absence of EADs, we analyzed data from the stochastic model using fixed diastolic interval pacing. An identical $B V R / C L$ dependence (determined via $C L=$ mean $A P D+D I$ ) was obtained with fixed $C L$ and fixed $D I$ pacing (Figure 7C), indicating that under these physiological conditions variations in DI do not contribute to $B V R$ rate dependence. In agreement with this conclusion, we found only a weak correlation between the DI and APD of the following beat in canine ventricular myocytes and simulations (correlation coefficients of $0.39 \pm 0.06$ and 0.15 at 1000 -ms CL, respectively; data not shown).

\section{Mechanisms contributing to exaggerated BVR in pathological conditions}

We previously reported that BVR is increased in pharmacological models of long-QT syndrome (LQT) type 2 (using the $I_{\mathrm{kr}}$-blocking drug dofetilide) and LQT3 (increased persistent $I_{\mathrm{Na}}$ due to ATXII) and that this BVR could be reduced by $\beta$-adrenergic stimulation ( $\beta A R S)$. In contrast, in a pharmacological LQT1 model, $\beta A R S+I_{K s}$ inhibition (using HMR1556) increased BVR, whereas HMR1556 alone had no effect on BVR. $^{8}$ The model was able to reproduce these experimental findings (Figure $8 A, B$ ), indicating that it can be employed to study the factors contributing to exaggerated BVR in LQT1-3.

The contribution of both average APD and CL-dependent changes in $I_{m}$ stochastics to BVR rate dependence (Figure 7) suggest that the increased BVR observed in pharmacological models of longQT syndrome (Figure 8) could be due to APD prolongation and/or alterations in ion-channel gating. Similarly, the rescue of BVR by $\beta A R S$ in the LQT2 and LQT3 models could be due solely to a reduction in APD or could be due to other factors. To investigate the effect of APD prolongation on BVR, we employed a deterministic current injection to reduce average APD back to baseline levels. When APD was reduced, BVR also reduced to control values (STV equaled $3.5 \pm 0.4,7.7 \pm 1.5$ and $3.9 \pm 0.6$ in control, LQT2, and LQT2 with reduced APD, respectively; Figure 9A). In contrast, removing the stochastic gating of $\mathrm{I}_{\mathrm{kr}}$ did not significantly alter BVR $(8.1 \pm 1.5 \mathrm{~ms})$. 


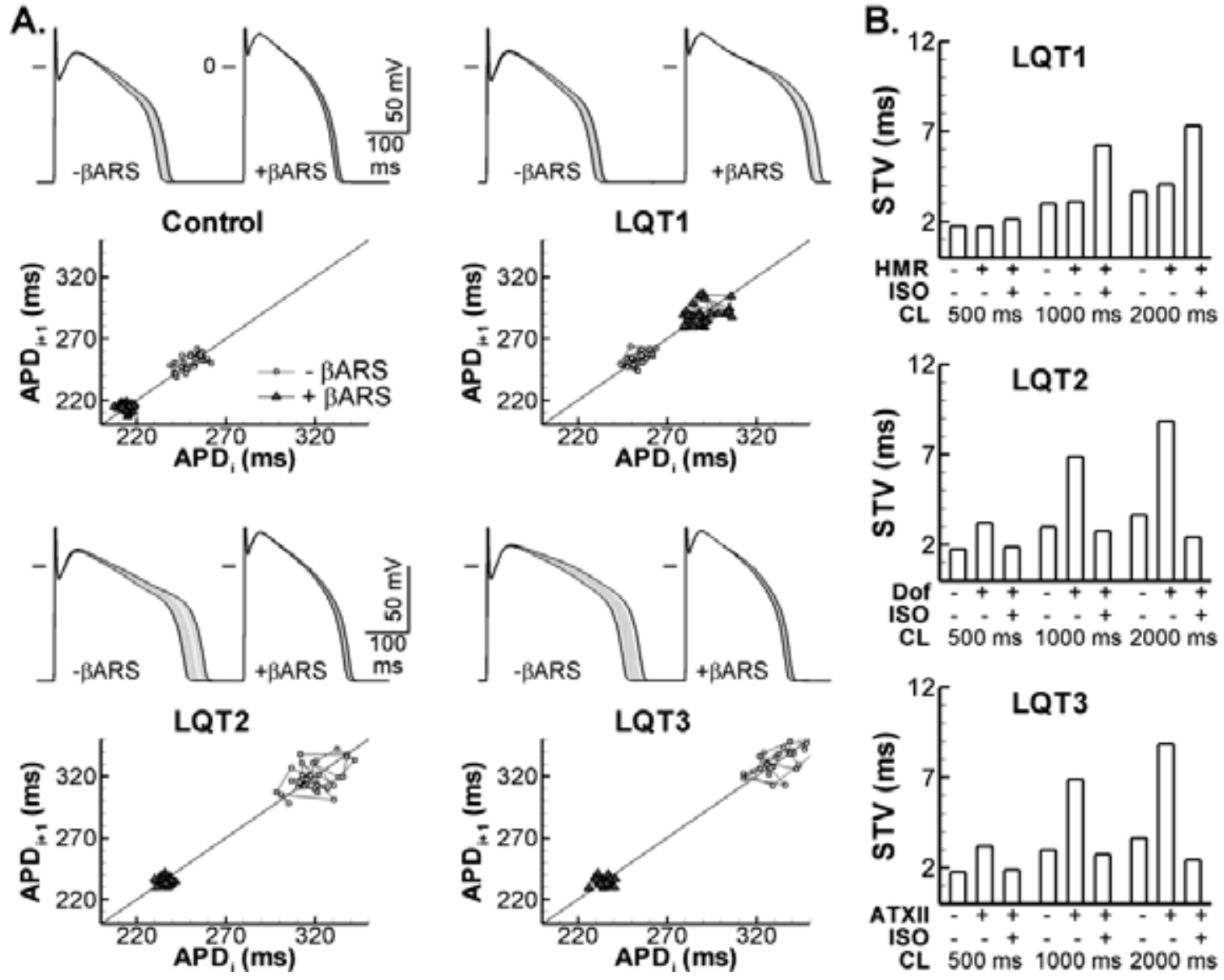

Figure 8. BVR in simulated LQT syndromes type 1-3 in the absence or presence of $\beta A R S$. A. Overlay of 30 consecutive APs in the absence (- $\beta A R S)$ or presence (+ $\beta A R S)$ of $\beta$-adrenergic receptor stimulation in control conditions (top-left panel) or simulated LQT1 (top-right panel), LQT2 (bottomleft panel), or LQT3 (bottom-right panel). Shortest and longest APs are shown in black, intermediate APs in grey. A Poincaré plot of the 30 APDs is shown below. B. Quantification of BVR in LQT1-3 at CL of 500,1000 or $2000 \mathrm{~ms}$ in the absence or presence of $\beta$ ARS. HMR indicates simulation of the $I_{\mathrm{Ks}}$ blocker HMR1556 (simulated LQT1), Dof simulation of the $I_{\mathrm{kr}}$ blocking drug dofetilide (LQT2) and ATXII indicates simulations with enhanced persistent $I_{\text {Na }}$ (LQT3). $\beta A R S$ reduces BVR significantly in LQT2 and LQT3, but not in LQT1, consistent with experimental results. ${ }^{8}$

When STV was plotted against average APD for individual canine ventricular myocytes or individual model cells generated based on a Gaussian distribution of conductances (similar to the approach for Figure 5A), an exponential relationship was obtained. There was no difference in the parameters for the STV vs. APD relationship between experiments and model or between control and LQT2 conditions (Figure 9B,C). These data indicate that the model is able to quantitatively reproduce experimental BVR characteristics covering a range of cell-to-cell differences. Moreover, these data suggest that the APD prolongation is the main determinant for the increased BVR in LQT2. 
A.

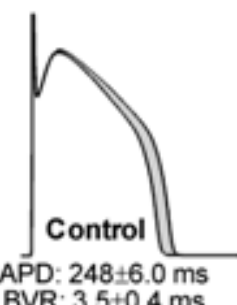

BVR: $3.5 \pm 0.4 \mathrm{~ms}$

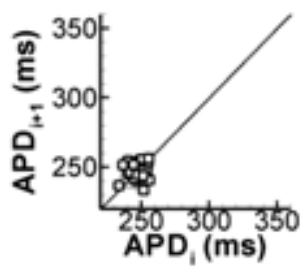

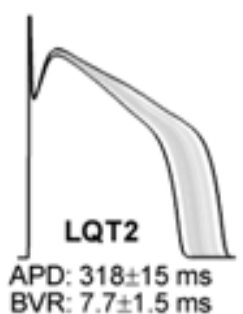
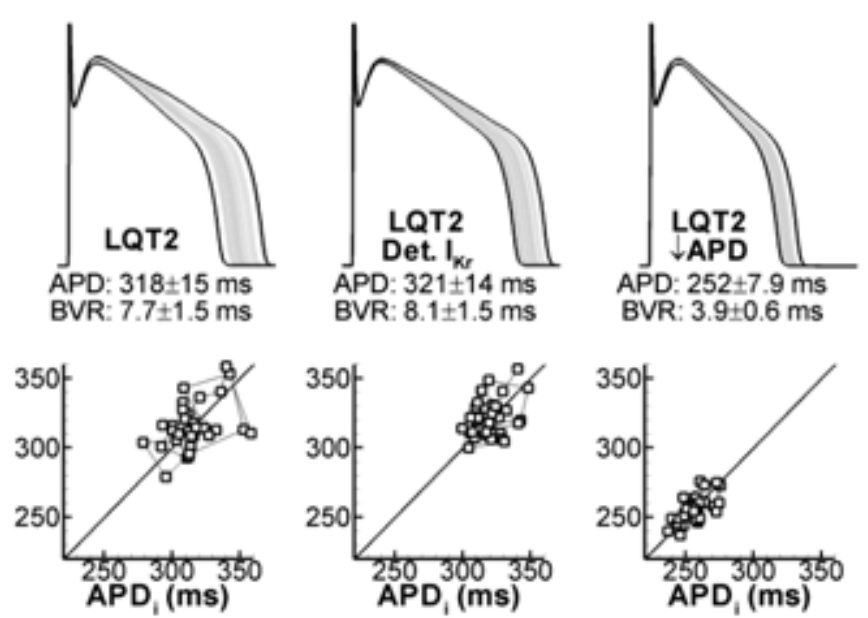
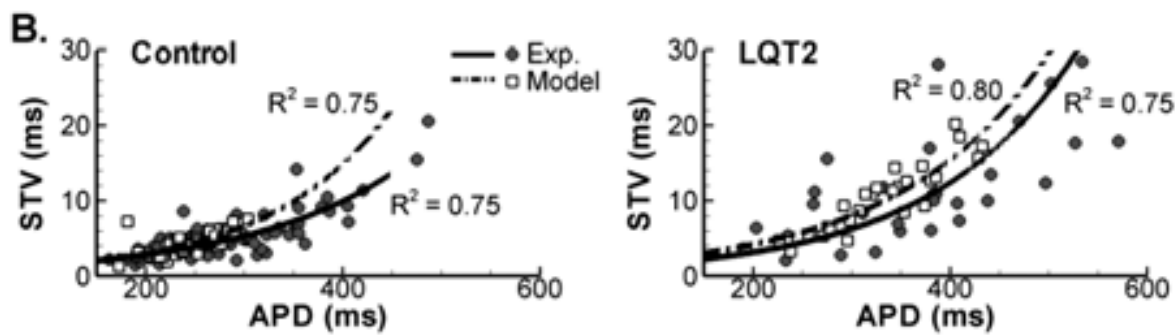

C.

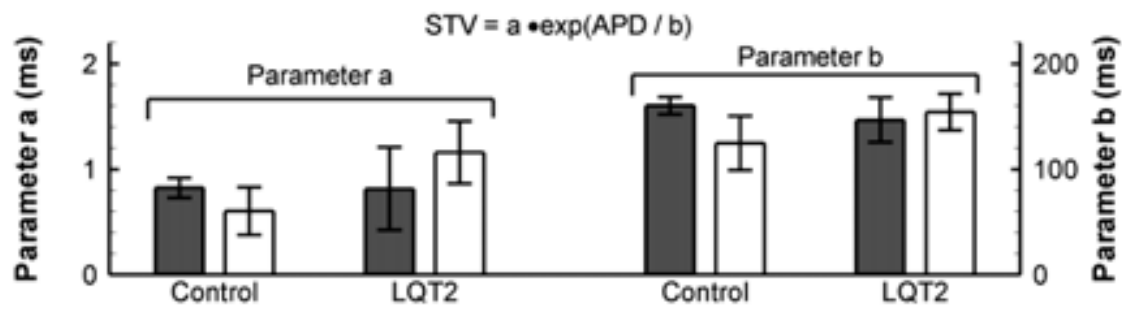

Figure 9. Role of APD in the observed increase in BVR under simulated LQT2 conditions. A. Overlay of 30 consecutive APs under control conditions, simulated LQT2, simulated LQT2 with deterministic $\mathrm{I}_{\mathrm{kr}}$ or simulated LQT2 with reduced APD due to injection of a deterministic stimulus current. Shortest and longest APs are shown in black, intermediate APs in grey. APD, STV and Poincaré plots are shown below each overlay. B. STV vs. APD relationship under control conditions (left panel) or LQT2 conditions (right panel) in individual canine ventricular myocytes (filled symbols) or individual model cells (open symbols; based on whole-cell conductances drawn from a Normal distribution, as in Figure 5A). Data were fit with a monoexponential function (lines). C. Parameters of the monoexponential fits of panel B under control and LQT2 conditions in experiments (grey bars) and model (white bars). The model shows a quantitatively similar STV vs. APD relationship as experiments and this relationship is not different between control and LQT2 conditions.

\section{DISCUSSION}

In this study, we developed a novel model of the canine ventricular myocyte electrophysiology including stochastic gating of all major ion currents and $\mathrm{SR} \mathrm{Ca}^{2+}$ handling processes. The model showed APD and BVR rate dependence consistent with experimental data from canine ventricular myocytes. Using this model, we obtained the following novel insights into the ionic contributors to BVR: i) stochastic channel gating (mainly of $I_{\mathrm{Na}}$ and $I_{\mathrm{Kr}}$ ) strongly contributes to baseline BVR; ii) BVR is more pronounced in cells with a strong plateau than in cells with triangular AP morphology; iii) BVR 
is reduced by cell-to-cell coupling, particularly in the case when one of the two cells has an increased BVR; iv) the rate dependence of BVR is due to active components and intrinsic components, but not dependent on variations in $\mathrm{DI}$; and v) APD prolongation strongly increases BVR but is not the sole determinant of exaggerated BVR in drug-related/-induced conditions.

\section{Relation to existing computational models}

Despite the experimental evidence of an important role for BVR as an indicator of proarrhythmic risk, $^{2,63}$ few computational models to date have incorporated temporal variability of repolarization. Wilders and Jongsma were among the first to examine stochastic channel gating in a computational cardiac cell model for their investigation of beating-rate variability in sinoatrial node cells. ${ }^{64}$ Subsequently, Tanskanen et al. employed a local control model of the canine ventricular myocyte to investigate the role of stochastic gating of L-type $\mathrm{Ca}^{2+}$ channels in EAD formation. ${ }^{24}$ These authors also provided a mathematical analysis suggesting/indicating that increased voltage noise skewed the distribution of APD towards longer APDs, enhancing the susceptibility to EADs. ${ }^{65}$ In contrast, Sato et al. have shown that the EADs observed in their model of the $\mathrm{H}_{2} \mathrm{O}_{2}$-treated rabbit ventricular myocyte were chaotic and not due to stochastic fluctuations. ${ }^{26}$ However, stochastic channel gating resulted in an increased variety in temporal dynamics of the chaotic model. ${ }^{25}$ Pueyo et al. also found that stochastic channel gating favored the occurrence of alternans and EAD generation during $I_{\mathrm{kr}}$ blockade. ${ }^{28}$ However, both Sato et al and Pueyo et al. only considered stochastic gating of $\mathrm{I}_{\mathrm{ks}}$. Lemay et al. adapted the Luo-Rudy dynamic model of the guinea-pig ventricular myocyte to investigate the role of stochastic gating and protein turnover of a selected number of currents on APD variability and intercellular conduction delays under physiological conditions. ${ }^{27}$

The results presented here provide a significant extension of these previously developed models by considering both stochastic gating of all major ion currents (except background currents) and $\mathrm{Ca}^{2+}$ handling processes. Moreover, we show that the stochastic model is quantitatively consistent with experimental measures of BVR in isolated canine ventricular myocytes and identify contributors to BVR in physiological and pathological conditions.

\section{BVR as a proarrhythmic marker}

Beat-to-beat variability of repolarization duration has been proposed as a more reliable proarrhythmic marker than prolongation of repolarization per se, at least for specific pathological conditions. ${ }^{2-4}$ However, its exact determinants remain incompletely understood. Our data indicate that BVR, determined largely by stochastic channel gating during baseline conditions, is modulated by a number of factors that may play a role in arrhythmogenesis.

We find that AP morphology and duration (Figure 6) affect BVR. Increased APD and a pronounced AP plateau result in a larger window in which depolarizing and repolarizing currents are in balance, creating a vulnerable window in which relatively small fluctuations could induce an EAD which may 
lead to triggered activity and/or regional dispersion of repolarization. Both Pueyo et al. ${ }^{28}$ and Tanskanen et al. $^{24}$ have shown that stochastic fluctuations in channel gating (of $I_{\mathrm{Ks}}$ and $I_{\text {CaL }}$, respectively) can indeed facilitate the development of EADs (however, see Sato et al. ${ }^{26}$ ). These data suggest that BVR reflects the robustness of repolarization and, when exaggerated, the risk of EAD development.

In addition to EADs induced by reactivation of $I_{\mathrm{CaL}}$ during a prolonged AP plateau, abnormal $\mathrm{Ca}^{2+}$ handling has been shown to be able to induce early- and delayed afterdepolarizations. ${ }^{66}$ As such, the consideration of both stochastic $\mathrm{Ca}^{2+}$ handling and ion-channel gating in the model presented here is important. Previous experimental data from our group have shown that buffering of intracellular $\mathrm{Ca}^{2+}$ (using BAPTA) can suppress BVR during $\beta$ ARS and $\mathrm{I}_{\mathrm{Ks}}$ blockade in single ventricular myocytes. ${ }^{8}$ Furthermore, in a pharmacological LQT2 model in intact rabbit hearts, abnormal $\mathrm{Ca}^{2+}$ handling also preceded fluctuations in membrane potential. ${ }^{67}$ We found no direct contribution of individual $\mathrm{Ca}^{2+}$ handling proteins to BVR under baseline conditions (Figure 4). However, alterations in $\mathrm{Ca}^{2+}$ homeostasis can have a significant impact on BVR, as is evident from the large impact of SERCA on STV observed in our regression analysis (Figure 5). Moreover, the strong positive feedback characteristics of $\mathrm{Ca}^{2+}$-induced $\mathrm{Ca}^{2+}$ release may amplify stochastic fluctuations within a subsarcolemmal microdomain and modulate BVR. Thus, BVR also reflects the stability of the intracellular $\mathrm{Ca}^{2+}$-handling system and $\mathrm{Ca}^{2+}$-sensitive currents.

Dispersion of repolarization has been shown to be arrhythmogenic in a variety of conditions. ${ }^{68}$ Cellto-cell coupling is able to suppress both temporal and spatial dispersion of repolarization (Figure 6), suggesting that BVR can indicate the degree of (un)coupling of the myocardium.

Combined, these data suggest that BVR reflects both the intrinsic temporal variability (stochastic channel gating and $\mathrm{Ca}^{2+}$ handling) as well as the sensitivity of the electrical system to these fluctuations (modulated by APD, AP morphology and cell-to-cell coupling). Our results highlight an important role for abnormal $\mathrm{Ca}^{2+}$ handling in BVR, consistent with experimental recordings, due to the presence of microdomains. Future experimental and computational studies should investigate the impact of $\mathrm{Ca}^{2+}$ on BVR at the subcellular level, providing a more extensive validation of local $\mathrm{Ca}^{2+}$ release and $\mathrm{Ca}^{2+}$ wave properties.

\section{Limitations and future directions}

Stochastic formulations of all 13 targets were based on the well-validated characteristics of the deterministic model ${ }^{12,22}$ using the methodology employed in local control models. ${ }^{24,36,39}$ This approach allows tracking of single-channel behavior, however a formal validation of single-channel characteristics based on dwell times, open probability distributions, etc. is beyond the scope of this study. Furthermore, since single-channel recordings are often performed in non-physiological 
solutions, it would be unclear whether any deviations in single-channel behavior observed in the model under these conditions would affect the stochastic properties relevant for BVR.

We estimated the effective number of channels in the model based on experimentally obtained single-channel conductance. For several targets, the single-channel conductance or expression density is not well constrained. For example, to the best of our knowledge, there are no data on $\mathrm{I}_{\mathrm{ks}}$ single-channel conductance from native tissue, and experimental data from heterologous expression systems show considerable variability (Table 1). We performed simulations over a range of channel densities to investigate the impact of this parameter (Figure 4). Because single-channel conductance has a large impact on BVR (Figure 4), the contribution of these targets may therefore be under- or overestimated.

In addition to single channel gating and $\mathrm{Ca}^{2+}$, other factors may modulate BVR. These factors include signaling pathways, changes in cell volume and $\mathrm{pH}$, etc. and are beyond the scope of the current investigation. Moreover, most of these factors will change on a time scale of minutes, whereas BVR reflects the changes in repolarization duration in the order of seconds. Thus, although these factors can affect BVR, they are likely to do so via changes in repolarization duration, $\mathrm{Ca}^{2+}$ handling, or stochastic channel gating that have been investigated here.

Finally, although the results presented here suggest that BVR reflects a combination of potentially proarrhythmic signals at the (sub)cellular level, its role as a marker for arrhythmogenesis can only be thoroughly investigated in a large multicellular model. The complexity of the cell model makes this computationally prohibitive for the current implementation. Our cell-pair simulations show that cellto-cell coupling will reduce but not eliminate BVR. Future studies should investigate the synchronization of variability during arrhythmogenesis in a multi-scale model.

\section{Conclusions}

We presented a novel stochastic model of the canine ventricular myocyte electrophysiology showing APD and BVR rate dependences consistent with experimental data from isolated canine ventricular myocytes under physiological conditions and in pharmacological models of LQT1-3. The model provides new insights into the (sub)cellular determinants of BVR and suggests modulating roles for several processes, including APD, AP morphology, $\mathrm{Ca}^{2+}$ handling, and cell-to-cell coupling. It provides a framework to further our understanding of the potential role that BVR can play as a proarrhythmic marker.

\section{ACKNOWLEDGEMENTS}

The authors thank members of the Volders team for helpful discussions. 


\section{SOURCES OF FUNDING}

P.G.A.V. is supported by a Vidi grant from the Netherlands Organization for Scientific Research (ZonMw 91710365). D.M.J. was financially supported by AstraZeneca Ltd, UK. Y.R. is supported by the National Institutes of Health-National Heart, Lung, and Blood Institute (Grants R01-HL049054-18 and R01-HLR01033343-26) and the National Science Foundation under Grant No. CBET-0929633. Any opinions, findings and conclusions or recommendations expressed in this material are those of the authors and do not necessarily reflect the views of the National Science Foundation (NSF).

\section{REFERENCES}

1. Zaniboni $M$, Pollard $A E$, Yang $L$, Spitzer $K W$. Beat-to-beat repolarization variability in ventricular myocytes and its suppression by electrical coupling. Am J Physiol Heart Circ Physiol. 2000;278:H677-687.

2. Hinterseer $M$, Beckmann $B M$, Thomsen $M B$, Pfeufer $A$, Ulbrich $M$, Sinner MF, Perz $S$, Wichmann HE, Lengyel C, Schimpf R, Maier SK, Várro A, Vos MA, Steinbeck G, Kääb S. Usefulness of short-term variability of QT intervals as a predictor for electrical remodeling and proarrhythmia in patients with nonischemic heart failure. Am J Cardiol. 2010;106:216220.

3. Tereshchenko LG, Han L, Cheng A, Marine JE, Spragg DD, Sinha S, Dalal D, Calkins H, Tomaselli GF, Berger RD. Beat-to-beat three-dimensional ECG variability predicts ventricular arrhythmia in ICD recipients. Heart Rhythm. 2010;7:1606-1613.

4. Thomsen MB, Verduyn SC, Stengl M, Beekman JD, de Pater G, van Opstal J, Volders PGA, Vos MA. Increased short-term variability of repolarization predicts $d$-sotalol-induced torsades de pointes in dogs. Circulation. 2004;110:2453-2459.

5. Gallacher DJ, Van de Water A, van der Linde H, Hermans AN, Lu HR, Towart R, Volders PGA. In vivo mechanisms precipitating torsades de pointes in a canine model of drug-induced long-QT1 syndrome. Cardiovasc Res. 2007;76:247-256.

6. Jacobson I, Carlsson L, Duker G. Beat-by-beat QT interval variability, but not QT prolongation per se, predicts drug-induced torsades de pointes in the anaesthetised methoxaminesensitized rabbit. J Pharmacol Toxicol Methods. 2011;63:40-46.

7. Hinterseer $M$, Beckmann BM, Thomsen MB, Pfeufer A, Dalla Pozza R, Loeff $M$, Netz $H$, Steinbeck G, Vos MA, Kääb S. Relation of increased short-term variability of QT interval to congenital long-QT syndrome. Am J Cardiol. 2009;103:1244-1248.

8. Johnson DM, Heijman J, Pollard CE, Valentin JP, Crijns HJ, Abi-Gerges N, Volders PGA. $\mathrm{I}_{\mathrm{Ks}}$ restricts excessive beat-to-beat variability of repolarization during beta-adrenergic receptor stimulation. J Mol Cell Cardiol. 2010;48:122-130.

9. Noble D, Rudy Y. Models of cardiac ventricular action potentials: iterative interaction between experiment and simulation. Philos Trans R Soc Lond A. 2001;359:1127-1142.

10. Courtemanche M, Ramirez RJ, Nattel S. Ionic mechanisms underlying human atrial action potential properties: insights from a mathematical model. Am J Physiol. 1998;275:H301-321.

11. Mahajan A, Shiferaw $Y$, Sato D, Baher A, Olcese R, Xie LH, Yang MJ, Chen PS, Restrepo JG, Karma A, Garfinkel A, Qu Z, Weiss JN. A rabbit ventricular action potential model replicating cardiac dynamics at rapid heart rates. Biophys J. 2008;94:392-410.

12. Decker KF, Heijman J, Silva JR, Hund TJ, Rudy Y. Properties and ionic mechanisms of action potential adaptation, restitution, and accommodation in canine epicardium. Am J Physiol Heart Circ Physiol. 2009;296:H1017-1026. 
13. O'Hara T, Virag L, Varro A, Rudy Y. Simulation of the undiseased human cardiac ventricular action potential: model formulation and experimental validation. PLoS Comput Biol. 2011;7:e1002061.

14. Grandi E, Pandit SV, Voigt N, Workman AJ, Dobrev D, Jalife J, Bers DM. Human atrial action potential and $\mathrm{Ca}^{2+}$ model: sinus rhythm and chronic atrial fibrillation. Circ Res. 2011;109:1055-1066.

15. Winslow RL, Rice J, Jafri S, Marban E, O'Rourke B. Mechanisms of altered excitationcontraction coupling in canine tachycardia-induced heart failure, II: model studies. Circ Res. 1999;84:571-586.

16. Clancy $\mathrm{CE}$, Rudy $\mathrm{Y} . \mathrm{Na}^{+}$channel mutation that causes both Brugada and long-QT syndrome phenotypes: a simulation study of mechanism. Circulation. 2002;105:1208-1213.

17. Pandit SV, Giles WR, Demir SS. A mathematical model of the electrophysiological alterations in rat ventricular myocytes in type-I diabetes. Biophys J. 2003;84:832-841.

18. Kneller J, Kalifa J, Zou R, Zaitsev AV, Warren M, Berenfeld O, Vigmond EJ, Leon LJ, Nattel S, Jalife J. Mechanisms of atrial fibrillation termination by pure sodium channel blockade in an ionically-realistic mathematical model. Circ Res. 2005;96:e35-47.

19. Wolf RM, Mitchell CC, Christensen MD, Mohler PJ, Hund TJ. Defining new insight into atypical arrhythmia: a computational model of ankyrin-B syndrome. Am J Physiol Heart Circ Physiol. 2010;299:H1505-1514.

20. Saucerman JJ, Brunton LL, Michailova AP, McCulloch AD. Modeling $\beta$-adrenergic control of cardiac myocyte contractility in silico. J Biol Chem. 2003;278:47997-48003.

21. Hund TJ, Rudy Y. Rate dependence and regulation of action potential and calcium transient in a canine cardiac ventricular cell model. Circulation. 2004;110:3168-3174.

22. Heijman J, Volders PGA, Westra RL, Rudy Y. Local control of $\beta$-adrenergic stimulation: Effects on ventricular myocyte electrophysiology and $\mathrm{Ca}^{2+}$-transient. J Mol Cell Cardiol. 2011;50:863871.

23. Tao T, Paterson DJ, Smith NP. A model of cellular cardiac-neural coupling that captures the sympathetic control of sinoatrial node excitability in normotensive and hypertensive rats. Biophys J. 2011;101:594-602.

24. Tanskanen AJ, Greenstein JL, O'Rourke B, Winslow RL. The role of stochastic and modal gating of cardiac L-type Ca2+ channels on early after-depolarizations. Biophys J. 2005;88:8595.

25. Sato D, Xie LH, Sovari AA, Tran DX, Morita N, Xie F, Karagueuzian H, Garfinkel A, Weiss JN, Qu Z. Synchronization of chaotic early afterdepolarizations in the genesis of cardiac arrhythmias. Proc Natl Acad Sci U S A. 2009;106:2983-2988.

26. Sato D, Xie LH, Nguyen TP, Weiss JN, Qu Z. Irregularly appearing early afterdepolarizations in cardiac myocytes: random fluctuations or dynamical chaos? Biophys J. 2010;99:765-773.

27. Lemay $M$, de Lange $E$, Kucera JP. Effects of stochastic channel gating and distribution on the cardiac action potential. Journal of Theoretical Biology. 2011;281:84-96.

28. Pueyo E, Corrias A, Virag L, Jost N, Szél T, Varro A, Szentandrassy N, Nanasi PP, Burrage K, Rodriguez B. A multiscale investigation of repolarization variability and its role in cardiac arrhtyhmogenesis. Biophys J. 2011;101:1-11.

29. Silva J, Rudy $Y$. Subunit interaction determines $I_{\mathrm{Ks}}$ participation in cardiac repolarization and repolarization reserve. Circulation. 2005;112:1384-1391.

30. Brennan $T$, Fink $M$, Rodriguez $B$. Multiscale modelling of drug-induced effects on cardiac electrophysiological activity. Eur J Pharm Sci. 2009;36:62-77.

31. Liu DW, Antzelevitch C. Characteristics of the delayed rectifier current ( $I_{\mathrm{Kr}}$ and $\left.I_{\mathrm{Ks}}\right)$ in canine ventricular epicardial, midmyocardial, and endocardial myocytes. A weaker $\mathrm{I}_{\mathrm{Ks}}$ contributes to the longer action potential of the M cell. Circ Res. 1995;76:351-365. 
32. Yang T, Snyders DJ, Roden DM. Ibutilide, a methanesulfonanilide antiarrhythmic, is a potent blocker of the rapidly activating delayed rectifier $\mathrm{K}+$ current $\left(\mathrm{I}_{\mathrm{Kr}}\right)$ in AT-1 cells. Concentration, time-, voltage-, and use-dependent effects. Circulation. 1995;91:1799-1806.

33. Jurkiewicz NK, Sanguinetti MC. Rate-dependent prolongation of cardiac action potentials by a methanesulfonanilide class III antiarrhythmic agent. Specific block of rapidly activating delayed rectifier K+ current by dofetilide. Circ Res. 1993;72:75-83.

34. Baba S, Dun W, Boyden PA. Can PKA activators rescue Na+ channel function in epicardial border zone cells that survive in the infarcted canine heart? Cardiovasc Res. 2004;64:260267.

35. Livshitz LM, Rudy Y. Regulation of $\mathrm{Ca}^{2+}$ and electrical alternans in cardiac myocytes: role of CAMKII and repolarizing currents. Am J Physiol Heart Circ Physiol. 2007;292:H2854-2866.

36. Restrepo JG, Weiss JN, Karma A. Calsequestrin-mediated mechanism for cellular calcium transient alternans. Biophys J. 2008;95:3767-3789.

37. Matsumoto $\mathrm{M}$, Nishimura T. Mersenne twister: a 623-dimensionally equidistributed uniform pseudo-random number generator. ACM Trans. Model. Comput. Simul. 1998;8:3-30.

38. Rao CV, Wolf DM, Arkin AP. Control, exploitation and tolerance of intracellular noise. Nature. 2002;420:231-237.

39. Gaur N, Rudy Y. Multiscale modeling of calcium cycling in cardiac ventricular myocyte: macroscopic consequences of microscopic dyadic function. Biophysical journal. 2011;100:2904-2912.

40. Rudy Y, Silva JR. Computational biology in the study of cardiac ion channels and cell electrophysiology. Q Rev Biophys. 2006;39:57-116.

41. Hille B. Ion channels of excitable membranes. 3rd ed. Sunderland, Mass.: Sinauer; 2001.

42. Tran K, Smith NP, Loiselle DS, Crampin EJ. A thermodynamic model of the cardiac sarcoplasmic/endoplasmic Ca ${ }^{2+}$ (SERCA) pump. Biophysical Journal. 2009;96:2029-2042.

43. Smith NP, Crampin EJ. Development of models of active ion transport for whole-cell modelling: cardiac sodium-potassium pump as a case study. Prog Biophys Mol Biol. 2004;85:387-405.

44. Guia A, Stern MD, Lakatta EG, Josephson IR. Ion concentration-dependence of rat cardiac unitary L-type calcium channel conductance. Biophys J. 2001;80:2742-2750.

45. Collier ML, Levesque PC, Kenyon JL, Hume JR. Unitary Cl- channels activated by cytoplasmic $\mathrm{Ca}^{2+}$ in canine ventricular myocytes. Circ Res. 1996;78:936-944.

46. Kääb S, Nuss HB, Chiamvimonvat N, O'Rourke B, Pak PH, Kass DA, Marban E, Tomaselli GF. Ionic mechanism of action potential prolongation in ventricular myocytes from dogs with pacing-induced heart failure. Circ Res. 1996;78:262-273.

47. Liu GX, Zhou J, Nattel S, Koren G. Single-channel recordings of a rapid delayed rectifier current in adult mouse ventricular myocytes: basic properties and effects of divalent cations. The Journal of physiology. 2004;556:401-413.

48. Veldkamp MW, van Ginneken AC, Bouman LN. Single delayed rectifier channels in the membrane of rabbit ventricular myocytes. Circ Res. 1993;72:865-878.

49. Yang $Y$, Sigworth FJ. Single-channel properties of $I_{\mathrm{Ks}}$ potassium channels. J Gen Physiol. 1998;112:665-678.

50. Romey G, Attali B, Chouabe C, Abitbol I, Guillemare E, Barhanin J, Lazdunski M. Molecular mechanism and functional significance of the MinK control of the KvLQT1 channel activity. $J$ Biol Chem. 1997;272:16713-16716.

51. Sesti F, Goldstein SA. Single-channel characteristics of wild-type $I_{K S}$ channels and channels formed with two minK mutants that cause long QT syndrome. J Gen Physiol. 1998;112:651663.

52. Yue L, Feng J, Li GR, Nattel S. Characterization of an ultrarapid delayed rectifier potassium channel involved in canine atrial repolarization. J Physiol. 1996;496:647-662. 
53. Sridhar A, da Cunha DN, Lacombe VA, Zhou Q, Fox JJ, Hamlin RL, Carnes CA. The plateau outward current in canine ventricle, sensitive to 4-aminopyridine, is a constitutive contributor to ventricular repolarization. Br J Pharmacol. 2007;152:870-879.

54. Ono K, Fozzard HA, Hanck DA. Mechanism of cAMP-dependent modulation of cardiac sodium channel current kinetics. Circ Res. 1993;72:807-815.

55. Blaustein MP, Lederer WJ. Sodium/calcium exchange: its physiological implications. Physiol Rev. 1999;79:763-854.

56. Dobretsov $\mathrm{M}$, Hastings $\mathrm{SL}$, Stimers JR. $\mathrm{Na}^{+}-\mathrm{K}^{+}$pump cycle during $\beta$-adrenergic stimulation of adult rat cardiac myocytes. J Physiol. 1998;507:527-539.

57. Fedida D, Giles WR. Regional variations in action potentials and transient outward current in myocytes isolated from rabbit left ventricle. J Physiol. 1991;442:191-209.

58. Volders PGA, Sipido KR, Carmeliet E, Spätjens RLHMG, Wellens HJ, Vos MA. Repolarizing K ${ }^{+}$ currents $I_{\mathrm{TO} 1}$ and $\mathrm{I}_{\mathrm{KS}}$ are larger in right than left canine ventricular midmyocardium. Circulation. 1999;99:206-210.

59. Volders PGA, StengI M, van Opstal JM, Gerlach U, Spätjens RL, Beekman JD, Sipido KR, Vos MA. Probing the contribution of $I_{\mathrm{Ks}}$ to canine ventricular repolarization: key role for $\beta$ adrenergic receptor stimulation. Circulation. 2003;107:2753-2760.

60. Sarkar AX, Sobie EA. Quantification of repolarization reserve to understand interpatient variability in the response to proarrhythmic drugs: A computational analysis. Heart Rhythm. 2011;8:1749-1755.

61. Shaw RM, Rudy Y. Ionic mechanisms of propagation in cardiac tissue. Roles of the sodium and L-type calcium currents during reduced excitability and decreased gap junction coupling. Circ Res. 1997;81:727-741.

62. Banyasz T, Horvath B, Virag L, Barandi L, Szentandrassy N, Harmati G, Magyar J, Marangoni S, Zaza A, Varro A, Nanasi PP. Reverse rate dependency is an intrinsic property of canine cardiac preparations. Cardiovasc Res. 2009;84:237-244.

63. Thomsen MB, Volders PG, Beekman JD, Matz J, Vos MA. Beat-to-Beat variability of repolarization determines proarrhythmic outcome in dogs susceptible to drug-induced torsades de pointes. J Am Coll Cardiol. 2006;48:1268-1276.

64. Wilders $\mathrm{R}$, Jongsma HJ. Beating irregularity of single pacemaker cells isolated from the rabbit sinoatrial node. Biophys J. 1993;65:2601-2613.

65. Tanskanen AJ, Alvarez LH. Voltage noise influences action potential duration in cardiac myocytes. Math Biosci. 2007;208:125-146.

66. Volders PGA, Kulcsár A, Vos MA, Sipido KR, Wellens HJ, Lazzara R, Szabo B. Similarities between early and delayed afterdepolarizations induced by isoproterenol in canine ventricular myocytes. Cardiovasc Res. 1997;34:348-359.

67. Nemec J, Kim JJ, Gabris B, Salama G. Calcium oscillations and T-wave lability precede ventricular arrhythmias in acquired long QT type 2. Heart Rhythm. 2010;7:1686-1694.

68. Antzelevitch C. Role of spatial dispersion of repolarization in inherited and acquired sudden cardiac death syndromes. Am J Physiol Heart Circ Physiol. 2007;293:H2024-H2038. 


\section{CHAPTER 8}

\section{GENERAL DisCUSSION}

8.1 Insights into the role of $\beta$-adrenergic receptor stimulation in arrhythmogenesis

8.2 Comparison of existing cell models

8.3 Applications of computational models of cardiac electrophysiology

8.3.1 Cellular applications

8.3.2 Clinical applications.....

8.4 Systems biology

8.4.1 Perspectives of computational electrophysiology 


\subsection{INSIGHTS INTO THE ROLE OF $\beta$-ADRENERGIC RECEPTOR STIMULATION IN}

\section{ARRHYTHMOGENESIS}

In the studies that led to this thesis we have developed a detailed computational model of the canine ventricular myocyte electrophysiology (Chapter 2 ) and have integrated it with a system of localized $\beta$-adrenergic receptor stimulation ( $\beta A R S$ ) and CaMKII signaling (Chapter 3 ) to obtain a better quantitative understanding of the complex local processes linking $\beta A R S$ and arrhythmogenesis (Figure 1). We have focused predominantly on the effects of $\beta A R S$ on ventricular myocyte electrophysiology and the proarrhythmic marker beat-to-beat variability of repolarization (BVR; Figure 1, grey boxes).

The sympathetic nervous system plays a crucial role in the modulation of cardiac output. Sympathetic stimulation results in an in increase in chronotropy, inotropy, lusitropy and dromotropy as a result of changes in cellular electrophysiology at various levels of the heart. In a number of pathological conditions sympathetic stimulation has been associated with an increased arrhythmogenic risk. ${ }^{1}$ The clinical importance of the connection between the autonomic nervous system and the heart is further supported by the fact that baroreflex sensitivity testing, ${ }^{2}$ exercise testing ${ }^{3}$ and epinephrine challenges ${ }^{4}$ are used for diagnostic stratification in clinical settings. At the therapeutic side, $\beta$-adrenergic receptor blockade is the mainstay of pharmacological treatment in various pathological conditions, and left or bilateral cardiac sympathetic denervation has proven valuable in the management of patients with intractable ventricular tachycardia (VT) in long-QT syndrome, ${ }^{5}$ catecholaminergic polymorphic $\mathrm{VT}^{6}$, and electrical storm by other causes. ${ }^{7,8}$

In animal models and experimental preparations, numerous groups have identified electrophysiological changes in response to $\beta A R S$ (as part of sympathetic stimulation) that contribute to arrhythmogenesis in the predisposed heart. ${ }^{9-13}$ These studies have highlighted a complex network of interacting components linking $\beta A R S$ and arrhythmogenesis at various levels of electrophysiological integration (Figure 1). ${ }^{14}$ 


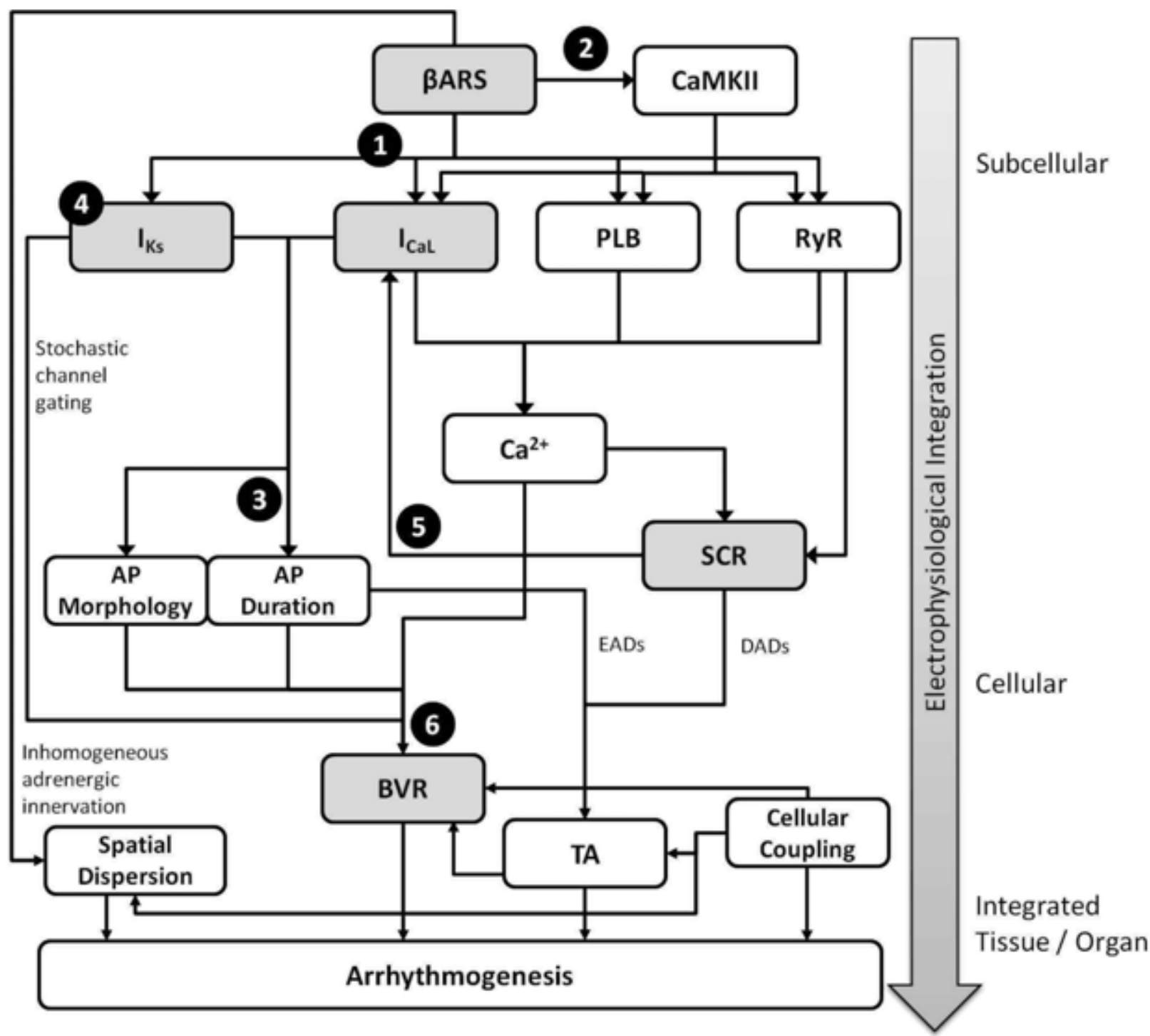

Figure 1. The effect of $\beta A R S$ on cardiac cellular electrophysiology and arrhythmogenesis. Vertical direction represents increasing level of electrophysiological integration from subcellular, macromolecular complexes to microdomains, cellular components and integrated tissue / organ. Grey boxes reflect focal points of the work presented in this thesis. Numbers 1-6 indicate interactions for which significant new insights were obtained (see text).

We have shown in Chapter 3 that localized cAMP signaling is critical for a precise control of the phosphorylation status of individual protein kinase A-substrates (\#1 in Figure 1). Moreover, localized $\beta A R S$ augmented $\mathrm{Ca}^{2+} / \mathrm{Calmodulin}$ kinase (CaMKII)-dependent signaling via $\left[\mathrm{Ca}^{2+}\right]_{i}$, inhibitor protein 1 and heart rate. These pathways had synergistic effects on cellular $\mathrm{Ca}^{2+}$ handling and spontaneous $\mathrm{Ca}^{2+}$ release (SCR) indicating that the link between $\beta$ ARS and arrhythmogenesis is, at least partly, mediated by CaMKII (\#2 in Figure 1). ${ }^{15-17}$

Advanced experimental techniques have facilitated the characterization of $\beta A R$ signaling at the subcellular level, as well as its electrophysiological effects. ${ }^{18,}{ }^{19}$ These studies have shown that localization of both signaling molecules and ions ensures precise control of substrate 
phosphorylation. Fluorescent indicators of cyclic AMP (cAMP) targeted to specific subcellular domains have shown that there is restricted diffusion of this second messenger which is controlled by (caveolar) microdomains incorporating different receptors, adenylyl cyclases, phosphodiesterases, etc. ${ }^{20-23}$ Disruption of these microdomains, e.g., by the cholesterol depleting agent methyl- $\beta$-cyclodextrin, results in altered electrophysiological responses to $\beta$ ARS compared to control conditions. ${ }^{24}$ Importantly, a similar disruption of local signaling environments was observed in pathological conditions. Nikolaev et al. have shown that a redistribution of $\beta 2$-adrenergic receptors occurs during heart failure ${ }^{25}$ and Kass and coworkers have shown that mutations in $K C N Q 1^{26}$ or the A-kinase anchoring protein $Y^{20 t i a o^{27}}$ can disrupt the macromolecular signaling complex of the slowly-activating delayed-rectifier $\mathrm{K}^{+}$current $\left(\mathrm{I}_{\mathrm{Ks}}\right)$. In all these conditions there is an important role for $\beta$ ARS in arrhythmogenesis.

In Chapter 3, we also quantified the contribution of individual electrophysiological PKA substrates to the $\beta$ ARS-dependent alterations in action potential (AP) and $\mathrm{Ca}^{2+}$ transient (\#3 in Figure 1). We showed, consistent with previous experimental work, ${ }^{28}$ that $\mathrm{I}_{\mathrm{Ks}}$ has only a minor impact on cardiac repolarization under basal, isolated-myocyte conditions. However, during $\beta A R S I_{K s}$ played a major role in the shortening of AP duration (APD). Consistent with these observations, we determined that the combination of reduced basal $I_{\mathrm{Ks}}$ and loss of cAMP-dependent $I_{\mathrm{Ks}}$ upregulation due to the longQT syndrome type-1 mutation KCNQ1-A341V results in a pronounced AP phenotype only during $\beta A R S$ (Chapter 4). The A341V data further underscored the importance of a correctly functioning local macromolecular complex in mediating the coupling between the BARS signaling pathway and the electrophysiological response. They provided novel evidence that the S6 transmembrane segment of KCNQ1 at and/or around residue A341 plays an important role in the BARS-dependent modulation of $I_{\mathrm{Ks}}$ (\#4 in Figure 1). Interestingly, a complete loss of cAMP-dependent $I_{\mathrm{Ks}}$ upregulation was also observed in heterozygous A341V conditions. Moreover, this effect was not unique for A341V, since the mutation G589D in the KCNQ1 C-terminus also resulted in a loss of cAMPdependent upregulation in heterozygous conditions. This observation has important implications for the arrhythmogenic risk of mutation carriers because even a heterozygous genotype will result in a homozygous phenotype with respect to cAMP-dependent $I_{K_{s}}$ upregulation. Consistent with this important role of $I_{\mathrm{Ks}}$ during $\beta A R S$, we found that an intact $I_{\mathrm{Ks}}$ is required to prevent excessive BVR during $\beta A R S$ in isolated canine ventricular myocytes (Chapter $\mathbf{5}$ ).

In addition to a direct effect on APD, a decreased function of $I_{\mathrm{Ks}}$ also reduces the heart's capabilities to withstand additional challenges on repolarization. This ability has previously been termed "repolarization reserve" and several studies have shown that $I_{\mathrm{Ks}}$ provides an important repolarization reserve in ventricular myocytes. ${ }^{28-30}$ This reserve is recruited when the balance between inward and outward currents is shifted, for example during pharmacological inhibition of the rapidly-activating delayed-rectifier $\mathrm{K}^{+}$current $\left(\mathrm{I}_{\mathrm{Kr}}\right) .{ }^{28}$ It has long been established that $\beta$ ARS can cause $\mathrm{Ca}^{2+}$ overload, SCR and triggered activity (TA). However, in most experimental studies SCR is investigated using a rapid-pacing protocol followed by a pause. Consequently, the effect of SCR on the following beat has received little attention. In Chapter 6, we provided novel evidence that SCR during regular pacing causes interspersed APD prolongation due to a reduced $\mathrm{Ca}^{2+}$-dependent inactivation of the L-type 
$\mathrm{Ca}^{2+}$ current $\left(\mathrm{I}_{\mathrm{CaL}}\right)$ during the following AP (\#5 in Figure 1). The role of $\mathrm{I}_{\mathrm{Ks}}$ in repolarization reserve was evident from the fact that the SCR-induced APD prolongation was significantly more pronounced during pharmacological $\mathrm{I}_{\mathrm{Ks}}$ blockade.

Several studies have shown that BVR of QT duration is a more reliable proarrhythmic marker than QT prolongation per se, at least under specific experimental conditions, ${ }^{31}$ or in selected human subjects. $^{32,33}$ The exact ionic mechanisms contributing to BVR under physiological and pathological conditions remain incompletely understood, but cellular BVR of APD likely contributes significantly to temporal variability of QT duration. Our results (Chapter 7) suggest that cellular BVR reflects multiple factors that can contribute to arrhythmogenesis, including AP morphology, APD, $\mathrm{Ca}^{2+}$ handling, stochastic ion-channel gating, and cell-to-cell coupling (\#6 in Figure 1). This indicates that BVR may be a potentially useful proarrhythmic marker but that additional data is required to identify the underlying (cellular) pathology and, consequently, optimal pharmacological treatment.

In summary, in this thesis we have provided data that indicate that $\beta A R S$ affects both repolarization and $\mathrm{Ca}^{2+}$ handling via localized effects on subcellular targets. In the (severely) predisposed heart, these characteristics may increase dispersion of repolarization and triggered activity, resulting in an increased susceptibility to ventricular tachyarrhythmias. The $\beta A R S$-dependent $I_{\mathrm{Ks}}$ upregulation plays a major role in both temporal and spatial dispersion of repolarization during $\beta A R S$, highlighting a key role for $\mathrm{I}_{\mathrm{Ks}}$ in the link between $\beta$ ARS and arrhythmogenesis.

\subsection{COMPARISON OF EXISTING CELL MODELS}

In recent years, numerous computational models of cardiac cellular electrophysiology have been developed. Although the models of the late 1990s and early 2000s were predominantly deterministic common-pool models, most of the recently developed models display mounting complexity, focusing on local stochastic aspects of cardiac electrophysiology and/or its regulation by signaling cascades. An overview of cellular models including their stochastic, $\beta$ ARS and CaMKII properties is provided in Table 1.

\begin{tabular}{|c|c|c|c|c|c|c|}
\hline Model $^{\text {Reference }}$ & Species & Cell-type & Model Type & $\begin{array}{l}\text { Stochastic } \\
\text { Targets }\end{array}$ & CaMKII Targets & $\beta A R S$ Targets \\
\hline Noble $1962^{34}$ & Generic & PF & $\begin{array}{l}\text { Constant } \\
\text { Concentr. }\end{array}$ & - & - & - \\
\hline $\begin{array}{c}\text { McAllister et al. } \\
1975^{35} \\
\end{array}$ & Generic & PF & $\begin{array}{l}\text { Constant } \\
\text { Concentr. }\end{array}$ & - & - & - \\
\hline $\begin{array}{c}\text { Beeler \& Reuter } \\
1977^{36} \\
\end{array}$ & Generic & VM & $\begin{array}{l}\text { Constant } \\
\text { Concentr. }\end{array}$ & - & - & - \\
\hline Kass \& Wiegers $1982^{37}$ & Calf & PF & $\begin{array}{l}\text { Constant } \\
\text { Concentr. }\end{array}$ & - & - & $\begin{array}{c}I_{s i} I_{x} \text { (by } \\
\text { noradrenaline) }\end{array}$ \\
\hline $\begin{array}{c}\text { Rasmusson et al. } \\
1990^{38,39}\end{array}$ & Bullfrog & AM / SAN & $\begin{array}{l}\text { Constant } \\
\text { Concentr. }\end{array}$ & - & - & - \\
\hline
\end{tabular}




\begin{tabular}{|c|c|c|c|c|c|c|}
\hline Model $^{\text {Reference }}$ & Species & Cell-type & Model Type & $\begin{array}{c}\text { Stochastic } \\
\text { Targets }\end{array}$ & CaMKII Targets & $\beta A R S$ Targets \\
\hline Liu et al. ${ }^{40}$ & Rabbit & AVN & $\begin{array}{l}\text { Constant } \\
\text { Concentr. }\end{array}$ & - & - & - \\
\hline Luo \& Rudy $1994^{41,42}$ & $\begin{array}{l}\text { Guinea- } \\
\text { Pig }\end{array}$ & VM & $\begin{array}{c}\text { Common } \\
\text { Pool }\end{array}$ & - & - & - \\
\hline Zeng \& Rudy $1995^{43}$ & $\begin{array}{c}\text { Guinea- } \\
\text { Pig }\end{array}$ & VM & $\begin{array}{c}\text { Common } \\
\text { Pool }\end{array}$ & - & - & $\begin{array}{c}I_{\mathrm{Ca},}, I_{\mathrm{K}}, I_{\mathrm{Na}}, I_{\mathrm{NaK}}, \\
\text { PLB }\end{array}$ \\
\hline $\begin{array}{c}\text { Courtemanche et al. } \\
1998^{44}\end{array}$ & Human & AM & $\begin{array}{c}\text { Common } \\
\text { Pool }\end{array}$ & - & - & - \\
\hline Nygren et al. $1998^{45}$ & Human & AM & $\begin{array}{c}\text { Common } \\
\text { Pool }\end{array}$ & - & - & - \\
\hline Winslow et al. $1999^{46}$ & Canine & VM (Mid) & $\begin{array}{c}\text { Common } \\
\text { Pool }\end{array}$ & - & - & - \\
\hline Pandit et al. $2001^{47}$ & Rat & $\begin{array}{c}\text { VM } \\
\text { (Transmural) } \\
\end{array}$ & $\begin{array}{c}\text { Common } \\
\text { Pool }\end{array}$ & - & - & - \\
\hline Puglisi et al. $2001^{48}$ & Rabbit & VM & $\begin{array}{c}\text { Common } \\
\text { Pool }\end{array}$ & - & - & - \\
\hline $\begin{array}{c}\text { Greenstein et al. } \\
2002^{49} \\
\end{array}$ & Canine & VM (Mid) & $\begin{array}{l}\text { Local } \\
\text { Control } \\
\end{array}$ & $\mathrm{I}_{\mathrm{CaL}}, \mathrm{RyR}$ & - & - \\
\hline Oehmen et al. $2002^{50}$ & Rabbit & SAN & $\begin{array}{c}\text { Common } \\
\text { Pool }\end{array}$ & - & - & - \\
\hline $\begin{array}{l}\text { Saucerman et al. } \\
\qquad 2003^{51}\end{array}$ & Rat & VM & $\begin{array}{l}\text { Common } \\
\text { Pool }\end{array}$ & - & - & 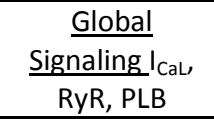 \\
\hline $\begin{array}{c}\text { Ten Tusscher et al. } \\
2004^{52}\end{array}$ & Human & VM & $\begin{array}{c}\text { Common } \\
\text { Pool }\end{array}$ & - & - & - \\
\hline $\begin{array}{c}\text { Bondarenko et al. } \\
2004^{53}\end{array}$ & Mouse & VM & $\begin{array}{c}\text { Common } \\
\text { Pool }\end{array}$ & - & - & - \\
\hline Hund \& Rudy $2004^{54}$ & Canine & VM (Epi) & $\begin{array}{c}\text { Common } \\
\text { Pool }\end{array}$ & - & $\begin{array}{l}\frac{\text { Local signaling }}{\mathrm{I}_{\text {CaL}}, \mathrm{RyR}, \mathrm{PLB}} \\
\end{array}$ & \\
\hline $\begin{array}{l}\text { Saucerman et al. } \\
\qquad 2004^{55}\end{array}$ & Rabbit & $\begin{array}{l}\text { VM (Multi- } \\
\text { scale) }\end{array}$ & $\begin{array}{l}\text { Common } \\
\text { Pool }\end{array}$ & - & - & $\begin{array}{c}\text { Global signaling } \\
I_{\text {CaL }}, I_{\text {Ks }}, T n I, \\
\text { RyR, PLB }\end{array}$ \\
\hline Shannon et al. $2004^{56}$ & Rabbit & VM & $\begin{array}{c}\text { Common } \\
\text { Pool }\end{array}$ & - & - & - \\
\hline $\begin{array}{c}\text { Tanskanen et al. } \\
2005^{57}\end{array}$ & Canine & VM (Mid) & $\begin{array}{c}\text { Local } \\
\text { Control } \\
\end{array}$ & $\mathrm{I}_{\mathrm{CaL}}, \mathrm{RyR}$ & - & $I_{\mathrm{CaL}}, I_{\mathrm{Ks}}, I_{\mathrm{Kr}}, \mathrm{PLB}$ \\
\hline $\begin{array}{c}\text { Terrenoire et al. } \\
2005^{58} \\
\end{array}$ & $\begin{array}{l}\text { Guinea- } \\
\text { Pig }\end{array}$ & VM & $\begin{array}{c}\text { Common } \\
\text { Pool }\end{array}$ & - & - & $\begin{array}{c}\frac{\text { Global signaling }}{I_{\text {CaL, }}, I_{\mathrm{Ks}}, P L B} \\
\end{array}$ \\
\hline $\begin{array}{c}\text { Saucerman et al. } \\
2006^{59} \\
\end{array}$ & Rat & VM & $\begin{array}{c}\text { Common } \\
\text { Pool }\end{array}$ & - & - & Local signaling \\
\hline Flaim et al. $2006^{60}$ & Canine & VM & $\begin{array}{c}\text { Common } \\
\text { Pool }\end{array}$ & - & - & - \\
\hline lyer et al. $2007^{61}$ & Human & VM & $\begin{array}{c}\text { Common } \\
\text { Pool }\end{array}$ & - & - & $\mathrm{I}_{\mathrm{CaL}}, \mathrm{PLB}$ \\
\hline lancu et al. $2007^{62}$ & - & - & - & - & - & $\underline{\text { Local signaling }}$ \\
\hline Faber et al. $2007^{63}$ & $\begin{array}{l}\text { Guinea- } \\
\text { Pig }\end{array}$ & VM & $\begin{array}{c}\text { Common } \\
\text { Pool }\end{array}$ & - & - & $\begin{array}{c}I_{\mathrm{CaL}}, I_{\mathrm{K} 1}, I_{\mathrm{KS}}, I_{\mathrm{NaK}}, \\
\text { PLB } \\
\end{array}$ \\
\hline Grandi et al. $2007^{64}$ & Rabbit & VM & $\begin{array}{c}\text { Common } \\
\text { Pool }\end{array}$ & - & $I_{\mathrm{CaL}}, \mathrm{I}_{\mathrm{Na}}, \mathrm{I}_{\mathrm{TO}}$ & - \\
\hline Fink et al. $2008^{65}$ & Human & VM & $\begin{array}{c}\text { Common } \\
\text { Pool }\end{array}$ & - & - & - \\
\hline $\begin{array}{l}\text { Kuzumoto et al. } \\
2008^{66}\end{array}$ & $\begin{array}{l}\text { Guinea- } \\
\text { Pig }\end{array}$ & VM & $\begin{array}{l}\text { Common } \\
\text { Pool }\end{array}$ & - & - & $\begin{array}{c}\text { Global signaling } \\
I_{\text {CaL, }} I_{\text {CTFR }}, I_{\mathrm{Ks}}, \\
I_{\text {Nak, }} I_{\text {pCa }}, T n I, \\
\text { PLB }\end{array}$ \\
\hline Mahajan et al. $2008^{67}$ & Rabbit & VM & $\begin{array}{c}\text { Common } \\
\text { Pool }\end{array}$ & - & - & - \\
\hline
\end{tabular}




\begin{tabular}{|c|c|c|c|c|c|c|}
\hline Model $^{\text {Reference }}$ & Species & Cell-type & Model Type & $\begin{array}{l}\text { Stochastic } \\
\text { Targets }\end{array}$ & CaMKII Targets & BARS Targets \\
\hline Violin et al. $2008^{68}$ & - & - & - & - & - & Global signaling \\
\hline Xin et al. $2008^{69}$ & - & - & - & - & - & Global signaling \\
\hline Hund et al. $2008^{70}$ & Canine & VM (Epi) & $\begin{array}{l}\text { Common } \\
\text { Pool }\end{array}$ & - & $\begin{array}{c}\frac{\text { Local signaling }}{\mathrm{I}_{\mathrm{CaL}}, I_{\mathrm{Na}}, I_{\mathrm{NaL}},} \\
\text { RyR, PLB }\end{array}$ & - \\
\hline Restrepo et al. $2008^{71}$ & Rabbit & VM & $\begin{array}{l}\text { Local } \\
\text { Control } \\
\end{array}$ & $\mathrm{I}_{\mathrm{CaL}}, \mathrm{RyR}$ & - & - \\
\hline $\begin{array}{l}\text { Saucerman \& Bers } \\
2008^{72}\end{array}$ & Rabbit & VM & $\begin{array}{l}\text { Common } \\
\text { Pool }\end{array}$ & - & $\begin{array}{c}\frac{\text { Local signaling }}{\text { (incl. }} \\
\text { calcineurin) I } I_{\text {CaL, }} \\
\text { RyR }\end{array}$ & - \\
\hline Sato et al. $2009^{73}$ & Rabbit & $\begin{array}{l}\text { VM (Multi- } \\
\text { scale) }\end{array}$ & $\begin{array}{c}\text { Common } \\
\text { Pool }\end{array}$ & $\mathrm{I}_{\mathrm{Ks}}$ (Langevin) & - & - \\
\hline Maleckar et al. $2009^{74}$ & Human & AM & $\begin{array}{c}\text { Common } \\
\text { Pool }\end{array}$ & - & - & - \\
\hline Decker et al. $2009^{75}$ & Canine & VM (Epi) & $\begin{array}{c}\text { Common } \\
\text { Pool }\end{array}$ & - & RyR, PLB & - \\
\hline $\begin{array}{c}\text { Ahrens-Nicklas et al. } \\
2009^{76}\end{array}$ & $\begin{array}{c}\text { Guinea- } \\
\text { Pig }\end{array}$ & $\begin{array}{c}\text { VM } \\
\text { (Transmural) } \\
\end{array}$ & $\begin{array}{c}\text { Common } \\
\text { Pool }\end{array}$ & - & - & $\frac{\text { Global signaling }}{\mathrm{I}_{\mathrm{CaL}}, \mathrm{I}_{\mathrm{Ks}}}$ \\
\hline $\begin{array}{l}\text { Koivumaki et al. } \\
2009^{77}\end{array}$ & Mouse & VM & $\begin{array}{l}\text { Common } \\
\text { Pool }\end{array}$ & - & $\begin{array}{l}\frac{\text { Local signaling }}{\text { (incl. }} \\
\text { calcineurin) I IaL, } \\
\text { RyR, PLB }\end{array}$ & 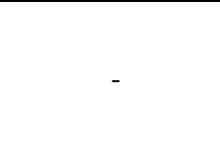 \\
\hline $\begin{array}{l}\text { Christensen et al. } \\
\qquad 2009^{78}\end{array}$ & Canine & VM (Epi) & $\begin{array}{l}\text { Common } \\
\text { Pool }\end{array}$ & - & $\begin{array}{c}\frac{\text { Local signaling }}{\text { (incl. oxidation) }} \\
I_{\mathrm{CaL}}, I_{\mathrm{Na}}, I_{\mathrm{NaL}} \\
\text { RyR, PLB }\end{array}$ & - \\
\hline Wolf et al. $2010^{79}$ & Mouse & VM & $\begin{array}{c}\text { Common } \\
\text { Pool }\end{array}$ & - & - & $I_{\mathrm{CaL}}, I_{\mathrm{Ks}}, \mathrm{PLB}$ \\
\hline Grandi et al. $2010^{80}$ & Human & VM & $\begin{array}{c}\text { Common } \\
\text { Pool }\end{array}$ & - & - & - \\
\hline Sampson et al. $2010^{81}$ & Canine & PF & $\begin{array}{c}\text { Common } \\
\text { Pool }\end{array}$ & - & - & - \\
\hline $\begin{array}{l}\text { Hashambhoy et al. } \\
2010^{82}\end{array}$ & Canine & VM (Mid) & $\begin{array}{l}\text { Local } \\
\text { Control } \\
\end{array}$ & $\mathrm{I}_{\mathrm{CaL}}, \mathrm{RyR}$ & $\frac{\text { Local signaling }}{\mathrm{I}_{\mathrm{CaL}}, \mathrm{RyR}, \mathrm{PLB}}$ & - \\
\hline $\begin{array}{l}\text { Soltis \& Saucerman } \\
\qquad 2010^{15}\end{array}$ & Rabbit & VM & $\begin{array}{l}\text { Common } \\
\text { Pool }\end{array}$ & - & $\begin{array}{c}\frac{\text { Local signaling }}{I_{\mathrm{CaL}}, I_{\mathrm{Na}}, I_{\mathrm{NaL}}, I_{\mathrm{To}}} \\
\text { RyR, PLB }\end{array}$ & $\begin{array}{c}\frac{\text { Global signaling }}{I_{\text {CaL, }} I_{\text {CFTR }} I_{\text {Ss, }}} \\
\text { Tnl, RyR, PLB }\end{array}$ \\
\hline $\begin{array}{c}\text { Koivumaki et al. } \\
2011^{83}\end{array}$ & Human & AM & $\begin{array}{l}\text { Peripheral } \\
\text { vs. Central }\end{array}$ & - & - & - \\
\hline Heijman et al. $2011^{16}$ & Canine & VM (Epi) & $\begin{array}{l}\text { Common } \\
\text { Pool }\end{array}$ & - & $\frac{\text { Local signaling }}{I_{\mathrm{CaL}}, I_{\mathrm{K} 1}, I_{\mathrm{Na}}, I_{\mathrm{NaL}}}$ & $\begin{array}{c}\text { Local signaling } \\
\mathrm{I}_{\text {CaL}}, I_{\mathrm{Ks}}, I_{\text {Kur }}, I_{\text {Naa }} \\
I_{\text {NaK }}, \text { Tnl, RyR, } \\
\text { PLB }\end{array}$ \\
\hline Li \& Rudy $2011^{84}$ & Canine & PF & $\begin{array}{c}\text { Common } \\
\text { Pool }\end{array}$ & - & $\frac{\text { Local signaling }}{\mathrm{I}_{\mathrm{CaL}}, \mathrm{RyR}, \mathrm{PLB}}$ & - \\
\hline Gaur \& Rudy $2011^{85}$ & $\begin{array}{l}\text { Guinea- } \\
\text { Pig }\end{array}$ & VM & $\begin{array}{l}\text { Local } \\
\text { Control } \\
\end{array}$ & $\mathrm{I}_{\mathrm{CaL}}, \mathrm{RyR}$ & - & - \\
\hline $\mathrm{O}^{\prime}$ Hara et al. $2011^{86}$ & Human & $\begin{array}{c}\text { VM } \\
\text { (Transmural) }\end{array}$ & $\begin{array}{l}\text { Common } \\
\text { Pool }\end{array}$ & - & $\begin{array}{c}\frac{\text { Local signaling }}{I_{\mathrm{CaL}}, I_{\mathrm{Na}}, I_{\mathrm{NaL}}, I_{\mathrm{TO}}} \\
\text { RyR, PLB }\end{array}$ & - \\
\hline Lemay et al. $2011^{87}$ & $\begin{array}{l}\text { Guinea- } \\
\text { Pig }\end{array}$ & VM & $\begin{array}{c}\text { Common } \\
\text { Pool }\end{array}$ & $\begin{array}{c}\mathrm{I}_{\mathrm{CaL}}, \mathrm{I}_{\mathrm{K} 1}, \mathrm{I}_{\mathrm{Kr}}, \mathrm{I}_{\mathrm{Ks}}, \\
\mathrm{I}_{\mathrm{Na}}\end{array}$ & - & - \\
\hline Tao et al. $2011^{88}$ & Rat & $\begin{array}{c}\text { SAN + } \\
\text { Cardiac } \\
\text { Neuron }\end{array}$ & $\begin{array}{l}\text { Common } \\
\text { Pool }\end{array}$ & - & - & $\begin{array}{c}\text { Global signaling } \\
I_{\text {CaL, }}, I_{f}, I_{\text {Ks }}, I_{s t} \\
\text { RyR, PLB }\end{array}$ \\
\hline
\end{tabular}




\begin{tabular}{|c|c|c|c|c|c|c|}
\hline Model $^{\text {Reference }}$ & Species & Cell-type & Model Type & $\begin{array}{l}\text { Stochastic } \\
\text { Targets }\end{array}$ & CaMKII Targets & $\beta A R S$ Targets \\
\hline Grandi et al. $2011^{89}$ & Human & AM & $\begin{array}{c}\text { Common } \\
\text { Pool }\end{array}$ & 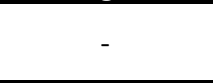 & - & $\begin{array}{c}I_{\text {CaL}}, I_{\mathrm{Ks}}, I_{\mathrm{Kur}}, I_{\mathrm{NaK}}, \\
\text { TnI, RyR, PLB }\end{array}$ \\
\hline Pueyo et al. 2011 ${ }^{90}$ & $\begin{array}{l}\text { Guinea } \\
\text { Pig \& } \\
\text { Human } \\
\end{array}$ & VM & $\begin{array}{l}\text { Common } \\
\text { Pool }\end{array}$ & $\mathrm{I}_{\mathrm{Ks}}$ (Langevin) & - & - \\
\hline Heijman et al. 2012 & Canine & VM (Epi) & $\begin{array}{l}\text { Common } \\
\text { Pool }\end{array}$ & $\begin{array}{c}I_{\mathrm{Ca} L}, I_{\mathrm{CI}(\mathrm{Ca})}, I_{\mathrm{K} 1}, \\
I_{\mathrm{Kr}}, I_{\mathrm{Ks}}, I_{\mathrm{Kur}}, I_{\mathrm{Na}}, \\
I_{\mathrm{NaCa}}, I_{\mathrm{NaK}}, I_{\mathrm{pCa}}, \\
I_{\text {To }}, \text { RyR, SERCA }\end{array}$ & 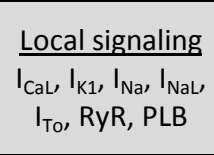 & $\begin{array}{c}\frac{\text { Local signaling }}{I_{\text {CaL }}, I_{\text {Ks }}, I_{\text {Kur }}, I_{\text {Na }}} \\
I_{\text {NaK }}, T n I, R y R, \\
\text { PLB }\end{array}$ \\
\hline
\end{tabular}

Table 1. Comparison of stochastic and signaling properties of existing computational myocyte models. Models are listed in chronological order. PF: Purkinje-fiber cell, VM: ventricular myocyte, SAN: sino-atrial node cell, AM: atrial myocyte, AVN: atrioventricular nodal cell.

The model developed in Chapter 7 of this thesis (last row of Table 1) represents the state-of-the-art in cardiac myocyte modeling, integrating both the BARS and CaMKII signaling cascades in a compartmental model with stochastic gating of 13 major ion channels and $\mathrm{Ca}^{2+}$ handling proteins. The model provides an integration of several important modeling contributions by other groups, including: i) the first integration of $\beta$ ARS signaling and electrophysiology by Saucerman et al. ${ }^{51,55}$ ii) the first model of localized $\beta$ ARS signaling by lancu et al. ${ }^{62,91}$ iii) the first integration of CaMKII signaling and electrophysiology by Hund \& Rudy ${ }^{54}$ and iv) several stochastic properties. ${ }^{57,71,85,87}$ Figure 2 also highlights this integration and presents a comparison of a selection of cellular computational models of cardiac electrophysiology over time, from 1993-2012. The complexity of each model was determined in four categories: i) electrophysiological complexity (quantified by the number of state variables); ii) complexity of the stochastic components; iii) complexity of the CaMKII components and iv) complexity of the $\beta A R S$ components. For categories ii-iv, the complexity of model $\mathrm{i}$ is determined by $\left(\mathrm{N}_{\mathrm{i}}^{\mathrm{X}}+1\right) \cdot \alpha^{\mathrm{X}}$, where $\mathrm{N}_{\mathrm{i}}^{\mathrm{X}}$ represents the number of electrophysiological targets affected by category $\mathrm{x}$ and $\alpha^{\mathrm{x}}$ is a scaling factor that is 1.0 in the absence of an underlying signaling cascade and 3.0 otherwise. For example, for the Heijman et al. 2011 model (Chapter 3) the CaMKII complexity equals 24 (7 targets, with cascade, Table 1 ) and the stochastic complexity 1 (no targets, no local control). To compare the individual categories, each complexity was normalized between 0 (for the lowest value among all models) and 1 (for the highest value). The overall complexity (Figure 2) represents the average normalized model complexity over the four categories. It can be appreciated that in recent years computational cell models of cardiac electrophysiology have become increasingly complex and now provide the ability to not only investigate purely electrical phenomena but also electromechanical (not shown) and electrophysiological modulation by signaling pathways. 


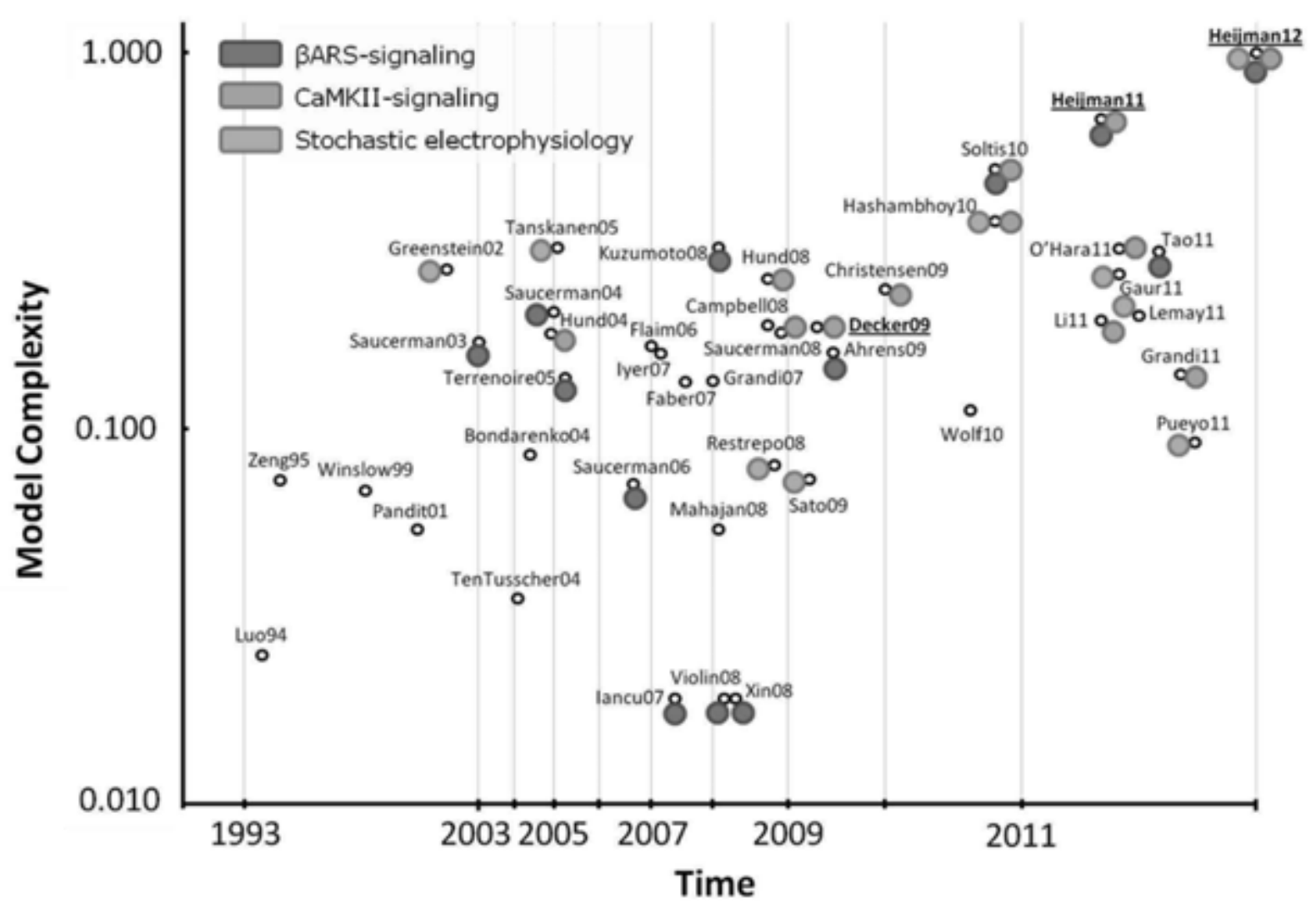

Figure 2. Comparison of cellular computational models of electrophysiology with a focus on the period 2000-2011. Models are identified based on the name of the first author and year of publication. Horizontal axis represents time on a logarithmic scale. Vertical axis represents average model complexity. Colored circles indentify models containing an integrated $\beta$ ARS cascade (blue), CaMKII-signaling cascade (orange) and stochastic electrophysiology (green). Models developed in this thesis (indicated with Decker09, Heijman11 and Heijman12 for Chapters 2, $\mathbf{3}$ and 7, respectively) are underlined. For color figure, see page 256.

\subsection{APPLICATIONS OF COMPUTATIONAL MODELS OF CARDiaC ELECTROPHYSIOLOGY}

\subsubsection{Cellular applications}

In this thesis, we have shown that cellular computational models of cardiac electrophysiology are powerful tools to extrapolate experimental results (Chapter 4), enabling to make predictions about the effect of mutations in the integrated environment of the cardiac myocyte in which multiple ion currents interact to shape the AP. Until recently, studies of the arrhythmogenic effects of genetic alterations in-situ were primarily performed in transgenic mouse models. Although these models have provided seminal insights into different pathologies, their application to electrophysiological conditions requires particular care due to the large differences of cardiac electrophysiology between mice and humans. ${ }^{92,93}$ Recent developments in research on induced pluripotent stem cells (IPS cells) 
have resulted in alternative electrophysiological preparations based on IPS-derived cardiomyocytes that can be used for patient-specific investigations of arrhythmogenesis. ${ }^{94-96}$ However, these preparations are methodologically complex, expensive and time-consuming. More importantly, there are still considerable difficulties with IPS-derived cells, including atypical maturation, lack of specific control of cell type (atrial versus ventricular versus nodal) and abnormal electrophysiological activity (e.g., significant spontaneous/pacemaker activity). ${ }^{97}$ These aspects may improve over time when more advanced methods become available. Computational models remain of paramount importance for the extrapolation of altered biophysical properties of an ion channel to changes at the level of the cardiac action potential and/or tissue. These insights are important to determine the consequences of ion-channel mutations under dynamic conditions and can help to explain pleiotropic effects of single mutations. For instance, Clancy and Rudy were among the first to employ Markov models of the cardiac $\mathrm{Na}^{+}$channel to study the 1795 insD mutation in SCN5A. ${ }^{98}$ They found that heart rate determined whether this mutation exhibited Brugada syndrome or long-QT syndrome type- 3 characteristics, extending on earlier experimental observations. ${ }^{99,}{ }^{100}$ Moreover, these authors as well as several other groups have shown that computational models have utility in the field of electropharmacology. Models can be used to predict the optimal characteristics of pharmacological agents for specific conditions (e.g., predominant open versus inactivated state block of an ion channel ${ }^{101}$ ) as well as assist in the drug-discovery process. ${ }^{102-105}$

Given the limitation that human cardiac preparations=are only rarely available for experiments, computer models allow for a detailed quantitative investigation of generic, human electrophysiological mechanisms by manipulation of individual model components. This ability can help to direct further experimental investigations, thereby allowing for a more efficient use of experimental resources and is facilitated by the recent human-specific models of the cardiac myocyte developed by several groups. ${ }^{80,83,86,89}$ We have employed our computational model for this purpose to study the mechanisms involved in APD prolongation after SCR (Chapter 6). The model corroborated preliminary experimental data and specifically identified reduced $\mathrm{Ca}^{2+}$-induced-Ca ${ }^{2+}$ release-dependent inactivation of the L-type $\mathrm{Ca}^{2+}$ current as the APD prolonging mechanism. This finding was subsequently confirmed in isolated ventricular myocytes under voltage-clamp conditions. This example demonstrates that computational modeling studies can lead the way in experimental design as well as help to identify arrhythmogenic mechanisms.

In addition to extrapolation and integration of experimental data, computational models can quantify the contribution of individual electrophysiological components to whole-cell properties, as was done in Chapter $\mathbf{3}$ for the determinants of localized cAMP signaling, and in Chapter $\mathbf{7}$ for the ionic contributors to BVR. Such an assessment can be performed by the selective inhibition of specific components, creating 'virtual knock-out' models. An alternative method was recently proposed by Sobie and coworkers based on multiple regression. ${ }^{106}$ In their approach, multiple parameter sets (effectively representing individual cells) were simulated and the output variables of interest were determined. Thereafter, a regression procedure was used to determine the impact of each parameter on the output variables. Such a modeling approach provides an overall picture of 
the determinants of a given process that is impossible to obtain experimentally since not all parameters can be quantified.

\subsubsection{Clinical applications}

Despite the important role that computational models have played in basic cardiovascular sciences, their direct clinical application is still in its infancy. Indirectly, together with extensive additional experimental research, computational models can influence clinical practice by suggesting novel therapeutic targets or biomarkers. ${ }^{103}$ For example, our computational (Chapter 3) and cellular experimental (Chapter 6) results, together with data from other groups ${ }^{107}$ suggest that targeting CaMKII could be a promising pathway to reduce $\mathrm{Ca}^{2+}$-dependent arrhythmogenesis, when the right pharmacological compound becomes available. ${ }^{108}$

An extremely interesting direct application of computational models in clinical practice would entail (arrhythmogenic) risk stratification of patients based on genotypic and phenotypic information. The major challenge is that cardiac pathologies are multifactorial and occur at all levels of electrophysiological integrity. Most computational models to date are concerned with cellular electrophysiology and as such cannot be applied directly to study arrhythmogenesis in patients, which is only visible at the level of the intact organ. However, (sub)cellular properties will likely determine the vulnerability of the organ to develop and sustain such arrhythmias. These properties can be evaluated in computational models and could in theory be used for risk-stratification purposes. However, recent research has indicated that even channelopathies which were originally considered monogenic diseases are not truly monogenic. ${ }^{109}$ Research in large founder populations has highlighted a remarkable diversity of clinical phenotypes, despite identical disease-causing mutations. ${ }^{110}$ These results have prompted the investigation of modifier genes that help to explain this phenotypic diversity. ${ }^{111-113}$ Often, these modifiers are common variants in non-protein coding regions of a gene, making it extremely difficult to include them in computational models. However, in order to accurately predict proarrhythmic risk in an individual patient these risk modifiers will have to be taken into account.

Investigating arrhythmogenesis using computational models is even more complex for acquired diseases, in which the underlying pathology is diverse, and multiple intrinsic and extrinsic processes affect the cellular electrophysiology. In several acquired conditions including heart failure, digitalis intoxication and myocardial ischemia, the adrenergic signaling pathway and/or its effects on ventricular repolarization are affected, resulting in an increased proarrhythmic risk during sympathetic stimulation. ${ }^{114-116}$ In the general population, these pathological conditions are significantly more common than inherited. Cellular models of the electrophysiological effects of several acquired conditions, including heart failure, ${ }^{46}$ hypertension, ${ }^{117}$ and of cells surviving the epicardial borderzone after myocardial infarction, ${ }^{118}$ have been described. However, to the best of our knowledge, none of these models are human-specific or have incorporated the effects of sympathetic stimulation. To study sympathetic-triggered arrhythmias in the human heart, these models will have to be significantly extended with the functional and structural alterations that 
occur under these conditions, including elements such as desensitization of the adrenergic signaling cascade, ${ }^{114}$ regional cardiac hyperinnervation, ${ }^{119}$ and arrhythmogenic ion-channel remodeling. ${ }^{120}$

Thus, although there certainly is a clear short-term clinical relevance of computational models via the facilitation of drug development and improved understanding of arrhythmogenesis, the integration of computational models into daily clinical practice to support diagnostic and therapeutic decisions (i.e., "personalized medicine") will still require a substantial concerted effort of multidisciplinary research teams.

\subsection{SYSTEMS BIOLOGY}

We consider the work presented in this thesis an example of a systems-biology approach to understand arrhythmogenesis at the level of the single cardiac myocyte. Kohl et al. ${ }^{121}$ stated that systems biology "necessarily involves the combined application of 'reductionist' and 'integrationist' research techniques, to allow identification and detailed characterization of the parts, investigation of their interaction with one another and with their wider environment, and elucidation of how parts and interactions give rise to maintenance of the entity" (Figure 3). Another major characteristic of systems biology according to Kohl et al. is that "it makes extensive use of mathematical modeling in order to represent and understand complex interactions of parts and biological entities." ${ }^{121}$
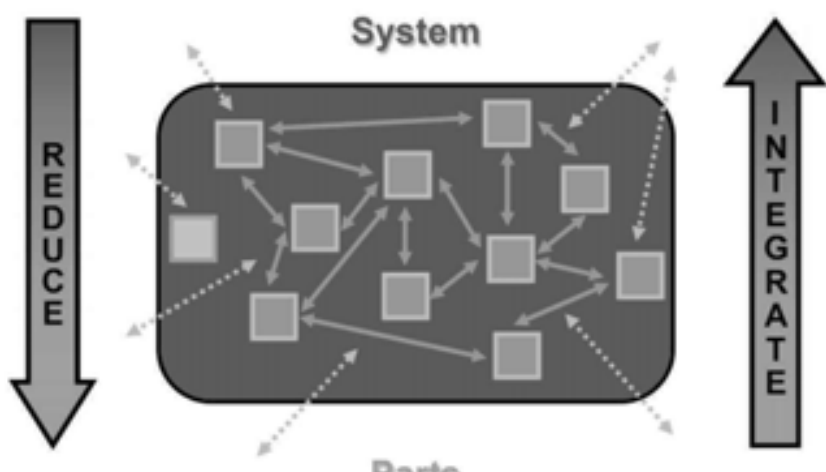

Figure 3. "A system as an 'entity that maintains its existence through the mutual interaction of its parts' (von Bertalanffy, 1968). Systems research must combine the (i) identification and (ii) detailed characterisation of parts (orange boxes, as opposed to 'look-alikes', pale blue box, which need to be identified and excluded), with the exploration of their interactions (iii) with each other (orange arrows), and (iv) with the environment (pale blue dashed arrows affecting parts either directly, or indirectly through modulation of internal interactions), to develop a (v) systemic understanding (an important, but often overlooked, aspect is that the system itself not only enables, but also restricts, the type and extent of functions and interactions that may occur; dark-blue box). Systems research 
therefore requires a combination of reductionist and integrative tools and techniques." Reproduced from Kohl and Noble. ${ }^{122}$ For color figure, see page 256.

Although several definitions of systems biology exist, we believe that the above definition exemplifies an optimal approach to improve understanding and treatment of cardiovascular disease. In this thesis, we employed a detailed computational model of the canine ventricular myocyte to identify the electrophysiological and signaling components operating during $\beta A R S$, their interaction, and their contribution to the whole-cell response during $\beta A R S$. This approach could be considered the starting point of a "middle-out" approach, in which comprehensive cellular models are extended towards the extremes of the spatial scales: from gene to organism. ${ }^{121}$

\subsubsection{Perspectives of computational electrophysiology}

Results such as those presented in this thesis have indicated that local subcellular processes are critical for the correct functioning of the heart, particularly during $\beta A R S$. Recent studies have started to combine cellular electrophysiology and molecular dynamics. ${ }^{123}$ In the future, such models will provide an important mechanistic link between genotypes and phenotypes. For example, the mechanisms underlying the loss of KCNQ1 phosphorylation in the presence of the S6 mutation KCNQ1-A341V (Chapter 4) are currently unclear, but may involve a defective transfer of PKA from the C-terminally-bound Yotiao to the $\mathrm{N}$-terminal phosphorylation site. Combined molecular and cellular computational models will provide information about the structural alterations due to A341V that could contribute to these biophysical alterations. In addition, some ion-channel mutations, particularly in the $\mathrm{Na}^{+}$channel, have been related to macroscopic structural alterations including dilated cardiomyopathy. ${ }^{124,}{ }^{125}$ The mechanisms relating these electrical and structural abnormalities are currently incompletely understood. Future computational modeling studies may help to investigate the abnormalities in $\mathrm{Na}^{+}$homeostasis as well as the downstream signaling effects caused by these mutations.

Similarly, there are many branches of the subcellular signaling pathways that require further integration in the computational cell models. Of particular interest in this respect are the interaction between $\beta A R S$ and CaMKII via the exchange protein activated by CAMP (EPAC) ${ }^{17,126}$ and the interaction between CAMP and cyclic GMP (cGMP). ${ }^{127}$ Both EPAC and cGMP have downstream effects on protein kinase $C$ which, like PKA, can phosphorylate many electrophysiological targets. The complex interplay between these networks will require novel computational approaches capable of simulating localized interactions within the cardiac myocyte to identify their role in arrhythmogenesis.

On the other hand, in order to apply these models to clinical problems, whole-heart stronglycoupled electromechanical simulations taking into account spatial heterogeneities in electrophysiology and signaling are required. Until now, the majority of computational models were 
concerned with the cellular level of cardiac electrophysiology. As computational power has increased, recent studies have expanded to multicellular simulations and several groups have described whole-heart models of cardiac electrophysiology. ${ }^{128-131}$

One of the major challenges in biomedical engineering in general and computational cardiac electrophysiology in particular, will be to bridge these microscopic and macroscopic worlds. In order to do this, both technical and theoretical advances will have to be made. Recently, several groups have reported on the use of graphics processing unit-based computations, ${ }^{132}$ or large multiprocessor systems ${ }^{133}$ to facilitate multi-scale simulations. In addition, more efficient computational techniques to solve the large systems of (partial) differential equations are being developed. Several simulation packages for cardiac electrophysiology are available. However, the example given by Plank et al. ${ }^{134}$ in which, using the CARP simulation package, 6.4 hours of CPU time were required to simulate 200-ms of electrical activity on a 862,515 node rabbit ventricular mesh using a simple ionic model, indicates that significant further improvements in simulation efficiency are required before detailed models can be employed at the whole-heart level.

The Virtual Physiological Human (VPH) Initiative has as one of its goals to "understand human physiology quantitatively, as a dynamic system, and at all relevant levels between genes and the organism" ${ }^{\prime 22}$ and, as such, incorporates the connection of microscopic and macroscopic research domains. Recently, well-validated models of the human ventricular ${ }^{80,86}$ and atrial ${ }^{83,89}$ myocyte electrophysiology have been published. Although several structural properties of the human heart can be obtained using non-invasive imaging modalities (e.g., magnetic resonance imaging and computed tomography scanning), local endocardial activation maps can only be obtained semiinvasively using catheter-based mapping techniques. Obtaining direct measurements of epicardial activation would require open-chest surgery and, consequently, relatively unphysiological conditions. Thus, the validation of whole heart-models will also present a major challenge for future research, particularly in the case of human-specific computational models. One promising approach could be to employ non-invasive reconstructions of epicardial electrical activity based on geometrical information and body-surface maps to validate whole-heart models. This technique, termed electrocardiographic imaging (ECGI), has been used successfully to characterize physiological and abnormal electrical activity in patients ${ }^{135-137}$ and has as a major advantage that it can provide a continuous reconstruction of electrical activity throughout the entire heart that could be superimposed on a whole-heart model employing the same geometrical information.

A comprehensive, systems-biology approach to human cardiac electrophysiology will provide a wealth of information that can be employed to improve the risk stratification and (pharmacological) treatment of patients at risk for sudden arrhythmic death and less malignant arrhythmias. Detailed cell models such as those developed in this thesis provide an important starting point for this approach. 


\subsection{REFERENCES}

1. Taggart $P$, Boyett MR, Logantha $S$, Lambiase PD. Anger, emotion, and arrhythmias: from brain to heart. Front Physiol. 2011;2:67.

2. La Rovere MT, Pinna GD, Hohnloser SH, Marcus FI, Mortara A, Nohara R, Bigger JT, Jr., Camm AJ, Schwartz PJ. Baroreflex sensitivity and heart rate variability in the identification of patients at risk for life-threatening arrhythmias: implications for clinical trials. Circulation. 2001;103:2072-2077.

3. Morise AP. Exercise testing in nonatherosclerotic heart disease: hypertrophic cardiomyopathy, valvular heart disease, and arrhythmias. Circulation. 2011;123:216-225.

4. Vyas $\mathrm{H}, \mathrm{Hejlik} J$, Ackerman MJ. Epinephrine QT stress testing in the evaluation of congenital long-QT syndrome: diagnostic accuracy of the paradoxical QT response. Circulation. 2006;113:1385-1392.

5. Schwartz PJ, Priori SG, Cerrone M, Spazzolini C, Odero A, Napolitano C, Bloise R, De Ferrari GM, Klersy C, Moss AJ, Zareba W, Robinson JL, Hall WJ, Brink PA, Toivonen L, Epstein AE, Li C, $\mathrm{Hu}$ D. Left cardiac sympathetic denervation in the management of high-risk patients affected by the long-QT syndrome. Circulation. 2004;109:1826-1833.

6. Wilde AA, Bhuiyan ZA, Crotti L, Facchini M, De Ferrari GM, Paul T, Ferrandi C, Koolbergen DR, Odero A, Schwartz PJ. Left cardiac sympathetic denervation for catecholaminergic polymorphic ventricular tachycardia. N Engl J Med. 2008;358:2024-2029.

7. Bourke T, Vaseghi M, Michowitz Y, Sankhla V, Shah M, Swapna N, Boyle NG, Mahajan A, Narasimhan C, Lokhandwala Y, Shivkumar K. Neuraxial modulation for refractory ventricular arrhythmias: value of thoracic epidural anesthesia and surgical left cardiac sympathetic denervation. Circulation. 2010;121:2255-2262.

8. Ajijola OA, Lellouche N, Bourke T, Tung R, Ahn S, Mahajan A, Shivkumar K. Bilateral cardiac sympathetic denervation for the management of electrical storm. J Am Coll Cardiol. 2012;59:91-92.

9. Priori SG, Mantica M, Schwartz PJ. Delayed afterdepolarizations elicited in vivo by left stellate ganglion stimulation. Circulation. 1988;78:178-185.

10. Tanabe $Y$, Inagaki M, Kurita $T$, Nagaya N, Taguchi A, Suyama $K$, Aihara N, Kamakura S, Sunagawa K, Nakamura K, Ohe T, Towbin JA, Priori SG, Shimizu W. Sympathetic stimulation produces a greater increase in both transmural and spatial dispersion of repolarization in LQT1 than LQT2 forms of congenital long QT syndrome. J Am Coll Cardiol. 2001;37:911-919.

11. Gallacher DJ, Van de Water A, van der Linde H, Hermans AN, Lu HR, Towart R, Volders PGA. In vivo mechanisms precipitating torsades de pointes in a canine model of drug-induced long-QT1 syndrome. Cardiovasc Res. 2007;76:247-256.

12. Hoeker GS, Katra RP, Wilson LD, Plummer BN, Laurita KR. Spontaneous calcium release in tissue from the failing canine heart. Am J Physiol Heart Circ Physiol. 2009;297:H1235-1242.

13. Kashimura T, Briston SJ, Trafford AW, Napolitano C, Priori SG, Eisner DA, Venetucci LA. In the RyR2(R4496C) mouse model of CPVT, beta-adrenergic stimulation induces Ca waves by increasing SR Ca content and not by decreasing the threshold for Ca waves. Circ Res. 2010;107:1483-1489.

14. Volders PGA. Novel insights into the role of the sympathetic nervous system in cardiac arrhythmogenesis. Heart Rhythm. 2010;7:1900-1906.

15. Soltis AR, Saucerman JJ. Synergy between CaMKII Substrates and beta-Adrenergic Signaling in Regulation of Cardiac Myocyte Ca ${ }^{2+}$ Handling. Biophys J. 2010;99:2038-2047.

16. Heijman J, Volders PGA, Westra RL, Rudy Y. Local control of $\beta$-adrenergic stimulation: Effects on ventricular myocyte electrophysiology and $\mathrm{Ca}^{2+}$-transient. J Mol Cell Cardiol. 2011;50:863871.

17. Grimm M, Brown JH. $\beta$-Adrenergic receptor signaling in the heart: Role of CaMKII. J Mol Cell Cardiol. 2010;48:322-330. 
18. Steinberg SF, Brunton LL. Compartmentation of $\mathrm{G}$ protein-coupled signaling pathways in cardiac myocytes. Annu Rev Pharmacol Toxicol. 2001;41:751-773.

19. Zaccolo M. Spatial control of cAMP signalling in health and disease. Curr Opin Pharmacol. 2011;11:649-655.

20. Fischmeister R, Castro LRV, Abi-Gerges A, Rochais F, Jurevicius J, Leroy J, Vandecasteele G. Compartmentation of cyclic nucleotide signaling in the heart: the role of cyclic nucleotide phosphodiesterases. Circ Res. 2006;99:816-828.

21. Nikolaev VO, Bunemann M, Schmitteckert E, Lohse MJ, Engelhardt S. Cyclic AMP imaging in adult cardiac myocytes reveals far-reaching $\beta 1$-adrenergic but locally confined $\beta 2$-adrenergic receptor-mediated signaling. Circ Res. 2006;99:1084-1091.

22. Vandecasteele $G$, Rochais $F$, Abi-Gerges A, Fischmeister R. Functional localization of cAMP signalling in cardiac myocytes. Biochem Soc Trans. 2006;34:484-488.

23. Zaccolo M, Di Benedetto G, Lissandron V, Mancuso L, Terrin A, Zamparo I. Restricted diffusion of a freely diffusible second messenger: mechanisms underlying compartmentalized cAMP signalling. Biochem Soc Trans. 2006;34:495-497.

24. Agarwal SR, MacDougall DA, Tyser R, Pugh SD, Calaghan SC, Harvey RD. Effects of cholesterol depletion on compartmentalized cAMP responses in adult cardiac myocytes. J Mol Cell Cardiol. 2011;50:500-509.

25. Nikolaev VO, Moshkov A, Lyon AR, Miragoli M, Novak P, Paur H, Lohse MJ, Korchev YE, Harding $\mathrm{SE}$, Gorelik J. $\beta 2$-adrenergic receptor redistribution in heart failure changes CAMP compartmentation. Science. 2010;327:1653-1657.

26. Marx SO, Kurokawa J, Reiken S, Motoike H, D'Armiento J, Marks AR, Kass RS. Requirement of a macromolecular signaling complex for $\beta$ adrenergic receptor modulation of the KCNQ1KCNE1 potassium channel. Science. 2002;295:496-499.

27. Chen L, Marquardt ML, Tester DJ, Sampson KJ, Ackerman MJ, Kass RS. Mutation of an Akinase-anchoring protein causes long-QT syndrome. Proc Natl Acad Sci U S A. 2007;104:20990-20995.

28. Volders PGA, StengI M, van Opstal JM, Gerlach U, Spätjens RL, Beekman JD, Sipido KR, Vos MA. Probing the contribution of $I_{\mathrm{Ks}}$ to canine ventricular repolarization: key role for $\beta$ adrenergic receptor stimulation. Circulation. 2003;107:2753-2760.

29. Jost N, Virág L, Bitay M, Takács J, Lengyel C, Biliczki P, Nagy Z, Bogáts G, Lathrop DA, Papp JG, Varró A. Restricting excessive cardiac action potential and QT prolongation: a vital role for $\mathrm{I}_{\mathrm{Ks}}$ in human ventricular muscle. Circulation. 2005;112:1392-1399.

30. Carmeliet E. Repolarization Reserve in Cardiac Cells. J Med Biol Eng. 2006;26:97-105.

31. Thomsen MB, Verduyn SC, StengI M, Beekman JD, de Pater G, van Opstal J, Volders PGA, Vos MA. Increased short-term variability of repolarization predicts $d$-sotalol-induced torsades de pointes in dogs. Circulation. 2004;110:2453-2459.

32. Hinterseer $M$, Thomsen MB, Beckmann BM, Pfeufer A, Schimpf $R$, Wichmann HE, Steinbeck $G$, Vos MA, Kaab S. Beat-to-beat variability of QT intervals is increased in patients with druginduced long-QT syndrome: a case control pilot study. Eur Heart J. 2008;29:185-190.

33. Hinterseer $M$, Beckmann $B M$, Thomsen MB, Pfeufer $A$, Ulbrich $M$, Sinner MF, Perz $S$, Wichmann HE, Lengyel C, Schimpf R, Maier SK, Várro A, Vos MA, Steinbeck G, Kääb S. Usefulness of short-term variability of QT intervals as a predictor for electrical remodeling and proarrhythmia in patients with nonischemic heart failure. Am J Cardiol. 2010;106:216220.

34. Noble D. A modification of the Hodgkin-Huxley equations applicable to Purkinje fibre action and pace-maker potentials. J Physiol. 1962;160:317-352.

35. McAllister RE, Noble D, Tsien RW. Reconstruction of the electrical activity of cardiac Purkinje fibres. J Physiol. 1975;251:1-59.

36. Beeler GW, Reuter $\mathrm{H}$. Reconstruction of the action potential of ventricular myocardial fibres. J Physiol. 1977;268:177-210. 
37. Kass RS, Wiegers SE. The ionic basis of concentration-related effects of noradrenaline on the action potential of calf cardiac purkinje fibres. J Physiol. 1982;322:541-558.

38. Rasmusson RL, Clark JW, Giles WR, Robinson K, Clark RB, Shibata EF, Campbell DL. A mathematical model of electrophysiological activity in a bullfrog atrial cell. Am J Physiol. 1990;259:H370-389.

39. Rasmusson RL, Clark JW, Giles WR, Shibata EF, Campbell DL. A mathematical model of a bullfrog cardiac pacemaker cell. Am J Physiol. 1990;259:H352-369.

40. Liu Y, Zeng W, Delmar M, Jalife J. Ionic mechanisms of electronic inhibition and concealed conduction in rabbit atrioventricular nodal myocytes. Circulation. 1993;88:1634-1646.

41. Luo $\mathrm{CH}$, Rudy Y. A dynamic model of the cardiac ventricular action potential. I. Simulations of ionic currents and concentration changes. Circ Res. 1994;74:1071-1096.

42. Luo $\mathrm{CH}$, Rudy Y. A dynamic model of the cardiac ventricular action potential. II. Afterdepolarizations, triggered activity, and potentiation. Circ Res. 1994;74:1097-1113.

43. Zeng J, Rudy Y. Early afterdepolarizations in cardiac myocytes: mechanism and rate dependence. Biophys J. 1995;68:949-964.

44. Courtemanche M, Ramirez RJ, Nattel S. Ionic mechanisms underlying human atrial action potential properties: insights from a mathematical model. Am J Physiol. 1998;275:H301-321.

45. Nygren A, Fiset C, Firek L, Clark JW, Lindblad DS, Clark RB, Giles WR. Mathematical model of an adult human atrial cell: the role of $\mathrm{K}^{+}$currents in repolarization. Circ Res. 1998;82:63-81.

46. Winslow RL, Rice J, Jafri S, Marban E, O'Rourke B. Mechanisms of altered excitationcontraction coupling in canine tachycardia-induced heart failure, II: model studies. Circ Res. 1999;84:571-586.

47. Pandit SV, Clark RB, Giles WR, Demir SS. A mathematical model of action potential heterogeneity in adult rat left ventricular myocytes. Biophys J. 2001;81:3029-3051.

48. Puglisi JL, Bers DM. LabHEART: an interactive computer model of rabbit ventricular myocyte ion channels and Ca transport. Am J Physiol Cell Physiol. 2001;281:C2049-2060.

49. Greenstein JL, Winslow RL. An integrative model of the cardiac ventricular myocyte incorporating local control of $\mathrm{Ca}^{2+}$ release. Biophys J. 2002;83:2918-2945.

50. Oehmen CS, Giles WR, Demir SS. Mathematical model of the rapidly activating delayed rectifier potassium current $\mathrm{I}_{\mathrm{kr}}$ in rabbit sinoatrial node. J Cardiovasc Electrophysiol. 2002;13:1131-1140.

51. Saucerman JJ, Brunton LL, Michailova AP, McCulloch AD. Modeling $\beta$-adrenergic control of cardiac myocyte contractility in silico. J Biol Chem. 2003;278:47997-48003.

52. ten Tusscher KH, Noble D, Noble PJ, Panfilov AV. A model for human ventricular tissue. Am J Physiol Heart Circ Physiol. 2004;286:H1573-1589.

53. Bondarenko VE, Szigeti GP, Bett GC, Kim SJ, Rasmusson RL. Computer model of action potential of mouse ventricular myocytes. Am J Physiol Heart Circ Physiol. 2004;287:H13781403.

54. Hund TJ, Rudy Y. Rate dependence and regulation of action potential and calcium transient in a canine cardiac ventricular cell model. Circulation. 2004;110:3168-3174.

55. Saucerman JJ, Healy SN, Belik ME, Puglisi JL, McCulloch AD. Proarrhythmic consequences of a KCNQ1 AKAP-binding domain mutation: computational models of whole cells and heterogeneous tissue. Circ Res. 2004;95:1216-1224.

56. Shannon TR, Wang F, Puglisi J, Weber C, Bers DM. A mathematical treatment of integrated Ca dynamics within the ventricular myocyte. Biophys J. 2004;87:3351-3371.

57. Tanskanen AJ, Greenstein JL, O'Rourke B, Winslow RL. The role of stochastic and modal gating of cardiac L-type $\mathrm{Ca}^{2+}$ channels on early after-depolarizations. Biophys J. 2005;88:8595.

58. Terrenoire C, Clancy CE, Cormier JW, Sampson KJ, Kass RS. Autonomic control of cardiac action potentials: role of potassium channel kinetics in response to sympathetic stimulation. Circ Res. 2005;96:e25--e34. 
59. Saucerman JJ, Zhang J, Martin JC, Peng LX, Stenbit AE, Tsien RY, McCulloch AD. Systems analysis of PKA-mediated phosphorylation gradients in live cardiac myocytes. Proc Natl Acad Sci U S A. 2006;103:12923-12928.

60. Flaim SN, Giles WR, McCulloch $A D$. Contributions of sustained $I_{\mathrm{Na}}$ and $I_{\mathrm{Kv} 43}$ to transmural heterogeneity of early repolarization and arrhythmogenesis in canine left ventricular myocytes. Am J Physiol Heart Circ Physiol. 2006;291:H2617-H2629.

61. Iyer V, Hajjar RJ, Armoundas AA. Mechanisms of abnormal calcium homeostasis in mutations responsible for catecholaminergic polymorphic ventricular tachycardia. Circ Res. 2007;100:e22-e31.

62. lancu RV, Jones SW, Harvey RD. Compartmentation of cAMP signaling in cardiac myocytes: a computational study. Biophys J. 2007;92:3317-3331.

63. Faber GM, Rudy Y. Calsequestrin mutation and catecholaminergic polymorphic ventricular tachycardia: a simulation study of cellular mechanism. Cardiovasc Res. 2007;75:79-88.

64. Grandi E, Puglisi JL, Wagner S, Maier LS, Severi S, Bers DM. Simulation of Ca-calmodulindependent protein kinase II on rabbit ventricular myocyte ion currents and action potentials. Biophys J. 2007;93:3835-3847.

65. Fink $M$, Noble $D$, Virag L, Varro A, Giles WR. Contributions of HERG $\mathrm{K}^{+}$current to repolarization of the human ventricular action potential. Prog Biophys Mol Biol. 2008;96:357-376.

66. Kuzumoto $M$, Takeuchi A, Nakai H, Oka C, Noma A, Matsuoka S. Simulation analysis of intracellular $\mathrm{Na}+$ and $\mathrm{Cl}$ - homeostasis during $\beta 1$-adrenergic stimulation of cardiac myocyte. Prog Biophys Mol Biol. 2008;96:171-186.

67. Mahajan A, Shiferaw $Y$, Sato D, Baher A, Olcese R, Xie LH, Yang MJ, Chen PS, Restrepo JG, Karma A, Garfinkel A, Qu Z, Weiss JN. A rabbit ventricular action potential model replicating cardiac dynamics at rapid heart rates. Biophys J. 2008;94:392-410.

68. Violin JD, DiPilato LM, Yildirim N, Elston TC, Zhang J, Lefkowitz RJ. $\beta 2$-adrenergic receptor signaling and desensitization elucidated by quantitative modeling of real time cAMP dynamics. J Biol Chem. 2008;283:2949-2961.

69. Xin W, Tran TM, Richter W, Clark RB, Rich TC. Roles of GRK and PDE4 activities in the regulation of $\beta 2$ adrenergic signaling. J Gen Physiol. 2008;131:349-364.

70. Hund TJ, Decker KF, Kanter E, Mohler PJ, Boyden PA, Schuessler RB, Yamada KA, Rudy Y. Role of activated CaMKII in abnormal calcium homeostasis and $I_{N a}$ remodeling after myocardial infarction: insights from mathematical modeling. J Mol Cell Cardiol. 2008;45:420-428.

71. Restrepo JG, Weiss JN, Karma A. Calsequestrin-mediated mechanism for cellular calcium transient alternans. Biophys J. 2008;95:3767-3789.

72. Saucerman JJ, Bers DM. Calmodulin mediates differential sensitivity of CaMKII and calcineurin to local $\mathrm{Ca}^{2+}$ in cardiac myocytes. Biophys J. 2008;95:4597-4612.

73. Sato D, Xie LH, Sovari AA, Tran DX, Morita N, Xie F, Karagueuzian H, Garfinkel A, Weiss JN, Qu Z. Synchronization of chaotic early afterdepolarizations in the genesis of cardiac arrhythmias. Proc Natl Acad Sci U S A. 2009;106:2983-2988.

74. Maleckar MM, Greenstein JL, Giles WR, Trayanova NA. $\mathrm{K}^{+}$current changes account for the rate dependence of the action potential in the human atrial myocyte. Am J Physiol Heart Circ Physiol. 2009;297:H1398-1410.

75. Decker KF, Heijman J, Silva JR, Hund TJ, Rudy Y. Properties and ionic mechanisms of action potential adaptation, restitution, and accommodation in canine epicardium. Am J Physiol Heart Circ Physiol. 2009;296:H1017-1026.

76. Ahrens-Nicklas RC, Clancy CE, Christini DJ. Re-evaluating the efficacy of beta-adrenergic agonists and antagonists in long QT-3 syndrome through computational modelling. Cardiovasc Res. 2009;82:439-447. 
77. Koivumaki JT, Korhonen T, Takalo J, Weckstrom M, Tavi P. Regulation of excitationcontraction coupling in mouse cardiac myocytes: integrative analysis with mathematical modelling. BMC Physiol. 2009;9:16.

78. Christensen MD, Dun W, Boyden PA, Anderson ME, Mohler PJ, Hund TJ. Oxidized calmodulin kinase II regulates conduction following myocardial infarction: a computational analysis. PLoS Comput Biol. 2009;5:e1000583.

79. Wolf RM, Mitchell CC, Christensen MD, Mohler PJ, Hund TJ. Defining new insight into atypical arrhythmia: a computational model of ankyrin-B syndrome. Am J Physiol Heart Circ Physiol. 2010;299:H1505-1514.

80. Grandi E, Pasqualini FS, Bers DM. A novel computational model of the human ventricular action potential and Ca transient. J Mol Cell Cardiol. 2010;48:112-121.

81. Sampson KJ, Iyer V, Marks AR, Kass RS. A computational model of Purkinje fibre single cell electrophysiology: implications for the long QT syndrome. J Physiol. 2010;588:2643-2655.

82. Hashambhoy YL, Greenstein JL, Winslow RL. Role of CaMKII in RyR leak, EC coupling and action potential duration: a computational model. J Mol Cell Cardiol. 2010;49:617-624.

83. Koivumaki JT, Korhonen T, Tavi P. Impact of sarcoplasmic reticulum calcium release on calcium dynamics and action potential morphology in human atrial myocytes: a computational study. PLoS Comput Biol. 2011;7:e1001067.

84. Li P, Rudy Y. A model of canine purkinje cell electrophysiology and $\mathrm{Ca}^{2+}$ cycling: rate dependence, triggered activity, and comparison to ventricular myocytes. Circ Res. 2011;109:71-79.

85. Gaur N, Rudy Y. Multiscale modeling of calcium cycling in cardiac ventricular myocyte: macroscopic consequences of microscopic dyadic function. Biophysical journal. 2011;100:2904-2912.

86. O'Hara T, Virag L, Varro A, Rudy Y. Simulation of the undiseased human cardiac ventricular action potential: model formulation and experimental validation. PLoS Comput Biol. 2011;7:e1002061.

87. Lemay $M$, de Lange $E$, Kucera JP. Effects of stochastic channel gating and distribution on the cardiac action potential. Journal of Theoretical Biology. 2011;281:84-96.

88. Tao T, Paterson DJ, Smith NP. A model of cellular cardiac-neural coupling that captures the sympathetic control of sinoatrial node excitability in normotensive and hypertensive rats. Biophys J. 2011;101:594-602.

89. Grandi E, Pandit SV, Voigt N, Workman AJ, Dobrev D, Jalife J, Bers DM. Human atrial action potential and $\mathrm{Ca}^{2+}$ model: sinus rhythm and chronic atrial fibrillation. Circ Res. 2011;109:1055-1066.

90. Pueyo E, Corrias A, Virag L, Jost N, Szél T, Varro A, Szentandrassy N, Nanasi PP, Burrage K, Rodriguez $B$. A multiscale investigation of repolarization variability and its role in cardiac arrhtyhmogenesis. Biophys J. 2011;101:1-11.

91. Iancu RV, Ramamurthy G, Warrier S, Nikolaev VO, Lohse MJ, Jones SW, Harvey RD. Cytoplasmic cAMP concentrations in intact cardiac myocytes. Am J Physiol Cell Physiol. 2008;295:C414-C422.

92. Rudy $Y$, Ackerman MJ, Bers DM, Clancy CE, Houser SR, London B, McCulloch AD, Przywara DA, Rasmusson RL, Solaro RJ, Trayanova NA, Wagoner DRV, Varro A, Weiss JN, Lathrop DA. Systems approach to understanding electromechanical activity in the human heart: a national heart, lung, and blood institute workshop summary. Circulation. 2008;118:12021211.

93. London B. Cardiac arrhythmias: from (transgenic) mice to men. J Cardiovasc Electrophysiol. 2001;12:1089-1091.

94. Moretti A, Bellin M, Welling A, Jung CB, Lam JT, Bott-Flugel L, Dorn T, Goedel A, Hohnke C, Hofmann F, Seyfarth M, Sinnecker D, Schomig A, Laugwitz KL. Patient-specific induced pluripotent stem-cell models for long-QT syndrome. N Engl J Med. 2010;363:1397-1409. 
95. Yazawa M, Hsueh B, Jia X, Pasca AM, Bernstein JA, Hallmayer J, Dolmetsch RE. Using induced pluripotent stem cells to investigate cardiac phenotypes in Timothy syndrome. Nature. 2011;471:230-234.

96. Itzhaki I, Maizels L, Huber I, Zwi-Dantsis L, Caspi O, Winterstern A, Feldman O, Gepstein A, Arbel G, Hammerman H, Boulos M, Gepstein L. Modelling the long QT syndrome with induced pluripotent stem cells. Nature. 2011;471:225-229.

97. Priori SG. Induced pluripotent stem cell-derived cardiomyocytes and long QT syndrome: is personalized medicine ready for prime time? Circ Res. 2011;109:822-824.

98. Clancy $\mathrm{CE}$, Rudy $\mathrm{Y}$. $\mathrm{Na}^{+}$channel mutation that causes both Brugada and long-QT syndrome phenotypes: a simulation study of mechanism. Circulation. 2002;105:1208-1213.

99. Bezzina C, Veldkamp MW, van Den Berg MP, Postma AV, Rook MB, Viersma JW, van Langen IM, Tan-Sindhunata G, Bink-Boelkens MT, van Der Hout AH, Mannens MM, Wilde AA. A single $\mathrm{Na}^{+}$channel mutation causing both long-QT and Brugada syndromes. Circ Res. 1999;85:1206-1213.

100. Veldkamp MW, Viswanathan PC, Bezzina C, Baartscheer A, Wilde AA, Balser JR. Two distinct congenital arrhythmias evoked by a multidysfunctional $\mathrm{Na}^{+}$channel. Circ Res. 2000;86:E9197.

101. Clancy CE, Zhu ZI, Rudy Y. Pharmacogenetics and anti-arrhythmic drug therapy: a theoretical investigation. Am J Physiol Heart Circ Physiol. 2007;292:H66-75.

102. Brennan $T$, Fink $M$, Rodriguez $B$. Multiscale modelling of drug-induced effects on cardiac electrophysiological activity. Eur J Pharm Sci. 2009;36:62-77.

103. Rodriguez B, Burrage K, Gavaghan D, Grau V, Kohl P, Noble D. The systems biology approach to drug development: application to toxicity assessment of cardiac drugs. Clin Pharmacol Ther. 2010;88:130-134.

104. Mirams GR, Cui Y, Sher A, Fink M, Cooper J, Heath BM, McMahon NC, Gavaghan DJ, Noble D. Simulation of multiple ion channel block provides improved early prediction of compounds' clinical torsadogenic risk. Cardiovasc Res. 2011;91:53-61.

105. Davies MR, Mistry HB, Hussein L, Pollard CE, Valentin JP, Swinton J, Abi-Gerges N. An in silico canine cardiac midmyocardial action potential duration model as a tool for early drug safety assessment. Am J Physiol Heart Circ Physiol. 2012; In Press.

106. Sarkar AX, Sobie EA. Quantification of repolarization reserve to understand interpatient variability in the response to proarrhythmic drugs: A computational analysis. Heart Rhythm. 2011;8:1749-1755.

107. Liu N, Ruan Y, Denegri M, Bachetti T, Li Y, Colombi B, Napolitano C, Coetzee WA, Priori SG. Calmodulin kinase II inhibition prevents arrhythmias in RyR2(R4496C+/-) mice with catecholaminergic polymorphic ventricular tachycardia. J Mol Cell Cardiol. 2011;50:214-222.

108. Nattel S, Carlsson L. Innovative approaches to anti-arrhythmic drug therapy. Nat Rev Drug Discov. 2006;5:1034-1049.

109. Kääb S, Schulze-Bahr E. Susceptibility genes and modifiers for cardiac arrhythmias. Cardiovasc Res. 2005;67:397-413.

110. Brink PA, Schwartz PJ. Of founder populations, long QT syndrome, and destiny. Heart Rhythm. 2009;6:S25-33.

111. Schwartz PJ, Vanoli E, Crotti L, Spazzolini C, Ferrandi C, Goosen A, Hedley P, Heradien M, Bacchini S, Turco A, La Rovere MT, Bartoli A, George AL, Jr., Brink PA. Neural control of heart rate is an arrhythmia risk modifier in long QT syndrome. J Am Coll Cardiol. 2008;51:920-929.

112. Crotti L, Monti MC, Insolia R, Peljto A, Goosen A, Brink PA, Greenberg DA, Schwartz PJ, George AL, Jr. NOS1AP is a genetic modifier of the long-QT syndrome. Circulation. 2009;120:1657-1663.

113. Tomas M, Napolitano C, De Giuli L, Bloise R, Subirana I, Malovini A, Bellazzi R, Arking DE, Marban E, Chakravarti A, Spooner PM, Priori SG. Polymorphisms in the NOS1AP gene 
modulate QT interval duration and risk of arrhythmias in the long QT syndrome. J Am Coll Cardiol. 2010;55:2745-2752.

114. El-Armouche A, Eschenhagen T. $\beta$-Adrenergic stimulation and myocardial function in the failing heart. Heart Fail Rev. 2009;14:225-241.

115. Roberts J, Kelliher GJ, Lathers CM. Role of adrenergic influences in digitalis-induced ventricular arrhythmia. Life Sci. 1976;18:665-677.

116. Schwartz PJ, Billman GE, Stone HL. Autonomic mechanisms in ventricular fibrillation induced by myocardial ischemia during exercise in dogs with healed myocardial infarction. An experimental preparation for sudden cardiac death. Circulation. 1984;69:790-800.

117. Padmala S, Demir SS. Computational model of the ventricular action potential in adult spontaneously hypertensive rats. J Cardiovasc Electrophysiol. 2003;14:990-995.

118. Decker KF, Rudy Y. Ionic mechanisms of electrophysiological heterogeneity and conduction block in the infarct border zone. Am J Physiol Heart Circ Physiol. 2010;299:H1588-1597.

119. Chen PS, Chen LS, Cao JM, Sharifi B, Karagueuzian HS, Fishbein MC. Sympathetic nerve sprouting, electrical remodeling and the mechanisms of sudden cardiac death. Cardiovasc Res. 2001;50:409-416.

120. Nattel S, Maguy A, Le Bouter S, Yeh YH. Arrhythmogenic ion-channel remodeling in the heart: heart failure, myocardial infarction, and atrial fibrillation. Physiol Rev. 2007;87:425456.

121. Kohl P, Crampin EJ, Quinn TA, Noble D. Systems biology: an approach. Clin Pharmacol Ther. 2010;88:25-33.

122. Kohl P, Noble D. Systems biology and the virtual physiological human. Mol Syst Biol. 2009;5.

123. Silva JR, Pan H, Wu D, Nekouzadeh A, Decker KF, Cui J, Baker NA, Sept D, Rudy Y. A multiscale model linking ion-channel molecular dynamics and electrostatics to the cardiac action potential. Proc Natl Acad Sci U S A. 2009;106:11102-11106.

124. McNair WP, Ku L, Taylor MR, Fain PR, Dao D, Wolfel E, Mestroni L. SCN5A mutation associated with dilated cardiomyopathy, conduction disorder, and arrhythmia. Circulation. 2004;110:2163-2167.

125. Ge J, Sun A, Paajanen V, Wang S, Su C, Yang Z, Li Y, Jia J, Wang K, Zou Y, Gao L, Fan Z. Molecular and clinical characterization of a novel SCN5A mutation associated with atrioventricular block and dilated cardiomyopathy. Circ Arrhythm Electrophysiol. 2008;1:8392.

126. Mangmool S, Shukla AK, Rockman HA. $\beta$-Arrestin-dependent activation of $\mathrm{Ca}^{2+} /$ calmodulin kinase II after $\beta 1$-adrenergic receptor stimulation. J Cell Biol. 2010;189:573-587.

127. Stangherlin A, Gesellchen F, Zoccarato A, Terrin A, Fields LA, Berrera M, Surdo NC, Craig MA, Smith G, Hamilton G, Zaccolo M. cGMP signals modulate cAMP levels in a compartmentspecific manner to regulate catecholamine-dependent signaling in cardiac myocytes. Circ Res. 2011;108:929-939.

128. Li W, Kohl P, Trayanova N. Myocardial ischemia lowers precordial thump efficacy: an inquiry into mechanisms using three-dimensional simulations. Heart Rhythm. 2006;3:179-186.

129. Keldermann RH, ten Tusscher KH, Nash MP, Hren R, Taggart P, Panfilov AV. Effect of heterogeneous APD restitution on VF organization in a model of the human ventricles. Am J Physiol Heart Circ Physiol. 2008;294:H764-774.

130. Clayton RH, Bernus O, Cherry EM, Dierckx H, Fenton FH, Mirabella L, Panfilov AV, Sachse FB, Seemann $\mathrm{G}$, Zhang $\mathrm{H}$. Models of cardiac tissue electrophysiology: progress, challenges and open questions. Prog Biophys Mol Biol. 2011;104:22-48.

131. Trayanova NA. Whole-heart modeling: applications to cardiac electrophysiology and electromechanics. Circulation research. 2011;108:113-128.

132. Sato D, Xie Y, Weiss JN, Qu Z, Garfinkel A, Sanderson AR. Acceleration of cardiac tissue simulation with graphic processing units. Med Biol Eng Comput. 2009;47:1011-1015. 
133. Bordas R, Carpentieri B, Fotia G, Maggio F, Nobes R, Pitt-Francis J, Southern J. Simulation of cardiac electrophysiology on next-generation high-performance computers. Philos Transact A Math Phys Eng Sci. 2009;367:1951-1969.

134. Plank G, Liebmann M, dos Santos RW, Vigmond EJ, Haase G. Algebraic multigrid preconditioner for the cardiac bidomain model. leee T Bio-Med Eng. 2007;54:585-596.

135. Cuculich PS, Zhang J, Wang Y, Desouza KA, Vijayakumar R, Woodard PK, Rudy Y. The electrophysiological cardiac ventricular substrate in patients after myocardial infarction: noninvasive characterization with electrocardiographic imaging. J Am Coll Cardiol. 2011;58:1893-1902.

136. Wang Y, Cuculich PS, Zhang J, Desouza KA, Vijayakumar R, Chen J, Faddis MN, Lindsay BD, Smith TW, Rudy Y. Noninvasive electroanatomic mapping of human ventricular arrhythmias with electrocardiographic imaging. Sci Transl Med. 2011;3:98ra84.

137. Wang $Y$, Rudy Y. Electrocardiographic imaging of normal human atrial repolarization. Heart Rhythm. 2009;6:582-583. 


\section{SUMMARY}

SAMENVATtING 


\section{SUMMARY}

The pump function of the heart is driven by a complex sequence of electrophysiological signals. Originating from the sinus node, electrical activation spreads rapidly through the atria. It is delayed in the atrioventricular node to allow time for the ventricles to fill with blood, after which the impulse travels through the specialized conduction system, initiating synchronized activation (and subsequent contraction) of the ventricular muscle. To meet varying demands, cardiac output is dynamically modulated by the autonomic nervous system via its effects on the electrophysiology of individual cardiac myocytes, including those of the impulse and conduction system. Sympathetic stimulation occurs during exercise or as part of the fight-or-flight response, and results in an increase in force of contraction, heart rate, rate of relaxation, and conduction velocity (called positive inotropy, chronotropy, lusitropy and dromotropy, respectively). In contrast, parasympathetic stimulation results in a decrease in these parameters. In a number of cardiovascular diseases, an increased sympathetic tone has been associated with the development of potentially lethal ventricular tachyarrhythmias.

At the level of the single ventricular myocyte, activation of $\beta$-adrenergic receptors by norepinephrine released from cardiac sympathetic nerve endings activates an intracellular pathway resulting in modulation of a number of ion channels and $\mathrm{Ca}^{2+}$ handling proteins. These changes form the basis of the altered electrical and contractile function during sympathetic stimulation.

Computational modeling has proven useful in enhancing our understanding of various components of normal cardiac electrophysiology but also arrhythmogenic mechanisms in a variety of pathological conditions. However, integrated models of sympathetic stimulation and cardiac electrophysiology have only recently become available. In this thesis we employ a combined computational and experimental approach to investigate $\beta$-adrenergic stimulation and its effects on ventricular electrophysiology in physiological and pathological conditions.

Chapter 1 represents a general introduction to this thesis and provides a brief summary of cardiac cellular electrophysiology and its modulation by adrenergic stimulation. The single cardiac myocyte is a complex entity in which numerous processes interact to modulate the action potential and $\mathrm{Ca}^{2+}$ transient. Computational models provide the opportunity to integrate a wide range of experimental data and make testable predictions about the effect of various interventions in such complex systems. They have been applied successfully since the early 1960s and their use has expanded rapidly in the past decade. We provide a historical overview of computational modeling of cardiac electrophysiology and highlight recent developments.

In Chapter 2, an improved computational model of the canine epicardial electrophysiology is presented. The model, an extension of the Hund-Rudy dynamic model (2004), is able to reproduce a wide range of experimentally observed rate-dependent behaviors in the single cardiac myocyte and 
in tissue, including action potential (AP) duration (APD) adaptation, restitution, and accommodation. Our simulations provide valuable insights into the mechanistic basis of rate-dependent phenomena important for determining the heart's response to rapid and irregular rates, such as those occurring during arrhythmias.

The electrophysiological model of the canine epicardial muscle cell provides the basis for Chapter 3, in which the model is extended with a framework to simulate localized B-adrenergic stimulation and its electrophysiological effects through a population-based modeling approach of nonphosphorylated and phosphorylated substrates. We show that localization of cyclic AMP is critical for a specific response of different substrates to stimulate different $\beta$-adrenergic-receptor isoforms. In addition, we determine the contribution of the different substrates to $\beta$-adrenergic-stimulationdependent changes in $\mathrm{AP}$ and $\mathrm{Ca}^{2+}$ transient in physiological conditions.

The $\beta$-adrenergic stimulation model is employed in Chapter $\mathbf{4}$ to predict the AP changes by the longQT syndrome type 1 mutation KCNQ1-A341V. We provide evidence that this mutation causes a dominant-negative reduction in CAMP-dependent upregulation of the slowly-activating delayedrectifier $K^{+}$current $\left(I_{K S}\right)$ on top of a dominant-negative reduction in baseline $I_{K s .}$. Combined, these alterations result in pronounced APD prolongation in the presence of $\beta$-adrenergic stimulation. The loss of cAMP-dependent $I_{\mathrm{Ks}}$ upregulation in the presence of heterozygous $A 341 \mathrm{~V}$ is due to reduced phosphorylation of residue S27 and is also present in heterozygous KCNQ1-G589D and KCNQ1-S27A. These data provide mechanistic insights into cAMP-dependent regulation of $I_{\mathrm{Ks}}$ and its role in arrhythmogenesis in long-QT syndrome type 1 , because heterozygous carriers will exhibit a homozygous phenotype with respect to $I_{\mathrm{Ks}}$ upregulation.

The link between $I_{K s}$ and beat-to-beat variability of repolarization duration in isolated canine ventricular myocytes is made in Chapter $\mathbf{5}$. We show that $\beta$-adrenergic stimulation is able to rescue the increased variability of repolarization which occurs during blockade of the rapidly-activating delayed-rectifier $\mathrm{K}^{+}$current $\left(\mathrm{I}_{\mathrm{Kr}}\right)$ or during augmentation of the late component of the $\mathrm{Na}^{+}$current $\left(I_{\mathrm{NaL}}\right)$. In contrast, during $\mathrm{I}_{\mathrm{Ks}}$ blockade, beat-to-beat variability of repolarization is only increased during $\beta$-adrenergic stimulation. The increased variability is sensitive to intracellular $\mathrm{Ca}^{2+}$ and, at least partly, mediated by the $\mathrm{Na}^{+}-\mathrm{Ca}^{2+}$ exchanger.

In Chapter 6, we identify pronounced APD prolongation following spontaneous $\mathrm{Ca}^{2+}$ release during $\mathrm{Ca}^{2+}$ overload induced by 6 -adrenergic stimulation. This prolongation is exacerbated by pharmacological inhibition of $I_{\mathrm{Ks}}$, resulting in a pharmacological model of long-QT syndrome type 1 . The interspersed occurrence of APD prolongation explains the large beat-to-beat variability of repolarization observed under these conditions. A combined computational and experimental analysis identifies reduced $\mathrm{Ca}^{2+}$-dependent inactivation of the L-type $\mathrm{Ca}^{2+}$ channel after spontaneous $\mathrm{Ca}^{2+}$ release as a major contributor to the APD prolongation. 
In Chapter 7, the B-adrenergic stimulation model is extended with detailed stochastic formulations of ion-channel gating to further investigate subcellular mechanisms of beat-to-beat variability of repolarization in physiological and pathological conditions. In addition to stochastic channel gating, we identify APD, AP morphology and beat-to-beat variations in $\mathrm{Ca}^{2+}$ as major modulators of singlecell APD variability. Cell-to-cell coupling is able to reduce variability. This model provides a state-ofthe-art tool to investigate cellular electrophysiology, and particularly beat-to-beat variability of repolarization, in a wide variety of conditions.

Finally, Chapter $\mathbf{8}$ contains a general discussion in which the results of these studies are discussed in a broader context. We provide potential applications of detailed cellular models of cardiac electrophysiology such as those developed for this thesis. The relevance of these models for clinical settings and limitations in their applicability are discussed, and we provide our definition of the term "systems biology" and its potential for personalized medicine. 


\section{SAMENVATTING}

De pompfunctie van het hart is het resultaat van een complex samenspel van elektrofysiologische signalen. De elektrische activatiegolf verspreidt zich vanuit de sinusknoop snel over de boezems om vervolgens te worden vertraagd in de atrioventrikulaire knoop zodat de kamers van het hart tijd hebben om te vullen met bloed. Hierna reist de elektrische impuls door het geleidingssysteem en zorgt voor een synchrone activatie (en de daaropvolgende contractie) van het spierweefsel in de hartkamers. Om aan wisselende energiebehoeften te kunnen voldoen, wordt het hart-minuutvolume gereguleerd door het onwillekeurige zenuwstelsel via de elektrofysiologische eigenschappen van individuele spiercellen, waaronder die van het impuls- en geleidingssyteem. Stimulatie van het sympathisch zenuwstelsel, bijvoorbeeld tijdens inspanning of als onderdeel van een 'vecht-of-vlucht' reactie, resulteert in een toename in contractiekracht, hartfrequentie, relaxatie- en geleidingssnelheid (respectievelijk inotropie, chronotropie, lusitropie en dromotropie genaamd). Stimulatie van het parasympathisch zenuwstelsel daarentegen resulteert in een afname van deze eigenschappen. In een aantal hart- en vaatziekten is er een verband aangetoond tussen een toename in sympathische activatie en het ontstaan van potentieel dodelijke kamerritmestoornissen.

Op het niveau van de individuele hartspiercel leidt sympathische stimulatie tot activatie van $\beta$ adrenerge receptoren via norepinefrine dat vrijkomt uit cardiale sympathische zenuwuiteinden. Stimulatie van $\beta$-adrenerge receptoren activeert een intracellulaire cascade die resulteert in veranderingen in de verschillende ionkanalen en eiwitten die de $\mathrm{Ca}^{2+}$-huishouding beïnvloeden. Deze veranderingen vormen de basis voor de veranderde elektrische activiteit en contractie tijdens sympathische stimulatie.

Computationele modellen hebben sterk bijgedragen aan ons begrip van de verschillende componenten die in de elektrofysiologie een rol spelen, waaronder ook het ontstaan van ritmestoornissen onder pathologische condities. Computermodellen die in staat zijn om sympathische stimulatie en cardiale elektrofysiologie te integreren zijn echter pas zeer recentelijk ontwikkeld. In dit proefschrift beschrijven we computationele en experimentele technieken om de effecten te bestuderen van $\beta$-adrenerge stimulatie op de elektrofysiologische eigenschappen van het hart onder normale en pathologische omstandigheden.

Hoofdstuk 1 is de introductie tot dit proefschrift en geeft een korte samenvatting van de cellulaire elektrofysiologie van het hart en de veranderingen ten gevolge van adrenerge stimulatie. De individuele hartspiercel is een zeer complex systeem. De bij activatie optredende actiepotentiaal en $\mathrm{Ca}^{2+}$ golf worden beïnvloed door meerdere processen. Computermodellen stellen ons in staat om een grote verscheidenheid aan experimentele gegevens te integreren en verifieerbare voorspellingen te doen over het effect van veranderingen in een dergelijk complex systeem. Dit soort modellen wordt al succesvol toegepast sinds de jaren '60 en in het afgelopen decennium is het gebruik ervan sterk toegenomen. We geven een historisch overzicht van computermodellen van de elektrofysiologie van het hart en beschrijven de recente ontwikkelingen in dit vakgebied. 
In Hoofdstuk 2 wordt een nieuw model van de elektrofysiologie van de epicardiale spiercel van het hondenhart gepresenteerd. Dit model, een uitbreiding van het Hund-Rudy model (2004), is in staat om een grote verscheidenheid aan experimentele frequentie-afhankelijke eigenschappen van individuele hartspiercellen na te bootsen. Hieronder vallen adaptatie, restitutie en accommodatie van de cardiale actiepotentiaal. De simulaties in dit hoofdstuk bieden nieuwe inzichten in de mechanismen die belangrijk zijn voor de respons van het hart tijdens snelle en onregelmatige hartritmestoornissen.

Met het model van de epicardiale spiercel als basis wordt in Hoofdstuk 3 een framework gepresenteerd om lokale b-adrenerge stimulatie en de gevolgen daarvan voor de cellulaire elektrofysiologie te simuleren. Lokaal cyclisch AMP blijkt essentieel te zijn voor een gerichte beïnvloeding van individuele eiwitten tijdens stimulatie van verschillende types adrenerge receptoren. Daarnaast wordt in dit hoofdstuk de bijdrage bepaald van ieder ionkanaal en eiwit van de $\mathrm{Ca}^{2+}$-huishouding aan de veranderingen in actiepotentiaal en $\mathrm{Ca}^{2+}$ golf tijdens $\beta$-adrenerge stimulatie.

Het $\beta$-adrenerge model wordt in Hoofdstuk 4 gebruikt om voorspellingen te doen over de actiepotentiaal-veranderingen in patiënten met het lange-QT syndroom type 1 door de genmutatie KCNQ1-A341V. We laten zien dat deze mutatie zorgt voor een dominant-negatief effect op zowel de basale stroom van het zogenaamde 'slowly-activating delayed-rectifier' kaliumkanaal $I_{K s}$ als op de cAMP-afhankelijke toename in $I_{k s}$. Samen zorgen deze veranderingen voor een sterke verlenging van de actiepotentiaal tijdens $\beta$-adrenerge stimulatie. Het verlies van de CAMP-afhankelijke $I_{\mathrm{Ks}}$-toename in heterozygote A341V condities komt door een verminderde fosforylatie van aminozuur S27 en komt ook voor in de heterozygote mutaties KCNQ1-G589D en KNQ1-S27A. Deze data geven nieuwe inzichten in de CAMP-afhankelijke regulatie van $I_{\mathrm{Ks}}$ en de rol hiervan bij de ontwikkeling van ritmestoornissen.

De invloed van $I_{\mathrm{Ks}}$ op de slag-op-slag veranderingen in repolarisatie in individuele kamerspiercellen van het hondenhart wordt onderzocht in Hoofdstuk 5. We laten zien dat adrenerge stimulatie in staat is om de toegenomen slag-op-slag variabiliteit in repolarisatie door inhibitie van de zogenaamde $\mathrm{I}_{\mathrm{kr}}$-stroom of door toename in de late component van de $\mathrm{Na}^{+}$-stroom te verminderen. De slag-op-slag variabiliteit in repolarisatie neemt daarentegen toe tijdens adrenerge stimulatie indien $I_{\mathrm{Ks}}$ geïnhibeerd is. $I_{\mathrm{ks}}$-inhibitie zonder adrenerge stimulatie heeft geen effect op de repolarisatievariabiliteit. Deze kan worden gereduceerd door manipulatie van het intracellulaire $\mathrm{Ca}^{2+}$ of de $\mathrm{Na}^{+}-\mathrm{Ca}^{2+}$ uitwisselaar.

In Hoofdstuk 6 worden de potentiële mechanismen waardoor $\beta$-adrenerge stimulatie ritmestoornissen opwekt verder onderzocht. Tijdens cellulaire $\mathrm{Ca}^{2+}$-overlading ten gevolge van $\mathrm{B}$ - 
adrenerge stimulatie ontstaat het spontane vrijkomen van $\mathrm{Ca}^{2+}$-ionen uit het sarcoplasmatisch reticulum dat resulteert in een sterke verlenging van de daaropvolgende actiepotentiaal. Deze verlenging neemt toe tijdens farmacologische inhibitie van $I_{\mathrm{ks}}$. De onregelmatige verlenging van de actiepotentiaal verklaart de grote slag-op-slag variabiliteit van repolarisatie die onder deze omstandigheden kan worden waargenomen. Een combinatie van computationele en experimentele analyses toont aan dat een verminderde $\mathrm{Ca}^{2+}$-afhankelijke inactivatie van het L-type $\mathrm{Ca}^{2+}$ kanaal in belangrijke mate bijdraagt aan de actiepotentiaal-verlenging.

In Hoofdstuk 7 wordt het $\mathbf{B}$-adrenerge model verder uitgebreid met een gedetailleerde stochastische beschrijving van de kinetiek van ionkanalen om de subcellulaire mechanismen van slag-op-slag variabiliteit in repolarisatie in fysiologische en pathologische condities verder te onderzoeken. Naast stochastische aspecten van ionkanalen, vinden wij belangrijke bijdragen van de actiepotentiaalmorfologie en -duur, alsmede van veranderingen in intracellulair $\mathrm{Ca}^{2+}$. Elektrische koppeling tussen twee cellen is in staat om deze repolarisatievariabiliteit te verminderen. Ons model biedt een stateof-the-art mogelijkheid om de cellulaire elektrofysiologie, en in het bijzonder de slag-op-slag variabiliteit in repolarisatie, te bestuderen onder verschillende omstandigheden.

Tot slot bevat Hoofdstuk 8 een algemene discussie waarin de resultaten van de verschillende studies van dit proefschrift in een bredere context worden geplaatst. We beschrijven toekomstige mogelijkheden van de gedetailleerde cellulaire computermodellen zoals wij die ontworpen hebben. De relevantie van deze modellen voor de kliniek, en hun voornaamste beperkingen, worden besproken en we geven onze definitie van de term "systeem-biologie" en haar potentiële rol in persoonspecifieke geneeskunde. 


\section{ACKNOWLEDGEMENTS DANKWOORD}


It is with great pleasure that I can finally start to write the most important chapter of this thesis (or at least the one that is in general the most widely read). Being a PhD student is somewhat like finding your way through a large maze: it is virtually impossible to find the exit without extensive help from others. Some draw a map, others give directions, some provide nourishment along the way, while others contribute to the entertainment when you are forced to camp at yet another dead-end. Each is vital to complete the process. When you have spent 4.5 years in 3 labs at 2 universities, it is impossible to thank everyone appropriately without doubling the size of this (already somewhat lengthy) thesis. Therefore, I would hereby like to thank everyone who has helped me to make this happen and stress that their contributions have not been forgotten even if they are not explicitly named in this chapter. However, some people should be thanked individually.

First and foremost, I would like to thank Dr. Paul Volders and Dr. Ronald Westra for their guidance. Ronald, no matter how busy you were, your enthusiasm for our project never lessened. You have always been able to provide a fresh perspective to our discussions, often aided by one of your favorite (Einstein) quotes. Many thanks for your support from my Bachelor thesis in 2006 until my $\mathrm{PhD}$ defense in 2012! Paul, during the first half of our collaboration (which also started with my Bachelor thesis in 2006), almost every meeting with you resulted in a headache. Your scientific standards and vision are admirable (and, as a PhD student, sometimes daunting) and your knowledge of the scientific literature is beyond comparison. Despite your busy clinical schedule I do not think that we ever had a scientific meeting that lasted less than 1.5-2.0 hours. I have learned a tremendous amount from your example and hope that we can continue to work together. Maybe one day I can then teach you the 'Track Changes' option in Word, as an alternative to your trademarked yellow highlighting. A special word of thanks should also go to my third scientific mentor: Prof. Yoram Rudy at Washington University in St. Louis. Dr. Rudy, your guidance, both scientifically and non-scientifically, has been extremely important. Your in-depth knowledge of the wide variety of cardiac electrophysiological topics that are being studied by the members of your team is amazing. I will never forget my time in St. Louis and hope that I can soon come back to visit you and Hadas (including a drink at Dressels, of course).

I would also like to thank my promotores Prof. Harry Crijns and Prof. Ralf Peeters for their support, particularly during the final stages of my $\mathrm{PhD}$, when the mountain of forms and letters seemed insurmountable. Even more important, of course, were your scientific comments during our progress meetings, which forced me to keep an eye on the big picture and helped to keep the project on track.

I am grateful and honored that Prof. Hein Wellens, Prof. Harry Struijker-Boudier and Prof. Leon de Windt agreed to be part of the thesis-assessment committee. A particular word of thanks should also go to the external members of the thesis-assessment committee: Prof. Mike Rosen, Prof. Rodolphe Sepulchre and Prof. Arthur Wilde for their critical reading of this thesis and their willingness to be present at the defense in Maastricht. 
Science in general and computational modeling in particular are multidisciplinary exercises and discussions with several collaborators have contributed substantially to this thesis. I would like to thank in particular Dr. Najah Abi-Gerges, Prof. David Eisner, Prof. Andrew Trafford and Prof. Antonio Zaza for their involvement in various chapters of this thesis.

My colleagues at the Department of Cardiology have had a large impact on my time in Maastricht, both scientifically and socially. I would like to thank Lucien Beckers; Inge Boulet; Rachel ter Bekke (I am looking forward to seeing what your next award will be. Your work is very impressive!); Michael Clerx (good luck with your own research in the wonderful DKE-Cardio joint venture!); Matthijs Cluitmans (thanks for being a great office-buddy and friend, I admire your combination of clinical and mathematical interests); Miren David (I am looking forward to seeing your future work, unraveling the mysteries of $I_{k s}$. Thanks for the patch-clamp lessons. Please come to Heidelberg for some more slacklining); Dan Johnson (thanks for the excellent discussions and cooperation in our joint projects and good luck finishing the last bits of your thesis!); Monique de Jong (thanks for all the cups of coffee, the early morning chats and many laughs); Helma Kuijpers; Viola Lentink (I hope this thesis finds its way to Peru!); Annerie Moers (thanks for everything!); Sandrine Seyen (all the best for you and the baby!); and Roel Spätjens (Roel, the lab would not run without you, whether it is administrative, experimental, or in the design of figures and posters, your hands are on everything that is published from our team. Thanks for all your help!). All the other colleagues at level 3 of UNS50 have, of course, also contributed to a pleasant working experience.

Likewise, I would like to thank my colleagues from the Rudy lab at Washington University in St. Louis for the warm welcome and wonderful time. In particular Tom O'Hara deserves a massive 'thank you' for helping me to get settled in St. Louis, letting me stay in his apartment and being a great friend. Tom, I am looking forward to your wedding with lunia Dadarlat this summer! Many thanks also to Keith Decker and Jon Silva for the interesting discussions in our joint project. The members of the 'cell group', Namit Gaur, Pan Li, Leonid Livshitz, Ashwin Mohan, Ali Nekouzadeh, Smiruthi Ramasubramanian and Jiajing $\mathbf{X u}$ have provided critical feedback on my work during many lab meetings, for which I am thankful. Thanks also to the members of the 'ECGI group' for many interesting scientific and non-scientific discussions. I am proud and grateful that I have had the opportunity to be part of such a high quality team. Maya Bera and Kimberly Smith have helped me with numerous organizational and technical aspects that were required for my stay at Wash. U. and my contact with the lab after my return to The Netherlands.

The support from everyone at the Department of Knowledge Engineering, from my very first day as an undergraduate student to the completion of my thesis, has been much appreciated. The members of the Biomathematics and Bioinformatics group in particular have helped through many discussions: Ivo Bleylevens (also as an office-buddy), Pietro Bonnizi, Pieter Collins, Martin Hoffmann, Stephan Janssen (good luck finishing your work!) and Joël Karel. 
My new colleagues in Mannheim have provided a warm welcome in the new lab. Many thanks in particular to Prof. Dobromir Dobrev for giving me the opportunity to work in his high-quality lab and Dr. Niels Voigt for the interesting collaborations during the first few months.

Many hours were spent at work, particularly in the last stages of my PhD. Fortunately, it is not the quantity but the quality of time spent with friends that matters. Therefore, I would like to thank the people who, at various times and places, have provided the more than necessary distraction from work. In Maastricht I would like to thank the old 'Heugem crew': Tyas Prevoo \& Tuulia Dau (also for all the discgolf and slackline sessions), Mark Jansen \& Ellen Derks (I will always remember our awesome trip to NYC), and Tom Starmans \& Philippa Gaijkema, as well as Roel, Rik \& Marianne, Frans and Sander \& Anne (I am already looking forward to our next pre-Christmas dinner). A special word of thanks goes to Tyas and Paul Smeets: I had a great time in our 'palace' at the stationsstraat. Tyas, we should plan some more sports sessions, both virtual and in real life. Paul, I really enjoyed all our discussion about science and everyday life and am looking forward to your defense in June. My fellow PhDAcademy Improv participants, in particular Burcu, Jan, Jasper, Jessie, Nevena, Nordin and Yeliz provided many hilarious moments of distraction. Eva Greiner has been a true friend since our first day as undergrads. Eva, good luck with your new job and please come visit us in Heidelberg. Finally, I would like to thank the people in St. Louis for making me feel at home and providing plenty of distraction besides work, in particular Karthik Divariam, Owen \& Lina Gentry (I am looking forward to meeting Edwin!), Dan Keesing (we should plan another climbing/hiking trip, this time without insane traffic jams or millions of ticks), Rob \& Kate Sauers, Akansha Saxena, Diana Wong and Dick Wu.

I am grateful that Matthijs Cluitmans and Paul Smeets agreed to stand beside me as paranimfs during the defense.

As is the custom, those who have been the most important are mentioned last. Dear Claire, after all these months in which we lived in different countries, the thesis is finally done and we can start our life together. There are no words that can accurately describe how important you are to me. Thank you for all your support. I am looking forward to our future together.

Mijn familie, in het bijzonder mijn ouders Huub en Marianne, zus Sasja en Oma hebben een onmisbare rol gespeeld in de totstandkoming van dit werk. Hun steun en liefde heeft mij gebracht waar ik nu ben en daarvoor kan ik hen niet genoeg bedanken. Huub, ik blijf een kind van mijn vader en prijs me zeer gelukkig dat ik mijn vader tevens mijn beste vriend kan noemen. Bedankt voor alles! 


\section{Curriculum VitaE Publications}




\section{CURRICULUM VITAE}

\section{Jordi Heijman}

Born in Sittard, The Netherlands on September 08

1997-2003

VWO, Trevianum Scholengroep, Sittard The Netherlands (Graduated Cum Laude)

2003-2006

Bachelor of Science in Knowledge Engineering / Computer Science, Maastricht University, The Netherlands (Graduated Summa Cum Laude)

2006-2007

Master of Science in Operations Research, Maastricht University, The Netherlands (Graduated Summa Cum Laude)

2007-2011

PhD, Depts. of Cardiology and Knowledge Engineering, Maastricht University, The Netherlands

Jordi Heijman was born on September 08, 1985 in Sittard, The Netherlands. In 2003, he started his Bachelor of Science degree in Knowledge Engineering at Maastricht University. During his studies he was an active member of the public-relations committee and participated in the FastTrac technical entrepeneurship program at Baylor University, Waco, TX, USA (July-August 2005). He graduated in 2006 summa cum laude. His Master of Science degree in Operations Research was also obtained summa cum laude from Maastricht University in 2007. For his master thesis "A parallel approach to polynomial optimization" he received the 2007 student prize for the Faculty of Humanities and Sciences. He started his PhD research in September 2007 on a joint project between the Departments of Cardiology and Knowledge Engineering at the same university. From May 2008 until September 2009 he was a visiting researcher at the Cardiac Bioelectricity and Arrhythmia Center at Washington University in St. Louis, MO, USA under the guidance of Prof. Yoram Rudy on a grant from the Hein Wellens Foundation. As of January 2012, he has started as a postdoctoral researcher in the Division of Experimental Cardiology, Medical Faculty Mannheim, Heidelberg University in the team of Prof. Dobromir Dobrev. 


\section{Publications}

\section{Journal articles}

7. J. Heijman, A. Zaza, D.M. Johnson, Y. Rudy, R.LM. Peeters, P.G.A. Volders" and R.L. Westra\#. "Computational Analysis of Beat-to-Beat Variability of Repolarization Duration in the Canine Ventricular Myocyte" (Manuscript in preparation)

6. R.L.H.M.G. Spätjens, M. Bébarová, I.R. Boulet, V. Lentink, H.J.H. Kuijpers, R. Jongbloed, Y.H.J.M. Arens, J. Heijman and P.G.A. Volders. "Preserved cAMP-Dependent Upregulation Despite Dominant-Negative Suppression of $\mathrm{I}_{\mathrm{Ks}}$ by the KCNQ1 Mutation K557E" (Manuscript in preparation)

5. D.M. Johnson\#, J Heijman\#, E.F. Bode, D.J. Greensmith, N. Abi-Gerges, D.A. Eisner, A.W. Trafford and P.G.A. Volders. "Diastolic Spontaneous Calcium Release from the Sarcoplasmic Reticulum Increases Beat-to-Beat Repolarization Variability in Calcium-Overloaded Canine Ventricular Myocytes" (Manuscript under revision)

4. J. Heijman, R.L.H.M.G. Spatjens, S.R.M. Seyen, V. Lentink, H.J.H. Kuijpers, I.R. Boulet, L.J. de Windt, M. David and P.G.A. Volders, "Dominant-Negative Control of cAMP-Dependent $I_{\mathrm{Ks}}$ Upregulation in Human Long-QT Syndrome Type 1" Circ. Res. 2012. 110. 211-219.

3. J. Heijman, P.G.A. Volders, R.L. Westra and Y. Rudy. "Local control of $\beta$-adrenergic stimulation: Effects on ventricular myocyte electrophysiology and $\mathrm{Ca}^{2+}$ transient", J. Mol. Cell. Cardiol. 2011. 50. 863-871.

2. D.M. Johnson, J. Heijman, C.E. Pollard, J-P Valentin, H.J.G.M. Crijns, N. Abi-Gerges and P.G.A. Volders, "I $\mathrm{Ks}_{\mathrm{s}}$ restricts excessive beat-to-beat variability of repolarization during betaadrenergic receptor stimulation", J. Mol. Cell. Cardiol. 2010. 48. 122-130.

1. K. F. Decker, J. Heijman, J. R. Silva, T. J. Hund and Y. Rudy, "Properties and Ionic Mechanisms of Action Potential Adaptation, Restitution and Accommodation in Canine Epicardium", Am. J. Physiol. Heart Circ. Physiol. 2009. 296. H1017-H1026.

\#: These authors contributed equally to this work

\section{Abstracts}

14. J. Heijman, A. Zaza, D.M. Johnson, Y. Rudy, R.L.M. Peeters, P.G.A. Volders", R.L. Westra\#, "Stochastic ion-channel gating contributes to beat-to-beat variability of repolarization duration and its rate dependence", Cardiovasc. Res. 2012. Frontiers in Cardiovascular Biology, Mar. $30-31$ 2012, London, UK.

13. D.M. Johnson", J Heijman\#, E.F. Bode, D.J. Greensmith, N. Abi-Gerges, D.A. Eisner, A.W. Trafford and P.G.A. Volders, "Increased $I_{\text {CaL }}$ Underlies Ventricular Action-Potential Prolongation After Spontaneous $\mathrm{Ca}^{2+}$ Release From The Sarcoplasmic Reticulum", Heart Rhythm. 2011. 8(11). 1823. Cardiac EP Society Meeting, Nov. 12 2011, Orlando FL, USA. 
12. J. Heijman, R.L.H.M.G. Spatjens, S.R.M. Seyen, V. Lentink, H.J.H. Kuijpers, I.R. Boulet, L.J. de Windt, M. David and P.G.A. Volders, "Dominant-Negative Control of cAMP-Dependent $\mathrm{I}_{\mathrm{Ks}}$ Upregulation in Human Long-QT Syndrome Type 1", Circulation. 2011. 124. A10189. AHA Scientific Sessions, Nov. 12-16 2011, Orlando, FL, USA.

11. M. David, R.L.H.M.G. Spätjens, S.R.M. Seyen, J. Heijman, V. Lentink, H.J.H. Kuijpers and P.G.A. Volders, "Mutation-specific differences on the $I_{\mathrm{ks}}$ macromolecular complex by the longQT1 mutations A341V and A341E", 35th Annual meeting of the European Working Group on Cardiac Cellular Electrophysiology, Sept. 17 - 19 2011, Oslo, Norway.

10. D.M. Johnson, J. Heijman and P.G.A. Volders, "Diastolic spontaneous calcium release from the sarcoplasmic reticulum provokes beat-to-beat repolarization instability in calciumoverloaded myocytes", Heart Rhythm. 2011. 8(5). S324. HRS Scientific Sessions, May 4-7 2011, San Francisco, CA, USA

9. R.L.H.M.G. Spatjens, J. Heijman, V. Lentink and P.G.A. Volders, "The Phosphomimetic Mutation S27D Rescues Suppressed Adrenergic Responsiveness in the Long-QT1 Hot-Spot Mutation A341V", Heart Rhythm. 2011. 8(5). S180. HRS Scientific Sessions, May 4-7 2011, San Francisco, CA, USA

8. J. Heijman, Y. Rudy, R.L. Westra and P.G.A. Volders, "Computational analysis of the downstream effects of $\beta$-adrenergic stimulation on CaMKII in the canine ventricular myocyte", Cardiovasc. Res. 2010. 87(Suppl 1). S47. Frontiers in Cardiovascular Biology, July 16-19 2010, Berlin, Germany

7. J. Heijman, R.L.H.M.G. Spatjens, V. Lentink and P.G.A. Volders, "Dominant-Negative Suppression of cAMP-Mediated I $\mathrm{Ks}_{\mathrm{s}}$ Upregulation by the Long-QT1 Hot-Spot Mutation A341V", Heart Rhythm. 2010. 7(5). S89. HRS Scientific Sessions, May 12-15 2010, Denver, CO, USA

6. J. Heijman, P.G.A. Volders, R.L. Westra and Y. Rudy, "A computational model of $\beta$-adrenergic signaling in cardiac myocytes: local control and rate-dependent action-potential effects", Heart Rhythm. 2009. 6(5). S97. HRS Scientific Sessions, May 11-14 2009, Boston, MA, USA

5. J. Heijman, J. R. Silva, D.M. Johnson, P.G.A. Volders, R.L.M. Peeters and R. L. Westra, "Analyzing the effects of pharmacological interventions on cardiac repolarization using Markov models for ion channels", Dutch Institute of Systems and Control Summerschool 2008, June 17 - 20 2008, Woudschoten, The Netherlands.

4. D.M. Johnson, J.Heijman, C.E. Pollard, J.P. Valentin, N. Abi-Gerges and P.G.A. Volders, "Differential Responses of Beat-to-Beat Variability of Repolarization to $\beta$-Adrenergic Receptor Stimulation in Canine Ventricular Myocytes: $I_{\mathrm{Ks}}$ Block Versus $I_{\mathrm{kr}}$ Block and Late $I_{\mathrm{Na}}$ Augmentation", Heart Rhythm. 2008. 5(5). S211, HRS Scientific Sessions, May 14 - 172008 , San Francisco, CA, USA.

3. J.Heijman, D.M. Johnson, P.G.A. Volders, R.L.M. Peeters and R.L. Westra, "Dynamics of cardiac cells: model vs. myocyte", 27th Beneluxmeeting on Systems and Control, Mar. 18 - 20 2008, Heeze, The Netherlands. 
2. D.M. Johnson, J.Heijman, C.E. Pollard, J.P. Valentin, N. Abi-Gerges and P.G.A. Volders, "Beatto-Beat Variability of Repolarization in Canine Ventricular Cell Models of LQT1, LQT2 and LQT3 Syndromes", 31st Annual meeting of the European Working Group on Cardiac Cellular Electrophysiology, Sept. 7 - 8 2007, Manchester, GB.

1. J. Heijman, D.M. Johnson, R.L. Westra and P.G.A. Volders, "Beat-to-beat variability of repolarization duration: intrinsic to native myocytes, but does it feature in ventricular cell models?", Biophys. J. 2007. 51st Biophysical Society Meeting, Mar. 3 - 7 2007, Baltimore, MD, USA.

\#: These authors contributed equally to this work. 
Curriculum Vitae \& Publications

240 | 
COLOR Figures 


\section{A. $\mathrm{I}_{\mathrm{CaL}} \mathrm{I}-\mathrm{V} \quad$ B. $\mathrm{I}_{\mathrm{CaL}}$ Steady-State C. $\mathrm{I}_{\mathrm{CaL}}$ Markov Model}
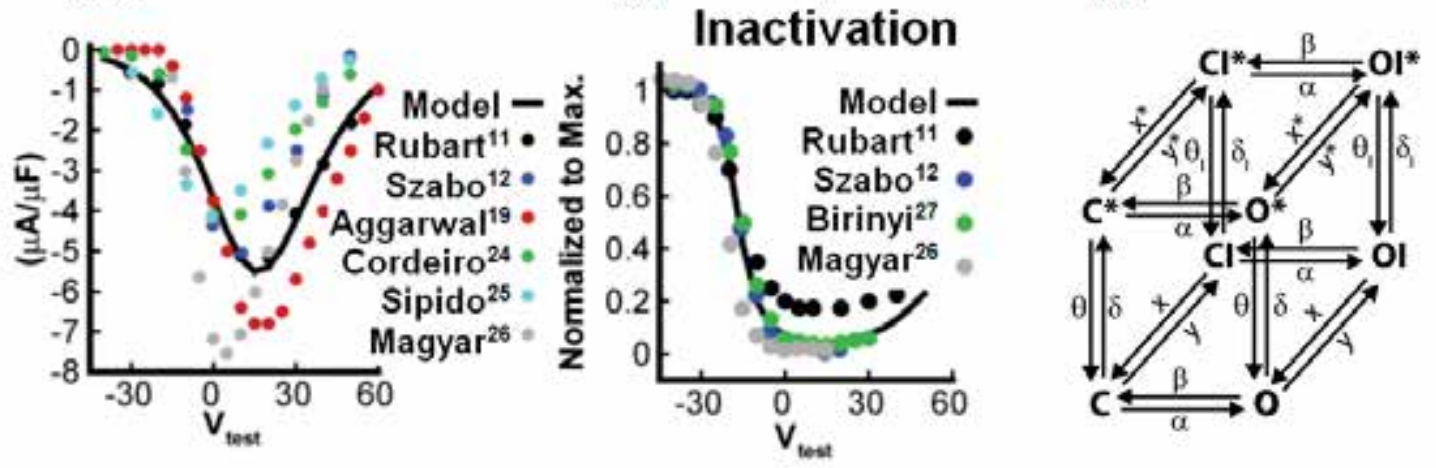

Chapter 2 - Figure 3 (Page 32). A. Model $\mathrm{I}_{\mathrm{CaL}}$ current-voltage (I-V) relationship and B. steady-state inactivation compared to multiple data sets from canine ventricular cells. ${ }^{11,12,19,24-27} \mathrm{C}$. I $\mathrm{CaL}$ Markov model structure.

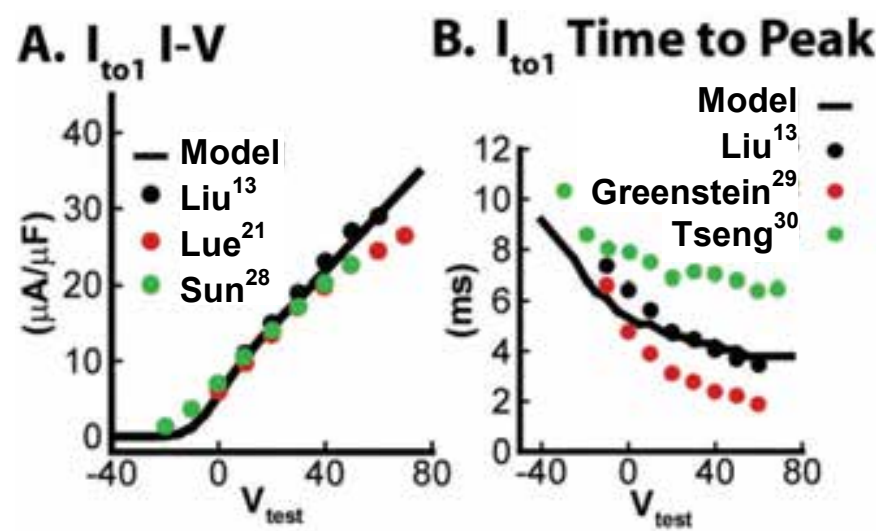

Chapter 2 - Figure 4 (Page 32). A. Model $I_{\text {to } 1} \mathrm{I}-\mathrm{V}$ curve. B. Time to peak compared to multiple data sets from canine left ventricular epicardial cells. ${ }^{13,21,28-30}$ 

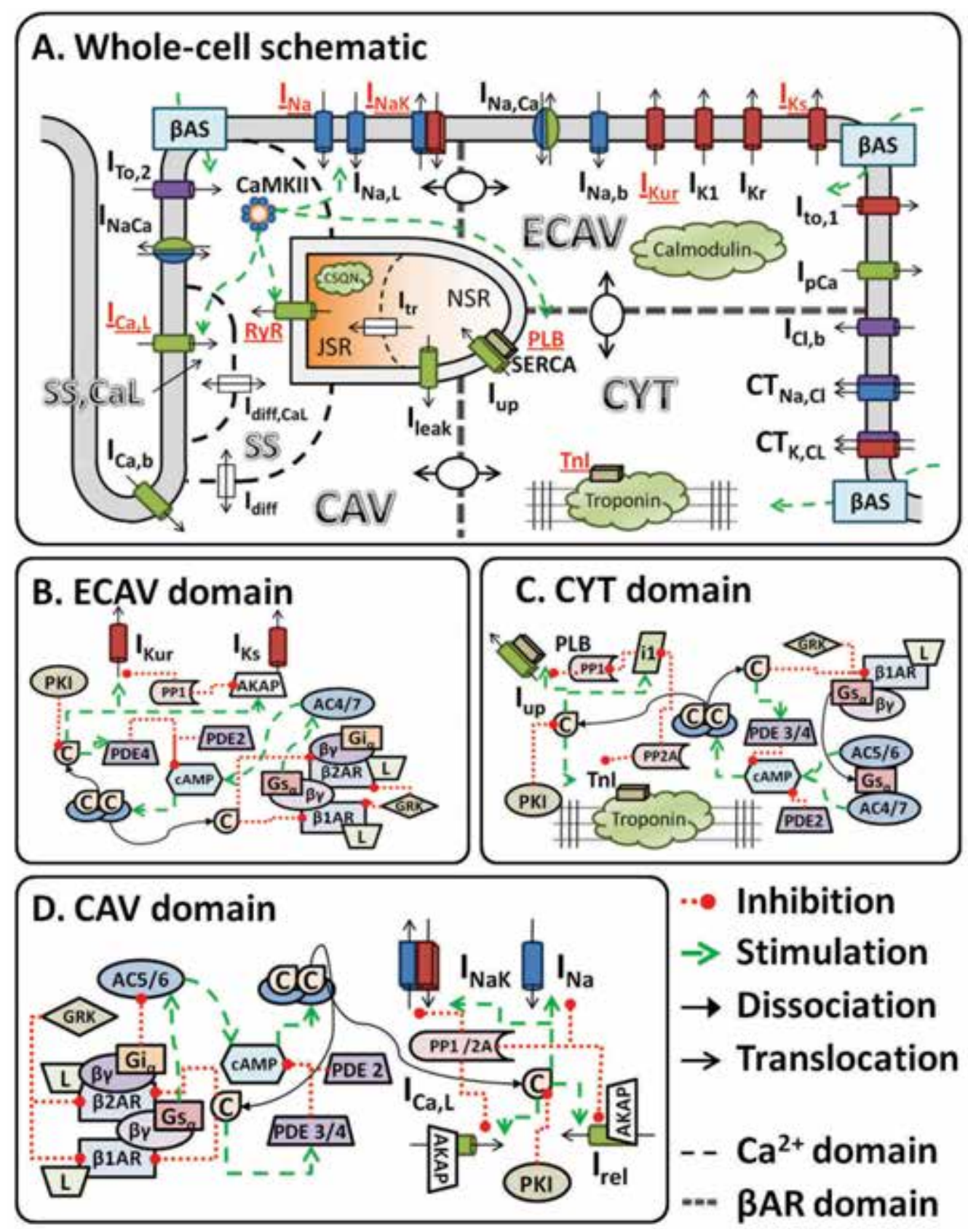

Chapter 3 - Figure 1 (Page 52). A. Schematic of the canine ventricular cell model including $\mathrm{Ca}^{2+}$ handling, electrophysiology, CaMKII signaling pathway and $\beta A R$ signaling domains. Substrates modulated by PKA phosphorylation are indicated in red. Model CaMKII targets include PLB/Serca and RyR. B-D, Detailed schematics of the interactions in the extracaveolar (ECAV; B), cytosolic (CYT; C) and caveolar (CAV; D) signaling domains. Abbreviations are defined in the appendix of this thesis. Model code is provided in the Research Section of http://rudylab.wustl.edu. 
A.

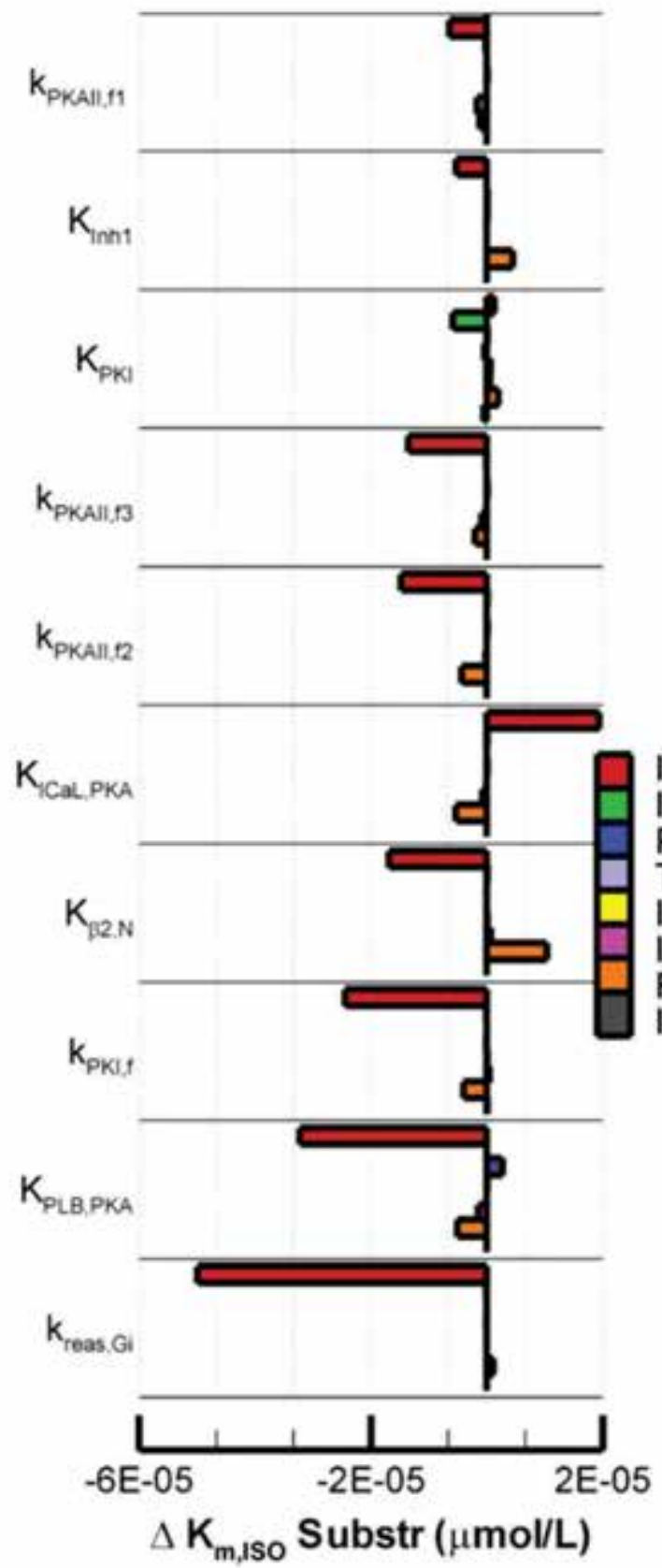

B.

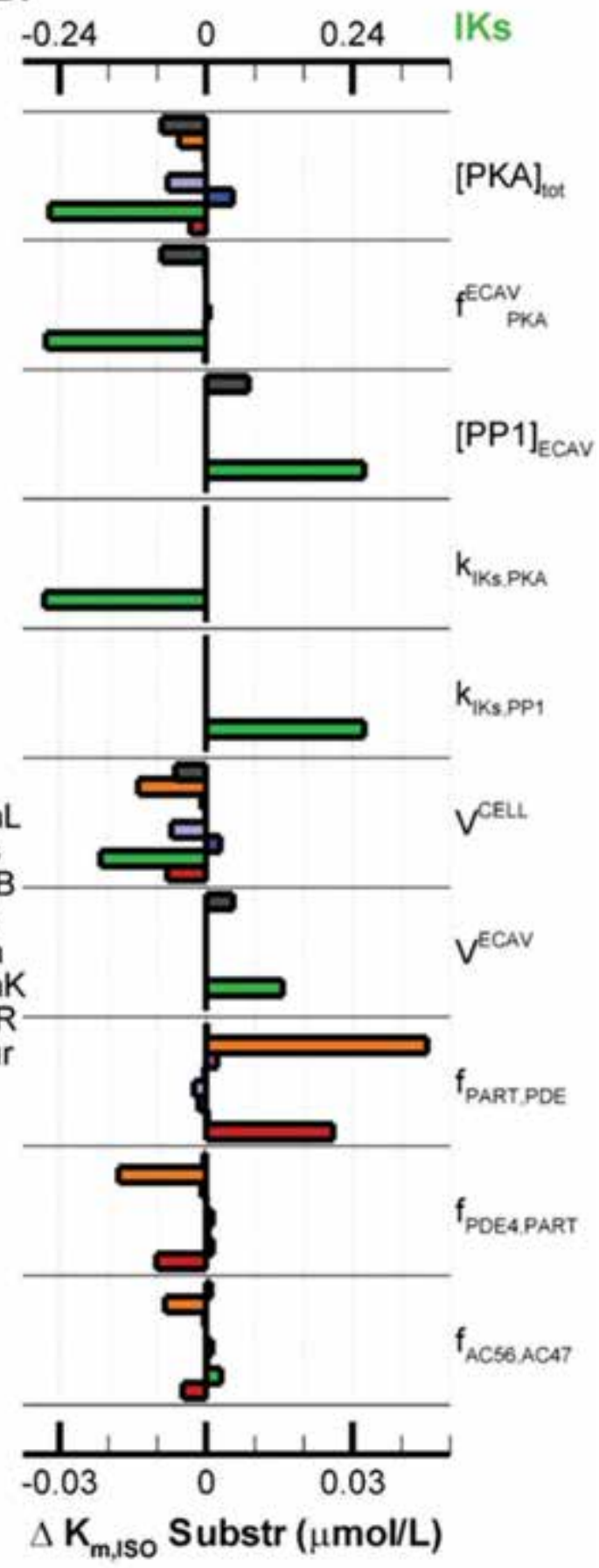

Chapter 3 - Figure 4 (Page 60). The 10 parameters with the smallest (A) and largest (B) impact on the mean change in ISO affinity over all substrates. Note different impact scale in (B) for $I_{\mathrm{ks}}$. 
A.

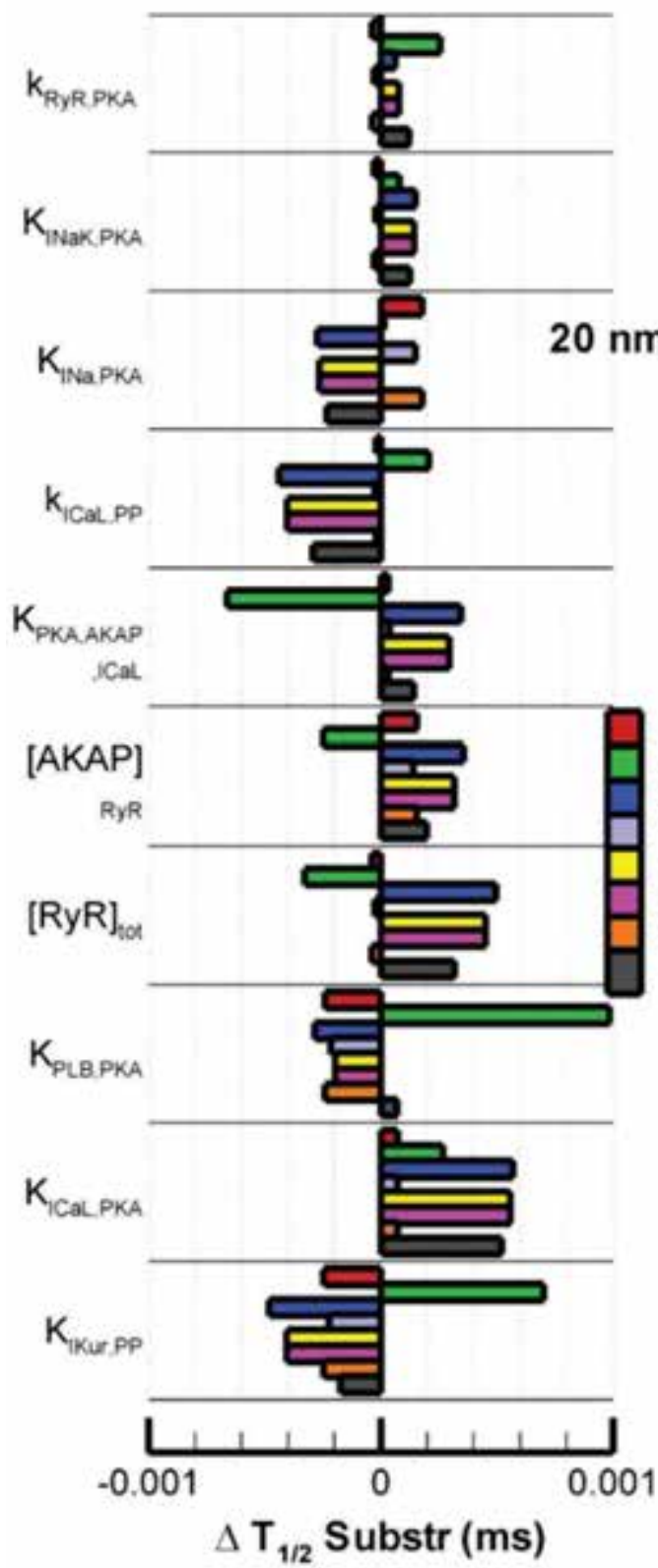

B.
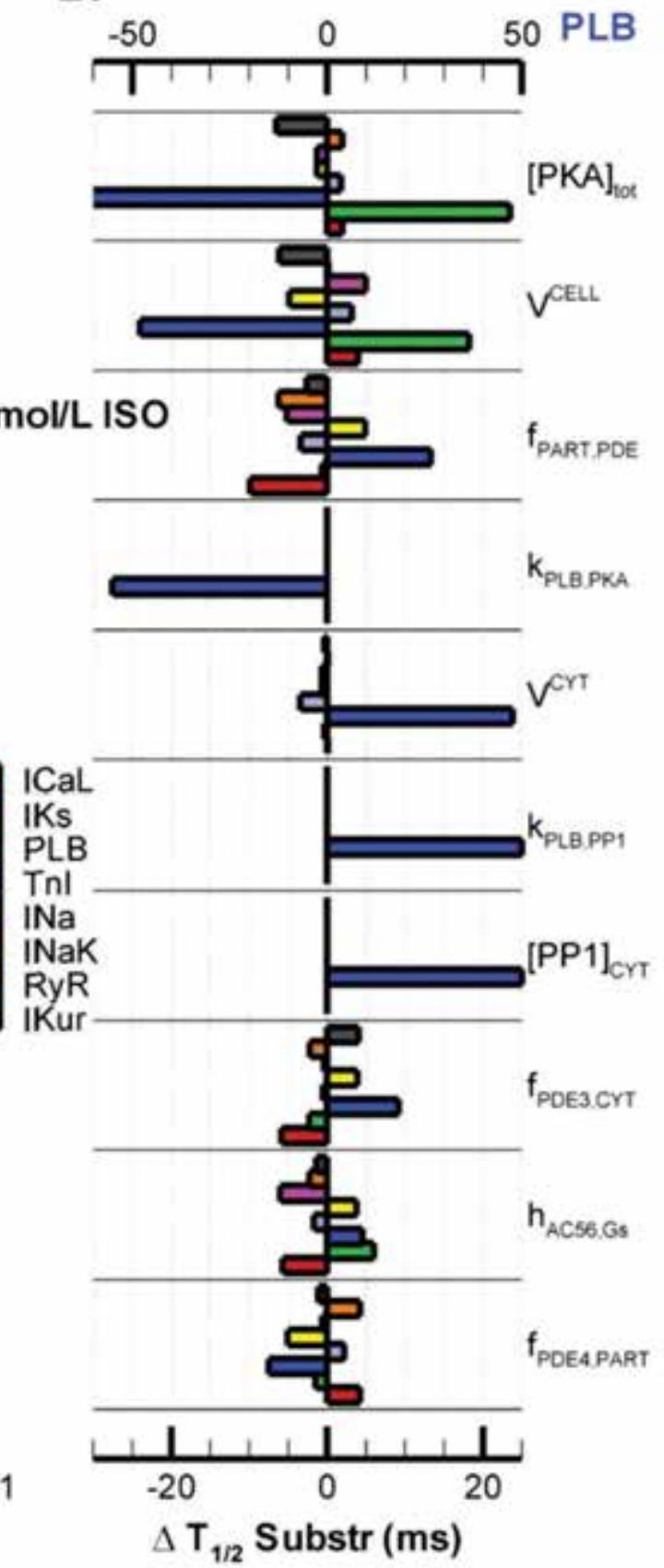

Chapter 3 - Figure 5 (Page 61). The 10 parameters with the smallest (A) and largest (B) impact on the mean change in time to half maximal activation in the presence of $20 \mathrm{nmol} / \mathrm{L}$ ISO over all substrates. Note different impact scale in (B) for PLB. 


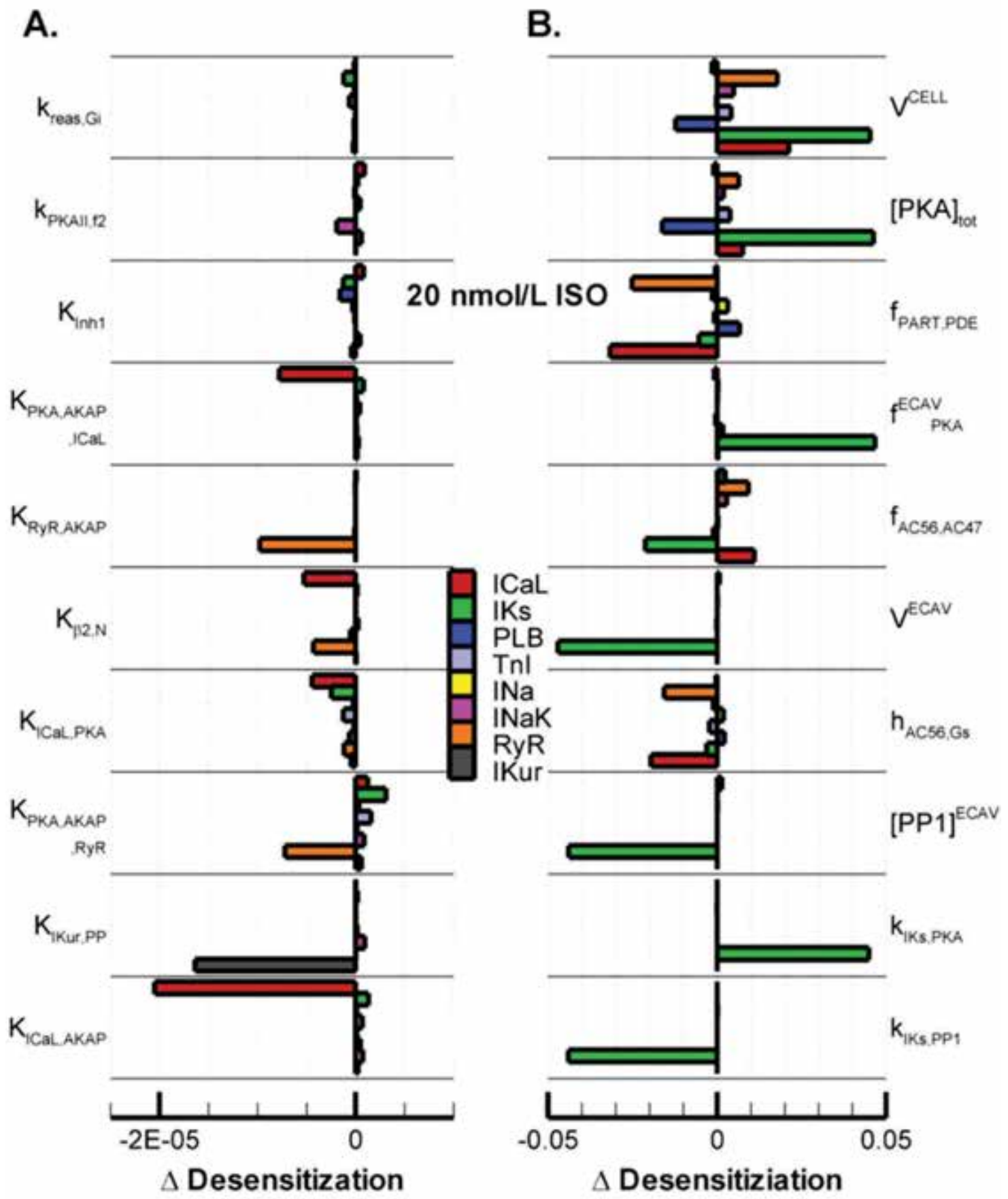

Chapter 3 - Figure 6 (Page 62). The 10 parameters with the smallest (A) and largest (B) impact on the mean change in the amount of desensitization (difference between peak phosphorylation level and level after $30 \mathrm{~min}$ ) in the presence of $20 \mathrm{nmol} / \mathrm{L}$ ISO over all substrates. 


\section{A. AC isoform activation}

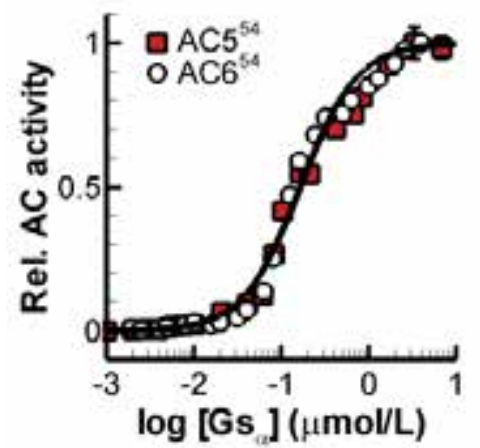

B. AC6 isoform inactivation

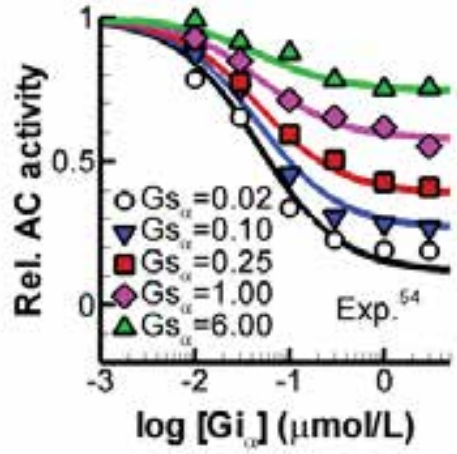

C. Global AC activity

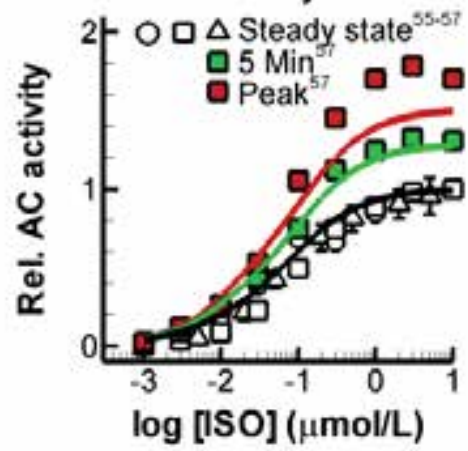

Chapter 3 - Figure 7 (Page 64). Comparison between adenylyl cyclase (AC) properties of model (lines) and experimental data (symbols). A. Gs-dependence of AC 5/6 in experiments and model. B. Inactivation of AC6 by $\mathrm{Gi}$ at various levels of Gs-stimulation in experiments ${ }^{54}$ and model. No error bars provided in experimental source. C. Simulated and measured whole-cell AC activity: (i) at peak (red), (ii) after $5 \mathrm{~min}$ (green) and (iii) at steady-state (white symbols; black line) in canine ventricular myocytes $^{55,56}$ (circles, triangles) and Chinese hamster fibroblasts with human $\beta 1 \mathrm{ARs}$ expressed (squares; no error bars provided in experimental source) ${ }^{57}$.

A. ICaL I-V

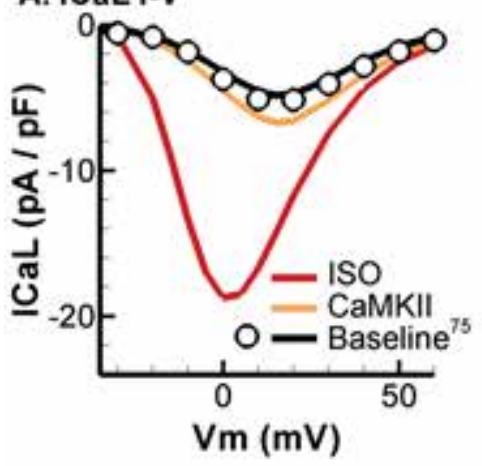

D. ICaL recovery from inactivation

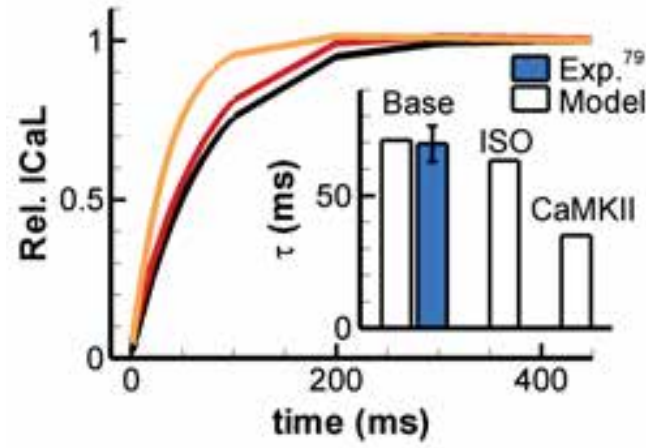

B. ICaL inactivation

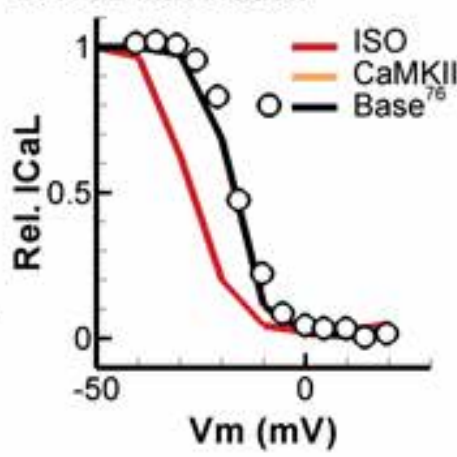

C. ICaL modulation
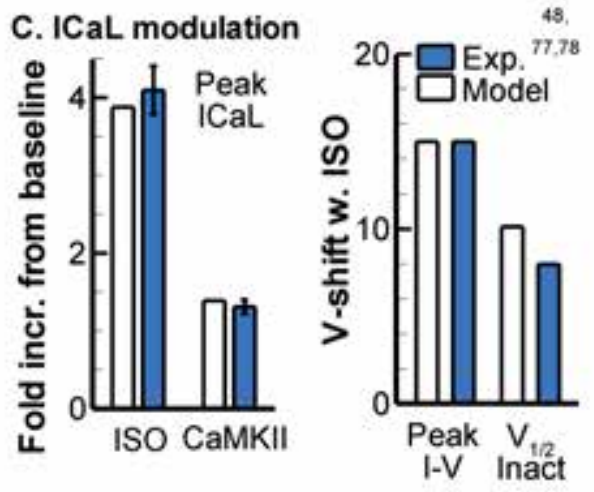

E. ICaL time course of inactivation

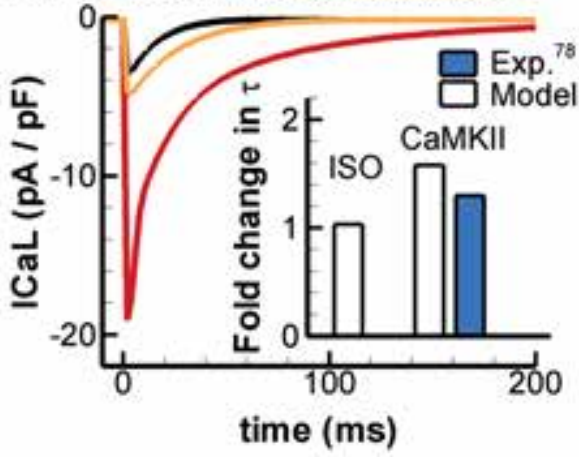

Chapter 3 - Figure 17 (Page 69). Simulated characteristics of PKA phosphorylated (red), CaMKII phosphorylated (orange) and non-phosphorylated (black) populations of $\mathrm{I}_{\mathrm{Cal}}$. Experimental data are shown as symbols, model output as lines. A. Peak I-V relationship. ${ }^{75}$ B. Voltage dependence of steady-state $I_{\text {CaL }}$ inactivation. ${ }^{76} \mathrm{C}$. Fold increase in peak $I_{\mathrm{CaL}} \mathrm{I}-\mathrm{V}$ with ISO or maximal CaMKII activation (left) and shift in peak I-V and midpoint of inactivation ( $\mathrm{V}_{1 / 2}$ Inact; right) during stimulation with ISO in model (white) and experiments ${ }^{48,77,78}$ (blue). D. Recovery from inactivation. Inset shows time- 
constants under baseline, ISO or CaMKII conditions based on a mono-exponential fit in model (white bars) and canine epicardial myocytes (blue bar). ${ }^{79} \mathrm{E}$. Time course of $\mathrm{I}_{\mathrm{CaL}}$ inactivation during a $200 \mathrm{~ms}$ depolarizing pulse to $0 \mathrm{mV}$. Inset shows the relative change in time constant of inactivation with 1.0 $\mu \mathrm{mol} / \mathrm{L}$ ISO or maximal CaMKII activation. CaMKII-dependent effects are consistent with experiments in rabbit ventricular myocytes. ${ }^{78}$

A. ITo recovery from inact.

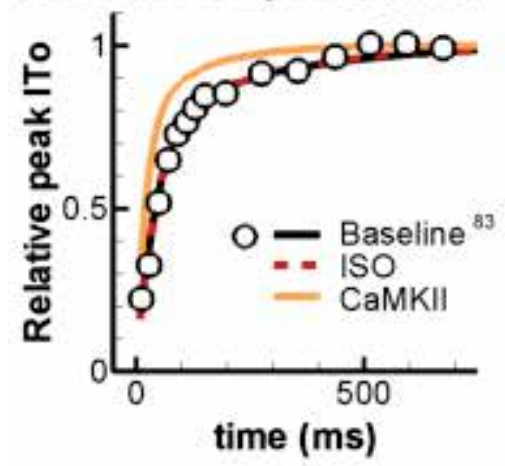

B. ITo time constants (model) C. Validation CaMKII Effect

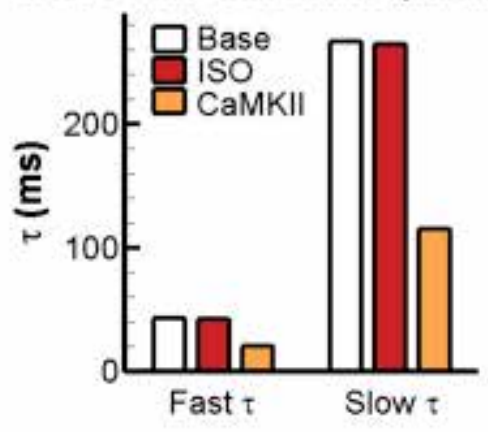

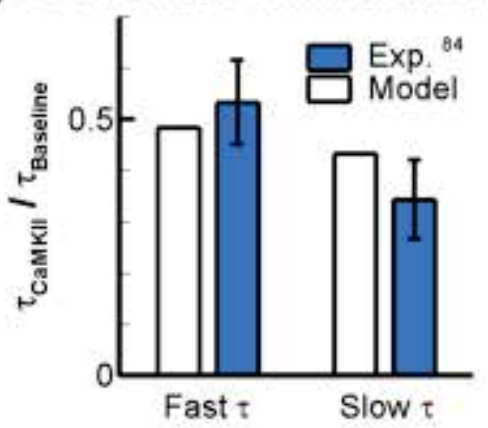

Chapter 3 - Figure 20 (Page 70). Characteristics of $I_{\text {To }}$ recovery from inactivation at baseline (black), with maximal CaMKII activity (orange) or in the presence of $1.0 \mu \mathrm{mol} / \mathrm{L}$ ISO (red; identical to baseline). A. Simulated (line) time course of $\mathrm{I}_{\mathrm{T}}$ recovery from inactivation (assessed using a doublepulse protocol to $+40 \mathrm{mV}$ for $200 \mathrm{~ms}$ from a holding potential of $-80 \mathrm{mV}$ ) compared to experimental data (white circles) from canine ventricular myocytes under baseline conditions. ${ }^{83} \mathrm{~B}$. Comparison of model fast and slow recovery time constants (based on a biexponential fit to the data in A). C. Validation of the effect of CaMKII on $\mathrm{I}_{\text {To }}$ recovery from inactivation in model (white) compared to data in rabbit ventricular myocytes acutely overexpressing CaMKII (blue). ${ }^{84}$

A. APD

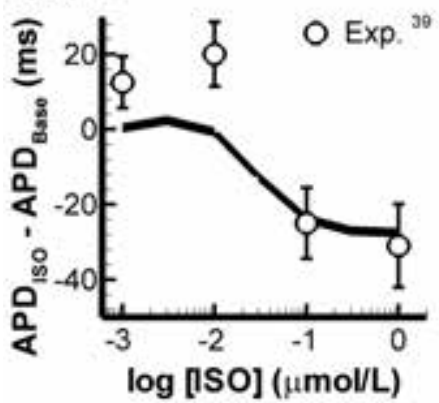

C. Time course APD

\section{B. CaT}

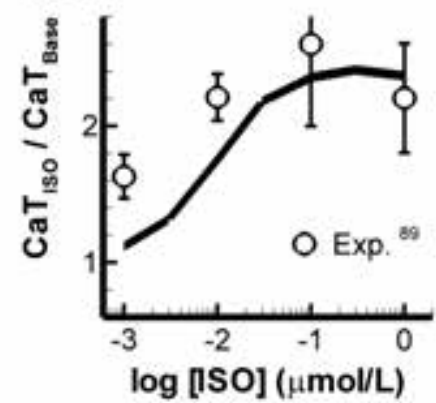

$100 \mathrm{nmol} / \mathrm{L}$ ISO

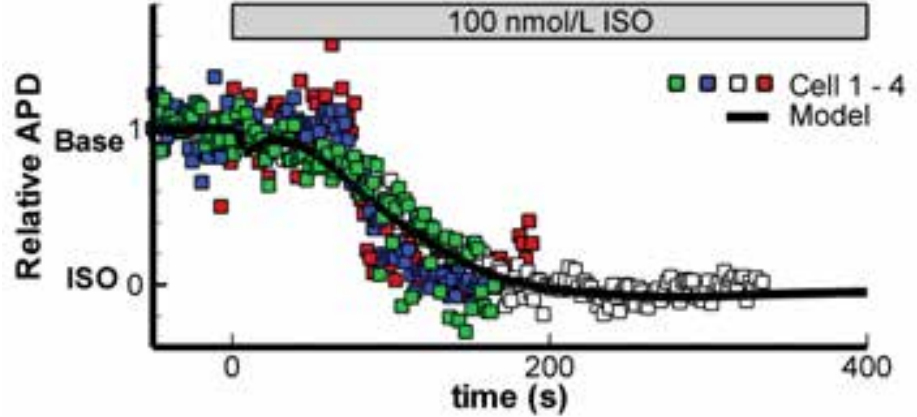


Chapter 3 - Figure 22 (Page 71). Concentration-dependent effects of ISO on APD (A;CL = $1000 \mathrm{~ms}$ ) and $\mathrm{CaT}$ amplitude $\left(\mathrm{B} ; \mathrm{CL}=2000 \mathrm{~ms}\right.$ ) in canine ventricular myocytes (symbols ${ }^{39}, 89$ ) and model (lines). C. Time course of APD changes during application of $100 \mathrm{nmol} / \mathrm{L}$ ISO in 4 canine ventricular myocytes (symbols) and model (line). APDs were normalized between 1 (baseline) and 0 (maximal response to ISO) to account for baseline cell to cell differences (see also Figure 26). $\mathrm{CL}=1000 \mathrm{~ms}$. For experimental protocol, see Johnson et al. ${ }^{90}$
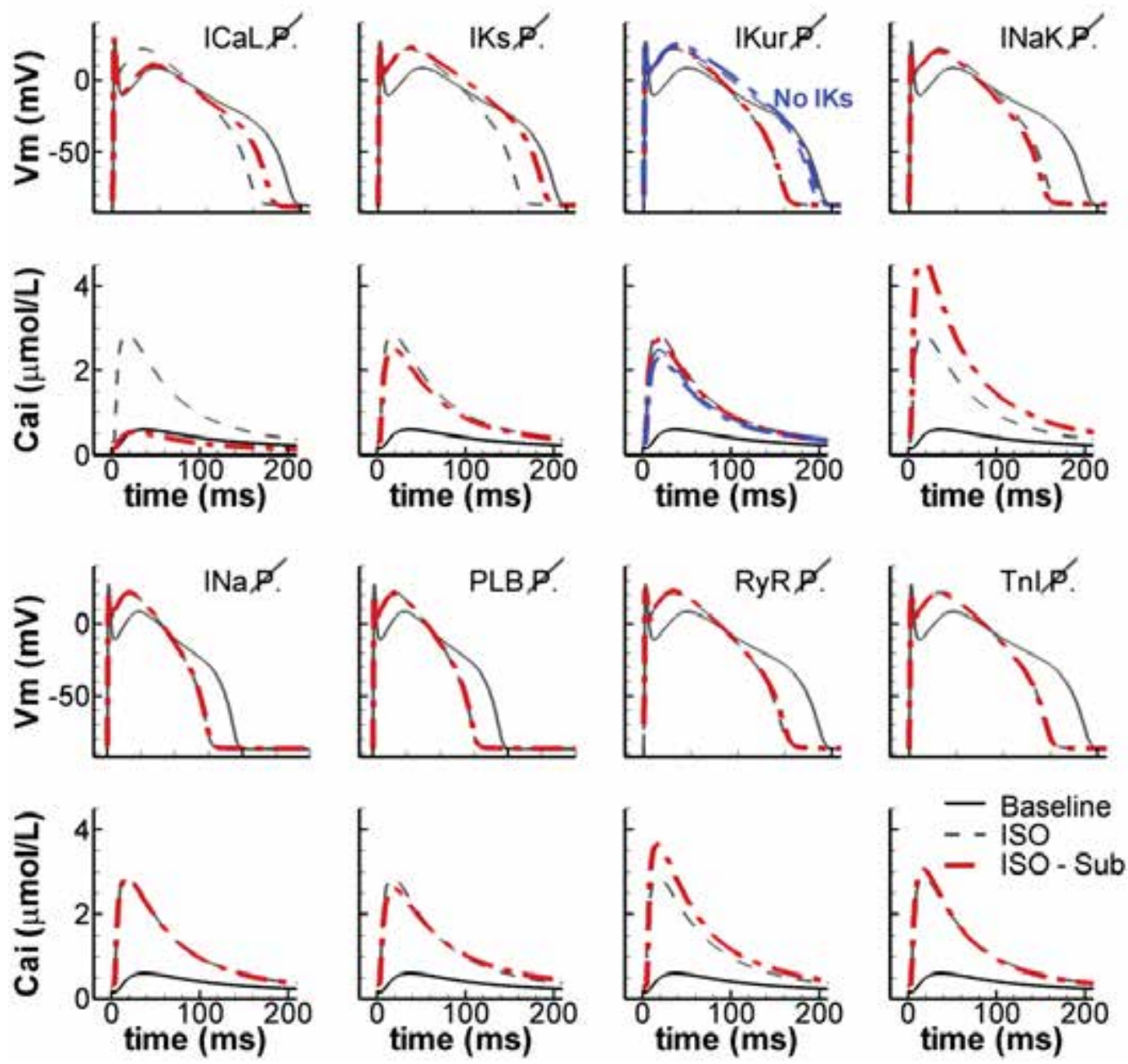

Chapter 3 - Figure 28 (Page 78). Contribution of individual substrates to APD and CaT changes in response to $\beta A R$ stimulation at $C L=350 \mathrm{~ms}$. Steady-state AP and CaT are shown in the absence of $\beta A R$ stimulation (black solid line), in the presence of a saturating dose of ISO (gray dashed line), or in the presence of $\beta A R$ stimulation without PKA-dependent phosphorylation (red dashed-dotted line) of $I_{\mathrm{CaL}}, I_{\mathrm{Ks}}, I_{\mathrm{Kur}}$, or $I_{\mathrm{NaK}}$ (top two rows; left to right) or without PKA-dependent phosphorylation of $I_{\mathrm{Na}}$ PLB, RyR or Tnl (bottom two rows; left to right). 

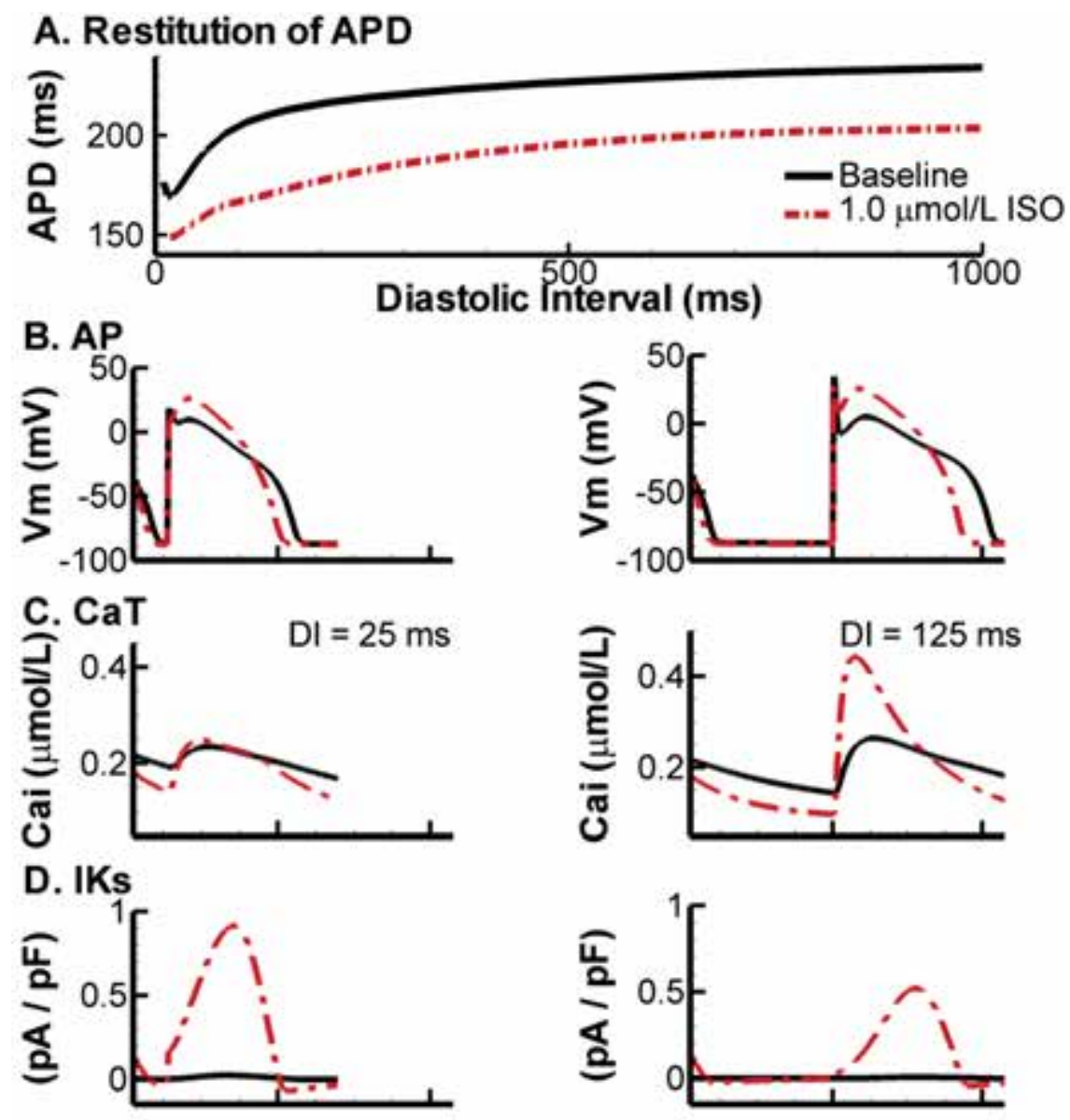

\section{E. IKs zone occupancy}
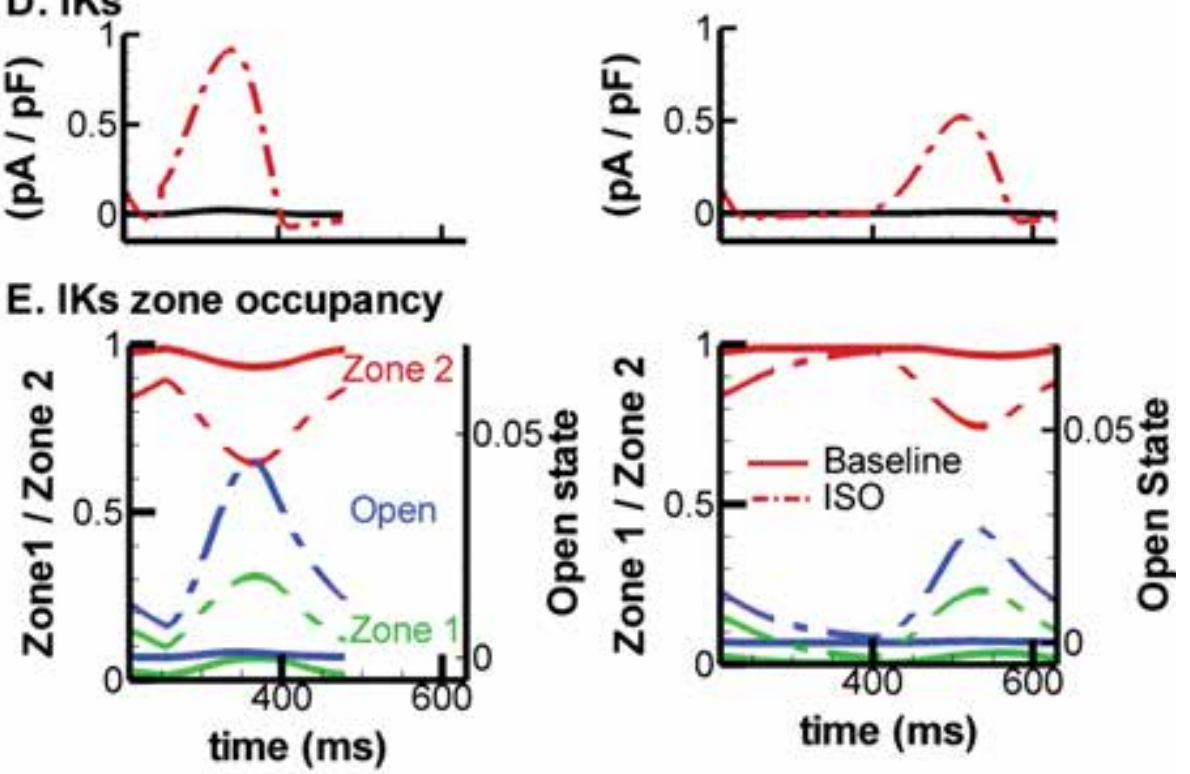

Chapter 3 - Figure 29 (Page 80). Kinetic changes of $I_{\mathrm{Ks}}$ due to $\beta$ ARS result in altered gating modes and increased available reserve for channel opening. A. APD restitution as function of diastolic interval after pacing to steady-state at $\mathrm{CL}=2000 \mathrm{~ms}$ in the absence (solid black) or presence (dashdotted red) of $1.0 \mu \mathrm{mol} / \mathrm{L}$ ISO. B. AP of premature beat at diastolic intervals of $25 \mathrm{~ms}$ (left) or $125 \mathrm{~ms}$ (right). C. CaT corresponding to these APs. D. Corresponding $\mathrm{I}_{\mathrm{ks}}$. E. Corresponding zone occupancies of $I_{K s}$ channels during the AP. Zone 2 (red) represents the deep closed states, Zone 1 (green) represents the closed states adjacent to the open state, termed "available reserve". Open state occupancy is shown in blue on a separate y-axis. Data in the absence of ISO are shown in solid lines, data in the presence of ISO in dashed-dotted lines. 


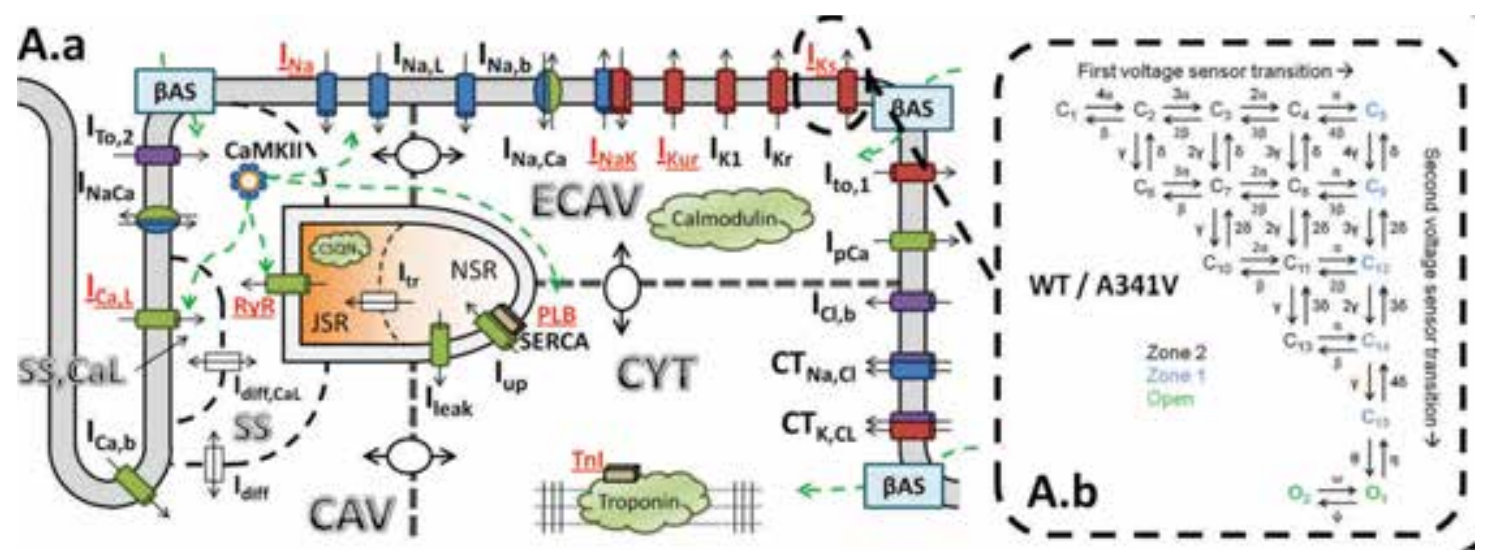

Chapter 4 - Figure 1 (Page 110). Computational modeling approach. A.a. Schematic overview of the structure of a single cell model, including all ion channels, pumps, exchangers, $\mathrm{Ca}^{2+}$-handling proteins, $\mathrm{Ca}^{2+} /$ Calmodulin-dependent kinase and compartmental $\beta A R$ signaling pathway, as published recently (Chapter 3). ${ }^{14}$ A.b. Structure of the 17 -state Markov model that was fitted to WT $\mathrm{I}_{\mathrm{Ks}}$, heterozygous or homozygous mutant channel characteristics in the absence and presence of $\beta A R$ stimulation.

A.

B.
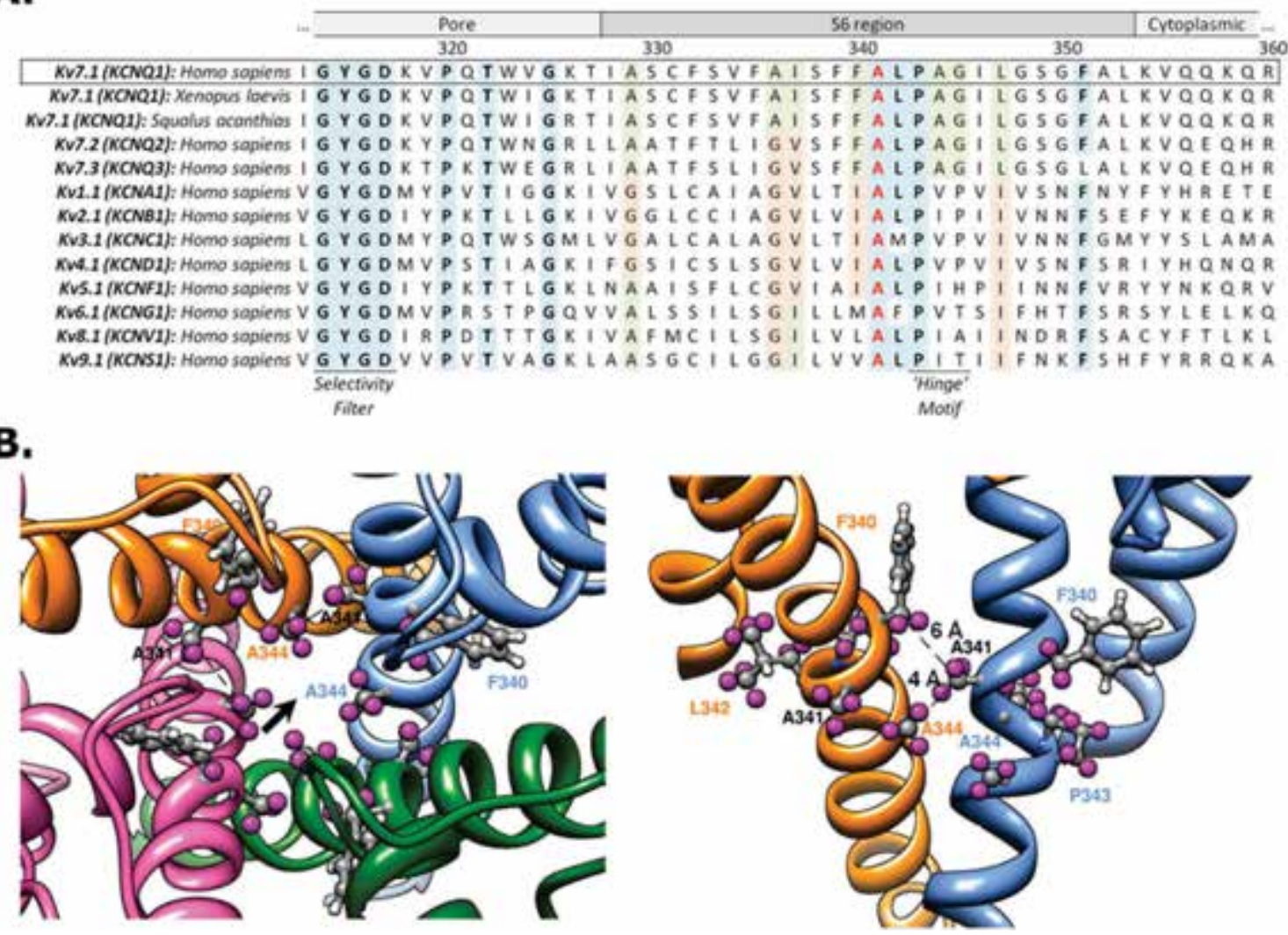

Chapter 4 - Figure 3 (Page 113). Location and conservation of A341 in KCNQ1. A. Multiple sequence alignment of the KCNQ1-S6 region with other members of the Kv7.x family and other voltage-gated potassium channels. Conserved residues are bold and highlighted in blue, partially conserved residues are highlighted in green and red. The conserved $\mathrm{K}^{+}$selectivity filter and the PAG (in Kv7.x) or 
PXP (in other Kv channels) hinge motifs are indicated below the alignment. A341 is indicated in red and is extremely conserved among all Kv channels. B. Location of A341 in the closed-state tetrameric KCNQ1 homology model described by Kang et al. ${ }^{21}$ Left panel shows top view from the extracellular side. Residues F340, A341 and A344 are shown as 'ball-and-stick' models. Black arrow indicates the viewing direction for the right panel. Right panel shows front view of two KCNQ1-subunits (only S5, pore and S6 region are shown). Residues F340 - A344 are shown. Distances between residue A341 of the right subunit and its two nearest residues are indicated with dashed lines.

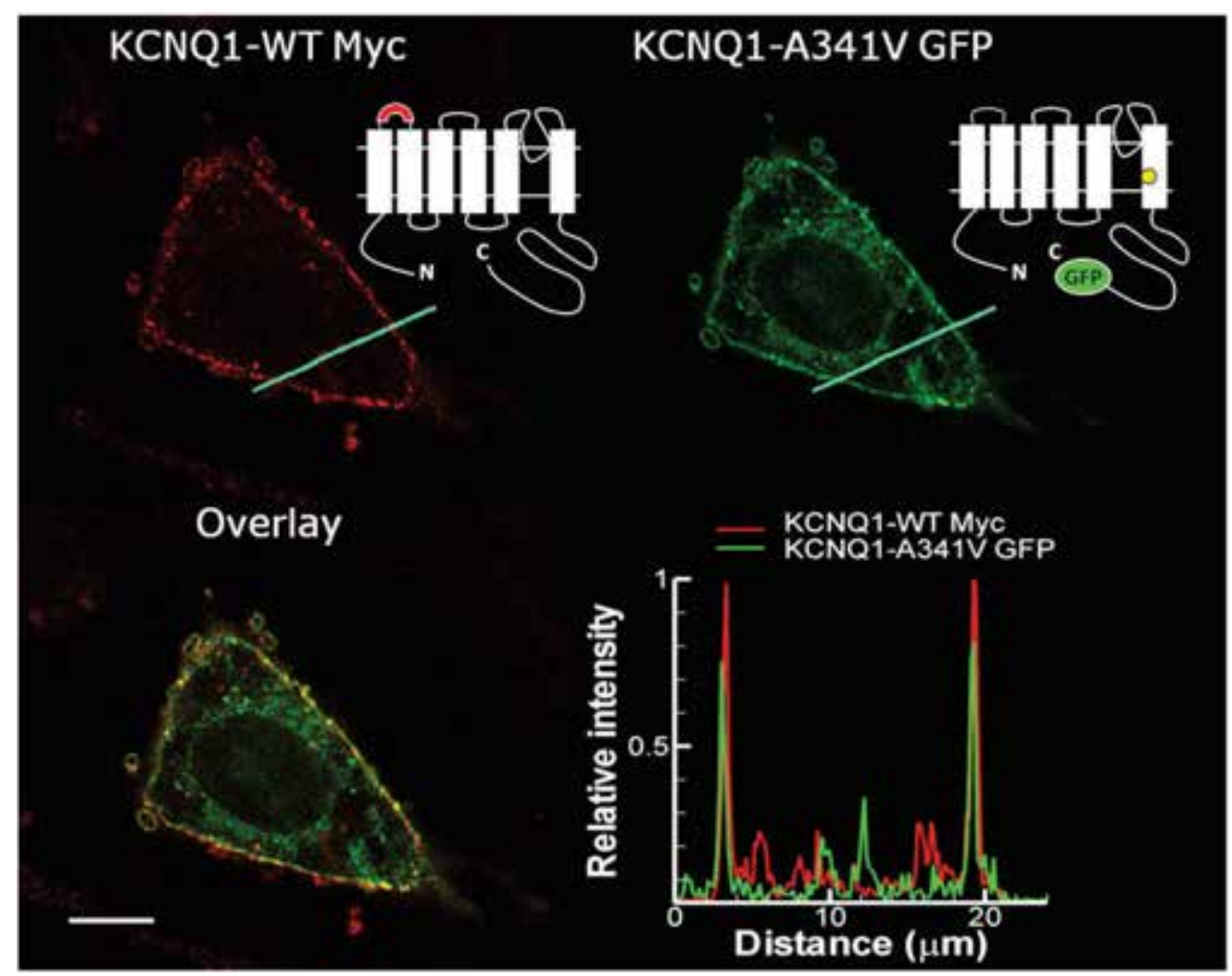

Chapter 4 - Figure 11 (Page 122). Localization of KCNQ1-WT and KCNQ1-A341V after co-transfection of both subunits with KCNE1 and Yotiao. A c-Myc epitope was inserted in the extracellular loop between transmembrane segments 1 and 2 of KCNQ1-WT and the KCNQ1-A341V was tagged with GFP at the C-terminus. Top-left panel shows a confocal image of KCNQ1-WT obtained with an anti-cMyc antibody in a representative $\mathrm{CHO}$ cell. Top-right panel shows GFP signal in the same cell. When the two signals are overlayed (bottom-left panel), a clear colocalization of WT and A341V KCNQ1 subunits can be observed at the cell membrane (yellow), confirming "heterozygous" channel expression. The bottom-right panel shows the relative signal-intensity profiles of KCNQ1-WT and KCNQ1-A341V along the cross-sections at the light-green line. Both signals show increased activity at the cell boundaries. The lower-left white scale-bar indicates $10 \mu \mathrm{m}$. 


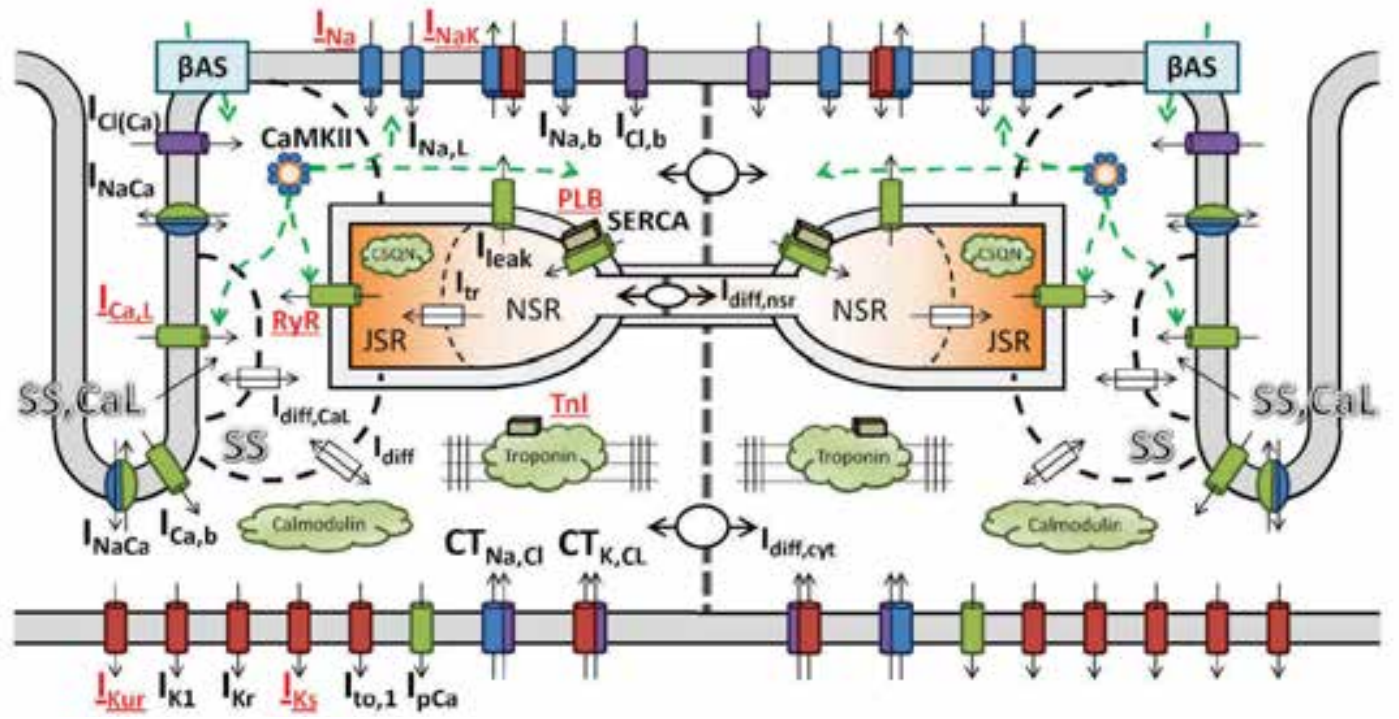

Chapter 6 - Figure 1 (Page 156). Schematic overview of the computational model of the canine ventricular myocyte. Adapted from Heijman et al. ${ }^{23}$ (Chapter 3 ). The model was divided into two identical domains to simulate the local origin of SCR. Only components on the left side of the model are labeled for clarity, identical components are located on the right side. The domains are coupled via diffusion of $\mathrm{Ca}^{2+}\left(\mathrm{I}_{\text {diff,cyt }}\right.$ and $\left.\mathrm{I}_{\text {diff,nsr }}\right)$. All other abbreviations are as previously described. ${ }^{23}$
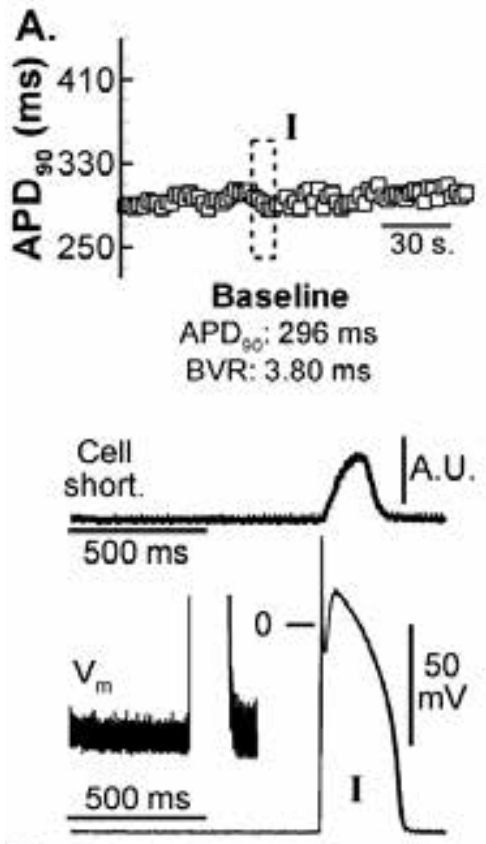

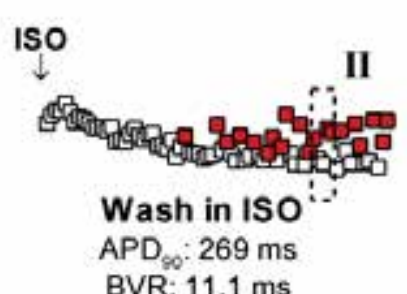

BVR: $11.1 \mathrm{~ms}$

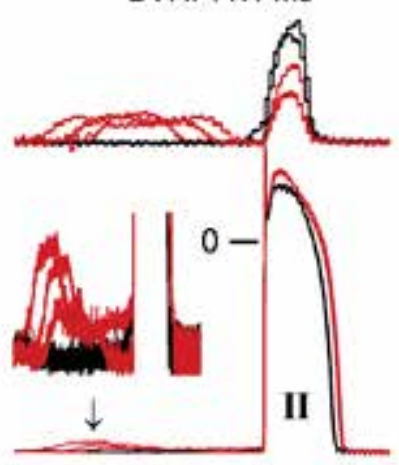

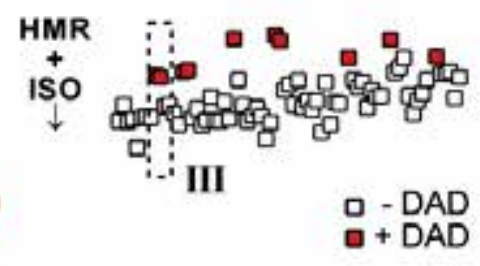

$\mathrm{HMR}+\mathrm{ISO}$

$\mathrm{APD}_{90}: 394 \mathrm{~ms}$

BVR: $18.9 \mathrm{~ms}$

B.

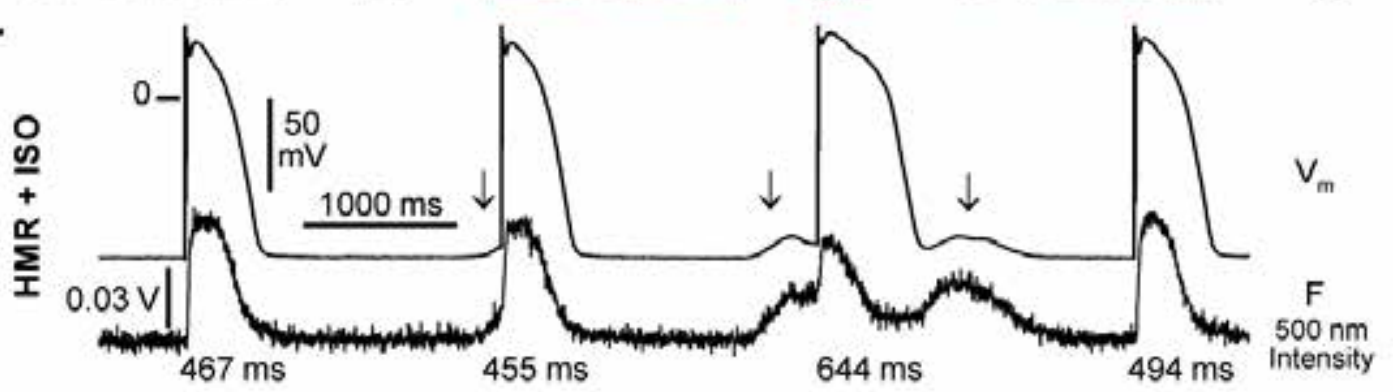


Chapter 6 - Figure 3 (Page 159). Occurrence of DADs in canine ventricular myocytes is associated with prolongation of subsequent APs and increased BVR. A. APD 90 for 75 consecutive beats at 2000ms CL at baseline (left panel: I), during wash-in of ISO (middle panel: II) and in the presence of HMR1556 and ISO (right panel: III). APDs preceded by a DAD are indicated with filled symbols, APDs without DADs depicted by open symbols. Dashed rectangle indicates a set of 5 beats for which cell shortening and membrane potential are depicted below. Insets show diastolic potentials at an expanded scale. Beats without prior SCR are indicated in black, beats with prior SCR in red. B. Membrane potential (top) and $\left[\mathrm{Ca}^{2+}\right]_{\mathrm{i}}$ for four consecutive beats. $\mathrm{APD}_{90} \mathrm{~S}$ are indicated below each $\mathrm{AP}$. SCR is indicated with arrows.

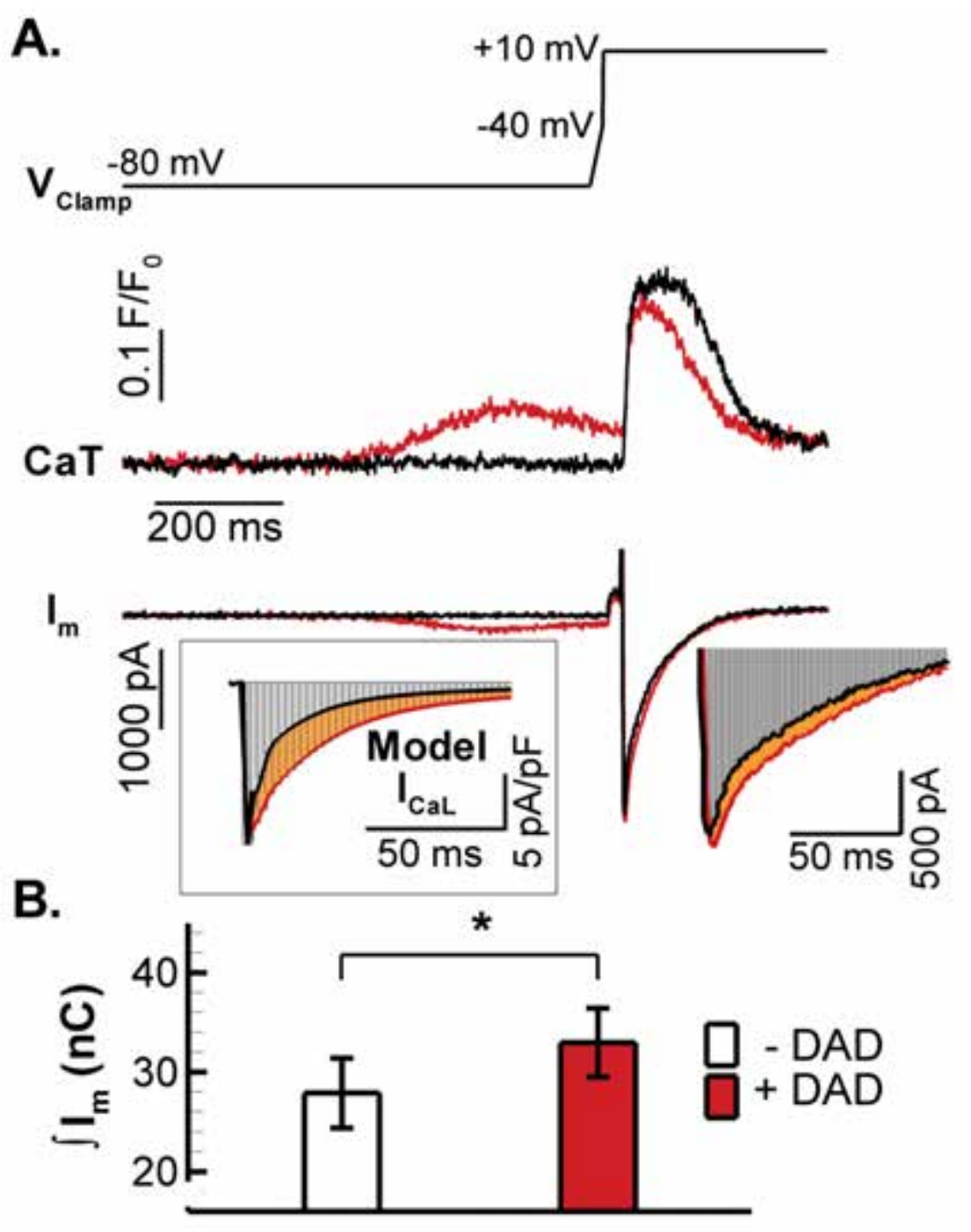

Chapter 6 - Figure 10 (Page 169). I CaL inactivation is slowed after SCR. A. Voltage-clamp protocol (top panel), intracellular $\mathrm{Ca}^{2+}$ (middle panel) and membrane current (bottom panel) in a representative canine ventricular myocyte in the presence of HMR1556 + ISO + increased $\left[\mathrm{Ca}^{2+}\right]_{0}(3.6$ or $5.0 \mathrm{mmol} / \mathrm{L})$ in the absence (black) or presence of prior SCR (red). Right inset shows membrane current at an expanded scale. 4-AP $(5.0 \mathrm{mmol} / \mathrm{L})$ and $\mathrm{BaCl}_{2}(0.1 \mathrm{mmol} / \mathrm{L})$ were used to isolate $\mathrm{I}_{\mathrm{CaL}}$. Left inset shows model results under similar conditions. B. Quantification of average membrane current integral 
(shaded area in right inset of panel A) in 6 canine ventricular myocytes in the absence or presence of SCR. Prior SCR significantly increases total inward current $(*$ : $P<0.05)$.
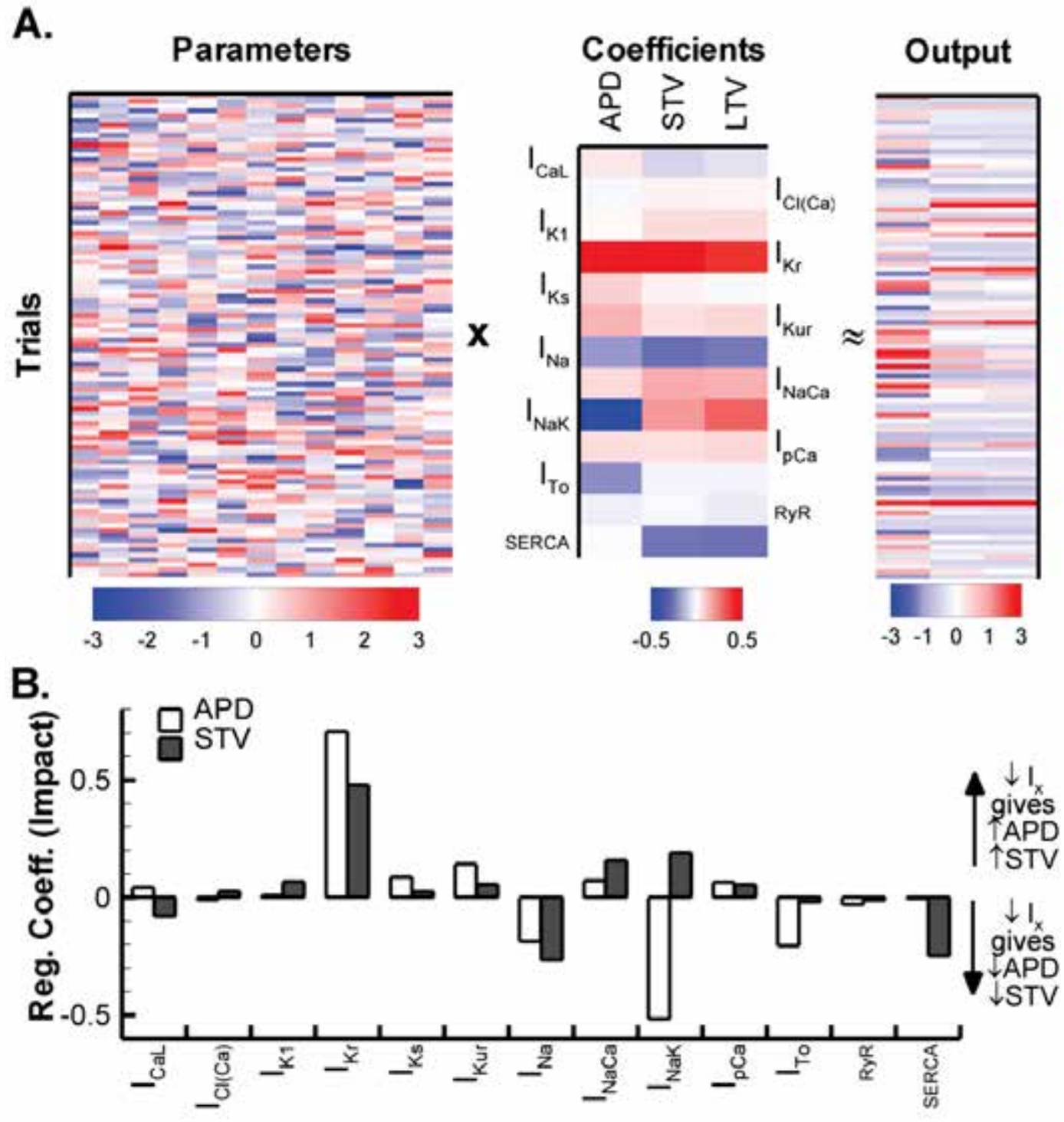

Chapter 7 - Figure 5 (Page 188). Contribution of currents to BVR determined via linear regression. A. Relative changes in the maximal conductance of the 13 currents/fluxes (lanes correspond to the column-pairs in panel B) for 100 (out of 300) trials (left panel) and corresponding changes in outputs (APD, STV and LTV) during steady-state pacing at $\mathrm{CL}=1000 \mathrm{~ms}$ (right panel). Middle panel shows the coefficients that indicate the contribution of each current to every output measure as determined via linear regression. B. Bar plot of the magnitude of the coefficients from panel A regarding their impact on APD (white bars) or STV (shaded bars). $I_{\mathrm{kr}}$ and $I_{\mathrm{Na}}$ have a large impact on both APD and $B V R$, consistent with the results from Figure 4. In addition, $I_{\text {Nak }}$ and $\mathrm{Ca}^{2+}$ uptake (SERCA) also affect STV. LTV showed similar pattern as STV and was not shown for the sake of clarity. 


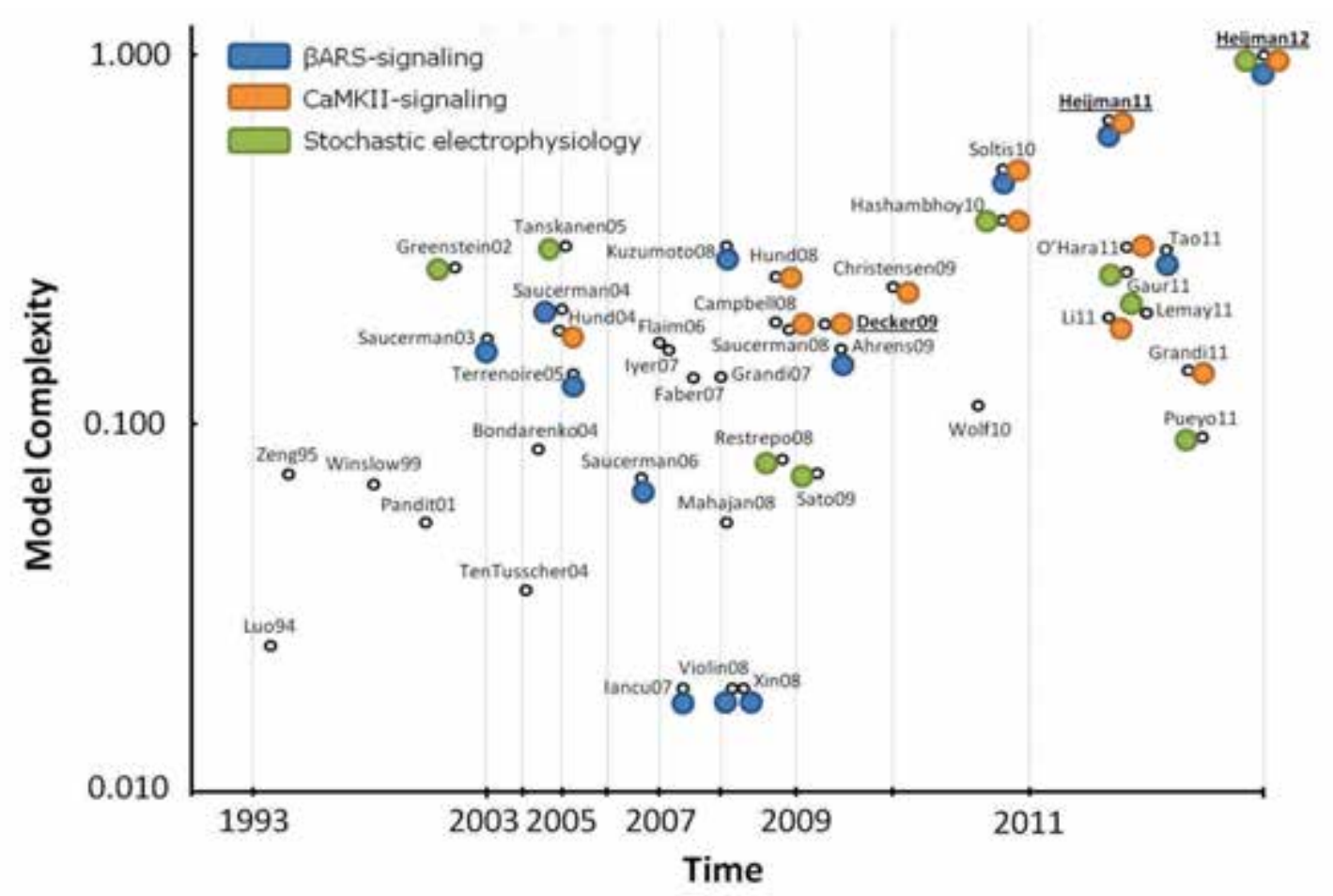

Chapter 8 - Figure 2 (Page 209). Comparison of cellular computational models of electrophysiology with a focus on the period 2000-2011. Models are identified based on the name of the first author and year of publication. Horizontal axis represents time on a logarithmic scale. Vertical axis represents average model complexity. Colored circles indentify models containing an integrated $\beta$ ARS cascade (blue), CaMKII-signaling cascade (orange) and stochastic electrophysiology (green). Models developed in this thesis (indicated with Decker09, Heijman11 and Heijman12 for Chapters 2, 3 and 7, respectively) are underlined.
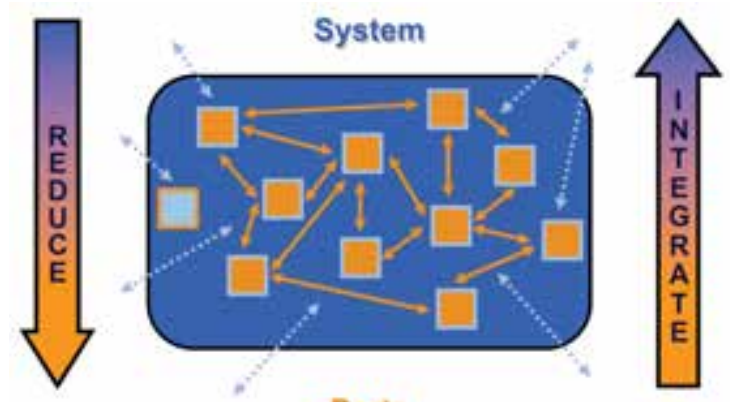

Chapter 8 - Figure 3 (Page 212). "A system as an 'entity that maintains its existence through the mutual interaction of its parts' (von Bertalanffy, 1968). Systems research must combine the (i) identification and (ii) detailed characterisation of parts (orange boxes, as opposed to 'look-alikes', pale blue box, which need to be identified and excluded), with the exploration of their interactions (iii) with each other (orange arrows), and (iv) with the environment (pale blue dashed arrows affecting parts either directly, or indirectly through modulation of internal interactions), to develop a (v) systemic understanding (an important, but often overlooked, aspect is that the system itself not only enables, but also restricts, the type and extent of functions and interactions that may occur; dark-blue box). Systems research therefore requires a combination of reductionist and integrative tools and techniques." Reproduced from Kohl and Noble. ${ }^{122}$ 


\section{APPENDIX}

MODEL DEFINITION AND EQUATIONS 


\section{CONTENTS}

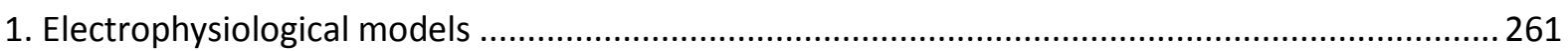

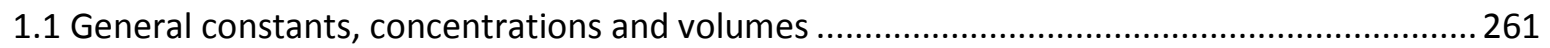

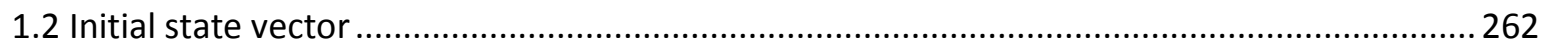

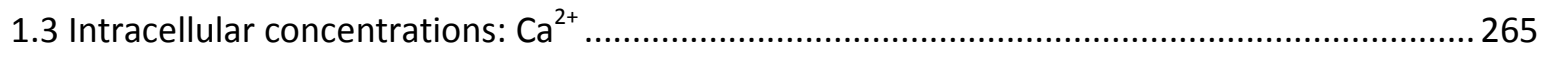

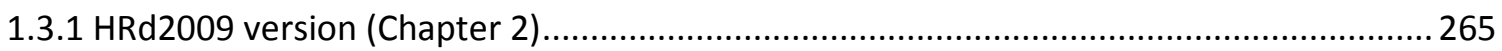

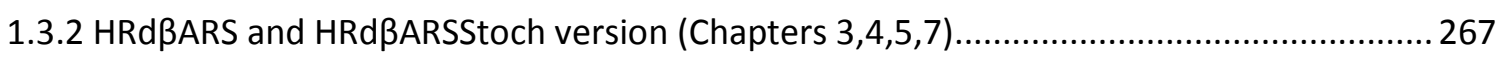

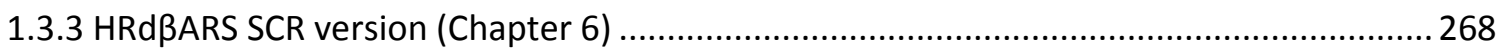

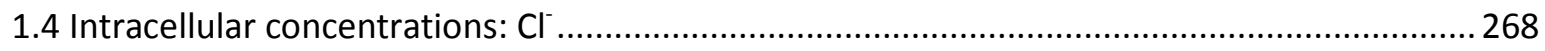

1.4.1 HRd2009, HRdßARS and HRdßARSStoch version (Chapters 2-7) ......................................268

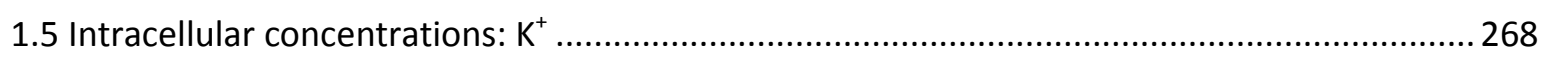

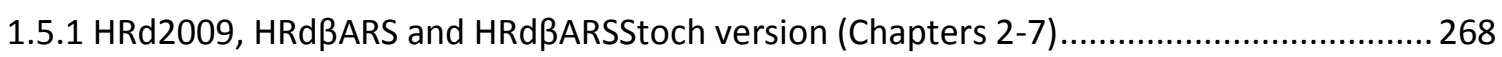

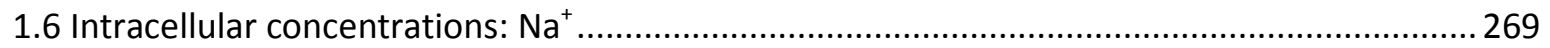

1.6.1 HRd2009, HRdßARS and HRdßARSStoch version (Chapters 2-7) .......................................269

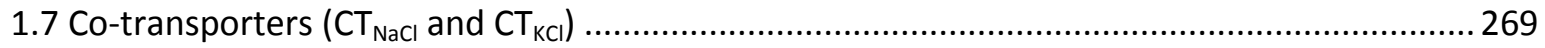

1.7.1 HRd2009, HRdßARS and HRdßARSStoch version (Chapters 2-7)....................................269

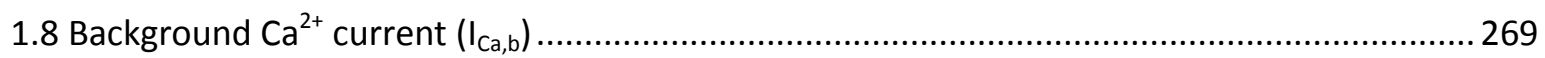

1.8.1 HRd2009, HRdßARS and HRdßARSStoch version (Chapters 2-7) .....................................269

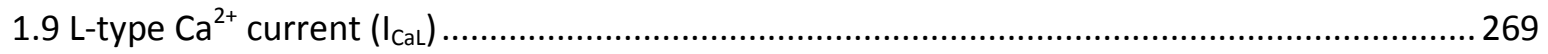

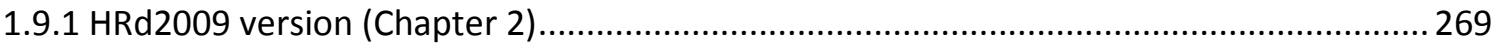

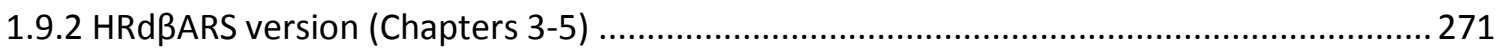

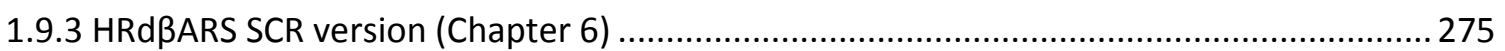

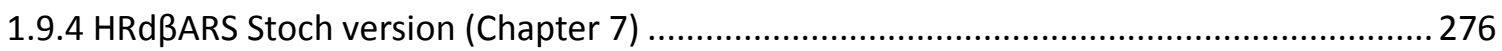

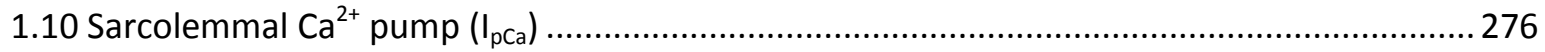

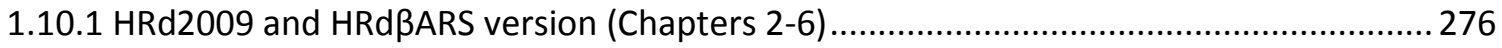

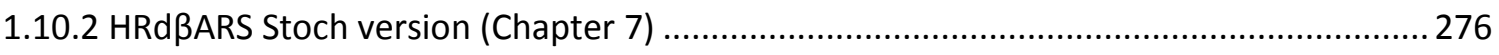

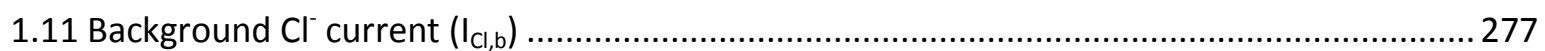

1.11.1 HRd2009, HRdßARS and HRdßARSStoch version (Chapters 2-7)..................................277

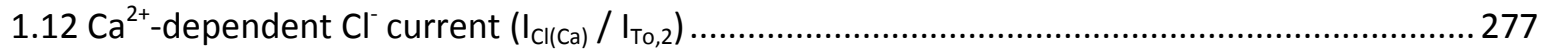

1.12.1 HRd2009, HRdßARS and HRdßARSStoch version (Chapters 2-7)................................... 277

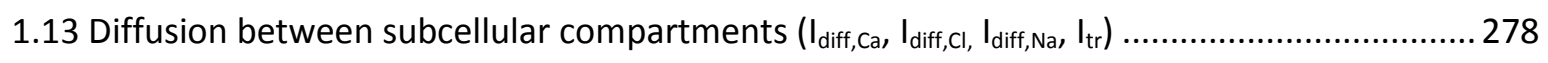

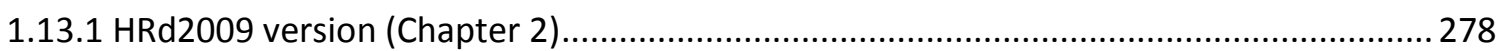

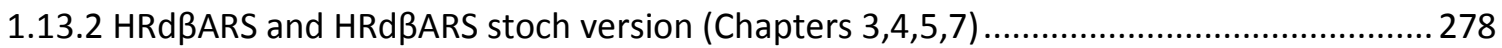

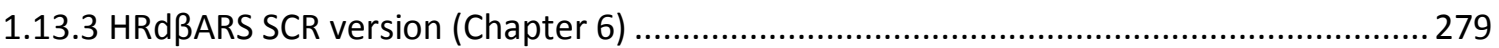

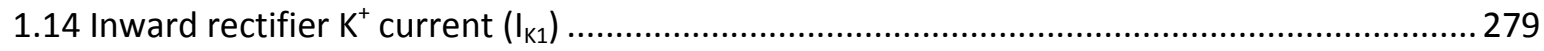

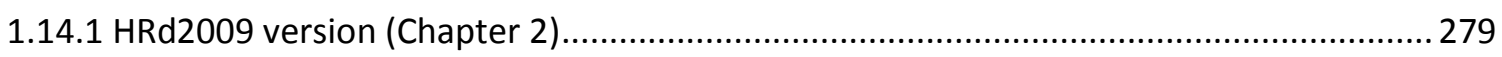

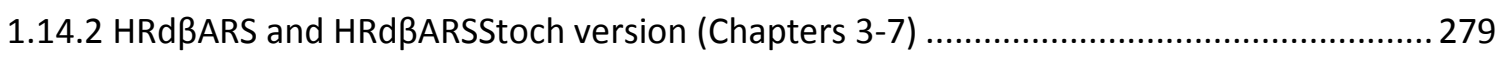




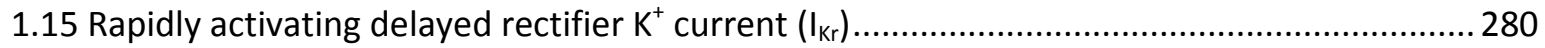

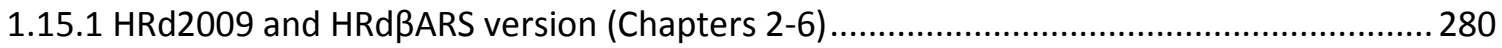

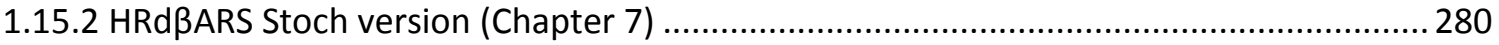

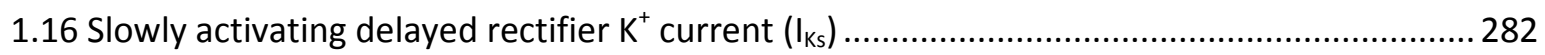

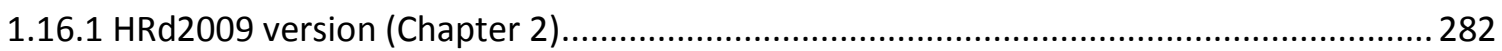

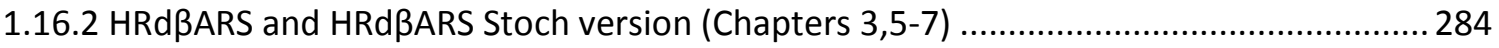

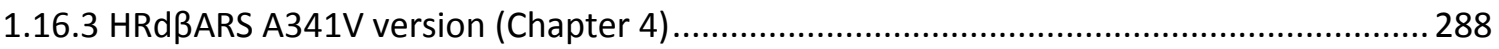

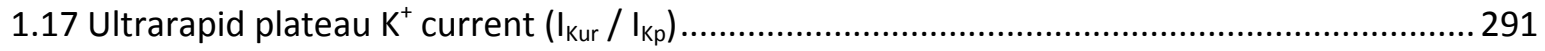

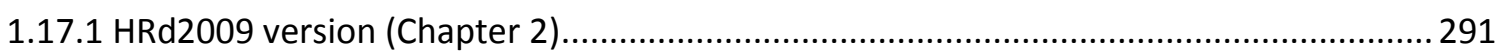

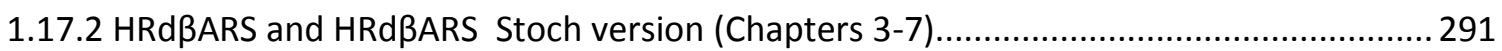

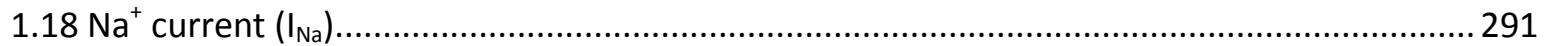

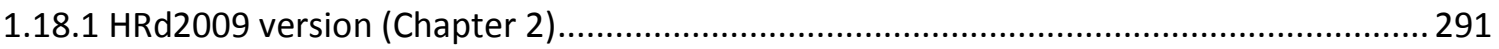

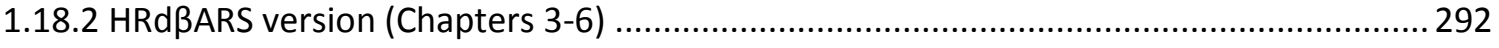

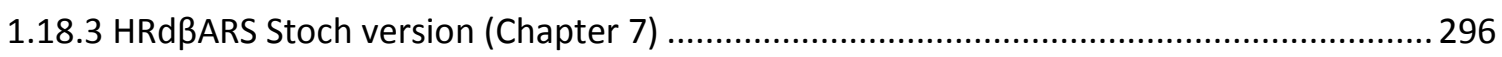

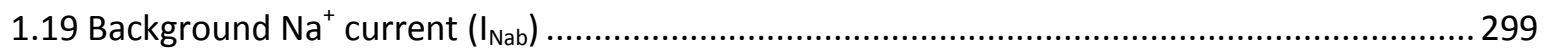

1.19.1 HRd2009, HRdßARS and HRdßARSStoch version (Chapters 2-7)...................................299

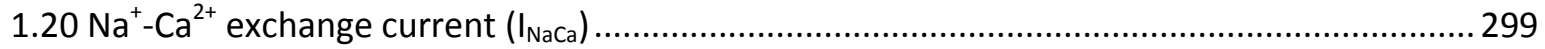

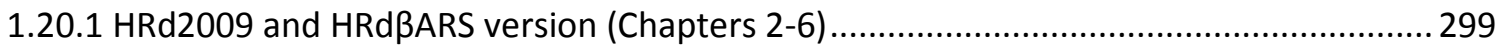

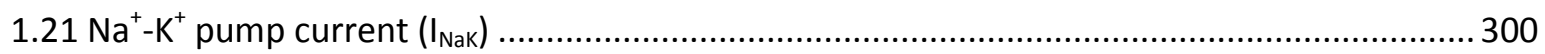

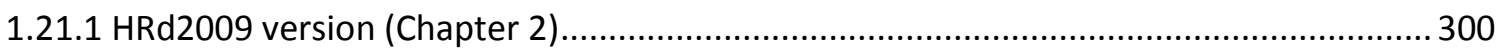

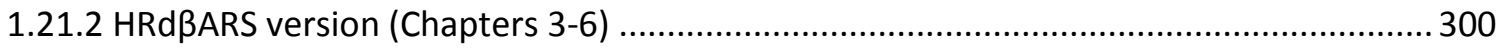

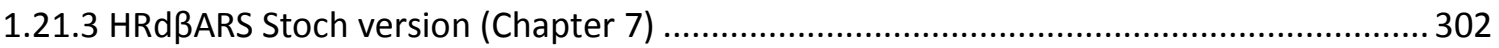

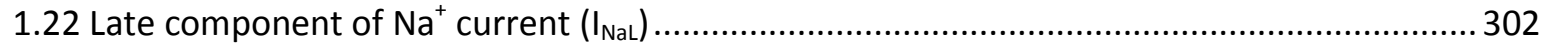

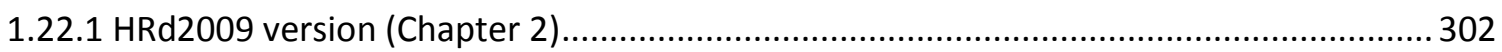

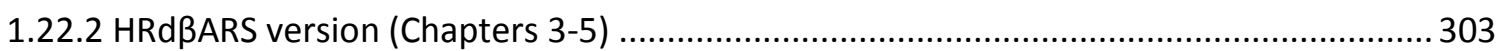

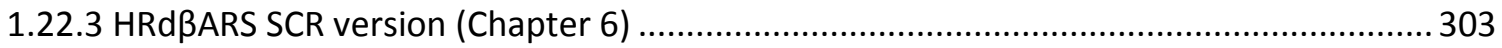

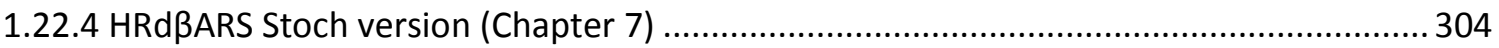

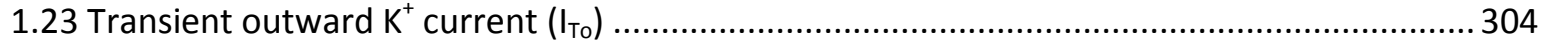

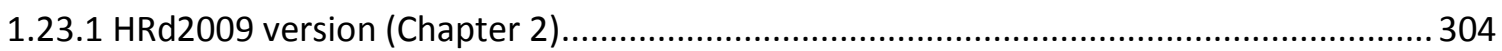

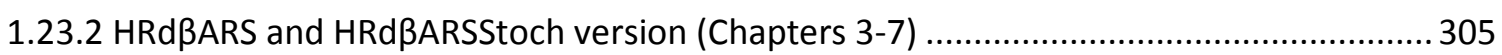

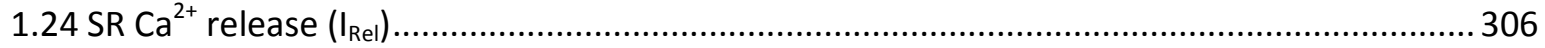

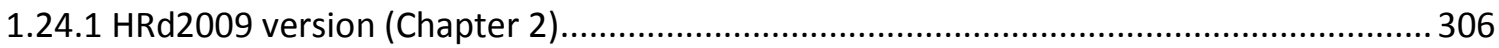

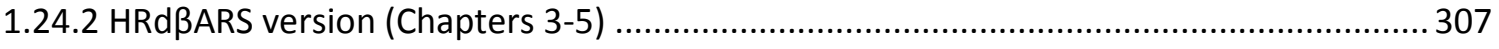

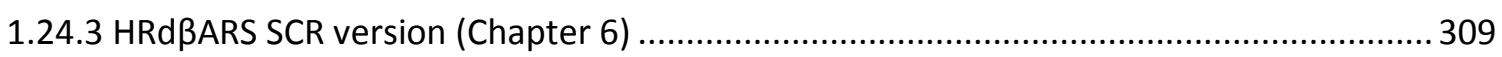

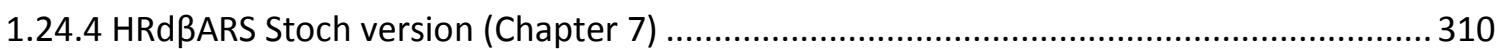

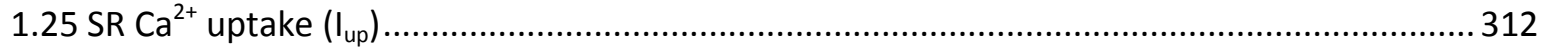

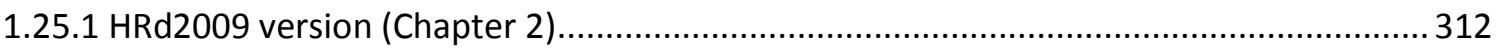

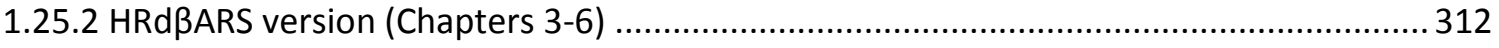




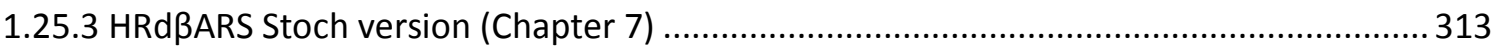

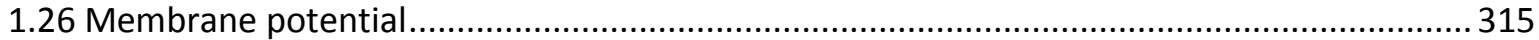

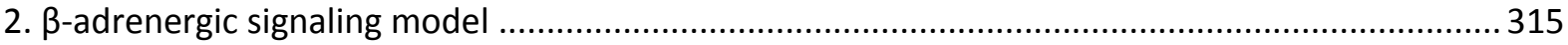

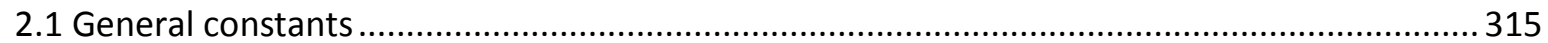

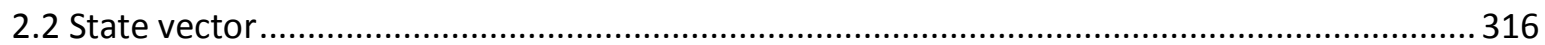

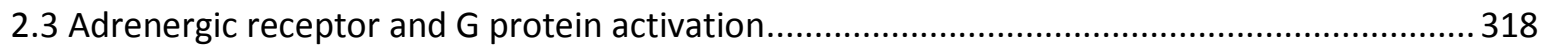

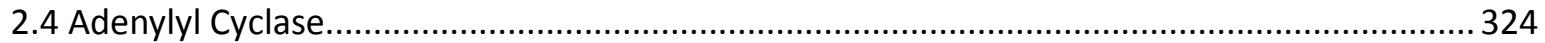

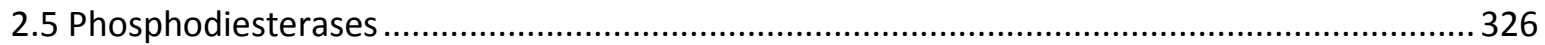

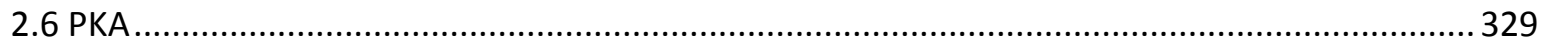

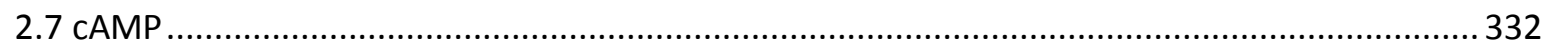

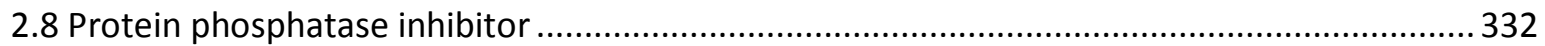

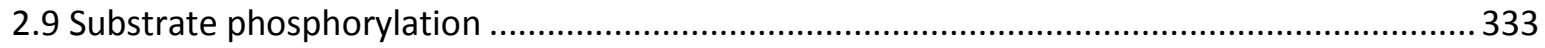

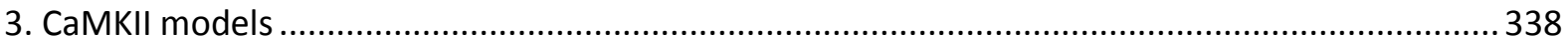

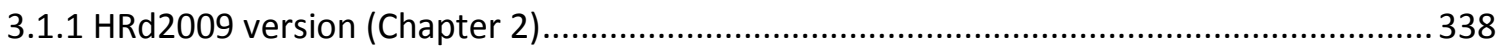

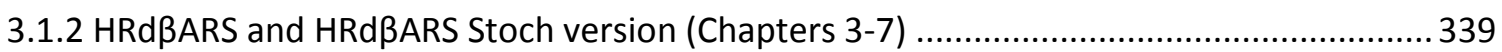

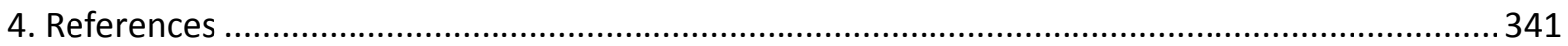




\section{ELECTROPHYSIOLOGICAL MODELS}

1.1 General constants, concentrations and volumes

\begin{tabular}{|ccc|}
\hline Symbol & Description & Value \\
\hline $\boldsymbol{F}$ & Faraday constant & $96487 \mathrm{C} / \mathrm{mol}$ \\
\hline $\boldsymbol{R}$ & Gas constant & $8314 \mathrm{~J} / \mathrm{kmol} / \mathrm{K}$ \\
\hline $\mathbf{C}_{\mathbf{m}}$ & Temperature & $310^{\circ} \mathrm{K}$ \\
\hline $\mathbf{z}_{\mathbf{C a}}$ & Specific membrane capacitance & $1 \mu \mathrm{F} / \mathrm{cm}^{2}$ \\
\hline $\mathbf{z}_{\mathbf{C l}}$ & Valence of $\mathrm{Ca}^{2+}$ ion & 2 \\
\hline $\mathbf{z}_{\mathbf{K}}$ & Valence of $\mathrm{Cl}^{-}$ion & -1 \\
\hline $\mathbf{z}_{\mathrm{Na}}$ & Valence of $\mathrm{K}^{+}$ion & 1 \\
\hline $\mathbf{N}_{\mathbf{A}}$ & Valence of Na ${ }^{+}$ion & 1 \\
\hline
\end{tabular}

Table 1: General constants

\begin{tabular}{|c|c|c|}
\hline Symbol & Description & Value \\
\hline 1 & Cell length & $0.010 \mathrm{~cm}$ \\
\hline $\mathbf{r}$ & Cell radius & $0.0011 \mathrm{~cm}$ \\
\hline $\mathbf{A}_{\text {geo }}$ & $\begin{array}{l}\text { Geometric membrane area }\left(2 \cdot \pi \cdot r^{2}+2 \cdot \pi \cdot r \cdot\right. \\
\text { l) }\end{array}$ & $0.767 \cdot 10^{-4} \mathrm{~cm}^{2}$ \\
\hline $\mathbf{R}_{\mathbf{C G}}$ & Capacitive to geometric ratio & 2 \\
\hline $\mathbf{A}_{\text {cap }}$ & Capacitive membrane area $\left(\mathrm{R}_{\mathrm{CG}} \cdot \mathrm{A}_{\text {geo }}\right)$ & $1.534 \cdot 10^{-4} \mathrm{~cm}^{2}$ \\
\hline $\mathrm{V}_{\text {cell }}$ & Cell volume $\left(\pi \cdot r^{2} \cdot l\right)$ & $3.801 \cdot 10^{-5} \mu \mathrm{L}$ \\
\hline$V_{\text {myo }}$ & Myoplasmic volume $\left(0.68 \cdot V_{\text {cell }}\right)$ & $2.584 \cdot 10^{-5} \mu \mathrm{L}$ \\
\hline $\mathbf{V}_{\text {mito }}$ & Mitochondrial volume $\left(0.238 \cdot V_{\text {cell }}\right)$ & $9.047 \cdot 10^{-6} \mu \mathrm{L}$ \\
\hline $\mathbf{V}_{\text {JSR }}$ & Junctional SR volume $\left(0.0048 \cdot \mathrm{V}_{\text {cell }}\right)$ & $1.820 \cdot 10^{-7} \mu \mathrm{L}$ \\
\hline $\mathbf{V}_{\text {NSR }}$ & Network SR volume $\left(0.0552 \cdot \mathrm{V}_{\text {cell }}\right)$ & $2.098 \cdot 10^{-6} \mu \mathrm{L}$ \\
\hline $\mathbf{V}_{\mathbf{S S}, \mathbf{S R}}$ & SR subspace volume $\left(0.02 \cdot V_{\text {cell }}\right)$ & $7.600 \cdot 10^{-7} \mu \mathrm{L}$ \\
\hline $\mathbf{V}_{\text {SS,CaL }}$ & $\begin{array}{c}\text { L-type } \mathrm{Ca}^{2+} \text { channel subspace volume }(0.002 \cdot \\
\left.\mathrm{V}_{\text {cell }}\right)\end{array}$ & $7.600 \cdot 10^{-8} \mu \mathrm{L}$ \\
\hline
\end{tabular}

Table 2: Geometric properties 


\begin{tabular}{|ccc|}
\hline Symbol & \multicolumn{1}{c|}{ Description } & Value \\
\hline$\left[\mathbf{C a}^{2+}\right]_{\mathbf{0}}$ & Extracellular $\mathrm{Ca}^{2+}$ concentration & $1.800 \mathrm{mmol} / \mathrm{L}$ \\
\hline$\left[\mathbf{C l}^{-}\right]_{\mathbf{o}}$ & Extracellular $\mathrm{Cl}^{-}$concentration & $100.0 \mathrm{mmol} / \mathrm{L}$ \\
\hline$\left[\mathrm{K}^{+}\right]_{\mathbf{o}}$ & Extracellular $\mathrm{K}^{+}$concentration & $5.400 \mathrm{mmol} / \mathrm{L}$ \\
\hline$\left[\mathrm{Na}^{+}\right]_{\mathbf{o}}$ & Extracellular $\mathrm{Na}^{+}$concentration & $140.0 \mathrm{mmol} / \mathrm{L}$ \\
\hline
\end{tabular}

Table 3: Composition of the extracellular solution

The reversal potential for the different currents is determined by their permeability to the ionic species, the (varying) intracellular and (constant) extracellular concentrations:

$$
\begin{aligned}
& \mathrm{E}_{\mathrm{Cl}}=-\frac{\mathrm{R} \cdot \mathrm{T}}{\mathrm{F}} \cdot \log \left(\frac{\left[\mathrm{Cl}^{-}\right]_{\mathrm{o}}}{\left[\mathrm{Cl}^{-}\right]_{\mathrm{i}}}\right) \\
& \mathrm{P}_{\mathrm{Na} / \mathrm{K}}=0.01833 \\
& \mathrm{E}_{\mathrm{Ks}}=\frac{\mathrm{R} \cdot \mathrm{T}}{\mathrm{F}} \cdot \log \left(\frac{\left[\mathrm{K}^{+}\right]_{\mathrm{o}}+\mathrm{P}_{\mathrm{Na} / \mathrm{K}} \cdot\left[\mathrm{Na}^{+}\right]_{\mathrm{o}}}{\left[\mathrm{K}^{+}\right]_{\mathrm{i}}+\mathrm{P}_{\mathrm{Na} / \mathrm{K}} \cdot\left[\mathrm{Na}^{+}\right]_{\mathrm{i}}}\right) \\
& \mathrm{E}_{\mathrm{K}}=\frac{\mathrm{R} \cdot \mathrm{T}}{\mathrm{F}} \cdot \log \left(\frac{\left[\mathrm{K}^{+}\right]_{\mathrm{o}}}{\left[\mathrm{K}^{+}\right]_{\mathrm{i}}}\right) \\
& \mathrm{E}_{\mathrm{Na}}=\frac{\mathrm{R} \cdot \mathrm{T}}{\mathrm{F}} \cdot \log \left(\frac{\left[\mathrm{Na}^{+}\right]_{\mathrm{o}}}{\left[\mathrm{Na}^{+}\right]_{\mathrm{i}}}\right)
\end{aligned}
$$

\begin{tabular}{|c|c|c|c|c|}
\hline \# & Variable & Description & Initial & $\begin{array}{l}\text { Applies } \\
\text { to }\end{array}$ \\
\hline 1 & $V_{\mathrm{m}}$ & Membrane potential (mV) & $-8.7491 \mathrm{E} 01$ & Ch. $1-6$ \\
\hline 2 & {$\left[\mathrm{Ca}^{2+}\right]_{\mathrm{i}, \text { tot }}$} & $\mathrm{Ca}^{2+}$ concentration in myoplasm $(\mathrm{mmol} / \mathrm{L})$ & 1.3394E-02 & \\
\hline 3 & {$\left[\mathrm{Ca}^{2+}\right]_{\mathrm{SS}}$} & $\mathrm{Ca}^{2+}$ concentration in restricted subspace $(\mathrm{mmol} / \mathrm{L})$ & $2.3413 \mathrm{E}-02$ & \\
\hline 4 & {$\left[\mathrm{Ca}^{2+}\right]_{\mathrm{SS}, \mathrm{CaL}}$} & $\begin{array}{l}\mathrm{Ca}^{2+} \text { concentration in subspace surrounding L-type } \\
\mathrm{Ca}^{2+} \text { channel (mmol/L) }\end{array}$ & $2.3413 \mathrm{E}-02$ & \\
\hline 5 & {$\left[\mathrm{Ca}^{2+}\right]_{\mathrm{JSR}}$} & $\begin{array}{l}\mathrm{Ca}^{2+} \text { concentration in junctional sarcoplasmic } \\
\text { reticulum }(\mathrm{mmol} / \mathrm{L})\end{array}$ & $6.8659 \mathrm{E}+00$ & \\
\hline 6 & {$\left[\mathrm{Ca}^{2+}\right]_{\mathrm{NSR}}$} & $\begin{array}{l}\mathrm{Ca}^{2+} \text { concentration in network sarcoplasmic reticulum } \\
(\mathrm{mmol} / \mathrm{L})\end{array}$ & $1.1910 \mathrm{E}+00$ & \\
\hline 7 & $\mathrm{CaMK}_{\text {trap }}$ & Fraction of CaMKII that has trapped calmodulin & $1.7546 \mathrm{E}-03$ & \\
\hline 8 & {$\left[\mathrm{Na}^{+}\right]_{\mathrm{i}}$} & $\mathrm{Na}^{+}$concentration in myoplasm $(\mathrm{mmol} / \mathrm{L})$ & $6.8909 E+00$ & \\
\hline 9 & {$\left[\mathrm{Na}^{+}\right]_{\mathrm{SS}}$} & $\mathrm{Na}^{+}$concentration in restricted subspace $(\mathrm{mmol} / \mathrm{L})$ & $6.8909 E+00$ & \\
\hline & {$\left[\mathrm{K}^{+}\right]_{\mathrm{i}}$} & $\mathrm{K}^{+}$concentration in myoplasm ( $\left.\mathrm{mmol} / \mathrm{L}\right)$ & $1.4562 \mathrm{E}+02$ & \\
\hline
\end{tabular}

\subsection{Initial state vector}




\begin{tabular}{|c|c|c|c|}
\hline 11 & {$\left[\mathrm{Cl}^{-}\right]_{\mathrm{i}}$} & $\mathrm{Cl}^{-}$concentration in myoplasm (mmol/L) & $2.0273 E+01$ \\
\hline 12 & {$\left[\mathrm{Cl}^{-}\right]_{\mathrm{SS}}$} & $\mathrm{Cl}^{-}$concentration in restricted subspace $(\mathrm{mmol} / \mathrm{L})$ & $2.0273 E+01$ \\
\hline 13 & $\mathrm{I}_{\mathrm{Rel}}^{\mathrm{NP}}$ & $\begin{array}{l}\mathrm{Ca}^{2+} \text { release flux from JSR through non- } \\
\text { phosphorylated ryanodine receptors }\end{array}$ & $3.6675 \mathrm{E}-09$ \\
\hline 14 & $\mathrm{~m}^{\mathrm{NP}}$ & $I_{\mathrm{Na}}$ activation gate for non-phosphorylated channels & $6.8172 \mathrm{E}-03$ \\
\hline 15 & $h^{N P}$ & $\begin{array}{l}I_{\mathrm{Na}} \text { fast inactivation gate for non-phosphorylated } \\
\text { channels }\end{array}$ & $9.0163 \mathrm{E}-01$ \\
\hline 16 & $\mathrm{j}^{\mathrm{NP}}$ & $\begin{array}{l}I_{\mathrm{Na}} \text { slow inactivation gate for non-phosphorylated } \\
\text { channels }\end{array}$ & $9.9709 E-01$ \\
\hline 17 & $\mathrm{~m}_{\mathrm{L}}$ & $\mathrm{I}_{\mathrm{Na}, \mathrm{L}}$ activation gate & $7.0530 \mathrm{E}-04$ \\
\hline 18 & $\mathrm{~h}_{\mathrm{L}}$ & $I_{\mathrm{Na}, \mathrm{L}}$ inactivation gate & $3.6003 \mathrm{E}-01$ \\
\hline 19 & $\mathrm{I}_{\mathrm{To}, \mathrm{a}}$ & $\begin{array}{l}\text { Activation gate of the 4-AP sensitive transient } \\
\text { outward } \mathrm{K}^{+} \text {current }\end{array}$ & $1.7687 \mathrm{E}-05$ \\
\hline 20 & $\mathrm{I}_{\mathrm{To}, \mathrm{if}}$ & $\begin{array}{l}\text { Fast inactivation gate of the 4-AP sensitive transient } \\
\text { outward } \mathrm{K}^{+} \text {current }\end{array}$ & $9.9798 \mathrm{E}-01$ \\
\hline 21 & $\mathrm{I}_{\mathrm{To}, \text { is }}$ & $\begin{array}{l}\text { Slow inactivation gate of the 4-AP sensitive transient } \\
\text { outward } \mathrm{K}^{+} \text {current }\end{array}$ & $9.8747 \mathrm{E}-01$ \\
\hline 22 & $\mathrm{X}_{\mathrm{r}}$ & $\mathrm{I}_{\mathrm{Kr}}$ activation gate & $1.2306 \mathrm{E}-08$ \\
\hline 23 & $\mathrm{i}_{2}$ & $\mathrm{I}_{\mathrm{T}, 2}$ inactivation gate & $9.9604 \mathrm{E}-01$ \\
\hline 24 & $\mathrm{C}_{\mathrm{CaL}}^{\mathrm{NP}}$ & $\begin{array}{l}\text { Fraction of NP I }{ }_{\text {CaL }} \text { channels in closed, non inactivated, } \\
\text { non } \mathrm{Ca}^{2+} \text { gated mode }\end{array}$ & 1.0000 \\
\hline 25 & $\mathrm{O}_{\mathrm{CaL}}^{\mathrm{NP}}$ & $\begin{array}{l}\text { Fraction of } \mathrm{NP} \mathrm{I}_{\mathrm{CaL}} \text { channels in open, non inactivated, } \\
\text { non } \mathrm{Ca}^{2+} \text { gated mode }\end{array}$ & 0.0000 \\
\hline 26 & $\mathrm{Cs}_{\mathrm{CaL}}^{\mathrm{NP}}$ & $\begin{array}{l}\text { Fraction of NP I }{ }_{\text {CaL }} \text { channels in closed, non inactivated, } \\
\mathrm{Ca}^{2+} \text { gated mode }\end{array}$ & 0.0000 \\
\hline 27 & $\mathrm{Os}_{\mathrm{CaL}}^{\mathrm{NP}}$ & $\begin{array}{l}\text { Fraction of NP I } \mathrm{CaL}_{\text {al }} \text { channels in open, non inactivated, } \\
\mathrm{Ca}^{2+} \text { gated mode }\end{array}$ & 0.0000 \\
\hline 28 & $\mathrm{CI}_{\mathrm{CaL}}^{\mathrm{NP}}$ & $\begin{array}{l}\text { Fraction of NP I }{ }_{\text {CaL }} \text { channels in closed, inactivated, non } \\
\mathrm{Ca}^{2+} \text { gated mode }\end{array}$ & 0.0000 \\
\hline 29 & $\mathrm{OI}_{\mathrm{CaL}}^{\mathrm{NP}}$ & $\begin{array}{l}\text { Fraction of NP I }{ }_{\text {CaL }} \text { channels in open, inactivated, non } \\
\mathrm{Ca}^{2+} \text { gated mode }\end{array}$ & 0.0000 \\
\hline 30 & $\mathrm{CIs}_{\mathrm{CaL}}^{\mathrm{NP}}$ & $\begin{array}{l}\text { Fraction of NP } \mathrm{I}_{\mathrm{CaL}} \text { channels in closed, inactivated, } \mathrm{Ca}^{2+} \\
\text { gated mode }\end{array}$ & 0.0000 \\
\hline 31 & $\mathrm{OIs}_{\mathrm{CaL}}^{\mathrm{NP}}$ & $\begin{array}{l}\text { Fraction of NP } \mathrm{I}_{\mathrm{CaL}} \text { channels in open, inactivated, } \mathrm{Ca}^{2+} \\
\text { gated mode }\end{array}$ & 0.0000 \\
\hline 32 & $\mathrm{C} 1_{\mathrm{Ks}}^{\mathrm{NP}}$ & $\begin{array}{l}\text { Fraction of non-phosphorylated } I_{\mathrm{Ks}} \text { channels in closed } \\
\text { state } 1\end{array}$ & $9.1141 \mathrm{E}-01$ \\
\hline 33 & $\mathrm{C} 2_{\mathrm{Ks}}^{\mathrm{NP}}$ & $\begin{array}{l}\text { Fraction of non-phosphorylated } I_{\mathrm{Ks}} \text { channels in closed } \\
\text { state } 2\end{array}$ & $8.4395 \mathrm{E}-02$ \\
\hline 34 & $\mathrm{C} 3 \mathrm{Ns}_{\mathrm{Ks}}^{\mathrm{NP}}$ & $\begin{array}{l}\text { Fraction of non-phosphorylated } I_{\mathrm{Ks}} \text { channels in closed } \\
\text { state } 3\end{array}$ & $2.9306 \mathrm{E}-03$ \\
\hline 35 & $\mathrm{C} 4 \mathrm{Ks}$ & $\begin{array}{l}\text { Fraction of non-phosphorylated } I_{\mathrm{Ks}} \text { channels in closed } \\
\text { state } 4\end{array}$ & $4.5228 \mathrm{E}-05$ \\
\hline 36 & $\mathrm{C}_{\mathrm{Ks}}^{\mathrm{NP}}$ & $\begin{array}{l}\text { Fraction of non-phosphorylated } \mathrm{I}_{\mathrm{Ks}} \text { channels in closed } \\
\text { state } 5\end{array}$ & $2.6175 \mathrm{E}-07$ \\
\hline
\end{tabular}




\begin{tabular}{|c|c|c|c|}
\hline 37 & $\mathrm{C} 6_{\mathrm{Ks}}^{\mathrm{NP}}$ & $\begin{array}{l}\text { Fraction of non-phosphorylated } I_{\mathrm{Ks}} \text { channels in closed } \\
\text { state } 6\end{array}$ & $1.1424 \mathrm{E}-03$ \\
\hline 38 & $\mathrm{C} 7_{\mathrm{Ks}}^{\mathrm{NP}}$ & $\begin{array}{l}\text { Fraction of non-phosphorylated } \mathrm{I}_{\mathrm{Ks}} \text { channels in closed } \\
\text { state } 7\end{array}$ & 7.9337E-05 \\
\hline 39 & $\mathrm{C} 8 \mathrm{NP}$ & $\begin{array}{l}\text { Fraction of non-phosphorylated } I_{\mathrm{Ks}} \text { channels in closed } \\
\text { state } 8\end{array}$ & $1.8366 \mathrm{E}-06$ \\
\hline 40 & $\mathrm{C} 9_{\mathrm{Ks}}^{\mathrm{NP}}$ & $\begin{array}{l}\text { Fraction of non-phosphorylated } \mathrm{I}_{\mathrm{Ks}} \text { channels in closed } \\
\text { state } 9\end{array}$ & $1.4172 \mathrm{E}-08$ \\
\hline 41 & $\mathrm{C} 10_{\mathrm{Ks}}^{\mathrm{NP}}$ & $\begin{array}{l}\text { Fraction of non-phosphorylated } I_{\mathrm{Ks}} \text { channels in closed } \\
\text { state } 10\end{array}$ & $5.3696 \mathrm{E}-07$ \\
\hline 42 & $\mathrm{C} 11_{\mathrm{Ks}}^{\mathrm{NP}}$ & $\begin{array}{l}\text { Fraction of non-phosphorylated } \mathrm{I}_{\mathrm{Ks}} \text { channels in closed } \\
\text { state } 11\end{array}$ & $2.4861 \mathrm{E}-08$ \\
\hline 43 & $\mathrm{C} 12_{\mathrm{Ks}}^{\mathrm{NP}}$ & $\begin{array}{l}\text { Fraction of non-phosphorylated } I_{\mathrm{Ks}} \text { channels in closed } \\
\text { state } 12\end{array}$ & $2.8776 \mathrm{E}-10$ \\
\hline 44 & $\mathrm{C} 13_{\mathrm{Ks}}^{\mathrm{NP}}$ & $\begin{array}{l}\text { Fraction of non-phosphorylated } \mathrm{I}_{\mathrm{Ks}} \text { channels in closed } \\
\text { state } 13\end{array}$ & $1.1217 \mathrm{E}-10$ \\
\hline 45 & $\mathrm{C} 14_{\mathrm{Ks}}^{\mathrm{NP}}$ & $\begin{array}{l}\text { Fraction of non-phosphorylated } \mathrm{I}_{\mathrm{Ks}} \text { channels in closed } \\
\text { state } 14\end{array}$ & $2.5967 \mathrm{E}-12$ \\
\hline 46 & $\mathrm{C} 15_{\mathrm{Ks}}^{\mathrm{NP}}$ & $\begin{array}{l}\text { Fraction of non-phosphorylated } \mathrm{I}_{\mathrm{Ks}} \text { channels in closed } \\
\text { state } 15\end{array}$ & $8.7874 \mathrm{E}-15$ \\
\hline 47 & $01_{\mathrm{Ks}}^{\mathrm{NP}}$ & $\begin{array}{l}\text { Fraction of non-phosphorylated } \mathrm{I}_{\mathrm{Ks}} \text { channels in open } \\
\text { state } 1\end{array}$ & $9.3722 \mathrm{E}-16$ \\
\hline 48 & $\mathrm{O} 2_{\mathrm{Ks}}^{\mathrm{NP}}$ & $\begin{array}{l}\text { Fraction of non-phosphorylated } \mathrm{I}_{\mathrm{Ks}} \text { channels in open } \\
\text { state } 2\end{array}$ & $1.6595 \mathrm{E}-17$ \\
\hline 49 & $\mathrm{C}_{\mathrm{CaL}}^{\mathrm{P}}$ & $\begin{array}{l}\text { Fraction of } \mathrm{P} \mathrm{I}_{\mathrm{CaL}} \text { channels in closed, non inactivated, } \\
\text { non } \mathrm{Ca}^{2+} \text { gated mode }\end{array}$ & 1.0000 \\
\hline 50 & $\mathrm{O}_{\mathrm{CaL}}^{\mathrm{P}}$ & $\begin{array}{l}\text { Fraction of } \mathrm{P} \mathrm{I}_{\mathrm{CaL}} \text { channels in open, non inactivated, } \\
\text { non } \mathrm{Ca}^{2+} \text { gated mode }\end{array}$ & 0.0000 \\
\hline 51 & $\mathrm{Cs}_{\mathrm{CaL}}^{\mathrm{P}}$ & $\begin{array}{l}\text { Fraction of } \mathrm{P} \mathrm{I}_{\mathrm{CaL}} \text { channels in closed, non inactivated, } \\
\mathrm{Ca}^{2+} \text { gated mode }\end{array}$ & 0.0000 \\
\hline 52 & $\mathrm{Os}_{\mathrm{CaL}}^{\mathrm{P}}$ & $\begin{array}{l}\text { Fraction of } \mathrm{P} \mathrm{I}_{\mathrm{CaL}} \text { channels in open, non inactivated, } \\
\mathrm{Ca}^{2+} \text { gated mode }\end{array}$ & 0.0000 \\
\hline 53 & $\mathrm{CI}_{\mathrm{CaL}}^{\mathrm{P}}$ & $\begin{array}{l}\text { Fraction of } \mathrm{P} \mathrm{I}_{\mathrm{CaL}} \text { channels in closed, inactivated, non } \\
\mathrm{Ca}^{2+} \text { gated mode }\end{array}$ & 0.0000 \\
\hline 54 & $\mathrm{OI}_{\mathrm{CaL}}^{\mathrm{P}}$ & $\begin{array}{l}\text { Fraction of } \mathrm{P} \mathrm{I}_{\mathrm{CaL}} \text { channels in open, inactivated, non } \\
\mathrm{Ca}^{2+} \text { gated mode }\end{array}$ & 0.0000 \\
\hline 55 & $\mathrm{CIs}_{\mathrm{CaL}}^{\mathrm{P}}$ & $\begin{array}{l}\text { Fraction of } \mathrm{P} \mathrm{I}_{\mathrm{CaL}} \text { channels in closed, inactivated, } \mathrm{Ca}^{2+} \\
\text { gated mode }\end{array}$ & 0.0000 \\
\hline 56 & $\mathrm{OIs}_{\mathrm{CaL}}^{\mathrm{P}}$ & $\begin{array}{l}\text { Fraction of } \mathrm{P} \mathrm{I}_{\mathrm{CaL}} \text { channels in open, inactivated, } \mathrm{Ca}^{2+} \\
\text { gated mode }\end{array}$ & 0.0000 \\
\hline 57 & $\mathrm{C} 1_{\mathrm{Ks}}^{\mathrm{P}}$ & $\begin{array}{l}\text { Fraction of phosphorylated } I_{\mathrm{ks}} \text { channels in closed state } \\
1\end{array}$ & $9.5624 \mathrm{E}-01$ \\
\hline 58 & $\mathrm{C} 2 \mathrm{Ks}_{\mathrm{Ks}}^{\mathrm{P}}$ & $\begin{array}{l}\text { Fraction of phosphorylated } I_{\mathrm{Ks}} \text { channels in closed state } \\
2\end{array}$ & $4.2127 \mathrm{E}-02$ \\
\hline 59 & $\mathrm{C} 3_{\mathrm{Ks}}^{\mathrm{P}}$ & $\begin{array}{l}\text { Fraction of phosphorylated } I_{\mathrm{ks}} \text { channels in closed state } \\
3\end{array}$ & $6.9597 \mathrm{E}-04$ \\
\hline 60 & $\mathrm{C} 4_{\mathrm{Ks}}^{\mathrm{P}}$ & $\begin{array}{l}\text { Fraction of phosphorylated } I_{\mathrm{Ks}} \text { channels in closed state } \\
4\end{array}$ & $5.1101 \mathrm{E}-06$ \\
\hline 61 & $\mathrm{C} 5_{\mathrm{Ks}}^{\mathrm{P}}$ & $\begin{array}{l}\text { Fraction of phosphorylated } I_{\mathrm{Ks}} \text { channels in closed state } \\
5\end{array}$ & $1.4070 \mathrm{E}-08$ \\
\hline
\end{tabular}




\begin{tabular}{|c|c|c|c|}
\hline 62 & $\mathrm{C} 6_{\mathrm{Ks}}^{\mathrm{P}}$ & $\begin{array}{l}\text { Fraction of phosphorylated } I_{\mathrm{ks}} \text { channels in closed state } \\
6\end{array}$ & $9.0269 \mathrm{E}-04$ \\
\hline 63 & $\mathrm{C} 7_{\mathrm{Ks}}^{\mathrm{P}}$ & $\begin{array}{l}\text { Fraction of phosphorylated } \mathrm{I}_{\mathrm{ks}} \text { channels in closed state } \\
7\end{array}$ & $2.9826 \mathrm{E}-05$ \\
\hline 64 & C8 $8_{\mathrm{Ks}}^{\mathrm{NP}}$ & $\begin{array}{l}\text { Fraction of phosphorylated } I_{\mathrm{Ks}} \text { channels in closed state } \\
8\end{array}$ & $3.2850 \mathrm{E}-07$ \\
\hline 65 & $\mathrm{C} 9 \mathrm{Ks}_{\mathrm{Ks}}^{\mathrm{P}}$ & $\begin{array}{l}\text { Fraction of phosphorylated } I_{\mathrm{Ks}} \text { channels in closed state } \\
9\end{array}$ & $1.2060 \mathrm{E}-09$ \\
\hline 66 & $\mathrm{C} 10_{\mathrm{Ks}}^{\mathrm{P}}$ & $\begin{array}{l}\text { Fraction of phosphorylated } I_{\mathrm{Ks}} \text { channels in closed state } \\
10\end{array}$ & $3.1956 \mathrm{E}-07$ \\
\hline 67 & $\mathrm{C} 11_{\mathrm{Ks}}^{\mathrm{P}}$ & $\begin{array}{l}\text { Fraction of phosphorylated } I_{\mathrm{ks}} \text { channels in closed state } \\
11\end{array}$ & 7.0390E-09 \\
\hline 68 & $\mathrm{C} 12_{\mathrm{Ks}}^{\mathrm{P}}$ & $\begin{array}{l}\text { Fraction of phosphorylated } I_{\mathrm{Ks}} \text { channels in closed state } \\
12\end{array}$ & $3.8763 \mathrm{E}-11$ \\
\hline 69 & $\mathrm{C} 13_{\mathrm{Ks}}^{\mathrm{P}}$ & $\begin{array}{l}\text { Fraction of phosphorylated } I_{\mathrm{Ks}} \text { channels in closed state } \\
13\end{array}$ & 5.0277E-11 \\
\hline 70 & $\mathrm{C} 14_{\mathrm{Ks}}^{\mathrm{P}}$ & $\begin{array}{l}\text { Fraction of phosphorylated } I_{\mathrm{Ks}} \text { channels in closed state } \\
14\end{array}$ & $5.5374 \mathrm{E}-13$ \\
\hline 71 & $\mathrm{C} 15_{\mathrm{Ks}}^{\mathrm{P}}$ & $\begin{array}{l}\text { Fraction of phosphorylated } I_{\mathrm{Ks}} \text { channels in closed state } \\
15\end{array}$ & $2.9664 \mathrm{E}-15$ \\
\hline 72 & $01_{\mathrm{Ks}}^{\mathrm{P}}$ & Fraction of phosphorylated $I_{\mathrm{Ks}}$ channels in open state 1 & $1.1201 \mathrm{E}-15$ \\
\hline 73 & $02_{\mathrm{Ks}}^{\mathrm{P}}$ & Fraction of phosphorylated $I_{\mathrm{Ks}}$ channels in open state 2 & $1.6613 \mathrm{E}-18$ \\
\hline 74 & $\mathrm{~m}^{\mathrm{P}, \mathrm{PKA}}$ & $I_{\mathrm{Na}}$ activation gate for PKA phosphorylated channels & $1.2360 \mathrm{E}-03$ \\
\hline 75 & $h^{\mathrm{P}, \mathrm{PKA}}$ & $\begin{array}{l}I_{\mathrm{Na}} \text { fast inactivation gate for PKA phosphorylated } \\
\text { channels }\end{array}$ & $7.9472 \mathrm{E}-01$ \\
\hline 76 & jP,PKA & $\begin{array}{l}I_{\text {Na }} \text { slow inactivation gate for PKA phosphorylated } \\
\text { channels }\end{array}$ & $9.9123 \mathrm{E}-01$ \\
\hline 77 & $\mathrm{~m}^{\text {P,CaMKII }}$ & $I_{\mathrm{Na}}$ activation gate for CaMKII phosphorylated channels & $6.8127 \mathrm{E}-04$ \\
\hline 78 & $\mathrm{~h}^{\mathrm{P}, \mathrm{CaMKII}}$ & $\begin{array}{l}\mathrm{I}_{\mathrm{Na}} \text { fast inactivation gate for CaMKII phosphorylated } \\
\text { channels }\end{array}$ & $8.3805 \mathrm{E}-01$ \\
\hline 79 & jP,CaMKII & $\begin{array}{l}I_{\mathrm{Na}} \text { slow inactivation gate for CaMKII phosphorylated } \\
\text { channels }\end{array}$ & $9.9281 \mathrm{E}-01$ \\
\hline 80 & $\mathrm{I}_{\mathrm{Rel}}^{\mathrm{P}}$ & $\begin{array}{l}\mathrm{Ca}^{2+} \text { release flux from JSR through phosphorylated } \\
\text { ryanodine receptors }\end{array}$ & 7.3074E-09 \\
\hline 81 & $\mathrm{I}_{\mathrm{To}, \mathrm{if}}^{\mathrm{p}, \mathrm{CaMKII}}$ & $\begin{array}{l}\text { Fast inactivation gate of the 4-AP sensitive transient } \\
\text { outward } \mathrm{K}^{+} \text {current for CaMKII phosphorylated } \\
\text { channels }\end{array}$ & $9.9798 \mathrm{E}-01$ \\
\hline 82 & $\mathrm{I}_{\mathrm{To}, \mathrm{is}}^{\mathrm{P}, \mathrm{CaMKII}}$ & $\begin{array}{l}\text { Slow inactivation gate of the 4-AP sensitive transient } \\
\text { outward } \mathrm{K}^{+} \text {current for CaMKII phosphorylated } \\
\text { channels }\end{array}$ & $9.8747 \mathrm{E}-01$ \\
\hline
\end{tabular}

\subsection{Intracellular concentrations: $\mathrm{Ca}^{2+}$}

\subsubsection{HRd2009 version (Chapter 2)}

$\frac{\mathrm{d}\left[\mathrm{Ca}^{2+}\right]_{\mathrm{i}, \text { tot }}}{\mathrm{dt}}=-\left(\frac{\left(\mathrm{I}_{\mathrm{Ca}, \mathrm{b}}+\mathrm{I}_{\mathrm{pCa}}-2 \cdot \mathrm{I}_{\mathrm{NaCa}, \mathrm{i}}\right) \cdot \mathrm{A}_{\mathrm{Cap}} \cdot \mathrm{C}_{\mathrm{m}}}{\mathrm{z}_{\mathrm{Ca}} \cdot \mathrm{F} \cdot \mathrm{V}_{\mathrm{myo}}}+\mathrm{I}_{\text {up }} \cdot \frac{\mathrm{V}_{\mathrm{NSR}}}{\mathrm{V}_{\mathrm{myo}}}-\mathrm{I}_{\text {Diff,Ca }} \cdot \frac{\mathrm{V}_{\mathrm{SS}, \mathrm{SR}}}{\mathrm{V}_{\mathrm{myo}}}\right)$ 
Buff $\mathrm{Ca}, \mathrm{i}^{\mathrm{b}}=[\overline{\mathrm{cmdn}}]+[\overline{\operatorname{trpn}}]-\left[\mathrm{Ca}^{2+}\right]_{\mathrm{i}, \mathrm{tot}}+\mathrm{K}_{\mathrm{m}, \mathrm{trpn}}+\mathrm{K}_{\mathrm{m}, \mathrm{cmdn}}$
$\mathrm{Buff}_{\mathrm{Ca}, \mathrm{i}}^{\mathrm{c}}=\mathrm{K}_{\mathrm{m}, \mathrm{trpn}} \cdot \mathrm{K}_{\mathrm{m}, \mathrm{cmdn}}-\left[\mathrm{Ca}^{2+}\right]_{\mathrm{i}, \mathrm{tot}} \cdot\left(\mathrm{K}_{\mathrm{m}, \mathrm{trpn}}+\mathrm{K}_{\mathrm{m}, \mathrm{cmdn}}\right)+[\overline{\mathrm{cmdn}}] \cdot \mathrm{K}_{\mathrm{m}, \mathrm{trpn}}+[\overline{\operatorname{trpn}}]$

$\cdot \mathrm{K}_{\mathrm{m}, \mathrm{cmdn}}$

Buff $_{\mathrm{Ca}, \mathrm{i}}^{\mathrm{d}}=-\mathrm{K}_{\mathrm{m}, \mathrm{trpn}} \cdot \mathrm{K}_{\mathrm{m}, \mathrm{cmdn}} \cdot\left[\mathrm{Ca}^{2+}\right]_{\mathrm{i}, \mathrm{tot}}$

$\left[\mathrm{Ca}^{2+}\right]_{\mathrm{i}}=-\frac{\mathrm{Buff}_{\mathrm{Ca}, \mathrm{i}}^{\mathrm{b}}}{3}+\frac{2}{3} \cdot \sqrt{\left(\mathrm{Buff}_{\mathrm{Ca}, \mathrm{i}}^{\mathrm{b}}\right)^{2}-3 \cdot \mathrm{Buff}_{\mathrm{Ca}, \mathrm{i}}^{\mathrm{c}}}$

$\cdot \cos \left(\frac{1}{3} \cdot \cos ^{-1}\left(\frac{9 \cdot \mathrm{Buff}_{\mathrm{Ca}, \mathrm{i}}^{\mathrm{b}} \cdot \mathrm{Buff}_{\mathrm{Ca}, \mathrm{i}}^{\mathrm{c}}-2 \cdot\left(\mathrm{Buff}_{\mathrm{Ca}, \mathrm{i}}^{\mathrm{b}}\right)^{3}-27 \cdot \mathrm{Buff}_{\mathrm{Ca}, \mathrm{i}}^{\mathrm{d}}}{2 \cdot\left(\left(\mathrm{Buff}_{\mathrm{Ca}, \mathrm{i}}^{\mathrm{b}}\right)^{2}-3 \cdot \mathrm{Buff}_{\mathrm{Ca}, \mathrm{i}}^{\mathrm{c}}\right)^{1.5}}\right)\right)$

$\frac{\mathrm{d}\left[\mathrm{Ca}^{2+}\right]_{\mathrm{Ss}, \mathrm{tot}}}{\mathrm{dt}}=\frac{2 \cdot \mathrm{I}_{\mathrm{NaCa}, \mathrm{ss}} \cdot \mathrm{A}_{\mathrm{Cap}} \cdot \mathrm{C}_{\mathrm{m}}}{\mathrm{z}_{\mathrm{Ca}} \cdot \mathrm{F} \cdot \mathrm{V}_{\mathrm{SS}, \mathrm{SR}}}+\mathrm{I}_{\mathrm{Rel}} \cdot \frac{\mathrm{V}_{\mathrm{JSR}}}{\mathrm{V}_{\mathrm{SS}, \mathrm{SR}}}-\mathrm{I}_{\text {Diff,Ca }}-\mathrm{I}_{\text {Diff,SS }}$

$\mathrm{Buff}_{\mathrm{Ca}, \mathrm{ss}}^{\mathrm{b}}=[\overline{\mathrm{BSL}}]+[\overline{\mathrm{BSR}}]-\left[\mathrm{Ca}^{2+}\right]_{\mathrm{ss}, \text { tot }}+\mathrm{K}_{\mathrm{m}, \mathrm{BSL}}+\mathrm{K}_{\mathrm{m}, \mathrm{BSR}}$

Buff $_{\mathrm{Ca}, \mathrm{ss}}^{\mathrm{c}}=\mathrm{K}_{\mathrm{m}, \mathrm{BSL}} \cdot \mathrm{K}_{\mathrm{m}, \mathrm{BSR}}-\left[\mathrm{Ca}^{2+}\right]_{\mathrm{ss}, \mathrm{tot}} \cdot\left(\mathrm{K}_{\mathrm{m}, \mathrm{BSL}}+\mathrm{K}_{\mathrm{m}, \mathrm{BSR}}\right)+[\overline{\mathrm{BSL}}] \cdot \mathrm{K}_{\mathrm{m}, \mathrm{BSR}}+[\overline{\mathrm{BSR}}] \cdot \mathrm{K}_{\mathrm{m}, \mathrm{BSL}}$

Buff $_{\mathrm{Ca}, \mathrm{ss}}^{\mathrm{d}}=-\mathrm{K}_{\mathrm{m}, \mathrm{BSL}} \cdot \mathrm{K}_{\mathrm{m}, \mathrm{BSR}} \cdot\left[\mathrm{Ca}^{2+}\right]_{\mathrm{ss}, \mathrm{tot}}$

$\left[\mathrm{Ca}^{2+}\right]_{\mathrm{ss}}=-\frac{\mathrm{Buff}_{\mathrm{Ca}, \mathrm{ss}}^{\mathrm{b}}}{3}+\frac{2}{3} \cdot \sqrt{\left(\mathrm{Buff}_{\mathrm{Ca}, \mathrm{ss}}^{\mathrm{b}}\right)^{2}-3 \cdot \mathrm{Buff}_{\mathrm{Ca}, \mathrm{ss}}^{\mathrm{c}}}$

$\cdot \cos \left(\frac{1}{3} \cdot \cos ^{-1}\left(\frac{9 \cdot \mathrm{Buff}_{\mathrm{Ca}, \mathrm{ss}}^{\mathrm{b}} \cdot \mathrm{Buff}_{\mathrm{Ca}, \mathrm{ss}}^{\mathrm{c}}-2 \cdot\left(\mathrm{Buff}_{\mathrm{Ca}, \mathrm{ss}}^{\mathrm{b}}\right)^{3}-27 \cdot \mathrm{Buff}_{\mathrm{Ca}, \mathrm{ss}}^{\mathrm{d}}}{2 \cdot\left(\left(\mathrm{Buff}_{\mathrm{Ca}, \mathrm{ss}}^{\mathrm{b}}\right)^{2}-3 \cdot \mathrm{Buff}_{\mathrm{Ca}, \mathrm{ss}}^{\mathrm{c}}\right)^{1.5}}\right)\right)$

$\frac{\mathrm{d}\left[\mathrm{Ca}^{2+}\right]_{\mathrm{SSCaL}, \text { tot }}}{\mathrm{dt}}=\frac{-\mathrm{I}_{\mathrm{CaL}} \cdot \mathrm{A}_{\mathrm{Cap}} \cdot \mathrm{C}_{\mathrm{m}}}{\mathrm{z}_{\mathrm{Ca}} \cdot \mathrm{F} \cdot \mathrm{V}_{\mathrm{SS}, \mathrm{CaL}}}+\mathrm{I}_{\mathrm{Diff}, \mathrm{SS}} \cdot \frac{\mathrm{V}_{\mathrm{SS}, \mathrm{SR}}}{\mathrm{V}_{\mathrm{SS}, \mathrm{CaL}}}$

Buff $\mathrm{Ca,ssCaL}=[\overline{\mathrm{BSL}}]+[\overline{\mathrm{BSR}}]-\left[\mathrm{Ca}^{2+}\right]_{\mathrm{ssCaL}, \mathrm{tot}}+\mathrm{K}_{\mathrm{m}, \mathrm{BSL}}+\mathrm{K}_{\mathrm{m}, \mathrm{BSR}}$

Buff $_{\mathrm{Ca}, \mathrm{sSCaL}}^{\mathrm{c}}=\mathrm{K}_{\mathrm{m}, \mathrm{BSL}} \cdot \mathrm{K}_{\mathrm{m}, \mathrm{BSR}}-\left[\mathrm{Ca}^{2+}\right]_{\mathrm{ssCaL}, \mathrm{tot}} \cdot\left(\mathrm{K}_{\mathrm{m}, \mathrm{BSL}}+\mathrm{K}_{\mathrm{m}, \mathrm{BSR}}\right)+[\overline{\mathrm{BSL}}] \cdot \mathrm{K}_{\mathrm{m}, \mathrm{BSR}}+[\overline{\mathrm{BSR}}]$

$\cdot \mathrm{K}_{\mathrm{m}, \mathrm{BSL}}$

Buff $_{\mathrm{Ca}, \text { ssCaL }}^{\mathrm{d}}=-\mathrm{K}_{\mathrm{m}, \mathrm{BSL}} \cdot \mathrm{K}_{\mathrm{m}, \mathrm{BSR}} \cdot\left[\mathrm{Ca}^{2+}\right]_{\mathrm{ssCaL}, \text { tot }}$

$\left[\mathrm{Ca}^{2+}\right]_{\mathrm{ssCaL}}=-\frac{\mathrm{Buff}_{\mathrm{Ca}, \mathrm{ssCaL}}^{\mathrm{b}}}{3}+\frac{2}{3} \cdot \sqrt{\left(\mathrm{Buff}_{\mathrm{Ca}, \mathrm{ssCaL}}^{\mathrm{b}}\right)^{2}-3 \cdot \mathrm{Buff}_{\mathrm{Ca}, \mathrm{ssCaL}}^{\mathrm{c}}}$

$\cdot \cos \left(\frac{1}{3}\right.$

$\left.\cdot \cos ^{-1}\left(\frac{9 \cdot \mathrm{Buff}_{\mathrm{Ca}, \mathrm{ssCaL}}^{\mathrm{b}} \cdot \mathrm{Buff}_{\mathrm{Ca}, \mathrm{ssCaL}}^{\mathrm{c}}-2 \cdot\left(\mathrm{Buff}_{\mathrm{Ca}, \mathrm{ssCaL}}^{\mathrm{b}}\right)^{3}-27 \cdot \mathrm{Buff}_{\mathrm{Ca}, \mathrm{ssCaL}}^{\mathrm{d}}}{2 \cdot\left(\left(\mathrm{Buff}_{\mathrm{Ca}, \mathrm{ssCaL}}^{\mathrm{b}}\right)^{2}-3 \cdot \mathrm{Buff}_{\mathrm{Ca}, \mathrm{ssCaL}}^{\mathrm{c}}\right)^{1.5}}\right)\right)$ 


$$
\begin{aligned}
& \frac{\mathrm{d}\left[\mathrm{Ca}^{2+}\right]_{\text {JSR,tot }}}{\mathrm{dt}}=\mathrm{I}_{\mathrm{tr}}-\mathrm{I}_{\mathrm{Rel}} \\
& \mathrm{Buff}_{\mathrm{Ca}, \mathrm{SR}}^{\mathrm{b}}=[\overline{\mathrm{CSQN}}]-\left[\mathrm{Ca}^{2+}\right]_{\mathrm{JSR}, \text { tot }}+\mathrm{K}_{\mathrm{m}, \mathrm{CSQN}} \\
& \mathrm{Buff}_{\mathrm{Ca}, \mathrm{SR}}^{\mathrm{c}}=\mathrm{K}_{\mathrm{m}, \mathrm{CSQN}} \cdot\left[\mathrm{Ca}^{2+}\right]_{\mathrm{JSR}, \text { tot }} \\
& {\left[\mathrm{Ca}^{2+}\right]_{\mathrm{JSR}}=-\frac{\mathrm{Buff}_{\mathrm{Ca}, \mathrm{SSR}}^{\mathrm{b}}}{2}+\frac{1}{2} \cdot \sqrt{\left(\mathrm{Buff}_{\mathrm{Ca}, \mathrm{JSR}}^{\mathrm{b}}\right)^{2}+4 \cdot \mathrm{Buff}_{\mathrm{Ca}, \mathrm{JSR}}^{\mathrm{c}}}} \\
& \frac{\mathrm{d}\left[\mathrm{Ca}^{2+}\right]_{\mathrm{NSR}}}{\mathrm{dt}}=\mathrm{I}_{\mathrm{up}}-\mathrm{I}_{\mathrm{tr}} \cdot \frac{\mathrm{V}_{\mathrm{JSR}}}{\mathrm{V}_{\mathrm{NSR}}}
\end{aligned}
$$

\begin{tabular}{|c|c|c|}
\hline Parameter & Value & Description \\
\hline$[\overline{\mathrm{cmdn}}]$ & $0.05 \mathrm{mmol} / \mathrm{L}$ & Maximum Calmodulin concentration \\
\hline$[\overline{\operatorname{trpn}}]$ & $0.07 \mathrm{mmol} / \mathrm{L}$ & Maximum Troponin concentration \\
\hline $\mathrm{K}_{\mathrm{m}}, \mathrm{cmdn}$ & $0.00238 \mathrm{mmol} / \mathrm{L}$ & Affinity of Calmodulin for $\mathrm{Ca}^{2+}$ \\
\hline $\mathrm{K}_{\mathrm{m}}, \operatorname{trpn}$ & $0.00050 \mathrm{mmol} / \mathrm{L}$ & Affinity of Troponin for $\mathrm{Ca}^{2+}$ \\
\hline$[\overline{\mathrm{BSL}}]$ & $1.124 \mathrm{mmol} / \mathrm{L}$ & Anionic $\mathrm{Ca}^{2+}$ binding sites on sarcolemma \\
\hline$[\overline{\mathrm{BSR}}]$ & $0.047 \mathrm{mmol} / \mathrm{L}$ & Anionic $\mathrm{Ca}^{2+}$ binding sites on SR \\
\hline $\mathrm{K}_{\mathrm{m}, \mathrm{BSL}}$ & $0.0087 \mathrm{mmol} / \mathrm{L}$ & Affinity of anionic $\mathrm{Ca}^{2+}$ binding sites on sarcolemma \\
\hline $\mathrm{K}_{\mathrm{m}, \mathrm{BSR}}$ & $0.00087 \mathrm{mmol} / \mathrm{L}$ & Affinity of anionic $\mathrm{Ca}^{2+}$ binding sites on SR \\
\hline$[\overline{\mathrm{CSQN}}]$ & $10.0 \mathrm{mmol} / \mathrm{L}$ & Maximum Calsequestrin concentration \\
\hline $\mathrm{K}_{\mathrm{m}, \mathrm{CSQN}}$ & $0.800 \mathrm{mmol} / \mathrm{L}$ & Affinity of Calsequestrin for $\mathrm{Ca}^{2+}$ \\
\hline
\end{tabular}

\subsubsection{HRd $\beta A R S$ and HRd $\beta A R S S t o c h$ version (Chapters 3,4,5,7)}

In addition to PLB phosphorylation, the lusitropic effect of $\beta A R$ stimulation is also mediated through phosphorylation of the inhibitory subunit of troponin ( $\mathrm{Tll}$ ), although the relative contribution of each process is still unclear. ${ }^{1,2}$ The phosphorylation of $\mathrm{Tnl}$ results in a $50 \%$ increase in $\mathrm{K}_{\mathrm{m}}$, thereby decreasing myofilament sensitivity. Because our model does not contain a mechanical component, $\mathrm{Tnl}$ phosphorylation only affects $\mathrm{Ca}^{2+}$ handling because of this altered affinity and its effect on the $\mathrm{Ca}^{2+}$ buffering property of troponin. The other equations regarding $\mathrm{Ca} 2+$ buffering and concentrations are identical to those in section 1.3.1.

$$
\begin{aligned}
\mathrm{K}_{\mathrm{m}, \text { trpn }}^{\mathrm{NP}} & =0.0005 \frac{\mathrm{mmol}}{\mathrm{L}}, \quad \mathrm{K}_{\mathrm{m}, \text { trpn }}^{\mathrm{P}}=1.5 \cdot 0.0005 \frac{\mathrm{mmol}}{\mathrm{L}}, \quad \mathrm{K}_{\mathrm{m}, \mathrm{cmdn}}=0.00238 \frac{\mathrm{mmol}}{\mathrm{L}} \\
\hat{\mathrm{f}}_{\mathrm{TnI}}^{\mathrm{P}} & =\frac{\mathrm{f}_{\mathrm{TnI}}^{\mathrm{P}}-0.6735}{0.9992-0.6735} \\
\mathrm{~K}_{\mathrm{m}, \text { trpn }} & =\left(1-\hat{\mathrm{f}}_{\mathrm{TnI}}^{\mathrm{P}}\right) \cdot \mathrm{K}_{\mathrm{m}, \text { trpn }}^{\mathrm{NP}}+\hat{\mathrm{f}}_{\mathrm{TnI}}^{\mathrm{P}} \cdot \mathrm{K}_{\mathrm{m}, \text { trpn }}^{\mathrm{P}}
\end{aligned}
$$




$$
\begin{aligned}
& \mathrm{Buff}_{\mathrm{Ca}, \mathrm{i}}^{\mathrm{b}}=[\overline{\mathrm{cmdn}}]+[\overline{\mathrm{trpn}}]-\left[\mathrm{Ca}^{2+}\right]_{\mathrm{i}, \mathrm{tot}}+\mathrm{K}_{\mathrm{m}, \mathrm{trpn}}+\mathrm{K}_{\mathrm{m}, \mathrm{cmdn}} \\
& \text { Buff }_{\mathrm{Ca}, \mathrm{i}}^{\mathrm{c}}=\mathrm{K}_{\mathrm{m}, \mathrm{trpn}} \cdot \mathrm{K}_{\mathrm{m}, \mathrm{cmdn}}-\left[\mathrm{Ca}^{2+}\right]_{\mathrm{i}, \mathrm{tot}} \cdot\left(\mathrm{K}_{\mathrm{m}, \mathrm{trpn}}+\mathrm{K}_{\mathrm{m}, \mathrm{cmdn}}\right)+[\overline{\mathrm{cmdn}}] \cdot \mathrm{K}_{\mathrm{m}, \mathrm{trpn}}+[\overline{\mathrm{trpn}}] \\
& \text { - } \mathrm{K}_{\mathrm{m}, \mathrm{cmdn}} \\
& \text { Buff }_{\mathrm{Ca}, \mathrm{i}}^{\mathrm{d}}=-\mathrm{K}_{\mathrm{m}, \mathrm{trpn}} \cdot \mathrm{K}_{\mathrm{m}, \mathrm{cmdn}} \cdot\left[\mathrm{Ca}^{2+}\right]_{\mathrm{i}, \text { tot }} \\
& {\left[\mathrm{Ca}^{2+}\right]_{\mathrm{i}}=-\frac{\mathrm{Buff}_{\mathrm{Ca}, \mathrm{i}}^{\mathrm{b}}}{3}+\frac{2}{3} \cdot \sqrt{\left(\mathrm{Buff}_{\mathrm{Ca}, \mathrm{i}}^{\mathrm{b}}\right)^{2}-3 \cdot \mathrm{Buff}_{\mathrm{C}, \mathrm{i}}^{\mathrm{c}}}} \\
& \cdot \cos \left(\frac{1}{3} \cdot \cos ^{-1}\left(\frac{9 \cdot \mathrm{Buff}_{\mathrm{Ca}, \mathrm{i}}^{\mathrm{b}} \cdot \mathrm{Buff}_{\mathrm{Ca}, \mathrm{i}}^{\mathrm{c}}-2 \cdot\left(\mathrm{Buff}_{\mathrm{Ca}, \mathrm{i}}^{\mathrm{b}}\right)^{3}-27 \cdot \mathrm{Buff}_{\mathrm{Ca}, \mathrm{i}}^{\mathrm{d}}}{2 \cdot\left(\left(\mathrm{Buff}_{\mathrm{Ca}, \mathrm{i}}^{\mathrm{b}}\right)^{2}-3 \cdot \mathrm{Buff}_{\mathrm{Ca}, \mathrm{i}}^{\mathrm{c}}\right)^{1.5}}\right)\right)
\end{aligned}
$$

\subsubsection{HRdßARS SCR version (Chapter 6)}

The HRdBARS equations (section 1.3.2) were extended to simulate two identical $\mathrm{Ca}^{2+}$ domains with $\mathrm{Ca}^{2+}$ diffusion between NSR and cytosolic domains. The superscript symbol $\mathrm{x}$ is used to designate one of the two identical $\mathrm{Ca}^{2+}$ domains. Whole-cell concentrations / currents are defined as the average of both domains and are indicated without superscript $x$.

$\mathrm{I}_{\text {Diff,cyt }}^{\mathrm{x}}=\frac{\left[\mathrm{Ca}^{2+}\right]_{\mathrm{i}}^{\mathrm{y}}-\left[\mathrm{Ca}^{2+}\right]_{\mathrm{i}}^{\mathrm{x}}}{\tau_{\text {Diff,cyt }}}, \quad \tau_{\text {Diff,cyt }}=1.00 \mathrm{~ms}, \quad \mathrm{y}$ : other $\mathrm{Ca}^{2+}$ domain $\mathrm{I}_{\mathrm{Diff}, \mathrm{nsr}}^{\mathrm{X}}=\frac{\left[\mathrm{Ca}^{2+}\right]_{\mathrm{NSR}}^{\mathrm{y}}-\left[\mathrm{Ca}^{2+}\right]_{\mathrm{NSR}}^{\mathrm{X}}}{\tau_{\mathrm{Diff}, \mathrm{nsr}}}, \quad \tau_{\mathrm{Diff}, \mathrm{nsr}}=25.0 \mathrm{~ms}, \quad \mathrm{y}$ : other $\mathrm{Ca}^{2+}$ domain $\frac{\mathrm{d}\left[\mathrm{Ca}^{2+}\right]_{\mathrm{i}, \text { tot }}^{\mathrm{X}}}{\mathrm{dt}}=-\left(\frac{\left(\mathrm{I}_{\mathrm{Ca}, \mathrm{b}}^{\mathrm{X}}+\mathrm{I}_{\mathrm{pCa}}^{\mathrm{X}}-2 \cdot \mathrm{I}_{\mathrm{NaCa}, \mathrm{i}}^{\mathrm{X}}\right) \cdot \mathrm{A}_{\mathrm{cap}} \cdot \mathrm{C}_{\mathrm{m}}}{\mathrm{z}_{\mathrm{Ca}} \cdot \mathrm{F} \cdot \mathrm{V}_{\text {myo }}}+\mathrm{I}_{\mathrm{up}}^{\mathrm{X}} \cdot \frac{\mathrm{V}_{\mathrm{NSR}}}{\mathrm{V}_{\mathrm{myo}}}-\mathrm{I}_{\mathrm{Diff,Ca}}^{\mathrm{X}} \cdot \frac{\mathrm{V}_{\mathrm{SS}, \mathrm{SR}}}{\mathrm{V}_{\mathrm{myo}}}-\mathrm{I}_{\text {Diff,cyt }}^{\mathrm{X}}\right)$ $\frac{\mathrm{d}\left[\mathrm{Ca}^{2+}\right]_{\mathrm{NSR}}^{\mathrm{x}}}{\mathrm{dt}}=\mathrm{I}_{\mathrm{up}}^{\mathrm{x}}-\mathrm{I}_{\mathrm{tr}}^{\mathrm{x}} \cdot \frac{\mathrm{V}_{\mathrm{JSR}}}{\mathrm{V}_{\mathrm{NSR}}}+\mathrm{I}_{\mathrm{Diff}, \mathrm{x} \text { r }}^{\mathrm{x}}$

\subsection{Intracellular concentrations: $\mathrm{Cl}^{-}$}

\subsubsection{HRd2009, HRdßARS and HRdßARSStoch version (Chapters 2-7)}

$$
\begin{aligned}
& \frac{\mathrm{d}\left[\mathrm{Cl}^{-}\right]_{\mathrm{i}}}{\mathrm{dt}}=-\left(\frac{\mathrm{I}_{\mathrm{Cl}, \mathrm{b}} \cdot \mathrm{A}_{\mathrm{Cap}} \cdot \mathrm{C}_{\mathrm{m}}}{\mathrm{z}_{\mathrm{Cl}} \cdot \mathrm{F} \cdot \mathrm{V}_{\mathrm{myo}}}-\mathrm{CT}_{\mathrm{NaCl}}-\mathrm{CT}_{\mathrm{KCl}}-\mathrm{I}_{\mathrm{Diff}, \mathrm{Cl}} \cdot \frac{\mathrm{V}_{\mathrm{SS}, \mathrm{SR}}}{\mathrm{V}_{\mathrm{myo}}}\right) \\
& \frac{\mathrm{d}\left[\mathrm{Cl}^{-}\right]_{\mathrm{ss}}}{\mathrm{dt}}=-\left(\frac{\mathrm{I}_{\mathrm{Cl}(\mathrm{Ca})} \cdot \mathrm{A}_{\mathrm{Cap}} \cdot \mathrm{C}_{\mathrm{m}}}{\mathrm{z}_{\mathrm{Cl}} \cdot \mathrm{F} \cdot \mathrm{V}_{\mathrm{SS}, \mathrm{SR}}}+\mathrm{I}_{\mathrm{Diff,Cl}}\right)
\end{aligned}
$$

\subsection{Intracellular concentrations: $\mathrm{K}^{+}$}

\subsubsection{HRd2009, HRdßARS and HRdßARSStoch version (Chapters 2-7)}

$$
\frac{\mathrm{d}\left[\mathrm{K}^{+}\right]_{\mathrm{i}}}{\mathrm{dt}}=-\left(\frac{\left(\mathrm{I}_{\mathrm{K} 1}+\mathrm{I}_{\mathrm{Kr}}+\mathrm{I}_{\mathrm{Ks}}+\mathrm{I}_{\mathrm{Kur}}+\mathrm{I}_{\mathrm{To}}+\mathrm{I}_{\mathrm{stim}}-2 \cdot \mathrm{I}_{\mathrm{NaK}}\right) \cdot \mathrm{A}_{\mathrm{Cap}} \cdot \mathrm{C}_{\mathrm{m}}}{\mathrm{z}_{\mathrm{K}} \cdot \mathrm{F} \cdot \mathrm{V}_{\mathrm{myo}}}-\mathrm{CT}_{\mathrm{KCl}}\right)
$$




\subsection{Intracellular concentrations: $\mathrm{Na}^{+}$}

\subsubsection{HRd2009, HRdßARS and HRdßARSStoch version (Chapters 2-7)}

$$
\begin{aligned}
& \frac{\mathrm{d}\left[\mathrm{Na}^{+}\right]_{\mathrm{i}}}{\mathrm{dt}}=-\left(\frac{\left(\mathrm{I}_{\mathrm{Na}}+\mathrm{I}_{\mathrm{Na}, \mathrm{b}}+\mathrm{I}_{\mathrm{NaL}}+3 \cdot \mathrm{I}_{\mathrm{NaK}}+3 \cdot \mathrm{I}_{\mathrm{NaCa}, \mathrm{i}}\right) \cdot \mathrm{A}_{\mathrm{Cap}} \cdot \mathrm{C}_{\mathrm{m}}}{\mathrm{z}_{\mathrm{Na}} \cdot \mathrm{F} \cdot \mathrm{V}_{\mathrm{myo}}}-\mathrm{CT}_{\mathrm{NaCl}}-\mathrm{I}_{\text {Diff,Na }} \cdot \frac{\mathrm{V}_{\mathrm{SS} . \mathrm{SR}}}{\mathrm{V}_{\mathrm{myo}}}\right) \\
& \frac{\mathrm{d}\left[\mathrm{Na}^{+}\right]_{\mathrm{SS}}}{\mathrm{dt}}=-\left(\frac{3 \cdot \mathrm{I}_{\mathrm{NaCa}, \mathrm{sS}} \cdot \mathrm{A}_{\mathrm{Cap}} \cdot \mathrm{C}_{\mathrm{m}}}{\mathrm{z}_{\mathrm{Na}} \cdot \mathrm{F} \cdot \mathrm{V}_{\mathrm{SS}, \mathrm{SR}}}+\mathrm{I}_{\mathrm{Diff}, \mathrm{Na}}\right)
\end{aligned}
$$

\subsection{Co-transporters $\left(\mathrm{CT}_{\mathrm{NaCl}}\right.$ and $\left.\mathrm{CT}_{\mathrm{KCl}}\right)$}

\subsubsection{HRd2009, HRd $\beta A R S$ and HRd $\beta A R S S t o c h$ version (Chapters 2-7)}

Background $\mathrm{Na}^{+}$current $\left(I_{\mathrm{Nab}}\right)$ and co-transporter fluxes were required to maintain a resting $\left[\mathrm{Na}^{+}\right]_{\mathrm{i}}$ concentration consistent with measurements in canine epicardial myocytes. ${ }^{3} \mathrm{~K}^{+}-\mathrm{Cl}^{-}\left(\mathrm{CT}_{\mathrm{KCl}}\right)$ and $\mathrm{Na}^{+}-\mathrm{Cl}^{-}$ $\left(\mathrm{CT}_{\mathrm{NaCl}}\right)$ cotransporter formulations were based on those in the original $\mathrm{HRd}$ model, ${ }^{4}$ with modified density.

$$
\begin{aligned}
\overline{\mathrm{CT}}_{\mathrm{NaCl}} & =2.461 \cdot 10^{-5} \frac{\mathrm{mmol}}{\mathrm{L} \cdot \mathrm{ms}}, \quad \overline{\mathrm{CT}}_{\mathrm{KCl}}=1.770 \cdot 10^{-5} \frac{\mathrm{mmol}}{\mathrm{L} \cdot \mathrm{ms}} \\
\mathrm{CT}_{\mathrm{NaCl}} & =\overline{\mathrm{CT}}_{\mathrm{NaCl}} \cdot \frac{\left(\mathrm{E}_{\mathrm{Na}}-\mathrm{E}_{\mathrm{Cl}}\right)^{4}}{\left(\mathrm{E}_{\mathrm{Na}}-\mathrm{E}_{\mathrm{Cl}}\right)^{4}+87.83^{4}} \\
\mathrm{CT}_{\mathrm{KCl}} & =\overline{\mathrm{CT}}_{\mathrm{KCl}} \cdot \frac{\mathrm{E}_{\mathrm{K}}-\mathrm{E}_{\mathrm{Cl}}}{\mathrm{E}_{\mathrm{K}}-\mathrm{E}_{\mathrm{Cl}}+87.83}
\end{aligned}
$$

\subsection{Background $\mathrm{Ca}^{2+}$ current $\left(\mathrm{I}_{\mathrm{Ca}, \mathrm{b}}\right)$}

\subsubsection{HRd2009, HRd $\beta A R S$ and HRd $\beta A R S S t o c h$ version (Chapters 2-7)}

The formulation for the background calcium current is equivalent to that in the original HRd model. ${ }^{4}$

$$
\begin{aligned}
& \mathrm{P}_{\mathrm{Ca}, \mathrm{b}}=1.9950 \cdot 10^{-7} \frac{\mathrm{cm}}{\mathrm{s}}, \quad \gamma_{\mathrm{Ca}, \mathrm{i}}=1.0, \quad \gamma_{\mathrm{Ca}, \mathrm{o}}=0.341 \\
& \mathrm{I}_{\mathrm{Ca}, \mathrm{b}}=\mathrm{P}_{\mathrm{Ca}, \mathrm{b}} \cdot\left(\mathrm{z}_{\mathrm{Ca}}\right)^{2} \cdot \frac{\mathrm{V}_{\mathrm{m}} \cdot \mathrm{F}^{2}}{\mathrm{R} \cdot \mathrm{T}} \cdot \frac{\gamma_{\mathrm{Ca}, \mathrm{i}} \cdot\left[\mathrm{Ca}^{2+}\right]_{\mathrm{i}} \cdot \exp \left(\mathrm{z}_{\mathrm{Ca}} \cdot \mathrm{V}_{\mathrm{m}} \cdot \frac{\mathrm{F}}{\mathrm{R} \cdot \mathrm{T}}\right)-\gamma_{\mathrm{Ca}, \mathrm{o}} \cdot\left[\mathrm{Ca}^{2+}\right]_{\mathrm{o}}}{\exp \left(\mathrm{z}_{\mathrm{Ca}} \cdot \mathrm{V}_{\mathrm{m}} \cdot \frac{\mathrm{F}}{\mathrm{R} \cdot \mathrm{T}}\right)-1}
\end{aligned}
$$

\subsection{L-type $\mathrm{Ca}^{2+}$ current (I $\left.\mathrm{CaL}\right)$}

\subsubsection{HRd2009 version (Chapter 2)}

A Markov model of $I_{\text {CaL }}$ was developed and fit to data from canine left ventricular epicardial myocytes wherever possible. The structure of the $I_{\text {cal }}$ Markov model is shown in Figure $\mathbf{3 C}$ of Chapter 2. Activation is voltage dependent ( $\alpha$ and $\beta$ transitions). In $\mathrm{Ca}^{2+}$ free conditions, inactivation 
and recovery from inactivation are voltage dependent ( $x$ and $y$ transitions), with constants fit to data on inactivation ${ }^{5}$ and recovery from inactivation ${ }^{6}$ using $\mathrm{Ba}^{2+}$ in canine ventricular myocytes. Elevation of $\mathrm{Ca}^{2+}$ in a local subspace $\left.\left(\left[\mathrm{Ca}^{2+}\right]_{\mathrm{ss}, \mathrm{CaL}}\right)\right)$ moves channels from the $\mathrm{Ca}^{2+}$-free gating tier to an upper tier ( $\theta$ and $\delta$ transitions) characterized by accelerated inactivation ( $x^{*}$ and $y^{*}$ transitions). This structure reflects the hypothesis that $\mathrm{Ca}^{2+}$ binding to calmodulin removes a "brake" and speeds up $\mathrm{I}_{\mathrm{CaL}}$ voltage dependent inactivation. ${ }^{7,8}\left[\mathrm{Ca}^{2+}\right]_{\mathrm{ss}, \mathrm{CaL}}$ elevation depends on $\mathrm{I}_{\mathrm{CaL}} \mathrm{Ca}^{2+}$ entry and diffusion of $\mathrm{Ca}^{2+}\left(\mathrm{I}_{\text {diff, ss }}\right)$ after SR $\mathrm{Ca}^{2+}$-induced $\mathrm{Ca}^{2+}$ release. This phenomenological approach allows reproduction of slowed $\mathrm{I}_{\mathrm{CaL}} \mathrm{Ca}^{2+}$ dependent inactivation when SR function is blocked or intracellular $\mathrm{Ca}^{2+}$ buffering is high. Model $I_{\text {CaL }} \mathrm{I}-\mathrm{V}$ curve is compared to several experimental data sets from canine ventricular cells $\mathrm{s}^{5}$, 913 (Figure 3A of Chapter 2. The Rubart I-V curve data ${ }^{11}$ was chosen for fitting because it falls in the middle range of data recorded at body temperature. Model $I_{\text {CaL }}$ steady state inactivation is compared to multiple experimental data sets in Figure 3B of Chapter 2. The Szabo steady state inactivation data ${ }^{13}$ chosen for model fitting has a slope and $V_{1 / 2}$ consistent with multiple experiments from canine ventricle at body temperature. ${ }^{10,11,13,14} \mathrm{I}_{\mathrm{CaL}}$ steady state inactivation data with lesser degrees of inactivation at steady state ${ }^{11}$ resulted in model APD outside the range observed in canine experiments.

$$
\begin{aligned}
& \mathrm{ACT}_{\tau}=0.59+0.8 \cdot \frac{\exp \left(0.052 \cdot\left(\mathrm{V}_{\mathrm{m}}+13\right)\right)}{1+\exp \left(0.132 \cdot\left(\mathrm{V}_{\mathrm{m}}+13\right)\right)} \\
& \mathrm{ACT}_{\infty}=\frac{1}{1+\exp \left(-\frac{\mathrm{V}_{\mathrm{m}}-13.56}{9.45}\right)} \\
& \alpha_{\mathrm{Ca}, \mathrm{L}}=\frac{\mathrm{ACT}_{\infty}}{\mathrm{ACT}_{\tau}}, \quad \beta_{\mathrm{Ca}, \mathrm{L}}=\frac{1-\mathrm{ACT}_{\infty}}{\mathrm{ACT}_{\tau}} \\
& \mathrm{I}_{\mathrm{V}, \tau}=\frac{1}{\frac{1}{24.828 \cdot\left(1+\exp \left(\left(\mathrm{V}_{\mathrm{m}}+49.10\right) / 10.349\right)\right)}+\frac{1}{30.553 \cdot\left(1+\exp \left(-\left(\mathrm{V}_{\mathrm{m}}+0.213\right) / 10.807\right)\right)}} \\
& \mathrm{I}_{\mathrm{V}, \infty}=\frac{1}{1.25} \cdot\left(0.25+\frac{1}{1+\exp \left(\left(\mathrm{V}_{\mathrm{m}}+17.5\right) / 3\right)}\right) \\
& \mathrm{x}_{\mathrm{Ca}, \mathrm{L}}=\frac{\mathrm{I}_{\mathrm{V}, \infty}}{\mathrm{I}_{\mathrm{V}, \tau}}, \quad \mathrm{y}_{\mathrm{Ca}, \mathrm{L}}^{\mathrm{NP}}=\frac{1-\mathrm{I}_{\mathrm{V}, \infty}}{\mathrm{I}_{\mathrm{V}, \tau}} \\
& \mathrm{Is}_{\mathrm{Ca}}=25-\frac{17.5}{1+\left(\frac{3.000 \cdot 10^{-3}}{\left[\mathrm{Ca}^{2+}\right]_{\mathrm{SS}, \mathrm{CaL}}}\right)^{4}} \\
& \mathrm{Is}_{\mathrm{V}, \tau}=\frac{1}{\frac{1}{24.828 \cdot\left(1+\exp \left(\left(\mathrm{V}_{\mathrm{m}}+49.10\right) / 10.349\right)\right)}+\frac{1}{\mathrm{Is}_{\mathrm{Ca}} \cdot\left(1+\exp \left(-\left(\mathrm{V}_{\mathrm{m}}+0.213\right) / 10.807\right)\right)}} \\
& I s_{V, \infty}=\frac{1}{1.0001} \cdot\left(0.0001+\frac{1}{1+\exp \left(\left(V_{m}+17.5\right) / 3\right)}\right) \\
& \mathrm{xs}_{\mathrm{Ca}, \mathrm{L}}=\frac{\mathrm{I} s_{\mathrm{V}, \infty}}{\mathrm{I} s_{\mathrm{V}, \tau}}, \quad y s_{\mathrm{Ca}, \mathrm{L}}=\frac{1-\mathrm{I} s_{\mathrm{V}, \infty}}{\mathrm{I} s_{\mathrm{V}, \tau}}
\end{aligned}
$$




$$
\begin{aligned}
& \delta_{\mathrm{Ca}, \mathrm{L}}=\frac{3}{1+\left(\frac{3.000 \cdot 10^{-3}}{\left[\mathrm{Ca}^{2+}\right]_{\mathrm{SS}, \mathrm{CaL}}}\right)^{4}, \quad \theta_{\mathrm{Ca}, \mathrm{L}}=1} \\
& \theta \mathrm{I}_{\mathrm{Ca}, \mathrm{L}}=1.0 \cdot 10^{-6}, \quad \delta \mathrm{I}_{\mathrm{Ca}, \mathrm{L}}=\theta \mathrm{I}_{\mathrm{Ca}, \mathrm{L}} \cdot \frac{\mathrm{x}_{\mathrm{Ca}, \mathrm{L}} \cdot \mathrm{ys}_{\mathrm{Ca}, \mathrm{L}} \cdot \delta_{\mathrm{Ca}, \mathrm{L}}}{\mathrm{y}_{\mathrm{Ca}, \mathrm{L}} \cdot \mathrm{xs}_{\mathrm{Ca}, \mathrm{L}} \cdot \theta_{\mathrm{Ca}, \mathrm{L}}}
\end{aligned}
$$

$\frac{\mathrm{dC}_{\mathrm{CaL}}}{\mathrm{dt}}=-\left(\alpha_{\mathrm{Ca}, \mathrm{L}}+\delta_{\mathrm{Ca}, \mathrm{L}}+\mathrm{y}_{\mathrm{Ca}, \mathrm{L}}\right) \cdot \mathrm{C}_{\mathrm{CaL}}+\beta_{\mathrm{Ca}, \mathrm{L}} \cdot \mathrm{O}_{\mathrm{CaL}}+\theta_{\mathrm{Ca}, \mathrm{L}} \cdot \mathrm{Cs}_{\mathrm{CaL}}+\mathrm{x}_{\mathrm{Ca}, \mathrm{L}} \cdot \mathrm{CI}_{\mathrm{CaL}}$

$\frac{\mathrm{dO}_{\mathrm{CaL}}}{\mathrm{dt}}=-\left(\beta_{\mathrm{Ca}, \mathrm{L}}+\delta_{\mathrm{Ca}, \mathrm{L}}+\mathrm{y}_{\mathrm{Ca}, \mathrm{L}}\right) \cdot \mathrm{O}_{\mathrm{CaL}}+\alpha_{\mathrm{Ca}, \mathrm{L}} \cdot \mathrm{C}_{\mathrm{CaL}}+\theta_{\mathrm{Ca}, \mathrm{L}} \cdot \mathrm{Os}_{\mathrm{CaL}}+\mathrm{x}_{\mathrm{Ca}, \mathrm{L}} \cdot \mathrm{OI}_{\mathrm{CaL}}$

$\frac{\mathrm{dCs}_{\mathrm{CaL}}}{\mathrm{dt}}=-\left(\alpha_{\mathrm{Ca}, \mathrm{L}}+\theta_{\mathrm{Ca}, \mathrm{L}}+\mathrm{ys}_{\mathrm{Ca}, \mathrm{L}}\right) \cdot \mathrm{Cs}_{\mathrm{CaL}}+\delta_{\mathrm{Ca}, \mathrm{L}} \cdot \mathrm{C}_{\mathrm{CaL}}+\beta_{\mathrm{Ca}, \mathrm{L}} \cdot \mathrm{Os}_{\mathrm{CaL}}+\mathrm{xs}_{\mathrm{Ca}, \mathrm{L}} \cdot \mathrm{CIs}_{\mathrm{CaL}}$

$\frac{\mathrm{dOs}_{\mathrm{CaL}}}{\mathrm{dt}}=-\left(\beta_{\mathrm{Ca}, \mathrm{L}}+\theta_{\mathrm{Ca}, \mathrm{L}}+\mathrm{ys}_{\mathrm{Ca}, \mathrm{L}}\right) \cdot \mathrm{Os}_{\mathrm{CaL}}+\delta_{\mathrm{Ca}, \mathrm{L}} \cdot \mathrm{O}_{\mathrm{CaL}}+\alpha_{\mathrm{Ca}, \mathrm{L}} \cdot \mathrm{Cs}_{\mathrm{CaL}}+\mathrm{xs}_{\mathrm{Ca}, \mathrm{L}} \cdot \mathrm{OIs}_{\mathrm{CaL}}$

$\frac{\mathrm{dCI}_{\mathrm{CaL}}}{\mathrm{dt}}=-\left(\alpha_{\mathrm{Ca}, \mathrm{L}}+\delta \mathrm{I}_{\mathrm{Ca}, \mathrm{L}}+\mathrm{x}_{\mathrm{Ca}, \mathrm{L}}\right) \cdot \mathrm{CI}_{\mathrm{CaL}}+\mathrm{y}_{\mathrm{Ca}, \mathrm{L}} \cdot \mathrm{C}_{\mathrm{CaL}}+\theta \mathrm{I}_{\mathrm{Ca}, \mathrm{L}} \cdot \mathrm{CIs}_{\mathrm{CaL}}+\beta_{\mathrm{Ca}, \mathrm{L}} \cdot \mathrm{OI}_{\mathrm{CaL}}$

$\frac{\mathrm{dOI}_{\mathrm{CaL}}}{\mathrm{dt}}=-\left(\beta_{\mathrm{Ca}, \mathrm{L}}+\delta \mathrm{I}_{\mathrm{Ca}, \mathrm{L}}+\mathrm{x}_{\mathrm{Ca}, \mathrm{L}}\right) \cdot \mathrm{OI}_{\mathrm{CaL}}+\mathrm{y}_{\mathrm{Ca}, \mathrm{L}} \cdot \mathrm{O}_{\mathrm{CaL}}+\theta \mathrm{I}_{\mathrm{Ca}, \mathrm{L}} \cdot \mathrm{OIs}_{\mathrm{CaL}}+\alpha_{\mathrm{Ca}, \mathrm{L}} \cdot \mathrm{CI}_{\mathrm{CaL}}$

$\frac{\mathrm{dCIs}_{\mathrm{CaL}}}{\mathrm{dt}}=-\left(\alpha_{\mathrm{Ca}, \mathrm{L}}+\theta \mathrm{I}_{\mathrm{Ca}, \mathrm{L}}+\mathrm{xs}_{\mathrm{Ca}, \mathrm{L}}\right) \cdot \mathrm{CIs}_{\mathrm{CaL}}+\delta \mathrm{I}_{\mathrm{Ca}, \mathrm{L}} \cdot \mathrm{CI}_{\mathrm{CaL}}+\mathrm{ys}_{\mathrm{Ca}, \mathrm{L}} \cdot \mathrm{Cs}_{\mathrm{CaL}}+\beta_{\mathrm{Ca}, \mathrm{L}} \cdot \mathrm{OIs}_{\mathrm{CaL}}$

$\frac{\mathrm{dOIs}_{\mathrm{CaL}}}{\mathrm{dt}}=-\left(\beta_{\mathrm{Ca}, \mathrm{L}}+\theta \mathrm{I}_{\mathrm{Ca}, \mathrm{L}}+\mathrm{xs}_{\mathrm{Ca}, \mathrm{L}}\right) \cdot \mathrm{OIs}_{\mathrm{CaL}}+\delta \mathrm{I}_{\mathrm{Ca}, \mathrm{L}} \cdot \mathrm{OI}_{\mathrm{CaL}}+\mathrm{ys}_{\mathrm{Ca}, \mathrm{L}} \cdot \mathrm{Os}_{\mathrm{CaL}}+\alpha_{\mathrm{Ca}, \mathrm{L}} \cdot \mathrm{CIs}_{\mathrm{CaL}}$

$\mathrm{P}_{\mathrm{Ca}, \mathrm{L}}=1.5552 \cdot 10^{-4} \frac{\mathrm{cm}}{\mathrm{s}}, \quad \gamma_{\mathrm{Ca}, \mathrm{i}}=1.0, \quad \gamma_{\mathrm{Ca}, \mathrm{o}}=0.341$

$\overline{\mathrm{I}}_{\mathrm{Ca}, \mathrm{L}}=\mathrm{P}_{\mathrm{Ca}, \mathrm{L}} \cdot\left(\mathrm{z}_{\mathrm{Ca}}\right)^{2} \cdot \frac{\mathrm{V}_{\mathrm{m}} \cdot \mathrm{F}^{2}}{\mathrm{R} \cdot \mathrm{T}} \cdot \frac{\gamma_{\mathrm{Ca}, \mathrm{i}} \cdot\left[\mathrm{Ca}^{2+}\right]_{\mathrm{SS}, \mathrm{CaL}} \cdot \exp \left(\mathrm{z}_{\mathrm{Ca}} \cdot \mathrm{V}_{\mathrm{m}} \cdot \frac{\mathrm{F}}{\mathrm{R} \cdot \mathrm{T}}\right)-\gamma_{\mathrm{Ca}, \mathrm{o}} \cdot\left[\mathrm{Ca}^{2+}\right]_{\mathrm{o}}}{\exp \left(\mathrm{z}_{\mathrm{Ca}} \cdot \mathrm{V}_{\mathrm{m}} \cdot \frac{\mathrm{F}}{\mathrm{R} \cdot \mathrm{T}}\right)-1}$

$\mathrm{I}_{\mathrm{Ca}, \mathrm{L}}=\overline{\mathrm{I}}_{\mathrm{Ca}, \mathrm{L}} \cdot\left(\mathrm{O}_{\mathrm{CaL}}+\mathrm{Os}_{\mathrm{CaL}}\right)$

\subsubsection{HRdßARS version (Chapters 3-5)}

The cardiac L-type $\mathrm{Ca}^{2+}$ channel is modulated through multiple pathways including phosphorylation by PKA. It plays a major role in the inotropic effect of $\beta$ AR stimulation. Both the $\alpha$ and $\beta$ subunits of the channel contain potential phosphorylation sites but the exact role of each subunit in mediating the PKA-dependent effects remains an area of active investigation. ${ }^{15,16}$ At the level of the channel, phosphorylation results in a mode of gating characterized by more frequent, longer lasting channel openings. ${ }^{17,18}$ The macroscopic effect in the (canine) ventricular myocyte is a 3-4 fold increase in peak $I_{\mathrm{CaL}}$, a 10-15 mV leftward shift in the activation I-V relationship and a $10 \mathrm{mV}$ leftward shift in the inactivation I-V relationship. ${ }^{19,20}$ These characteristics are all reproduced by the phosphorylated version of the eight state Markov model that was adapted based on the baseline model. ${ }^{21}$ 
Furthermore, it has been shown that $\beta A R$ stimulation shifted the inactivation of L-type $\mathrm{Ca}^{2+}$ channels from largely voltage-dependent to mainly $\mathrm{Ca}^{2+}$-dependent. ${ }^{22}$ This is incorporated in our model by increasing the rate from the lower to the upper, $\mathrm{Ca}^{2+}$ controlled tier such that, upon activation, more channels reside in this upper tier of the Markov model during $\beta A R$ stimulation compared to baseline conditions. Namiki et al. performed a detailed analysis of the time-course of recovery from inactivation of $\mathrm{I}_{\mathrm{CaL}}$ in adult and newborn rabbit ventricular myocytes. ${ }^{23}$ They concluded that, in the presence of $\mathrm{Ca}^{2+}$ buffers EGTA or BAPTA, Forskolin did not alter $\mathrm{I}_{\mathrm{CaL}}$ recovery from inactivation in adult myocytes, although with the perforated patch technique differences were seen. These differences are likely due to altered $\left[\mathrm{Ca}^{2+}\right]_{1}$ and/or CaMKII (see below), and not due to direct PKA dependent phosphorylation (since they were not present with EGTA or BAPTA). As such, I $I_{\text {cat }}$ recovery from inactivation is identical in non-phosphorylated and PKA-phosphorylated populations.

The $\mathrm{I}_{\mathrm{CaL}}$ formulation for both populations was extended to include CaMKII-dependent alterations in channel kinetics based on a previous computational model. ${ }^{24}$ Parameters were chosen to obtain a $30 \%$ increase in peak $\mathrm{I}_{\mathrm{CaL}}$ and slowed $\mathrm{Ca}^{2+}$-dependent inactivation during maximal CaMKII activation under baseline conditions, consistent with experimental observations in rabbit ventricular myocytes. ${ }^{25}$ In addition, CaMKII-phosphorylated I $I_{\text {cal }}$ channels were modeled with accelerated recovery from inactivation, consistent with several experimental studies that showed slowed recovery of inactivation with pharmacological ${ }^{26}$ or genetic ${ }^{27,}{ }^{28}$ CaMKII inhibition. The time constant of diffusion between the subspace (SS) and L-type $\mathrm{Ca}^{2+}$ channel subspace $\left(\mathrm{SS}_{\mathrm{ICaL}}\right.$ ) was increased to have a larger contribution of SR-Ca ${ }^{2+}$ release to $\mathrm{Ca}^{2+}$-dependent inactivation of $\mathrm{I}_{\mathrm{CaL}}$, consistent with experimental results in rabbit ventricular myocytes ${ }^{7} . \mathrm{Ca}^{2+}$-dependent inactivation of $\mathrm{I}_{\mathrm{CaL}}$ also plays a major role in APD responses during altered $\mathrm{Ca}^{2+}$-cycling. For example, Grandi et al. show that $\mathrm{Ca}^{2+}-$ dependent inactivation is critical for the APD shortening observed when extracellular $\mathrm{Ca}^{2+}$ is increased. ${ }^{29}$ Papp et al. show that an increased CaT (as a result of caffeine-induced $\mathrm{Ca}^{2+}$ release from the SR) corresponds to a reduced APD, whereas the subsequent APD in the presence of a small CaT is prolonged. ${ }^{30}$ The model presented here is able to qualitatively reproduce these experimental observations. Baseline $I_{\text {CaL }}$ parameters were adjusted such that rate-dependent APD and CaT properties and $\mathrm{I}_{\mathrm{CaL}} \mathrm{I}-\mathrm{V}$ characteristics validated in the baseline model ${ }^{21}$ remain intact.

\section{Nonphosphorylated Channels:}

$$
\begin{aligned}
& \mathrm{ACT}_{\tau}=0.59+0.8 \cdot \frac{\exp \left(0.052 \cdot\left(\mathrm{V}_{\mathrm{m}}+13\right)\right)}{1+\exp \left(0.132 \cdot\left(\mathrm{V}_{\mathrm{m}}+13\right)\right)} \\
& \mathrm{ACT}_{\infty}^{\mathrm{NP}}=\frac{1}{\left(1+\exp \left(-\frac{\mathrm{V}_{\mathrm{m}}-13.56}{9.45}\right)\right) \cdot\left(1+\exp \left(-\frac{\mathrm{V}_{\mathrm{m}}+25}{5}\right)\right)} \\
& \alpha_{\mathrm{Ca}, \mathrm{L}}^{\mathrm{NP}}=\frac{\mathrm{ACT}_{\infty}^{\mathrm{NP}}}{\mathrm{ACT}_{\tau}}, \quad \beta_{\mathrm{Ca}, \mathrm{L}}^{\mathrm{NP}}=\frac{1-\mathrm{ACT}_{\infty}^{\mathrm{NP}}}{\mathrm{ACT}_{\tau}} \\
& \mathrm{I}_{\mathrm{V}, \tau}^{\mathrm{NP}}=\frac{1}{\frac{1}{70.0 \cdot\left(1+\exp \left(\left(\mathrm{V}_{\mathrm{m}}+49.10\right) / 10.349\right)\right)}+\frac{1}{26.553 \cdot\left(1+\exp \left(-\left(\mathrm{V}_{\mathrm{m}}+0.213\right) / 10.807\right)\right)}}
\end{aligned}
$$




$$
\begin{aligned}
& \mathrm{I}_{\mathrm{V}, \infty}^{\mathrm{NP}}=\frac{1}{1.2474} \cdot\left(0.2472+\frac{1}{1+\exp \left(\left(\mathrm{V}_{\mathrm{m}}+17.5\right) / 3\right)}\right) \\
& \mathrm{x}_{\mathrm{Ca}, \mathrm{L}}^{\mathrm{NP}}=\frac{\mathrm{I}_{\mathrm{V}, \infty}^{\mathrm{NP}}}{\mathrm{I}_{\mathrm{V}, \tau}^{\mathrm{NP}}}, \quad \mathrm{y}_{\mathrm{Ca}, \mathrm{L}}^{\mathrm{NP}}=\frac{1-\mathrm{I}_{\mathrm{V}, \infty}^{\mathrm{NP}}}{\mathrm{I}_{\mathrm{V}, \tau}^{\mathrm{NP}}} \\
& \Delta \tau_{\mathrm{Ca}, \mathrm{CaMK}}=5.0 \cdot \mathrm{f}_{\mathrm{ICaL}}^{\mathrm{P}, \mathrm{CaMKI}}
\end{aligned}
$$$$
\mathrm{Is}_{\mathrm{Ca}}^{\mathrm{NP}}=13.825-\frac{6.3836-\Delta \tau_{\mathrm{Ca}, \mathrm{CaMK}}}{1+\left(\frac{1.100 \cdot 10^{-3}}{\left[\mathrm{Ca}^{2+}\right]_{\mathrm{SS}, \mathrm{CaL}}}\right)^{4}}-\frac{3.3696}{1+\left(\frac{1.200 \cdot 10^{-2}}{\left[\mathrm{Ca}^{2+}\right]_{\mathrm{SS}, \mathrm{CaL}}}\right)^{10}}
$$$$
\mathrm{Is}_{\mathrm{V}, \tau}^{\mathrm{NP}}=\frac{1}{\frac{1}{70.0 \cdot\left(1+\exp \left(\left(\mathrm{V}_{\mathrm{m}}+49.10\right) / 10.349\right)\right)}+\frac{1}{\mathrm{Is}_{\mathrm{Ca}}^{\mathrm{NP}} \cdot\left(1+\exp \left(-\left(\mathrm{V}_{\mathrm{m}}+0.213\right) / 10.807\right)\right)}}
$$$$
\mathrm{Is}_{\mathrm{V}, \infty}^{\mathrm{NP}}=\frac{1}{1.001} \cdot\left(0.001+\frac{1}{1+\exp \left(\left(\mathrm{V}_{\mathrm{m}}+17.5\right) / 3\right)}\right)
$$$$
\mathrm{Xs}_{\mathrm{Ca}, \mathrm{L}}^{\mathrm{NP}}=\frac{\mathrm{Is} \mathrm{V}_{\mathrm{V}, \infty}^{\mathrm{NP}}}{\mathrm{I} s_{\mathrm{V}, \tau}^{\mathrm{NP}}}, \quad \mathrm{ys} \mathrm{Ca}, \mathrm{L}^{\mathrm{NP}}=\frac{1-\mathrm{I} s_{\mathrm{V}, \infty}^{\mathrm{NP}}}{\mathrm{I} s_{\mathrm{V}, \tau}^{\mathrm{N}}}
$$

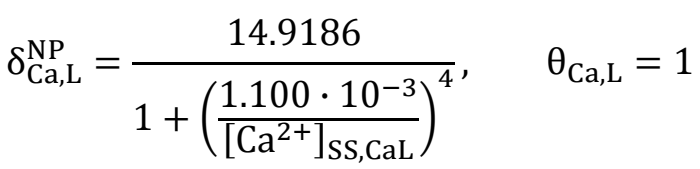

$\theta \mathrm{I}_{\mathrm{Ca}, \mathrm{L}}=1.0 \cdot 10^{-6}, \quad \delta \mathrm{I}_{\mathrm{Ca}, \mathrm{L}}^{\mathrm{NP}}=\theta \mathrm{I}_{\mathrm{Ca}, \mathrm{L}} \cdot \frac{\mathrm{x}_{\mathrm{Ca}, \mathrm{L}}^{\mathrm{NP}} \cdot \mathrm{ys}_{\mathrm{Ca}, \mathrm{L}}^{\mathrm{NP}} \cdot \delta_{\mathrm{Ca}, \mathrm{L}}^{\mathrm{NP}}}{\mathrm{y}_{\mathrm{Ca}, \mathrm{L}}^{\mathrm{NP}} \cdot \mathrm{xs}_{\mathrm{Ca}, \mathrm{L}}^{\mathrm{NP}} \cdot \theta_{\mathrm{Ca}, \mathrm{L}}}$

$$
\frac{\mathrm{dC}_{\mathrm{Ca}}^{\mathrm{NP}}}{\mathrm{dt}}=-\left(\alpha_{\mathrm{Ca}, \mathrm{L}}^{\mathrm{NP}}+\delta_{\mathrm{Ca}, \mathrm{L}}^{\mathrm{NP}}+\mathrm{y}_{\mathrm{Ca}, \mathrm{L}}^{\mathrm{NP}}\right) \cdot \mathrm{C}_{\mathrm{CaL}}^{\mathrm{NP}}+\beta_{\mathrm{Ca}, \mathrm{L}}^{\mathrm{NP}} \cdot \mathrm{O}_{\mathrm{CaL}}^{\mathrm{NP}}+\theta_{\mathrm{Ca}, \mathrm{L}} \cdot \mathrm{Cs}_{\mathrm{CaL}}^{\mathrm{NP}}+\mathrm{x}_{\mathrm{Ca}, \mathrm{L}}^{\mathrm{NP}} \cdot \mathrm{CI}_{\mathrm{CaL}}^{\mathrm{NP}}
$$$$
\frac{\mathrm{dO}_{\mathrm{CaL}}^{\mathrm{NP}}}{\mathrm{dt}}=-\left(\beta_{\mathrm{Ca}, \mathrm{L}}^{\mathrm{NP}}+\delta_{\mathrm{Ca}, \mathrm{L}}^{\mathrm{NP}}+\mathrm{y}_{\mathrm{Ca}, \mathrm{L}}^{\mathrm{NP}}\right) \cdot \mathrm{O}_{\mathrm{CaL}}^{\mathrm{NP}}+\alpha_{\mathrm{Ca}, \mathrm{L}}^{\mathrm{NP}} \cdot \mathrm{C}_{\mathrm{CaL}}^{\mathrm{NP}}+\theta_{\mathrm{Ca}, \mathrm{L}} \cdot \mathrm{Os}_{\mathrm{CaL}}^{\mathrm{NP}}+\mathrm{x}_{\mathrm{Ca}, \mathrm{L}}^{\mathrm{NP}} \cdot \mathrm{OI}_{\mathrm{CaL}}^{\mathrm{NP}}
$$$$
\frac{\mathrm{dCs}_{\mathrm{CaL}}^{\mathrm{NP}}}{\mathrm{dt}}=-\left(\alpha_{\mathrm{Ca}, \mathrm{L}}^{\mathrm{NP}}+\theta_{\mathrm{Ca}, \mathrm{L}}+\mathrm{ys}_{\mathrm{Ca}, \mathrm{L}}^{\mathrm{NP}}\right) \cdot \mathrm{Cs}_{\mathrm{CaL}}^{\mathrm{NP}}+\delta_{\mathrm{Ca}, \mathrm{L}}^{\mathrm{NP}} \cdot \mathrm{C}_{\mathrm{CaL}}^{\mathrm{NP}}+\beta_{\mathrm{Ca}, \mathrm{L}}^{\mathrm{NP}} \cdot \mathrm{Os}_{\mathrm{CaL}}^{\mathrm{NP}}+\mathrm{xs}_{\mathrm{Ca}, \mathrm{L}}^{\mathrm{NP}} \cdot \mathrm{CIs}_{\mathrm{CaL}}^{\mathrm{NP}}
$$$$
\frac{\mathrm{dOs}_{\mathrm{CaL}}^{\mathrm{NP}}}{\mathrm{dt}}=-\left(\beta_{\mathrm{Ca}, \mathrm{L}}^{\mathrm{NP}}+\theta_{\mathrm{Ca}, \mathrm{L}}+\mathrm{ys}_{\mathrm{Ca}, \mathrm{L}}^{\mathrm{NP}}\right) \cdot \mathrm{Os}_{\mathrm{CaL}}^{\mathrm{NP}}+\delta_{\mathrm{Ca}, \mathrm{L}}^{\mathrm{NP}} \cdot \mathrm{O}_{\mathrm{CaL}}^{\mathrm{NP}}+\alpha_{\mathrm{Ca}, \mathrm{L}}^{\mathrm{NP}} \cdot \mathrm{Cs}_{\mathrm{CaL}}^{\mathrm{NP}}+\mathrm{xs}_{\mathrm{Ca}, \mathrm{L}}^{\mathrm{NP}} \cdot \mathrm{OIs}_{\mathrm{CaL}}^{\mathrm{NP}}
$$$$
\frac{\mathrm{dCI}_{\mathrm{CaL}}^{\mathrm{NP}}}{\mathrm{dt}}=-\left(\alpha_{\mathrm{Ca}, \mathrm{L}}^{\mathrm{NP}}+\delta \mathrm{I}_{\mathrm{Ca}, \mathrm{L}}^{\mathrm{NP}}+\mathrm{x}_{\mathrm{Ca}, \mathrm{L}}^{\mathrm{NP}}\right) \cdot \mathrm{CI}_{\mathrm{CaL}}^{\mathrm{NP}}+\mathrm{y}_{\mathrm{Ca}, \mathrm{L}}^{\mathrm{NP}} \cdot \mathrm{C}_{\mathrm{CaL}}^{\mathrm{NP}}+\theta \mathrm{I}_{\mathrm{Ca}, \mathrm{L}} \cdot \mathrm{CIs}_{\mathrm{CaL}}^{\mathrm{NP}}+\beta_{\mathrm{Ca}, \mathrm{L}}^{\mathrm{NP}} \cdot \mathrm{OI}_{\mathrm{CaL}}^{\mathrm{NP}}
$$$$
\frac{\mathrm{dOI}_{\mathrm{CaL}}^{\mathrm{NP}}}{\mathrm{dt}}=-\left(\beta_{\mathrm{Ca}, \mathrm{L}}^{\mathrm{NP}}+\delta \mathrm{I}_{\mathrm{Ca}, \mathrm{L}}^{\mathrm{NP}}+\mathrm{x}_{\mathrm{Ca}, \mathrm{L}}^{\mathrm{NP}}\right) \cdot \mathrm{OI}_{\mathrm{CaL}}^{\mathrm{NP}}+\mathrm{y}_{\mathrm{Ca}, \mathrm{L}}^{\mathrm{NP}} \cdot \mathrm{O}_{\mathrm{CaL}}^{\mathrm{NP}}+\theta \mathrm{I}_{\mathrm{Ca}, \mathrm{L}} \cdot \mathrm{OIs}_{\mathrm{CaL}}^{\mathrm{NP}}+\alpha_{\mathrm{Ca}, \mathrm{L}}^{\mathrm{NP}} \cdot \mathrm{CI}_{\mathrm{CaL}}^{\mathrm{NP}}
$$$$
\frac{d \mathrm{dIs}_{\mathrm{CaL}}^{\mathrm{NP}}}{\mathrm{dt}}=-\left(\alpha_{\mathrm{Ca}, \mathrm{L}}^{\mathrm{NP}}+\theta \mathrm{I}_{\mathrm{Ca}, \mathrm{L}}+\mathrm{xs}_{\mathrm{Ca}, \mathrm{L}}^{\mathrm{NP}}\right) \cdot \mathrm{CIs}_{\mathrm{CaL}}^{\mathrm{NP}}+\delta \mathrm{I}_{\mathrm{Ca}, \mathrm{L}}^{\mathrm{NP}} \cdot \mathrm{CI}_{\mathrm{CaL}}^{\mathrm{NP}}+\mathrm{ys}_{\mathrm{Ca}, \mathrm{L}}^{\mathrm{NP}} \cdot \mathrm{Cs}_{\mathrm{CaL}}^{\mathrm{NP}}+\beta_{\mathrm{Ca}, \mathrm{L}}^{\mathrm{NP}} \cdot \mathrm{OIs}_{\mathrm{CaL}}^{\mathrm{NP}}
$$$$
\frac{d O I s_{C a L}^{N P}}{d t}=-\left(\beta_{C a, L}^{N P}+\theta I_{C a, L}+x s_{C a, L}^{N P}\right) \cdot \operatorname{OIs}_{C a L}^{N P}+\delta I_{C a, L}^{N P} \cdot \mathrm{OI}_{C a L}^{N P}+y s_{C a, L}^{N P} \cdot O s_{C a L}^{N P}+\alpha_{C a, L}^{N P} \cdot C I s_{C a L}^{N P}
$$ 
Model definition and equations

$$
\begin{aligned}
& \mathrm{P}_{\mathrm{Ca}, \mathrm{L} / \mathrm{NP}}=1.5552 \cdot 10^{-4} \cdot\left(1+0.4 \cdot \mathrm{f}_{\mathrm{ICaL}}^{\mathrm{P}, \mathrm{CaMKII}}\right) \frac{\mathrm{cm}}{\mathrm{s}}, \quad \gamma_{\mathrm{Ca}, \mathrm{i}}=1.0, \quad \gamma_{\mathrm{Ca}, \mathrm{o}}=0.341 \\
& \overline{\mathrm{I}}_{\mathrm{Ca}, \mathrm{L}}^{\mathrm{NP}}=\mathrm{P}_{\mathrm{Ca}, \mathrm{L} / \mathrm{NP}} \cdot\left(\mathrm{z}_{\mathrm{Ca}}\right)^{2} \cdot \frac{\mathrm{V}_{\mathrm{m}} \cdot \mathrm{F}^{2}}{\mathrm{R} \cdot \mathrm{T}} \cdot \frac{\gamma_{\mathrm{Ca}, \mathrm{i}} \cdot\left[\mathrm{Ca}^{2+}\right]_{\mathrm{SS}, \mathrm{CaL}} \cdot \exp \left(\mathrm{z}_{\mathrm{Ca}} \cdot \mathrm{V}_{\mathrm{m}} \cdot \frac{\mathrm{F}}{\mathrm{R} \cdot \mathrm{T}}\right)-\gamma_{\mathrm{Ca}, \mathrm{o}} \cdot\left[\mathrm{Ca}^{2+}\right]_{\mathrm{o}}}{\exp \left(\mathrm{z}_{\mathrm{Ca}} \cdot \mathrm{V}_{\mathrm{m}} \cdot \frac{\mathrm{F}}{\mathrm{R} \cdot \mathrm{T}}\right)-1} \\
& \mathrm{I}_{\mathrm{Ca}, \mathrm{L}}^{\mathrm{NP}}=\overline{\mathrm{I}}_{\mathrm{Ca}, \mathrm{L}}^{\mathrm{NP}} \cdot\left(\mathrm{O}_{\mathrm{CaL}}^{\mathrm{NP}}+\mathrm{Os}_{\mathrm{CaL}}^{\mathrm{NP}}\right)
\end{aligned}
$$

Phosphorylated Channels:

$$
\begin{aligned}
& \operatorname{ACT}_{\infty}^{\mathrm{P}}=\frac{1}{\left(1+\exp \left(-\frac{\mathrm{V}_{\mathrm{m}}--4.798}{7.5699}\right)\right) \cdot\left(1+\exp \left(-\frac{\mathrm{V}_{\mathrm{m}}+25}{5}\right)\right)} \\
& \alpha_{\mathrm{Ca}, \mathrm{L}}^{\mathrm{P}}=\frac{\mathrm{ACT}_{\infty}^{\mathrm{P}}}{\mathrm{ACT}_{\tau}}, \quad \beta_{\mathrm{Ca}, \mathrm{L}}^{\mathrm{P}}=\frac{1-\mathrm{ACT}_{\infty}^{\mathrm{P}}}{\mathrm{ACT}_{\tau}} \\
& \mathrm{I}_{\mathrm{V}, \tau}^{\mathrm{P}}=\frac{1}{\frac{1}{70.0 \cdot\left(1+\exp \left(\left(\mathrm{V}_{\mathrm{m}}+49.10\right) / 10.349\right)\right)}+\frac{1}{30 \cdot\left(1+\exp \left(-\left(\mathrm{V}_{\mathrm{m}}+0.213\right) / 10.807\right)\right)}} \\
& \mathrm{I}_{\mathrm{V}, \infty}^{\mathrm{P}}=\frac{1}{1.100} \cdot\left(0.100+\frac{1}{1+\exp \left(\left(\mathrm{V}_{\mathrm{m}}+29.979\right) / 3.1775\right)}\right) \\
& \mathrm{x}_{\mathrm{Ca}, \mathrm{L}}^{\mathrm{P}}=\frac{\mathrm{I}_{\mathrm{V}, \infty}^{\mathrm{P}}}{\mathrm{I}_{\mathrm{V}, \tau}^{\mathrm{P}}}, \quad \mathrm{y}_{\mathrm{Ca}, \mathrm{L}}^{\mathrm{P}}=\frac{1-\mathrm{I}_{\mathrm{V}, \infty}^{\mathrm{P}}}{\mathrm{I}_{\mathrm{V}, \tau}^{\mathrm{P}}} \\
& \Delta \tau_{\mathrm{Ca}, \mathrm{CaMK}}=0.1 \cdot \mathrm{f}_{\mathrm{ICaL}}^{\mathrm{P}, \mathrm{CaMKII}} \\
& \operatorname{Is}_{\mathrm{Ca}}^{\mathrm{P}}=32.5-\frac{18.0-\Delta \tau_{\mathrm{Ca}, \mathrm{CaMK}}}{1+\left(\frac{0.002}{\left[\mathrm{Ca}^{2+}\right]_{\mathrm{SS}, \mathrm{CaL}}}\right)^{4}}-\frac{10.0}{1+\left(\frac{0.010}{\left[\mathrm{Ca}^{2+}\right]_{\mathrm{Ss}, \mathrm{CaL}}}\right)^{10}} \\
& \mathrm{Is}_{\mathrm{V}, \tau}^{\mathrm{P}}=\frac{1}{\frac{1}{70.0 \cdot\left(1+\exp \left(\left(\mathrm{V}_{\mathrm{m}}+49.10\right) / 10.349\right)\right)}+\frac{1}{\mathrm{Is}_{\mathrm{Ca}}^{\mathrm{P}} \cdot\left(1+\exp \left(-\left(\mathrm{V}_{\mathrm{m}}+0.213\right) / 10.807\right)\right)}} \\
& \operatorname{Is}_{\mathrm{V}, \infty}^{\mathrm{P}}=\frac{1}{1.0001} \cdot\left(0.0001+\frac{1}{1+\exp \left(\left(\mathrm{V}_{\mathrm{m}}+29.979\right) / 3.1775\right)}\right) \\
& \mathrm{xs}_{\mathrm{Ca}, \mathrm{L}}^{\mathrm{P}}=\frac{\mathrm{Is}}{\mathrm{I} \mathrm{s}_{\mathrm{V}, \tau}^{\mathrm{P}}}, \quad \mathrm{ys} \mathrm{s}_{\mathrm{Ca}, \mathrm{L}}^{\mathrm{P}}=\frac{1-\mathrm{Is} \mathrm{s}_{\mathrm{V}, \infty}^{\mathrm{P}}}{\mathrm{Is} \mathrm{V}, \tau} \\
& \delta_{\mathrm{Ca}, \mathrm{L}}^{\mathrm{P}}=\frac{6.000}{1+\left(\frac{0.002}{\left[\mathrm{Ca}^{2+}\right]_{\mathrm{SS}, \mathrm{CaL}}}\right)^{4}} \\
& \delta I_{\mathrm{Ca}, \mathrm{L}}^{\mathrm{P}}=\theta \mathrm{I}_{\mathrm{Ca}, \mathrm{L}} \cdot \frac{\mathrm{x}_{\mathrm{Ca}, \mathrm{L}}^{\mathrm{P}} \cdot \mathrm{ys}_{\mathrm{Ca}, \mathrm{L}}^{\mathrm{P}} \cdot \delta_{\mathrm{Ca}, \mathrm{L}}^{\mathrm{P}}}{\mathrm{y}_{\mathrm{Ca}, \mathrm{L}}^{\mathrm{P}} \cdot \mathrm{xs}_{\mathrm{Ca}, \mathrm{L}}^{\mathrm{P}} \cdot \theta_{\mathrm{Ca}, \mathrm{L}}} \\
& \frac{\mathrm{dC}_{\mathrm{CaL}}^{\mathrm{P}}}{\mathrm{dt}}=-\left(\alpha_{\mathrm{Ca}, \mathrm{L}}^{\mathrm{P}}+\delta_{\mathrm{Ca}, \mathrm{L}}^{\mathrm{P}}+\mathrm{y}_{\mathrm{Ca}, \mathrm{L}}^{\mathrm{P}}\right) \cdot \mathrm{C}_{\mathrm{CaL}}^{\mathrm{P}}+\beta_{\mathrm{Ca}, \mathrm{L}}^{\mathrm{P}} \cdot \mathrm{O}_{\mathrm{CaL}}^{\mathrm{P}}+\theta_{\mathrm{Ca}, \mathrm{L}} \cdot \mathrm{Cs}_{\mathrm{CaL}}^{\mathrm{P}}+\mathrm{x}_{\mathrm{Ca}, \mathrm{L}}^{\mathrm{P}} \cdot \mathrm{CI}_{\mathrm{CaL}}^{\mathrm{P}}
\end{aligned}
$$

274 | 
$\frac{\mathrm{dO}_{\mathrm{CaL}}^{\mathrm{P}}}{\mathrm{dt}}=-\left(\beta_{\mathrm{Ca}, \mathrm{L}}^{\mathrm{P}}+\delta_{\mathrm{Ca}, \mathrm{L}}^{\mathrm{P}}+\mathrm{y}_{\mathrm{Ca}, \mathrm{L}}^{\mathrm{P}}\right) \cdot \mathrm{O}_{\mathrm{CaL}}^{\mathrm{P}}+\alpha_{\mathrm{Ca}, \mathrm{L}}^{\mathrm{P}} \cdot \mathrm{C}_{\mathrm{CaL}}^{\mathrm{P}}+\theta_{\mathrm{Ca}, \mathrm{L}} \cdot \mathrm{Os}_{\mathrm{CaL}}^{\mathrm{P}}+\mathrm{x}_{\mathrm{Ca}, \mathrm{L}}^{\mathrm{P}} \cdot \mathrm{OI}_{\mathrm{CaL}}^{\mathrm{P}}$ $\frac{\mathrm{dCs}}{\mathrm{dt}}=-\left(\alpha_{\mathrm{Ca}, \mathrm{L}}^{\mathrm{P}}+\theta_{\mathrm{Ca}, \mathrm{L}}+\mathrm{ys}_{\mathrm{Ca}, \mathrm{L}}^{\mathrm{P}}\right) \cdot \mathrm{Cs}_{\mathrm{CaL}}^{\mathrm{P}}+\delta_{\mathrm{Ca}, \mathrm{L}}^{\mathrm{P}} \cdot \mathrm{C}_{\mathrm{CaL}}^{\mathrm{P}}+\beta_{\mathrm{Ca}, \mathrm{L}}^{\mathrm{P}} \cdot \mathrm{Os}_{\mathrm{CaL}}^{\mathrm{P}}+\mathrm{xs}_{\mathrm{Ca}, \mathrm{L}}^{\mathrm{P}} \cdot \mathrm{CIs}_{\mathrm{CaL}}^{\mathrm{P}}$ $\frac{\mathrm{dOs}_{\mathrm{CaL}}^{\mathrm{P}}}{\mathrm{dt}}=-\left(\beta_{\mathrm{Ca}, \mathrm{L}}^{\mathrm{P}}+\theta_{\mathrm{Ca}, \mathrm{L}}+\mathrm{ys}_{\mathrm{Ca}, \mathrm{L}}^{\mathrm{P}}\right) \cdot \mathrm{Os}_{\mathrm{CaL}}^{\mathrm{P}}+\delta_{\mathrm{Ca}, \mathrm{L}}^{\mathrm{P}} \cdot \mathrm{O}_{\mathrm{CaL}}^{\mathrm{P}}+\alpha_{\mathrm{Ca}, \mathrm{L}}^{\mathrm{P}} \cdot \mathrm{Cs}_{\mathrm{CaL}}^{\mathrm{P}}+\mathrm{xs}_{\mathrm{Ca}, \mathrm{L}}^{\mathrm{P}} \cdot \mathrm{OIs}_{\mathrm{CaL}}^{\mathrm{P}}$ $\frac{\mathrm{dCI}_{\mathrm{CaL}}^{\mathrm{P}}}{\mathrm{dt}}=-\left(\alpha_{\mathrm{Ca}, \mathrm{L}}^{\mathrm{P}}+\delta \mathrm{I}_{\mathrm{Ca}, \mathrm{L}}^{\mathrm{P}}+\mathrm{x}_{\mathrm{Ca}, \mathrm{L}}^{\mathrm{P}}\right) \cdot \mathrm{CI}_{\mathrm{CaL}}^{\mathrm{P}}+\mathrm{y}_{\mathrm{Ca}, \mathrm{L}}^{\mathrm{P}} \cdot \mathrm{C}_{\mathrm{CaL}}^{\mathrm{P}}+\theta \mathrm{II}_{\mathrm{Ca}, \mathrm{L}} \cdot \mathrm{CIs}_{\mathrm{CaL}}^{\mathrm{P}}+\beta_{\mathrm{Ca}, \mathrm{L}}^{\mathrm{P}} \cdot \mathrm{OI}_{\mathrm{CaL}}^{\mathrm{P}}$ $\frac{\mathrm{dOI}}{\mathrm{CaL}}=-\left(\beta_{\mathrm{Ca}, \mathrm{L}}^{\mathrm{P}}+\delta \mathrm{I}_{\mathrm{Ca}, \mathrm{L}}^{\mathrm{P}}+\mathrm{x}_{\mathrm{Ca}, \mathrm{L}}^{\mathrm{P}}\right) \cdot \mathrm{OI}_{\mathrm{CaL}}^{\mathrm{P}}+\mathrm{y}_{\mathrm{Ca}, \mathrm{L}}^{\mathrm{P}} \cdot \mathrm{O}_{\mathrm{CaL}}^{\mathrm{P}}+\theta \mathrm{I}_{\mathrm{Ca}, \mathrm{L}} \cdot \mathrm{OII}_{\mathrm{CaL}}^{\mathrm{P}}+\alpha_{\mathrm{Ca}, \mathrm{L}}^{\mathrm{P}} \cdot \mathrm{CI}_{\mathrm{CaL}}^{\mathrm{P}}$ $\frac{\mathrm{dCIs}_{\mathrm{CaL}}^{\mathrm{P}}}{\mathrm{dt}}=-\left(\alpha_{\mathrm{Ca}, \mathrm{L}}^{\mathrm{P}}+\theta \mathrm{I}_{\mathrm{Ca}, \mathrm{L}}+\mathrm{xs}_{\mathrm{Ca}, \mathrm{L}}^{\mathrm{P}}\right) \cdot \mathrm{CIs}_{\mathrm{CaL}}^{\mathrm{P}}+\delta \mathrm{I}_{\mathrm{Ca}, \mathrm{L}}^{\mathrm{P}} \cdot \mathrm{CI}_{\mathrm{CaL}}^{\mathrm{P}}+\mathrm{ys}_{\mathrm{Ca}, \mathrm{L}}^{\mathrm{P}} \cdot \mathrm{Cs}_{\mathrm{CaL}}^{\mathrm{P}}+\beta_{\mathrm{Ca}, \mathrm{L}}^{\mathrm{P}} \cdot \mathrm{OIs}_{\mathrm{CaL}}^{\mathrm{P}}$ $\frac{\mathrm{dOIs}_{\mathrm{CaL}}^{\mathrm{P}}}{\mathrm{dt}}=-\left(\beta_{\mathrm{Ca}, \mathrm{L}}^{\mathrm{P}}+\theta \mathrm{I}_{\mathrm{Ca}, \mathrm{L}}+\mathrm{xs}_{\mathrm{Ca}, \mathrm{L}}^{\mathrm{P}}\right) \cdot \mathrm{OIs}_{\mathrm{CaL}}^{\mathrm{P}}+\delta \mathrm{I}_{\mathrm{Ca}, \mathrm{L}}^{\mathrm{P}} \cdot \mathrm{OI}_{\mathrm{CaL}}^{\mathrm{P}}+\mathrm{ys}_{\mathrm{Ca}, \mathrm{L}}^{\mathrm{P}} \cdot \mathrm{Os}_{\mathrm{CaL}}^{\mathrm{P}}+\alpha_{\mathrm{Ca}, \mathrm{L}}^{\mathrm{P}} \cdot \mathrm{CIs}_{\mathrm{CaL}}^{\mathrm{P}}$ $\mathrm{P}_{\mathrm{Ca}, \mathrm{L} / \mathrm{P}}=2.579 \cdot 10^{-4} \cdot\left(1+0.1 \cdot \mathrm{f}_{\mathrm{ICaL}}^{\mathrm{P}, \mathrm{CaMKII}}\right) \frac{\mathrm{cm}}{\mathrm{s}}$ $\overline{\mathrm{I}}_{\mathrm{Ca}, \mathrm{L}}^{\mathrm{P}}=\mathrm{P}_{\mathrm{Ca}, \mathrm{L} / \mathrm{P}} \cdot\left(\mathrm{z}_{\mathrm{Ca}}\right)^{2} \cdot \frac{\mathrm{V}_{\mathrm{m}} \cdot \mathrm{F}^{2}}{\mathrm{R} \cdot \mathrm{T}} \cdot \frac{\gamma_{\mathrm{Ca}, \mathrm{i}} \cdot\left[\mathrm{Ca}^{2+}\right]_{\mathrm{SS}, \mathrm{CaL}} \cdot \exp \left(\mathrm{z}_{\mathrm{Ca}} \cdot \mathrm{V}_{\mathrm{m}} \cdot \frac{\mathrm{F}}{\mathrm{R} \cdot \mathrm{T}}\right)-\gamma_{\mathrm{Ca}, \mathrm{o}} \cdot\left[\mathrm{Ca}^{2+}\right]_{\mathrm{o}}}{\exp \left(\mathrm{z}_{\mathrm{Ca}} \cdot \mathrm{V}_{\mathrm{m}} \cdot \frac{\mathrm{F}}{\mathrm{R} \cdot \mathrm{T}}\right)-1}$ $\mathrm{I}_{\mathrm{Ca}, \mathrm{L}}^{\mathrm{P}}=\overline{\mathrm{I}}_{\mathrm{Ca}, \mathrm{L}}^{\mathrm{P}} \cdot\left(\mathrm{O}_{\mathrm{CaL}}^{\mathrm{P}}+\mathrm{Os}_{\mathrm{CaL}}^{\mathrm{P}}\right)$

\section{Combined current:}

$\hat{\mathrm{f}}_{\mathrm{Ca}, \mathrm{L}}^{\mathrm{P}}=\frac{\mathrm{f}_{\mathrm{ICa}, \mathrm{L}}^{\mathrm{p}}-\left(0.0269+\frac{[\mathrm{ICaL}]_{\mathrm{ARN}}}{[\mathrm{ICaL}]_{\text {tot }}}\right)}{0.9273-\left(0.0269+\frac{[\mathrm{ICaL}]_{\mathrm{ARN}}}{[\mathrm{ICaL}]_{\text {tot }}}\right)}$

$\mathrm{I}_{\mathrm{Ca}, \mathrm{L}}=\hat{\mathrm{f}}_{\mathrm{Ca}, \mathrm{L}}^{\mathrm{P}} \cdot \mathrm{I}_{\mathrm{Ca}, \mathrm{L}}^{\mathrm{P}}+\left(1-\hat{\mathrm{f}}_{\mathrm{Ca}, \mathrm{L}}^{\mathrm{P}}\right) \cdot \mathrm{I}_{\mathrm{Ca}, \mathrm{L}}^{\mathrm{NP}}$

\subsubsection{HRdßARS SCR version (Chapter 6)}

The model structure and majority of parameters are identical to those in section 1.9.2. Parameters that control $\mathrm{V}_{\mathrm{m}}$ and $\mathrm{Ca}^{2+}$-dependent inactivation are as follows:

$$
\begin{aligned}
& \mathrm{I}_{\mathrm{V}, \tau}^{\mathrm{P}}=\frac{1}{\frac{1}{70.0 \cdot\left(1+\exp \left(\left(\mathrm{V}_{\mathrm{m}}+49.10\right) / 10.349\right)\right)}+\frac{1}{75 \cdot\left(1+\exp \left(-\left(\mathrm{V}_{\mathrm{m}}+0.213\right) / 10.807\right)\right)}} \\
& \mathrm{I}_{\mathrm{V}, \infty}^{\mathrm{P}}=\frac{1}{1.02} \cdot\left(0.02+\frac{1}{1+\exp \left(\left(\mathrm{V}_{\mathrm{m}}+29.979\right) / 3.1775\right)}\right) \\
& \mathrm{Is}_{\mathrm{V}, \infty}^{\mathrm{P}}=\frac{1}{1.0004} \cdot\left(0.0004+\frac{1}{1+\exp \left(\left(\mathrm{V}_{\mathrm{m}}+29.979\right) / 3.1775\right)}\right)
\end{aligned}
$$




\subsubsection{HRdßARS Stoch version (Chapter 7)}

The model structure and majority of parameters are identical to those in section 1.9.2. Parameters that control $\mathrm{V}_{\mathrm{m}}$ and $\mathrm{Ca}^{2+}$-dependent inactivation of the non-phosphorylated and phosphorylated $\mathrm{I}_{\mathrm{CaL}}$ formulations were adjusted slightly to reduce the sensitivity of the model for the generation of early afterdepolarizations (EADs) in response to $\mathrm{I}_{\mathrm{kr}}$ blockade (requiring $80 \% \mathrm{I}_{\mathrm{kr}}$ reduction to generate EADs compared to $25 \%$ in the absence of these changes) and to match the amount of action potential duration (APD) prolongation after $I_{\mathrm{Ks}}$ blockade in the presence of adrenergic stimulation, while maintaining peak I-V and inactivation characteristics similar to experimental recordings.

$$
\begin{aligned}
& \mathrm{I}_{\mathrm{V}, \tau}^{\mathrm{NP}}=\frac{1}{\frac{70.0 \cdot\left(1+\exp \left(\left(\mathrm{V}_{\mathrm{m}}+49.10\right) / 10.349\right)\right)}{26.553 \cdot\left(1+\exp \left(-\left(\mathrm{V}_{\mathrm{m}}+0.213\right) / 10.807\right)\right)}} \\
& \mathrm{I}_{\mathrm{V}, \infty}^{\mathrm{NP}}=\frac{1}{1.2474} \cdot\left(0.2474+\frac{1}{1+\exp \left(\left(\mathrm{V}_{\mathrm{m}}+19.0\right) / 2.5\right)}\right) \\
& \mathrm{Is}_{\mathrm{V}, \infty}^{\mathrm{NP}}=\frac{1}{1.04} \cdot\left(0.04+\frac{1}{1+\exp \left(\left(\mathrm{V}_{\mathrm{m}}+19.0\right) / 2.5\right)}\right) \\
& \mathrm{I}_{\mathrm{V}, \tau}^{\mathrm{P}}=\frac{\frac{1}{70.0 \cdot\left(1+\exp \left(\left(\mathrm{V}_{\mathrm{m}}+49.10\right) / 10.349\right)\right)}+\frac{1}{75 \cdot\left(1+\exp \left(-\left(\mathrm{V}_{\mathrm{m}}+0.213\right) / 10.807\right)\right)}}{1} \\
& \mathrm{I}_{\mathrm{V}, \infty}^{\mathrm{P}}=\frac{1}{1.02} \cdot\left(0.02+\frac{1}{1+\exp \left(\left(\mathrm{V}_{\mathrm{m}}+29.979\right) / 3.1775\right)}\right) \\
& \mathrm{Is}_{\mathrm{V}, \infty}^{\mathrm{P}}=\frac{1}{1.0007} \cdot\left(0.0007+\frac{1}{1+\exp \left(\left(\mathrm{V}_{\mathrm{m}}+29.979\right) / 3.1775\right)}\right)
\end{aligned}
$$

\subsection{Sarcolemmal Ca ${ }^{2+}$ pump $\left(I_{\mathrm{pCa}}\right)$}

\subsubsection{HRd2009 and HRdßARS version (Chapters 2-6)}

The formulation for the sarcolemmal calcium pump is equivalent to that in the original HRd model. ${ }^{4}$

$\overline{\mathrm{G}}_{\mathrm{pCa}}=0.0575 \mathrm{mS} / \mu \mathrm{F}, \quad \mathrm{K}_{\mathrm{m}, \mathrm{pCa}}=5.00 \cdot 10^{-4} \mathrm{mmol} / \mathrm{L}$

$\mathrm{I}_{\mathrm{pCa}}=\overline{\mathrm{G}}_{\mathrm{pCa}} \cdot \frac{\left[\mathrm{Ca}^{2+}\right]_{\mathrm{i}}}{\mathrm{K}_{\mathrm{m}, \mathrm{pCa}}+\left[\mathrm{Ca}^{2+}\right]_{\mathrm{i}}}$

\subsubsection{HRdßARS Stoch version (Chapter 7)}

The SR and sarcolemmal $\mathrm{Ca}^{2+}$-ATPases (underlying $\mathrm{I}_{\mathrm{up}}$ and $\mathrm{I}_{\mathrm{pCa}}$, respectively) were simulated using the two-state, four-transition model structure of Tran et al. ${ }^{31}$ Parameters of the models were adjusted to reproduce the $\mathrm{Ca}^{2+}$ dependence of the deterministic formulations (section 1.10.1). We assumed that the state distribution changes more rapidly than the changes in ionic concentrations on both sides of the pump, resulting in a quasi-equilibrium situation in which individual rates are decoupled. The 
number of ions transported via each transition was derived from a Binomial distribution and the net ion flux (or current) in the interval $\Delta \mathrm{t}$ was determined.

$$
\begin{aligned}
& \overline{\mathrm{N}}_{\mathrm{pCa}}=2.00 \cdot 10^{6} \text { pumps } \\
& \mathrm{k}_{\mathrm{pCa}, 1+}=5.2272 \cdot 10^{3} \cdot\left[\mathrm{Ca}^{2+}\right]_{\mathrm{i}} \cdot \frac{1.00 \cdot 10^{5}}{\overline{\mathrm{N}}_{\mathrm{pCa}}}, \quad \mathrm{k}_{\mathrm{pCa}, 1-}=1.7362 \cdot \frac{1.00 \cdot 10^{5}}{\overline{\mathrm{N}}_{\mathrm{pCa}}} \\
& \mathrm{k}_{\mathrm{pCa}, 2-}=0.2503 \cdot \frac{1.00 \cdot 10^{5}}{\overline{\mathrm{N}}_{\mathrm{pCa}}} \\
& \mathrm{n}_{\mathrm{pCa}}=\frac{\mathrm{k}_{\mathrm{pCa}, 2-}}{\mathrm{k}_{\mathrm{pCa}, 1+}+\mathrm{k}_{\mathrm{pCa}, 1-}+\mathrm{k}_{\mathrm{pCa}, 2-}} \cdot \overline{\mathrm{N}}_{\mathrm{pCa}} \\
& \mathrm{I}_{\mathrm{pCa}}=\frac{\mathrm{B}\left(\mathrm{n}_{\mathrm{pCa}}, \mathrm{k}_{\mathrm{pCa}, 1+} \cdot \Delta \mathrm{t}\right) \cdot \mathrm{z}_{\mathrm{Ca}} \cdot 10^{9} \cdot \mathrm{F}}{\Delta \mathrm{t} \cdot \mathrm{N}_{\mathrm{A}} \cdot \mathrm{C}_{\mathrm{m}} \cdot \mathrm{A}_{\mathrm{cap}}}
\end{aligned}
$$

\subsection{Background $\mathrm{Cl}^{-}$current $\left(\mathrm{I}_{\mathrm{Cl}, \mathrm{b}}\right)$}

\subsubsection{HRd2009, HRdßARS and HRdßARSStoch version (Chapters 2-7)}

The formulation for the background chloride current is equivalent to that in the original HRd model. ${ }^{4}$

$\mathrm{G}_{\mathrm{Cl}, \mathrm{b}}=2.2500 \mathrm{pA} / \mathrm{pF}$

$\mathrm{I}_{\mathrm{Cl}, \mathrm{b}}=\mathrm{G}_{\mathrm{Cl}, \mathrm{b}} \cdot\left(\mathrm{V}_{\mathrm{m}}-\mathrm{E}_{\mathrm{Cl}}\right)$

\section{$1.12 \mathrm{Ca}^{2+}$-dependent $\mathrm{Cl}^{-}$current $\left(\mathrm{I}_{\mathrm{Cl}(\mathrm{Ca})} / \mathrm{I}_{\mathrm{To}, 2}\right)$}

\subsubsection{HRd2009, HRdßARS and HRdßARSStoch version (Chapters 2-7)}

A formulation for $I_{\mathrm{Cl}\left(\mathrm{Ca}_{\mathrm{a}} /\right.} / \mathrm{I}_{\mathrm{To}, 2}$ was developed and fit to data from canine epicardial myocytes. ${ }^{32}$ The formulation reflects the following properties of $\mathrm{I}_{\mathrm{CI}(\mathrm{Ca})}$ : i) $\mathrm{Cl}^{-}$carrying current ii) activation by SR $\left[\mathrm{Ca}^{2+}\right.$ ] release and iii) current voltage relationship ${ }^{32}$.

$$
\begin{aligned}
\mathrm{k}_{\mathrm{Ca}, \mathrm{Cl}} & =0.4, \quad \tau_{\mathrm{Cl}(\mathrm{Ca})}=8 \mathrm{~ms}, \quad \mathrm{P}_{\mathrm{Cl}}=9.0 \cdot 10^{-7} \mathrm{~cm} / \mathrm{s} \\
\mathrm{K}_{\mathrm{Cl}(\mathrm{Ca})} & =\frac{1}{1+\left(\frac{\mathrm{J}_{\mathrm{Rel}}}{\mathrm{k}_{\mathrm{Ca}, \mathrm{Cl}}}\right)^{2}} \\
\alpha_{\mathrm{Cl}(\mathrm{Ca})} & =\frac{0.025}{1+\exp \left(\frac{\mathrm{V}_{\mathrm{m}}+58}{5.0}\right)} \\
\beta_{\mathrm{Cl}(\mathrm{Ca})} & =\frac{1}{5 \cdot\left(1+\exp \left(\frac{\mathrm{V}_{\mathrm{m}}+19}{-9.0}\right)\right)} \\
\mathrm{i}_{\mathrm{Cl}(\mathrm{Ca}), \infty} & =\frac{\alpha_{\mathrm{Ca}, \mathrm{Cl}}}{\alpha_{\mathrm{Ca}, \mathrm{Cl}}+\beta_{\mathrm{Ca}, \mathrm{Cl}}}
\end{aligned}
$$


$\frac{\mathrm{di}_{\mathrm{Cl}(\mathrm{Ca})}}{\mathrm{dt}}=\frac{\mathrm{i}_{\mathrm{Cl}(\mathrm{Ca}), \infty}-\mathrm{i}_{\mathrm{Cl}(\mathrm{Ca})}}{\tau_{\mathrm{Cl}(\mathrm{Ca})}}$

$\overline{\mathrm{I}}_{\mathrm{Cl}(\mathrm{Ca})}=\mathrm{P}_{\mathrm{Cl}} \cdot\left(\mathrm{z}_{\mathrm{Cl}}\right)^{2} \cdot \frac{\mathrm{V}_{\mathrm{m}} \cdot \mathrm{F}^{2}}{\mathrm{R} \cdot \mathrm{T}} \cdot \frac{\left[\mathrm{Cl}^{-}\right]_{\mathrm{i}}-\left[\mathrm{Cl}^{-}\right]_{\mathrm{o}} \cdot \exp \left(-\mathrm{z}_{\mathrm{Cl}} \cdot \mathrm{V}_{\mathrm{m}} \cdot \frac{\mathrm{F}}{\mathrm{R} \cdot \mathrm{T}}\right)}{1-\exp \left(-\mathrm{z}_{\mathrm{Cl}} \cdot \mathrm{V}_{\mathrm{m}} \cdot \frac{\mathrm{F}}{\mathrm{R} \cdot \mathrm{T}}\right)}$

$\mathrm{I}_{\mathrm{Cl}(\mathrm{Ca})}=\overline{\mathrm{I}}_{\mathrm{Cl}(\mathrm{Ca})} \cdot \mathrm{i}_{\mathrm{Cl}(\mathrm{Ca})} \cdot \mathrm{K}_{\mathrm{Cl}(\mathrm{Ca})}$

\subsection{Diffusion between subcellular compartments ( $\left.I_{\text {diff,Ca, }} I_{\text {diff,Cl, }} I_{\text {diff,Na, }} I_{t r}\right)$}

\subsubsection{HRd2009 version (Chapter 2)}

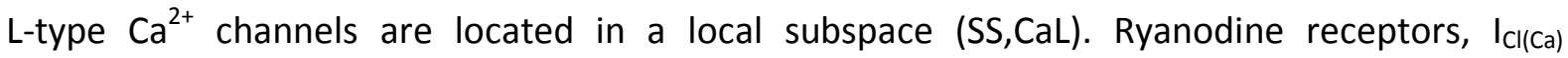
channels and $20 \%$ of $\mathrm{Na}^{+}-\mathrm{Ca}^{2+}$ exchangers are located in a $\mathrm{Ca}^{2+}$ release subspace (SS,SR). $\mathrm{Ca}^{2+}$ diffusion occurs between SS,CaL and SS,SR ( $\left.\mathrm{I}_{\text {diff,SS}}\right)$ and diffusion of $\mathrm{Ca}^{2+}, \mathrm{Na}^{+}$and $\mathrm{Cl}^{-}$occur between the SS,SR and the bulk myoplasm (I $I_{\text {diff,Ca, }} I_{\text {diff,Na }}$ and $\left.I_{\text {diff,Cl }}\right)$. The time constant of NSR/JSR $\mathrm{Ca}^{2+}$ transfer $\left(\mathrm{I}_{\mathrm{tr}}\right)$ was adjusted to $25 \mathrm{~ms}$.

$\mathrm{I}_{\mathrm{tr}}=\frac{\left[\mathrm{Ca}^{2+}\right]_{\mathrm{NSR}}-\left[\mathrm{Ca}^{2+}\right]_{\mathrm{JSR}}}{\tau_{\mathrm{tr}}}, \quad \tau_{\mathrm{tr}}=25 \mathrm{~ms}$

$\mathrm{I}_{\text {Diff,SS }}=\frac{\left[\mathrm{Ca}^{2+}\right]_{\mathrm{SS}, \mathrm{SR}}-\left[\mathrm{Ca}^{2+}\right]_{\mathrm{SS}, \mathrm{CaL}}}{\tau_{\text {Diff,SS }}}, \quad \tau_{\text {Diff,SS }}=2.0 \mathrm{~ms}$

$\mathrm{I}_{\text {Diff,Ca }}=\frac{\left[\mathrm{Ca}^{2+}\right]_{\mathrm{SS}, \mathrm{SR}}-\left[\mathrm{Ca}^{2+}\right]_{\mathrm{i}}}{\tau_{\text {Diff }}}, \quad \tau_{\text {Diff }}=0.2 \mathrm{~ms}$

$\mathrm{I}_{\text {Diff,Na }}=\frac{\left[\mathrm{Na}^{+}\right]_{\mathrm{SS}, \mathrm{SR}}-\left[\mathrm{Na}^{+}\right]_{\mathrm{i}}}{\tau_{\text {Diff }}}$

$\mathrm{I}_{\text {Diff,Cl }}=\frac{\left[\mathrm{Cl}^{-}\right]_{\mathrm{SS}, \mathrm{SR}}-\left[\mathrm{Cl}^{-}\right]_{\mathrm{i}}}{\tau_{\text {Diff }}}$

\subsubsection{HRd $\beta A R S$ and HRd $\beta A R S$ stoch version (Chapters $3,4,5,7$ )}

Model structure in the HRdBARS models is identical to that of the HRd2009 model. The time constant of recovery for the SR $\mathrm{Ca}^{2+}$ system, represented by $\tau_{\text {tr }}$ which is independent of RyR phosphorylation, is increased to $75 \mathrm{~ms}$, closer to the value in the original HRd model ${ }^{4}$ in order to prevent premature secondary $\mathrm{Ca}^{2+}$ induced $\mathrm{Ca}^{2+}$ release events due to the high $\mathrm{SR} \mathrm{Ca}^{2+}$ load and large $\mathrm{I}_{\mathrm{CaL}}$ during $\beta$-adrenergic stimulation which would result in nonmonotonic decay of the $\mathrm{Ca}^{2+}$ transient, which is not observed experimentally. The time constant of $\mathrm{Ca}^{2+}$ diffusion is also adjusted.

$\tau_{\text {Diff,SS }}=0.02 \mathrm{~ms}$

$\tau_{\mathrm{tr}}=75 \mathrm{~ms}$ 


\subsubsection{HRdßARS SCR version (Chapter 6)}

The time constant of $\mathrm{Ca}^{2+}$ transfer from NSR to JSR was further slowed to $100 \mathrm{~ms}$ to match experimental data on the amount of APD prolongation after spontaneous $\mathrm{Ca}^{2+}$ release (Chapter 5 ).

$\tau_{\text {Diff,SS }}=0.02 \mathrm{~ms}$

$\tau_{\mathrm{tr}}=100 \mathrm{~ms}$

\subsection{Inward rectifier $\mathrm{K}^{+}$current $\left(\mathrm{I}_{\mathrm{K} 1}\right)$}

\subsubsection{HRd2009 version (Chapter 2)}

The formulation for the inward rectifier potassium current is equivalent to that in the original HRd model. ${ }^{4}$

$\alpha_{\mathrm{K} 1}=\frac{1.02}{1+\exp \left(2.385 \cdot 10^{-1} \cdot\left(\mathrm{V}_{\mathrm{m}}-\mathrm{E}_{\mathrm{K}}-59.22\right)\right)}$

$\beta_{\mathrm{K} 1}$

$=\frac{0.4912 \cdot \exp \left(8.032 \cdot 10^{-2} \cdot\left(\mathrm{V}_{\mathrm{m}}-\mathrm{E}_{\mathrm{K}}+5.476\right)\right)+\exp \left(6.175 \cdot 10^{-2} \cdot\left(\mathrm{V}_{\mathrm{m}}-\mathrm{E}_{\mathrm{K}}-5.943 \cdot 10^{2}\right)\right)}{1+\exp \left(-0.5143 \cdot\left(\mathrm{V}_{\mathrm{m}}-\mathrm{E}_{\mathrm{K}}+4.753\right)\right)}$

$\mathrm{K}_{1}=\frac{\alpha_{\mathrm{K} 1}}{\alpha_{\mathrm{K} 1}+\beta_{\mathrm{K} 1}}$

$\overline{\mathrm{G}}_{\mathrm{K} 1}=0.500 \cdot \sqrt{\frac{\left[\mathrm{K}^{+}\right]_{\mathrm{o}}}{5.400}}$

$\mathrm{I}_{\mathrm{K} 1}=\overline{\mathrm{G}}_{\mathrm{K} 1} \cdot \mathrm{K}_{1} \cdot\left(\mathrm{V}_{\mathrm{m}}-\mathrm{E}_{\mathrm{K}}\right)$

\subsubsection{HRdßARS and HRdßARSStoch version (Chapters 3-7)}

Wagner et al recently described complex regulation of $\mathrm{I}_{\mathrm{K} 1}$ by CaMKII. ${ }^{33}$ CaMKII overexpression (either transgenic or acutely with adenovirus) resulted in $30 \%$ decrease in $I_{K 1}$ channel protein. However, $I_{K 1}$ current was increased in rabbit ventricular myocytes acutely overexpressing CaMKII, and this effect was abolished by CaMKII inhibition with AIP. Thus, it is likely that CaMKII phosphorylation increases $I_{K 1}$ and that this effect can offset the reduction in channel protein expression resulting from long term CaMKII activity. Based on this mechanism, we included a $20 \%$ upregulation of $I_{\mathrm{K} 1}$ in CaMKII phosphorylated channels.

Nonphosphorylated Channels:

$$
\begin{aligned}
& \alpha_{\mathrm{K} 1}=\frac{1.02}{1+\exp \left(0.2385 \cdot\left(\mathrm{V}_{\mathrm{m}}-\mathrm{E}_{\mathrm{K}}-59.215\right)\right)} \\
& \beta_{\mathrm{K} 1}=\frac{0.49124 \cdot \exp \left(0.08032 \cdot\left(\mathrm{V}_{\mathrm{m}}-\mathrm{E}_{\mathrm{K}}+5.476\right)\right)+\exp \left(0.06175 \cdot\left(\mathrm{V}_{\mathrm{m}}-\mathrm{E}_{\mathrm{K}}-594.31\right)\right)}{1+\exp \left(-0.5143 \cdot\left(\mathrm{V}_{\mathrm{m}}-\mathrm{E}_{\mathrm{K}}+4.753\right)\right)}
\end{aligned}
$$


$\overline{\mathrm{G}}_{\mathrm{K} 1}=0.500 \cdot \sqrt{\frac{\left[\mathrm{K}^{+}\right]_{o}}{5.400}}$
$\mathrm{I}_{\mathrm{K} 1}^{\mathrm{NP}}=\overline{\mathrm{G}}_{\mathrm{K} 1} \cdot \frac{\alpha_{\mathrm{K} 1}}{\alpha_{\mathrm{K} 1}+\beta_{\mathrm{K} 1}} \cdot\left(\mathrm{V}_{\mathrm{m}}-\mathrm{E}_{\mathrm{K}}\right)$

CaMKII phosphorylated Channels:

$\mathrm{I}_{\mathrm{K} 1}^{\mathrm{CaMKII}}=1.200 \cdot \overline{\mathrm{G}}_{\mathrm{K} 1} \cdot \frac{\alpha_{\mathrm{K} 1}}{\alpha_{\mathrm{K} 1}+\beta_{\mathrm{K} 1}} \cdot\left(\mathrm{V}_{\mathrm{m}}-\mathrm{E}_{\mathrm{K}}\right)$

Combined I 1 c current:

$\mathrm{I}_{\mathrm{K} 1}=\left(1-\mathrm{f}_{\mathrm{K} 1}^{\mathrm{P} \text {,CaMKII }}\right) \cdot \mathrm{I}_{\mathrm{K} 1}^{\mathrm{NP}}+\mathrm{f}_{\mathrm{IK} 1}^{\mathrm{P}, \text { CaMKII }} \cdot \mathrm{I}_{\mathrm{K} 1}^{\mathrm{P}, \text { CaMKII }}$

\subsection{Rapidly activating delayed rectifier $\mathrm{K}^{+}$current $\left(\mathrm{I}_{\mathrm{Kr}}\right)$}

\subsubsection{HRd2009 and HRdßARS version (Chapters 2-6)}

The formulation for the rapid component of the delayed rectifier potassium current is equivalent to that in the original HRd model. ${ }^{4}$

$$
\begin{aligned}
& \tau_{\mathrm{Xr}}=\frac{1}{\frac{6.000 \cdot 10^{-4} \cdot\left(\mathrm{V}_{\mathrm{m}}-1.738\right)}{1-\exp \left(-0.136 \cdot\left(\mathrm{V}_{\mathrm{m}}-1.738\right)\right)}+\frac{3.000 \cdot 10^{-4} \cdot\left(\mathrm{V}_{\mathrm{m}}+38.36\right)}{\exp \left(0.152 \cdot\left(\mathrm{V}_{\mathrm{m}}+38.36\right)\right)-1}} \\
& \mathrm{X}_{\mathrm{r}, \infty}=\frac{1}{1+\exp \left(-\left(\mathrm{V}_{\mathrm{m}}+10.08\right) / 4.250\right)} \\
& \frac{1}{\mathrm{dt}}=\frac{\mathrm{X}_{\mathrm{Kr}, \infty}-\mathrm{X}_{\mathrm{r}}}{1+\exp \left(\left(\mathrm{V}_{\mathrm{m}}+10.00\right) / 15.40\right)} \\
& \overline{\mathrm{G}}_{\mathrm{Kr}}=1.385 \cdot 10^{-2} \cdot \sqrt{\frac{\left[\mathrm{K}^{+}\right]_{\mathrm{o}}}{5.400}} \\
& \mathrm{I}_{\mathrm{Kr}}=\overline{\mathrm{G}}_{\mathrm{Kr}} \cdot \mathrm{X}_{\mathrm{r}} \cdot \mathrm{R}_{\mathrm{Kr}} \cdot\left(\mathrm{V}_{\mathrm{m}}-\mathrm{E}_{\mathrm{K}}\right)
\end{aligned}
$$

\subsubsection{HRdßARS Stoch version (Chapter 7)}

The original Hodgkin-Huxley based formulation of $I_{\mathrm{kr}}$ was replaced with a 10-state Markov model based on the model structure described by Silva and Rudy ${ }^{34}$ extended with state-dependent block of $\mathrm{I}_{\mathrm{kr}}$ channels by dofetilide (Figure 1A of Chapter 7 ). Model parameters were adjusted to reproduce $\mathrm{I}_{\mathrm{kr}}$ peak and tail I-V relationships, time constants of activation and time constants of deactivation measured in canine ventricular myocytes ${ }^{35}$ (Figure 1B-D of Chapter 7). Channels can enter the 
blocked mode via open and inactivated states, based on a $V_{m}$-dependent modulation of the dofetilide $\mathrm{EC} 50 .{ }^{36}$ The model reproduces dofetilide dose-response relationship measured in rabbit ventricular myocytes, ${ }^{37}$ use-dependence of relative inhibition as determined in AT-1 cells ${ }^{36}$ and $V_{m}$ dependence of dofetilide $\mathrm{EC}^{36}{ }^{36}$ (Figure 1E-G of Chapter 7).

$$
\begin{aligned}
& \alpha_{\mathrm{Kr}, 2}=4.8712 \cdot 10^{-3} \cdot \exp \left(2.5082 \cdot \mathrm{V}_{\mathrm{m}} \cdot \frac{\mathrm{F}}{\mathrm{R} \cdot \mathrm{T}}\right) \\
& \beta_{\mathrm{Kr}, 2}=5.0582 \cdot 10^{-4} \cdot \exp \left(0.8035 \cdot \mathrm{V}_{\mathrm{m}} \cdot \frac{\mathrm{F}}{\mathrm{R} \cdot \mathrm{T}}\right) \\
& \alpha_{\mathrm{Kr}, 1}=3.1224, \beta_{\mathrm{Kr}, 1}=2.3685 \\
& \alpha_{\mathrm{Kr}}=1.1070 \cdot 10^{-2} \cdot \exp \left(1.5816 \cdot \mathrm{V}_{\mathrm{m}} \cdot \frac{\mathrm{F}}{\mathrm{R} \cdot \mathrm{T}}\right) \\
& \beta_{\mathrm{Kr}}=3.7892 \cdot 10^{-3} \cdot \exp \left(1.1872 \cdot \mathrm{V}_{\mathrm{m}} \cdot \frac{\mathrm{F}}{\mathrm{R} \cdot \mathrm{T}}\right) \\
& \alpha_{\mathrm{Kr}, \mathrm{i}}=4.8457 \cdot 10^{-1} \cdot \exp \left(0.7296 \cdot \mathrm{V}_{\mathrm{m}} \cdot \frac{\mathrm{F}}{\mathrm{R} \cdot \mathrm{T}}\right) \cdot \frac{4.5}{\left[\mathrm{~K}^{+}\right]_{\mathrm{o}}} \\
& \beta_{\mathrm{Kr}, \mathrm{i}}=1.2120 \cdot \exp \left(0.5816 \cdot \mathrm{V}_{\mathrm{m}} \cdot \frac{\mathrm{F}}{\mathrm{R} \cdot \mathrm{T}}\right) \cdot\left(\frac{4.5}{\left[\mathrm{~K}^{+}\right]_{\mathrm{o}}}\right)^{0.3} \\
& \mu_{\mathrm{Kr}}=\frac{\alpha_{\mathrm{Kr}, \mathrm{i}} \cdot \beta_{\mathrm{Kr}, 2}}{\beta_{\mathrm{Kr}, \mathrm{i}}} \\
& \mathrm{k}_{\text {Dof,on }}=5.2353 \cdot 10^{-3} \cdot[\text { Dof }], \quad \mathrm{k}_{\text {Dof,off }}=3.2172 \cdot 10^{-4} \\
& \mathrm{k}_{\text {Dof,on,i }}=2.7898 \cdot 10^{-2} \cdot[\text { Dof }], \quad \mathrm{k}_{\text {Dof,off,i }}=8.7055 \cdot 10^{-4} \\
& \alpha_{\mathrm{Kr}, \mathrm{i}, \mathrm{Dof}}=\frac{\mathrm{k}_{\mathrm{Dof}, \mathrm{off}, \mathrm{i}} \cdot \alpha_{\mathrm{Kr}, \mathrm{i}} \cdot \mathrm{k}_{\mathrm{Dof}, \mathrm{on}}}{\mathrm{k}_{\text {Dof,off }} \cdot \mathrm{k}_{\mathrm{Dof}, \mathrm{on}, \mathrm{i}}} \\
& \mu_{\mathrm{Kr}, \mathrm{Dof}}=\frac{\alpha_{\mathrm{Kr}, \mathrm{i}, \mathrm{Dof}} \cdot \beta_{\mathrm{Kr}, 2}}{\beta_{\mathrm{Kr}, \mathrm{i}}} \\
& \frac{\mathrm{dC} 3_{\mathrm{Kr}}}{\mathrm{dt}}=\beta_{\mathrm{Kr}} \cdot \mathrm{C} 2_{\mathrm{Kr}}-\alpha_{\mathrm{Kr}} \cdot \mathrm{C} 3_{\mathrm{Kr}} \\
& \frac{\mathrm{dC} 2_{\mathrm{Kr}}}{\mathrm{dt}}=\beta_{\mathrm{Kr}, 1} \cdot \mathrm{C} 1_{\mathrm{Kr}}+\alpha_{\mathrm{Kr}} \cdot \mathrm{C} 3_{\mathrm{Kr}}-\left(\alpha_{\mathrm{Kr}, 1}+\beta_{\mathrm{Kr}}\right) \cdot \mathrm{C} 2_{\mathrm{Kr}} \\
& \frac{\mathrm{dC} 1_{\mathrm{Kr}}}{\mathrm{dt}}=\alpha_{\mathrm{Kr}, 1} \cdot \mathrm{C} 2_{\mathrm{Kr}}+\beta_{\mathrm{Kr}, 2} \cdot \mathrm{O}_{\mathrm{Kr}}+\mu_{\mathrm{Kr}} \cdot \mathrm{In}_{\mathrm{Kr}}-\left(2 \cdot \alpha_{\mathrm{Kr}, 2}+\beta_{\mathrm{Kr}, 1}\right) \cdot \mathrm{C} 1_{\mathrm{Kr}} \\
& \frac{\mathrm{dO}_{\mathrm{Kr}}}{\mathrm{dt}}=\alpha_{\mathrm{Kr}, \mathrm{i}} \cdot \mathrm{In}_{\mathrm{Kr}}+\alpha_{\mathrm{Kr}, 2} \cdot \mathrm{C} 1_{\mathrm{Kr}}+\mathrm{k}_{\text {Dof,off }} \cdot \mathrm{B}_{\mathrm{Kr}}-\left(\beta_{\mathrm{Kr}, \mathrm{i}}+\beta_{\mathrm{Kr}, 2}+\mathrm{k}_{\mathrm{Dof}, \mathrm{on}}\right) \cdot \mathrm{O}_{\mathrm{Kr}} \\
& \frac{\mathrm{dIn}}{\mathrm{dt}}=\alpha_{\mathrm{Kr}, 2} \cdot \mathrm{C} 1_{\mathrm{Kr}}+\beta_{\mathrm{Kr}, \mathrm{i}} \cdot \mathrm{O}_{\mathrm{Kr}}+\mathrm{k}_{\mathrm{Dof}, \mathrm{off}, \mathrm{i}} \cdot \operatorname{InB}_{\mathrm{Kr}}-\left(\mu_{\mathrm{Kr}}+\alpha_{\mathrm{Kr}, \mathrm{i}}+\mathrm{k}_{\mathrm{Dof}, \mathrm{on}, \mathrm{i}}\right) \cdot \operatorname{In}_{\mathrm{Kr}} \\
& \frac{\mathrm{dC} 3 \mathrm{~B}_{\mathrm{Kr}}}{\mathrm{dt}}=\beta_{\mathrm{Kr}} \cdot \mathrm{C} 2 \mathrm{~B}_{\mathrm{Kr}}-\alpha_{\mathrm{Kr}} \cdot \mathrm{C} 3 \mathrm{~B}_{\mathrm{Kr}} \\
& \frac{\mathrm{dC} 2 \mathrm{~B}_{\mathrm{Kr}}}{\mathrm{dt}}=\beta_{\mathrm{Kr}, 1} \cdot \mathrm{C} 1 \mathrm{~B}_{\mathrm{Kr}}+\alpha_{\mathrm{Kr}} \cdot \mathrm{C} 3 \mathrm{~B}_{\mathrm{Kr}}-\left(\alpha_{\mathrm{Kr}, 1}+\beta_{\mathrm{Kr}}\right) \cdot \mathrm{C} 2 \mathrm{~B}_{\mathrm{Kr}}
\end{aligned}
$$




$$
\begin{aligned}
& \frac{\mathrm{dC} 1 \mathrm{~B}_{\mathrm{Kr}}}{\mathrm{dt}}=\alpha_{\mathrm{Kr}, 1} \cdot \mathrm{C} 2 \mathrm{~B}_{\mathrm{Kr}}+\beta_{\mathrm{Kr}, 2} \cdot \mathrm{B}_{\mathrm{Kr}}+\mu_{\mathrm{Kr}, \mathrm{Dof}} \cdot \mathrm{InB}_{\mathrm{Kr}}-\left(2 \cdot \alpha_{\mathrm{Kr}, 2}+\beta_{\mathrm{Kr}, 1}\right) \cdot \mathrm{C} 1 \mathrm{~B}_{\mathrm{Kr}} \\
& \frac{\mathrm{dB} \mathrm{Kr}}{\mathrm{dt}}=\alpha_{\mathrm{Kr}, \mathrm{i}, \mathrm{Dof}} \cdot \mathrm{InB}_{\mathrm{Kr}}+\alpha_{\mathrm{Kr}, 2} \cdot \mathrm{C} \mathrm{B}_{\mathrm{Kr}}+\mathrm{k}_{\mathrm{Dof}, \mathrm{on}} \cdot \mathrm{O}_{\mathrm{Kr}}-\left(\beta_{\mathrm{Kr}, \mathrm{i}}+\beta_{\mathrm{Kr}, 2}+\mathrm{k}_{\mathrm{Dof}, \mathrm{off}}\right) \cdot \mathrm{B}_{\mathrm{Kr}} \\
& \frac{\mathrm{dInB}}{\mathrm{dt}}=\alpha_{\mathrm{Kr}, 2} \cdot \mathrm{C} 1 \mathrm{~B}_{\mathrm{Kr}}+\beta_{\mathrm{Kr}, \mathrm{i}} \cdot \mathrm{B}_{\mathrm{Kr}}+\mathrm{k}_{\mathrm{Dof}, \mathrm{on}, \mathrm{i}} \cdot \operatorname{In}_{\mathrm{Kr}}-\left(\mu_{\mathrm{Kr}, \mathrm{Dof}}+\alpha_{\mathrm{Kr}, \mathrm{i}, \mathrm{Dof}}+\mathrm{k}_{\mathrm{Dof}, \mathrm{off}, \mathrm{i}}\right) \cdot \mathrm{InB}_{\mathrm{Kr}} \\
& \mathrm{G}_{\mathrm{Kr}}=5.2530 \cdot 10^{-3} \cdot\left(\left[\mathrm{K}^{+}\right]_{\mathrm{o}}\right)^{0.59} \\
& \mathrm{I}_{\mathrm{Kr}}=\mathrm{G}_{\mathrm{Kr}} \cdot \mathrm{O}_{\mathrm{Kr}} \cdot\left(\mathrm{V}_{\mathrm{m}}-\mathrm{E}_{\mathrm{K}}\right)
\end{aligned}
$$

\subsection{Slowly activating delayed rectifier $\mathrm{K}^{+}$current $\left(\mathrm{I}_{\mathrm{Ks}}\right)$}

\subsubsection{HRd2009 version (Chapter 2)}

The Hodgkin-Huxley based formulation for $I_{\mathrm{Ks}}$ used in a previous model of the canine epicardial myocyte ${ }^{4}$ was replaced with the 17 state Markov model proposed by Silva and Rudy ${ }^{34}$. The structure of the $I_{\mathrm{Ks}}$ model is shown in Figure $\mathbf{1 1}$ of Chapter 2. The original Silva-Rudy model accurately reproduces the kinetics of $I_{\mathrm{Ks}}$ in guinea pig and human based on underlying voltage sensor transitions and has provided insight into the role of $I_{\mathrm{Ks}}$ in APD adaptation in these species. ${ }^{34}$ Transition rate parameters were adjusted to fit the following data from canine epicardial and midmyocardial myocytes: i) tail current amplitudes at holding potentials from -20 to $+70 \mathrm{mV}$ after $300 \mathrm{~ms}$ and 3000 $\mathrm{ms}$ of activation ${ }^{38}$; ii) tail currents at holding potentials from -10 to $60 \mathrm{mV}$ after $5000 \mathrm{~ms}$ of activation ${ }^{35}, 39$; iii) activation half-time on depolarization to $+20 \mathrm{mV}^{35}$, 39; iv) deactivation half-times for tail currents to $-25,-50$ and $-80 \mathrm{mV}^{35,39}$ and v) accumulation characteristics for inter-pulse intervals between 20 and $150 \mathrm{~ms}$ after $200 \mathrm{~ms}$ depolarizing steps ${ }^{39}$. Current density was scaled to that of canine epicardial myocytes. ${ }^{35,39}$

$$
\begin{aligned}
& \alpha_{\mathrm{Ks}}=1.4864 \cdot 10^{-2} \cdot \exp \left(2.9877 \cdot 10^{-2} \cdot \mathrm{V}_{\mathrm{m}} \cdot \frac{\mathrm{F}}{\mathrm{R} \cdot \mathrm{T}}\right) \\
& \beta_{\mathrm{Ks}}=8.3986 \cdot 10^{-2} \cdot \exp \left(-5.5461 \cdot 10^{-2} \cdot \mathrm{V}_{\mathrm{m}} \cdot \frac{\mathrm{F}}{\mathrm{R} \cdot \mathrm{T}}\right) \\
& \gamma_{\mathrm{Ks}}=1.4601 \cdot 10^{-2} \cdot \exp \left(2.4465 \cdot 10^{-1} \cdot \mathrm{V}_{\mathrm{m}} \cdot \frac{\mathrm{F}}{\mathrm{R} \cdot \mathrm{T}}\right) \\
& \delta_{\mathrm{Ks}}=3.1173 \cdot 10^{-3} \cdot \exp \left(-4.2625 \cdot 10^{-1} \cdot \mathrm{V}_{\mathrm{m}} \cdot \frac{\mathrm{F}}{\mathrm{R} \cdot \mathrm{T}}\right) \\
& \eta_{\mathrm{Ks}}=7.7320 \cdot 10^{-2} \cdot \exp \left(-6.4726 \cdot 10^{-2} \cdot \mathrm{V}_{\mathrm{m}} \cdot \frac{\mathrm{F}}{\mathrm{R} \cdot \mathrm{T}}\right) \\
& \theta_{\mathrm{Ks}}=8.9538 \cdot 10^{-2} \\
& \omega_{\mathrm{Ks}}=7.9405 \cdot 10^{-1} \cdot \exp \left(-8.0174 \cdot 10^{-2} \cdot \mathrm{V}_{\mathrm{m}} \cdot \frac{\mathrm{F}}{\mathrm{R} \cdot \mathrm{T}}\right) \\
& \psi_{\mathrm{Ks}}=5.8638 \cdot 10^{-1} \cdot \exp \left(2.8206 \cdot 10^{-1} \cdot \mathrm{V}_{\mathrm{m}} \cdot \frac{\mathrm{F}}{\mathrm{R} \cdot \mathrm{T}}\right)
\end{aligned}
$$




$$
\begin{aligned}
& \frac{\mathrm{dC} 1_{\mathrm{Ks}}}{\mathrm{dt}}=-\left(4 \cdot \alpha_{\mathrm{Ks}}\right) \cdot \mathrm{C} 1_{\mathrm{Ks}}+\beta_{\mathrm{KS}} \cdot \mathrm{C} 2_{\mathrm{Ks}} \\
& \frac{\mathrm{dC} 2_{\mathrm{Ks}}}{\mathrm{dt}}=-\left(3 \cdot \alpha_{\mathrm{Ks}}+\beta_{\mathrm{Ks}}+\gamma_{\mathrm{Ks}}\right) \cdot \mathrm{C} 2_{\mathrm{Ks}}+4 \cdot \alpha_{\mathrm{Ks}} \cdot \mathrm{C} 1_{\mathrm{Ks}}+2 \cdot \beta_{\mathrm{Ks}} \cdot \mathrm{C} 3_{\mathrm{Ks}}+\delta_{\mathrm{Ks}} \cdot \mathrm{C} 6_{\mathrm{Ks}} \\
& \frac{\mathrm{dC} 3_{\mathrm{Ks}}}{\mathrm{dt}}=-\left(2 \cdot \alpha_{\mathrm{Ks}}+2 \cdot \beta_{\mathrm{Ks}}+2 \cdot \gamma_{\mathrm{Ks}}\right) \cdot \mathrm{C} 3_{\mathrm{Ks}}+3 \cdot \alpha_{\mathrm{Ks}} \cdot \mathrm{C} 2_{\mathrm{Ks}}+3 \cdot \beta_{\mathrm{Ks}} \cdot \mathrm{C} 4_{\mathrm{Ks}}+\delta_{\mathrm{Ks}} \cdot \mathrm{C} 7_{\mathrm{Ks}} \\
& \frac{\mathrm{dC} 4_{\mathrm{Ks}}}{\mathrm{dt}}=-\left(\alpha_{\mathrm{Ks}}+3 \cdot \beta_{\mathrm{Ks}}+3 \cdot \gamma_{\mathrm{Ks}}\right) \cdot \mathrm{C} 4_{\mathrm{Ks}}+2 \cdot \alpha_{\mathrm{Ks}} \cdot \mathrm{C} 3_{\mathrm{Ks}}+4 \cdot \beta_{\mathrm{Ks}} \cdot \mathrm{C} 5_{\mathrm{Ks}}+\delta_{\mathrm{Ks}} \cdot \mathrm{C} 8_{\mathrm{Ks}}^{\mathrm{NP}} \\
& \frac{\mathrm{dC} 5_{\mathrm{Ks}}}{\mathrm{dt}}=-\left(4 \cdot \beta_{\mathrm{Ks}}+4 \cdot \gamma_{\mathrm{Ks}}\right) \cdot \mathrm{C} 5_{\mathrm{Ks}}+\alpha_{\mathrm{Ks}} \cdot \mathrm{C} 4_{\mathrm{Ks}}+\delta_{\mathrm{Ks}} \cdot \mathrm{C} 9_{\mathrm{Ks}} \\
& \frac{\mathrm{dC} 6_{\mathrm{Ks}}}{\mathrm{dt}}=-\left(3 \cdot \alpha_{\mathrm{Ks}}+\delta_{\mathrm{Ks}}\right) \cdot \mathrm{C} 6_{\mathrm{Ks}}+\beta_{\mathrm{Ks}} \cdot \mathrm{C} 7_{\mathrm{Ks}}+\gamma_{\mathrm{Ks}} \cdot \mathrm{C} 2_{\mathrm{Ks}} \\
& \frac{\mathrm{dC} 7_{\mathrm{Ks}}}{\mathrm{dt}}=-\left(2 \cdot \alpha_{\mathrm{Ks}}+\beta_{\mathrm{Ks}}+\gamma_{\mathrm{Ks}}+\delta_{\mathrm{Ks}}\right) \cdot \mathrm{C} 7_{\mathrm{Ks}}+3 \cdot \alpha_{\mathrm{Ks}} \cdot \mathrm{C} 6_{\mathrm{Ks}}+2 \cdot \beta_{\mathrm{Ks}} \cdot \mathrm{C} 8_{\mathrm{Ks}}+2 \cdot \gamma_{\mathrm{Ks}} \cdot \mathrm{C} 3_{\mathrm{Ks}}+2 \\
& \cdot \delta_{\mathrm{Ks}} \cdot \mathrm{C} 10_{\mathrm{Ks}} \\
& \frac{\mathrm{dC} 8_{\mathrm{Ks}}}{\mathrm{dt}}=-\left(\alpha_{\mathrm{Ks}}+2 \cdot \beta_{\mathrm{Ks}}+2 \cdot \gamma_{\mathrm{Ks}}+\delta_{\mathrm{Ks}}\right) \cdot \mathrm{C} 8_{\mathrm{Ks}}+2 \cdot \alpha_{\mathrm{Ks}} \cdot \mathrm{C} 7_{\mathrm{Ks}}+3 \cdot \beta_{\mathrm{Ks}} \cdot \mathrm{C} 9_{\mathrm{Ks}}+3 \cdot \gamma_{\mathrm{Ks}} \cdot \mathrm{C} 4_{\mathrm{Ks}} \\
& +2 \cdot \delta_{\mathrm{Ks}} \cdot \mathrm{C} 11_{\mathrm{Ks}} \\
& \frac{\mathrm{dC} 9_{\mathrm{Ks}}}{\mathrm{dt}}=-\left(3 \cdot \beta_{\mathrm{Ks}}+3 \cdot \gamma_{\mathrm{Ks}}+\delta_{\mathrm{Ks}}\right) \cdot \mathrm{C} 9_{\mathrm{Ks}}+\alpha_{\mathrm{Ks}} \cdot \mathrm{C} 8_{\mathrm{Ks}}+4 \cdot \gamma_{\mathrm{Ks}} \cdot \mathrm{C} 5_{\mathrm{Ks}}+2 \cdot \delta_{\mathrm{Ks}} \cdot \mathrm{C} 12_{\mathrm{Ks}} \\
& \frac{\mathrm{dC} 10_{\mathrm{Ks}}}{\mathrm{dt}}=-\left(2 \cdot \alpha_{\mathrm{Ks}}+2 \cdot \delta_{\mathrm{Ks}}\right) \cdot \mathrm{C} 10_{\mathrm{Ks}}+\beta_{\mathrm{Ks}} \cdot \mathrm{C} 11_{\mathrm{Ks}}+\gamma_{\mathrm{Ks}} \cdot \mathrm{C} 7_{\mathrm{Ks}} \\
& \frac{\mathrm{dC} 11_{\mathrm{Ks}}}{\mathrm{dt}}=-\left(\alpha_{\mathrm{Ks}}+\beta_{\mathrm{Ks}}+\gamma_{\mathrm{Ks}}+2 \cdot \delta_{\mathrm{Ks}}\right) \cdot \mathrm{C} 11_{\mathrm{Ks}}+2 \cdot \alpha_{\mathrm{Ks}} \cdot \mathrm{C} 10_{\mathrm{Ks}}+2 \cdot \beta_{\mathrm{Ks}} \cdot \mathrm{C} 12_{\mathrm{Ks}}+2 \cdot \gamma_{\mathrm{Ks}} \\
& \cdot \mathrm{C} 8_{\mathrm{Ks}}+3 \cdot \delta_{\mathrm{Ks}} \cdot \mathrm{C} 13_{\mathrm{Ks}} \\
& \frac{\mathrm{dC} 12_{\mathrm{Ks}}}{\mathrm{dt}}=-\left(2 \cdot \beta_{\mathrm{Ks}}+2 \cdot \gamma_{\mathrm{Ks}}+2 \cdot \delta_{\mathrm{Ks}}\right) \cdot \mathrm{C} 12_{\mathrm{Ks}}+\alpha_{\mathrm{Ks}} \cdot \mathrm{C} 11_{\mathrm{Ks}}+3 \cdot \gamma_{\mathrm{Ks}} \cdot \mathrm{C} 9_{\mathrm{Ks}}+3 \cdot \delta_{\mathrm{Ks}} \cdot \mathrm{C} 14_{\mathrm{Ks}} \\
& \frac{\mathrm{dC} 13_{\mathrm{Ks}}}{\mathrm{dt}}=-\left(\alpha_{\mathrm{Ks}}+3 \cdot \delta_{\mathrm{Ks}}\right) \cdot \mathrm{C} 13_{\mathrm{Ks}}+\beta_{\mathrm{Ks}} \cdot \mathrm{C} 14_{\mathrm{Ks}}+\gamma_{\mathrm{Ks}} \cdot \mathrm{C} 11_{\mathrm{Ks}} \\
& \frac{\mathrm{dC} 14_{\mathrm{Ks}}}{\mathrm{dt}}=-\left(\beta_{\mathrm{Ks}}+\gamma_{\mathrm{Ks}}+3 \cdot \delta_{\mathrm{Ks}}\right) \cdot \mathrm{C} 14_{\mathrm{Ks}}+\alpha_{\mathrm{Ks}} \cdot \mathrm{C} 13_{\mathrm{Ks}}+2 \cdot \gamma_{\mathrm{Ks}} \cdot \mathrm{C} 12_{\mathrm{Ks}}+4 \cdot \delta_{\mathrm{Ks}} \cdot \mathrm{C} 15_{\mathrm{Ks}} \\
& \frac{\mathrm{dC} 15_{\mathrm{Ks}}}{\mathrm{dt}}=-\left(4 \cdot \delta_{\mathrm{Ks}}+\theta_{\mathrm{Ks}}\right) \cdot \mathrm{C} 15_{\mathrm{Ks}}+\gamma_{\mathrm{Ks}} \cdot \mathrm{C} 14_{\mathrm{Ks}}+\eta_{\mathrm{Ks}} \cdot 01_{\mathrm{Ks}} \\
& \frac{\mathrm{d} 01_{\mathrm{Ks}}}{\mathrm{dt}}=-\left(\eta_{\mathrm{Ks}}+\psi_{\mathrm{Ks}}\right) \cdot 01_{\mathrm{Ks}}+\theta_{\mathrm{Ks}} \cdot \mathrm{C} 15_{\mathrm{Ks}}+\omega_{\mathrm{Ks}} \cdot 02_{\mathrm{Ks}} \\
& \frac{\mathrm{dO} 2_{\mathrm{Ks}}}{\mathrm{dt}}=-\left(\omega_{\mathrm{Ks}}\right) \cdot 02_{\mathrm{Ks}}+\psi_{\mathrm{Ks}} \cdot 01_{\mathrm{Ks}}
\end{aligned}
$$




$$
\begin{aligned}
& \overline{\mathrm{G}}_{\mathrm{Ks}}=0.19561 \cdot\left(1+\frac{0.6}{1+\left(3.8 \cdot \frac{10^{-5}}{\left[\mathrm{Ca}^{2+}\right]_{\mathrm{i}}}\right)^{1.4}}\right) \\
& \mathrm{I}_{\mathrm{Ks}}=\overline{\mathrm{G}}_{\mathrm{Ks}} \cdot\left(01_{\mathrm{Ks}}+02_{\mathrm{Ks}}\right) \cdot\left(\mathrm{V}_{\mathrm{m}}-\mathrm{E}_{\mathrm{Ks}}\right)
\end{aligned}
$$

\subsubsection{HRdßARS and HRd $\beta A R S$ Stoch version (Chapters 3,5-7)}

$I_{\mathrm{Ks}}$ kinetics, current amplitude and adrenergic modulation are species and age dependent. ${ }^{40}$ In large mammals (dog, human), $I_{\mathrm{Ks}}$ is small under baseline conditions with slow activation kinetics and fast deactivation kinetics. ${ }^{39}$ Phosphorylation by PKA significantly increases $I_{\mathrm{Ks}}$ in most environments, including guinea pig ventricular myocytes, ${ }^{41}$ human $\mathrm{I}_{\mathrm{Ks}}$ expressed in HEK cells ${ }^{42}$ and (adult) canine ventricular myocytes ${ }^{38}$. In the dog a 3-fold increase in current amplitude, left-ward shift in tail current amplitude I-V curve, increased activation kinetics and decreased deactivation kinetics are observed in the presence of isoproterenol. ${ }^{38,39}$ The 17-state Markov model (Figure 11 of Chapter 2), which represent 2 transitions of the voltage sensor for each of the 4 subunits $^{34}$ is used here. Both its baseline and $\beta A R$ versions reproduce experimental data from canine ventricular myocytes in the absence or presence of isoproterenol. ${ }^{38,39}$ The increased $I_{\mathrm{Ks}}$ observed during adrenergic stimulation can result from increased channel density, increased single channel conductance and/or increased channel open probability. Recently, Severi et al. used the same Markov model structure to simulate $\mathrm{I}_{\mathrm{Ks}}$ and its modulation by adrenergic stimulation in guinea pig ventricular myocytes. ${ }^{41}$ They showed that an increase in total conductance is not required to simulate all $I_{\mathrm{Ks}}$ properties during adrenergic stimulation. Similarly, we are able to reproduce both baseline and adrenergic stimulated characteristics of canine $I_{\mathrm{ks}}$ without altering the total conductance.

Nonphosphorylated Channels:

$$
\begin{aligned}
& \alpha_{\mathrm{Ks}}^{\mathrm{NP}}=\frac{7.3990 \cdot 10^{-3}}{1+\exp \left(-\frac{\mathrm{V}_{\mathrm{m}}-3.1196 \cdot 10^{-2}}{8.0019 \cdot 10^{-1}} \cdot \frac{\mathrm{F}}{\mathrm{R} \cdot \mathrm{T}}\right)} \\
& \beta_{\mathrm{Ks}}^{\mathrm{NP}}=\frac{5.6992 \cdot 10^{-3}}{1+\exp \left(\frac{\mathrm{V}_{\mathrm{m}}-4.1520 \cdot 10^{-2}}{1.3489} \cdot \frac{\mathrm{F}}{\mathrm{R} \cdot \mathrm{T}}\right)} \\
& \gamma_{\mathrm{Ks}}^{\mathrm{NP}}=\frac{3.8839 \cdot 10^{-1}}{1+\exp \left(-\frac{\mathrm{V}_{\mathrm{m}}+1.5019 \cdot 10^{-1}}{6.0693 \cdot 10^{-1}} \cdot \frac{\mathrm{F}}{\mathrm{R} \cdot \mathrm{T}}\right)} \\
& \delta_{\mathrm{Ks}}^{\mathrm{NP}}=9.0654 \cdot 10^{-2} \cdot \exp \left(-1.1157 \cdot 10^{-1} \cdot \frac{\mathrm{V}_{\mathrm{m}} \cdot \mathrm{F}}{\mathrm{R} \cdot \mathrm{T}}\right) \\
& \eta_{\mathrm{Ks}}^{\mathrm{NP}}=3.1124 \cdot 10^{-3}+\frac{2.8330 \cdot 10^{-2}-3.1124 \cdot 10^{-3}}{1+\exp \left(\frac{\mathrm{V}_{\mathrm{m}}+5.1660 \cdot 10^{-2}}{1.5522} \cdot \frac{\mathrm{F}}{\mathrm{R} \cdot \mathrm{T}}\right)} \\
& \theta_{\mathrm{Ks}}^{\mathrm{NP}}=2.7304 \cdot 10^{-3} \\
& \omega_{\mathrm{Ks}}^{\mathrm{NP}}=4.4198 \cdot 10^{-4} \cdot \exp \left(-1.2022 \cdot \frac{\mathrm{V}_{\mathrm{m}} \cdot \mathrm{F}}{\mathrm{R} \cdot \mathrm{T}}\right)
\end{aligned}
$$


$\Psi_{\mathrm{Ks}}^{\mathrm{NP}}=4.0173 \cdot 10^{-4} \cdot \exp \left(2.0873 \cdot 10^{-4} \cdot \frac{\mathrm{V}_{\mathrm{m}} \cdot \mathrm{F}}{\mathrm{R} \cdot \mathrm{T}}\right)$

$\frac{\mathrm{dC} 1 \mathrm{Ks}}{\mathrm{dt}}=-\left(4 \cdot \alpha_{\mathrm{Ks}}^{\mathrm{NP}}\right) \cdot \mathrm{C} 1_{\mathrm{Ks}}^{\mathrm{NP}}+\beta_{\mathrm{Ks}}^{\mathrm{NP}} \cdot \mathrm{C} 2_{\mathrm{Ks}}^{\mathrm{NP}}$

$\frac{\mathrm{dC} 2_{\mathrm{Ks}}^{\mathrm{NP}}}{\mathrm{dt}}=-\left(3 \cdot \alpha_{\mathrm{Ks}}^{\mathrm{NP}}+\beta_{\mathrm{Ks}}^{\mathrm{NP}}+\gamma_{\mathrm{Ks}}^{\mathrm{NP}}\right) \cdot \mathrm{C} 2_{\mathrm{Ks}}^{\mathrm{NP}}+4 \cdot \alpha_{\mathrm{Ks}}^{\mathrm{NP}} \cdot \mathrm{C} 1_{\mathrm{Ks}}^{\mathrm{NP}}+2 \cdot \beta_{\mathrm{Ks}}^{\mathrm{NP}} \cdot \mathrm{C} 3_{\mathrm{Ks}}^{\mathrm{NP}}+\delta_{\mathrm{Ks}}^{\mathrm{NP}} \cdot \mathrm{C} 6_{\mathrm{Ks}}^{\mathrm{NP}}$

$\frac{\mathrm{dC} 3{ }_{\mathrm{Ks}}^{\mathrm{NP}}}{\mathrm{dt}}=-\left(2 \cdot \alpha_{\mathrm{Ks}}^{\mathrm{NP}}+2 \cdot \beta_{\mathrm{Ks}}^{\mathrm{NP}}+2 \cdot \gamma_{\mathrm{Ks}}^{\mathrm{NP}}\right) \cdot \mathrm{C} 3_{\mathrm{Ks}}^{\mathrm{NP}}+3 \cdot \alpha_{\mathrm{Ks}}^{\mathrm{NP}} \cdot \mathrm{C} 2_{\mathrm{Ks}}^{\mathrm{NP}}+3 \cdot \beta_{\mathrm{Ks}}^{\mathrm{NP}} \cdot \mathrm{C} 4_{\mathrm{Ks}}^{\mathrm{NP}}+\delta_{\mathrm{Ks}}^{\mathrm{NP}} \cdot \mathrm{C} 7_{\mathrm{Ks}}^{\mathrm{NP}}$ $\frac{\mathrm{dC} 4_{\mathrm{Ks}}^{\mathrm{NP}}}{\mathrm{dt}}=-\left(\alpha_{\mathrm{Ks}}^{\mathrm{NP}}+3 \cdot \beta_{\mathrm{Ks}}^{\mathrm{NP}}+3 \cdot \gamma_{\mathrm{Ks}}^{\mathrm{NP}}\right) \cdot \mathrm{C} 4_{\mathrm{Ks}}^{\mathrm{NP}}+2 \cdot \alpha_{\mathrm{Ks}}^{\mathrm{NP}} \cdot \mathrm{C} 3_{\mathrm{Ks}}^{\mathrm{NP}}+4 \cdot \beta_{\mathrm{Ks}}^{\mathrm{NP}} \cdot \mathrm{C} 5_{\mathrm{Ks}}^{\mathrm{NP}}+\delta_{\mathrm{Ks}}^{\mathrm{NP}} \cdot \mathrm{C} 8_{\mathrm{Ks}}^{\mathrm{NP}}$ $\frac{\mathrm{dC} 5_{\mathrm{Ks}}^{\mathrm{NP}}}{\mathrm{dt}}=-\left(4 \cdot \beta_{\mathrm{Ks}}^{\mathrm{NP}}+4 \cdot \gamma_{\mathrm{Ks}}^{\mathrm{NP}}\right) \cdot \mathrm{C} 5_{\mathrm{Ks}}^{\mathrm{NP}}+\alpha_{\mathrm{Ks}}^{\mathrm{NP}} \cdot \mathrm{C} 4_{\mathrm{Ks}}^{\mathrm{NP}}+\delta_{\mathrm{Ks}}^{\mathrm{NP}} \cdot \mathrm{C} 9_{\mathrm{Ks}}^{\mathrm{NP}}$ $\frac{\mathrm{dC} 6_{\mathrm{Ks}}^{\mathrm{NP}}}{\mathrm{dt}}=-\left(3 \cdot \alpha_{\mathrm{Ks}}^{\mathrm{NP}}+\delta_{\mathrm{Ks}}^{\mathrm{NP}}\right) \cdot \mathrm{C} 6_{\mathrm{Ks}}^{\mathrm{NP}}+\beta_{\mathrm{Ks}}^{\mathrm{NP}} \cdot \mathrm{C} 7_{\mathrm{Ks}}^{\mathrm{NP}}+\gamma_{\mathrm{Ks}}^{\mathrm{NP}} \cdot \mathrm{C} 2_{\mathrm{Ks}}^{\mathrm{NP}}$ $\frac{\mathrm{dC} 7 \mathrm{Ks}^{\mathrm{NP}}}{\mathrm{dt}}=-\left(2 \cdot \alpha_{\mathrm{Ks}}^{\mathrm{NP}}+\beta_{\mathrm{Ks}}^{\mathrm{NP}}+\gamma_{\mathrm{Ks}}^{\mathrm{NP}}+\delta_{\mathrm{Ks}}^{\mathrm{NP}}\right) \cdot \mathrm{C} 7_{\mathrm{Ks}}^{\mathrm{NP}}+3 \cdot \alpha_{\mathrm{Ks}}^{\mathrm{NP}} \cdot \mathrm{C} 6_{\mathrm{Ks}}^{\mathrm{NP}}+2 \cdot \beta_{\mathrm{Ks}}^{\mathrm{NP}} \cdot \mathrm{C} 8_{\mathrm{Ks}}^{\mathrm{NP}}+2 \cdot \gamma_{\mathrm{Ks}}^{\mathrm{NP}} \cdot \mathrm{C} 3_{\mathrm{Ks}}^{\mathrm{NP}}$ $+2 \cdot \delta_{\mathrm{Ks}}^{\mathrm{NP}} \cdot \mathrm{C} 10_{\mathrm{Ks}}^{\mathrm{NP}}$

$\frac{\mathrm{dC} 8 \mathrm{Ks}^{\mathrm{NP}}}{\mathrm{dt}}=-\left(\alpha_{\mathrm{Ks}}^{\mathrm{NP}}+2 \cdot \beta_{\mathrm{Ks}}^{\mathrm{NP}}+2 \cdot \gamma_{\mathrm{Ks}}^{\mathrm{NP}}+\delta_{\mathrm{Ks}}^{\mathrm{NP}}\right) \cdot \mathrm{C} 8_{\mathrm{Ks}}^{\mathrm{NP}}+2 \cdot \alpha_{\mathrm{Ks}}^{\mathrm{NP}} \cdot \mathrm{C} 7_{\mathrm{Ks}}^{\mathrm{NP}}+3 \cdot \beta_{\mathrm{Ks}}^{\mathrm{NP}} \cdot \mathrm{C}_{\mathrm{Ks}}^{\mathrm{NP}}+3 \cdot \gamma_{\mathrm{Ks}}^{\mathrm{NP}}$ $\cdot \mathrm{C} 4 \mathrm{Ks}+2 \cdot \delta_{\mathrm{Ks}}^{\mathrm{NP}} \cdot \mathrm{C} 11_{\mathrm{Ks}}^{\mathrm{NP}}$

$\frac{\mathrm{dC} 9_{\mathrm{Ks}}^{\mathrm{NP}}}{\mathrm{dt}}=-\left(3 \cdot \beta_{\mathrm{Ks}}^{\mathrm{NP}}+3 \cdot \gamma_{\mathrm{Ks}}^{\mathrm{NP}}+\delta_{\mathrm{Ks}}^{\mathrm{NP}}\right) \cdot \mathrm{C} 9_{\mathrm{Ks}}^{\mathrm{NP}}+\alpha_{\mathrm{Ks}}^{\mathrm{NP}} \cdot \mathrm{C} 8_{\mathrm{Ks}}^{\mathrm{NP}}+4 \cdot \gamma_{\mathrm{Ks}}^{\mathrm{NP}} \cdot \mathrm{C} 5_{\mathrm{Ks}}^{\mathrm{NP}}+2 \cdot \delta_{\mathrm{Ks}}^{\mathrm{NP}} \cdot \mathrm{C} 12_{\mathrm{Ks}}^{\mathrm{NP}}$ $\frac{\mathrm{dC} 10_{\mathrm{Ks}}^{\mathrm{NP}}}{\mathrm{dt}}=-\left(2 \cdot \alpha_{\mathrm{Ks}}^{\mathrm{NP}}+2 \cdot \delta_{\mathrm{Ks}}^{\mathrm{NP}}\right) \cdot \mathrm{C} 10_{\mathrm{Ks}}^{\mathrm{NP}}+\beta_{\mathrm{Ks}}^{\mathrm{NP}} \cdot \mathrm{C} 11_{\mathrm{Ks}}^{\mathrm{NP}}+\gamma_{\mathrm{Ks}}^{\mathrm{NP}} \cdot \mathrm{C} 77_{\mathrm{Ks}}^{\mathrm{NP}}$ $\frac{\mathrm{dC} 11_{\mathrm{Ks}}^{\mathrm{NP}}}{\mathrm{dt}}=-\left(\alpha_{\mathrm{Ks}}^{\mathrm{NP}}+\beta_{\mathrm{Ks}}^{\mathrm{NP}}+\gamma_{\mathrm{Ks}}^{\mathrm{NP}}+2 \cdot \delta_{\mathrm{Ks}}^{\mathrm{NP}}\right) \cdot \mathrm{C} 11_{\mathrm{Ks}}^{\mathrm{NP}}+2 \cdot \alpha_{\mathrm{Ks}}^{\mathrm{NP}} \cdot \mathrm{C} 10_{\mathrm{Ks}}^{\mathrm{NP}}+2 \cdot \beta_{\mathrm{Ks}}^{\mathrm{NP}} \cdot \mathrm{C} 12_{\mathrm{Ks}}^{\mathrm{NP}}+2 \cdot \gamma_{\mathrm{Ks}}^{\mathrm{NP}}$ $\cdot \mathrm{C} 8{ }_{\mathrm{Ks}}^{\mathrm{NP}}+3 \cdot \delta_{\mathrm{Ks}}^{\mathrm{NP}} \cdot \mathrm{C} 13_{\mathrm{Ks}}^{\mathrm{NP}}$

$\frac{\mathrm{dC} 12_{\mathrm{Ks}}^{\mathrm{NP}}}{\mathrm{dt}}=-\left(2 \cdot \beta_{\mathrm{Ks}}^{\mathrm{NP}}+2 \cdot \gamma_{\mathrm{Ks}}^{\mathrm{NP}}+2 \cdot \delta_{\mathrm{Ks}}^{\mathrm{NP}}\right) \cdot \mathrm{C} 12_{\mathrm{Ks}}^{\mathrm{NP}}+\alpha_{\mathrm{Ks}}^{\mathrm{NP}} \cdot \mathrm{C} 11_{\mathrm{Ks}}^{\mathrm{NP}}+3 \cdot \gamma_{\mathrm{Ks}}^{\mathrm{NP}} \cdot \mathrm{C} 9_{\mathrm{Ks}}^{\mathrm{NP}}+3 \cdot \delta_{\mathrm{Ks}}^{\mathrm{NP}}$ $\cdot \mathrm{C} 14_{\mathrm{Ks}}^{\mathrm{NP}}$

$\frac{\mathrm{dC} 13_{\mathrm{Ks}}^{\mathrm{NP}}}{\mathrm{dt}}=-\left(\alpha_{\mathrm{Ks}}^{\mathrm{NP}}+3 \cdot \delta_{\mathrm{Ks}}^{\mathrm{NP}}\right) \cdot \mathrm{C} 13_{\mathrm{Ks}}^{\mathrm{NP}}+\beta_{\mathrm{Ks}}^{\mathrm{NP}} \cdot \mathrm{C} 14_{\mathrm{Ks}}^{\mathrm{NP}}+\gamma_{\mathrm{Ks}}^{\mathrm{NP}} \cdot \mathrm{C} 11_{\mathrm{Ks}}^{\mathrm{NP}}$ $\frac{\mathrm{dC} 14_{\mathrm{Ks}}^{\mathrm{NP}}}{\mathrm{dt}}=-\left(\beta_{\mathrm{Ks}}^{\mathrm{NP}}+\gamma_{\mathrm{Ks}}^{\mathrm{NP}}+3 \cdot \delta_{\mathrm{Ks}}^{\mathrm{NP}}\right) \cdot \mathrm{C} 14_{\mathrm{Ks}}^{\mathrm{NP}}+\alpha_{\mathrm{Ks}}^{\mathrm{NP}} \cdot \mathrm{C} 13_{\mathrm{Ks}}^{\mathrm{NP}}+2 \cdot \gamma_{\mathrm{Ks}}^{\mathrm{NP}} \cdot \mathrm{C} 12_{\mathrm{Ks}}^{\mathrm{NP}}+4 \cdot \delta_{\mathrm{Ks}}^{\mathrm{NP}} \cdot \mathrm{C} 15_{\mathrm{Ks}}^{\mathrm{NP}}$ $\frac{\mathrm{dC} 15_{\mathrm{Ks}}^{\mathrm{NP}}}{\mathrm{dt}}=-\left(4 \cdot \delta_{\mathrm{Ks}}^{\mathrm{NP}}+\theta_{\mathrm{Ks}}^{\mathrm{NP}}\right) \cdot \mathrm{C} 15_{\mathrm{Ks}}^{\mathrm{NP}}+\gamma_{\mathrm{Ks}}^{\mathrm{NP}} \cdot \mathrm{C} 14_{\mathrm{Ks}}^{\mathrm{NP}}+\eta_{\mathrm{Ks}}^{\mathrm{NP}} \cdot 01_{\mathrm{Ks}}^{\mathrm{NP}}$ $\frac{\mathrm{d} 01_{\mathrm{Ks}}^{\mathrm{NP}}}{\mathrm{dt}}=-\left(\eta_{\mathrm{Ks}}^{\mathrm{NP}}+\psi_{\mathrm{Ks}}^{\mathrm{NP}}\right) \cdot 01_{\mathrm{Ks}}^{\mathrm{NP}}+\theta_{\mathrm{Ks}}^{\mathrm{NP}} \cdot \mathrm{C} 15_{\mathrm{Ks}}^{\mathrm{NP}}+\omega_{\mathrm{Ks}}^{\mathrm{NP}} \cdot 02_{\mathrm{Ks}}^{\mathrm{NP}}$ $\frac{\mathrm{dO} 2_{\mathrm{Ks}}^{\mathrm{NP}}}{\mathrm{dt}}=-\left(\omega_{\mathrm{Ks}}^{\mathrm{NP}}\right) \cdot 02_{\mathrm{Ks}}^{\mathrm{NP}}+\psi_{\mathrm{Ks}}^{\mathrm{NP}} \cdot 01_{\mathrm{Ks}}^{\mathrm{NP}}$ 
$\overline{\mathrm{G}}_{\mathrm{Ks}}^{\mathrm{NP}}=0.19561 \cdot\left(1+\frac{0.6}{1+\left(3.8 \cdot \frac{10^{-5}}{\left[\mathrm{Ca}^{2+}\right]_{\mathrm{i}}}\right)^{1.4}}\right)$

$\mathrm{P}_{\mathrm{Na} / \mathrm{K}}=0.01833$

$\mathrm{E}_{\mathrm{Ks}}=\frac{\mathrm{R} \cdot \mathrm{T}}{\mathrm{F}} \cdot \log \left(\frac{\left[\mathrm{K}^{+}\right]_{\mathrm{o}}+\mathrm{P}_{\mathrm{Na} / \mathrm{K}} \cdot\left[\mathrm{Na}^{+}\right]_{\mathrm{o}}}{\left[\mathrm{K}^{+}\right]_{\mathrm{i}}+\mathrm{P}_{\mathrm{Na} / \mathrm{K}} \cdot\left[\mathrm{Na}^{+}\right]_{\mathrm{i}}}\right)$

$\mathrm{I}_{\mathrm{Ks}}^{\mathrm{NP}}=\overline{\mathrm{G}}_{\mathrm{Ks}}^{\mathrm{NP}} \cdot\left(\mathrm{O} 1_{\mathrm{Ks}}^{\mathrm{NP}}+02_{\mathrm{Ks}}^{\mathrm{NP}}\right) \cdot\left(\mathrm{V}_{\mathrm{m}}-\mathrm{E}_{\mathrm{Ks}}\right)$

Phosphorylated Channels:

$\alpha_{\mathrm{Ks}}^{\mathrm{NP}}=\frac{9.9415 \cdot 10^{-3}}{1+\exp \left(-\frac{\mathrm{V}_{\mathrm{m}}-4.4809 \cdot 10^{-2}}{5.8172 \cdot 10^{-1}} \cdot \frac{\mathrm{F}}{\mathrm{R} \cdot \mathrm{T}}\right)}$

$\beta_{\mathrm{Ks}}^{\mathrm{NP}}=\frac{3.3201 \cdot 10^{-3}}{1+\exp \left(\frac{\mathrm{V}_{\mathrm{m}}-9.4217 \cdot 10^{-2}}{0.9536} \cdot \frac{\mathrm{F}}{\mathrm{R} \cdot \mathrm{T}}\right)}$

$\gamma_{\mathrm{Ks}}^{\mathrm{NP}}=\frac{5.6356 \cdot 10^{-1}}{1+\exp \left(-\frac{\mathrm{V}_{\mathrm{m}}+1.7986 \cdot 10^{-1}}{5.8381 \cdot 10^{-1}} \cdot \frac{\mathrm{F}}{\mathrm{R} \cdot \mathrm{T}}\right)}$

$\delta_{\mathrm{Ks}}^{\mathrm{NP}}=6.5700 \cdot 10^{-2} \cdot \exp \left(-1.1899 \cdot 10^{-1} \cdot \frac{\mathrm{V}_{\mathrm{m}} \cdot \mathrm{F}}{\mathrm{R} \cdot \mathrm{T}}\right)$

$\eta_{\mathrm{Ks}}^{\mathrm{NP}}=3.8525 \cdot 10^{-4}+\frac{1.2406 \cdot 10^{-2}-3.8525 \cdot 10^{-4}}{1+\exp \left(\frac{\mathrm{V}_{\mathrm{m}}+6.4118 \cdot 10^{-2}}{7.7992 \cdot 10^{-1}} \cdot \frac{\mathrm{F}}{\mathrm{R} \cdot \mathrm{T}}\right)}$

$\theta_{\mathrm{Ks}}^{\mathrm{NP}}=4.6171 \cdot 10^{-3}$

$\omega_{\mathrm{Ks}}^{\mathrm{NP}}=2.3730 \cdot 10^{-4} \cdot \exp \left(-1.9742 \cdot \frac{\mathrm{V}_{\mathrm{m}} \cdot \mathrm{F}}{\mathrm{R} \cdot \mathrm{T}}\right)$

$\psi_{\mathrm{Ks}}^{\mathrm{NP}}=2.2652 \cdot 10^{-4} \cdot \exp \left(2.4689 \cdot 10^{-4} \cdot \frac{\mathrm{V}_{\mathrm{m}} \cdot \mathrm{F}}{\mathrm{R} \cdot \mathrm{T}}\right)$

$\frac{\mathrm{dC} 1_{\mathrm{Ks}}^{\mathrm{P}}}{\mathrm{dt}}=-\left(4 \cdot \alpha_{\mathrm{Ks}}^{\mathrm{P}}\right) \cdot \mathrm{C} 1_{\mathrm{Ks}}^{\mathrm{P}}+\beta_{\mathrm{Ks}}^{\mathrm{P}} \cdot \mathrm{C} 2_{\mathrm{Ks}}^{\mathrm{P}}$

$\frac{\mathrm{dC} 2_{\mathrm{Ks}}^{\mathrm{P}}}{\mathrm{dt}}=-\left(3 \cdot \alpha_{\mathrm{Ks}}^{\mathrm{P}}+\beta_{\mathrm{Ks}}^{\mathrm{P}}+\gamma_{\mathrm{Ks}}^{\mathrm{P}}\right) \cdot \mathrm{C} 2_{\mathrm{Ks}}^{\mathrm{P}}+4 \cdot \alpha_{\mathrm{Ks}}^{\mathrm{P}} \cdot \mathrm{C} 1_{\mathrm{Ks}}^{\mathrm{P}}+2 \cdot \beta_{\mathrm{Ks}}^{\mathrm{P}} \cdot \mathrm{C} 3_{\mathrm{Ks}}^{\mathrm{P}}+\delta_{\mathrm{Ks}}^{\mathrm{P}} \cdot \mathrm{C} 6_{\mathrm{Ks}}^{\mathrm{P}}$

$\frac{\mathrm{dC} 3_{\mathrm{Ks}}^{\mathrm{P}}}{\mathrm{dt}}=-\left(2 \cdot \alpha_{\mathrm{Ks}}^{\mathrm{P}}+2 \cdot \beta_{\mathrm{Ks}}^{\mathrm{P}}+2 \cdot \gamma_{\mathrm{Ks}}^{\mathrm{P}}\right) \cdot \mathrm{C} 3_{\mathrm{Ks}}^{\mathrm{P}}+3 \cdot \alpha_{\mathrm{Ks}}^{\mathrm{P}} \cdot \mathrm{C} 2_{\mathrm{Ks}}^{\mathrm{P}}+3 \cdot \beta_{\mathrm{Ks}}^{\mathrm{P}} \cdot \mathrm{C} 4_{\mathrm{Ks}}^{\mathrm{P}}+\delta_{\mathrm{Ks}}^{\mathrm{P}} \cdot \mathrm{C} 7_{\mathrm{Ks}}^{\mathrm{P}}$

$\frac{\mathrm{dC} 4_{\mathrm{Ks}}^{\mathrm{P}}}{\mathrm{dt}}=-\left(1 \cdot \alpha_{\mathrm{Ks}}^{\mathrm{P}}+3 \cdot \beta_{\mathrm{Ks}}^{\mathrm{P}}+3 \cdot \gamma_{\mathrm{Ks}}^{\mathrm{P}}\right) \cdot \mathrm{C} 4_{\mathrm{Ks}}^{\mathrm{P}}+2 \cdot \alpha_{\mathrm{Ks}}^{\mathrm{P}} \cdot \mathrm{C} 3_{\mathrm{Ks}}^{\mathrm{P}}+4 \cdot \beta_{\mathrm{Ks}}^{\mathrm{P}} \cdot \mathrm{C} 5_{\mathrm{Ks}}^{\mathrm{P}}+\delta_{\mathrm{Ks}}^{\mathrm{P}} \cdot \mathrm{C} 8_{\mathrm{Ks}}^{\mathrm{P}}$ 


$$
\begin{aligned}
& \frac{\mathrm{dC} 5_{\mathrm{Ks}}^{\mathrm{P}}}{\mathrm{dt}}=-\left(4 \cdot \beta_{\mathrm{Ks}}^{\mathrm{P}}+4 \cdot \gamma_{\mathrm{Ks}}^{\mathrm{P}}\right) \cdot \mathrm{C} 5_{\mathrm{Ks}}^{\mathrm{P}}+\alpha_{\mathrm{Ks}}^{\mathrm{P}} \cdot \mathrm{C} 4_{\mathrm{Ks}}^{\mathrm{P}}+\delta_{\mathrm{Ks}}^{\mathrm{P}} \cdot \mathrm{C} 9_{\mathrm{Ks}}^{\mathrm{P}} \\
& \frac{\mathrm{dC} 6_{\mathrm{Ks}}^{\mathrm{P}}}{\mathrm{dt}}=-\left(3 \cdot \alpha_{\mathrm{Ks}}^{\mathrm{P}}+\delta_{\mathrm{Ks}}^{\mathrm{P}}\right) \cdot \mathrm{C} 6_{\mathrm{Ks}}^{\mathrm{P}}+\beta_{\mathrm{Ks}}^{\mathrm{P}} \cdot \mathrm{C} 7_{\mathrm{Ks}}^{\mathrm{P}}+\gamma_{\mathrm{Ks}}^{\mathrm{P}} \cdot \mathrm{C} 2_{\mathrm{Ks}}^{\mathrm{P}} \\
& \frac{\mathrm{dC} 77_{\mathrm{Ks}}^{\mathrm{P}}}{\mathrm{dt}}=-\left(2 \cdot \alpha_{\mathrm{Ks}}^{\mathrm{P}}+\beta_{\mathrm{Ks}}^{\mathrm{P}}+\gamma_{\mathrm{Ks}}^{\mathrm{P}}+\delta_{\mathrm{Ks}}^{\mathrm{P}}\right) \cdot \mathrm{C} 7_{\mathrm{Ks}}^{\mathrm{P}}+3 \cdot \alpha_{\mathrm{Ks}}^{\mathrm{P}} \cdot \mathrm{C} 6_{\mathrm{Ks}}^{\mathrm{P}}+2 \cdot \beta_{\mathrm{Ks}}^{\mathrm{P}} \cdot \mathrm{C} 8_{\mathrm{Ks}}^{\mathrm{P}}+2 \cdot \gamma_{\mathrm{Ks}}^{\mathrm{P}} \cdot \mathrm{C} 3_{\mathrm{Ks}}^{\mathrm{P}}+2 \\
& \cdot \delta_{\mathrm{Ks}}^{\mathrm{P}} \cdot \mathrm{C} 10_{\mathrm{Ks}}^{\mathrm{P}} \\
& \frac{\mathrm{dC} 8_{\mathrm{Ks}}^{\mathrm{P}}}{\mathrm{dt}}=-\left(\alpha_{\mathrm{Ks}}^{\mathrm{P}}+2 \cdot \beta_{\mathrm{Ks}}^{\mathrm{P}}+2 \cdot \gamma_{\mathrm{Ks}}^{\mathrm{P}}+\delta_{\mathrm{Ks}}^{\mathrm{P}}\right) \cdot \mathrm{C} 8_{\mathrm{Ks}}^{\mathrm{P}}+2 \cdot \alpha_{\mathrm{Ks}}^{\mathrm{P}} \cdot \mathrm{C} 7_{\mathrm{Ks}}^{\mathrm{P}}+3 \cdot \beta_{\mathrm{Ks}}^{\mathrm{P}} \cdot \mathrm{C} 9_{\mathrm{Ks}}^{\mathrm{P}}+3 \cdot \gamma_{\mathrm{Ks}}^{\mathrm{P}} \cdot \mathrm{C} 4_{\mathrm{Ks}}^{\mathrm{P}} \\
& +2 \cdot \delta_{\mathrm{Ks}}^{\mathrm{P}} \cdot \mathrm{C} 11_{\mathrm{Ks}}^{\mathrm{P}} \\
& \frac{\mathrm{dC} 9}{\mathrm{dt}}=-\left(3 \cdot \beta_{\mathrm{Ks}}^{\mathrm{P}}+3 \cdot \gamma_{\mathrm{Ks}}^{\mathrm{P}}+\delta_{\mathrm{Ks}}^{\mathrm{P}}\right) \cdot \mathrm{C} 9_{\mathrm{Ks}}^{\mathrm{P}}+\alpha_{\mathrm{Ks}}^{\mathrm{P}} \cdot \mathrm{C} 8_{\mathrm{Ks}}^{\mathrm{P}}+4 \cdot \gamma_{\mathrm{Ks}}^{\mathrm{P}} \cdot \mathrm{C} 5_{\mathrm{Ks}}^{\mathrm{P}}+2 \cdot \delta_{\mathrm{Ks}}^{\mathrm{P}} \cdot \mathrm{C} 12_{\mathrm{Ks}}^{\mathrm{P}} \\
& \frac{\mathrm{dC} 10_{\mathrm{Ks}}^{\mathrm{P}}}{\mathrm{dt}}=-\left(2 \cdot \alpha_{\mathrm{Ks}}^{\mathrm{P}}+2 \cdot \delta_{\mathrm{Ks}}^{\mathrm{P}}\right) \cdot \mathrm{C} 10_{\mathrm{Ks}}^{\mathrm{P}}+\beta_{\mathrm{Ks}}^{\mathrm{P}} \cdot \mathrm{C} 11_{\mathrm{Ks}}^{\mathrm{P}}+\gamma_{\mathrm{Ks}}^{\mathrm{P}} \cdot \mathrm{C} 7 \mathrm{Ks}^{\mathrm{P}} \\
& \frac{\mathrm{dC} 11_{\mathrm{Ks}}^{\mathrm{P}}}{\mathrm{dt}}=-\left(\alpha_{\mathrm{Ks}}^{\mathrm{P}}+\beta_{\mathrm{Ks}}^{\mathrm{P}}+\gamma_{\mathrm{Ks}}^{\mathrm{P}}+2 \cdot \delta_{\mathrm{Ks}}^{\mathrm{P}}\right) \cdot \mathrm{C} 11_{\mathrm{Ks}}^{\mathrm{P}}+2 \cdot \alpha_{\mathrm{Ks}}^{\mathrm{P}} \cdot \mathrm{C} 10_{\mathrm{Ks}}^{\mathrm{P}}+2 \cdot \beta_{\mathrm{Ks}}^{\mathrm{P}} \cdot \mathrm{C} 12_{\mathrm{Ks}}^{\mathrm{P}}+2 \cdot \gamma_{\mathrm{Ks}}^{\mathrm{P}} \\
& \cdot \mathrm{C} 8{ }_{\mathrm{Ks}}^{\mathrm{P}}+3 \cdot \delta_{\mathrm{Ks}}^{\mathrm{P}} \cdot \mathrm{C} 13_{\mathrm{Ks}}^{\mathrm{P}} \\
& \frac{\mathrm{dC} 12_{\mathrm{Ks}}^{\mathrm{P}}}{\mathrm{dt}}=-\left(2 \cdot \beta_{\mathrm{Ks}}^{\mathrm{P}}+2 \cdot \gamma_{\mathrm{Ks}}^{\mathrm{P}}+2 \cdot \delta_{\mathrm{Ks}}^{\mathrm{P}}\right) \cdot \mathrm{C} 12_{\mathrm{Ks}}^{\mathrm{P}}+\alpha_{\mathrm{Ks}}^{\mathrm{P}} \cdot \mathrm{C} 11_{\mathrm{Ks}}^{\mathrm{P}}+3 \cdot \gamma_{\mathrm{Ks}}^{\mathrm{P}} \cdot \mathrm{C} 9_{\mathrm{Ks}}^{\mathrm{P}}+3 \cdot \delta_{\mathrm{Ks}}^{\mathrm{P}} \cdot \mathrm{C} 14_{\mathrm{Ks}}^{\mathrm{P}} \\
& \frac{\mathrm{dC} 13_{\mathrm{Ks}}^{\mathrm{P}}}{\mathrm{dt}}=-\left(\alpha_{\mathrm{Ks}}^{\mathrm{P}}+3 \cdot \delta_{\mathrm{Ks}}^{\mathrm{P}}\right) \cdot \mathrm{C} 13_{\mathrm{Ks}}^{\mathrm{P}}+\beta_{\mathrm{Ks}}^{\mathrm{P}} \cdot \mathrm{C} 14_{\mathrm{Ks}}^{\mathrm{P}}+\gamma_{\mathrm{Ks}}^{\mathrm{P}} \cdot \mathrm{C} 11_{\mathrm{Ks}}^{\mathrm{P}} \\
& \frac{\mathrm{dC} 14_{\mathrm{Ks}}^{\mathrm{P}}}{\mathrm{dt}}=-\left(\beta_{\mathrm{Ks}}^{\mathrm{P}}+\gamma_{\mathrm{Ks}}^{\mathrm{P}}+3 \cdot \delta_{\mathrm{Ks}}^{\mathrm{P}}\right) \cdot \mathrm{C} 14_{\mathrm{Ks}}^{\mathrm{P}}+\alpha_{\mathrm{Ks}}^{\mathrm{P}} \cdot \mathrm{C} 13_{\mathrm{Ks}}^{\mathrm{P}}+2 \cdot \gamma_{\mathrm{Ks}}^{\mathrm{P}} \cdot \mathrm{C} 12_{\mathrm{Ks}}^{\mathrm{P}}+4 \cdot \delta_{\mathrm{Ks}}^{\mathrm{P}} \cdot \mathrm{C} 15_{\mathrm{Ks}}^{\mathrm{P}} \\
& \frac{\mathrm{dC} 15_{\mathrm{Ks}}^{\mathrm{P}}}{\mathrm{dt}}=-\left(4 \cdot \delta_{\mathrm{Ks}}^{\mathrm{P}}+\theta_{\mathrm{Ks}}^{\mathrm{P}}\right) \cdot \mathrm{C} 15_{\mathrm{Ks}}^{\mathrm{P}}+\gamma_{\mathrm{Ks}}^{\mathrm{P}} \cdot \mathrm{C} 14_{\mathrm{Ks}}^{\mathrm{P}}+\eta_{\mathrm{Ks}}^{\mathrm{P}} \cdot 01_{\mathrm{Ks}}^{\mathrm{P}} \\
& \frac{\mathrm{d} 01_{\mathrm{Ks}}^{\mathrm{P}}}{\mathrm{dt}}=-\left(\eta_{\mathrm{Ks}}^{\mathrm{P}}+\psi_{\mathrm{Ks}}^{\mathrm{P}}\right) \cdot 01_{\mathrm{Ks}}^{\mathrm{P}}+\theta_{\mathrm{Ks}}^{\mathrm{P}} \cdot \mathrm{C} 15_{\mathrm{Ks}}^{\mathrm{P}}+\omega_{\mathrm{Ks}}^{\mathrm{P}} \cdot 02_{\mathrm{Ks}}^{\mathrm{P}} \\
& \frac{\mathrm{dO} 2_{\mathrm{Ks}}^{\mathrm{P}}}{\mathrm{dt}}=-\left(\omega_{\mathrm{Ks}}^{\mathrm{P}}\right) \cdot 02_{\mathrm{Ks}}^{\mathrm{P}}+\psi_{\mathrm{Ks}}^{\mathrm{P}} \cdot 01_{\mathrm{Ks}}^{\mathrm{P}} \\
& \overline{\mathrm{G}}_{\mathrm{Ks}}^{\mathrm{P}}=0.19561 \cdot\left(1+\frac{0.6}{1+\left(3.8 \cdot \frac{10^{-5}}{\left[\mathrm{Ca}^{2+}\right]_{\mathrm{i}}}\right)^{1.4}}\right) \\
& \mathrm{I}_{\mathrm{Ks}}^{\mathrm{P}}=\overline{\mathrm{G}}_{\mathrm{Ks}}^{\mathrm{P}} \cdot\left(\mathrm{O} 1_{\mathrm{Ks}}^{\mathrm{P}}+02_{\mathrm{Ks}}^{\mathrm{P}}\right) \cdot\left(\mathrm{V}_{\mathrm{m}}-\mathrm{E}_{\mathrm{Ks}}\right)
\end{aligned}
$$

\section{Combined current:}

$\left.\hat{\mathrm{f}}_{\mathrm{Ks}}^{\mathrm{P}}=\frac{\mathrm{f}_{\mathrm{IKs}}^{\mathrm{P}}-\left(0.0306+\frac{[\mathrm{IKs}]_{\mathrm{ARN}}}{[\mathrm{IKs}]_{\mathrm{tot}}}\right)}{0.7850-\left(0.0306+\frac{[\mathrm{IKs}]_{\mathrm{ARN}}}{[\mathrm{IKs}]_{\mathrm{tot}}}\right.}\right)$ 
$\mathrm{I}_{\mathrm{Ks}}=\hat{\mathrm{f}}_{\mathrm{Ks}}^{\mathrm{P}} \cdot \mathrm{I}_{\mathrm{Ks}}^{\mathrm{P}}+\left(1-\hat{\mathrm{f}}_{\mathrm{Ks}}^{\mathrm{P}}\right) \cdot \mathrm{I}_{\mathrm{Ks}}^{\mathrm{NP}}$

\subsubsection{HRdßARS A341V version (Chapter 4)}

Model structure, differential equations and calculation of $\mathrm{I}_{\mathrm{ks}}$ are identical to that in section 1.16.2. Model parameters were adjusted based on data obtained in $\mathrm{CHO}$ cells expressing WT or mutant $\mathrm{I}_{\mathrm{Ks}}$ channels in the absence or presence of cAMP/OA (Chapter 4):

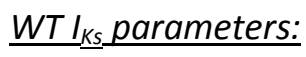

$$
\begin{aligned}
& \alpha_{\mathrm{Ks}}^{\mathrm{NP}}=\frac{8.7313 \cdot 10^{-3}}{1+\exp \left(-\frac{\mathrm{V}_{\mathrm{m}}-3.3032 \cdot 10^{1}}{1.1946} \cdot \frac{\mathrm{F}}{\mathrm{R} \cdot \mathrm{T}}\right)} \\
& \beta_{\mathrm{Ks}}^{\mathrm{NP}}=\frac{1.1068 \cdot 10^{-2}}{1+\exp \left(\frac{\mathrm{V}_{\mathrm{m}}-1.9519 \cdot 10^{-2}}{1.4447} \cdot \frac{\mathrm{F}}{\mathrm{R} \cdot \mathrm{T}}\right)} \\
& \gamma_{\mathrm{Ks}}^{\mathrm{NP}}=\frac{1.5226 \cdot 10^{-1}}{1+\exp \left(-\frac{\mathrm{V}_{\mathrm{m}}+5.3866 \cdot 10^{-2}}{5.3191 \cdot 10^{-1}} \cdot \frac{\mathrm{F}}{\mathrm{R} \cdot \mathrm{T}}\right)} \\
& \delta_{\mathrm{Ks}}^{\mathrm{NP}}=1.0372 \cdot 10^{-2} \cdot \exp \left(4.7872 \cdot 10^{-3} \cdot \frac{\mathrm{V}_{\mathrm{m}} \cdot \mathrm{F}}{\mathrm{R} \cdot \mathrm{T}}\right) \\
& \eta_{\mathrm{Ks}}^{\mathrm{NP}}=1.5447 \cdot 10^{-2}+\frac{6.9977 \cdot 10^{-2}-1.5447 \cdot 10^{-2}}{1+\exp \left(\frac{\mathrm{V}_{\mathrm{m}}+1.0318 \cdot 10^{2}}{5.2369 \cdot 10^{-1}} \cdot \frac{\mathrm{F}}{\mathrm{R} \cdot \mathrm{T}}\right)} \\
& \theta_{\mathrm{Ks}}^{\mathrm{NP}}=1.7695 \cdot 10^{-3} \\
& \omega_{\mathrm{Ks}}^{\mathrm{NP}}=5.6517 \cdot 10^{-4} \cdot \exp \left(-1.5643 \cdot \frac{\mathrm{V}_{\mathrm{m}} \cdot \mathrm{F}}{\mathrm{R} \cdot \mathrm{T}}\right) \\
& \psi_{\mathrm{Ks}}^{\mathrm{NP}}=2.8148 \cdot 10^{-5} \cdot \exp \left(1.9762 \cdot 10^{-4} \cdot \frac{\mathrm{V}_{\mathrm{m}} \cdot \mathrm{F}}{\mathrm{R} \cdot \mathrm{T}}\right) \\
& \alpha_{\mathrm{Ks}}^{\mathrm{P}}=\frac{6.9994 \cdot 10^{-3}}{1+\exp \left(-\frac{\mathrm{V}_{\mathrm{m}}-5.6717 \cdot 10^{-1}}{5.7717 \cdot 10^{-1}} \cdot \frac{\mathrm{F}}{\mathrm{R} \cdot \mathrm{T}}\right)} \\
& \beta_{\mathrm{Ks}}^{\mathrm{P}}=\frac{1.3731 \cdot 10^{-2}}{1+\exp \left(\frac{\mathrm{V}_{\mathrm{m}}-3.8608 \cdot 10^{-3}}{1.7486} \cdot \frac{\mathrm{F}}{\mathrm{R} \cdot \mathrm{T}}\right)} \\
& \gamma_{\mathrm{Ks}}^{\mathrm{P}}=\frac{1.6490 \cdot 10^{-1}}{1+\exp \left(-\frac{\mathrm{V}_{\mathrm{m}}-2.0329 \cdot 10^{-2}}{4.8136 \cdot 10^{-1}} \cdot \frac{\mathrm{F}}{\mathrm{R} \cdot \mathrm{T}}\right)} \\
& \delta_{\mathrm{Ks}}^{\mathrm{P}}=4.7241 \cdot 10^{-3} \cdot \exp \left(1.6283 \cdot 10^{-2} \cdot \frac{\mathrm{V}_{\mathrm{m}} \cdot \mathrm{F}}{\mathrm{R} \cdot \mathrm{T}}\right) \\
& \eta_{\mathrm{Ks}}^{\mathrm{P}}=9.6896 \cdot 10^{-3}+\frac{1.4242 \cdot 10^{-1}-9.6896 \cdot 10^{-3}}{1+\exp \left(\frac{\mathrm{V}_{\mathrm{m}}+1.1338 \cdot 10^{2}}{5.5965 \cdot 10^{-1}} \cdot \frac{\mathrm{F}}{\mathrm{R} \cdot \mathrm{T}}\right)} \\
& \theta_{\mathrm{Ks}}^{\mathrm{P}}=3.2528 \cdot 10^{-3} \\
& \omega_{\mathrm{Ks}}^{\mathrm{P}}=2.3813 \cdot 10^{-3} \cdot \exp \left(-1.9602 \cdot \frac{\mathrm{V}_{\mathrm{m}} \cdot \mathrm{F}}{\mathrm{R} \cdot \mathrm{T}}\right)
\end{aligned}
$$


$\psi_{\mathrm{Ks}}^{\mathrm{P}}=4.4670 \cdot 10^{-5} \cdot \exp \left(8.9068 \cdot 10^{-5} \cdot \frac{\mathrm{V}_{\mathrm{m}} \cdot \mathrm{F}}{\mathrm{R} \cdot \mathrm{T}}\right)$

\section{$\underline{A 341 V_{\text {Het }} \underline{I}_{K s} \text { parameters: }}$}

$\begin{aligned} \alpha_{\mathrm{Ks}}^{\mathrm{NP}} & =\frac{7.8749 \cdot 10^{-3}}{1+\exp \left(-\frac{\mathrm{V}_{\mathrm{m}}-5.1876 \cdot 10^{1}}{1.0212} \cdot \frac{\mathrm{F}}{\mathrm{R} \cdot \mathrm{T}}\right)} \\ \beta_{\mathrm{Ks}}^{\mathrm{NP}} & =\frac{4.5532 \cdot 10^{-3}}{1+\exp \left(\frac{\mathrm{V}_{\mathrm{m}}-1.4922 \cdot 10^{-2}}{4.2919} \cdot \frac{\mathrm{F}}{\mathrm{R} \cdot \mathrm{T}}\right)}\end{aligned}$

$\gamma_{\mathrm{Ks}}^{\mathrm{NP}}=\frac{1.0993 \cdot 10^{-1}}{1+\exp \left(-\frac{\mathrm{V}_{\mathrm{m}}+1.1599 \cdot 10^{-3}}{6.2763 \cdot 10^{-1}} \cdot \frac{\mathrm{F}}{\mathrm{R} \cdot \mathrm{T}}\right)}$

$\delta_{\mathrm{Ks}}^{\mathrm{NP}}=2.6854 \cdot 10^{-2} \cdot \exp \left(2.3346 \cdot 10^{-3} \cdot \frac{\mathrm{V}_{\mathrm{m}} \cdot \mathrm{F}}{\mathrm{R} \cdot \mathrm{T}}\right)$

$\eta_{\mathrm{Ks}}^{\mathrm{NP}}=9.0527 \cdot 10^{-3}+\frac{6.5743 \cdot 10^{-2}-9.0527 \cdot 10^{-3}}{1+\exp \left(\frac{\mathrm{V}_{\mathrm{m}}+3.0409 \cdot 10^{1}}{0.7658} \cdot \frac{\mathrm{F}}{\mathrm{R} \cdot \mathrm{T}}\right)}$

$\theta_{\mathrm{Ks}}^{\mathrm{NP}}=1.2691 \cdot 10^{-3}$

$\omega_{\mathrm{Ks}}^{\mathrm{NP}}=5.6517 \cdot 10^{-4} \cdot \exp \left(-1.5643 \cdot \frac{\mathrm{V}_{\mathrm{m}} \cdot \mathrm{F}}{\mathrm{R} \cdot \mathrm{T}}\right)$

$\psi_{\mathrm{Ks}}^{\mathrm{NP}}=2.8148 \cdot 10^{-5} \cdot \exp \left(1.9762 \cdot 10^{-4} \cdot \frac{\mathrm{V}_{\mathrm{m}} \cdot \mathrm{F}}{\mathrm{R} \cdot \mathrm{T}}\right)$

$\alpha_{\mathrm{Ks}}^{\mathrm{P}}=\frac{1.1355 \cdot 10^{-2}}{1+\exp \left(-\frac{\mathrm{V}_{\mathrm{m}}-6.8892 \cdot 10^{1}}{1.4039} \cdot \frac{\mathrm{F}}{\mathrm{R} \cdot \mathrm{T}}\right)}$

$\beta_{\mathrm{Ks}}^{\mathrm{P}}=\frac{3.8524 \cdot 10^{-3}}{1+\exp \left(\frac{\mathrm{V}_{\mathrm{m}}-1.0399 \cdot 10^{-2}}{3.3478} \cdot \frac{\mathrm{F}}{\mathrm{R} \cdot \mathrm{T}}\right)}$

$\gamma_{\mathrm{Ks}}^{\mathrm{P}}=\frac{1.2805 \cdot 10^{-1}}{1+\exp \left(-\frac{\mathrm{V}_{\mathrm{m}}+1.1741 \cdot 10^{-3}}{0.4837} \cdot \frac{\mathrm{F}}{\mathrm{R} \cdot \mathrm{T}}\right)}$

$\delta_{\mathrm{Ks}}^{\mathrm{P}}=2.1670 \cdot 10^{-2} \cdot \exp \left(2.4782 \cdot 10^{-3} \cdot \frac{\mathrm{V}_{\mathrm{m}} \cdot \mathrm{F}}{\mathrm{R} \cdot \mathrm{T}}\right)$

$\eta_{\mathrm{Ks}}^{\mathrm{P}}=5.8191 \cdot 10^{-3}+\frac{4.4396 \cdot 10^{-2}-5.8191 \cdot 10^{-3}}{1+\exp \left(\frac{\mathrm{V}_{\mathrm{m}}+4.2618 \cdot 10^{1}}{1.1263} \cdot \frac{\mathrm{F}}{\mathrm{R} \cdot \mathrm{T}}\right)}$

$\theta_{\mathrm{Ks}}^{\mathrm{P}}=6.2849 \cdot 10^{-4}$

$\omega_{\mathrm{Ks}}^{\mathrm{P}}=5.6517 \cdot 10^{-4} \cdot \exp \left(-1.5643 \cdot \frac{\mathrm{V}_{\mathrm{m}} \cdot \mathrm{F}}{\mathrm{R} \cdot \mathrm{T}}\right)$

$\psi_{\mathrm{Ks}}^{\mathrm{P}}=2.8148 \cdot 10^{-5} \cdot \exp \left(1.9762 \cdot 10^{-4} \cdot \frac{\mathrm{V}_{\mathrm{m}} \cdot \mathrm{F}}{\mathrm{R} \cdot \mathrm{T}}\right)$

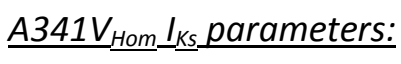




$$
\begin{aligned}
& \alpha_{\mathrm{Ks}}^{\mathrm{NP}}=\frac{6.4929 \cdot 10^{-3}}{1+\exp \left(-\frac{\mathrm{V}_{\mathrm{m}}-8.4272 \cdot 10^{1}}{1.1093} \cdot \frac{\mathrm{F}}{\mathrm{R} \cdot \mathrm{T}}\right)} \\
& \beta_{\mathrm{Ks}}^{\mathrm{NP}}=\frac{2.6793 \cdot 10^{-3}}{1+\exp \left(\frac{\mathrm{V}_{\mathrm{m}}-1.4346 \cdot 10^{-2}}{3.1158} \cdot \frac{\mathrm{F}}{\mathrm{R} \cdot \mathrm{T}}\right)} \\
& \gamma_{\mathrm{Ks}}^{\mathrm{NP}}=\frac{6.6132 \cdot 10^{-2}}{1+\exp \left(-\frac{\mathrm{V}_{\mathrm{m}}+1.5849 \cdot 10^{-3}}{0.2105} \cdot \frac{\mathrm{F}}{\mathrm{R} \cdot \mathrm{T}}\right)} \\
& \delta_{\mathrm{Ks}}^{\mathrm{NP}}=3.0593 \cdot 10^{-2} \cdot \exp \left(3.8659 \cdot 10^{-3} \cdot \frac{\mathrm{V}_{\mathrm{m}} \cdot \mathrm{F}}{\mathrm{R} \cdot \mathrm{T}}\right) \\
& \eta_{\mathrm{Ks}}^{\mathrm{NP}}=7.7273 \cdot 10^{-3}+\frac{8.7107 \cdot 10^{-2}-7.7273 \cdot 10^{-3}}{1+\exp \left(\frac{\mathrm{V}_{\mathrm{m}}+1.3602 \cdot 10^{1}}{0.8158} \cdot \frac{\mathrm{F}}{\mathrm{R} \cdot \mathrm{T}}\right)}
\end{aligned}
$$

$\theta_{\mathrm{Ks}}^{\mathrm{NP}}=3.3856 \cdot 10^{-4}$

$\omega_{\mathrm{Ks}}^{\mathrm{NP}}=5.6517 \cdot 10^{-4} \cdot \exp \left(-1.5643 \cdot \frac{\mathrm{V}_{\mathrm{m}} \cdot \mathrm{F}}{\mathrm{R} \cdot \mathrm{T}}\right)$

$\psi_{\mathrm{Ks}}^{\mathrm{NP}}=2.8148 \cdot 10^{-5} \cdot \exp \left(1.9762 \cdot 10^{-4} \cdot \frac{\mathrm{V}_{\mathrm{m}} \cdot \mathrm{F}}{\mathrm{R} \cdot \mathrm{T}}\right)$

$\alpha_{\mathrm{Ks}}^{\mathrm{P}}=\frac{7.2469 \cdot 10^{-3}}{1+\exp \left(-\frac{\mathrm{V}_{\mathrm{m}}-8.0876 \cdot 10^{1}}{0.9005} \cdot \frac{\mathrm{F}}{\mathrm{R} \cdot \mathrm{T}}\right)}$

$\beta_{\mathrm{Ks}}^{\mathrm{P}}=\frac{3.3092 \cdot 10^{-3}}{1+\exp \left(\frac{\mathrm{V}_{\mathrm{m}}-1.4431 \cdot 10^{-2}}{3.4604} \cdot \frac{\mathrm{F}}{\mathrm{R} \cdot \mathrm{T}}\right)}$

$\gamma_{\mathrm{Ks}}^{\mathrm{P}}=\frac{6.6278 \cdot 10^{-2}}{1+\exp \left(-\frac{\mathrm{V}_{\mathrm{m}}+1.4175 \cdot 10^{-3}}{0.1937} \cdot \frac{\mathrm{F}}{\mathrm{R} \cdot \mathrm{T}}\right)}$

$\delta_{\mathrm{Ks}}^{\mathrm{P}}=3.0039 \cdot 10^{-2} \cdot \exp \left(3.6233 \cdot 10^{-3} \cdot \frac{\mathrm{V}_{\mathrm{m}} \cdot \mathrm{F}}{\mathrm{R} \cdot \mathrm{T}}\right)$

$\eta_{\mathrm{Ks}}^{\mathrm{P}}=7.7647 \cdot 10^{-3}+\frac{7.7303 \cdot 10^{-2}-7.7647 \cdot 10^{-3}}{1+\exp \left(\frac{\mathrm{V}_{\mathrm{m}}+1.3807 \cdot 10^{1}}{0.9041} \cdot \frac{\mathrm{F}}{\mathrm{R} \cdot \mathrm{T}}\right)}$

$\theta_{\mathrm{Ks}}^{\mathrm{P}}=3.4785 \cdot 10^{-4}$

$\omega_{\mathrm{Ks}}^{\mathrm{P}}=5.6517 \cdot 10^{-4} \cdot \exp \left(-1.5643 \cdot \frac{\mathrm{V}_{\mathrm{m}} \cdot \mathrm{F}}{\mathrm{R} \cdot \mathrm{T}}\right)$

$\psi_{\mathrm{Ks}}^{\mathrm{P}}=2.8148 \cdot 10^{-5} \cdot \exp \left(1.9762 \cdot 10^{-4} \cdot \frac{\mathrm{V}_{\mathrm{m}} \cdot \mathrm{F}}{\mathrm{R} \cdot \mathrm{T}}\right)$

And $\overline{\mathrm{G}}_{\mathrm{Ks}}=0.6603 \cdot\left(1+\frac{0.6}{1+\left(3.8 \frac{10^{-5}}{\left[\mathrm{Ca}^{2+}\right]_{\mathrm{i}}}\right)^{1.4}}\right)$ for all conditions. 


\subsection{Ultrarapid plateau $\mathrm{K}^{+}$current $\left(\mathrm{I}_{\mathrm{Kur}} / \mathrm{I}_{\mathrm{Kp}}\right)$}

\subsubsection{HRd2009 version (Chapter 2)}

The formulation for the plateau potassium current is equivalent to that in the original HRd model. ${ }^{4}$

$\overline{\mathrm{G}}_{\mathrm{Kur}}=2.760 \cdot 10^{-3}$

$\mathrm{I}_{\text {Kur }}=\overline{\mathrm{G}}_{\text {Kur }} \cdot \frac{1}{1+\exp \left(\left(7.488-\mathrm{V}_{\mathrm{m}}\right) / 5.980\right)} \cdot\left(\mathrm{V}_{\mathrm{m}}-\mathrm{E}_{\mathrm{K}}\right)$

\subsubsection{HRdßARS and HRdßARS Stoch version (Chapters 3-7)}

In many species and cell types, there exists an ultrarapid delayed rectifier potassium current activating at plateau membrane potentials $\left(I_{K u r}\right)^{43}$ Although $I_{K u r}$ was originally thought to be functionally absent in ventricular myocytes from larger mammals including the dog, recent reports describe a plateau activated potassium current sensitive to micromolar concentrations of 4aminopyridine. ${ }^{44,45}$ This current is increased in the presence of isoproterenol. In addition, the nonstimulated I-V relationship of this current is consistent with the plateau potassium current that is present in the baseline model. ${ }^{21}$ Therefore, adrenergic stimulation-dependent changes in I-V of $I_{\text {Kur }}$ are included in the model and replace the original formulation of $I_{\mathrm{kp}}$.

\section{Nonphosphorylated Channels:}

$$
\begin{aligned}
& \mathrm{G}_{\mathrm{Kur}}^{\mathrm{NP}}=3.48 \cdot 10^{-3} \\
& \mathrm{I}_{\mathrm{Kur}}^{\mathrm{NP}}=\mathrm{G}_{\mathrm{Kur}}^{\mathrm{NP}} \cdot \frac{1}{1+\exp \left(\left(15.0-\mathrm{V}_{\mathrm{m}}\right) / 17\right)} \cdot\left(\mathrm{V}_{\mathrm{m}}-\mathrm{E}_{\mathrm{K}}\right)
\end{aligned}
$$

Phosphorylated Channels:

$$
\begin{aligned}
& \mathrm{G}_{\mathrm{Kur}}^{\mathrm{P}}=3.62 \cdot 3.48 \cdot 10^{-3} \\
& \mathrm{I}_{\mathrm{Kur}}^{\mathrm{P}}=\mathrm{G}_{\mathrm{Kur}}^{\mathrm{P}} \cdot \frac{1}{1+\exp \left(\left(36.0-\mathrm{V}_{\mathrm{m}}\right) / 17\right)} \cdot\left(\mathrm{V}_{\mathrm{m}}-\mathrm{E}_{\mathrm{K}}\right)
\end{aligned}
$$

\section{Combined I Kurcurrent:}

$\hat{\mathrm{f}}_{\mathrm{Kur}}^{\mathrm{P}}=\frac{\mathrm{f}_{\mathrm{IKur}}^{\mathrm{P}}-0.3937}{0.5894-0.3937}$

$\mathrm{I}_{\text {Kur }}=\hat{\mathrm{f}}_{\mathrm{Kur}}^{\mathrm{P}} \cdot \mathrm{I}_{\mathrm{Kur}}^{\mathrm{P}}+\left(1-\hat{\mathrm{f}}_{\mathrm{Kur}}^{\mathrm{P}}\right) \cdot \mathrm{I}_{\mathrm{Kur}}^{\mathrm{NP}}$

\section{$1.18 \mathrm{Na}^{+}$current $\left(I_{\mathrm{Na}}\right)$}

\subsubsection{HRd2009 version (Chapter 2)}

Fast $\mathrm{Na}^{+}$current density $\left(\mathrm{I}_{\mathrm{Na}}\right)$ was adjusted to achieve conduction velocity, ${ }^{46}$ maximum upstroke velocity ${ }^{47}$ and maximum upstroke voltage ${ }^{47}$ consistent with data in canine epicardial tissue. $\mathrm{Na}^{+}$ 
current recovery from inactivation was adjusted to fit data on recovery of upstroke velocity in canine epicardial myocytes ${ }^{48}$ :

$$
\begin{aligned}
\alpha_{\mathrm{m}} & =\frac{0.32 \cdot\left(\mathrm{V}_{\mathrm{m}}+47.13\right)}{1-\exp \left(-0.1 \cdot\left(\mathrm{V}_{\mathrm{m}}+47.13\right)\right)} \\
\beta_{\mathrm{m}} & =0.08 \cdot \exp \left(-\frac{\mathrm{V}_{\mathrm{m}}}{11}\right) \\
\text { If } \mathrm{V}_{\mathrm{m}} & >-40.0 \mathrm{mV} \\
\alpha_{\mathrm{h}} & =0.0, \quad \alpha_{\mathrm{j}}=0.0 \\
\beta_{\mathrm{h}} & =\frac{1}{0.13 \cdot\left(1+\exp \left(\frac{\mathrm{V}_{\mathrm{m}}+10.66}{-11.1}\right)\right)} \\
\beta_{\mathrm{j}} & =\frac{0.3 \cdot \exp \left(-2.535 \cdot 10^{-7} \cdot \mathrm{V}_{\mathrm{m}}\right)}{1+\exp \left(-0.1 \cdot\left(\mathrm{V}_{\mathrm{m}}+32\right)\right)}
\end{aligned}
$$

Else

$$
\begin{array}{rl}
\alpha_{\mathrm{h}} & =0.135 \cdot \exp \left(\frac{80+\mathrm{V}_{\mathrm{m}}}{-6.8}\right) \\
\alpha_{\mathrm{j}} & =\frac{\left(-1.2714 \cdot 10^{5} \cdot \exp \left(0.2444 \cdot \mathrm{V}_{\mathrm{m}}\right)-6.948 \cdot 10^{-5} \cdot \exp \left(-0.04391 \cdot \mathrm{V}_{\mathrm{m}}\right)\right) \cdot\left(\mathrm{V}_{\mathrm{m}}+37.78\right)}{1+\exp \left(0.311 \cdot\left(\mathrm{V}_{\mathrm{m}}+79.23\right)\right)} \\
\beta_{\mathrm{h}} & =3.56 \cdot \exp \left(0.079 \cdot \mathrm{V}_{\mathrm{m}}\right)+3.1 \cdot 10^{5} \cdot \exp \left(0.35 \cdot \mathrm{V}_{\mathrm{m}}\right) \\
\beta_{\mathrm{j}} & =\frac{0.1212 \cdot \exp \left(-0.01052 \cdot \mathrm{V}_{\mathrm{m}}\right)}{1+\exp \left(-0.1378 \cdot\left(\mathrm{V}_{\mathrm{m}}+40.14\right)\right)} \\
\frac{\mathrm{dm}}{\mathrm{dt}} & =\alpha_{\mathrm{m}} \cdot(1-\mathrm{m})-\beta_{\mathrm{m}} \cdot \mathrm{m} \\
\frac{\mathrm{dh}}{\mathrm{dt}}=\alpha_{\mathrm{h}} \cdot(1-\mathrm{h})-\beta_{\mathrm{h}} \cdot \mathrm{h} & \mathrm{dj} \\
\frac{\mathrm{dt}}{2}=\alpha_{\mathrm{j}} \cdot(1-\mathrm{j})-\beta_{\mathrm{j}} \cdot \mathrm{j} \\
\overline{\mathrm{G}}_{\mathrm{Na}}=9.075 \frac{\mathrm{mS}}{\mu \mathrm{F}} \\
\mathrm{I}_{\mathrm{Na}}=\overline{\mathrm{G}}_{\mathrm{Na}} \cdot \mathrm{m}^{3} \cdot \mathrm{h} \cdot \mathrm{j} \cdot\left(\mathrm{V}_{\mathrm{m}}-\mathrm{E}_{\mathrm{Na}}\right)
\end{array}
$$

\subsubsection{HRdßARS version (Chapters 3-6)}

$\beta A R$ stimulation alters $I_{\mathrm{Na}}$ through both increase channel trafficking as well as phosphorylation of a number of consensus phosphorylation sites. This results in increased current amplitude and a hyperpolarizing shift of the activation and inactivation I-V relationships. ${ }^{49,50}$ The model reproduces the $3.7 \mathrm{mV}$ and $4.9 \mathrm{mV}$ shifts in respectively the activation and inactivation curves measured in 5 
$\mathrm{mmol} / \mathrm{L}$ extracullar $\mathrm{Na}^{+}$in canine ventricular myocytes in the presence of a PKA activator 'cocktail' (8-cpt-cAMP, IBMX and forskolin). ${ }^{50} \mathrm{~A}$ similar modulation of $\mathrm{I}_{\mathrm{Na}}$ by CaMKII has been reported in rabbit myocytes overexpressing CaMKII $\delta^{51}$ and was incorporated in a previous model ${ }^{24}$. We incorporate four populations of $I_{\mathrm{Na}}$ (non-phosphorylated, phosphorylated by PKA, phosphorylated by CaMKII or phosphorylated by both kinases).

\section{Nonphosphorylated Channels:}

$$
\begin{aligned}
& \alpha_{\mathrm{m}}^{\mathrm{NP}}=\frac{0.32 \cdot\left(\mathrm{V}_{\mathrm{m}}+58.5029\right)}{1-\exp \left(-0.1 \cdot\left(\mathrm{V}_{\mathrm{m}}+58.5029\right)\right)} \\
& \beta_{\mathrm{m}}^{\mathrm{NP}}=0.08 \cdot \exp \left(-\frac{\mathrm{V}_{\mathrm{m}}-13.7299}{11}\right) \\
& \text { If } \mathrm{V}_{\mathrm{m}}>-40.0 \mathrm{mV} \\
& \alpha_{\mathrm{h}}^{\mathrm{NP}}=0.0, \quad \alpha_{\mathrm{j}}^{\mathrm{NP}}=0.0 \\
& \beta_{\mathrm{h}}^{\mathrm{NP}}=\frac{1}{0.13 \cdot\left(1+\exp \left(\frac{\mathrm{V}_{\mathrm{m}}+27.4034}{-11.1}\right)\right)} \\
& \beta_{\mathrm{j}}^{\mathrm{NP}}=\frac{0.3 \cdot \exp \left(-2.535 \cdot 10^{-7} \cdot \mathrm{V}_{\mathrm{m}}\right)}{1+\exp \left(-0.1 \cdot\left(\mathrm{V}_{\mathrm{m}}+32\right)\right)}
\end{aligned}
$$

Else

$$
\begin{aligned}
& \alpha_{\mathrm{h}}^{\mathrm{NP}}=0.135 \cdot \exp \left(\frac{87+\mathrm{V}_{\mathrm{m}}}{-6.8}\right) \alpha_{\mathrm{j}}^{\mathrm{NP}} \\
&=\frac{\left(-1.2714 \cdot 10^{5} \cdot \exp \left(0.2444 \cdot \mathrm{V}_{\mathrm{m}}\right)-6.948 \cdot 10^{-5} \cdot \exp \left(-0.04391 \cdot \mathrm{V}_{\mathrm{m}}\right)\right) \cdot\left(\mathrm{V}_{\mathrm{m}}+37.78\right)}{1+\exp \left(0.311 \cdot\left(\mathrm{V}_{\mathrm{m}}+79.23\right)\right)} \\
& \beta_{\mathrm{h}}^{\mathrm{NP}}=3.56 \cdot \exp \left(0.079 \cdot\left(\mathrm{V}_{\mathrm{m}}+7\right)\right)+3.1 \cdot 10^{5} \cdot \exp \left(0.35 \cdot\left(\mathrm{V}_{\mathrm{m}}+7\right)\right) \\
& \frac{\beta_{\mathrm{j}}^{\mathrm{NP}}}{\mathrm{dm}}=\frac{0.1212 \cdot \exp \left(-0.01052 \cdot \mathrm{V}_{\mathrm{m}}\right)}{1+\exp \left(-0.1378 \cdot\left(\mathrm{V}_{\mathrm{m}}+40.14\right)\right)} \\
& \frac{\mathrm{dt}}{\mathrm{dt}}=\alpha_{\mathrm{m}}^{\mathrm{NP}} \cdot\left(1-\mathrm{m}^{\mathrm{NP}}\right)-\beta_{\mathrm{m}}^{\mathrm{NP}} \cdot \mathrm{m}^{\mathrm{NP}} \\
& \frac{\mathrm{dj}^{\mathrm{NP}}}{\mathrm{dt}}=\alpha_{\mathrm{h}}^{\mathrm{NP}} \cdot\left(1-\mathrm{h}^{\mathrm{NP}}\right)-\beta_{\mathrm{h}}^{\mathrm{NP}} \cdot \mathrm{h}^{\mathrm{NP}} \cdot\left(1-\mathrm{j}^{\mathrm{NP}}\right)-\beta_{\mathrm{j}}^{\mathrm{NP}} \cdot \mathrm{j}^{\mathrm{NP}} \\
& \overline{\mathrm{G}}_{\mathrm{Na}}^{\mathrm{NP}}=19.5112 \frac{\mathrm{mS}}{\mu \mathrm{F}} \\
& \mathrm{I}_{\mathrm{Na}}^{\mathrm{NP}}=\overline{\mathrm{G}}_{\mathrm{Na}}^{\mathrm{NP}} \cdot\left(\mathrm{m}^{\mathrm{NP}}\right)^{3} \cdot \mathrm{h}^{\mathrm{NP}} \cdot \mathrm{j}^{\mathrm{NP}} \cdot\left(\mathrm{V}_{\mathrm{m}}-\mathrm{E}_{\mathrm{Na}}\right)
\end{aligned}
$$




\section{PKA Phosphorylated Channels:}

$$
\begin{aligned}
& \Delta \mathrm{V}_{\mathrm{Na}, \mathrm{Act}}=3.7 \mathrm{mV}, \quad \Delta \mathrm{V}_{\mathrm{Na} \text {,Inact }}^{\mathrm{PKA}}=4.9 \mathrm{mV} \\
& \alpha_{\mathrm{m}}^{\mathrm{P}, \mathrm{PKA}}=\frac{0.32 \cdot\left(\mathrm{V}_{\mathrm{m}}+58.5029+\Delta \mathrm{V}_{\mathrm{Na}, \mathrm{Act}}\right)}{1-\exp \left(-0.1 \cdot\left(\mathrm{V}_{\mathrm{m}}+58.5029+\Delta \mathrm{V}_{\mathrm{Na}, \mathrm{Act}}\right)\right)} \\
& \beta_{\mathrm{m}}^{\mathrm{P}, \mathrm{PKA}}=0.08 \cdot \exp \left(-\frac{\mathrm{V}_{\mathrm{m}}-13.7299+\Delta \mathrm{V}_{\mathrm{Na}, \mathrm{Act}}}{11}\right) \\
& \text { If } \mathrm{V}_{\mathrm{m}}>-40.0 \mathrm{mV} \\
& \alpha_{\mathrm{h}}^{\mathrm{P}, \mathrm{PKA}}=0.0, \quad \alpha_{\mathrm{j}}^{\mathrm{P}, \mathrm{PKA}}=0.0 \\
& \beta_{\mathrm{h}}^{\mathrm{P} \text {,PKA }}=\frac{1}{0.13 \cdot\left(1+\exp \left(\frac{\mathrm{V}_{\mathrm{m}}+\Delta \mathrm{V}_{\mathrm{Na}, \text { Inact }}^{\mathrm{PKA}}+27.4034}{-11.1}\right)\right)} \\
& \beta_{\mathrm{j}}^{\mathrm{P}, \mathrm{PKA}}=\frac{0.3 \cdot \exp \left(-2.535 \cdot 10^{-7} \cdot\left(\mathrm{V}_{\mathrm{m}}+\Delta \mathrm{V}_{\mathrm{Na}, \text { Inact }}^{\mathrm{PKA}}\right)\right)}{1+\exp \left(-0.1 \cdot\left(\mathrm{V}_{\mathrm{m}}+\Delta \mathrm{V}_{\mathrm{Na}, \text { Inact }}^{\mathrm{PKA}}+32\right)\right)}
\end{aligned}
$$

Else

$$
\begin{aligned}
& \alpha_{\mathrm{h}}^{\mathrm{P} \text {,PKA }}=0.135 \cdot \exp \left(\frac{87+\mathrm{V}_{\mathrm{m}}+\Delta \mathrm{V}_{\mathrm{Na}, \text { Inact }}^{\mathrm{PKA}}}{-6.8}\right) \\
& \alpha_{\mathrm{j}}^{\mathrm{P}, \mathrm{PKA}}=\frac{1}{1+\exp \left(0.311 \cdot\left(\mathrm{V}_{\mathrm{m}}+\Delta \mathrm{V}_{\text {Na,Inact }}^{\mathrm{PKA}}+79.23\right)\right)} \\
& \cdot\left(-1.2714 \cdot 10^{5} \cdot \exp \left(0.2444 \cdot\left(\mathrm{V}_{\mathrm{m}}+\Delta \mathrm{V}_{\mathrm{Na}, \text { Inact }}^{\mathrm{PKA}}\right)\right)-6.948 \cdot 10^{-5}\right. \\
& \left.\cdot \exp \left(-0.04391 \cdot\left(\mathrm{V}_{\mathrm{m}}+\Delta \mathrm{V}_{\mathrm{Na}, \text { Inact }}^{\mathrm{PKA}}\right)\right)\right) \cdot\left(\mathrm{V}_{\mathrm{m}}+\Delta \mathrm{V}_{\mathrm{Na} \text {,Inact }}^{\mathrm{PKA}}+37.78\right) \\
& \beta_{\mathrm{h}}^{\mathrm{P} \text { PKA }}=3.56 \cdot \exp \left(0.079 \cdot\left(\mathrm{V}_{\mathrm{m}}+\Delta \mathrm{V}_{\mathrm{Na} \text {,Inact }}^{\mathrm{PKA}}+7\right)\right)+3.1 \cdot 10^{5} \\
& \cdot \exp \left(0.35 \cdot\left(\mathrm{V}_{\mathrm{m}}+\Delta \mathrm{V}_{\mathrm{Na}, \text { Inact }}^{\mathrm{PKA}}+7\right)\right) \\
& \beta_{\mathrm{j}}^{\mathrm{P}, \mathrm{PKA}}=\frac{0.1212 \cdot \exp \left(-0.01052 \cdot\left(\mathrm{V}_{\mathrm{m}}+\Delta \mathrm{V}_{\mathrm{Na}, \text { Inact }}^{\mathrm{PKA}}\right)\right)}{1+\exp \left(-0.1378 \cdot\left(\mathrm{V}_{\mathrm{m}}+\Delta \mathrm{V}_{\mathrm{Na}, \text { Inact }}^{\mathrm{PKA}}+40.14\right)\right)} \\
& \frac{\mathrm{dm}^{\mathrm{P}, \mathrm{PKA}}}{\mathrm{dt}}=\alpha_{\mathrm{m}}^{\mathrm{P}, \mathrm{PKA}} \cdot\left(1-\mathrm{m}^{\mathrm{P}, \mathrm{PKA}}\right)-\beta_{\mathrm{m}}^{\mathrm{P}, \mathrm{PKA}} \cdot \mathrm{m}^{\mathrm{P}, \mathrm{PKA}} \\
& \frac{d h^{\mathrm{P}, \mathrm{PKA}}}{\mathrm{dt}}=\alpha_{\mathrm{h}}^{\mathrm{P}, \mathrm{PKA}} \cdot\left(1-\mathrm{h}^{\mathrm{P}, \mathrm{PKA}}\right)-\beta_{\mathrm{h}}^{\mathrm{P}, \mathrm{PKA}} \cdot \mathrm{h}^{\mathrm{P}, \mathrm{PKA}} \\
& \frac{d \mathrm{~d}^{\mathrm{P}, \mathrm{PKA}}}{\mathrm{dt}}=\alpha_{\mathrm{j}}^{\mathrm{P}, \mathrm{PKA}} \cdot\left(1-\mathrm{j}^{\mathrm{P}, \mathrm{PKA}}\right)-\beta_{\mathrm{j}}^{\mathrm{P}, \mathrm{PKA}} \cdot \mathrm{j}^{\mathrm{P}, \mathrm{PKA}}
\end{aligned}
$$




$$
\begin{aligned}
& \overline{\mathrm{G}}_{\mathrm{Na}}^{\mathrm{P}}=1.25 \cdot 19.5112 \frac{\mathrm{mS}}{\mu \mathrm{F}} \\
& \mathrm{I}_{\mathrm{Na}}^{\mathrm{P}, \mathrm{PKA}}=\overline{\mathrm{G}}_{\mathrm{Na}}^{\mathrm{P}} \cdot\left(\mathrm{m}^{\mathrm{P}, \mathrm{PKA}}\right)^{3} \cdot \mathrm{h}^{\mathrm{P}, \mathrm{PKA}} \cdot \mathrm{j}^{\mathrm{P}, \mathrm{PKA}} \cdot\left(\mathrm{V}_{\mathrm{m}}-\mathrm{E}_{\mathrm{Na}}\right)
\end{aligned}
$$

\section{CaMKII Phosphorylated Channels:}

$$
\begin{aligned}
& \Delta \mathrm{V}_{\mathrm{Na}, \text { Inact }}^{\mathrm{CaMK}}=3.25 \mathrm{mV} \\
& \alpha_{\mathrm{m}}^{\mathrm{P}, \mathrm{CaMK}}=\alpha_{\mathrm{m}}^{\mathrm{NP}}, \quad \beta_{\mathrm{m}}^{\mathrm{P}, \mathrm{CaMK}}=\beta_{\mathrm{m}}^{\mathrm{NP}} \\
& \text { If } \mathrm{V}_{\mathrm{m}}+\Delta \mathrm{V}_{\mathrm{Na}, \text { Inact }}^{\mathrm{CaMK}}>-40.0 \mathrm{mV} \\
& \alpha_{\mathrm{h}}^{\mathrm{P}, \mathrm{CaMK}}=0.0, \quad \alpha_{\mathrm{j}}^{\mathrm{P}, \mathrm{CaMK}}=0.0 \\
& \beta_{\mathrm{h}}^{\mathrm{P}, \text { СaMK }}=\frac{1}{0.13 \cdot\left(1+\exp \left(\frac{\mathrm{V}_{\mathrm{m}}+\Delta \mathrm{V}_{\mathrm{Na}, \text { Inact }}^{\mathrm{CaMK}}+27.4034}{-11.1}\right)\right)} \\
& \beta_{\mathrm{j}}^{\mathrm{P}, \mathrm{CaMK}}=\frac{0.3 \cdot \exp \left(-2.535 \cdot 10^{-7} \cdot\left(\mathrm{V}_{\mathrm{m}}+\Delta \mathrm{V}_{\mathrm{Na}, \text { Inact }}^{\mathrm{CaMK}}\right)\right)}{1+\exp \left(-0.1 \cdot\left(\mathrm{V}_{\mathrm{m}}+\Delta \mathrm{V}_{\mathrm{Na}, \text { Inact }}^{\mathrm{CaMK}}+32\right)\right)}
\end{aligned}
$$

Else

$$
\begin{aligned}
& \alpha_{\mathrm{h}}^{\mathrm{P}, \text { CaMK }}=0.135 \cdot \exp \left(\frac{87+\mathrm{V}_{\mathrm{m}}+\Delta \mathrm{V}_{\mathrm{Na}, \text { Inact }}^{\mathrm{CaMK}}}{-6.8}\right) \\
& \alpha_{\mathrm{j}}^{\mathrm{P}, \mathrm{CaMK}}=0.82 \cdot \frac{1}{1+\exp \left(0.311 \cdot\left(\mathrm{V}_{\mathrm{m}}+\Delta \mathrm{V}_{\mathrm{Na}, \text { Inact }}^{\mathrm{CaMK}}+79.23\right)\right)} \\
& \cdot\left(-1.2714 \cdot 10^{5} \cdot \exp \left(0.2444 \cdot\left(\mathrm{V}_{\mathrm{m}}+\Delta \mathrm{V}_{\mathrm{Na}, \text { Inact }}^{\mathrm{CaMK}}\right)\right)-6.948 \cdot 10^{-5}\right. \\
& \left.\cdot \exp \left(-0.04391 \cdot\left(\mathrm{V}_{\mathrm{m}}+\Delta \mathrm{V}_{\mathrm{Na}, \text { Inact }}^{\mathrm{CaMK}}\right)\right)\right) \cdot\left(\mathrm{V}_{\mathrm{m}}+\Delta \mathrm{V}_{\mathrm{Na}, \text { Inact }}^{\mathrm{CaMK}}+37.78\right) \\
& \beta_{\mathrm{h}}^{\text {P,CaMK }}=3.56 \cdot \exp \left(0.079 \cdot\left(\mathrm{V}_{\mathrm{m}}+\Delta \mathrm{V}_{\mathrm{Na}, \text { Inact }}^{\mathrm{CaMK}}+7\right)\right)+3.1 \cdot 10^{5} \\
& \cdot \exp \left(0.35 \cdot\left(\mathrm{V}_{\mathrm{m}}+\Delta \mathrm{V}_{\mathrm{Na}, \text { Inact }}^{\mathrm{CaMK}}+7\right)\right) \\
& \beta_{\mathrm{j}}^{\mathrm{P}, \mathrm{CaMK}}=\frac{0.1212 \cdot \exp \left(-0.01052 \cdot\left(\mathrm{V}_{\mathrm{m}}+\Delta \mathrm{V}_{\mathrm{Na}, \text { Inact }}^{\mathrm{CaMK}}\right)\right)}{1+\exp \left(-0.1378 \cdot\left(\mathrm{V}_{\mathrm{m}}+\Delta \mathrm{V}_{\text {Na,Inact }}^{\mathrm{CaMK}}+40.14\right)\right)} \\
& \frac{\mathrm{dm}^{\mathrm{P}, \text { CaMKII }}}{\mathrm{dt}}=\alpha_{\mathrm{m}}^{\mathrm{P}, \text { CAMK }} \cdot\left(1-\mathrm{m}^{\mathrm{P}, \mathrm{CAMK}}\right)-\beta_{\mathrm{m}}^{\mathrm{P}, \mathrm{CaMK}} \cdot \mathrm{m}^{\mathrm{P}, \mathrm{CaMK}} \\
& \frac{\mathrm{dh}^{\mathrm{P}, \mathrm{CaMKII}}}{\mathrm{dt}}=\alpha_{\mathrm{h}}^{\mathrm{P}, \text { CaMK }} \cdot\left(1-\mathrm{h}^{\mathrm{P}, \mathrm{CaMK}}\right)-\beta_{\mathrm{h}}^{\mathrm{P}, \mathrm{CaMK}} \cdot \mathrm{h}^{\mathrm{P}, \mathrm{CaMK}} \\
& \frac{\mathrm{dj}^{\mathrm{P}, \mathrm{CaMKII}}}{\mathrm{dt}}=\alpha_{\mathrm{j}}^{\mathrm{P}, \mathrm{CaMK}} \cdot\left(1-\mathrm{j}^{\mathrm{P}, \mathrm{CaMK}}\right)-\beta_{\mathrm{j}}^{\mathrm{P}, \mathrm{CaMK}} \cdot \mathrm{j}^{\mathrm{P}, \mathrm{CaMK}}
\end{aligned}
$$


$\mathrm{I}_{\mathrm{Na}}^{\mathrm{P}, \mathrm{CaMK}}=\overline{\mathrm{G}}_{\mathrm{Na}}^{\mathrm{NP}} \cdot\left(\mathrm{m}^{\mathrm{P}, \mathrm{CaMK}}\right)^{3} \cdot \mathrm{h}^{\mathrm{P}, \mathrm{CaMK}} \cdot \mathrm{j}^{\mathrm{P}, \mathrm{CaMK}} \cdot\left(\mathrm{V}_{\mathrm{m}}-\mathrm{E}_{\mathrm{Na}}\right)$

\section{PKA and CaMKII Phosphorylated Channels:}

$\overline{\mathrm{G}}_{\mathrm{Na}}^{\mathrm{P}}=19.5112 \frac{\mathrm{mS}}{\mu \mathrm{F}}$

$\mathrm{I}_{\mathrm{Na}}^{\mathrm{P} \text {,Both }}=\overline{\mathrm{G}}_{\mathrm{Na}}^{\mathrm{P}} \cdot\left(\mathrm{m}^{\mathrm{P}, \mathrm{PKA}}\right)^{3} \cdot \mathrm{h}^{\mathrm{P}, \mathrm{CaMK}} \cdot \mathrm{j}^{\mathrm{P}, \mathrm{CaMK}} \cdot\left(\mathrm{V}_{\mathrm{m}}-\mathrm{E}_{\mathrm{Na}}\right)$

Combined I $I_{\text {Na Current: }}$

$$
\begin{aligned}
& \hat{\mathrm{f}}_{\mathrm{INa}}^{\mathrm{PKA}}=\frac{\mathrm{f}_{\mathrm{INa}}^{\mathrm{P}}-0.2395}{0.9501-0.2395} \\
& \mathrm{f}_{\text {INa }}^{\text {Both }}=\hat{\mathrm{f}}_{\text {INa }}^{\text {PKA }} \cdot \mathrm{f}_{\text {INa }}^{\text {P,CaMKII }} \\
& \mathrm{f}_{\text {INa }}^{\text {PKA,only }}=\hat{\mathrm{f}}_{\text {INa }}^{\text {PKA }}-\mathrm{f}_{\text {INa }}^{\text {Both }} \\
& \mathrm{f}_{\text {INa }}^{\text {CaMKII,only }}=\mathrm{f}_{\text {INa }}^{\text {P,CaMKII }}-\mathrm{f}_{\text {INa }}^{\text {Both }} \\
& \mathrm{I}_{\mathrm{Na}}=\left(1-\mathrm{f}_{\mathrm{INa}}^{\text {PKA,only }}-\mathrm{f}_{\mathrm{INa}}^{\text {CaMKII,only }}-\mathrm{f}_{\mathrm{INa}}^{\mathrm{Both}}\right) \cdot \mathrm{I}_{\mathrm{Na}}^{\mathrm{NP}}+\mathrm{f}_{\mathrm{INa}}^{\mathrm{PKA} \text {,only }} \cdot \mathrm{I}_{\mathrm{Na}}^{\mathrm{P}, \text { PKA }}+\mathrm{f}_{\mathrm{INa}}^{\text {CaMKII,only }} \\
& \cdot \mathrm{I}_{\mathrm{Na}}^{\mathrm{P}, \mathrm{CaMK}}+\mathrm{f}_{\mathrm{INa}}^{\mathrm{Both}} \cdot \mathrm{I}_{\mathrm{Na}}^{\mathrm{P}, \text { Both }}
\end{aligned}
$$

\subsubsection{HRdßARS Stoch version (Chapter 7)}

The Markov model structure of Clancy and Rudy ${ }^{52}$ was employed for both normal inactivation and slow inactivation populations (Figure $\mathbf{2}$ of Chapter 7). Model parameters were adjusted to reproduce characteristics of $I_{\mathrm{Na}}$ and $I_{\mathrm{NaL}}$ from the original Hodgkin-Huxley-based formulation, including PKA- and CaMKII-dependent alterations (section 1.18.2). Model $I_{\text {Na }}$ peak I-V relationship and steady-state inactivation are consistent with those measured in isolated canine ventricular myocytes. ${ }^{50}$

$$
\begin{aligned}
& \alpha_{\mathrm{Na}, 11}^{\mathrm{NP}}=\frac{4.5170}{6.6161 \cdot 10^{-2} \cdot \exp \left(-\frac{\mathrm{V}_{\mathrm{m}}}{18.033}\right)+2.1276 \cdot 10^{-1} \cdot \exp \left(-\frac{\mathrm{V}_{\mathrm{m}}}{167.77}\right)} \\
& \alpha_{\mathrm{Na}, 12}^{\mathrm{NP}}=\frac{4.5170}{6.6161 \cdot 10^{-2} \cdot \exp \left(-\frac{\mathrm{V}_{\mathrm{m}}}{24.237}\right)+1.7127 \cdot 10^{-1} \cdot \exp \left(-\frac{\mathrm{V}_{\mathrm{m}}}{167.77}\right)} \\
& \alpha_{\mathrm{Na}, 13}^{\mathrm{NP}}=\frac{4.5170}{6.6161 \cdot 10^{-2} \cdot \exp \left(-\frac{\mathrm{V}_{\mathrm{m}}}{13.038}\right)+2.7731 \cdot 10^{-1} \cdot \exp \left(-\frac{\mathrm{V}_{\mathrm{m}}}{167.77}\right)} \\
& \beta_{\mathrm{Na}, 11}^{\mathrm{NP}}=1.7687 \cdot 10^{-1} \cdot \exp \left(-\frac{\mathrm{V}_{\mathrm{m}}}{15.905}\right) \\
& \beta_{\mathrm{Na}, 12}^{\mathrm{NP}}=2.2240 \cdot 10^{-1} \cdot \exp \left(-\frac{\mathrm{V}_{\mathrm{m}}-6.1967}{15.905}\right) \\
& \beta_{\mathrm{Na}, 13}^{\mathrm{NP}}=2.9500 \cdot 10^{-1} \cdot \exp \left(-\frac{\mathrm{V}_{\mathrm{m}}-10.891}{15.905}\right) \\
& \alpha_{\mathrm{Na}, 2}^{\mathrm{NP}}=6.3736 \cdot \exp \left(\frac{\mathrm{V}_{\mathrm{m}}+3.3271 \cdot \mathrm{f}_{\mathrm{INa}}^{\mathrm{P}, \mathrm{CaMKII}}}{109.64}\right), \quad \alpha_{\mathrm{Na}, 2 \mathrm{l}}^{\mathrm{NP}}=\alpha_{\mathrm{Na}, 2}^{\mathrm{NP}} \cdot 1.8366 \cdot 10^{-4}
\end{aligned}
$$




$$
\begin{aligned}
& \beta_{\mathrm{Na}, \mathrm{i}}^{\mathrm{NP}}=8.0991 \cdot 10^{-8} \cdot \exp \left(-\frac{\mathrm{V}_{\mathrm{m}}+3.3271 \cdot \mathrm{f}_{\mathrm{INa}}^{\mathrm{P}, \mathrm{CaMKII}}}{5.6611}\right), \quad \beta_{\mathrm{Na}, \mathrm{il}}^{\mathrm{NP}}=\beta_{\mathrm{Na}, \mathrm{i}}^{\mathrm{NP}} \cdot 1.1014 \cdot 10^{-2} \\
& \alpha_{\mathrm{Na}, 4}^{\mathrm{NP}}=\frac{\beta_{\mathrm{Na}, \mathrm{i}}^{\mathrm{NP}}}{9.2183}, \quad \alpha_{\mathrm{Na}, 4 \mathrm{l}}^{\mathrm{NP}}=\alpha_{\mathrm{Na}, 4}^{\mathrm{NP}} \cdot 1.1014 \cdot 10^{-2} \\
& \alpha_{\mathrm{Na}, \mathrm{i}}^{\mathrm{NP}}=3.1367 \cdot 10^{-2}+1.6356 \cdot 10^{-5} \cdot\left(\mathrm{V}_{\mathrm{m}}+3.3271 \cdot \mathrm{f}_{\mathrm{INa}}^{\mathrm{P}, \mathrm{CaMKII}}\right), \\
& \alpha_{\mathrm{Na}, \mathrm{il}}^{\mathrm{NP}}=\alpha_{\mathrm{Na}, \mathrm{i}}^{\mathrm{NP}} \cdot 1.1014 \cdot 10^{-2} \\
& \beta_{\mathrm{Na}, 2}^{\mathrm{NP}}=\frac{\alpha_{\mathrm{Na}, 13}^{\mathrm{NP}} \cdot \alpha_{\mathrm{Na}, 2}^{\mathrm{NP}} \cdot \beta_{\mathrm{Na}, \mathrm{i}}^{\mathrm{NP}}}{\beta_{\mathrm{Na}, 13}^{\mathrm{NP}} \cdot \alpha_{\mathrm{Na}, \mathrm{i}}^{\mathrm{NP}}} \quad \beta_{\mathrm{Na}, 2 \mathrm{~N}}^{\mathrm{NP}}=\frac{\alpha_{\mathrm{Na}, 13}^{\mathrm{NP}} \cdot \alpha_{\mathrm{Na}, 21}^{\mathrm{NP}} \cdot \beta_{\mathrm{Na}, \mathrm{ll}}^{\mathrm{NP}}}{\beta_{\mathrm{Na}, 13}^{\mathrm{NP}} \cdot \alpha_{\mathrm{Na}, \mathrm{il}}^{\mathrm{NP}}} \\
& \gamma_{\mathrm{Na}}^{\mathrm{NP}}=1.3053 \cdot 10^{-7}+2.4015 \cdot 10^{-7} \cdot \mathrm{f}_{\mathrm{INa}}^{\mathrm{P}, \mathrm{CaMKII}}+\frac{2.90 \cdot 10^{-7}}{1+\left(\frac{4.0}{[\mathrm{ATXII}]}\right)^{2}} \\
& \delta_{\mathrm{Na}}^{\mathrm{NP}}=4.8365 \cdot 10^{-4} \\
& \mathrm{f}_{\mathrm{Na}, \text { slow }}^{\mathrm{NP}}=\frac{\gamma_{\mathrm{Na}}^{\mathrm{NP}}}{\gamma_{\mathrm{Na}}^{\mathrm{NP}}+\delta_{\mathrm{Na}}^{\mathrm{NP}}}
\end{aligned}
$$$$
\alpha_{\mathrm{Na}, 11}^{\mathrm{P}}=\frac{8.3241}{6.6161 \cdot 10^{-2} \cdot \exp \left(-\frac{\mathrm{V}_{\mathrm{m}}}{18.033}\right)+2.1276 \cdot 10^{-1} \cdot \exp \left(-\frac{\mathrm{V}_{\mathrm{m}}}{167.77}\right)}
$$$$
\alpha_{\mathrm{Na}, 12}^{\mathrm{P}}=\frac{8.3241}{6.6161 \cdot 10^{-2} \cdot \exp \left(-\frac{\mathrm{V}_{\mathrm{m}}}{24.237}\right)+1.7127 \cdot 10^{-1} \cdot \exp \left(-\frac{\mathrm{V}_{\mathrm{m}}}{167.77}\right)}
$$$$
\alpha_{\mathrm{Na}, 13}^{\mathrm{P}}=\frac{8.3241}{6.6161 \cdot 10^{-2} \cdot \exp \left(-\frac{\mathrm{V}_{\mathrm{m}}}{13.038}\right)+2.7731 \cdot 10^{-1} \cdot \exp \left(-\frac{\mathrm{V}_{\mathrm{m}}}{167.77}\right)}
$$$$
\beta_{\mathrm{Na}, 11}^{\mathrm{P}}=1.0336 \cdot 10^{-1} \cdot \exp \left(-\frac{\mathrm{V}_{\mathrm{m}}}{15.905}\right)
$$$$
\beta_{\mathrm{Na}, 12}^{\mathrm{P}}=2.2972 \cdot 10^{-1} \cdot \exp \left(-\frac{\mathrm{V}_{\mathrm{m}}-6.1967}{15.905}\right)
$$$$
\beta_{\mathrm{Na}, 13}^{\mathrm{P}}=2.6387 \cdot 10^{-1} \cdot \exp \left(-\frac{\mathrm{V}_{\mathrm{m}}-10.891}{15.905}\right)
$$$$
\alpha_{\mathrm{Na}, 2}^{\mathrm{P}}=5.3095 \cdot \exp \left(\frac{\mathrm{V}_{\mathrm{m}}+0.5625 \cdot \mathrm{f}_{\mathrm{INa}}^{\mathrm{P}, \mathrm{CaMKII}}}{109.64}\right), \quad \alpha_{\mathrm{Na}, 2 \mathrm{l}}^{\mathrm{P}}=\alpha_{\mathrm{Na}, 2}^{\mathrm{P}} \cdot 1.4827 \cdot 10^{-4}
$$$$
\beta_{\mathrm{Na}, \mathrm{i}}^{\mathrm{P}}=2.9162 \cdot 10^{-8} \cdot \exp \left(-\frac{\mathrm{V}_{\mathrm{m}}+0.5625 \cdot \mathrm{f}_{\mathrm{INa}}^{\mathrm{P}, \mathrm{CaMKII}}}{5.6779}\right), \quad \beta_{\mathrm{Na}, \mathrm{il}}^{\mathrm{NP}}=\beta_{\mathrm{Na}, \mathrm{i}}^{\mathrm{NP}} \cdot 2.6707 \cdot 10^{-2}
$$$$
\alpha_{\mathrm{Na}, 4}^{\mathrm{P}}=\frac{\beta_{\mathrm{Na}, \mathrm{i}}^{\mathrm{P}}}{6.0179}, \quad \alpha_{\mathrm{Na}, 4 \mathrm{l}}^{\mathrm{P}}=\alpha_{\mathrm{Na}, 4}^{\mathrm{P}} \cdot 2.6707 \cdot 10^{-2}
$$$$
\alpha_{\mathrm{Na}, \mathrm{i}}^{\mathrm{P}}=3.1367 \cdot 10^{-2}+7.9063 \cdot 10^{-6} \cdot\left(\mathrm{V}_{\mathrm{m}}+0.5625 \cdot \mathrm{f}_{\mathrm{INa}}^{\mathrm{P}, \mathrm{CaMKII}}\right) \text {, }
$$

$$
\alpha_{\mathrm{N} a, i \mathrm{l}}^{\mathrm{P}}=\alpha_{\mathrm{Na}, \mathrm{i}}^{\mathrm{P}} \cdot 2.6707 \cdot 10^{-2}
$$

$\beta_{\mathrm{Na}, 2}^{\mathrm{P}}=\frac{\alpha_{\mathrm{Na}, 13}^{\mathrm{P}} \cdot \alpha_{\mathrm{Na}, 2}^{\mathrm{P}} \cdot \beta_{\mathrm{Na}, \mathrm{i}}^{\mathrm{P}}}{\beta_{\mathrm{Na}, 13}^{\mathrm{P}} \cdot \alpha_{\mathrm{Na}, \mathrm{i}}^{\mathrm{P}}}, \quad \beta_{\mathrm{Na}, 21}^{\mathrm{NP}}=\frac{\alpha_{\mathrm{Na}, 13}^{\mathrm{P}} \cdot \alpha_{\mathrm{Na}, 21}^{\mathrm{P}} \cdot \beta_{\mathrm{Na}, \mathrm{il}}^{\mathrm{P}}}{\beta_{\mathrm{Na}, 13}^{\mathrm{P}} \cdot \alpha_{\mathrm{Na}, \mathrm{il}}^{\mathrm{P}}}$ 


$$
\begin{aligned}
& \gamma_{\mathrm{Na}}^{\mathrm{P}}=1.4099 \cdot 10^{-7}+2.1616 \cdot 10^{-7} \cdot \mathrm{f}_{\mathrm{INa}}^{\mathrm{P}, \mathrm{CaMKII}}+\frac{2.90 \cdot 10^{-7}}{1+\left(\frac{4.0}{[\text { ATXII }]}\right)^{2}} \\
& \delta_{\mathrm{Na}}^{\mathrm{P}}=4.6975 \cdot 10^{-4} \\
& \mathrm{f}_{\mathrm{Na} \text {,slow }}^{\mathrm{P}}=\frac{\gamma_{\mathrm{Na}}^{\mathrm{P}}}{\gamma_{\mathrm{Na}}^{\mathrm{P}}+\delta_{\mathrm{Na}}^{\mathrm{P}}}
\end{aligned}
$$

For $x \in\{N P, P\}:$

$$
\begin{aligned}
& \frac{\mathrm{dLC} 3_{\mathrm{Na}}^{\mathrm{X}}}{\mathrm{dt}}=\beta_{\mathrm{Na}, 11}^{\mathrm{x}} \cdot \mathrm{LC}_{\mathrm{Na}}^{\mathrm{x}}+\beta_{\mathrm{Na}, \mathrm{il}}^{\mathrm{X}} \cdot \mathrm{LIC}_{\mathrm{Na}}^{\mathrm{x}}-\left(\alpha_{\mathrm{Na}, 11}^{\mathrm{x}}+\alpha_{\mathrm{Na}, \mathrm{il}}^{\mathrm{x}}\right) \cdot \operatorname{LC} 3_{\mathrm{Na}}^{\mathrm{X}} \\
& \frac{\mathrm{dLC} 2_{\mathrm{Na}}^{\mathrm{X}}}{\mathrm{dt}}=\alpha_{\mathrm{Na}, 11}^{\mathrm{x}} \cdot \mathrm{LC}_{\mathrm{Na}}^{\mathrm{x}}+\beta_{\mathrm{Na}, 12}^{\mathrm{x}} \cdot \mathrm{LC}_{\mathrm{Na}}^{\mathrm{x}}+\beta_{\mathrm{Na}, \mathrm{il}}^{\mathrm{x}} \cdot \mathrm{LIC}_{\mathrm{Na}}^{\mathrm{x}}-\left(\alpha_{\mathrm{Na}, 12}^{\mathrm{x}}+\beta_{\mathrm{Na}, 11}^{\mathrm{x}}+\alpha_{\mathrm{Na}, \mathrm{il}}^{\mathrm{x}}\right) \cdot \mathrm{LC}_{\mathrm{Na}}^{\mathrm{x}} \\
& \frac{\mathrm{dLC} 1_{\mathrm{Na}}^{\mathrm{x}}}{\mathrm{dt}}=\alpha_{\mathrm{Na}, 12}^{\mathrm{x}} \cdot \mathrm{LC}_{\mathrm{Na}}^{\mathrm{x}}+\beta_{\mathrm{Na}, 13}^{\mathrm{x}} \cdot \mathrm{LO}_{\mathrm{Na}}^{\mathrm{x}}+\beta_{\mathrm{Na}, \mathrm{il}}^{\mathrm{x}} \cdot \mathrm{LIC1}_{\mathrm{Na}}^{\mathrm{x}}-\left(\alpha_{\mathrm{Na}, 13}^{\mathrm{x}}+\beta_{\mathrm{Na}, 12}^{\mathrm{x}}+\alpha_{\mathrm{Na}, \mathrm{il}}^{\mathrm{x}}\right) \cdot \mathrm{LC}_{\mathrm{Na}}^{\mathrm{x}} \\
& \frac{\mathrm{dLO}_{\mathrm{Na}}^{\mathrm{X}}}{\mathrm{dt}}=\alpha_{\mathrm{Na}, 13}^{\mathrm{x}} \cdot \mathrm{LC}_{\mathrm{Na}}^{\mathrm{x}}+\beta_{\mathrm{Na}, 21}^{\mathrm{x}} \cdot \mathrm{LIF}_{\mathrm{Na}}^{\mathrm{x}}-\left(\beta_{\mathrm{Na}, 13}^{\mathrm{x}}+\alpha_{\mathrm{Na}, 21}^{\mathrm{x}}\right) \cdot \mathrm{LO}_{\mathrm{Na}}^{\mathrm{x}} \\
& \frac{\mathrm{dUC}_{\mathrm{Na}}^{\mathrm{x}}}{\mathrm{dt}}=\beta_{\mathrm{Na}, 11}^{\mathrm{x}} \cdot \mathrm{LC}_{\mathrm{Na}}^{\mathrm{x}}+\beta_{\mathrm{Na}, \mathrm{i}}^{\mathrm{x}} \cdot \mathrm{LIC}_{\mathrm{Na}}^{\mathrm{x}}-\left(\alpha_{\mathrm{Na}, 11}^{\mathrm{x}}+\alpha_{\mathrm{Na}, \mathrm{i}}^{\mathrm{x}}\right) \cdot \mathrm{UC}_{\mathrm{Na}}^{\mathrm{x}} \\
& \frac{\mathrm{dUC} 2_{\mathrm{Na}}^{\mathrm{x}}}{\mathrm{dt}}=\alpha_{\mathrm{Na}, 11}^{\mathrm{x}} \cdot \mathrm{LC} 3_{\mathrm{Na}}^{\mathrm{x}}+\beta_{\mathrm{Na}, 12}^{\mathrm{x}} \cdot \mathrm{LC}_{\mathrm{Na}}^{\mathrm{X}}+\beta_{\mathrm{Na}, \mathrm{i}}^{\mathrm{x}} \cdot \mathrm{LIC} 2_{\mathrm{Na}}^{\mathrm{X}}-\left(\alpha_{\mathrm{Na}, 12}^{\mathrm{x}}+\beta_{\mathrm{Na}, 11}^{\mathrm{X}}+\alpha_{\mathrm{Na}, \mathrm{i}}^{\mathrm{x}}\right) \cdot \mathrm{UC} 2_{\mathrm{Na}}^{\mathrm{X}} \\
& \frac{d U C 1}{d t}=\alpha_{N a, 12}^{x} \cdot L_{N}^{x} 2_{N a}^{x}+\beta_{N a, 13}^{x} \cdot L_{N a}^{x}+\beta_{N a, i}^{x} \cdot \operatorname{LIF}_{N a}^{x}-\left(\alpha_{N a, 13}^{x}+\beta_{N a, 12}^{x}+\alpha_{N a, i}^{x}\right) \cdot U C 1_{N a}^{x} \\
& \frac{\mathrm{dUO}_{\mathrm{Na}}^{\mathrm{x}}}{\mathrm{dt}}=\alpha_{\mathrm{Na}, 13}^{\mathrm{x}} \cdot \mathrm{LC}_{\mathrm{Na}}^{\mathrm{x}}+\beta_{\mathrm{Na}, 2}^{\mathrm{x}} \cdot \mathrm{LIF}_{\mathrm{Na}}^{\mathrm{x}}-\left(\beta_{\mathrm{Na}, 13}^{\mathrm{x}}+\alpha_{\mathrm{Na}, 2}^{\mathrm{x}}\right) \cdot \mathrm{UO}_{\mathrm{Na}}^{\mathrm{x}}
\end{aligned}
$$

$$
\begin{aligned}
& \frac{\mathrm{dLIC} 3_{\mathrm{Na}}^{\mathrm{x}}}{\mathrm{dt}}=\beta_{\mathrm{Na}, 11}^{\mathrm{x}} \cdot \mathrm{LIC}_{\mathrm{Na}}^{\mathrm{x}}+\alpha_{\mathrm{Na}, \mathrm{il}}^{\mathrm{x}} \cdot \mathrm{LC}_{\mathrm{Na}}^{\mathrm{x}}-\left(\alpha_{\mathrm{Na}, 11}^{\mathrm{x}}+\beta_{\mathrm{Na}, \mathrm{il}}^{\mathrm{x}}\right) \cdot \mathrm{LIC}_{\mathrm{Na}}^{\mathrm{X}} \\
& \frac{\mathrm{dLIC} 2_{\mathrm{Na}}^{\mathrm{x}}}{\mathrm{dt}}=\alpha_{\mathrm{Na}, 11}^{\mathrm{x}} \cdot \mathrm{LIC}_{\mathrm{Na}}^{\mathrm{x}}+\beta_{\mathrm{Na}, 12}^{\mathrm{x}} \cdot \operatorname{LIF}_{\mathrm{Na}}^{\mathrm{x}}+\alpha_{\mathrm{Na}, \mathrm{il}}^{\mathrm{x}} \cdot \mathrm{LC}_{\mathrm{Na}}^{\mathrm{x}}-\left(\alpha_{\mathrm{Na}, 12}^{\mathrm{x}}+\beta_{\mathrm{Na}, 11}^{\mathrm{x}}+\beta_{\mathrm{Na}, \mathrm{il}}^{\mathrm{x}}\right) \cdot \mathrm{LIC}_{\mathrm{Na}}^{\mathrm{x}} \\
& \frac{\mathrm{dLIF}_{\mathrm{Na}}^{\mathrm{X}}}{\mathrm{dt}}=\alpha_{\mathrm{Na}, 12}^{\mathrm{x}} \cdot \mathrm{LIC}_{\mathrm{Na}}^{\mathrm{x}}+\beta_{\mathrm{Na}, \mathrm{il}}^{\mathrm{x}} \cdot \operatorname{LIM}_{\mathrm{Na}}^{\mathrm{x}}+\alpha_{\mathrm{Na}, \mathrm{il}}^{\mathrm{x}} \cdot \mathrm{LC}_{\mathrm{Na}}^{\mathrm{x}}+\alpha_{\mathrm{Na}, 2}^{\mathrm{x}} \cdot \mathrm{LO}_{\mathrm{Na}}^{\mathrm{x}} \\
& -\left(\alpha_{\mathrm{Na}, 41}^{\mathrm{X}}+\beta_{\mathrm{Na}, 12}^{\mathrm{x}}+\beta_{\mathrm{Na}, 2 \mathrm{l}}^{\mathrm{x}}+\beta_{\mathrm{Na}, \mathrm{il}}^{\mathrm{X}}\right) \cdot \mathrm{LIF}_{\mathrm{Na}}^{\mathrm{X}} \\
& \frac{\mathrm{dLIM}_{\mathrm{Na}}^{\mathrm{x}}}{\mathrm{dt}}=\alpha_{\mathrm{Na}, 4 \mathrm{l}}^{\mathrm{x}} \cdot \mathrm{LIF}_{\mathrm{Na}}^{\mathrm{x}}-\beta_{\mathrm{Na}, \mathrm{il}}^{\mathrm{x}} \cdot \mathrm{LIM}_{\mathrm{Na}}^{\mathrm{x}} \\
& \frac{\mathrm{dUIC} 3_{\mathrm{Na}}^{\mathrm{x}}}{\mathrm{dt}}=\beta_{\mathrm{Na}, 11}^{\mathrm{x}} \cdot \mathrm{UIC}_{\mathrm{Na}}^{\mathrm{x}}+\alpha_{\mathrm{Na}, \mathrm{i}}^{\mathrm{x}} \cdot \mathrm{UC} 3_{\mathrm{Na}}^{\mathrm{x}}-\left(\alpha_{\mathrm{Na}, 11}^{\mathrm{x}}+\beta_{\mathrm{Na}, \mathrm{i}}^{\mathrm{x}}\right) \cdot \mathrm{UIC}_{\mathrm{Na}}^{\mathrm{x}} \\
& \frac{\mathrm{dUIC} 2_{\mathrm{Na}}^{\mathrm{x}}}{\mathrm{dt}}=\alpha_{\mathrm{Na}, 11}^{\mathrm{x}} \cdot \mathrm{UIC}_{\mathrm{Na}}^{\mathrm{x}}+\beta_{\mathrm{Na}, 12}^{\mathrm{x}} \cdot \mathrm{UIF}_{\mathrm{Na}}^{\mathrm{x}}+\alpha_{\mathrm{Na}, \mathrm{i}}^{\mathrm{x}} \cdot \mathrm{UC} 2_{\mathrm{Na}}^{\mathrm{x}}-\left(\alpha_{\mathrm{Na}, 12}^{\mathrm{x}}+\beta_{\mathrm{Na}, 11}^{\mathrm{x}}+\beta_{\mathrm{Na}, \mathrm{i}}^{\mathrm{x}}\right) \cdot \mathrm{UIC} 2_{\mathrm{Na}}^{\mathrm{x}}
\end{aligned}
$$




$$
\begin{aligned}
& \frac{\mathrm{dUIF}_{\mathrm{Na}}^{\mathrm{x}}}{\mathrm{dt}}=\alpha_{\mathrm{Na}, 12}^{\mathrm{x}} \cdot \mathrm{UIC}_{\mathrm{Na}}^{\mathrm{x}}+\beta_{\mathrm{Na}, \mathrm{i}}^{\mathrm{x}} \cdot \mathrm{UIM}_{\mathrm{Na}}^{\mathrm{x}}+\alpha_{\mathrm{Na}, \mathrm{i}}^{\mathrm{x}} \cdot \mathrm{UC1}_{\mathrm{Na}}^{\mathrm{x}}+\alpha_{\mathrm{Na}, 2}^{\mathrm{x}} \cdot \mathrm{UO}_{\mathrm{Na}}^{\mathrm{x}} \\
& -\left(\alpha_{\mathrm{Na}, 4}^{\mathrm{x}}+\beta_{\mathrm{Na}, 12}^{\mathrm{x}}+\beta_{\mathrm{Na}, 2}^{\mathrm{x}}+\beta_{\mathrm{Na}, \mathrm{i}}^{\mathrm{x}}\right) \cdot \mathrm{UIF}_{\mathrm{Na}}^{\mathrm{x}}
\end{aligned}
$$

$$
\begin{aligned}
& \mathrm{I}_{\mathrm{Na}}^{\mathrm{NP}}=11.188 \cdot\left(\left(1-\mathrm{f}_{\mathrm{Na}, \text { slow }}^{\mathrm{NP}}\right) \cdot \mathrm{UO}_{\mathrm{Na}}^{\mathrm{NP}}+\mathrm{f}_{\mathrm{Na}, \text { slow }}^{\mathrm{NP}} \cdot \mathrm{LO}_{\mathrm{Na}}^{\mathrm{NP}}\right) \cdot\left(\mathrm{V}_{\mathrm{m}}-\mathrm{E}_{\mathrm{Na}}\right) \\
& \mathrm{I}_{\mathrm{Na}}^{\mathrm{P}}=11.188 \cdot\left(\left(1-\mathrm{f}_{\mathrm{Na}, \text { slow }}^{\mathrm{P}}\right) \cdot \mathrm{UO}_{\mathrm{Na}}^{\mathrm{P}}+\mathrm{f}_{\mathrm{Na} \text {,slow }}^{\mathrm{P}} \cdot \mathrm{LO}_{\mathrm{Na}}^{\mathrm{P}}\right) \cdot\left(\mathrm{V}_{\mathrm{m}}-\mathrm{E}_{\mathrm{Na}}\right) \\
& \mathrm{I}_{\mathrm{Na}}=\left(1-\hat{\mathrm{f}}_{\mathrm{INa}}^{\mathrm{PKA}}\right) \cdot \mathrm{I}_{\mathrm{Na}}^{\mathrm{NP}}+\hat{\mathrm{f}}_{\mathrm{INa}}^{\mathrm{PKA}} \cdot \mathrm{I}_{\mathrm{Na}}^{\mathrm{P}}
\end{aligned}
$$

\subsection{Background $\mathrm{Na}^{+}$current $\left(\mathrm{I}_{\mathrm{Nab}}\right)$}

\subsubsection{HRd2009, HRdßARS and HRdßARSStoch version (Chapters 2-7)}

Background $\mathrm{Na}^{+}$current $\left(I_{\mathrm{Nab}}\right)$ and co-transporter fluxes were required to maintain a resting $\left[\mathrm{Na}^{+}\right]_{\mathrm{i}}$ concentration consistent with measurements in canine epicardial myocytes. ${ }^{3} \mathrm{Na}^{+}$permeability for the background $\mathrm{Na}^{+}$current was given by the constant field equation, following the formulation used by Gao et al. ${ }^{3}$ :

$\mathrm{P}_{\mathrm{Nab}}=0.32 \cdot 10^{-8} \mathrm{~cm} / \mathrm{s}$

$I_{N a b}=P_{N a b} \cdot \frac{F^{2} \cdot V_{m}}{R \cdot T} \cdot \frac{\left[\mathrm{Na}^{+}\right]_{i} \cdot \exp \left(V_{m} \cdot \frac{F}{R \cdot T}\right)-\left[\mathrm{Na}^{+}\right]_{o}}{\exp \left(V_{m} \cdot \frac{F}{R \cdot T}\right)-1}$

\section{$1.20 \mathrm{Na}^{+}-\mathrm{Ca}^{2+}$ exchange current $\left(\mathrm{I}_{\mathrm{NaCa}}\right)$}

\subsubsection{HRd2009 and HRdßARS version (Chapters 2-6)}

The $\mathrm{Na}^{+}-\mathrm{Ca}^{2+}$ exchanger formulation was adopted from Bers and Weber. ${ }^{53} 20 \%$ of exchangers were located in the restricted space, consistent with experimental results and previous work from our lab. ${ }^{54}$

$$
\begin{aligned}
& \mathrm{v}_{\mathrm{max}, \mathrm{NaCa}}=4.50 \mathrm{pA} / \mathrm{pF}, \quad \eta=0.27, \quad \mathrm{~K}_{\mathrm{mCa}, \mathrm{act}}=1.24 \cdot 10^{-4} \mathrm{mmol} / \mathrm{L} \\
& \mathrm{K}_{\mathrm{mCa}, \mathrm{o}}=1.30 \mathrm{mmol} / \mathrm{L}, \quad \mathrm{K}_{\mathrm{mCa}, \mathrm{i}}=3.60 \cdot 10^{-3} \mathrm{mmol} / \mathrm{L}, \quad \mathrm{K}_{\mathrm{mNa}, \mathrm{o}}=87.5 \mathrm{mmol} / \mathrm{L} \\
& \mathrm{K}_{\mathrm{mNa}, \mathrm{i}}=12.3 \mathrm{mmol} / \mathrm{L}, \quad \mathrm{k}_{\mathrm{sat}}=0.32 \\
& \mathrm{~A}_{\mathrm{NaCa}, \mathrm{ss}}=\mathrm{K}_{\mathrm{mCa}, \mathrm{o}} \cdot\left[\mathrm{Na}^{+}\right]_{\mathrm{ss}}^{3}+\mathrm{K}_{\mathrm{mNa}, \mathrm{o}}^{3} \cdot\left[\mathrm{Ca}^{2+}\right]_{\mathrm{ss}}+\mathrm{K}_{\mathrm{mNa}, \mathrm{i}}^{3} \cdot\left[\mathrm{Ca}^{2+}\right]_{\mathrm{o}} \cdot\left(1+\frac{\left[\mathrm{Ca}^{2+}\right]_{\mathrm{ss}}}{\mathrm{K}_{\mathrm{mCa}, \mathrm{i}}}\right)+\mathrm{K}_{\mathrm{mCa}, \mathrm{i}} \\
& \cdot\left[\mathrm{Na}^{+}\right]_{\mathrm{o}}^{3} \cdot\left(1+\frac{\left[\mathrm{Na}^{+}\right]_{\mathrm{ss}}^{3}}{\mathrm{~K}_{\mathrm{mNa}, \mathrm{i}}^{3}}\right)+\left[\mathrm{Na}^{+}\right]_{\mathrm{ss}}^{3} \cdot\left[\mathrm{Ca}^{2+}\right]_{\mathrm{o}}+\left[\mathrm{Na}^{+}\right]_{\mathrm{o}}^{3} \cdot\left[\mathrm{Ca}^{2+}\right]_{\mathrm{ss}}
\end{aligned}
$$




$$
\begin{aligned}
& \mathrm{D}_{\mathrm{NaCa}, \mathrm{ss}}=\left[\mathrm{Na}^{+}\right]_{\mathrm{ss}}^{3} \cdot\left[\mathrm{Ca}^{2+}\right]_{\mathrm{o}} \cdot \exp \left(\eta \cdot \mathrm{V}_{\mathrm{m}} \cdot \frac{\mathrm{F}}{\mathrm{R} \cdot \mathrm{T}}\right)-\left[\mathrm{Na}^{+}\right]_{\mathrm{o}}^{3} \cdot\left[\mathrm{Ca}^{2+}\right]_{\mathrm{ss}} \cdot \exp \left((\eta-1) \cdot \mathrm{V}_{\mathrm{m}} \cdot \frac{\mathrm{F}}{\mathrm{R} \cdot \mathrm{T}}\right) \\
& \mathrm{I}_{\mathrm{NaCa}, \mathrm{ss}}=\frac{0.2 \cdot \mathrm{v}_{\mathrm{max}, \mathrm{NaCa}} \cdot \mathrm{D}_{\mathrm{NaCa}, \mathrm{ss}}}{\left(1+\left(\frac{\mathrm{K}_{\mathrm{mCa}, \mathrm{act}}}{\left[\mathrm{Ca}^{2+}\right]_{\mathrm{ss}}}\right)^{2}\right) \cdot\left(1+\mathrm{k}_{\mathrm{sat}} \cdot \exp \left((\eta-1) \cdot \mathrm{V}_{\mathrm{m}} \cdot \frac{\mathrm{F}}{\mathrm{R} \cdot \mathrm{T}}\right)\right) \cdot \mathrm{A}_{\mathrm{NaCa}, \mathrm{ss}}} \\
& \mathrm{A}_{\mathrm{NaCa}, \mathrm{i}}=\mathrm{K}_{\mathrm{mCa}, \mathrm{o}} \cdot\left[\mathrm{Na}^{+}\right]_{\mathrm{i}}^{3}+\mathrm{K}_{\mathrm{mNa}, \mathrm{o}}^{3} \cdot\left[\mathrm{Ca}^{2+}\right]_{\mathrm{i}}+\mathrm{K}_{\mathrm{mNa}, \mathrm{i}}^{3} \cdot\left[\mathrm{Ca}^{2+}\right]_{\mathrm{o}} \cdot\left(1+\frac{\left[\mathrm{Ca}^{2+}\right]_{\mathrm{i}}}{\mathrm{K}_{\mathrm{mCa}, \mathrm{i}}}\right)+\mathrm{K}_{\mathrm{mCa}, \mathrm{i}} \cdot\left[\mathrm{Na}^{+}\right]_{\mathrm{o}}^{3} \\
& \mathrm{D}_{\mathrm{NaCa}, \mathrm{i}}=\left[\mathrm{Na}^{+}\right]_{\mathrm{i}}^{3} \cdot\left[\mathrm{Ca}^{2+}\right]_{\mathrm{o}} \cdot \exp \left(\eta \cdot \mathrm{V}_{\mathrm{m}} \cdot \frac{\mathrm{F}}{\mathrm{R} \cdot \mathrm{T}}\right)-\left[\mathrm{Na}^{+}\right]_{\mathrm{o}}^{3} \cdot\left[\mathrm{Ca}^{2+}\right]_{\mathrm{i}} \cdot \exp \left((\eta-1) \cdot \mathrm{V}_{\mathrm{m}} \cdot \frac{\mathrm{F}}{\mathrm{R} \cdot \mathrm{T}}\right) \\
& \mathrm{I}_{\mathrm{NaCa}, \mathrm{i}}=\frac{0.8 \cdot \mathrm{Na}_{\mathrm{max}, \mathrm{NaCa}} \cdot \mathrm{D}_{\mathrm{NaCa}, \mathrm{i}}}{\left.\left(1+\left(\frac{\mathrm{K}_{\mathrm{mCa}, \mathrm{act}}}{\left[\mathrm{Ca}^{2+}\right]_{\mathrm{i}}}\right)^{2}\right) \cdot\left(1+\mathrm{Ca}^{2+}\right]_{\mathrm{o}}+\left[\mathrm{Na}^{+}\right]_{\mathrm{s}}^{3} \cdot\left[\mathrm{Ca}^{2+}\right]_{\mathrm{i}} \cdot \exp \left((\eta-1) \cdot \mathrm{V}_{\mathrm{m}} \cdot \frac{\mathrm{F}}{\mathrm{R} \cdot \mathrm{T}}\right)\right) \cdot \mathrm{A}_{\mathrm{NaCa}, \mathrm{i}}}
\end{aligned}
$$

\section{$1.21 \mathrm{Na}^{+}-\mathrm{K}^{+}$pump current $\left(\mathrm{I}_{\mathrm{NaK}}\right)$}

\subsubsection{HRd2009 version (Chapter 2)}

A recently developed $I_{\mathrm{NaK}}$ formulation based on data from canine left ventricular epicardial myocytes ${ }^{3,55}$ was incorporated into the model. Pump density was adjusted to $37^{\circ} \mathrm{C}$ using $Q_{10}=2.1^{56}$

$$
\begin{aligned}
\mathrm{K}_{\mathrm{NaK}, \mathrm{Ko}} & =1.5 \mathrm{mmol} / \mathrm{L}, \quad \mathrm{K}_{\mathrm{NaK}, \mathrm{Nai}}=2.6 \mathrm{mmol} / \mathrm{L}, \quad \overline{\mathrm{I}}_{\mathrm{NaK}}=1.4 \mathrm{mS} / \mu \mathrm{F} \\
\varphi_{\mathrm{NaK}} & =\frac{1}{1+\exp \left(-\left(\mathrm{V}_{\mathrm{m}}+92\right) \cdot \frac{\mathrm{F}}{\mathrm{R} \cdot \mathrm{T}}\right)} \\
\mathrm{P}_{\mathrm{K}}^{\mathrm{NaK}} & =\frac{\left[\mathrm{K}^{+}\right]_{\mathrm{o}}}{\left[\mathrm{K}^{+}\right]_{\mathrm{o}}+\mathrm{K}_{\mathrm{NaK}, \mathrm{Ko}}^{\mathrm{NP}}} \\
\mathrm{P}_{\mathrm{Na}}^{\mathrm{NaK}} & =\left(\frac{\left[\mathrm{Na}^{+}\right]_{\mathrm{i}}}{\left[\mathrm{Na}^{+}\right]_{\mathrm{i}}+\mathrm{K}_{\mathrm{NaK}, \mathrm{Nai}}^{\mathrm{NP}}}\right)^{3} \\
\mathrm{I}_{\mathrm{NaK}} & =\overline{\mathrm{I}}_{\mathrm{NaK}} \cdot \varphi_{\mathrm{NaK}} \cdot \mathrm{P}_{\mathrm{K}}^{\mathrm{NaK}} \cdot \mathrm{P}_{\mathrm{Na}}^{\mathrm{NaK}}
\end{aligned}
$$

\subsubsection{HRd $\beta$ ARS version (Chapters 3-6)}

Complex results regarding the regulation of the $\mathrm{Na}^{+}-\mathrm{K}^{+}-\mathrm{ATPase}$ current $\left(\mathrm{I}_{\mathrm{NaK}}\right)$ by PKA-dependent phosphorylation have been reported in various species including rat, ${ }^{57}$ guinea $\mathrm{pig}^{58}$ and $\mathrm{dog}^{59}$ showing either an increase or decrease in pump current depending on intracellular $\mathrm{Ca}^{2+}$ concentrations. More recently, it has been shown that the PKA dependent stimulation of $I_{\mathrm{NaK}}$ is isoform specific and mediated by the associated protein phospholemman (PLM). ${ }^{60,61}$ PLM has long 
been recognized as a major PKA and PKC substrate in the heart and is proposed to have a similar role as PLB for SERCA (i.e., inhibition relieved by phosphorylation). In the model we assume that the PKAdependent increase in $I_{\mathrm{NaK}}$ results from an increased $\mathrm{Na}^{+}$affinity mediated through PLM phosphorylation. The $30 \%$ increase in $\mathrm{Na}^{+}$affinity (as found in mouse ventricular myocytes ${ }^{61}$ ) results in a 17\%-33\% increase in pump current for intracellular $\mathrm{Na}^{+}$levels in the physiological range, consistent with the increase in $I_{\text {NaK }}$ measured experimentally. ${ }^{60-62}$ We assume that under normal physiological conditions the $\mathrm{Ca}^{2+}$ concentrations are such that only stimulation of $\mathrm{I}_{\text {Nak }}$ occurs and hence do not incorporate the $\mathrm{Ca}^{2+}$ dependence explicitly in our model. Other groups have reported that PLM also affects $\mathrm{Na}^{+}-\mathrm{Ca}^{2+}$ exchange, ${ }^{63}$ but disparate results exist. ${ }^{64}$ Because we do not incorporate direct effects of $\beta A R$ stimulation on $\mathrm{Na}^{+}-\mathrm{Ca}^{2+}$ exchange, ${ }^{65}$ we also do not consider interactions between PLM and $\mathrm{Na}^{+}-\mathrm{Ca}^{2+}$ exchanger.

Nonphosphorylated Channels:

$$
\begin{aligned}
& \mathrm{K}_{\mathrm{NaK}, \mathrm{Ko}}^{\mathrm{NP}}=1.5 \mathrm{mmol} / \mathrm{L}, \quad \mathrm{K}_{\mathrm{NaK}, \mathrm{Nai}}^{\mathrm{NP}}=2.6 \mathrm{mmol} / \mathrm{L}, \quad \overline{\mathrm{I}}_{\mathrm{NaK}}^{\mathrm{NP}}=1.4 \mathrm{mS} / \mu \mathrm{F} \\
& \varphi_{\mathrm{NaK}}^{\mathrm{NP}}=\frac{1}{1+\exp \left(-\left(\mathrm{V}_{\mathrm{m}}+92\right) \cdot \frac{\mathrm{F}}{\mathrm{R} \cdot \mathrm{T}}\right)} \\
& \mathrm{P}_{\mathrm{K}}^{\mathrm{NaK}, \mathrm{NP}}=\frac{\left[\mathrm{K}^{+}\right]_{\mathrm{o}}}{\left[\mathrm{K}^{+}\right]_{\mathrm{o}}+\mathrm{K}_{\mathrm{NaK}, \mathrm{Ko}}^{\mathrm{NP}}} \\
& \mathrm{P}_{\mathrm{Na}}^{\mathrm{NaK}, \mathrm{NP}}=\left(\frac{\left[\mathrm{Na}^{+}\right]_{\mathrm{i}}}{\left[\mathrm{Na}^{+}\right]_{\mathrm{i}}+\mathrm{K}_{\mathrm{NaK}, \mathrm{Nai}}^{\mathrm{NP}}}\right)^{3} \\
& \mathrm{I}_{\mathrm{NaK}}^{\mathrm{NP}}=\overline{\mathrm{I}}_{\mathrm{NaK}}^{\mathrm{NP}} \cdot \varphi_{\mathrm{NaK}}^{\mathrm{NP}} \cdot \mathrm{P}_{\mathrm{K}}^{\mathrm{NaK}, \mathrm{NP}} \cdot \mathrm{P}_{\mathrm{Na}}^{\mathrm{NaK}, \mathrm{NP}} \\
& \mathrm{K}_{\mathrm{NaK}, \mathrm{Ko}}^{\mathrm{P}}=1.5 \mathrm{mmol} / \mathrm{L}, \quad \mathrm{K}_{\mathrm{NaK}, \mathrm{Nai}}^{\mathrm{P}}=1.846 \mathrm{mmol} / \mathrm{L}, \quad \overline{\mathrm{I}}_{\mathrm{NaK}}^{\mathrm{P}}=1.4 \mathrm{mS} / \mu \mathrm{F} \\
& \varphi_{\mathrm{NaK}}^{\mathrm{P}}=\frac{1}{1+\exp \left(-\left(\mathrm{V}_{\mathrm{m}}+92\right) \cdot \frac{\mathrm{F}}{\mathrm{R} \cdot \mathrm{T}}\right)} \\
& \mathrm{P}_{\mathrm{K}}^{\mathrm{NaK}, \mathrm{P}}=\frac{\left[\mathrm{K}^{+}\right]_{\mathrm{o}}}{\left[\mathrm{K}^{+}\right]_{\mathrm{o}}+\mathrm{K}_{\mathrm{NaK}, \mathrm{Ko}}^{\mathrm{P}}} \\
& \mathrm{P}_{\mathrm{Na}}^{\mathrm{NaK}, \mathrm{P}}=\left(\frac{\left[\mathrm{Na}^{+}\right]_{\mathrm{i}}}{\left[\mathrm{Na}^{+}\right]_{\mathrm{i}}+\mathrm{K}_{\mathrm{NaK}, \mathrm{Nai}}^{\mathrm{P}}}\right)^{3} \\
& \mathrm{I}_{\mathrm{NaK}}^{\mathrm{P}}=\overline{\mathrm{I}}_{\mathrm{NaK}}^{\mathrm{P}} \cdot \varphi_{\mathrm{NaK}}^{\mathrm{P}} \cdot \mathrm{P}_{\mathrm{K}}^{\mathrm{NaK}, \mathrm{P}} \cdot \mathrm{P}_{\mathrm{Na}}^{\mathrm{NaK}, \mathrm{P}}
\end{aligned}
$$

\section{Combined current:}

$\hat{\mathrm{f}}_{\mathrm{NaK}}^{\mathrm{P}}=\frac{\mathrm{f}_{\mathrm{INaK}}^{\mathrm{P}}-0.1263}{0.9980-0.1263}$

$\mathrm{I}_{\mathrm{NaK}}=\hat{\mathrm{f}}_{\mathrm{NaK}}^{\mathrm{P}} \cdot \mathrm{I}_{\mathrm{NaK}}^{\mathrm{P}}+\left(1-\hat{\mathrm{f}}_{\mathrm{NaK}}^{\mathrm{P}}\right) \cdot \mathrm{I}_{\mathrm{NaK}}^{\mathrm{NP}}$ 


\subsubsection{HRdßARS Stoch version (Chapter 7)}

Smith and Crampin have previously described a detailed model of the $\mathrm{Na}^{+}-\mathrm{K}^{+}$-ATPase based on a consecutive ("ping-pong") model for $\mathrm{Na}^{+}$and $\mathrm{K}^{+}$binding. ${ }^{66}$ In their model, 'slippage' of ions is negligible due to a tight coupling between conformational changes of the pump and ion binding, thereby ensuring a strict $3 \mathrm{Na}^{+}: 2 \mathrm{~K}^{+}$stoichiometry. Based on these assumptions and since both pumps belong to the family of P-type cation transporters, ${ }^{66}$ we employed a similar two-state model structure as that used for SERCA. We set the 'backward' (counter-clockwise) rates to zero to ensure the strict stoichiometry. Forward rates were fitted to reproduce $\mathrm{Na}^{+}, \mathrm{K}^{+}$and $\mathrm{V}_{\mathrm{m}}$ dependence of the original $I_{\text {Nak }}$ formulation for both the non-phosphorylated and phosphorylated populations.

$$
\begin{aligned}
& \varphi_{\mathrm{NaK}}=\frac{1}{1+\exp \left(-\left(\mathrm{V}_{\mathrm{m}}+92\right) \cdot \frac{\mathrm{F}}{\mathrm{R} \cdot \mathrm{T}}\right)} \\
& \mathrm{k}_{\mathrm{NaK}, 1+}^{\mathrm{NP}}=1.1893 \cdot \varphi_{\mathrm{NaK}} \cdot\left(\frac{\left[\mathrm{Na}^{+}\right]_{\mathrm{i}}}{11.5767+\left[\mathrm{Na}^{+}\right]_{\mathrm{i}}}\right)^{1.5298} \cdot \frac{2.00 \cdot 10^{6}}{\overline{\mathrm{N}}_{\mathrm{NaK}}}, \quad \mathrm{k}_{\mathrm{NaK}, 1-}^{\mathrm{NP}}=0 \\
& \mathrm{k}_{\mathrm{NaK}, 2+}^{\mathrm{NP}}=1.6425 \cdot \varphi_{\mathrm{NaK}} \cdot\left(\frac{\left[\mathrm{K}^{+}\right]_{\mathrm{o}}}{2.3694+\left[\mathrm{K}^{+}\right]_{\mathrm{o}}}\right)^{1.5298} \cdot \frac{2.00 \cdot 10^{6}}{\overline{\mathrm{N}}_{\mathrm{NaK}}}, \quad \mathrm{k}_{\mathrm{NaK}, 2-}^{\mathrm{NP}}=0 \\
& \mathrm{n}_{\mathrm{NaK}}^{\mathrm{NP}}=\frac{\mathrm{k}_{\mathrm{NaK}, 2+}^{\mathrm{NP}}+\mathrm{k}_{\mathrm{NaK}, 2-}^{\mathrm{NP}}}{\mathrm{k}_{\mathrm{NaK}, 1+}^{\mathrm{NP}}+\mathrm{k}_{\mathrm{NaK}, 1-}^{\mathrm{NP}}+\mathrm{k}_{\mathrm{NaK}, 2+}^{\mathrm{NP}}+\mathrm{k}_{\mathrm{NaK}, 2-}^{\mathrm{NP}}} \cdot\left(1-\mathrm{f}_{\mathrm{INaK}}^{\mathrm{P}}\right) \cdot \overline{\mathrm{N}}_{\mathrm{NaK}} \\
& \mathrm{F}_{\mathrm{NaK}}^{\mathrm{NP}}=3 \cdot \mathrm{B}\left(\mathrm{n}_{\mathrm{NaK}}^{\mathrm{NP}}, \mathrm{k}_{\mathrm{NaK}, 1+}^{\mathrm{NP}} \cdot \Delta \mathrm{t}\right)-2 \cdot \mathrm{B}\left(\left(1-\mathrm{f}_{\mathrm{INaK}}^{\mathrm{P}}\right) \cdot \overline{\mathrm{N}}_{\mathrm{NaK}}-\mathrm{n}_{\mathrm{NaK}}^{\mathrm{NP}}, \mathrm{k}_{\mathrm{NaK}, 2+}^{\mathrm{NP}} \cdot \Delta \mathrm{t}\right) \\
& \mathrm{k}_{\mathrm{NaK}, 1+}^{\mathrm{P}}=1.1112 \cdot \varphi_{\mathrm{NaK}} \cdot\left(\frac{\left[\mathrm{Na}^{+}\right]_{\mathrm{i}}}{7.6962+\left[\mathrm{Na}^{+}\right]_{\mathrm{i}}}\right)^{1.5206} \cdot \frac{2.00 \cdot 10^{6}}{\overline{\mathrm{N}}_{\mathrm{NaK}}}, \quad \mathrm{k}_{\mathrm{NaK}, 1-}^{\mathrm{P}}=0 \\
& \mathrm{k}_{\mathrm{NaK}, 2+}^{\mathrm{P}}=1.7324 \cdot \varphi_{\mathrm{NaK}} \cdot\left(\frac{\left[\mathrm{K}^{+}\right]_{\mathrm{o}}}{2.3694+\left[\mathrm{K}^{+}\right]_{\mathrm{o}}}\right)^{1.5206} \cdot \frac{2.00 \cdot 10^{6}}{\overline{\mathrm{N}}_{\mathrm{NaK}}}, \quad \mathrm{k}_{\mathrm{NaK}, 2-}^{\mathrm{P}}=0 \\
& \mathrm{n}_{\mathrm{NaK}}^{\mathrm{P}}=\frac{\mathrm{k}_{\mathrm{NaK}, 2+}^{\mathrm{P}}+\mathrm{k}_{\mathrm{NaK}, 2-}^{\mathrm{P}}}{\mathrm{k}_{\mathrm{NaK}, 1+}^{\mathrm{P}}+\mathrm{k}_{\mathrm{NaK}, 1-}^{\mathrm{P}}+\mathrm{k}_{\mathrm{NaK}, 2+}^{\mathrm{P}}+\mathrm{k}_{\mathrm{NaK}, 2-}^{\mathrm{P}}} \cdot \mathrm{f}_{\mathrm{INaK}}^{\mathrm{P}} \cdot \overline{\mathrm{N}}_{\mathrm{NaK}} \\
& \mathrm{F}_{\mathrm{NaK}}^{\mathrm{P}}=3 \cdot \mathrm{B}\left(\mathrm{n}_{\mathrm{NaK}}^{\mathrm{P}}, \mathrm{k}_{\mathrm{NaK}, 1+}^{\mathrm{P}} \cdot \Delta \mathrm{t}\right)-2 \cdot \mathrm{B}\left(\mathrm{f}_{\mathrm{INaK}}^{\mathrm{P}} \cdot \overline{\mathrm{N}}_{\mathrm{NaK}}-\mathrm{n}_{\mathrm{NaK}}^{\mathrm{P}}, \mathrm{k}_{\mathrm{NaK}, 2+}^{\mathrm{P}} \cdot \Delta \mathrm{t}\right) \\
& \mathrm{I}_{\mathrm{NaK}}=\frac{\left(\mathrm{F}_{\mathrm{NaK}}^{\mathrm{NP}}+\mathrm{F}_{\mathrm{NaK}}^{\mathrm{P}}\right) \cdot 10^{9} \cdot \mathrm{F}}{\Delta \mathrm{t} \cdot \mathrm{N}_{\mathrm{A}} \cdot \mathrm{C}_{\mathrm{m}} \cdot \mathrm{A}_{\mathrm{cap}}}
\end{aligned}
$$

\subsection{Late component of $\mathrm{Na}^{+}$current $\left(\mathrm{I}_{\mathrm{NaL}}\right)$}

\subsubsection{HRd2009 version (Chapter 2)}

The formulation for the late $\mathrm{Na}^{+}$current is equivalent to that in the original HRd model. ${ }^{4}$

$$
\begin{aligned}
& \alpha_{\mathrm{mL}}=\frac{0.32 \cdot\left(\mathrm{V}_{\mathrm{m}}+47.13\right)}{1-\exp \left(-0.1 \cdot\left(\mathrm{V}_{\mathrm{m}}+47.13\right)\right)} \\
& \beta_{\mathrm{mL}}=0.08 \cdot \exp \left(-\frac{\mathrm{V}_{\mathrm{m}}}{11}\right)
\end{aligned}
$$




$$
\begin{aligned}
& \mathrm{h}_{\mathrm{L}, \infty}=\frac{1}{1+\exp \left(\frac{\mathrm{V}_{\mathrm{m}}+91}{6.1}\right)} \\
& \tau_{\mathrm{hL}}=600 \mathrm{~ms} \\
& \frac{\mathrm{dm}_{\mathrm{L}}}{\mathrm{dt}}=\alpha_{\mathrm{mL}} \cdot\left(1-\mathrm{m}_{\mathrm{L}}\right)-\beta_{\mathrm{mL}} \cdot \mathrm{m}_{\mathrm{L}} \\
& \frac{\mathrm{dh}_{\mathrm{L}}}{\mathrm{dt}}=\frac{\mathrm{h}_{\mathrm{L}, \infty}-\mathrm{h}_{\mathrm{L}}}{\tau_{\mathrm{hL}}} \\
& \mathrm{I}_{\mathrm{NaL}}=6.500 \cdot 10^{-3} \cdot\left(\mathrm{m}_{\mathrm{L}}\right)^{3} \cdot \mathrm{h}_{\mathrm{L}} \cdot\left(\mathrm{V}_{\mathrm{m}}-\mathrm{E}_{\mathrm{Na}}\right)
\end{aligned}
$$

\subsubsection{HRdßARS version (Chapters 3-5)}

A small number of $\mathrm{Na}^{+}$channels fail to inactivate rapidly and enter a "burst-mode" which in the model is represented by the late $\mathrm{Na}^{+}$current $\left(\mathrm{I}_{\mathrm{NaL}}\right)$. It has been shown that PKA does not affect the sustained component of wild-type $I_{\mathrm{Na}}$ channels ${ }^{67}$ and hence $I_{\mathrm{NaL}}$ is not modulated by PKA in our model. CaMKII, on the other hand, increases the fraction of channels gating in this burst mode, ${ }^{24}$ which is represented by an increase in maximal conductance in the model.

$$
\begin{aligned}
& \alpha_{\mathrm{mL}}=\frac{0.32 \cdot\left(\mathrm{V}_{\mathrm{m}}+47.13\right)}{1-\exp \left(-0.1 \cdot\left(\mathrm{V}_{\mathrm{m}}+47.13\right)\right)} \\
& \beta_{\mathrm{mL}}=0.08 \cdot \exp \left(-\frac{\mathrm{V}_{\mathrm{m}}}{11}\right) \\
& \mathrm{h}_{\mathrm{L}, \infty}=\frac{1}{1+\exp \left(\frac{\mathrm{V}_{\mathrm{m}}+91}{6.1}\right)} \\
& \tau_{\mathrm{hL}}=600 \mathrm{~ms} \\
& \frac{\mathrm{dm}_{\mathrm{L}}}{\mathrm{dt}}=\alpha_{\mathrm{mL}} \cdot\left(1-\mathrm{m}_{\mathrm{L}}\right)-\beta_{\mathrm{mL}} \cdot \mathrm{m}_{\mathrm{L}} \\
& \frac{\mathrm{dh}_{\mathrm{L}}}{\mathrm{dt}}=\frac{\mathrm{h}_{\mathrm{L}, \infty}-\mathrm{h}_{\mathrm{L}}}{\tau_{\mathrm{hL}}} \\
& \mathrm{I}_{\mathrm{NaL}}^{\mathrm{NP}}=6.500 \cdot 10^{-3} \cdot\left(\mathrm{m}_{\mathrm{L}}\right)^{3} \cdot \mathrm{h}_{\mathrm{L}} \cdot\left(\mathrm{V}_{\mathrm{m}}-\mathrm{E}_{\mathrm{Na}}\right) \\
& \mathrm{I}_{\mathrm{NaL}}^{\mathrm{P}, \mathrm{CaMK}}=1.600 \cdot 10^{-2} \cdot\left(\mathrm{m}_{\mathrm{L}}\right)^{3} \cdot \mathrm{h}_{\mathrm{L}} \cdot\left(\mathrm{V}_{\mathrm{m}}-\mathrm{E}_{\mathrm{Na}}\right) \\
& \mathrm{I}_{\mathrm{NaL}}=\left(1-\mathrm{f}_{\mathrm{Na}}^{\mathrm{P}, \mathrm{CaMKII}}\right) \cdot \mathrm{I}_{\mathrm{NaL}}^{\mathrm{NP}}+\mathrm{f}_{\mathrm{Na}}^{\mathrm{P}, \mathrm{CaMKII}} \cdot \mathrm{I}_{\mathrm{NaL}}^{\mathrm{P}, \mathrm{CaMK}}
\end{aligned}
$$

\subsubsection{HRdßARS SCR version (Chapter 6)}

The maximal conductance of $I_{\text {NaL }}$ was increased to represent the midmyocardial origin of the myocytes ${ }^{68}$ and to match experimental APD rate dependence (Figure 2 in Chapter 6). 
$\mathrm{I}_{\mathrm{NaL}}^{\mathrm{NP}}=1.6 \cdot 6.500 \cdot 10^{-3} \cdot\left(\mathrm{m}_{\mathrm{L}}\right)^{3} \cdot \mathrm{h}_{\mathrm{L}} \cdot\left(\mathrm{V}_{\mathrm{m}}-\mathrm{E}_{\mathrm{Na}}\right)$

$\mathrm{I}_{\mathrm{NaL}}^{\mathrm{P}, \mathrm{CaMK}}=1.6 \cdot 1.600 \cdot 10^{-2} \cdot\left(\mathrm{m}_{\mathrm{L}}\right)^{3} \cdot \mathrm{h}_{\mathrm{L}} \cdot\left(\mathrm{V}_{\mathrm{m}}-\mathrm{E}_{\mathrm{Na}}\right)$

\subsubsection{HRdßARS Stoch version (Chapter 7)}

In the stochastic model, the sustained (or late) component of $I_{\mathrm{Na}}$, including its CaMKII-dependent regulation, is included in the Markov model of the $\mathrm{Na}^{+}$channel. As such the $\mathrm{I}_{\mathrm{NaL}}$ current is set to zero.

$\mathrm{I}_{\mathrm{NaL}}=0$

\subsection{Transient outward $\mathrm{K}^{+}$current $\left(\mathrm{I}_{\mathrm{TO}}\right)$}

\subsubsection{HRd2009 version (Chapter 2)}

Model $\mathrm{I}_{\text {To }}$ is derived from a previous formulation. ${ }^{69}$ Modifications were made to better fit experimental measurements from the canine (Figure 5 of Chapter 2). Model $\mathrm{I}_{\mathrm{TO}}$ density is consistent with multiple experiments from canine left ventricular epicardial cells. ${ }^{48,} 70,71$ Activation and inactivation kinetics were adjusted to reproduce experimentally measured time to peak ${ }^{48,72,73}$ and onset of inactivation ${ }^{48,71,72}$. Model time to peak falls in the middle range of published data from canine epicardial cells and is a significant improvement on other published Hodgkin- Huxley formulations ${ }^{71,74}$, in which time to peak is much faster ( $\left.<1 \mathrm{~ms}\right)$ than that observed experimentally. A slow inactivation gate was included to reproduce the slow recovery from inactivation observed in canine $^{70}$.

$$
\begin{aligned}
& \mathrm{R}_{\mathrm{To}}=\exp \left(\frac{\mathrm{V}_{\mathrm{m}}}{550}\right), \quad \overline{\mathrm{G}}_{\mathrm{To}}=0.4975 \mathrm{mS} / \mu \mathrm{F} \\
& \tau_{\mathrm{To}, \mathrm{a}}=\left(\frac{1}{1.2089 \cdot\left(1+\exp \left(\frac{\mathrm{V}_{\mathrm{m}}-18.4099}{-29.3814}\right)\right)}+\frac{3.5}{1+\exp \left(\frac{\mathrm{V}_{\mathrm{m}}+100}{29.3814}\right)}\right)^{-1} \\
& \mathrm{To}_{\mathrm{a}, \infty}=\frac{1}{1+\exp \left(\left(\mathrm{V}_{\mathrm{m}}+9.437\right) /-7.133\right)} \\
& \alpha_{\mathrm{To}, \text { if }}=\frac{0.025}{1+\exp \left(\left(\mathrm{V}_{\mathrm{m}}+58\right) / 5\right)} \\
& \beta_{\mathrm{To}, \text { if }}=\frac{1}{9.7953 \cdot\left(1+\exp \left(\left(\mathrm{V}_{\mathrm{m}}+19\right) /-9\right)\right)} \\
& \alpha_{\mathrm{To}, \text { is }}=\frac{1}{250 \cdot\left(1+\exp \left(\left(\mathrm{V}_{\mathrm{m}}+60\right) / 5\right)\right)} \\
& \beta_{\mathrm{To}, \text { is }}=\beta_{\mathrm{To}, \text { if }}
\end{aligned}
$$




$$
\begin{aligned}
& \frac{\mathrm{dTo}_{\mathrm{a}}}{\mathrm{dt}}=\frac{\mathrm{To}_{\mathrm{a}, \mathrm{o}}-\mathrm{To}_{\mathrm{a}}}{\tau_{\mathrm{To}, \mathrm{a}}} \\
& \frac{\mathrm{dTo} \mathrm{T}_{\text {if }}}{\mathrm{dt}}=\alpha_{\mathrm{To}, \mathrm{if}} \cdot\left(1-\mathrm{To}_{\mathrm{if}}\right)-\beta_{\mathrm{To}, \mathrm{if}} \cdot \mathrm{To}_{\text {if }} \\
& \frac{\mathrm{dTo} \mathrm{is}_{\mathrm{is}}}{\mathrm{dt}}=\alpha_{\mathrm{To}, \mathrm{is}} \cdot\left(1-\mathrm{To}_{\text {is }}\right)-\beta_{\mathrm{To}, \text { is }} \cdot \mathrm{To}_{\text {is }} \\
& \mathrm{I}_{\mathrm{To}}=\overline{\mathrm{G}}_{\mathrm{To}} \cdot \mathrm{To}_{\mathrm{a}}^{3} \cdot \mathrm{To}_{\text {if }} \cdot \mathrm{To}_{\text {is }} \cdot \mathrm{R}_{\mathrm{To}} \cdot\left(\mathrm{V}_{\mathrm{m}}-\mathrm{E}_{\mathrm{K}}\right)
\end{aligned}
$$

\subsubsection{HRd $\beta A R S$ and HRd $\beta A R S S t o c h$ version (Chapters 3-7)}

Currently there is no clear evidence for PKA-dependent modulation of the 4-AP sensitive transient outward $\mathrm{K}^{+}$current $\left(\mathrm{I}_{\mathrm{T}}\right.$; see ${ }^{75}$ for a recent review). Recently, however, Wagner et al. found that both chronic (transgenic) and acute (adenovirus-mediated) CaMKII overexpression altered $\mathrm{I}_{\mathrm{TO}_{\mathrm{O}}}{ }^{33}$ Both acute and chronic CaMKII overexpression increased the slow component of $\mathrm{I}_{\text {To }}$ via increased KV1.4 expression, and chronic (but not acute) CaMKII overexpression caused downregulation of Kv4.2 and KChIP2. However, these changes were not sensitive to CaMKIl-inhibtion by AIP, implicating mechanisms other than direct phosphorylation of channel subunits. In contrast, both acute and chronic CaMKII overexpression accelerated $I_{\text {To }}$ recovery from inactivation and this effect was reversible by AIP. Consistent with these data, ${ }^{33}$ we increased the fast and slow rates of $I_{\text {To }}$ recovery from inactivation by 1.9 and 2.9 fold, respectively for CaMKII-phosphorylated channels. The HodgkinHuxley formulation with two independent inactivation gates was adapted to a combined biexponential formulation to match the biexponential time course of recovery from inactivation observed in canine ventricular myocytes. ${ }^{70}$

Nonphosphorylated Channels:

$$
\begin{aligned}
& \mathrm{R}_{\mathrm{To}}=\exp \left(\frac{\mathrm{V}_{\mathrm{m}}}{550}\right), \quad \overline{\mathrm{G}}_{\mathrm{To}}=0.4975 \mathrm{mS} / \mu \mathrm{F} \\
& \tau_{\mathrm{To}, \mathrm{a}}=\left(\frac{1}{1.2089 \cdot\left(1+\exp \left(\frac{\mathrm{V}_{\mathrm{m}}-18.4099}{-29.3814}\right)\right)}+\frac{3.5}{1+\exp \left(\frac{\mathrm{V}_{\mathrm{m}}+100}{29.3814}\right)}\right)^{-1} \\
& \mathrm{To}_{\mathrm{a}, \infty}=\frac{1}{1+\exp \left(\left(\mathrm{V}_{\mathrm{m}}+9.437\right) /-7.133\right)} \\
& \alpha_{\mathrm{To}, \text { if }}=\frac{0.02144}{1+\exp \left(\left(\mathrm{V}_{\mathrm{m}}+58\right) / 5\right)} \\
& \beta_{\mathrm{To}, \text { if }}=\frac{1}{0.5 \cdot 9.7953 \cdot\left(1+\exp \left(\left(\mathrm{V}_{\mathrm{m}}+19\right) /-9\right)\right)} \\
& \alpha_{\mathrm{To}, \text { is }}=\frac{0.56034}{250 \cdot\left(1+\exp \left(\left(\mathrm{V}_{\mathrm{m}}+60\right) / 5\right)\right)} \\
& \beta_{\mathrm{To}, \text { is }}=\beta_{\mathrm{To}, \text { if }}
\end{aligned}
$$




$$
\begin{aligned}
& \frac{\mathrm{dTo}_{\mathrm{a}}}{\mathrm{dt}}=\frac{\mathrm{To}_{\mathrm{a}, \mathrm{o}}-\mathrm{To}_{\mathrm{a}}}{\tau_{\mathrm{To}, \mathrm{a}}} \\
& \frac{\mathrm{dTo}_{\mathrm{if}}}{\mathrm{dt}}=\alpha_{\mathrm{To}, \text { if }} \cdot\left(1-\mathrm{To}_{\text {if }}\right)-\beta_{\mathrm{To}, \mathrm{if}} \cdot \mathrm{To}_{\text {if }} \\
& \frac{\mathrm{dTo} \mathrm{is}_{\mathrm{is}}}{\mathrm{dt}}=\alpha_{\mathrm{To}, \text { is }} \cdot\left(1-\mathrm{To}_{\text {is }}\right)-\beta_{\mathrm{To}, \text { is }} \cdot \mathrm{To}_{\text {is }} \\
& \mathrm{I}_{\mathrm{To}}^{\mathrm{NP}}=\overline{\mathrm{G}}_{\mathrm{To}} \cdot \mathrm{To}_{\mathrm{a}}^{3} \cdot\left(0.7356 \cdot \mathrm{To}_{\text {if }}+0.2644 \cdot \mathrm{To}_{\text {is }}\right) \cdot\left(\mathrm{V}_{\mathrm{m}}-\mathrm{E}_{\mathrm{K}}\right)
\end{aligned}
$$

\section{CaMKIl phosphorylated Channels:}

$$
\begin{aligned}
& \alpha_{\mathrm{To}, \mathrm{if}}^{\mathrm{P}, \mathrm{CaMK}}=\frac{0.04796}{1+\exp \left(\left(\mathrm{V}_{\mathrm{m}}+58\right) / 5\right)} \\
& \beta_{\mathrm{To}, \mathrm{if}}^{\mathrm{P}, \mathrm{CaMK}}=\beta_{\mathrm{To}, \mathrm{if}} \\
& \alpha_{\mathrm{To}, \mathrm{is}}^{\mathrm{P}, \mathrm{CaMK}}=\frac{2.4600}{250 \cdot\left(1+\exp \left(\left(\mathrm{V}_{\mathrm{m}}+60\right) / 5\right)\right)} \\
& \beta_{\mathrm{To}, \text { is }}^{\mathrm{P}, \mathrm{CaMK}}=\beta_{\mathrm{To}, \mathrm{if}}^{\mathrm{P}, \mathrm{CaMK}}
\end{aligned}
$$

$\frac{\mathrm{dTo}_{\text {if }}^{\mathrm{P}, \mathrm{CaMKII}}}{\mathrm{dt}}=\alpha_{\mathrm{To}, \mathrm{if}}^{\mathrm{P}, \mathrm{CaMK}} \cdot\left(1-\mathrm{To}_{\text {if }}^{\mathrm{P}, \mathrm{CaMKII}}\right)-\beta_{\mathrm{To}, \mathrm{if}}^{\mathrm{P}, \mathrm{CaMK}} \cdot \mathrm{To}_{\text {if }}^{\mathrm{P}, \mathrm{CaMKII}}$

$\frac{\mathrm{dTo}_{\mathrm{is}}^{\mathrm{P}, \text { CaMKII }}}{\mathrm{dt}}=\alpha_{\mathrm{To}, \mathrm{is}}^{\mathrm{P} \text {,CaMK }} \cdot\left(1-\mathrm{To}_{\text {is }}^{\mathrm{P}, \mathrm{CaMKII}}\right)-\beta_{\mathrm{To}, \mathrm{is}}^{\mathrm{P}, \mathrm{CaMK}} \cdot \mathrm{To}_{\mathrm{is}}^{\mathrm{P} \text {,CaMKII }}$

$\mathrm{I}_{\mathrm{To}}^{\mathrm{P} \text {,CaMKII }}=\overline{\mathrm{G}}_{\mathrm{To}} \cdot \mathrm{To}_{\mathrm{a}}^{3} \cdot\left(0.7356 \cdot \mathrm{To}_{\mathrm{if}}^{\mathrm{P}, \mathrm{CaMKII}}+0.2644 \cdot \mathrm{To}_{\mathrm{is}}^{\mathrm{P}, \mathrm{CaMKII}}\right) \cdot\left(\mathrm{V}_{\mathrm{m}}-\mathrm{E}_{\mathrm{K}}\right)$

Combined I Io current:

$\mathrm{I}_{\text {To }}=\left(1-\mathrm{f}_{\text {ITo }}^{\mathrm{P} \text {,CaMKII }}\right) \cdot \mathrm{I}_{\text {To }}^{\text {NP }}+\mathrm{f}_{\text {ITo }}^{\text {P,CaMKII }} \cdot \mathrm{I}_{\text {To }}^{\text {P,CaMKII }}$

\subsection{4 $\mathrm{SR} \mathrm{Ca}^{2+}$ release $\left(\mathrm{I}_{\mathrm{Rel}}\right)$}

\subsubsection{HRd2009 version (Chapter 2)}

Model SR release formulation is equivalent to that in Livshitz et al. ${ }^{76}$

$$
\begin{aligned}
& \Delta \beta_{0}=1.000 \mathrm{mmol} / \mathrm{L}, \quad \mathrm{K}_{\beta}=0.2800 \mathrm{mmol} / \mathrm{L}, \quad \mathrm{h}_{\beta}=10 \\
& \kappa=0.1125 \mathrm{pA} / \mathrm{pF}, \quad \mathrm{K}_{\mathrm{Rel}, \infty}=1.000 \mathrm{mmol} / \mathrm{L}, \quad \mathrm{h}_{\mathrm{Rel}}=8 \\
& \mathrm{~K}_{\mathrm{Rel}, \tau}=0.0123 \mathrm{mmol} / \mathrm{L}, \quad \beta_{0}=4.7500 \mathrm{~ms} \\
& \Delta \beta_{\tau, \text { CaMK }}=\frac{\Delta \beta_{0}}{1+\left(\frac{\mathrm{K}_{\beta}}{\text { CaMK }_{\text {active }}}\right)^{\mathrm{h}_{\beta}}} \\
& \beta_{\tau}=\beta_{0} \cdot\left(1+\Delta \beta_{\tau, \text { CaMK }}\right) \\
& \alpha_{\text {Rel }}=\kappa \cdot \beta_{\tau}
\end{aligned}
$$




$$
\begin{aligned}
& \mathrm{I}_{\mathrm{Rel}, \tau}=\frac{\beta_{\tau}}{1+\frac{\mathrm{K}_{\mathrm{Rel}, \tau}}{\left[\mathrm{Ca}^{2+}\right]_{\mathrm{JRR}}}} \\
& \mathrm{I}_{\mathrm{Rel}, \infty}=\frac{\alpha_{\mathrm{Rel}} \cdot \mathrm{I}_{\mathrm{Ca}, \mathrm{L}}}{1+\left(\frac{\mathrm{K}_{\mathrm{Rel}, \infty}}{\left[\mathrm{Ca}^{2+}\right]_{\mathrm{JSR}}}\right)^{\mathrm{h}_{\mathrm{R}}}} \\
& \frac{\mathrm{dI}_{\mathrm{Rel}}}{\mathrm{dt}}=-\frac{\mathrm{I}_{\mathrm{Rel}, \infty}+\mathrm{I}_{\mathrm{Rel}}}{\mathrm{I}_{\mathrm{Rel}, \tau}}
\end{aligned}
$$

\subsubsection{HRdßARS version (Chapters 3-5)}

The exact mechanisms and functional effects of ryanodine receptor (RyR) phosphorylation are still actively debated. ${ }^{77}$ Marks and colleagues showed increased RyR open probability after PKAdependent phosphorylation of Ser 2809 (Ser 2808 in mice) and dissociation of the regulatory protein FKBP12.6. ${ }^{78}$ Furthermore, they argued that the RyR may become hyperphosphorylated during heart failure, leading to increased sensitivity to $\mathrm{Ca}^{2+}$-induced RyR activation. Other groups have questioned these results, showing no differences in adrenergic response of S2808A knock-in mice ${ }^{79}$ and no differences between RyR phosphorylation levels in normal and failing canine hearts. ${ }^{80}$ The mechanisms of RyR phosphorylation are further complicated by the fact that there are multiple consensus phosphorylation sites for multiple kinases. Xiao and others provided evidence that not S2809 but S2030 is the major phosphorylation site activated by $\beta A R$ stimulation and that phosphorylation increased the sensitivity of the RyR to luminal $\mathrm{Ca}^{2+} \cdot{ }^{81}$ Other confounding effects include the effects of adrenergic stimulation on PLB and $\mathrm{I}_{\mathrm{CaL}}$, which change the trigger strength and $\mathrm{Ca}^{2+}$ load that affect RyR properties indirectly. Ginsburg and Bers analyzed the effects of isoproterenol on SR-Ca ${ }^{2+}$ release in rabbit and mouse ventricular myocytes with controlled $\mathrm{Ca}^{2+}$ load and trigger strength and found no difference in the total amount of $\mathrm{Ca}^{2+}$ released compared to control conditions. However, this amount of $\mathrm{Ca}^{2+}$ was released faster during isoproterenol. ${ }^{82}$ Similar findings were reported recently by Ogrodnik and Niggli in guinea pig ventricular myocytes. ${ }^{83}$

Because the model does not distinguish individual phosphorylation sites on a substrate, we are only interested in the overall functional response of RyRs to $\beta A R$ stimulation. Here, we adopt the results from Ginsburg and Bers. ${ }^{82}$ The phosphorylated channels release approximately the same total amount of $\mathrm{Ca}^{2+}$ when the $\mathrm{I}_{\mathrm{CaL}}$ trigger and $\mathrm{Ca}^{2+}$ load are matched to baseline conditions (same initial $\mathrm{Ca}^{2+}$ concentration in the JSR and $\mathrm{I}_{\mathrm{CaL}}$ clamped) but the maximum release is approximately 1.8 fold higher in phosphorylated channels than in non-phosphorylated channels. In addition, the RyR can be phosphorylated by CaMKII. Formulations for the changes caused by CaMKII phosphorylation were included in our baseline model and remained unchanged. ${ }^{21,76}$

Besides effects on the triggered, $\mathrm{Ca}^{2+}$ induced $\mathrm{Ca}^{2+}$ release, $\beta$ AR stimulation also increases the leak of $\mathrm{Ca}^{2+}$ from the SR. ${ }^{83,84}$ This effect is independent of CAMP but sensitive to CaMKII inhibition and 
results in a much steeper dependence of leak amplitude on SR $\mathrm{Ca}^{2+}$ load. ${ }^{84}$ We incorporate an explicit JSR $\mathrm{Ca}^{2+}$ leak formulation with phosphorylation as a direct function of ISO, because of the cAMP independence and as yet unclear mechanism resulting in cAMP-independent increases in CaMKII phosphorylation of RyR.

Nonphosphorylated Channels:

$$
\begin{aligned}
& \Delta \beta_{0}^{\mathrm{NP}}=2.0 \mathrm{mmol} / \mathrm{L}, \quad \beta_{0}=3.167 \mathrm{~ms}, \quad \kappa=0.1125 \mu \mathrm{A} / \mathrm{uF} \\
& \mathrm{K}_{\mathrm{Rel}, \tau}=0.0123 \mathrm{mmol} / \mathrm{L}, \quad \mathrm{K}_{\mathrm{Rel}, \infty}=1.0 \mathrm{mmol} / \mathrm{L}, \quad \mathrm{h}_{\mathrm{R}}=8.0 \\
& \Delta \beta_{\tau, \mathrm{CaMK}}=\Delta \beta_{0}^{\mathrm{NP}} \cdot \mathrm{f}_{\mathrm{RyR}}^{\mathrm{P}, \mathrm{CaMKII}} \\
& \beta_{\tau}=\beta_{0} \cdot\left(1+\Delta \beta_{\tau, \mathrm{CaMK}}\right) \\
& \alpha_{\mathrm{Rel}}=\kappa \cdot \beta_{\tau} \\
& \mathrm{I}_{\mathrm{Rel}, \tau}^{\mathrm{NP}}=\frac{\beta_{\tau}}{1+\frac{\mathrm{K}_{\mathrm{Rel}, \tau}}{\left[\mathrm{Ca}^{2+}\right]_{\mathrm{JRR}}}} \\
& \mathrm{I}_{\mathrm{Rel}, \infty}^{\mathrm{NP}}=\frac{\alpha_{\mathrm{Rel}} \cdot \mathrm{I}_{\mathrm{Ca}, \mathrm{L}}}{1+\left(\frac{\mathrm{K}_{\mathrm{Rel}, \infty}}{\left[\mathrm{Ca}^{2+}\right]_{\mathrm{JSR}}}\right)^{\mathrm{h}_{\mathrm{R}}}} \\
& \frac{\mathrm{dI}_{\mathrm{Rel}}^{\mathrm{NP}}=}{\mathrm{dt}}=\frac{\mathrm{I}_{\mathrm{Rel}, \infty}^{\mathrm{NP}}+\mathrm{I}_{\mathrm{Rel}}^{\mathrm{NP}}}{\mathrm{I}_{\mathrm{Rel}, \tau}^{\mathrm{NP}}} \\
& \mathrm{I}_{\mathrm{RyR}, \mathrm{leak}}^{\mathrm{NP}}=1.75 \cdot 10^{-4} \cdot \exp \left(\frac{\left[\mathrm{Ca}^{2+}\right]_{\mathrm{SR}}}{20}\right) \cdot\left(\left[\mathrm{Ca}^{2+}\right]_{\mathrm{JSR}}-\left[\mathrm{Ca}^{2+}\right]_{\mathrm{SS}}\right)
\end{aligned}
$$

\section{Phosphorylated Channels:}

$$
\Delta \beta_{0}^{\mathrm{P}}=0.0 \mathrm{mmol} / \mathrm{L}
$$

$$
\begin{aligned}
& \Delta \beta_{\tau, \mathrm{CaMK}}=\Delta \beta_{0}^{\mathrm{P}} \cdot \mathrm{f}_{\mathrm{RyR}}^{\mathrm{P}, \mathrm{CaMKII}} \\
& \beta_{\tau}=\beta_{0} \cdot\left(1+\Delta \beta_{\tau, \mathrm{CaMK}}\right) \\
& \mathrm{I}_{\mathrm{Rel}, \tau}^{\mathrm{P}}=0.4 \cdot \frac{\beta_{\tau}}{1+\frac{\mathrm{K}_{\mathrm{Rel}, \tau}}{\left[\mathrm{Ca}^{2+}\right]_{\mathrm{JSR}}}} \\
& \mathrm{I}_{\mathrm{Rel}, \infty}^{\mathrm{P}}=1.8 \cdot \frac{\alpha_{\mathrm{Rel}} \cdot \mathrm{I}_{\mathrm{Ca}, \mathrm{L}}}{1+\left(\frac{\mathrm{K}_{\mathrm{Rel}, \infty}}{\left[\mathrm{Ca}^{2+}\right]_{\mathrm{JSR}}}\right)^{\mathrm{h}_{\mathrm{R}}}} \\
& \frac{\mathrm{dI}_{\mathrm{Rel}}^{\mathrm{P}}}{\mathrm{dt}}=-\frac{\mathrm{I}_{\mathrm{Rel}, \infty}^{\mathrm{P}}+\mathrm{I}_{\mathrm{Rel}}^{\mathrm{P}}}{\mathrm{I}_{\mathrm{Rel}, \tau}^{\mathrm{P}}}
\end{aligned}
$$


$\mathrm{I}_{\mathrm{RyR}, \text { leak }}^{\mathrm{P}}=5.0 \cdot 10^{-4} \cdot \exp \left(\frac{\left[\mathrm{Ca}^{2+}\right]_{\mathrm{JSR}}}{1.1}\right) \cdot\left(\left[\mathrm{Ca}^{2+}\right]_{\mathrm{JSR}}-\left[\mathrm{Ca}^{2+}\right]_{\mathrm{SS}}\right)$

\section{Combined current:}

$$
\begin{aligned}
& \hat{\mathrm{f}}_{\mathrm{RyR}}^{\mathrm{P}}=\frac{\mathrm{f}_{\mathrm{RyR}}^{\mathrm{p}}-\left(0.0329+\frac{[\mathrm{RyR}]_{\mathrm{ARN}}}{[\mathrm{RyR}]_{\text {tot }}}\right)}{0.9586-\left(0.0329+\frac{[\mathrm{RyR}]_{\mathrm{ARN}}}{[\mathrm{RyR}]_{\text {tot }}}\right)} \\
& \frac{\mathrm{d} \hat{\mathrm{f}}_{\mathrm{RyR}, \text { leak }}^{\mathrm{P}}}{\mathrm{dt}}=\frac{\left(\frac{[\mathrm{ISO}]}{[\mathrm{ISO}]+0.05}-\hat{\mathrm{f}}_{\mathrm{RyR}, \text { leak }}^{\mathrm{P}}\right)}{\tau_{\mathrm{RyR}, \text { leak }}}, \quad \tau_{\text {RyR,leak }}=100 \mathrm{~s} \\
& \mathrm{I}_{\text {Rel }}=\left(1-\hat{\mathrm{f}}_{\mathrm{RyR}}^{\mathrm{P}}\right) \cdot \mathrm{I}_{\mathrm{Rel}}^{\mathrm{NP}}+\hat{\mathrm{f}}_{\mathrm{RyR}}^{\mathrm{P}} \cdot \mathrm{I}_{\text {Rel }}^{\mathrm{P}}+\left(1-\hat{\mathrm{f}}_{\mathrm{RyR}, \text { leak }}^{\mathrm{P}}\right) \cdot \mathrm{I}_{\mathrm{RyR}, \text { leak }}^{\mathrm{NP}}+\hat{\mathrm{f}}_{\mathrm{RyR}, \text { leak }}^{\mathrm{P}} \cdot \mathrm{I}_{\mathrm{RyR}, \text { leak }}^{\mathrm{P}}
\end{aligned}
$$

Store-overload induced $\mathrm{Ca}^{2+}$ release:

$$
\begin{aligned}
\alpha_{\text {SOICR }} & =\frac{0.0078}{1+\exp \left(-\frac{\left[\mathrm{Ca}^{2+}\right]_{\mathrm{JSR}}-2.3814}{1.0 \cdot 10^{-4}}\right)} \\
\beta_{\mathrm{SOICR}} & =7.7525 \cdot 10^{-4}+\frac{7.6084 \cdot 10^{-2}}{1+\exp \left(\frac{\left[\mathrm{Ca}^{2+}\right]_{\mathrm{JSR}}-0.7410}{1.0 \cdot 10^{-4}}\right)}
\end{aligned}
$$

$\frac{\mathrm{dSwitch}_{\text {On }}}{\mathrm{dt}}=\alpha_{\mathrm{SOICR}} \cdot\left(1-\right.$ Switch $\left._{\mathrm{On}}\right)-\beta_{\mathrm{SOICR}} \cdot$ Switch $_{\mathrm{On}}$

$$
\begin{gathered}
I_{\text {RelSpon }, \infty}=\frac{G_{\text {RelSpon }}}{1+\exp \left(-\frac{\text { Switch }_{\text {On }}-0.75}{0.0001}\right)}, \quad G_{\text {RelSpon }}=0.6655 \\
\tau_{\text {RelSpon }}=\frac{7.0153}{1+\frac{\mathrm{K}_{\text {Rel }, \tau}}{\left[\mathrm{Ca}^{2+}\right]_{\text {JSR }}}} \\
\frac{\mathrm{dI}_{\text {Rel,SOICR }}}{d t}=\frac{\mathrm{I}_{\text {RelSpon, }, 0}-\mathrm{I}_{\text {Rel,SOICR }}}{\tau_{\text {RelSpon }}} \\
\mathrm{I}_{\text {Rel }}=\left(1-\hat{\mathrm{f}}_{\mathrm{RyR}}^{\mathrm{P}}\right) \cdot \mathrm{I}_{\mathrm{Rel}}^{\mathrm{NP}}+\hat{\mathrm{f}}_{\mathrm{RyR}}^{\mathrm{P}} \cdot \mathrm{I}_{\mathrm{Rel}}^{\mathrm{P}}+\left(1-\hat{\mathrm{f}}_{\mathrm{RyR}, \text { leak }}^{\mathrm{P}}\right) \cdot \mathrm{I}_{\mathrm{RyR}, \text { leak }}^{\mathrm{NP}}+\hat{\mathrm{f}}_{\mathrm{RyR}, \text { leak }}^{\mathrm{P}} \cdot \mathrm{I}_{\mathrm{RyR}, \text { leak }}^{\mathrm{P}}+\mathrm{I}_{\text {Rel,SoICR }}
\end{gathered}
$$

\subsubsection{HRdßARS SCR version (Chapter 6)}

To initiate diastolic $\mathrm{Ca}^{2+}$ release, steady-state RyR activation was set to a constant value in one of the two domains (indicated with superscript $\mathrm{x}$ ) for the interval of diastolic SR $\mathrm{Ca}^{2+}$ release. In addition, the steepness of the dependence on SR $\mathrm{Ca}^{2+}$ load and $\mathrm{I}_{\mathrm{CaL}}$ trigger strength were adjusted to reduce the incidence of secondary activation of RyR during repolarization.

$\mathrm{h}_{\mathrm{R}}=10$ 


$$
\begin{aligned}
& \mathrm{I}_{\mathrm{Rel}, \infty}^{\mathrm{NP}}=\frac{\alpha_{\mathrm{Rel}}}{1+\left(\frac{\mathrm{K}_{\mathrm{Rel}, \infty}}{\left[\mathrm{Ca}^{2+}\right]_{\mathrm{JSR}}^{\mathrm{X}}}\right)^{\mathrm{h}_{\mathrm{R}}}} \cdot \frac{\mathrm{I}_{\mathrm{ICaL}}^{\mathrm{X}}}{1+\exp \left(\frac{\left(\mathrm{I}_{\mathrm{ICaL}}^{\mathrm{X}}+1.5\right)}{0.0001}\right)}-\mathrm{SCR}_{\text {level }} \\
& \mathrm{I}_{\mathrm{Rel}, \infty}^{\mathrm{P}}=1.9925 \cdot \frac{\alpha_{\mathrm{Rel}}}{1+\left(\frac{\mathrm{K}_{\mathrm{Rel}, \infty}}{\left[\mathrm{Ca}^{2+}\right]_{\mathrm{JSR}}^{\mathrm{X}}}\right)^{\mathrm{h}_{\mathrm{R}}}} \cdot \frac{\mathrm{I}_{\mathrm{ICaL}}^{\mathrm{X}}}{1+\exp \left(\frac{\left(\mathrm{I}_{\mathrm{ICaL}}^{\mathrm{X}}+1.5\right)}{0.0001}\right)}-\mathrm{SCR}_{\text {level }}
\end{aligned}
$$

\subsubsection{HRdßARS Stoch version (Chapter 7)}

The original phosphorylated and non-phosphorylation CICR formulations were replaced by two 4state Markov Models proposed by Restrepo et al. in their local control model of $\mathrm{Ca}^{2+}$ handling in the rabbit ventricular myocyte. ${ }^{85}$ Model parameters were adjusted to reproduce rate-dependent $\mathrm{Ca}^{2+}$ transient (CaT) properties (amplitude, time to peak, and time constant of decay) of the original HRd $\beta A R$ model in the absence or presence of $\beta$-adrenergic stimulation ( $\beta A R S$ ).

Nonphosphorylated Channels:

$$
\begin{aligned}
& \widehat{\mathrm{M}}_{\mathrm{RyR}}^{\mathrm{NP}}=\frac{1}{1+\left(\frac{\left[\mathrm{Ca}^{2+}\right]_{\mathrm{JSR}}}{0.4163}\right)^{15.24}} \\
& \mathrm{Ac}_{\mathrm{RyR}, \infty}^{\mathrm{NP}}=3.9665 \cdot 10^{-5} \cdot\left(\exp \left(\frac{\left[\mathrm{Ca}^{2+}\right]_{\mathrm{JSR}}}{2.4898}\right)-1\right)+\frac{0.032}{1+\left(\frac{1.1960 \cdot 10^{-3} \cdot\left(1+2.0 \cdot \mathrm{f}_{\mathrm{RYR}}^{\mathrm{P}, \mathrm{CaMKII}}\right)}{\left[\mathrm{Ca}^{2+}\right]_{\mathrm{ss}, \mathrm{CaL}}}\right)^{8}} \\
& \operatorname{Acl}_{\mathrm{RyR}, \infty}^{\mathrm{NP}}=3.9665 \cdot 10^{-5} \cdot\left(\exp \left(\frac{\left[\mathrm{Ca}^{2+}\right]_{\mathrm{JSR}}}{2.4898}\right)-1\right)+\frac{0.0005}{1+\left(\frac{0.003 \cdot\left(1+2.0 \cdot \mathrm{f}_{\mathrm{RYR}}^{\mathrm{P}, \mathrm{CaMKII}}\right)}{\left[\mathrm{Ca}^{2+}\right]_{\mathrm{Ss}, \mathrm{CaL}}}\right)^{8}} \\
& \tau_{\mathrm{RyR}, \mathrm{Ac}}^{\mathrm{NP}}=0.2+0.5000 \cdot \widehat{\mathrm{M}}_{\mathrm{RyR}}^{\mathrm{NP}} \\
& \mathrm{k}_{\mathrm{RyR}, 12}^{\mathrm{NP}}=\frac{\mathrm{Ac}_{\mathrm{RyR}, \infty}^{\mathrm{NP}}}{\tau_{\mathrm{RyR}, \mathrm{Ac}}^{\mathrm{NP}}}, \quad \mathrm{k}_{\mathrm{RyR}, 21}^{\mathrm{NP}}=\frac{1-\mathrm{Ac}_{\mathrm{RyR}, \infty}^{\mathrm{NP}}}{\tau_{\mathrm{RyR}, \mathrm{Ac}}^{\mathrm{NP}}} \\
& \mathrm{k}_{\mathrm{RyR}, 43}^{\mathrm{NP}}=\frac{\mathrm{Acl}_{\mathrm{RyR}, \infty}^{\mathrm{NP}}}{\tau_{\mathrm{RyR}, \mathrm{Ac}}^{\mathrm{NP}}}, \quad \mathrm{k}_{\mathrm{RyR}, 34}^{\mathrm{NP}}=\frac{1-\mathrm{Acl}_{\mathrm{RyR}, \infty}^{\mathrm{NP}}}{\tau_{\mathrm{RyR}, \mathrm{Ac}}^{\mathrm{NP}}} \\
& \mathrm{k}_{\mathrm{RyR}, 14,23}^{\mathrm{NP}}=\frac{\widehat{\mathrm{M}}_{\mathrm{RyR}}^{\mathrm{NP}}}{0.5250}, \quad \mathrm{k}_{\mathrm{RyR}, 41}^{\mathrm{NP}}=\frac{1}{395.19} \\
& \mathrm{k}_{\mathrm{RyR}, 32}^{\mathrm{NP}}=\frac{\mathrm{k}_{\mathrm{RyR}, 41}^{\mathrm{NP}} \cdot \mathrm{k}_{\mathrm{RyR}, 12}^{\mathrm{NP}} \cdot \mathrm{k}_{\mathrm{RyR}, 34}^{\mathrm{NP}}}{\mathrm{k}_{\mathrm{RyR}, 43}^{\mathrm{NP}} \cdot \mathrm{k}_{\mathrm{RyR}, 21}^{\mathrm{NP}}} \\
& \frac{\mathrm{dCB}_{\mathrm{RyR}}^{\mathrm{NP}}}{\mathrm{dt}}=\mathrm{k}_{\mathrm{RyR}, 21}^{\mathrm{NP}} \cdot \mathrm{OB}_{\mathrm{RyR}}^{\mathrm{NP}}+\mathrm{k}_{\mathrm{RyR}, 41}^{\mathrm{NP}} \cdot \mathrm{CU}_{\mathrm{RyR}}^{\mathrm{NP}}-\left(\mathrm{k}_{\mathrm{RyR}, 12}^{\mathrm{NP}}+\mathrm{k}_{\mathrm{RyR}, 14,23}^{\mathrm{NP}}\right) \cdot \mathrm{CB}_{\mathrm{RyR}}^{\mathrm{NP}}
\end{aligned}
$$


$\frac{\mathrm{dOB}_{\mathrm{RyR}}^{\mathrm{NP}}}{\mathrm{dt}}=\mathrm{k}_{\mathrm{RyR}, 12}^{\mathrm{NP}} \cdot \mathrm{CB}_{\mathrm{RyR}}^{\mathrm{NP}}+\mathrm{k}_{\mathrm{RyR}, 32}^{\mathrm{NP}} \cdot \mathrm{OU}_{\mathrm{RyR}}^{\mathrm{NP}}-\left(\mathrm{k}_{\mathrm{RyR}, 21}^{\mathrm{NP}}+\mathrm{k}_{\mathrm{RyR}, 14,23}^{\mathrm{NP}}\right) \cdot \mathrm{OB}_{\mathrm{RyR}}^{\mathrm{NP}}$ $\frac{\mathrm{dOU}}{\mathrm{Ry}} \mathrm{NP}_{\mathrm{R}}^{\mathrm{NP}}=\mathrm{k}_{\mathrm{RyR}, 14,23}^{\mathrm{NP}} \cdot \mathrm{OB}_{\mathrm{RyR}}^{\mathrm{NP}}+\mathrm{k}_{\mathrm{RyR}, 43}^{\mathrm{NP}} \cdot \mathrm{CU}_{\mathrm{RyR}}^{\mathrm{NP}}-\left(\mathrm{k}_{\mathrm{RyR}, 32}^{\mathrm{NP}}+\mathrm{k}_{\mathrm{RyR}, 34}^{\mathrm{NP}}\right) \cdot \mathrm{OU}_{\mathrm{RyR}}^{\mathrm{NP}}$ $\frac{\mathrm{dCU}_{\mathrm{RyR}}^{\mathrm{NP}}}{\mathrm{dt}}=\mathrm{k}_{\mathrm{RyR}, 14,23}^{\mathrm{NP}} \cdot \mathrm{CB}_{\mathrm{RyR}}^{\mathrm{NP}}+\mathrm{k}_{\mathrm{RyR}, 34}^{\mathrm{NP}} \cdot \mathrm{OU}_{\mathrm{RyR}}^{\mathrm{NP}}-\left(\mathrm{k}_{\mathrm{RyR}, 41}^{\mathrm{NP}}+\mathrm{k}_{\mathrm{RyR}, 43}^{\mathrm{NP}}\right) \cdot \mathrm{CU}_{\mathrm{RyR}}^{\mathrm{NP}}$

$\mathrm{I}_{\mathrm{Rel}}^{\mathrm{NP}}=\frac{3.5289 \cdot 10^{-5}}{\mathrm{~V}_{\mathrm{ss}}} \cdot\left(\mathrm{OB}_{\mathrm{RyR}}^{\mathrm{NP}}+\mathrm{OU}_{\mathrm{RyR}}^{\mathrm{NP}}\right) \cdot\left(\left[\mathrm{Ca}^{2+}\right]_{\mathrm{JSR}}-\left[\mathrm{Ca}^{2+}\right]_{\mathrm{Ss}, \mathrm{CaL}}\right)$

Phosphorylated Channels:

$\widehat{\mathrm{M}}_{\mathrm{RyR}}^{\mathrm{P}}=\frac{1}{1+\left(\frac{\left[\mathrm{Ca}^{2+}\right]_{\mathrm{JSR}}}{0.4240}\right)^{15.24}}$

$\mathrm{Ac}_{\mathrm{RyR}, \infty}^{\mathrm{P}}=1.8891 \cdot 10^{-5} \cdot\left(\exp \left(\frac{\left[\mathrm{Ca}^{2+}\right]_{\mathrm{JSR}}}{1.4257}\right)-1\right)+\frac{0.07}{1+\left(\frac{0.003 \cdot\left(1+2.0 \cdot \mathrm{f}_{\mathrm{RYR}}^{\mathrm{P}, \mathrm{CaMKII}}\right)}{\left[\mathrm{Ca}^{2+}\right]_{\mathrm{Ss}, \mathrm{CaL}}}\right)^{8}}$

$\operatorname{Acl}_{\mathrm{RyR}, \infty}^{\mathrm{P}}=1.8891 \cdot 10^{-5} \cdot\left(\exp \left(\frac{\left[\mathrm{Ca}^{2+}\right]_{\mathrm{JSR}}}{1.4257}\right)-1\right)+\frac{0.0005}{1+\left(\frac{0.006 \cdot\left(1+2.0 \cdot \mathrm{f}_{\mathrm{RYR}}^{\mathrm{P}, \mathrm{CaMKII}}\right)}{\left[\mathrm{Ca}^{2+}\right]_{\mathrm{Ss}, \mathrm{CaL}}}\right)^{8}}$

$\tau_{\mathrm{RyR}, \mathrm{Ac}}^{\mathrm{P}}=0.3026+3.0000 \cdot \widehat{\mathrm{M}}_{\mathrm{RyR}}^{\mathrm{P}}$

$\mathrm{k}_{\mathrm{RyR}, 12}^{\mathrm{P}}=\frac{\mathrm{Ac}_{\mathrm{RyR}, \infty}^{\mathrm{P}}}{\tau_{\mathrm{RyR}, \mathrm{Ac}}^{\mathrm{P}}}, \quad \mathrm{k}_{\mathrm{RyR}, 21}^{\mathrm{P}}=\frac{1-\mathrm{Ac}_{\mathrm{RyR}, \infty}^{\mathrm{P}}}{\tau_{\mathrm{RyR}, \mathrm{Ac}}^{\mathrm{P}}}$

$\mathrm{k}_{\mathrm{RyR}, 43}^{\mathrm{P}}=\frac{\operatorname{Acl}_{\mathrm{RyR}, \infty}^{\mathrm{P}}}{\tau_{\mathrm{RyR}, \mathrm{Ac}}^{\mathrm{P}}}, \quad \mathrm{k}_{\mathrm{RyR}, 34}^{\mathrm{P}}=\frac{1-\operatorname{Acl}_{\mathrm{RyR}, \infty}^{\mathrm{P}}}{\tau_{\mathrm{RyR}, \mathrm{Ac}}^{\mathrm{P}}}$

$\mathrm{k}_{\mathrm{RyR}, 14,23}^{\mathrm{P}}=\frac{\widehat{\mathrm{M}}_{\mathrm{RyR}}^{\mathrm{P}}}{0.6000}, \quad \mathrm{k}_{\mathrm{RyR}, 41}^{\mathrm{P}}=\frac{1}{409.16}$

$\mathrm{k}_{\mathrm{RyR}, 32}^{\mathrm{P}}=\frac{\mathrm{k}_{\mathrm{RyR}, 41}^{\mathrm{P}} \cdot \mathrm{k}_{\mathrm{RyR}, 12}^{\mathrm{P}} \cdot \mathrm{k}_{\mathrm{RyR}, 34}^{\mathrm{P}}}{\mathrm{k}_{\mathrm{RyR}, 43}^{\mathrm{P}} \cdot \mathrm{k}_{\mathrm{RyR}, 21}^{\mathrm{P}}}$

$\frac{\mathrm{dCB}_{\mathrm{RyR}}^{\mathrm{P}}}{\mathrm{dt}}=\mathrm{k}_{\mathrm{RyR}, 21}^{\mathrm{P}} \cdot \mathrm{OB}_{\mathrm{RyR}}^{\mathrm{P}}+\mathrm{k}_{\mathrm{RyR}, 41}^{\mathrm{P}} \cdot \mathrm{CU}_{\mathrm{RyR}}^{\mathrm{P}}-\left(\mathrm{k}_{\mathrm{RyR}, 12}^{\mathrm{P}}+\mathrm{k}_{\mathrm{RyR}, 14,23}^{\mathrm{P}}\right) \cdot \mathrm{CB}_{\mathrm{RyR}}^{\mathrm{P}}$ $\frac{\mathrm{dOB}}{\mathrm{RyR}}=\mathrm{k}_{\mathrm{RyR}, 12}^{\mathrm{P}} \cdot \mathrm{CB}_{\mathrm{RyR}}^{\mathrm{P}}+\mathrm{k}_{\mathrm{RyR}, 32}^{\mathrm{P}} \cdot \mathrm{OU}_{\mathrm{RyR}}^{\mathrm{P}}-\left(\mathrm{k}_{\mathrm{RyR}, 21}^{\mathrm{P}}+\mathrm{k}_{\mathrm{RyR}, 14,23}^{\mathrm{P}}\right) \cdot \mathrm{OB}_{\mathrm{RyR}}^{\mathrm{P}}$ $\frac{\mathrm{dOU}}{\mathrm{dt}}=\mathrm{k}_{\mathrm{RyR}, 14,23}^{\mathrm{P}} \cdot \mathrm{OB}_{\mathrm{RyR}}^{\mathrm{P}}+\mathrm{k}_{\mathrm{RyR}, 43}^{\mathrm{P}} \cdot \mathrm{CU}_{\mathrm{RyR}}^{\mathrm{P}}-\left(\mathrm{k}_{\mathrm{RyR}, 32}^{\mathrm{P}}+\mathrm{k}_{\mathrm{RyR}, 34}^{\mathrm{P}}\right) \cdot \mathrm{OU}_{\mathrm{RyR}}^{\mathrm{P}}$ $\frac{\mathrm{dCU}_{\mathrm{RyR}}^{\mathrm{P}}}{\mathrm{dt}}=\mathrm{k}_{\mathrm{RyR}, 14,23}^{\mathrm{P}} \cdot \mathrm{CB}_{\mathrm{RyR}}^{\mathrm{P}}+\mathrm{k}_{\mathrm{RyR}, 34}^{\mathrm{P}} \cdot \mathrm{OU}_{\mathrm{RyR}}^{\mathrm{P}}-\left(\mathrm{k}_{\mathrm{RyR}, 41}^{\mathrm{P}}+\mathrm{k}_{\mathrm{RyR}, 43}^{\mathrm{P}}\right) \cdot \mathrm{CU}_{\mathrm{RyR}}^{\mathrm{P}}$ 
$\mathrm{I}_{\mathrm{Rel}}^{\mathrm{P}}=\frac{3.5289 \cdot 10^{-5}}{\mathrm{~V}_{\mathrm{Ss}}} \cdot\left(\mathrm{OB}_{\mathrm{RyR}}^{\mathrm{P}}+\mathrm{OU}_{\mathrm{RyR}}^{\mathrm{P}}\right) \cdot\left(\left[\mathrm{Ca}^{2+}\right]_{\mathrm{JSR}}-\left[\mathrm{Ca}^{2+}\right]_{\mathrm{ss}, \mathrm{CaL}}\right)$

\section{Combined current:}

$$
\begin{aligned}
& \hat{\mathrm{f}}_{\mathrm{RyR}}^{\mathrm{P}}=\frac{\mathrm{f}_{\mathrm{RyR}}^{\mathrm{p}}-\left(0.0329+\frac{[\mathrm{RyR}]_{\mathrm{ARN}}}{[\mathrm{RyR}]_{\text {tot }}}\right)}{0.9586-\left(0.0329+\frac{[\mathrm{RyR}]_{\mathrm{ARN}}}{[\mathrm{RyR}]_{\text {tot }}}\right)} \\
& \mathrm{I}_{\text {Rel }}=\left(1-\hat{\mathrm{f}}_{\mathrm{RyR}}^{\mathrm{P}}\right) \cdot \mathrm{I}_{\mathrm{Rel}}^{\mathrm{NP}}+\hat{\mathrm{f}}_{\mathrm{RyR}}^{\mathrm{P}} \cdot \mathrm{I}_{\mathrm{Rel}}^{\mathrm{P}}
\end{aligned}
$$

\section{$1.25 \mathrm{SR} \mathrm{Ca}^{2+}$ uptake $\left(\mathrm{I}_{\mathrm{up}}\right)$}

\subsubsection{HRd2009 version (Chapter 2)}

Model SR uptake formulation is equivalent to that in Livshitz et al. ${ }^{76}$

$$
\begin{aligned}
& \bar{\Delta} \mathrm{K}_{\mathrm{m}, \mathrm{PLB}}=1.700 \cdot 10^{-4} \mathrm{mmol} / \mathrm{L}, \quad \mathrm{K}_{\mathrm{m}, \mathrm{CaMK}}=0.15, \quad \overline{\mathrm{NSR}}=15 \mathrm{mmol} / \mathrm{L} \\
& \bar{\Delta} \mathrm{I}_{\text {up,CaMK }}=0.75, \quad \mathrm{~K}_{\mathrm{m}, \text { up }}=9.200 \cdot 10^{-4} \mathrm{mmol} / \mathrm{L}, \quad \overline{\mathrm{I}}_{\text {up }}=4.3750 \cdot 10^{-3} \mathrm{mmol} / \mathrm{L} / \mathrm{ms} \\
& \Delta \mathrm{K}_{\mathrm{m}, \mathrm{PLB}}=\bar{\Delta} \mathrm{K}_{\mathrm{m}, \mathrm{PLB}} \cdot \frac{\text { CaMK }_{\text {active }}}{\mathrm{K}_{\mathrm{m}, \mathrm{CaMK}}+\text { CaMK }_{\text {active }}} \\
& \Delta \mathrm{I}_{\text {up,CaMK }}=\bar{\Delta} \mathrm{I}_{\mathrm{up}, \mathrm{CaMK}} \cdot \frac{\text { CaMK }_{\text {active }}}{\mathrm{K}_{\mathrm{m}, \mathrm{CaMK}}+\text { CaMK }_{\text {active }}} \\
& \mathrm{I}_{\mathrm{up}}=\overline{\mathrm{I}}_{\mathrm{up}} \cdot\left(1+\Delta \mathrm{I}_{\mathrm{up}, \mathrm{CaMK}}\right) \cdot \frac{\left[\mathrm{Ca}^{2+}\right]_{\mathrm{i}}}{\left[\mathrm{Ca}^{2+}\right]_{\mathrm{i}}+\mathrm{K}_{\mathrm{m}, \mathrm{up}}-\Delta \mathrm{K}_{\mathrm{m}, \mathrm{PLB}}}-\frac{\overline{\mathrm{I}}_{\mathrm{up}}}{\overline{\mathrm{NSR}}} \cdot\left[\mathrm{Ca}^{2+}\right]_{\mathrm{NSR}}
\end{aligned}
$$

\subsubsection{HRdßARS version (Chapters 3-6)}

Phospholamban (PLB), the inhibitor protein of the SR-Ca ${ }^{2+}$ uptake pump (SERCA2a) can be phosphorylated at Ser 10 by PKC, Ser 16 by PKA and at Thr 17 by CaMKII. Ser 10 phosphorylation is physiologically not relevant, but phosphorylation of Ser 16 and Thr 17 relieves PLB inhibition of SERCA2a, ${ }^{86}$ thereby increasing the affinity of SERCA2a to $\mathrm{Ca}^{2+87}$ and increasing the $\mathrm{Ca}^{2+}$ uptake into the SR and contributing to the lusitropic effect of $\beta A R$ stimulation. Phosphorylation of PLB by CaMKII was already incorporated in our baseline model, ${ }^{4,21}$ and we use the formulation presented there. We assume that phosphorylation of PLB by PKA and CaMKII occurs independently, as suggested by Hagemann et al. ${ }^{88}$ Other studies have claimed that Ser 16 phosphorylation by PKA is required for Thr 17 phosphorylation by CaMKII in situ. ${ }^{86}$ However, this could be because $\beta A R$ stimulation indirectly enhances the CaMKII signaling pathway through increased $\mathrm{Ca}^{2+}$-cycling, thereby making the extent of Thr 17 phosphorylation strongly influenced by Ser 16 phosphorylation. This is also suggested by the rightward shift in Thr 17 isoproterenol dose-response curve in comparison with Ser $16 .{ }^{89}$ The two 
separate phosphorylation processes result in 4 populations of channels: non-phosphorylated, phosphorylated by PKA only, phosphorylated by CaMKII only and phosphorylated by both kinases.

In addition, we have adapted the modulation of SERCA2a by CaMKII that was present in the baseline model $^{21}$ to enhance the frequency dependent increase in CaT decay. DeSantiago et al. showed that frequency dependent acceleration of relaxation (FDAR) and CaT decay are mediated by CaMKII but are independent of PLB. ${ }^{90}$ In the model, time constants of CaT decay are $188 \mathrm{~ms}$ and $239 \mathrm{~ms}$ at $\mathrm{CL}=$ $1000 \mathrm{~ms}$ and $\mathrm{CL}=6000 \mathrm{~ms}$, respectively, consistent with experimental observations in canine ventricular myocytes $(147 \pm 22 \mathrm{~ms}$ and $237 \pm 55 \mathrm{~ms}) .{ }^{91} \mathrm{FDAR}$, as quantified by the ratio of CaT decay time-constants at CLs of 500 and 5000 ms, is 0.56 in the model. This is qualitatively similar to the value of $0.36 \pm 0.03$ observed by DeSantiago et al. in rat ventricular myocytes, considering the well known differences in $\mathrm{Ca}^{2+}$ handling between rodents and large mammals. Consistent with the data of DeSantiago et al., FDAR is significantly reduced (ratio of 0.95) when CaMKII is inhibited. As in previous modeling studies, ${ }^{92}$ we combined the NSR leak flux $l_{\text {leak, }}$, which represents reverse mode SERCA2a, in the formulation of $I_{\text {up }}$.

$$
\begin{aligned}
& \mathrm{f}_{\mathrm{up}}^{\mathrm{PKA}}=\frac{\mathrm{f}_{\mathrm{PLB}}^{\mathrm{P}}-0.6591}{0.9945-0.6591} \\
& \mathrm{f}_{\mathrm{up}}^{\text {Both }}=\mathrm{f}_{\mathrm{up}}^{\mathrm{PKA}} \cdot \mathrm{f}_{\mathrm{PLB}}^{\mathrm{P}, \mathrm{CaMKII}} \\
& \mathrm{f}_{\text {up }}^{\text {PKA,only }}=\mathrm{f}_{\text {up }}^{\text {PKA }}-\mathrm{f}_{\text {up }}^{\text {Both }} \\
& \mathrm{f}_{\text {up }}^{\text {CaMKII,only }}=\mathrm{f}_{\text {PLB }}^{\text {P,CaMKII }}-\mathrm{f}_{\text {up }}^{\text {Both }} \\
& \mathrm{K}_{\mathrm{m} \text {,up }}^{\text {Baseline }}=9.2 \cdot 10^{-4} \mathrm{mmol} / \mathrm{L}, \quad \mathrm{K}_{\mathrm{m} \text {,up }}^{\mathrm{PKA}}=4.97 \cdot 10^{-4} \mathrm{mmol} / \mathrm{L}, \\
& \mathrm{K}_{\mathrm{m}, \mathrm{up}}^{\mathrm{CaMKI}}=7.5 \cdot 10^{-4} \mathrm{mmol} / \mathrm{L}, \quad \mathrm{K}_{\mathrm{m}, \mathrm{up}}^{\text {Both }}=4.97 \cdot 10^{-4} \mathrm{mmol} / \mathrm{L} \\
& \overline{\mathrm{I}}_{\mathrm{up}}=4.375 \cdot 10^{-3} \frac{\mathrm{mmol} / \mathrm{L}}{\mathrm{ms}}, \quad\left[\mathrm{Ca}^{2+}\right]_{\mathrm{NSR}, \mathrm{Max}}=15 \mathrm{mmol} / \mathrm{L} \\
& \mathrm{K}_{\mathrm{m}, \mathrm{up}}=\left(1-\mathrm{f}_{\text {up }}^{\text {PKA,only }}-\mathrm{f}_{\text {up }}^{\text {CaMKII,only }}-\mathrm{f}_{\text {up }}^{\text {Both }}\right) \cdot \mathrm{K}_{\mathrm{m}, \mathrm{up}}^{\text {Baseline }}+\mathrm{f}_{\text {up }}^{\text {PKA,only }} \cdot \mathrm{K}_{\mathrm{m}, \mathrm{up}}^{\text {PKA }}+\mathrm{f}_{\text {up }}^{\text {CaMKII,only }} \\
& \cdot \mathrm{K}_{\mathrm{m}, \mathrm{up}}^{\text {CaMKII }}+\mathrm{f}_{\mathrm{up}}^{\text {Both }} \cdot \mathrm{K}_{\mathrm{m}, \mathrm{up}}^{\text {Both }} \\
& \mathrm{f}_{\mathrm{SERCA} 2 \mathrm{a}}^{\mathrm{CaMKI}}=\frac{1}{1+\left(\frac{0.03}{\text { CaMK }_{\text {active }}}\right)^{2}} \\
& \mathrm{I}_{\text {up }}=\left(1+2.25 \cdot \mathrm{f}_{\mathrm{SERCA} 2 \mathrm{a}}^{\mathrm{CaMKII}}\right) \cdot \overline{\mathrm{I}}_{\mathrm{up}} \cdot\left(\frac{\left[\mathrm{Ca}^{2+}\right]_{\mathrm{i}}}{\mathrm{K}_{\mathrm{m}, \mathrm{up}}+\left[\mathrm{Ca}^{2+}\right]_{\mathrm{i}}}-\frac{\left[\mathrm{Ca}^{2+}\right]_{\mathrm{NSR}}}{\left[\mathrm{Ca}^{2+}\right]_{\mathrm{NSR}, \mathrm{Max}}}\right)
\end{aligned}
$$

\subsubsection{HRdßARS Stoch version (Chapter 7)}

The SR and sarcolemmal $\mathrm{Ca}^{2+}$-ATPases (underlying $\mathrm{I}_{\mathrm{up}}$ and $\mathrm{I}_{\mathrm{pCa}}$, respectively) were simulated using the two-state, four-transition model structure of Tran et al. ${ }^{31}$ Parameters of the models were adjusted to 
reproduce the $\mathrm{Ca}^{2+}$ dependence of the deterministic formulations (section 1.25.2). We assumed that the state distribution changes more rapidly than the changes in ionic concentrations on both sides of the pump, resulting in a quasi-equilibrium situation in which individual rates are decoupled. The number of ions transported via each transition was derived from a Binomial distribution and the net ion flux (or current) in the interval $\Delta \mathrm{t}$ was determined.

$\overline{\mathrm{N}}_{\mathrm{SERCA}}=2.20 \cdot 10^{6}$ pumps

$$
\begin{aligned}
& \mathrm{f}_{\mathrm{up}}^{\mathrm{NP}}=1-\mathrm{f}_{\mathrm{up}}^{\mathrm{PKA}, \text { only }}-\mathrm{f}_{\mathrm{up}}^{\mathrm{CaMKII}, \text { only }}-\mathrm{f}_{\mathrm{up}}^{\text {Both }} \\
& \mathrm{k}_{\mathrm{SERCA}, 1+}^{\mathrm{NP}}=4.6571 \cdot 10^{3} \cdot\left[\mathrm{Ca}^{2+}\right]_{\mathrm{i}} \cdot \frac{2.00 \cdot 10^{6}}{\overline{\mathrm{N}}_{\mathrm{SERCA}}}, \quad \mathrm{k}_{\mathrm{SERCA}, 1-}^{\mathrm{NP}}=1.1618 \cdot \frac{2.00 \cdot 10^{6}}{\overline{\mathrm{N}}_{\mathrm{SERCA}}} \\
& \mathrm{k}_{\mathrm{SERCA}, 2+}^{\mathrm{NP}}=0.7514 \cdot\left[\mathrm{Ca}^{2+}\right]_{\mathrm{NSR}} \cdot \frac{2.00 \cdot 10^{6}}{\overline{\mathrm{N}}_{\mathrm{SERCA}}}, \quad \mathrm{k}_{\mathrm{SERCA}, 2-}^{\mathrm{NP}}=2.5205 \cdot \frac{2.00 \cdot 10^{6}}{\overline{\mathrm{N}}_{\mathrm{SERCA}}} \\
& \mathrm{n}_{\mathrm{SERCA}}^{\mathrm{NP}}=\frac{\mathrm{k}_{\mathrm{SERCA}, 2+}^{\mathrm{NP}}+\mathrm{k}_{\mathrm{SERCA}, 2-}^{\mathrm{NP}}}{\mathrm{k}_{\mathrm{SERCA}, 1+}^{\mathrm{NP}}+\mathrm{k}_{\mathrm{SERCA}, 1-}^{\mathrm{NP}}+\mathrm{k}_{\mathrm{SERCA}, 2+}^{\mathrm{NP}}+\mathrm{k}_{\mathrm{SERCA}, 2-}^{\mathrm{NP}}} \cdot \mathrm{f}_{\mathrm{up}}^{\mathrm{NP}} \cdot \overline{\mathrm{N}}_{\mathrm{SERCA}} \\
& \mathrm{J}_{\mathrm{up}}^{\mathrm{NP}}=\mathrm{B}\left(\mathrm{n}_{\mathrm{SERCA}}^{\mathrm{NP}}, \mathrm{k}_{\mathrm{SERCA}, 1+}^{\mathrm{NP}} \cdot \Delta \mathrm{t}\right)-\mathrm{B}\left(\mathrm{f}_{\mathrm{up}}^{\mathrm{NP}} \cdot \overline{\mathrm{N}}_{\mathrm{SERCA}}-\mathrm{n}_{\mathrm{SERCA}}^{\mathrm{NP}}, \mathrm{k}_{\mathrm{SERCA}, 2+}^{\mathrm{NP}} \cdot \Delta \mathrm{t}\right)
\end{aligned}
$$

$$
\begin{aligned}
& \mathrm{k}_{\mathrm{SERCA}, 1+}^{\mathrm{P}}=7.5269 \cdot 10^{3} \cdot\left[\mathrm{Ca}^{2+}\right]_{\mathrm{i}} \cdot \frac{2.00 \cdot 10^{6}}{\overline{\mathrm{N}}_{\mathrm{SERCA}}}, \quad \mathrm{k}_{\mathrm{SERCA}, 1-}^{\mathrm{P}}=0.7188 \cdot \frac{2.00 \cdot 10^{6}}{\overline{\mathrm{N}}_{\mathrm{SERCA}}} \\
& \mathrm{k}_{\mathrm{SERCA}, 2+}^{\mathrm{P}}=0.8456 \cdot\left[\mathrm{Ca}^{2+}\right]_{\mathrm{NSR}} \cdot \frac{2.00 \cdot 10^{6}}{\overline{\mathrm{N}}_{\mathrm{SERCA}}}, \quad \mathrm{k}_{\mathrm{SERCA}, 2-}^{\mathrm{P}}=2.6131 \cdot \frac{2.00 \cdot 10^{6}}{\overline{\mathrm{N}}_{\mathrm{SERCA}}} \\
& \mathrm{n}_{\mathrm{SERCA}}^{\mathrm{P}}=\frac{\mathrm{k}_{\mathrm{SERCA}, 2+}^{\mathrm{P}}+\mathrm{k}_{\mathrm{SERCA}, 2-}^{\mathrm{P}}}{\mathrm{k}_{\mathrm{SERCA}, 1+}^{\mathrm{P}}+\mathrm{k}_{\mathrm{SERCA}, 1-}^{\mathrm{P}}+\mathrm{k}_{\mathrm{SERCA}, 2+}^{\mathrm{P}}+\mathrm{k}_{\mathrm{SERCA}, 2-}^{\mathrm{P}}} \cdot\left(\mathrm{f}_{\mathrm{up}}^{\mathrm{PKA}, \text { only }}+\mathrm{f}_{\mathrm{up}}^{\text {Both }}\right) \cdot \overline{\mathrm{N}}_{\text {SERCA }} \\
& \mathrm{J}_{\mathrm{up}}^{\mathrm{PKA}}=\mathrm{B}\left(\mathrm{n}_{\text {SERCA }}^{\mathrm{P}}, \mathrm{k}_{\text {SERCA }, 1+}^{\mathrm{P}} \cdot \Delta \mathrm{t}\right)-\mathrm{B}\left(\left(\mathrm{f}_{\text {up }}^{\mathrm{PKA}, \text { only }}+\mathrm{f}_{\mathrm{up}}^{\text {Both }}\right) \cdot \overline{\mathrm{N}}_{\text {SERCA }}-\mathrm{n}_{\text {SERCA }}^{\mathrm{P}}, \mathrm{k}_{\text {SERCA }, 2+}^{\mathrm{P}} \cdot \Delta \mathrm{t}\right)
\end{aligned}
$$

$$
\begin{aligned}
& \mathrm{k}_{\mathrm{SERCA}, 1+}^{\mathrm{CaMKIIP}}=5.6542 \cdot 10^{3} \cdot\left[\mathrm{Ca}^{2+}\right]_{\mathrm{i}} \cdot \frac{2.00 \cdot 10^{6}}{\overline{\mathrm{N}}_{\mathrm{SERCA}}}, \quad \mathrm{k}_{\mathrm{SERCA}, 1-}^{\mathrm{CaMKII}}=0.7009 \cdot \frac{2.00 \cdot 10^{6}}{\overline{\mathrm{N}}_{\mathrm{SERCA}}} \\
& \mathrm{k}_{\mathrm{SERCA}, 2+}^{\mathrm{CaMKIIP}}=1.1779 \cdot\left[\mathrm{Ca}^{2+}\right]_{\mathrm{NSR}} \cdot \frac{2.00 \cdot 10^{6}}{\overline{\mathrm{N}}_{\mathrm{SERCA}}}, \quad \mathrm{k}_{\mathrm{SERCA}, 2-}^{\mathrm{CaMKIP}}=2.6034 \cdot \frac{2.00 \cdot 10^{6}}{\overline{\mathrm{N}}_{\mathrm{SERCA}}} \\
& \mathrm{n}_{\text {SERCA }}^{\text {CaMKIIP }}=\frac{\mathrm{k}_{\text {SERCA,2+ }}^{\text {CaMKIIP }}+\mathrm{k}_{\text {SERCA,2- }}^{\text {CaMKIIP }}}{\mathrm{k}_{\text {SERCA,1+ }}^{\text {CaMKIIP }}+\mathrm{k}_{\text {SERCA,1- }}^{\text {CaMKIIP }}+\mathrm{k}_{\text {SERCA,2+ }}^{\text {CaMKIP }}+\mathrm{k}_{\text {SERCA }, 2-}^{\text {CaMKIP }}} \cdot \mathrm{f}_{\text {up }}^{\text {CaMKII,only }} \cdot \overline{\mathrm{N}}_{\text {SERCA }} \\
& \mathrm{J}_{\mathrm{up}}^{\text {CaMKII }}=\mathrm{B}\left(\mathrm{n}_{\mathrm{SERCA}}^{\text {CaMKIP }}, \mathrm{k}_{\mathrm{SERCA}, 1+}^{\text {CaMKIIP }} \cdot \Delta \mathrm{t}\right)-\mathrm{B}\left(\mathrm{f}_{\mathrm{up}}^{\text {CaMKII,only }} \cdot \overline{\mathrm{N}}_{\text {SERCA }}-\mathrm{n}_{\text {SERCA }}^{\text {CaMKIIP }}, \mathrm{k}_{\text {SERCA }, 2+}^{\text {CaMKIP }} \cdot \Delta \mathrm{t}\right) \\
& \mathrm{I}_{\mathrm{up}}=\left(1+2.25 \cdot \mathrm{f}_{\mathrm{SERCA2a}}^{\mathrm{CaMMII}}\right) \cdot \frac{\left(\mathrm{Jup}_{\mathrm{up}}^{\mathrm{NP}}+\mathrm{J}_{\mathrm{up}}^{\mathrm{PKA}}+\mathrm{J}_{\mathrm{up}}^{\mathrm{CaMKII}}\right) \cdot 10^{9}}{\Delta \mathrm{t} \cdot \mathrm{N}_{\mathrm{A}} \cdot \mathrm{V}_{\mathrm{NSR}}}
\end{aligned}
$$




\subsection{Membrane potential}

A detailed description of computational methods for strand simulations has been described previously. ${ }^{93}$ Briefly, propagation in a strand of serially connected HRd model cells was simulated via a finite difference approximation of the cable equation and no-flux boundary conditions were employed.

$$
\begin{gathered}
\mathrm{I}_{\text {ion }}=\mathrm{I}_{\mathrm{CaL}}+\mathrm{I}_{\mathrm{Cab}}+\mathrm{I}_{\mathrm{pCa}}+\mathrm{I}_{\mathrm{Cl}, \mathrm{b}}+\mathrm{I}_{\mathrm{Cl}(\mathrm{Ca})}+\mathrm{I}_{\mathrm{K} 1}+\mathrm{I}_{\mathrm{Kr}}+\mathrm{I}_{\mathrm{Ks}}+\mathrm{I}_{\mathrm{Kur}}+\mathrm{I}_{\mathrm{NaCa}}+\mathrm{I}_{\mathrm{NaCa}, \mathrm{ss}}+\mathrm{I}_{\mathrm{Na}}+\mathrm{I}_{\mathrm{NaK}} \\
+\mathrm{I}_{\mathrm{Nab}}+\mathrm{I}_{\mathrm{NaL}}+\mathrm{I}_{\mathrm{To}}
\end{gathered}
$$

For single cell simulations:

$\frac{\mathrm{dV}_{\mathrm{m}}}{\mathrm{dt}}=-\frac{1}{\mathrm{C}_{\mathrm{m}}} \cdot\left(\mathrm{I}_{\text {ion }}+\mathrm{I}_{\text {stim }}\right)$

For 1-dimensional strand simulations with spatial step-size $\Delta \mathrm{x}$ :

$$
\begin{aligned}
& \frac{d V_{m}}{d t}=\frac{1}{C_{m}} \cdot\left(\frac{\left(V_{m}^{i-1}-V_{m}^{i}\right)-\left(V_{m}^{i+1}-V_{m}^{i}\right)}{\Delta x^{2}} \cdot \frac{1000 \cdot r}{2 \cdot R_{C G} \cdot R_{i}}-\left(I_{\text {ion }}+I_{\text {stim }}\right)\right) \\
& \mathrm{R}_{\mathrm{i}}=\mathrm{R}_{\text {myo }}+\frac{\mathrm{R}_{\mathrm{g}}}{\Delta \mathrm{x}}, \quad \mathrm{R}_{\text {myo }}=150 \Omega \cdot \mathrm{cm}, \quad \mathrm{R}_{\mathrm{g}}=1.5 \Omega \cdot \mathrm{cm}^{2}
\end{aligned}
$$

\section{2. $\beta$-ADRENERGIC SIGNALING MODEL}

\subsection{General constants}

The tables in this section provide an overview of the parameters used in the $\beta A R$ signaling model. Parameters are taken directly from the cited literature (indicated by \#), converted from the cited literature (indicated by \&), result from a direct fit from the data in the cited literature (@) or result from a global fit to more macroscopic data (\%). Species specificity is indicated by (C) for dog, (R) for rat, (A) for Rabbit, (M) for mouse, (N) for expressions in Chinese hamster ovary cells, (E) for expressions in insect cells, $(G)$ for guinea pig and $(H)$ for human.

It should be noted that all rate constants and derivatives in the adrenergic signaling component of the model are given in $\mathrm{s}^{-1}$, whereas the electrophysiological model operates in $\mathrm{ms}^{-1}$. As such, derivatives of the signaling component have to be scaled by a factor 1000 when both components are implemented. 


\begin{tabular}{|cclc|}
\hline Parameter & Value & Description & Ref \\
\hline$[\mathrm{L}]$ & $0 \ldots 1 \mu \mathrm{mol} / \mathrm{L}$ & Concentration of isoproterenol & \\
\hline$[\mathrm{IBMX}]$ & $0 \ldots 100 \mu \mathrm{mol} / \mathrm{L}$ & Concentration of IBMX & $21,94^{\#}$ \\
\hline $\mathrm{V}^{\mathrm{CELL}}$ & $3.801 \mathrm{E}-5 \mu \mathrm{L}$ & Cell volume & $21^{\#}$ \\
\hline $\mathrm{V}^{\mathrm{CAV}}$ & $0.02 \cdot \mathrm{V}^{\mathrm{CELL}}$ & Caveolar volume & $94^{\text {\& }}$ \\
\hline $\mathrm{V}^{\mathrm{ECAV}}$ & $0.04 \cdot \mathrm{V}^{\mathrm{CELL}}$ & Extracaveolar volume & $21^{\#}$ \\
\hline $\mathrm{V}^{\mathrm{CYT}}$ & $0.678 \cdot \mathrm{V}^{\mathrm{CELL}}$ & Cytosolic volume & \\
\hline
\end{tabular}

\subsection{State vector}

\begin{tabular}{|c|c|c|c|}
\hline$\#$ & Variable & Description & Initial \\
\hline 1 & {$[\mathrm{Gs}]_{\alpha, \mathrm{GTP}}^{\mathrm{CAV}}$} & $\begin{array}{l}\text { Concentration of active } \mathrm{Gs}_{\alpha} \text { subunit in caveolar } \\
\text { compartment }(\mu \mathrm{mol} / \mathrm{L})\end{array}$ & $6.8504 \mathrm{E}-03$ \\
\hline 2 & {$[\mathrm{Gs}]_{\alpha, \mathrm{GTP}}^{\mathrm{ECAV}}$} & $\begin{array}{l}\text { Concentration of active } \mathrm{Gs}_{\alpha} \text { subunit in extracaveolar } \\
\text { compartment }(\mu \mathrm{mol} / \mathrm{L})\end{array}$ & $1.8463 \mathrm{E}-02$ \\
\hline 3 & {$[\mathrm{Gs}]_{\alpha, \mathrm{GTP}}^{\mathrm{CYT}}$} & $\begin{array}{l}\text { Concentration of active } \mathrm{Gs}_{\alpha} \text { subunit in cytoplasmic } \\
\text { compartment }(\mu \mathrm{mol} / \mathrm{L})\end{array}$ & $7.3142 \mathrm{E}-04$ \\
\hline 4 & {$[\mathrm{Gs}]_{\beta \gamma}^{\mathrm{CAV}}$} & $\begin{array}{l}\text { Concentration of } \mathrm{Gs}_{\beta \gamma} \text { subunit in caveolar compartment } \\
(\mu \mathrm{mol} / \mathrm{L})\end{array}$ & 7.4577E-03 \\
\hline 5 & {$[\mathrm{Gs}]_{\beta \gamma}^{\mathrm{ECAV}}$} & $\begin{array}{l}\text { Concentration of } \mathrm{Gs}_{\beta \gamma} \text { subunit in extracaveolar } \\
\text { compartment }(\mu \mathrm{mol} / \mathrm{L})\end{array}$ & $1.9102 \mathrm{E}-02$ \\
\hline 6 & {$[\mathrm{Gs}]_{\beta \gamma}^{\mathrm{CYT}}$} & $\begin{array}{l}\text { Concentration of } \mathrm{Gs}_{\beta \gamma} \text { subunit in cytoplasmic compartment } \\
(\mu \mathrm{mol} / \mathrm{L})\end{array}$ & $1.1514 \mathrm{E}-03$ \\
\hline 7 & {$[\mathrm{Gs}]_{\alpha, \mathrm{GDP}}^{\mathrm{CAV}}$} & $\begin{array}{l}\text { Concentration of inactive } \mathrm{Gs}_{\alpha} \text { subunit in caveolar } \\
\text { compartment }(\mu \mathrm{mol} / \mathrm{L})\end{array}$ & $6.0732 \mathrm{E}-04$ \\
\hline 8 & {$[\mathrm{Gs}]_{\alpha, G D P}^{\mathrm{ECAV}}$} & $\begin{array}{l}\text { Concentration of inactive } \mathrm{Gs}_{\alpha} \text { subunit in extracaveolar } \\
\text { compartment }(\mu \mathrm{mol} / \mathrm{L})\end{array}$ & $6.3904 \mathrm{E}-04$ \\
\hline 9 & {$[\mathrm{Gs}]_{\alpha, \mathrm{GDP}}^{\mathrm{CYT}}$} & $\begin{array}{l}\text { Concentration of inactive } \mathrm{Gs}_{\alpha} \text { subunit in cytoplasmic } \\
\text { compartment }(\mu \mathrm{mol} / \mathrm{L})\end{array}$ & 4.1999E-04 \\
\hline 10 & {$[\mathrm{Gi}]_{\alpha, \mathrm{GTP}}^{\mathrm{CAV}}$} & $\begin{array}{l}\text { Concentration of active } \mathrm{Gi}_{\alpha} \text { subunit in caveolar } \\
\text { compartment }(\mu \mathrm{mol} / \mathrm{L})\end{array}$ & $1.5963 \mathrm{E}-03$ \\
\hline 11 & {$[\mathrm{Gi}]_{\alpha, \mathrm{GTP}}^{\mathrm{ECAV}}$} & $\begin{array}{l}\text { Concentration of active } \mathrm{Gi}_{\alpha} \text { subunit in extracaveolar } \\
\text { compartment }(\mu \mathrm{mol} / \mathrm{L})\end{array}$ & $3.6432 \mathrm{E}-04$ \\
\hline 12 & {$[\mathrm{Gi}]_{\beta \gamma}^{\mathrm{CAV}}$} & $\begin{array}{l}\text { Concentration of active } \mathrm{Gi}_{\beta \gamma} \text { subunit in caveolar } \\
\text { compartment }(\mu \mathrm{mol} / \mathrm{L})\end{array}$ & $2.0991 \mathrm{E}-03$ \\
\hline 13 & {$[\mathrm{Gi}]_{\beta \gamma}^{\mathrm{ECAV}}$} & $\begin{array}{l}\text { Concentration of active } \mathrm{Gi}_{\beta \gamma} \text { subunit in extracaveolar } \\
\text { compartment }(\mu \mathrm{mol} / \mathrm{L})\end{array}$ & 7.0566E-04 \\
\hline 14 & {$[\mathrm{Gi}]_{\alpha, \mathrm{GDP}}^{\mathrm{CAV}}$} & $\begin{array}{l}\text { Concentration of inactive } \mathrm{Gi}_{\alpha} \text { subunit in caveolar } \\
\text { compartment }(\mu \mathrm{mol} / \mathrm{L})\end{array}$ & $5.0279 \mathrm{E}-04$ \\
\hline 15 & {$[\mathrm{Gi}]_{\alpha, \mathrm{GDP}}^{\mathrm{ECAV}}$} & $\begin{array}{l}\text { Concentration of inactive } \mathrm{Gi}_{\alpha} \text { subunit in extracaveolar } \\
\text { compartment }(\mu \mathrm{mol} / \mathrm{L})\end{array}$ & $3.4134 \mathrm{E}-04$ \\
\hline 16 & {$[\mathrm{cAMP}]^{\mathrm{CAV}}$} & Concentration of cAMP in caveolar compartment $(\mu \mathrm{mol} / \mathrm{L})$ & $3.4710 \mathrm{E}-01$ \\
\hline 17 & {$[\mathrm{CAMP}]^{\mathrm{ECAV}}$} & $\begin{array}{l}\text { Concentration of cAMP in extracaveolar compartment } \\
(\mu \mathrm{mol} / \mathrm{L})\end{array}$ & $9.6236 \mathrm{E}+00$ \\
\hline
\end{tabular}




\begin{tabular}{|c|c|c|c|}
\hline 18 & {$[\mathrm{cAMP}]^{\mathrm{CYT}}$} & Concentration of cAMP in cytosolic compartment $(\mu \mathrm{mol} / \mathrm{L})$ & $4.7408 \mathrm{E}-01$ \\
\hline 19 & {$\left[\mathrm{R}_{\beta 1}\right]_{\mathrm{PKA}, \text { tot }}^{\mathrm{CAV}}$} & $\begin{array}{l}\text { Concentration of total PKA-phosphorylated } \beta 1 \text { receptors } \\
\text { (caveolar; } \mu \mathrm{mol} / \mathrm{L} \text { ) }\end{array}$ & 1.4904E-02 \\
\hline 20 & {$\left[\mathrm{R}_{\beta 1}\right]_{\text {PKA,tot }}^{\mathrm{ECAV}}$} & $\begin{array}{l}\text { Concentration of total PKA-phosphorylated } \beta 1 \text { receptors } \\
\text { (extracaveolar; } \mu \mathrm{mol} / \mathrm{L} \text { ) }\end{array}$ & $2.0302 \mathrm{E}-02$ \\
\hline 21 & {$\left[\mathrm{R}_{\beta 1}\right]_{\mathrm{PKA}, \mathrm{tot}}^{\mathrm{CYT}}$} & $\begin{array}{l}\text { Concentration of total PKA-phosphorylated receptors } \\
\text { (cytoplasmic; } \mu \mathrm{mol} / \mathrm{L} \text { ) }\end{array}$ & $9.4446 \mathrm{E}-03$ \\
\hline 22 & {$\left[\mathrm{R}_{\beta 1}\right]_{\mathrm{GRK}, \mathrm{tot}}^{\mathrm{CAV}}$} & $\begin{array}{l}\text { Concentration of total GRK-phosphorylated receptors } \\
\text { (caveolar; } \mu \mathrm{mol} / \mathrm{L} \text { ) }\end{array}$ & $0.0000 E+00$ \\
\hline 23 & {$\left[\mathrm{R}_{\beta 1}\right]_{\mathrm{GRK}, \text { tot }}^{\mathrm{ECAV}}$} & $\begin{array}{l}\text { Concentration of total GRK-phosphorylated receptors } \\
\text { (extracaveolar; } \mu \mathrm{mol} / \mathrm{L} \text { ) }\end{array}$ & $0.0000 \mathrm{E}+00$ \\
\hline 24 & {$\left[\mathrm{R}_{\beta 1}\right]_{\mathrm{GRK}, \mathrm{tot}}^{\mathrm{CYT}}$} & $\begin{array}{l}\text { Concentration of total GRK-phosphorylated receptors } \\
\text { (cytoplasmic; } \mu \mathrm{mol} / \mathrm{L} \text { ) }\end{array}$ & $0.0000 \mathrm{E}+00$ \\
\hline 25 & {$\left[\mathrm{R}_{\beta 2}\right]_{\mathrm{PKA}, \mathrm{tot}}^{\mathrm{CAV}}$} & $\begin{array}{l}\text { Concentration of total PKA-phosphorylated } \beta 2 \text { receptors } \\
\text { (caveolar; } \mu \mathrm{mol} / \mathrm{L} \text { ) }\end{array}$ & $2.7546 \mathrm{E}-02$ \\
\hline 26 & {$\left[\mathrm{R}_{\beta 2}\right]_{\mathrm{PKA}, \mathrm{tot}}^{\mathrm{ECAV}}$} & $\begin{array}{l}\text { Concentration of total PKA-phosphorylated } \beta 2 \text { receptors } \\
\text { (extracaveolar; } \mu \mathrm{mol} / \mathrm{L} \text { ) }\end{array}$ & $1.1025 \mathrm{E}-02$ \\
\hline 27 & {$\left[\mathrm{R}_{\beta 2}\right]_{\mathrm{GRK}, \mathrm{tot}}^{\mathrm{CAV}}$} & $\begin{array}{l}\text { Concentration of total GRK-phosphorylated } \beta 2 \text { receptors } \\
\text { (caveolar; } \mu \mathrm{mol} / \mathrm{L} \text { ) }\end{array}$ & $0.0000 E+00$ \\
\hline 28 & {$\left[\mathrm{R}_{\beta 2}\right]_{\mathrm{GRK}, \mathrm{tot}}^{\mathrm{ECAV}}$} & $\begin{array}{l}\text { Concentration of total GRK-phosphorylated } \beta 2 \text { receptors } \\
\text { (extracaveolar; } \mu \mathrm{mol} / \mathrm{L} \text { ) }\end{array}$ & $0.0000 E+00$ \\
\hline 29 & {$[\mathrm{ARC}]^{\mathrm{CAV}}$} & $\begin{array}{l}\text { Concentration of PKA RC dimer with } 1 \text { cAMP molecule } \\
\text { bound (caveolar; } \mu \mathrm{mol} / \mathrm{L} \text { ) }\end{array}$ & $9.0480 \mathrm{E}-02$ \\
\hline 30 & {$\left[\mathrm{~A}_{2} \mathrm{RC}\right]^{\mathrm{CAV}}$} & $\begin{array}{l}\text { Concentration of PKA RC dimer with } 2 \text { cAMP molecules } \\
\text { bound (caveolar; } \mu \mathrm{mol} / \mathrm{L} \text { ) }\end{array}$ & 2.7649E-03 \\
\hline 31 & {$\left[\mathrm{~A}_{2} \mathrm{R}\right]^{\mathrm{CAV}}$} & $\begin{array}{l}\text { Concentration of PKA R subunit with } 2 \text { cAMP molecules } \\
\text { bound (caveolar; } \mu \mathrm{mol} / \mathrm{L} \text { ) }\end{array}$ & $2.2548 \mathrm{E}-01$ \\
\hline 32 & {$[\mathrm{C}]^{\mathrm{CAV}}$} & $\begin{array}{l}\text { Concentration of free PKA catalytic subunit (caveolar; } \\
\mu \mathrm{mol} / \mathrm{L} \text { ) }\end{array}$ & 3.2657E-02 \\
\hline 33 & {$[\mathrm{PKIC}]^{\mathrm{CAV}}$} & $\begin{array}{l}\text { Concentration of PKI inactivated PKA C subunit (caveolar; } \\
\mu \mathrm{mol} / \mathrm{L} \text { ) }\end{array}$ & $1.9282 \mathrm{E}-01$ \\
\hline 34 & {$[\mathrm{ARC}]^{\mathrm{ECAV}}$} & $\begin{array}{l}\text { Concentration of PKA RC dimer with } 1 \text { cAMP molecule } \\
\text { bound (extracaveolar; } \mu \mathrm{mol} / \mathrm{L} \text { ) }\end{array}$ & 2.0544E-01 \\
\hline 35 & {$\left[\mathrm{~A}_{2} \mathrm{RC}\right]^{\mathrm{ECAV}}$} & $\begin{array}{l}\text { Concentration of PKA RC dimer with } 2 \text { cAMP molecules } \\
\text { bound (extracaveolar; } \mu \mathrm{mol} / \mathrm{L} \text { ) }\end{array}$ & $1.7406 \mathrm{E}-01$ \\
\hline & {$\left[\mathrm{A}_{2} \mathrm{R}\right]^{\mathrm{ECAV}}$} & $\begin{array}{l}\text { Concentration of PKA R subunit with } 2 \text { cAMP molecules } \\
\text { bound (extracaveolar; } \mu \mathrm{mol} / \mathrm{L} \text { ) }\end{array}$ & $8.1716 \mathrm{E}-01$ \\
\hline 37 & {$[\mathrm{C}]^{\mathrm{ECAV}}$} & $\begin{array}{l}\text { Concentration of free PKA catalytic subunit (extracaveolar; } \\
\mu \mathrm{mol} / \mathrm{L} \text { ) }\end{array}$ & $5.6725 \mathrm{E}-01$ \\
\hline 38 & {$[\mathrm{PKIC}]^{\mathrm{ECAV}}$} & $\begin{array}{l}\text { Concentration of PKI inactivated PKA C subunit } \\
\text { (extracaveolar) }\end{array}$ & $2.4991 \mathrm{E}-01$ \\
\hline 39 & {$[\mathrm{ARC}]^{\mathrm{CYT}}$} & $\begin{array}{l}\text { Concentration of PKA RC dimer with } 1 \text { cAMP molecule } \\
\text { bound (cytoplasmic; } \mu \mathrm{mol} / \mathrm{L} \text { ) }\end{array}$ & $6.4693 \mathrm{E}-02$ \\
\hline & {$\left[\mathrm{A}_{2} \mathrm{RC}\right]^{\mathrm{CYT}}$} & $\begin{array}{l}\text { Concentration of PKA RC dimer with } 2 \text { cAMP molecules } \\
\text { bound (cytoplasmic; } \mu \mathrm{mol} / \mathrm{L} \text { ) }\end{array}$ & $6.6500 \mathrm{E}-02$ \\
\hline 41 & {$\left[\mathrm{~A}_{2} \mathrm{R}\right]^{\mathrm{CYT}}$} & $\begin{array}{l}\text { Concentration of PKA R subunit with } 2 \text { cAMP molecules } \\
\text { bound (cytoplasmic; } \mu \mathrm{mol} / \mathrm{L} \text { ) }\end{array}$ & 4.8906E-01 \\
\hline 42 & {$[\mathrm{C}]^{\mathrm{CYT}}$} & $\begin{array}{l}\text { Concentration of free PKA catalytic subunit (cytoplasmic; } \\
\mu \mathrm{mol} / \mathrm{L} \text { ) }\end{array}$ & $3.6211 \mathrm{E}-01$ \\
\hline
\end{tabular}




\begin{tabular}{|c|c|c|c|}
\hline 43 & {$[\mathrm{PKIC}]^{\mathrm{CYT}}$} & $\begin{array}{l}\text { Concentration of PKI inactivated PKA C subunit } \\
\text { (cytoplasmic; } \mu \mathrm{mol} / \mathrm{L} \text { ) }\end{array}$ & $1.2695 \mathrm{E}-01$ \\
\hline 44 & {$[\mathrm{PDE} 3]_{\mathrm{P}}^{\mathrm{CAV}}$} & Concentration of phosphorylated PDE3 (caveolar; $\mu \mathrm{mol} / \mathrm{L}$ ) & $2.3692 \mathrm{E}-02$ \\
\hline 45 & {$[\mathrm{PDE} 3]_{\mathrm{P}}^{\mathrm{CYT}}$} & $\begin{array}{l}\text { Concentration of phosphorylated PDE3 (cytoplasmic; } \\
\mu \mathrm{mol} / \mathrm{L} \text { ) }\end{array}$ & $1.2840 \mathrm{E}-02$ \\
\hline 46 & {$[\mathrm{PDE} 4]_{\mathrm{P}}^{\mathrm{CAV}}$} & Concentration of phosphorylated PDE4 (caveolar; $\mu \mathrm{mol} / \mathrm{L}$ ) & $6.3736 \mathrm{E}-03$ \\
\hline 47 & {$[\mathrm{PDE} 4]_{\mathrm{P}}^{\mathrm{ECAV}}$} & $\begin{array}{l}\text { Concentration of phosphorylated PDE4 (extracaveolar; } \\
\mu \mathrm{mol} / \mathrm{L} \text { ) }\end{array}$ & 4.2917E-05 \\
\hline 48 & {$[\mathrm{PDE} 4]_{\mathrm{P}}^{\mathrm{CYT}}$} & $\begin{array}{l}\text { Concentration of phosphorylated PDE4 (cytoplasmic; } \\
\mu \mathrm{mol} / \mathrm{L} \text { ) }\end{array}$ & $9.1704 \mathrm{E}-03$ \\
\hline 49 & {$[\text { Inhib1 }]_{\mathrm{P}}^{\mathrm{CYT}}$} & $\begin{array}{l}\text { Concentration of phosphorylated PP1 Inhibitor } 1 \\
\text { (cytoplasmic; } \mu \mathrm{mol} / \mathrm{L} \text { ) }\end{array}$ & $2.8266 \mathrm{E}-02$ \\
\hline 50 & {$[\mathrm{ICaL}]^{\mathrm{P}}$} & Concentration of phosphorylated L-type $\mathrm{Ca}^{2+}$ channels & $6.7371 \mathrm{E}-04$ \\
\hline 51 & {$[\mathrm{IKs}]^{\mathrm{P}}$} & Concentration of phosphorylated $I_{\mathrm{Ks}}$ channels & 7.6599E-04 \\
\hline 52 & $\mathrm{f}_{\mathrm{PLB}}^{\mathrm{p}}$ & Fraction of phosphorylated PLB & 5.9217E-01 \\
\hline 53 & $\mathrm{f}_{\mathrm{TnI}}^{\mathrm{p}}$ & Fraction of phosphorylated Tnl & $6.7351 \mathrm{E}-01$ \\
\hline 54 & $\mathrm{f}_{\mathrm{INa}}^{\mathrm{p}}$ & Fraction of phosphorylated $I_{\mathrm{Na}}$ channels & $2.3948 \mathrm{E}-01$ \\
\hline 55 & $\mathrm{f}_{\mathrm{INaK}}^{\mathrm{p}}$ & Fraction of phosphorylated $I_{\mathrm{NaK}}$ & $1.2635 \mathrm{E}-01$ \\
\hline 56 & {$[\mathrm{RyR}]^{\mathrm{P}}$} & Concentration of phosphorylated RyR & $4.1069 \mathrm{E}-03$ \\
\hline 57 & $\mathrm{f}_{\mathrm{IKur}}^{\mathrm{p}}$ & Fraction of phosphorylated $I_{\text {Kur }}$ & $5.8938 \mathrm{E}-02$ \\
\hline 58 & $\mathrm{f}_{\mathrm{ICaL}}^{\mathrm{P}, \mathrm{CaMKII}}$ & Fraction of CaMKII-phosphorylated $I_{\text {CaL }}$ & $0.0000 E+00$ \\
\hline 59 & $\mathrm{f}_{\mathrm{PLB}}^{\mathrm{P}, \mathrm{CaMKII}}$ & Fraction of CaMKII-phosphorylated PLB & $0.0000 E+00$ \\
\hline 60 & $\mathrm{f}_{\mathrm{INa}}^{\mathrm{P}, \mathrm{CaMKII}}$ & Fraction of CaMKII-phosphorylated I $\mathrm{Na}$ & $0.0000 E+00$ \\
\hline 61 & $\mathrm{f}_{\text {RyR }}^{\text {P,CaMKII }}$ & Fraction of CaMKII-phosphorylated RyR & $0.0000 E+00$ \\
\hline 62 & $\mathrm{f}_{\text {ITo }}^{\text {P,CaMKII }}$ & Fraction of CaMKII-phosphorylated $\mathrm{I}_{\text {To }}$ & $0.0000 E+00$ \\
\hline 63 & $\mathrm{f}_{\mathrm{IK} 1}^{\mathrm{P}, \mathrm{CaMKII}}$ & Fraction of CaMKII-phosphorylated $I_{\mathrm{K} 1}$ & $0.0000 E+00$ \\
\hline
\end{tabular}

\subsection{Adrenergic receptor and G protein activation}

Both $\beta 1$ and $\beta 2$ adrenergic receptor isoforms are incorporated in the model. The former make up $80 \%$ of the $\beta A R$ in healthy ventricular myocytes and are distributed throughout the whole cell. ${ }^{95,96}$ In contrast, $\beta 2 A R$ are highly localized in the CAV domain. ${ }^{97} \beta 2 A R$ in the model have higher affinity for isoproterenol, consistent with experimental observations. ${ }^{98}$ Upon phosphorylation by PKA, $\beta 2 A R$ switch from predominantly Gs-coupled to Gi-coupled, thereby providing an attenuating signal during intense $\beta A R$ stimulation. ${ }^{99}$ The exact mechanism by which the Gi pathway localizes the effects of $\beta 2 A R$ stimulation is not known. Jo et al. ${ }^{100}$ have shown that the $G_{\beta \gamma}$ subunits of $G i$ and phosphatidylinositol 3 kinase play a major role in limiting the effect of $\beta 2 A R$ stimulation on PLB phosphorylation in adult rat ventricular myocytes. The exact link between phosphatidylinositol 3 
kinase and PLB phosphorylation, however is unknown. On the other hand the main adenylyl cyclase (AC) isoform in the heart is known to be inhibited by Gi. In line with the adenylyl cyclase mediated mechanism, Zama et al. ${ }^{99}$ have shown that pertussis toxin (PTX) mediated inhibition of Gi results in enhanced AC activity in pseudo- phosphorylated $\beta 2 A R$. Because of the incomplete understanding of the phosphatidylinositol 3 kinase pathway, we have currently only modeled the effect of $\beta 2 A R-$ mediated Gi activation on AC.

The complex formation between receptor, G-protein (Gs for $\beta 1 A R$, either Gs or Gi for $\beta 2 A R$ ) and ligand is assumed to be very fast ${ }^{94,101}$ and hence is given by algebraic, steady-state equations. This requires finding the unique nonnegative real root of a second order (for CYT and Gi binding to $\beta 2 A R$ in CAV and ECAV) or third order ( $\beta 1 A R / \beta 2 A R / G s / L i g a n d$ binding in CAV and ECAV) polynomial to determine the distribution of free and complexed proteins. For both receptor isoforms, desensitization due to PKA phosphorylation is independent of ligand and/or G-protein binding, whereas desensitization through GRK only affects the ligand bound states. ${ }^{101,}{ }^{102}$ G-proteins are activated through a slow process by the RG complex and a fast process by the LRG complex. Inhibition of either $\beta 1 A R$ or $\beta 2 A R$ is simulated by reducing the active fraction of the LRG complex of each respective receptor isoform.

\section{For $x \in\{C A V, E C A V\}:$}

$\left[\mathrm{R}_{\beta 1}\right]_{\text {tot }}^{x}=\mathrm{f}_{\mathrm{R}, \beta 1}^{\mathrm{X}} \cdot\left[\mathrm{R}_{\beta 1}\right]_{\mathrm{tot}} \cdot \frac{\mathrm{V}^{\mathrm{CELL}}}{\mathrm{V}^{\mathrm{x}}}$

$\left[\mathrm{R}_{\beta 2}\right]_{\text {tot }}^{x}=\mathrm{f}_{\mathrm{R}, \beta 2}^{\mathrm{x}} \cdot\left[\mathrm{R}_{\beta 2}\right]_{\mathrm{tot}} \cdot \frac{\mathrm{V}^{\mathrm{CELL}}}{\mathrm{V}^{\mathrm{x}}}$

$[\mathrm{Gs}]_{\alpha \beta \gamma}^{\mathrm{x}}=\mathrm{f}_{\mathrm{Gs}}^{\mathrm{x}} \cdot[\mathrm{Gs}]_{\mathrm{tot}} \cdot \frac{\mathrm{V}^{\mathrm{CELL}}}{\mathrm{V}^{\mathrm{x}}}-[\mathrm{Gs}]_{\alpha, \mathrm{GTP}}^{\mathrm{x}}-[\mathrm{Gs}]_{\alpha, \mathrm{GDP}}^{\mathrm{x}}$

$[\mathrm{Gi}]_{\alpha \beta \gamma}^{\mathrm{x}}=\mathrm{f}_{\mathrm{Gi}}^{\mathrm{x}} \cdot[\mathrm{Gi}]_{\mathrm{tot}} \cdot \frac{\mathrm{V}^{\mathrm{CELL}}}{\mathrm{V}^{\mathrm{x}}}-[\mathrm{Gi}]_{\alpha, \mathrm{GTP}}^{\mathrm{x}}-[\mathrm{Gi}]_{\alpha, \mathrm{GDP}}^{\mathrm{x}}$

$\left[\mathrm{R}_{\beta 1}\right]_{\mathrm{n} \text {,tot }}^{\mathrm{X}}=\left[\mathrm{R}_{\beta 1}\right]_{\text {tot }}^{\mathrm{X}}-\left[\mathrm{R}_{\beta 1}\right]_{\mathrm{PKA}, \text { tot }}^{\mathrm{X}}-\left[\mathrm{R}_{\beta 1}\right]_{\mathrm{GRK}, \text { tot }}^{\mathrm{X}}$

$\left[\mathrm{R}_{\beta 2}\right]_{\text {np,tot }}^{\mathrm{X}}=\left[\mathrm{R}_{\beta 2}\right]_{\text {tot }}^{\mathrm{X}}-\left[\mathrm{R}_{\beta 2}\right]_{\mathrm{PKA}, \text { tot }}^{\mathrm{X}}-\left[\mathrm{R}_{\beta 2}\right]_{\mathrm{GRK}, \text { tot }}^{\mathrm{X}}$

$$
\begin{aligned}
\mathrm{a}_{\mathrm{LRGi}} & =\frac{1}{\mathrm{~K}_{\beta 2, \mathrm{~N}}} \cdot\left(\mathrm{K}_{\beta 2, \mathrm{~F}}+[\mathrm{L}]\right) \cdot\left(\mathrm{K}_{\beta 2, \mathrm{~N}}+[\mathrm{L}]\right) \\
\mathrm{b}_{\mathrm{LRGi}} & =[\mathrm{Gi}]_{\alpha \beta \gamma}^{\mathrm{x}} \cdot\left(\mathrm{L}+\mathrm{K}_{\beta 2, \mathrm{~F}}\right)-\left(\mathrm{K}_{\beta 2, \mathrm{~F}}+[\mathrm{L}]\right) \cdot\left[\mathrm{R}_{\beta 2}\right]_{\mathrm{PKA,tot}}^{\mathrm{x}}+\mathrm{K}_{\beta 2, \mathrm{~A}} \cdot \mathrm{K}_{\beta 2, \mathrm{~F}}+\frac{\mathrm{K}_{\beta 2, \mathrm{~A}} \cdot \mathrm{K}_{\beta 2, \mathrm{~F}} \cdot[\mathrm{L}]}{\mathrm{K}_{\beta 2, \mathrm{~N}}} \\
\mathrm{c}_{\mathrm{LRGi}} & =-\left[\mathrm{R}_{\beta 2}\right]_{\mathrm{PKA}, \mathrm{tot}}^{\mathrm{x}} \cdot \mathrm{K}_{\beta 2, \mathrm{~A}} \cdot \mathrm{K}_{\beta 2, \mathrm{~F}} \\
{\left[\mathrm{R}_{\beta 2}\right]_{\mathrm{PKA}, \mathrm{f}}^{\mathrm{x}} } & =\frac{-\mathrm{b}_{\mathrm{LRGi}}+\sqrt{\mathrm{b}_{\mathrm{LRGi}}^{2}-4 \cdot \mathrm{a}_{\mathrm{LRGi}} \cdot \mathrm{c}_{\mathrm{LRGi}}}}{2 \cdot \mathrm{a}_{\mathrm{LRGi}}}
\end{aligned}
$$




$$
\begin{aligned}
& {[\mathrm{Gi}]_{\mathrm{f}}^{\mathrm{x}}=\frac{[\mathrm{Gi}]_{\alpha \beta \gamma}^{\mathrm{X}}}{1+\frac{\left[\mathrm{R}_{\beta 2}\right]_{\mathrm{PKA}, \mathrm{f}}^{\mathrm{x}}}{\mathrm{K}_{\beta 2, \mathrm{~A}}}+\frac{[\mathrm{L}] \cdot\left[\mathrm{R}_{\beta 2}\right]_{\mathrm{PKA}, \mathrm{f}}^{\mathrm{X}}}{\mathrm{K}_{\beta 2, \mathrm{~A}} \cdot \mathrm{K}_{\beta 2, \mathrm{~F}}}}} \\
& {\left[\mathrm{R}_{\beta 2} \mathrm{Gi}\right]_{\mathrm{PKA}}^{\mathrm{X}}=\frac{\left[\mathrm{R}_{\beta 2}\right]_{\mathrm{PKA}, \mathrm{f}}^{\mathrm{X}} \cdot[\mathrm{Gi}]_{\mathrm{f}}^{\mathrm{X}}}{\mathrm{K}_{\beta 2, \mathrm{~A}}^{\mathrm{X}}}} \\
& {\left[\mathrm{LR}_{\beta 2} \mathrm{Gi}\right]_{\mathrm{PKA}}^{\mathrm{X}}=\frac{[\mathrm{L}] \cdot\left[\mathrm{R}_{\beta 2}\right]_{\mathrm{PKA}, \mathrm{f}}^{\mathrm{X}} \cdot[\mathrm{Gi}]_{\mathrm{f}}^{\mathrm{X}}}{\mathrm{K}_{\beta 2, \mathrm{~A}} \cdot \mathrm{K}_{\beta 2, \mathrm{~F}}}} \\
& \mathrm{~b}_{\mathrm{LRG}}=\left[\mathrm{R}_{\beta 1}\right]_{\mathrm{np}, \mathrm{tot}}^{\mathrm{x}} \\
& +\left(\mathrm{K}_{\beta 1, \mathrm{C}} \cdot \mathrm{K}_{\beta 1, \mathrm{H}} \cdot \mathrm{K}_{\beta 2, \mathrm{~L}} \cdot[\mathrm{L}]^{2}-[\mathrm{Gs}]_{\alpha \beta \gamma}^{\mathrm{X}}\right. \\
& \cdot\left(\mathrm{K}_{\beta 1, \mathrm{~L}} \cdot \mathrm{K}_{\beta 2, \mathrm{~L}} \cdot[\mathrm{L}]^{2}+\mathrm{K}_{\beta 1, \mathrm{H}} \cdot \mathrm{K}_{\beta 2, \mathrm{H}} \cdot \mathrm{K}_{\beta 1, \mathrm{~L}} \cdot \mathrm{K}_{\beta 2, \mathrm{~L}}+\mathrm{K}_{\beta 1, \mathrm{H}} \cdot \mathrm{K}_{\beta 1, \mathrm{~L}} \cdot \mathrm{K}_{\beta 2, \mathrm{~L}} \cdot[\mathrm{L}]\right. \\
& \left.+\mathrm{K}_{\beta 2, \mathrm{H}} \cdot \mathrm{K}_{\beta 1, \mathrm{~L}} \cdot \mathrm{K}_{\beta 2, \mathrm{~L}} \cdot[\mathrm{L}]\right)+\mathrm{K}_{\beta 2, \mathrm{C}} \cdot \mathrm{K}_{\beta 2, \mathrm{H}} \cdot \mathrm{K}_{\beta 1, \mathrm{~L}} \cdot[\mathrm{L}]^{2}+\mathrm{K}_{\beta 1, \mathrm{~L}} \cdot \mathrm{K}_{\beta 2, \mathrm{~L}} \cdot[\mathrm{L}]^{2} \\
& \cdot\left[\mathrm{R}_{\beta 2}\right]_{\mathrm{np}, \mathrm{tot}}^{\mathrm{X}}+\mathrm{K}_{\beta 1, \mathrm{C}} \cdot \mathrm{K}_{\beta 1, \mathrm{H}} \cdot \mathrm{K}_{\beta 1, \mathrm{~L}} \cdot \mathrm{K}_{\beta 2, \mathrm{H}} \cdot \mathrm{K}_{\beta 2, \mathrm{~L}}+\mathrm{K}_{\beta 1, \mathrm{H}} \cdot \mathrm{K}_{\beta 1, \mathrm{~L}} \cdot \mathrm{K}_{\beta 2, \mathrm{C}} \cdot \mathrm{K}_{\beta 2, \mathrm{H}} \\
& \cdot \mathrm{K}_{\beta 2, \mathrm{~L}}+\mathrm{K}_{\beta 1, \mathrm{C}} \cdot \mathrm{K}_{\beta 1, \mathrm{H}} \cdot \mathrm{K}_{\beta 2, \mathrm{H}} \cdot \mathrm{K}_{\beta 2, \mathrm{~L}} \cdot[\mathrm{L}]+\mathrm{K}_{\beta 1, \mathrm{H}} \cdot \mathrm{K}_{\beta 1, \mathrm{~L}} \cdot \mathrm{K}_{\beta 2, \mathrm{C}} \cdot \mathrm{K}_{\beta 2, \mathrm{H}} \cdot[\mathrm{L}] \\
& +\mathrm{K}_{\beta 1, \mathrm{C}} \cdot \mathrm{K}_{\beta 1, \mathrm{~L}} \cdot \mathrm{K}_{\beta 2, \mathrm{H}} \cdot \mathrm{K}_{\beta 2, \mathrm{~L}} \cdot[\mathrm{L}]+\mathrm{K}_{\beta 1, \mathrm{~L}} \cdot \mathrm{K}_{\beta 2, \mathrm{C}} \cdot \mathrm{K}_{\beta 2, \mathrm{H}} \cdot \mathrm{K}_{\beta 2, \mathrm{~L}} \cdot[\mathrm{L}]+\left[\mathrm{R}_{\beta 2}\right]_{\text {np,tot }}^{\mathrm{X}} \\
& \cdot\left(\mathrm{K}_{\beta 1, \mathrm{H}} \cdot \mathrm{K}_{\beta 1, \mathrm{~L}} \cdot \mathrm{K}_{\beta 2, \mathrm{H}} \cdot \mathrm{K}_{\beta 2, \mathrm{~L}}+\mathrm{K}_{\beta 1, \mathrm{H}} \cdot \mathrm{K}_{\beta 1, \mathrm{~L}} \cdot \mathrm{K}_{\beta 2, \mathrm{~L}} \cdot[\mathrm{L}]+\mathrm{K}_{\beta 1, \mathrm{~L}} \cdot \mathrm{K}_{\beta 2, \mathrm{H}} \cdot \mathrm{K}_{\beta 2, \mathrm{~L}}\right. \\
& \cdot[\mathrm{L}])) \\
& /\left(\mathrm{K}_{\beta 1, \mathrm{~L}} \cdot \mathrm{K}_{\beta 2, \mathrm{~L}} \cdot[\mathrm{L}]^{2}+\mathrm{K}_{\beta 1, \mathrm{H}} \cdot \mathrm{K}_{\beta 1, \mathrm{~L}} \cdot \mathrm{K}_{\beta 2, \mathrm{H}} \cdot \mathrm{K}_{\beta 2, \mathrm{~L}}+\mathrm{K}_{\beta 1, \mathrm{H}} \cdot \mathrm{K}_{\beta 1, \mathrm{~L}} \cdot \mathrm{K}_{\beta 2, \mathrm{~L}} \cdot[\mathrm{L}]\right. \\
& \left.+\mathrm{K}_{\beta 1, \mathrm{~L}} \cdot \mathrm{K}_{\beta 2, \mathrm{H}} \cdot \mathrm{K}_{\beta 2, \mathrm{~L}} \cdot[\mathrm{L}]\right) \\
& c_{\mathrm{LRG}}=\left(\left(\left[\mathrm{R}_{\beta 2}\right]_{\mathrm{np}, \mathrm{tot}}^{\mathrm{X}}-[\mathrm{Gs}]_{\alpha \beta \gamma}^{\mathrm{x}}\right)\right. \\
& \cdot\left(\mathrm{K}_{\beta 2, \mathrm{~L}} \cdot \mathrm{K}_{\beta 1, \mathrm{C}} \cdot \mathrm{K}_{\beta 1, \mathrm{H}} \cdot[\mathrm{L}]^{2}+\mathrm{K}_{\beta 1, \mathrm{C}} \cdot \mathrm{K}_{\beta 1, \mathrm{H}} \cdot \mathrm{K}_{\beta 1, \mathrm{~L}} \cdot \mathrm{K}_{\beta 2, \mathrm{~L}} \cdot \mathrm{K}_{\beta 2, \mathrm{H}}+\mathrm{K}_{\beta 1, \mathrm{H}} \cdot \mathrm{K}_{\beta 1, \mathrm{C}}\right. \\
& \left.\cdot \mathrm{K}_{\beta 2, \mathrm{H}} \cdot \mathrm{K}_{\beta 2, \mathrm{~L}} \cdot[\mathrm{L}]+\mathrm{K}_{\beta 1, \mathrm{H}} \cdot \mathrm{K}_{\beta 1, \mathrm{~L}} \cdot \mathrm{K}_{\beta 1, \mathrm{C}} \cdot \mathrm{K}_{\beta 2, \mathrm{~L}} \cdot[\mathrm{L}]\right)+\left(\left[\mathrm{R}_{\beta 1}\right]_{\text {np,tot }}^{\mathrm{x}}-[\mathrm{Gs}]_{\alpha \beta \gamma}^{\mathrm{x}}\right) \\
& \cdot\left(\mathrm{K}_{\beta 1, \mathrm{H}} \cdot \mathrm{K}_{\beta 1, \mathrm{~L}} \cdot \mathrm{K}_{\beta 2, \mathrm{H}} \cdot \mathrm{K}_{\beta 2, \mathrm{~L}} \cdot \mathrm{K}_{\beta 2, \mathrm{C}}+\mathrm{K}_{\beta 2, \mathrm{H}} \cdot \mathrm{K}_{\beta 1, \mathrm{~L}} \cdot \mathrm{K}_{\beta 2, \mathrm{C}} \cdot \mathrm{K}_{\beta 2, \mathrm{~L}} \cdot[\mathrm{L}]+\mathrm{K}_{\beta 2, \mathrm{C}}\right. \\
& \left.\cdot \mathrm{K}_{\beta 2, \mathrm{H}} \cdot \mathrm{K}_{\beta 1, \mathrm{~L}} \cdot[\mathrm{L}]^{2}+\mathrm{K}_{\beta 1, \mathrm{~L}} \cdot \mathrm{K}_{\beta 1, \mathrm{H}} \cdot \mathrm{K}_{\beta 2, \mathrm{H}} \cdot \mathrm{K}_{\beta 2, \mathrm{C}} \cdot[\mathrm{L}]\right)+\mathrm{K}_{\beta 1, \mathrm{C}} \cdot \mathrm{K}_{\beta 1, \mathrm{H}} \cdot \mathrm{K}_{\beta 1, \mathrm{~L}} \\
& \cdot \mathrm{K}_{\beta 2, \mathrm{H}} \cdot \mathrm{K}_{\beta 2, \mathrm{~L}} \cdot \mathrm{K}_{\beta 2, \mathrm{C}}+\mathrm{K}_{\beta 1, \mathrm{H}} \cdot \mathrm{K}_{\beta 1, \mathrm{C}} \cdot \mathrm{K}_{\beta 2, \mathrm{C}} \cdot \mathrm{K}_{\beta 2, \mathrm{H}} \cdot \mathrm{K}_{\beta 2, \mathrm{~L}} \cdot[\mathrm{L}]+\mathrm{K}_{\beta 1, \mathrm{H}} \cdot \mathrm{K}_{\beta 1, \mathrm{C}} \\
& \left.\cdot \mathrm{K}_{\beta 2, \mathrm{C}} \cdot \mathrm{K}_{\beta 2, \mathrm{H}} \cdot \mathrm{K}_{\beta 1, \mathrm{~L}} \cdot[\mathrm{L}]+\mathrm{K}_{\beta 1, \mathrm{C}} \cdot \mathrm{K}_{\beta 1, \mathrm{H}} \cdot \mathrm{K}_{\beta 2, \mathrm{C}} \cdot \mathrm{K}_{\beta 2, \mathrm{H}} \cdot[\mathrm{L}]^{2}\right) \\
& /\left(\mathrm{K}_{\beta 1, \mathrm{~L}} \cdot \mathrm{K}_{\beta 2, \mathrm{~L}} \cdot\left(\mathrm{K}_{\beta 2, \mathrm{H}}+[\mathrm{L}]\right) \cdot\left(\mathrm{K}_{\beta 1, \mathrm{H}}+[\mathrm{L}]\right)\right) \\
& \mathrm{d}_{\mathrm{LRG}}=\frac{\left([\mathrm{Gs}]_{\alpha \beta \gamma}^{\mathrm{x}} \cdot \mathrm{K}_{\beta 1, \mathrm{H}} \cdot \mathrm{K}_{\beta 1, \mathrm{C}} \cdot \mathrm{K}_{\beta 2, \mathrm{H}} \cdot \mathrm{K}_{\beta 2, \mathrm{C}} \cdot\left(\mathrm{K}_{\beta 2, \mathrm{~L}}+[\mathrm{L}]\right) \cdot\left(\mathrm{K}_{\beta 1, \mathrm{~L}}+[\mathrm{L}]\right)\right)}{\left(\mathrm{K}_{\beta 1, \mathrm{~L}} \cdot \mathrm{K}_{\beta 2, \mathrm{~L}} \cdot\left(\mathrm{K}_{\beta 2, \mathrm{H}}+[\mathrm{L}]\right) \cdot\left(\mathrm{K}_{\beta 1, \mathrm{H}}+[\mathrm{L}]\right)\right)}
\end{aligned}
$$




$$
\begin{aligned}
& \varphi_{\mathrm{LRG}}=\left(\frac{1}{2} \cdot \mathrm{d}_{\mathrm{LRG}}+\frac{1}{6} \cdot \mathrm{b}_{\mathrm{LRG}} \cdot \mathrm{c}_{\mathrm{LRG}}-\frac{1}{27} \cdot\left(\mathrm{b}_{\mathrm{LRG}}\right)^{3}\right. \\
& +\left(-\frac{1}{27} \cdot\left(b_{\mathrm{LRG}}\right)^{3} \cdot \mathrm{d}_{\mathrm{LRG}}-\frac{1}{108} \cdot\left(\mathrm{b}_{\mathrm{LRG}}\right)^{2} \cdot\left(\mathrm{c}_{\mathrm{LRG}}\right)^{2}+\frac{1}{6} \cdot \mathrm{b}_{\mathrm{LRG}} \cdot \mathrm{c}_{\mathrm{LRG}} \cdot \mathrm{d}_{\mathrm{LRG}}+\frac{1}{27}\right. \\
& \left.\left.\cdot\left(\mathrm{c}_{\mathrm{LRG}}\right)^{3}+\frac{1}{4} \cdot\left(\mathrm{d}_{\mathrm{LRG}}\right)^{2}\right)^{\frac{1}{2}}\right)^{\frac{1}{3}} \\
& {[\mathrm{Gs}]_{\mathrm{f}}^{\mathrm{X}}=\varphi_{\mathrm{LRG}}-\frac{1}{\varphi_{\mathrm{LRG}}} \cdot\left(\frac{\mathrm{c}_{\mathrm{LRG}}}{3}-\frac{\left(\mathrm{b}_{\mathrm{LRG}}\right)^{2}}{9}\right)-\frac{\mathrm{b}_{\mathrm{LRG}}}{3}} \\
& {\left[\mathrm{R}_{\beta 1}\right]_{\mathrm{np}, \mathrm{f}}^{\mathrm{x}}=\frac{\left[\mathrm{R}_{\beta 1}\right]_{\mathrm{np}, \mathrm{tot}}^{\mathrm{x}}}{1+\frac{[\mathrm{L}]}{\mathrm{K}_{\beta 1, \mathrm{~L}}}+[\mathrm{Gs}]_{\mathrm{f}}^{\mathrm{x}} \cdot\left(\frac{1}{\mathrm{~K}_{\beta 1, \mathrm{C}}}+\frac{[\mathrm{L}]}{\mathrm{K}_{\beta 1, \mathrm{C}} \cdot \mathrm{K}_{\beta 1, \mathrm{H}}}\right)}} \\
& {\left[\mathrm{R}_{\beta 2}\right]_{\mathrm{np}, \mathrm{f}}^{\mathrm{x}}=\frac{\left[\mathrm{R}_{\beta 2}\right]_{\mathrm{np}, \mathrm{tot}}^{\mathrm{x}}}{1+\frac{[\mathrm{L}]}{\mathrm{K}_{\beta 2, \mathrm{~L}}}+[\mathrm{Gs}]_{\mathrm{f}}^{\mathrm{x}} \cdot\left(\frac{1}{\mathrm{~K}_{\beta 2, \mathrm{C}}}+\frac{[\mathrm{L}]}{\mathrm{K}_{\beta 2, \mathrm{C}} \cdot \mathrm{K}_{\beta 2, \mathrm{H}}}\right)}} \\
& {\left[\mathrm{LR}_{\beta 1}\right]_{\mathrm{np}}^{\mathrm{X}}=\frac{[\mathrm{L}] \cdot\left[\mathrm{R}_{\beta 1}\right]_{\mathrm{np}, \mathrm{f}}^{\mathrm{X}}}{\mathrm{K}_{\beta 1, \mathrm{~L}}}} \\
& {\left[\mathrm{R}_{\beta 1} \mathrm{Gs}\right]_{\mathrm{np}}^{\mathrm{X}}=\frac{\left[\mathrm{R}_{\beta 1}\right]_{\mathrm{np}, \mathrm{f}}^{\mathrm{X}} \cdot[\mathrm{Gs}]_{\mathrm{f}}^{\mathrm{X}}}{\mathrm{K}_{\beta 1, \mathrm{C}}}} \\
& {\left[\mathrm{LR}_{\beta 1} \mathrm{Gs}\right]_{\mathrm{np}}^{\mathrm{x}}=\frac{[\mathrm{L}] \cdot\left[\mathrm{R}_{\beta 1}\right]_{\mathrm{np}, \mathrm{f}}^{\mathrm{x}} \cdot[\mathrm{Gs}]_{\mathrm{f}}^{\mathrm{X}}}{\mathrm{K}_{\beta 1, \mathrm{C}} \cdot \mathrm{K}_{\beta 1, \mathrm{H}}}} \\
& {\left[\mathrm{LR}_{\beta 2}\right]_{\mathrm{np}}^{\mathrm{X}}=\frac{[\mathrm{L}] \cdot\left[\mathrm{R}_{\beta 2}\right]_{\mathrm{np}, \mathrm{f}}^{\mathrm{X}}}{\mathrm{K}_{\beta 2, \mathrm{~L}}}} \\
& {\left[\mathrm{R}_{\beta 2} \mathrm{Gs}\right]_{\mathrm{np}}^{\mathrm{X}}=\frac{\left[\mathrm{R}_{\beta 2}\right]_{\mathrm{np}, \mathrm{f}}^{\mathrm{X}} \cdot[\mathrm{Gs}]_{\mathrm{f}}^{\mathrm{X}}}{\mathrm{K}_{\beta 2, \mathrm{C}}}} \\
& {\left[\mathrm{LR}_{\beta 2} \mathrm{Gs}\right]_{\mathrm{np}}^{\mathrm{x}}=\frac{[\mathrm{L}] \cdot\left[\mathrm{R}_{\beta 2}\right]_{\mathrm{np}, \mathrm{f}}^{\mathrm{x}} \cdot[\mathrm{Gs}]_{\mathrm{f}}^{\mathrm{X}}}{\mathrm{K}_{\beta 2, \mathrm{C}} \cdot \mathrm{K}_{\beta 2, \mathrm{H}}}}
\end{aligned}
$$

$\frac{\mathrm{d}\left[\mathrm{R}_{\beta 1}\right]_{\text {PKA,tot }}^{\mathrm{X}}}{\mathrm{dt}}=\mathrm{k}_{\text {PKAp }} \cdot[\mathrm{C}]^{\mathrm{X}} \cdot\left[\mathrm{R}_{\beta 1}\right]_{\text {np,tot }}^{\mathrm{X}}-\mathrm{k}_{\text {PKAdp }} \cdot\left[\mathrm{R}_{\beta 1}\right]_{\text {PKA,tot }}^{\mathrm{X}}$$$
\frac{\mathrm{d}\left[\mathrm{R}_{\beta 1}\right]_{\mathrm{GRK}, \mathrm{tot}}^{\mathrm{x}}}{\mathrm{dt}}=\mathrm{k}_{\mathrm{GRKp}} \cdot[\mathrm{GRK}] \cdot\left(\left[\mathrm{LR}_{\beta 1}\right]_{\mathrm{np}}^{\mathrm{x}}+\left[\mathrm{LR}_{\beta 1} \mathrm{Gs}\right]_{\mathrm{np}}^{\mathrm{x}}\right)-\mathrm{k}_{\mathrm{GRKdp}} \cdot\left[\mathrm{R}_{\beta 1}\right]_{\mathrm{GRK}, \text { tot }}^{\mathrm{x}}
$$$$
\frac{\mathrm{d}\left[\mathrm{R}_{\beta 2}\right]_{\mathrm{PKA}, \mathrm{tot}}^{\mathrm{x}}}{\mathrm{dt}}=\mathrm{k}_{\mathrm{PKAp}} \cdot[\mathrm{C}]^{\mathrm{X}} \cdot\left[\mathrm{R}_{\beta 2}\right]_{\text {np,tot }}^{\mathrm{x}}-\mathrm{k}_{\mathrm{PKAdp}} \cdot\left[\mathrm{R}_{\beta 2}\right]_{\mathrm{PKA}, \text { tot }}^{\mathrm{x}}
$$ 


$$
\begin{aligned}
& \frac{\mathrm{d}\left[\mathrm{R}_{\beta 2}\right]_{\mathrm{GRK}, \mathrm{tot}}^{\mathrm{x}}}{\mathrm{dt}}= \mathrm{k}_{\mathrm{GRKp}} \cdot[\mathrm{GRK}] \cdot\left(\left[\mathrm{LR}_{\beta 2}\right]_{\mathrm{np}}^{\mathrm{x}}+\left[\mathrm{LR}_{\beta 2} \mathrm{Gs}\right]_{\mathrm{np}}^{\mathrm{x}}\right)-\mathrm{k}_{\mathrm{GRKdp}} \cdot\left[\mathrm{R}_{\beta 2}\right]_{\mathrm{GRK}, \mathrm{tot}}^{\mathrm{x}} \\
& \frac{\mathrm{d}[\mathrm{Gs}]_{\alpha, \mathrm{GTP}}^{\mathrm{x}}}{\mathrm{dt}}= \mathrm{k}_{\mathrm{act} 2, \mathrm{Gs}} \cdot\left(\left[\mathrm{R}_{\beta 1} \mathrm{Gs}\right]_{\mathrm{np}}^{\mathrm{x}}+\left[\mathrm{R}_{\beta 2} \mathrm{Gs}\right]_{\mathrm{np}}^{\mathrm{x}}\right)+\mathrm{k}_{\mathrm{act} 1, \mathrm{Gs}} \cdot\left(\left[\mathrm{LR}_{\beta 1} \mathrm{Gs}\right]_{\mathrm{np}}^{\mathrm{x}}+\left[\mathrm{LR}_{\beta 2} \mathrm{Gs}\right]_{\mathrm{np}}^{\mathrm{x}}\right) \\
&-\mathrm{k}_{\mathrm{hydr}, \mathrm{Gs}} \cdot[\mathrm{Gs}]_{\alpha, \mathrm{GTP}}^{\mathrm{x}} \\
& \frac{\mathrm{d}[\mathrm{Gs}]_{\beta \gamma}^{\mathrm{x}}}{\mathrm{dt}}= \mathrm{k}_{\mathrm{act} 2, \mathrm{Gs}} \cdot\left(\left[\mathrm{R}_{\beta 1} \mathrm{Gs}\right]_{\mathrm{np}}^{\mathrm{x}}+\left[\mathrm{R}_{\beta 2} \mathrm{Gs}\right]_{\mathrm{np}}^{\mathrm{x}}\right)+\mathrm{k}_{\mathrm{act} 1, \mathrm{Gs}} \cdot\left(\left[\mathrm{LR}_{\beta 1} \mathrm{Gs}\right]_{\mathrm{np}}^{\mathrm{x}}+\left[\mathrm{LR}_{\beta 2} \mathrm{Gs}\right]_{\mathrm{np}}^{\mathrm{x}}\right) \\
& \frac{\mathrm{d}[\mathrm{Gs}]_{\alpha, \mathrm{GDP}}^{\mathrm{x}}}{\mathrm{dt}}= \mathrm{k}_{\mathrm{hydr}, \mathrm{Gs}} \cdot[\mathrm{Gs}]_{\alpha, \mathrm{GTP}}^{\mathrm{x}}-\mathrm{k}_{\mathrm{reas}, \mathrm{Gs}} \cdot[\mathrm{Gs}]_{\beta \gamma}^{\mathrm{x}} \cdot[\mathrm{Gs}]_{\alpha, \mathrm{GDP}}^{\mathrm{x}} \cdot[\mathrm{Gs}]_{\beta \gamma}^{\mathrm{x}} \cdot[\mathrm{Gs}]_{\alpha, \mathrm{GDP}}^{\mathrm{x}} \\
& \frac{\mathrm{d}[\mathrm{Gi}]_{\alpha, \mathrm{GTP}}^{\mathrm{x}}=}{\mathrm{dt}}=\mathrm{k}_{\mathrm{act} 2, \mathrm{Gi}} \cdot\left[\mathrm{R}_{\beta 2} \mathrm{Gi}\right]_{\mathrm{PKA}}^{\mathrm{x}}+\mathrm{k}_{\mathrm{act} 1, \mathrm{Gi}} \cdot\left[\mathrm{LR}_{\beta 2} \mathrm{Gi}\right]_{\mathrm{PKA}}^{\mathrm{x}}-\mathrm{k}_{\mathrm{hydr}, \mathrm{Gi}} \cdot[\mathrm{Gi}]_{\alpha, \mathrm{GTP}}^{\mathrm{x}} \\
& \frac{\mathrm{d}[\mathrm{Gi}]_{\beta \gamma}^{\mathrm{x}}}{\mathrm{dt}}= \mathrm{k}_{\mathrm{act} 2, \mathrm{Gi}} \cdot\left[\mathrm{R}_{\beta 2} \mathrm{Gi}\right]_{\mathrm{PKA}}^{\mathrm{x}}+\mathrm{k}_{\mathrm{act} 1, \mathrm{Gi}} \cdot\left[\mathrm{LR}_{\beta 2} \mathrm{Gi}\right]_{\mathrm{PKA}}^{\mathrm{x}}-\mathrm{k}_{\mathrm{reas}, \mathrm{Gi}} \cdot[\mathrm{Gi}]_{\beta \gamma}^{\mathrm{x}} \cdot[\mathrm{Gi}]_{\alpha, \mathrm{GDP}}^{\mathrm{x}} \\
& \frac{\mathrm{d}[\mathrm{Gi}]_{\alpha, \mathrm{GDP}}^{\mathrm{x}}}{\mathrm{dt}}= \mathrm{k}_{\mathrm{hydr}, \mathrm{Gi}} \cdot[\mathrm{Gi}]_{\alpha, \mathrm{GTP}}^{\mathrm{x}}-\mathrm{k}_{\mathrm{reas}, \mathrm{Gi}} \cdot[\mathrm{Gi}]_{\beta \gamma}^{\mathrm{x}} \cdot[\mathrm{Gi}]_{\alpha, \mathrm{GDP}}^{\mathrm{x}}
\end{aligned}
$$

\section{For $x \in\{C Y T\}:$}

$$
\begin{aligned}
& {\left[\mathrm{R}_{\beta 1}\right]_{\text {tot }}^{\mathrm{x}}=\mathrm{f}_{\mathrm{R}, \beta 1}^{\mathrm{x}} \cdot\left[\mathrm{R}_{\beta 1}\right]_{\text {tot }} \cdot \frac{\mathrm{V}^{\mathrm{CELL}}}{\mathrm{V}^{\mathrm{x}}}} \\
& {[\mathrm{Gs}]_{\alpha \beta \gamma}^{\mathrm{X}}=\mathrm{f}_{\mathrm{Gs}}^{\mathrm{X}} \cdot[\mathrm{Gs}]_{\mathrm{tot}} \cdot \frac{\mathrm{V}^{\mathrm{CELL}}}{\mathrm{V}^{\mathrm{x}}}-[\mathrm{Gs}]_{\alpha, \mathrm{GTP}}^{\mathrm{x}}-[\mathrm{Gs}]_{\alpha, \mathrm{GDP}}^{\mathrm{X}}} \\
& {\left[\mathrm{R}_{\beta 1}\right]_{\text {np,tot }}^{\mathrm{X}}=\left[\mathrm{R}_{\beta 1}\right]_{\text {tot }}^{\mathrm{X}}-\left[\mathrm{R}_{\beta 1}\right]_{\mathrm{PKA}, \text { tot }}^{\mathrm{X}}-\left[\mathrm{R}_{\beta 1}\right]_{\mathrm{GRK}, \text { tot }}^{\mathrm{X}}} \\
& \mathrm{a}_{\mathrm{LRG}}=\frac{1}{\mathrm{~K}_{\beta 1, \mathrm{~L}}} \cdot\left(\mathrm{K}_{\beta 1, \mathrm{H}}+[\mathrm{L}]\right) \cdot\left(\mathrm{K}_{\beta 1, \mathrm{~L}}+[\mathrm{L}]\right) \\
& \mathrm{b}_{\mathrm{LRG}}=[\mathrm{Gs}]_{\alpha \beta \gamma}^{\mathrm{x}} \cdot\left(\mathrm{L}+\mathrm{K}_{\beta 1, \mathrm{H}}\right)-\left(\mathrm{K}_{\beta 2, \mathrm{H}}+[\mathrm{L}]\right) \cdot\left[\mathrm{R}_{\beta 1}\right]_{\mathrm{np}, \mathrm{tot}}^{\mathrm{x}}+\mathrm{K}_{\beta 1, \mathrm{C}} \cdot \mathrm{K}_{\beta 1, \mathrm{H}} \\
& +\frac{\mathrm{K}_{\beta 1, \mathrm{C}} \cdot \mathrm{K}_{\beta 1, \mathrm{H}} \cdot[\mathrm{L}]}{\mathrm{K}_{\beta 1, \mathrm{~L}}} \\
& \mathrm{c}_{\mathrm{LRG}}=-\left[\mathrm{R}_{\beta 1}\right]_{\mathrm{np}, \mathrm{tot}}^{\mathrm{x}} \cdot \mathrm{K}_{\beta 1, \mathrm{H}} \cdot \mathrm{K}_{\beta 1, \mathrm{C}} \\
& {\left[\mathrm{R}_{\beta 1}\right]_{\mathrm{np}, \mathrm{f}}^{\mathrm{x}}=\frac{-\mathrm{b}_{\mathrm{LRG}}+\sqrt{\mathrm{b}_{\mathrm{LRG}}^{2}-4 \cdot \mathrm{a}_{\mathrm{LRG}} \cdot \mathrm{c}_{\mathrm{LRG}}}}{2 \cdot \mathrm{a}_{\mathrm{LRG}}}} \\
& {[\mathrm{Gs}]_{\mathrm{f}}^{\mathrm{X}}=\frac{[\mathrm{Gs}]_{\alpha \beta \gamma}^{\mathrm{x}}}{1+\frac{\left[\mathrm{R}_{\beta 1}\right]_{\mathrm{np}, \mathrm{f}}^{\mathrm{X}}}{\mathrm{K}_{\beta 1, \mathrm{C}}}+\frac{[\mathrm{L}] \cdot\left[\mathrm{R}_{\beta 1}\right]_{\mathrm{np}, \mathrm{f}}^{\mathrm{X}}}{\mathrm{K}_{\beta 1, \mathrm{H}} \cdot \mathrm{K}_{\beta 1, \mathrm{C}}}}} \\
& {\left[\mathrm{LR}_{\beta 1}\right]_{\mathrm{np}}^{\mathrm{x}}=\frac{[\mathrm{L}] \cdot\left[\mathrm{R}_{\beta 1}\right]_{\mathrm{np}, \mathrm{f}}^{\mathrm{x}}}{\mathrm{K}_{\beta 1, \mathrm{~L}}}}
\end{aligned}
$$




$$
\begin{aligned}
& {\left[\mathrm{R}_{\beta 1} \mathrm{Gs}\right]_{\mathrm{np}}^{\mathrm{X}}=\frac{\left[\mathrm{R}_{\beta 1}\right]_{\mathrm{np}, \mathrm{f}}^{\mathrm{X}} \cdot[\mathrm{Gs}]_{\mathrm{f}}^{\mathrm{X}}}{\mathrm{K}_{\beta 1, \mathrm{C}}}} \\
& {\left[\mathrm{LR}_{\beta 1} \mathrm{Gs}\right]_{\mathrm{np}}^{\mathrm{X}}=\frac{[\mathrm{L}] \cdot\left[\mathrm{R}_{\beta 1}\right]_{\mathrm{np}, \mathrm{f}}^{\mathrm{X}} \cdot[\mathrm{Gs}]_{\mathrm{f}}^{\mathrm{X}}}{\mathrm{K}_{\beta 1, \mathrm{C}} \cdot \mathrm{K}_{\beta 1, \mathrm{H}}}} \\
& \frac{\mathrm{d}\left[\mathrm{R}_{\beta 1}\right]_{\mathrm{PKA}, \mathrm{tot}}^{\mathrm{X}}}{\mathrm{dt}}=\mathrm{k}_{\beta 1, \mathrm{PKAp}} \cdot[\mathrm{C}]^{\mathrm{X}} \cdot\left[\mathrm{R}_{\beta 1}\right]_{\text {np,tot }}^{\mathrm{X}}-\mathrm{k}_{\beta 1, \text { PKAdp }} \cdot\left[\mathrm{R}_{\beta 1}\right]_{\text {PKA,tot }}^{\mathrm{X}} \\
& \frac{\mathrm{d}\left[\mathrm{R}_{\beta 1}\right]_{\mathrm{GRK}, \mathrm{tot}}^{\mathrm{x}}}{\mathrm{dt}}=\mathrm{k}_{\beta 1, \mathrm{GRKp}} \cdot[\mathrm{GRK}] \cdot\left(\left[\mathrm{LR}_{\beta 1}\right]_{\mathrm{np}}^{\mathrm{x}}+\left[\mathrm{LR}_{\beta 1} \mathrm{Gs}\right]_{\mathrm{np}}^{\mathrm{x}}\right)-\mathrm{k}_{\beta 1, \mathrm{GRKdp}} \cdot\left[\mathrm{R}_{\beta 1}\right]_{\mathrm{GRK}, \text { tot }}^{\mathrm{x}} \\
& \frac{\mathrm{d}[\mathrm{Gs}]_{\alpha, \mathrm{GTP}}^{\mathrm{X}}}{\mathrm{dt}}=\mathrm{k}_{\mathrm{act} 2, \mathrm{Gs}} \cdot\left[\mathrm{R}_{\beta 1} \mathrm{Gs}\right]_{\mathrm{np}}^{\mathrm{x}}+\mathrm{k}_{\mathrm{act} 1, \mathrm{Gs}} \cdot\left[\mathrm{LR}_{\beta 1} \mathrm{Gs}\right]_{\mathrm{np}}^{\mathrm{x}}-\mathrm{k}_{\mathrm{hydr}, \mathrm{Gs}} \cdot[\mathrm{Gs}]_{\alpha, \mathrm{GTP}}^{\mathrm{x}} \\
& \frac{\mathrm{d}[\mathrm{Gs}]_{\beta \gamma}^{\mathrm{x}}}{\mathrm{dt}}=\mathrm{k}_{\mathrm{act} 2, \mathrm{Gs}} \cdot\left[\mathrm{R}_{\beta 1} \mathrm{Gs}\right]_{\mathrm{np}}^{\mathrm{x}}+\mathrm{k}_{\mathrm{act} 1, \mathrm{Gs}} \cdot\left[\mathrm{LR}_{\beta 1} \mathrm{Gs}\right]_{\mathrm{np}}^{\mathrm{x}}-\mathrm{k}_{\mathrm{reas}, \mathrm{Gs}} \cdot[\mathrm{Gs}]_{\beta \gamma}^{\mathrm{x}} \cdot[\mathrm{Gs}]_{\alpha, \mathrm{GDP}}^{\mathrm{x}} \\
& \frac{\mathrm{d}[\mathrm{Gs}]_{\alpha, \mathrm{GDP}}^{\mathrm{x}}}{\mathrm{dt}}=\mathrm{k}_{\mathrm{hydr}, \mathrm{Gs}} \cdot[\mathrm{Gs}]_{\alpha, \mathrm{GTP}}^{\mathrm{x}}-\mathrm{k}_{\mathrm{reas}, \mathrm{Gs}} \cdot[\mathrm{Gs}]_{\beta \gamma}^{\mathrm{x}} \cdot[\mathrm{Gs}]_{\alpha, \mathrm{GDP}}^{\mathrm{x}}
\end{aligned}
$$

\begin{tabular}{|c|c|c|c|}
\hline Parameter & Value & Description & Ref \\
\hline$\left[\mathrm{R}_{\beta 1}\right]_{\text {tot }}$ & $\begin{array}{c}0.85^{*} 0.025 \\
\mu \mathrm{mol} / \mathrm{L}\end{array}$ & Total cellular $\beta 1 A R$ concentration & $95,103^{\#,(C)}$ \\
\hline$\left[\mathrm{R}_{\beta 2}\right]_{\text {tot }}$ & $\begin{array}{c}0.15^{*} 0.025 \\
\mu \mathrm{mol} / \mathrm{L}\end{array}$ & Total cellular $\beta 2 A R$ concentration & $103^{\#,(C)}$ \\
\hline $\mathrm{f}_{\mathrm{R}, \beta 1}^{\mathrm{CAV}}$ & 0.0812 & Fraction of receptors located in CAV & $\%$ \\
\hline $\mathrm{f}_{\mathrm{R}, \beta 1}^{\mathrm{ECAV}}$ & 0.4874 & Fraction of receptors located in ECAV & $\%$ \\
\hline $\mathrm{f}_{\mathrm{R}, \beta 1}^{\mathrm{CYT}}$ & $1-\mathrm{f}_{\mathrm{R}, \beta 1}^{\mathrm{CAV}}-\mathrm{f}_{\mathrm{R}, \beta 1}^{\mathrm{ECAV}}$ & Fraction of receptors located in CYT & \\
\hline $\mathrm{f}_{\mathrm{R}, \beta 2}^{\mathrm{CAV}}$ & 0.850 & Fraction of $\beta 2 A R$ located in CAV & $\begin{array}{c}97 \\
104^{\&,(R)}\end{array}$ \\
\hline $\mathrm{f}_{\mathrm{R}, \beta 2}^{\mathrm{ECAV}}$ & $1-\mathrm{f}_{\mathrm{R}, \beta 2}^{\mathrm{CAV}}$ & Fraction of $\beta 2 A R$ located in ECAV & \\
\hline$[\mathrm{Gs}]_{\text {tot }}$ & $224 \cdot\left[\mathrm{R}_{\beta 1}\right]_{\text {tot }}$ & Total Gs protein concentration & $105^{\#,(R)}$ \\
\hline$[\mathrm{Gi}]_{\text {tot }}$ & $0.500 \mu \mathrm{mol} / \mathrm{L}$ & Total Gi protein concentration & $94^{\&}$ \\
\hline $\mathrm{f}_{\mathrm{Gs}}^{\mathrm{CAV}}$ & $1.1071 \mathrm{E}-3$ & Fraction of G-proteins located in CAV & $\%$ \\
\hline $\mathrm{f}_{\mathrm{Gs}}^{\mathrm{ECAV}}$ & 0.5664 & Fraction of G-proteins located in ECAV & $\%$ \\
\hline $\mathrm{f}_{\mathrm{Gs}}^{\mathrm{CYT}}$ & $1-\mathrm{f}_{\mathrm{Gs}}^{\mathrm{CAV}}-\mathrm{f}_{\mathrm{GS}}^{\mathrm{ECAV}}$ & Fraction of G-proteins located in CYT & \\
\hline $\mathrm{f}_{\mathrm{Gi}}^{\mathrm{CAV}}$ & 0.850 & Fraction of Gi located in CAV & $\begin{array}{c}97 \\
104^{\&,(R)} \\
\end{array}$ \\
\hline $\mathrm{f}_{\mathrm{Gi}}^{\mathrm{ECAV}}$ & $1-\mathrm{f}_{\mathrm{Gi}}^{\mathrm{CAV}}$ & Fraction of Gi located in ECAV & \\
\hline $\mathrm{K}_{\beta 1, \mathrm{~L}}$ & $0.567 \mu \mathrm{mol} / \mathrm{L}$ & $\beta 1$ Receptor / ligand low affinity constant & $98^{\#,(\mathrm{~N})}$ \\
\hline $\mathrm{K}_{\beta 1, \mathrm{H}}$ & $0.062 \mu \mathrm{mol} / \mathrm{L}$ & $\beta 1$ Receptor / ligand high affinity constant & $98^{\#,(N)}$ \\
\hline
\end{tabular}




\begin{tabular}{|c|c|c|c|}
\hline $\mathrm{K}_{\beta 1, \mathrm{C}}$ & $2.4490 \mu \mathrm{mol} / \mathrm{L}$ & $\beta 1$ Receptor / G-protein affinity constant & $\%$ \\
\hline $\mathrm{K}_{\beta 2, \mathrm{~L}}$ & $1.053 \mu \mathrm{mol} / \mathrm{L}$ & $\beta 2$ Receptor / ligand low affinity constant & $98^{\#,(N)}$ \\
\hline $\mathrm{K}_{\beta 2, \mathrm{H}}$ & $0.012 \mu \mathrm{mol} / \mathrm{L}$ & $\beta 2$ Receptor / ligand high affinity constant & $98^{\#,(\mathrm{~N})}$ \\
\hline $\mathrm{K}_{\beta 2, \mathrm{C}}$ & $1.8463 \mu \mathrm{mol} / \mathrm{L}$ & B2 Receptor / G-protein affinity constant & $\%$ \\
\hline $\mathrm{K}_{\beta 2, \mathrm{~N}}$ & $\mathrm{~K}_{\beta 2, \mathrm{~L}}$ & Phosph. $\beta 2$ receptor/ligand low affinity constant & \\
\hline $\mathrm{K}_{\beta 2, \mathrm{~F}}$ & $0.100 \mu \mathrm{mol} / \mathrm{L}$ & Phosph. $\beta 2$ receptor/ligand high affinity constant & $\%$ \\
\hline $\mathrm{K}_{\beta 2, \mathrm{~A}}$ & $1.6655 \mu \mathrm{mol} / \mathrm{L}$ & Phosph. $\beta 2$ Receptor/Gi-protein affinity constant & $\%$ \\
\hline $\begin{array}{l}\mathrm{k}_{\mathrm{GRKp}} \\
\cdot[\mathrm{GRK}] \\
\end{array}$ & 4.655E-4 s $\mathrm{s}^{-1}$ & Rate for GRK dependent receptor desensitization & $\%$ \\
\hline $\mathrm{k}_{\mathrm{GRKdp}}$ & $3.442 \mathrm{E}-4 \mathrm{~s}^{-1}$ & Rate for GRK dephosphorylation & $\%$ \\
\hline $\mathrm{k}_{\mathrm{PKAp}}$ & $\begin{array}{c}2.275 \mathrm{E}-3 \mu \mathrm{mol} / \mathrm{L}^{-} \\
{ }^{1} \mathrm{~s}^{-1}\end{array}$ & Rate for PKA dependent receptor desensitization & $\%$ \\
\hline $\mathrm{k}_{\text {PKAdp }}$ & $3.5556 \mathrm{E}-4 \mathrm{~s}^{-1}$ & Rate for PKA dephosphorylation & $\%$ \\
\hline $\mathrm{k}_{\mathrm{act} 1, \mathrm{Gs}}$ & $4.9054 \mathrm{~s}^{-1}$ & Activation rate for Gs by high affinity complex & $\%$ \\
\hline $\mathrm{k}_{\mathrm{act} 2, \mathrm{Gs}}$ & $0.2595 \mathrm{~s}^{-1}$ & Activation rate for Gs by low affinity complex & $\%$ \\
\hline $\mathrm{k}_{\mathrm{hydr}, \mathrm{Gs}}$ & $0.800 \mathrm{~s}^{-1}$ & Gs GTP to GDP hydrolysis constant & $\begin{array}{c}94,102, \\
106^{\#}\end{array}$ \\
\hline $\mathrm{k}_{\text {reas,Gs }}$ & $1.210 \mathrm{E} 3 \underset{1}{\mu \mathrm{mol} / \mathrm{L}^{-1} \mathrm{~s}^{-}}$ & Reassociation rate for Gs subunits & $\begin{array}{c}94,102, \\
106^{\#} \\
\end{array}$ \\
\hline $\mathrm{k}_{\mathrm{act} 1, \mathrm{Gi}}$ & $4.000 \mathrm{~s}^{-1}$ & Activation rate for Gi by high affinity complex & $94^{\&}$ \\
\hline $\mathrm{k}_{\mathrm{act} 2, \mathrm{Gi}}$ & $0.050 \mathrm{~s}^{-1}$ & Activation rate for Gi by low affinity complex & $94^{\&}$ \\
\hline $\mathrm{k}_{\mathrm{hydr,Gi}}$ & $\mathrm{k}_{\mathrm{hydr}, \mathrm{Gs}}$ & Gi GTP to GDP hydrolysis constant & \\
\hline $\mathrm{k}_{\text {reas,Gi }}$ & $\mathrm{k}_{\text {reas,Gs }}$ & Reassociation rate for Gi subunits & \\
\hline
\end{tabular}

\subsection{Adenylyl Cyclase}

Two distinct groups of adenylyl cyclases (ACs) are distinguished in the model. AC types 5 and 6 are the most common ACs In the mammalian heart. ${ }^{107}$ Both are stimulated by $\mathrm{Gs}_{\alpha}$ with similar affinities and show significant sequence similarity. AC types 4 and 7 make up approximately $25 \%$ of the ACs in the ventricle and have a higher affinity for Gs than types 5 and $6 .{ }^{94,108}$ Furthermore, there is a difference in the regulation by $\mathrm{Gi}$ of types $5 / 6$ versus $4 / 7$ with the former being inhibited by the $\mathrm{Gi}_{\alpha}$ subunit and the latter being stimulated by the $\mathrm{Gi}_{\beta \gamma}$ subunits. ${ }^{94}$ Because of the pronounced caveolar localization of Gi-coupled $\beta 2$-receptors and the absence of $A C 4 / 7$ from this domain, we only modeled Gi regulation of $A C 5 / 6$. All types are modeled with a Hill equation and fixed affinity for ATP. AC activity is scaled by a constant factor to produce the desired intracellular cAMP concentration during maximal $\beta A R$ stimulation $\left(2.5-3.0 \mu \mathrm{mol} / \mathrm{L}\right.$ in canine ventricular myocytes, ${ }^{109}$ also consistent with $20.5 \mathrm{pmol} / 2 * 10^{5}$ rat ventricular myocytes $=20.5 \mathrm{pmol} / 7.6 \mu \mathrm{L}=2.7 \mu \mathrm{mol} / \mathrm{L}$ as in Post et al. ${ }^{105}$ ). Parameters for types 5 and 6 were derived from human AC expressed in Sf9 cells. ${ }^{110}$ 
It should be noted, however, that significant species differences have been reported for these types. Other parameters were determined by fitting experimental AC activity dose response curves. ${ }^{111-113}$

In neurons it is known that overall AC activity is modulated by free intracellular $\mathrm{Ca}^{2+}$, but this effect is much less pronounced in the heart. ${ }^{114}$ Some influence of local $\mathrm{Ca}^{2+}$ entrance through L-type $\mathrm{Ca}^{2+}$ channels has been reported in chick embryonic ventricular myocytes, ${ }^{115}$ but to the best of our knowledge no detailed characterization of the physiological importance of this regulation has been performed in adult mammalian ventricular myocytes. Disparate results also exist about the functional effect of phosphorylation of AC types 5 and 6 by PKA. Several reports show an inhibitory effect of PKA-dependent phosphorylation on AC activity in expression systems. ${ }^{107,}{ }^{114}$ However, experiments in crude membrane preparations of rat myocardium showed an increased AC activity when PKA catalytic subunits were added in the presence of isoproterenol. ${ }^{116} \mathrm{Both}^{\mathrm{Ca}^{2+}}$ and PKAdependent inhibition of $A C$ were proposed as negative feedback mechanisms limiting CAMP accumulation during intense adrenergic stimulation. However, other negative feedback loops exist, and are incorporated into the model. Because of the lack of clear experimental evidence about the functional relevance of $\mathrm{AC}$ inhibition by $\mathrm{Ca}^{2+}$ and PKA, these mechanisms are not included in this model.

$$
\begin{aligned}
& {[\mathrm{AC} 56]^{\mathrm{CAV}}=\mathrm{f}_{\mathrm{AC} 66}^{\mathrm{CAV}} \cdot \mathrm{f}_{\mathrm{AC} 56, \mathrm{AC} 47} \cdot[\mathrm{AC}]_{\text {tot }} \cdot \frac{\mathrm{V}^{\mathrm{CELL}}}{\mathrm{V}^{\mathrm{CAV}}}} \\
& {[\mathrm{AC} 56]^{\mathrm{CYT}}=\left(1-\mathrm{f}_{\mathrm{AC} 56}^{\mathrm{CAV}}\right) \cdot \mathrm{f}_{\mathrm{AC} 56, \mathrm{AC} 47} \cdot[\mathrm{AC}]_{\text {tot }} \cdot \frac{\mathrm{V}^{\mathrm{CELL}}}{\mathrm{V}^{\mathrm{CYT}}}} \\
& {[\mathrm{AC} 47]^{\mathrm{ECAV}}=\mathrm{f}_{\mathrm{AC} 47}^{\mathrm{ECAV}} \cdot\left(1-\mathrm{f}_{\mathrm{AC} 56, \mathrm{AC} 47}\right) \cdot[\mathrm{AC}]_{\text {tot }} \cdot \frac{\mathrm{V}^{\mathrm{CELL}}}{\mathrm{V}^{\mathrm{ECAV}}}} \\
& {[\mathrm{AC} 47]^{\mathrm{CYT}}=\left(1-\mathrm{f}_{\mathrm{AC} 47}^{\mathrm{ECAV}}\right) \cdot\left(1-\mathrm{f}_{\mathrm{AC} 56, \mathrm{AC} 47}\right) \cdot[\mathrm{AC}]_{\text {tot }} \cdot \frac{\mathrm{V}^{\mathrm{CELL}}}{\mathrm{V}^{\mathrm{CYT}}}} \\
& {[\mathrm{Gi}]_{\alpha, \mathrm{GTP}}^{\mathrm{CYT}}=0.0}
\end{aligned}
$$

For $x \in\{C A V, C Y T\}:$

$$
\begin{aligned}
& \mathrm{k}_{\mathrm{AC} 56}^{\mathrm{x}}=\mathrm{AF}_{56} \cdot\left(\mathrm{AC5} 6_{\text {basal }}+\frac{\left([\mathrm{Gs}]_{\alpha, \mathrm{GTP}}^{\mathrm{x}}\right)^{\mathrm{h}_{\mathrm{AC} 56, \mathrm{Gs}}}}{\mathrm{K}_{\mathrm{m}, \mathrm{Gs}}^{\mathrm{AC} 56}+\left([\mathrm{Gs}]_{\alpha, \mathrm{GTP}}^{\mathrm{x}}\right)^{\mathrm{h}_{\mathrm{AC} 56, \mathrm{Gs}}}}\right) \\
& \cdot\left(1-\left(1-\frac{\mathrm{V}_{\mathrm{GsGi}}^{\mathrm{AC5}} \cdot\left([\mathrm{Gs}]_{\alpha, \mathrm{GTP}}^{\mathrm{x}}\right)^{\mathrm{h}_{\mathrm{AC} 56, \mathrm{GsGi}}}}{\mathrm{K}_{\mathrm{m}, \mathrm{GSGi}}^{\mathrm{AC5}}+\left([\mathrm{Gs}]_{\alpha, \mathrm{GTP}}^{\mathrm{x}}\right)^{\mathrm{h}_{\mathrm{AC} 56, \mathrm{GsGi}}}}\right) \cdot \frac{[\mathrm{Gi}]_{\alpha, \mathrm{GTP}}^{\mathrm{x}}}{\mathrm{K}_{\mathrm{m}, \mathrm{Gi}}^{\mathrm{AC5} 6}+[\mathrm{Gi}]_{\alpha, \mathrm{GTP}}^{\mathrm{x}}}\right) \\
& \frac{\mathrm{dcAMP}_{\mathrm{AC} 56}^{\mathrm{X}}}{\mathrm{dt}}=\frac{\mathrm{k}_{\mathrm{AC} 56}^{\mathrm{x}} \cdot[\mathrm{AC} 56]^{\mathrm{x}} \cdot[\mathrm{ATP}]}{\mathrm{K}_{\mathrm{m}, \mathrm{ATP}}+[\mathrm{ATP}]}
\end{aligned}
$$

For $x \in\{E C A V, C Y T\}:$ 


$$
\begin{aligned}
\mathrm{k}_{\mathrm{AC} 47}^{\mathrm{x}} & =\mathrm{AF}_{47} \cdot\left(\mathrm{AC} 47_{\text {basal }}+\frac{\left([\mathrm{Gs}]_{\alpha, \mathrm{GTP}}^{\mathrm{x}}\right)^{\mathrm{h}_{\mathrm{AC} 47}}}{\mathrm{~K}_{\mathrm{m}, \mathrm{AC} 47}+\left([\mathrm{Gs}]_{\alpha, \mathrm{GTP}}^{\mathrm{x}}\right)^{\mathrm{h}_{\mathrm{AC} 47}}}\right) \\
\frac{\mathrm{dcAMP}_{\mathrm{AC} 47}^{\mathrm{x}}}{\mathrm{dt}} & =\frac{\mathrm{k}_{\mathrm{AC} 47}^{\mathrm{x}} \cdot[\mathrm{AC} 47]^{\mathrm{x}} \cdot[\mathrm{ATP}]}{\mathrm{K}_{\mathrm{m}, \mathrm{ATP}}+[\mathrm{ATP}]}
\end{aligned}
$$

\begin{tabular}{|c|c|c|c|}
\hline Parameter & Value & Description & Ref \\
\hline $\mathrm{K}_{\mathrm{m}, \mathrm{ATP}}$ & $315 \mu \mathrm{mol} / \mathrm{L}$ & AC affinity for ATP & $117^{\#}$ \\
\hline$[\mathrm{ATP}]$ & $5.00 \mathrm{E} 3 \mu \mathrm{mol} / \mathrm{L}$ & Concentration of ATP & $117^{\#}$ \\
\hline$[\mathrm{AC}]_{\text {tot }}$ & $3 \cdot\left[\mathrm{R}_{\beta 1}\right]_{\text {tot }}$ & Total cellular AC concentration & $105^{\#,(R)}$ \\
\hline $\mathrm{f}_{\mathrm{AC} 56, \mathrm{AC} 47}$ & 0.740 & Fraction of $A C$ that is of type $5 / 6$ & $108^{\mathrm{Q},(\mathrm{H})}$ \\
\hline $\mathrm{f}_{\mathrm{AC} 56}^{\mathrm{CAV}}$ & 0.0875 & Fraction of AC56 located in caveolae & $\%$ \\
\hline $\mathrm{f}_{\mathrm{AC} 47}^{\mathrm{ECAV}}$ & 0.1648 & Fraction of AC47 in extracaveolar space & $\%$ \\
\hline $\mathrm{K}_{\mathrm{m}, \mathrm{Gs}}^{\mathrm{AC56}}$ & $0.0852 \mu \mathrm{mol} / \mathrm{L}$ & AC56 affinity for Gs & $110^{@,(\mathrm{E})}$ \\
\hline $\mathrm{h}_{\mathrm{AC} 56, \mathrm{Gs}}$ & 1.357 & Hill coefficient for AC56 activation & $110^{@,(\mathrm{E})}$ \\
\hline $\mathrm{K}_{\mathrm{m}, \mathrm{GSGi}}^{\mathrm{AC56}}$ & $0.482 \mu \mathrm{mol} / \mathrm{L}$ & Gs-dependence of inactivation by Gi for AC56 & $110^{@,(\mathrm{E})}$ \\
\hline $\mathrm{h}_{\mathrm{AC} 56, \mathrm{GsGi}}$ & 0.662 & Hill coefficient for Gs/Gi interaction of AC56 & $110^{@,(\mathrm{E})}$ \\
\hline $\mathrm{V}_{\mathrm{GsGi}}^{\mathrm{AC56}}$ & 0.857 & Maximum reduction in Gi inhibition by Gs & $110^{@,(\mathrm{E})}$ \\
\hline $\mathrm{K}_{\mathrm{m}, \mathrm{Gi}}^{\mathrm{AC} 56}$ & $0.0465 \mu \mathrm{mol} / \mathrm{L}$ & AC56 affinity for inhibition by Gi & $110^{@,(\mathrm{E})}$ \\
\hline AC56 $6_{\text {basal }}$ & $0.0377 \mu \mathrm{mol} / \mathrm{L}$ & Basal AC56 activity & $\%$ \\
\hline AF56 & $41.320 \mathrm{~s}^{-1}$ & Amplification factor for AC56 & $\%$ \\
\hline $\mathrm{K}_{\mathrm{m}, \mathrm{AC} 47}$ & $0.0314 \mu \mathrm{mol} / \mathrm{L}$ & AC47 affinity for Gs & $94^{\#,(\mathrm{E})}$ \\
\hline $\mathrm{h}_{\mathrm{AC} 47}$ & 1.004 & Hill coefficient for $\mathrm{AC} 47$ activation & $94^{\#,(E)}$ \\
\hline AC47 basal & 0.0315 & Basal AC47 activity & $94^{\#}$ \\
\hline AF47 & $3.3757 \mathrm{~s}^{-1}$ & Amplification factor for AC47 & $\%$ \\
\hline
\end{tabular}

\subsection{Phosphodiesterases}

Four phosphodiesterase isoforms (PDE1-4) have been identified in the mammalian heart. PDE1 is mostly found in non-myocyte cardiac cells ${ }^{118,119}$ and is therefore not included in our model. Considerable information is available about the relative distribution and activity of PDE2-4 in the canine ventricle. ${ }^{119} 80-90 \%$ of the PDE cAMP-hydrolizing activity in the dog is present in the cytosol. In the particulate fraction, PDE3 and PDE4 are present in a $78 \%$ to $22 \%$ ratio, whereby PDE3 is mostly associated with T-tubule and sarcoplasmic reticulum and PDE4 is associated with the sarcolemmal membrane. In the cytosol, PDE3 is responsible for $30-40 \%$ of the hydrolyzing activity. Furthermore, 
in dog myocardium PDE4 levels are 7-fold higher in cytosolic compared to particulate fractions. These relative activities are used to determine the subcellular distribution of PDEs in the model. IBMX is a non-specific pharmacological PDE inhibitor with different affinities for PDE2-4. ${ }^{120}$ We assume that IBMX inhibits PDEs equivalently in each compartment. The relative activities of the different PDE isoforms under basal conditions are similar to those reported by Rochais et al. in adult rat ventricular myocytes. ${ }^{121}$ However, opposite to the experimental data from the rat, the model shows a slightly larger contribution of PDE3 versus PDE4. This is consistent with experimental observations highlighting species differences between PDE isoforms in rodents (PDE4 dominant in mouse, similar PDE3/PDE4 in rat) and larger mammals (PDE3 dominant). ${ }^{119}$ Finally, the rate of cAMP hydrolysis by long isoforms of PDE3 and PDE4 is stimulated between 2 and 3-fold after phosphorylation by PKA. ${ }^{118}$ This results in an approximately $50 \%$ increase in whole-cell PDE activity in the presence of ISO. ${ }^{122}$

$$
\begin{aligned}
& \mathrm{r}_{\mathrm{PDE} 3, \mathrm{CYT}}=\frac{\mathrm{f}_{\mathrm{PDE} 3, \mathrm{CYT}}}{1-\mathrm{f}_{\mathrm{PDE} 3, \mathrm{CYT}}} \\
& \mathrm{f}_{\mathrm{PDE} 2, \mathrm{PART}}=\mathrm{f}_{\mathrm{PDE} 2}^{\mathrm{CAV}}+\mathrm{f}_{\mathrm{PDE} 2}^{\mathrm{ECAV}} \\
& \alpha_{\mathrm{PDE} 3}=\mathrm{r}_{\mathrm{PDE} 3, \mathrm{CYT}} \\
& \cdot\left(\mathrm{f}_{\mathrm{PDE} 4, \mathrm{PART}} \cdot\left(1+\mathrm{r}_{\mathrm{PDE} 3 . \mathrm{PDE} 4}-\mathrm{r}_{\mathrm{PDE} 3 . \mathrm{PDE} 4} \cdot \mathrm{f}_{\mathrm{PDE} 2, \mathrm{PART}}-\mathrm{f}_{\mathrm{PART}, \mathrm{PDE}}\right)\right. \\
& \left.+\mathrm{f}_{\mathrm{PDE} 2, \mathrm{PART}} \cdot\left(\mathrm{f}_{\mathrm{PART}, \mathrm{PDE}}-1\right)\right)+\mathrm{r}_{\mathrm{PDE} 3 . \mathrm{PDE} 4} \cdot \mathrm{f}_{\mathrm{PDE} 4, \mathrm{PART}} \\
& \text { - }\left(\mathrm{f}_{\text {PART,PDE }}-\mathrm{f}_{\text {PDE2,PART }}\right) \\
& \beta_{\text {PDE3 }}=f_{\text {PDE4,PART }} \cdot\left(1+r_{\text {PDE3.PDE4 }}+f_{\text {PART,PDE }} \cdot\left(r_{\text {PDE3,CYT }}-r_{\text {PDE3.PDE4 }}\right)\right)-f_{\text {PART,PDE }} \\
& \cdot\left(1+\mathrm{r}_{\mathrm{PDE} 3, \mathrm{CYT}}\right) \\
& {[\mathrm{PDE} 3]_{\mathrm{tot}}=\frac{\alpha_{\mathrm{PDE} 3}}{\beta_{\mathrm{PDE} 3}} \cdot[\mathrm{PDE} 2]_{\mathrm{tot}}} \\
& {[\text { PDE } 4]_{\text {tot }}=\frac{\left(\mathrm{f}_{\text {PART,PDE }}-\mathrm{f}_{\text {PDE2,PART }}\right) \cdot[\text { PDE2 }]_{\text {tot }}+f_{\text {PART,PDE }} \cdot[\text { PDE3 }]_{\text {tot }}}{\left(1+\mathrm{r}_{\text {PDE3.PDE } 4}\right) \cdot f_{\text {PDE4,PART }}-f_{\text {PART,PDE }}}} \\
& \mathrm{f}_{\mathrm{PDE} 4}^{\mathrm{ECAV}}=\mathrm{f}_{\mathrm{PDE} 4, \mathrm{PART}}-\mathrm{f}_{\mathrm{PDE} 4}^{\mathrm{CAV}} \\
& \mathrm{f}_{\mathrm{PDE} 3}^{\mathrm{CAV}}=\frac{\mathrm{r}_{\mathrm{PDE} 3 . \mathrm{PDE} 4} \cdot \mathrm{f}_{\mathrm{PDE} 4, \mathrm{PART}} \cdot[\mathrm{PDE} 4]_{\mathrm{tot}}}{[\mathrm{PDE} 3]_{\mathrm{tot}}} \\
& \mathrm{f}_{\mathrm{PDE} 3}^{\mathrm{CYT}}=1-\mathrm{f}_{\mathrm{PDE} 3}^{\mathrm{CAV}}
\end{aligned}
$$

For $x \in\{C A V, E C A V, C Y T\}:$

$$
\begin{aligned}
& {[\mathrm{PDE} 2]^{\mathrm{x}}=\left(1-\frac{[\mathrm{IBMX}]^{h_{I B M X}, P D E 2}}{\mathrm{~K}_{\mathrm{m}, \mathrm{PDE} 2}^{\mathrm{IBMX}}+[\mathrm{IBMX}]^{h_{I B M X, P D E 2}}}\right) \cdot \mathrm{f}_{\mathrm{PDE} 2}^{\mathrm{X}} \cdot[\mathrm{PDE} 2]_{\mathrm{tot}} \cdot \frac{\mathrm{V}^{\mathrm{CELL}}}{\mathrm{V}^{\mathrm{x}}}} \\
& {[\mathrm{PDE} 4]_{\mathrm{tot}}^{\mathrm{x}}=\left(1-\frac{[\mathrm{IBMX}]^{h_{I B M X}, P D E 4}}{\mathrm{~K}_{\mathrm{m}, \mathrm{PDE} 4}^{\mathrm{IBMX}}+[\mathrm{IBMX}]^{h_{I B M X, P D E 4}}}\right) \cdot \mathrm{f}_{\mathrm{PDE} 4}^{\mathrm{x}} \cdot[\mathrm{PDE} 4]_{\mathrm{tot}} \cdot \frac{\mathrm{V}^{\mathrm{CELL}}}{\mathrm{V}^{\mathrm{x}}}} \\
& \frac{\mathrm{d}[\mathrm{PDE} 4]_{\mathrm{p}}^{\mathrm{x}}}{\mathrm{dt}}=\mathrm{k}_{\mathrm{f}, \mathrm{PDEp}} \cdot[\mathrm{C}]^{\mathrm{x}} \cdot\left([\mathrm{PDE} 4]_{\mathrm{tot}}^{\mathrm{x}}-[\mathrm{PDE} 4]_{\mathrm{p}}^{\mathrm{x}}\right)-\mathrm{k}_{\mathrm{b}, \mathrm{PDEp}} \cdot[\mathrm{PDE} 4]_{\mathrm{p}}^{\mathrm{x}}
\end{aligned}
$$


$\frac{\mathrm{dcAMP}_{\mathrm{PDE} 2}^{\mathrm{x}}}{\mathrm{dt}}=\frac{\mathrm{k}_{\mathrm{PDE} 2} \cdot[\mathrm{PDE} 2]^{\mathrm{x}} \cdot[\mathrm{cAMP}]^{\mathrm{x}}}{\mathrm{K}_{\mathrm{m}, \mathrm{PDE} 2}+[\mathrm{cAMP}]^{\mathrm{x}}}$

$\frac{\mathrm{dcAMP}}{\mathrm{Pt}}=\frac{\mathrm{k}_{\mathrm{PDE} 4}^{\mathrm{X}} \cdot\left([\mathrm{PDE} 4]_{\mathrm{tot}}^{\mathrm{X}}-[\mathrm{PDE} 4]_{\mathrm{p}}^{\mathrm{x}}\right) \cdot[\mathrm{cAMP}]^{\mathrm{x}}+\Delta_{\mathrm{k}, \mathrm{PDE} 3 / 4} \cdot \mathrm{k}_{\mathrm{PDE} 4} \cdot[\mathrm{PDE} 4]_{\mathrm{p}}^{\mathrm{X}} \cdot[\mathrm{cAMP}]^{\mathrm{x}}}{\mathrm{K}_{\mathrm{m}, \mathrm{PDE} 4}+[\mathrm{CAMP}]^{\mathrm{x}}}$

For $x \in\{C A V, C Y T\}:$

$$
\begin{aligned}
{[\mathrm{PDE} 3]_{\mathrm{tot}}^{\mathrm{x}} } & =\left(1-\frac{[\mathrm{IBMX}]^{h_{I B M X}, \text {PDE3}}}{\mathrm{K}_{\mathrm{m}, \mathrm{PDE} 3}^{\mathrm{IBMX}}+[\mathrm{IBMX}]^{h_{I B M X, P D E 3}}}\right) \cdot \mathrm{f}_{\mathrm{PDE} 3}^{\mathrm{x}} \cdot[\mathrm{PDE} 3]_{\mathrm{tot}} \cdot \frac{\mathrm{V}^{\mathrm{CELL}}}{\mathrm{V}^{\mathrm{x}}} \\
\frac{\mathrm{d}[\mathrm{PDE} 3]_{\mathrm{p}}^{\mathrm{x}}}{\mathrm{dt}} & =\mathrm{k}_{\mathrm{f}, \mathrm{PDEp}} \cdot[\mathrm{C}]^{\mathrm{x}} \cdot\left([\mathrm{PDE} 3]_{\mathrm{tot}}^{\mathrm{x}}-[\mathrm{PDE} 3]_{\mathrm{p}}^{\mathrm{x}}\right)-\mathrm{k}_{\mathrm{b}, \mathrm{PDEp}} \cdot[\mathrm{PDE} 3]_{\mathrm{p}}^{\mathrm{x}} \\
\frac{\mathrm{dcAMP} \mathrm{PDE} 3}{\mathrm{dt}} & =\frac{\mathrm{k}_{\mathrm{PDE} 3} \cdot\left([\mathrm{PDE} 3]_{\mathrm{tot}}^{\mathrm{x}}-[\mathrm{PDE} 3]_{\mathrm{p}}^{\mathrm{x}}\right) \cdot[\mathrm{cAMP}]^{\mathrm{x}}+\Delta_{\mathrm{k}, \mathrm{PDE} 3 / 4} \cdot \mathrm{k}_{\mathrm{PDE} 3} \cdot[\mathrm{PDE} 3]_{\mathrm{p}}^{\mathrm{x}} \cdot[\mathrm{cAMP}]^{\mathrm{x}}}{\mathrm{K}_{\mathrm{m}, \mathrm{PDE} 3}+[\mathrm{cAMP}]^{\mathrm{x}}}
\end{aligned}
$$

\begin{tabular}{|c|c|c|c|}
\hline Parameter & Value & Description & Ref \\
\hline$[\mathrm{PDE} 2]_{\text {tot }}$ & $\begin{array}{l}2.9268 \mathrm{E}-2 \\
\mu \mathrm{mol} / \mathrm{L}\end{array}$ & Total cellular concentration of PDE2 & $\%$ \\
\hline $\mathrm{f}_{\mathrm{PDE} 2}^{\mathrm{CAV}}$ & 0.1696 & Fraction of PDE2 located in caveolar compartment & $\%$ \\
\hline $\mathrm{f}_{\mathrm{PDE} 2}^{\mathrm{ECAV}}$ & $2.1257 \mathrm{E}-4$ & $\begin{array}{l}\text { Fraction of PDE2 located in extracaveolar } \\
\text { compartment }\end{array}$ & $\%$ \\
\hline $\mathrm{f}_{\mathrm{PDE} 2}^{\mathrm{CYT}}$ & $\begin{array}{l}1-\mathrm{f}_{\mathrm{PDE}}^{\mathrm{CAV}} \\
-\mathrm{f}_{\mathrm{PDE}}^{\mathrm{ECAV}} \\
\end{array}$ & Fraction of PDE2 located in cytosolic compartment & \\
\hline $\mathrm{f}_{\mathrm{PDE} 3, \mathrm{CYT}}$ & 0.35 & Fraction of PDE in cytosol that is of type 3 & $119^{\#,(C)}$ \\
\hline $\mathrm{f}_{\mathrm{PDE} 4, \mathrm{PART}}$ & 0.125 & Fraction of PDE4 located in the particulate fraction & $119^{\#,(C)}$ \\
\hline $\mathrm{f}_{\text {PART,PDE }}$ & 0.2 & Fraction of total PDE located in the particulate fraction & $119^{\#,(C)}$ \\
\hline $\mathrm{r}_{\mathrm{PDE} 3, \mathrm{PDE} 4}$ & 3.71 & Ratio of PDE3 to PDE4 in the particulate fraction & $119^{\#,(C)}$ \\
\hline $\mathrm{f}_{\mathrm{PDE} 4}^{\mathrm{CAV}}$ & 0.1248 & Fraction of PDE4 located in caveolar compartment & $\%$ \\
\hline $\mathrm{f}_{\mathrm{PDE} 4}^{\mathrm{CYT}}$ & $1-\mathrm{f}_{\mathrm{PDE} 4, \text { part }}$ & Fraction of PDE4 located in cytosolic compartment & \\
\hline [IBMX] & $0 \ldots 100 \mu \mathrm{mol} / \mathrm{L}$ & Concentration of IBMX & \\
\hline $\mathrm{h}_{\text {IBMX,PDE2 }}$ & 1.167 & Hill coefficient for inhibition of PDE2 by IBMX & $120^{@,(G)}$ \\
\hline $\mathrm{K}_{\mathrm{PDE} 2}^{\mathrm{IBMX}}$ & $21.58 \mu \mathrm{mol} / \mathrm{L}$ & Affinity of IBMX for PDE2 & $120^{@,(G)}$ \\
\hline $\mathrm{h}_{\text {IBMX,PDE3 }}$ & 0.7629 & Hill coefficient for inhibition of PDE3 by IBMX & $120^{@,(G)}$ \\
\hline $\mathrm{K}_{\mathrm{PDE} 3}^{\mathrm{IBMX}}$ & $2.642 \mu \mathrm{mol} / \mathrm{L}$ & Affinity of IBMX for PDE3 & $120^{@,(G)}$ \\
\hline $\mathrm{h}_{\text {IBMX,PDE4 }}$ & 0.9024 & Hill coefficient for inhibition of PDE4 by IBMX & $120^{@,(G)}$ \\
\hline $\mathrm{K}_{\mathrm{PDE} 4}^{\mathrm{IBMX}}$ & $11.89 \mu \mathrm{mol} / \mathrm{L}$ & Affinity of IBMX for PDE4 & $120^{@,(G)}$ \\
\hline $\mathrm{k}_{\mathrm{f}, \mathrm{PDEp}}$ & $\begin{array}{c}0.0196 \mu \mathrm{mol} / \mathrm{L}^{-} \\
{ }_{1} \mathrm{~s}^{-1}\end{array}$ & Rate of phosphorylation by PKA of PDE3 and PDE4 & $123^{\&}$ \\
\hline
\end{tabular}




\begin{tabular}{|cclc|}
\hline $\mathrm{k}_{\mathrm{b}, \text { PDEp }}$ & $0.0102 \mathrm{~s}^{-1}$ & Rate of dephosphorylation of PDE3 and PDE4 & $123^{\&}$ \\
\hline$\Delta_{\mathrm{k}, \mathrm{PDE} / 4}$ & 3.0 & Increase in PDE3 / PDE4 activity after phosphorylation & $118^{\#}$ \\
\hline $\mathrm{k}_{\mathrm{PDE} 2}$ & $20 \mathrm{~s}^{-1}$ & Rate of cAMP hydrolysis by PDE2 & $106^{\&}$ \\
\hline $\mathrm{K}_{\mathrm{m}, \mathrm{PDE} 2}$ & $50 \mu \mathrm{mol} / \mathrm{L}$ & Affinity of PDE2 for cAMP & $119^{\#,(\mathrm{C})}$ \\
\hline $\mathrm{k}_{\mathrm{PDE} 3}$ & $2.5 \mathrm{~s}^{-1}$ & Rate of cAMP hydrolysis by PDE3 & 106, \\
\hline $\mathrm{K}_{\mathrm{m}, \mathrm{PDE} 3}$ & $0.8 \mu \mathrm{mol} / \mathrm{L}$ & Affinity of PDE3 for cAMP & $124^{\&}$ \\
\hline $\mathrm{k}_{\mathrm{PDE} 4}$ & $4.0 \mathrm{~s}^{-1}$ & Rate of cAMP hydrolysis by PDE4 & $119^{\#,(\mathrm{C})}$ \\
\hline $\mathrm{K}_{\mathrm{m}, \mathrm{PDE} 4}$ & $1.4 \mu \mathrm{mol} / \mathrm{L}$ & Affinity of PDE4 for cAMP & 106, \\
\hline
\end{tabular}

\subsection{PKA}

A concentration of protein kinase A (PKA) is located in each compartment. We assume that a cAMP molecule can only bind to the first binding site of a regulatory subunit, which causes a conformational change that makes it possible for a second CAMP molecule to bind to the second binding site. Once both binding sites are occupied, the $\mathrm{C}$ subunit can dissociate. ${ }^{125}$ Furthermore, we assume that both $\mathrm{R}$ subunits are identical and operate independently. ${ }^{102,}{ }^{126}$ Therefore, only R-C dimer states are modeled. Inhibition of PKA catalytic subunit by PKI is included with an apparent affinity of $0.2 \mathrm{nM}^{127}$ The total amount of PKI in the cell is sufficient to inhibit at most $20 \%$ of the $\mathrm{PKA}^{127}$ and is assumed to have the same distribution as PKA.

In the heart, PKA is made up of two different isoforms which differ in their regulatory subunits (RI and RII). Distribution of the isoforms is species-dependent, but in general PKA type I is assumed to be mainly cytosolic whereas PKA type II is mainly found in the particulate fraction. ${ }^{128,129}$ Accordingly, we define the PKA in the caveolar and extracaveolar compartments to be of type II, and the PKA in the cytosolic compartment to be of type I. The different isoforms have similar affinities for the binding between $\mathrm{R}$ and $\mathrm{C}$ subunits (although rate constants for type II are approximately six times faster than type I), ${ }^{130}$ but differ in their affinities for activation by cAMP. ${ }^{131}$ Therefore, the affinities for the two CAMP binding reactions are significantly higher in the cytosolic compartment in order to reproduce the cAMP dose-response curve for PKA type I. In contrast to the RI subunit, the RII subunit can be phosphorylated by the catalytic subunit on Ser-96, which affects RII-C binding affinity, affinity of binding between PKA and A-kinase anchoring proteins (AKAPs) and substrate phosphorylation. ${ }^{132}$ However, under normal physiological conditions, RII is almost completely phosphorylated both in the absence and presence of $\beta A R$ stimulation by isoproterenol ${ }^{132,}{ }^{133}$ and therefore (de)phosphorylation of RII is not explicitly incorporated in our model.

For $x \in\{C A V, E C A V\}:$

$$
[\mathrm{PKA}]^{\mathrm{x}}=\mathrm{f}_{\mathrm{PKA}}^{\mathrm{x}} \cdot[\mathrm{PKA}]_{\mathrm{tot}} \cdot \frac{\mathrm{V}^{\mathrm{CELL}}}{\mathrm{V}^{\mathrm{x}}}
$$




$$
\begin{aligned}
& {[\mathrm{RC}]_{\mathrm{f}}^{\mathrm{x}}=2 \cdot[\mathrm{PKA}]^{\mathrm{x}}-[\mathrm{ARC}]^{\mathrm{x}}-\left[\mathrm{A}_{2} \mathrm{RC}\right]^{\mathrm{x}}-\left[\mathrm{A}_{2} \mathrm{R}\right]^{\mathrm{x}}} \\
& {[\mathrm{PKI}]_{\mathrm{f}}^{\mathrm{x}}=\mathrm{f}_{\mathrm{PKI}}^{\mathrm{x}} \cdot[\mathrm{PKI}]_{\mathrm{tot}} \cdot \frac{\mathrm{V}^{\mathrm{CELL}}}{\mathrm{V}^{\mathrm{x}}}-[\mathrm{PKIC}]^{\mathrm{x}}} \\
& \mathrm{k}_{\text {PKAII,b1 }}=\mathrm{k}_{\text {PKAII,f1 }} \cdot \mathrm{K}_{\text {PKAII,1 }} \\
& \mathrm{k}_{\mathrm{PKAII}, \mathrm{b} 2}=\mathrm{k}_{\mathrm{PKAII}, \mathrm{f} 2} \cdot \mathrm{K}_{\mathrm{PKAII}, 2} \\
& \mathrm{k}_{\mathrm{PKAII}, \mathrm{b} 3}=\mathrm{k}_{\mathrm{PKAII}, \mathrm{f3}} \cdot \mathrm{K}_{\mathrm{PKAII}, 3} \\
& \mathrm{k}_{\mathrm{PKI}, \mathrm{b}}=\mathrm{k}_{\mathrm{PKI}, \mathrm{f}} \cdot \mathrm{K}_{\mathrm{PKI}} \\
& \frac{\mathrm{d}[\mathrm{cAMP}]_{\mathrm{PKA}}^{\mathrm{x}}}{\mathrm{dt}}=-\mathrm{k}_{\mathrm{PKAII}, \mathrm{f} 1} \cdot[\mathrm{RC}]_{\mathrm{f}}^{\mathrm{x}} \cdot[\mathrm{cAMP}]^{\mathrm{x}}+\mathrm{k}_{\mathrm{PKAII}, \mathrm{b} 1} \cdot[\mathrm{ARC}]^{\mathrm{x}}-\mathrm{k}_{\mathrm{PKAII}, \mathrm{f} 2} \cdot[\mathrm{ARC}]^{\mathrm{x}} \cdot[\mathrm{cAMP}]^{\mathrm{x}} \\
& +\mathrm{k}_{\mathrm{PKAI}, \mathrm{b} 2} \cdot\left[\mathrm{A}_{2} \mathrm{RC}\right]^{\mathrm{x}} \\
& \frac{\mathrm{d}[\mathrm{ARC}]^{\mathrm{x}}}{\mathrm{dt}}=\mathrm{k}_{\mathrm{PKAII}, \mathrm{f} 1} \cdot[\mathrm{RC}]_{\mathrm{f}}^{\mathrm{x}} \cdot[\mathrm{CAMP}]^{\mathrm{x}}-\mathrm{k}_{\mathrm{PKAII}, \mathrm{b} 1} \cdot[\mathrm{ARC}]^{\mathrm{x}}-\mathrm{k}_{\mathrm{PKAII}, \mathrm{f2} 2} \cdot[\mathrm{ARC}]^{\mathrm{x}} \cdot[\mathrm{cAMP}]^{\mathrm{x}} \\
& +\mathrm{k}_{\mathrm{PKAII}, \mathrm{b} 2} \cdot\left[\mathrm{A}_{2} \mathrm{RC}\right]^{\mathrm{x}} \\
& \frac{\mathrm{d}\left[\mathrm{A}_{2} \mathrm{RC}\right]^{\mathrm{x}}}{\mathrm{dt}}=\mathrm{k}_{\mathrm{PKAII}, \mathrm{f2} 2} \cdot[\mathrm{ARC}]^{\mathrm{x}} \cdot[\mathrm{cAMP}]^{\mathrm{x}}-\left(\mathrm{k}_{\mathrm{PKAII}, \mathrm{b} 2}+\mathrm{k}_{\mathrm{PKAII}, \mathrm{f3}}\right) \cdot\left[\mathrm{A}_{2} \mathrm{RC}\right]^{\mathrm{x}}+\mathrm{k}_{\mathrm{PKAII}, \mathrm{b} 3} \cdot\left[\mathrm{A}_{2} \mathrm{R}\right]^{\mathrm{x}} \\
& \cdot[\mathrm{C}]^{\mathrm{x}} \\
& \frac{\mathrm{d}\left[\mathrm{A}_{2} \mathrm{R}\right]^{\mathrm{x}}}{\mathrm{dt}}=\mathrm{k}_{\text {PKAII, }, 33} \cdot\left[\mathrm{A}_{2} \mathrm{RC}\right]^{\mathrm{x}}-\mathrm{k}_{\text {PKAII }, \mathrm{b} 3} \cdot\left[\mathrm{A}_{2} \mathrm{R}\right]^{\mathrm{x}} \cdot[\mathrm{C}]^{\mathrm{x}} \\
& \frac{\mathrm{d}[\mathrm{C}]^{\mathrm{x}}}{\mathrm{dt}}=\mathrm{k}_{\mathrm{PKAII}, \mathrm{f3}} \cdot\left[\mathrm{A}_{2} \mathrm{RC}\right]^{\mathrm{x}}-\mathrm{k}_{\mathrm{PKAII}, \mathrm{b} 3} \cdot\left[\mathrm{A}_{2} \mathrm{R}\right]^{\mathrm{x}} \cdot[\mathrm{C}]^{\mathrm{x}}+\mathrm{k}_{\mathrm{PKI}, \mathrm{b}} \cdot[\mathrm{PKIC}]^{\mathrm{x}}-\mathrm{k}_{\mathrm{PKI}, \mathrm{f}} \cdot[\mathrm{PKI}]_{\mathrm{f}}^{\mathrm{x}} \cdot[\mathrm{C}]^{\mathrm{x}} \\
& \frac{\mathrm{d}[\mathrm{PKIC}]^{\mathrm{x}}}{\mathrm{dt}}=-\mathrm{k}_{\mathrm{PKI}, \mathrm{b}} \cdot[\mathrm{PKIC}]^{\mathrm{x}}+\mathrm{k}_{\mathrm{PKI}, \mathrm{f}} \cdot[\mathrm{PKI}]_{\mathrm{f}}^{\mathrm{x}} \cdot[\mathrm{C}]^{\mathrm{x}}
\end{aligned}
$$

For $x=C Y T:$

$$
\begin{aligned}
{[\mathrm{PKA}]^{\mathrm{x}}=} & \mathrm{f}_{\mathrm{PKA}}^{\mathrm{x}} \cdot[\mathrm{PKA}]_{\mathrm{tot}} \cdot \frac{\mathrm{V}^{\mathrm{CELL}}}{\mathrm{V}^{\mathrm{x}}} \\
{[\mathrm{RCC}]_{\mathrm{f}}^{\mathrm{x}}=} & 2 \cdot[\mathrm{PKA}]^{\mathrm{x}}-[\mathrm{ARC}]^{\mathrm{x}}-\left[\mathrm{A}_{2} \mathrm{RC}\right]^{\mathrm{x}}-\left[\mathrm{A}_{2} \mathrm{R}\right]^{\mathrm{x}} \\
{[\mathrm{PKI}]_{\mathrm{f}}^{\mathrm{x}}=} & \mathrm{f}_{\mathrm{PKI}}^{\mathrm{x}} \cdot[\mathrm{PKI}]_{\mathrm{tot}} \cdot \frac{\mathrm{V}^{\mathrm{CELL}}}{\mathrm{V}^{\mathrm{x}}-[\mathrm{PKIC}]^{\mathrm{x}}} \\
\mathrm{k}_{\mathrm{PKAI}, \mathrm{b} 1}= & \mathrm{k}_{\mathrm{PKAI}, \mathrm{f} 1} \cdot \mathrm{K}_{\mathrm{PKAI}, 1} \\
\mathrm{k}_{\mathrm{PKAI}, \mathrm{b} 2=}= & \mathrm{k}_{\mathrm{PKAI}, \mathrm{f} 2} \cdot \mathrm{K}_{\mathrm{PKAI}, 2} \\
\mathrm{k}_{\mathrm{PKAI}, \mathrm{b} 3=}= & \mathrm{k}_{\mathrm{PKAI}, \mathrm{f3} 3} \cdot \mathrm{K}_{\mathrm{PKAI}, 3} \\
\frac{\mathrm{d}[\mathrm{cAMP}]_{\mathrm{PKA}}^{\mathrm{x}}=}{\mathrm{dt}} & -\mathrm{k}_{\mathrm{PKAI}, \mathrm{f} 1} \cdot[\mathrm{RC}]_{\mathrm{f}}^{\mathrm{x}} \cdot[\mathrm{cAMP}]^{\mathrm{x}}+\mathrm{k}_{\mathrm{PKAI}, \mathrm{b} 1} \cdot[\mathrm{ARC}]^{\mathrm{x}}-\mathrm{k}_{\mathrm{PKAI}, \mathrm{f} 2} \cdot[\mathrm{ARC}]^{\mathrm{x}} \cdot[\mathrm{CAMP}]^{\mathrm{x}} \\
& +\mathrm{k}_{\mathrm{PKAI}, \mathrm{b} 2} \cdot\left[\mathrm{A}_{2} \mathrm{RC}\right]^{\mathrm{x}} \\
\frac{\mathrm{d}[\mathrm{ARC}]^{\mathrm{x}}}{\mathrm{dt}}= & \mathrm{k}_{\mathrm{PKAI}, \mathrm{f} 1} \cdot[\mathrm{RC}]_{\mathrm{f}}^{\mathrm{x}} \cdot[\mathrm{cAMP}]^{\mathrm{x}}-\mathrm{k}_{\mathrm{PKAI}, \mathrm{b} 1} \cdot[\mathrm{ARC}]^{\mathrm{x}}-\mathrm{k}_{\mathrm{PKAI}, \mathrm{f} 2} \cdot[\mathrm{ARC}]^{\mathrm{x}} \cdot[\mathrm{cAMP}]^{\mathrm{x}} \\
& +\mathrm{k}_{\mathrm{PKAI}, \mathrm{b} 2} \cdot\left[\mathrm{A}_{2} \mathrm{RC}\right]^{\mathrm{x}}
\end{aligned}
$$




$$
\begin{aligned}
\frac{\mathrm{d}\left[\mathrm{A}_{2} \mathrm{RC}\right]^{\mathrm{x}}}{\mathrm{dt}}= & \mathrm{k}_{\mathrm{PKAI}, \mathrm{f} 2} \cdot[\mathrm{ARC}]^{\mathrm{x}} \cdot[\mathrm{CAMP}]^{\mathrm{x}}-\left(\mathrm{k}_{\mathrm{PKAI}, \mathrm{b} 2}+\mathrm{k}_{\mathrm{PKAI}, \mathrm{f3}}\right) \cdot\left[\mathrm{A}_{2} \mathrm{RC}\right]^{\mathrm{x}}+\mathrm{k}_{\mathrm{PKAI}, \mathrm{b} 3} \cdot\left[\mathrm{A}_{2} \mathrm{R}\right]^{\mathrm{x}} \\
& \cdot[\mathrm{C}]^{\mathrm{x}} \\
\frac{\mathrm{d}\left[\mathrm{A}_{2} \mathrm{R}\right]^{\mathrm{x}}}{\mathrm{dt}}= & \mathrm{k}_{\mathrm{PKAI}, \mathrm{f3}} \cdot\left[\mathrm{A}_{2} \mathrm{RC}\right]^{\mathrm{x}}-\mathrm{k}_{\mathrm{PKAI}, \mathrm{b} 3} \cdot\left[\mathrm{A}_{2} \mathrm{R}\right]^{\mathrm{x}} \cdot[\mathrm{C}]^{\mathrm{x}} \\
\frac{\mathrm{d}[\mathrm{C}]^{\mathrm{x}}}{\mathrm{dt}}= & \mathrm{k}_{\mathrm{PKAI}, \mathrm{f3}} \cdot\left[\mathrm{A}_{2} \mathrm{RC}\right]^{\mathrm{x}}-\mathrm{k}_{\mathrm{PKAI}, \mathrm{b} 3} \cdot\left[\mathrm{A}_{2} \mathrm{R}\right]^{\mathrm{x}} \cdot[\mathrm{C}]^{\mathrm{x}}+\mathrm{k}_{\mathrm{PKI}, \mathrm{b}} \cdot[\mathrm{PKIC}]^{\mathrm{x}}-\mathrm{k}_{\mathrm{PKI}, \mathrm{f}} \cdot[\mathrm{PKI}]_{\mathrm{f}}^{\mathrm{x}} \\
& \cdot[\mathrm{C}]^{\mathrm{x}} \\
\frac{\mathrm{d}[\mathrm{PKIC}]^{\mathrm{x}}}{\mathrm{dt}}= & -\mathrm{k}_{\mathrm{PKI}, \mathrm{b}} \cdot[\mathrm{PKIC}]^{\mathrm{x}}+\mathrm{k}_{\mathrm{PKI}, \mathrm{f}} \cdot[\mathrm{PKI}]_{\mathrm{f}}^{\mathrm{x}} \cdot[\mathrm{C}]^{\mathrm{x}}
\end{aligned}
$$

\begin{tabular}{|cclc|}
\hline Parameter & Value & Description & Ref \\
\hline$[\text { PKA }]_{\text {tot }}$ & $0.25 \mu \mathrm{mol} / \mathrm{L}$ & Total cellular concentration of PKA holoenzyme & 134, \\
$135^{\#,(A)}$
\end{tabular}




\begin{tabular}{|cclc|}
\hline $\mathrm{K}_{\mathrm{PKAI}, 3}$ & $\mathrm{~K}_{\mathrm{PKAII}, 3}$ & Equilibrium value for dissociation of $\mathrm{C}$ subunit & $130^{\#}$ \\
\hline $\mathrm{k}_{\mathrm{PKI}, \mathrm{f}}$ & $50 \mu \mathrm{mol} / \mathrm{L}^{-1} \mathrm{~s}^{-}$ & Forward rate for inhibition of $\mathrm{C}$ subunit by PKI & $136^{\&}$ \\
\hline $\mathrm{K}_{\mathrm{PKI}}$ & $2.0 \mathrm{E}-4 \mu \mathrm{mol} / \mathrm{L}$ & Equilibrium value for inhibition of C subunit by PKI & $127^{\#}$ \\
\hline
\end{tabular}

\section{7 cAMP}

As in lancu et al., ${ }^{94}$ CAMP can diffuse between the three compartments. The rate of change in free cAMP concentration in each compartment depends on the production of CAMP by ACs, its breakdown by PDEs, buffering by PKA and transfer between compartments.

$$
\begin{aligned}
& \frac{d[c A M P]^{C A V}}{d t}=\frac{d c A M P_{P K A}^{C A V}}{d t}+\frac{d c A M P_{A C 56}^{C A V}}{d t}-\frac{d c A M P_{P D E 2}^{C A V}}{d t}-\frac{d c A M P_{P D E 3}^{C A V}}{d t}-\frac{d c A M P_{P D E 4}^{C A V}}{d t}-J_{C A V / E C A V} \\
& \cdot \frac{[\mathrm{CAMP}]^{\mathrm{CAV}}-[\mathrm{CAMP}]^{\mathrm{ECAV}}}{\mathrm{V}^{\mathrm{CAV}}}-\mathrm{J}_{\mathrm{CAV} / \mathrm{CYT}} \cdot \frac{[\mathrm{CAMP}]^{\mathrm{CAV}}-\text { [CAMP }^{\mathrm{CYT}}}{\mathrm{V}^{\mathrm{CAV}}} \\
& \frac{d[c A M P]^{E C A V}}{d t}=\frac{d c A M P_{P K A}^{E C A V}}{d t}+\frac{d c A M P_{A C 47}^{E C A V}}{d t}-\frac{d c A M P_{P D E 2}^{E C A V}}{d t}-\frac{d c A M P_{P D E 4}^{E C A V}}{d t}+J_{C A V / E C A V} \\
& \cdot \frac{[\mathrm{CAMP}]^{\mathrm{CAV}}-[\mathrm{cAMP}]^{\mathrm{ECAV}}}{\mathrm{V}^{\mathrm{ECAV}}}-\mathrm{J}_{\mathrm{ECAV} / \mathrm{CYT}} \cdot \frac{[\mathrm{CAMP}]^{\mathrm{ECAV}}-[\mathrm{cAMP}]^{\mathrm{CYT}}}{\mathrm{V}^{\mathrm{ECAV}}} \\
& \frac{\mathrm{d}[\mathrm{cAMP}]^{\mathrm{CYT}}}{\mathrm{dt}}=\frac{\mathrm{dcAMP}_{\mathrm{PKA}}^{\mathrm{CYT}}}{\mathrm{dt}}+\frac{\mathrm{dcAMP}_{\mathrm{AC56}}^{\mathrm{CYT}}}{\mathrm{dt}}+\frac{\mathrm{dcAMP}_{\mathrm{AC} 47}^{\mathrm{CYT}}}{\mathrm{dt}}-\frac{\mathrm{dcAMP}_{\mathrm{PDE} 2}^{\mathrm{CYT}}}{\mathrm{dt}}-\frac{\mathrm{dcAMP}_{\mathrm{PDE} 3}^{\mathrm{CYT}}}{\mathrm{dt}}-\frac{\mathrm{dcAMP}_{\mathrm{PDE} 4}^{\mathrm{CYT}}}{\mathrm{dt}} \\
& +\mathrm{J}_{\mathrm{CAV} / \mathrm{CYT}} \cdot \frac{[\mathrm{cAMP}]^{\mathrm{CAV}}-[\mathrm{cAMP}]^{\mathrm{CYT}}}{\mathrm{V}^{\mathrm{CYT}}}-\mathrm{J}_{\mathrm{ECAV} / \mathrm{CYT}} \cdot \frac{[\mathrm{cAMP}]^{\mathrm{ECAV}}-[\mathrm{cAMP}]^{\mathrm{CYT}}}{\mathrm{V}^{\mathrm{CYT}}}
\end{aligned}
$$

\begin{tabular}{|cclc|}
\hline Parameter & Value & Description & Ref \\
\hline $\mathrm{J}_{\mathrm{CAV} / \mathrm{CYT}}$ & $\begin{array}{c}7.500 \mathrm{E}-8 \mu \mathrm{L} \mathrm{s} \\
1\end{array}$ & $\begin{array}{l}\text { Rate of cAMP diffusion between caveolar and cytosolic } \\
\text { compartments }\end{array}$ & $106^{\#}$ \\
\hline $\mathrm{J}_{\mathrm{CAV} / \mathrm{ECAV}}$ & $\begin{array}{c}5.000 \mathrm{E}-9 \mu \mathrm{L} \mathrm{s} \\
1\end{array}$ & $\begin{array}{l}\text { Rate of cAMP diffusion between caveolar and } \\
\text { extracaveolar compartments }\end{array}$ & $106^{\#}$ \\
\hline $\mathrm{J}_{\mathrm{ECAV} / \mathrm{CYT}}$ & $\begin{array}{c}0.900 \mathrm{E}-8 \mu \mathrm{L} \mathrm{s} \\
1\end{array}$ & $\begin{array}{l}\text { Rate of cAMP diffusion between extracaveolar and } \\
\text { cytosolic compartments }\end{array}$ & $106^{\#}$ \\
\hline
\end{tabular}

\subsection{Protein phosphatase inhibitor}

PP1 is inhibited by the phosphorylated form of inhibitor protein $1,{ }^{137}$ thereby providing a positive feedback loop for PKA-dependent substrate phosphorylation. Inhibitor protein 1 is only found in the cytosolic compartment and has a very high affinity for PP1 $(1 \mathrm{nM}) .{ }^{137}$ As such, PP1-Inhibitor binding is assumed to be instantaneous and modeled by an algebraic equation. Knockout of inhibitor protein 1 resulted in a $23 \%$ increase in PP1 activity in the isoproterenol-stimulated mouse heart. ${ }^{138}$ In the model, total concentration of inhibitor 1 is chosen to produce a similar effect. Inhibitor protein 1 does not alter $\mathrm{I}_{\mathrm{CaL}}$ or $\mathrm{Tnl}$ phosphorylation. ${ }^{138} \mathrm{In}$ the model, the absence of $\mathrm{I}_{\mathrm{CaL}}$ effects results from the 
different subcellular localization whereas the absence of Tnl effects can be explained by the fact that PP2A (which is not inhibited by inhibitor protein 1 ) is the major phosphatase dephosphorylating Tnl.

$$
\begin{aligned}
& {[\text { Inhib1 }]_{\text {tot }}^{\text {CYT }}=\frac{f_{P P 1, I n h 1}}{1-f_{P P 1, I n h 1}} \cdot \mathrm{K}_{\text {Inh1 }}+f_{\text {PP1,Inh1 }} \cdot[\text { PP1 }]_{\text {tot }}^{\text {CYT }}} \\
& \frac{\mathrm{d}[\text { Inhib1 }]_{\mathrm{p}}^{\mathrm{CYT}}}{\mathrm{dt}}=\frac{\mathrm{k}_{\text {Inh1p }} \cdot[\mathrm{C}]^{\mathrm{CYT}} \cdot\left([\text { Inhib1 }]_{\mathrm{tot}}^{\mathrm{CYT}}-[\text { Inhib1 }]_{\mathrm{p}}^{\mathrm{CYT}}\right)}{\mathrm{K}_{\text {Inh1p }}+\left([\text { Inhib1 }]_{\mathrm{tot}}^{\mathrm{CYT}}-[\text { Inhib1 }]_{\mathrm{p}}^{\mathrm{CYT}}\right)}-\frac{\mathrm{k}_{\text {Inh1dp }} \cdot[\mathrm{PP} 2 \mathrm{~A}]^{\mathrm{CYT}} \cdot[\text { Inhib1 }]_{\mathrm{p}}^{\mathrm{CYT}}}{\mathrm{K}_{\text {Inh1dp }}+[\text { Inhib1 }]_{\mathrm{p}}^{\mathrm{CYT}}} \\
& {[\mathrm{PP} 1]^{\mathrm{CYT}}=\frac{1}{2} \cdot \sqrt{\left(\mathrm{K}_{\mathrm{Inh} 1}-[\mathrm{PP} 1]_{\mathrm{tot}}^{\mathrm{CYT}}+[\mathrm{Inhib} 1]_{\mathrm{p}}^{\mathrm{CYT}}\right)^{2}+4 \cdot \mathrm{K}_{\mathrm{Inh} 1} \cdot[\mathrm{PP} 1]_{\mathrm{tot}}^{\mathrm{CYT}}}-\frac{1}{2}} \\
& \cdot\left(\mathrm{K}_{\mathrm{Inh} 1}-[\mathrm{PP} 1]_{\mathrm{tot}}^{\mathrm{CYT}}+[\text { Inhib1 }]_{\mathrm{p}}^{\mathrm{CYT}}\right)
\end{aligned}
$$

\begin{tabular}{|c|c|c|c|}
\hline Parameter & Value & Description & Ref \\
\hline $\mathrm{f}_{\mathrm{PP} 1, \mathrm{Inh} 1}$ & 0.3 & Fractional increase in PP1 after Inh1 knockout & $138^{\#,(\mathrm{M})}$ \\
\hline $\mathrm{k}_{\text {Inh1,p }}$ & $1.0145 \mathrm{E}-2 \mathrm{~s}^{-1}$ & Rate of phosphorylation of inhibitor 1 by PKA & $140^{@,(G)}$ \\
\hline $\begin{array}{l}\mathrm{k}_{\text {Inh1,dp }} \\
\cdot[\mathrm{PP} 2 \mathrm{~A}]^{\mathrm{CYT}}\end{array}$ & $\begin{array}{l}3.5731 \mathrm{E}-3 \\
\mu \mathrm{mol} / \mathrm{L} \mathrm{s}^{-1}\end{array}$ & Rate of dephosphorylation of inhibitor 1 & $140^{@,(G)}$ \\
\hline $\mathrm{K}_{\mathrm{Inh} 1, \mathrm{p}}$ & $\begin{array}{c}1.4690 \mathrm{E}-3 \\
\mu \mathrm{mol} / \mathrm{L}\end{array}$ & Affinity of inhibitor 1 for PKA catalytic subunit & $140^{@,(G)}$ \\
\hline $\mathrm{K}_{\mathrm{Inh} 1, \mathrm{dp}}$ & $\begin{array}{c}1.9526 \mathrm{E}-5 \\
\mu \mathrm{mol} / \mathrm{L}\end{array}$ & Affinity of inhibitor 1 for PP2A & $140^{@,(G)}$ \\
\hline$[\mathrm{PP} 1]_{\mathrm{tot}}^{\mathrm{CYT}}$ & $0.2 \mu \mathrm{mol} / \mathrm{L}$ & Total concentration of PP1 in the cytosolic compartment & $\%$ \\
\hline $\mathrm{K}_{\mathrm{Inh} 1}$ & $1.0 \mathrm{E}-3 \mu \mathrm{mol} / \mathrm{L}$ & Affinity for PP1 - Inhibitor 1 binding & $137^{\#}$ \\
\hline
\end{tabular}

\subsection{Substrate phosphorylation}

The substrates are divided into those with and without AKAPs. For the substrates without AKAPs, a standard Michaelis-Menten formalism for phosphorylation is implemented. For substrates with AKAPs, a steady-state distribution is calculated for AKAP / Channel complexes, AKAP / Channel / PKA complexes, AKAP / Channel / phosphatase complexes and AKAP / Channel / PKA / phosphatase complexes. Although some AKAPs also bind PDEs, ${ }^{141}$ we do not incorporate this explicitly in our model and only consider the effects of an AKAP on its associated channel. Different AKAPs bind PKA with different affinities in the range of $2-90 \mathrm{nM} .{ }^{142}$ We use an intermediate affinity of $10 \mathrm{nM}$ for all AKAPs and assume this affinity is similar for the binding of PP1. AKAPs and channels are assumed to have a 1:1 stoichiometry and high affinity $(0.1 \mathrm{nM})$ under normal physiological conditions ${ }^{124}$ (although mutations in both channel ${ }^{143}$ and AKAP ${ }^{144}$ have been described that greatly reduce this affinity). 
AKAPs are critical for reliable (de)phosphorylation of their associated channels. Moreover, it is known for the $I_{K s}$ channel that the AKAP yotiao also has an active role in mediating the phosphorylation effect on channel gating and phosphomimetic mutations in the $I_{\mathrm{Ks}}$ channel do not result in gating changes in the absence of yotiao. ${ }^{143,145}$ Based on this data, the model assumes that, for substrates with AKAPs ( $\left.I_{\mathrm{Ks}}, I_{\mathrm{CaL}}, \mathrm{RyR}\right)$, phosphorylation and dephosphorylation only occur in channels with AKAP. Channels with AKAP and PKA are constitutively phosphorylated whereas channels with AKAP and phosphatase are always dephosphorylated. Channels with AKAP, PKA and phosphatase follow a standard Michaelis-Menten formalism.

Affinities and rate constants for phosphorylation are derived by fitting the model to the isoproterenol dose-response curves and the time course of phosphorylation obtained experimentally in ventricular myocytes. All substrates have half-maximal isproterenol concentrations in the nanomolar range and reach maximum phosphorylation levels in 2-4 minutes.

$$
\begin{aligned}
& \frac{\mathrm{df}_{\mathrm{IKur}}^{\mathrm{P}}}{\mathrm{dt}}=\frac{\mathrm{k}_{\mathrm{IKur}, \mathrm{PKA}} \cdot[\mathrm{C}]^{\mathrm{ECAV}} \cdot\left(1-\mathrm{f}_{\mathrm{IKur}}^{\mathrm{P}}\right)}{\mathrm{K}_{\mathrm{IKur}, \mathrm{PKA}}+\left(1-\mathrm{f}_{\mathrm{IKur}}^{\mathrm{P}}\right)}-\frac{\mathrm{k}_{\mathrm{IKur}, \mathrm{PP}} \cdot[\mathrm{PP} 1]^{\mathrm{ECAV}} \cdot \mathrm{f}_{\mathrm{IKur}}^{\mathrm{P}}}{\mathrm{K}_{\mathrm{IKur}, \mathrm{PP}}+\mathrm{f}_{\mathrm{IKur}}^{\mathrm{P}}} \\
& \frac{\mathrm{df}_{\mathrm{INa}}^{\mathrm{P}}}{\mathrm{dt}}=\frac{\mathrm{k}_{\mathrm{INa}, \mathrm{PKA}} \cdot[\mathrm{C}]^{\mathrm{CAV}} \cdot\left(1-\mathrm{f}_{\mathrm{INa}}^{\mathrm{P}}\right)}{\mathrm{K}_{\mathrm{INa}, \mathrm{PKA}}+\left(1-\mathrm{f}_{\mathrm{INa}}^{\mathrm{P}}\right)}-\frac{\mathrm{k}_{\mathrm{INa}, \mathrm{PP}} \cdot[\mathrm{PP}]^{\mathrm{CAV}} \cdot \mathrm{f}_{\mathrm{INa}}^{\mathrm{P}}}{\mathrm{K}_{\mathrm{INa}, \mathrm{PP}}+\mathrm{f}_{\mathrm{INa}}^{\mathrm{P}}} \\
& \frac{\mathrm{df}_{\mathrm{INaK}}^{\mathrm{P}}}{\mathrm{dt}}=\frac{\mathrm{k}_{\mathrm{INaK}, \mathrm{PKA}} \cdot[\mathrm{C}]^{\mathrm{CAV}} \cdot\left(1-\mathrm{f}_{\mathrm{INaK}}^{\mathrm{P}}\right)}{\mathrm{K}_{\mathrm{INaK}, \mathrm{PKA}}+\left(1-\mathrm{f}_{\mathrm{INaK}}^{\mathrm{P}}\right)}-\frac{\mathrm{k}_{\mathrm{INaK}, \mathrm{PP}} \cdot[\mathrm{PP}]^{\mathrm{CAV}} \cdot \mathrm{f}_{\mathrm{INaK}}^{\mathrm{P}}}{\mathrm{K}_{\mathrm{INaK}, \mathrm{PP}}+\mathrm{f}_{\mathrm{INaK}}^{\mathrm{P}}} \\
& \frac{\mathrm{df}_{\mathrm{PLB}}^{\mathrm{P}}}{\mathrm{dt}}=\frac{\mathrm{k}_{\mathrm{PLB}, \mathrm{PKA}} \cdot[\mathrm{C}]^{\mathrm{CYT}} \cdot\left(1-\mathrm{f}_{\mathrm{PLB}}^{\mathrm{P}}\right)}{\mathrm{K}_{\mathrm{PLB}, \mathrm{PKA}}+\left(1-\mathrm{f}_{\mathrm{PLB}}^{\mathrm{P}}\right)}-\frac{\mathrm{k}_{\mathrm{PLB}, \mathrm{PP}} \cdot[\mathrm{PP} 1]^{\mathrm{CYT}} \cdot \mathrm{f}_{\mathrm{PLB}}^{\mathrm{P}}}{\mathrm{K}_{\mathrm{PLB}, \mathrm{PP}}+\mathrm{f}_{\mathrm{PLB}}^{\mathrm{P}}} \\
& \frac{\mathrm{df}_{\mathrm{TnI}}^{\mathrm{P}}}{\mathrm{dt}}=\frac{\mathrm{k}_{\mathrm{TnI}, \mathrm{PKA}} \cdot[\mathrm{C}]^{\mathrm{CYT}} \cdot\left(1-\mathrm{f}_{\mathrm{TnI}}^{\mathrm{P}}\right)}{\mathrm{K}_{\mathrm{TnI}, \mathrm{PKA}}+\left(1-\mathrm{f}_{\mathrm{TnI}}^{\mathrm{P}}\right)}-\frac{\mathrm{k}_{\mathrm{TnI}, \mathrm{PP}} \cdot[\mathrm{PP} 2 \mathrm{~A}]^{\mathrm{CYT}} \cdot \mathrm{f}_{\mathrm{TnI}}^{\mathrm{P}}}{\mathrm{K}_{\mathrm{TnI}, \mathrm{PP}}+\mathrm{f}_{\mathrm{TnI}}^{\mathrm{P}}}
\end{aligned}
$$

For $I_{K S}$ :

$$
\begin{aligned}
& \mathrm{K}=\mathrm{K}_{\mathrm{PP} 1, \text { Yotiao }} \quad \mathrm{L}=\mathrm{K}_{\mathrm{IKs}, \text { Yotiao }} \quad \mathrm{M}=\mathrm{K}_{\mathrm{PKA}, \text { Yotiao }} \\
& {[\mathrm{PP} 1]_{\mathrm{f}}=\frac{1}{2 \cdot \mathrm{K}^{-1}}} \\
& \cdot\left(-\left(1+\mathrm{K}^{-1} \cdot\left([\text { Yotiao }]_{\mathrm{tot}}-[\mathrm{PP} 1]^{\mathrm{ECAV}}\right)\right)\right. \\
& \left.+\sqrt{\left(1+\mathrm{K}^{-1} \cdot\left([\text { Yotiao }]_{\mathrm{tot}}-[\mathrm{PP} 1]^{\mathrm{ECAV}}\right)\right)^{2}+4 \cdot \mathrm{K}^{-1} \cdot[\mathrm{PP} 1]^{\mathrm{ECAV}}}\right)
\end{aligned}
$$

$[\mathrm{IKs}]_{\mathrm{NNN}}=\frac{1}{2 \cdot \mathrm{L}^{-1}}$

$$
\begin{aligned}
& \cdot\left(-\left(1+\mathrm{L}^{-1} \cdot\left([\text { Yotiao }]_{\text {tot }}-[\mathrm{IKs}]_{\mathrm{tot}}\right)\right)\right. \\
& \left.+\sqrt{\left(1+\mathrm{L}^{-1} \cdot\left([\text { Yotiao }]_{\mathrm{tot}}-[\mathrm{IKs}]_{\mathrm{tot}}\right)\right)^{2}+4 \cdot \mathrm{L}^{-1} \cdot[\mathrm{IKs}]_{\mathrm{tot}}}\right)
\end{aligned}
$$




$$
\begin{aligned}
{[\mathrm{R}]_{\mathrm{f}}=\frac{1}{2 \cdot \mathrm{M}^{-1}} } & \left(-\left(1+\mathrm{M}^{-1} \cdot\left([\text { Yotiao }]_{\mathrm{tot}}-[\mathrm{PKA}]^{\mathrm{ECAV}}\right)\right)\right. \\
& \left.+\sqrt{\left(1+\mathrm{M}^{-1} \cdot\left([\text { Yotiao }]_{\mathrm{tot}}-[\mathrm{PKA}]^{\mathrm{ECAV}}\right)\right)^{2}+4 \cdot \mathrm{M}^{-1} \cdot[\mathrm{PKA}]^{\mathrm{ECAV}}}\right)
\end{aligned}
$$

$[\text { Yotiao }]_{\mathrm{f}}=\frac{\left[\text { Yotiao }_{\text {tot }}-[\mathrm{IKs}]_{\mathrm{tot}}+[\mathrm{IKs}]_{\mathrm{NNN}}\right.}{\mathrm{K}^{-1} \cdot[\mathrm{PP} 1]_{\mathrm{f}}+\mathrm{M}^{-1} \cdot\left(\mathrm{K}^{-1} \cdot[\mathrm{PP} 1]_{\mathrm{f}}+1\right) \cdot[\mathrm{R}]_{\mathrm{f}}+1}$

$[\mathrm{IKs}]_{\mathrm{ARN}}=\mathrm{L}^{-1} \cdot \mathrm{M}^{-1} \cdot[\mathrm{IKs}]_{\mathrm{NNN}} \cdot\left[\mathrm{Yotiao}_{\mathrm{f}} \cdot[\mathrm{R}]_{\mathrm{f}}\right.$

$[\mathrm{IKs}]_{\mathrm{ARP}}=[\mathrm{IKs}]_{\mathrm{ARN}} \cdot \mathrm{K}^{-1} \cdot[\mathrm{PP} 1]_{\mathrm{f}}$

$$
\begin{aligned}
& \frac{\mathrm{d}[\mathrm{IKs}]^{\mathrm{P}}}{\mathrm{dt}}=\frac{\mathrm{k}_{\mathrm{IKs}, \mathrm{PKA}} \cdot[\mathrm{C}]^{\mathrm{ECAV}} \cdot\left([\mathrm{IKs}]_{\mathrm{ARP}}-[\mathrm{IKs}]^{\mathrm{P}}\right)}{\mathrm{K}_{\mathrm{IKs}, \mathrm{PKA}}+\left([\mathrm{IKs}]_{\mathrm{ARP}}-[\mathrm{IKs}]^{\mathrm{P}}\right)}-\frac{\mathrm{k}_{\mathrm{IKs}, \mathrm{PP}} \cdot[\mathrm{PP} 1]^{\mathrm{ECAV}} \cdot[\mathrm{IKs}]^{\mathrm{P}}}{\mathrm{K}_{\mathrm{IKs}, \mathrm{PP}}+[\mathrm{IKs}]^{\mathrm{P}}} \\
& \mathrm{f}_{\mathrm{IKs}}^{\mathrm{P}}=\frac{[\mathrm{IKs}]^{\mathrm{P}}+[\mathrm{IKs}]_{\mathrm{ARN}}}{[\mathrm{IKs}]_{\mathrm{tot}}}
\end{aligned}
$$

\section{For $y \in\left\{I_{C a L}, R y R\right\}:$}

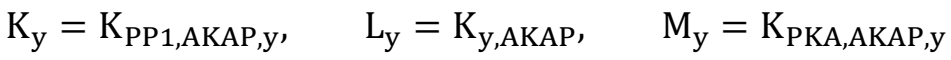

$$
\begin{aligned}
& b_{P P}=[A K A P]_{\text {tot }}^{\text {ICaL }}+[A K A P]_{\text {tot }}^{\text {RyR }}+K_{I C a L}+K_{\text {RyR }}-[P P]^{\mathrm{CAV}} \\
& c_{\mathrm{PP}}=[\mathrm{AKAP}]_{\mathrm{tot}}^{\mathrm{ICaL}} \cdot \mathrm{K}_{\mathrm{RyR}}+[\mathrm{AKAP}]_{\mathrm{tot}}^{\mathrm{RyR}} \cdot \mathrm{K}_{\mathrm{ICaL}}+\mathrm{K}_{\mathrm{ICaL}} \cdot \mathrm{K}_{\mathrm{RyR}}-[\mathrm{PP}]^{\mathrm{CAV}} \cdot\left(\mathrm{K}_{\mathrm{ICaL}}+\mathrm{K}_{\mathrm{RyR}}\right) \\
& \mathrm{d}_{\mathrm{PP}}=[\mathrm{PP}]^{\mathrm{CAV}} \cdot \mathrm{K}_{\mathrm{ICaL}} \cdot \mathrm{K}_{\mathrm{RyR}} \\
& \varphi_{\mathrm{PP}}=\left(\frac{1}{2} \cdot \mathrm{d}_{\mathrm{PP}}+\frac{1}{6} \cdot \mathrm{b}_{\mathrm{PP}} \cdot \mathrm{c}_{\mathrm{PP}}-\frac{1}{27} \cdot\left(\mathrm{b}_{\mathrm{PP}}\right)^{3}\right. \\
& +\left(-\frac{1}{27} \cdot\left(b_{P P}\right)^{3} \cdot d_{P P}-\frac{1}{108} \cdot\left(b_{P P}\right)^{2} \cdot\left(c_{P P}\right)^{2}+\frac{1}{6} \cdot b_{P P} \cdot c_{P P} \cdot d_{P P}+\frac{1}{27} \cdot\left(c_{P P}\right)^{3}\right. \\
& \left.\left.+\frac{1}{4} \cdot\left(\mathrm{d}_{\mathrm{PP}}\right)^{2}\right)^{\frac{1}{2}}\right)^{\frac{1}{3}} \\
& {[\mathrm{PP}]_{\mathrm{f}}=\varphi_{\mathrm{PP}}-\frac{1}{\varphi_{\mathrm{PP}}} \cdot\left(\frac{\mathrm{c}_{\mathrm{PP}}}{3}-\frac{\left(\mathrm{b}_{\mathrm{PP}}\right)^{2}}{9}\right)-\frac{\mathrm{b}_{\mathrm{PP}}}{3}} \\
& \mathrm{~b}_{\mathrm{R}}=[\mathrm{AKAP}]_{\text {tot }}^{\mathrm{ICaL}}+[\mathrm{AKAP}]_{\text {tot }}^{\mathrm{RyR}}+\mathrm{M}_{\mathrm{ICaL}}+\mathrm{M}_{\mathrm{RyR}}-[\mathrm{PKA}]^{\mathrm{CAV}} \\
& \mathrm{c}_{\mathrm{R}}=[\mathrm{AKAP}]_{\mathrm{tot}}^{\mathrm{ICaL}} \cdot \mathrm{M}_{\mathrm{RyR}}+[\mathrm{AKAP}]_{\mathrm{tot}}^{\mathrm{RyR}} \cdot \mathrm{M}_{\mathrm{ICaL}}+\mathrm{M}_{\mathrm{ICaL}} \cdot \mathrm{M}_{\mathrm{RyR}}-[\mathrm{PKA}]^{\mathrm{CAV}} \cdot\left(\mathrm{M}_{\mathrm{ICaL}}+\mathrm{M}_{\mathrm{RyR}}\right) \\
& \mathrm{d}_{\mathrm{R}}=[\mathrm{PKA}]^{\mathrm{CAV}} \cdot \mathrm{M}_{\mathrm{ICaL}} \cdot \mathrm{M}_{\mathrm{RyR}}
\end{aligned}
$$




$$
\begin{aligned}
& \varphi_{R}=\left(\frac{1}{2} \cdot d_{R}+\frac{1}{6} \cdot b_{R} \cdot c_{R}-\frac{1}{27} \cdot\left(b_{R}\right)^{3}\right. \\
& +\left(-\frac{1}{27} \cdot\left(b_{R}\right)^{3} \cdot d_{R}-\frac{1}{108} \cdot\left(b_{R}\right)^{2} \cdot\left(c_{R}\right)^{2}+\frac{1}{6} \cdot b_{R} \cdot c_{R} \cdot d_{R}+\frac{1}{27} \cdot\left(c_{R}\right)^{3}+\frac{1}{4}\right. \\
& \left.\left.\cdot\left(\mathrm{d}_{\mathrm{R}}\right)^{2}\right)^{\frac{1}{2}}\right)^{\frac{1}{3}} \\
& {[\mathrm{R}]_{\mathrm{f}}=\varphi_{\mathrm{R}}-\frac{1}{\varphi_{\mathrm{R}}} \cdot\left(\frac{\mathrm{c}_{\mathrm{R}}}{3}-\frac{\left(\mathrm{b}_{\mathrm{R}}\right)^{2}}{9}\right)-\frac{\mathrm{b}_{\mathrm{R}}}{3}} \\
& {[\mathrm{y}]_{\mathrm{NNN}}=\frac{1}{2 \cdot\left(\mathrm{L}_{\mathrm{y}}\right)^{-1}}} \\
& \cdot\left(-\left(\left(\mathrm{L}_{\mathrm{y}}\right)^{-1} \cdot\left([\mathrm{AKAP}]_{\text {tot }}^{\mathrm{y}}-[\mathrm{y}]_{\mathrm{tot}}\right)+1\right)\right. \\
& \left.+\sqrt{\left(\left(\mathrm{L}_{\mathrm{y}}\right)^{-1} \cdot\left([\mathrm{AKAP}]_{\mathrm{tot}}^{\mathrm{y}}-[\mathrm{y}]_{\mathrm{tot}}\right)+1\right)^{2}+4 \cdot\left(\mathrm{L}_{\mathrm{y}}\right)^{-1} \cdot[\mathrm{y}]_{\mathrm{tot}}}\right) \\
& {[\mathrm{AKAP}]_{\mathrm{f}}^{\mathrm{y}}=\frac{[\mathrm{AKAP}]_{\mathrm{tot}}^{\mathrm{y}}-[\mathrm{y}]_{\mathrm{tot}}+[\mathrm{y}]_{\mathrm{NNN}}}{\left(\mathrm{K}_{\mathrm{y}}\right)^{-1} \cdot[\mathrm{PP}]_{\mathrm{f}}+\left(\mathrm{M}_{\mathrm{y}}\right)^{-1} \cdot\left(\left(\mathrm{K}_{\mathrm{y}}\right)^{-1} \cdot[\mathrm{PP}]_{\mathrm{f}}+1\right) \cdot[\mathrm{R}]_{\mathrm{f}}+1}} \\
& {[\mathrm{y}]_{\mathrm{ARN}}=\left(\mathrm{L}_{\mathrm{y}}\right)^{-1} \cdot\left(\mathrm{M}_{\mathrm{y}}\right)^{-1} \cdot[\mathrm{y}]_{\mathrm{NNN}} \cdot[\mathrm{AKAP}]_{\mathrm{f}}^{\mathrm{y}} \cdot[\mathrm{R}]_{\mathrm{f}}} \\
& {[\mathrm{y}]_{\mathrm{ARP}}=[\mathrm{y}]_{\mathrm{ARN}} \cdot\left(\mathrm{K}_{\mathrm{y}}\right)^{-1} \cdot[\mathrm{PP}]_{\mathrm{f}}} \\
& \frac{\mathrm{d}[\mathrm{y}]^{\mathrm{p}}}{\mathrm{dt}}=\frac{\mathrm{k}_{\mathrm{y}, \mathrm{PKA}} \cdot[\mathrm{C}]^{\mathrm{CAV}} \cdot\left([\mathrm{y}]_{\mathrm{ARP}}-[\mathrm{y}]^{\mathrm{P}}\right)}{\mathrm{K}_{\mathrm{y}, \mathrm{PKA}}+\left([\mathrm{y}]_{\text {tot }}-[\mathrm{y}]^{\mathrm{P}}\right)}-\frac{\mathrm{k}_{\mathrm{y}, \mathrm{PP}} \cdot[\mathrm{PP}]^{\mathrm{CAV}} \cdot[\mathrm{y}]^{\mathrm{p}}}{\mathrm{K}_{\mathrm{y}, \mathrm{PP}}+[\mathrm{y}]^{\mathrm{P}}} \\
& \mathrm{f}_{\mathrm{y}}^{\mathrm{P}}=\frac{[\mathrm{y}]^{\mathrm{P}}+[\mathrm{y}]_{\text {ARN }}}{[\mathrm{y}]_{\text {tot }}}
\end{aligned}
$$

\begin{tabular}{|c|c|c|c|}
\hline Parameter & Value & Description & Ref \\
\hline $\mathrm{k}_{\mathrm{ICaL}, \mathrm{PKA}}$ & 5.1009E-4 s ${ }^{-1}$ & Rate of $\mathrm{I}_{\text {CaL }}$ phosphorylation & $20,{ }_{\text {(C) }}^{146^{@, ~}}$ \\
\hline $\mathrm{K}_{\mathrm{ICaL}, \mathrm{PKA}}$ & $\begin{array}{c}1.2702 \mathrm{E}-6 \\
\mu \mathrm{mol} / \mathrm{L}\end{array}$ & Affinity of $I_{\text {CaL }}$ for phosphorylation by PKA & $\begin{array}{l}20,146^{@}, \\
\text { (C) }\end{array}$ \\
\hline $\mathrm{k}_{\mathrm{ICaL}, \mathrm{PP}}$ & $6.9030 \mathrm{E}-4 \mathrm{~s}^{-1}$ & Rate of $\mathrm{I}_{\text {CaL }}$ dephosphorylation & $20,{ }_{\text {(C) }}^{146^{@, ~}}$ \\
\hline $\mathrm{K}_{\mathrm{ICaL}, \mathrm{PP}}$ & $\begin{array}{c}6.3064 \mathrm{E}-3 \\
\mu \mathrm{mol} / \mathrm{L}\end{array}$ & $\begin{array}{l}\text { Affinity of } I_{\text {CaL }} \text { for dephosphorylation by } \\
\text { phosphatases }\end{array}$ & $20,{ }_{\text {(C) }}^{146^{@, ~}}$ \\
\hline $\mathrm{k}_{\mathrm{IKs}, \mathrm{PKA}}$ & $1.6305 \mathrm{E}-1 \mathrm{~s}^{-1}$ & Rate of $I_{\mathrm{Ks}}$ phosphorylation & $147^{@, ~(C)}$ \\
\hline $\mathrm{K}_{\mathrm{IKs}, \mathrm{PKA}}$ & $\begin{array}{c}9.9794 \mathrm{E}-05 \\
\mu \mathrm{mol} / \mathrm{L}\end{array}$ & Affinity of $I_{\mathrm{ks}}$ for phosphorylation by PKA & $147^{@,(C)}$ \\
\hline
\end{tabular}




\begin{tabular}{|c|c|c|c|}
\hline $\mathrm{k}_{\mathrm{IKs}, \mathrm{PP}}$ & $1.0542 \mathrm{~s}^{-1}$ & Rate of $I_{\mathrm{Ks}}$ dephosphorylation & $147^{@, ~(C)}$ \\
\hline $\mathrm{K}_{\mathrm{IKs}, \mathrm{PP}}$ & $\begin{array}{c}1.1147 \mathrm{E}-4 \\
\mu \mathrm{mol} / \mathrm{L}\end{array}$ & $\begin{array}{l}\text { Affinity of } I_{\mathrm{Ks}} \text { for dephosphorylation by } \\
\text { phosphatases }\end{array}$ & $147^{@, ~(c) ~}$ \\
\hline $\mathrm{k}_{\mathrm{IKur}, \mathrm{PKA}}$ & $\begin{array}{l}6.9537 \mathrm{E}-2 \mathrm{~s}^{-1} \\
(\mu \mathrm{mol} / \mathrm{L})^{-1}\end{array}$ & Rate of $I_{\text {Kur }}$ phosphorylation & $43^{@, ~(C)}$ \\
\hline $\mathrm{K}_{\mathrm{IKur}, \mathrm{PKA}}$ & $2.7623 \mathrm{E}-1$ & Affinity of $I_{\text {Kur }}$ for phosphorylation by PKA & $43^{@, ~(C)}$ \\
\hline $\mathrm{k}_{\mathrm{IKur}, \mathrm{PP}}$ & $\begin{array}{l}3.1700 \mathrm{E}-1 \mathrm{~s}^{-1} \\
(\mu \mathrm{mol} / \mathrm{L})^{-1}\end{array}$ & Rate of $I_{\text {Kur }}$ dephosphorylation & $43^{@, ~(C)}$ \\
\hline $\mathrm{K}_{\mathrm{IKur}, \mathrm{PP}}$ & $2.3310 \mathrm{E}-3$ & $\begin{array}{l}\text { Affinity of } I_{\text {Kur }} \text { for dephosphorylation by } \\
\text { phosphatases }\end{array}$ & $43^{@, ~(C)}$ \\
\hline $\mathrm{k}_{\mathrm{INa}, \mathrm{PKA}}$ & $\begin{array}{l}1.3680 \mathrm{E}-2 \mathrm{~s}^{-1} \\
(\mu \mathrm{mol} / \mathrm{L})^{-1}\end{array}$ & Rate of $I_{N a}$ phosphorylation & $148^{@,(R)}$ \\
\hline $\mathrm{K}_{\mathrm{INa}, \mathrm{PKA}}$ & $1.09883 \mathrm{E}-1$ & Affinity of $I_{\mathrm{Na}}$ for phosphorylation by PKA & $148^{@,(R)}$ \\
\hline $\mathrm{k}_{\mathrm{INa}, \mathrm{PP}}$ & $\begin{array}{l}5.2811 \mathrm{E}-2 \mathrm{~s}^{-1} \\
(\mu \mathrm{mol} / \mathrm{L})^{-1}\end{array}$ & Rate of $I_{\mathrm{Na}}$ dephosphorylation & $148^{@,(R)}$ \\
\hline $\mathrm{K}_{\mathrm{INa}, \mathrm{PP}}$ & 7.8605 & $\begin{array}{l}\text { Affinity of } I_{\mathrm{Na}} \text { for dephosphorylation by } \\
\text { phosphatases }\end{array}$ & $148^{@,(R)}$ \\
\hline $\mathrm{k}_{\mathrm{INaK}, \mathrm{PKA}}$ & $\begin{array}{l}1.5265 \mathrm{E}-2 \mathrm{~s}^{-1} \\
(\mu \mathrm{mol} / \mathrm{L})^{-1}\end{array}$ & Rate of $I_{\text {NaK }}$ phosphorylation & $62^{@,(G)}$ \\
\hline $\mathrm{K}_{\mathrm{INaK,PKA}}$ & $1.1001 \mathrm{E}-3$ & Affinity of $I_{\mathrm{NaK}}$ for phosphorylation by PKA & $62^{@,(G)}$ \\
\hline $\mathrm{k}_{\mathrm{INaK}, \mathrm{PP}}$ & $\begin{array}{l}9.2455 \mathrm{E}-2 \mathrm{~s}^{-1} \\
(\mu \mathrm{mol} / \mathrm{L})^{-1}\end{array}$ & Rate of $I_{\text {Nak }}$ dephosphorylation & $62^{@, ~(G)}$ \\
\hline $\mathrm{K}_{\mathrm{INaK}, \mathrm{PP}}$ & 5.7392 & $\begin{array}{l}\text { Affinity of } I_{\mathrm{Nak}} \text { for dephosphorylation by } \\
\text { phosphatases }\end{array}$ & $62^{@, ~(G)}$ \\
\hline $\mathrm{k}_{\mathrm{PLB}, \mathrm{PKA}}$ & $\begin{array}{l}1.1316 \mathrm{E}-1 \mathrm{~s}^{-1} \\
(\mu \mathrm{mol} / \mathrm{L})^{-1}\end{array}$ & Rate of PLB phosphorylation & $\begin{array}{l}1,89 \\
149^{@} \\
(\mathrm{R}) /(\mathrm{G})\end{array}$ \\
\hline $\mathrm{K}_{\mathrm{PLB}, \mathrm{PKA}}$ & 4.9097E-4 & Affinity of PLB for phosphorylation by PKA & $\begin{array}{l}1,89 \\
149^{@} \\
(\mathrm{R}) /(\mathrm{G})\end{array}$ \\
\hline $\mathrm{k}_{\mathrm{PLB}, \mathrm{PP}}$ & $\begin{array}{l}2.5755 \mathrm{E}-1 \mathrm{~s}^{-1} \\
(\mu \mathrm{mol} / \mathrm{L})^{-1}\end{array}$ & Rate of PLB dephosphorylation & $\begin{array}{l}1,89 \\
149^{@} \\
(\mathrm{R}) /(\mathrm{G})\end{array}$ \\
\hline $\mathrm{K}_{\mathrm{PLB}, \mathrm{PP}}$ & $4.8393 \mathrm{E}-2$ & $\begin{array}{l}\text { Affinity of PLB for dephosphorylation by } \\
\text { phosphatases }\end{array}$ & $\begin{array}{l}1,89 \\
149^{@} \\
(\mathrm{R}) /(\mathrm{G})\end{array}$ \\
\hline $\mathrm{k}_{\mathrm{RyR}, \mathrm{PKA}}$ & $2.5548 \mathrm{E}-3 \mathrm{~s}^{-1}$ & Rate of RyR phosphorylation & $81^{@,(R)}$ \\
\hline $\mathrm{K}_{\mathrm{RyR}, \mathrm{PKA}}$ & $\begin{array}{c}6.6298 \mathrm{E}-5 \\
\mu \mathrm{mol} / \mathrm{L}\end{array}$ & Affinity of RyR for phosphorylation by PKA & $81^{@,(R)}$ \\
\hline $\mathrm{k}_{\mathrm{RyR}, \mathrm{PP}}$ & $3.8257 \mathrm{E}-3 \mathrm{~s}^{-1}$ & Rate of RyR dephosphorylation & $81^{@,(R)}$ \\
\hline $\mathrm{K}_{\mathrm{RyR}, \mathrm{PP}}$ & $\begin{array}{l}4.3003 \mathrm{E}-2 \\
\mu \mathrm{mol} / \mathrm{L}\end{array}$ & $\begin{array}{l}\text { Affinity of RyR for dephosphorylation by } \\
\text { phosphatases }\end{array}$ & $81^{@,(R)}$ \\
\hline $\mathrm{k}_{\mathrm{TnI}, \mathrm{PKA}}$ & $\begin{array}{l}1.0408 \mathrm{E}-1 \mathrm{~s}^{-1} \\
(\mu \mathrm{mol} / \mathrm{L})^{-1}\end{array}$ & Rate of Tnl phosphorylation & $1, \underset{(\mathrm{R})}{150^{@,}}$ \\
\hline $\mathrm{K}_{\mathrm{TnI}, \mathrm{PKA}}$ & $2.7143 E-5$ & Affinity of Tnl for phosphorylation by PKA & $1, \underset{(R)}{150^{@, ~}}$ \\
\hline
\end{tabular}




\begin{tabular}{|c|c|c|c|}
\hline $\begin{array}{l}\mathrm{k}_{\mathrm{TnI}, \mathrm{PP}} \\
\cdot[\mathrm{PP} 2 \mathrm{~A}]^{\mathrm{CYT}}\end{array}$ & $\begin{array}{l}5.2633 \mathrm{E}-2 \mathrm{~s}^{-1} \\
(\mu \mathrm{mol} / \mathrm{L})^{-1}\end{array}$ & Rate of Tnl dephosphorylation & $1,{ }_{(\mathrm{R})}^{150^{@, ~}}$ \\
\hline $\mathrm{K}_{\mathrm{TnI}, \mathrm{PP}}$ & $2.6714 \mathrm{E}-1$ & $\begin{array}{l}\text { Affinity of Tnl for dephosphorylation by } \\
\text { phosphatases }\end{array}$ & $1,150^{@}$ \\
\hline $\mathrm{K}_{\mathrm{PKA}, \text { Yotiao }}$ & $0.01 \mu \mathrm{mol} / \mathrm{L}$ & Binding affinity between PKA and yotiao & $133^{\&}$ \\
\hline $\mathrm{K}_{\mathrm{IKs}, \text { Yotiao }}$ & $1 \mathrm{E}-4 \mu \mathrm{mol} / \mathrm{L}$ & Binding affinity between $I_{\mathrm{Ks}}$ channel andyYotiao & $124^{\#}$ \\
\hline $\mathrm{K}_{\mathrm{PP} 1, Y o t i a o}$ & $\mathrm{~K}_{\mathrm{PKA}, \text { Yotiao }}$ & Binding affinity between PP1 and yotiao & \\
\hline$[\text { Yotiao }]_{\text {tot }}$ & $0.025 \mu \mathrm{mol} / \mathrm{L}$ & Total concentration of yotiao & $124^{\#}$ \\
\hline$[\mathrm{IKs}]_{\text {tot }}$ & $0.025 \mu \mathrm{mol} / \mathrm{L}$ & Total concentration of $I_{\mathrm{Ks}}$ channels & $124^{\#}$ \\
\hline $\mathrm{K}_{\mathrm{PKA}, \mathrm{AKAP}, \mathrm{ICaL}}$ & $\mathrm{K}_{\mathrm{PKA}, \text { Yotiao }}$ & Binding affinity between PKA and $I_{\mathrm{CaL}}$ AKAP & \\
\hline $\mathrm{K}_{\mathrm{PP} 1, \mathrm{AKAP.ICaL}}$ & $\mathrm{K}_{\mathrm{PKA}, \text { Yotiao }}$ & Binding affinity between PP1 and $I_{\mathrm{CaL}}$ AKAP & \\
\hline $\mathrm{K}_{\mathrm{ICaL}, \mathrm{AKAP}}$ & $\mathrm{K}_{\mathrm{IKs}, Y o t i a o}$ & Binding affinity between $I_{\mathrm{CaL}}$ channel and AKAP & \\
\hline $\mathrm{K}_{\mathrm{PKA}, \mathrm{AKAP}, \mathrm{RyR}}$ & $\mathrm{K}_{\mathrm{PKA}, \text { Yotiao }}$ & Binding affinity between PKA and RyR AKAP & \\
\hline $\mathrm{K}_{\mathrm{PP} 1, \mathrm{AKAP} . \mathrm{RyR}}$ & $\mathrm{K}_{\mathrm{PKA}, \text { Yotiao }}$ & Binding affinity between PP1 and RyR AKAP & \\
\hline $\mathrm{K}_{\mathrm{RyR}, \mathrm{AKAP}}$ & $\mathrm{K}_{\mathrm{IKs}, Y o t i a o}$ & Binding affinity between RyR channel and AKAP & \\
\hline$[\mathrm{ICaL}]_{\text {tot }}$ & $0.025 \mu \mathrm{mol} / \mathrm{L}$ & Total concentration of $\mathrm{I}_{\mathrm{CaL}}$ channels & $124^{\#}$ \\
\hline$[\mathrm{AKAP}]_{\mathrm{tot}}^{\mathrm{ICaL}}$ & $0.025 \mu \mathrm{mol} / \mathrm{L}$ & Total concentration of $\mathrm{I}_{\mathrm{CaL}}$ AKAP & \\
\hline$[\mathrm{RyR}]_{\text {tot }}$ & $0.125 \mu \mathrm{mol} / \mathrm{L}$ & Total concentration of RyRs & $124^{\#}$ \\
\hline$[\mathrm{AKAP}]_{\text {tot }}^{\mathrm{RyR}}$ & $0.125 \mu \mathrm{mol} / \mathrm{L}$ & Total concentration of RyR AKAP & \\
\hline$[\mathrm{PP}]^{\mathrm{CAV}}$ & $0.25 \mu \mathrm{mol} / \mathrm{L}$ & PP concentration in caveolar compartment & $\%$ \\
\hline$[\mathrm{PP} 1]^{\mathrm{ECAV}}$ & $0.1 \mu \mathrm{mol} / \mathrm{L}$ & PP1 concentration in extracaveolar compartment & $\%$ \\
\hline
\end{tabular}

\section{CAMKII MODELS}

\subsubsection{HRd2009 version (Chapter 2)}

Model CaMKII formulation is equivalent to that in the original HRd model. ${ }^{4}$

$$
\begin{aligned}
& \mathrm{CaMK}_{\text {bound }}=\mathrm{CaMK}_{0} \cdot\left(1-\mathrm{CaMK}_{\text {trap }}\right) \cdot \frac{1}{1+\frac{\mathrm{K}_{\mathrm{m}, \mathrm{CaM}}}{\left[\mathrm{Ca}^{2+}\right]_{\mathrm{ss}}}} \\
& \frac{\mathrm{dCaMK}_{\text {trap }}}{\mathrm{dt}}=\alpha_{\mathrm{CaMK}} \cdot \mathrm{CaMK}_{\text {bound }} \cdot\left(\mathrm{CaMK}_{\text {bound }}+\mathrm{CaMK}_{\text {trap }}\right)-\beta_{\mathrm{CaMK}} \cdot \mathrm{CaMK}_{\text {trap }} \\
& \mathrm{CaMK}_{\text {active }}=\mathrm{CaMK}_{\text {bound }}+\mathrm{CaMK}_{\text {trap }}
\end{aligned}
$$




\begin{tabular}{|cclc|}
\hline Parameter & Value & Description & Ref \\
\hline $\mathrm{CaMK}_{0}$ & 0.05 & Equilibrium fraction of active CaMKIl binding sites & $4^{\#}$ \\
\hline $\mathrm{K}_{\mathrm{m}, \mathrm{CaM}}$ & $0.0015 \mathrm{mmol} / \mathrm{L}$ & CaMKII affinity for $\mathrm{Ca}^{2+} / \mathrm{CaM}$ activation & $4^{\#}$ \\
\hline$\alpha_{\mathrm{CaMK}}$ & $50 \mathrm{~s}^{-1}$ & Rate of phosphorylation of CaMKII & $4^{\#}$ \\
\hline$\beta_{\mathrm{CaMK}}$ & $0.68 \mathrm{~s}^{-1}$ & Rate of dephosphorylation of CaMKII & $4^{\#}$ \\
\hline
\end{tabular}

\subsubsection{HRdßARS and HRdßARS Stoch version (Chapters 3-7)}

In the model, activation of CaMKII is localized to the $\mathrm{Ca}^{2+}$ subspace, where $\mathrm{Ca}^{2+}$ levels are sufficiently high for activation of CaMKII, consistent with previous modeling studies. ${ }^{4,151,152}$ In contrast to cAMP compartmentation, where different FRET sensors (PKA or EPAC based) and cyclic-nucleotide-gated channels can be used to measure CAMP concentrations in different parts of the cell, there are currently no experimental techniques to measure localized CaMKII activity. Therefore, we did not include detailed localization of CaMKII, other than its dependence on subspace $\mathrm{Ca}^{2+}$. Nonetheless, a distinction is made between the substrates localized in or near the T-tubules and those further away with respect to the time constant of phosphorylation. Time constants for phosphorylation of RyR and $I_{\text {CaL }}$ phosphorylation are based on the time-course of CaMKII-dependent RyR phosphorylation at S2815 by Huke et al, whereas time constants for the other substrates $\left(I_{K 1}, I_{T O}, I_{N a}, P L B\right)$ are based on the time-course of PLB T17 phosphorylation reported by the same group. ${ }^{153}$ Steady-state CaMKII phosphorylation levels are based on those previously published. ${ }^{4,24}$ Based on experimental data, we have included an additional interaction between the CAMP/PKA signaling pathway via PP1dependent dephosphorylation of CaMKII activity. Huke et al. found that CaMKII activity is strongly regulated by $\mathrm{PP} 1 .^{153}$ Consistent with their observations, we made the deactivation rate of autonomous CaMKII dependent on whole cell PP1 activity. This PP1 activity can subsequently be modulated by $\beta A R S$ via PKA-dependent activation of Inh1 and inhibition of PP1.

$$
\begin{aligned}
\mathrm{CaMK}_{\text {bound }}= & \mathrm{CaMK}_{0} \cdot\left(1-\mathrm{CaMK}_{\text {trap }}\right) \cdot \frac{1}{1+\frac{\mathrm{K}_{\mathrm{m}, \mathrm{CaM}}}{\left[\mathrm{Ca}^{2+}\right]_{\mathrm{ss}}}} \\
\frac{\mathrm{dCaMK}}{\mathrm{trap}}= & \alpha_{\mathrm{CaMK}} \cdot \mathrm{CaMK}_{\mathrm{bound}} \cdot\left(\mathrm{CaMK}_{\text {bound }}+\mathrm{CaMK}_{\text {trap }}\right)-\mathrm{CaMK}_{\text {trap }} \\
& \cdot\left(0.1 \cdot \beta_{\mathrm{CaMK}}+0.9 \cdot \beta_{\mathrm{CaMK}}\right. \\
& \left.\cdot \frac{\mathrm{V}^{\mathrm{CAV}} \cdot[\mathrm{PP}]^{\mathrm{CAV}}+\mathrm{V}^{\mathrm{ECAV}} \cdot[\mathrm{PP} 1]^{\mathrm{ECAV}}+\mathrm{V}^{\mathrm{CYT}} \cdot[\mathrm{PP} 1]^{\mathrm{CYT}}}{\mathrm{V}^{\mathrm{CELL}} \cdot 0.1371}\right)
\end{aligned}
$$

$\mathrm{CaMK}_{\text {active }}=\mathrm{CaMK}_{\text {bound }}+\mathrm{CaMK}_{\text {trap }}$

$\frac{\mathrm{df}_{\text {ICaL }}^{\text {P,CaMKII }}}{\mathrm{dt}}=\left(\frac{\text { CaMK }_{\text {active }}}{\text { CaMK }_{\text {active }}+\mathrm{K}_{\text {ICaL,CaMKII }}}-\mathrm{f}_{\text {ICaL }}^{\text {P,CaMKII }}\right) / \tau_{\text {ICaL,CaMKII }}$ 
$\frac{\mathrm{df}_{\mathrm{PLB}}^{\mathrm{P}, \text { CaMKII }}}{\mathrm{dt}}=\left(\frac{\text { CaMK }_{\text {active }}}{\text { CaMK }_{\text {active }}+\mathrm{K}_{\mathrm{PLB}, \text { CaMKII }}}-\mathrm{f}_{\mathrm{PLB}}^{\mathrm{P}, \text { CaMKII }}\right) / \tau_{\mathrm{PLB}, \text { CaMKII }}$

$\frac{\mathrm{df}_{\text {INa }}^{\text {P,CaMKII }}}{\mathrm{dt}}=\left(\frac{\text { CaMK }_{\text {active }}}{\text { CaMK }_{\text {active }}+\mathrm{K}_{\text {INa,CaMKII }}}-\mathrm{f}_{\text {INa }}^{\text {P,CaMKII }}\right) / \tau_{\text {INa,CaMKII }}$

$\frac{\mathrm{df}_{\text {RyR }}^{\text {P,CaMKII }}}{\mathrm{dt}}=\left(\frac{\text { CaMK }_{\text {active }}{ }^{2}}{\text { CaMK }_{\text {active }}{ }^{2}+\mathrm{K}_{\text {RyR,CaMKII }}^{2}}-\mathrm{f}_{\text {RyR }}^{\text {P,CaMKII }}\right) / \tau_{\text {RyR,CaMKII }}$

$\frac{\mathrm{df}_{\text {ITo }}^{\text {P,CaMKII }}}{\mathrm{dt}}=\left(\frac{\text { CaMK }_{\text {active }}}{\text { CaMK }_{\text {active }}+\mathrm{K}_{\text {ITo,CaMKII }}}-\mathrm{f}_{\text {ITo }}^{\text {P,CaMKII }}\right) / \tau_{\text {ITo,CaMKII }}$

$\frac{\mathrm{df}_{\text {IK1 }}^{\text {P,CaMKII }}}{\mathrm{dt}}=\left(\frac{\text { CaMK }_{\text {active }}}{\text { CaMK }_{\text {active }}+\mathrm{K}_{\text {IK1,CaMKII }}}-\mathrm{f}_{\text {IK1 }}^{\text {P,CaMKII }}\right) / \tau_{\text {IK1,CaMKII }}$

\begin{tabular}{|c|c|c|c|}
\hline Parameter & Value & Description & Ref \\
\hline $\mathrm{CaMK}_{0}$ & 0.05 & Equilibrium fraction of active CaMKII binding sites & $21^{\#}$ \\
\hline $\mathrm{K}_{\mathrm{m}, \mathrm{CaM}}$ & $0.0015 \mathrm{mmol} / \mathrm{L}$ & CaMKII affinity for $\mathrm{Ca}^{2+} / \mathrm{CaM}$ activation & $21^{\#}$ \\
\hline$\alpha_{\text {CaMK }}$ & $50 \mathrm{~s}^{-1}$ & Rate of phosphorylation of CaMKII & $21^{\#}$ \\
\hline$\beta_{\text {СаМK }}$ & $0.68 \mathrm{~s}^{-1}$ & Rate of dephosphorylation of CaMKII & $21^{\#}$ \\
\hline $\mathrm{K}_{\mathrm{ICaL}, \mathrm{CaMKII}}$ & $\mathrm{K}_{\mathrm{PLB}, \mathrm{CaMKII}}$ & Affinity of $I_{\mathrm{CaL}}$ for CaMKII & \\
\hline$\tau_{\text {ICaL,CaMKII }}$ & $\tau_{\text {RyR,CaMKII }}$ & Time constant of CaMKII I CaL phosphorylation & \\
\hline $\mathrm{K}_{\text {PLB,CaMKII }}$ & 0.15 & Affinity of PLB for CaMKII & $21^{\#}$ \\
\hline$\tau_{\text {PLB,CaMKII }}$ & $100 \mathrm{~s}$ & Time constant of CaMKII PLB phosphorylation & $153^{@,(R)}$ \\
\hline $\mathrm{K}_{\mathrm{INa}, \mathrm{CaMKII}}$ & $\mathrm{K}_{\text {PLB,CaMKII }}$ & Affinity of $I_{\mathrm{Na}}$ for CaMKII & \\
\hline$\tau_{\text {INa,CaMKII }}$ & $\tau_{\text {PLB,CaMKII }}$ & Time constant of CaMKII I I $I_{\mathrm{Na}}$ phosphorylation & \\
\hline $\mathrm{K}_{\mathrm{RyR}, \mathrm{CaMKII}}$ & $\mathrm{K}_{\text {PLB,CaMKII }}$ & Affinity of RyR for CaMKII & \\
\hline$\tau_{\text {RyR,CaMKII }}$ & $50 \mathrm{~s}$ & Time constant of CaMKII RyR phosphorylation & $153^{@,(R)}$ \\
\hline $\mathrm{K}_{\text {ITo,CaMKII }}$ & $\mathrm{K}_{\mathrm{PLB}, \mathrm{CaMKII}}$ & Affinity of $\mathrm{I}_{\mathrm{To}}$ for CaMKII & \\
\hline$\tau_{\text {ITo,CaMKII }}$ & $\tau_{\mathrm{PLB}, \mathrm{CaMKII}}$ & Time constant of CaMKII I To $_{\text {o }}$ phosphorylation & \\
\hline $\mathrm{K}_{\mathrm{IK} 1, \mathrm{CaMKII}}$ & $\mathrm{K}_{\text {PLB,CaMKII }}$ & Affinity of $I_{\mathrm{K} 1}$ for CaMKII & \\
\hline$\tau_{\text {IK1,CaMKII }}$ & $\tau_{\mathrm{PLB}, \mathrm{CaMKII}}$ & Time constant of CaMKII I $\mathrm{K}_{\mathrm{K} 1}$ phosphorylation & \\
\hline
\end{tabular}




\section{REFERENCES}

1. Li L, Desantiago J, Chu G, Kranias EG, Bers DM. Phosphorylation of phospholamban and troponin I in beta-adrenergic-induced acceleration of cardiac relaxation. Am J Physiol Heart Circ Physiol. 2000;278:H769-779.

2. Yasuda S-i, Coutu P, Sadayappan S, Robbins J, Metzger JM. Cardiac transgenic and gene transfer strategies converge to support an important role for troponin I in regulating relaxation in cardiac myocytes. Circ Res. 2007;101:377--386.

3. Gao J, Wang $\mathrm{W}$, Cohen IS, Mathias RT. Transmural gradients in $\mathrm{Na} / \mathrm{K}$ pump activity and $\left[\mathrm{Na}^{+}\right]_{\mathrm{i}}$ in canine ventricle. Biophys J. 2005;89:1700-1709.

4. Hund TJ, Rudy Y. Rate dependence and regulation of action potential and calcium transient in a canine cardiac ventricular cell model. Circulation. 2004;110:3168-3174.

5. Aggarwal $\mathrm{R}$, Boyden PA. Diminished $\mathrm{Ca}^{2+}$ and $\mathrm{Ba}^{2+}$ currents in myocytes surviving in the epicardial border zone of the 5-day infarcted canine heart. Circ Res. 1995;77:1180-1191.

6. Tseng GN. Calcium current restitution in mammalian ventricular myocytes is modulated by intracellular calcium. Circ Res. 1988;63:468-482.

7. Mahajan A, Shiferaw Y, Sato D, Baher A, Olcese R, Xie LH, Yang MJ, Chen PS, Restrepo JG, Karma A, Garfinkel A, Qu Z, Weiss JN. A rabbit ventricular action potential model replicating cardiac dynamics at rapid heart rates. Biophys J. 2008;94:392-410.

8. Pitt GS. Calmodulin and CaMKII as molecular switches for cardiac ion channels. Cardiovasc Res. 2007;73:641-647.

9. Cordeiro JM, Greene L, Heilmann C, Antzelevitch D, Antzelevitch C. Transmural heterogeneity of calcium activity and mechanical function in the canine left ventricle. Am J Physiol Heart Circ Physiol. 2004;286:H1471-1479.

10. Magyar J, Szentandrassy N, Banyasz T, Fulop L, Varro A, Nanasi PP. Effects of thymol on calcium and potassium currents in canine and human ventricular cardiomyocytes. $\mathrm{Br} \mathrm{J}$ Pharmacol. 2002;136:330-338.

11. Rubart M, Lopshire JC, Fineberg NS, Zipes DP. Changes in left ventricular repolarization and ion channel currents following a transient rate increase superimposed on bradycardia in anesthetized dogs. J Cardiovasc Electrophysiol. 2000;11:652-664.

12. Sipido KR, Volders PGA, de Groot SHM, Verdonck F, Van de Werf F, Wellens HJJ, Vos MA. Enhanced $\mathrm{Ca}^{2+}$ release and $\mathrm{Na} / \mathrm{Ca}$ exchange activity in hypertrophied canine ventricular myocytes: potential link between contractile adaptation and arrhythmogenesis. Circulation. 2000;102:2137-2144.

13. Szabó G, Szentandrássy N, Bíró T, Tóth BI, Czifra G, Magyar J, Bányász T, Varró A, Kovács L, Nánási PP. Asymmetrical distribution of ion channels in canine and human left-ventricular wall: epicardium versus midmyocardium. Pflugers Arch. 2005;450:307-316.

14. Birinyi P, Acsai K, Banyasz T, Toth A, Horvath B, Virag L, Szentandrassy N, Magyar J, Varro A, Fulop F, Nanasi PP. Effects of SEA0400 and KB-R7943 on Na+/Ca2+ exchange current and Ltype $\mathrm{Ca} 2+$ current in canine ventricular cardiomyocytes. Naunyn Schmiedebergs Arch Pharmacol. 2005;372:63-70.

15. Kamp TJ, Hell JW. Regulation of cardiac L-type calcium channels by protein kinase A and protein kinase C. Circ Res. 2000;87:1095-1102.

16. Bers DM. Calcium cycling and signaling in cardiac myocytes. Annu Rev Physiol. 2008;70:2349. 
17. Herzig S, Patil P, Neumann J, Staschen CM, Yue DT. Mechanisms of $\beta$-adrenergic stimulation of cardiac $\mathrm{Ca} 2+$ channels revealed by discrete-time Markov analysis of slow gating. Biophys J. 1993;65:1599-1612.

18. Yue DT, Herzig S, Marban E. Beta-adrenergic stimulation of calcium channels occurs by potentiation of high-activity gating modes. Proc Natl Acad Sci U S A. 1990;87:753-757.

19. Antoons G, Volders PG, Stankovicova T, Bito V, Stengl M, Vos MA, Sipido KR. Window Ca2+ current and its modulation by $\mathrm{Ca} 2+$ release in hypertrophied cardiac myocytes from dogs with chronic atrioventricular block. J Physiol. 2007;579:147-160.

20. Nagykaldi Z, Kem D, Lazzara R, Szabo B. Canine ventricular myocyte $\beta 2$-adrenoceptors are not functionally coupled to L-type calcium current. J Cardiovasc Electrophysiol. 1999;10:1240-1251.

21. Decker KF, Heijman J, Silva JR, Hund TJ, Rudy Y. Properties and ionic mechanisms of action potential adaptation, restitution, and accommodation in canine epicardium. Am J Physiol Heart Circ Physiol. 2009;296:H1017-1026.

22. Findlay I. $\beta$-Adrenergic stimulation modulates $\mathrm{Ca} 2+-$ and voltage-dependent inactivation of L-type Ca2+ channel currents in guinea-pig ventricular myocytes. J Physiol. 2002;541:741751.

23. Namiki T, Joyner RW, Wagner MB. Developmental changes in time course of recovery from inactivation in L-type calcium currents of rabbit ventricular myocytes. Am J Physiol Heart Circ Physiol. 2007;292:H295-303.

24. Hund TJ, Decker KF, Kanter E, Mohler PJ, Boyden PA, Schuessler RB, Yamada KA, Rudy Y. Role of activated CaMKII in abnormal calcium homeostasis and $I_{N a}$ remodeling after myocardial infarction: insights from mathematical modeling. J Mol Cell Cardiol. 2008;45:420-428.

25. Kohlhaas $M$, Zhang $T$, Seidler $T$, Zibrova D, Dybkova N, Steen $A$, Wagner $S$, Chen $L$, Brown JH, Bers DM, Maier LS. Increased sarcoplasmic reticulum calcium leak but unaltered contractility by acute CaMKII overexpression in isolated rabbit cardiac myocytes. Circ Res. 2006;98:235244.

26. Guo J, Duff HJ. Calmodulin kinase II accelerates L-type Ca2+ current recovery from inactivation and compensates for the direct inhibitory effect of [Ca2+]i in rat ventricular myocytes. J Physiol. 2006;574:509-518.

27. Picht E, DeSantiago J, Huke S, Kaetzel MA, Dedman JR, Bers DM. CaMKII inhibition targeted to the sarcoplasmic reticulum inhibits frequency-dependent acceleration of relaxation and Ca2+ current facilitation. J Mol Cell Cardiol. 2007;42:196-205.

28. Xu L, Lai D, Cheng J, Lim HJ, Keskanokwong T, Backs J, Olson EN, Wang Y. Alterations of Ltype calcium current and cardiac function in CaMKII\{delta\} knockout mice. Circ Res. 2010;107:398-407.

29. Grandi E, Pasqualini FS, Pes C, Corsi C, Zaza A, Severi S. Theoretical investigation of action potential duration dependence on extracellular $\mathrm{Ca} 2+$ in human cardiomyocytes. J Mol Cell Cardiol. 2009;46:332-342.

30. Papp Z, Peineau N, Szigeti G, Argibay J, Kovacs L. Calcium-dependent modulation of the plateau phase of action potential in isolated ventricular cells of rabbit heart. Acta Physiol Scand. 1999;167:119-129.

31. Tran K, Smith NP, Loiselle DS, Crampin EJ. A thermodynamic model of the cardiac sarcoplasmic/endoplasmic Ca ${ }^{2+}$ (SERCA) pump. Biophysical Journal. 2009;96:2029-2042. 
32. Aggarwal R, Pu J, Boyden PA. $\mathrm{Ca}^{2+}$-dependent outward currents in myocytes from epicardial border zone of 5-day infarcted canine heart. Am J Physiol. 1997;273:H1386-1394.

33. Wagner S, Hacker E, Grandi E, Weber SL, Dybkova N, Sossalla S, Sowa T, Fabritz L, Kirchhof P, Bers DM, Maier LS. Ca/calmodulin kinase II differentially modulates potassium currents. Circ Arrhythm Electrophysiol. 2009;2:285-294.

34. Silva J, Rudy Y. Subunit interaction determines $I_{\mathrm{ks}}$ participation in cardiac repolarization and repolarization reserve. Circulation. 2005;112:1384-1391.

35. Liu DW, Antzelevitch C. Characteristics of the delayed rectifier current $\left(I_{\mathrm{Kr}}\right.$ and $\left.I_{\mathrm{Ks}}\right)$ in canine ventricular epicardial, midmyocardial, and endocardial myocytes. A weaker $I_{\mathrm{Ks}}$ contributes to the longer action potential of the M cell. Circ Res. 1995;76:351-365.

36. Yang T, Snyders DJ, Roden DM. Ibutilide, a methanesulfonanilide antiarrhythmic, is a potent blocker of the rapidly activating delayed rectifier $\mathrm{K}+$ current $\left(\mathrm{I}_{\mathrm{Kr}}\right)$ in AT-1 cells. Concentration, time-, voltage-, and use-dependent effects. Circulation. 1995;91:1799-1806.

37. Jurkiewicz NK, Sanguinetti MC. Rate-dependent prolongation of cardiac action potentials by a methanesulfonanilide class III antiarrhythmic agent. Specific block of rapidly activating delayed rectifier K+ current by dofetilide. Circulation research. 1993;72:75-83.

38. Volders PGA, StengI M, van Opstal JM, Gerlach U, Spätjens RL, Beekman JD, Sipido KR, Vos $\mathrm{MA}$. Probing the contribution of $\mathrm{I}_{\mathrm{Ks}}$ to canine ventricular repolarization: key role for $\beta$ adrenergic receptor stimulation. Circulation. 2003;107:2753-2760.

39. StengI M, Volders PGA, Thomsen MB, Spätjens RLHMG, Sipido KR, Vos MA. Accumulation of slowly activating delayed rectifier potassium current $I_{\mathrm{Ks}}$ in canine ventricular myocytes. $J$ Physiol. 2003;551:777-786.

40. Charpentier F, Liu QY, Rosen MR, Robinson RB. Age-related differences in $\beta$-adrenergic regulation of repolarization in canine epicardial myocytes. Am J Physiol. 1996;271:H1174H1181.

41. Severi $S$, Corsi $C$, Rocchetti $M$, Zaza A. Mechanisms of beta-adrenergic modulation of $I_{\mathrm{Ks}}$ in the guinea-pig ventricle: insights from experimental and model-based analysis. Biophys $J$. 2009;96:3862-3872.

42. Imredy JP, Penniman JR, Dech SJ, Irving WD, Salata JJ. Modeling of the adrenergic response of the human IKs current (hKCNQ1/hKCNE1) stably expressed in HEK-293 cells. Am J Physiol Heart Circ Physiol. 2008;295:H1867-H1881.

43. Yue L, Feng J, Wang Z, Nattel S. Adrenergic control of the ultrarapid delayed rectifier current in canine atrial myocytes. J Physiol. 1999;516 (Pt 2):385-398.

44. Sridhar A, da Cunha DN, Lacombe VA, Zhou Q, Fox JJ, Hamlin RL, Carnes CA. The plateau outward current in canine ventricle, sensitive to 4-aminopyridine, is a constitutive contributor to ventricular repolarization. Br J Pharmacol. 2007;152:870-879.

45. Wettwer $\mathrm{E}$. Is there a functional correlate of Kv1.5 in the ventricle of canine heart and what would it mean for the use of $I_{\text {Kur }}$ blockers? Br J Pharmacol. 2007;152:835-837.

46. Spach MS, Heidlage JF, Dolber PC, Barr RC. Electrophysiological effects of remodeling cardiac gap junctions and cell size: experimental and model studies of normal cardiac growth. Circ Res. 2000;86:302-311.

47. Diego JMD, Sun ZQ, Antzelevitch C. $I_{\text {to }}$ and action potential notch are smaller in left vs. right canine ventricular epicardium. Am J Physiol. 1996;271:H548-H561. 
48. Lue WM, Boyden PA. Abnormal electrical properties of myocytes from chronically infarcted canine heart. Alterations in Vmax and the transient outward current. Circulation. 1992;85:1175-1188.

49. Ono K, Fozzard HA, Hanck DA. Mechanism of cAMP-dependent modulation of cardiac sodium channel current kinetics. Circ Res. 1993;72:807-815.

50. Baba S, Dun W, Boyden PA. Can PKA activators rescue $\mathrm{Na}^{+}$channel function in epicardial border zone cells that survive in the infarcted canine heart? Cardiovasc Res. 2004;64:260267.

51. Wagner S, Dybkova N, Rasenack ECL, Jacobshagen C, Fabritz L, Kirchhof P, Maier SKG, Zhang T, Hasenfuss G, Brown JH, Bers DM, Maier LS. $\mathrm{Ca}^{2+} /$ calmodulin-dependent protein kinase II regulates cardiac $\mathrm{Na}+$ channels. J Clin Invest. 2006;116:3127--3138.

52. Clancy $\mathrm{CE}$, Rudy $\mathrm{Y}$. $\mathrm{Na}^{+}$channel mutation that causes both Brugada and long-QT syndrome phenotypes: a simulation study of mechanism. Circulation. 2002;105:1208-1213.

53. Bers DM, Weber CR. Na/Ca exchange function in intact ventricular myocytes. Ann $N$ Y Acad Sci. 2002;976:500-512.

54. Faber GM, Rudy Y. Calsequestrin mutation and catecholaminergic polymorphic ventricular tachycardia: a simulation study of cellular mechanism. Cardiovasc Res. 2007;75:79-88.

55. Gao J, Mathias RT, Cohen IS, Baldo GJ. Two functionally different $\mathrm{Na} / \mathrm{K}$ pumps in cardiac ventricular myocytes. J Gen Physiol. 1995;106:995-1030.

56. Sakai R, Hagiwara N, Matsuda N, Kassanuki H, Hosoda S. Sodium-potassium pump current in rabbit sino-atrial node cells. J Physiol. 1996;490 ( Pt 1):51-62.

57. Dobretsov $\mathrm{M}$, Hastings $\mathrm{SL}$, Stimers JR. $\mathrm{Na}^{+}-\mathrm{K}^{+}$pump cycle during $\beta$-adrenergic stimulation of adult rat cardiac myocytes. J Physiol. 1998;507:527-539.

58. Gao J, Wymore R, Wymore RT, Wang Y, McKinnon D, Dixon JE, Mathias RT, Cohen IS, Baldo GJ. Isoform-specific regulation of the sodium pump by $\alpha$ - and $\beta$-adrenergic agonists in the guinea-pig ventricle. J Physiol. 1999;516 (Pt 2):377-383.

59. Charpentier F, Legato MJ, Steinberg SF, Cohen IS, Rosen MR. $\beta$-adrenergic modulation of Na$\mathrm{K}$ pump activity in young and adult canine cardiac Purkinje fibers. Am J Physiol. 1996;271:H706-H712.

60. Silverman BDZ, Fuller W, Eaton P, Deng J, Moorman JR, Cheung JY, James AF, Shattock MJ. Serine 68 phosphorylation of phospholemman: acute isoform-specific activation of cardiac $\mathrm{Na} / \mathrm{K}$ ATPase. Cardiovasc Res. 2005;65:93--103.

61. Despa S, Bossuyt J, Han F, Ginsburg KS, Jia L-G, Kutchai H, Tucker AL, Bers DM. Phospholemman-phosphorylation mediates the $\beta$-adrenergic effects on $\mathrm{Na} / \mathrm{K}$ pump function in cardiac myocytes. Circ Res. 2005;97:252-259.

62. Gao J, Mathias RT, Cohen IS, Baldo GJ. Isoprenaline, $\mathrm{Ca} 2+$ and the $\mathrm{Na}(+)-\mathrm{K}+$ pump in guineapig ventricular myocytes. J Physiol. 1992;449:689-704.

63. Zhang XQ, Ahlers BA, Tucker AL, Song J, Wang J, Moorman JR, Mounsey JP, Carl LL, Rothblum $\mathrm{LI}$, Cheung JY. Phospholemman inhibition of the cardiac $\mathrm{Na}+\mathrm{Ca} 2+$ exchanger. Role of phosphorylation. J Biol Chem. 2006;281:7784-7792.

64. Despa S, Tucker AL, Bers DM. Phospholemman-mediated activation of Na/K-ATPase limits $[\mathrm{Na}] \mathrm{i}$ and inotropic state during $\beta$-adrenergic stimulation in mouse ventricular myocytes. Circulation. 2008;117:1849-1855.

65. Ginsburg KS, Bers DM. Isoproterenol does not enhance $\mathrm{Ca}$-dependent $\mathrm{Na} / \mathrm{Ca}$ exchange current in intact rabbit ventricular myocytes. J Mol Cell Cardiol. 2005;39:972-981. 
66. Smith NP, Crampin EJ. Development of models of active ion transport for whole-cell modelling: cardiac sodium-potassium pump as a case study. Prog Biophys Mol Biol. 2004;85:387-405.

67. Tateyama M, Rivolta I, Clancy CE, Kass RS. Modulation of cardiac sodium channel gating by protein kinase A can be altered by disease-linked mutation. J Biol Chem. 2003;278:46718-46726.

68. Zygmunt AC, Eddlestone GT, Thomas GP, Nesterenko VV, Antzelevitch C. Larger late sodium conductance in $\mathrm{M}$ cells contributes to electrical heterogeneity in canine ventricle. Am J Physiol Heart Circ Physiol. 2001;281:H689-697.

69. Dong $M$, Sun X, Prinz AA, Wang HS. Effect of simulated I(to) on guinea pig and canine ventricular action potential morphology. Am J Physiol Heart Circ Physiol. 2006;291:H631637.

70. Liu DW, Gintant GA, Antzelevitch C. Ionic bases for electrophysiological distinctions among epicardial, midmyocardial, and endocardial myocytes from the free wall of the canine left ventricle. Circ Res. 1993;72:671-687.

71. Sun X, Wang HS. Role of the transient outward current (Ito) in shaping canine ventricular action potential--a dynamic clamp study. J Physiol. 2005;564:411-419.

72. Greenstein JL, Wu R, Po S, Tomaselli GF, Winslow RL. Role of the calcium-independent transient outward current $I_{\text {to } 1}$ in shaping action potential morphology and duration. Circ Res. 2000;87:1026-1033.

73. Tseng GN, Hoffman BF. Two components of transient outward current in canine ventricular myocytes. Circ Res. 1989;64:633-647.

74. Dumaine $R$, Towbin JA, Brugada $P$, Vatta $M$, Nesterenko DV, Nesterenko VV, Brugada J, Brugada R, Antzelevitch C. Ionic mechanisms responsible for the electrocardiographic phenotype of the Brugada syndrome are temperature dependent. Circ Res. 1999;85:803809.

75. van der Heyden MA, Wijnhoven TJ, Opthof T. Molecular aspects of adrenergic modulation of the transient outward current. Cardiovasc Res. 2006;71:430-442.

76. Livshitz LM, Rudy Y. Regulation of $\mathrm{Ca}^{2+}$ and electrical alternans in cardiac myocytes: role of CAMKII and repolarizing currents. Am J Physiol Heart Circ Physiol. 2007;292:H2854-2866.

77. Bridge JHB, Savio-Galimberti E. What are the consequences of phosphorylation and hyperphosphorylation of ryanodine receptors in normal and failing heart? Circ Res. 2008;102:995-997.

78. Marx SO, Reiken S, Hisamatsu Y, Jayaraman T, Burkhoff D, Rosemblit N, Marks AR. PKA phosphorylation dissociates FKBP12.6 from the calcium release channel (ryanodine receptor): defective regulation in failing hearts. Cell. 2000;101:365-376.

79. MacDonnell SM, García-Rivas G, Scherman JA, Kubo H, Chen X, Valdivia H, Houser SR. Adrenergic regulation of cardiac contractility does not involve phosphorylation of the cardiac ryanodine receptor at serine 2808. Circ Res. 2008;102:e65-e72.

80. Jiang MT, Lokuta AJ, Farrell EF, Wolff MR, Haworth RA, Valdivia HH. Abnormal Ca2+ release, but normal ryanodine receptors, in canine and human heart failure. Circ Res. 2002;91:10151022.

81. Xiao B, Zhong G, Obayashi M, Yang D, Chen K, Walsh MP, Shimoni Y, Cheng H, Keurs HT, Chen SRW. Ser-2030, but not Ser-2808, is the major phosphorylation site in cardiac 
ryanodine receptors responding to protein kinase $A$ activation upon beta-adrenergic stimulation in normal and failing hearts. Biochem J. 2006;396:7--16.

82. Ginsburg KS, Bers DM. Modulation of excitation-contraction coupling by isoproterenol in cardiomyocytes with controlled SR Ca2+ load and Ca2+ current trigger. J Physiol. 2004;556:463-480.

83. Ogrodnik J, Niggli E. Increased $\mathrm{Ca}(2+)$ leak and spatiotemporal coherence of $\mathrm{Ca}(2+)$ release in cardiomyocytes during $\beta$-adrenergic stimulation. J Physiol. 2010;588:225-242.

84. Curran J, Hinton MJ, Ríos E, Bers DM, Shannon TR. $\beta$-Adrenergic enhancement of sarcoplasmic reticulum calcium leak in cardiac myocytes is mediated by calcium/calmodulindependent protein kinase. Circ Res. 2007;100:391-398.

85. Restrepo JG, Weiss JN, Karma A. Calsequestrin-mediated mechanism for cellular calcium transient alternans. Biophys J. 2008;95:3767-3789.

86. Mattiazzi A, Mundiña-Weilenmann C, Guoxiang C, Vittone L, Kranias E. Role of phospholamban phosphorylation on Thr17 in cardiac physiological and pathological conditions. Cardiovasc Res. 2005;68:366-375.

87. Odermatt A, Kurzydlowski K, MacLennan DH. The vmax of the Ca2+-ATPase of cardiac sarcoplasmic reticulum (SERCA2a) is not altered by Ca2+/calmodulin-dependent phosphorylation or by interaction with phospholamban. J Biol Chem. 1996;271:1420614213.

88. Hagemann D, Kuschel M, Kuramochi T, Zhu W, Cheng H, Xiao RP. Frequency-encoding Thr17 phospholamban phosphorylation is independent of Ser16 phosphorylation in cardiac myocytes. J Biol Chem. 2000;275:22532-22536.

89. Kuschel M, Karczewski P, Hempel P, Schlegel WP, Krause EG, Bartel S. Ser16 prevails over Thr17 phospholamban phosphorylation in the $\beta$-adrenergic regulation of cardiac relaxation. Am J Physiol. 1999;276:H1625-H1633.

90. DeSantiago J, Maier LS, Bers DM. Frequency-dependent acceleration of relaxation in the heart depends on CaMKII, but not phospholamban. J Mol Cell Cardiol. 2002;34:975-984.

91. O'Rourke B, Kass DA, Tomaselli GF, Kaab S, Tunin R, Marban E. Mechanisms of altered excitation-contraction coupling in canine tachycardia-induced heart failure, I: experimental studies. Circ Res. 1999;84:562-570.

92. Shannon TR, Wang F, Bers DM. Regulation of cardiac sarcoplasmic reticulum Ca release by luminal [Ca] and altered gating assessed with a mathematical model. Biophys J. 2005;89:4096--4110.

93. Shaw RM, Rudy Y. Ionic mechanisms of propagation in cardiac tissue. Roles of the sodium and L-type calcium currents during reduced excitability and decreased gap junction coupling. Circ Res. 1997;81:727-741.

94. lancu RV, Jones SW, Harvey RD. Compartmentation of cAMP signaling in cardiac myocytes: a computational study. Biophys J. 2007;92:3317-3331.

95. Madamanchi A. $\beta$-Adrenergic receptor signaling in cardiac function and heart failure. Mcgill J Med. 2007;10:99-104.

96. Nikolaev VO, Bunemann M, Schmitteckert E, Lohse MJ, Engelhardt S. Cyclic AMP imaging in adult cardiac myocytes reveals far-reaching $\beta 1$-adrenergic but locally confined $\beta 2$-adrenergic receptor-mediated signaling. Circ Res. 2006;99:1084-1091. 
97. Rybin VO, $\mathrm{Xu} X$, Lisanti MP, Steinberg SF. Differential targeting of $\beta$-adrenergic receptor subtypes and adenylyl cyclase to cardiomyocyte caveolae. A mechanism to functionally regulate the cAMP signaling pathway. J Biol Chem. 2000;275:41447-41457.

98. Green SA, Holt BD, Liggett SB. $\beta 1$ - and $\beta 2$-adrenergic receptors display subtype-selective coupling to Gs. Mol Pharmacol. 1992;41:889-893.

99. Zamah AM, Delahunty $M$, Luttrell LM, Lefkowitz RJ. Protein kinase A-mediated phosphorylation of the beta 2-adrenergic receptor regulates its coupling to $\mathrm{Gs}$ and $\mathrm{Gi}$. Demonstration in a reconstituted system. J Biol Chem. 2002;277:31249-31256.

100. Jo SH, Leblais V, Wang PH, Crow MT, Xiao RP. Phosphatidylinositol 3-kinase functionally compartmentalizes the concurrent $\mathrm{G}(\mathrm{s})$ signaling during beta2-adrenergic stimulation. Circ Res. 2002;91:46-53.

101. Violin JD, DiPilato LM, Yildirim N, Elston TC, Zhang J, Lefkowitz RJ. $\beta 2$-adrenergic receptor signaling and desensitization elucidated by quantitative modeling of real time cAMP dynamics. J Biol Chem. 2008;283:2949-2961.

102. Saucerman JJ, Brunton LL, Michailova AP, McCulloch AD. Modeling $\beta$-adrenergic control of cardiac myocyte contractility in silico. J Biol Chem. 2003;278:47997-48003.

103. Endoh M, Hiramoto $T$, Ishihata A, Takanashi M, Inui J. Myocardial $\alpha 1$-adrenoceptors mediate positive inotropic effect and changes in phosphatidylinositol metabolism. Species differences in receptor distribution and the intracellular coupling process in mammalian ventricular myocardium. Circ Res. 1991;68:1179-1190.

104. Nikolaev VO, Moshkov A, Lyon AR, Miragoli M, Novak P, Paur H, Lohse MJ, Korchev YE, Harding SE, Gorelik J. $\beta 2$-adrenergic receptor redistribution in heart failure changes cAMP compartmentation. Science. 2010;327:1653-1657.

105. Post SR, Hilal-Dandan R, Urasawa K, Brunton LL, Insel PA. Quantification of signalling components and amplification in the beta-adrenergic-receptor-adenylate cyclase pathway in isolated adult rat ventricular myocytes. Biochem J. 1995;311 (Pt 1):75-80.

106. lancu RV, Ramamurthy G, Warrier S, Nikolaev VO, Lohse MJ, Jones SW, Harvey RD. Cytoplasmic cAMP concentrations in intact cardiac myocytes. Am J Physiol Cell Physiol. 2008;295:C414-C422.

107. Sunahara RK, Dessauer CW, Gilman AG. Complexity and diversity of mammalian adenylyl cyclases. Annu Rev Pharmacol Toxicol. 1996;36:461-480.

108. Wang T, Brown MJ. Differential expression of adenylyl cyclase subtypes in human cardiovascular system. Mol Cell Endocrinol. 2004;223:55-62.

109. Hohl CM, Li QA. Compartmentation of CAMP in adult canine ventricular myocytes. Relation to single-cell free Ca2+ transients. Circ Res. 1991;69:1369-1379.

110. Chen-Goodspeed M, Lukan AN, Dessauer CW. Modeling of Galpha(s) and Galpha(i) regulation of human type V and VI adenylyl cyclase. J Biol Chem. 2005;280:1808-1816.

111. Steinberg SF, Zhang H, Pak E, Pagnotta G, Boyden PA. Characteristics of the $\beta$-adrenergic receptor complex in the epicardial border zone of the 5-day infarcted canine heart. Circulation. 1995;91:2824-2833.

112. Yu X, Zhang M, Kyker K, Patterson E, Benovic JL, Kem DC. Ischemic inactivation of G proteincoupled receptor kinase and altered desensitization of canine cardiac $\beta$-adrenergic receptors. Circulation. 2000;102:2535-2540. 
113. Freedman NJ, Liggett SB, Drachman DE, Pei G, Caron MG, Lefkowitz RJ. Phosphorylation and desensitization of the human $\beta 1$-adrenergic receptor. Involvement of $G$ protein-coupled receptor kinases and cAMP-dependent protein kinase. J Biol Chem. 1995;270:17953-17961.

114. Willoughby $D$, Cooper DMF. Organization and Ca2+ regulation of adenylyl cyclases in cAMP microdomains. Physiol Rev. 2007;87:965-1010.

115. Yu HJ, Ma H, Green RD. Calcium entry via L-type calcium channels acts as a negative regulator of adenylyl cyclase activity and cyclic AMP levels in cardiac myocytes. Mol Pharmacol. 1993;44:689-693.

116. Dobson JG, Shea LG, Fenton RA. $\beta$-adrenergic and antiadrenergic modulation of cardiac adenylyl cyclase is influenced by phosphorylation. Am J Physiol Heart Circ Physiol. 2003;285:H1471-H1478.

117. Dessauer CW, Scully TT, Gilman AG. Interactions of forskolin and ATP with the cytosolic domains of mammalian adenylyl cyclase. J Biol Chem. 1997;272:22272-22277.

118. Fischmeister R, Castro LRV, Abi-Gerges A, Rochais F, Jurevicius J, Leroy J, Vandecasteele G. Compartmentation of cyclic nucleotide signaling in the heart: the role of cyclic nucleotide phosphodiesterases. Circ Res. 2006;99:816-828.

119. Osadchii OE. Myocardial phosphodiesterases and regulation of cardiac contractility in health and cardiac disease. Cardiovasc Drugs Ther. 2007;21:171-194.

120. Bethke T, Meyer W, Schmitz W, Scholz H, Stein B, Thomas K, Wenzlaff H. Phosphodiesterase inhibition in ventricular cardiomyocytes from guinea-pig hearts. $\mathrm{Br} J$ Pharmacol. 1992;107:127-133.

121. Rochais F, Abi-Gerges A, Horner K, Lefebvre F, Cooper DM, Conti M, Fischmeister R, Vandecasteele G. A specific pattern of phosphodiesterases controls the cAMP signals generated by different Gs-coupled receptors in adult rat ventricular myocytes. Circ Res. 2006;98:1081-1088.

122. Leroy J, Abi-Gerges A, Nikolaev VO, Richter W, Lechêne P, Mazet J-L, Conti M, Fischmeister R, Vandecasteele G. Spatiotemporal dynamics of $\beta$-adrenergic cAMP signals and L-type Ca2+ channel regulation in adult rat ventricular myocytes: role of phosphodiesterases. Circ Res. 2008;102:1091-1100.

123. Xin W, Tran TM, Richter W, Clark RB, Rich TC. Roles of GRK and PDE4 activities in the regulation of $\beta 2$ adrenergic signaling. J Gen Physiol. 2008;131:349-364.

124. Saucerman JJ, Healy SN, Belik ME, Puglisi JL, McCulloch AD. Proarrhythmic consequences of a KCNQ1 AKAP-binding domain mutation: computational models of whole cells and heterogeneous tissue. Circ Res. 2004;95:1216-1224.

125. Tasken K, Aandahl EM. Localized effects of cAMP mediated by distinct routes of protein kinase A. Physiol Rev. 2004;84:137-167.

126. Rich TC, Karpen JW. Review article: cyclic AMP sensors in living cells: what signals can they actually measure? Ann Biomed Eng. 2002;30:1088-1099.

127. Krebs EG, Beavo JA. Phosphorylation-dephosphorylation of enzymes. Annu Rev Biochem. 1979;48:923-959.

128. Corbin JD, Sugden PH, Lincoln TM, Keely SL. Compartmentalization of adenosine 3':5'monophosphate and adenosine 3':5'-monophosphate-dependent protein kinase in heart tissue. J Biol Chem. 1977;252:3854-3861. 
129. Reinitz CA, Bianco RA, Shabb JB. Compartmentation of the type I regulatory subunit of cAMP-dependent protein kinase in cardiac ventricular muscle. Arch Biochem Biophys. 1997;348:391-402.

130. Cheng X, Phelps C, Taylor SS. Differential binding of cAMP-dependent protein kinase regulatory subunit isoforms lalpha and llbeta to the catalytic subunit. I Biol Chem. 2001;276:4102-4108.

131. Dostmann WR, Taylor SS. Identifying the molecular switches that determine whether (Rp)CAMPS functions as an antagonist or an agonist in the activation of CAMP-dependent protein kinase I. Biochemistry. 1991;30:8710-8716.

132. Manni S, Mauban JH, Ward CW, Bond M. Phosphorylation of the CAMP-dependent Protein Kinase (PKA) Regulatory Subunit Modulates PKA-AKAP Interaction, Substrate Phosphorylation, and Calcium Signaling in Cardiac Cells. J Biol Chem. 2008;283:24145-24154.

133. Zakhary DR, Moravec CS, Bond M. Regulation of PKA binding to AKAPs in the heart: alterations in human heart failure. Circulation. 2000;101:1459-1464.

134. Hofmann F, Bechtel PJ, Krebs EG. Concentrations of cyclic AMP-dependent protein kinase subunits in various tissues. J Biol Chem. 1977;252:1441-1447.

135. Beavo JA, Bechtel PJ, Krebs EG. Activation of protein kinase by physiological concentrations of cyclic AMP. Proc Natl Acad Sci U S A. 1974;71:3580-3583.

136. Saucerman JJ, Zhang J, Martin JC, Peng LX, Stenbit AE, Tsien RY, McCulloch AD. Systems analysis of PKA-mediated phosphorylation gradients in live cardiac myocytes. Proc Natl Acad Sci U S A. 2006;103:12923-12928.

137. Herzig S, Neumann J. Effects of serine/threonine protein phosphatases on ion channels in excitable membranes. Physiol Rev. 2000;80:173-210.

138. Carr AN, Schmidt AG, Suzuki Y, del Monte F, Sato Y, Lanner C, Breeden K, Jing SL, Allen PB, Greengard P, Yatani A, Hoit BD, Grupp IL, Hajjar RJ, DePaoli-Roach AA, Kranias EG. Type 1 phosphatase, a negative regulator of cardiac function. Mol Cell Biol. 2002;22:4124-4135.

139. Layland J, Solaro RJ, Shah AM. Regulation of cardiac contractile function by troponin I phosphorylation. Cardiovasc Res. 2005;66:12-21.

140. Neumann J, Gupta RC, Schmitz W, Scholz H, Nairn AC, Watanabe AM. Evidence for isoproterenol-induced phosphorylation of phosphatase inhibitor-1 in the intact heart. Circ Res. 1991;69:1450-1457.

141. Mauban JRH, O'Donnell M, Warrier S, Manni S, Bond M. AKAP-Scaffolding Proteins and Regulation of Cardiac Physiology. Physiology (Bethesda). 2009;24:78-87.

142. Alto NM, Soderling SH, Hoshi N, Langeberg LK, Fayos R, Jennings PA, Scott JD. Bioinformatic design of A-kinase anchoring protein-in silico: a potent and selective peptide antagonist of type II protein kinase A anchoring. Proc Natl Acad Sci U S A. 2003;100:4445-4450.

143. Marx SO, Kurokawa J, Reiken S, Motoike H, D'Armiento J, Marks AR, Kass RS. Requirement of a macromolecular signaling complex for $\beta$ adrenergic receptor modulation of the KCNQ1KCNE1 potassium channel. Science. 2002;295:496-499.

144. Chen L, Marquardt ML, Tester DJ, Sampson KJ, Ackerman MJ, Kass RS. Mutation of an Akinase-anchoring protein causes long-QT syndrome. Proc Natl Acad Sci U $S$ A. 2007;104:20990-20995.

145. Chen L, Kass RS. Dual roles of the A kinase-anchoring protein Yotiao in the modulation of a cardiac potassium channel: a passive adaptor versus an active regulator. Eur J Cell Biol. 2006;85:623-626. 
146. Yatani A, Shen YT, Yan L, Chen W, Kim SJ, Sano K, Irie K, Vatner SF, Vatner DE. Down regulation of the L-type Ca2+ channel, GRK2, and phosphorylated phospholamban: protective mechanisms for the denervated failing heart. J Mol Cell Cardiol. 2006;40:619-628.

147. Stengl M, Ramakers C, Donker DW, Nabar A, Rybin AV, Spatjens RLHMG, van der Nagel T, Wodzig WKWH, Sipido KR, Antoons G, Moorman AFM, Vos MA, Volders PGA. Temporal patterns of electrical remodeling in canine ventricular hypertrophy: focus on IKs downregulation and blunted beta-adrenergic activation. Cardiovasc Res. 2006;72:90-100.

148. Kirstein M, Eickhorn R, Kochsiek K, Langenfeld H. Dose-dependent alteration of rat cardiac sodium current by isoproterenol: results from direct measurements on multicellular preparations. Pflugers Arch. 1996;431:395-401.

149. Gupta RC, Neumann J, Durant P, Watanabe AM. A1-adenosine receptor-mediated inhibition of isoproterenol-stimulated protein phosphorylation in ventricular myocytes. Evidence against a cAMP-dependent effect. Circ Res. 1993;72:65-74.

150. Sulakhe PV, Vo XT. Regulation of phospholamban and troponin-I phosphorylation in the intact rat cardiomyocytes by adrenergic and cholinergic stimuli: roles of cyclic nucleotides, calcium, protein kinases and phosphatases and depolarization. Mol Cell Biochem. 1995;149150:103--126.

151. Saucerman JJ, Bers DM. Calmodulin mediates differential sensitivity of CaMKII and calcineurin to local Ca2+ in cardiac myocytes. Biophys J. 2008;95:4597-4612.

152. Hashambhoy YL, Greenstein JL, Winslow RL. Role of CaMKII in RyR leak, EC coupling and action potential duration: a computational model. J Mol Cell Cardiol. 2010;49:617-624.

153. Huke S, Bers DM. Temporal dissociation of frequency-dependent acceleration of relaxation and protein phosphorylation by CaMKII. J Mol Cell Cardiol. 2007;42:590-599. 
352 | 